\title{
IntechOpen
}

\section{Composites and Their Properties}

Edited by Ning Hu

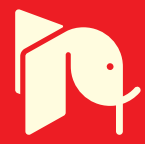

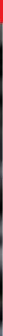
Wha W 



\section{COMPOSITES AND \\ THEIR PROPERTIES}

Edited by Ning $\mathrm{Hu}$ 
Edited by Ning $\mathrm{Hu}$

\section{Contributors}

M. Sayuti, Shamsuddin Sulaiman, B.T Hang Tuah Baharudin, Mohd Khairol Anuar Mohd Ariffin, Dr.T.R Raghavan Vijayaram, Biplab Sanyal, Sumanta Bhandary, Zainab Shakir Radeef, Asst. Prof. Ali Sabea Hammood, David_Alejandro Arellano_Escarpita, Diego Ernesto Cardenas Fuentes, Hugo Ramón Elizalde Siller, Ricardo A. Ramirez-Mendoza, Oliver Probst, Cheng Yan, Nor Bahiyah Baba, Pavel Andreevich Radchenko, Andrey Vasilievich Radchenko, Susanna Laurenzi, Mario Marchetti, Karel Frydrýšek, Pawel Kostial, (vice-Professor = Docent) Jan Krmela, Ph.D., Head Of Department Of Numerical Methods And Computational, llya Mazov, Vladimir Kuznetsov, Anatoly Romanenko, Valentin Suslyaev, Dewan Muhammad Nuruzzaman, Asaduzzaman Chowdhury, Milan Zmindak, Martin Dudinsky, Marcin Molenda, Michał Świętosławski, Roman Dziembaj, Junqian Zhang, Fang Wang, Mohamed Altenaiji, Go Yamamoto, Toshiyuki Hashida, Edwin Dado, Eddie Koenders, Dárlinton Carvalho, Konstantin Rozanov, Marina Koledintseva, Eugene Yelsukov, Jinxiang Chen, Yuan Li, Rafic Younes

\section{(c) The Editor(s) and the Author(s) 2012}

The moral rights of the and the author(s) have been asserted. All rights to the book as a whole are reserved by INTECH. The book as a whole (compilation) cannot be reproduced, distributed or used for commercial or non-commercial purposes without INTECH's written permission. Enquiries concerning the use of the book should be directed to INTECH rights and permissions department (permissions@intechopen.com).

Violations are liable to prosecution under the governing Copyright Law.

\section{(cc) BY}

Individual chapters of this publication are distributed under the terms of the Creative Commons Attribution 3.0 Unported License which permits commercial use, distribution and reproduction of the individual chapters, provided the original author(s) and source publication are appropriately acknowledged. If so indicated, certain images may not be included under the Creative Commons license. In such cases users will need to obtain permission from the license holder to reproduce the material. More details and guidelines concerning content reuse and adaptation can be foundat http://www.intechopen.com/copyright-policy.html.

\section{Notice}

Statements and opinions expressed in the chapters are these of the individual contributors and not necessarily those of the editors or publisher. No responsibility is accepted for the accuracy of information contained in the published chapters. The publisher assumes no responsibility for any damage or injury to persons or property arising out of the use of any materials, instructions, methods or ideas contained in the book.

First published in Croatia, 2012 by INTECH d.o.o.

eBook (PDF) Published by IN TECH d.o.o.

Place and year of publication of eBook (PDF): Rijeka, 2019.

IntechOpen is the global imprint of IN TECH d.o.o.

Printed in Croatia

Legal deposit, Croatia: National and University Library in Zagreb

Additional hard and PDF copies can be obtained from orders@intechopen.com

Composites and Their Properties

Edited by Ning Hu

p. cm.

ISBN 978-953-51-0711-8

eBook (PDF) ISBN 978-953-51-6228-5 


\section{We are IntechOpen, \\ the world's leading publisher of Open Access books}

Built by scientists, for scientists

\section{$4,100+$}

Open access books available

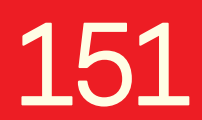

Countries delivered to
$116,000+$

International authors and editors
$120 \mathrm{M}+$

Downloads

Our authors are among the

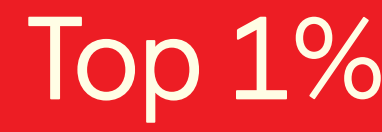

most cited scientists

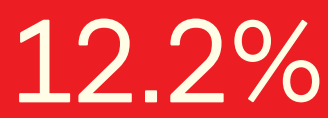

Contributors from top 500 universities

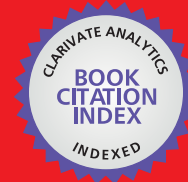

WEB OF SCIENCE ${ }^{\mathrm{TM}}$

Selection of our books indexed in the Book Citation Index in Web of Science ${ }^{\mathrm{TM}}$ Core Collection (BKCI)

Interested in publishing with us?

Contact book.department@intechopen.com

Numbers displayed above are based on latest data collected.

For more information visit www.intechopen.com

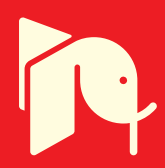





\section{Meet the editor}

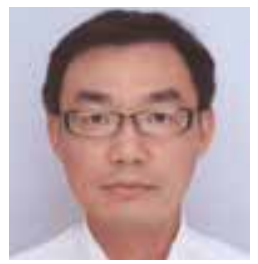

$\mathrm{Dr} \mathrm{Hu}$ is currently a full professor in the Department of Mechanical Engineering, Chiba University, Japan. He has worked on a broad range of research topics including: structural and functional composites, computational solids mechanics, structural engineering. Recently, his research has been mainly focused on: impact behaviours of FRP laminated composites, structural health monitoring and non-destructive damage evaluation techniques, structural and functional nanocomposites, multi-scale simulations of various physical phenomena in nanocomposites. To date, he has generated over 120 papers on various journals with a high citation number, and over 80 papers on various international conferences. He is now an editorial board member of 4 journals. He has also delivered over 20 Keynote and Invited Talks on various conferences. He has been a reviewer for Carbon, Polymer, CST and other over 60 various journals. 



\section{Contents}

\section{Preface XIII}

Section 1 Nanocomposites 1

Chapter 1 Graphene-Boron Nitride Composite: A Material with Advanced Functionalities $\mathbf{3}$

Sumanta Bhandary and Biplab Sanyal

Chapter 2 Graphene Nanocomposites 17

Mingchao Wang, Cheng Yan and Lin Ma

Chapter 3 Properties of MWNT-Containing Polymer

Composite Materials Depending on Their Structure 37

llya Mazov, Vladimir Kuznetsov,

Anatoly Romanenko and Valentin Suslyaev

Chapter $4 \quad \mathrm{C} \mathrm{Li}_{2} \mathrm{MnSiO}_{4}$ Nanocomposite

Cathode Material for Li-Ion Batteries $\mathbf{6 1}$

Marcin Molenda, Michał Świętosławski and Roman Dziembaj

Section 2 Damages and Fractures -

Theoretical and Numerical Modeling 81

Chapter 5 Biaxial Tensile Strength Characterization

of Textile Composite Materials $\mathbf{8 3}$

David Alejandro Arellano Escárpita, Diego Cárdenas,

Hugo Elizalde, Ricardo Ramirez and Oliver Probst

Chapter 6 Modelling of Fracture of Anisotropic Composite

Materials Under Dynamic Loads 107

Andrey Radchenko and Pavel Radchenko

Chapter 7 Finite Element Implementation of Failure and Damage Simulation in Composite Plates 131

Milan Žmindák and Martin Dudinský 
Chapter 8 Numerical Modelling of Damage Evolution and Failure Behavior of Continuous Fiber Reinforced Composites 153 F. Wang and J. Q. Zhang

Chapter 9 Molecular Simulations on Interfacial Sliding of Carbon Nanotube Reinforced Alumina Composites 173

Yuan Li, Sen Liu, Ning Hu, Weifeng Yuan and Bin Gu

Section 3 Design, Processing, and Manufacturing Technologies

Chapter 10 Advanced Composite Materials by Resin

Transfer Molding for Aerospace Applications 197

Susanna Laurenzi and Mario Marchetti

Chapter 11 Netcentric Virtual Laboratories for Composite Materials

E. Dado, E.A.B. Koenders and D.B.F. Carvalho

Section 4 Mechanical and Physical Properties of Composites

Chapter 12 Characterizations of Environmental Composites 247

Ali Hammood and Zainab Radeef

Chapter 13 The Chosen Aspects of Materials and Construction Influence on the Tire Safety 265

Pavel Koštial, Jan Krmela, Karel Frydrýšek and Ivan Ružiak

Chapter 14 Friction and Wear of Polymer and Composites 299

Dewan Muhammad Nuruzzaman

and Mohammad Asaduzzaman Chowdhury

Chapter 15 Frequency-Dependent Effective Material Parameters of Composites as a Function of Inclusion Shape 331

Konstantin N. Rozanov, Marina Y. Koledintseva

and Eugene P. Yelsukov

Chapter 16 The Lightweight Composite Structure and Mechanical Properties of the Beetle Forewing 359

Jinxiang Chen, Qing-Qing Ni and Juan Xie

Chapter 17 Comparative Review Study on Elastic Properties Modeling for Unidirectional Composite Materials 391

Rafic Younes, Ali Hallal, Farouk Fardoun and Fadi Hajj Chehade

Section 5 Metal and Ceramic Matrix Composites 409

Chapter 18 Manufacturing and Properties of Quartz $\left(\mathrm{SiO}_{2}\right)$

Particulate Reinforced Al-11.8\%Si Matrix Composites 411

M. Sayuti, S. Sulaiman, T.R. Vijayaram,

B.T.H.T Baharudin and M.K.A. Arifin 
Chapter 19 Characterisation of Aluminium Matrix Syntactic Foams Under Static and Dynamic Loading 437

M. Altenaiji, G.K. Schleyer and Y.Y. Zhao

Chapter 20 YSZ Reinforced Ni-P Composite by Electroless Nickel Co-Deposition 457

Nor Bahiyah Baba

Chapter 21 Carbon Nanotube Reinforced

Alumina Composite Materials $\mathbf{4 8 3}$

Go Yamamoto and Toshiyuki Hashida 



\section{Preface}

Composites are engineered or naturally occurring materials made from two or more constituent materials with significantly different physical or chemical properties which remain separate and distinct within the finished structure. Basically, they can be categorized into two major types, i.e., structural composites with outstanding mechanical properties and functional composites with various outstanding physical, chemical or electrochemical properties. They have been widely used in a wide variety of products, e.g., advanced spacecraft and aircraft components, boat and scull hulls, sporting goods, sensor/actuator, catalysts and pollution processing materials, biomedical materials, and batteries, etc.

This book focuses on the fabrication, properties and their evaluation or modelling in various composites, e.g., the recently developed nanocomposites. The book has been divided into five parts, which deal with: functional and structural nanocomposites, numerical and theoretical modelling of various damages in textile and long fiber reinforced composites, design, processing and manufacturing technologies and their effects on mechanical properties of composites, characterization of mechanical and physical properties of various composites, and metal and ceramic matrix composites, respectively.

A list of chapters is given below along with short descriptions by providing a glimpse on the content of each chapter.

\section{Part 1. Nanocomposites}

Chapter 1. Graphene-Boron Nitride Composite: A Material with Advanced Functionalities In this chapter, thermodynamics stability and electronic properties of boron nitridegraphene nanocomposite have been presented. Among several possible isomers, stability of a desired composite are discussed and analysed.

\section{Chapter 2. Graphene Nanocomposites}

This chapter focuses on the recent development in the research field of graphene and graphene-polymer nanocomposites. The description of mechanical, electrical and thermal properties of graphene and graphene-polymer nanocomposites have been presented along with the detailed discussion on the influences of some important 
factors. Some fabrication techniques of graphene-polymer nanocomposites are also briefly introduced.

Chapter 3. Properties of MWNT-containing Polymer Composite Materials Depending on Their Structure

In this chapter, the electrical and electromagnetic properties of polymethylmethacrylate and polystyrene matrix based composites using multiwall carbon nanotubes as fillers are investigated and discussed in detail.

\section{Chapter 4. C/Li2 MnSiO4 Nanocomposite Cathode Material for Li-ion Batteries}

In this chapter, synthesis of $\mathrm{C} / \mathrm{Li}_{2} \mathrm{MnSiO}_{4}$ nanocomposite and investigation of its structural and electrochemical properties have been presented. Excellent electrical conductivity and electrochemical properties of this new nanocomposite are confirmed, leading to its hopeful use in Li-ion batteries.

\section{Part 2 Damages and Fractures-Theoretical and Numerical Modelling}

\section{Chapter 5. Biaxial Tensile Strength Characterization of Textile Composite Materials}

This chapter presents a review of biaxial testing methods and some important experimental techniques developed and the corresponding results obtained by the authors to address the issue of accurate strength prediction of textile composites.

\section{Chapter 6. Modelling of Fracture of Anisotropic Composite Materials under Dynamic Loads}

This chapter presents a powerful numerical approach for simulating the impact problems of composites with foreign objects, by considering various complex damage phenomena. This approach is further applied to investigate the properties of composites in impact processes.

Chapter 7. Finite Element Implementation of Failure and Damage Simulation in Composite Plates

The review on several powerful numerical approaches for modelling and simulating the delamination propagation in laminated composite materials has been presented, and a new damage model is further proposed with the numerical verifications.

Chapter 8. Numerical Modelling of Damage Evolution and Failure Behavior of Continuous Fiber Reinforced Composites

The chapter comprehensively presents a work about an authors' model for simulating the damage evolution of continuous fiber reinforced composites under cyclically thermomechanical loading.

Chapter 9. Molecular Simulations on Interfacial Sliding of Carbon Nanotube Reinforced Alumina Composites

This chapter focuses on the interfacial mechanical properties between walls in a multiwall carbon nanotube, and between a carbon nanotube (CNT) and an alumina matrix by performing a series of pull-out simulations based on molecular mechanics. The significant contribution of CNT cap area on the pull-out behaviours is emphasized. 


\section{Part 3 Design, Processing, and Manufacturing Technologies}

\section{Chapter 10. Advanced Resin Transfer Molding in Aerospace}

This chapter presents a comprehensive description on the resin transfer molding (RTM), which is one of the most promising technologies available today. The properties of the composites prepared by RTM and their current applications in the aerospace field are focused on from the aspects of both numerical and experimental explorations.

\section{Chapter 11. Netcentric Virtual Laboratories for Composite Materials}

The main focus of this book chapter is on a new concept for establishing virtual laboratories which is based on a 'netcentric' approach. In this approach, a netcentric virtual laboratory is a system considered as a part of devices, information and services, etc., that are interconnected by the internet. Accessing the system enables the possibility to conduct virtual experiments by means of designing and evaluating composite materials and their associating properties.

\section{Part 4 Mechanical and Physical Properties of Composites}

\section{Chapter 12. Characterizations of Environmental Composites}

In this chapter, the erosion and corrosion properties of composites made from polyester resin-matrix and Kevlar reinforced fiber and ramie reinforced fiber are reported by performing a massive amount of experiments.

Chapter 13. The Chosen Aspects of Rubber Composite Influence on the Tire Safety

In this chpater, the influence of both rubber blends and rubber composites on the tire safety is explored. The special attention is paid to the influence of breaker angle on tire deformation and potential risks resulting from improper breaker construction and rubber blend.

\section{Chapter 14. Friction and Wear of Polymer and Composites}

In this chapter, friction coefficient and wear rate of different types of polymer and composite materials sliding against steel counterface are described. Effects of duration of rubbing, normal load, sliding speed, vertical vibration, horizontal vibration, natural frequency of vibration on friction coefficient are explored.

Chapter 15. Frequency-dependent effective material parameters of composites as a function of inclusion shape

This chapter provides a comprehensive review on the theoretical background for modeling frequency-dependent permittivity and permeability of composites, especially by focusing on available mixing rules along with the analysis of their advantages and drawbacks for particular applications.

Chapter 16. Light Weight Composites Structure of Beetle Forewing and Its Mechanical Properties In this chapter, the light weight composites structure of beetle forewing, its mechanical properties and their applications are dealt with. Moreover, a new type of lightweight biomimetic composite that is more complicated and delicate than the present honeycomb structure is presented. 
Chapter 17. Comparative Review Study on Elastic Properties Modelling for Unidirectional Composite Materials

This chapter presents an exploration on the effectiveness of various analytical and numerical models for evaluating the effective material properties of fiber reinforced composites.

\section{Part 5. Metal and Ceramic Matrix Composites}

Chapter 18. Manufacturing and Properties of Quartz (SiO2) Particulate Reinforced Al11.8\% Si Matrix Composites

This chapter describes the process of manufacturing, as well as the properties of quartz-silicon dioxide particulate reinforced LM6 aluminium alloy composites. The tensile strength, impact, hardness, density, thermal diffusivity, and thermal conductivity are explored in detail by conducting mechanical and physical tests.

Chapter 19. YSZ Reinforced Ni-P Composite by Electroless Nickel Co-deposition

This chapter provides an overview on physical characteristic, e.g., electrical conductivity, of YSZ ceramic reinforced Ni-P matrix composite being fabricated by electroless nickel co-deposition.

Chapter 20. Characterisation of aluminium matrix syntactic foams under static and dynamic loading

In this chapter, the performance of energy absorption capability for aluminium matrix syntactic foams under static and dynamic loading is explored using experimental and numerical techniques.

\section{Chapter 21. Carbon Nanotube Reinforced Alumina Composite Materials}

This chapter presents a novel processing approach based on the precursor method to fabricate the nanocomposites of multiwall carbon nanotubes (MWCNTs) and alumina. The MWCNTs used in this study are modified with an acid treatment, which leads to improved mechanical properties.

\section{Chapter 22. Particulate Reinforced Metal Matrix Composites}

In this chapter, fabrication methods, mechanical properties, and industrial applications of different types of metal matrix composites are discussed comprehensively.

\section{Acknowledgements}

I would like to express my sincere appreciation to the authors of the chapters in this book for their excellent contributions and for their efforts involved in the publication process. I do believe that the contents in this book will be helpful to many researchers in this field around the world.

Ning Hu, Ph.D.

Professor, Department of Mechanical Engineering, Chiba University, 1-33 Yayoi-cho, Inage-ku, Chiba 263-8522, 


Section 1

Nanocomposites 



\title{
Graphene-Boron Nitride Composite: A Material with Advanced Functionalities
}

\author{
Sumanta Bhandary and Biplab Sanyal \\ Additional information is available at the end of the chapter
}

http://dx.doi.org/10.5772/50729

\section{Introduction}

The discovery of two dimensional materials is extremely exciting due to their unique properties, resulting from the lowering of dimensionality. Physics in 2D is quite rich (e.g., high temperature superconductivity, fractional quantum Hall effect etc.) and is different from its other dimensional counterparts. A 2D material acts as the bridge between bulk 3D systems and $0 \mathrm{D}$ quantum dots or 1D chain materials. This can well be the building block for materials with other dimensions. The discovery of graphene, the 2D allotrope of carbon by Geim and Novoselov [1] made an enormous sensation owing to a plethora of exciting properties. They were awarded the Nobel prize in Physics in 2010. Graphene, an atomically thick C layer, has broken the jinx of impossibility of the formation of a 2D structure at a finite temperature, as argued by Landau et al. $[2,3]$. The argument that a $2 \mathrm{D}$ material is thermodynamically unstable due to the out-of-plane thermal distortion, which is comparable to its bond length, was proven invalid with this discovery. One of the recent interests is to understand this apparent discrepancy by considering rippled structures of graphene at finite temperatures.

Graphene, with its exciting appearance, has won the crowns of the thinnest, the strongest, the most stretchable material along with extremely high electron mobility and thermal conductivity [4]. The linear dispersion curve at the Dirac point gives rise to exciting elementary electronic properties. Electrons in graphene behave like massless Dirac fermions, similar to the relativistic particles in quantum electrodynamics and hence has brought different branches of science together under a truly interdisciplinary platform. At low temperature and high magnetic field, a fascinating phenomenon, called the half integer quantum hall effect, is observed. The relativistic nature of carriers in graphene shows $100 \%$ tunneling through a potential barrier by changing its chirality. The phenomenon is known as "Klein-Paradox". Minimum conductivity of a value of conductivity quantum $\left(e^{2} / h\right.$ per spin per valley) is measured at zero field, which makes graphene unique. "The CERN on table top" is thus a significant naming of the experiments performed with this fascinating material [5].

An infinite pristine graphene is a semi metal, i.e., a metal with zero band gap [6]. Inversion symmetry provided by $P 6 / \mathrm{mmm}$ space group results in a band degeneracy at the Dirac points 
( $K$ and $K^{\prime}$ ) in the hexagonal Brillouin zone (BZ). This limits its most anticipated application in electronics as the on-off current ratio becomes too small to be employed in a device. The opening of a band gap is thus essential from electronics point of view retaining a high carrier mobility. Several approaches have been already made by modifying graphene, either chemically [7, 8] or by structural confinement [9-11] to improve its application possibilities, both from theory and experiment. It should be noted that in theoretical studies, the use of density functional theory (DFT) [12] has always played an instrumental role in understanding and predicting the properties of materials, often in a quantitative way.

Boron Nitride (BN), on the other hand, can have different forms of structures like bulk hexagonal BN with $s p^{2}$ bond, cubic BN with $s p^{3}$ bond, analogous to graphite and diamond respectively. A $2 \mathrm{D}$ sheet with strong $s p^{2}$ bonds can also be derived from it, which resembles its carbon counterpart, graphene. But two different chemical species in the two sublattices of BN forbid the inversion symmetry, which results in the degeneracy lifting at Dirac points in the BZ. Hexagonal BN sheet thus turns out to be an insulator with a band gap of $5.97 \mathrm{eV}$.

This opens up a possibility of alloying these neighboring elements in the periodic table to form another interesting class of materials. Possibilities are bright and so are the promises. B-N bond length is just 1.7\% larger than the $\mathrm{C}-\mathrm{C}$ bond, which makes them perfect for alloying with minimal internal stress. At the same time, introduction of $\mathrm{BN}$ in graphene, breaks the inversion symmetry, which can result in the opening up of a band gap in graphene. On top of that, the electronegativities of $B, C$, and $N$ are respectively 2.0, 2.5 and 3.0 [13], which means that the charge transfer in different kinds of $\mathrm{BCN}$ structures is going to play an interesting role both in stability and electronic properties.

Hexagonal BNC (h-BNC) films have been recently synthesized [14] on a $\mathrm{Cu}$ substrate by thermal catalytic chemical vapor deposition method. For the synthesis, ammonia borane $\left(\mathrm{NH}_{3}-\mathrm{BH}_{3}\right)$ and methane were used as precursors for $\mathrm{BN}$ and $\mathrm{C}$ respectively. In the experimental situation, it is possible to control the relative percentage of $\mathrm{C}$ and $\mathrm{BN}$. The interesting point is that the h-BNC films can be lithographically patterned for fabrication of devices. The atomic force microscopy images indicated the formation of 2-3 layers of h-BNC. The structures and compositions of the films were characterized by atomic high resolution transmission electron microscopy and electron energy-loss spectroscopy. Electrical measurements in a four-probe device showed that the electrical conductivity of h-BNC ribbons increased with an increase in the percentage of graphene. The h-BNC field effect transistor showed ambipolar behavior similar to graphene but with reduced carrier mobility of $5-20 \mathrm{~cm}^{2} \mathrm{~V}^{-1} \mathrm{~s}^{-1}$. From all these detailed analysis, one could conclude that in h-BNC films, hybridized h-BN and graphene domains were formed with unique electronic properties. Therefore, one can imagine the h-BN domains as extended impurities in the graphene lattice.

The structure and composition of BN-graphene composite are important issues to consider. As mentioned before, substitution of $\mathrm{C}$ in graphene by $\mathrm{B}$ and $\mathrm{N}$ can give an alloyed $\mathrm{BCN}$ configuration. Considering the possibilities of thermodynamic non-equilibrium at the time of growth process, one can think of several ways of alloying. The potential barrier among those individual structures can be quite high and that can keep these relatively high energetic structures stable at room temperature. For example, a huge potential barrier has to be crossed to reach a phase segregated alloy from a normal alloy, which makes normal alloy stable at room temperature. Now, depending on the growth process, different types of alloying are possible. Firstly, one can think of an even mixture of boron nitride and carbon, where one $C_{2}$ 
block is replaced by B-N. In this case, the formula unit will be $\mathrm{BC}_{2} \mathrm{~N}$. Secondly, a whole area of graphene can be replaced by boron nitride, which makes them phase separated. This we call as phase segregated alloy. The formula unit of phase segregated alloy can change depending on the percentage of doping. The final part of the following section will be devoted to the phase segregated BCN alloys. Apart from those, a distributed alloying is possible with different $\mathrm{BN}$ :graphene ratios.

The substitution of $\mathrm{C}_{2}$ with $\mathrm{B}-\mathrm{N}$ introduces several interesting features. Firstly, B-N bond length is $1.7 \%$ bigger that C-C bond but C-B bond is $15 \%$ bigger than $\mathrm{C}-\mathrm{N}$ bond. So, this is going to create intra-layer strain, which is going to affect its stability. Secondly, the difference in electro negativity in $B(2.0)$ and $N(3.0)$ will definitely cause a charge transfer. The orientation of charged pair B-N do have a major contribution in cohesive energy. Thirdly, as mentioned earlier, this will break inversion symmetry in graphene, which brings a significant change in electronic properties. Keeping these in mind, we are now going to discuss stability and electronic structure of $\mathrm{BC}_{2} \mathrm{~N}$.

\section{Stability of $\mathrm{BC}_{2} \mathbf{N}$}

In this section we will mainly focus on the stability issues for various $\mathrm{BC}_{2} \mathrm{~N}$ structures $[13,15]$. To demonstrate the factors for structural stability, we have chosen five different structures of $\mathrm{BC}_{2} \mathrm{~N}$ (Fig. 1). Let's first have a closer look at structure I. Every $\mathrm{C}$ atom has one $\mathrm{C}, \mathrm{N}$ and $\mathrm{B}$ as nearest neighbors while $\mathrm{B}(\mathrm{N})$ has two $\mathrm{Cs}$ and one $\mathrm{N}(\mathrm{B})$ as their nearest neighbors. There is a possibility of all bonds to be relaxed, retaining the hexagonal structure. Stress is thus minimized in this structure, which helps obviously in the stability. In structure II, each $C$ has two $\mathrm{C}$ and either one $\mathrm{B}$ or $\mathrm{N}$ as nearest neighbors. $\mathrm{C}_{2}$ and $\mathrm{BN}$ form own striped regions, which lie parallel to each other in this structure. Now C-B bond is much larger that C-C. So this is definitely going to put some internal stress. From the point of view of intra-layer stress, this structure is definitely less stable than the previous one. Looking at structure III, one can see this structure looks similar to structure I but B-N bond orientations are different. Each $\mathrm{C}$ atom now has either two $N$ or $B$ and one $C$ in its neighboring position while $N(B)$ has two $C$ and one $\mathrm{B}(\mathrm{N})$ as neighbors. This obviously adds some uncompensated strain in the structure. Structure III, thus consists of two parallel C-N and C-B chains and as C-B bond length is much larger than C-N (15\%), this mismatch is going to introduce a large strain in the interface. On the other hand in structure I, C-N and C-B are lined up making the structural energy lower compared to structure III. Structure IV does not contain any C-C bond. C-B and C-N chains are lined parallel to each other. Finally in structure V, B-N bonds are placed in such a way that they make $60^{\circ}$ angle to each other. Both of the last two structures thus have uncompensated strain, which increases their structural energies.

Bond energy is another key factor in stability. When the bond energies are counted, the ordering of the bonds is the following [13]:

$$
\begin{gathered}
B-N(4.00 \mathrm{eV})>C-C(3.71 \mathrm{eV})>N-C(2.83 \mathrm{eV})> \\
B-C(2.59 \mathrm{eV})>B-B(2.32 \mathrm{eV})>N-N(2.11 \mathrm{eV})
\end{gathered}
$$

The maximization of stable bonds like B-N and C-C will thus stabilize the structure as a whole. Now, a structure like II, with a striped pattern of $\mathrm{C}$ and B-N chains has maximum number of such bonds. This makes it most stable even though a structural strain is present. In this case bond energy wins over structural stress. For the structures like I and III, number 


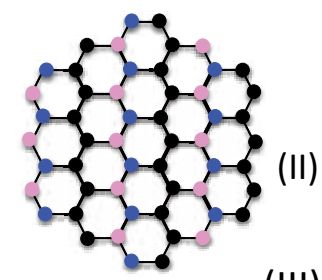

(III)

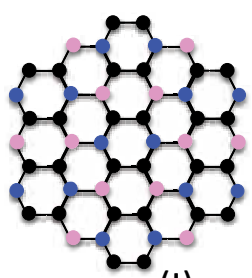

(I)
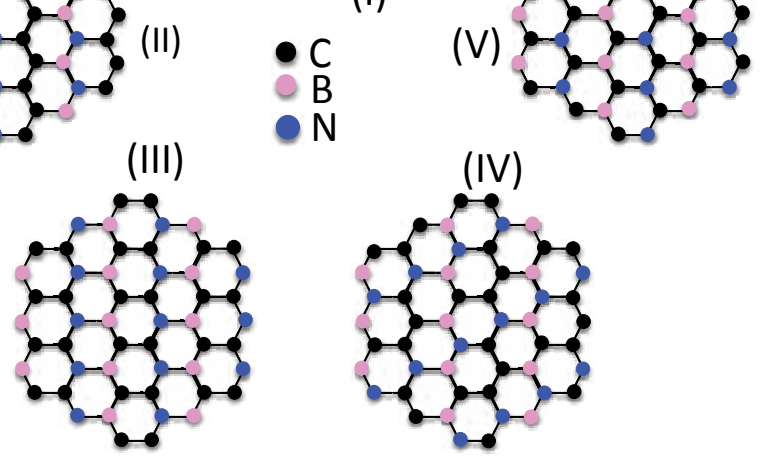

(IV)

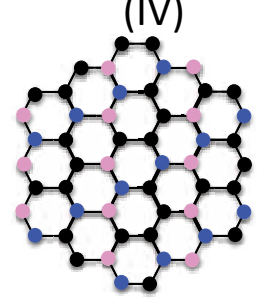

Figure 1. Crystal structure of different isomers of $\mathrm{BC}_{2} \mathrm{~N}$. Filled black, pink, and blue circles represent carbon, boron and nitrogen respectively.

of such bonds is equal. In that case, intra-layer stress acts as the deciding factor. Structure IV, on the other hand does not have any C-C or B-N bond but only C-B or C-N. Therefore, the issue of stability is the most prominent here. The number of strong bonds is sufficiently large in structure $\mathrm{V}$ for the stabilization despite of $60^{\circ}$ arrangement of $\mathrm{B}-\mathrm{N}$ bonds.

Another important issue is the charge transfer as there is a difference between the electronegativities of $\mathrm{B}, \mathrm{C}$, and $\mathrm{N}$. As mentioned earlier, $\mathrm{N}$ is the most electronegative and $\mathrm{B}$ is the least one while $\mathrm{C}$ behaves as a neutral atom. This also adds an ionic character in the bond formation. B (N) always gains some +ve (-ve) charges. So, the gain in the electrostatic energy only happens if these $+/$ - charges are situated in an alternative manner. Otherwise the electrostatic repulsion makes the structure unstable. From this point of view, structures II, III, and $\mathrm{V}$ are more stable than the other two following the trend shown from bond energies. Thus, as reported by Itoh et al.[13], ordering of the structures will be: II $>$ V $>$ (I,III) $>$ IV . Stability of other possible isomers can as well be anticipated with the same arguments.

So far we have talked about the substitutional alloying of $\mathrm{BN}$ and graphene, where $\mathrm{BN}$ to $\mathrm{C}_{2}$ ratio is 1:1. Another group of structures, which can be formed by alloying $\mathrm{BN}$ and graphene is the phase segregated BCN. In this kind of structure, BN (graphene) retains its own phase, separated by graphene $(\mathrm{BN})$. The experimental evidence of these kinds of structures have been shown [14]. The size of the graphene or BN phase has an impact on the stability and electronic properties. Here, the $\mathrm{BN}: \mathrm{C}_{2}$ ratio is thus not only $1: 1$ but can be varied and if varied controllably, one can control the electronic properties such as band gap [16]. Lam et al.[16] have shown that, by controlling the graphene phase, one can control the band gap according to the desired values for technological applications. The phase segregated $(\mathrm{BN})_{m}\left(\mathrm{C}_{2}\right)_{n}$ alloys 
are also found to be stable over the first kind of alloying, which indicates a transformation due to thermal vibration. Yuge et al.[17] with DFT studies and Monte Carlo simulations have shown a tendency of phase separation between $\mathrm{BN}$ and graphene.

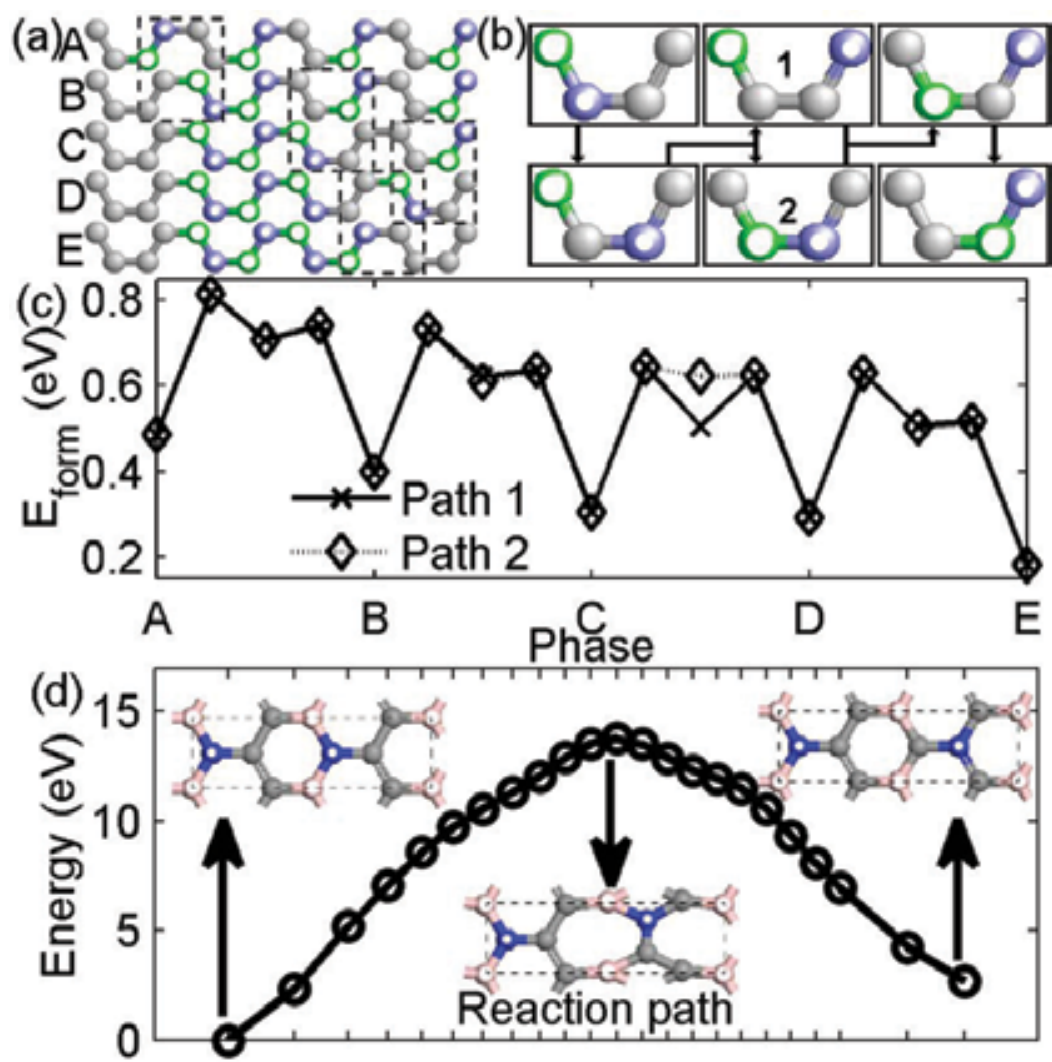

Figure 2. (a) Different steps (A-E) of phase separation process, (b) Swapping of BN and C dimers, (c) Formation energies for different steps shown in (a) for two different paths demonstrated in (b), (d) Activation energy in going from left to right configuration in the initial step of phase segregation. Reprinted with permission from Appl. Phys. Lett. 98, 022101 (2011). Copyright (2011) American Institute of Physics.

Even though a tendency is indicated, a recent calculation by Lam et al., have shown that this possibility is hindered as the activation energy required for phase segregation is extremely high. As shown in Fig. 2, they have chosen a possible path for phase separation by swapping $\mathrm{B}-\mathrm{N}$ bond to $\mathrm{C}-\mathrm{C}$ bonds. This kind of swapping can also happen in two ways (Fig. 2(b)). Calculated formation energies for these two process are shown in Fig. 2(c), which basically demonstrates that the intermediate structures are quite high in energy compared to evenly distributed and phase separated structures. The authors also performed nudged elastic band (NEB) calculations to determine the activation barriers for the first step to occur, i. e. to change a B-N bond to B-C and N-C bonds (Fig. 2(d)). Activation energy required is $1.63 \mathrm{eV} /$ atom suggesting that this process can happen only at elevated temperatures. At room temperature that is why the pristine $(\mathrm{BN})_{m}\left(\mathrm{C}_{2}\right)_{n}$ should be stable and so are the phase separated ones. 
There can be two different patterns for phase segregated $\mathrm{BCN}$ alloys. One is the phase separated island-like and the other one is a striped pattern. The island-like pattern consists of larger graphene-BN interface region than that in the striped pattern. This means that the number of $\mathrm{B}-\mathrm{C}$ and $\mathrm{N}-\mathrm{C}$ bonds are less in striped pattern than in an island form. As we have discussed earlier, the maximization of $\mathrm{C}-\mathrm{C}$ and B-N bonds thus favors a striped pattern [13].

Till now, we have discussed mainly the stability issues of $(B N)_{m}\left(C_{2}\right)_{n}$ with 1:1 ratio and phase separated BCN alloys. A distributed mixture of BN and graphene with different $m: n$ ratios can also form depending on the growth condition. Different isomeric structures are also possible for a particular $m: n$ ratio. In the following section we are going to present a DFT study to analyze the stability and electronic properties of $(\mathrm{BN})_{m}\left(\mathrm{C}_{2}\right)_{n}$ with different $m: n$ ratios. Utilizing the concept of aromaticity, the aim is to find out stable isomers for a particular $m: n$ ratio and also to explore the possibilities of achieving desired electronic properties.

Aromaticity, as extensively used to determine the stability of organic molecules, can provide us a working principle for determining stability of the structures as well. Benzene $\left(\mathrm{C}_{6} \mathrm{H}_{6}\right)$ is the prototype for the organic molecules, which are stabilized by aromaticity. Borazine $\left(\mathrm{B}_{3} \mathrm{~N}_{3} \mathrm{H}_{6}\right)$, an isoelectric $\mathrm{BN}$ analogue of benzene on the other hand has one-third stability of benzene from the point of view of aromaticity [18-21]. This is particularly interesting in $(\mathrm{BN})_{m}\left(\mathrm{C}_{2}\right)_{n}$, as the admixture of two not only changes the electronic property but also affects its stability. To investigate a stable isomer, our first working principle thus is to maximize the carbon hexagons, which essentially mimic benzene rings. A carbon-hexagon again can be surrounded by $\mathrm{BN}$ and each hexagon can be kept aloof or all hexagons can form a carbon-pathway. In a carbon pathway, $\pi$-conjugation is allowed whereas it is hindered in isolated C-hexagons.

To look for reasonable isomers, we consider that the following structural possibilities will not occur. Firstly, a hexagon will not contain $\mathrm{B}$ and $\mathrm{N}$ in 1 and 3 positions with respect to each other. These kind of structures are described by zwitterionic and biradical resonance structures, which basically result in an odd number of $\pi$-electrons on two of the Cs in the hexagon (Fig. 3).

Hence, a B-N pair should be placed either in 1,4 or 1,2 position in the hexagon with respect to each other. $\pi$-electrons will thus be distributed over a $\mathrm{C}-\mathrm{C}$ bond and form a resonance structure. Second kind of structural constraint, that we consider, is the absence of B-B or N-N bonds. As discussed earlier, these kind of bonds result in the lowering of $\pi$-bonds and thus decreased relative stability of an isomer.

The relative positions of $\mathrm{B}$ and $\mathrm{N}$ around an all $\mathrm{C}$ hexagon is also a key factor that controls the electronic properties. To illustrate the phenomenon, let's consider the following two isomers. As in Fig. 3, the isomer I and isomer II, both have similar chemical configuration. But in Isomer $\mathrm{I}, \mathrm{B}$ and $\mathrm{N}$ are connected to $\mathrm{C}$ at position 1 and 4 in the hexagon, which we can call B-ring-N para-arrangement. A donor- acceptor (D-A) interaction is thus established in this kind of structural arrangement. On the other hand in isomer II, B and N are connected to $1^{\text {st }} \& 2^{\text {nd }}\left(4^{\text {th }} \& 5^{\text {th }}\right)$ positioned $C$ atoms in the hexagon. Although a D-A interaction occurs between neighboring $\mathrm{B}$ and $\mathrm{N}, \mathrm{B}-$ ring-N interaction is forbidden. The local D-A interaction around a C-hexagon, as shown in Fig. 4, increases the HOMO-LUMO gap whereas N-ring-N (or B-ring-B) para arrangement results in the lowering of the HOMO-LUMO gap. 


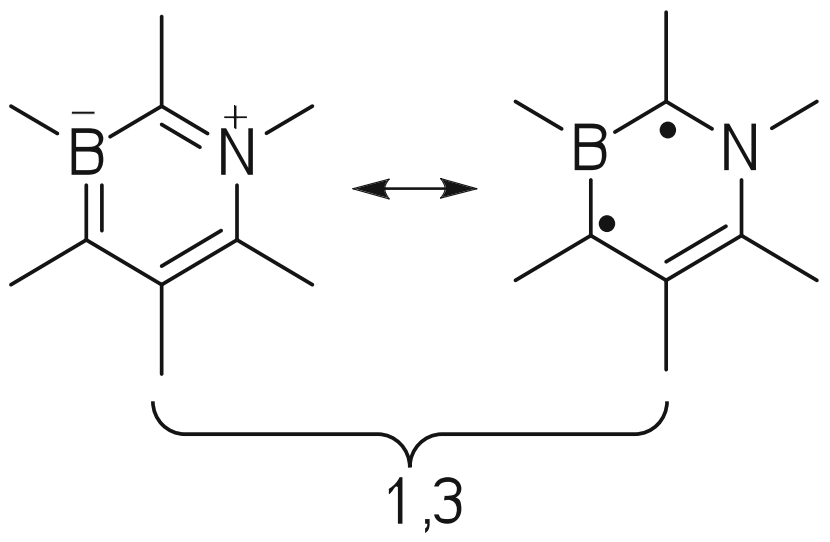

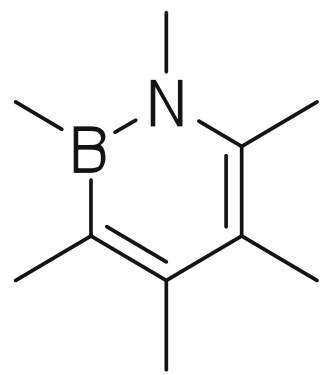

12

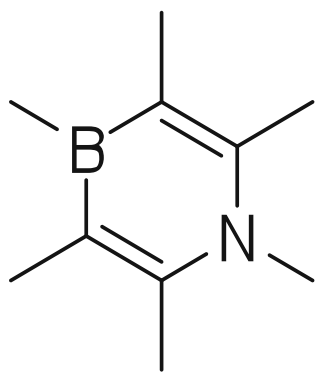

1,4

Figure 3. Schematic representation of zwitterionic and biradical resonance structures. Reprinted with permission from J. Phys. Chem. C 115, 10264 (2011). Copyright (2011) American Chemical Society.

We have performed density functional calculations to investigate the isomers of $(B N)_{m}\left(C_{2}\right)_{n}$ [22]. All the structures are optimized with both (Perdew-Burke-Ernzerhof) PBE [23] and (Heyd-Scuseria-Ernzerhof) HSE [24] functionals. The functionals based on local spin density approximation or generalized gradient approximations reproduce the structural parameters reasonably well, whereas the band gaps come out to be much smaller compared to experiments. The reason behind this is the self interaction error. HSE, with a better description of exchange and correlation within hybrid DFT, yields a band gap, which is much closer to the experimental value.

The degree of aromaticity is calculated quantitatively, with a harmonic oscillator model of aromaticity (HOMA) prescribed by Krygowski et al.[25]. The HOMA value of an ideal aromatic compound (Benzene) will be 1, whereas the value will be close to zero for non aromatic compounds. Anti-aromatic compound with the least stability will have a negative HOMA value. As mentioned earlier, the aim is to find the important isomers with relatively high stability and reasonable band gaps among $(\mathrm{BN})_{m}\left(\mathrm{C}_{2}\right)_{n}$ compounds with $m: n$ ratios 1:1, 2:1, 1:3 and 2:3. Let's focus on each type separately. 


\subsection{1:1 h-BN:Graphene $\left(\mathrm{BC}_{2} \mathrm{~N}\right)$}

We have considered six isomers for $\mathrm{BC}_{2} \mathrm{~N}$, among which two structures $\mathrm{BC}_{2} \mathrm{~N}-\mathrm{I}$ and $\mathrm{BC}_{2} \mathrm{~N}-\mathrm{II}$ consist of all $\mathrm{C}$-hexagon pathways. In the third one, $\mathrm{BC}_{2} \mathrm{~N}$-III, all $\mathrm{C}$-hexagons are connected linearly as in polyacenes where as the fourth one , $\mathrm{BC}_{2} \mathrm{~N}-\mathrm{IV}$, has disconnected all-C-hexagons. The other two structures, $\mathrm{BC}_{2} \mathrm{~N}-\mathrm{V} \& \mathrm{BC}_{2} \mathrm{~N}-\mathrm{VI}$ do not have any all-C hexagon but $\mathrm{BC}_{2} \mathrm{~N}-\mathrm{VI}$ has at least polyacetylene paths whereas $\mathrm{BC}_{2} \mathrm{~N}-\mathrm{V}$ has only isolated $\mathrm{C}-\mathrm{C}$ bonds. Although there

Isomer I<smiles>[2H]c1cc([2H])c(N)cc1N</smiles>

$(2 \times B-$ ring-N)<smiles>[2H]C1=CC(=[OH+])C([2H])=CC1=[NH2+]</smiles>

\section{Isomer ||}<smiles>[2H]c1cc(N)c([2H])cc1N</smiles>

(B-ring-B + N-ring-N)
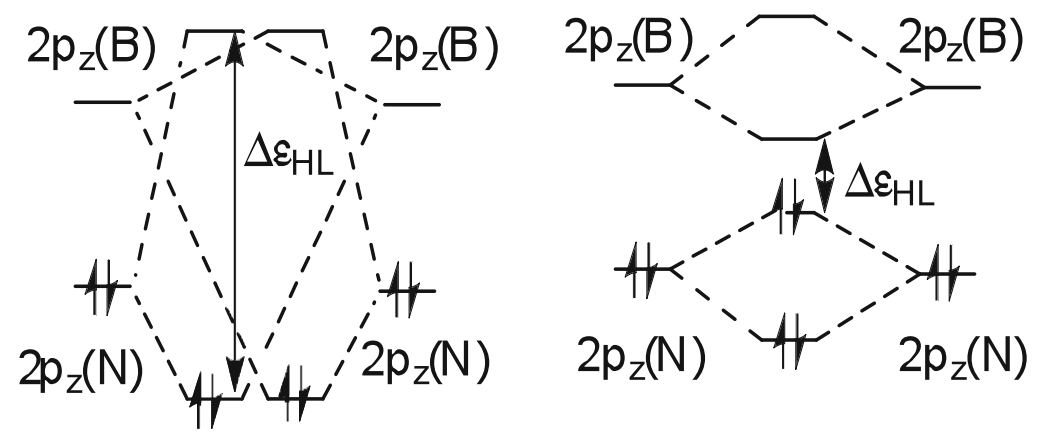

Figure 4. Qualitative representation of opening up a band gap and D-A interaction in isomer I and reduction of band gap in isomer II, with molecular orbital diagrams and valence bond representation. Reprinted with permission from J. Phys. Chem. C 115, 10264 (2011). Copyright (2011) American Chemical Society.

are several other isomers possible, we limit ourselves with these and try to understand the properties with the knowledge of aromaticity and conjugation. Firstly, the first three isomers, among all six are most stable and the relative energies differ by at most $0.15 \mathrm{eV}$ (PBE) and $0.07 \mathrm{eV}$ (HSE). The presence of all C-hexagons connected to each other not only increases the 
stable C-C and B-N bonds but also helps in the $\pi$-conjugation. The result is reflected in the HOMA values of first two structures, which are 0.842 and 0.888 respectively. This suggests the formation of aromatic benzene like all-C hexagons. The HOMA value of $\mathrm{BC}_{2} \mathrm{~N}-\mathrm{III}$ is little less (0.642) but this structure in particular is not stable due to aromaticity rather due to the formation of polyacetylene paths. A slightly lower HOMA value observed in $\mathrm{BC}_{2} \mathrm{~N}-\mathrm{I}$ compared to $\mathrm{BC}_{2} \mathrm{~N}-\mathrm{II}$ is due to the difference in $\mathrm{B}-\mathrm{C}$ bond $(0.02 \AA)$, which leads to a change in D-A interaction.

If we look at the formation energies of $\mathrm{BC}_{2} \mathrm{~N}-\mathrm{IV} \& \mathrm{BC}_{2} \mathrm{~N}-\mathrm{VI}$, the values are quite close. $\mathrm{BC}_{2} \mathrm{~N}-\mathrm{IV}$ consists of completely isolated all-C hexagons. This is the reason of having high aromaticity of 0.88 . But at the same time this increases $\mathrm{N}-\mathrm{C} \& \mathrm{~B}-\mathrm{C}$ bonds and restricts $\pi$-conjugation. Therefore, this structure is less probable thermodynamically. $\mathrm{BC}_{2} \mathrm{~N}$-VI, which was suggested to be the most stable $\mathrm{BC}_{2} \mathrm{~N}$ structure by Liu et al.[15], on the other hand has no aromatic all $\mathrm{C}$-hexagon. But this structure contains all-C polyacetylene paths with $\mathrm{C}-\mathrm{C}$ bond length $1.42 \AA$, which explains its low formation energy. $\mathrm{BC}_{2} \mathrm{~N}-\mathrm{V}$ is the least stable among all, which has neither all-C hexagon nor polyacetylene C-paths. Obviously most unstable $\mathrm{B}-\mathrm{C}$ and $\mathrm{B}-\mathrm{N}$ bonds are maximized here creating an enormous strain in the structure. The presence of only C-C bond of $1.327 \AA$ explains that. These factors make this compound thermodynamically most unstable among all five structures.

All these results give us a stand point from where we can judge the thermodynamic stability of other $(\mathrm{BN})_{m}\left(\mathrm{C}_{2}\right)_{n}$ structures with the following working principles in hand:

(a) $\pi$-Conjugation within all $\mathrm{C}$ path increases stability.

(b) The formation of aromatic all C-hexagons also does the same, while this is more effective when hexagons are connected.

(c) There is not much contribution of B-ring-B or B-ring- $\mathrm{N}$ arrangement of $\mathrm{B}$ and $\mathrm{N}$ around poly(para-phenylene) (PPP) path in total energies. But indeed these, as discussed earlier, will affect the band gap, which we will present in the following section.

Coming to the band gap issue, the first three structures, which are close in energy, have band gaps ranging from 1.6 to $2.3 \mathrm{eV}$ in $\mathrm{HSE}$ calculations ( 0.7 to $1.7 \mathrm{eV}$ in $\mathrm{PBE}$ ). The difference in $\mathrm{BC}_{2} \mathrm{~N}-\mathrm{I} \& \mathrm{BC}_{2} \mathrm{~N}-\mathrm{II}$ comes from the arrangement of $\mathrm{B}$ and $\mathrm{N}$ around all- $\mathrm{C}$ hexagon. As discussed in Fig. 3, D-A interaction increases for para-positions (i.e.1,4 or 2,6), which is observed in $\mathrm{BC}_{2} \mathrm{~N}-\mathrm{I}$. The band gap is $0.5 \mathrm{eV}\left(0.65 \mathrm{eV}\right.$ in PBE), higher than that in $\mathrm{BC}_{2} \mathrm{~N}-\mathrm{II}$, where $\mathrm{B}$ and $\mathrm{N}$ are oriented in ortho-position (i.e.1,2 or 4,5). Quite obviously, $\mathrm{BC}_{2} \mathrm{~N}-\mathrm{III}$ has all-C chain, which resembles a graphene nanoribbon and has the least value of the band gap.

\subsection{2:1 h-BN:Graphene (BCN)}

We have investigated three structures of $\mathrm{BCN}$, which have recently been synthesized [26]. The first one (BCN-I) has aromatic all-C hexagons connected in PPP path whereas the second one (BCN-III) contains all-C hexagon but connected in zigzag polyacene bonds. The final one (BCN-IV) consists of neither all-C hexagon nor a stripe of all-C region. BCN-I \& BCN-II are iso-energetic, which is expected and is $\sim 0.5 \mathrm{eV}$ lower than BCN-IV. This again explains the importance of aromatic all- $C$ hexagon and $\pi$-conjugation. The absence of these and also the increased B-C, N-C bonds make BCN-IV relatively unstable. Another key point in BCN-I \& $\mathrm{BCN}-\mathrm{I}$ is the position of $\mathrm{B}$ and $\mathrm{N}$ around the hexagon. The stability may not be affected but 
the band gap is definitely changed by this. As expected, from the discussion in Fig. 4, BCN-I has a quite high value of the band gap. Aromaticity is higher in BCN-I (0.846) compared to $\mathrm{BCN}$-III (0.557), which is also seen in $\mathrm{BC}_{2} \mathrm{~N}$ structures. Iso-energetic $\mathrm{BCN}-\mathrm{I} \& \mathrm{BCN}-\mathrm{II}$ are equally probable during growth process but with a band gap range 1.3 to $2.7 \mathrm{eV}$.

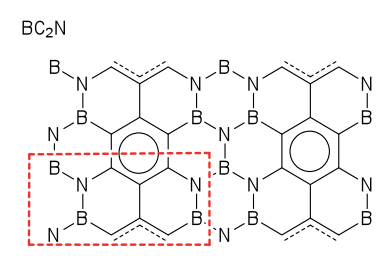

I $\underline{0.06} / 0.11(2.31 / 1.70)$ HOMA $=0.842$

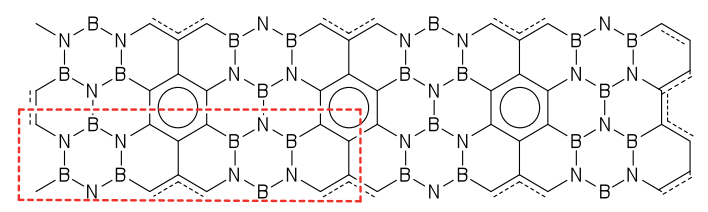

II $\underline{0.07 / 0.07}(1.80 / 1.05)$ HOMA $=0.888$
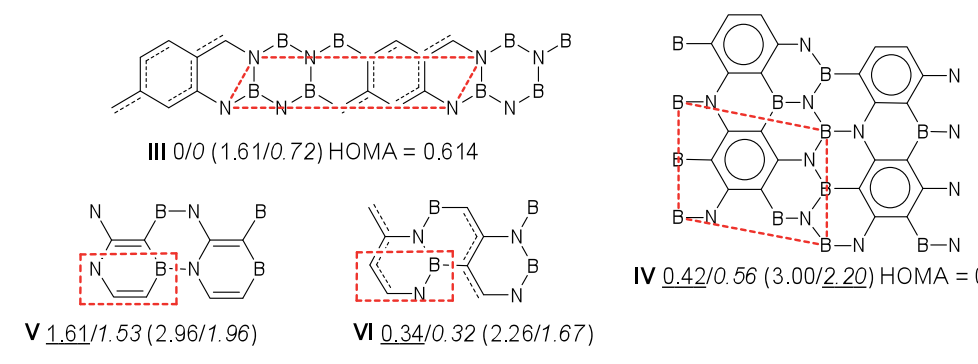

IV $\underline{0.42} / 0.56(3.00 / \underline{2} .20)$ HOMA $=0.880$

VI $\underline{0.34 / 0.32}(2.26 / 1.67)$

Figure 5. Six isomers of $\mathrm{BC}_{2} \mathrm{~N}$ are considered for this study. Relative energy (per formula unit) with respect to most stable structures and band gaps (in parentheses) are shown from HSE (normal print) and PBE (italic) calculations. HOMA values of all-C hexagons, obtained from HSE calculations are also provided. Reprinted with permission from J. Phys. Chem. C 115, 10264 (2011). Copyright (2011) American Chemical Society.

\subsection{2:3 \& 1:3 h-BN:Graphene $\left(\mathrm{BC}_{3} \mathrm{~N} \& \mathrm{BC}_{6} \mathrm{~N}\right)$}

We now gradually increase the C-percentage with the anticipation of lowering the band gap because of increased graphene region. Two structures of $\mathrm{BC}_{3} \mathrm{~N}$ and three structures of $\mathrm{BC}_{6} \mathrm{~N}$ have been examined. Both the structures of $\mathrm{BC}_{3} \mathrm{~N}$ consist of aromatic hexagonal all- $\mathrm{C}$ rings connected in PPP path. The similarity in HOMA values depicts that picture. The position of $\mathrm{B}$ and $\mathrm{N}$ around $\mathrm{C}$-ring is different though in $\mathrm{BC}_{3} \mathrm{~N}-\mathrm{I}$ and $\mathrm{BC}_{3} \mathrm{~N}$-II. The para arrangement of B-ring- $\mathrm{N}$ results in a large band gap in $\mathrm{BC}_{3} \mathrm{~N}-\mathrm{I}(2.14 \mathrm{eV})$ while the ortho arrangement of the same in $\mathrm{BC}_{3} \mathrm{~N}-\mathrm{II}$ lowers the value of the band gap. The stability is not affected by that fact as aromaticity and $\pi$-band formation are quite similar, which make those structures iso-energetic. A similar situation is also seen in $\mathrm{BC}_{6} \mathrm{~N}$ structures. $\mathrm{BC}_{6} \mathrm{~N}-\mathrm{I}$ and $\mathrm{BC}_{6} \mathrm{~N}-\mathrm{II}$ are iso-energetic mainly due to a similarity in structures. Both of them contain all- $\mathrm{C}$ rings connected in PPP path. But in the third one $\left(\mathrm{BC}_{6} \mathrm{~N}-\mathrm{III}\right)$, all-C rings are separated, which makes this structure relatively unstable due to restricted $\pi$-conjugation. The HOMA value is maximum (0.93) in $\mathrm{BC}_{6} \mathrm{~N}-\mathrm{III}$, whereas the values are 0.78 and 0.86 respectively for $\mathrm{BC}_{6} \mathrm{~N}-\mathrm{I}$ and $\mathrm{BC}_{6} \mathrm{~N}$-II. The para arrangement of $\mathrm{B}$-ring- $\mathrm{N}$ in $\mathrm{BC}_{6} \mathrm{~N}-\mathrm{I}$ and $\mathrm{BC}_{6} \mathrm{~N}-\mathrm{III}$ leads to large band gaps (1.58 and $1.34 \mathrm{eV}$ respectively) while ortho-positioning of the same results in a reduced band gap in $\mathrm{BC}_{6} \mathrm{~N}-\mathrm{II}$. Finally, we have summarized the calculated (HSE and PBE) band gaps for all $(\mathrm{BN})_{m}\left(\mathrm{C}_{2}\right)_{n}$ isomers (Fig. 7A and Fig. 7B). As expected, the band gaps are increased for HSE functional. Apart from that, both HSE and PBE -level calculations show a similar trend. A 


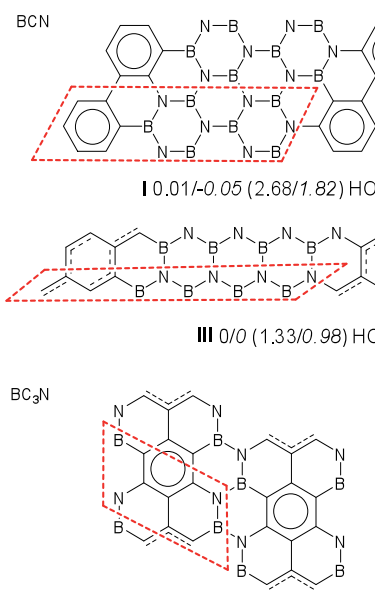

I $0 / 0(2.14 / 1.50)$ HOMA $=0.876$

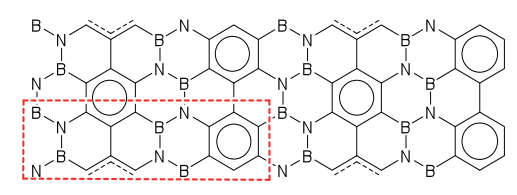

II $0.07 / 0.03(0.94 / 0.50)$ HOMA $=0.890$

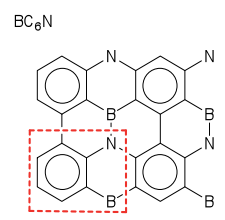

$10.07 / 0.08(1.56 / 1.02)$ HOMA $=0.781$

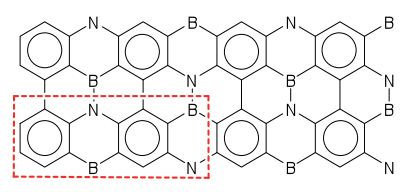

II $0 / 0(0.05 / 0.17)$ HOMA $=0.858$

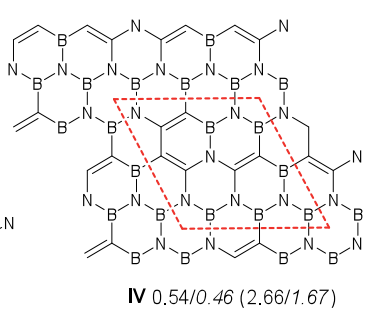

IV $0.54 / 0.46(2.66 / 1.67)$

Figure 6. The isomers of $\mathrm{BCN}, \mathrm{BC}_{3} \mathrm{~N}, \mathrm{BC}_{6} \mathrm{~N}$. Relative energy (per formula unit) with respect to most stable structures and band gaps (in parentheses) are shown from HSE (normal print) and PBE (italic) calculations. HOMA values of all-C hexagons, obtained from HSE calculations are also provided. Reprinted with permission from J. Phys. Chem. C 115, 10264 (2011). Copyright (2011) American Chemical Society.

general trend is observed that an increase in the graphene region reduces the band gap. All the lowest energy structures for different compositions of $(\mathrm{BN})_{m}\left(\mathrm{C}_{2}\right)_{n}$ have band gaps around $1 \mathrm{eV}$, which is a desired value for technological applications. One very important thing that we learnt from this study is that the position of $\mathrm{B}$ and $\mathrm{N}$ around $\mathrm{C}$-ring controls the band gap without affecting the stability.

\section{Functionalization}

Incorporation of magnetism in 2D sp-materials has been an important point of discussion in recent times. The combination of localized moments of $3 d$ transition metal atoms and the $s p$ electrons of the host $2 \mathrm{D}$ lattice can give rise to interesting magnetic properties relevant to nano devices based on the principle of magnetoresistance, for example. Ferromagnetic long-ranged order, half metallicity, large magnetic anisotropy, electric field driven switching of magnetization etc. are being studied for transition metal atoms adsorbed on graphene and 2D BN sheets. Another important point is the adsorption of these species at the interface between $\mathrm{BN}$ and graphene. A recent study [27] based on first principles electronic structure calculations has revealed some interesting electronic and magnetic properties of $\mathrm{Fe}, \mathrm{Co}$ and $\mathrm{Ni}$ adatoms adsorbed on a h- $\mathrm{BC}_{2} \mathrm{~N}$ sheet. A hexagonal site at the interface between $\mathrm{BN}$ and graphene 

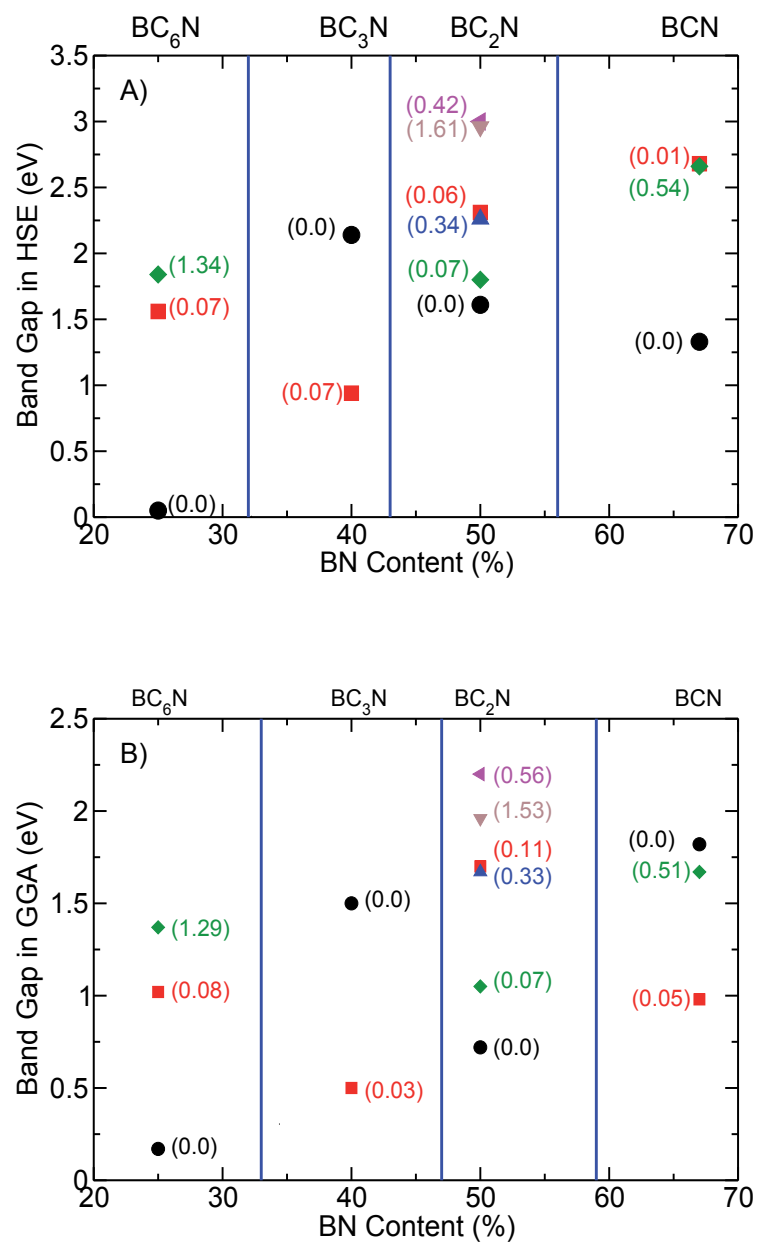

Figure 7. Band gaps of different isomers of $(\mathrm{BN})_{m}\left(\mathrm{C}_{2}\right)_{n}$ are plotted against $\mathrm{BN}$ density, obtained from both (A) HSE and (B) PBE calculations. Relative energies are given in parentheses.

turns out to be a favorable site for adsorption. The presence of $\mathrm{Fe}, \mathrm{Co}$ and $\mathrm{Ni}$ makes the system a magnetic semiconductor, a magnetic semi-metal and a non magnetic semiconductor respectively. Another interesting observation was that the adatoms are highly mobile on the surface and hence have the possibility to have a clustered configuration among themselves. It is interesting to note that the properties of this hybrid system are tunable in the sense that they can be modified by having different combinations of the width of each subsystem, BN and graphene.

Not only by magnetic adatoms, but by intrinsic edge properties, one can render magnetism in these 2D sheets. By DFT calculations, Dutta et al. [28] predicted interesting magnetic properties of H-passivated zigzag nanoribbons (ZGNRs) of various widths, doped by boron and nitrogen, keeping the whole system isoelectronic with $C$ atoms in graphene. In the 
extreme case, all $\mathrm{C}$ atoms of ZGNRs are replaced by $\mathrm{B}$ and $\mathrm{N}$ atoms and zigzag $\mathrm{BN}$ nanoribbons are formed. In the ground state, the two edges are antiferromagnetically coupled and remain so for all dopings. However, the application of an external electric field affects the electronic structure of the nanoribbon giving rise to semiconducting and half-metallic properties. Electric-field induced changes in the magnetic properties are very interesting from a technological point of view. Other related studies [29] based on DFT revealed energetics, electronic structure and magnetism of quantum dots and nanorods of graphene embedded in $\mathrm{BN}$ sheet. It was showed that the formation energies and the HOMO-LUMO gaps of quantum dots vary as $1 / \sqrt{n}$, where $n$ is the number of carbon atoms in the dots.

Adsorption of gases on 2D materials is an important topic from the technological and environmental points of view. Materials for clean energy are always sought for and in this respect, efficient hydrogen uptake of suitable materials is an important issue. Raidongia et al. [26] have studied $\mathrm{H}_{2}$ adsorption on $\mathrm{BCN}$ at $77 \mathrm{~K}$ and $1 \mathrm{~atm}$. pressure. From their experiments, $\mathrm{H}_{2}$ uptake of $2.6 \mathrm{wt} \%$ was observed. Also, $\mathrm{CO}_{2}$ adsorption is very important for environmental issues. In their study, $\mathrm{BCN}$ was found to have a very high $\mathrm{CO}_{2}$ uptake of $100 \mathrm{wt} \%$ at $195 \mathrm{~K}$ and $1 \mathrm{~atm}$. pressure. It should be noted that the uptake is only $58 \%$ by the activated charcoal under identical conditions. At room temperature and 40 bar pressure, the $\mathrm{CO}_{2}$ uptake was found to be $44 \mathrm{wt} \%$.

In a recent theoretical study, Cao et al. [30] showed that a zigzag interface between BN and graphene can have a strong capability of adsorbing hydrogen, much stronger than pure graphene, $\mathrm{BN}$ or the armchair interface between them. Moreover, the adsorption of hydrogen induces a semiconductor to metal transition. As the mobility of hydrogen on the surface is rather high, the hydrogen atoms can migrate to the zigzag interface and hence will increase the density of hydrogen storage with the added functionality of band gap engineering.

\section{Summary and outlook}

Graphene-BN nanocomposites offer a huge potential in various technological sectors, e.g., nano electronics, gas sensing, hydrogen storage, nanomagnetic storage devices, to name a few. The unique combination of these two materials with different electronic properties, forming a 2D network, offers many possibilities for studying fundamental science and applications for nanotechnology. However, many challenges, both in the domains of experiment and theory, will come in the way. Experimental synthesis of samples of good quality and state-of-the-art characterization techniques to reveal the atomic scale physics will be the issues. From the point of view of theory, one faces difficulties in having a correct description of the band gaps and electronic structures in standard approximations of materials-specific theories. However, with the availability of powerful supercomputing facilities, it is nowadays possible to treat large systems by sophisticated many body theories to have a much better quantitative descriptions. Nevertheless, one may envisage many interesting directions for the applications of these nanocomposites to utilize the interface properties of BN and graphene. One of them is the spin switching properties of organometallics adsorbed at the interface, similar to what has been studied recently [31] for a 2D graphene sheet. The other application can be the adsorption of amino acids [32] at the interface to increase the activity by their immobilization. Hopefully, in near future, we will observe many applications of these nanocomposites, useful for the human society. 


\section{Author details}

Sumanta Bhandary and Biplab Sanyal

Department of Physics and Astronomy, Uppsala University, Box 516, 75120 Uppsala, Sweden

\section{References}

[1] K. S. Novoselov, A. K. Geim, S. V. Morozov, D. Jiang, Y. Zhang, S. V. Dubonos, I. V. Grigorieva, A. A. Firsov, Science 306, 666 (2004).

[2] L. D. Landau, Phys. Z. Sowjetunion 11, 26 (1937).

[3] R. E. Peierls, Ann. I. H. Poincare 5, 177 (1935).

[4] A.K. Geim and K.S. Novoselov, Nat. Mater. 6, 183 (2007); A.H. Castro Neto et al., Rev. Mod. Phys. 81, 109 (2009); A.K. Geim, Science 324, 1530 (2009).

[5] M. I. Katsnelson, Materials Today 10, 20 (2007).

[6] P. R. Wallace, Phys. Rev. 71 622-634 (1947).

[7] J. O. Sofo, A. S. Chaudhari and G. D. Barber, Phys. Rev. B 75, 153401 (2007); D. C. Elias et al., 323, 610 (2009); O. Leenaerts, H. Peelaers, A. D. HernĞndez-Nieves, B. Partoens, and F. M. Peeters, Phys. Rev. B 82, 195436 (2010).

[8] M. Klintenberg, S. Lebegue, M. I. Katsnelson, and O. Eriksson, Phys. Rev. B 81, 085433(2010).

[9] M. Y. Han, B. Özyilmaz, Y. Zhang, and P. Kim, Phys. Rev. Lett. 98, 206805 (2007).

[10] Y.-W. Son, M. L. Cohen and S. G. Louie, Nature 444, 347 (2006).

[11] S. Bhandary, O. Eriksson, B. Sanyal and M I. Katsnelson, Phys. Rev. B 82,165405 (2010).

[12] P. Hohenberg and W. Kohn, Phys. Rev. B 136, 864 (1964); W. Kohn and L. J. Sham, Phys. Rev. A 140, 1133 (1965).

[13] H. Nozaki and S. Itoh, J. Phys. Chem. Solids, 57, 41 (1996).

[14] L. Ci, L. Song, C. Jin, D. Jariwala, D. Wu, Y. Li, A. Srivastava, Z. F. Wang, K. Storr, L. Balicas, F. Liu and P. M. Ajayan, Nat. Mater. 9, 430 (2010).

[15] A. Y. Liu, R. M. Wentzcovitch and M. L. Cohen, Phys. Rev. B 39,1760 (1989).

[16] K.-T. Lam, Y. Lu, Y. P. Feng and G. Liang, Appl. Phys. Lett. 98, 022101 (2011).

[17] K. Yuge, Phys. Rev. B 79, 144109 (2009).

[18] P. v. R. Schleyer, H. Jiao, Pure Appl. Chem 68, 209 (1996).

[19] E. D. Jemmis and B. Kiran, Inorg. Chem. 37, 2110 (1998).

[20] P. W. Fowler and E. J. Steiner, J. Phys. Chem. A 101, 1409 (1997).

[21] W. H. Fink, J. C. Richards, J. Am. Chem. Soc. 113, 3393 (1991).

[22] J. Zhu, S. Bhandary, B. Sanyal and H. Ottosson, J. Phys. Chem. C 115, 10264 (2011).

[23] J. P. Perdew and Y. Wang, Phys. Rev. B 45, 13244 (1992).

[24] J. Heyd and G. E. Scuseria, J. Chem. Phys. 120, 7274 (2004); J. Heyd, G. E. Scuseria and M. Ernzerhof, J. Chem. Phys. 118, 8207 (2003).

[25] T. M. Krygowsky, J. Chem. Inf. Comput. Sci. 33, 70 (1993).

[26] K. Raidongia, A. Nag, K. P. S. S. Hembram, U. V. Waghmare, R. Datta and C. N. R. Rao, Chem. Eur. J. 16, 149 (2010).

[27] P. Srivastava, M. Deshpande and P. Sen, Phys. Chem. Chem. Phys. 13, 21593 (2011).

[28] S. Dutta, A. K. Manna and S. K. Pati, Phys. Rev. Lett. 102, 096601 (2009).

[29] S. Bhowmick, A. K. Singh and B. I. Yakobson, J. Phys. Chem. C 115, 9889 (2011).

[30] T. Cao, J. Feng and E. G. Wang, Phys. Rev. B 84, 205447 (2011).

[31] S. Bhandary, S. Ghosh, H. Herper, H. Wende, O. Eriksson and B. Sanyal, Phys. Rev. Lett. 107, 257202 (2011).

[32] S. Mukhopadhyay, R. H. Scheicher, R. Pandey and S. P. Karna, J. Phys. Chem. Lett. 2, 2442 (2011). 


\title{
Graphene Nanocomposites
}

\author{
Mingchao Wang, Cheng Yan and Lin Ma
}

Additional information is available at the end of the chapter

http://dx.doi.org/10.5772/50840

\section{Introduction}

Graphene, one of the allotropes (diamond, carbon nanotube, and fullerene) of carbon, is a monolayer of honeycomb lattice of carbon atomsdiscovered in 2004. The Nobel Prize in Physics 2010 was awarded to Andre Geim and Konstantin Novoselov for their ground breaking experiments on the two-dimensional graphene [1]. Since its discovery, the research communities have shown a lot of interest in this novel material owing to its unique properties. As shown in Figure 1, the number of publications on graphene has dramatically increased in recent years. It has been confirmed that graphene possesses very peculiar electrical properties such as anomalous quantum hall effect, and high electron mobility at room temperature $\left(250000 \mathrm{~cm}^{2} / \mathrm{Vs}\right)$. Graphene is also one of the stiffest (modulus $\sim 1 \mathrm{TPa}$ ) and strongest (strength $\sim 100 \mathrm{GPa}$ ) materials. In addition, it has exceptional thermal conductivity $\left(5000 \mathrm{Wm}^{-1} \mathrm{~K}^{-1}\right)$. Based on these exceptional properties, graphene has found its applications in various fields such as field effect devices, sensors, electrodes, solar cells, energy storage devices and nanocomposites. Only adding 1 volume per cent graphene into polymer (e.g. polystyrene), the nanocomposite has a conductivity of $\sim 0.1 \mathrm{Sm}^{-1}$ [2], sufficient for many electrical applications. Significant improvement in strength, fracture toughness and fatigue strength has also been achieved in these nanocomposites [3-5]. Therefore, graphene-polymer nanocomposites have demonstrated a great potential to serve as next generation functional or structural materials.

Relatively, limited research has been conducted to understand the intrinsic structureproperty relationship in graphene based composites such as graphene-polymer nanocomposites. The mechanical property enhancement observed in graphene-polymer nanocomposites is generally attributed to the high specific surface area, excellent mechanical properties of graphene, and its capacity to deflect crack growth in a far more effectively way than one-dimensional (e.g. nanotube) and zero-dimensional (e.g. nanoparticle) fillers [5]. On the other hand, the graphene sheets or thin platelets dispersed in polymer matrix may create wavy or wrinkled structures that tend to unfold rather than stretch under applied loading. 
This may severely reduce their stiffness due to weak adhesion at the graphene-polymer interfaces [6]. However, a wrinkled surface texture could create mechanical interlocking and load transfer between graphene and polymer matrix, leading to improved mechanical strength [7]. Furthermore, structural defects and stability of graphene can significantly influence the graphene-polymer interfacial behaviour. Therefore, further work is required to understand the structure-property relationship in graphene and the graphene-polymer interface behaviour.

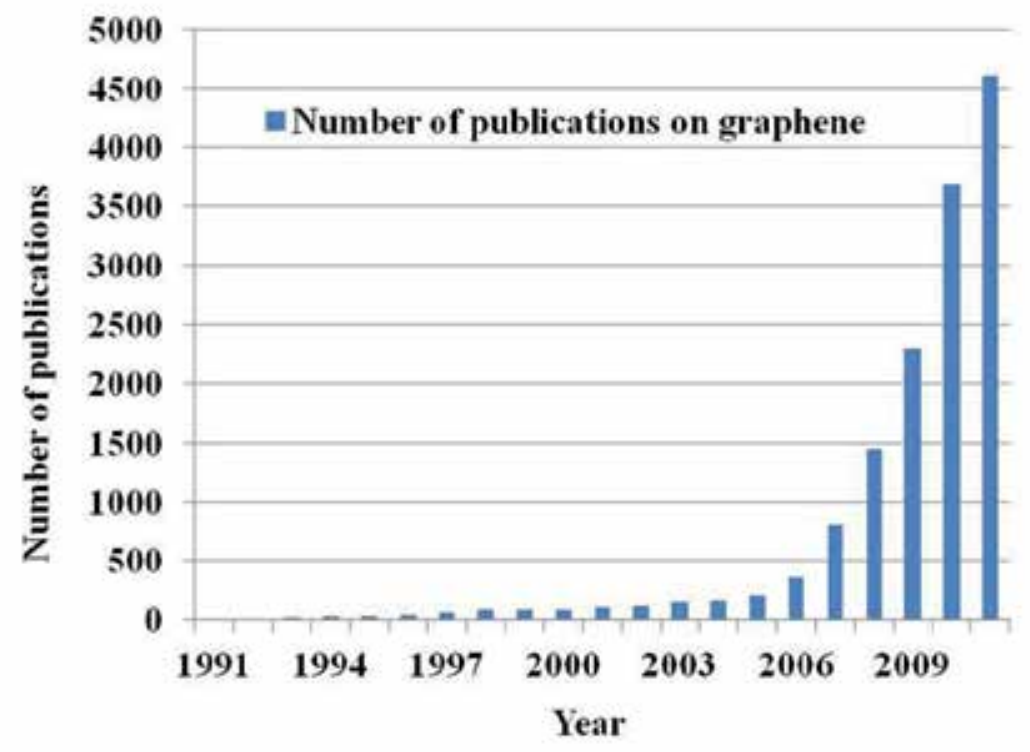

Figure 1. Number of publications on graphene in past 20 years.

\section{Graphene}

\subsection{Mechanical and electrical properties}

Mechanical properties

Graphene, a special monolayer of hexagon-lattice, are even stiffer and stronger than carbon nanotube (CNT). By nanoindentation on a free-standing monolayer graphene, the Young's modulus and intrinsic strength were estimated as $\sim 1.0 \mathrm{TPa}$ and $\sigma_{\text {int }}=130 \mathrm{GPa}$ at a strain of $\varepsilon_{\text {int }}=0.25[8,9]$. Atomistic simulations demonstrated size and chirality dependent elastic properties in graphene nanoribbons [10,11]. The size effect on Young's modulus is negligible when the diagonal length of a graphene nanoribbon is over $10.0 \mathrm{~nm}$. The maximum Cauchy (true) stress and fracture strain for graphene loaded in the armchair direction were estimated as $102 \mathrm{GPa}$ and 0.13 , respectively. Higher values were observed in the zigzag direction, i.e., $129 \mathrm{GPa}$ and 0.20 , respectively. Besides size and chirality dependence, temperature also shows significant influence on the mechanical properties of graphene. Zhao et al. suggested[12] that Young's modulus does not vary significantly with temperature until 
about $1200 \mathrm{~K}$, beyond which graphene becomes softer. The fracture strength and fracture strain decrease significantly with the increase with temperature [12]. Even though monolayer graphene is generally regarded as an ideal structure for practical applications, graphene flakes with few layers are often present in the routine of synthesis, such as mechanical exfoliation. It has been confirmed the layer number is another noticeable factor in dictating the mechanical properties. Table 1 summarizes the intrinsic mechanical properties of the single, bilayer and trilayer graphene.

\begin{tabular}{|llll|}
\hline Method & Material & Mechanical properties & References \\
\hline AFM & Monolayer graphene & $\mathrm{E}=1 \pm 0.1 \mathrm{TPa}$ & {$[8]$} \\
\hline & & $\sigma$ int $=130 \pm 10 \mathrm{GPa}$ at \&int $=0.25$ & \\
\hline & & & {$[13]$} \\
\hline Raman & Graphene & Strain $\sim 1.3 \%$ in tension & \\
\hline & & Strain $~ 0.7 \%$ in compression & {$[14]$} \\
\hline AFM & Monolayer & $\mathrm{E}=1.02 \mathrm{TPa} ; \sigma=130 \mathrm{GPa}$ & \\
\hline & Bilayer & $\mathrm{E}=1.04 \mathrm{TPa} ; \sigma=126 \mathrm{GPa}$ & \\
\hline & Trilayer graphene & $\mathrm{E}=0.98 \mathrm{TPa} ; \sigma=101 \mathrm{GPa}$ & \\
\hline
\end{tabular}

Table 1. Mechanical properties of graphene.

In a single graphene sheet, the $\mathrm{sp}^{2}$ hybridized carbon atoms are arranged in hexagonal fashion. A single hexagonal ring comprises of three strong in-plane sigma bonds $\mathrm{p}_{z}$ orbitals perpendicular to the planes. Different graphene layers are bonded by weak $\mathrm{p}_{z}$ interaction. As a result, the hexagonal structure is generallystable but delamination can occur between the graphene layers when subjected to shear stresses. For example, scotch tape was used to obtain single graphene sheet by peeling bulk graphite layer by layer [1]. In general, the interaction between graphene and other material is considered to be in the form of nonbonded van der Waals attraction. For example, the graphene- $\mathrm{SiO}_{2}$ adhesion energy estimated by pressurized blister tests is about $0.45 \pm 0.02 \mathrm{Jm}^{-2}$ for monolayer graphene and $0.31 \pm 0.03$ $\mathrm{Jm}^{-2}$ for two- to five-layer graphene sheets [15]. These values are greater than the adhesion energies measured in typical micromechanical structures and are comparable to solid-liquid adhesion energies. This can be attributed to the extreme flexibility of graphene, which allows it to conform to the topography of even the smoothest substrates, thus making its interaction with the substrate more liquid-like rather than solid-like.

\section{Electrical transport properties}

As a semiconductor with zero band gap, graphene has unusual charge carriers that behave as massless relativistic particles (Dirac fermions), which is different from electrons when subjected to magnetic fields and has the anomalous integer quantum Hall Effect (QHE) [16]. This effect was even observed at room temperature [17]. The band structure of single layer graphene exhibits two bands which intersect at two in equivalent point $\mathrm{K}$ and $\mathrm{K}_{0}$ in the reciprocal space. Near these points electronic dispersion resembles that of the relativistic Dirac electrons. $\mathrm{K}$ and $\mathrm{K}_{0}$ are referred as Dirac points where valence and conduction bands are degenerated, making graphene a zero band gap semiconductor. 
Another important characteristic of single-layer graphene is its ambipolar electric field effect at room temperature, which is charge carriers can be tuned between electrons and holes by applying a required gate voltage $[1,18]$. In positive gate bias the Fermi level rises above the Dirac point, which promotes electrons populating into conduction band, whereas in negative gate bias the Fermi level drops below the Dirac point promoting the holes in valence band.

\subsection{Structural defect}

Recently, different synthesis methods have been developed to produce high quality graphene such as chemical vapor-deposition (CVD)[19-21] and epitaxial growth[22,23] on metal or SiC substrates. However, various defects and impurities are often introduced into graphene during the processing. The second law of thermodynamics also indicates the presence of a certain amount of disorder in crystalline materials. Like other crystalline materials, it is expected the defects and impurities may strongly influence the electrical, mechanical and thermal properties of graphene. Structural defects, such as Stone-Wales (S-W) defect and vacancies in graphene, can significantly reduce its intrinsic strength. Quantized fracture mechanics (QFM) as well as molecular dynamics (MD) simulations demonstrated that even one vacancy can lead to strength loss by $20 \%$ of pristine graphene [11]. Zheng et al. [24] found that Young's modulus depends largely on the degree of functionalization and molecular structure of the functional groups attached to a graphene sheet, attributed to the binding energy between the functional groups and the graphene, as well as $\mathrm{sp}^{2}$-to-sp $\mathrm{s}^{3}$ bond transition. This was also confirmed in the graphene with hydrogen function groups [25].

On the other hand, imperfection in graphene can be used to tailor the properties of graphene and achieve new functions[26,27]. Defects in graphene are divided into two different types, namely intrinsic and extrinsic. Imperfection without the presence of foreign atoms is referred to as intrinsic type, and other is referred to as extrinsic type. In terms of dimensionality, defects in graphene can also be categorized as point defect (0D) and line defect (1D). In this section, we will review the formation of several typical intrinsic lattice defects in graphene.

\section{Point defects}

One of the unique properties of graphene is its ability to reconstruct the atom arrangement by forming non-hexagonal rings. The simplest example is SW defect [28], which does not involve any removed or added atoms. Four hexagons are transformed into two pentagons and two heptagons [SW(55-77) defect] by rotating one C-C bond by $90^{\circ}$. The existing SW defect was observed in recent experimental research [29], as shown in Figure 2a. The estimated formation energy $\left(E_{\mathrm{f}}\right)$ for $\mathrm{SW}(55-77)$ defect is $5 \mathrm{eV}$ by density functional theory (DFT) calculation [30,31], and $20 \mathrm{eV}$ by molecular dynamics (MD) simulation [11]. Besides these atomic simulations, a topological continuum framework was proposed to evaluate the formation energy of associated and dissociated SW defects in graphene [32]. The high formation energy indicates a negligible kinetic formation rate of SW defect below $1000{ }^{\circ} \mathrm{C}$. In addition, it has been reported that low mechanical strain (less than failure strain) cannot lead to the formation of SW defects [11]. 

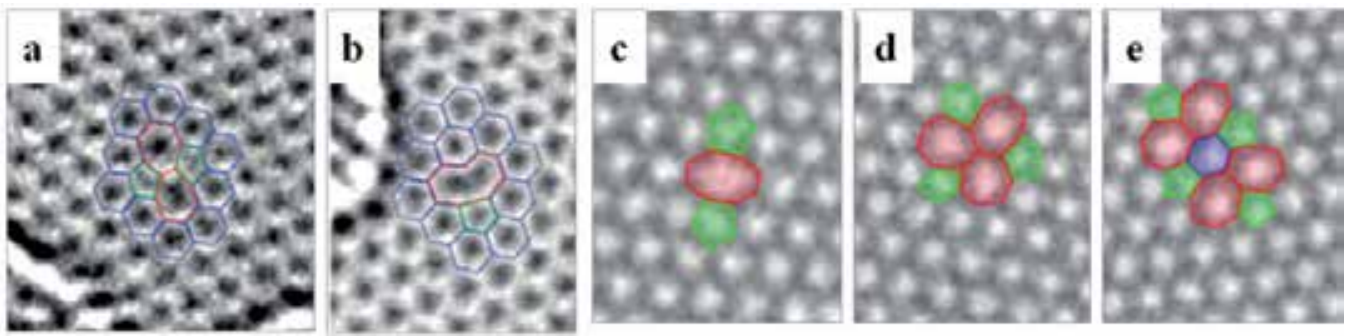

Figure 2. (a) Stone-Wales (SW55-77) defect, (b) Single vacancy (SV) defect ([29]. Reprinted with permission from American Chemical Society, Copyright 2008), (c) double vacancies (DV5-8-5), (d) double vacancies (DV555-777), (e) double vacancies (DV5555-6-7777) defect (Reprinted with permission from Ref [37] Copyright 2010 American Chemical Society).

Besides SW defect, another simple defect in graphene is missing lattice atoms. Single vacancy (SV) in graphene was experimentally observed using TEM $[29,33]$ and scanning tunnelling microscope (STM) [34]. As shown in Figure 2b, one dangling bond remains toward the missing atom, which leads to the formation of a five-member ring and a nine-member ring. Such SV defect has formation energy Ef $7.5 \mathrm{eV}$ [35], which is much higher than that in many other materials (i.e. less than $3.0 \mathrm{eV}$ in most metals). Double vacancies (DV) can also be created either by the combination of two SVs or by removing two neighbouring atoms. As shown in Figure 2c, two pentagons and one octagon (DV(5-8-5) defect) appear instead of four hexagons. Simulations [35] show that the formation energy of a DV (about $8 \mathrm{eV}$ ) is of the same order for a SV. In fact, the DV(5-8-5) is not even the energetically favour one. There are also other possible ways for a graphene lattice to arrange two missing atoms. For example, one C-C bond in the octagon of DV(5-8-5) defect transforms it into three pentagons and three heptagons (DV(555-777) defect) (Figure 2d). After rotating another C-C bond, DV (555777) defect is transformed into DV(5555-6-7777) defect (Figure 2e). Multiple vacancies (MV) are created by removing more than 2 atoms. Generally, DV with even number of missing atoms are energetically favoured than that with odd number of missing atoms, where a dangling bond exists in the vicinity of defect [36].

Line defects

One-dimensional line defects have been observed in recent experimental studies [27,38,39]. Generally, these line defects have tilted boundaries separating two domains of different lattice orientations [37]. For example, a domain boundary has been observed to appear due to lattice mismatch in graphene grown on a Ni surface [27]. It is well-known that the properties of polycrystalline materials are governed by the size of grains as well as the atomic structure of grain boundaries, especially in two-dimensional graphene. In particular, grain boundaries may dominate the electronic transport in graphene [40].

\subsection{Morphology}

Generally, it is believed that long-range order does not exist according to Mermin-Wagner theorem [41]. Thus, dislocation should appear in 2D crystals at any finite temperature. 
However, over the past two decades, researchers have demonstrated that long-range order can present due to anharmonic coupling between bending and stretching modes [42,43]. As a result, $2 \mathrm{D}$ membranes can exist but tend to be rippled. The typical height of roughness fluctuation scales with sample size $L$ as $L^{\xi}$, with $\xi \approx 0.6$. Indeed, ripples in freestanding graphene were observed in recent experiments [44,45]. This kind of geometrical feature is generally referred as intrinsic morphology. In contrast, the morphology of substrate-supported graphene is regulated by the graphene-substrate interaction and is referred as extrinsic morphology. In this section, both intrinsic and extrinsic morphologies of graphene are reviewed.

\section{Intrinsic morphology}

As mentioned above, the shape of $2 \mathrm{D}$ graphene in $3 \mathrm{D}$ space is affected by its random intrinsic corrugations. The out-of-plane corrugations lead to increased strain energy but stabilize the random thermal fluctuation [46]. TEM observation indicates that suspended graphene sheets are generally not flat and the surface roughness can reach to about $1 \mathrm{~nm}$ [44]. In atomic force microscopy (AFM) measurements, nanometre-high buckles were observed in a single layer of graphene. The buckles in multi-layer graphene can penetrate from one layer to another [45]. To verify the experimental observation, simulations has been conducted to investigate the morphology of graphene and good agreement with the experiment has been achieved [47,48]. Atomistic Monte Carlo simulations also indicates that thermal fluctuation can create ripples with a ridge length around $8 \mathrm{~nm}$ [47], which is compatible with experimental findings $(5-10 \mathrm{~nm})$ [44].

Besides the effect of thermal fluctuation, sample size, aspect ratio, free edges and structural defects can also significantly affect the intrinsic morphologies of graphene. The constraint condition at the edge (e.g. periodic boundary or open edge) also affects the out-of-plane displacement [49]. As the aspect ratio $(n)$ increases, its morphology changes from planar membrane, worm-like nanoribbons, and above the critical value $\mathrm{n}_{\mathrm{cr}}=50$, the nanoribbons self-fold into nanoscrolls, forming another structural phase, as shown in Figure 3. This implies that low aspect ratio in graphene nanoribbons is preferred for electronic applications as self-folding can be avoided.
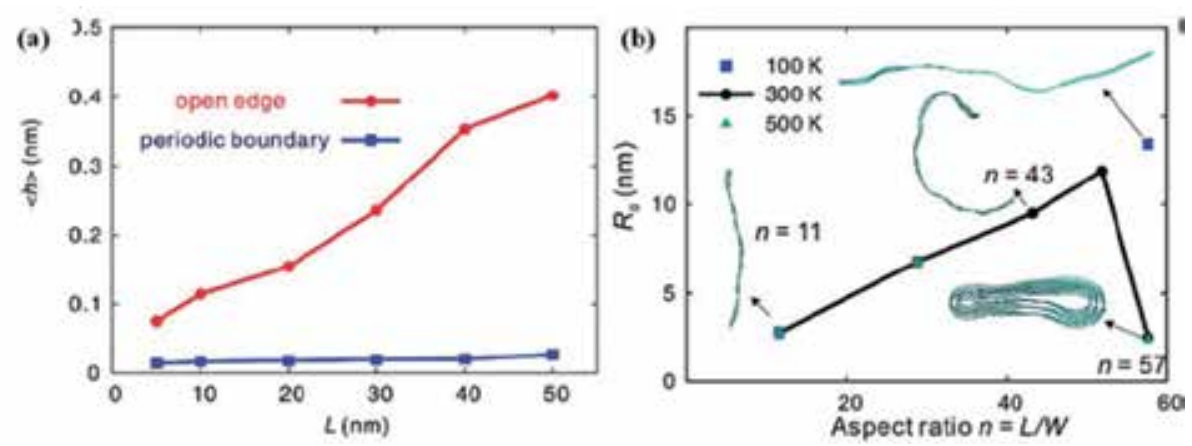

Figure 3. (a) Averaged out-of-plane displacement amplitude $<\mathrm{h}>$ of both graphene sheets with periodic boundary condition (red line) and open edges (blue line), and (b) Dependence of graphene sheet conformation on aspect ratio $\mathrm{n}=\mathrm{L} / \mathrm{W}$ (Reprinted with permission from Ref. [49] Copyright 2010 American Chemical Society). 
For finite-sized graphene with open edges, the reconstruction of free edges results in nonzero edge stress. For regular (armchair and zigzag) and reconstructed edges terminated with hydrogen ( $\mathrm{r}-\mathrm{H}$ edge), they are subjected to compressive stresses [50-52]. Corresponding to the compressive stress, out-of-plane ripples are primarily confined to the edge areas. The influence of edge stresses is more dramatic in the nanoribbons than that in the sheets. Tensile stress is often associated with reconstructed edges terminated with pentagonshexagons ring ( $r-5-6$ edge) and pentagons-heptagons ring (r-5-7 edge)[53]. Such edge stress leads to large-scale curling of graphene sheets into cylindrical surfaces with their ends arching inward. Furthermore, attached chemical groups on graphene surface can change its morphology as a result of bond transition from $\mathrm{sp}^{2}$ type to $\mathrm{sp}^{3}$ type [54].

\section{Extrinsic morphology}

Graphene is also found to appear corrugations when fabricated on a substrate, which is often referred as the intrinsic morphology of graphene. Recent experiments indicate that unwanted photo-resist residue under the graphene can lead to such random corrugations. After removal of the resist residue, atomic-resolution images of graphene show that the graphene corrugations stem from its partial conformation to its substrate [55]. In addition, it has been demonstrated that single and few-layer graphene partially follow the surface morphology of the substrates [56-58]. These experimental studies suggest that the regulated extrinsic morphology of substrate-supported graphene is essentially different from that of free-standing graphene.

In terms of energy, the extrinsic morphology of graphene regulated by the supporting substrate is governed by the interplay among three types of energetics: (1) graphene strain energy, (2) graphene-substrate interaction energy and (3) substrate strain energy [46]. As graphene conforms to a substrate, the strain energy in the graphene and substrate increases but the graphene-substrate interaction energy decreases. By minimizing the total energy of the system, the equilibrium extrinsic morphology can be determined. In practice, the underlying substrate can be patterned with different features such as nanowires (1D), nanotubes (1D) or nanoparticles (0D). Graphene on a patterned substrate will conform to a regular extrinsic morphology.

For the substrate with 1D periodic sinusoidal surface, the regulated graphene is expected to have a similar morphology that can be described by

$$
w_{g}(x)=A_{g} \cos \left(\frac{2 \pi x}{\lambda}\right), \quad w_{s}(x)=A_{s} \cos \left(\frac{2 \pi x}{\lambda}\right)-h
$$

where $\lambda$ is the wavelength; $h$ is the distance between the middle planes of the graphene and the substrate surface; $\mathrm{Ag}$ and $\mathrm{As}_{\mathrm{s}}$ are the amplitudes of the graphene morphology and the substrate surface, respectively. The graphene-substrate interaction energy is given by summing up all interaction energies between carbon and the substrate atoms via van der Waals force, i.e., [59]

$$
E_{\text {int }}=\int_{S} \int_{V_{S}} V(r) \rho_{S} d V_{S} \rho_{C} d S
$$


The strain energy of graphene sheet is given by

$$
E_{g}=\frac{1}{\lambda / 2} \int_{0}^{\lambda / 2} D / 2\left(\partial^{2} w_{g} / \partial x^{2}\right)^{2} d x=\frac{4 \pi^{4} D A_{g}^{2}}{\lambda^{4}}
$$
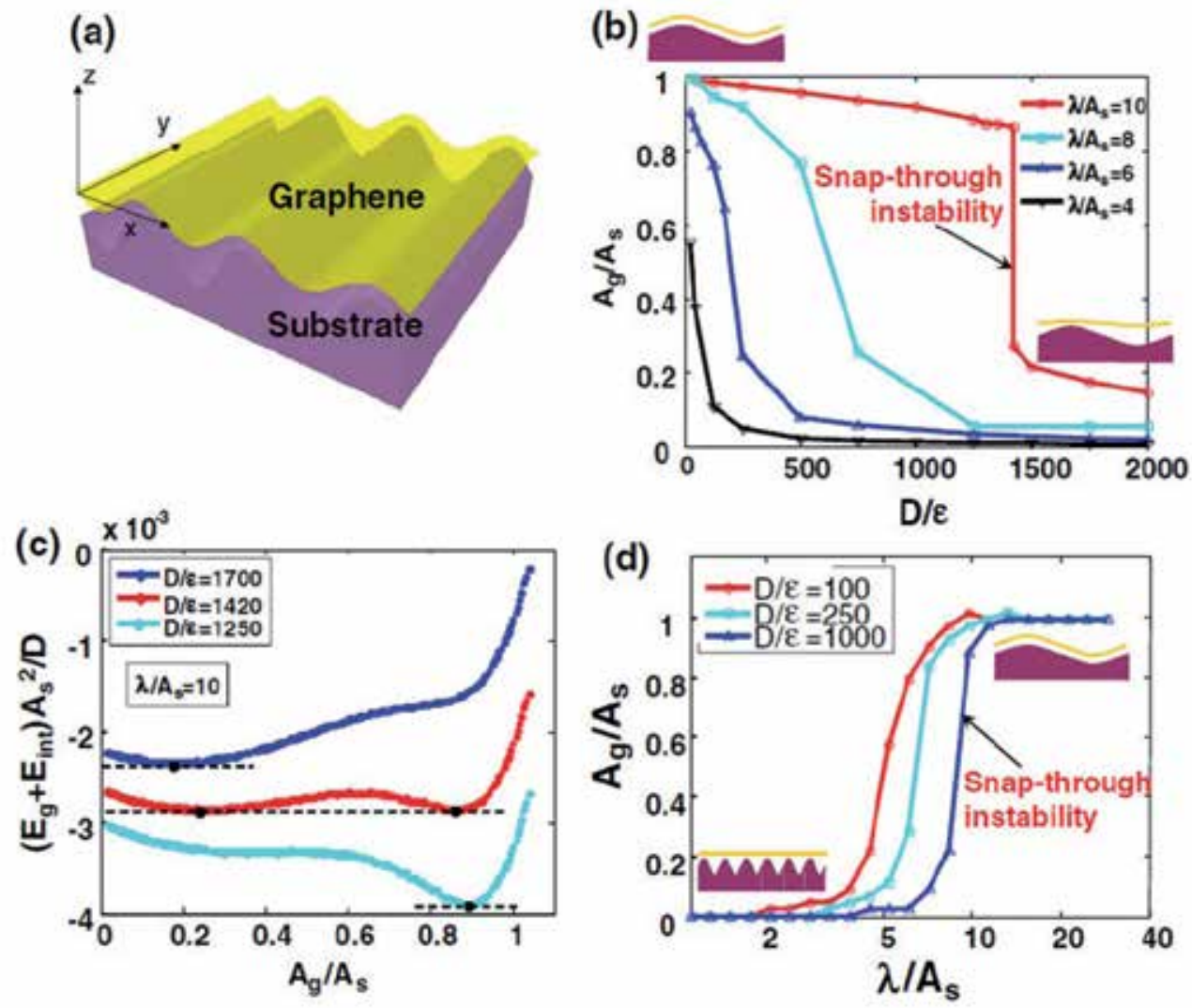

Figure 4. (a) Schematics of a graphene sheet on the corrugated substrate. (b) and (d) The normalized equilibrium amplitude of the graphene corrugation $A_{g} / A_{s}$ as a function of $D / \varepsilon$ for various $\lambda / A_{s}$. (c) Normalized total energy as a function of $\mathrm{A}_{\mathrm{g}} / \mathrm{A}_{s}$ for various $\mathrm{D} / \varepsilon$ (Reprinted with permission from Ref. [59] Copyright 2010 IOP Publishing).

In terms of the minimum potential energy, there exists a minimum value of $\left(E_{g}+E_{s}\right)$ where $\mathrm{Ag}$ and $h$ define the equilibrium morphology of the graphene on the substrate. Figure 4 shows the normalized equilibrium amplitude of the graphene corrugation $\mathrm{Ag}_{\mathrm{g}} / \mathrm{As}_{\mathrm{s}}$ as a function of $\mathrm{D} / \varepsilon$ for various $\lambda / \mathrm{A}_{\mathrm{s}}$. By analysing given substrate surface roughness $\left(\lambda / \mathrm{A}_{s}\right)$ and graphene-substrate interfacial bonding $(D / \varepsilon)$ respectively, it was found that there is a sharp transition in the normalized equilibrium amplitude of the graphene corrugation. Such snapthrough instability of the extrinsic morphology of graphene on the substrate can be understood by the energetic parameter shown in Figure 4c. Besides the interfacial bonding energy, the substrate surface roughness can also influence the extrinsic morphology graphene, as 
shown in Figure $4 \mathrm{~d}$. Similar to the effect of substrate on the morphology of mounted graphene, $0 \mathrm{D}$ and $1 \mathrm{D}$ patterned nanoscale array can determine the equilibrium extrinsic morphology of graphene on the substrate $[60,61]$.

\section{Graphene-polymer nanocomposites}

Polymer matrix nanocomposites with graphene and its derivatives as fillers have shown a great potential for various important applications, such as electronics, green energy, aerospace and automotiveindustries. As mentioned before, 2-D graphene possesses better electrical, mechanical and thermal properties as well as other unique features, including higher aspect ratio and larger specific surface area as compared to other reinforcements such as CNTs and carbon and Kevlar fibres. It is reasonable to expect some significant improvement in a range of properties in the composites with graphene as nanofiller. The recent success in synthesis of large amount of graphene further promotes the development of graphene based composite and hybrid materials.

\subsection{Synthesis of graphene-polymer nanocomposites}

Similar to processing other polymer matrix composites, solution blending, melt mixing and in-situ polymerization are the commonly used approaches to produce graphene-polymer composites.

\section{Solution blending}

Solution blending is the most popular technique to fabricate polymer-based composites in that the polymer is readily soluble in common aqueous and organic solvents, such as water, acetone, dimethylformamide (DMF), chloroform, dichloromethane (DCM) and toluene. This technique includes the solubilisation of the polymer in suitable solvents, and mixing with the solution of the dispersed suspension of graphene or graphene oxide (GO) platelets. The polymers including PS [2], polycarbonate [62], polyacrylamide, polyimides [63] and poly(methyl methacrylate) (PMMA) [64] have been successfully mixed with GO in solution blending where the GO surface was usually functionalized using isocyanates, alkylamine and alkyl-chlorosilanes to enhance its dispensability in organic solvents. In addition, the facile production of aqueous GO platelet suspensions via sonication makes this technique particularly appealing for water-soluble polymers such as poly(vinyl alcohol) (PVA) [65] and poly(allylamine), composites of which can be produced via simple filtration [65].

For solution blending methods, the extent of exfoliation of GO platelets usually governs the dispersion of GO platelets in the composite. Thus, solution blending offers a promising approach to dispersing GO platelets into certain polymer matrix. Specifically, small molecule functionalization and grafting-to/from methods have been reported to achieve stable GO platelet suspensions prior to mixing with polymer matrix. Some techniques, including Lyophilizations methods [66], phase transfer techniques [67], and surfactants [68] have been employed to facilitate solution blending of graphene-polymer nanocomposites. Nevertheless, surfactants may deteriorate composite properties. For example, the matrix-filler interfa- 
cial thermal resistance in SWNT/polymer nanocomposites was increased by employing surfactants [69].

Melt mixing

Melt mixing technique utilizes a high temperature and shear forces to disperse the fillers in the polymer matrix. This process prevents the use of toxic solvents. Furthermore, compared with solution blending, melt mixing is often believed to be more cost effective. For graphene-polymer nanocomposites, the high temperature liquefies the polymer phase and allows easy dispersion or intercalation of GO platelets. However, the melt mixing is less effective in dispersing graphene sheets compared to solvent blending or in situ polymerization due to the increased viscosity at a high filler loading. The process can be applicable to both polar and non-polar polymers. Various graphene-based nanocomposites such as, exfoliated graphite-PMMA, graphene-polypropylene (PP), GO-poly (ethylene-2, 6-naphthalate) (PEN) and graphene-polycarbonate, can be fabricated by this technique. Even though the utility of graphene nanofiller is constrained by the low throughput of chemically reduced graphene in the melt mixing process, graphene production in bulk quantity in thermal reduction can be an appropriate choice for industrial scale production. However, the loss of the functional group in thermal reduction may be an obstacle in obtaining homogeneous dispersion in polymeric matrix melts especially in non-polar polymers.

In situ polymerization

This fabrication technique starts with mixing of filler in neat monomer (or multiple monomers), followed by polymerization in the presence of the dispersed filler. Then, precipitation/extraction or solution casting follows to generate samples for testing. In situ polymerization methods have produced composites with covalent crosslink between the matrix and filler. In addition, in situ polymerization has also produced non-covalent composites of a variety of polymers, such as poly (ethylene), PMMA and poly (pyrrole).

Unlike solution blending or melt mixing techniques, in situ polymerization technique achieves a high level of dispersion of graphene-based filler without prior exfoliation. It has been reported that monomer is intercalated between the layers of graphite or GO, followed by polymerization to separate the layers. This technique has been widely investigated for graphite or GO-derived polymer nanocomposites. For example, graphite can be intercalated by an alkali metal and a monomer, followed by polymerization initiated by thenegatively charged graphene sheets [70]. Although the polymerization may exfoliate the graphite nanoplatelets (GNPs), single-layer graphene platelets were not observed. TEM observation showed $3.6 \mathrm{~nm}$ thickness of graphene platelets with relatively low aspect ratio of about 30 dispersed in the PE matrix [71].

\subsection{Fundamental properties}

\section{Mechanical properties}

Higher mechanical properties of graphene sheets have attracted increasing attention worldwide. Similar to other composites, the mechanical properties depend on the 
concentration, aspect ratio and distribution of the nanofiller in the matrix and the interface bonding. For example, at a nanofiller weight fraction of $0.1 \pm 0.002 \%$, the graphene-epoxy nanocompositesshow noticeable enhancement in the mechanical properties [3-5]. The Young's modulus, fracture strength of the nanocomposites are about $\sim 31 \%$ and $\sim 40 \%$ greater than the pristine epoxy, more efficient than the composites reinforced by multi-walled CNTs.The increase in fracture strength of the nanocomposites (graphene-PS) with $0.9 \mathrm{wt} \%$ graphene sheets is attributed to effective load transfer between the graphene layers and polymer matrix [72].

Besides simple reinforcing effects (Young's modulus and fracture strength), improvements in fracture toughness, fatigue strength and buckling resistance have also been reported in graphene-polymer nanocomposites[3,5,73-75]. For example, in situ polymerized grapheneepoxy nanocomposites show much higher buckling strength, fracture energy and fatigue strength than single- or multi-walled carbon nanotube-epoxy nanocomposites.However, the underlying strengthening and toughening mechanisms are still not well understood. Several factors, such as interfacial adhesion, spatial distribution and alignment of graphene nanofiller are considered to be crucial for effective reinforcement in the nanocomposites. Benefiting from improved interfacial adhesion, $76 \%$ and $62 \%$ the increase in elastic modulus and strength were achieved in the $0.7 \mathrm{wt} \%$ GO-PVA nanocomposite, respectively [76]. It was reported that the elastic modulus and fracture strength of Nylon- 6 can be greatly improved by adding only $0.1 \mathrm{wt} \% \mathrm{GO}$ [77]. The covalent bonding formed between the filler and matrix is attributed to the improved mechanical properties in the epoxy and polyurethane with GO-derived fillers [78-80]. Polymer nanocomposites with low loadings of functionalized graphene sheets (FGS) were reported to have a large shift in the glass transition temperature $\mathrm{T}_{\mathrm{g}}$ [64]. In FGS-poly (acrylonitrile) nanocomposite, the shift in $\mathrm{T}_{\mathrm{g}}$ is over $40^{\circ} \mathrm{C}$ when adding 1 $w \mathrm{t} \%$ FGS filler loading. This behaviour can be attributed to the altered mobility of polymer chains at the filler-matrix interfaces $[81,82]$. Generally, a weak filler-matrix interface can constrain the chain mobility and thus increase the $\mathrm{T}_{\mathrm{g}}$.

\section{Electrical properties}

One of the most fascinating properties of graphene is its excellent electrical conductivity. When used as fillers in an insulating polymer matrix, conductive graphene may greatly improve the electrical conductivity of the composite. When the added graphene loading exceeds the electrical percolation threshold, a conductive network is expected in the polymer matrix. The conductivity $\sigma_{c}$ as a function of filler loading can be described using a simple power-law expression, i.e.,

$$
\sigma_{c}=\sigma_{f}\left(\phi-\phi_{c}\right)^{t}
$$

where $\phi$ is the filler volume fraction; $\phi_{\mathrm{c}}$ is the percolation threshold; $\sigma_{\mathrm{f}}$ is the filler conductivity, and $t$ is a scaling exponent. The overall electrical performance is dependent on the processing and dispersion, aggregation and alignment of the filler. The intrinsic characteristics of the filler such as aspect ratio and morphology also play a role in dictating the conductivity. The inter-sheet junction formed may affect the conductivity as well. 
A high level of dispersion may not necessarily promote the onset of electrical percolation [83]. The polymer reason is a thin layer of polymer may coat on the well-dispersed fillers and prevent direct the formation of a conductive network. In fact, the lowest electrical percolation threshold for graphene-polymer nanocomposites was reported for the nanocomposite with heterogeneously dispersed graphene (about 0.15 wt \%) [84]. For example, compression moulded polycarbonate and GO-polyester nanocomposites with aligned platelets showed an increased percolation threshold that isabout twice as high as the annealed samples with randomly oriented platelets $[85,86]$. Therefore, slight aggregation of the filler may lower the percolation threshold and improve the electrical conductivity of these nanocomposites [87]. Both theoretical analysis [88,89] and experiments demonstrated that the electrical conductivity of the nanocomposites correlates strongly with the aspect ratio of the platelets and higher aspect ratio leads to a higher conductivity. On the other hand, wrinkled, folded, or other non-flat morphologies may increase the electrical percolation threshold [90].

\section{Thermal properties}

The exceptional thermal properties of graphene have been used to improve the thermal stability and conductivity in nanocomposites. The 2D geometry of graphene-base materials may offer lower interfacial thermal resistance and thus provide higher thermal conductivity in the nanocomposites. The 2D geometry of graphene also introduces anisotropy into the thermal conductivity of graphene-polymer nanocomposites. For instance, the measured in-plane thermal conductivity is as much as ten times higher than the cross-plane conductivity [91]. Generally, thermal conductivity in nanocomposites can be analysed by percolation theory. Since phonons are the major mode of thermal conduction in amorphous polymers, covalent bonding between the filler and matrix can reduce phonon scattering at the filler-matrix interface, and subsequently enhance the thermal conductivity of nanocomposites [92]. In recent research, significant enhancements in thermal conductivity have been achieved in graphene-epoxy nanocomposites, with conductivities increasing to $3 \sim 6 \mathrm{~W} / \mathrm{mK}$ from $0.2 \mathrm{~W} / \mathrm{mK}$ for neat epoxy. However, such significant improvement often needs a high filler loading, about $20 \mathrm{wt} \%$ and even higher. Some research has also reported improvement in thermal stability of graphene-polymer nanocomposites [93,94]. Furthermore, the negative coefficient of thermal expansion (CTE) [95] and high surface of graphene can lower the CTE of polymer matrix [96]. For instance, the CTE of a GOepoxy nanocomposite with $5 \%$ filler loading decreases by nearly $32 \%$ for temperature below the polymer glass transition Temperature $\left(\mathrm{T}_{\mathrm{g}}\right)$ [97].

\section{Structure-property relationship}

\subsection{Microstructure effect}

TEM and wide-angle X-ray scattering (WAXS), are often utilized to examine the dispersion of graphene fillers in composites. Sometimes, the morphological features of dispersed fillers can be missed out due to the tiny platelet thickness and intensity scattering. Recently, smallangle X-ray scattering (SAXS) and ultra-small-angle X-ray scattering (USAXS) have been increasingly used to examine the aggregation of filler at large material length scale. 


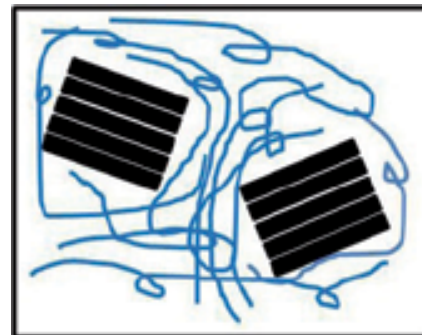

a

Separated

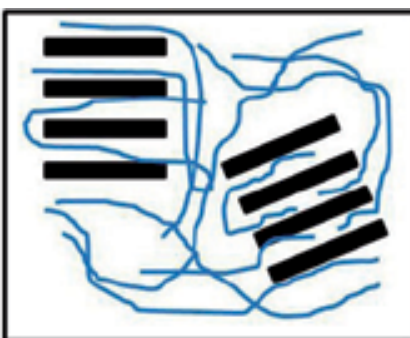

b Intercalated

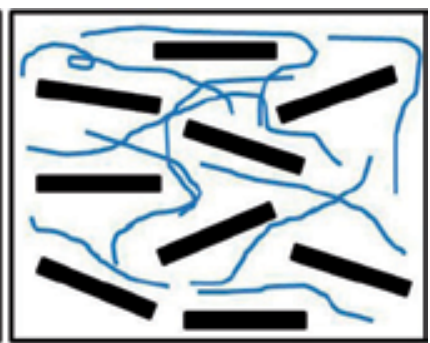

c

Exfoliated

Figure 5. Filler dispersion in graphene-based nanocomposites: (a) separated, (b) intercalated, and (c) exfoliated phases.

In GO-derived and GNP-polymer nanocomposites, the fillers can exist in different forms such as stacked, intercalated or exfoliated, as shown in Figure 5. As compared with the separated phase, increased interlayer spacing (in the order of few nanometres) can be achieved in the intercalated structures. In the exfoliated structure, exfoliated platelets have the largest interfacial contact with the polymer matrix, generally ideal for improvement of various properties of the composites. Due to increased interaction with the polymer matrix, exfoliated phase normally has a curved shape when embedded into a polymer matrix. The rumpled shape of filler then can result in mechanical interlocking, which is one the possible strengthening mechanisms. However, low modulus was also observed in the composite with wrinkled platelets [6]. The material processing methods can also influence the microstructure in nanocomposites. Randomly oriented exfoliated platelets can be achieved using solution blending or in situ polymerization [78]. Platelet restacking or incomplete exfoliation can also result in lower modulus due to decreased aspect ratio.

\subsection{Interfacial behaviour}

Although graphene-polymer nanocomposites exhibit excellent mechanical properties, the underlying strengthening and toughening mechanisms have not been well understood. Generally, it is believed that interfacial adhesion plays a key role in determining the improvements in mechanical properties of graphene-polymer nanocomposites. Low interfacial adhesion may lead to lower load transfer between the filler and matrix. Both AFM and Raman spectroscopy $[98,99]$ can be utilized to measure the graphene-polymer interfacial adhesion. Raman spectra and their Raman bands were found to shift with stress, which enables stress-transfer to be monitored between the matrix and reinforcing phase. Moreover, a universal calibration has been established between the rate of shift of the $G^{\prime}$ band with strain[100]. Recently, interfacial shear stress [98] and effective Young's modulus [101] were successfully determined using Raman spectroscopy. The relationship between matrix strain $\varepsilon$ mand strain in the graphene flake\&fcan be described by 


$$
\begin{gathered}
\varepsilon_{f}=\varepsilon_{m}\left[1-\frac{\cosh \left(n s \frac{x}{l}\right)}{\cosh (n s / 2)}\right] \\
n=\sqrt{\frac{2 G_{m}}{E_{f}}}
\end{gathered}
$$

where $n$ and $\mathrm{Gm}$ is the matrix shear modulus, Ef is the Young's modulus of the graphene filler. The variation of shear stress $\tau$, at the graphene-polymer interface is given by

$$
\tau_{i}=n E_{f} \varepsilon_{m} \frac{\sinh \left(n s \frac{l}{x}\right)}{\cosh (n s / 2)}
$$

Corresponding to $\varepsilon \mathrm{m}=0.4 \%$, there is a good agreement between the measured and predicted (Equation 5) variation of fibre strain with position on the monolayer, validating the use of the shear lag analysis. At $\varepsilon_{m}=0.6 \%$, however, the interface failure occurs between the filler and polymer and stress transfer is taking place through interfacial friction. The interfacial shear stress (ISS) between graphene and polymer is determined in the range of $\sim 0.3-0.8 \mathrm{MPa}$, much lower than that between CNTs and polymer ( 20-40 MPa). The low ISS was attributed to the poor interface adhesion.

Raman spectroscopy analysis [98,102] confirmed the reinforcement effect of graphene and its dependence with crystallographic orientations and the layer number of graphene. It is demonstrated that monolayer or bilayer graphene has better load transfer than tri-layer or multi-layer [103]. Without compromising an even dispersion, higher filler loading is easy to achieve with multilayer graphene. There is therefore a balance in design of graphenepolymer nanocomposites between a higher filler loading and decreased load transfer as the number of layers in graphene filler increases. Raman G-band analysis suggested that load transfer at the GPL-PDMS interface is more effective in comparison to that along single wall carbon nanotube/PDMS interface [7]. In terms of loading mode, it is interesting to note that the GPL fillers went into compression under tensile loading and vice versa. Up to now, interface load transfer, mechanical interlocking caused by wrinkled surface and defects in graphene are main factor in controlling the reinforcement mechanisms. Due to the complex interactions between graphene, functional groups attached and the polymer, controversial results are often observed in the load transfer analysis. Further theoretical and numerical analysis is much needed.

\section{Conclusion}

In summary, the interesting properties of graphene and its composites mentioned above have led to the exploration of numerous applications such as transistors, chemical and biosensors, energy storage devices, nanoelectro-mechanical systems and others, just as the research 
community has done with carbon nanotubes previously. The past decade has witnessed the rapid growth of carbon-based nanotechnology. Further research in the area will assist the development of next generation graphene based composites and hybrid materials.

\section{Author details}

Mingchao Wang, Cheng Yan and Lin Ma

School of Chemistry, Physics and Mechanical Engineering, Science and Engineering Faculty,

Queensland University of Technology, Brisbane, Australia

\section{References}

[1] Novoselov KS, Geim AK, Morozov SV, Jiang D, Zhang Y, Dubonos SV, Grigorieva IV, Firsov AA, Science. 306 (2004).

http://www.sciencemag.org/content/306/5696/666.abstract.

[2] Stankovich S, Dikin DA, Dommett GHB, Kohlhaas KM, Zimney EJ, Stach EA, Piner RD, Nguyen ST, Ruoff RS, Nature. 442 (2006).

http://dx.doi.org/10.1038/nature04969.

[3] Rafiee MA, Rafiee J, Wang Z, Song H, Yu Z-Z, Koratkar N, ACS Nano. 3 (2009). http://dx.doi.org/10.1021/nn9010472.

[4] Rafiee MA, Lu W, Thomas AV, Zandiatashbar A, Rafiee J, Tour JM, Koratkar NA, ACS Nano. 4 (2010). http://dx.doi.org/10.1021/nn102529n.

[5] Rafiee MA, Rafiee J, Srivastava I, Wang Z, Song HH, Yu ZZ, Koratkar N, Small. 6 (2010). http://dx.doi.org/10.1002/smll.200901480.

[6] Wakabayashi K, Pierre C, Dikin DA, Ruoff RS, Ramanathan T, Brinson LC, Torkelson JM, Macromolecules. 41 (2008). http://dx.doi.org/10.1021/ma071687b.

[7] Srivastava I, Mehta RJ, Yu Z-Z, Schadler L, Koratkar N, Appl. Phys. Lett. 98 (2011). http://link.aip.org/link/?APL/98/063102/1.

[8] Lee C, Wei X, Kysar JW, Hone J, Science. 321 (2008). http://www.sciencemag.org/content/321/5887/385.abstract.

[9] Scharfenberg S, Rocklin DZ, Chialvo C, Weaver RL, Goldbart PM, Mason N, Appl. Phys. Lett. 98 (2011). http://link.aip.org/link/?APL/98/091908/1.

[10] Zhao H, Min K, Aluru NR, Nano Lett. 9 (2009). http://dx.doi.org/10.1021/n1901448z.

[11] Wang MC, Yan C, Ma L, Hu N, Chen MW, Comp. Mater. Sci. 54 (2012). http://www.sciencedirect.com/science/article/pii/S0927025611006033.

[12] Zhao H, Aluru NR, J. Appl. Phys. 108 (2010). http://link.aip.org/link/?JAP/108/064321/1.

[13] Tsoukleri G, Parthenios J, Papagelis K, Jalil R, Ferrari AC, Geim AK, Novoselov KS, Galiotis C, Small. 5 (2009). 
http://dx.doi.org/10.1002/smll.200900802.

[14] Lee C, Wei X, Li Q, Carpick R, Kysar JW, Hone J, physica status solidi (b). 246 (2009). http://dx.doi.org/10.1002/pssb.200982329.

[15] Koenig SP, Boddeti NG, Dunn ML, Bunch JS, Nat Nano. 6 (2011). http://dx.doi.org/10.1038/nnano.2011.123.

[16] Zhang Y, Tan Y-W, Stormer HL, Kim P, Nature. 438 (2005). http://dx.doi.org/10.1038/nature04235.

[17] Novoselov KS, Jiang Z, Zhang Y, Morozov SV, Stormer HL, Zeitler U, Maan JC, Boebinger GS, Kim P, Geim AK, Science. 315 (2007).

http://www.sciencemag.org/content/315/5817/1379.abstract.

[18] Geim AK, Novoselov KS, Nat. Mater. 6 (2007). http://dx.doi.org/10.1038/nmat1849.

[19] Somani PR, Somani SP, Umeno M, Chem. Phys. Lett. 430 (2006). http://www.sciencedirect.com/science/article/pii/S0009261406009018.

[20] Bae S, Kim H, Lee Y, Xu X, Park J-S, Zheng Y, Balakrishnan J, Lei T, Ri Kim H, Song YI, Kim Y-J, Kim KS, Ozyilmaz B, Ahn J-H, Hong BH, Iijima S, Nat Nano. 5 (2010). http://dx.doi.org/10.1038/nnano.2010.132.

[21] Kim KS, Zhao Y, Jang H, Lee SY, Kim JM, Kim KS, Ahn J-H, Kim P, Choi J-Y, Hong BH, Nature. 457 (2009). http://dx.doi.org/10.1038/nature07719.

[22] Berger C, Song Z, Li T, Li X, Ogbazghi AY, Feng R, Dai Z, Marchenkov AN, Conrad EH, First PN, de Heer WA, The Journal of Physical Chemistry B. 108 (2004). http://dx.doi.org/10.1021/jp040650f.

[23] Berger C, Song Z, Li X, Wu X, Brown N, Naud C, Mayou D, Li T, Hass J, Marchenkov AN, Conrad EH, First PN, de Heer WA, Science. 312 (2006). http://www.sciencemag.org/content/312/5777/1191.abstract.

[24] Zheng Q, Geng Y, Wang S, Li Z, Kim J-K, Carbon. 48 (2010). http://www.sciencedirect.com/science/article/B6TWD-50NBNTH1/2/f9d91c8358333a00160e505b972e0cd3.

[25] Pei QX, Zhang YW, Shenoy VB, Carbon. 48 (2010). http://www.sciencedirect.com/science/article/B6TWD-4XNF8DY3/2/d59770660b4ae737f9385b89c3439a0c.

[26] OuYang F, Huang B, Li Z, Xiao J, Wang H, Xu H, J. Phys. Chem. C. 112 (2008). http://dx.doi.org/10.1021/jp710547x.

[27] Lahiri J, Lin Y, Bozkurt P, Oleynik II, Batzill M, Nat Nano. 5 (2010). http://dx.doi.org/10.1038/nnano.2010.53.

[28] Stone AJ, Wales DJ, Chem. Phys. Lett. 128 (1986). http://www.sciencedirect.com/science/article/pii/0009261486806613.

[29] Meyer JC, Kisielowski C, Erni R, Rossell MD, Crommie MF, Zettl A, Nano Lett. 8 (2008). http://dx.doi.org/10.1021/n1801386m.

[30] Li L, Reich S, Robertson J, Phys. Rev. B. 72 (2005). http://link.aps.org/doi/10.1103/PhysRevB.72.184109.

[31] Ma J, Alfè D, Michaelides A, Wang E, Phys. Rev. B. 80 (2009). 
http://link.aps.org/doi/10.1103/PhysRevB.80.033407.

[32] Ertekin E, Chrzan DC, Daw MS, Phys. Rev. B. 79 (2009).

http://link.aps.org/doi/10.1103/PhysRevB.79.155421.

[33] Gass MH, Bangert U, Bleloch AL, Wang P, Nair RR, Geim AK, Nat Nano. 3 (2008). http://dx.doi.org/10.1038/nnano.2008.280.

[34] Ugeda MM, Brihuega I, Guinea F, Gómez-Rodríguez JM, Phys. Rev. Lett. 104 (2010). http://link.aps.org/doi/10.1103/PhysRevLett.104.096804.

[35] Krasheninnikov AV, Lehtinen PO, Foster AS, Nieminen RM, Chem. Phys. Lett. 418 (2006). http://www.sciencedirect.com/science/article/pii/S0009261405016544.

[36] Kotakoski J, Krasheninnikov AV, Nordlund K, Phys. Rev. B. 74 (2006). http://link.aps.org/doi/10.1103/PhysRevB.74.245420.

[37] Banhart F, Kotakoski J, Krasheninnikov AV, ACS Nano. 5 (2010). http://dx.doi.org/10.1021/nn102598m.

[38] Coraux J, N’Diaye AT, Busse C, Michely T, Nano Lett. 8 (2008). http://dx.doi.org/10.1021/nl0728874.

[39] Cervenka J, Katsnelson MI, Flipse CFJ, Nat Phys. 5 (2009). http://dx.doi.org/10.1038/nphys1399.

[40] Yazyev OV, Louie SG, Phys. Rev. B. 81 (2010). http://link.aps.org/doi/10.1103/PhysRevB.81.195420.

[41] Mermin ND, Physical Review. 176 (1968). http://link.aps.org/doi/10.1103/PhysRev.176.250.

[42] Nelson DR, Peliti L, Journal De Physique. 48 (1987). http://hal.archives-ouvertes.fr/jpa-00210530/en/.

[43] Le Doussal P, Radzihovsky L, Phys. Rev. Lett. 69 (1992). http://link.aps.org/doi/10.1103/PhysRevLett.69.1209.

[44] Meyer JC, Geim AK, Katsnelson MI, Novoselov KS, Booth TJ, Roth S, Nature. 446 (2007). http://dx.doi.org/10.1038/nature05545.

[45] Li Z, Cheng Z, Wang R, Li Q, Fang Y, Nano Lett. 9 (2009). http://dx.doi.org/10.1021/n1901815u.

[46] Teng L, Model. Simul. Mater. Sc. 19 (2011). http://stacks.iop.org/0965-0393/19/i=5/a=054005.

[47] Fasolino A, Los JH, Katsnelson MI, Nature Materials. 6 (2007). http://www.nature.com/nmat/journal/v6/n11/full/nmat2011.html.

[48] Duan WH, Gong K, Wang Q, Carbon. 49 (2011). http://www.sciencedirect.com/science/article/pii/S0008622311002247.

[49] Xu Z, Buehler MJ, ACS Nano. 4 (2010). http://dx.doi.org/10.1021/nn100575k.

[50] Shenoy VB, Reddy CD, Ramasubramaniam A, Zhang YW, Phys. Rev. Lett. 101 (2008). http://link.aps.org/doi/10.1103/PhysRevLett.101.245501.

[51] Huang B, Liu M, Su N, Wu J, Duan W, Gu B-l, Liu F, Phys. Rev. Lett. 102 (2009). http://link.aps.org/doi/10.1103/PhysRevLett.102.166404.

[52] Bets KV, Yakobson BI, Nano Research. 2 (2009). 
http://www.nanoarchive.org/8385/.

[53] Reddy CD, Ramasubramaniam A, Shenoy VB, Zhang Y-W, Appl. Phys. Lett. 94 (2009). http://link.aip.org/link/?APL/94/101904/1

http://dx.doi.org/10.1063/1.3094878.

[54] Pei QX, Zhang YW, Shenoy VB, Nanotechnology. 21 (2010). http://dx.doi.org/10.1088/0957-4484/21/11/115709.

[55] Ishigami M, Chen JH, Cullen WG, Fuhrer MS, Williams ED, Nano Lett. 7 (2007). http://dx.doi.org/10.1021/n1070613a.

[56] Stolyarova E, Rim KT, Ryu S, Maultzsch J, Kim P, Brus LE, Heinz TF, Hybertsen MS, Flynn GW, P. Natl. Acad. Sci. USA. 104 (2007). http://www.pnas.org/content/104/22/9209.abstract.

[57] Stoberl U, Wurstbauer U, Wegscheider W, Weiss D, Eroms J, Appl. Phys. Lett. 93 (2008). http://dx.doi.org/10.1063/1.2968310.

[58] Geringer V, Liebmann M, Echtermeyer T, Runte S, Schmidt M, Rückamp R, Lemme MC, Morgenstern M, Phys. Rev. Lett. 102 (2009). http://link.aps.org/doi/10.1103/PhysRevLett.102.076102.

[59] Teng L, Zhao Z, J. Phys. D Appl. Phys. 43 (2010). http://stacks.iop.org/0022-3727/43/i=7/a=075303.

[60] Zhang Z, Li T, J. Appl. Phys. 107 (2010). http://dx.doi.org/10.1063/1.3427551.

[61] Zhang Z, Li T, J. Nanomater. 2011 (2011). http://dx.doi.org/10.1155/2011/374018.

[62] Higginbotham AL, Lomeda JR, Morgan AB, Tour JM, ACS Appl. Mater. Inter. 1 (2009). http://dx.doi.org/10.1021/am900419m.

[63] Chen D, Zhu H, Liu T, ACS Appl. Mater. Inter. 2 (2010). http://dx.doi.org/10.1021/am1008437.

[64] RamanathanT, Abdala AA, StankovichS, Dikin DA, Herrera Alonso M, Piner RD, Adamson DH, Schniepp HC, ChenX, Ruoff RS, Nguyen ST, Aksay IA, Prud'Homme RK, Brinson LC, Nat Nano. 3 (2008). http://dx.doi.org/10.1038/nnano.2008.96.

[65] Xu Y, Hong W, Bai H, Li C, Shi G, Carbon. 47 (2009). http://www.sciencedirect.com/science/article/pii/S0008622309005296.

[66] Cao Y, Feng J, Wu P, Carbon. 48 (2010). http://www.sciencedirect.com/science/article/pii/S0008622310004549.

[67] Wei T, Luo G, Fan Z, Zheng C, Yan J, Yao C, Li W, Zhang C, Carbon. 47 (2009). http://www.sciencedirect.com/science/article/pii/S0008622309002565.

[68] Lee HB, Raghu AV, Yoon KS, Jeong HM, J. Macromol. Sci. B. 49 (2010). http://www.tandfonline.com/doi/abs/10.1080/00222341003603701.

[69] Bryning MB, Milkie DE, Islam MF, Kikkawa JM, Yodh AG, Appl. Phys. Lett. 87 (2005). http://dx.doi.org/10.1063/1.2103398.

[70] Shioyama H, Synthetic Metals. 114 (2000). http://www.sciencedirect.com/science/article/pii/S0379677900002228.

[71] Fim FdC, Guterres JM, Basso NRS, Galland GB, J. Polym. Sci. Pol. Chem. 48 (2010). 
http://dx.doi.org/10.1002/pola.23822.

[72] Fang M, Wang K, Lu H, Yang Y, Nutt S, J. Mater. Chem. 19 (2009). http://dx.doi.org/10.1039/B908220D.

[73] Rafiee MA, Rafiee J, Yu ZZ, Koratkar N, Appl. Phys. Lett. 95 (2009). http://apl.aip.org/applab/v95/i22/p223103_s1?isAuthorized=no.

[74] Yavari F, Rafiee MA, Rafiee J, Yu ZZ, Koratkar N, ACS Appl. Mater. Inter. 2 (2010). http://dx.doi.org/10.1021/am100728r.

[75] Rafiq R, Cai D, Jin J, Song M, Carbon. 48 (2010). http://www.sciencedirect.com/science/article/pii/S0008622310005476.

[76] Liang J, Huang Y, Zhang L, Wang Y, Ma Y, Guo T, Chen Y, Adv. Funct. Mater. 19 (2009). http://dx.doi.org/10.1002/adfm.200801776.

[77] Xu Z, Gao C, Macromolecules. 43 (2010). http://dx.doi.org/10.1021/ma1009337.

[78] Kim H, Miura Y, Macosko CW, Chem. Mater. 22 (2010). http://dx.doi.org/10.1021/cm100477v.

[79] Lee YR, Raghu AV, Jeong HM, Kim BK, Macromol. Chem. Physic. 210 (2009). http://dx.doi.org/10.1002/macp.200900157.

[80] Miller SG, Bauer JL, Maryanski MJ, Heimann PJ, Barlow JP, Gosau J-M, Allred RE, Compos. Sci. Technol. 70 (2010). http://www.sciencedirect.com/science/article/pii/S0266353810000825.

[81] Bansal A, Yang H, Li C, Cho K, Benicewicz BC, Kumar SK, Schadler LS, Nat. Mater. 4 (2005). http://dx.doi.org/10.1038/nmat1447.

[82] Priestley RD, Ellison CJ, Broadbelt LJ, Torkelson JM, Science. 309 (2005). http://www.sciencemag.org/content/309/5733/456.abstract.

[83] Schaefer DW, Justice RS, Macromolecules. 40 (2007). http://dx.doi.org/10.1021/ma070356w.

[84] Pang H, Chen T, Zhang G, Zeng B, Li Z-M, Mater. Lett. 64 (2010). http://www.sciencedirect.com/science/article/pii/S0167577X10005379.

[85] Kim H, Macosko CW, Macromolecules. 41 (2008). http://dx.doi.org/10.1021/ma702385h.

[86] Kim H, Macosko CW, Polymer. 50 (2009). http://www.sciencedirect.com/science/article/pii/S0032386109004558.

[87] Bauhofer W, Kovacs JZ, Compos. Sci. Technol. 69 (2009). http://www.sciencedirect.com/science/article/pii/S026635380800239X.

[88] Hicks J, Behnam A, Ural A, Appl. Phys. Lett. 95 (2009). http://dx.doi.org/10.1063/1.3267079.

[89] Li J, Kim J-K, Compos. Sci. Technol. 67 (2007). http://www.sciencedirect.com/science/article/pii/S0266353806004386.

[90] Yi YB, Tawerghi E, Phys. Rev. E. 79 (2009). http://link.aps.org/doi/10.1103/PhysRevE.79.041134. 
[91] Veca LM, Meziani MJ, Wang W, Wang X, Lu F, Zhang P, Lin Y, Fee R, Connell JW, Sun Y-P, Adv. Mater. 21 (2009). http://dx.doi.org/10.1002/adma.200802317.

[92] Ganguli S, Roy AK, Anderson DP, Carbon. 46 (2008). http://www.sciencedirect.com/science/article/pii/S000862230800078X.

[93] Kim I-H, Jeong YG, Journal of Polymer Science Part B: Polymer Physics. 48 (2010). http://dx.doi.org/10.1002/polb.21956.

[94] Bao Q, Zhang H, Yang J-x, Wang S, Tang DY, Jose R, Ramakrishna S, Lim CT, Loh KP, Adv. Funct. Mater. 20 (2010). http://dx.doi.org/10.1002/adfm.200901658.

[95] Bao W, Miao F, Chen Z, Zhang H, Jang W, Dames C, Lau CN, Nat Nano. 4 (2009). http://dx.doi.org/10.1038/nnano.2009.191.

[96] Paul DR, Robeson LM, Polymer. 49 (2008). http://www.sciencedirect.com/science/article/pii/S0032386108003157.

[97] Wang S, Tambraparni M, Qiu J, Tipton J, Dean D, Macromolecules. 42 (2009). http://dx.doi.org/10.1021/ma900631c.

[98] Gong L, Kinloch IA, Young RJ, Riaz I, Jalil R, Novoselov KS, Adv. Mater. 22 (2010). http://onlinelibrary.wiley.com/doi/10.1002/adma.200904264/abstract.

[99] Kranbuehl DE, Cai M, Glover AJ, Schniepp HC, J. Appl. Pol. Sci. 122 (2011). http://dx.doi.org/10.1002/app.34787.

[100] Cooper CA, Young RJ, Halsall M, Compos A Appl. Sci. Manu. 32 (2001). http://www.sciencedirect.com/science/article/pii/S1359835X0000107X.

[101] Frank O, Tsoukleri G, Riaz I, Papagelis K, Parthenios J, Ferrari AC, Geim AK, Novoselov KS, Galiotis C, Nat Commun. 2 (2011). http://dx.doi.org/10.1038/ncomms1247.

[102] Young RJ, Gong L, Kinloch IA, Riaz I, Jalil R, Novoselov KS, ACS Nano. 5 (2011). http://pubs.acs.org/doi/abs/10.1021/nn2002079.

[103] Gong L, Young RJ, Kinloch IA, Riaz I, Jalil R, Novoselov KS, ACS Nano. (2012). http://dx.doi.org/10.1021/nn203917d. 


\title{
Properties of MWNT-Containing Polymer Composite Materials Depending on Their Structure
}

\author{
Ilya Mazov, Vladimir Kuznetsov, Anatoly Romanenko and Valentin Suslyaev
}

Additional information is available at the end of the chapter

http://dx.doi.org/10.5772/48245

\section{Introduction}

Carbon nanotubes (CNTs) are tubular structures composed of curved graphene sheets with diameter up to several tens of nanometers with typical length up to several micrometers. Single- and doublewall CNTs have diameters from 1.2 to ca. $3 \mathrm{~nm}$ and are usually packed in relatively dense structures ("ropes"). Multiwall carbon nanotubes can contain up to tens of concentrically aligned tubules and have diameter from 3-4 to tens of nanometers. Carbon nanotubes, both single- and multiwall, show outstanding mechanical and electrical properties $\left[{ }^{1},{ }^{2}\right]$. Nowadays CNTs are regarded as one of the key materials for development of various nanotechnology applications - new materials, sensors, actuators, field emitters etc. $\left[{ }^{3}, 4,5,6\right]$. In the last decade great effort was done in this field by many research groups, investigating structural, physical, mechanical, and electrical properties of CNTs.

Among all types of nanotubes single-wall nanotubes (SWNTs) were widely recognized as most perspective in regard of their predicted properties. Depending on chirality and diameter SWNTs can show significantly different electronic structure thus revealing metallic of semiconducting properties $\left[{ }^{7}, 8\right]$. Mechanical properties of SWNTs were investigated both theoretically and experimentally and were shown to outstand all other construction materials such as steel, carbon fibers etc [ $\left.{ }^{9}\right]$.

Extremely remarkable properties of SWNTs are strongly limited in usage by their high cost and low yield of production methods. Commonly used methods of SWNT synthesis include arc discharge $\left[{ }^{10}\right]$, laser evaporation of carbon targets $\left[{ }^{11}\right]$, or catalytic decomposition of gaseous carbonaceous species (carbon monoxide $\left[{ }^{12}\right]$, alcohols $\left[{ }^{13}\right]$, various hydrocarbons $\left[{ }^{14}\right]$ ) by CVD technique [15]. As-produced SWNTs need purification from amorphous carbon and other graphene-like species (fullerenes, multiwall CNTs etc.) which is usually performed by 
strong oxidative media and/or by selective surfactants (followed by ultra-centrifugation etc.). Involvement of such complex techniques results in high cost of resulting material, especially in the case of production of CNTs with tailored mechanical and electronic properties. According to market analysis the average price of highly purified SWNTs (90-99 wt. \%) lays in range 200-600 \$ per gram depending on purity, chirality and surface composition. Note that high amounts of SWNTs are still less available.

Multiwall carbon nanotubes (MWNTs) were firstly described in 1953 and now are one of the most common and widely used nanotubes allotrope. Multiwall nanotubes are composed with several concentrically aligned tubular graphene sheets, with typical diameter in range 8-30 nm. Physical and mechanical properties of MWNTs are significantly lower than that for SWNTs but still are higher than properties of commonly used construction materials and reinforcement additives.

Multiwall carbon nanotubes can be synthesized in the same way as SWNTs by arc discharge, graphite evaporation by laser irradiation or by catalytic decomposition of gaseous carbon-containing species $\left.{ }^{[16}\right]$ by CVD. The last one is the most perspective due to possibility to regulate CNT diameter and length; due to high yield and high selectivity of the process less or even no purification by aggressive oxidation is needed to achieve MWNT with high purity (higher than $90 \mathrm{wt}$. \%) [ $\left.{ }^{17}\right]$. CVD process has high scaling potential, e.g. by realization of the fluidized bed technique $\left[{ }^{18},{ }^{19}{ }^{20}\right]$.

In the last few years significant progress was achieved in the scaling of synthesis of MWNTs by catalytic CVD route. Several companies have demonstrated large-scale facilities for the process, for example, Bayer AG (Germany), Nanocyl (Belgium), Arkema (France), Hyperion Catalyst (USA), CheapTubes Inc. (China). Development of the large-scale synthesis route for MWNTs with high purity and relatively low defectiveness allowed to significantly lower market price for such product which is in range 1-15 \$ per one gram depending on purity, mean diameter and surface functionalization.

Relatively high availability of MWNTs and their remarkable properties result in great interest of their usage in various nanotechnology applications. At the present time high amount of research work was done in the field of MWNT investigation and application. Multiwall carbon nanotubes can be used as components of composite materials with polymer, metal or ceramic matrices $\left[{ }^{21}\right]$; as chemical sensors $\left[{ }^{22},{ }^{23}\right]$; as components of catalytic systems $\left[{ }^{24},{ }^{25}\right]$; as electromagnetic shielding materials $\left[{ }^{26}\right]$; for biomedical applications such as selective drug delivery $\left[{ }^{27},{ }^{28}\right]$ etc.

One of the most perspective approaches of usage of MWNT's superior properties is development of new multi-functional composite materials with improved and tailorable properties. Such composites can be used in various applications, for example as construction materials $\left[{ }^{29}\right]$, anti-static coatings $\left[{ }^{30}\right]$, low-weight electromagnetic shielding $\left[{ }^{31}\right]$, conductive polymers [32] etc. Polymer matrices are mostly used for development of such composite due to their light weight, low price, good processability and controllable chemical, physical and mechanical properties as well as good scaling perspectives. 
To date several tens of polymer matrices were investigated for the synthesis of MWNT-and SWNT-loaded polymeric composites. These are epoxy resins [33], polyurethanes [ $\left.{ }^{34}\right]$, polyolefines $\left[{ }^{35},{ }^{36}\right]$, polymethylmethacrylate $\left[{ }^{37}\right]$, polystyrene $\left[{ }^{31}\right]$, and others.

Systematic investigation of properties of novel multifunctional composite materials, containing carbon nanotubes is of essential importance to understand and improve their properties.

It is known [24, $\left.{ }^{38}\right]$ that CNTs of same type but produced by different vendors often show significant difference in chemical and physical properties depending on their diameter, length, defectiveness, agglomeration state, surface chemistry etc. Variation in properties of the filler may result in non-linear changes in properties of resulting composite. Thus investigation of properties of CNT-containing composites depending on properties of incorporated nanotubes and polymer host matrix is an important task.

In this chapter we describe an attempt to systematic investigation of structural, physicalchemical, and electrophysical and electromagnetic properties of thermoplastic composite materials, comprising multiwall carbon nanotubes with different mean diameters and morphology.

\section{MWNT-containing polymer composites}

\subsection{Approaches to synthesis, main problems}

As it was mentioned above, CNT (and, first of all, MWNT)-containing polymer-based composite materials attract great interest in the last decade. Great work was done in this field and tens of various host matrices were investigated. Several main problems in the area of design and preparation of nanotube-filled composite materials can be outlined according to literature analysis.

1. As-synthesized carbon nanotubes are usually arranged either in dense aligned arrays ("ropes") or tangled "furballs", composed of several tens of closely matted CNTs. First type is more typical for SWNTs, and the second one is most typical for CVD-produced MWNTs. Dense entangled MWNT arrays should be destructed during composite synthesis in order to achieve maximum dispersion state of nanotubes and subsequent maximum increase in properties of the composite. Also in this case it is possible to reach electrical percolation threshold at relatively low concentration of the filler due to intensive linking of highdispersed nanotubes between each other. See review $\left[{ }^{39}\right]$ for details of dispersion of MWNTs in various liquids using different technique.

2. Carbon nanotube fillers in the polymer matrix (and, in common sense, in all types of composite materials) can act as reinforcement material in several ways.

The first, incorporated nanotubes with high mechanical properties and high electrical conductivity, may act in the same way as macroscopic fillers (carbon fiber, glass wool etc.) providing stress transfer from the low-strength matrix in the case of mechanical load or charge transfer through continuous linked conductive network in the case of electrical load $\left[{ }^{40},{ }^{41}\right]$. 
The second, CNT filler is providing nucleation sites for the growth of polymer nanosized crystallites. Introduction of high amount of nanotubes with high surface chemical potential results in significant reduction of the grain size of resulting composite $\left[{ }^{42},{ }^{43}\right]$ thus leading to increase of its mechanical properties.

Thus it is of crucial importance to obtain high dispersion degree of nanotube filler in the bulk volume of the polymer matrix and provide intensive interaction between CNTs and polymer. These two tasks should be resolved during composite synthesis in order to obtain material with increased properties. This can be done in several ways depending on the type of the polymer used as matrix material.

All polymer materials can be roughly divided in two parts - thermoplastic and thermoreactive polymers. Both types were used for synthesis of MWNT-containing composites with certain success.

Thermoreactive matrices, such as epoxy resins and polyurethanes, were one of the first used for preparation of MWNT-loaded composites. The main route of preparation of such type of materials involves mixing of carbon filler with resin or with chosen intermediate solvent (acetone, dimethylformamide etc. $\left[{ }^{44}\right]$ ) which is later mixed with the resin. The mixing process is often assisted with ultrasonic treatment which results in higher dispersion degree of the nanotube filler in the matrix $\left[{ }^{45}\right]$. Obtained mixture is molded to form necessary shape and cured. The technique described is quite experimentally simple and scalable. However, due to high viscosity of epoxy resin it is hard to disperse entangled nanotubes uniformly in whole volume of the polymer. In the case of usage of intermediate solvents such as acetone, the last must be evaporated before curing process. Carbon nanotubes tend to spontaneous agglomeration while staying in suspension, thus destroying achieved dispersive state and resulting in lowering of composite's properties as compared with theoretically predicted.

Several special procedures can improve the process, such as chemical surface functionalization $\left[{ }^{46},{ }^{47}\right]$ or usage of short aligned CNT arrays as starting material $\left[{ }^{48},{ }^{49}\right]$, allowing to obtain good distribution of CNTs with low electrical percolation threshold and increased mechanical properties.

Thermoplastic polymers can be used as matrix materials for design and synthesis of nanotube-filled composites. These matrices can be processed by variety of techniques, such as solution casting, extrusion, pressure molding, hot pressing etc. Processing methods can be divided in two main parts - temperature-assisted and solution-assisted technique.

Twin-screw extrusion, pressure molding, hot pressing, liquid casting and similar methods can be described as temperature-assisted. The main step of these techniques involves melting of mixture of the polymer matrix material and CNT filler with subsequent processing of the melt blend ${ }^{35}$. Such approaches are relatively cheap, scalable and experimentally simple, but still have several disadvantages.

Usually the polymer blend has high viscosity in molten state thus preventing disaggregation of entangled agglomerates of CNTs. Usage of ultrasonic treatment is this case is complicated 
by high temperatures and closed volume of experimental setup. Moreover, increase of CNT loading results in further sharp increase of viscosity of polymer-nanotube blend, preventing achieving high dispersion of incorporated nanotubes.

These problems can be partially solved by using of additional mixing procedure, such as mechanical activation of the solid polymer powder with CNTs, or using of high CNT shear flow mixers for the molten mixture providing high dispersion degree. However, these procedures can result in breaking of nanotubes, especially in the case of multiwall CNTs with defective walls, with corresponding CNT shortening and decrease in mechanical and electrophysical properties of the composite.

Solution-assisted technique of preparation of nanotube-filled polymer composites includes dissolving of polymer material in appropriate solvent, mixing of the resulting solution with CNTs and subsequent evaporation of the solvent with formation of the polymer-nanotube film [50]. High disaggregation state of CNTs can be achieved by using ultrasonication and/or high-intensive mixing [51] of the nanotube-polymer suspension due to reasonably low viscosity of the solution. Thin films can be produced by this technique, allowing one to design functionally grade materials, electrostatic coatings, polarizing films etc.

However, this technique has some disadvantages - it is hard to obtain massive samples by solution casting and also special precautions must be applied in order to avoid CNT agglomeration during drying of the composite film (for example, surface functionalization, shortening of nanotubes etc. $[52,53])$.

There are some specific methods of synthesis of CNT-loaded composite materials, which cannot be ascribed to abovementioned types, for example in situ polymerization and coagulation precipitation techniques. The first one includes deposition of the catalyst on the surface of CNTs with further polymerization [ ${ }^{54}$ ] or radical polymerization of the monomer (e.g. polystyrene, polymethylmethacrylate) in presence of carbon nanotubes or other nanosized fillers or monomers $[37,55,56,57]$. High dispersion degree of nanotubes can be achieved by such methods, with following processing using conventional techniques, mentioned above.

The second method of CNT/polymer composite synthesis, coagulation precipitation, was firstly developed by $\mathrm{Du}$ et al. ${ }^{[58}$ for SWNT/PMMA composites. The coagulation precipitation $(\mathrm{CP})$ technique involves dissolution of the polymer in appropriate solvent, mixing of this solution with carbon nanotubes (or other filler materials). The resulting slurry is mixed with the second solution in which the first solvent is soluble, but the polymer is not. As a result the polymer/CNT mixture immediately precipitates, forming disperse composite material which can be later processed in usual ways.

Coagulation precipitation technique has several remarkable advantages as compared with abovementioned methods. By right choice of the first solvent it is possible to obtain both dissolution of the polymer and good wetting of nanotubes. For example, in the case of MWNT/PMMA or MWNT/PS composites dimethylformamide of N-methylpyrrolidone can be used for this task. These solvents are known to provide very stable CNT suspensions [ $\left.{ }^{59}\right]$ 
and can dissolve corresponding polymers in high concentrations. The second solvent for this system is water.

High CNT dispersion state is produced by ultrasonic treatment of the polymer-solvent mixture and is quite stable during minutes. It is of crucial importance that such high dispersion can be "frozen" on the second step by mixing with the second solvent, thus there is no reason to obtain super-stable CNT suspension.

The precipitation of polymer starts immediately after mixing with the second solvent and this process proceeds at high rate, producing small particles. Dispersed carbon nanotubes act as nucleation sites allowing reaching intimate interaction between polymer and individual CNTs.

$\mathrm{CP}$ technique still has certain inconveniences - for example, lack of scalability potential. Solution pair should be chosen carefully, providing both mutual solubility, and partial solubility of the polymer matrix material.

In the present chapter we describe preparation of MWNT-containing composite materials using coagulation precipitation technique and polymethylmethacrylate (PMMA) and polystyrene (PS) as matrices. All these polymers are thermoplastics and can be processed using common pressing and extrusion techniques.

\subsection{Experimental: Synthesis of MWNTs and MWNT-loaded composites}

Multiwall carbon nanotubes were synthesized in-lab by ethylene decomposition over bimetallic FeCo catalyst in hot-wall CVD reactor at $680{ }^{\circ} \mathrm{C}$. Details of preparation technique can be found elsewhere $\left.{ }^{60}\right]$. As-prepared MWNTs were additionally purified by reflux in $\mathrm{HCl}$ (15 wt. \%) during 3 hours, washed with distilled water and dried in air at $80^{\circ} \mathrm{C}$ for 24 hours.

Preparation of MWNT-loaded composites was performed using coagulation precipitation technique.

MWNT/PMMA and MWNT/PS composites were synthesized in similar way as follows. Polymer powder (PMMA, m.w. 100000; Polystyrene, m.w. 120000) was dissolved in dimethylformamide with concentration $0.1 \mathrm{~g} / \mathrm{ml}$. Calculated amount of air-dried MWNTs was loaded in water-cooled glass reactor and poured with polymer/DMF solution $(40 \mathrm{ml})$ diluted with pure DMF $(40 \mathrm{ml})$.

Resulting mixture was sonicated by Ti horn ultrasonicator (output power $8.5 \mathrm{~W} / \mathrm{cm}^{2}$ ) during 15 minutes under constant water cooling (the temperature of mixture was not higher than $\left.50{ }^{\circ} \mathrm{C}\right)$. Resulting slurry was poured in $\sim 1.5$ liters of distilled water $\left(\mathrm{t}=60-70{ }^{\circ} \mathrm{C}\right)$ under vigorous stirring immediately after US treatment. Precipitation of the polymer-MWNT composite proceeds immediately, resulting in formation of spongy-like deposit, which was left to stay overnight to complete coagulation.

The precipitate was filtered using Buchner funnel, washed with water $(3 \times 500 \mathrm{ml})$ and dried in air at $60{ }^{\circ} \mathrm{C}$ overnight. Residual water was removed by drying in vacuum $\left(10^{-2}\right.$ torr $)$ at $60^{\circ} \mathrm{C}$ for 2 hours. 
Composite powder can be processed using common technique. In our case hot pressing was chosen as one of the most simple ways to make polymer films. Powder was placed between two polished steel plates, which were heated up to melting temperature of the polymer and pressed with hydraulic press with pressure ca. $400 \mathrm{~kg} / \mathrm{cm}^{2}$. Copper ring with $0.5 \mathrm{~mm}$ thickness was used as spaces. Produced composite films were $\varnothing 60 \times 0.5 \mathrm{~mm}^{3}$ in dimensions.

Scanning and transmission electron microscopy was used for investigation of the structure of composite powder and films (JSM6460LV and JEM 2010 electron microscopes were used). Powder samples were placed on the conductive carbon adhesive tape, films were cut in plates with size ca. $8 \times 3 \times 0.5 \mathrm{~mm}^{3}$, which were broken and glued to the copper stand with breaks upwards using silver glue. In order to avoid surface charging during SEM investigations all samples were additionally covered with 5-10 nm gold layer.

Electrophysical properties of composite films were investigated using four-probe technique with silver wires connected to sample surface with silver glue.

Electromagnetic response properties of free-standing polymer composite films were investigated in frequency range $0.01-12 \mathrm{GHz}, 26-37 \mathrm{GHz}$. Array of experimental setups was used: quasi-optical setup based on panoramic meter KSvN R2-65 (Russia), Mach-Zehnder interferometer based on backward wave oscillator (Russia), HP Agilent PNA 8363B network analyzer (Agilent, USA) with multimode resonators (for dielectric permittivity spectra), and R2M-04 reflectance/transmission meter (Mikran, Russia). For $\varepsilon$ spectra measurement samples were cut in pieces with size $0.5 \times 2.5 \times 30 \mathrm{~mm}^{3}$ and placed in antinode of electric field parallel to electric field lines. Transmission coefficient was measured using polyfoam gasket, samples were cut in rings with an outer diameter $\varnothing 16.5 \mathrm{~mm}$ and inner diameter $\varnothing 6.95 \mathrm{~mm}$ and glued to the gasket. The measuring setup was calibrated using pure polyfoam gasket covered with glue.

\subsection{Properties of pure MWNTs}

Macro-scale properties of MWNTs and MWNT-based composites depend strongly on their nanoscale parameters, such as diameter, particle size distribution, morphology of agglomerates. In this work we have investigated three types of MWNTs differencing in main diameter (and diameter distribution), defectiveness and morphology of agglomerates. On Figure 1 TEM data and diameter distribution for all types of MWNTs is shown.

Mean diameter of MWNT (as obtained by statistical analysis of TEM images) makes the value of 7-9 nm for MWNT', $10-12 \mathrm{~nm}$ for MWNT ${ }^{12}$, and $\sim 22-24 \mathrm{~nm}$ for MWNT22. From TEM images one can roughly evaluate defectiveness of MWNT (amount of amorphous species on the surface of CNT, opened walls etc.) which is increasing with decreasing of their mean diameter.

According to SEM data the morphology of MWNT secondary agglomerates varies, changing from rope-like structure for "thin" tubes (MWNT', MWNT'2) to tangled furball-like structure for "thick" tubes (MWNT22). On figure 2 SEM images of typical MWNT agglomerates are presented. 


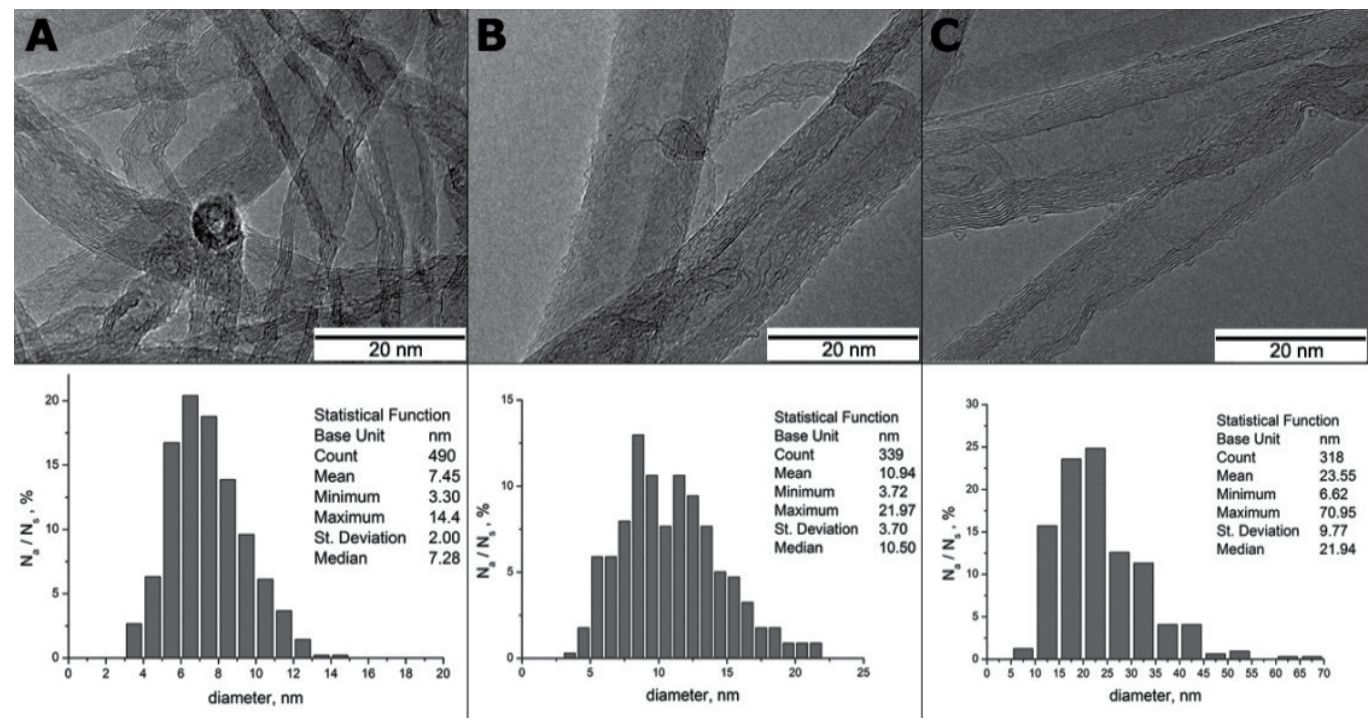

Figure 1. HRTEM images of MWNT samples used for preparation of MWNT/PMMA composites and their statistically calculated diameter distribution. A-MWNT ${ }^{8}, \mathrm{~B}-\mathrm{MWNT}^{12}, \mathrm{C}-\mathrm{MWNT}^{22}$.
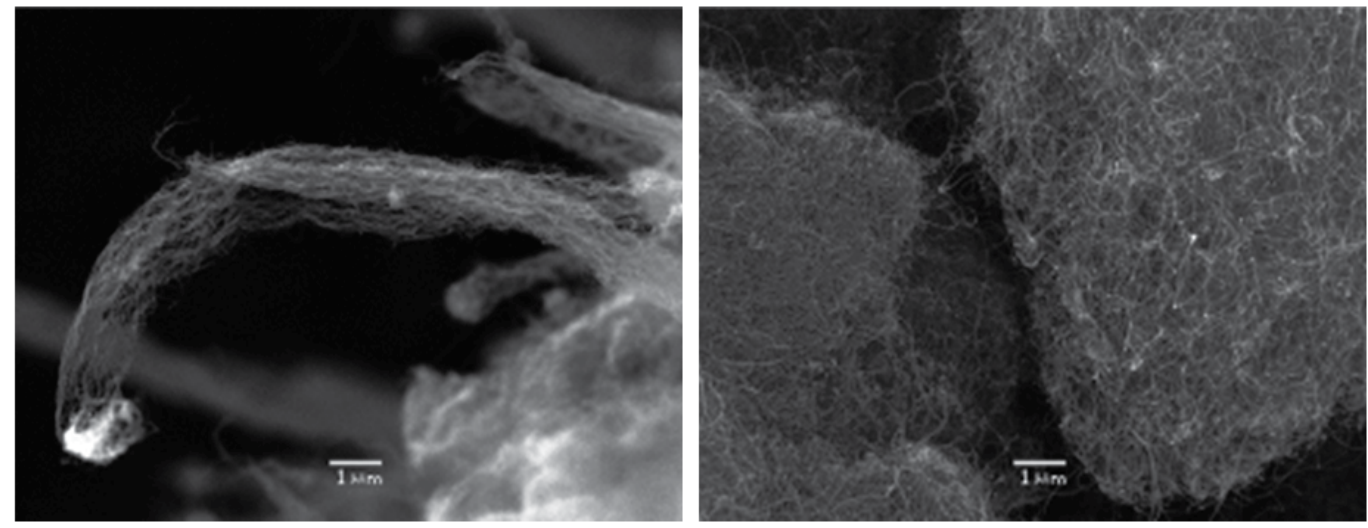

Figure 2. SEM micrographs of secondary aggregates of MWNTs. Left - MWNT8, "rope-like" structure; right - MWNT"12, MWNT22, "furball" structure.

Entangled nanotubes, which are forming "furball" structure, are hard to disperse in liquid media. MWNTs of all types tend to agglomerate in liquid dispersion, thus it is necessary to achieve certain conditions, allowing to "freeze" dispersed state of nanotubes. In our case usage of the coagulation precipitation technique allows to do this, as coagulation proceeds almost immediately after sonication and dispergation.

\subsection{PMMA and PS composites - Influence of matrix type}

Polymethylmethacrylate (PMMA) and polystyrene (PS) both are cheap, large-scale and easily processable polymer matrices, which are often used as model systems for synthesis of polymer composites. Polymers with similar average molecular weight were used for 
synthesis of composites, both PMMA and PS are soluble in DMF and precipitate using water as secondary solvent.

Principal physical-chemical properties of polymethylmethacrylate and polystyrene polymers are quite similar (see Table 1 for details) [ $\left.{ }^{61}\right]$.

From the Table 1 it can be clearly seen that PMMA and PS have very similar physical and chemical properties and the main difference is their surface composition and polarity of the surface $\left[{ }^{62},{ }^{63}\right]$. Carboxylic functions are present in PMMA structure thus resulting in increased oxygen content and increased polarity of the surface. Polystyrene has no oxygencontaining groups, showing lower polarity and surface tension [ $\left.{ }^{64}\right]$.

\begin{tabular}{|c|c|c|c|c|c|c|c|}
\hline Polymer & Formula & $\begin{array}{l}\text { Surface } \\
\text { free } \\
\text { energy }\end{array}$ & $\begin{array}{c}\text { Surface } \\
\text { oxygen } \\
(\mathrm{XPS})\end{array}$ & Polarity ${ }^{1}$ & $\begin{array}{c}\text { Density, } \\
\mathrm{g} / \mathrm{cm}^{3}\end{array}$ & $\begin{array}{l}\text { Thermal } \\
\text { conductivity, } \\
\mathrm{W} / \mathrm{m} \times \mathrm{K}\end{array}$ & $\begin{array}{c}\text { Dielectric } \\
\text { constant, } \\
\varepsilon^{\prime} @ 1 \mathrm{MHz} @ 2 \\
5^{\circ} \mathrm{C}\end{array}$ \\
\hline PMMA & & $\begin{array}{c}41.2 \\
\mathrm{~mJ} / \mathrm{m}^{2} \\
{[65]}\end{array}$ & $\begin{array}{c}38.6 \text { at. } \% \\
{\left[{ }^{66}\right]}\end{array}$ & $\begin{array}{c}0.28 \\
{\left[{ }^{67}\right]}\end{array}$ & 1.17 & 0.19 & $2.80\left[{ }^{68}\right]$ \\
\hline PS & & $\begin{array}{c}40.1 \\
\mathrm{~mJ} / \mathrm{m}^{2}\end{array}$ & 0 at. $\%$ & 0.17 & 1.04 & 0.22 & 2.55 \\
\hline
\end{tabular}

Table 1. Physical-chemical properties of polymethylmethacrylate (PMMA) and polystyrene (PS).

Thus investigation of these model polymer systems allows one to reveal basic principles of the influence of the matrix type on the properties of MWNT-loaded composites.

Transmission electron microscopy analysis of MWNT/PMMA and MWNT/PS composites was performed to investigate internal structure of materials. Corresponding micrographs are shown on figure 3. Interaction of carbon nanotubes with polymer matrix results in surface wetting of MWNTs with polymer, which depends strongly both on the surface composition of CNTs and on surface properties of the polymer. As-prepared nanotubes have low amount of polar (oxygen-containing) groups, thus surface is mostly hydrophobic. Chemical functionalization (e.g. oxidation) can modify surface composition of the materials, making it hydrophilic. According to our previous study [ $\left.{ }^{69}\right]$, as-prepared CNTs have ca. 0.3-0.5 oxygen-containing groups per $\mathrm{nm}^{2}$, so their surface is mostly hydrophobic, but still can show several hydrophilic behavior.

${ }^{1}$ Polarity calculated according to surface tension values, measured by sessile drop technique. 


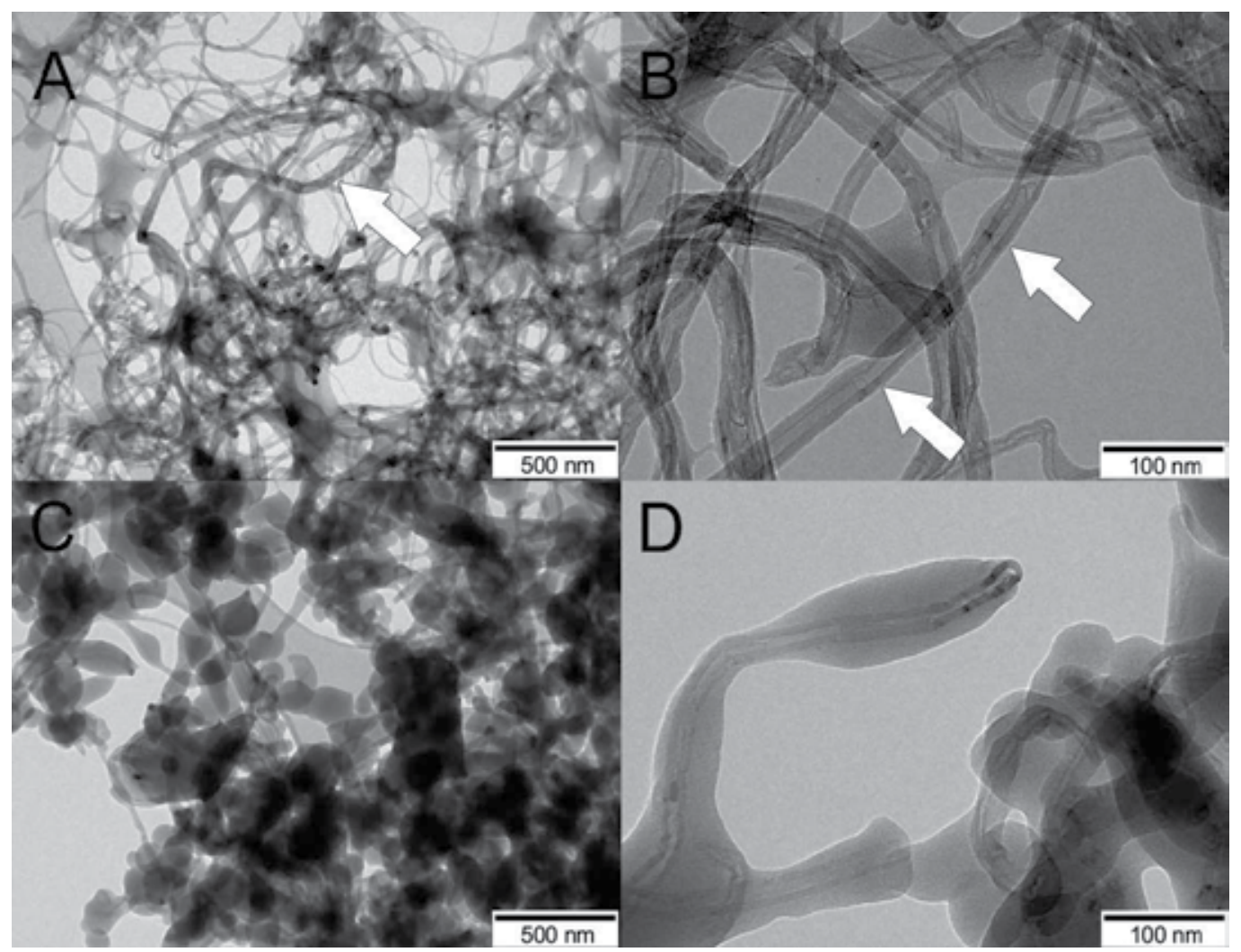

Figure 3. TEM images of MWNT-containing composites. A, B - MWNT22/PMMA (5 wt. \%), C, D MWNT $22 / P S(10$ wt. \%). Arrows are indicating naked parts of MWNTs (not covered with PMMA).

It is reasonable to suggest that hydrophilic surface would have higher affinity to polar polymer matrices, and pristine nanotubes would be better wetted by low-polar polymers.

Such behavior was observed for both compared MWNT/PMMA and MWNT/PS samples. As it can be seen from Figure 3, the surface of CNTs is covered with polymer. In the case of PMMA as a matrix, nanotubes are not fully covered with polymer. Both covered and naked surface areas are observed (marked with arrows on Fig. 3 A, B) for PMMA-treated composite. It is important to note that observed contact angles between nanotube surface and PMMA layer are still lower than $35^{\circ}$.

The possible reason for partial surface coverage with PMMA is certain irregularity of the functional composition of MWNT surface, for example, PMMA-covered areas are observed due to higher polarity of that nanotube part (e.g. due to partial oxidation), providing better wetting and lowering of the contact angle.

In contrast, PS-covered composite sample reveal significantly different structure where the surface of MWNTs is completely covered with thin continuous layer of the polymer (with thickness up to several nanometers). In the case of low CNT loading in composite one can observe disaggregated nanotubes, completely wrapped and wetted by PS (fig. 3, D). For 
higher nanotube content polystyrene "drops" are observed settled on the surface of nanotubes covered still with undestructured PS layer. As soon as polystyrene shows lower polarity as compared with PMMA and moreover has aromatic rings in structure, which have high affinity to the conjugated $\pi$-system of pristine (low-oxidized) nanotube, the wetting is more ease and coverage is higher in this case.

The contact angle between PS drops and CNT surface is in range $22-25^{\circ}$ indicating high wetting ability of PS towards untreated surface of nanotubes.

Interfacial interaction between polymer matrix and incorporated nanotubes significantly affects structural and physical properties of composite films. Formation of dispersed nanotube array or network in the volume of the polymer matrix is essential to drastical change of material properties. Carbon nanotubes tend to agglomerate during synthesis of composites, thus decreasing dispersion degree.

Formation of CNT agglomerates in composite powder was observed for both PMMA- and PS-based materials using transmission electron microscopy. TEM micrographs of different nanotubes agglomerates are shown on Figure 4.

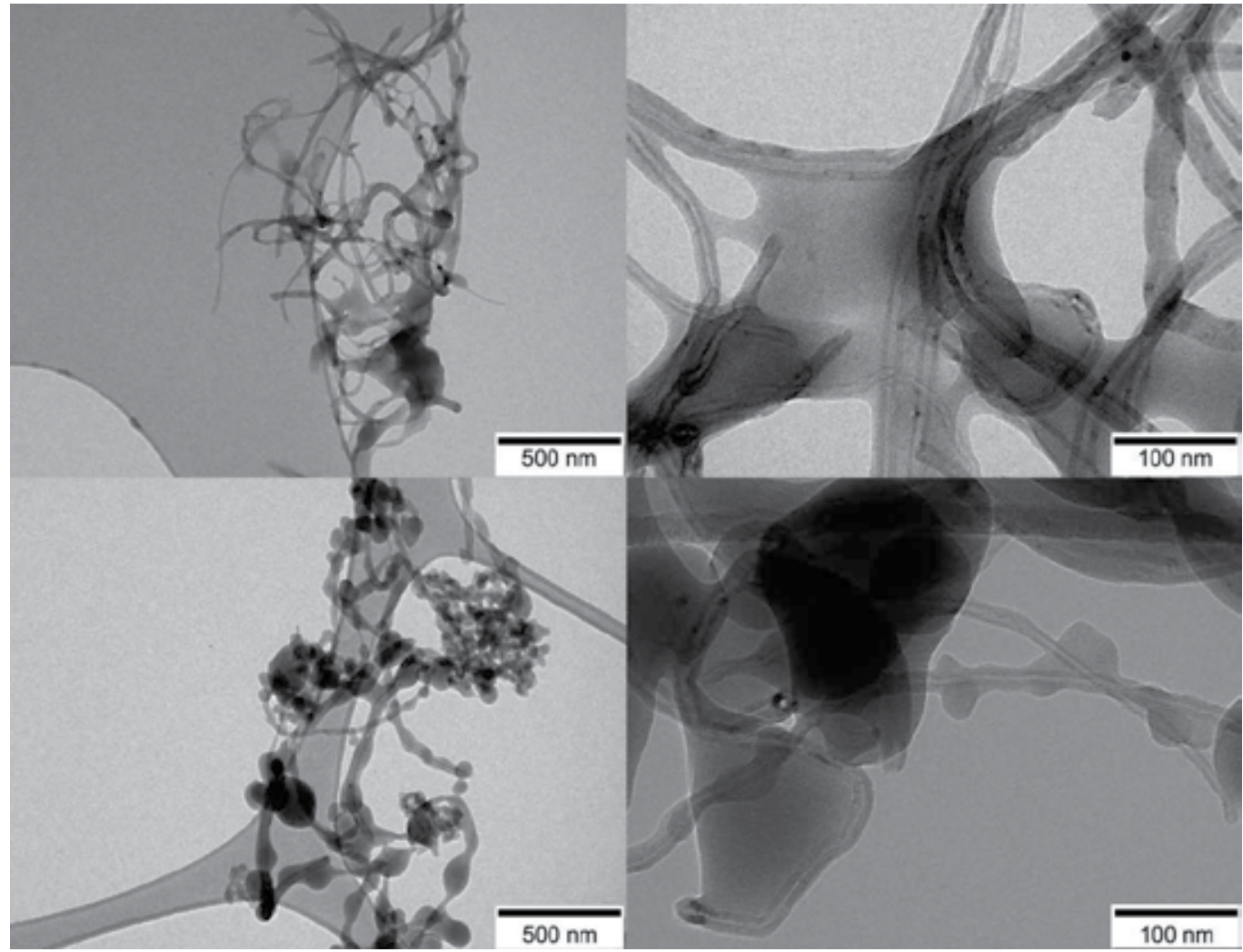

Figure 4. TEM images of agglomerated nanotubes in composite powders. A, B - MWNT22/PMMA (5 wt. \%), C, D - MWNT22/PS (10 wt. \%). 
Two types of nanotube agglomerates can be distinguished in polymer-based composite materials. These agglomerates have been identified as "primary" and "secondary", according to their origin. Primary agglomerates are formed mainly during synthesis of carbon nanotubes themselves and can be avoided in growth-aligned nanotube arrays $\left[{ }^{70}\right]$. These aggregates must be destroyed during composite synthesis by ultrasonic treatment, high-shear flow mixing etc. Destruction of such particles in necessary to achieve high dispersion degree and, for example, correspondingly low percolation threshold for composites. Nevertheless, for CVD-grown nanotubes these aggregates are hard to destroy and they are still occurring in composites, covered with polymer layer. Such aggregates are typical for systems with low wetting of CNTs with polymer, e.g. for MWNT-PMMA (fig. 3A \& 4-A).

Agglomerates of secondary type are formed during the process of composite synthesis due to re-aggregation of dispersed nanotubes in solution due to van der Walls forces. In this case individual nanotubes are separated from each other in solution and linked by the polymer particle in solid composite (fig. 4-B\&D). Strong wetting of carbon nanotubes with polymer assists destruction of primary agglomerates with subsequent formation of secondary-type agglomerates. Such phenomenon is observed for PS-based composites and is clearly seen on TEM micrographs - even for high MWNT loading (up to $10 \mathrm{wt}$. \%) almost none primary agglomerates were observed. MWNTs are dispersed and separated from each other, forming secondary agglomerates, clued by polymer particles (fig. 4-C\&D).

Investigation of composite films using scanning electron microscopy (SEM) was performed in order to elucidate influence of CNT-polymer interface on structural properties of composites. Corresponding SEM images of fresh breaks of composite films with different MWNT loading are shown on fig. 5.

For both types of polymer matrices separated nanotubes can be clearly seen on the surface of film breaks.

Difference in polymer-nanotube interface properties results in significant changes of the dispersion state of nanotubes in PMMA- and PS-based composites. For PMMA-based samples only single nanotubes can be seen for samples with 1 wt. \% of nanotubes (fig. 5-A). Increase of MWNT content to $4 \mathrm{wt}$. \% results in more uniform and dense dispersion of nanotubes in the volume of the polymer (fig. 5-C). Nevertheless, low-filled areas can be observed indicating relatively low dispersion degree of nanotubes. Certain amount of MWNTs can still occur in the agglomerated form, preventing formation of highlydisaggregated nanotube "network" in the polymer matrix.

Such phenomenon can be observed for the highest investigated MWNT loading in the composite (10 wt. \%, fig. 5-E). Dense and uniform nanotube array can be clearly seen on the surface of composite film break. Note that the length of MWNT residues on the surface of the film is $1-2 \mu \mathrm{m}$, moreover, the surface of nanotubes is not covered with the polymer. This may be attributed to stretching of CNTs from the volume of the polymer during breakage of the film, which can be easily assumed taking into consideration low wetting ability of PMMA towards hydrophobic surface of untreated carbon nanotubes. 
Polystyrene-based composite materials show opposite phenomenon of high wetting and high dispersion degree even at low MWNT loadings. Nanotubes in all MWNT/PS composite film samples are well-dispersed and covered with polymer layer. Uniform CNT distribution can be observed for samples with both low and high nanotube loading. According to SEM data nanotubes are randomly and evenly distributed on whole breakage area, which can be clearly seen on fig. 5-B, D, F.

Note that protruding parts of MWNT for PS-based composites are shorter as compared with similar PMMA-based samples and are wrapped with polymer layer. Such phenomenon can be attributed to higher wetting of CNTs with polystyrene and higher adhesion of filler to the material of the polymer matrix.

Thereby according to TEM and SEM data significant difference in wetting ability of investigated polymers towards MWNTs results in drastic changes of structural properties of composite powders and films. Highly-wetting aromatic polystyrene matrix allows one to reach higher dispersion degree at lower filler concentrations as compared with more polar polymethylmethacrylate due to mainly hydrophobic surface character of untreated nanotubes.

Area of application of novel materials is strongly dependent on their physical properties. As it was shown above, MWNT-based composites are perspective as tailorable materials for electrical and electromagnetic applications. Structure and dispersion state of nanotubes in composite, as well as their interconnections (i.e. formation of connected array of CNTs) strongly affect physical properties of composite materials. It is well known that introduction of continuous carbon nanotubes in the dielectric polymer matrix allows to increase conductivity of the resulting composite by several orders of magnitude. Conductive composite materials can be characterized by the percolation threshold which for multiwall carbon nanotubes lays in range from ca. 0.005 wt. \% [11] to 3-4 wt. \% [72] depending on the electrophysical properties of initial nanotubes and peculiarities of the composites' preparation (alignment and dispersion of nanotubes etc.).

Electrical conductivity measurements reveal significant difference between PMMA- and PSbased composites with similar MWNT loading. Conductivity data for PMMA-based composites with different types of MWNTs is shown on figure 6.

Electrical percolation threshold for PMMA-based composites was estimated as 1-2 wt. \%, reaching maximum value of $10^{-1}-10^{-2} \mathrm{~S} / \mathrm{m}$ for $3-5 \mathrm{wt}$. \% of CNT content.

PS-based composite materials show surprisingly low electrical conductivity. Significant conductivity $\left(\sim 4.5 \times 10^{-4} \mathrm{~S} / \mathrm{cm}\right)$ was observed for composite sample with $10.0 \mathrm{wt}$. \% loading of CNTs, all other samples show electrical resistivity higher than $10^{9} \mathrm{Ohm} / \mathrm{cm}$ which was the sensitivity threshold for the setup used for measurements.

As it was shown by electron microscopy investigations, PS-based composites reveal higher wetting and higher dispersion degree of nanotubes in the volume of the polymer as compared with PMMA-based materials. Unusually high percolation threshold and low 
conductivity value can be explained taking into consideration insulation of conductive nanotubes with wrapped polystyrene layer. In this case perfectly dispersed threedimensional nanotube network in the bulk volume of the polymer is formed by insulating objects, preventing current flow through composite even at high CNT loadings.

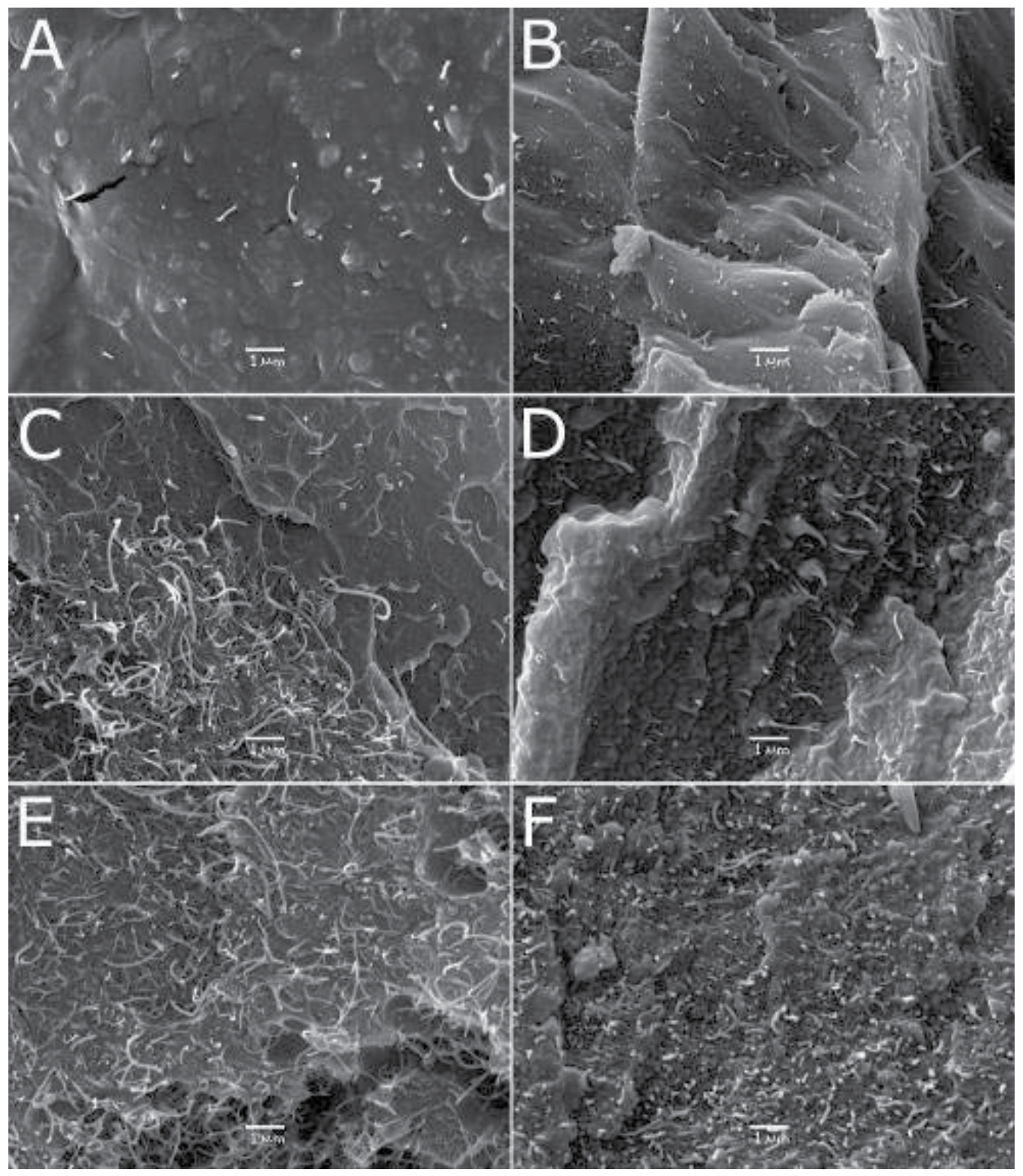

Figure 5. SEM images of MWNT/PMMA (left column) and MWNT/PS (right column) composite films. A, B - 1 wt. \%, C, D - 4 wt. \%, E, F - 10 wt. \%.

In the case of poorly-wetted PMMA-based composites the surface of nanotubes is not completely insulated, thus allowing reaching of electrical saturation at relatively low CNT 
loadings. Moreover, destruction of primary agglomerates of nanotubes, observed for PS and not for PMMA-based samples, results in diminishing of electrical contacts between nanotubes due to their insulation. Residual CNT agglomerates in the case of PMMA-based composites facilitate formation of conductive paths in the volume of the polymer matrix. As a result overall electrical conductivity of MWNT/PMMA materials is higher as compared with PS-based samples.

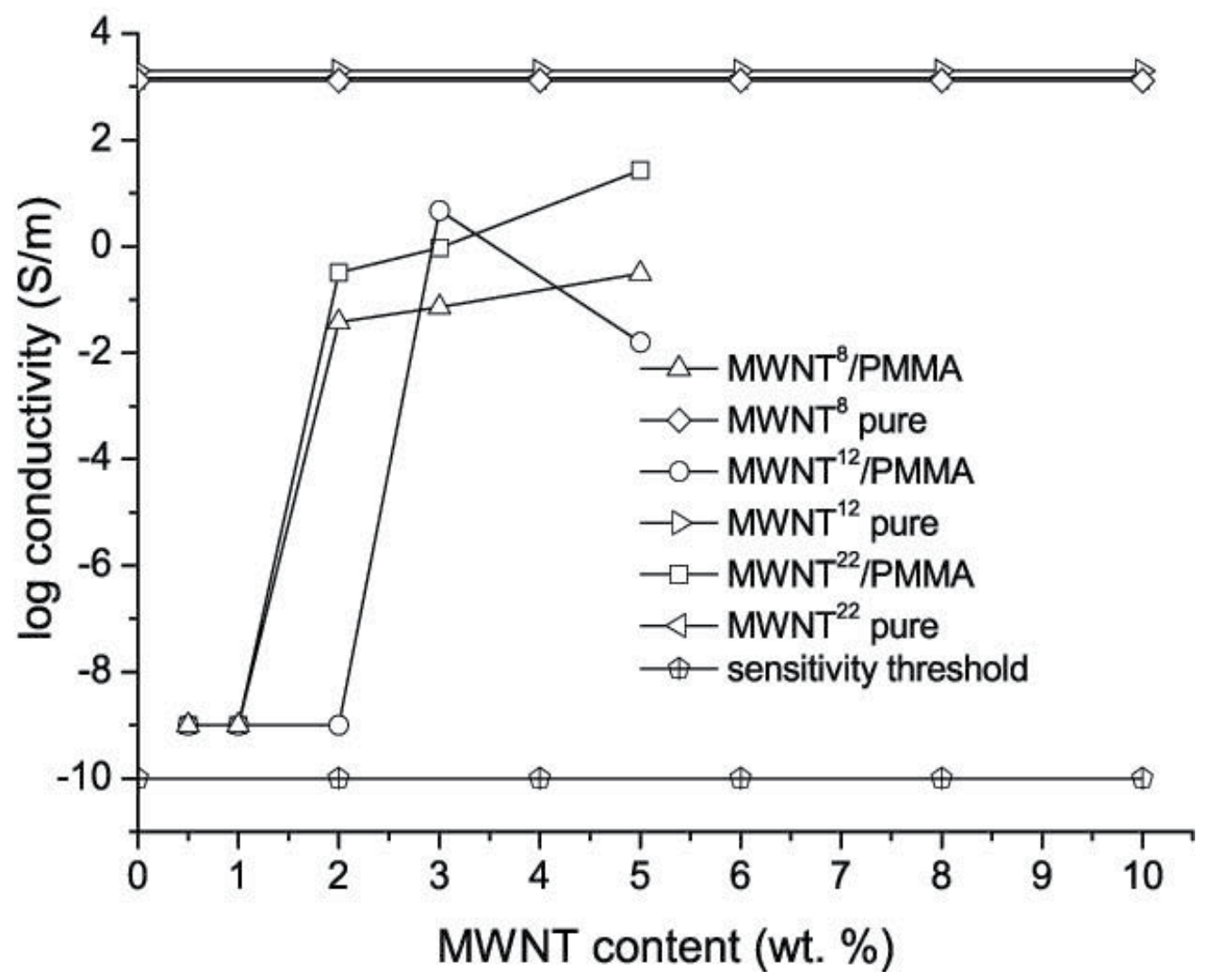

Figure 6. Electrical conductivity of MWNT/PMMA composites with various types of nanotubes versus filler concentration.

Electromagnetic response properties of composite materials play significant role in their application area. Incorporation of conductive media, such as carbon nanotubes, affects strongly the way of interaction of certain material with electromagnetic irradiation (EMI). Conjugated $\pi$-system, occurring in carbon nanotubes, allows both to dissipate and reflect electromagnetic wave, thus providing two possible mechanisms of EM response reflectance and absorbance.

Electromagnetic response properties of MWNT-containing composites were investigated in broadband region (2-36 GHz) and was found to be strongly dependent on MWNT type and diameter as well as on polymer matrix type. 
Influence of structural properties of MWNTs on EM shielding properties of composites was investigated for MWNT/PMMA materials due to their high electrical conductivity. Structure of initial CNT affects electrical and electromagnetic properties of CNT-based composites. We have investigated EM response properties of MWNT-based composites in frequency range 3-11 GHz (complex dielectric permittivity for MWNT/PMMA samples). Transmission $(T)$ and reflection $(R)$ coefficients can be easily calculated using following equations. Absorption coefficient of the EM radiation in the sample can be calculated as $A=1-R-T$.

$$
R=\left|\rho \frac{1-e^{-i 2 k d}}{1-\rho^{2} e^{-i 2 k d}}\right| ; \quad T=\left|\frac{\left(1-\rho^{2}\right) e^{-i k d}}{1-\rho^{2} e^{-i 2 k d}}\right|,
$$

where $\rho=\frac{Z-1}{Z+1}, Z=\sqrt{\mu^{*} / \varepsilon^{*}}$ is wave impedance, and $k=\frac{2 \pi f \sqrt{\varepsilon^{*} \mu^{*}}}{c}$ is the wavenumber, $f$ is the frequency of the EM wave, $c$ is the speed of light, $d$ is the thickness of the sample, $\varepsilon^{*}=\varepsilon^{\prime}-i \varepsilon^{\prime \prime}$ and $\mu^{*}=\mu^{\prime}-i \mu^{\prime \prime}$ are complex permittivity and magnetic permeability of the investigated material correspondingly. Dielectric losses $\left(\varepsilon^{\prime}\right.$ and $\left.\varepsilon^{\prime \prime}\right)$ are measured experimentally, and $\mu^{\prime}=1, \mu^{\prime \prime}=0$ (no magnetic losses are observed in the sample). Observed values of $\varepsilon^{\prime}$ and $\varepsilon^{\prime \prime}$ are growing with increase of the CNT loading in the composite and lay in range 20-70 for MWNT22/PMMA and 5-40 for MWNT ${ }^{12}$ and MWNT8/PMMA composites.

On the figure 7 data on measured transmission and reflections coefficients are shown.

Increase of the conductivity of the composites with MWNT loading in all samples leads to growth of $R$ and diminishing of $T$ parameters. For example for the MWNT 8 PMMA composite one can see higher $R$ and lower $T$ values for the sample with 3 wt.\% of MWNT whereas for the sample with 5 wt. \% these values are lower and higher, correspondingly, correlating with its conductivity. It should be mentioned that even composites with the lowest electrical conductivity (with CNT loading 0.5-2 wt.\%) show high values of the $R$, which may be due to formation of isolated conductive MWNT structures in the volume of the polymer that cannot be registered by macroscopic measurements of the electrical conductivity but still can interact with electromagnetic field. Polarization of such isolated structures gives contribution in EM response of non-conductive materials with subsequent growth of the permittivity.

Note that transmission and reflection coefficients do not depend directly on the electrical conductivity for different MWNT types. The highest values of $R$ are observed for the samples MWNT22/PMMA which show the lowest conductivity among other composites. This phenomenon may be described taking into account significant difference between number of individual nanotubes of each type incorporated into polymer matrix. Relationship between CNT number can be roughly estimated as relationship between $r_{o j}{ }^{2}-r_{i j}{ }^{2}$, where $r_{o j}$ is the outer diameter of CNT type $j, r_{i j}$ is the inner diameter of CNT type $j$

Simple math gives rough approximate of relationship between CNT number in the composite with the same weight loading as $N\left(M W N T^{22}\right): N\left(M W N T^{12}\right): N\left(M W N T^{8}\right) \approx 8: 4: 1$. 
Thus it is possible to propose that despite of the lower macroscopic electrical conductivity, composite materials comprising MWNT with lower diameter possess higher amount of polarizable species, giving higher values of dielectric permittivity and reflection coefficient.

All samples with low CNT content (0.5 wt. \%) are almost transparent in all frequency range. Increase of MWNT loading leads to reduction of transmission coefficient. Transmission is strongly affected by MWNT type and is changing unidirectionally with the dependence of permittivity - the lowest transmission is observed for $\mathrm{MWNT}^{22}$-containing composites which have higher $\varepsilon$ value as compared with MWNT'12/PMMA, MWNT//PMMA and composites.

For both matrices percolation-like concentration dependence can be observed with sharp decrease of EM transmission coefficient (and corresponding increase of EM shielding of composites) after 1-2 wt. \% of MWNTs in materials.

Nevertheless, the most significant difference for investigated matrices is that in case of PS matrix EM shielding is provided mainly by absorption, growing with increase of MWNT loading, and for the case of PMMA matrix main part of EM shielding is constituted by EM reflectance. Principal changes in mechanisms of EM attenuation in polymer composites filled with same type MWNTs can be explained taking into consideration abovementioned differences in their structural and electrophysical properties.

PMMA-based composites show high electrical conductivity and relatively low percolation threshold therefore above percolation threshold such materials interact with incident EM radiation as typical conductor, reflecting most part of electromagnetic wave.

Sharp increase in EM absorbance for PMMA-based composites at MWNT loading lower than 2 wt. \% may be attributed to absence of reflectance allowing propagation of incident EM wave through the sample and its interaction with conjugated $\pi$-system of incorporated carbon nanotubes.

The value of EM absorbance reaches saturation for MWNT loadings higher than 2 wt. \%, corresponding to electrical percolation threshold value. This phenomenon indicates formation of interconnected array (or cluster) of carbon nanotubes in the volume of the polymer, providing conductive paths for electrical current, decreasing electrical resistivity of the composite with subsequent increase in EM reflectance. Further increase of EM reflectance with growing MWNT loading is caused by formation of new conductive paths in linked nanotube network, increasing its electrical conductivity and reflective properties, however not affecting or diminishing absorption of EM radiation.

Polystyrene-based composites with same MWNTs as filler show surprisingly high shielding efficiency, as they are almost insulating at low nanotube loadings. In contrast to PMMAbased materials main part of shielding efficiency of PS-based composites is provided by absorption, growing with increase of MWNT concentration, especially for higher frequencies. At the same time reflection of EM radiation is increasing slowly, showing similar behavior as compared with EM absorbance for MWNT/PMMA composites. 

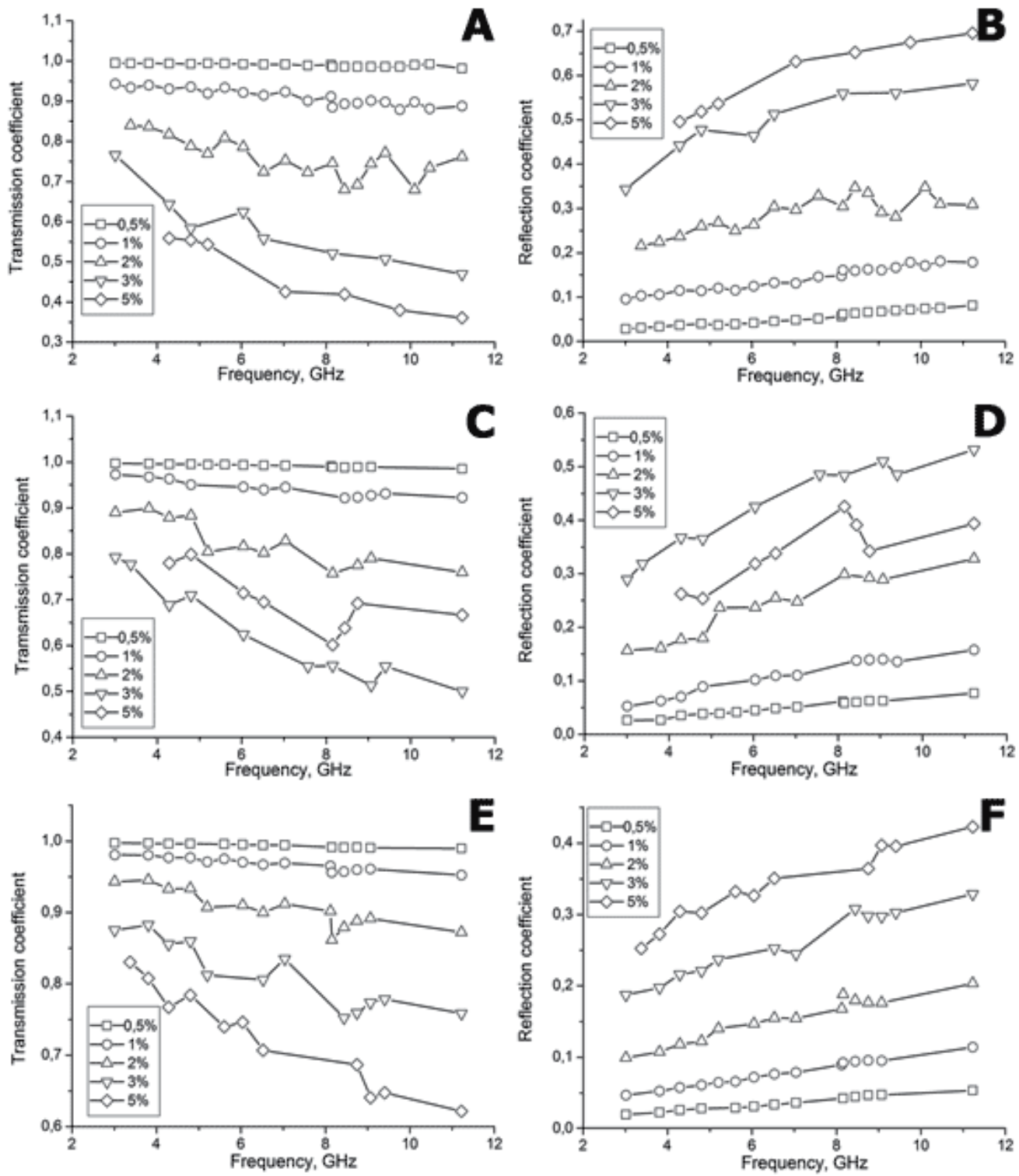

Figure 7. Transmission (A, C, E) and reflection (B, D, F) coefficients for MWNT\#1, \#2, \#3/PMMA composites respectively.

Internal structure of composite materials and interaction between MWNTs and polystyrene matrix show $i$ ) strong wetting of MWNT surface with polymer, providing certain insulation of nanotubes from each other; ii) high dispersion degree of MWNT without primary agglomerates; iii) random uniform distribution state of individual nanotubes in the volume of the polymer even for high filler concentrations. All these factors affect changes in EMI shielding mechanism of MWNT/PS composites as compared with relatively poorlydispersed highly-conductive PMMA-based materials. 

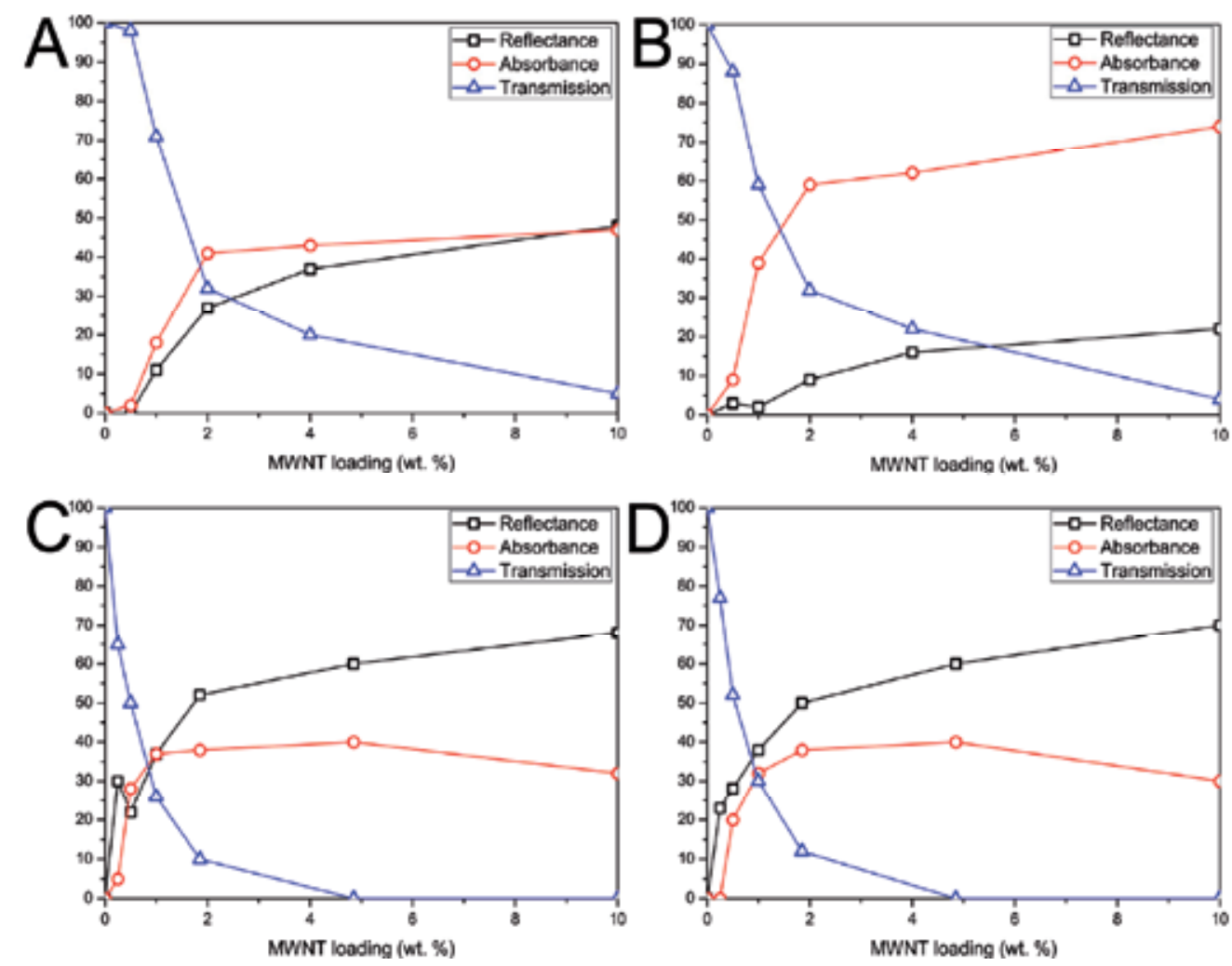

Figure 8. Concentration dependence of electromagnetic response properties of PS-based (A, B) and PMMA-based (C, D) composites in Ka-band. A, C - 29 GHz, B, D - 34 GHz.

Low-conductive PS composites possess correspondingly low EMI reflectance from the surface, allowing electromagnetic radiation to propagate in-depth of the sample. The characteristic wavelength for the EM radiation in microwave region is $1-10 \mathrm{~mm}$, which is higher than the size of the individual nanotube and MWNT agglomerate and is comparable with the macroscopic size of the composite sample. Thus incident EM wave may interact with whole volume of the composite sample and this interaction is strongly facilitated due to the absence of surface conductivity and reflectance due to skin-effects.

Propagating EM wave interacts with individual nanotubes uniformly dispersed in the volume of the composite with corresponding attenuation. High dispersion degree of MWNTs in polymer results in uniform density of attenuating particles, providing correspondingly high "extinction coefficient" of composite material.

\section{Conclusion}

Properties of multiwall carbon nanotubes and MWNT-containing composite materials are strongly dependent on their structure and morphology and on the type of polymer matrix. 
Variations of mean diameter distribution and morphology of agglomerates results in significant changes of their dispersive and electrical properties, affecting electromagnetic response of composites.

MWNT-filled composites with PMMA matrix show higher EM reflectance, higher electrical conductivity with low percolation threshold due to relatively low wetting of hydrophobic MWNTs with polar PMMA molecules and corresponding low dispersion degree of individual nanotubes.

In contrast for low-polar polystyrene matrix high wetting was observed resulting in formation of uniformly dispersed array of PS-covered MWNTs in the matrix. Coverage of MWNT surface with polystyrene results in insulation of nanotubes, thus resulting in high electrical resistivity with high percolation threshold. High shielding efficiency was observed for such composites, provided mainly by absorbance of EM radiation with low-conductive media.

Thus variation of main properties of polymer matrices and incorporated nanotubes allows to obtain composite materials with predictable tailorable properties, which may be used in various applications.

\section{Author details}

Ilya Mazov

Boreskov Institute of Catalysis, Novosibirsk, Russia

National Research Technical University "MISIS", Moscow, Russia

Vladimir Kuznetsov

Boreskov Institute of Catalysis, Novosibirsk, Russia

Anatoly Romanenko

Nikolaev Institute of Inorganic Chemistry, Novosibirsk, Russia

Valentin Suslyaev

National Research Tomsk State University, Tomsk, Russia

\section{Acknowledgement}

This work was partially supported by RFBR grant \#11-03-00351, ISTC project B-1708, and RNP projects \#2.1.1/10256, \#16.740.11.0016 \& \#16.740.11.0146.

\section{References}

[1] J.-P. Salvetat, J.-M. Bonard, N.H. Thomson, A.J. Kulik, L. Forro, W. Benoit, L. Zuppiroli, Mechanical properties of carbon nanotubes, Appl. Phys. A 69, 255-260 (1999)

[2] M. Terrones, Science And Technology Of The Twenty-First Century: Synthesis, Properties, And Applications Of Carbon Nanotubes, Annu. Rev. Mater.Res. (2003) 33 419-501

[3] P.M. Ajayan, O.Z. Zhou, Applications of Carbon Nanotubes in M. S. Dresselhaus, G. Dresselhaus, Ph. Avouris (Eds.): Carbon Nanotubes, Topics Appl. Phys. 80, 391-425 (2001) 
[4] D. Srivastava, C. Wei, K. Cho, Nanomechanics of carbon nanotubes and composites, Appl. Mech. Rev. 56 (2003) 215-230

[5] J.R. Stetter, G.J. Maclay Carbon nanotubes and sensors: a review, Advanced Micro and Nanosystems, Vol. 1, edited by: H. Baltes, O. Brand, G.K. Fedder, C. Hierold, J. Korvink, O. Tabata 357-382 (2004)

[6] J. Wang, Carbon-Nanotube Based Electrochemical Biosensors: A Review, Electroanalysis 17 (2005) 7-14

[7] J.W.G. Wilder, L.C. Venema, A.G. Rinzler, R.E. Smalley, C. Dekker Electronic structure of atomically resolved carbon nanotubes Nature 391 (1998) 59-62

[8] Q. Liu, W. Ren, Z.-G. Chen, L. Yin, F. Li, H. Cong, H.-M. Cheng, Semiconducting properties of cup-stacked carbon nanotubes, Carbon 47 (2009) 731-736

[9] R.S. Ruoff, D. Qian, W.K. Liu Mechanical properties of carbon nanotubes: theoretical predictions and experimental measurements C.R. Physique 4 (2003) 993-1008

[10] T. W. Ebbesen, P. M. Ajayan, Large scale synthesis of carbon nanotubes, Nature, 358, 220 (1992).

[11] A. Thess, R. Lee, P. Nikolaev, H. Dai, P. Petit, J. Robert, C. Xu, Y.H. Lee, S.G. Kim, A.G. Rinzler, D.T. Colbert, G.E. Scuseria, D.Tománek, J.E. Fischer, R.E. Smalley Crystalline Ropes of Metallic Carbon Nanotubes, Science 273 (1996) 483-487

[12] B. Kitiyanan, W.E. Alvarez, J.H. Harwell, D.E. Resasco, Controlled production of singlewall carbon nanotubes by catalytic decomposition of $\mathrm{CO}$ on bimetallic Co-Mo catalysts, Chemical Physics Letters 317 (2000) 497-503

[13] Y. Murakami, S. Chiashi, Y. Miyauchi, M. Hu, M. Ogura, T. Okubo, S. Maruyama, Growth of vertically aligned single-walled carbon nanotube films on quartz substrates and their optical anisotropy, Chemical Physics Letters 385 (2004) 298-303

[14] G. Zhang, D. Mann, L. Zhang, A. Javey, Y. Li, E. Yenilmez, Q. Wang, J.P. McVittie Y. Nishi, J. Gibbons, H. Dai Ultra-high-yield growth of vertical single-walled carbon nanotubes: Hidden roles of hydrogen and oxygen PNAS 102 (2005) 16141-16145

[15] M. Kumar, Y. Ando, Chemical Vapor Deposition of Carbon Nanotubes: A Review on Growth Mechanism and Mass Production Journal of Nanoscience and Nanotechnology 10 (2010) 3739-3758

[16] Y. Li, X.B. Zhang, X.Y. Tao, J.M. Xu, W.Z. Huang, J.H. Luo, Z.Q. Luo, T. Li, F. Liu, Y. Bao, H.J. Geise, Mass production of high-quality multi-walled carbon nanotube bundles on a Ni/Mo/MgO catalyst, Carbon 43 (2005) 295-301

[17] K.B. Hong, A.A.B. Ismail, M.E. Mahayuddin, A.R. Mohamed, S.H.S. Zeiri, Production of High Purity Multi-Walled Carbon Nanotubes from Catalytic Decomposition of Methane Journal of Natural Gas Chemistry 15 (2006) 266-270

[18] D. Venegoni, Ph. Serp, R. Feurer , Y. Kihn, C. Vahlas, Ph. Kalck, Parametric study for the growth of carbon nanotubes by catalytic chemical vapor deposition in a fluidized bed reactor, Carbon 40 (2002) 1799-1807

[19] M. Corrias, B. Caussat, A. Ayral, J. Durand, Y. Kihn, Ph. Kalck, Ph. Serp, Carbon nanotubes produced by fuidized bed catalytic CVD: first approach of the process, Chemical Engineering Science 58 (2003) 4475 - 4482

[20] S.W. Jeong, S.Y. Son, D.H. Lee, Synthesis of multi-walled carbon nanotubes using Co$\mathrm{Fe}-\mathrm{Mo} / \mathrm{Al}_{2} \mathrm{O}_{3}$ catalytic powders in a fluidized bed reactor, Advanced Powder Technology 21 (2010) 93-99 
[21] J. Wang, H. Kou, X. Liu, Y. Pan, J. Guo, Reinforcement of mullite matrix with multiwalled carbon nanotubes, Ceramics International 33 (2007) 719-722

[22] Y.-T. Jang, S.-I. Moon, J.-H. Ahn, Y.-H. Lee, B.-K. Ju A simple approach in fabricating chemical sensor using laterally grown multi-walled carbon nanotubes, Sensors and Actuators B: Chemical 99 (2004) 118-122

[23] N. Sinha, J. Ma, J.T.W. Yeow, Carbon Nanotube-Based Sensors, Journal of Nanoscience and Nanotechnology 6 (2006) 573-590

[24] N. Maksimova, G. Mestl, R. Schlogl Catalytic activity of carbon nanotubes and other carbon materials for oxidative dehydrogenation of ethylbenzene to styrene, Studies in Surface Science and Catalysis, G.F. Froment and K.C. Waugh (Editors) 133 (2001) 383-389

[25] M.S. Saha, R. Li, X. Sun, High loading and monodispersed Pt nanoparticles on multiwalled carbon nanotubes for high performance proton exchange membrane fuel cells, Journal of Power Sources 177 (2008) 314-322

[26] M.H. Al-Saleh, U. Sundararaj, Electromagnetic interference shielding mechanisms of CNT/polymer composites, Carbon 47 (2009) 1738-1746

[27] T.A. Hilder, J.M. Hill Carbon nanotubes as drug delivery nanocapsules, Current Applied Physics 8 (2008) 258-261

[28] C. Tripisciano, K. Kraemer, A. Taylor, E. Borowiak-Palen Single-wall carbon nanotubes based anticancer drug delivery system, Chemical Physics Letters 478 (2009) 200-205

[29] Makar, J. \& Beaudoin, J. Carbon nanotubes and their application in the construction industry. Nanotechnology 331-341 (2003

[30] M. Sangermano, S. Pegel, P. Potschke, B. Voit Antistatic Epoxy Coatings With Carbon Nanotubes Obtained by Cationic Photopolymerization, Macromol. Rapid Commun. 29 (2008) 396-400

[31] Y. Yang, M.C. Gupta, K.L. Dudley, R.W. Lawrence A Comparative Study of EMI Shielding Properties of Carbon Nanofiber and Multi-Walled Carbon Nanotube Filled Polymer Composites Journal of Nanoscience and Nanotechnology 5 (2005) 927-931

[32] X. Zhang, J. Zhang, Z. Liu, Conducting polymer/carbon nanotube composite films made by in situ electropolymerization using an ionic surfactant as the supporting electrolyte, Carbon 43 (2005) 2186-2191

[33] Sun, L. and Sue, H.-J. (2010) Epoxy/Carbon Nanotube Nanocomposites, in Epoxy Polymers: New Materials and Innovations (eds J.-P. Pascault and R. J. J. Williams), Wiley-VCH Verlag GmbH \& Co. KGaA, Weinheim, Germany

[34] S. Shang, W. Zeng, X. Tao, High stretchable MWNTs/polyurethane conductive nanocomposites J. Mater. Chem., 21 (2011) 7274-7280

[35] Y. Zou, Y. Feng, L. Wang, X. Liu, Processing and properties of MWNT/HDPE composites, Carbon 42 (2004) 271-277

[36] W. Tang, M.H. Santare, S.G. Advani, Melt processing and mechanical property characterization of multi-walled carbon nanotube /high density polyethylene (MWNT/HDPE) composite films, Carbon 41 (2003) 2779-2785

[37] S. Shang, L. Li, X. Yang, Y. Wei Polymethylmethacrylate-carbon nanotubes composites prepared by microemulsion polymerization for gas sensor, Composites Science and Technology 69 (2009) 1156-1159 
[38] J.H. Lehman, M. Terrones, E. Mansfield, K.E. Hurst, V. Meunier, Evaluating the characteristics of multiwall carbon nanotubes, Carbon 49 (2011) 2581-2602

[39] J. Hilding, E.A. Grulke, Z.G. Zhang, F. Lockwood, Dispersion of Carbon Nanotubes in Liquids, Journal Of Dispersion Science And Technology 24 (2003) 1-41

[40] L.S. Schadler, S.C. Giannaris,P.M. Ajayan, Load transfer in carbon nanotube epoxy composites, Appl. Phys. Lett. 73, 3842 (1998)

[41] L.R. Xu, S. Sengupta, Interfacial stress transfer and property mismatch in discontinuous nanofiber/nanotube composite materials, J. Nanosci Nanotechnol. 4 (2005) 620-626

[42] K.T. Kim, J. Eckert, S.B. Menzel, T. Gemming, S.H. Hong, Grain refinement assisted strengthening of carbon nanotube reinforced copper matrix nanocomposites Appl. Phys. Lett. 92, 121901 (2008)

[43] X. Zeng, G. Zhou, Q. Xu, Y. Xiong, C. Luo, J. Wu, A new technique for dispersion of carbon nanotube in a metal melt, Materials Science and Engineering: A 527 (2010) 5335-5340

[44] K. Q. Xiao, L.C. Zhang, Effective separation and alignment of long entangled carbon nanotubes in epoxy, Journal of Materials Science 40 (2005) 6513-6516

[45] S. Bal, S.S. Samal Carbon nanotube reinforced polymer composites-A state of the art, Bull.Mater. Sci. 30 (2007) 379-386

[46] Y.J. Kim, T.S. Shin, H.D. Choi, J.H. Kwon, Y.-C. Chung, H.G. Yoon Electrical conductivity of chemically modified multiwalled carbon nanotube/epoxy composites, Carbon 43 (2005) 23-30

[47] R.E. Gorga, K.K.S. Lau, K.K. Gleason, R.E. Cohen, The Importance of Interfacial Design at the Carbon Nanotube/Polymer Composite Interface, Journal of Applied Polymer Science, 102 (2006) 1413-1418

[48] Z. Yang, Z. Cao, H. Sun, Y. Li Composite Films Based on Aligned Carbon Nanotube Arrays and a Poly(N-Isopropyl Acrylamide) Hydrogel, Adv. Mater. 2008, 20, 2201-2205

[49] E.J. Garcia, A.J. Hart, B.L. Wardle, A.H. Slocum, Fabrication and Nanocompression Testing of Aligned Carbon-Nanotube-Polymer Nanocomposites, Adv. Mater. 2007, 19, 2151-2156

[50] W. Yuan, J. Che, M.B. Chan-Park A Novel Polyimide Dispersing Matrix for Highly Electrically Conductive Solution-Cast Carbon Nanotube-Based Composite, Chem. Mater. 23 (2011) 4149-4157

[51] P. Ciselli, R. Zhang, Z. Wang, C.T. Reynolds, M. Baxendale, T. Peijs, Oriented UHMW$\mathrm{PE} / \mathrm{CNT}$ composite tapes by a solution casting-drawing process using mixed-solvents European Polymer Journal 45 (2009) 2741-2748

[52] N.G. Sahoo, Y.C. Jung, H.J. Yoo, J.W. Cho, Effect of Functionalized Carbon Nanotubes on Molecular Interaction and Properties of Polyurethane Composites, Macromol. Chem. Phys. 207 (2006) 1773-1780

[53] K. Zhang, J.Y.Lim , B.J.Park, H.J. Jin, H.J.Choi Carboxylic Acid Functionalized MultiWalled Carbon Nanotube-Adsorption onto Poly(methyl methacrylate) Microspheres Journal of Nanoscience and Nanotechnology 9 (2009) 1058-1061

[54] L. Cui, N.H. Tarte, S.I. Woo, Synthesis and Characterization of PMMA/MWNT Nanocomposites Prepared by in Situ Polymerization with Ni(acac)2 Catalyst, Macromolecules 2009, 42, 8649-8654

[55] H.-J. Jin, H.J. Choi, S.H. Yoon, S.J. Myung, S.E. Shim, Carbon Nanotube-Adsorbed Polystyrene and Poly(methyl methacrylate) Microspheres Chem. Mater. 2005, 17, 4034-4037 
[56] D. Wang, J. Zhu, Q. Yao, C.A. Wilkie, A Comparison of Various Methods for the Preparation of Polystyrene and Poly(methyl methacrylate) Clay Nanocomposites, Chem. Mater.2002, 14, 3837-3843

[57] T. Wang, S. Shi, F. Yang, L. M. Zhou, S. Kuroda, Poly(methyl methacrylate)/polystyrene composite latex particles with a novel core/shell morphology, J Mater Sci (2010) 45:33923395

[58] F. Du, J.E. Fischer, K.I. Winey, Coagulation Method for Preparing Single-Walled Carbon Nanotube/Poly(methyl methacrylate) Composites and Their Modulus, Electrical Conductivity, and Thermal Stability, Journal of Polymer Science: Part B: Polymer Physics 41 (2003) 3333-3338

[59] T. Gabor, D. Aranyi, K. Papp, F.H. Karman, E. Kalman, Dispersibility of Carbon Nanotubes, Materials Science Forum 161 (2007) 537-538

[60] A. Usoltseva, V. Kuznetsov, N. Rudina, E. Moroz, M. Haluska, S. Roth, Influence of catalysts' ctivation on their activity and selectivity in carbon nanotubes synthesis, Phys. Stat. Sol. 11 (2007) 3920-3924.

[61] M. Sikka, N.N. Pellegrini, E.A. Schmitt, K.I. Winey, Modifying a Polystyrene/Poly (methyl methacrylate) Interface with Poly(styrene-co-methyl methacrylate) Random Copolymers, Macromolecules 30 (1997) $445-455$

[62] S. Varennes, H. P. Schreiber, On Origins of Time-Dependence in Contact Angle Measurements, The Journal of Adhesion, 76:293-306, 2001

[63] Y. Li, Y. Yang, F. Yu, L. Dong, Surface and Interface Morphology of Polystyrene/Poly (methyl methacrylate) Thin-Film Blends and Bilayers, Journal of Polymer Science: Part B: Polymer Physics, Vol. 44, 9-21 (2006)

[64] Y.T. Sung, W.J. Seo, Y.H. Kim, H.S. Lee, W.N. Kim Evaluation of interfacial tension for poly(methyl methacrylate) and polystyrene by rheological measurements and interaction parameter of the two polymers, Korea-Australia Rheology Journal 16 (2004) 135-140

[65] Tanaka K, Takahara A, Kajiyama T. Macromolecules 1996;29:3232-9.

[66] C. Ton-That, A.G. Shard, D.O.H. Teare, R.H. Bradley, XPS and AFM surface studies of solvent-cast PS/PMMA blends, Polymer 42 (2001) 1121-1129

[67] S. Wu, Polar and Nonpolar Interactions in Adhesion, J. Adhesion, 1973. Vol. 5, pp. 39-55

[68] P.K.C. Pillai, P. Khurana, A. Tripathi, Dielectric studies of poly(methyl methacrylate) /polystyrene double layer system Journal of Materials Science Letters 5 (1986) 629-632

[69] I. Mazov, V.L. Kuznetsov, I.A. Simonova, A.I. Stadnichenko, A.V. Ishchenko, A.I. Romanenko, E.N. Tkachev, O.B. Anikeeva "Oxidation behavior of multiwall carbon nanotubes with different diameters and morphology", Applied Surface Science, 258, 17 (2012) 6272-6280

[70] L. Ci, J. Suhr, V. Pushparaj, X. Zhang, P. M. Ajayan, Continuous Carbon Nanotube Reinforced Composites, Nano Letters 8 (2008) 2762-2766

[71] A. Moisala, Q. Li, I.A. Kinloch, A.H. Windle, Thermal and electrical conductivity of single- and multi-walled carbon nanotube-epoxy composites, Compos. Sci. Technol., 66, 10 (2006), pp 1285-1288

[72] W.K. Park, J.H. Kim Effect of Carbon Nanotube Pre-treatment on Dispersion and Electrical Properties of Melt Mixed Multi-Walled Carbon Nanotubes / Poly(methyl methacrylate) Composites, Macromolecular Research, Vol. 13, No. 3, pp 206-211 (2005) 


\title{
$\mathrm{C} / \mathrm{Li}_{2} \mathrm{MnSiO}_{4}$ Nanocomposite Cathode Material for Li-Ion Batteries
}

\author{
Marcin Molenda, Michał Świętosławski and Roman Dziembaj
}

Additional information is available at the end of the chapter

http://dx.doi.org/10.5772/48319

\section{Introduction}

Technological development of portable devices, e.g, mobile phones, laptops, etc., as well as progress in electrical vehicles (EV) and hybrid electrical vehicles (HEV) technologies require batteries efficient in volumetric and gravimetric energy storage, exhibiting large number of charge/discharge cycles and being cheap and safe for users. Moreover, materials used in energy storage and conversion systems should be environmentally friendly and recyclable. Currently, rechargeable lithium-ion batteries (LIBs) are the most popular portable energy storage system, mostly due to their highest energy density among all others rechargeable battery technologies, like $\mathrm{Ni}-\mathrm{Cd}$ or $\mathrm{Ni}-\mathrm{MH}$ cells which reached the theoretical limit of performance. Commercially available LIBs are based on layered lithium cobalt oxide $\left(\mathrm{LiCoO}_{2}\right)$ or related systems, which are expensive and toxic. These materials are unstable in an overcharged state, thus the battery safety is affected, especially in high power (20-100 $\mathrm{kWh}$ ) applications for EV, HEV and renewable energy systems. The bigger battery capacity results in more energy accumulated, thus operational safety is a key issue. On the other hand, lifetime and capacity retention of LIBs in changeable operation conditions (from $-30^{\circ} \mathrm{C}$ to $+60^{\circ} \mathrm{C}$, average lifetime 2-4 years) are a challenges to develop new materials and cell assembly technologies.

\section{Li-ion battery technology}

First rechargeable lithium cells taking advantages of intercalation process were developed in year 1972 [1]. The cells $\mathrm{Li} / \mathrm{Li}^{+} / \mathrm{Li}_{x} \mathrm{TiS}_{2}$ revealed $2 \mathrm{~V}$ potential and relatively low gravimetric capacity. Applications of metallic lithium as anode material resulted in common cell breakdown due to formation of dendritic structures on anode during cell cycling. The problems forced research and development of new intercalation materials for lithium batteries. In the eighties a new conception of lithium cell was proposed (so called Li-ion 
batteries or "rocking-chair batteries"), which consisted of application of two different lithium intercalation compounds as anode and cathode materials [2-4]. As anode material a graphite intercalated with lithium was used while cathode materials were based on layered 3d transition metal oxides.

\subsection{Layered $\mathrm{LiCoO}_{2}$ oxide and related systems}

The first $\mathrm{Li}_{\times} \mathrm{C}_{6} / \mathrm{Li}^{+} / \mathrm{Li}_{1-x} \mathrm{CoO}_{2} \mathrm{Li}$-ion battery system was commercialized in 1993 by Sony Co. In Fig. 1 a working mechanism during discharge cycle of Li-ion cell is presented.

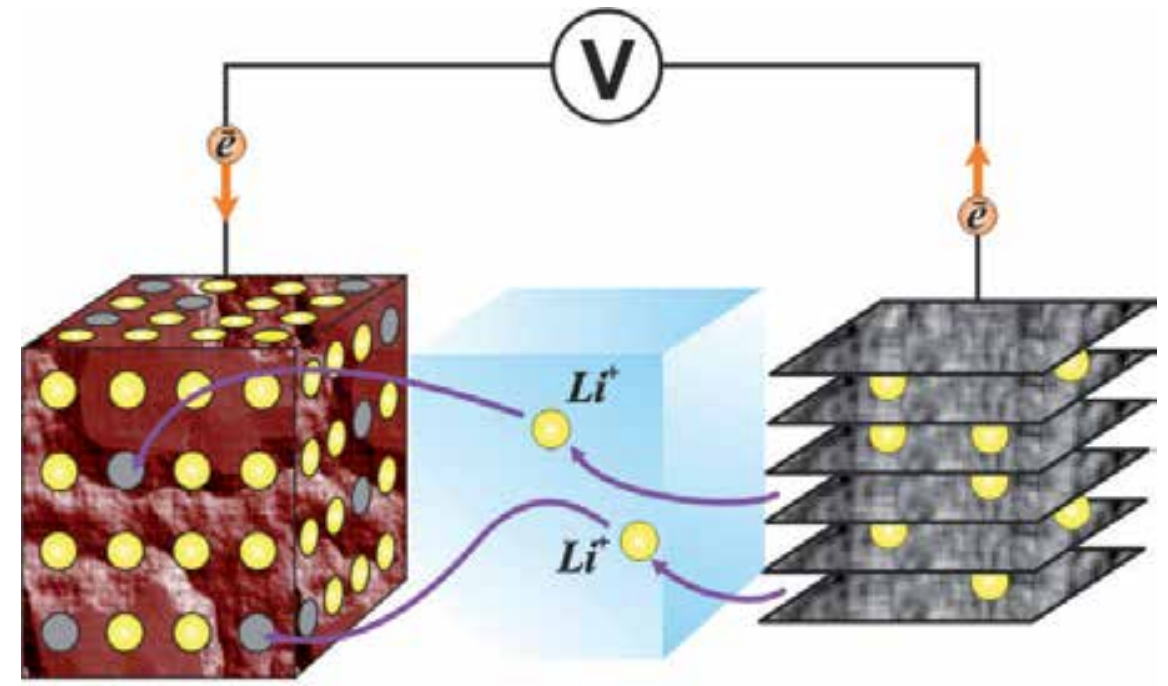

\section{CATHODE $(+) \quad$ ELECTROLYTE $\quad(-)$ ANODE}

Figure 1. Working mechanism of Li-ion batteries (discharge cycle).

The electrochemical reaction at the graphite anode side can be written as (1):

$$
\mathrm{C}_{6}+\mathrm{xLi}^{+}+\mathrm{xe}^{-} \leftrightarrow \mathrm{Lix}_{6}
$$

and suitably at $\mathrm{LiCoO}_{2}$ cathode side (2):

$$
\mathrm{LiCoO}_{2} \leftrightarrow \mathrm{Li}_{1-x} \mathrm{CoO}_{2}+\mathrm{xLi}^{+}+\mathrm{xe}^{-}
$$

Commercially available LIBs based on layered lithium cobalt oxide $\left(\mathrm{LiCoO}_{2}\right)$ or related systems ( $\mathrm{LiNi}_{y} \mathrm{CO}_{1-y} \mathrm{O}_{2}, \mathrm{LiMn}_{\mathrm{y}} \mathrm{Co}_{-\mathrm{y}} \mathrm{O}_{2}, \mathrm{LiMn}_{1 / 3} \mathrm{Ni}_{1 / 3} \mathrm{Co}_{1 / 3} \mathrm{O}_{2}$ ) reveal reversible capacity 130-150 $\mathrm{mAh} / \mathrm{g}$ and working potential 3.6-3.7 V. The oxide materials are expensive and toxic due to cobalt content and are unstable in an overcharged state, thus the safety is strongly affected. This is related to strong oxidizing behavior of the charged layered oxide cathode in contact with organic electrolyte what may lead to combustion or even explosion [4, 5]. Unfortunately, this effect may be also increased by application of nanosized materials with high surface area. 


\subsection{Spinel $\mathrm{LiMn}_{2} \mathrm{O}_{4}$ and related systems}

An alternative material for cathode based on layered oxides $\mathrm{LiM}_{\mathrm{y}} \mathrm{Co}_{1-\mathrm{y}} \mathrm{O}_{2}(\mathrm{M}=\mathrm{Ni}, \mathrm{Mn}, \mathrm{Fe})$ is $\mathrm{LiMn}_{2} \mathrm{O}_{4}$ spinel. $\mathrm{LiMn}_{2} \mathrm{O}_{4}$ reveals a little lower reversible capacity $120 \mathrm{mAh} / \mathrm{g}$ at working potential $4 \mathrm{~V}$, but the material is distinctly cheaper and nontoxic. However, application of spinel as cathode material in commercial Li-ion batteries is retarded by phase transition observed near room temperature, i.e. battery operation temperature, and related to JahnTeller distortion of $\mathrm{Mn}^{3+}$ ions, what resulted in capacity fading. Stabilization of cubic spinel structure is possible by controlled formation of cationic defects [6], by lithium [7] or 3d metal substitution [8] into spinel lattice as well as by isoelectronic sulfur substitution $[9,10]$. Instability of high oxidation state of transition metal in spinel structure observed in charged state of cathode leads to oxygen evolution, similarly to the layered oxide cathodes, and reaction with electrolyte. Application of spinel based materials is limited to cheap battery packs for EV [11].

\subsection{Olivine $\mathrm{LiFePO}_{4}$ cathode material}

An interesting and very promising group of insertion materials are $\mathrm{LiMXO}_{4}(\mathrm{M}=$ metal $3 \mathrm{~d}$, $\mathrm{X}=\mathrm{S}, \mathrm{P}, \mathrm{As}, \mathrm{Mo}, \mathrm{W}$ ) type compounds [12], with $\mathrm{LiFePO}_{4}$ among them. $\mathrm{LiFePO}_{4}$ lithium iron phosphate of olivine structure is chemically and thermally stable material with relatively high gravimetric capacity $170 \mathrm{mAh} / \mathrm{g}$ at working potential $3.5 \mathrm{~V}$. The material is cheap, nontoxic and environmental friendly. Its high chemical stability towards electrolyte, related to strong P-O bonds, significantly improves safety of LIBs. However, very low electrical conductivity of $\mathrm{LiFePO}_{4}$ system $\left(10^{-9} \mathrm{~S} / \mathrm{cm} @ \mathrm{RT}\right)$ requires application of carbon coatings and composite formation $[13,14]$.

\section{Orthosilicates $\mathrm{Li}_{2} \mathrm{MSiO}_{4}-\mathrm{New}$ high capacity cathode materials}

Application of orthosilicates $\mathrm{Li}_{2} \mathrm{MSiO}_{4}(\mathrm{M}=\mathrm{Fe}, \mathrm{Mn}, \mathrm{Co}, \mathrm{Ni})$ compounds as insertion materials for LIBs was firstly proposed by Prof. Goodenough [12, 15]. The materials mainly crystallizes in orthorhombic system of Pmn21 space group in olivine structure [16-18]. The $\mathrm{Li}_{2} \mathrm{MSiO}_{4}$ olivine structure may be described as wavy layers of [SiMO$\left.]_{\infty}\right]_{\infty}$ on $a c$ axis plane and connected along $b$ axis by $\mathrm{LiO}_{4}$ tetrahedra [16]. Within the layers every tetrahedron $\mathrm{SiO}_{4}$ shares its corner with four next $\mathrm{MO}_{4}$ tetrahedra. Lithium ions occupy the tetrahedral sites $\left(\mathrm{LiO}_{4}\right)$ between two layers and share 3 and 1 oxygen atoms with the layers. In fact, diffusion of lithium ions in this structure is possible only through the canals formed by $\mathrm{LiO}_{4}$ tetrahedra. $\mathrm{Li}_{2} \mathrm{MSiO}_{4}$ silicates reveal a possibility of reversible insertion of two lithium ions per molecule, so this leads to exchange of two electrons by transition metal. As a results the silicates reveal very high theoretical gravimetric capacity, up to $330 \mathrm{~mA} / \mathrm{g}$. However, structural limitation due to sharing of $\mathrm{LiO}_{4}$ between two wavy layers, should affect a little the capacity. Calculations of electrochemical potential of lithium insertion/deinsertion show a two stage process:

$$
\mathrm{Li}_{2} \mathrm{M}^{2+} \mathrm{SiO}_{4} \rightarrow \square \mathrm{LiM}^{3+} \mathrm{SiO}_{4}+\mathrm{Li}^{+}
$$




$$
\mathrm{rLiM}^{3+} \mathrm{SiO}_{4} \rightarrow \Upsilon_{2} \mathrm{M}^{4+} \mathrm{SiO}_{4}+\mathrm{Li}^{+}
$$

Depending on transition metal potential of the reaction (3) vary from 3.2 V (for Fe) to 4.1-4.4 $\mathrm{V}$ (for $\mathrm{Mn}, \mathrm{Co}, \mathrm{Ni}$ ), while the potential of reaction (4) is in range 4.5-5.0 V. Deinsertion of the second lithium ion requires applying high potential above $4.5 \mathrm{~V}$, and this is a challenge for electrolyte. Orthosilicates $\mathrm{Li}_{2} \mathrm{MSiO}_{4}(\mathrm{M}=\mathrm{Fe}, \mathrm{Mn}, \mathrm{Co}, \mathrm{Ni})$ materials reveal very low electrical conductivity $\left(10^{-12}-10^{-15} \mathrm{~S} / \mathrm{cm} @ \mathrm{RT}\right)$ and carbon coating of the materials is required. On the other hand, downsizing of material grains should improve electrochemical performance [18]. Very strong covalent bonding of $\mathrm{Si}-\mathrm{O}$ results in high chemical stability towards electrolyte, strongly increasing the safety of LIBs based on silicates. The materials are nontoxic, environmental friendly and cheap. The properties mentioned above provide a challenge for developing a composite cathode materials based on $\mathrm{Li}_{2} \mathrm{MSiO}_{4}$ orthosilicates.

\section{4. $\mathrm{Li}_{2} \mathrm{MnSiO}_{4}$ nanostructured cathode material}

$\mathrm{Li}_{2} \mathrm{MnSiO}_{4}$ is a member of dilithiumorthosilicates $\mathrm{Li}_{2} \mathrm{MSiO}_{4}(\mathrm{M}=\mathrm{Fe}, \mathrm{Mn}, \mathrm{Co})$ family, thanks to strong covalent $\mathrm{Si}-\mathrm{O}$ bond, it shows high thermal and chemical stability. High theoretical capacity $333 \mathrm{mAh} / \mathrm{g}$, low production costs, safety and optimal working potential make them an attractive cathode material. Very low electrical conductivity $\left(10^{-12}-10^{-15} \mathrm{~S} / \mathrm{cm} @ \mathrm{RT}\right)$ can be improved by coating with conductive carbon layers (CCL) and by grains size downsizing [18-20]. The properties of $\mathrm{Li}_{2} \mathrm{MnSiO}_{4}$ material comes to a conclusion that cathode material for LIBs based on this compound should be prepared as $\mathrm{C} / \mathrm{Li}_{2} \mathrm{MnSiO}_{4}$ nanocomposite. Thus, the special preparation techniques have to be applied in terms to obtain nanocrystaline grains of $\mathrm{Li}_{2} \mathrm{MnSiO}_{4}$ cathode material coated by conductive carbon layers (CCL). During last few years several different technics of $\mathrm{Li}_{2} \mathrm{MnSiO}_{4}$ synthesis were proposed. Hydrothermal synthesis [21-24] as well as solid-state reactions [25-28] can lead to one phase product but the control of the grain size is significantly limited. Sol-gel method is one of the soft chemistry techniques which can be used in synthesis of nanosized lithium orthosilicates [29-33]. Sol-gel processes, especially Pechini's method is a simple technic, characterized by low cost and low temperature of treatment, resulting in homogenous, high purity materials.

\subsection{Preparation of nanostructured $\mathrm{Li}_{2} \mathrm{MnSiO}_{4}$}

$\mathrm{Li}_{2} \mathrm{MnSiO}_{4}$ was produced using sol-gel synthesis - Pechini type. Starting reagents were: lithium acetate dihydrate (Aldrich), manganese acetate tetrahydrate (Aldrich), tetraethoxysilane (TEOS) (98\%, Aldrich) as a source of silicon, ethylene glycol (POCh), citric acid (POCh) and ethanol (POCh). Thanks to chelating metal ions in solution by citric acid the cations can be mixed at the molecular level and the stoichiometric composition can be achived. The reactants were mixed in a molar ratio 1:1:18:6:4:16 $\mathrm{Mn}: \mathrm{Si}_{2} \mathrm{C}_{2} \mathrm{H}_{6} \mathrm{O}_{2}: \mathrm{C}_{6} \mathrm{H}_{8} \mathrm{O}_{7}: \mathrm{C}_{2} \mathrm{H}_{5} \mathrm{OH}: \mathrm{H}_{2} \mathrm{O}$. Based on previous studies it was affirmed, that using $20 \%$ excess of lithium acetate leads to one-phase product. All reagents were dissolved in glass reactor under constant argon flow (Ar 5.7). As a solvent, only stoichiometric amount of distilled water was used. Heating water to $35{ }^{\circ} \mathrm{C}$ assure fast and complete dissolution of 
metal acetates. Prepared mixture was heated to $60{ }^{\circ} \mathrm{C}$ and few drops of concentrated hydrochloric acid was added to initiate polymerization of metal citrates using ethylene glycol and TEOS. Reaction was conducted for $24 \mathrm{~h}$ in close reactor. Obtained gel was aged for 3 days at $60{ }^{\circ} \mathrm{C}$ in close reactor (Ar atmosphere) and for 3 days at $60{ }^{\circ} \mathrm{C}$ in an air-drier (Air atmosphere).

Thermogravimetric analysis (TGA) coupled with simultaneous differential thermal analysis (SDTA) and mass spectrometry evolved gas analysis (MS-EGA) of precursor were performed in Mettler-Toledo 851 $1^{\mathrm{e}}$ thermo-analyzer using $150 \mu \mathrm{l}$ corundum crucibles under flow of air/argon $(80 \mathrm{ml} / \mathrm{min})$, within temperature range $20-800{ }^{\circ} \mathrm{C}$ with heating rate of 10 ${ }^{\circ} \mathrm{C} / \mathrm{min}$. The simultaneous MS-EGA was performed in on-line joined quadruple mass spectrometer (QMS) (Thermostar-Balzers). The 17, 18 and $44 \mathrm{~m} / \mathrm{z}$ mass lines, ascribed to $\mathrm{OH}$, $\mathrm{H}_{2} \mathrm{O}$ and $\mathrm{CO}_{2}$ species respectively, were collected during the TGA experiments. TGA of the $\mathrm{Li}_{2} \mathrm{MnSiO}_{4}$ precursor are shown in the Fig. 2 and Fig. 3.
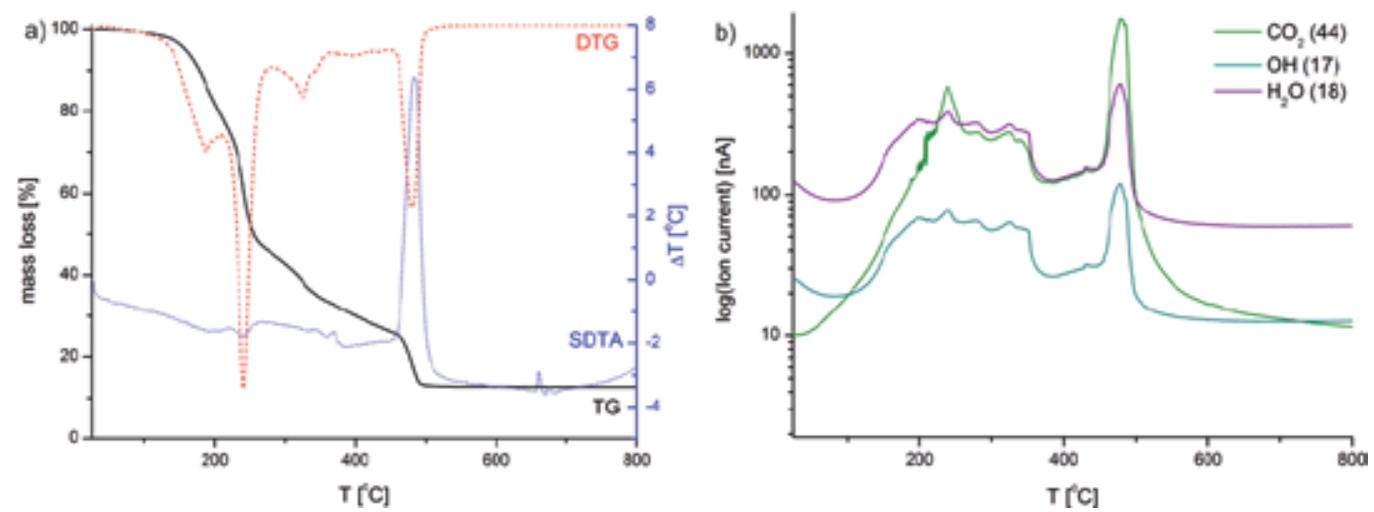

Figure 2. Thermal analysis (TGA/DTG/SDTA) conducted under air atmosphere (a) with evolved gas analysis (EGA-QMS) (b) of $\mathrm{Li}_{2} \mathrm{MnSiO}_{4}$ precursor.

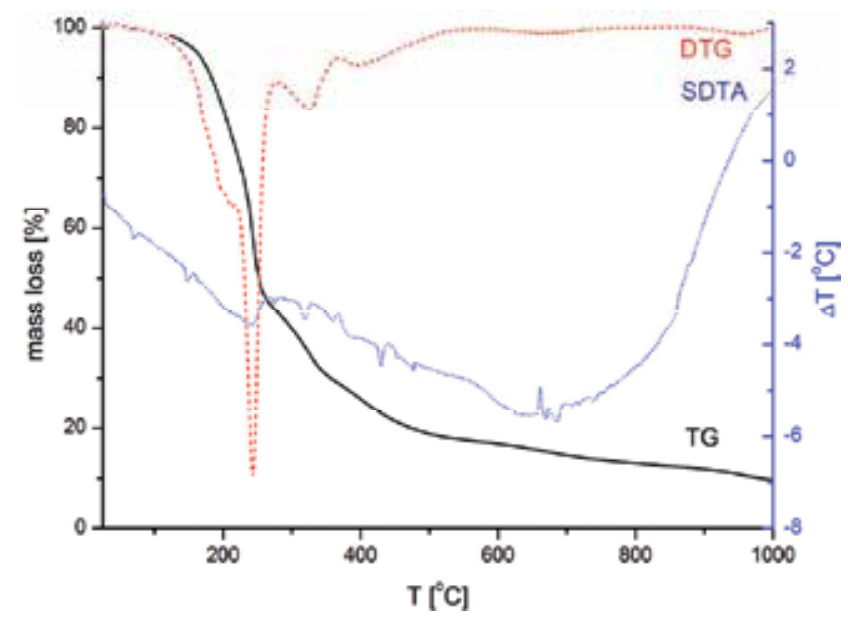

Figure 3. Thermal analysis (TGA/DTG/SDTA) of $\mathrm{Li}_{2} \mathrm{MnSiO}_{4}$ precursor performed under inert atmosphere (Ar 5.0). 
According to TGA curve (Fig. 2a) a complete decomposition of gel organic matrix occurs above $500{ }^{\circ} \mathrm{C}$. Evolved gas analysis confirmed the disintegration of organic components. Amount of active material can be estimated at about $12 \mathrm{wt} . \%$ of the precursor. Basing on TGA results calcination conditions were chosen. $\mathrm{Li}_{2} \mathrm{MnSiO}_{4}$ precursor was calcined under $\mathrm{Ar}$ flow at $600,700,800$ and $900{ }^{\circ} \mathrm{C}$.

\subsection{Properties of nanostructured $\mathrm{Li}_{2} \mathrm{MnSiO}_{4}$}

X-ray powder diffraction patterns of the samples were collected on BRUKER D2 PHASER using $\mathrm{Cu} \mathrm{K} \alpha$ radiation $=1.5418 \AA$ A. In Fig. 4 XRD patterns of $\mathrm{Li}_{2} \mathrm{MnSiO}_{4}$ obtained at different temperatures $\left(600,700,800\right.$ and $\left.900{ }^{\circ} \mathrm{C}\right)$ are collected.

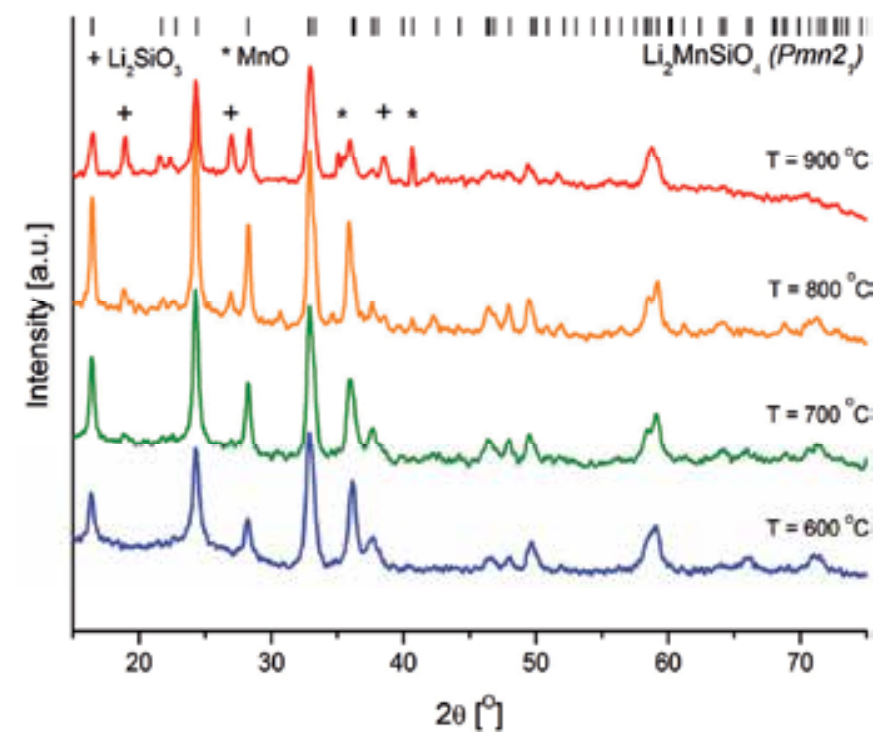

Figure 4. XRD patterns of $\mathrm{Li}_{2} \mathrm{MnSiO}_{4}$ after calcination at $600{ }^{\circ} \mathrm{C}, 700{ }^{\circ} \mathrm{C}, 800{ }^{\circ} \mathrm{C}$ and $900{ }^{\circ} \mathrm{C}$. $\mathrm{Li}_{2} \mathrm{MnSiO}_{4}$ (Pmn21 phase) diffraction lines positions are marked in the top of the figure.

In the sample calcined at $600{ }^{\circ} \mathrm{C}$ all diffraction lines can be attributed to pure $\mathrm{Li}_{2} \mathrm{MnSiO}_{4}$ phase $\left(P m n 2_{1}\right)$. In other specimens obtained at higher temperatures, $\mathrm{MnO}$ and $\mathrm{Li}_{2} \mathrm{SiO}_{3}$ impurities starts to appear. Formation of $\mathrm{MnO}$ and $\mathrm{Li}_{2} \mathrm{SiO}_{3}$ in samples calcined at temperatures above $700{ }^{\circ} \mathrm{C}$ can be connected, with $\mathrm{Li}_{2} \mathrm{MnSiO}_{4}$ decomposition. Average crystallites sizes calculated from diffraction lines broadening based on Scherrer equation are in the range of 17-19 $\mathrm{nm}$ depending on calcination temperature of the sample (Tab. 1). The calcination under carefully selected conditions leads to a transformation of a part of organic compounds from the gel matrix into thick carbon layer covering the active material. The temperature programmed oxidation (TPO) performed using TGA equipment confirmed presence of carbon in samples. Fig. $5 b$ presents exemplary TG/DTG/SDTA curves from TPO conducted under air flow in temperature range of $20-1000{ }^{\circ} \mathrm{C}$. The amounts of carbon in samples calcined in different temperatures were calculated from TPO measurements and are collected in Table 1. 
Measurements of the specific surface area of the samples were performed in Micrometrics ASAP 2010 using BET isotherm method. About $500 \mathrm{mg}$ of each sample was preliminary degassed at $250-300{ }^{\circ} \mathrm{C}$ for $3 \mathrm{~h}$ under pressure $0.26-0.4 \mathrm{~Pa}$. Then, $\mathrm{N}_{2}$ sorption was performed at pressure of $8 \cdot 10^{4} \mathrm{~Pa}$. One of pore size distribution plot with the adsorption-desorption curves inset is presented in Fig. 5a. Exact values of specific surface area and average pores diameters are presented in table 1.
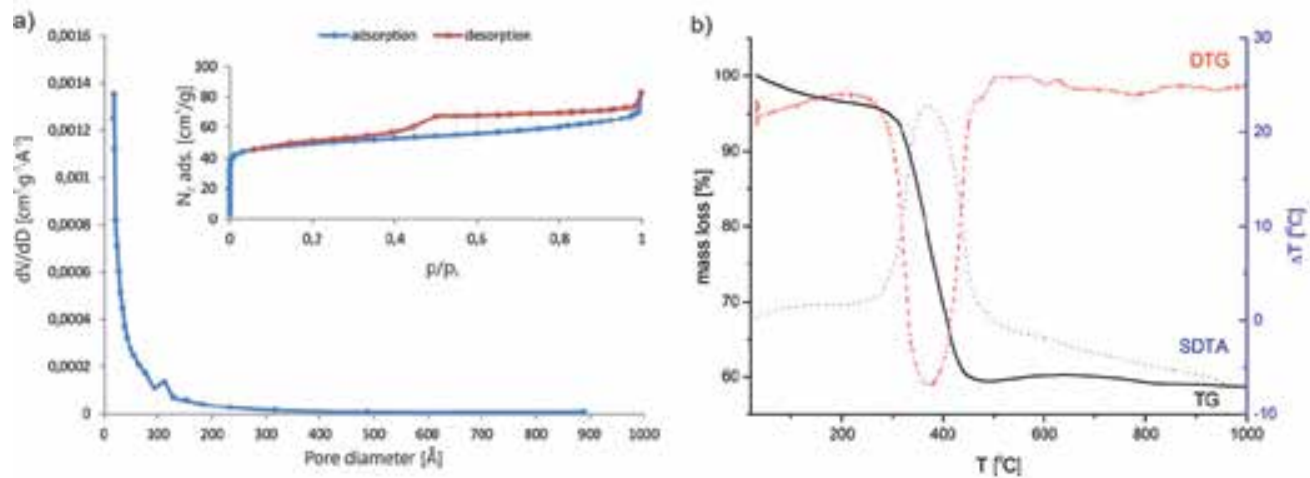

Figure 5. Adsorption-desorption isotherm and pore diameter distribution of $\mathrm{C} / \mathrm{Li}_{2} \mathrm{MnSiO}_{4}$ calcined at $600^{\circ} \mathrm{C}$, b) TGA/DTG/SDTA curves of $\mathrm{C} / \mathrm{Li}_{2} \mathrm{MnSiO}_{4}$ calcined at $600{ }^{\circ} \mathrm{C}$.

Low temperature $\mathrm{N}_{2}$-adsorption (BET) measurements of $\mathrm{C} / \mathrm{Li}_{2} \mathrm{MnSiO}_{4}$ composites show that specific surface area of samples decrease with increasing calcination temperature. Decrease of specific surface area is connected with graphitization of carbon during calcination at higher temperatures.

\begin{tabular}{|c|c|c|c|c|c|}
\hline Sample name & $\begin{array}{c}\text { Precursor } \\
\text { calcination } \\
\text { temperature }\end{array}$ & $\begin{array}{c}\text { Carbon } \\
\text { content }\end{array}$ & $\begin{array}{c}\text { Specific } \\
\text { surface } \\
\text { area (BET) }\end{array}$ & $\begin{array}{c}\text { Average } \\
\text { pore } \\
\text { dimension } \\
(\mathrm{BJH})\end{array}$ & $\begin{array}{c}\text { Crystallite } \\
\text { size (from } \\
\text { XRD) }\end{array}$ \\
\hline $\mathrm{C} / \mathrm{Li}_{2} \mathrm{MnSiO}_{4} @ 600$ & $600^{\circ} \mathrm{C}$ & $34 \%$ & $169 \mathrm{~m}^{2} / \mathrm{g}$ & $50 \AA$ & $17 \mathrm{~nm}$ \\
\hline $\mathrm{C} / \mathrm{Li}_{2} \mathrm{MnSiO}_{4} @ 700$ & $700^{\circ} \mathrm{C}$ & $32 \%$ & $152 \mathrm{~m}^{2} / \mathrm{g}$ & $72 \AA$ & $18 \mathrm{~nm}$ \\
\hline $\mathrm{C} / \mathrm{Li}_{2} \mathrm{MnSiO}_{4} @ 800$ & $800^{\circ} \mathrm{C}$ & $32 \%$ & $144 \mathrm{~m}^{2} / \mathrm{g}$ & $73 \AA$ & $19 \mathrm{~nm}$ \\
\hline $\mathrm{C} / \mathrm{Li}_{2} \mathrm{MnSiO}_{4} @ 900$ & $900^{\circ} \mathrm{C}$ & $35 \%$ & $57 \mathrm{~m}^{2} / \mathrm{g}$ & $73 \AA$ & $19 \mathrm{~nm}$ \\
\hline
\end{tabular}

Table 1. Morphology parameters of $\mathrm{C} / \mathrm{Li}_{2} \mathrm{MnSiO}_{4}$ composites calcined at different temperatures.

Electrical conductivity was measured using the $\mathrm{AC}(33 \mathrm{~Hz})$ 4-probe method within temperature range of $-40 \div 55{ }^{\circ} \mathrm{C}$. The carbon coated composite powders were so elastic that the standard preparation of pellets was impossible. The fine powder samples were placed into a glass tube and pressed by a screw-press between parallel gold disc electrodes $(\varnothing=5$ $\mathrm{mm}$ ) till the measured resistance remained constant. The results of electrical conductivity measurements are gather in Fig. 6. All composites exhibit good electrical conductivity (up to 0,36 S/cm for $\left.\mathrm{C} / \mathrm{Li}_{2} \mathrm{MnSiO}_{4} @ 900\right)$ in comparison with $\mathrm{Li}_{2} \mathrm{MnSiO}_{4}$ itself $\left(\sim 10^{-15} \mathrm{~S} / \mathrm{cm}\right.$ at room 
temperature - RT). It can be observed that temperature dependence of conductivity for composites calcined at higher temperatures (700, 800 and $900{ }^{\circ} \mathrm{C}$ ) seem unaffected, by temperature (metallic-like behavior). Conductivity value and its invariability against temperature indicate that carbon layers consist of graphite-like domains. In case of $\mathrm{C} / \mathrm{Li}_{2} \mathrm{MnSiO}_{4} @ 600^{\circ} \mathrm{C}$ the carbon layers is more disordered and probably consist of an activated-like carbon. Those results are consistent with BET measurements in case of graphitization process occurring at higher temperatures.

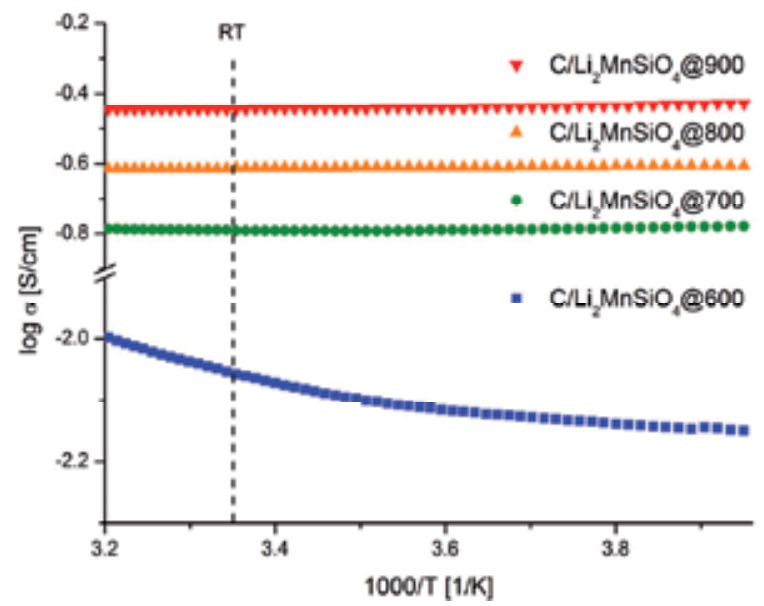

Figure 6. Electrical conductivity measurement of $\mathrm{C} / \mathrm{Li}_{2} \mathrm{MnSiO}_{4}$ (temp. range: -20 to $40^{\circ} \mathrm{C}$ ). Dash line represent room temperature $(\mathrm{RT})$.

Selected samples were investigated using transmission electron microscope (TEM) (Fig. 7 and Fig. 8). Micrographs were collected on TECNAI G2 F20 (200 kV) coupled with an energy dispersive $\mathrm{X}$-ray spectrometer (EDAX).

TEM micrographs reveal well dispersed lithium manganese orthosilicate grains (dark grey and black dots) in the carbon matrix. Figures 7a; 7c; 7d present C/Li2 $\mathrm{MnSiO}_{4} @ 600$ at different magnifications. Fig. 7b shows STEM-HAADF (Scanning Transmission Electron Microscopy High Angular Annular Dark Field) image of the $\mathrm{C} / \mathrm{Li}_{2} \mathrm{MnSiO}_{4} @ 600$ composite. Energy dispersive X-ray spectroscopy (EDS) (Fig. 7b1; 7b2) confirmed presence of lithium manganese silicate in the spots marked in the micrograph $7 \mathrm{~b}(\mathrm{Cu}$ peaks in all EDS spectra originate from TEM copper grid). High resolution electron microscopy image (HREM) shows single grain of $\mathrm{Li}_{2} \mathrm{MnSiO}_{4}$ (Fig. 7e). Corresponding to that IFFT (Inverse Fast Furrier Transform) image (Fig. 7f) clearly shows a crystalline structure of obtained material which can be identified as $\mathrm{Li}_{2} \mathrm{MnSiO}_{4}$. Grains in the composite have sizes in a range of $5-10 \mathrm{~nm}$. Microstructure of a $\mathrm{C} / \mathrm{Li}_{2} \mathrm{MnSiO}_{4}$ composite calcined at $700{ }^{\circ} \mathrm{C}$ is shown in Fig. 8 . $\mathrm{C} / \mathrm{Li}_{2} \mathrm{MnSiO}_{4} @ 700{ }^{\circ} \mathrm{C}$ composite has a bimodal distribution of a crystallite size. TEM micrographs show presence of small $(5-10 \mathrm{~nm}) \mathrm{Li}_{2} \mathrm{MnSiO}_{4}$ crystallites, analogues of $\mathrm{C} / \mathrm{Li}_{2} \mathrm{MnSiO}_{4} @ 600{ }^{\circ} \mathrm{C}$ and a bigger, well crystallized silicates grains, in a range of $50-150 \mathrm{~nm}$ (Fig. 8a, c, d). Both types of crystallites in the sample have the same composition which is confirmed by EDS analysis presented in fig. $8 b_{1}, b_{2}, b_{3}$. 


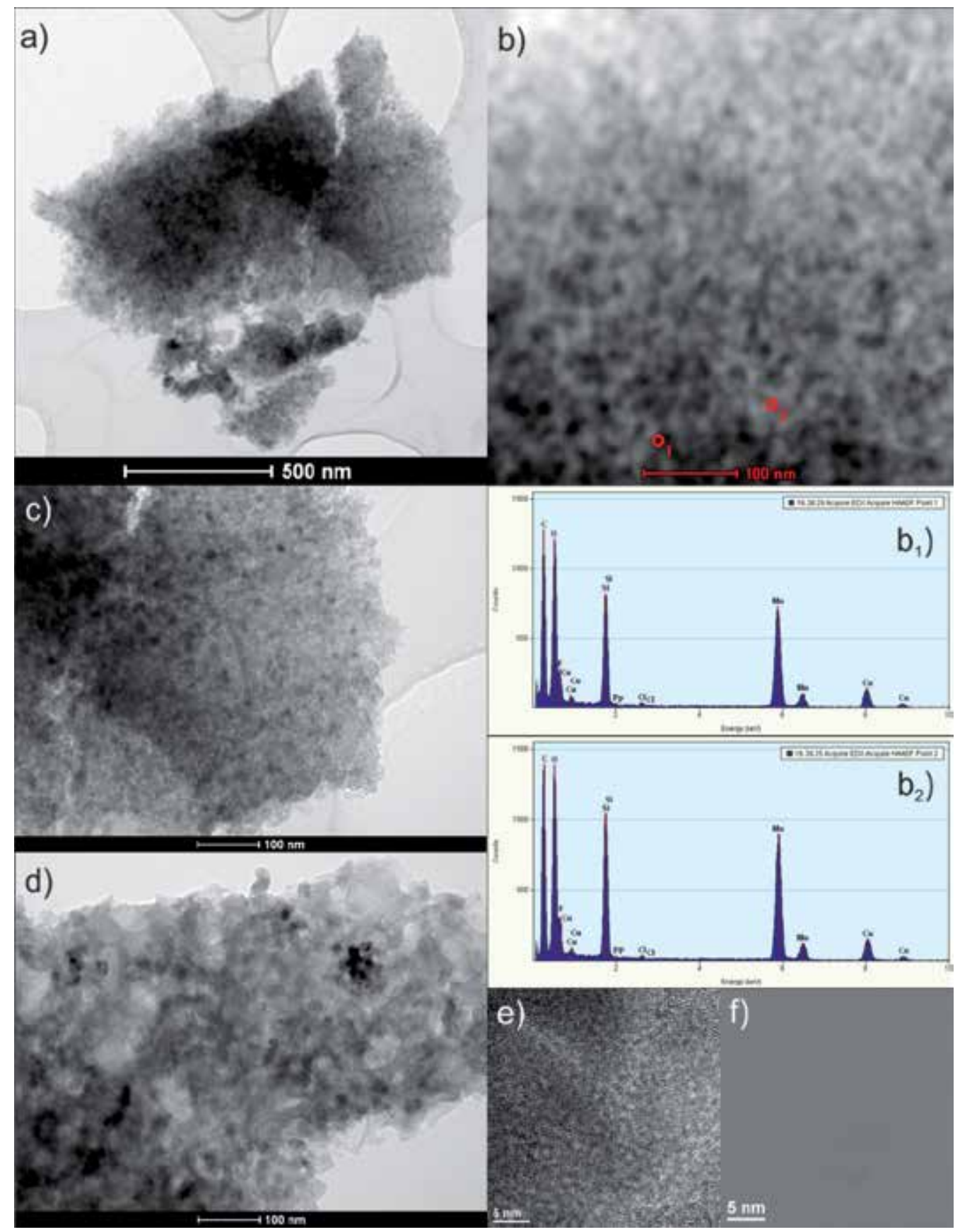

Figure 7. TEM micrographs of $\mathrm{C} / \mathrm{Li}_{2} \mathrm{MnSiO}_{4} @ 600$. Micrographs a), c) and d) present bright field images of $\mathrm{C} / \mathrm{Li}_{2} \mathrm{MnSiO}_{4} @ 600$ composite in different magnification; b) microstructure image observed in STEMHAADF with EDS analysis ( $b_{1}$; $b_{2}$ ) from marked points; e) high resolution micrograph (HREM) of a single grain and IFFT image $f$ ) from the same region. 

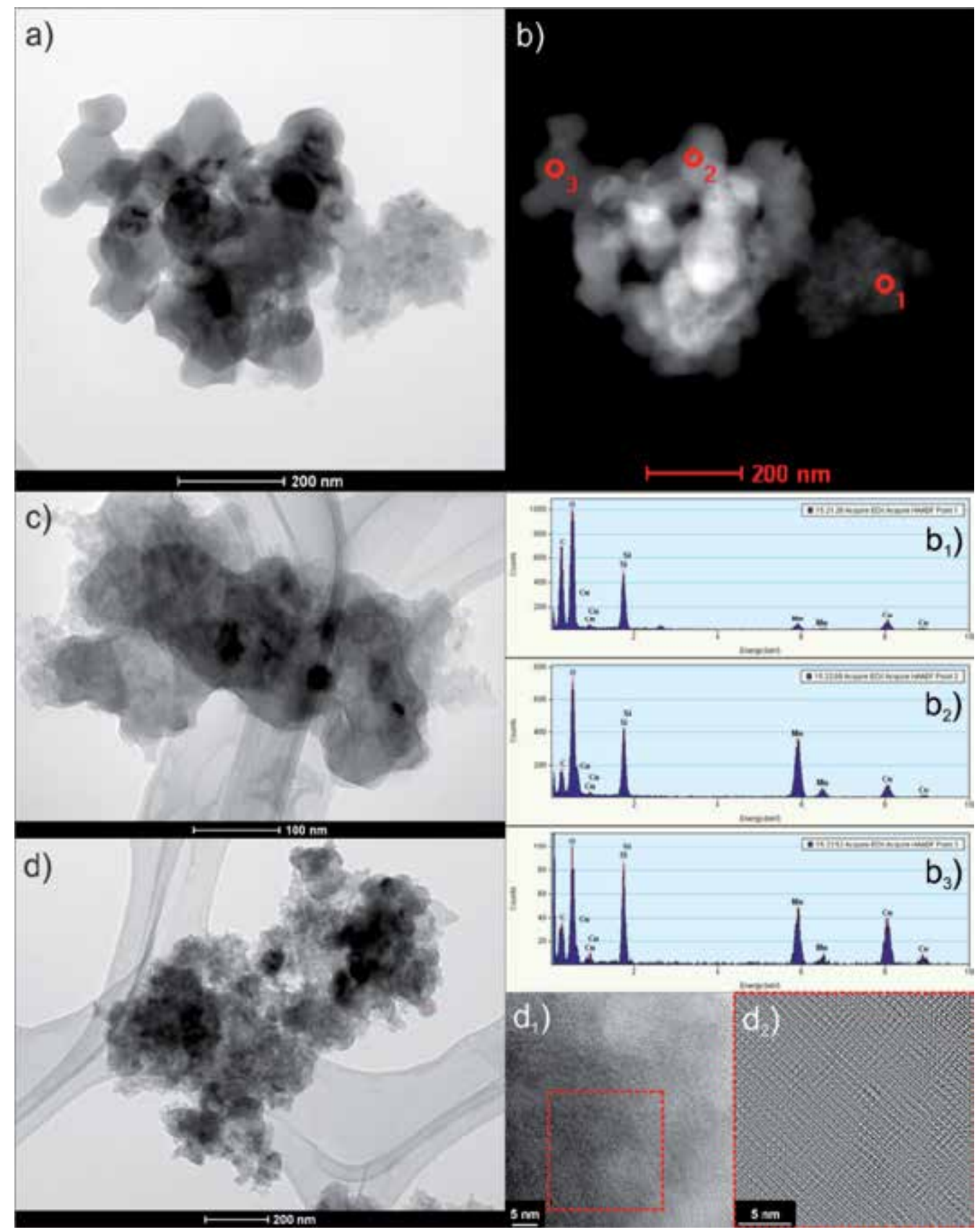

Figure 8. TEM micrographs of $\mathrm{C} / \mathrm{Li}_{2} \mathrm{MnSiO}_{4} @ 700$. Micrographs a), c) and d) presents bright field images of $\mathrm{C} / \mathrm{Li}_{2} \mathrm{MnSiO}_{4} @ 700$ composite in different magnification; b) microstructure image observed in STEMHAADF with EDS analysis $\left(b_{1} ; b_{2} ; b_{3}\right)$ from selected points; $\left.d_{1}\right)$ high resolution micrograph (HREM) of region marked in Fig. 8d; $d_{2}$ ) IFFT image from square marked in $d_{1}$ ). 
STEM-HAADF image (Fig. 8b) from the same region as a bright field Fig. 8a shows points from where EDS analysis was conducted. All of lithium manganese orthosilicate crystallites in $\mathrm{C} / \mathrm{Li}_{2} \mathrm{MnSiO}_{4} @ 700{ }^{\circ} \mathrm{C}$ are well dispersed and fully covered in amorphous carbon. HREM image in Fig. $8 \mathrm{~d}_{1}$ with IFFT in Fig. $8 \mathrm{~d}_{2}$ shows crystalline structure of $\mathrm{Li}_{2} \mathrm{MnSiO}_{4}$ grain.

\section{5. $\mathrm{C} / \mathrm{Li}_{2} \mathrm{MnSiO}_{4}$ composite cathode material}

Nevertheless obtained composites show homogeneous distribution of particles in carbon matrix and exhibit good electrical conductivity they work poorly in a battery cell (see paragraph 5.2). Thickness of primary carbon coating on active material grains strongly limits the electrochemical performance of composite.

\section{1. $\mathrm{CCL} / \mathrm{Li}_{2} \mathrm{MnSiO}_{4}$ composite preparation and properties}

Preparation of composites with well define morphology of carbon layers and optimal carbon content was achieved by burning out the primary carbon and recoating $\mathrm{Li}_{2} \mathrm{MnSiO}_{4}$ nanosized grains with conductive carbon layers (CCL/Li2 $\mathrm{MnSiO}_{4}$ composites). Burning out of primary carbon formed in the sample during synthesis process was carried through calcination of $\mathrm{C} / \mathrm{Li}_{2} \mathrm{MnSiO}_{4} @ 600$ and $\mathrm{C} / \mathrm{Li}_{2} \mathrm{MnSiO}_{4} @ 700$ under air flow at $300{ }^{\circ} \mathrm{C}$ for $3 \mathrm{~h}$. CCL/ $/ \mathrm{Li}_{2} \mathrm{MnSiO}_{4}$ composites were produced by wet polymer precursor deposition on active material grains and subsequent controlled pyrolysis [14, 20, 34]. Poly-N-vinylformamide (PNVF) obtained by radical-free polymerization of $\mathrm{N}$ vinylformamide (Aldrich) with pyromellitic acid (PMA) additive (5-10 $\mathrm{wt} \%$ ) was used as a carbon polymer precursor [34]. To achieve an impregnation, $\mathrm{Li}_{2} \mathrm{MnSiO}_{4}$ grains were suspended in water polymer solution (8-15 wt\%). Finally, samples were dried up in an air drier at $90{ }^{\circ} \mathrm{C}$ for $24 \mathrm{~h}$. Prepared samples were pyrolyzed at $600{ }^{\circ} \mathrm{C}$ for $6 \mathrm{~h}$ under inert atmosphere (Ar).

Burning out primary carbon from the surface of lithium manganese silicate in air atmosphere leads to partial decomposition of active material. Fig. 9 shows XRD patterns of C/Li2 $\mathrm{MnSiO}_{4} @ 600, \mathrm{Li}_{2} \mathrm{MnSiO}_{4} @ 600$ without carbon and CCL/Li2 $\mathrm{MnSiO}_{4} @ 600$ composite after coating with CCL from polymer precursor.

In diffraction patterns of $\mathrm{Li}_{2} \mathrm{MnSiO}_{4} @ 600$ it is clearly visible that after burning out primary carbon layer new phase appears in the sample $\left(\mathrm{LiMn}_{2} \mathrm{O}_{4}\right)$. Proposed carbon coating process can reverse decomposition of $\mathrm{Li}_{2} \mathrm{MnSiO}_{4}$. After coating $\mathrm{Li}_{2} \mathrm{MnSiO}_{4}\left(P m n 2_{1}\right)$ is a dominant phase in CCL/Li2 $\mathrm{MnSiO}_{4} @ 600$ and all the lines, except for very low intensity line around $19^{\circ}$, can be associated with $\mathrm{Li}_{2} \mathrm{MnSiO}_{4}$ phase. CCL preparation procedure influence active material grain size. Average crystallite size calculated using Scherer equation in $\mathrm{CCL} / \mathrm{Li}_{2} \mathrm{MnSiO}_{4} @ 600$ is ca. $25 \mathrm{~nm}$. TEM analysis confirms increase of crystallites diameters. $\mathrm{Li}_{2} \mathrm{MnSiO}_{4}$ grains observed under TEM are in the range 25-75 nm. TEM micrographs of CCL/ $\mathrm{Li}_{2} \mathrm{MnSiO}_{4} @ 600$ are presented in Fig. 10. 


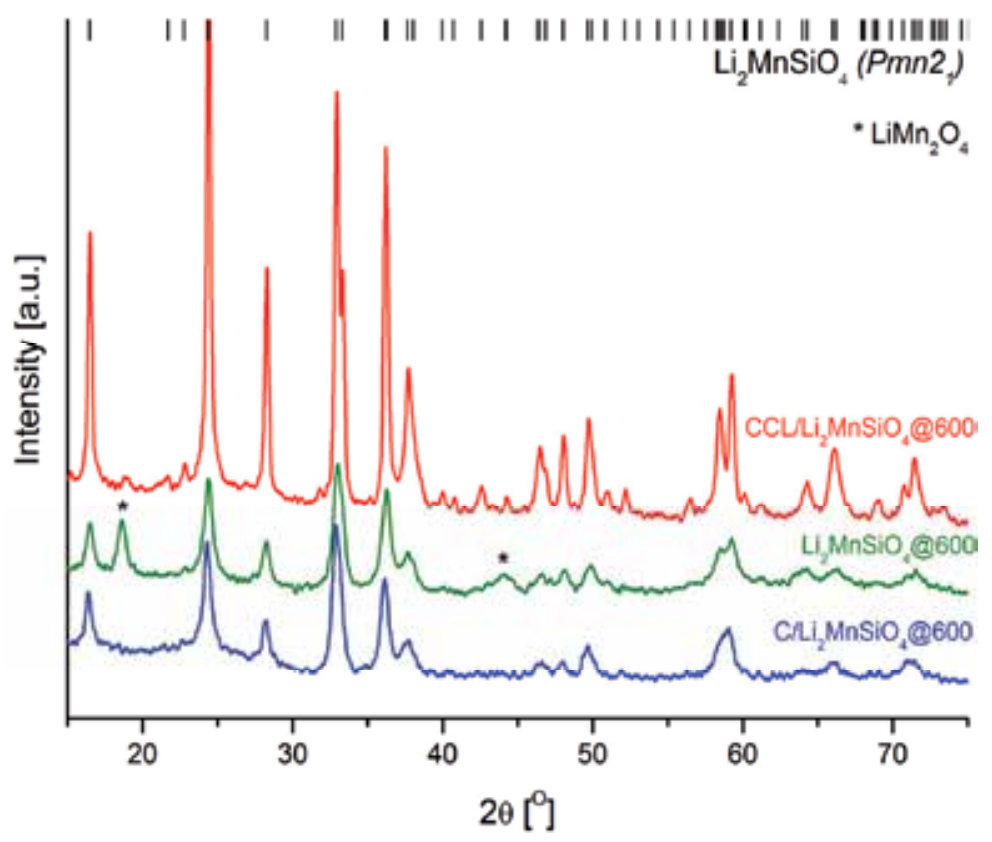

Figure 9. XRD patterns of $\mathrm{C} / \mathrm{Li}_{2} \mathrm{MnSiO}_{4} @ 600, \mathrm{Li}_{2} \mathrm{MnSiO}_{4} @ 600$ and $\mathrm{CCL} / \mathrm{Li}_{2} \mathrm{MnSiO}_{4} @ 600$.

Bright field micrographs (Fig. 10a, c, d) shows crystallites of $\mathrm{Li}_{2} \mathrm{MnSiO}_{4}$ covered with conductive carbon layers (CCL). Formed carbon coatings adhere well to the surface of active material grains, no voids are visible at the $\mathrm{CCL} / \mathrm{Li}_{2} \mathrm{MnSiO}_{4}$ interface (Fig. 10e). Silicate grains are uniformly covered with approximately 4-5 nm thick CCL. EDS analysis once again confirm presence of lithium manganese silicate in the composite (Fig. 10b, $b_{1}$ ). Carbon content in samples was calculated from TPO measurements and they are displayed in Table 2 .

\begin{tabular}{|c|c|c|}
\hline Sample name & $\begin{array}{c}\text { Precursor calcination } \\
\text { temperature }\end{array}$ & Carbon content \\
\hline $\mathrm{CCL} / \mathrm{Li}_{2} \mathrm{MnSiO}_{4} @ 600 \_7.5 \%$ & $600{ }^{\circ} \mathrm{C}$ & $7.5 \%$ \\
\hline $\mathrm{CCL} / \mathrm{Li}_{2} \mathrm{MnSiO}_{4} @ 600 \_12 \%$ & $600^{\circ} \mathrm{C}$ & $12 \%$ \\
\hline $\mathrm{CCL} / \mathrm{Li}_{2} \mathrm{MnSiO}_{4} @ 700 \_5 \%$ & $700{ }^{\circ} \mathrm{C}$ & $5 \%$ \\
\hline $\mathrm{CCL} / \mathrm{Li}_{2} \mathrm{MnSiO}_{4} @ 700 \_10 \%$ & $700{ }^{\circ} \mathrm{C}$ & $10 \%$ \\
\hline
\end{tabular}

Table 2. Carbon content of $\mathrm{CCL} / \mathrm{Li}_{2} \mathrm{MnSiO}_{4}$ composites. 


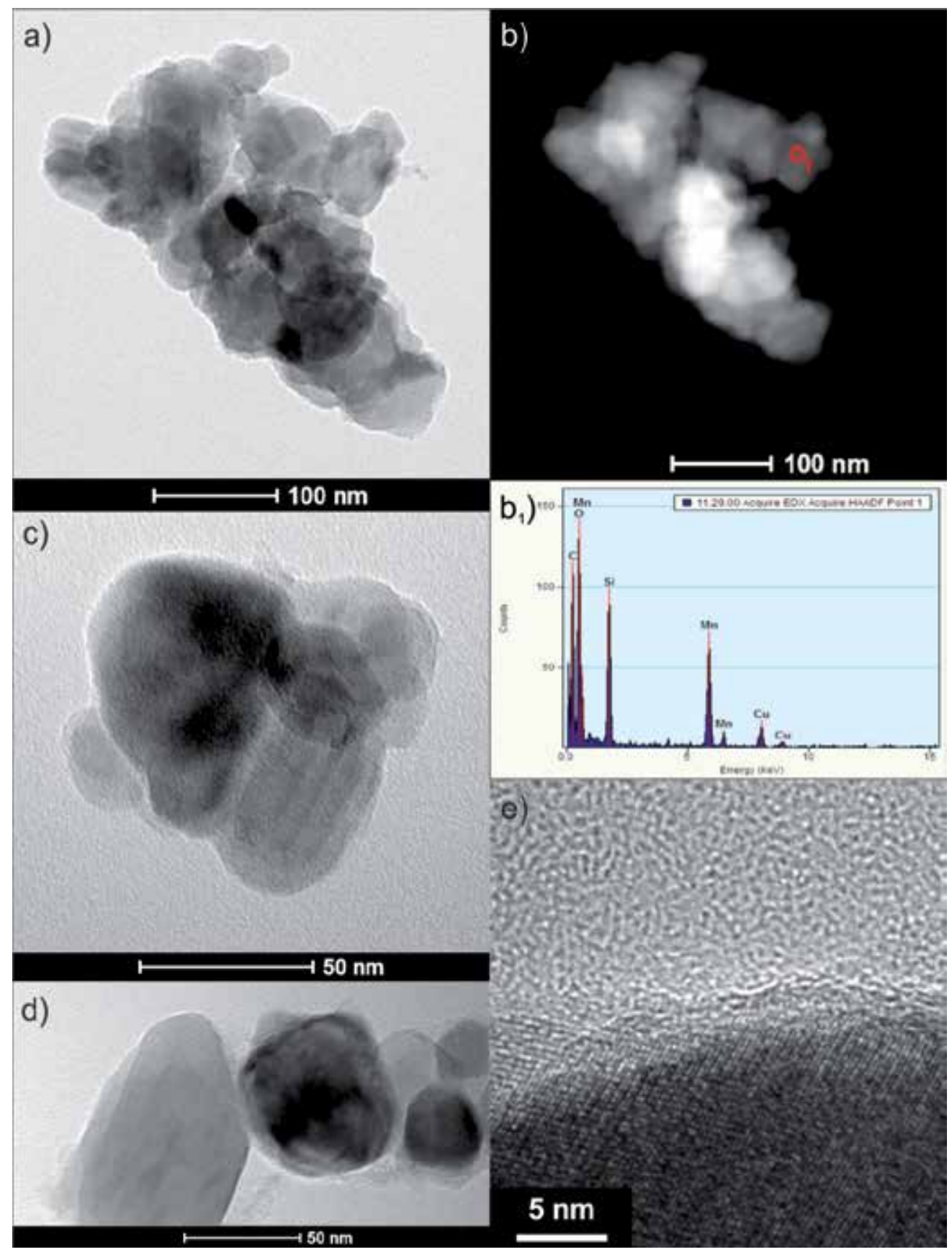

Figure 10. TEM micrographs of CCL/Li2 $\mathrm{MnSiO}_{4} @ 600$. Micrographs a), c) and d) presents bright field images of $\mathrm{CCL} / \mathrm{Li}_{2} \mathrm{MnSiO}_{4} @ 600$ composite; b) microstructure image observed in STEM-HAADF with EDS analysis ( $\left.b_{1}\right)$ from selected point; e) HREM micrograph of carbon coating on the active material grain surface. 


\subsection{Electrochemical properties of $\mathrm{C} / \mathrm{Li}_{2} \mathrm{MnSiO}_{4}$ composite cathode}

The charge-discharge cycling studies of $\mathrm{Li}^{2} \mathrm{Li}^{+} /\left(\mathrm{C} / \mathrm{Li}_{2} \mathrm{MnSiO}_{4}\right)$ cells were conducted in a four electrode configuration using CR2032 assembly between 2.7 and $4.7 \mathrm{~V}$ at C/200 rate at room temperature. $\mathrm{LiPF}_{6}$ solution $1 \mathrm{M}$ in EC/DEC (1:1) was used in the cells as an electrolyte. The galvanostatic measurements were carried out using ATLAS 0961 MBI test system. Chargedischarge tests were conducted for all CCL composites listed in table 2, composites with primary carbon $\left(\mathrm{C} / \mathrm{Li}_{2} \mathrm{MnSiO}_{4} @ 600, \mathrm{C} / \mathrm{Li}_{2} \mathrm{MnSiO}_{4} @ 700\right.$ and $\left.\mathrm{C} / \mathrm{Li}_{2} \mathrm{MnSiO}_{4} @ 800\right)$ and standard composites ( $\mathrm{Li}_{2} \mathrm{MnSiO}_{4} @ 600 \_\mathrm{CB}$ and $\mathrm{Li}_{2} \mathrm{MnSiO}_{4} @ 700 \_\mathrm{CB}$ ). Standard composites were prepared by mixing $\mathrm{Li}_{2} \mathrm{MnSiO}_{4}$ powder with commercial carbon additive - carbon black (CB). 15 wt.\% of carbon was used. Results collected from charge/discharge tests are presented in fig. 11-16.

$\mathrm{C} / \mathrm{Li}_{2} \mathrm{MnSiO}_{4} @ 600$ sample did not show any reversible capacity (Fig. 11a). Lack of electrochemical activity of $\mathrm{C} / \mathrm{Li}_{2} \mathrm{MnSiO}_{4} @ 600$ is connected with too high carbon loading. High carbon content (34\%) is responsible for limiting of ionic conductivity in the composite and for surface polarization. Galvanostatic cycling studies of $\mathrm{C} / \mathrm{Li}_{2} \mathrm{MnSiO}_{4} @ 700$ and $\mathrm{C} / \mathrm{Li}_{2} \mathrm{MnSiO}_{4} @ 800$ revealed reversible capacity in a range of 30 $\mathrm{mAh} \cdot \mathrm{g}^{-1}$. Fig. 11b show charge and discharge capacity for 7 cycles of $\mathrm{C} / \mathrm{Li}_{2} \mathrm{MnSiO}_{4} @ 700$ composite. After first four cycles reversible capacity stabilizes at about $30 \mathrm{mAh} \cdot \mathrm{g}^{-1}$. Very poor performance of this sample is also connected with high amount of carbon in prepared composite $(32 \%)$.

Standard composites obtained by mixing active silicate materials with carbon black (15\%) show extremely low reversible capacity as well (Fig. 12a and 12b). In this case, carbon additive does not provide sufficient electrical contact between active material grains. Due to this fact, even under low $\mathrm{C} / 200$ rate samples performed very poorly in charge/discharge tests.

a)

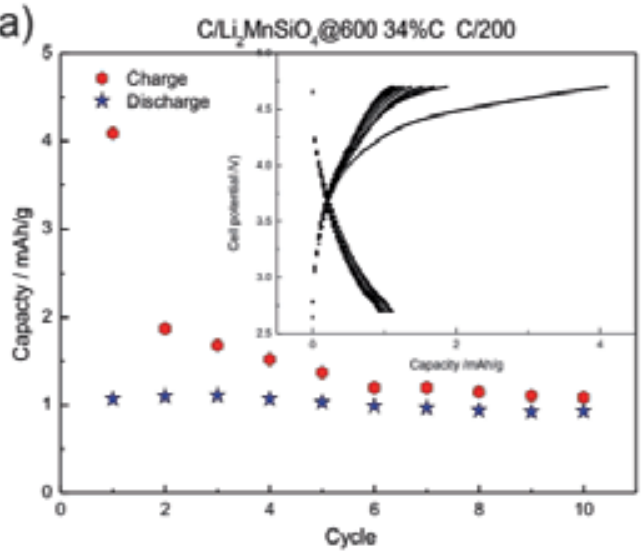

b)

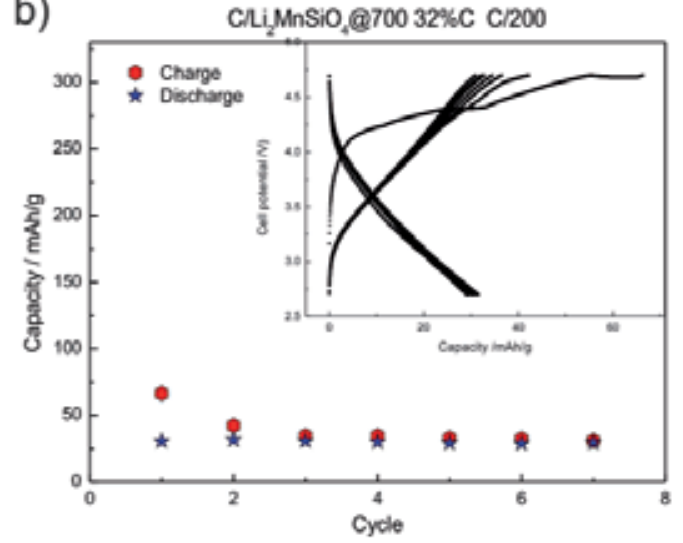

Figure 11. Cell cycling behavior of: a) C/Li2 $\mathrm{MnSiO}_{4} @ 600$, b) C/Li2 $\mathrm{MnSiO}_{4} @ 700$. 
a)

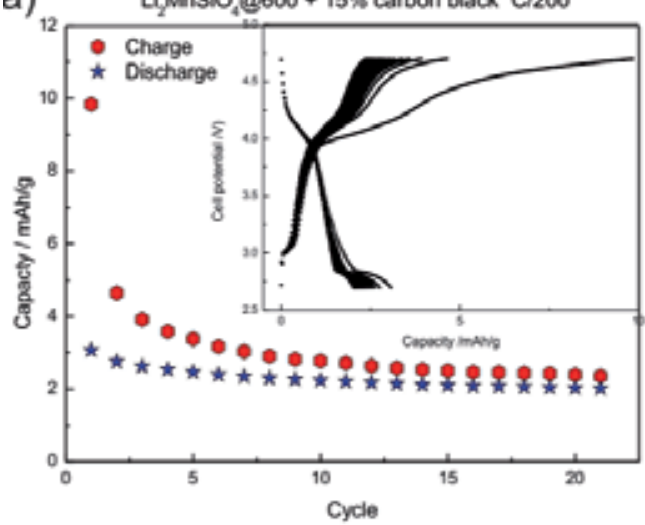

b)

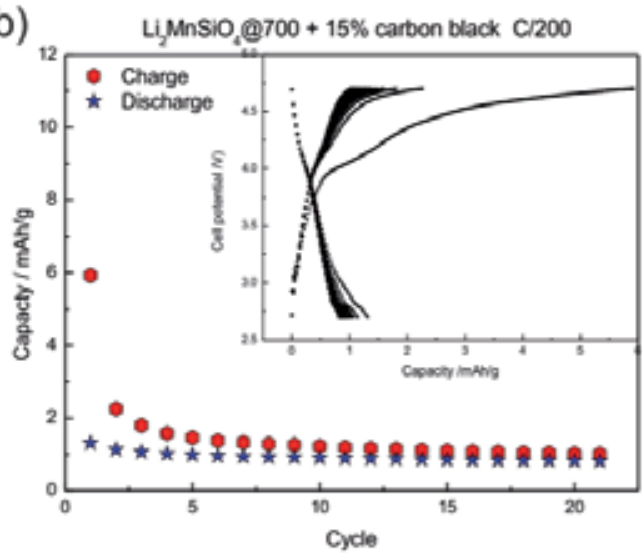

Figure 12. Cell cycling behavior of standard composites $\mathrm{Li} 2 \mathrm{MnSiO} 4+$ carbon black $(15 \%$ carbon content)

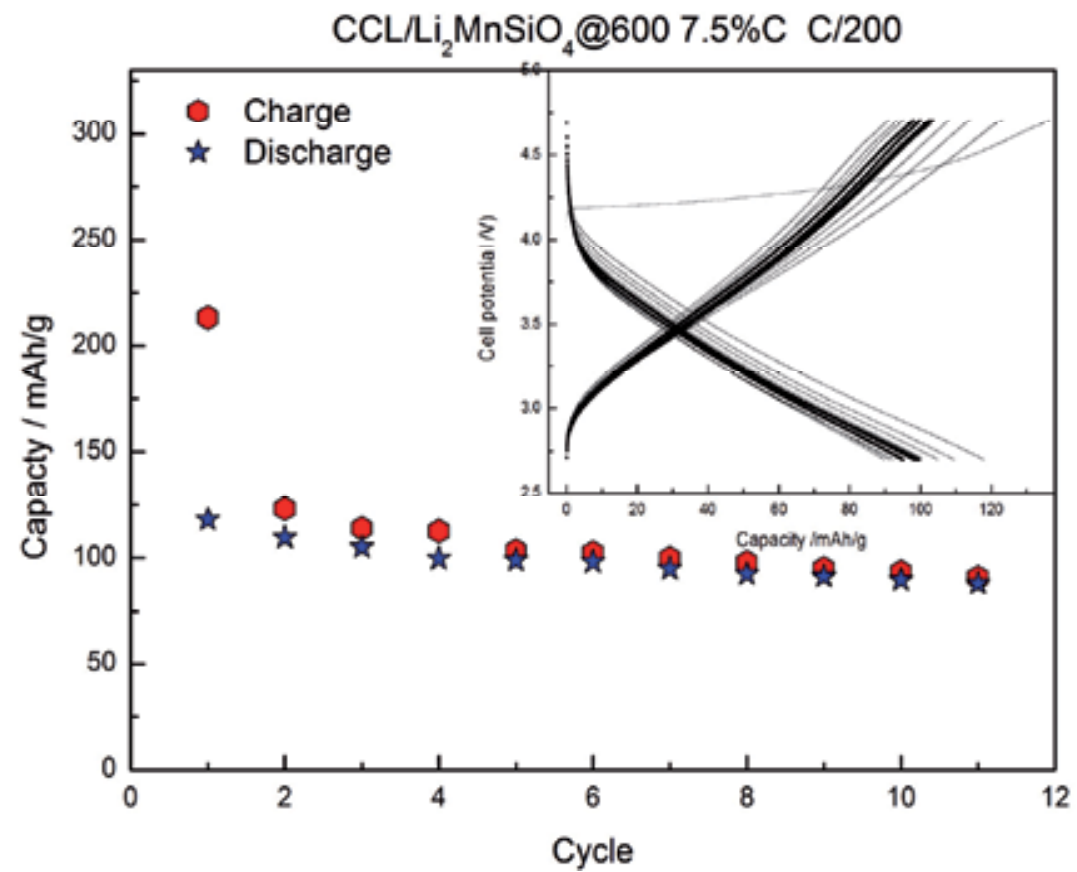

Figure 13. Cell cycling behavior of CCL/ $\mathrm{Li}_{2} \mathrm{MnSiO}_{4} @ 600$ (7.5\% carbon content) 


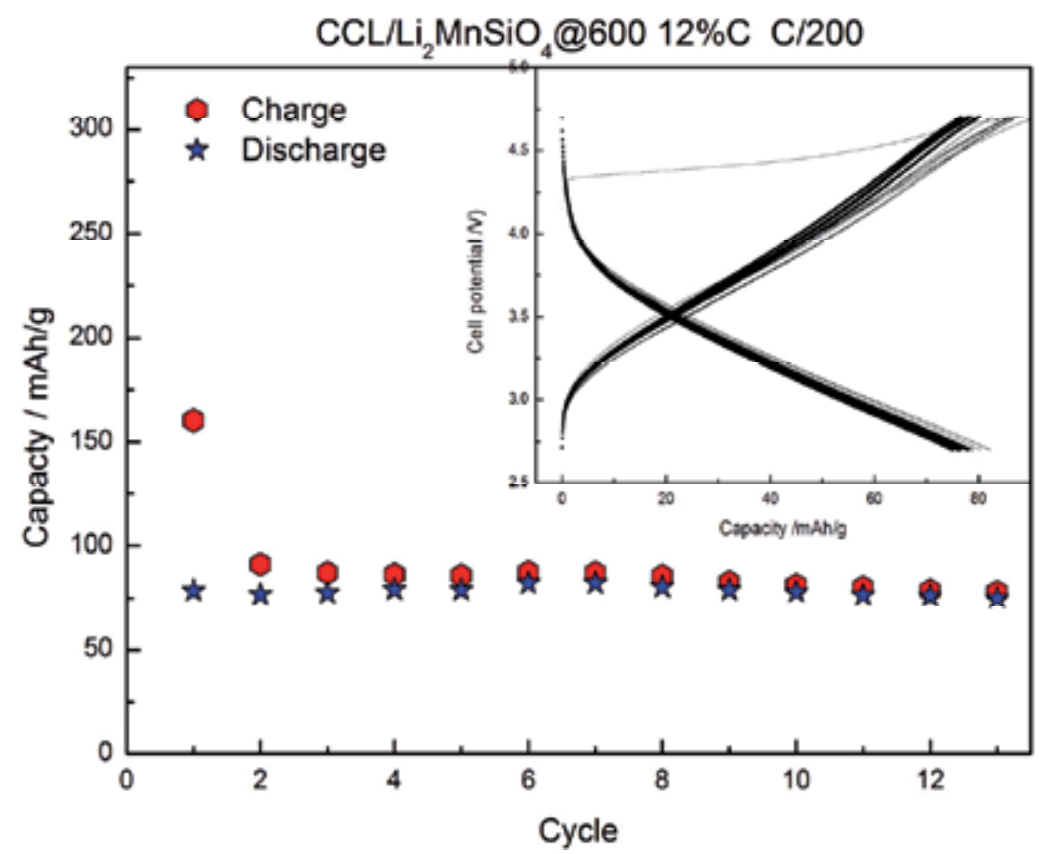

Figure 14. Cell cycling behavior of CCL/Li2 $\mathrm{MnSiO}_{4} @ 600$ (12\% carbon content)

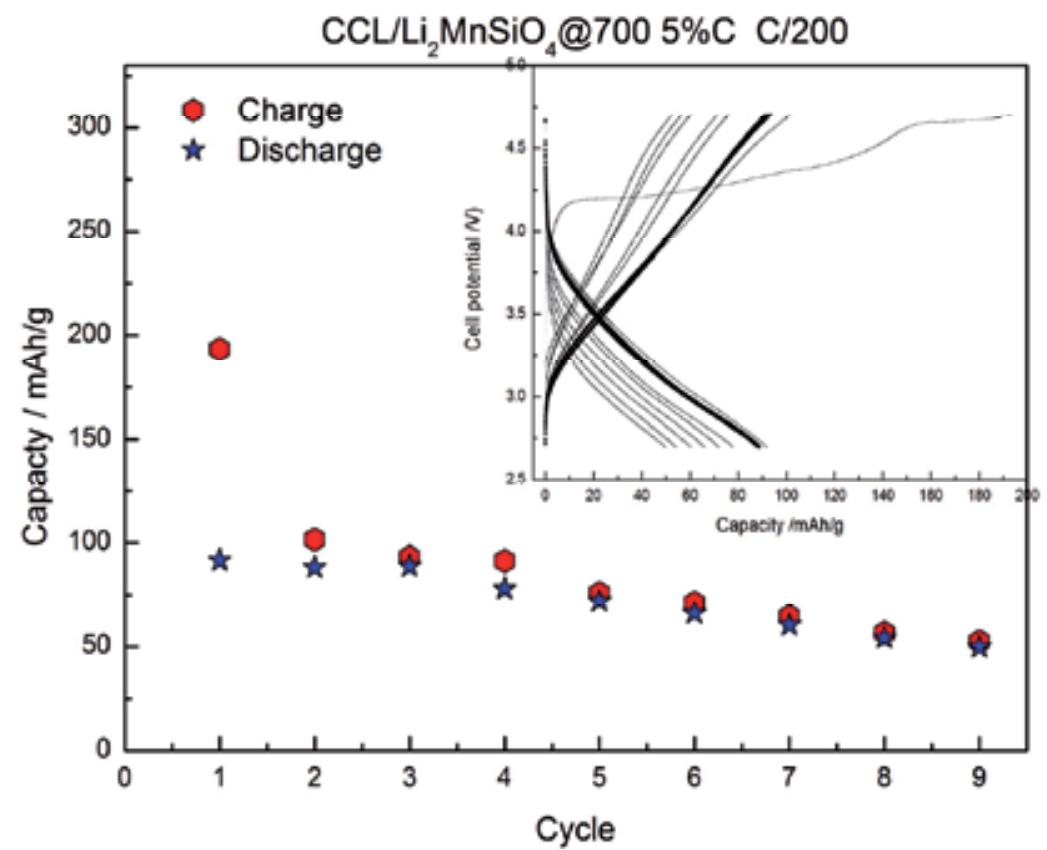

Figure 15. Cell cycling behavior of $\mathrm{CCL} / \mathrm{Li}_{2} \mathrm{MnSiO}_{4} @ 700$ (5\% carbon content) 


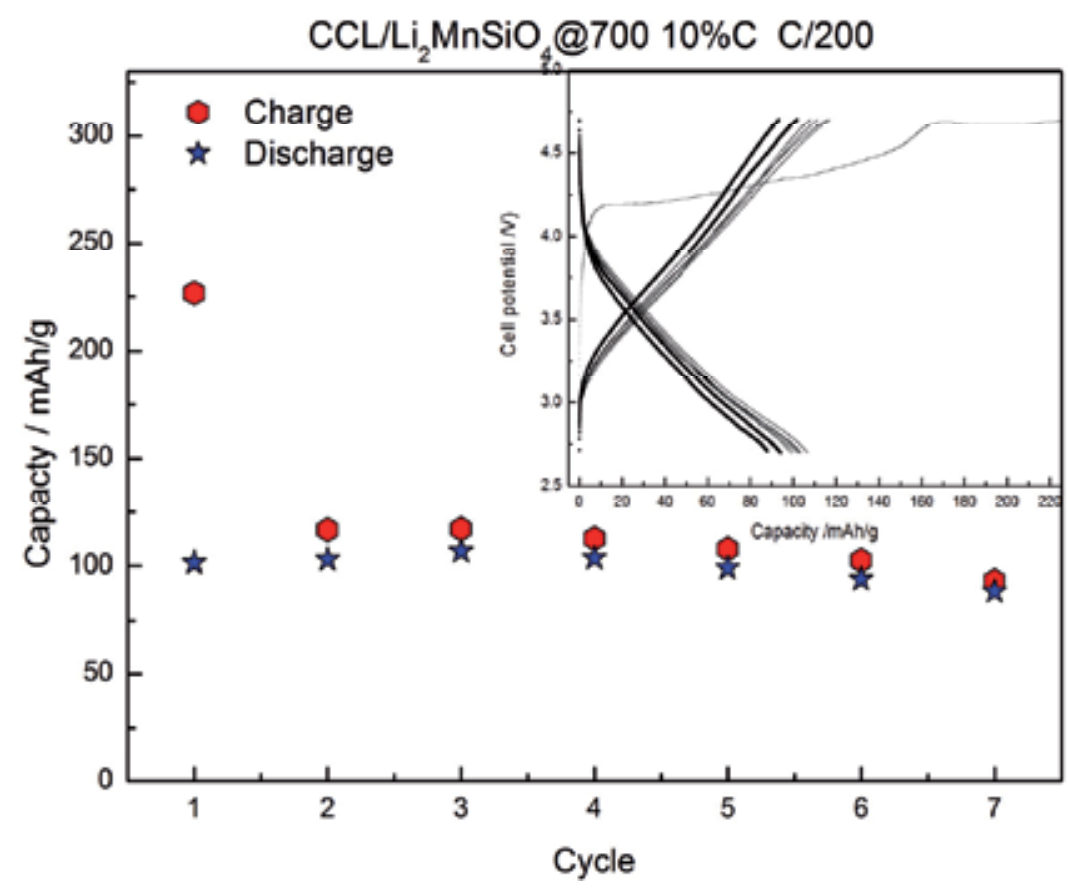

Figure 16. Cell cycling behavior of $\mathrm{CCL} / \mathrm{Li}_{2} \mathrm{MnSiO}_{4} @ 700$ (10\% carbon content)

Fig. 13-16 show cycling behavior of CCL composites with different amounts of carbon. Delithation of CCL composites after initial charging is in the range of $48-68 \%$ (charged CCL/Li2 $\mathrm{MnSiO}_{4} @ 600 \_12 \% \mathrm{C}$ - correspond to composition $\mathrm{Li}_{1.04} \mathrm{MnSiO}_{4}$ and charged CCL/Li2 $\mathrm{MnSiO}_{4} @ 700 \_10 \% \mathrm{C}$ - correspond to composition $\left.\mathrm{Li} 0.64 \mathrm{MnSiO}_{4}\right)$. The observed capacity loss in the first cycles is related to SEI formation in CCL. During lithium extraction, $\mathrm{Mn}^{3+}$ and $\mathrm{Mn}^{4+}$ appear, and at the same time $\mathrm{Li}_{2} \mathrm{MnSiO}_{4}$ undergoes decomposition caused by Jahn-Teller distortion associated with changes in lattice parameters during $\mathrm{Mn}^{3+} \rightarrow \mathrm{Mn}^{4+}$ transition [30]. Despite the fact that crystalline structure collapses, discharge capacity is around $90-110 \mathrm{mAh} / \mathrm{g}$. CCL composites produced from $\mathrm{Li}_{2} \mathrm{MnSiO}_{4}$ calcined at $600{ }^{\circ} \mathrm{C}$ exhibit better coulombic efficiency than $\mathrm{Li}_{2} \mathrm{MnSiO}_{4}$ calcined at $700{ }^{\circ} \mathrm{C}$, the reversible capacity after 10 cycle is close to $100 \mathrm{mAh} / \mathrm{g}$. The results indicate that coulombic efficiency of cathode material depends on grain size and homogeneity in grain size distribution, while cell capacity is limited by carbon coating performance.

\section{Conclusions}

Dilithium manganese orthosilicate - a high energy density cathode material - was successfully synthesized by sol-gel Pechini method. Encapsulation of nanosized grains of $\mathrm{Li}_{2} \mathrm{MnSiO}_{4}$ in carbon matrix, resulted from organic precursor, avoided further sintering. Different crystallite size was obtained, in particular, nanoparticles within range of 5-10 nm. High chemical stability of this material under a highly reductive environment was observed. Application of carbon coating improved electrical conductivity of cathode material to the 
satisfactory level of $\sim 10^{-0.5} \mathrm{~S} / \mathrm{cm}$. Thickness of carbon coating on active material grains strongly limits the electrochemical performance of composite. Formation of $\mathrm{CCL} / \mathrm{Li}_{2} \mathrm{MnSiO}_{4}$ composites significantly improved electrochemical performance of cathode materials, showing a reversible capacity of $90-110 \mathrm{mAh} / \mathrm{g}$ after 10 cycles. Electrochemical tests indicated that composite preparation should be optimized in terms of carbon content, CCL performance and homogeneity of a crystallite size. It was found that coulombic efficiency of cathode material depends on grain size and homogeneity in grain size distribution, while cell capacity is limited by carbon coating performance.

\section{Author details}

Marcin Molenda*, Michał Świętosławski and Roman Dziembaj

Jagiellonian University, Faculty of Chemistry, Krakow, Poland

\section{Acknowledgement}

This work has been financially supported by the Polish National Science Centre under research grant no. N N209 088638, and by the European Institute of Innovation and Technology under the KIC InnoEnergy NewMat project. The part of the measurements was carried out with the equipment purchased thanks to the financial support of the European Regional Development Fund in the framework of the Polish Innovation Economy Operational Program (POIG.02.01.00-12-023/08). One of the authors (M.Ś.) acknowledge a financial support from the International PhD-studies programme at the Faculty of Chemistry Jagiellonian University within the Foundation for Polish Science MPD. TEM analysis were carried out in Laboratory of Transmission Analytical Electron Microscopy at the Institute of Metallurgy and Material Science, Polish Academy of Sciences.

\section{References}

[1] Whittingham M.S. Electrical Energy Storage and Intercalation Chemistry. Science 1976;192 1126-1127.

[2] Nagaura T., Tozawa K. Lithium ion rechargeable battery. Progress in Batteries \& Solar Cells 1990;9 209-217.

[3] Tarascon J.-M., Guyomard D. The $\mathrm{Li}_{1+x} \mathrm{Mn}_{2} \mathrm{O}_{4} / \mathrm{C}$ rocking-chair system: a review Electrochimica Acta 1993;38(9), 1221-1231.

[4] Tarascon J.-M., Armand M. Issues and challenges facing rechargeable lithium batteries. Nature 2001;414(6861) 359-367.

[5] Zhang Z., Gong Z., Yang Y. Electrochemical Performance and Surface Properties of Bare and $\mathrm{TiO}_{2}$-Coated Cathode Materials in Lithium-Ion Batteries. The Journal of Physical Chemistry B 2004;108(45) 17546-17552.

\footnotetext{
" Corresponding Author
} 
[6] Molenda M., Dziembaj R., Podstawka E., Proniewicz L.M. Changes in local structure of lithium manganese spinels (Li:Mn=1:2) characterised by XRD, DSC, TGA, IR, and Raman spectroscopy. Journal of Physics and Chemistry of Solids 2005;66(10) 1761-1768.

[7] Dziembaj R., Molenda M. Stabilization of the spinel structure in $\mathrm{Li}_{1}+\delta 7 \mathrm{Mn}_{2}-\delta 7 \mathrm{O}_{4}$ obtained by sol-gel method. Journal of Power Sources 2003;119-121C 121-124.

[8] Molenda J., Marzec J., Świerczek K., Ojczyk W., Ziemnicki M., Wilk P., Molenda M., Drozdek M., Dziembaj R. The effect of 3d substitutions in the manganese sublattice on the charge transport mechanism and electrochemical properties of manganese spinel. Solid State Ionics 2004;171(3-4) 215-227.

[9] Molenda M., Dziembaj R., Podstawka E., Proniewicz L.M., Piwowarska Z. An attempt to improve electrical conductivity of the pyrolysed carbon-LiMn2 $\mathrm{O}_{4-\mathrm{y}} \mathrm{S}_{\mathrm{y}} \quad(0 \leq \mathrm{y} \leq 0.5)$ composites. Journal of Power Sources 2007;174(2) 613-618.

[10] Molenda M., Dziembaj R., Podstawka E., Łasocha W., Proniewicz L.M. Influence of sulphur substitution on structural and electrical properties of lithium-manganese spinels. J. Phys. Chem. Solids 2006;67(5-6) 1348-1350.

[11] Chung K.Y., Yoon W.-S., Lee H.S., Yang X.-Q., McBreen J., Deng B.H., Wang X.Q., Yoshio M., Wang R., Gui J., Okada M. Comparative studies between oxygen-deficient $\mathrm{LiMn}_{2} \mathrm{O}_{4}$ and Al-doped $\mathrm{LiMn}_{2} \mathrm{O}_{4}$. Journal of Power Sources 2005;146(1-2) 226-231.

[12] Padhi A.K., Nanjundaswamy K.S., Goodenough J.B. Phospho-olivines as PositiveElectrode Materials for Rechargeable Lithium Batteries. Journal of the Electrochemical Society 1997;144(4) 1188-1194.

[13] Goodenough J.B., Kim Y. Challenges for Rechargeable Li Batteries. Chemistry of Materials 2010;22(3) 587-603.

[14] Molenda J., Molenda M. Composite Cathode Material for Li-Ion Batteries Based on $\mathrm{LiFePO}_{4}$ System. In: Cuppoletti J. (ed.), Metal, Ceramic and Polymeric Composites for Various Uses. InTech; 2011.

[15] Padhi A.K., Nanjundaswamy K.S., Masquelier C., Okada S., Goodenough J.B. Effect of structure on the $\mathrm{Fe}^{3+} / \mathrm{Fe}^{2+}$ redox couple in iron phosphates. Journal of the Electrochemical Society 1997;144(5) 1609-1613.

[16] Arroyo-de Dompablo M.E., Armand M., Tarascon J.M., Amator U. On-demand design of polyoxianionic cathode materials based on electronegativity correlations: An exploration of the $\mathrm{Li}_{2} \mathrm{MSiO}_{4}$ system $(\mathrm{M}=\mathrm{Fe}, \mathrm{Mn}, \mathrm{Co}, \mathrm{Ni})$. Electrochemistry Communication 2006;8 1292-1298.

[17] Gong Z.L., Li Y.X., Yang Y. Synthesis and electrochemical performance of $\mathrm{Li}_{2} \mathrm{CoSiO}_{4}$ as cathode material for lithium ion batteries. Journal of Power Sources 2007;174(2) 524-527.

[18] Nyten A., Abouimrane A., Armand M., Gustafsson T., Thomas J.O. Electrochemical performance of $\mathrm{Li}_{2} \mathrm{FeSiO}_{4}$ as a new Li-battery cathode material. Electrochemistry Communication 2005;7(2) 156-160.

[19] Kokalj A., Dominko R., Mali G., Meden A., Gaberscek M., Jamnik J. Beyond OneElectron Reaction in Li Cathode Materials: Designing $\mathrm{Li}_{2} \mathrm{Mn}_{\times} \mathrm{Fe}_{1-\mathrm{x}} \mathrm{SiO}_{4}$. Chemistry of Materials 2007;19(15) 3633-3640.

[20] Molenda M., Dziembaj R., Drozdek M., Podstawka E., Proniewicz L.M. Direct preparation of conductive carbon layer (CCL) on alumina as a model system for direct 
preparation of carbon coated particles of the composite Li-ion electrodes. Solid State Ionics 2008; 179(1-6) 197-201.

[21] Sirisopanaporn C., Boulineau A., Hanzel D., Dominko R., Budic B., Armstrong A.R., Bruce P.G., Masquelier C. Crystal Structure of a New Polymorph of $\mathrm{Li}_{2} \mathrm{FeSiO}_{4}$. Inorganic Chemistry 2010;49(16) 7446-7451.

[22] Boulineau A., Sirisopanaporn C., Dominko R., Armstrong A.R., Bruce P.G., Masquelier C. Polymorphism and structural defects in $\mathrm{Li}_{2} \mathrm{FeSiO}_{4}$. Dalton Transactions 2010;39(27) 6310-6316.

[23] Sirisopanaporn C., Masquelier C., Bruce P.G., Armstrong A.R., Dominko R. Dependence of $\mathrm{Li}_{2} \mathrm{FeSiO}_{4}$ Electrochemistry on Structure. Journal of the American Chemical Society 2011;133(5) 1263-1265.

[24] Mali G., Rangusa M., Sirisopanaporn C., Dominko R. Understending 'Li MAS NMR spectra of $\mathrm{Li}_{2} \mathrm{MSiO}_{4}$ materials $(\mathrm{M}=\mathrm{Mn}, \mathrm{Fe}, \mathrm{Zn})$. Solid State Nuclear Magnetic Resonance 2012;42 33-41.

[25] Liu W., Xu Y., Yang R. Synthesis, characterization and electrochemical performance of $\mathrm{Li}_{2} \mathrm{MnSiO}_{4} / \mathrm{C}$ cathode material by solid-state reaction. Journal of Alloys and Compounds 2009;480(2) L1-L4.

[26] Karthikeyan K., Aravindan V., Lee S.B., Jang I.C., Lim H.H., Park G.J., Yoshio M., Lee Y.S. Electrochemical performance of carbon-coated lithium manganese silicate for asymmetric hybrid supercapacitors. Journal of Power Sources 2010;195(11) 3761-3764.

[27] Gummow R.J., Sharma N., Peterson V.K., He Y. Crystal chemistry of the Pmnb polymorph of $\mathrm{Li}_{2} \mathrm{MnSiO}_{4}$. Journal of Solid State Chemistry 2012;188 32-37.

[28] Gummow R.J., Sharma N., Peterson V.K., He Y. Synthesis, structure, and electrochemical performance of magnesium-substituted lithium manganese orthosilicate cathode materials for lithium-ion batteries. Journal of Power Sources 2012;197 231-237.

[29] Molenda M., Swietoslawski M., Rafalska-Lasocha A., Dziembaj R. Synthesis and Properties of $\mathrm{Li}_{2} \mathrm{MnSiO}_{4}$ Composite Cathode Material for Safe Li-ion Batteries. Functional Material Letters 2011;4(2) 135-138.

[30] Dominko R., Bele M., Kokalj A., Gaberscek M., Jamnik J. $\mathrm{Li}_{2} \mathrm{MnSiO}_{4}$ as a potential Libattery cathode material. Journal of Power Sources 2007;174(2) 457-461.

[31] Li Y.X., Gong Z.L., Yang Y. Synthesis and characterization of $\mathrm{Li}_{2} \mathrm{MnSiO}_{4} / \mathrm{C}$ nanocomposite cathode material for lithium ion batteries. Journal of Power Sources 2007;174(2) 528-532.

[32] Aravindan V., Ravi S., Kim W.S., Lee S.Y.,. Lee Y.S. Size controlled synthesis of $\mathrm{Li}_{2} \mathrm{MnSiO}_{4}$ nanoparticles: effect of calcination temperature and carbon content for high performance lithium batteries. Journal of Colloid and Interface Science 2011;355(2) 472477.

[33] Swietoslawski M., Molenda M., Zaitz M., Dziembaj R. C/Li2 $\mathrm{MnSiO}_{4}$ as a Composite Cathode Material for Li-ion Batteries, ECS Transaction 2012;41 - in press

[34] Molenda M., Dziembaj R., Kochanowski A., Bortel E., Drozdek M., Piwowarska Z. Process for the preparation of conductive carbon layers on powdered supports Int. Patent Appl. No. WO 2010/021557, US Patent Application 20110151112. 


\section{Damages and Fractures -}

\section{Theoretical and Numerical Modeling}





\title{
Biaxial Tensile Strength Characterization of Textile Composite Materials
}

\author{
David Alejandro Arellano Escárpita, Diego Cárdenas, Hugo Elizalde, \\ Ricardo Ramirez and Oliver Probst
}

Additional information is available at the end of the chapter

http://dx.doi.org/10.5772/48105

\section{Introduction}

Woven architecture confers textile composites (TC) multidirectional reinforcement while the undulating nature of fibres also provides a certain degree of out-of-plane reinforcement and good impact absorption; furthermore, fibre entanglement provides cohesion to the fabric and makes mould placement an easy task, which is advantageous for reducing production times [1]. These features make TC an attractive alternative for the manufacture of highperformance, lightweight structural components. Another interesting feature of TC is that they can be entangled on a variety of patterns, depending of the specific applications intended. Despite the wide interest of textile composites for industry and structural applications, most of the research efforts for strength characterization has focused on unidirectional composites (UDC), resulting in a large number of failure theories developed for UDC (around 20, as inferred from the conclusions of the World-Wide Failure Excercise (WWFE) [2]); some of the most popular failure models are used indistinctly for UDC and TC: most designers use Maximum Strain, Maximum Stress, Tsai-Hill and Tsai-Wu both for UDC or TC as stated in reference [3], despite the fact that none of the aforementioned failure criteria has been developed specifically for TC, which has led to use of high safety factors in critical structural applications to overcome associated uncertainties [4]. The most successful approaches to predict TC strength are based on phenomenological modeling of interactions between constituents at different scales (matrix-yarn-fiber), providing new insight into TC failure mechanisms. However, the implementation of phenomenological models as design tools is considerably more complex than that of traditional failure criteria, while still exhibiting significant deviation from the scarce experimental data [5] available. This scarcity of experimental data to validate or reject failure theories continues to be a major obstacle for improving TC models. Recent investigations reporting biaxial tensile strength tests in 2D- 
triaxial TC employing tubular specimens suggested that the failure envelope predicted by the maximum strain criterion fits the experimental data in the tension-tension (T-T) quadrant [6] fairly well. Other tests performed on cruciform specimens indicated that the maximum stress criterion is more adequate [5]; however, the authors of ref. [5] expressed some concerns about the generality of the experimental methodology for the case of non quasi-isotropic lay-up configurations, such as the one studied in their work. In view of the lack of consensus for accurate TC strength prediction [7],[8], and as stated by researchers who participated in the World Wide Failure Exercises (WWFE) [2] more experimental data, better testing methods and properly designed specimens are needed to generate reliable biaxial strength models.

\section{Biaxial testing review}

Combined multi-axial strength characterization of composites is far from straightforward, as three basic elements are required: i) An apparatus capable of applying multi-axial loads, ii) a specimen capable of generating a homogeneous stress and strain field in a predefined gauge zone, producing failure inside this zone for correct strength characterization, and iii) a measurement system capable of acquiring the applied loads and resulting specimen strains. Although the general procedure is similar to that for uni-axial testing, significant complications arise due to the requirements outlined above; moreover, the required equipment is costly and available generally only at large specialized research centres. Regarding the specimens, the ability of generating a homogeneous multi-axial strain field inside a pre-specified gauge zone is not straightforward mainly due to geometric stress concentrations. Finally, the data acquisition system requires a free surface in order to perform direct measurements. In practice these factors limit the number of combined loads that can be applied to a single specimen to only two, although some researchers have proposed apparatuses designed to apply tri-axial loads, albeit at the expense of limiting the access for full field strain measurements. Efforts on multi-axial testing have been disperse and rather unsuccessful in defining adequate testing methodologies, as evidenced by the lack of standardization by international organisms which have otherwise generated wellknown standards for uni-axial characterization of composites, such the ASTM D3039 (standard testing procedures for obtaining tensile properties of polymer matrix composites), British Standard: BS 2782: Part 3: Method 320A-F: Method for obtaining mechanical properties of plastics, BS EN ISO 527 Part 5: Plastics. Determination of tensile properties and test conditions for unidirectional fibre-reinforced plastic composites, CRAG (Composite Research Advisory Group) Test Methods for the Measurement of the Engineering Properties of Fibre Reinforced Plastics, Standard ASTM D6856: Testing procedures for textile composite materials, Japanese Industrial Standard JIS K7054: Tensile Test Method for Plastics Reinforced by Glass Fibre, Russian Standards GOST 25.601-80: Design Calculation and Strength Testing Methods of mechanical testing of polymeric composite materials. In brief, there exist at least seven standards for tensile uni-axial characterization, while none specific standard for bi-axial testing. This demonstrates the need for developing biaxial 
testing methodologies. In this chapter a review is presented of the state of the art of multiaxial testing with emphasis on biaxial tensile specimens, testing machines and data measurement systems. The reasons for concentrating on biaxial loads are: i) The complexity of testing systems increases considerably with the the number of independent applied. ii) Most structural applications of composites uses thin skins, resulting in shell structures, in which the thickness of the laminates is significantly smaller than the other dimensions. One characteristic of shell structures is that buckling failure modes are the limiting factors in the case compressive loads [9]; consequently, the structural strength depends little on the materials strength and mostly on the geometry and stiffness. On the other hand, when tensile loads are applied to shell, the structures tend to be stable, and the final failure does depend on the materials strength. Evidently, given these fundamentally different failure modes in the cases of compressive and tensile loads, respectively, a combination of biaxial load conditions (compressive-compressive, compressive-tensile, tensile-compressive, and tensile-tensile) can lead to a quite complex behaviour and the need for developing predictive failure models that can account for this complexity.

\subsection{Biaxial and multiaxial specimens}

To generate useful strength data, a biaxial specimen must be capable of meeting a set of requirements [10],[11],[12],[13]: i) A sufficiently wide homogeneous biaxially-stressed zone must be generated for strain measurements, ii) Failure must occur within this zone. iii) No spurious loads (other than tension/compression) should be acting on the gauge specimen. iv) The specimen should accept arbitrary biaxial load ratios. The very design of specimens that recreate biaxially loaded components has become a constantly evolving field, aiming to provide optimal geometry, manufacture and general arrangement for a valid and reliable test [14]. Specimens designed for biaxial testing can be classified into three main groups: i) tubes, ii) thin plates and iii) cruciforms. A review of these groups and their main features is given below.

\subsubsection{Tubular specimens}

Multi-axial stress states were formerly created with thin-walled tubes subjected to internal pressure, torsion and axial loads [10],[11],[15]. These specimens allow simultaneous application of tensile and compressive longitudinal loads, as well as tangential and shear loads, therefore representing a versatile scheme for the conduction of multi-axial characterization (Figure 1).

However, the existence of stress gradients across the tubular wall makes this method less accurate than setups based on flat plates, which are also more representative of common industrial applications than the tubular geometry. Some studies also reveal high stress concentrations on the gripping ends. A further disadvantage is a pressure leakage after the onset of matrix failure, although some correction can be provided by internal linings [15]. 


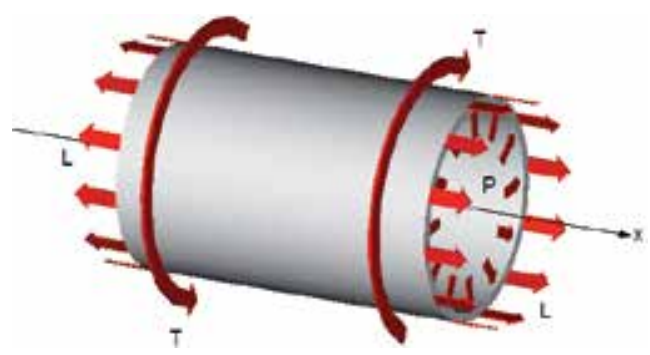

Figure 1. Thin-walled tube specimen.

\subsubsection{Thin plates}

Round or elliptical flat sheets subject to pressure in the hydraulic bulge test [16], as shown in Figure 2, can develop a biaxial stress state, although the technique has several disadvantages, for example, non-homogeneous stress distributions induced by gripping of the edges [17]. Also, just like the rhomboidal plate case, the loading ratio is shapedependant [18] and can therefore not be varied during the test to obtain a full characterization.

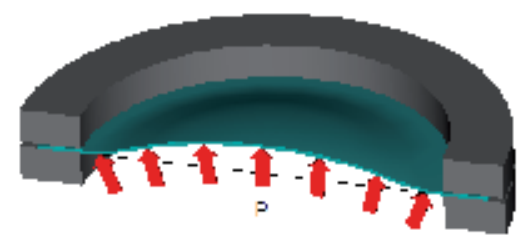

Figure 2. Elliptical flat sheet used in the bulge test.

\subsubsection{Cruciform specimens}

Testing biaxially-loaded cruciform specimens represent a more direct approach for obtaining true biaxial stress states, and consequently this method has gained wide acceptance [7],[8],[10],[11],[15]. As suggested by many researchers in the field [7],[10],[13], an ideal cruciform specimen should accomplish the following features: i) It should be capable of generating a sufficiently wide and homogenous biaxial stress/strain field in the gauge area, ii) failure must occur in the predefined gauge zone, iii) the cruciform should accept arbitrary biaxial load ratios for generating a complete failure envelope (within a desired range), iv) both the tested and the reinforcement layers should be of the same material, $v$ ) the transition between the gauge zone and the reinforced regions should be gradual enough as to avoid undesirable high stress concentrations, vi) the cruciform fillet radius should be as small as possible in order to reduce stress coupling effects, and vii) stress measurements in the test area should be comparable to nominal values obtained by dividing each applied load by its corresponding cross-sectional area. Although various cruciform geometries containing a central-square thinned gauge zone have been proposed in the literature, none can claim full satisfaction of the above requirements due to difficulties inherent to biaxial tests [10]. A cruciform with a thinned central region and a series of limbs 
separated by slots is presented in Figure 3a. [19]; the slotted configuration allows greater deformations to occur in the thinned section, thus enforcing failure there. Nevertheless, thickness-change can induce undesirable stress concentrations that usually lead to premature failure outside the gauge zone. Also, the extensive machining required for thinning is an undesirable feature.

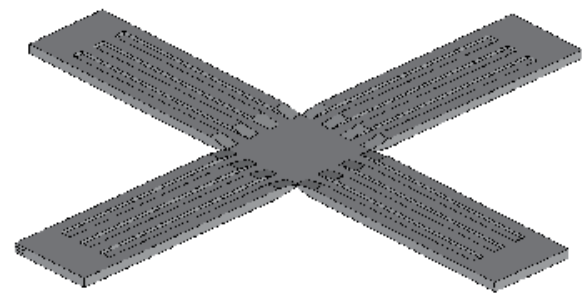

a)

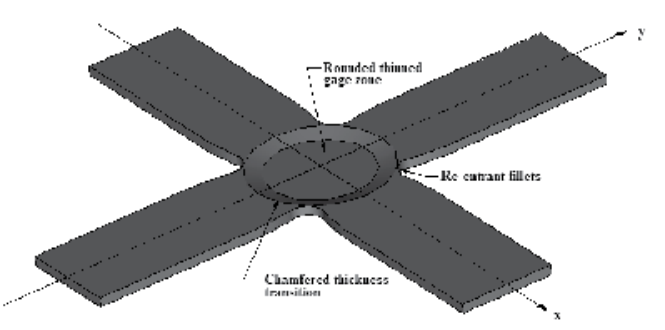

b)

Figure 3. a). Slotted configuration[19] b). Thinned circular zone in the gauge zone [13].

Another cruciform, shown in Figure 3b, with a thinned circular zone in the gauge area [13] exhibits failure outside it, mainly because manufacturing defects caused unexpected higher strength in one axis. The implementation of a rhomboidal shaped test zone is suggested in [20], although, to the authors knowledge, no results with this geometry have been reported so far. Some experiments concluded that loading must be orthogonal to the fibre orientation to produce failure in the test zone [12]. The main difficulty in obtaining an optimal configuration is eliminating stress concentrations in the arms joints. To solve this, an iterative optimization process (numerical/experimental) yielded optimum geometric parameters of the specimen [21]. Results from this study led to a configuration characterized by a thinned square test zone and filleted corners between arms. Given that failure is prone to occur in the arms, reference [23] presented a design where a small cruciform slot is placed in the centre to cause load transfer from the arms to this region (figure $4 \mathrm{a}$ ).

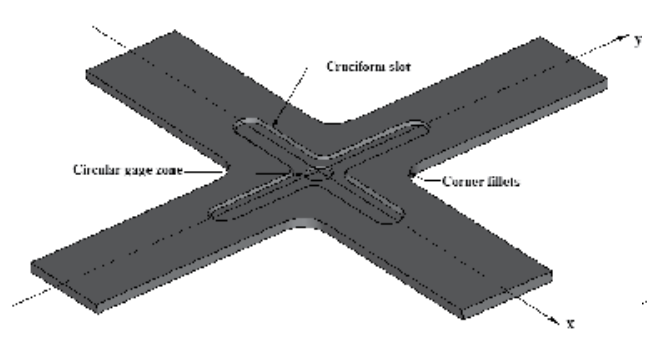

a)

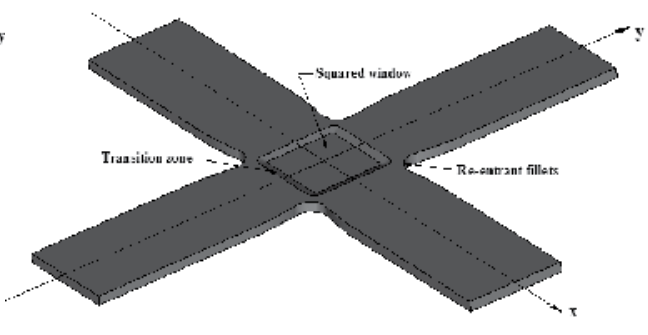

b)

Figure 4. a). Inner cruciform slot [23]. b). Cruciform with thinned rounded square gauge zone and filleted corners [11].

Nevertheless the gauge zone is much reduced, and this makes this specimen useless for TC characterization. In the cruciform proposed by Ebrahim et al [10] failure in the gauge zone is 
achieved. The design is characterized by a thinned rounded square gauge zone and considers a gradual thickness reduction in the biaxially loaded zone, and also filleted corners as shown in Figure $4 \mathrm{~b}$. Results were satisfactory, but it was found that the top and bottom edges of the depression presented high strain gradients. Based on the aforementioned references, a comprehensive study was conducted by the authors to obtain an improved cruciform design. A main feature of this new design is a rhomboid-shaped gauge zone which led to a much more homogeneous strain/strain distribution because of the alleviation of stress concentrations which occur in other designs due to the short distance between the gauge zone and the corners of the arms. Additionally, the corners are filleted to avoid another zone of stress concentration. The specimen is comprised of different layers where the inner layer is under study, whereas the outer ones (equal quantity on each side) are only for reinforcement.

\subsubsection{Enhanced rhomboid-windowed cruciform specimen}

In order to avoid premature failure due stress concentrations, a modified cruciform was proposed by considering this design concepts: i) Given that fillets are prime examples of stress concentrators, both the cruciform and gauge zone fillets should be as far apart as possible from each other, thus favouring a rhomboid-windowed gauge zone. This modification also intends to minimize regions of stress interactions, which cause lack of homogeneity in the strain field and even premature failure, as reported for some squarewindowed specimens [22],[24]. Traditional (instead of re-entrant) fillets were preferred to maintain this stress concentrator as separated as possible from the gauge zone. ii) Since the focus of this research are textile composites (TC), the proposed specimen also features wider arms and a larger gauge zone, seeking to reduce the textile unit cell vs. gauge zone length ratio. This modification is in tune with ASTM standards on testing procedures for textile composites [25]. iii) To avoid polluting the obtained strength data with in-situ effects, adhesion between adjacent layers and other multilayer-related uncertainties, characterization is performed for a single-layer central gauge zone, while a number of reinforcement layers are added outside the gauge zone to enforce failure inside it. The resulting rhomboid windowed cruciform shape was similar to other specimens employed for fatigue characterization of ABS plastic, which report a smooth biaxial strain field at the gauge zone [26]. Basic dimensions were selected from a specimen reported in literature [27]: arm width $w=50 \mathrm{~mm}$ and cruciform fillets $R=25 \mathrm{~mm}$. The rhomboid window length $l$ was set identical to the arm width, $l=50 \mathrm{~mm}$ while the window's fillet radius $r$ was set as $10 \%$ of $l$; the geometry is sketched in figure 5. Finite-element (FE) analysis demonstrated that this geometry generates a more uniform strain distribution, while the maximum shear strain in the cruciform fillet is relatively slow.

Once the suitability of a rhomboid windowed cruciform specimen for creating a biaxial strain state was established, a geometrical optimization process based on the experiment design methodology was conducted. Suitable objective functions were defined in order to homogenize the $\varepsilon_{x}$ and $\varepsilon_{y}$ strain fields inside the rhomboid gauge zone while maintaining shear strain $\gamma_{x y}$ field close to zero. Details of the optimization process can be found in reference [29] 


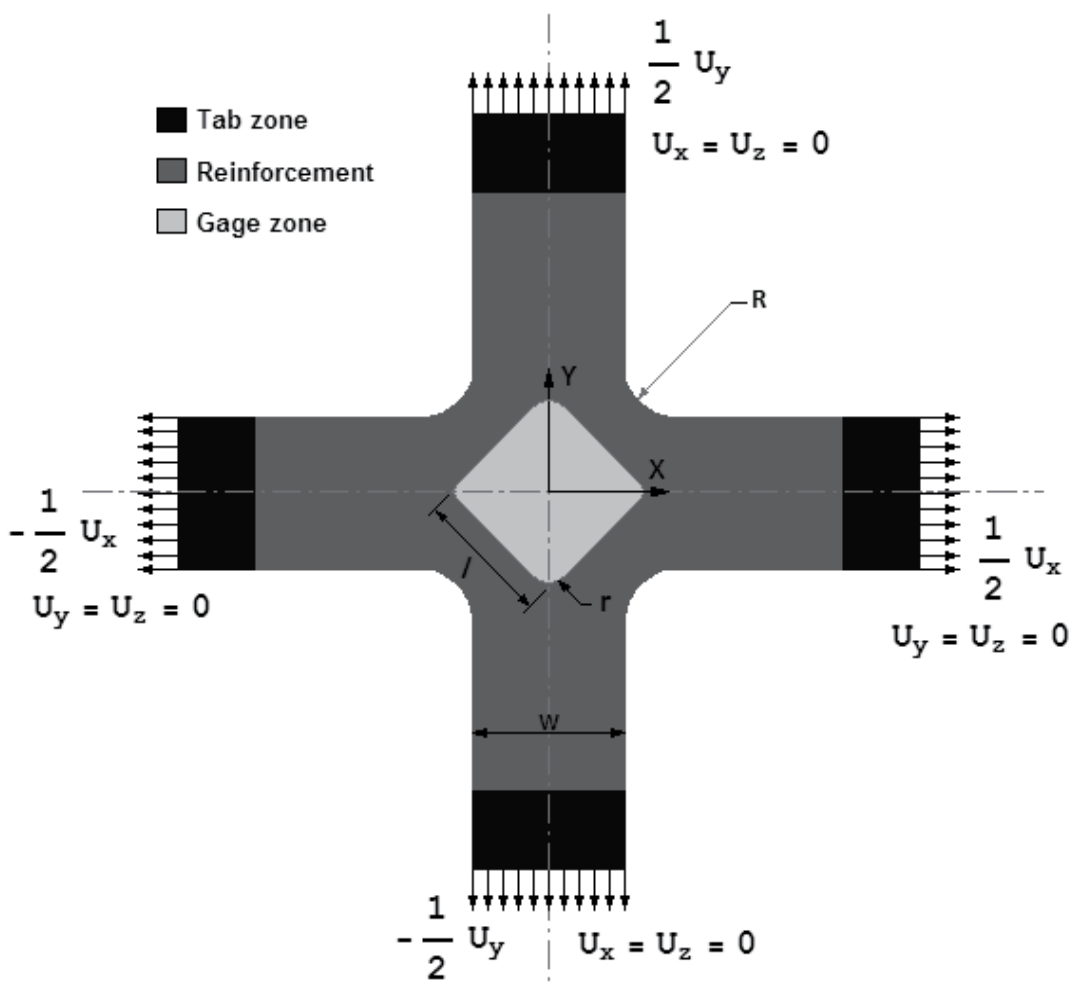

Figure 5. Geometry of the proposed cruciform specimen. Lay up for the reinforcement region is $[0] 5$, while for the gauge zone is [0] (that is, a single layer). All dimensions are given in $\mathrm{mm}$.

\begin{tabular}{cll}
\hline E$_{11}$ & {$[\mathrm{GPa}]$} & 25.0 \\
$\mathrm{E}_{22}$ & {$[\mathrm{GPa}]$} & 25.0 \\
$\mathrm{~V}_{12}$ & {$[-]$} & 0.2 \\
$\mathrm{G}_{12}$ & {$[\mathrm{GPa}]$} & 4.0 \\
\hline
\end{tabular}

Table 1. In-plane measured mechanical properties for a TC conformed of: Epoxy West System 105/206 reinforced with fibreglass cloth style \#7520, bidirectional plain weave 8.5 oz./sq. yd, with $18 \mathrm{~L} \times 18 \mathrm{~W}$ threads per inch count.

Evaluation of specimen using finite element analysis was carried by applying boundary conditions as defined in figure 5 , with $U_{x}$ and $U_{y}$ chosen to produce a maximum strain ( $\varepsilon_{x}$ or $\left.\varepsilon_{y}\right)$ of $2 \%$ inside the gauge zone, corresponding to typical failure strain values reported for glass-epoxy TC [5]. The materials properties correspond to a generic plain weave bidirectional textile, as presented in Table 1.

The optimized geometry is defined in Figure 6, while the results of the FE analysis are shown in Figure 7, which splits the geometry into top and bottom sections for simultaneously illustrating the $\varepsilon_{x}$ and $\gamma_{x}$ strain fields, respectively, in a single graph; due to full symmetry, the $\varepsilon_{y}$ strain field is identical to the $\varepsilon_{x}$ field when rotated by $90^{\circ}$. The resulting geometry generates a very homogeneous strain field in the gauge zone and keeps shear strains near zero, while keeping shear strains in the fillet regions below the failure value. 
These results are believed to represent a great improvement if compared with other specimens reported in the literature.

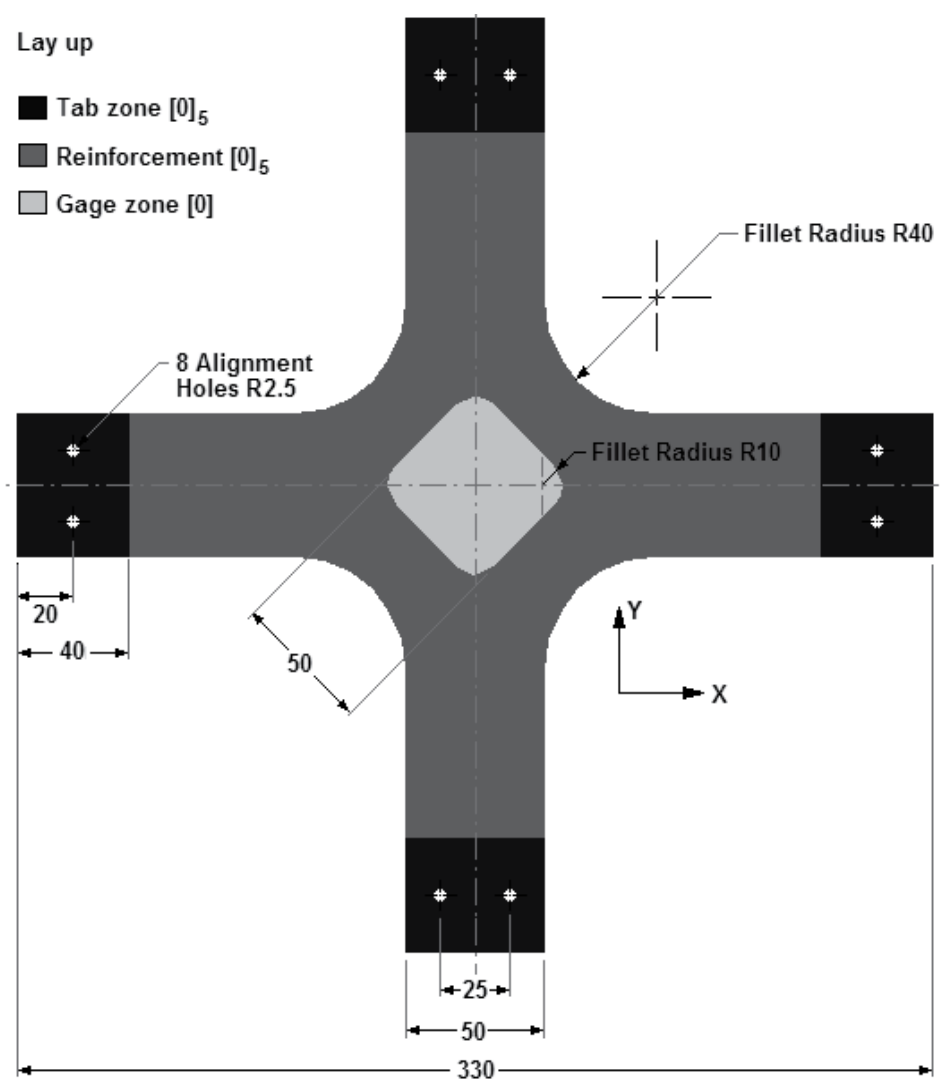

Figure 6. Optimized specimen specifications Dimensions are in $\mathrm{mm}$.

\subsection{Biaxial testing machines}

To apply biaxial loads on cruciform specimens a specific device is required, which can meet the following requirements [28],[30],[12],[31]: i) The loads applied to a cruciform specimen must be strictly in tension or compression, avoiding spurious shear or bending loads. ii)The restriction previously stated implies that orthogonality among load axes must be guaranteed at all times during the test, and, consequently iii) the centre of the specimen must remain either still or the load axes must displace with it. An efficient method to ensure the previous condition is to apply equal displacements in the loaded axis. These requirements can be accomplished by using an active control system, or by passive mechanical methods, such that the one described later. A review of the most common biaxial testing systems is presented next. 


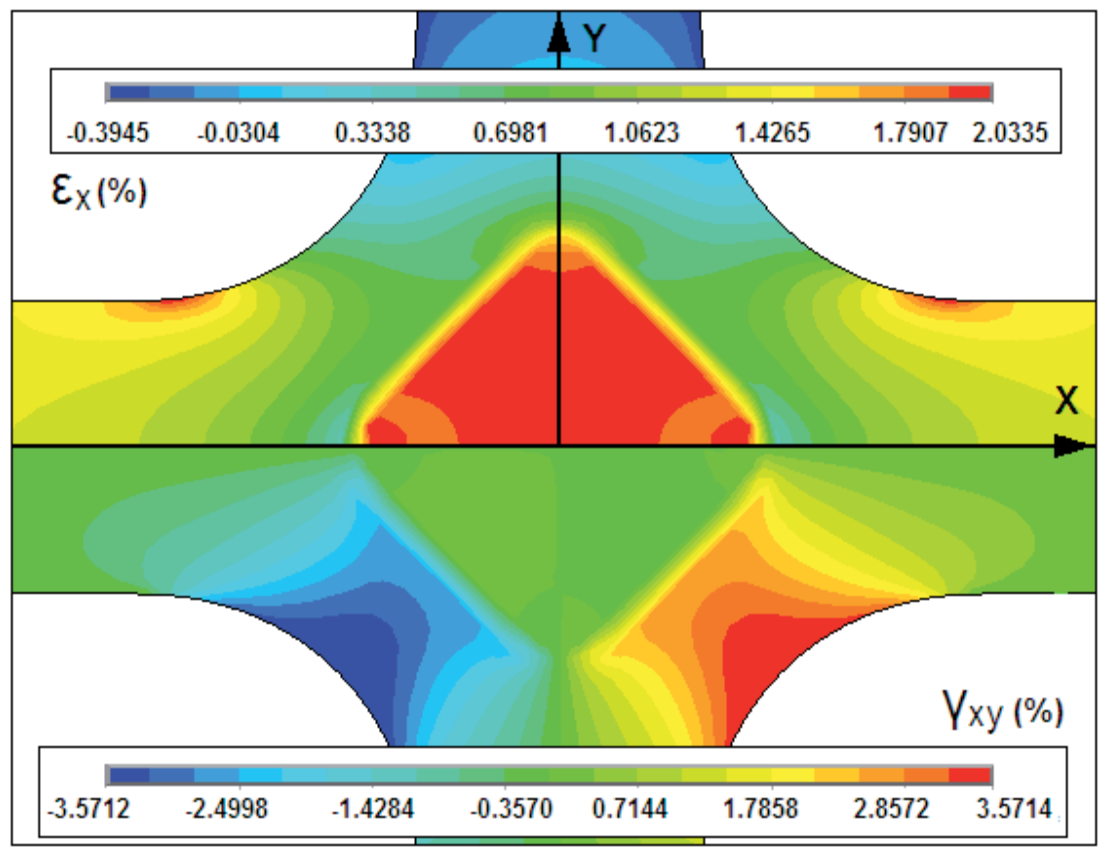

Figure 7. Linear strain field (upper part of the graph) and shear strain field (lower part) within and near the gauge zone of the optimized specimen.

\subsubsection{Hydraulic systems}

Hydraulic systems rely on hydraulic actuators to apply loads to the specimen; they typically use double-acting pistons with a closed-loop servo control system which sense displacements and/or loads as feedback, as implemented in the design by Pascoe and de Villiers [32]. This configuration (sketched on figure 8) which comprises the use of independent actuator for each applied load, allows the centre of the specimen to move during the test, which is an undesirable condition; this adverse feature can be avoided by implementing a control system that ensures synchronization of opposite actuators [33],[18] thereby avoiding motion of the centre of the specimen.

This configuration also allows the load ratios to be varied in order to obtain a full failure envelope. None of the systems mentioned could ensure equal displacement in both extremes of each axis, even the one using synchronization control, therefore allowing the centre of the specimen to move. If systems are implemented to correct this problem, the design and manufacturing costs inevitably increase. Fessler [34] proposed a machine in which motion is allowed only in one direction at one arm for each cruciform axis. This is the most common basic configuration found in the literature related to biaxial characterization of composites [35],[33]. In an attempt to simplify the previous concept while maintaining symmetric load conditions, some modifications have been proposed; for example, each loading axis, consisting of a pair of opposite hydraulic actuators, can be connected to a common hydraulic line so the force exerted by each side is the same and thus movements of the 

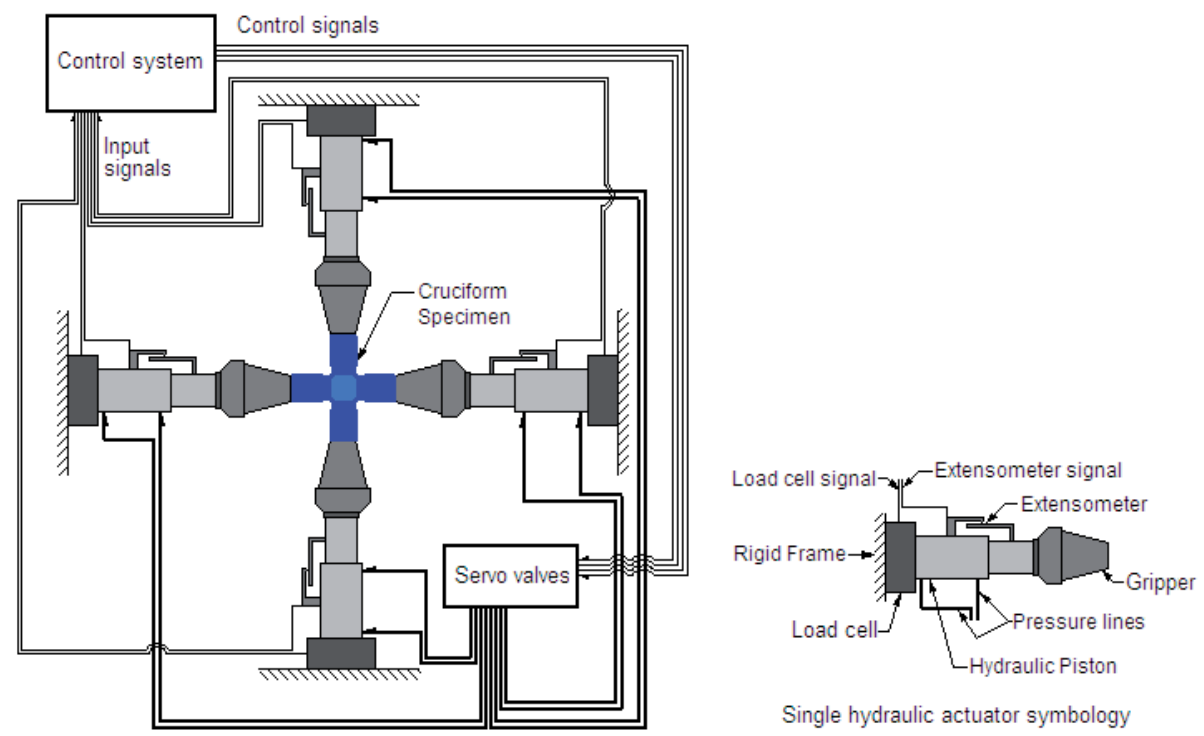

Figure 8. Use of independent actuator per load applied.

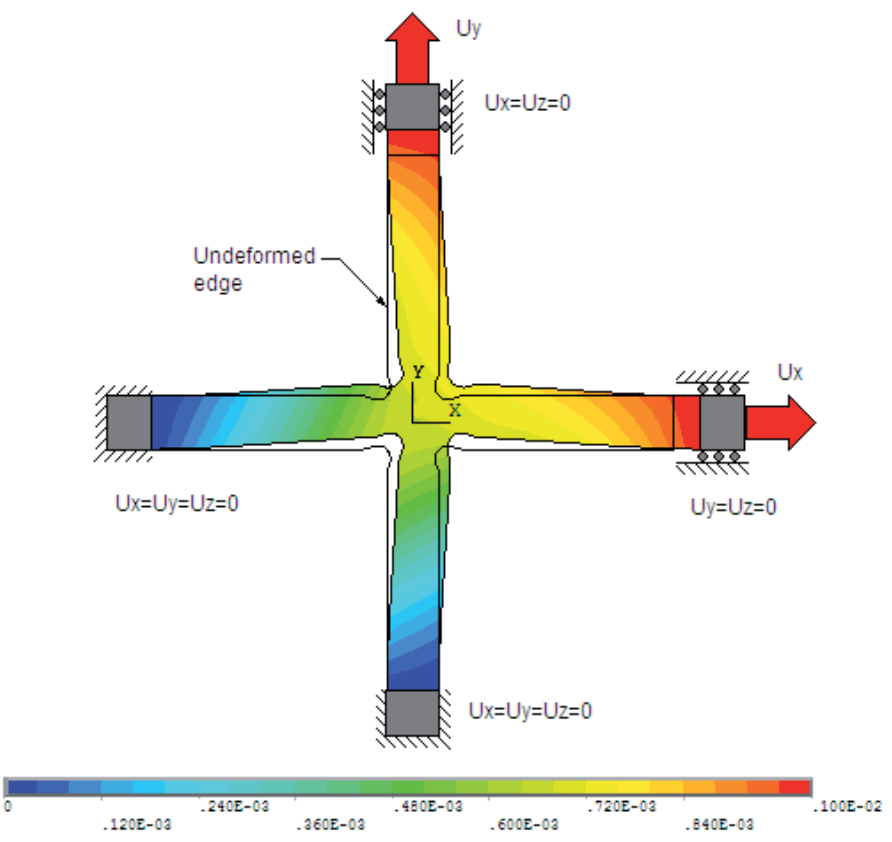

Figure 9. Contour plots of the magnitude of the displacement vector for the case of a configuration where one end of each axis is fixed and the other is displaced.

centre of the specimen are eliminated. Although the common hydraulic line ensures equal force in both extremes of one axis this does not ensure equal displacements. Another variation to hydraulic systems is described in the US Patent No. 5279166, which describes a biaxial testing machine consisting of two independently orthogonal loading axes capable of 
applying tension and/or compression loads; two ends of the specimen are gripped to fixed ends while the complementary ends are fixed to grips attached to actuators that apply the load, made in an attempt to reduce the complexity and hence the costs of biaxial testing machines (fig. 9). This configuration results in significant displacements of the centre of the specimen, although it is stated that the machine has a mechanism that helps maintain the centre of the specimen and ensure that the loads are always orthogonal. In spite of these features, under large displacements the mechanism used is not capable of maintaining the orthogonally of the loads as shown by a quick finite-element evaluation, whose results are shown Figure 8; moreover, the resulting displacement field is completely asymmetric, a condition which generates undesirable shear stress. While most of the biaxial testing hydraulic machines are original developments, a commercial biaxial testing machine has been developed by the company MTS in conjunction with NASA. It uses four independent hydraulic actuators, each with a load cell and hydraulic grippers, and an active alignment system for the specimen. While solving most of the problems mentioned above, the cost of this system is too high for entry-level composites development laboratories.

\subsubsection{Mechanical systems}

Mechanical systems owe their name to the fact that they are based on the kinematics of their mechanisms to maintain load symmetry, no matter if the actuators are hydraulic or mechanic; even the application of deadweight to the specimen through systems of ropes, pulleys, levers and bearings has been considered, as presented by Hayhurst et al [36]. In practice, the mechanical systems proposed for the characterization of composite materials are mainly test rigs designed to be adapted to conventional uniaxial testing machines; basically, they are mechanisms consisting of coupled jointed-arms capable of applying inplane biaxial loads to cruciform specimens. The load ratio is dependent on the geometrical configuration of the device [31] and can therefore be varied only by changing the length of one element, an impractical solution. Similar devices are found in French Patent No. 2579327 [37] and US Patent No. 7204160. A simpler mechanism is presented in US Patent No. 5905205 [30] which uses a four-bar rhomboid-shaped mechanism on which the loading ratios are changed before the test by certain variations in the assembly of the members. One of most practical mechanical systems found consist of four arms, joined at one side to a common block fixed via revolute joints to an universal test machine actuator through a load cell [26] which permits monitoring the applied force, while the other sides are linked, also with revolute joints, to a sliding block each; those blocks slide over a flat plate, fixed to the universal machine's frame. The sliding blocks assemble the grippers which hold the specimen.

\subsubsection{A novel biaxial testing apparatus}

After reviewing the existing machines and mechanisms on which biaxial tests can be carried out, some conclusions can be drawn; in the case of some of them, the lack of a mechanism that automatically corrects any load difference that could lead to the displacement of the 
centre of the specimen makes them unsuitable for reliable tests; in those case where such mechanism does exist, it is controlled by means of an active system that increases design complexity and costs.

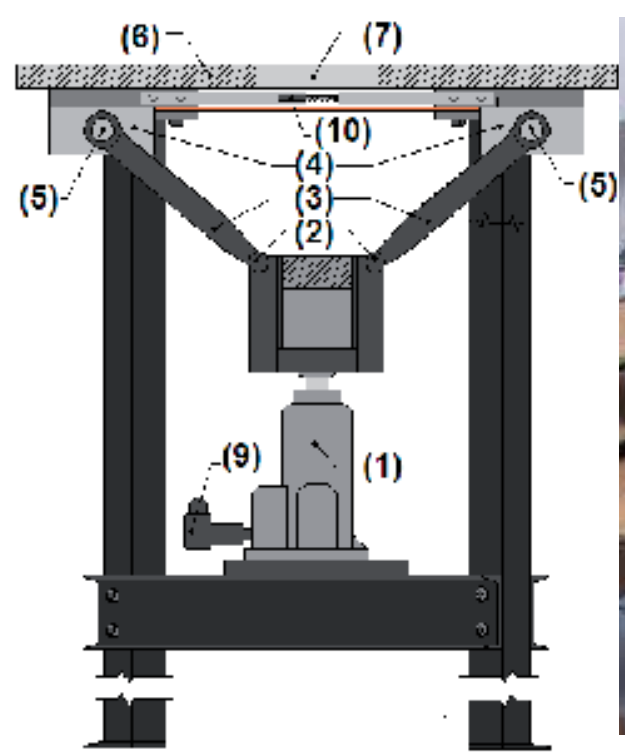

a.

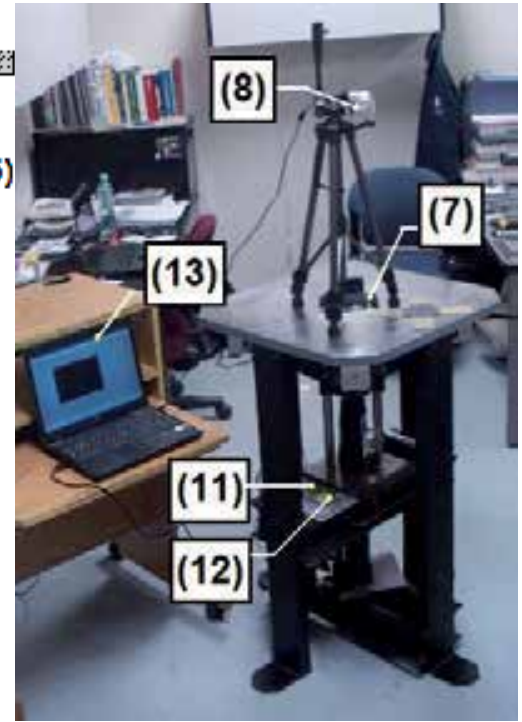

b.

Figure 10. a). Sketch of the biaxial testing machine, showing one load/displacement axis sketch. b). System general arrangement.

The design proposed by the authors considers these drawbacks, as well as the testing requirements previously stated; in addition, construction costs for the novel proposed design are considerably lower compared to other systems. The resulting apparatus is sketched in Figure 10a and a photograph of the completed machine in Figure 10b. The operational principles are described in the following (numbers in parentheses refer to the components identified in the corresponding figures): The loads are applied through a symmetrical slider-crank-slider mechanism meeting the following requirements: The hydraulic piston (1), which is the first slider, is attached on its base to the machine frame and provides the load drive, while its piston is linked by a revolute joint (2) to a pair of arms (3) arranged symmetrically, which in turn are connected by cylindrical joints (5) to the blocks where the grips holding the tab zone of the specimen are installed (4); these cylindrical joints allow to absorb small misalignment in the loads, as established in $i v$.

The grip blocks are lubricated and slide on the lower side of a flat plate (6) featuring a rectangular window (7) allowing a full-field view from the top of the machine, where a high-definition digital camera (HDDC) was installed (8), thereby satisfying the requirement iii. A similar arrangement was installed at a right angle with respect the first one, ensuring the independence of the load axes as required by $i$. Data acquisition is conducted by measuring the pressure in the hydraulic cylinders (9) and correcting this information by 
considering the geometry of the mechanism, while the displacements are measured directly at the grips through resistive displacement sensors (10); all sensors are powered by a power board to provide a common voltage reference (11), and the signals are acquired through a National Instruments 8-channel analogical data acquisition board (12). The information was stored and processed on a laptop (13) by using a Lab View routine.

\subsection{Data acquisition techniques}

Unlike uniaxial tensile tests in which ultimate failure stress and strains data can be straightforwardly obtained from the collected load and displacement data, in the case of biaxial tests the strength values cannot be calculated directly in this way because the stress and strain fields are not necessarily homogeneous along the specimen and generally depend on the load in a non-trivial way due the complex geometry. For this reason biaxial testing requires a method capable of measuring the full strain field in the biaxially loaded zone of the specimen. Given that strain cannot be measured directly it is necessary to measure the displacement field, from which the strain field can be easily calculated. Using the strain field and a constitutive model the stress field can also be calculated. However, full-field measurement techniques are not standard data acquisition methods and in order to identify the most suitable technique for this research a survey was realized.

\subsubsection{Full field strain measurement methods}

The first method considered was reflective photo-elasticity; it is based on birefringence, a physical property which consists of the change of the refraction index of a material when shear stresses are applied. It has been used since decade of the 1950s [38], so is a well characterized technique. However, some limiting factors have been identified for the purposes of the curent project: 1) The preparation of the samples is extremely laborious and requires the application of a layer of birefringent material on the surface to be observed, with a thickness of a few millimetres [38].If compared with the thickness of the composite layer under study which is of the orderof about $0.2-0.3 \mathrm{~mm}$ it is clear that the application of the measurement layer significantly affects the test results. Another technique considered was Moiré interferometry. This technique requires the printing of a pattern of lines on a transparent medium, which is then illuminated by a LASER source, generating an interference pattern which depends on the deformation of the specimen [39]. However, this method has the disadvantage that the data reduction process is tedious and complex [40], and the results heavily depend on the analyst's experience. After considering these options, a technique called digital image correlation (CDI) was identified from biaxial testing literature [10], [11], [41]. The basic concept consists of obtaining digital images of studied geometry on it initial, non-deformed state and after being subjected to a deformation. The surface of the part under study is pre-printed with a random speckle pattern, so that the displacements between corresponding points on photographs of the non-deformed and deformed states, respectively, can be identified by a computer algorithm. This method has some advantage over the ones mentioned above [42]: 
i) The experimental setup and specimen preparation are relatively simple; only one fixed CCD camera is needed to record the digital images of the test specimen surface before and after deformation. ii) Low requirements as to the measurement environment: 2D DIC does not require a laser source. A white light source or natural light can be used for illumination during loading. Thus, it is suitable for both laboratory and field applications. iii) Wide range of measurement sensitivity and resolution: Since the 2D DIC method deals with digital images, the digital images recorded by various high spatial-resolution digital image acquisition devices can be directly processed by the 2D DIC method. For the reasons stated above the 2D DIC method is currently one of the most actively used optical measurement techniques and demonstrates increasingly broad application prospects. Nevertheless, the 2D DIC method also has some disadvantages: i) The surface of the planar test object must have a random grey intensity distribution. ii)The measurements depend heavily on the quality of the imaging system. iii) At present, the strain measurement accuracy of the 2D DIC method is lower than that of interferometric techniques, and is not recommended for the measurement of very small and non-homogeneous deformations. Despite these restrictions the low cost associated with equipment and the low specimen preparation requirements makes Digital Image Correlation the preferred technique for the purposes of this study. The drawbacks can be largely avoided by using the highest-definition camcorder commercially available, using an established Digital Image Correlation program and using a specimen that generates a relatively homogeneous strain field. It was shown above that by proper design and optimization a very homogeneous strain field can indeed be obtained in the gauge zone, so this restriction of the DIC technique was of no concern to this project. Finally, the expected strain values were large enough to be safely detected by the DIC technique.

\section{Experimental setup}

\subsection{Specimen manufacture}

As stated by recent research [11] the milling process typically employed to thin the gauge zone produces undesirable damage and stress concentrations in unidirectional (UD) composites; for textile composites (TC), milling would exacerbate this problem due to its more complex 3D structure, making milling an unacceptable choice. The main concern is to preserve the integrity of the textile structure, especially when characterizing a single lamina. To generate a damage-free cruciform specimen with a single-layered gauge zone, a novel manufacturing process was developed by the authors, explained below: 1). Nonimpregnated fabric sheets were fixed to a $6 \mathrm{~mm}$ thick plywood base to ensure dimensional stability, with a printed grid to help proper fibre alignment of each cloth. The whole arrangement was cut into a square pre-form using a water jet, also cutting away the rhomboidal window corresponding to the gauge zone, as shown in the Figure 11. Afterwards, the material was oven-dried at $60^{\circ} \mathrm{C}$ during 12 hours to eliminate moisture.

2) The following numeric values inside brackets refer to indications given in Figure 12. Two reinforcement layers (1) corresponding to the bottom side of the cruciform specimen were placed in a lamination frame, consisting of a flat surface (2) surrounded by a square border 
(3) with a side length equal to that of the specimen. A pre-formed 2-layer rhomboid step (4) was located at the centre, corresponding to the location of the gauge zone, to ensure planarity of the central layer (5). The reinforcement layers were manually resin-impregnated and, immediately after this, the central layer (5) was placed and impregnated. Finally, the process was repeated for the last two reinforcement layers (6), as shown. Room environment was controlled during the lamination process at a temperature of $80 \pm 2^{\circ} \mathrm{C}$ and $50-60 \%$ relative humidity. Immediately after the impregnation process was completed, the laminate was placed in a vacuum bag consisting of a peel ply (7), perforated film (8), bleeder cloth (9) and the bag itself (10), using sealing tape to ensure vacuum seal (11). 0.8 bar vacuum pressure was applied through a valve located at a corner (12), sufficiently away from where the final shape would be cut. The whole arrangement was cured during 4 hours inside a pre-heated oven at $80 \pm 2^{\circ} \mathrm{C}$, as measured by a thermo-couple (13) located at the gauge zone, as shown in Figure 12. 3) After curing, the final cruciform geometry was obtained through water jet cutting. Nine specimens were prepared meeting the dimensional specifications in Figure 6.

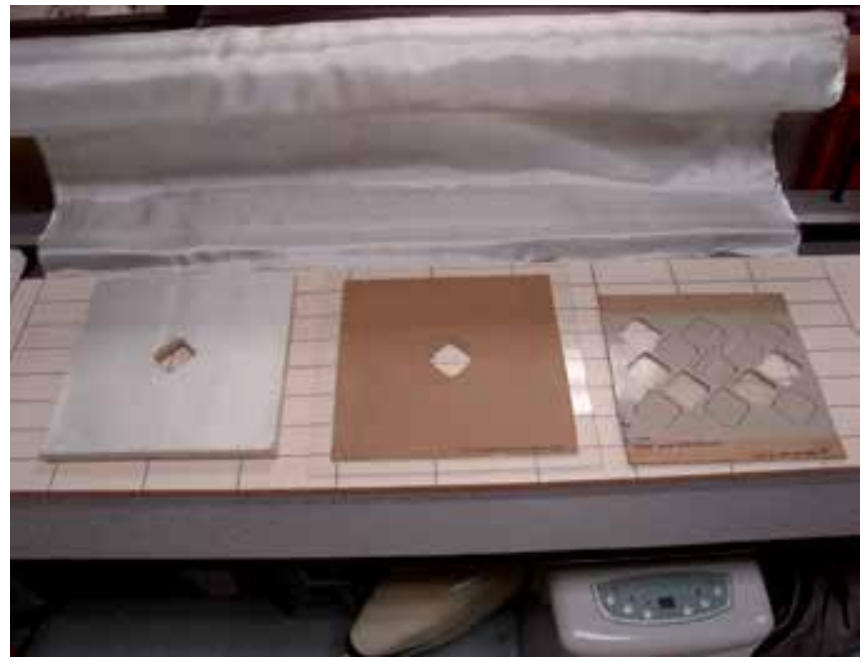

Figure 11. Rhomboid window cutted on the reinforcement layers and other auxiliary tools.

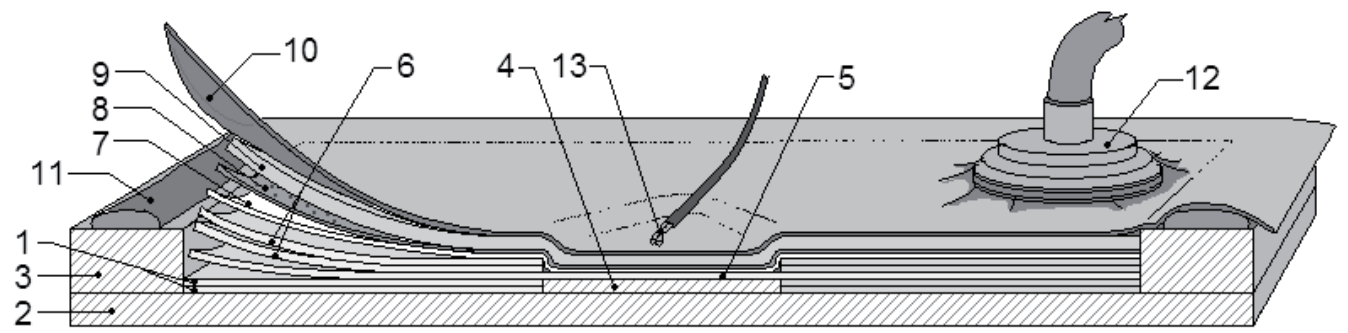

Figure 12. Arrangement for specimen manufacture.

To provide visual reference for the digital image correlation (DIC) strain field measurement [24][41], specimens were painted with a black-dot random speckle pattern over a whitemate primer, as shown in Figure 13. This technique was preferred over the spraying 
technique reported in [41], as it might result in an inadequate control of the dot size distribution, leading to uncertainty in the DIC measurements. Additionally, five uniaxial, [0] $]_{5}$ layup specimens were prepared in order to perform uniaxial tests to provide precise input data for the development of failure criteria.

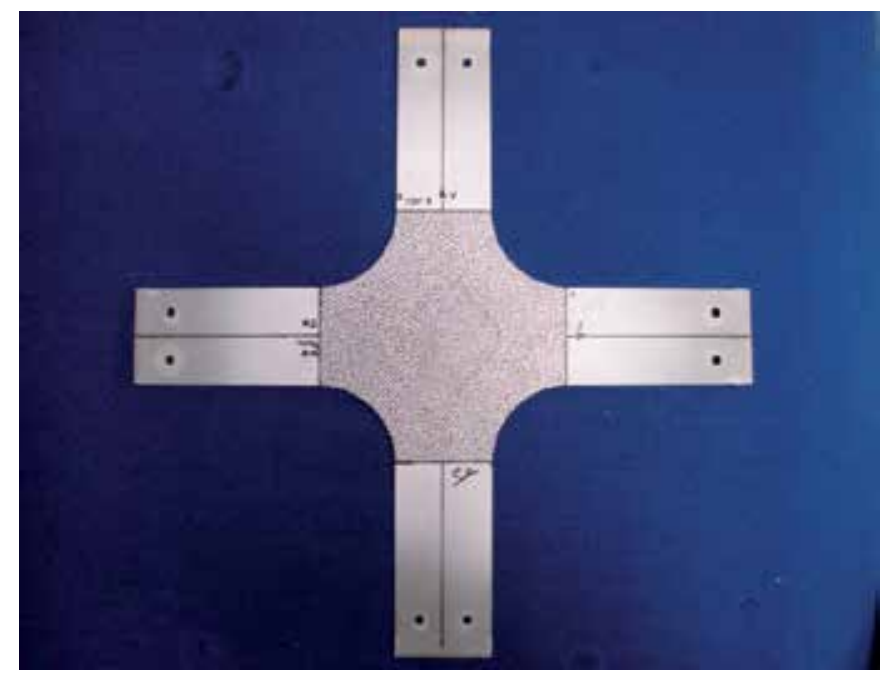

Figure 13. Finished specimen exhibiting its speckle pattern used with the digital image correlation technique

\subsection{Biaxial testing}

Experimental validation of the optimized cruciform was conducted with the biaxial testing apparatus described in section 2.2.3 as follows: after mounting the specimen in the grippers, a pre-load of $500 \mathrm{~N}$ was applied to each axis prior to tightening the mounting bolts. (Figure 14) Then, preload and alignment bolts were removed, setting the measured displacements and loads to zero. A high-quality video of the specimen was recorded with a high definition cam coder with adjustable focus and exposure parameters functions for subsequent DIC analyses.

A chronometer synchronized with the computer clock was placed near the specimen and inside the camera vision field, to ensure its inclusion in the captured images; this provided a time reference to relate each video frame with correspondent load data. After starting video recording and the data capture routine, biaxial displacements were applied at a rate of $1 \mathrm{~mm} / \mathrm{min}$ until final failure. This load rate was selected based on the ASTM 3039 standard [43], which recommends a displacement speed such that failure occurs 1 to 10 minutes after the start of the test.

Data acquisition and reduction was conducted as follows: two video frames were taken from the recorded video sequence, one corresponding to the beginning of the test and another just prior to final failure, as shown in figure 15. Both images were fed into the open access software DIC2D (developed by Dr. Wang's team at the Catholic University of America) to obtain the full strain field $\left(\varepsilon_{x}, \varepsilon_{y}\right.$ and $\left.\gamma_{x y}\right)$. The three tests performed covered a 
range of biaxial ratio $B R$ values in the vicinity of the critical condition $B R=1$ : $B R=1.5$ (Test \#1), $B R=1.25$ (Test \#3), and $B R=1$ (Test \#5). Figure 15 shows the final failure sequence representative of the tests conducted. It should be noted that the failure occurred well within the gauge zone as expected from the FE-predicted strain fields.

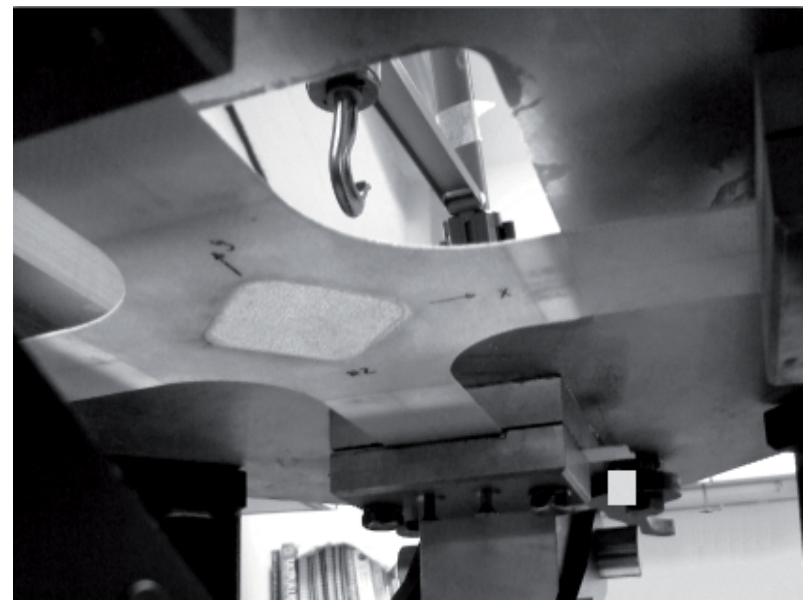

Figure 14. Cruciform specimen mounted in the biaxial testing machine.
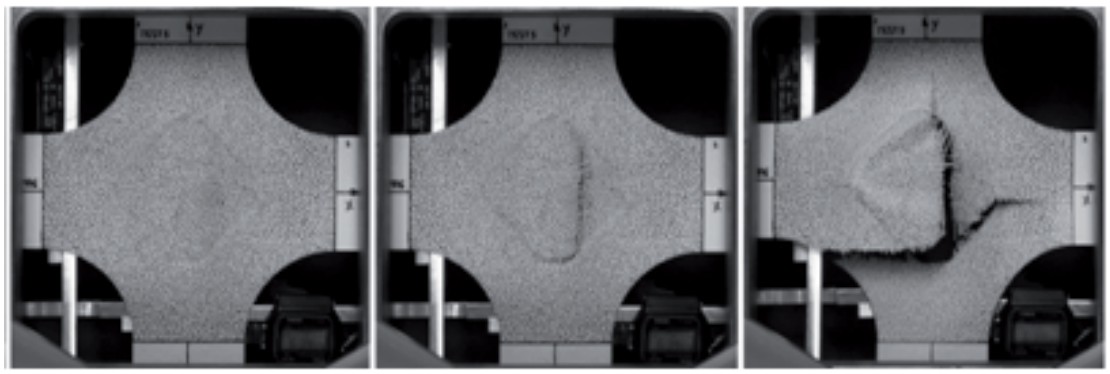

Figure 15. Final failure sequence recorded at 30 frames per second.

The final failure is clearly fibre-dominated, due to its catastrophic nature; it is possible to identify the final failure onset region inside the rhomboidal gauge zone, as required for a successful test. Regarding the strain field, it can be seen from Figure 16 that the agreement between the experimentally results (obtained from DIC) and the FE prediction is remarkably good. The DIC and the FE images show the same symmetry of the experimental shear strain pattern and similar homogeneity and smoothness, and the absolute strain values cover a similar range. This can be considered an additional indicator of the success of the experimental procedure presented in this work.

The same procedure used to characterize the ultimate strain can be used to obtain the matrix onset failure envelope (as opposed to fibre failure), but due to the fact that this phenomenon cannot be deduced visually a different approach was used for this purpose. The load vs. displacement plots were used to identify the change in the slope which evidences matrix damage, as shown in the Figure 17. This method is proposed as an extrapolation of the 
method employed for uniaxial tests defined by the ASTM 3039 standard for the uniaxial tensile characterization of composites [43]. Linear fits were obtained for every linear segment of curves corresponding to every perpendicular axis, and the intersections were calculated solving the resulting equations, which allowed to quantify the strain values corresponding to the onset of matrix damage, considering that the latter occurs at the first observed slope change. Once the displacement and strain were identified, the digital image corresponding were used to perform a DIC analysis and to get the full field strain in the same fashion described previously.

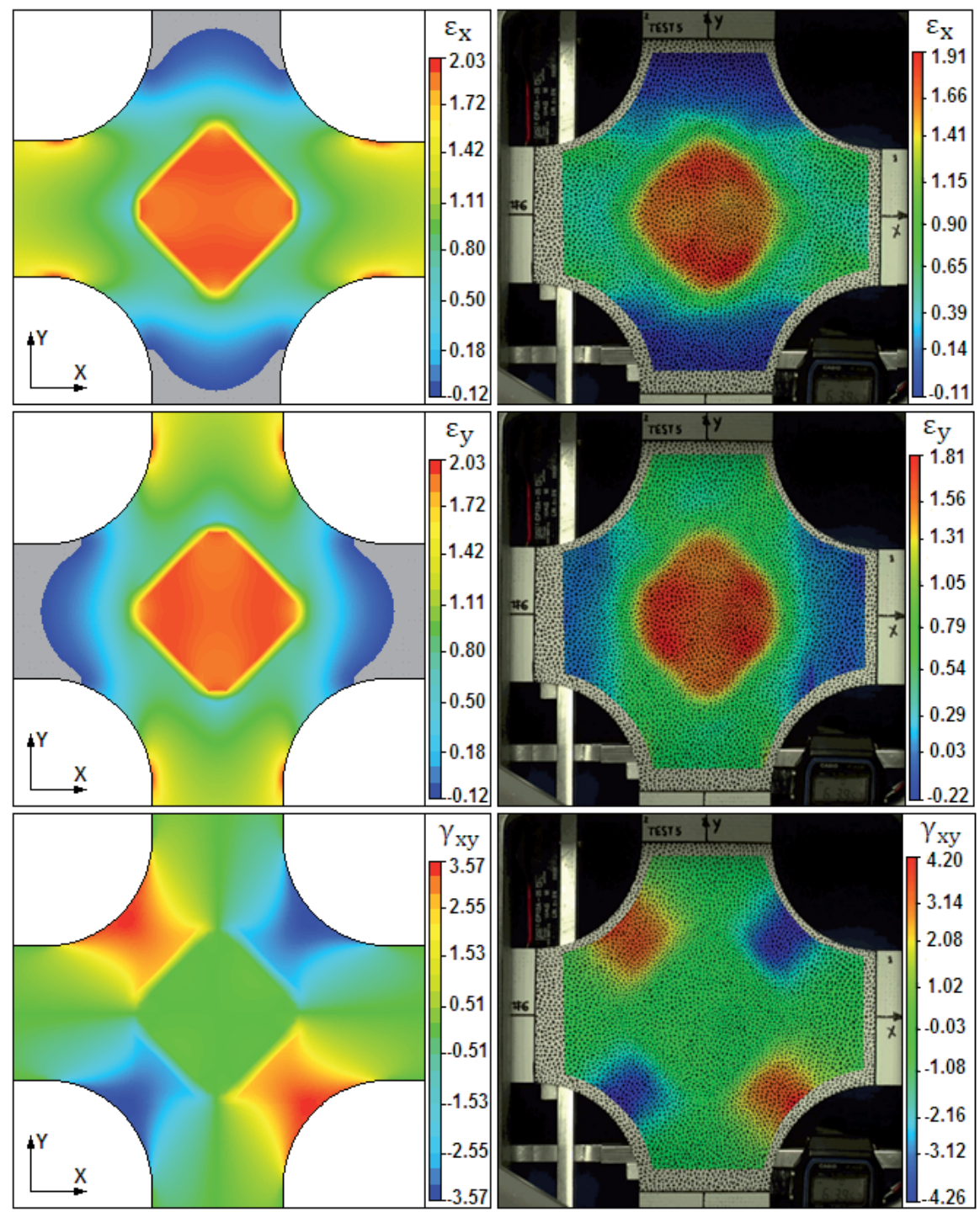

Figure 16. FE vs DIC strain field comparison for Test \#5. The first column corresponds to the FE results, while the second column exhibits the results of the digital image correlation (DIC) process. The first and second row show the linear strain field, while the last row exhibits the shear strain stress field. 


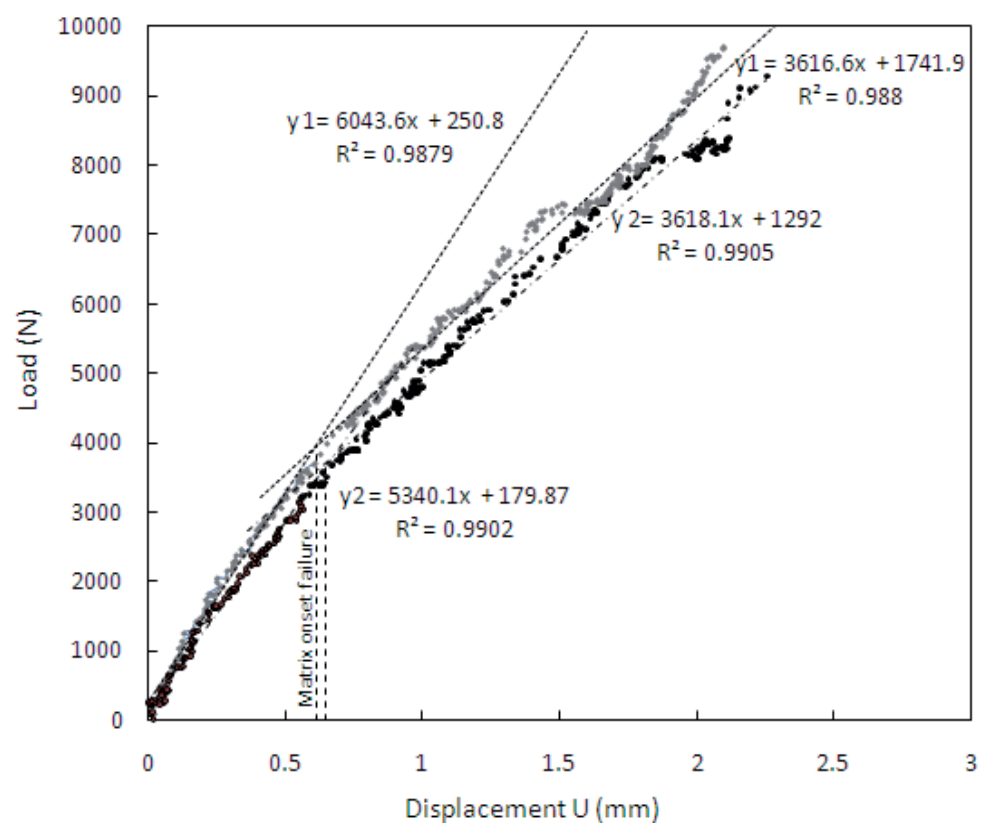

Figure 17. Load vs diplacement for biaxial test \#3. The location where the change of slope occurs is interpreted as the onset of matrix failure.

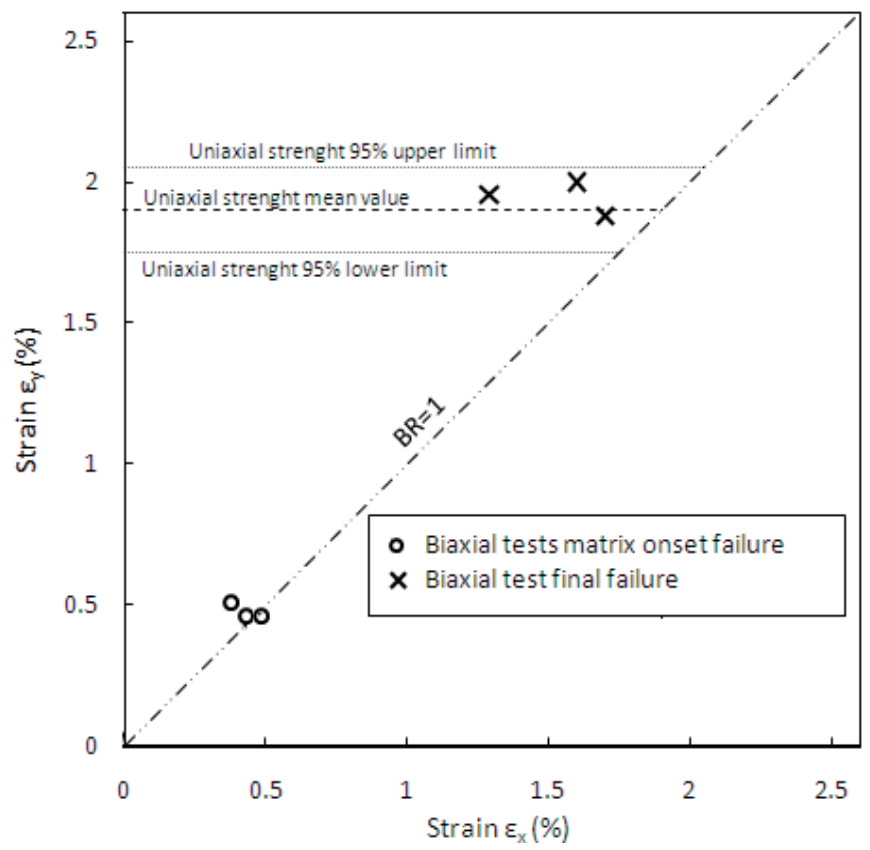

Figure 18. Failure envelope data obtained from the experimental data

Is important to remark that the use of the slope change in the load vs. displacements curves can be significantly influenced by geometrical effects and materials non-linearity, and other 
auxiliary techniques such as sonic emission or in-situ x-ray scanning should be employed to verify that this change can be effectively used as a matrix damage onset indication.

Experimental strength data for single layer biaxial strength obtained from the experimental program are presented in Figure 18 as well as data from uniaxial test performed on five layer specimens. Confidence intervals calculated for the failure strains observed on uniaxial tests are presented in the figure. It should be noted that single-layer strength data fall inside the $95 \%$ confidence limits which suggest that interactions between $\varepsilon_{1}$ and $\varepsilon_{2}$ strains are significant for single layer laminates. This finding should not be used as design criterion before more experimental data are obtained, but it gives a good indication of the feasibility of the methodology presented for the purposes of failure analysis.

\section{Conclusions}

Improvement over existing cruciform specimens for biaxial testing was achieved by proposing a specimen with rhomboidal thinned gauge zone, based on conclusions from a qualitative stress concentration analysis. An optimization based on the experiment design methodology was performed to achieve a highly homogeneous strain distribution withinu the rhomboidal gage zone while shear strains in the cruciform fillets were kept well below the failure values in order to avoid the premature failure typically affecting this kind of specimens. The resulting geometry generates very homogeneous strain field within the gauge zone and keeps shear strains near zero, while keeping shear strains in fillets below the failure value; this is believed to represent a great improvement over other specimens reported in the literature. In addition to meeting the requirements for equi-biaxial tests, the specimen was evaluated under various biaxial ratios, demonstrating that is practically insensitive to biaxial ratio, and hence can be used without any modification to obtain the full tension-tension failure envelope.

A manufacturing process which avoids machining operations normally required to generate the thinned gauge zone was developed, in an attempt to preserve the textile architecture from machining micro-damage. It consists in cutting the rhomboidal windows from the reinforcement layers prior to its matrix impregnation by using a water jet cutting machine. Despite the highly manual work involved in the specimens manufacturing process, it measured specifications were according to those extrapolated from ASTM 3039 for composite materials unidirectional samples.

Validation of the specimen's geometry and manufacturing technique was made through experimental testing, which were conducted on the in-house-developed biaxial machine. The cruciform's full strain field was measured via digital image correlation; the results demonstrate, in close agreement with the results obtained from finite-element(FE) simulations, that the specimen generates a significantly more homogeneous biaxial load state in the gauge zone than others reported in literature, and failure occurs, for all the tests, inside the gauge zone, as intended. 


\section{Author details}

David Alejandro Arellano Escárpita*, Diego Cárdenas, Hugo Elizalde and Ricardo Ramirez

Mechatronics Engineering Department, Instituto Tecnológico y de Estudios

Superiores de Monterrey, Campus Ciudad de México, Col. Ejidos de Huipulco, Tlalpan, México D.F., Mexico

Oliver Probst

Physics Department, Instituto Tecnológico y de Estudios Superiores de Monterrey, Campus Monterrey, Monterrey, N.L., Mexico

\section{References}

[1] Crookston JJ, Long AC, Jones IA. A summary review of mechanical properties prediction methods for textile reinforced polymer composites. Proceedings of the Institution of Mechanical Engineers, Part L: Journal of Materials: Design and Applications 2005; 219(2):91-109.

[2] Soden PD, Kaddour AS, Hinton M.J. Recommendations for designers and researchers resulting from the world-wide failure exercise. Composites Science and Technology 2004; 64(3-4): 589-604.

[3] Kaddour AS, Hinton MJ. Instructions To Contributors Of The Second World-Wide Failure Exercise (Wwfe-II): Part(A).

[4] Lomov SV, Huysmans G, Luo Y, Parmas RS, Prodromou A, Verpoest I, Phelan FR. Textile composites: modelling strategies. Composites: Part A 2001; 32(10): 1379-1394.

[5] Welsh JS, Mayes JS, Key CT, McLaughlin RN. Comparison of MCT failure prediction techniques and experimental verification for biaxially loaded glass fabric-reinforced composite laminates. Journal of Composite Materials 2004; 38(24):2165-2181.

[6] Swanson SR, Smith LV. Comparison of the biaxial strength properties of braided and laminated carbon fibre composites. Composites: Part B 1996; 27(1):71-77.

[7] Welsh JS, Adams DF. An experimental investigation of the biaxial strength of IM6/35016 carbon/epoxy cross-ply laminates using cruciform specimens. Composites: Part A 2002; 33(6):829-839.

[8] Welsh JS, Mayes JS, Biskner A. 2-D biaxial testing and failure predictions of IM7/977-2 carbon/epoxy quasi-isotropic laminates. Composite Structures 2006; 75(1-4):60-66.

[9] Ng RK, Yousefpour A, Uyema M, Ghasemi MN. Design, Analysis, Manufacture, and Test of Shallow Water Pressure Vessels Using E-Glass/Epoxy Woven Composite Material for a Semi-Autonomous Underwater Vehicle. Journal of Composite Materials 2002; 36(21): 2443-2478.

\footnotetext{
" Corresponding Author
} 
[10] Ebrahim L, Van Paepegem W, Degrieck J, Ramault C, Makris A, Van Hemelrijck D. Strain distribution in cruciform specimens subjected to biaxial loading conditions. Part 1: Two-dimensional versus three-dimensional finite element model. Polymer Testing 2010; 29(1):7-13.

[11] Ebrahim L, Van Paepegem W, Degrieck J, Ramault C, Makris A, Van Hemelrijck D. Strain distribution in cruciform specimens subjected to biaxial loading conditions. Part 2: Influence of geometrical discontinuities. Polymer Testing 2010; 29(1):132-138.

[12] Fawaz, Z. Étude analytique, numérique et expérimentale portant sur la rupture et la fatigue biaxiales des lamelles renforcées de fibres. PhD Thesis, Sherbrooke: Université de Sherbrooke, 1992.

[13] Gupte AA. Optimization of Cruciform Biaxial Composite Specimen. In: Master on Science Thesis. South Dakota State University, 2003.

[14] Sepúlveda CG. Biaxial Testing of Composite Materials: Technical Specifications and Experimental Set-up Maestría en Ciencias con Especialidad en Sistemas de Manufactura México Mayo 2009

[15] Soden PD, Hinton MJ, Kaddour AS. Biaxial test results for strength and deformation of a range of E-glass and carbon fibre reinforced composite laminates: failure exercise benchmark data. In: Failure Criteria in Fibre Reinforced Polymer Composites: the World-Wide Failure Exercise. Oxford: Elsevier Science LTD, 2004, p. 52-96.

[16] Bird JE, Duncan J. Strain hardening at high strain in aluminum alloys and its effect on strain localization. Metallurgical and Materials Transactions A 1981; 12(2): 235-241.

[17] Dudderar TF, Koch, Doerries DE. Measurement of the shapes of foil bulge-test samples. Experimental Mechanics 1977; 17(4): 133-140.

[18] Boehler JP, Demmerle S, Koss S. A New Direct Biaxial Testing Machine for Anisotropic Materials. Experimental Mechanics 1944; 34(1): 1-9.

[19] Mönch, E., and D. Galster. “A Method for Producing a Defined Uniform Biaxial Tensile Stress Field." British Journal Of Applied Physics 14, no. 11 (1963).

[20] Sacharuk, Z. Critères de rupture et optimisation des éléments en matériaux composites. PhD Thesis. Université de Sherbrooke. Sherbrooke 1990

[21] Youssef, Y. Résistance des composites stratifiés sous chargement biaxial: validation expérimentale des prédictions théoriques. PhD Thesis, Sherbrooke: Université de Sherbrooke, 1995.

[22] Arellano D, Sepúlveda G, Elizalde H, Ramírez R. "Enhanced cruciform specimen for biaxial testing of fibre-reinforced composites." International Materials Research Congress. Cancun, 2007.

[23] Yong Yu, Min Wan, Xiang-Dong Wu, Xian-Bin Zhou. Design of a cruciform biaxial tensile specimen for limit strain analysis by FEM. Journal of Materials Processing Technology 2002; 123:(67-70)

[24] Antoniou AE, Van Hemelrijck D, Philippidis P. Failure prediction for a glass/epoxy cruciform specimen under static biaxial loading. Composite Science and Technology 2010; 70(8):1232-1241. 
[25] ASTM Standard D6856-03, Standard Guide for Testing Fabric-Reinforced Textile Composite Materials, 2003.

[26] Kwon HJ, Jar PYB, Xia Z. Characterization of bi-axial fatigue resistance of polymer plates. Journal of Materials Science 2005; 40(4):965- 972.

[27] Gdoutos EE, Gower M, Shaw R, Mera R. Development of a Cruciform Specimen Geometry for the Characterisation of Biaxial Material Performance for Fibre Reinforced Plastics. Experimental Analysis of Nano and Engineering Materials and Structures. Springer Netherlands, 2007. p. 937-938.

[28] [28] Chaudonneret M, Gilles P, Labourdette R, Policella H. Machine d'essais de traction biaxiale pour essais statiques et dynamiques. La Recherche Aérospatiale 1977:299-305.

[29] David Alejandro Arellano Escárpita. Experimental investigation of textile composites strength subject to biaxial tensile loads. Ph.D. Thesis. Instituto Tecnológico y de Estudios Superiores de Monterrey. Monterrey 2011.

[30] Clay SB. Biaxial Testing Apparatus. United States of America Patent 5905205. 18 May 1999.

[31] Ferron G, Makinde A. Design and Development of a Biaxial Strength Testing Device. Journal of Testing and Evaluation (ASTM) 16, no. 3 (May 1988).

[32] [32] Pascoe KJ, de Villiers JWR. Low Cycle Fatigue of Steels Under Biaxial Straining. Journal of Strain Analysis 1967; 2(2).

[33] Welsh JS, Adams DF. Development of an Electromechanical Triaxial Test Facility for Composite Materials. Experimental Mechanics 2000; 40(3): 312-320.

[34] Fessler H, Musson JK. A 30 Ton Biaxial Tensile Testing Machine. Professional Engineering Publishing 1969; 4(1): 22-26.

[35] Makinde A, Thibodeau L, Neale K. Development of an apparatus for biaxial testing using cruciform specimens. Experimental Mechanics 1992; 32(2): 138-144.

[36] Hayhurst DR. A Biaxial-Tension Creep-Rupture Testing Machine. Professional Engineering Publishing 1973; 8(2): 119-123.

[37] Ferron, G. Dispositif perfectionné d'essais de traction biaxiale. France. 26 Septembre 1986.

[38] Doyle JF, Phillips JWP. Manual on experimental stress analysis. Society for Experimental Mechanics (U.S.). Society for Experimental Mechanics, 1989.

[39] Post D, Han B. Chap 22, "Moire Interferometry," Handbook on Experimental Mechanics, W. N. Sharpe, Jr., ed., Springer-Verlag, NY, 2008.

[40] Gre'diac M. The use of full-field measurement methods in composite material characterization: interest and limitations. Composites: Part A 2004; 35(7-8):751-761.

[41] Lecompte D, Smits A, Bossuyt S, Sol H, Vantomme J, Van Hemelrijck D, Habraken AM. Quality assessment of speckle patterns for digital image correlation. Optics and Lasers in Engineering 2006; 44(11):1132-1145. 
[42] Bing P, Kemao Q, Huimin X, Anand A. Two-dimensional digital image correlation for in-plane displacement and strain measurement: a review. Measurement Science and Technology 2009; 20(6): 1-17.

[43] ASTM Standard D 3039/D 3039M, Standard Test Method for Tensile Properties of Polymer Matrix Composite Materials, 2000. 


\title{
Modelling of Fracture of Anisotropic Composite Materials Under Dynamic Loads
}

\author{
Andrey Radchenko and Pavel Radchenko \\ Additional information is available at the end of the chapter
}

http://dx.doi.org/10.5772/48549

\section{Introduction}

At present, the wide application of the materials with preset directivity of properties in various fields of engineering defines the increased interest to the investigations of anisotropic materials behaviour under various conditions. But in Russia as well as abroad such investigations are conducted mainly for static conditions. The behaviour of anisotropic materials under dynamic loads is practically not investigated. This is especially the case with experimental investigations as well as with mathematical and numerical modelling. The impact interaction of the solids in a wide range of kinematic and geometric conditions is the complex problem of mechanics. The difficulties, connected with the theoretic study of the fracture and deformation of materials on impact by analytical methods force to introduce member of simplifying hypothesis which distort the real picture in majority of cases. In this connection it should be accepted that the leading role in the investigation of phenomena, connected with high-speed interaction of solids belongs to the experimental and numeric investigations at present. The investigations of the material damage under impact show that the fracture mechanisms change with the interaction conditions. The experiments strongly testifiers that in a number of case the resulting fracture is determined by the combination of several mechanisms. But in the experiments we fail to trace sequence, operation time and the contribution of various fracture mechanisms. Besides, the distractions, obtained at the initial stages of the process can't always be identified in the analysis of the resulting fracture of the materials. For anisotropic materials the strength itself is multivalued and uncertain notion due to the polymorphism of behaviour of these materials under the load. The limiting state of anisotropic bodies may be of different physical nature in dependence on load orientation, stressed state type and other factors. The dependence of the physical nature of limiting states is revealed in the sturdy of the experimental data. The investigations of hydrostatic pressure effect upon the strength of isotropic materials show that comprehensively the compression exerts a weak action on the 
resistance of isotropic materials under static loads. Therefore, the classic theories of strength, plasticity and creep are based on assumption about the lack of the effect of fall stress tensor upon strength isotropic materials. In the experiments with anisotropic materials it was state that flow phenomenon may arise only under the action of hydrostatic pressure. Upon the materials, strength is due to the anisotropy. The shape of anisotropic bodies changes under the action of hydrostatic pressure. If these changes reach such values that, they don't disappear under relief, the limiting state should come. Therefore, the postulate of classic strength that hydrostatic pressure can't transfer the material to the dangerous state is not valid for anisotropic materials. The phenomenological approach to the investigation of the dynamics of deformation and fracture of anisotropic and isotropic materials is used in the project. The phenomenological approach to the materials strength requires that the conditions of the transition into the limiting state of various physical natures should be determinated by one equation (criterion). The necessity of such an approach results from fracture polymorphism, being deduced experimentally. For anisotropic bodies the phenomenological approach has many advantages, since there appears the possibility to use general condition of strength for the material different in composition and technology but similar in symmetry of properties, and also for the materials with substantial anisotropy, for which one and the same stressed state can result in limiting conditions, different in physical nature.

\section{Equations of the model}

\subsection{Basic equations}

The system of the equations describing non-stationary adiabatic movements of the compressed media in the Cartesian coordinate system $X Y Z$, includes following equations (Johnson, 1977):

- continuity equation

$$
\dot{\rho}+\operatorname{div} \rho \vec{v}=0
$$

- motion equation

$$
\begin{aligned}
& \rho \dot{u}=\sigma_{x x, x}+\sigma_{x y, y}+\sigma_{x z, z} \\
& \rho \dot{v}=\sigma_{y x, x}+\sigma_{y y, y}+\sigma_{y z, z} \\
& \rho \dot{w}=\sigma_{z x, x}+\sigma_{z y, y}+\sigma_{z z, z}
\end{aligned}
$$

- $\quad$ energy equation

$$
\dot{E}=\frac{1}{\rho} \sigma_{i j} e_{i j} ; i, j=x, y, z
$$

Here $\rho$-density of media; $\vec{v}$ - velocity vector, $u, v, w$ - components of velocity vector on axes $x, y, z$ accordingly; $\sigma_{i j}$-components of a symmetric stress tensor; $E$ - specific 
internal energy; $e_{i j}$ - components of a symmetric strain rate tensor; the point over a symbol means a time derivative; a comma after a symbol - a derivative on corresponding coordinate.

The behavior of the aluminum isotropic cylinder at high-velocity impact is described by elastic-plastic media, in which communication between components of strain velocity tensor and components of stress deviator are defined by Prandtl-Reuss equation:

$$
2 G\left(e_{i j}-\frac{1}{3} e_{k k} \delta_{i j}\right)=\frac{D S^{i j}}{D t}+\lambda S^{i j},(\lambda \geq 0) ; \frac{D S^{i j}}{D t}=\frac{d S^{i j}}{d t}-S^{i k} \omega_{j k}-S^{j k} \omega_{i k}
$$

where $\omega_{i j}=\frac{1}{2}\left(\nabla_{i} v_{j}-\nabla_{j} v_{i}\right), G-$ shear modulus. Parameter $\lambda=0$ at elastic deformation, and at elastic $(\lambda>0)$ is defined by means of a Mises condition:

$$
S^{i j} S_{i j}=\frac{2}{3} \sigma_{d}^{2}
$$

where $\sigma_{d}$-dynamic yield point. The ball part of stress tensor (pressure) is calculated on the Mi-Gruneisen equation as function of specific internal energy $E$ and density $\rho$ :

$$
P=\sum_{n=1}^{3} K_{n}\left(\frac{V_{0}}{V}-1\right)^{n}\left[\frac{1-K_{0}\left(\frac{V_{0}}{V}-1\right)}{2}\right]+K_{0} \rho E
$$

where $K_{0}, K_{1}, K_{2}, K_{3}$ - constants of material.

\subsection{Model of deformation and fracture of anisotropic materials}

The behavior of an anisotropic material of targets is described within the limits of elasticfragile model. Before fracture components of a stress tensor in a target material were defined from equations of the generalized Hooke's law which have been written down in terms of strain rate:

$$
\dot{\sigma}_{\mathrm{ij}}=C_{i j k l} e_{k l}
$$

where $C_{i j k l}$ - elastic constants.

Thus components of a tensor of elastic constants possess, owing to symmetry of stress tensors and strain tensors and presence of the elastic potential, following properties of symmetry:

$$
C_{i j k l}=C_{j i k l}=C_{i j l k}=C_{i j l k} ; \quad C_{i j k l}=C_{k l i j}
$$

At transition to another, also orthogonal, coordinate system, elastic constants will be transformed by equations: 


$$
C_{a b c d}^{\prime}=C_{i j k l} q_{i a} q_{j b} q_{k c} q_{l d}
$$

where $q_{i j}$ - cosine of the angle between corresponding axes $i$ and $j$. In three-dimensional space transformation of the component of a tensor of the fourth rank demands summation of the compositions, containing as multipliers 4 cosines of angles of rotation of axes.

Fracture of an anisotropic material is described within the limits of model with use of Tsai$\mathrm{Wu}$ fracture criterion with various ultimate strengths of pressure and tension (Tsai \& $\mathrm{Wu}$, 1971). This criterion, which has been written down by scalar functions from components of a stress tensor, has the following appearance:

$$
f\left(\sigma_{i j}\right)=F_{i j} \sigma_{i j}+F_{i j k l} \sigma_{i j} \sigma_{k l}+\ldots \geq 1 ; i, j, k, l=1,2,3
$$

Here $F_{i j}$ and $F_{i j k l}$ are components of tensor of the second and the fourth rank respectively, and obey transformation laws:

$$
F_{a b}^{\prime}=F_{\mathrm{ij}} q_{i a} q_{j b} ; \quad F_{a b c d}^{\prime}=F_{\mathrm{ijk} 1} q_{i a} q_{j b} q_{k c} q_{l d}
$$

Components of tensors of strength for criterion are defined by following equations:

$$
F_{i i}=\frac{1}{X_{i i}}-\frac{1}{X_{i i}^{\prime}} ; \quad F_{i i i i}=\frac{1}{X_{i i} X_{i i}^{\prime}} ; \quad F_{i j}=\frac{1}{2}\left(\frac{1}{X_{i j}}-\frac{1}{X_{i j}^{\prime}}\right) ; \quad F_{i j i j}=\frac{1}{4 X_{i j} X_{i j}^{\prime}} ; i \neq j
$$

where $X_{i i}, X_{i i}^{\prime}$ - limits of strength on pressure and tension along the direction $i ; X_{i j}, X_{i j}^{\prime}-$ shear strength along the two opposite directions with $i \neq j$. Coefficients $F_{1122}, F_{2233}, F_{3311}$ are defined at carrying out the experiments on biaxial tension in planes 1-2, 2-3, 1-3 accordingly. The remained coefficients are defined similarly at combined stressing in corresponding planes (Radchenko et al., 2012).

It is supposed that fracture of anisotropic materials in the conditions of intensive dynamic loads occurs as follows:

- $\quad$ if strength criterion is violated in the conditions of pressure $\left(e_{k k} \leq 0\right)$, the material loses anisotropy of properties, and its behaviour is described by hydrodynamic model, thus the material keeps its strength only on pressure; the stress tensor becomes in this case spherical $\left(\sigma_{i j}=-P\right)$;

- if the criterion is violated in the conditions of tension $\left(e_{k k}>0\right)$, the material is considered completely fractured, and components of a stress tensor are appropriate to be equal to zero $\left(\sigma_{i j}=0\right)$.

Pressure in orthotropic materials of targets is calculated by means of the equation of a condition (Kanel et al., 1996):

$$
P=\left[\exp \left(4 \beta \frac{V_{0}-V}{V_{0}}\right)-1\right] \frac{\rho_{0} \alpha^{2}}{4 \beta}
$$


Here $\rho_{0}$ is initial density of a material; $V_{0}, V$ - relative initial and current volumes. Coefficients of the given equation are calculated from a shock adiabat: $D=\alpha+\beta u$, where $\alpha=1400 \mathrm{~m} / \mathrm{s}, \beta=2.25$, and $u$ - mass velocity.

\subsection{Initial and boundary conditions}

It is considered (fig. 1) a three-dimensional task of high-speed interaction of compact (diameter of the projectile is equal to its height) cylindrical projectile (area $D_{1}$ ) with one or several targets (areas $D_{2}, D_{3}, D_{4}$ ). In this paper we consider the materials with the following mechanical characteristics (Ashkenazi \& Ganov, 1980): Steel St3 with $\rho_{0}=7850$ $\mathrm{kg} / \mathrm{m}^{3}, E=204 \mathrm{GPa}, \mu=0.3, G=79 \mathrm{GPa}, \sigma_{0.2}=1.01 \mathrm{GPa}, K_{0}=1.91, K_{1}=153 \mathrm{GPa}, K_{2}=176 \mathrm{GPa}$, $K_{3}=53.1 \mathrm{GPa}$; aluminum with $\rho_{0}=2700 \mathrm{~kg} / \mathrm{m}^{3}, E=70 \mathrm{GPa}, \mu=0.3, \mathrm{G}=27 \mathrm{GPa}, \sigma_{0.2}=310 \mathrm{MPa}$, $K_{0}=2.13, K_{1}=74.4 \mathrm{GPa}, K_{2}=53.2 \mathrm{GPa}, K_{3}=30.5 \mathrm{GPa}$; organoplastic with $\rho_{0}=1350 \mathrm{~kg} / \mathrm{m}^{3}$, $E_{1}=48.6 \mathrm{GPa}, E_{2}=21.3 \mathrm{GPa}, E_{3}=7.1 \mathrm{GPa}, \mu_{12}=0.28, \mu_{23}=0.26, \mu_{31}=0.037, G_{12}=930 \mathrm{GPa}$, $G_{23}=900 \mathrm{GPa}, G_{31}=850 \mathrm{GPa}, X_{11}=2.67 \mathrm{GPa}, X_{22}=1.18 \mathrm{GPa}, X_{33}=0.395 \mathrm{GPa}, X_{11}^{\prime}=0.37 \mathrm{GPa}$, $X_{22}^{\prime}=0.5 \mathrm{GPa}, X_{33}^{\prime}=1.94 \mathrm{GPa}, X_{12}=0.975 \mathrm{GPa}, X_{23}=0.8 \mathrm{GPa}, X_{31}=0.607 \mathrm{GPa}, X_{11}^{(12)}=2.3 \mathrm{GPa}$, $X_{11}^{(31)}=2 \mathrm{GPa}, X_{22}^{(12)}=1 \mathrm{GPa}, X_{22}^{(23)}=0.9 \mathrm{GPa}, X_{33}^{(23)}=0.35 \mathrm{GPa}, X_{33}^{(31)}=0.31 \mathrm{GPa}, c_{x}=6000 \mathrm{~m} / \mathrm{s}$, $c_{y}=3970 \mathrm{~m} / \mathrm{s}, c_{z}=2300 \mathrm{~m} / \mathrm{s}$. The meeting corner (between a normal to a target and a longitudinal axis of the projectile) made a corner $\alpha=0^{\circ}$ (normal impact).

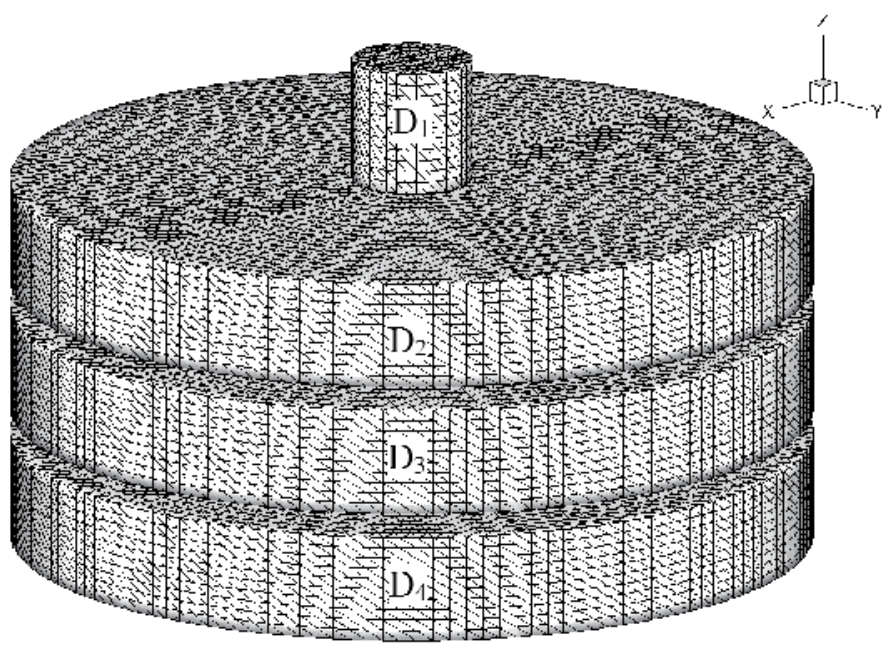

Figure 1. Three-dimensional formulation of the task

Initial conditions $(t=0)$ :

$$
\begin{gathered}
\sigma_{i j}=E=u=v=0, \quad w=v_{0}, \quad i, j=x, y, z, \quad x, y, z \in D_{1} \\
\sigma_{i j}=E=u=v=w=0, \quad i, j=x, y, z, \quad x, y, z \in D_{2}, D_{3}, D_{4} \\
\rho=\rho_{i}, \quad x, y, z \in D_{i}, \quad i=1,2,3,4
\end{gathered}
$$


Boundary conditions:

On free surfaces conditions of free border are realized:

$$
\bar{T}_{n n}=\bar{T}_{n \tau 1}=\bar{T}_{n \tau 2}=0
$$

On contact surface sliding condition without a friction is realized:

$$
\bar{T}_{n n}^{+}=\bar{T}_{n n}^{-}, \bar{T}_{n \tau}^{+}=\bar{T}_{n \tau}^{-}=\bar{T}_{n s}^{+}=\bar{T}_{n s}^{-}=0, \bar{v}_{n}^{+}=\bar{v}_{n}^{-}
$$

Here $\bar{n}$ - a unit vector of a normal to a surface in a considered point, $\bar{\tau}$ and $\bar{s}$ - unit vectors, tangents to a surface in this point, $\bar{T}_{n}-$ a force vector on a platform with a normal $\bar{n}, \bar{v}-$ a velocity vector. The subscripts at vectors $\bar{T}_{n}$ and $\bar{v}$ also mean projections on corresponding basis vectors; the badge plus "+" characterizes value of parameters in a material on the top border of a contact surface, a badge a minus "-" - on bottom.

The problem solves numerically using the finite element method in the explicit formulation by Johnson G.R. (Johnson, 1977).

\section{Check of adequacy of the model}

The series of test calculations for check convergence of the solution (independence of the solution from a spatial interval) has been passed. Dependence of the $\sigma_{x x}$ component in the central point of a target from total number of elements $N_{E}$ in a calculating grid was considered. The received curve shows fast convergence of the decision at a grid compaction (fig. 2).

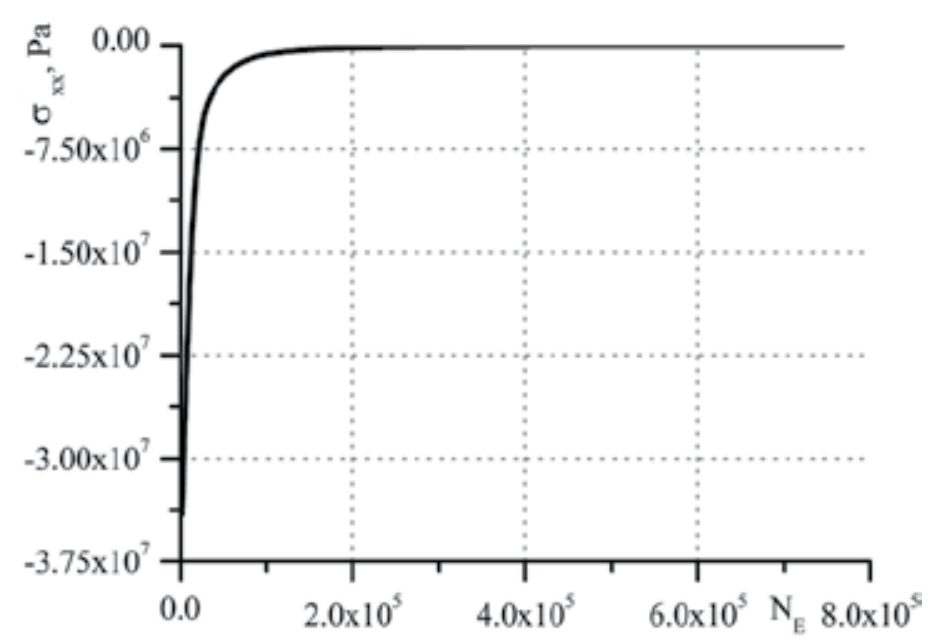

Figure 2. Convergence of the solution 


\subsection{Experiments with isotropic materials}

In connection with a considerable quantity of works on a problem of impact on rigid target, numerical experiments for check of the numerical methods have been made. The problem about normal impact of the cylindrical projectile with length $L_{0}=23.47 \mathrm{~mm}$ and diameter $D_{0}$ $=7.62 \mathrm{~mm}$ on rigid target with initial velocity $v_{0}$ is considered. Material of the projectile steel of St3 mark. In the table 1 the received results of calculations in comparison with experiment and the data of calculations from M.L. Wilkins works on residual length of the drummer $L$ are presented at various velocities of impact (Wilkins \& Giunan, 1973). In the table $1 \delta_{l}$ is the relative divergence between spent calculations and experiments.

\begin{tabular}{|c|c|c|c|c|}
\hline$v_{0}, \mathrm{~m} / \mathrm{sec}$ & Experiment, $\frac{L}{L_{0}}$ & M.L. Wilkins, $\frac{L}{L_{0}}$ & Calculation, $\frac{L}{L_{0}}$ & $\delta_{l}, \%$ \\
\hline 175 & 0.911 & 0.911 & 0.915 & 0.4 \\
\hline 252 & 0.842 & 0.842 & 0.839 & 0.4 \\
\hline 311 & 0.766 & 0.766 & 0.760 & 0.8 \\
\hline 402 & 0.635 & 0.667 & 0.619 & 2.5 \\
\hline
\end{tabular}

Table 1. Comparison with experiments and calculations

For check of adequacy of model the number of comparisons of numerical calculations with experimental data has been spent. Table 2 shown the results of experiments and calculations at the interaction of the steel projectile with mass of 20 grams with the glass-fibre plastic isotropic targets $\left(\rho_{0}=1930 \mathrm{~kg} / \mathrm{m}^{3}\right)$. The following symbols are introduced into the table: $h$ is the target thickness, $v_{0}$ is the initial projectile velocity, $v_{1}$ is the post-perforation velocity of projectile, $\varepsilon$ is the relative decrease of the projectile height after target piercing, $\delta_{v}$ is the relative divergence between post-perforation velocity of projectile in the experiment and calculation.

\begin{tabular}{|c|c|c|c|c|}
\hline \multirow{2}{*}{$\mathrm{h}, \mathrm{mm}$} & \multirow{2}{*}{$v_{0}, \mathrm{~m} / \mathrm{sec}$} & Experiment & Calculation & $\delta_{v}, \%$ \\
\cline { 3 - 5 } & & $v_{1}, \mathrm{~m} / \mathrm{sec}$ & $v_{1}, \mathrm{~m} / \mathrm{sec}$ & \\
\hline 5 & 992 & 928 & 970 & 6.3 \\
\hline 9 & 1163 & 1013 & 950 & 6.2 \\
\hline 14 & 1064 & 812 & 780 & 4.0 \\
\hline
\end{tabular}

Table 2. Comparison with experiments for isotropic targets

\subsection{Experiments with anisotropic materials}

A number of experiments of penetration for check of the offered model of behavior of anisotropic materials was similarly carried out (Radchenko et al., 1999). The results on beyond barrier speeds of striker at the interaction of 20-gram striker with transtropic barrier are presented in table 3 . 


\begin{tabular}{|c|c|c|c|c|}
\hline \multirow{2}{*}{$\mathrm{h}, \mathrm{mm}$} & \multirow{2}{*}{$v_{0}, \mathrm{~m} / \mathrm{s}$} & Experiment & Calculation & $\delta_{v}, \%$ \\
\cline { 3 - 5 } & & $v_{1}, \mathrm{~m} / \mathrm{s}$ & $v_{1}, \mathrm{~m} / \mathrm{s}$ & \\
\hline 26 & 1054 & 698 & 640 & 8.3 \\
\hline 26 & 1077 & 695 & 638 & 8.2 \\
\hline 18 & 1012 & 897 & 836 & 6.8 \\
\hline 18 & 956 & 838 & 792 & 5.5 \\
\hline
\end{tabular}

Table 3. Comparison with experiments for anisotropic targets

Comparison of numerical and experimental results allows conclude that the proposed model satisfactorily describes the process of breaking through the isotropic and transtropic plates. A deviation of calculated values of the post-penetration velocities from the experimental values does not exceed $8.5 \%$.

\section{Deformation and fracture of anisotropic composite materials and designs under dynamic loading}

\subsection{Pulse effect}

Problems of dynamic deformation of the ball from organoplastic under effect of omnidirectional compression pulse are considered in three-dimensional statement. Homogeneous orthotropic ball with diameter of $10 \mathrm{~mm}$ was subjected to compression with pulse pressure of $1 \mathrm{GPa}$ during $3 \mu \mathrm{sec}$ (fig. 3). It is supposed that the failure of anisotropic material in condition of intense dynamic loads happens in accordance with (Radchenko et al., 1999). The material of the ball is orthotropic organoplastic. Already at the time moment of $0.6 \mu \mathrm{sec}$ in $Z X$ cross section (where there is most significant difference between characteristics), the distribution of stress and field of velocities (fig. 4) illustrate the origin of heterogeneous picture of Strain-stress State of the ball. In ZY section the distribution of stress as well as field of velocities is close to one-dimension Strain-stress State of isotropic ball under the effect of omnidirectional compression (fig. 5). At this moment, the stresses achieves maximum values (-2 GPa) near at ball poles on $Z$ axis.

In this case the ball failure arises in the region of maximum stresses. With time the Strainstress State of anisotropic ball differs from Strain-stress State of isotropic ball more strongly. Isolines of stress and field of rates at the shown in fig. 6, fig. 7. Evident sliding lines are seen in fig. 6a and fig. 7a. Only $\sigma_{x}$ distribution in $Z Y$ cross section (fig. $7 \mathrm{~b}$ ) is closest to the distribution of stress in isotropic ball, maximum stress are achieved in the centers.

Up to $1 \mu \mathrm{sec}$ in all directions, mass velocities are directed into the insight of the ball, but with of $1 \mu \mathrm{sec}$, in direction $X$ rates change the sign and the increase in ball size begins in this direction. In other directions, mass velocities are directed inside of the ball up to the moment of load removal $(3 \mu \mathrm{sec})$. This, maximum decrease in ball dimensions in $X$ directions is $11 \%$ and is achieved to $1 \mu \mathrm{sec}$, and in $Z$ direction it is $24 \%$ and is achieved in $3 \mu \mathrm{sec}$. 
Substantial change in the shape of the ball is observed to the moment of cessation of compression pulse action (fig. 8) it acquires dump - bells shape due to the compression along $\mathrm{Z}$ axis.

The expansion of the ball in all directions begins after the release of the load. Fig. 9 shows the field of velocities in $6 \mu \mathrm{sec}$, to that moment the material of the ball has been completely fractured.

The ball under the effect of omnidirectional compression pulse may transform not to ellipsoid but to the dump - bells under the certain relations of its mechanical characteristics of the value of pressure pulse.

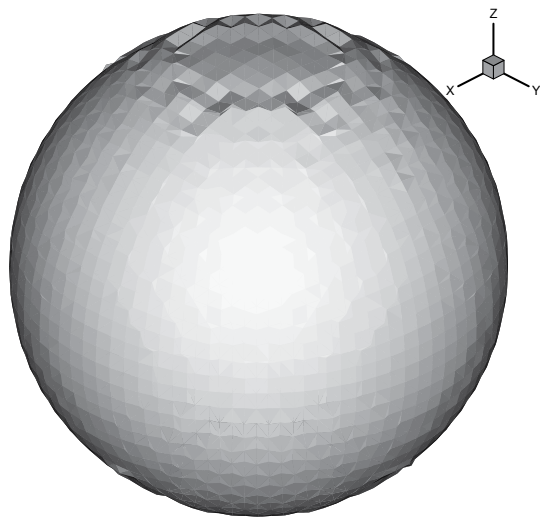

$\mathrm{t}=1.2 \mu \mathrm{sec}$

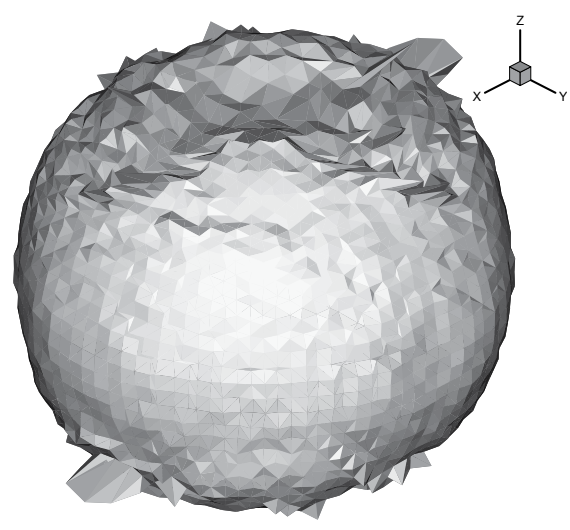

$\mathrm{t}=4 \mu \mathrm{sec}$

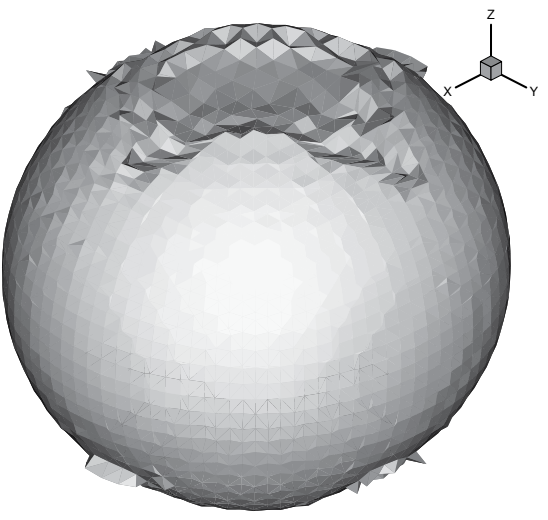

$\mathrm{t}=2.8 \mu \mathrm{sec}$

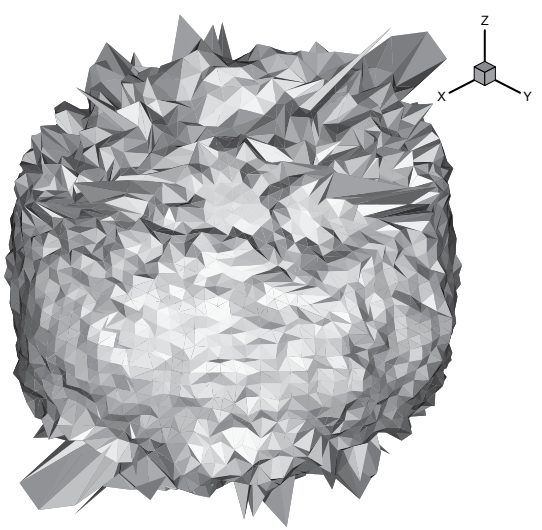

$\mathrm{t}=6 \mu \mathrm{sec}$

Figure 3. Volume configurations of orthotropic ball at loading by the pulse of pressure: $P=P_{0}$ if $t \leq \tau$ and $P=0$ if $t>\tau . P=1 \mathrm{GPa}, \tau=3 \mu \mathrm{sec}$ 


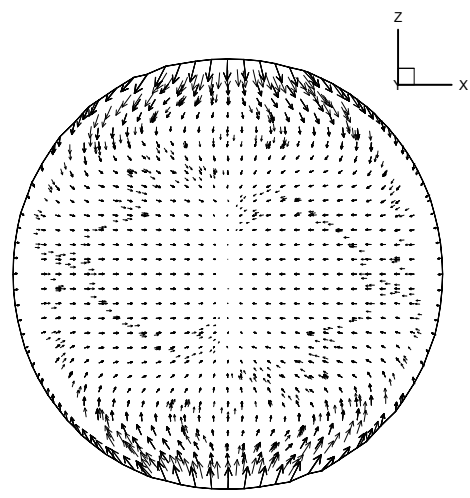

(a)

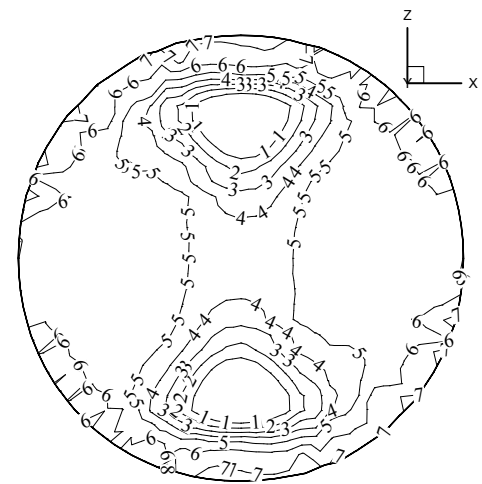

(b)

Figure 4. Field of mass velocities (a) and distribution of isolines of stress $\sigma_{x}(b) . t=0.6 \mu$ sec.1: $-2,2:-1.8$, 3: -1.6, 4: -1.4, 5: -1.2, 6: -1, 7: -0.8, 8: -0.6 GPa

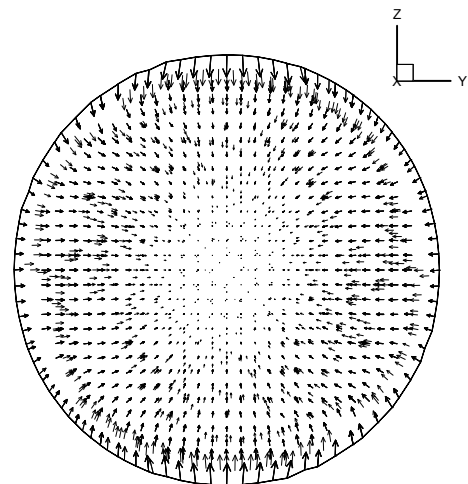

(a)

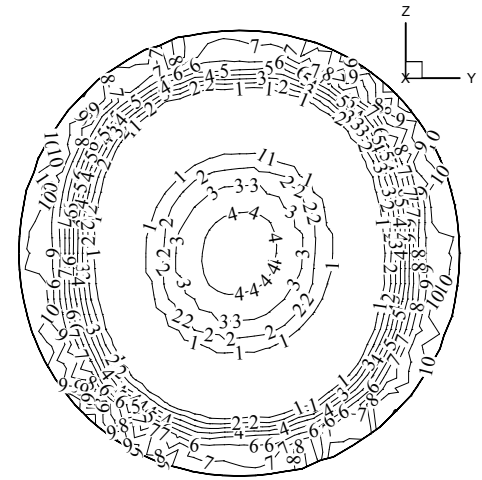

(b)

Figure 5. Field of mass velocities (a) and distribution of isolines of stress $\sigma_{x}(b) . t=0.6 \mu$ sec. $1:-2,2:-1.8$, 3: $-1.6,4:-1.4,5:-1.2,6:-1,7:-0.8,8:-0.6 \mathrm{GPa}$

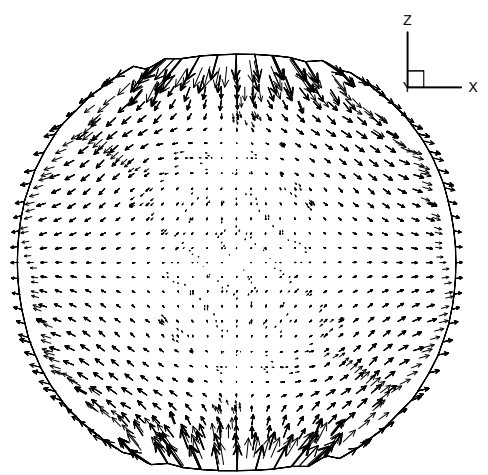

(a)

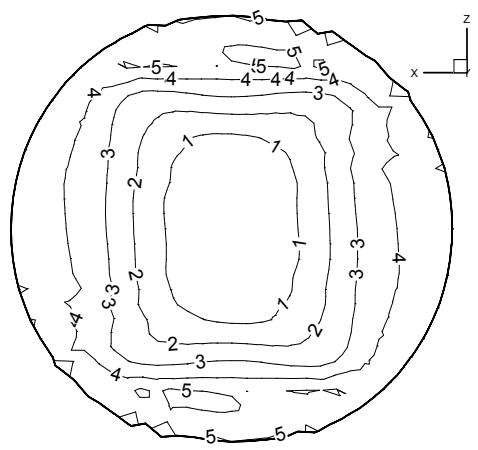

(b)

Figure 6. Field of mass velocities (a) and distribution of isolines of stress $\sigma_{\mathrm{x}}(\mathrm{b}) . t=1.2 \mu \mathrm{sec} .1:-2.4,2:-2$, 3: $-1.6,4:-1.2,5:-0.8,6:-0.4,7: 0,8: 0.4,9: 0.8,10: 1.2 \mathrm{Gpa}$ 


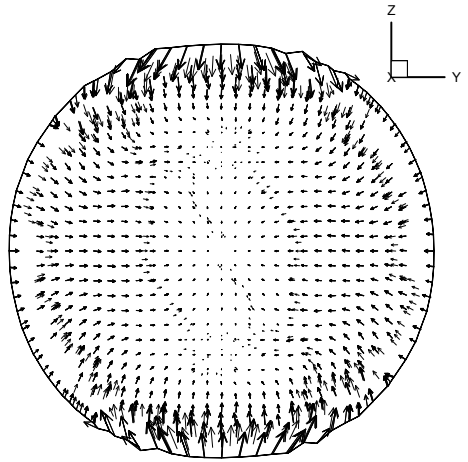

(a)

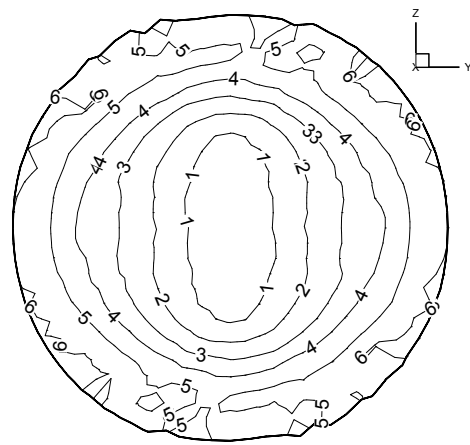

(b)

Figure 7. Field of mass velocities (a) and distribution of isolines of stress $\sigma_{x}(b) . t=1.2 \mu s e c .1:-2.4,2:-2$, 3: $-1.6,4:-1.2,5:-0.8,6:-0.4,7: 0,8: 0.4,9: 0.8,10: 1.2 \mathrm{GPa}$
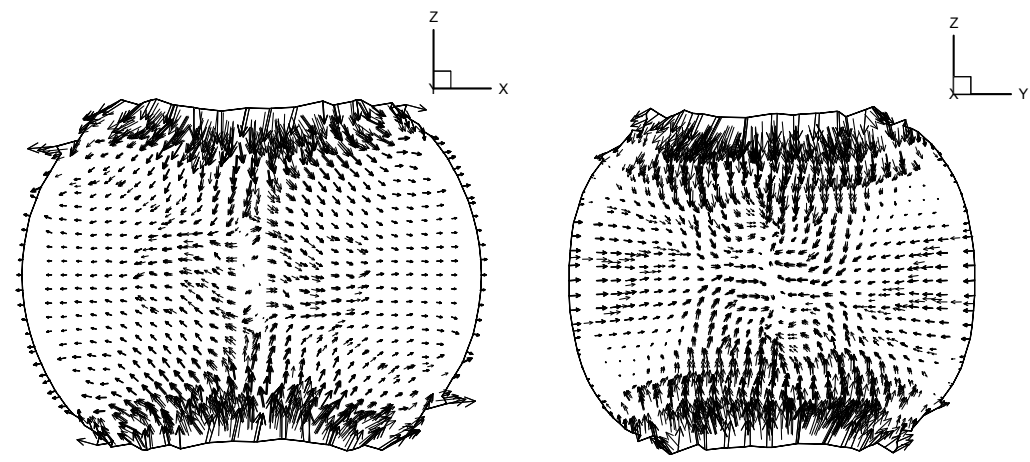

Figure 8. Field of mass velocities. $t=3 \mu \mathrm{sec}$.

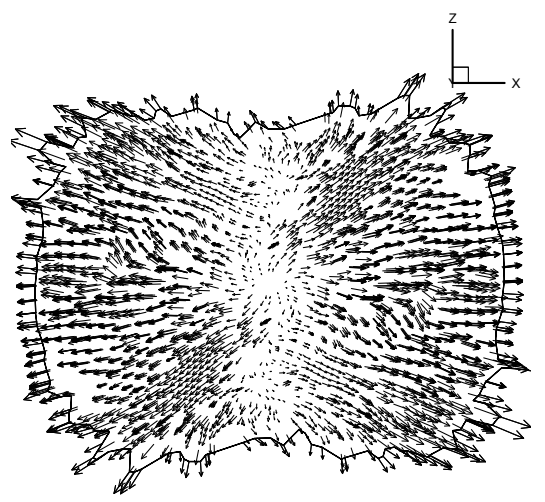

Figure 9. Field of mass velocities. $t=6 \mu$ sec. 


\subsection{Influence of orientation of elastic and strength properties on fracture of anisotropic materials under dynamic loading}

We studied the penetration of a barrier with the initial orientation of the properties, as well as penetration of a barrier with properties reoriented by $90^{\circ}$ about the axis $O Y$. In the direction of the axis $Z$ initial material has the highest strength on compression and the lowest strength on tension. Refocused the material, on the contrary, along the $Z$ axis has the lowest compressive strength and the highest tensile strength. In addition to the various limits of the tensile and compression on the dynamics of fracture will affect significantly the velocity of propagation of waves of compression and unloading, which in an anisotropic material depend on the direction.

A material of the projectile is isotropic steel, a material of targets - orthotropic organoplastic. Orientation of properties of orthotropic material changes by turn of axes of symmetry of an initial material round an axis on an angle $\beta=90^{\circ}$.

On fig. 10 and fig. 11 configurations of the projectile and targets with distribution of isolines of relative volume of fractures for various velocities of interaction at the moment of time $t$ $=40 \mu \mathrm{sec}$ are presented. To the left of a symmetry axis configurations for initial orientation of a material of a target, to the right - for the reoriented material are given.

For a case of initial orientation of properties of organoplastic at velocity $50 \mathrm{~m} / \mathrm{s}$ (fig. 10a, to the left of a symmetry axis) on an obverse surface of a target on perimeter of the projectile and on a contact surface in the target center the conic zones of fracture focused at an angle $45^{\circ}$ to a direction of impact are formed. These zones arise in an initial stage of interaction at the expense of action of tensile stress in the unloading waves extending from an obverse surface of a target and a lateral surface of the projectile. The further development of these zones of fracture is caused by action of tensile stress as a result of introduction of the projectile. At initial velocity $50 \mathrm{~m} / \mathrm{s}$ there is no perforation of a target. To $t=30 \mu \mathrm{sec}$ velocity of the projectile reduces to zero and the kickback of the projectile from a target is observed. Values of vertical component of the velocity of the center of projectile weights $v_{z}$ and a part of the fractured material of a target are presented in table 4 (at tension $D_{t}$ and pressure $D_{p}$ at the moment of time $t=50 \mu \mathrm{sec}$ ).

\begin{tabular}{|c|c|c|c|c|c|c|c|c|}
\hline$v_{0}, \mathrm{~m} / \mathrm{s}$ & \multicolumn{2}{|c|}{50} & \multicolumn{2}{c|}{100} & \multicolumn{2}{c|}{200} & \multicolumn{2}{c|}{400} \\
\hline$\beta$ & $0^{\circ}$ & $90^{\circ}$ & $0^{\circ}$ & $90^{\circ}$ & $0^{\circ}$ & $90^{\circ}$ & $0^{\circ}$ & $90^{\circ}$ \\
\hline$v_{z}, \mathrm{~m} / \mathrm{s}$ & -5.17 & 4.08 & 9.05 & 12.97 & 49.97 & 127.6 & 191.55 & 303.69 \\
\hline$D_{t}$ & 0.012 & 0.005 & 0.056 & 0.011 & 0.162 & 0.128 & 0.502 & 0.282 \\
\hline$D_{p}$ & 0.006 & 0.002 & 0.043 & 0.029 & 0.104 & 0.021 & 0.112 & 0.019 \\
\hline
\end{tabular}

Table 4. Velocity of the center of projectile weights and part of the break material in targets

In case of the reoriented material (fig. 10a, to the right of a symmetry axis) a picture of development of fracture is qualitative other. In this case, strength of a material on pressure 
in a direction of axis $Z$ (an impact direction) is minimal. It leads to that the material break in the wave of pressure formed at the moment of impact and extending on a thickness of a target. Penetration of the projectile thus occurs in already weakened material. Though perforation in this case also isn't present, the projectile gets deeply, and its full braking is observed in $50 \mu \mathrm{sec}$. With increase in velocity of impact the volume of areas of fracture grows. At velocity $100 \mathrm{~m} / \mathrm{s}$ (fig. 10b) fracture areas extend to a greater depth on a thickness of a target. And for an initial material of a target the marked orientation $\left(45^{\circ}\right)$ was kept only by a crack extending from an obverse surface on perimeter of the projectile. The crack located near to an axis of symmetry isn't identified any more. It is caused by that with increase in velocity of impact the amplitude of the pressure wave grows - its size is already sufficient for material fracture in the top half of target.

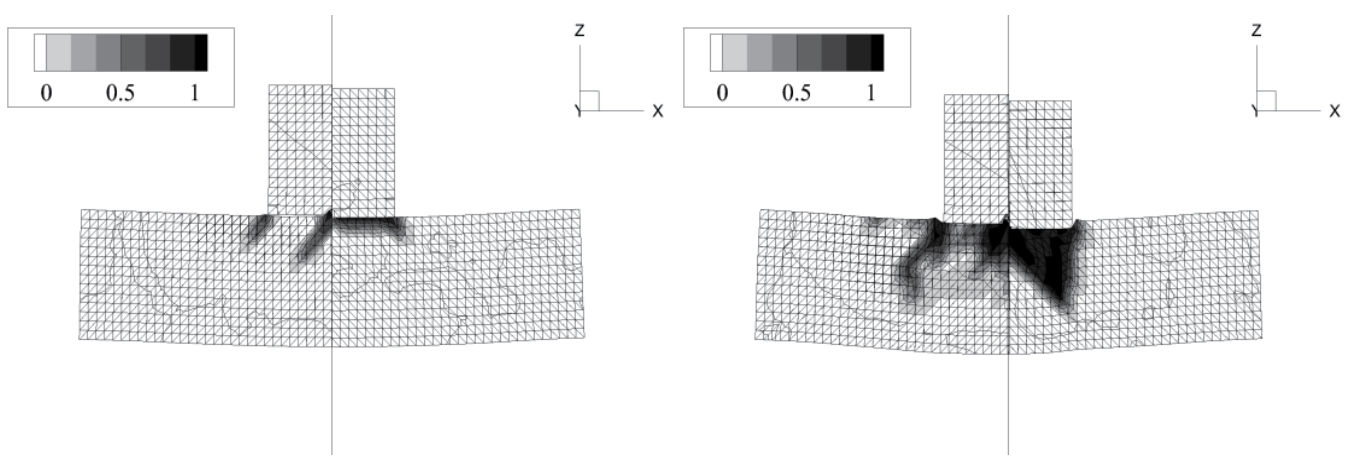

(a)

(b)

Figure 10. Relative volume of fractures in target. $v_{0}=50 \mathrm{~m} / \mathrm{s}(\mathrm{a})$ and $v_{0}=100 \mathrm{~m} / \mathrm{s}(\mathrm{b}), \mathrm{t}=40 \mu \mathrm{sec}$.

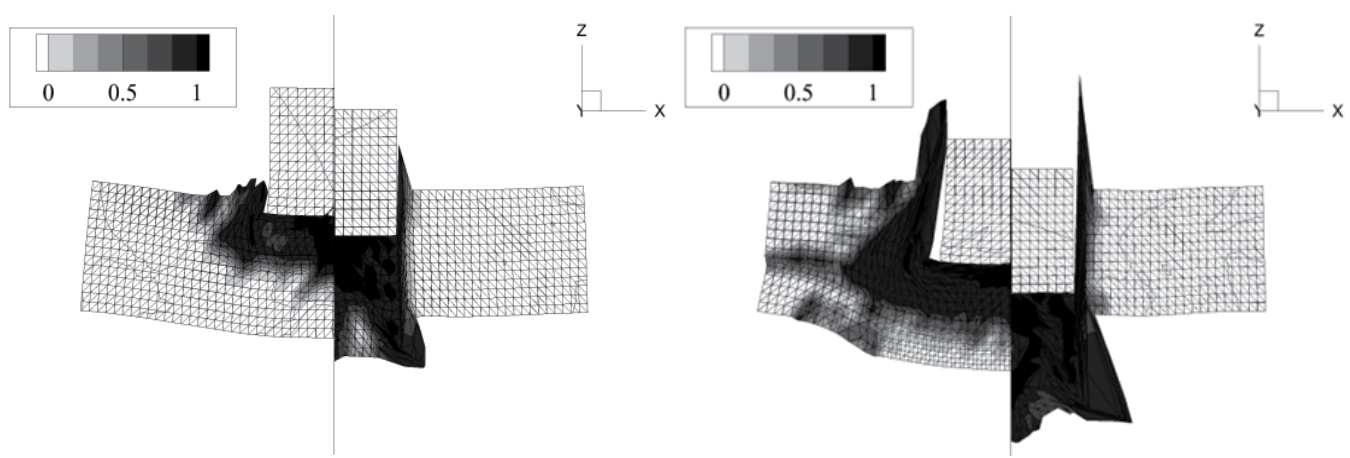

(a)

(b)

Figure 11. Relative volume of fractures in target. $v_{0}=200 \mathrm{~m} / \mathrm{s}$ (a) and $v_{0}=400 \mathrm{~m} / \mathrm{s}(\mathrm{b}), \mathrm{t}=40 \mu \mathrm{sec}$.

In case of the reoriented material the unloading wave extending from a back surface of a barrier, lowers level of compressive stresses that leads to smaller distribution of fracture area on a thickness near to a symmetry axis (fig. 10b). For velocity $100 \mathrm{~m} / \mathrm{s}$ also it is not observed perforation of targets, thus in case of an initial material velocity of the projectile reduced to zero at $45 \mu \mathrm{sec}$, in case of the reoriented material - at $60 \mu \mathrm{sec}$. 
For velocities of impact $200 \mathrm{~m} / \mathrm{s}$ and above (fig. 11) it is already observed perforation of targets from both types of materials. But thus the plate from an initial material greater maintains resistance to penetration of the projectile in comparison with a plate from the reoriented material. For example, at initial velocities $200 \mathrm{~m} / \mathrm{s}$ (fig. 11a) and $400 \mathrm{~m} / \mathrm{s}$ (fig. 11b) post-perforation velocity of the projectile after perforation of plates from an initial material makes $37 \mathrm{~m} / \mathrm{s}$ and $187 \mathrm{~m} / \mathrm{s}$ accordingly, and post-perforation velocity after perforation plates of the reoriented material $125 \mathrm{~m} / \mathrm{s}$ and $300 \mathrm{~m} / \mathrm{s}$. Greater resistance to penetration of the projectile in plates from an initial material is caused by a various picture of fracture which is defined by orientation of elastic and strength properties in relation to external loading. For velocities of impact above $200 \mathrm{~m} / \mathrm{s}$ there is fracture of the reoriented material in the unloading wave extending from a back surface of a target (fig. 11), that increases volume of the break material in front of the projectile, essentially reducing resistance to penetration. Such dynamics of fracture become clear by various velocities of wave distribution in the initial and reoriented materials.

In an initial material velocity of wave distribution the greatest in a direction of an axis $X$ perpendicular to an impact direction, therefore unloading waves from an obverse surface of a target and a lateral surface of the projectile lower stresses in a pressure wave to its exit on a back surface that doesn't lead to material fracture in a pressure wave in the bottom half of plate and a unloading wave from a back surface of the barrier having small amplitude at the expense of easing of a pressure wave.

In the reoriented material velocity of distribution of waves is maximal in a direction of axis $Z$, therefore the pressure wave loses energy only on fracture of a material and being reflected from a back surface by an intensive unloading wave breaking a material.

\subsection{Numerical modeling of deformation and fracture of the composite spaced targets under impact}

This paper presents a comparative analysis of fracture in monolithic and spaced barriers during high-velocity interaction with compact projectiles. The material of barriers is orthotropic organoplastic with a high degree of anisotropy of elastic and strength properties. We investigate the fracture efficiency of the protective properties of monolithic and spaced barriers depending on the orientation of anisotropic material properties in a range of velocities of impact from 750 to $3000 \mathrm{~m} / \mathrm{s}$.

At high-velocity impact on spaced designs the defining role in the fracture of the projectile and barriers is played by shock wave processes. As a result of the extension of these processes there is a fracture of the projectile and thin screens protecting the main design. Thin screens are effective for protection of space vehicles against particles of small space debris, moving at velocities more than $3 \mathrm{~km} / \mathrm{s}$ at which intensive fracture of particles begins. At interaction with more massive particles with velocities not over $3 \mathrm{~km} / \mathrm{s}$ thin screens are not so effective. In space similar situations are possible at the interaction of devices with space debris on catching up courses. At velocities of impact in a range not over $3 \mathrm{~km} / \mathrm{s}$ an important role belongs to the strength characteristics of materials. Earlier conducted 
researches of the fracture of the spaced barriers made from isotropic metal materials have shown that their efficiency, in comparison with efficiency of the monolithic barrier, increases with increase in velocity of interaction. Now for manufacturing of aircrafts elements the various types of composite materials having a high degree of anisotropy of the elastic and strength properties are used, and without such property as anisotropy it is impossible to describe and predict the behavior of design elements and of the design as a whole (Radchenko \& Radchenko, 2011).

On fig. 12, 13 computational configurations of the aluminum projectile, of the monolithic barrier and also of the two-layer and three-layer spaced barriers made from anisotropic organoplastic for velocities of impact from 750 to $2000 \mathrm{~m} / \mathrm{s}$ accordingly are presented.
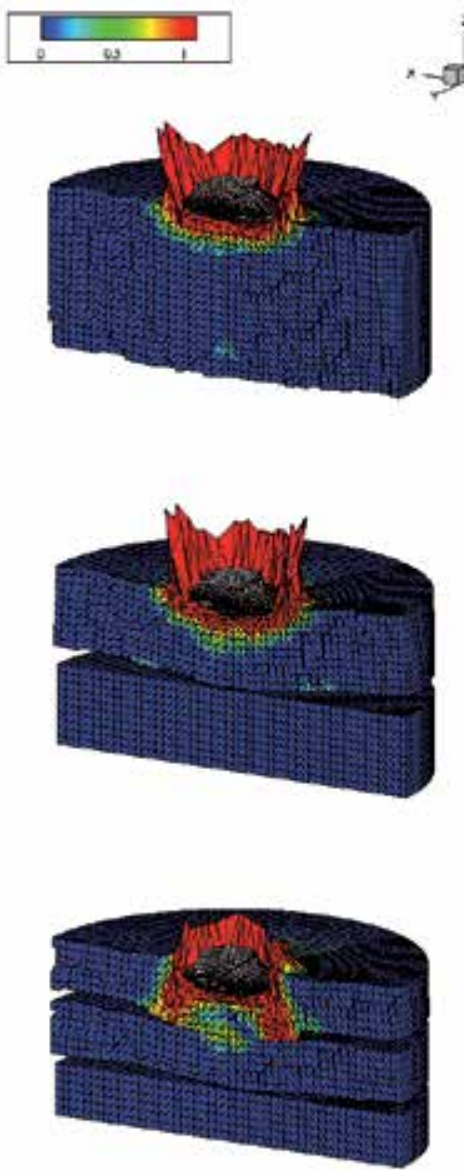

(a)
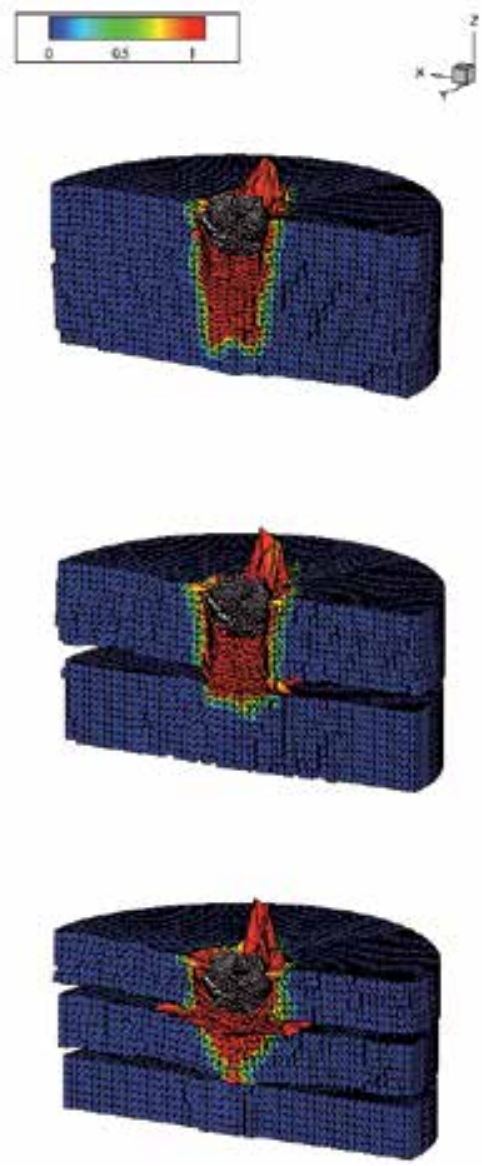

(b)

Figure 12. Calculated configurations of cooperating bodies and isolines of relative volume of fractures $\left(V / V_{0}\right)$ in targets. a: $v_{0}=750 \mathrm{~m} / \mathrm{s}, \beta=0^{\circ}, \mathrm{b}: v_{0}=750 \mathrm{~m} / \mathrm{s}, \beta=90^{\circ} ; \mathrm{t}=40 \mu \mathrm{sec}$

On fig. 12a, fig. 13b barriers made from an initial material are given, on a fig. 12b, fig. $13 b$ there are barriers made from reoriented material. It is necessary to explain that values of $V$ 
and $V_{0}$ are given in node of a mesh: $V$ is a volume of incorporating elements of node in which the fracture condition was satisfied; $V_{0}$ is the total volume of the elements containing this node. Value of 1 for $V / V_{0}$ corresponds to the full fracture of the material in node of a mesh. As researches showed (Radchenko et al., 1999), a picture of the fracture observing in barriers depends on orientation of a material properties in relation to the direction of action of external loading, in this case to an impact direction. As a result the dynamic nature of fractures in a barrier defines an efficiency of its protective properties.

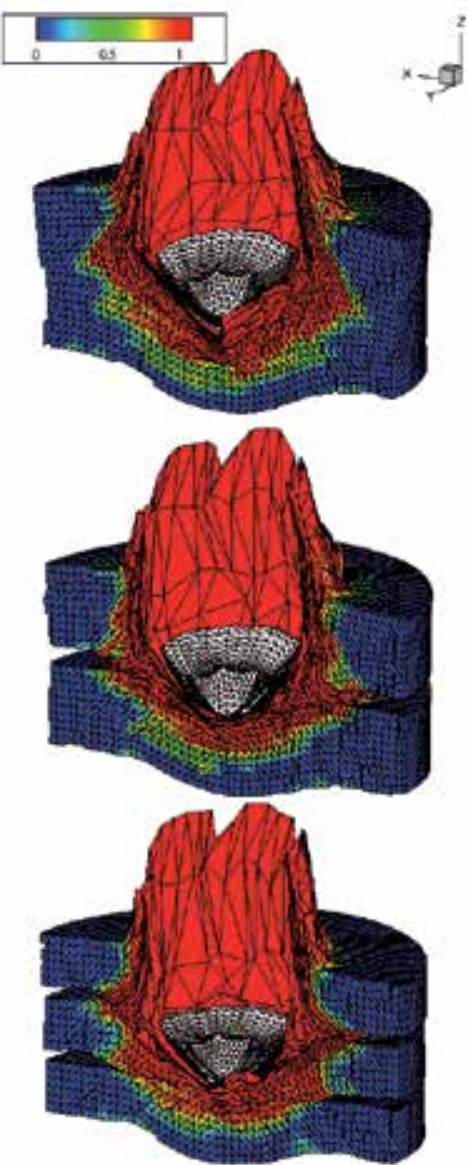

(a)

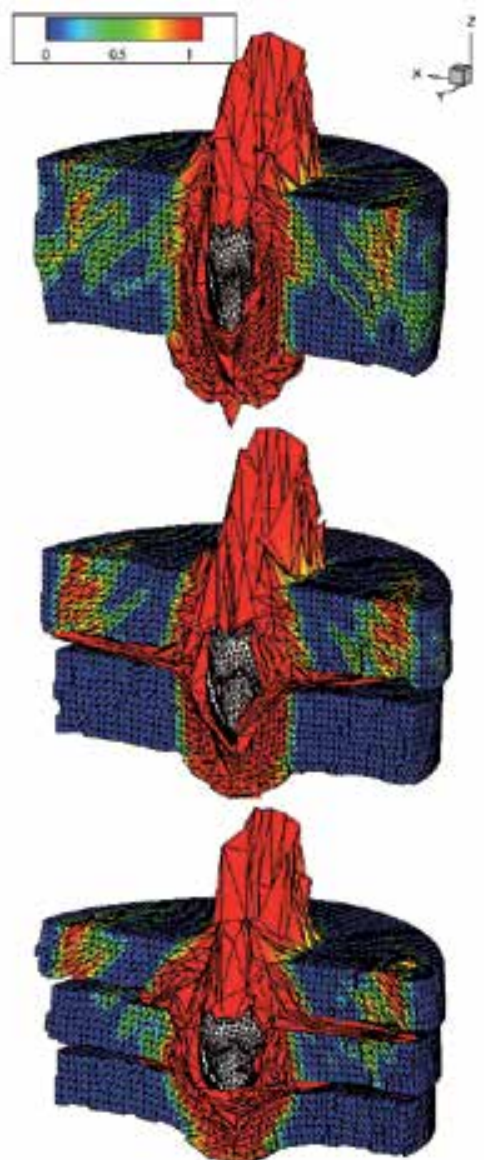

(b)

Figure 13. Calculated configurations of cooperating bodies and isolines of relative volume of fractures $\left(V / V_{0}\right)$ in targets. a: $v_{0}=2000 \mathrm{~m} / \mathrm{s}, \beta=0^{\circ}, \mathrm{b}: v_{0}=2000 \mathrm{~m} / \mathrm{s}, \beta=90^{\circ} ; \mathrm{t}=40 \mu \mathrm{sec}$

The fracture of barriers material begins in a compression wave $\left(e_{k k} \leq 0\right)$, initiated at the moment of impact. Fracture development in barriers depends not only on value of the strength material characteristics, but also on velocities of propagation of compression and unloading waves. 
In barriers made from the reoriented material (fig. 12b, fig. 13b) at the expense of greater velocity of wave propagation in an impact direction (along the axis $Z$ ) and smaller value of strength on pressure in this direction, the fracture occurring in a compression wave gets deeply on a thickness of a barrier. The unloading waves moving from free surfaces, reaching areas in which the material was already weakened at pressure, completely destroy it. In this case the projectile has before itself an extended area of the fractured material, which doesn't resist to a projectile introduction $\left(\sigma_{i j}=0\right)$.

In barriers made from an initial material (fig. 12a, fig. 13a) we have other picture: the areas of fracture realized in a compression wave, have more extended sizes in directions which are perpendicular to an impact direction. Before the projectile the area of not fractured material providing greater resistance to the projectile introduction remains.

The dynamics of fracture in barriers is estimated fully on fig. 14, which shows changes in time of the total (on a barrier) relative volume of fractures at pressure $\left(V_{p} / V_{0}\right)$ or tension ( $\left.V_{t} / V_{0}\right)$ ( $V_{0}$ is total volume of barrier) for various initial velocities of impact. Curves with $V_{p}$ characterize change in time of relative volume of the material destroyed in conditions of pressure $\left(e_{k k} \leq 0\right)$ and keeping resistance to loading only on pressure, for monolithic and spaced (two- or three-layer) barriers. Curves with $V_{t}$ characterize change in time of relative volume completely fractured material $\left(e_{k k}>0\right)$, not resisting to loading $\left(\sigma_{i j}=0\right)$. As the material fracture begins in a compression wave, at the beginning of the process volume of the fractured material being in a condition of pressure, is more than volume of the fractured material at tension. Eventually volume of the material, fractured at tension, increases at simultaneous reduction of volume of the material fractured at pressure. It is caused by influence of unloading waves, extending from free surfaces of the projectile and barriers. If for projectile velocity of $750 \mathrm{~m} / \mathrm{s}$ the volume of completely fractured material $\left(e_{k k}>0\right)$ in the monolithic barrier was the smallest, with increase in impact velocity the picture changes the volume of the fractured material in a monolithic barrier begins to exceed the corresponding values for the spaced barriers.

The comparative analysis of protective properties of the monolithic and spaced barriers made from an initial material can be spent analogically on dependences of velocity of the center of the projectile weights from time, resulted on a fig. 15 for various orientations of a material properties. For an initial orientation of a material properties at velocities of impact to $2000 \mathrm{~m} / \mathrm{s}$ the more intensive braking of the projectile on a monolithic barrier occurs (fig. 15a, fig. 15b, fig. 15c). And at velocity of $750 \mathrm{~m} / \mathrm{s}$ the through penetration for the monolithic and for the spaced barriers is absent. In this case during all the process of the interaction the projectile velocity falls more at interaction with the monolithic barrier. Following on efficiency is the spaced barrier made from two plates, and the least effective is a barrier made from three plates.

With increase in initial velocity of impact the picture changes. For the impact velocity of $1500 \mathrm{~m} / \mathrm{s}$ the monolithic barrier is still more effective - braking of the projectile on it occurs more intensively. Post-penetration velocity of the projectile after penetration of the 
monolithic barrier makes $50 \mathrm{~m} / \mathrm{s}$, after penetration of the spaced barriers $-150 \mathrm{~m} / \mathrm{s}$, but for the initial velocity of $1500 \mathrm{~m} / \mathrm{s}$ in $36 \mu \mathrm{sec}$ after the impact start the projectile velocities for cases of two-layer and three-layer spaced designs are leveled. The increase in velocity to $3000 \mathrm{~m} / \mathrm{s}$ leads to that after $26 \mu \mathrm{sec}$ after the process start the projectile begins to brake more intensively on the spaced targets, thus post-penetration velocity after penetration of the monolithic barrier is already on $10-15 \%$ above, than after penetration of the spaced barriers. For the reoriented material the tendency of increase of the spaced designs efficiency remains - with growth of velocity of interaction the difference in post-penetration velocities of the projectile after penetration of the monolithic and the spaced barriers decreases.
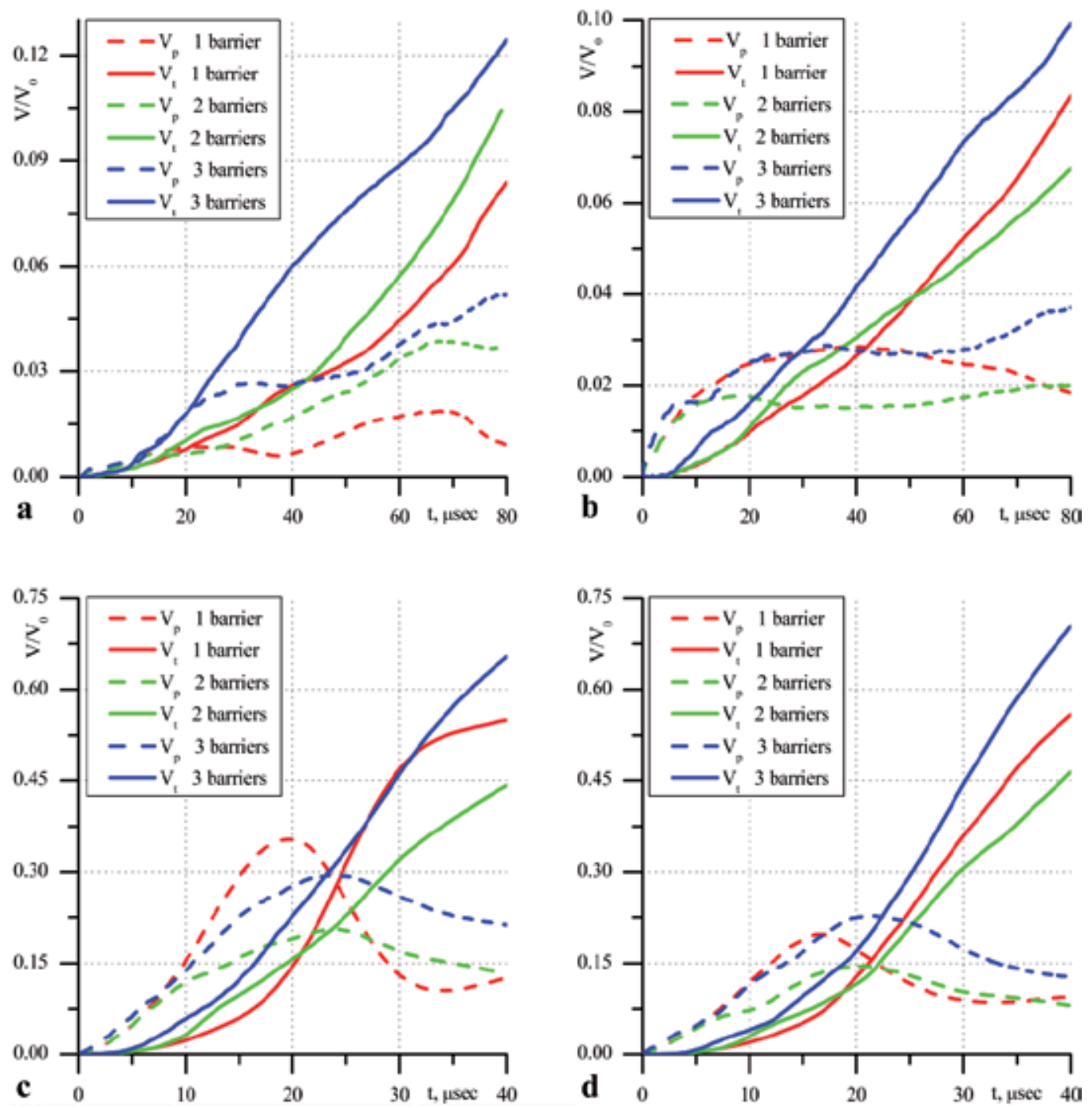

Figure 14. Relative volume of fractures at pressure and tension. 

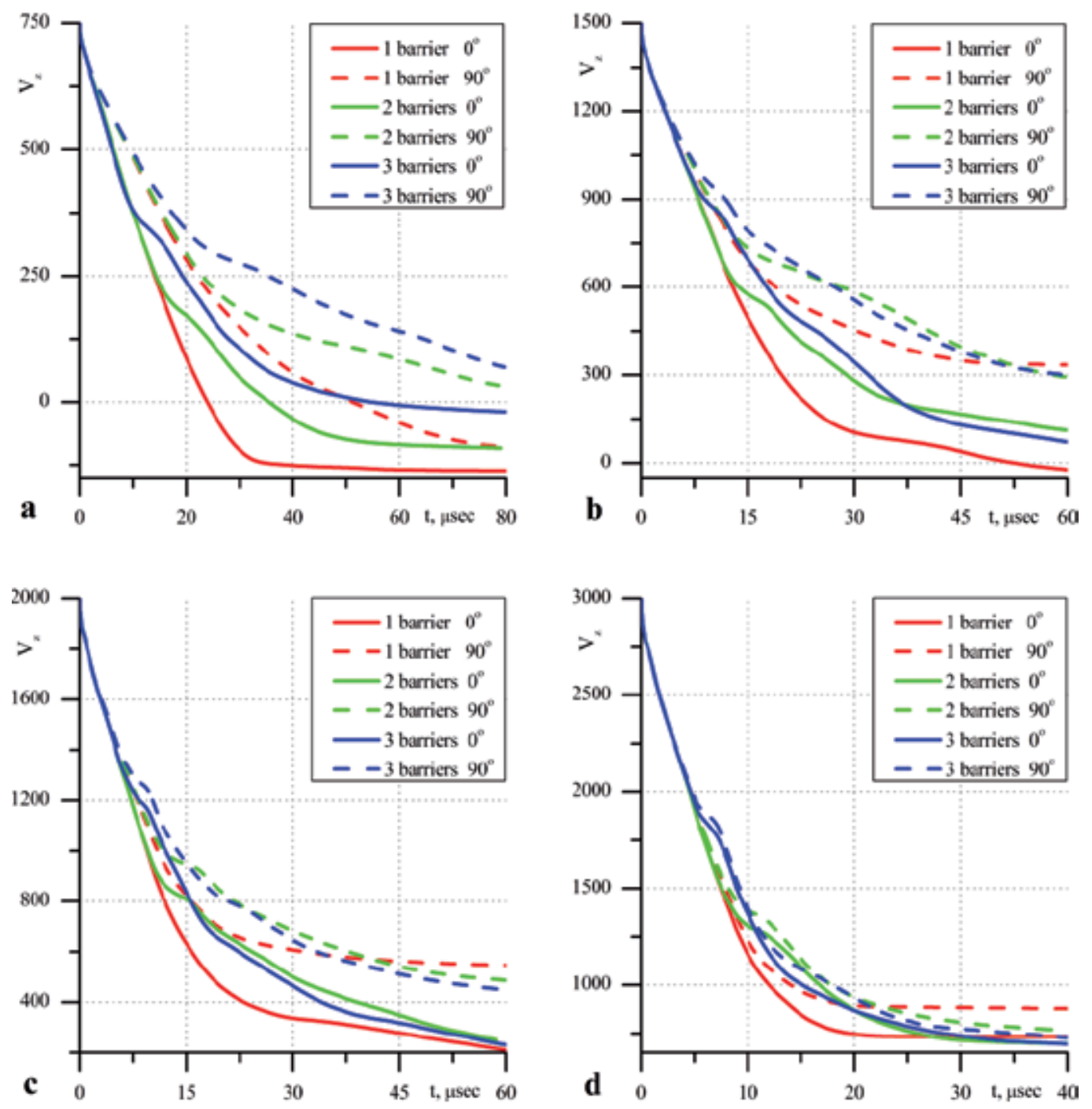

Figure 15. Velocity of the center of weights of the projectile at different initial velocities.

\subsection{Numerical analysis of impact interaction of two anisotropic solids}

Let's consider normal impact of orthotropic projectile with diameter $15 \mathrm{~mm}$ and length 75 $\mathrm{mm}$ and orthotropic barrier with thickness $60 \mathrm{~mm}$ with initial velocity $v_{0}=700 \mathrm{M} / \mathrm{c}$ along axis Z (Radchenko \& Radchenko, 2010). Orientation of properties of orthotropic material changes by turn of axes of symmetry of an initial material round an axis $O Y$ on a corner $\beta$, counted from a positive direction of an axis $Z$. Following cases of orientation of properties of a material of a projectile and a barrier are investigated: $0-0,0-90,90-0$, and 90-90. Here numbers correspond to values $\beta$ for a material of a projectile and a barrier in degrees, accordingly. Cases when the top and bottom half of a projectile and a barrier consist of 
materials reoriented on $90^{\circ}$ are also considered (conditionally these cases are designated as 0-90 1/2 and 45-135 1/2).

On fig. 16 settlement configurations of the projectile and a barrier and as are presented an isolines of relative volume of fracture for various cases of orientation of properties of a material. The fig. 16a corresponds to a case $(0-901 / 2)$ when the lower half of projectile and a barrier consists of an initial material in which the greatest strength characteristics of tension are oriented in an axis direction $X$, perpendicular to a impact direction, and in the upper half of projectile and a barrier of property of a material are reoriented on $90^{\circ}$ by turn concerning an axis $Y$. The Fig. 16b corresponds to a case (45-1351/2) when the lower half of projectile and a barrier consist of a material in which maximum strength characteristics of tension are oriented at an angle $45^{\circ}$ to an axis $X$ in a plane $Z X$, and in the upper halves properties of a material are reoriented by turn on $90^{\circ}$ concerning an axis $Y$. In the first case (Fig. 16a), besides fracture of a head part, fracture of the projectile on boundary of materials with various orientation of properties at reaching by a wave of compression of the upper half of projectile (in which the material has the minimum strength on compression in an axis direction $Z$ ), that leads to its sharing on two parts is observed. In the second case (Fig. 16b) because axes of symmetry of materials of the projectile and a barrier do not coincide with coordinate axises and, accordingly, with a direction of propagation of shock waves, fracture process in the projectile and a barrier passes not symmetrically. In the upper half of barrier the fracture area is oriented in a direction of the minimum values of strength on compression $\left(\beta=135^{\circ}\right)$. In the projectile the fracture area as is formed on boundary of section of materials with various orientation of elastic and strength properties, but in this case fracture extends from a side surface of the projectile at an angle $135^{\circ}$ and volume of fracture in a head part of the projectile much more.

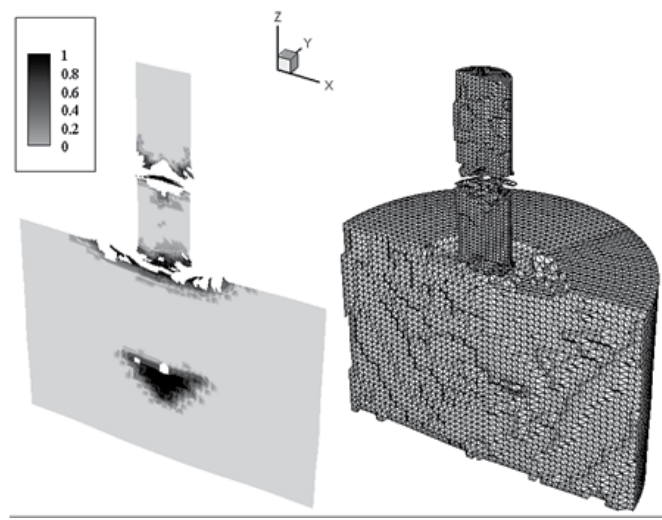

(a)

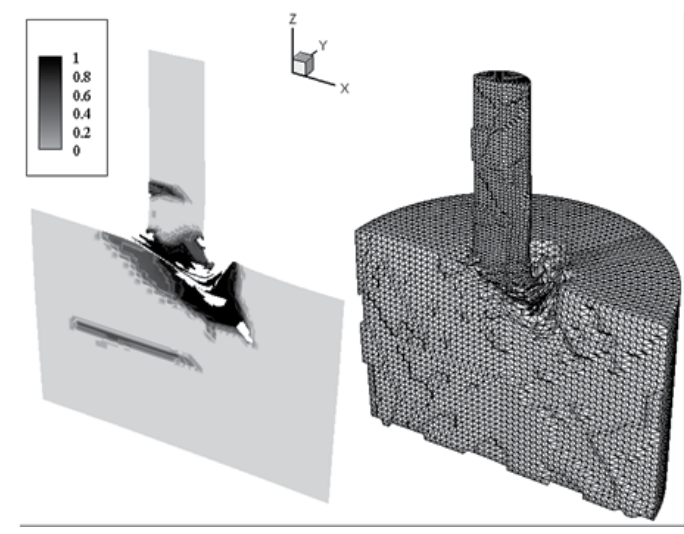

(b)

Figure 16. Configurations of interacting bodies and allocation of isolines of relative volume of fracture. $v_{0}=700 \mathrm{~m} / \mathrm{s}, t=36 \mu \mathrm{sec}$.

Dynamics of fracture in the projectile is illustrated by fig. 17 where changes in time of relative volume of the fracture derivated in the conditions of compression and tension are 
resulted. The analysis of curves on Fig. 17 allows to draw a conclusion that for all considered cases of interaction the least levels of irreversible fracture (Fig. 17b) are realized in the projectile from a material with orientation of axes of symmetry $\beta=0^{\circ}$. It is caused by that in this case the material of the projectile has the greatest strength properties on compression in an axis direction $Z$. Therefore the fracture at the initial stage of process of interaction in a wave of compression (fig. 17a), extending on length of the projectile, are minimal. For other cases of orientation of properties of a material of the projectile the volume of the fractured material in a wave of compression is more, in this connection the most part of a material of the projectile appears weakened, and further does not offer resistance at occurrence of tension powers. Curves on fig. 17a have a maximum in a range from 16 to $20 \mu \mathrm{sec}$, and then the volume of the material fracture at compression, decreases, after that instant the volume of irreversible fracture of a material increases at tension.

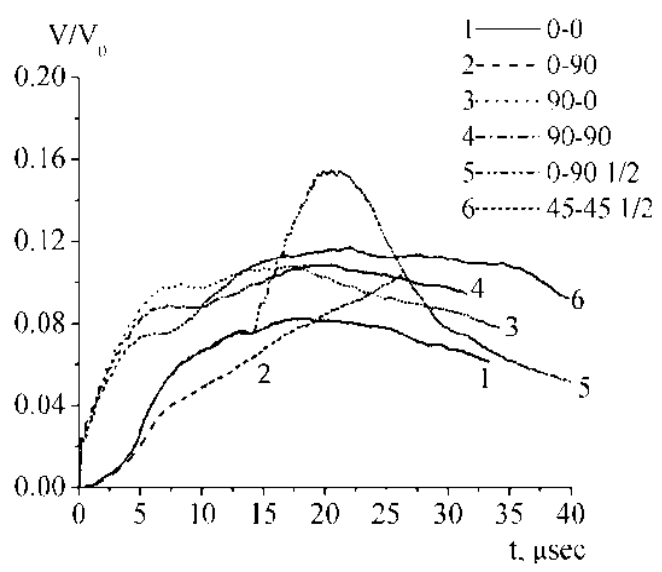

(a)

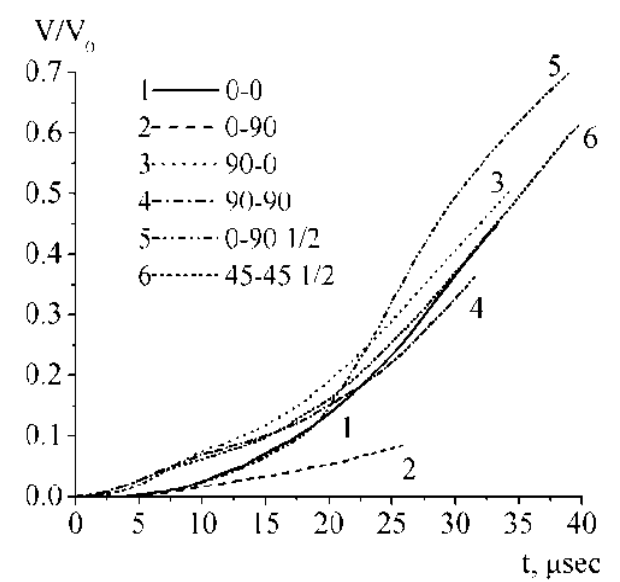

(b)

Figure 17. Change in time of relative volume of fracture in the projectile at compression (a) and tension (b) for various orientations of properties of a material.

Penetration power of the projectile depending on orientation of properties of a material at its interaction with various barriers can be estimated on the curves characterizing change in time of velocity of centre of mass the projectile (fig. 18). The most intensive braking of the projectile is observed for a case of orientation of properties $0-0$ (a curve 1) and 0-90 1/2 (a curve 5). In the first of these two cases of property of a material of the projectile and a barrier are oriented equally and correspond to a case of maximum strength on compression in a direction of impact (axis Z), that stipulates high firmness of a barrier to impact. In the second case the projectile has extensive fracture and is divided on two parts (fig. 16a) that leads to loss of its penetration power. 


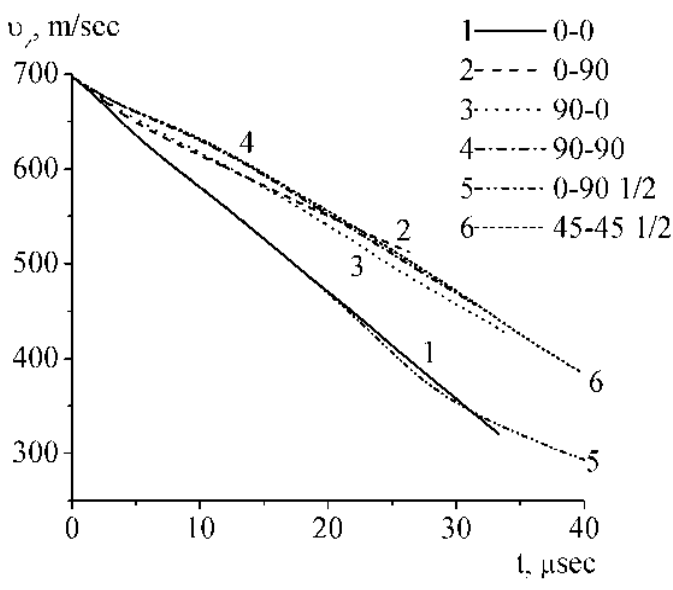

Figure 18. Change in time of velocity of centre of mass of the projectile.

\section{Conclusion}

1. The offered model allows to describe adequately main laws of the fracture processes of anisotropic materials under dynamic loads. The carried out researches have shown, that anisotropy of properties is the essential factor which is necessary for taking into account for the adequate description and the prediction of development of shock-wave processes and fracture in the materials under dynamic loadings. The influence of anisotropic properties orientation increases with decrease in the velocity interaction.

2. The qualitative and quantitative discrepancies in the fracture of isotropic and anisotropic materials under the dynamic loads are defined not only by strength parameters but either by the interaction of the compression and tension waves. Different velocities of waves propagation along the directions in anisotropic barriers provide the discharge of the impact wave and the narrowing in the fracture region.

3. The influence of hydrostatic pressure on the fracture of anisotropic materials under dynamic loads is shown. Full compression leads to a fragmentation of an orthotropic ball without causing the fracture of an isotropic ball. That is why adequately description of the dynamic behavior of anisotropic solids required accounting of the hydrostatic pressure.

4. It is established that at low-velocity impact formation and a direction of development of fracture zone in a target is defined by orientation of elastic and strength properties of an anisotropic material in relation to an impact direction. Depending on orientation of properties development of the conic cracks caused by combined action of tensile stresses in waves of unloading and at the expense of penetration of the projectile, or fracture of material in a pressure and unloading wave is probable.

5. A comparative analysis of the effectiveness of the protective properties of monolithic and spaced barriers from anisotropic materials for various cases of orientation of the 
material properties is carried out. It is established that the effectiveness of spaced designs increases with the velocity of interaction and determined by the development of fracture in barriers, depending on the velocities of wave propagation and the orientation of the elastic and strength properties of anisotropic material with relationship to the direction of impact.

Orientation of properties of an anisotropic material of the projectile and a barrier essentially influences dynamics of fracture and on process of propagation of shock waves in the projectile and a barrier. The offered model of behavior of anisotropic materials at dynamic loads allow to carry out researches by definition of optimization of properties of a material of the projectile and a barrier at any orientation of axes of symmetry.

\section{Author details}

Andrey Radchenko

Tomsk State University of Architecture and Building, Russia

Pavel Radchenko

Institute of Strength Physics and Materials Science of SB RAS, Russia

\section{References}

Ashkenazi, E. K., \& Ganov, A.V. (1980) Anisotropy of the construction materials, Leningrad

Johnson, G. R. Three-dimensional analysis of sliding surface during high velocity impact. (1977) Journal of Applied Mechanics, No. 6, pp. 771-773

Johnson, G.R. High Velocity Impact Calculations in Three Dimension. (1977) Journal Applied Mechanics, Vol. 3, p. 95-100

Kanel, G.I., Razorenov, S.V., Utkin, A.V., \& Fortov, V.E. (1996) Shock wave phenomena in condensed media, Yanus-K, Moscow

Radchenko, A. V., Kobenko, S.V., Marcenuk, I.N., Khorev, I.E., Kanel, G.I., \& Fortov, V.E. (1999) Research on features of behaviour of isotropic and anisotropic materials under impact. International Journal of Impact Engineering, Vol. 23, No. 1, pp. 745-756

Radchenko, A., Radchenko, P., Tuch, E., Krivosheina, M., \& Kobenko, S. (2012) Comparison of Application of Various Strength Criteria on Modeling of Behavior of Composite Materials at Impact. Journal of Material Science and Engineering A, Vol. 2, No. 1, pp. 112120

Radchenko, A.V., \& Radchenko, P.A. (2010) Numerical analysis of impact of two anisotropic solids. Journal of Materials Science and Engineering, Vol. 4, No. 3, pp. 74-79

Radchenko, A.V., \& Radchenko, P.A. (2011) Numerical modeling of development of fracture in anisotropic composite materials at low-velocity loading. Journal of Materials Science, Vol. 46, No. 8, pp. 2720-2725 
Tsai, S.W., \& Wu, E.M. (1971) A General Theory of Strength for Anisotropic Materials. Journal of Composite Materials, Vol. 5, No. 1, pp. 58-80

Wilkins, M.L., \& Guinan, M.W. (1973) Impact of cylinders on a rigid boundary. Journal of Applied Physics, Vol. 44, No. 3, pp. 1200-1206 


\title{
Finite Element Implementation of Failure and Damage Simulation in Composite Plates
}

\author{
Milan Žmindák and Martin Dudinský \\ Additional information is available at the end of the chapter
}

http://dx.doi.org/10.5772/46498

\section{Introduction}

Composite materials are now common engineering materials used in a wide range of applications. They play an important role in the aviation, aerospace and automotive industry, and are also used in the construction of ships, submarines, nuclear and chemical facilities, etc.

The meaning of the word damage is quite broad in everyday life. In continuum mechanics the term damage is referred to as the reduction of the internal integrity of the material due to the generation, spreading and merging of small cracks, cavities and similar defects. Damage is called elastic, if the material deforms only elastically (in macroscopic level) before the occurrence of damage, as well as during its evolution. This damage model can be used if the ability of the material to deform plastically is low. Fiber-reinforced polymer matrix composites can be considered as such materials.

The use of composite materials in the design of constructions is increasing in traditional structures such as for example development of airplanes or in the automotive industry. Recently this kind of materials is used in development of special technique and rotating systems such as propellers, compressor turbine blades etc. Other applications are in electronics, electrochemical industry, environmental and biomedical engineering (Chung, 2003).

The costs for designing of composite structures is possible partially eliminate by numerical simulation of solving problem. In this case the simulation is not accepted as universal tool for analyzing of systems behaviour but it is an effective alternative to processes of experimental sciences. Simulations support development of new theories and suggestion of new experiments for testing these theories. Experiments are necessary for obtaining of input data into simulation programs and for verification of numerical programs and models. 
Laminated composites have a lot of advantages but in some cases they show different limitations that are caused by stress concentrations between layers. Discontinuous change of material properties is reason for occurrence of interlaminar stresses that often cause delamination failure (Zhang \& Wang, 2009). Delamination may originate from manufacturing imperfections, cracks produced by fatigue or low velocity impact, stress concentration near geometrical/material discontinuity such as joints and free edges, or due to high interlaminar stresses (Elmarakbi, 2009)

Delaminations in layered plates and beams have been analyzed by using both cohesive damage models and fracture mechanics. Cohesive elements are widely used, in both forms of continuous interface elements and point cohesive elements (Cui, 1993), at the interface between solid finite elements to predict and to understand the damage behaviour in the interfaces of different layers in composite laminates. In the context of the fracture mechanics approach (Sládek, et al. , 2002), it allows us to predict the growth of a preexisting crack or defect. In a homogeneous and isotropic body subjected to a general loading condition, a crack tends to grow by kinking in a direction such a pure mode I condition at its tip is maintained. On the contrary, delaminations in laminated composites are constrained to propagate in its own plane because the toughness of the interface is relatively low in comparison to that of the adjoining material. Therefore a delamination crack propagates with its advancing tip in mixed mode condition and, consequently, requires a fracture criterion including all three mode components.

The theory of crack growth may be developed by using one of two approaches. First, the Griffith energetic (or global) approach introduces the concept of energy release rate (ERR) $G$ as the energy available for fracture on one hand, and the critical surface energy $G_{r}$ as the energy necessary for fracture on the other hand. Alternatively, the Irwin (local) approach is based on the stress intensity factor concept, which represents the energy stress field in the neighborhood of the crack tip. These two approaches are equivalent and, therefore, the energy criterion may be rewritten in terms of stress intensity factors.

Microcracking in a material is almost always associated with changes in mechanical behavior of the material. The problem of microcracking in fiber-reinforced composites is complicated due to the multitude of different microcracking modes which may initiate and evolve independently or simultaneously. Continuum Damage Mechanics (CDM) considers damaged materials as a continuum, in spite of heterogenity, micro-cavities, and microdefects and is based on expressing of stiffness reduction caused by damage, by establishing effective damage parameters which represent cumulative degradation of material. There are basically two categories of CDM models used for estimating the constitutive behavior of composite materials containing microcracks -phenomenological models and micromechanics models.

The phenomenological CDM models employ scalar, second order or fourth order tensors using mathematically and thermodynamically consistent formulations of damage mechanics. Damage parameters are identified through macroscopic experiments and in general, they do not explicitly account for damage mechanism in the microstructure. On the 
other hand the micromechanics-based approaches conduct micromechanical analysis of representative volume element (RVE) with subsequent homogenization to predict evolving material damage behavior (Mishnaevsky, 2007). Most damage models do not account for the evolution of damage or the effect of loading history (Jain \& Ghosh, 2009). Significant error can consequently accrue in the solution of problems, especially those that involve nonproportional loading. Some of these homogenization studies have overcome this shortcoming through the introduction of simultaneous RVE-based microscopic and macroscopic analysis in each load step. However, such approaches can be computationally very expensive since detailed micro-mechanical analyses need to be conducted in each load step at every integration point in elements of the macroscopic structure.

Jain \& Ghosh (2009) have developed a 3D homogenization-based continuum damage mechanics (HCDM) model for fiber-reinforced composites undergoing micro-mechanical damage. Micromechanical damage in the RVE is explicitly incorporated in the form of fibermatrix interfacial debonding. The model uses the evolving principal damage coordinate system as its reference in order to represent the anisotropic coefficients, which is necessary for retaining accuracy with nonproportional loading. The HCDM model parameters are calibrated by using homogenized micromechanical solutions for the RVE for a few strain histories.

There are many works written about damage of composite plates and many models for various types of damage, plates or loading have been developed. Shu (2006) presented generalized model for laminated composite plates with interfacial damage. This model deals with three kinds of interfacial debonding conditions: perfect bonding, weak bonding and delamination. Iannucci \& Ankersen (2006) described unconventional energy based composite damage model for woven and unidirectional composite materials. This damage model has been implemented into FE codes for shell elements, with regard to tensile, compressive and shear damage failure modes. Riccio \& Pietropaoli (2008) dealt with modeling damage propagation in composite plates with embedded delamination under compressive load. The influence of different failure mechanisms on the compressive behavior of delaminated composite plates was assessed, by comparing numerical results obtained with models characterized by different degrees of complexity. Tiberkak et al. (2008) studied damage prediction in composite plates subjected to low velocity impact. Fiber-reinforced composite plates subjected to low velocity impact were studied by use of finite element analysis where Mindlin's plate theory and 9-node Lagrangian element were considered. Clegg et al. (2006) worked out interesting study of hypervelocity impact damage prediction in composites. This study reports on the development of an extended orthotropic continuum material model and associated material characterization techniques for the simulation and validation of impacts onto fiber-reinforced composite materials. The model allows to predict the extent of damage and residual strength of the fiber-reinforced composite material after impact.

Many studies of an effect of various aspects of damage process on behavior of composite plates can be found in the literature (e.g. Gayathri et al. , 2010). Many authors have been 
dealing with problematic of damage of composite plated under cyclic loading or problematic of impact fatigue damage (e.g. Azouaoui et al. , 2010). Composite materials are becoming more and more used for important structural elements and structures, so the problematic of fatigue damage of composites is becoming more and more actual. Numerical implementation of damage is not simple. Finite element method (FEM) is the most utilized method for modeling damage. Fast Multipole BEM method or various meshless methods are also establishing at the present time.

Firstly, the goal of this chapter is to present the numerical results of the delamination analysis of two laminae with different thickness with two orthotropic material properties and subjected to a pair of opposed forces. For this goal we used commercial FEM software ANSYS and the mode I, II, and III components of energy release rate (ERR) were calculated. Secondly, the goal is to present the numerical results of elastic damage of thin composite plates. The analysis was performed by user own software, created in MATLAB programming language. This software can perform numerical analysis of elastic damage using FEM layered plate finite elements based on the Kirchhoff plate theory.

This chapter is organized in three sections: Second section is focused on failure modeling in laminates by using standard shear deformable elements, whereas interface elements were used for the interface model. The delamination propagation is controlled by the critical ERR. In third section, in first, a general description of damage is provided, then damage model used is examined. Finally, some numerical results obtained for damage of plate are presented.

\section{Theoretical background of failure modeling in laminates}

The mechanisms that lead to failure in composite materials are not yet fully understood, especially for matrix or fiber compression. Strength-based failure criteria are commonly used with the FEM to predict failure events in composite structures. Numerous continuumbased criteria have been derived to relate internal stresses and experimental measures of material strength to the onset of failure (Dávila et al., 2005). In Fig. 1 a laminate contains a single in-plane delamination crack of area $\Omega_{D}$ with a smooth front $\partial \Omega_{D}$. The laminate thickness is denoted by $h_{0}$. The $x-y$ plane is taken to be the mid-plane of the laminate, and the $\mathrm{z}$-axis is taken positive downwards from the mid-plane.

\subsection{Plate finite elements for sublaminate modeling}

Each sublaminate is represented by an assembly of first order shear deformable (FSDT) plate elements bonded by zero-thickness interfaces in the transverse direction as shown in Fig. 2 . The delamination plane separates the delaminated structure into two sublaminates of thickness $h_{1}, h_{2}$ and each sublaminate consist the upper $n_{u}$ plates and the lower $n_{\imath}$ plates. Each plate element is composed from one or few physical fiber-reinforced plies with their material axes arbitrarily oriented. Lagrangian multipliers through constraint equations (CE) are used for enforcing adhesion between the plates inside each sublaminate. Accordingly, 
the displacements in the $z$-th plate element, in terms of a global reference system located at the laminate mid-surface, are expressed by (e.g. Carrera 2002; Reddy 1995)

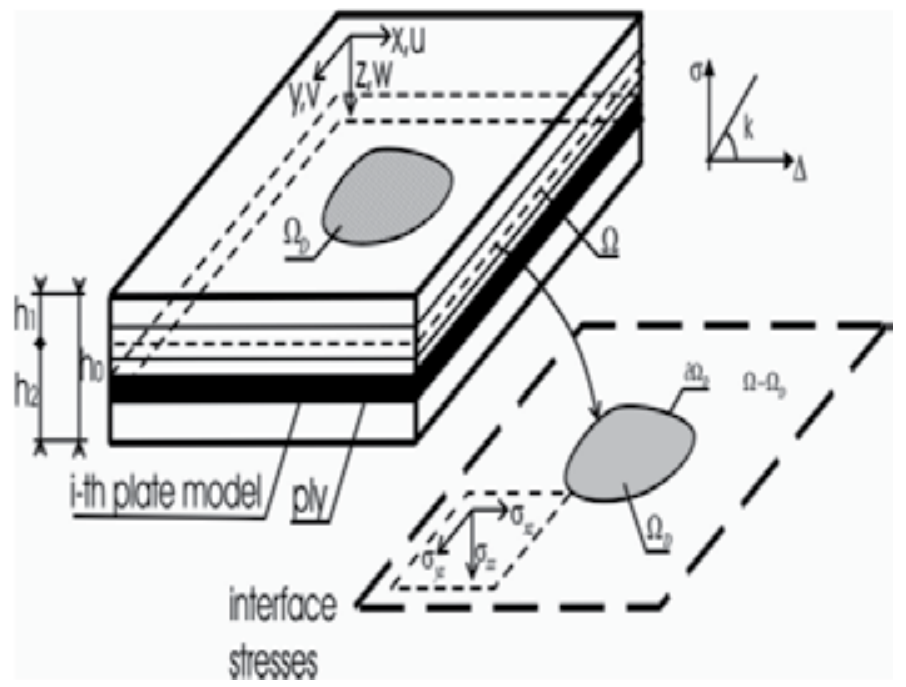

Figure 1. Delaminated composite plate

$$
\begin{aligned}
& u_{i}(x, y, z)=u_{i}^{0}(x, y)+\left(z-z_{i}\right) \psi_{x i}(x, y) \\
& v_{i}(x, y, z)=v_{i}^{0}(x, y)+\left(z-z_{i}\right) \psi_{y i}(x, y) \\
& w_{i}(x, y, z)=w_{i}^{0}(x, y)
\end{aligned}
$$

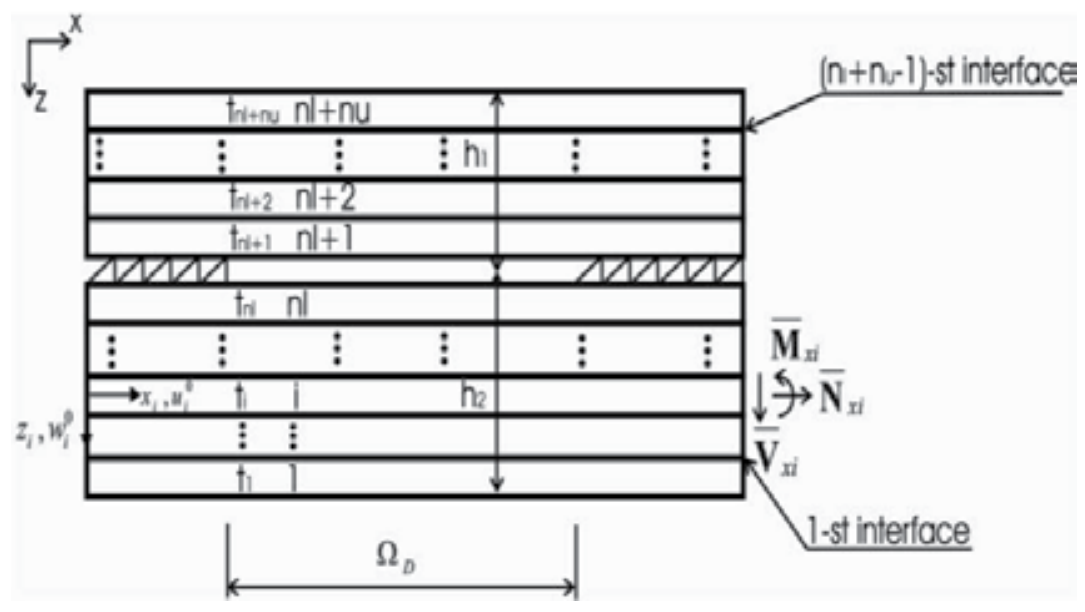

Figure 2. Laminate subdivision in plate elements 
where $u_{i}, v_{i}$ refer to the in-plane displacements, and $w_{i}$ to the transverse displacements through the thickness of the $i$-th plate element, $u_{i}^{0}, v_{i}^{0}, w_{i}^{0}$, are the displacements at the midsurface of the $i$-th plate element and $\psi_{\mathrm{xi}}(\mathrm{x}, \mathrm{y}), \psi_{\mathrm{yi}}(\mathrm{x}, \mathrm{y})$ denote rotations of transverse normals about $y$ and $x$, respectively.

At the reference surfaces, the membrane strain vector $\varepsilon_{i}$, the curvature $\kappa_{i}$, and the transverse shear strain $\gamma_{i}$, respectively are defined as

$$
\left\{\begin{array}{c}
\varepsilon_{x x i} \\
\varepsilon_{y y i} \\
\gamma_{x y i}
\end{array}\right\}=\left\{\begin{array}{c}
\frac{\partial u_{i}^{0}}{\partial x} \\
\frac{\partial v_{i}^{0}}{\partial y} \\
\frac{\partial u_{i}^{0}}{\partial y}+\frac{\partial v_{i}^{0}}{\partial x}
\end{array}\right\},\left\{\begin{array}{c}
\kappa_{x x i} \\
\kappa_{y y i} \\
\kappa_{x y i}
\end{array}\right\}=\left\{\begin{array}{c}
\frac{\partial \psi_{x i}^{0}}{\partial x} \\
\frac{\partial \psi_{\text {yi }}^{0}}{\partial y} \\
\frac{\partial \psi_{x i}^{0}}{\partial y}+\frac{\partial \psi_{y i}^{0}}{\partial x}
\end{array}\right\},\left\{\begin{array}{c}
\gamma_{y z i} \\
\gamma_{x z i}
\end{array}\right\}=\left\{\begin{array}{c}
\psi_{x i}^{0}+\frac{\partial w_{i}^{0}}{\partial y} \\
\psi_{x i}^{0}+\frac{\partial w_{i}^{0}}{\partial x}
\end{array}\right\}
$$

The constitutive relations between stress resultants and corresponding strains are given in (Reddy \& Miravete, 1995; Žmindák, 2010). In these works standard FSDT finite elements available in ANSYS software are used (ANSYS, 2007) to join these elements at the interfaces inside each sublaminate using CE or rigid links characterized by two nodes and three degrees of freedom at each node.

\subsubsection{Interface elements for delamination modeling}

Delamination is defined as the fracture of the plane separating two plies of a laminated composite structure (Fenske, et al., 2001). This fracture occurs within the thin resin-rich layer that forms between plies during the manufacturing process. Perfect adhesion is assumed in the undelaminated region $\Omega-\Omega_{D}$, whereas sub-laminates are free to deflect along the delaminated region $\Omega_{\mathrm{D}}$ but not to penetrate each other. A linear interface model, is introduced along $\Omega-\Omega_{\mathrm{D}}$ to enforce adhesion. The constitutive equation of the interface involves two stiffness parameters, $k_{z}, k_{x y}$, imposing displacement continuity in the thickness and in-plane directions, respectively, by treating them as penalty parameters. The relationship between the components of the traction vector $\sigma$ acting at the lower surface of the upper sublaminate, $\sigma_{z x}, \sigma_{z y}$ and $\sigma_{z z}$, in the out-of-plane $(z)$ and in the in-plane ( $x$ and $y$ ) directions, respectively, and the corresponding components of relative interface displacement vector $\Delta, \Delta u, \Delta v$ and $\Delta w$ is expressed as

$$
\mathbf{\sigma}=\mathbf{K} \boldsymbol{\Delta}
$$

Interface elements are implemented using COMBIN14 element. Relative opening and sliding displacements are evaluated as the difference between displacements at the interface between the lower and the upper sublaminate. 


\subsubsection{Contact formulation for damage interface}

In order to avoid interpenetration between delaminated sublaminates in the delaminated region $\Omega_{\mathrm{D}}$, a unilateral frictionless contact interface can be introduced, characterized by a zero stiffness for opening relative displacements $(\Delta w \geq 0)$ and a positive stiffness for closing relative displacements $(\Delta w \leq 0)$, then the contact stress $\sigma_{\mathrm{zz}}$ is

$$
\left.\sigma_{\mathrm{zz}}=\frac{1}{2}(1-\operatorname{sign}(\Delta \mathrm{w}))\right) \mathrm{k}_{\mathrm{z}} \Delta \mathrm{w}
$$

where $k_{z}$ is the penalty number imposing contact constraint and sign is the signum function. A very large value for $k z$ restricts sublaminate overlapping and simulates the contact condition. Unilateral contact conditions may be implemented in ANSYS using COMBIN39. This element is a unidirectional element with nonlinear constitutive relationships with appropriate specialization of the nonlinear constitutive law according to (4).

If we introduce a scalar damage variable $D$ with the value of 1 for no adhesion and the value of 0 for perfect adhesion, we get a single extended interface model with constitutive law valid both for undelaminated $\Omega-\Omega_{D}$ and delaminated $\Omega_{D}$ areas. Consequently constitutive law can be expressed as

$$
\sigma=(1-\mathrm{D}) \mathbf{K} \Delta
$$

In this work we use the formulation via FEs, related to plate elements, interface elements and Lagrange multipliers. It is worth noting that in commercial FEA packages the Lagrange multipliers are represented by either CE or rigid links, whereas interface elements are implemented by the analyst using a combination of spring elements (COMBIN14) and CE.

\subsubsection{Mixed mode analysis}

In order to predict crack propagation in laminates for general loading conditions, ERR distributions along the delamination front are needed. Fracture mechanics assumes that delamination propagation is controlled by the critical ERR. Delamination grows on the region of the delamination front where the following condition is satisfied and is of the form

$$
\left(\frac{G_{I}(s)}{G_{I}^{c}}\right)^{\alpha}+\left(\frac{G_{I I}(s)}{G_{I I}^{c}}\right)^{\beta}+\left(\frac{G_{I I I}(s)}{G_{I I I}^{c}}\right)^{\gamma}=1
$$

where $\alpha, \beta$ and $\gamma$ are mixed mode fracture parameters determined by fitting experimental test results.

The critical ERR $\left(G_{I}^{c}, G_{I I}^{c}, G_{I I I}^{c}\right)$ material properties can be evaluated from experimental procedures. The closed-form expressions for the ERR are (Barbero, 2008) 


$$
\begin{aligned}
& \mathrm{G}(\mathrm{s})=\frac{1}{2} \mathrm{k}_{\mathrm{z}, \mathrm{k}_{\mathrm{xy}} \rightarrow \infty}\left[\mathrm{k}_{\mathrm{z}} \Delta \mathrm{w}^{2}(\mathrm{~s})+\mathrm{k}_{\mathrm{xy}} \Delta \mathrm{u}^{2}(\mathrm{~s})+\mathrm{k}_{\mathrm{xy}} \Delta \mathrm{v}^{2}(\mathrm{~s})\right], \Delta \mathrm{w}(\mathrm{s}) \geq 0 \\
& \mathrm{G}=\mathrm{G}_{\mathrm{I}}(\mathrm{s})+\mathrm{G}_{\mathrm{II}}(\mathrm{s})+\mathrm{G}_{\mathrm{III}}(\mathrm{s}) \\
& G_{I}(s)=\left\{\begin{array}{ccc}
\lim _{k_{z}, k_{x y} \rightarrow \infty} \frac{1}{2} k_{z} \Delta w^{2}(s) & \text { if } \quad \Delta w(s) \geq 0 \\
0 & \text { if } & \Delta w(s)<0
\end{array}\right\} \\
& \mathrm{G}_{\mathrm{II}}(\mathrm{s})=\lim _{\mathrm{k}_{\mathrm{z}}, \mathrm{k}_{\mathrm{xy}} \rightarrow \infty} \frac{1}{2} \mathrm{k}_{\mathrm{xy}} \Delta \mathrm{u}_{\mathrm{n}}^{2}(\mathrm{~s}) \\
& \mathrm{G}_{\mathrm{III}}(\mathrm{s})=\lim _{\mathrm{k}_{\mathrm{z}}, \mathrm{k}_{\mathrm{xy}} \rightarrow \infty} \frac{1}{2} \mathrm{k}_{\mathrm{xy}} \Delta \mathrm{u}_{\mathrm{t}}^{2}(\mathrm{~s})
\end{aligned}
$$

They are obtained by means of the interface model, using FE code to check whether propagation occurs. Once made a global FEA of the laminate, then the calculation of $G(s)$ along the delamination front reduces to a simple post-computation. The extent of the propagation of the delamination area may be established by releasing the node in which the relation (6) is first satisfied, leading to a modification of the delamination front, which in turn requires another equilibrium solution. It follows the fact that the delamination growth analysis must be accomplished iteratively. For simplicity, only the computation of ERR is described here. The study of the propagation for a 3D planar delamination requires the use of nonlinear incremental numerical computation.

The delaminated laminate is represented using two sublaminates (Fig. 2). In this case, the model is called a two-layer plate model. Multilayer plate model in each sublaminate is necessary to achieve sufficient accuracy when the mode components are needed. Sublaminates are modeled using standard shear deformable elements (SHELL181), whereas interface elements can be used for the interface model. Available interface elements (INTER204) are only compatible with solid elements, therefore interface elements are simulated here by coupling CE with spring elements (COMBIN14). Plate and interface models must be described by the same in-plane mesh.

The FE model of the plates adjacent to the delamination plane in proximity of the delamination front is illustrated in Fig. 3. Interface elements model the undelaminated region $\Omega-\Omega_{\mathrm{D}}$ up to the delamination front. The mesh of interface and plate elements must be sufficiently refined in order to capture the high interface stress gradient in the neighborhood of the delamination front, which occurs because high values for interface stiffness must be used to simulate perfect adhesion. The individual ERR at the general node $\mathrm{A}$ of the delamination front are calculated using the reactions obtained from spring elements and the relative displacements between the nodes already delaminated and located along the normal direction.

ERRs are computed by using (9), which is a modified version of (8) in order to avoid excessive mesh refining at the delamination front. This leads to the following expressions 


$$
\mathrm{G}_{\mathrm{I}}(\mathrm{A})=\left(\frac{1}{2} \frac{\mathrm{R}_{\mathrm{A}}^{\mathrm{z}}{ }^{\mathrm{w}}{ }_{\mathrm{B}-\mathrm{B}^{\prime}}}{\Delta_{\mathrm{n}} \Delta_{\mathrm{t}}}\right), \mathrm{G}_{\mathrm{II}}(\mathrm{A})=\left(\frac{1}{2} \frac{\mathrm{R}_{\mathrm{A}}^{\mathrm{n}} \Delta_{\mathrm{nB}-\mathrm{u}^{\prime}}}{\Delta_{\mathrm{n}} \Delta_{\mathrm{t}}}\right), \mathrm{G}_{\mathrm{III}}(\mathrm{A})=\left(\frac{1}{2} \frac{\mathrm{R}_{\mathrm{A}}^{\mathrm{t}} \Delta_{\mathrm{tB}-\mathrm{u}^{\prime}}}{\Delta_{\mathrm{n}} \Delta_{\mathrm{t}}}\right)
$$

where $R_{A}^{z}$ is the reaction in the spring element connecting node $A$ in the $z$-direction, $\Delta \mathrm{w}_{\mathrm{B}-\mathrm{B}^{\prime}}$ is the relative $\mathrm{z}$-displacement between the nodes $B$ and $B^{\prime}$. These are located immediately ahead of the delamination front along its normal direction passing through $A$.

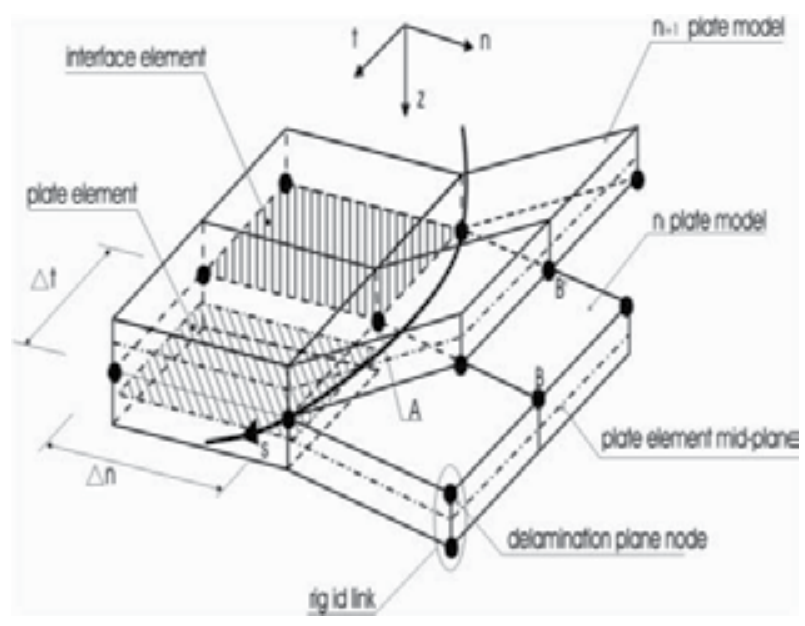

Figure 3. Plate assembly in the neighborhood of the delamination front

Similar definitions apply for reactions and relative displacement related to modes II and III. The characteristic mesh sizes in the normal and tangential directions of the delamination front are denoted by $\Delta_{\mathrm{n}}$ and $\Delta_{\mathrm{t}}$. In (9), the same element size is assumed for elements ahead of and behind the delamination front. Value of $\Delta_{t} / 2$ must be used in (9) instead of $\Delta_{t}$ when the node is placed at a free edge.

In order to simplify the FE modeling procedure, it is possible to introduce spring elements only along the delamination front instead of the entire undelaminated region. The perfect adhesion along the remaining portion of the undelaminated region can be imposed by CE. However, when the delamination propagation must be simulated, it is necessary to introduce interface elements in the whole undelaminated region $\Omega-\Omega_{D}$.

In the next example, the delamination modeling techniques presented so far are applied to analyze typical 3D delamination problems in laminated plates. The ERR distribution along the delamination front are computed for different laminates and loading conditions.

\subsection{Finite element modeling and numerical example}

One of the most powerful computational methods for structural analysis of composites is the FEM. The starting point would be a "validated" FE model, with a reasonably fine mesh, 
correct boundary conditions, material properties, etc. (Bathe, 1996). As a minimum requirement, the model is expected to produce stress and strains that have reasonable accuracy to those of the real structure prior to failure initiation. In spite of the great success of the FEM and BEM as effective numerical tools for the solution of boundary-value problems on complex domains, there is still a growing interest in the development of new advanced methods. Many meshless formulations are becoming popular due to their high adaptivity and a low cost to prepare input data for numerical analysis (Guiamatsia et al., 2009).

The results of the delamination analysis of two laminae with different thickness and material are processed in this section. The laminae are fixed on one side and free on the other side. Loads are applied on the free side depending on the analyzed type of delamination.

The upper sublamina has these properties: $E_{x}=35000 \mathrm{MPa}, E_{y}=E_{z}=10500 \mathrm{MPa}, G_{y z}=10500$ $\mathrm{MPa}, G_{x y}=G_{x z}=1167 \mathrm{MPa}, v_{x y}=v_{x z}=v_{y z}=0.3$. The lower sublamina has these properties: $E_{x}=$ $70000 \mathrm{MPa}, E_{y}=E_{z}=21000 \mathrm{MPa}, G_{y z}=2100 \mathrm{MPa}, G_{x y}=G_{x z}=2333 \mathrm{MPa}, v_{x y}=v_{x z}=v_{y z}=0.3$. The pair of forces applied on the laminae is $T=1 \mathrm{~N} / \mathrm{mm}$ and the dimensions of the laminate are: a $=10 \mathrm{~mm}, \mathrm{~B}=20 \mathrm{~mm}, L=20 \mathrm{~mm}, h_{1}=0.5 \mathrm{~mm}, h_{2}=1 \mathrm{~mm}$.
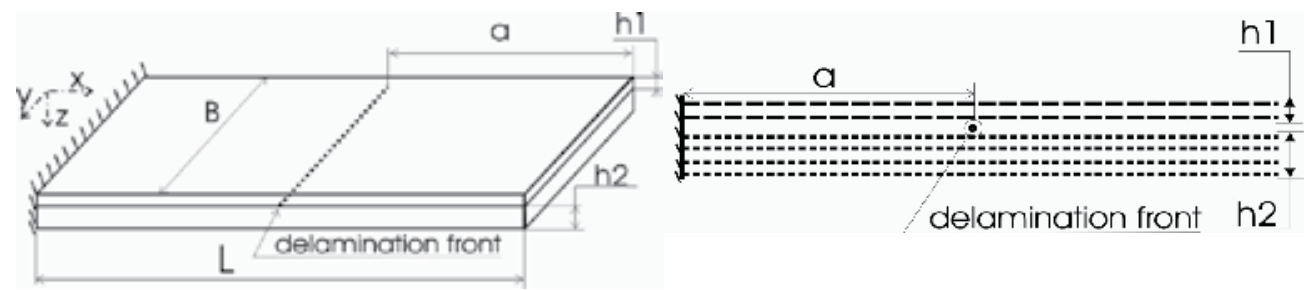

Figure 4. a) Scheme of boundary conditions and laminate dimensions,

b) scheme of FEM model

The upper sublaminate is composed by four plates $n_{u}=4$ and the lower by two $n_{l}=2$. The plates are meshed by SHELL181 elements. The zone of mesh refinement has these dimensions $5 * 20 \mathrm{~mm}$, it is centered around of the delamination front which is placed in the middle of the laminate. The interface between the sublaminates is modeled without stiffness for opening displacements and with positive stiffness for closing displacements. The interface between sublaminates is modeled by means of CE (Constrain Equation), since it is easier to apply than beam elements and delamination propagation is not solved. The delamination front is created by spring elements COMBIN 14, in each node of the delamination front by three elements. The stiffness of the spring elements binding the laminae is chosen as $k_{z} \mathrm{a} k_{x y}=$ $10^{8} \mathrm{~N} / \mathrm{mm}^{3}$. These elements are oriented in different directions, they were created always from a pair of nodes placed on the surface of the lower sublamina. One of the pair nodes is bounded to the upper plate by means of CE and the second one is bounded to the lower plate. ERR is calculated by using deformation along the delamination front.

Model I

At model I the ERR for delamination type I is analyzed. Model I is loaded with opening forces $T$ of magnitude $1 \mathrm{~N} / \mathrm{mm}$, which are parallel to $\mathrm{z}$ axis, displayed on Fig. 5a. For the calculation 
of the ERR the equation (9) was used for the type I. As the biggest ERR is in the middle of the model, it is expected that the beginning of the delamination is in the middle of the model. The distribution of ERR through the width of the laminate is displayed on the Fig.5b.

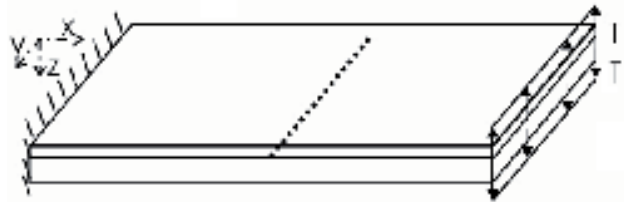

(a)

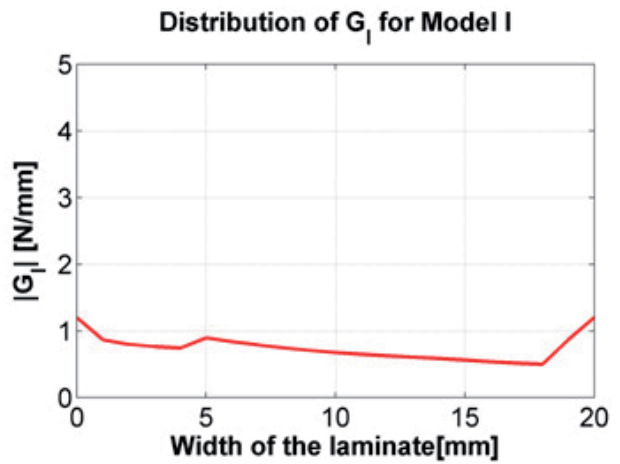

(b)

Figure 5. Scheme of FEM Model I: a) Forces applied on the laminate model, b) ERR distribution of $G_{I}$ for delamination type I

Model II

In this model the delamination type II (sliding type)was simulated. The applied forces are parallel to the $x$ axis, Fig. 6 a). Two types of ERR were analyzed in this model, $G_{I I}$ in the $x$ direction and $G_{\text {III }}$ in the $y$ direction. ERRs were calculated separately for each direction. The reaction of the spring elements are used for the calculation of $G_{I I}$ and the y reactions are used for the calculation of $\mathrm{G}_{\mathrm{III}}$. Both distributions are displaying the absolute values of ERR, at both distributions the values of ERR are smaller then the values of $G_{I}$. The values of GII are in the range of $(0.5,2) \cdot 10^{-4}$ and the values of GIII are in the range of $(0,4) \cdot 10^{-5}$ (Fig. 7$)$

Model III

At this model the delamination type III (tearing type) was analyzed. The geometry of model I was used, with the mesh and its refinement around the delamination front, boundary conditions and the linking between the shell plates, but the direction of the applied forces has changed, Fig.8a). Both types of ERR are analyzed here, $G_{I I}$ in the $x$ direction and $G_{\text {III }}$ the $y$ direction. The values of ERR are in these ranges: value of $G_{I I}$ in the range of $(0,14) \cdot 10^{-3}$ and the value of GIII in the range of $(0,0.02)$. It is possible that better results could be achieved by increasing of the number of plate elements layers simulating the sublamina. These models can be also modeled by solid elements, but there is greater number of elements needed for accurately simulating of the stress and ERR gradients. Thereby the number of equations and computing time increase. 


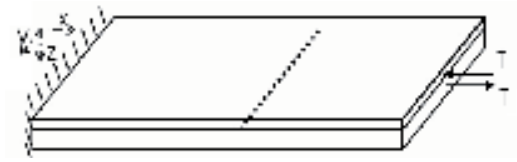

(a)

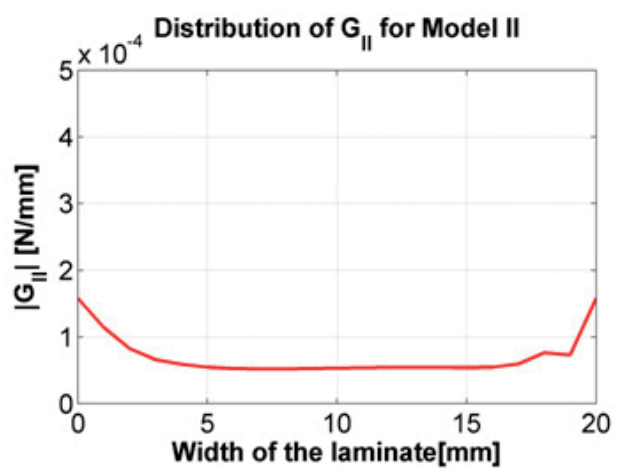

(b)

Figure 6. Scheme of FEM Model II: a) direction of loads for delamination type II, b) distribution of ERR for type II delamination of $G_{I I}$

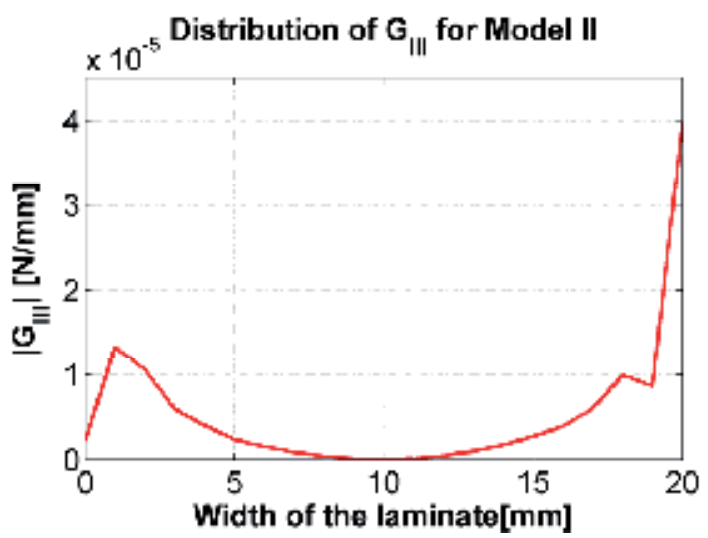

Figure 7. ERR distribution of GIII for delamination type II

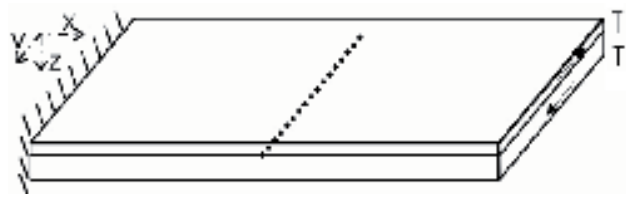

(a)

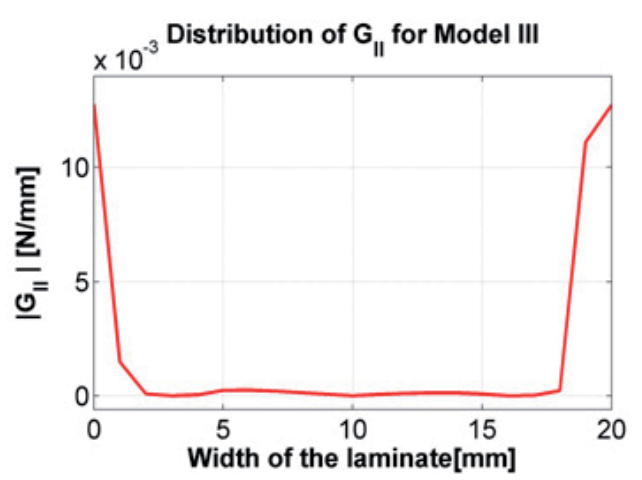

(b)

Figure 8. Scheme of FEM Model III: a) definitions of loads for delamination type III, b) distribution of $\mathrm{G}_{\mathrm{II}}$ for model III 


\section{Continuum damage mechanics}

There are many material modeling strategies to predict damage in laminated composites subjected to static or impulsive loads. Broadly, they can be classified as (Jain \& Ghosh, 2009):

- failure criteria approach (Kormaníková, 2011),

- fracture mechanics approach (based on energy release rates),

- plasticity or yield surface approach,

- damage mechanics approach

We consider a volume of material free of damage if no cracks or cavities can be observed at the microscopic scale. The opposite state is the fracture of the volume element. Theory of damage describes the phenomena between the virgin state of material and the macroscopic onset of crack (Jain \& Ghosh, 2009; Tumino et al., 2007). The volume element must be of sufficiently large size compared to the inhomogenities of the composite material. In Fig. 9 this volume is depicted. One section of this element is related to its normal and to its area $S$. Due to the presence of defects, an effective area $\tilde{S}$ for resistance can be found. Total area of defects is therefore:

$$
\mathrm{S}_{\mathrm{D}}=\mathrm{S}-\tilde{\mathrm{S}}
$$

The local damage related to the direction $\mathbf{n}$ is defined as:

$$
\mathrm{D}=\frac{\mathrm{S}_{\mathrm{D}}}{\mathrm{S}}
$$

For isotropic damage, the dependence on the normal $\mathrm{n}$ can be neglected, i.e.

$$
\mathrm{D}=\mathrm{D}_{\mathrm{n}} \forall \mathrm{n}
$$

We note that damage $D$ is a scalar assuming values between 0 and 1 . For $D=0$ the material is undamaged, for $0<D<1$ the material is damaged, for $D=1$ complete failure occurs. The quantitative evaluation of damage is not a trivial issue, it must be linked to a variable that is able to characterize the phenomenon.

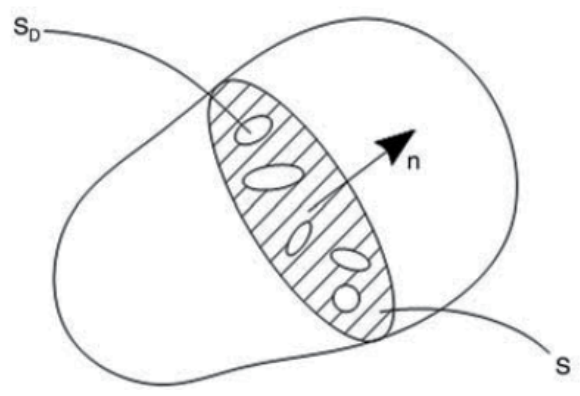

Figure 9. Representative volume element for damage mechanics 
We note that several papers can be found in literature where the constitutive equations of the materials are a function of a scalar variable of damage (Barbero, 2008). For the formulation of a general multidimensional damage model it is necessary to generalize the scalar damage variables. It is therefore necessary to define corresponding tensorial damage variables that can be used in general states of deformation and damage (Tumino at al., 2007).

In this part, we focused on presenting the methodology of numerical solving of elastic damage of thin composite plates reinforced by long fibers based on continuum damage mechanics by means of the finite element method.

\subsection{Damage model used}

The model for fiber-reinforced lamina mentioned next was presented by Barbero and de Vivo (Barbero, 2001) and is suitable for fiber - reinforced composite material with polymer matrix. On the lamina level these composites are considered as ideal homogenous and transversely isotropic. All parameters of this model can be easily identified from available experimental data. It is assumed that damage in principal directions is identical with the principal material directions throughout the damage process. Therefore the evolution of damage is solved in the lamina coordinate system. The model predicts the evolution of damage and its effect on stiffness and subsequent redistribution of stress.

\subsubsection{Damage surface and damage potential}

Damage surface is similar to the Tsai-Wu damage surface and is defined by tensors $\mathbf{J}$ and $\mathbf{H}$ (Barbero, 2008 and it is commonly used for predicting failure of fiber-reinforced lamina with respect to experimental material strength values. Damage surface and damage potential have the form of (Barbero \& de Vivo, 2001)

$$
\begin{gathered}
g(Y, \gamma)=\sqrt{J_{11} Y_{1}^{2}+J_{22} Y_{2}^{2}+J_{33} Y_{3}^{2}}+\sqrt{H_{1} Y_{1}^{2}+H_{2} Y_{2}^{2}+H_{3} Y_{3}^{2}}-\left(\gamma+\gamma_{0}\right) \\
f(Y, \gamma)=\sqrt{J_{11} Y_{1}^{2}+J_{22} Y_{2}^{2}+J_{33} Y_{3}^{2}}-\left(\gamma+\gamma_{0}\right)
\end{gathered}
$$

where the thermodynamic forces $Y_{1}, Y_{2}$ and $Y_{3}$ can be calculated by means of relations

$$
\begin{gathered}
Y_{1}=\frac{1}{\Omega_{1}^{2}}\left(\frac{\bar{S}_{11}}{\Omega_{1}^{4}} \sigma_{1}^{2}+\frac{\bar{S}_{12}}{\Omega_{1}^{2} \Omega_{2}^{2}} \sigma_{1} \sigma_{2}+\frac{\bar{S}_{66}}{\Omega_{1}^{2} \Omega_{2}^{2}} \sigma_{6}^{2}\right) \\
Y_{2}=\frac{1}{\Omega_{2}^{2}}\left(\frac{\bar{S}_{22}}{\Omega_{2}^{4}} \sigma_{2}^{2}+\frac{\bar{S}_{12}}{\Omega_{1}^{2} \Omega_{2}^{2}} \sigma_{1} \sigma_{2}+\frac{\bar{S}_{66}}{\Omega_{1}^{2} \Omega_{2}^{2}} \sigma_{6}^{2}\right) \\
Y_{3}=0
\end{gathered}
$$

where stresses and components of matrix $\overline{\mathbf{S}}$ are defined in the lamina coordinate system. Matrix $\overline{\mathbf{S}}$ gives the strain-stress relations in the effective configuration (Barbero, 2007). 
Equation (13) and (14) can be written for different simple stress states: tension and compression in fiber direction, tension in transverse direction, in-plane shear. Tensors $J$ and $\boldsymbol{H}$ can be derived in terms of material strength values.

\subsubsection{Hardening parameters}

In the present model for damage isotropic hardening is considered and the hardening function was used in the form of

$$
\gamma=c_{1}\left[\exp \left(\frac{\delta}{c_{2}}\right)+1\right]
$$

The hardening parameters $\gamma_{0}, \mathrm{c}_{1}$ and $\mathrm{c}_{2}$ are determined by approximating the experimental stress-strain curves for in-plane shear loading. If this curve is not available, we can reconstruct it using function

$$
\sigma_{6}=F_{6} \tanh \left(\frac{G_{12}}{F_{6}} \gamma_{6}\right)
$$

where $F_{6}$ is the in-plane shear strength, $G_{12}$ is the in-plane initial elasticity modulus and $\gamma_{6}$ is the in-plane shear strain (in the lamina coordinate system). This function represents experimental data very well.

\subsubsection{Critical damage level}

The reaching of critical damage level is dependent on stresses in points of lamina. If in a point of lamina only normal stresses in the fiber direction or transverse direction (i.e., normal stress in lamina coordinate system) occur, then simply comparing the values of damaged variables with critical values of damage variables for given material at this point is sufficient. The damage has reached critical level if at least one of the values $D_{1}$, $D_{2}$ in the point of lamina is greater or equal to its critical value. If in a given point of lamina also shear stress occurs (in lamina coordinate system), it is additionally necessary to compare the value of the product of $\left(1-D_{1}\right)\left(1-D_{2}\right)$ with $k_{s}$ value for given material. If the value of this product is less or equal to $k_{s}$ value, the damage has reached a critical level.

\subsection{Implementation of numerical method}

The Newton-Raphson method was used for solving the system of nonlinear equations. Evolution of damage has been solved using return-mapping algorithm described in (Neto, 2008). The input values are strains and strain increments in lamina coordinate system, state variables $D_{1}, D_{2}$, and $\delta$ in integration point from the start of last performed iteration, $\overline{\mathbf{C}}$ matrix (gives the stress-strain relations in the effective configuration (Barbero, 2007) and 
damage parameters related to damage model. The output variables are $D_{1}, D_{2}$, and $\delta$, stresses and strains in lamina coordinate system in this integration point at the end of the last performed iteration. Another output is constitutive damage matrix $\mathrm{C}_{\mathrm{ED}}$ in lamina coordinate system, which reflects the effect of damage on the behavior of structure. Flowchart of this algorithm is described in Fig. 10.

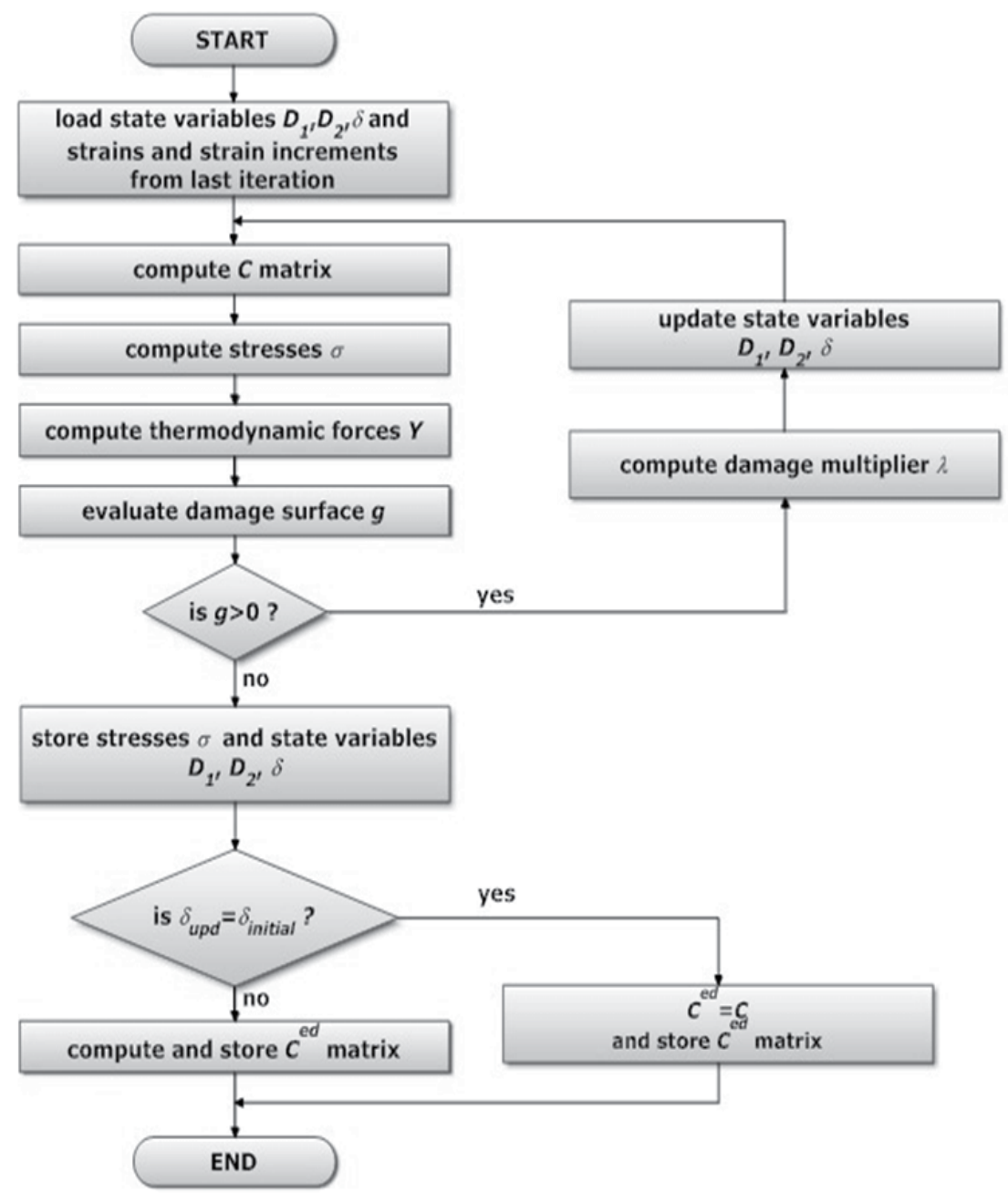

Figure 10. Flowchart of return-mapping algorithm used for solving damage evolution in particular integration points

\subsection{Numerical example}

One problem for two different materials was simulated in order to study the damage of laminated fiber-reinforced composite plates. The composites are reinforced by carbon fibers embedded in epoxy matrix. The simply supported composite plate with laminate stacking sequence of $[0,45,-45,90] \mathrm{s}$ with dimensions of $125 \times 125 \times 2.5 \mathrm{~mm}$ was loaded by transverse 
force $F=-4000 \mathrm{~N}$ in the middle of the plate. Own program created in MATLAB language was used for this analysis. Four-node layered plate finite elements based on Kirchhoff (classical) plate theory were used.

Material properties, damage parameters and hardening parameters and critical damage values are given in Table 1 - Table 3 . The parameters $J_{33}$ and $H_{3}$ are equal to zero. The plate model was divided into $8 \times 8$ elements and was analyzed in fifty load substeps. The linear static analysis shows that the largest magnitudes of stress are in parallel direction with fibers and transverse to fibers and they occur in the outer layers in the middle of the plate.

\begin{tabular}{|c|c|c|c|c|}
\hline & $\mathrm{E}_{1}[\mathrm{GPa}]$ & $\mathrm{E}_{2}[\mathrm{GPa}]$ & $\mathrm{G}_{12}[\mathrm{GPa}]$ & $\boldsymbol{v}_{12}$ \\
\hline $\mathbf{M 3 0 / 9 4 9}$ & 167 & 8.13 & 4.41 & 0.27 \\
\hline $\mathbf{M 4 0 / 9 4 8}$ & 228 & 7.99 & 4.97 & 0.292 \\
\hline
\end{tabular}

Table 1. Material properties

\begin{tabular}{|c|c|c|c|c|c|c|c|}
\hline & $\mathbf{J}_{\mathbf{1}}$ & $\mathbf{J}_{\mathbf{2}}$ & $\mathbf{H}_{\mathbf{1}}$ & $\mathbf{H}_{\mathbf{2}}$ & $\mathrm{V}_{\mathbf{0}}$ & $\mathrm{c}_{1}$ & $\mathrm{c}_{\mathbf{2}}$ \\
\hline $\mathbf{M} 30 / 949$ & $0.952 .10^{-3}$ & 0.438 & $25.585 .10^{-3}$ & $21.665 .10^{-3}$ & 0.6 & 0.30 & 0.395 \\
\hline $\mathbf{M 4 0 / 9 4 8}$ & $2.208 .10^{-3}$ & 0.214 & $10.503 .10^{-3}$ & $8.130 .10^{-3}$ & 0.12 & 0.10 & 0.395 \\
\hline
\end{tabular}

Table 2. Damage and hardening parameters

\begin{tabular}{|c|c|c|c|c|}
\hline & $\mathbf{D}_{\mathbf{1 t}}^{\mathrm{cr}}$ & $\mathbf{D}_{1 \mathrm{c}}^{\mathrm{cr}}$ & $\mathbf{D}_{2 \mathrm{c}}^{\mathrm{cr}}$ & $\mathbf{k}_{\mathbf{s}}$ \\
\hline $\mathbf{M 3 0 / 9 4 9}$ & 0.105 & 0.111 & 0.5 & 0.944 \\
\hline $\mathbf{M 4 0 / 9 4 8}$ & 0.105 & 0.111 & 0.5 & 0.908 \\
\hline
\end{tabular}

Table 3. Critical values of damage variables

The largest magnitudes of shear stress occur in the outer layers in the corner nodes. The largest magnitudes of stress in layers 2, 3, 6 and 7 occur in the center of the plate. According to the results of linear static analysis it can be expected that damage will reach the critical level in some of the above points.

Fig. 11 shows the analysis results of elastic damage of the plate made from material M30/949. Fig. 11a shows the evolution of individual stress components in dependence on strains (both in lamina coordinate system) in the midsurface of layer 1 (first layer from the bottom) in integration point (IP) 1 (in element 1, nearest to the corner). Fig. 11b shows the evolution of individual stress components in the midsurface of layer 2 in IP 872 (in element 28 , nearest to the center of the plate). Fig. 12 plots described damage variables evolution in these IPs. The analysis results show that reaching the critical level is caused not by normal stresses in the lamina coordinate system, but by shear stress (in the lamina coordinate system). The analysis results of the plate made from material M30/949 show that for given load the critical level of damage was reached in layers 2 and 7 in the center of the plate and its vicinity. In IPs that are closest to the center of the plate in these layers, the critical level of damage was reached between 13th and 14th load substep. However, it is not postulated that 
used damage model predicts failure: it only predicts damage evolution and its effect on stiffness and consequent stress redistribution (Barbero \& de Vivo, 2001). In some cases, failure can occur before the critical level of damage is reached. For plate made from material $\mathrm{M} 30 / 949$ load $\mathrm{F}=-1096 \mathrm{~N}$ is already critical.

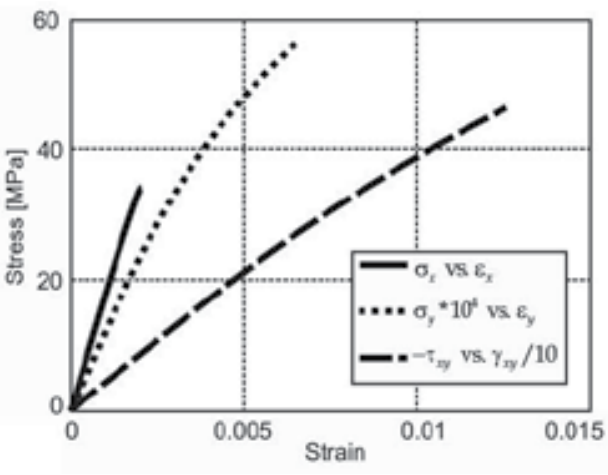

(a)

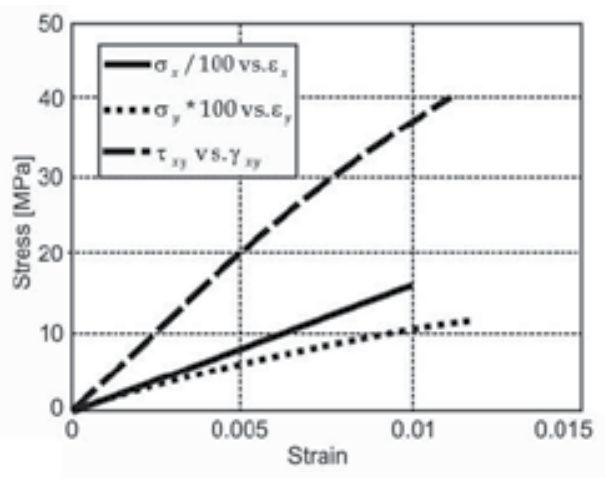

(b)

Figure 11. Stress and strain evolution for plate from material M30/949 in (a) IP 1, (b) IP 872

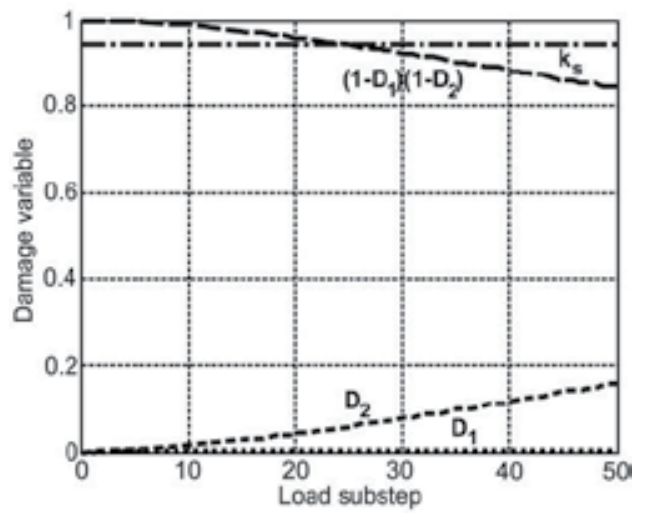

(a)

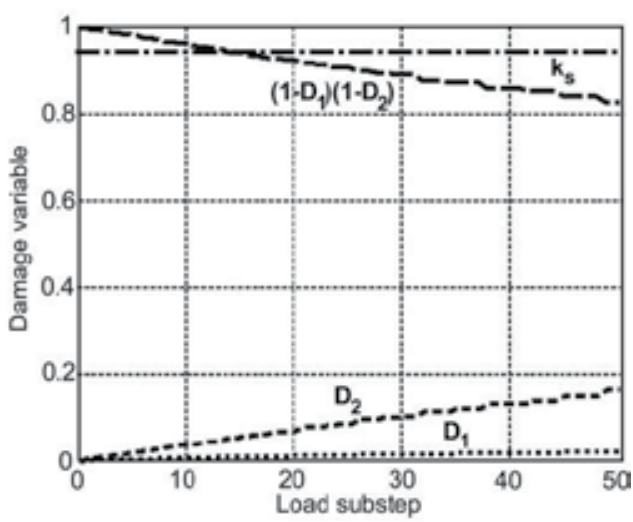

(b)

Figure 12. Damage variables evolution for plate from material M30/949 in (a) IP 1, (b) IP 872

Fig. 13 shows the analysis results of elastic damage of the plate made from material M40/948. Fig. 13a shows the evolution of individual stress components in dependence on strains (both in lamina coordinate system) in the midsurface of layer 1 in IP 868 (in element 28 , nearest to the center of the plate). Fig. $13 \mathrm{~b}$ shows the evolution of individual stress components in the midsurface of layer 2 in IP 872 (in element 28, nearest to the center of the plate). The results show that reaching the critical level of damage will be also caused by shear stress in lamina coordinate system. However, the critical damage level was reached in layers 1 and 7 in the center of plate and its vicinity. The critical level of damage was reached 
between 12th and 13th load substep in the nearest IPs. The critical level of damage would be also reached in the center of the plate and its vicinity in layers 2 and 7 (in the nearest IPs it would be reached between 16th and 17th load substep) and also in layers 3 and 6 (in the nearest IPs it would be reached between 27th and 28th load substep). Fig. 14 shows damage variables evolution in IP 868 and IP 872. For plate made from material M40/948 load F = $990 \mathrm{~N}$ is already critical.

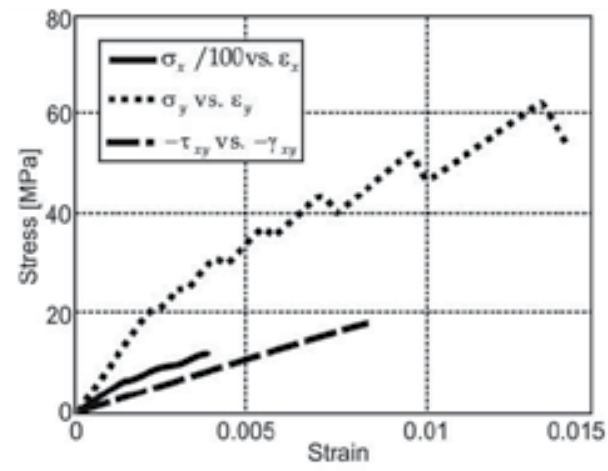

(a)

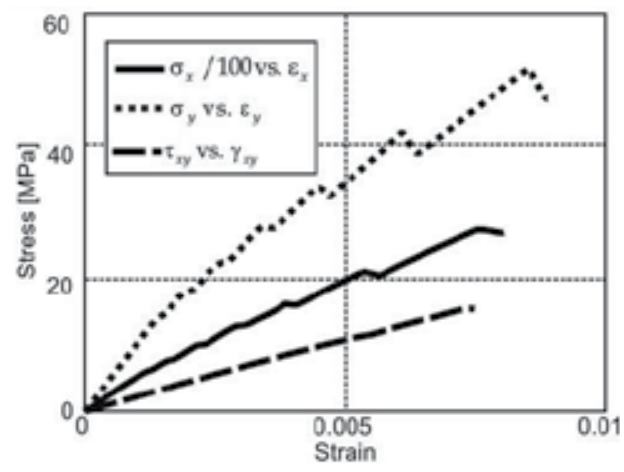

(b)

Figure 13. Stress and strain evolution for plate from material M40/948 in (a) IP 868, (b) IP 872

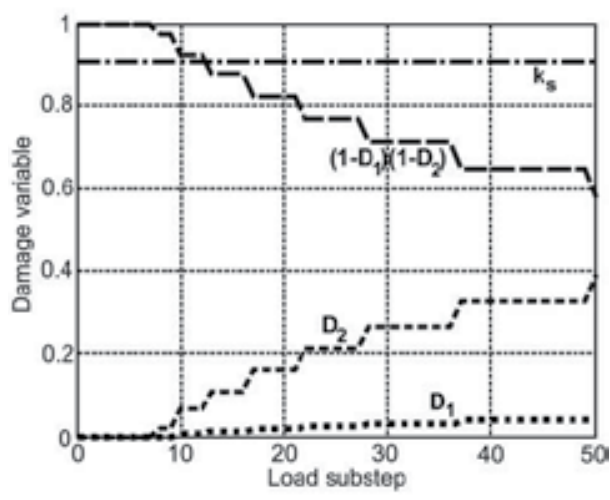

(a)

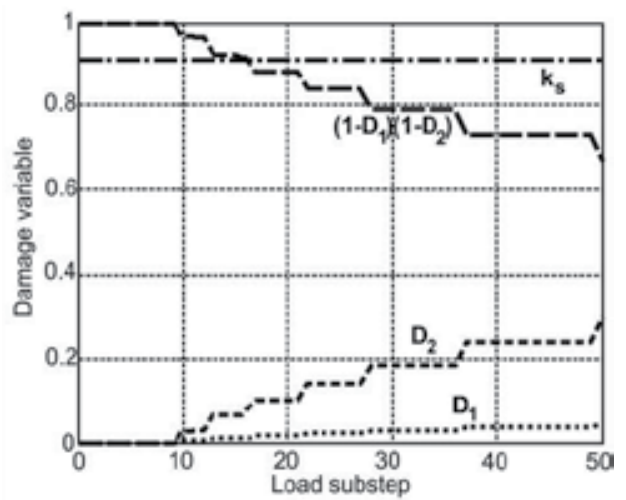

(b)

Figure 14. Damage variables evolution for plate from material M40/948 in (a) IP 868, (b) IP 872

\section{Conclusion}

The methodology of delamination calculation in laminated plates was applied in this chapter. The analyses shows that if mixed mode conditions are involved, a double plate model is suitable to accurately capture the mode decomposition in region near the midpoint 
of delamination front. The solution converges quickly because a small number of plates is needed to obtain a reasonable approximation

Damage model presented in this chapter has been utilized in this solution. This damage model is suitable for elastic damage of fiber-reinforced composite materials with polymer matrix. The postulated damage surface reduces to the Tsai-Wu surface in stress space. The problem of elastic damage is considered as material nonlinearity, so we get system of nonlinear equations. The Newton-Raphson method has been used for solving this system of nonlinear equations. Evolution of damage has been solved using the return-mapping algorithm. Flowchart of this algorithm was also presented. Numerical example of one problem for two different materials was presented next. Own program created in MATLAB language was used for this analysis. Four-node layered plate finite elements based on Kirchhoff (classical) plate theory were used. The analysis results show that change of material as well as the presence and values of shear stress have significant influence on the evolution of damage as well as on location of critical damage and load at which the critical level of damage will be reached. Critical damage level has not necessary to be reached in places with maximum magnitude of equivalent stress, but can be reached in other places.

\section{Author details}

Milan Žmindák and Martin Dudinský

University of Žilina, Slovakia

\section{Acknowledgement}

The authors gratefully acknowledge the support by the Slovak Grant Agency VEGA 1/1226/12 and Slovak Science and Technology Assistance Agency registered under number APVV-0169-07.

\section{References}

Ansys v.11, Theory manual (2007), ANSYS, Inc., Southpointe, PA.

Azouaoui, K. et al. 2010. Evaluation of impact fatigue damage in glass/epoxy composite laminate. International Journal of Fatigue, Vol. 32, No. 2, pp. 443-452, ISSN 0142-1123

Bathe, K. J. (1996). Finite Element Procedures, Prentice Hall, ISBN 0-13-301458-4, New Jersey

Barbero, E. J. (2007). Finite Element Analysis of Composite Materials, CRC Press, ISBN 1-42005433-3, Boca Raton

Barbero, E. J. \& De Vivo, L. (2001). A Constitutive Model for Elastic Damage in FiberReinforced PMC Laminae. International Journal of Damage Mechanics. Vol. 10, No. 1, pp. 73-93, ISSN 1056-7895

Carrera, E. (2002). Theories and Finite Elements for Multilayered, Anisotropic, Composite Plates and Shells. Arch. Comput. Meth. Engng. Vol. 9, Nr. 2, 2002, pp. 87-140, ISSN 11343060. 
Clegg, R. A. et al. (2006). Hypervelocity impact damage prediction in composites: Part Imaterial model and characterisation. International Journal of Impact Engineering. Vol. 33, No. 1-12, pp. 190-200, ISSN 0734-743X

Cui W, Wisnom M. A. (1993). Combined stress-based and fracture mechanics-based model for predicting delamination in composites. Composites 1993; Vol. 24, pp. 467-74, ISSN 1359-835X.

Dávila, C.G., Camanho, P.P., Rose, Ch. A. (2005), Failure criteria for FRP Laminates. Journal of Composite Materials. Vol. 39, No.4, pp. 323-345. ISSN 0021-9983

Elmarakbi, A., Hu, N., Fukunaga, H., (2009), Finite element simulation of delamination growth in composite materials using LS-DYNA. Composites Science E Technology, 69 pp. 2383-2391, ISSN 0266-3538

Fenske, M.T., Vizzini, A.J. (2001), The inclusion of in-plane stresses in delamination Criteria. Journal of Composite Materials. Vol. 35, No.15, pp. 1325-1342. ISSN 0021-9983

Gayathri, P. et al. (2010). Effect of matrix cracking and material uncertainty on composite plates. Reliability Engineering \& System Safety. Vol. 95, No. 7, pp. 716-728, ISSN 0951-8320

Guiamatsia, I. et al. (2009). Element-free Galerkin modelling of composite damage. Composites Science and Technology. Vol. 69, No. 15-16, pp. 2640-2648, ISSN 0266-3538

Chung Deborah, D.L. (2003). Composite Materials: Functional Materials for Modern Technology, ISBN 1-85233-665-X, Springer.

Iannucci, L. \& Ankersen, J. (2006). An energy based damage model for thin laminated composites. Composites Science and Technology, Vol. 66, No. 7-8, pp. 934-951. ISSN 02663538

Jain, J.R. \& Ghosh, S. (2009). Damage Evolution in Composites with a Homogenizationbased Continuum Damage Mechanics Model. International Journal of Damage Mechanics, Vol. 18, No. 6, pp. 533-568, ISSN 1056-7895

Kormaníková, E., Žmindák, M., Riecky, D. (2011). Strength of composites with fibres. In: Computational Modelling and Advanced Simulations, Murín, J., et al., pp. 167-184, ISBN 978-94-007-0316-2 Springer Science + Business Media B.V., Dordercht

Laš, V. \& Zemčík, R. (2008). Progressive Damage of Unidirectional Composite Panels. Journal of Composite Materials, Vol. 42, No. 1, pp. 25-44, ISSN 0021-9983

Mishnaevsky, L., Jr (2007). Computational mesomechanics of Composites, Numerical analysis of the effect of microstructures of composites on their strength and damage resistance.

Neto, E.A. de Souza, Peric, D., Owen, D.R.J. (2008). Computational Methods for Plasticity: Theory and Applications, ISBN 978-0-470-69452-7, John Wiley \& Sons, Ltd.

Reddy, J.N., Miravete, A. (1995), Practical analysis of composite laminates, ISBN 0-84939401-5, CRC Press

Riccio, A. \& Pietropaoli, E. (2008). Modeling Damage Propagation in Composite Plates with Embedded Delamination under Compressive Load. Journal of Composite Materials, Vol. 42, No. 13, pp. 1309-1335, ISSN 0021-9983

Sládek, J., Sládek, V., Jakubovičová, L (2002). Application of Boundary Element Methods in Fracture Mechanics, ISBN 80-968823-0-9, University of Žilina, Faculty of Mechanical Engineering, Žilina 
Shu, X. (2006). A generalised model of laminated composite plates with interfacial damage. Composite Structures, Vol. 74, No. 2, pp. 237-246, ISSN 0263-8223

Tiberkak, R. et al. (2008). Damage prediction in composite plates subjected to low velocity impact. Composite Structures, Vol. 83, No. 1, pp. 73-82, ISSN 0263-8223

Tumino, D., Campello, F., Catalanotti, G. (2007). A continuum damage model to simulate failure in composite plates under uniaxial compression. Express Polymer Letters 1, No.1, pp. 15-23, ISSN 1788-618X

Zhang, Z., Wang, S., (2009). Buckling, post-buckling and delamination propagation in debonded composite laminates. Part 1: Theoretical development, Composite Structures, Vol. 88, pp. 121-130, ISSN: 0263-8223.

Žmindák, M., Riecky, D., Danišovič, S. (2010). Finite element implementation of failure and damage models for composite structures, Proceedings of the XV international conference " Machine Modelling and Simulation, Warszaw university of technology, Krasiczyn, August, 2010.

Žmindák, M., Riecky,D., Soukup, J. (2010). Failure of Composites with Short Fibers. Communications. 4/2010, pp. 33-39, ISSN-13354205. 


\title{
Numerical Modelling of Damage Evolution and Failure Behavior of Continuous Fiber Reinforced Composites
}

\author{
F. Wang and J. Q. Zhang \\ Additional information is available at the end of the chapter
}

http://dx.doi.org/10.5772/48481

\section{Introduction}

Demands on high performance materials for use in a spectrum of structural and nonstructural applications are increasing to the point that monolithic materials cannot fully satisfy multifunctional requirements. One approach that has emerged is to develop advanced fiber composites whose properties are tailored for wide applications in engineering, such as turbine generators and ultrasonic aircrafts (Miracle, 2005). Composite materials are composed of reinforcements and matrices, in which mechanical behaviors can be seriously affected by their micro-structures. Therefore, it becomes necessary to deal with these materials from a micromechanical view point. Most importantly, an urgent task in the field of fiber-reinforced composites is to develop a relationship between the material structure and its performance under more severe loads and environments.

Damage and failure in fiber-reinforced composites evolves at several different length scales. At the smallest scale, pre-existing defects in fibers grow. Due to statistical distribution of such defect, the fiber strength exhibits large variability. Therefore, as increasing load is applied to the composite, the weakest fiber will first break. The loads carried by the broken fiber are redistributed among the remaining unbroken fibers and matrix as determined by the constitutive response of the fibers, matrix and interface. And this then causes other fibers near the failure site to fail and thus shed further load to intact fibers. Consequently, the failure of fibrous composites goes through a very complicated damage evolution, which is a combination of fiber fracture, matrix deformation and interfacial debonding and slipping around the fiber breaks, before it reaches ultimate failure. It is obvious that the connection between the microstructural scale and the macroscopic scale is nontrivial and involves mechanics, stochastics, and volume scaling (Curtin, 1999). 
Local damage evolution at the scale of fiber diameter in composites under loading determines its fracture toughness, strength, and eventually lifetime. The mechanical behavior of such a composite depends on the evolution of multi-damages throughout the application of loading, and then modelling composites undergoing progressive damage becomes a complex procedure due to many mechanisms involved (Mishnaevsky \& Brøndsted, 2008). The development of a micromechanical damage model has to take different aspects into account such as (i) the proposition of a micromechanics-based approach that describes the influence of the damage variable on material properties (Blassiau et al., 2008), (ii) the definition of a pertinent damage variable and its law of evolution (Kruch et al., 2006), and (iii) the use of an appropriate and efficient experimental technique for the evaluation of damage, such as neutron diffraction (Hanan et al., 2005) and acoustic emission (Bussiba et al., 2008), etc.

Reliability concerns in utilizing fibrous composites in structural application have motivated the development of many numerical and analytical failure models in the presence of multidamages. Different from the phenomenological approaches based upon the macroscopic level, the progressive model is needed to consider local damage mechanisms, such as fiber breakage, matrix deformation, interfacial debonding, etc (Kabir et al., 2006) and predict the dominant failure modes. This method seems to be more accurate but computationally complicated because it accounts for many failure mechanisms and is also related to damage accumulation correlated with material properties degradation. Recently computational micromechanics is also emerging as an accurate tool to study the mechanical behaviors of composites because of the sophistication of the modeling tools and the everincreasing power of digital computers (González, 2004). Within the framework, the macroscopic properties of a composite can be obtained by means of numerical simulation of the deformation and failure of the microstructure (Xia et al., 2001; F. Zhang et al., 2009).

In our previous work, the local cyclic shear plasticity of the interface around a broken fiber in ductile matrix composites under the in-phase and out-of-phase thermo-mechanical fatigue loads was analyzed by using the single-fiber shear-lag model (Zhang et al., 2002). A multifiber shear-lag model including matrix tensile modulus based upon an influence function superimposition technique was developed to simulate the nonlinear stress-strain response and the progressive failure of continuous fiber reinforced metal matrix composites under static tensile loading (Zhang \& Wang, 2009) as well as thermomechanical fatigue loading (Zhang \& Wang, 2010). This chapter will summarize our work and be organized as follows: an analytical model of the fibrous composite will be presented in Section II, in which fiber strength statistics, matrix behavior, and interfacial mechanics are explained in details. In Section III, we will introduce an influence function superimposition technique to derive stress profiles for any configuration of breaks, by considering local matrix plasticity and interface yield. In Section IV, numerical models combined with Monte-Carlo method will be developed to simulate progressive damage. In Section $\mathrm{V}$, we will investigate failure behaviors of continuous fiber reinforced composites under cyclically thermomechanical loading. Finally, we will discuss limitations of the existing models, and aspects of the existing theories that require improvement. 


\section{Analytical model for multiple damage events}

In the real multifiber composites of practical interest, the evolution of the fiber fragmentation during loading is, in principle, different because each individual fiber experiences a nonuniform stress due to the uniform applied stress plus stresses transferred from other broken fibers in the composite. The evolution of fiber damage thus depends crucially on the nature of the load transfer from broken or slipping fibers to unbroken fibers.

Consider an infinite, two-dimensional (2D) unidirectional composites reinforced with parallel, evenly-spaced fibers embedded in matrix material, in which the fibers and matrix regions are numbered in the serial number, shown in Fig. 1. The lamina is loaded in simple tension along the fiber direction. The width of fibers is $D$, and the width of each matrix region $W$ can be related to the fiber volume fraction, $V_{\mathrm{f}}$ (Zhang et al., 2002).

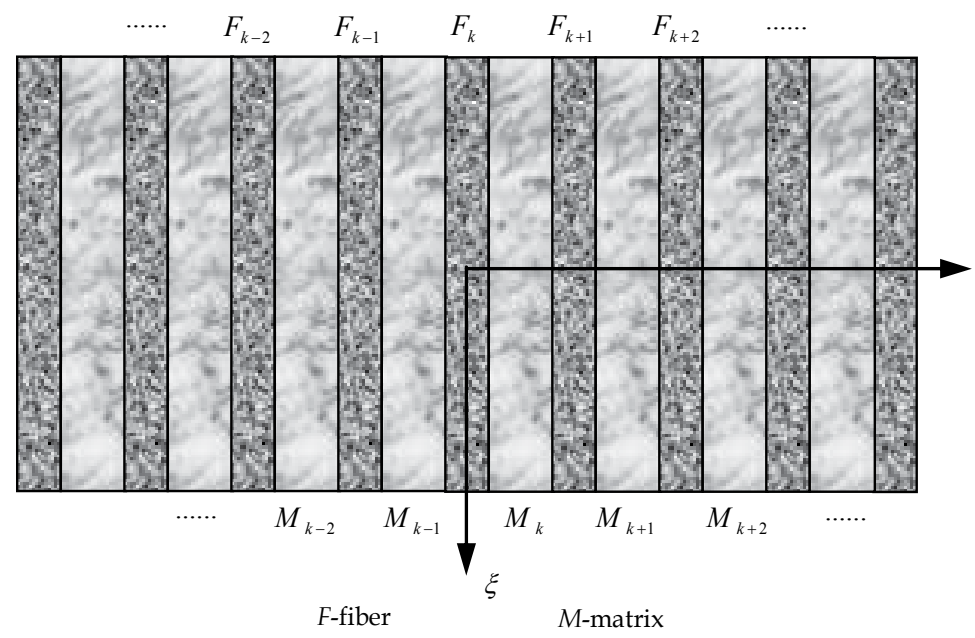

Figure 1. Schematic of the 2D fiber-matrix arrangement.

In many typical applications, fibrous composites are subjected to a cyclic mechanical loading with a superimposed variation in temperature. This type loading condition is referred to as thermomechanical fatigue (TMF) and can be regarded as one of the most severe types because the significant difference in the coefficient of the thermal expansion between the matrix and the fiber causes high thermal stresses and stress amplitudes raising irreversible deformation in the composites (Mall \& Schubbe, 1994). In-phase and out-of-phase thermomechanical fatigue (TMF) loads are illustrated in Fig. 2. The tensile stress applied to composites varies between $\left(\sigma_{\infty}\right)$ min and $\left(\sigma_{\infty}\right)$ max while the temperature changes between $T_{\max }$ and $T_{\min }$. For convenience, the state ' $\mathrm{A}$ ' is used to represent the state of TMF loads with the maximum applied stress, and the state ' $\mathrm{B}$ ' denotes the state of TMF load with the minimum mechanical load. It is clearly illustrated that in-phase conditions subject the composite to high stresses at hot temperatures and low stresses at low temperatures. Conversely, out-ofphase TMF conditions subject the composite to high stresses at low temperatures and vice versa since the peaks in the waveforms are $180^{\circ}$ apart (Williams \& Pindera, 1995). 


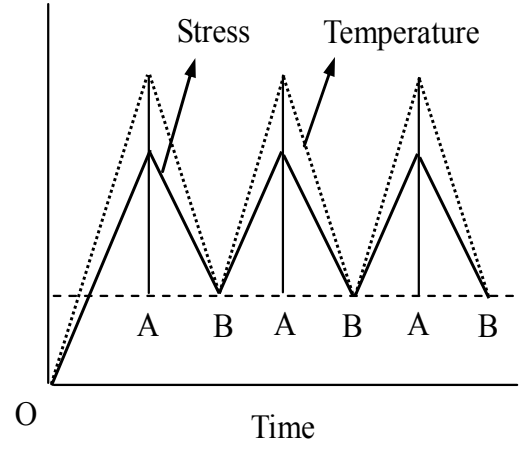

(a)

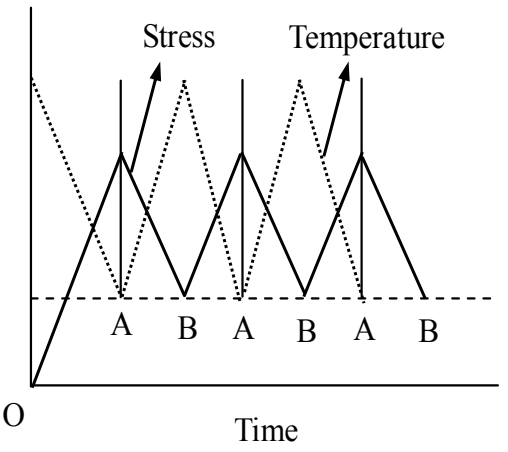

(b)

Figure 2. Stress and temperature for, (a) in-phase, (b) out-of-phase thermomechanical cycling.

Since the fiber strength exhibits large variability due to statistic distribution of defect, the weakest fiber break usually takes place at very early loading stage. After some loading level there are $N$ fiber breaks occurred in the composite. The coordinates of the $n$-th fiber break, which locates in the $k_{n}$-th fiber with vertical position $\xi_{n}$, are given by $\left(k_{n}, \xi_{n}\right)(n=1,2, \ldots, N)$.

If the interfacial strength is higher than the matrix yield stress in shear, the plastic deformation of the matrix will occur before fiber/matrix debonding (Beyerlein \& Phoenix, 1996). This was observed in ductile resin and some metal matrix composites with a strong interface. Some experiments show that the microdamage and deformation modes include the fiber breakage, fiber pullout, debonding, and local plasticity around fiber breaks (Liu \& He, 2001).

In order to analyze the complex combination of microdamage and deformation, we may propose a micromechanical model, shown in Fig. 3., in which a broken fiber, accompanying with its (reserve) tensile yielding matrix and its (reverse) shear yielding interface, and its debonding interface is called as a damage-plasticity event. Essentially the nonlinear cyclic behavior and thermomechanical fatigue failure of the composite is the result of the interactions among these damage-plasticity events under TMF loads.

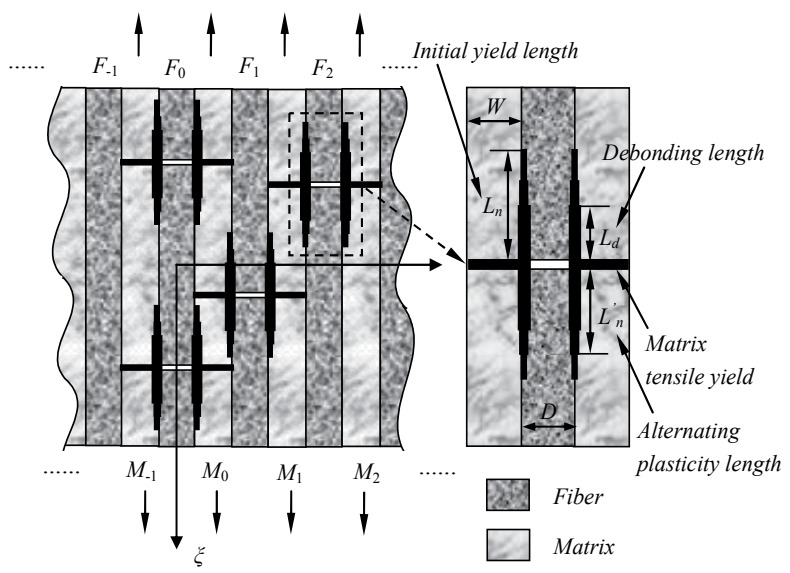

Figure 3. Multi-damage modes in composites. 
In the loading phase the local interface shear yield and the matrix tensile yield around the fiber break could take place due to stress concentration as the load increases. The initial interface yield length caused during the loading phase is represented by, $2 \mathrm{Ln}$, associated with the $n$-th fiber break. For fatigue case, when the load is released from state ' $\mathrm{A}$ ' to state ' $\mathrm{B}$ ' in the unloading phase, the yielded interface and the yielded matrix around the fiber breaks start the elastic unloading. The interface shear stress at broken sites relaxes faster than that at any other positions. Thus, the shear stress of broken sites will change sign. Subsequently, there are two possible cases: the complete elastic unloading and the reverse shear plastic yielding within a certain length, depending on the load range. If the load range is not such large that the shear stress of the broken site does not reach the reverse yield stress before state ' $\mathrm{B}$ ', the unloading is completely elastic. In this case the unloadingreloading will be totally elastic, that is, shakedown occurs. If the load range is sufficiently large, the interface shear stress around the broken sites will change sign and will reach the reverse yield stress at some moment before the load reached state ' $\mathrm{B}$ '. The interface plasticity is modeled by the elastic, perfectly plastic shear stress-strain relation, ignoring the effect of the interface tensile stress, that is,

$$
\tau=\left\{\begin{array}{cc}
G_{c} \gamma & |\tau|<\tau_{s} \\
\tau_{s}(T) & \text { yielded }
\end{array},\right.
$$

where $G_{c}$ and $\tau_{\mathrm{s}}$ are the shear modulus of the interface and the yield shear stress, respectively; $\tau$ and $\gamma$ denote the interfacial shear stress and shear strain, respectively. This interface constitutive law allows the interface to undergo the cyclic plasticity under TMF loading. The yield shear stress is related to the temperature by

$$
\tau_{s}=c_{1}+c_{2}\left[1-\exp \left(c_{3} T\right)\right]
$$

where $c_{1}, c_{2}$ and $c_{3}$ are material constants.

With continuous mechanical unloading of composite, the reverse plastic yield of the interface will extend forward from the fiber break tip. Up to the loading moment ' $\mathrm{B}$ ', the reverse plastic yield has occurred within a certain length, $2 L^{\prime}$ n. With the repeated loading and unloading processes, the cyclic interface shear plasticity takes place within the length, $2 L_{n}^{\prime}$, leading to debonding. In other words, the interface is debonded in the length of $2 L_{\mathrm{d}}$ because of cyclic plastic shear strain accumulation. It must be pointed out that material parameters in cyclic interface shear plasticity and debonding growth are difficult to measure directly. For the strong interface considered here, it is assumed that the interface shear yield is governed by the shear yield of matrix in a very thin layer. In other words, the material parameters of the interface are assumed to the same as those of the matrix.

Matrix tensile yield in the matrix regions neighboring with the fiber break sites could take place due to matrix tensile stress transferred from the tensile load released from the broken fiber. It is assumed that the $M$ breaks of the $N$ fiber breaks $(M \leq N)$ cause the associated matrix tensile yielding. Each matrix tensile yield zone takes the shape of a narrow strip 
spanning the matrix width, and is colinear with the associated fiber break. The constitutive behavior of matrix in the yield zones obeys the uniaxial cyclic elastic, perfectly plastic stressstrain relation. The yield tensile stress $\sigma_{\mathrm{s}}$ is related to the yield shear stress $\tau_{\mathrm{s}}$ by von Mises yield criterion, that is, $\sigma_{s}=\sqrt{3} \tau_{s}$.

\section{Stress profiles for multiple damage events}

Accurate prediction of the mechanical properties of the composite materials requires detailed knowledge of the micromechanical stress state in and around broken fibers as a function of the constituent material properties (Xia et al., 2002). Stress transfer between fiber and matrix is one of fundamental issues for a composite system (Hanan et al., 2003; Beyerlein \& Landis, 1999). In order to obtain solutions for deformation in the composites containing multiple damage events, a theoretical model under the framework of $2 \mathrm{D}$ shearlag arguments will be used to derive stress profiles for any configuration of breaks in the presence of matrix tensile yield and interface shear yield. It is assumed that the tensile load in a fiber-reinforced composite loaded in the fiber direction is mainly carried by the fibers and matrix regions, where the load between fiber and matrix is transferred through the fiber/matrix interface shear stress. The transverse deformation in the fibers and matrix regions is neglected. The interface is assumed to be in as state of shear behavior as controlled by the axial displacements of the fiber and matrix materials. Shear-lag analysis of stresses and deformations, involving the cyclic thermoplasticity, must be carefully carried out by applying TMF loads to the composites incrementally.

\subsection{Stress solution in loading phase}

Let us consider the TMF loading phase. As the thermomechanical loads increase, $N$ fiber breaks have appeared where each fiber break is accompanied by the interface yielding of length, $2 L \mathrm{n}$, and where $M$ breaks $(M \leq N)$ cause the associated matrix tensile yielding simultaneously. The interfacial shear stress within the yield zone $0 \leq\left|\xi-\xi_{\mathrm{n}}\right| \leq L_{\mathrm{n}} / l_{\mathrm{c}}$ associated with the $n$-th fiber-break has to be equal to the yield shear stress, it follows

$$
\tau\left(k_{n}, \xi\right)=\tau_{s}, \text { for } x=x_{\mathrm{n}}+L_{\mathrm{n}} / l_{\mathrm{c}} \text { and } n=1,2,3, \ldots, N,
$$

where $\tau\left(k_{\mathrm{n}}, \xi\right)$ is the interface shear stress at the location, $\left(k_{\mathrm{n}}, \xi\right)$. Since the shear stress is constant $\left(\tau_{\mathrm{s}}\right)$ within the interface yield region, the fiber stress can be obtained simply from the equilibrium condition of fiber, that is,

$$
\sigma^{f}\left(k_{n}, \xi\right)=\frac{2 \tau_{s}}{D}\left|l_{c}\left(\xi-\xi_{n}\right)\right|, \text { for } 0 \leq 1 x-x_{\mathrm{n}} \mid \leq L_{\mathrm{n}} / l_{\mathrm{c}} \text { and } n=1,2,3, \ldots, N,
$$

where $\sigma^{\mathrm{f}}\left(k_{\mathrm{n}}, \xi\right)$ is fiber stress at the location, $\left(k_{\mathrm{n}}, \xi\right)$. Matrix tensile stress in the narrow matrix yield zone is

$$
\sigma^{m}\left(k_{t}, \xi_{t}\right)=\sigma_{s}, t=1,2,3, \ldots, M,
$$


In order to obtain solutions for fiber stress and stress in matrix outside the yield zones the superposition method (Beyerlein \& Phoenix, 1996; Landis et al., 2000) has been further extended in our previous work (Zhang \& Wang, 2009, 2010). Original superposition method of Beyerlein excludes the axial load-bearing capacity of matrix, which is suitable for polymer matrix composites. Our extended superposition method includes the axial load-bearing capacity of matrix, which is applicable to fiber reinforced metal matrix composites. The fundamental concepts behind influence superimposition techniques are to obtain analytical solutions for the influence of a 'unit' failure on the stress and displacement fields and then use a weighted superposition technique to obtain the solution for the full problem. Indeed, the units solutions for a single fiber-break and a single matrix-break, called the influence functions, are used as building blocks for the solutions for fiber stresses and matrix stresses in the composites.

In the method, the narrow matrix yield zones is modeled as fictitious matrix breaks with surface tensile traction $\sigma_{\mathrm{s}}$, and the interface yield length is considered as a fictitious debonding crack with surface shear traction $\tau_{\mathrm{s}}$. The $M$ fictitious matrix breaks (matrix yield zones) can be decomposed into the $M$ problems of single matrix-break with opening displacements, $w_{t}^{m}(t=1,2,3, \ldots, M)$, that are yet to be determined by stress condition, eq.(5). For an interface yield length $0 \leq\left|\xi-\xi_{\mathrm{n}}\right| \leq L_{\mathrm{n}} / l_{\mathrm{c}}$ associated with the $n$-th fiber break, the fiber segment with the known fiber stress given by eq. (4) can be modeled as a continuous distribution of fictitious fiber break with non-zero opening displacement $w_{n}^{f}(\xi)$ which is to be determined through the stress conditions, eqs.(3-4).

Therefore, the axial stresses in fibers and matrix regions and the interfacial shear stress, $\sigma^{\mathrm{f}}(k$, $\xi), \sigma^{\mathrm{m}}(k, \xi)$ and $\tau(k, \xi)$ at any position $(k, \xi)$ can be obtained by using the superposition method as follows,

$$
\begin{gathered}
\sigma^{f}(k, \xi)=\bar{\sigma}_{f}+\sum_{j=1}^{N} \int_{\xi_{j}-L_{j} / l_{c}}^{\xi_{j}+L_{j} / l_{c}} w_{j}^{f}\left(\xi^{\prime}\right) p_{k-k_{j}}^{f}\left(\xi-\xi^{\prime}\right) d \xi^{\prime}+\sum_{i=1}^{M} w_{i}^{m} q_{k-k_{i}}^{f}\left(\xi-\xi_{i}\right), \\
\sigma^{m}(k, \xi)=\bar{\sigma}_{m}+\sum_{j=1}^{N} \int_{\xi_{j}-L_{j} / l_{c}}^{\xi_{j}+L_{j} / l_{c}} w_{j}^{f}\left(\xi^{\prime}\right) p_{k-k_{j}}^{m}\left(\xi-\xi^{\prime}\right) d \xi^{\prime}+\sum_{i=1}^{M} w_{i}^{m} q_{k-k_{i}}^{m}\left(\xi-\xi_{i}\right), \\
\tau(k, \xi)=\sum_{j=1}^{N} \int_{\xi_{j}-L_{j} / l_{c}}^{\xi_{j}+L_{j} / l_{c}} w_{j}^{f}\left(\xi^{\prime}\right) s_{k-k_{j}}\left(\xi-\xi^{\prime}\right) d \xi^{\prime}+\sum_{i=1}^{M} w_{i}^{m} t_{k-k_{i}}\left(\xi-\xi_{i}\right),
\end{gathered}
$$

where $\bar{\sigma}_{f}$ and $\bar{\sigma}_{m}$ denote the remote stress in fiber and matrix, respectively. The influence functions $p_{k-k_{j}}^{f}\left(\xi-\xi^{\prime}\right), p_{k-k_{j}}^{m}\left(\xi-\xi^{\prime}\right)$ and $s_{k-k_{j}}\left(\xi-\xi^{\prime}\right)$ represents fiber stress, matrix stress and interface shear stress at position $(k, \xi)$ due to a unit opening displacement applied at the fiber-break with position $\left(k_{j}, \xi^{\prime}\right)$, respectively. The influence functions $q_{k-k_{i}}^{f}\left(\xi-\xi_{i}\right)$, $q_{k-k_{i}}^{m}\left(\xi-\xi_{i}\right)$ and $t_{k-k_{i}}\left(\xi-\xi_{i}\right)$ stand for fiber stress, matrix stress and interface shear stress at position $(k, \xi)$ due to a unit opening displacement applied at the fictitious matrix break $\left(k_{\mathrm{i}}\right.$, 
$\left.\xi_{i}\right)$, respectively. The expressions for the influence functions are given in the appendix. The undetermined coefficient functions $w_{j}^{f}(j=1,2, \ldots, N)$, the unknown coefficient constant $w_{i}^{m}$ $(i=1,2, \ldots, M)$ and the interface yield lengths $L_{j}(j=1,2, \ldots, N)$ can be obtained by the $(2 N+M)$ stress conditions given by eqs. (3-5). Substitution of eqs. (6-8) into eqs. (3-5) gives

$$
\begin{gathered}
\sum_{j=1}^{N} \int_{\xi_{j}-L_{j} / l_{c}}^{\xi_{j}+L_{j} / l_{c}} w_{j}^{f}\left(\xi^{\prime}\right) s_{k_{n}-k_{j}}\left(\xi_{n}+L_{n} / l_{c}-\xi^{\prime}\right) d \xi^{\prime}+\sum_{i=1}^{M} w_{i}^{m} t_{k_{n}-k_{i}}\left(\xi_{n}+L_{n} / l_{c}-\xi_{i}\right)=\tau_{s^{\prime}} \text { for } n=1,2,3, \ldots, N, \\
\bar{\sigma}_{f}+\sum_{j=1}^{N} \int_{\xi_{j}-L_{j} / l_{c}}^{\xi_{j}+L_{j} / l_{c}} w_{j}^{f}\left(\xi^{\prime}\right) p_{k_{n}-k_{j}}^{f}\left(\xi-\xi^{\prime}\right) d \xi^{\prime}+\sum_{i=1}^{M} w_{i}^{m} q_{k_{n}-k_{i}}^{f}\left(\xi-\xi_{i}\right)=\frac{2 \tau_{s}}{D}\left|l_{c}\left(\xi-\xi_{n}\right)\right|, \\
\text { for } 0 \leq \leq x-x_{\mathrm{n}} \leq L_{\mathrm{n}} / l_{\mathrm{c}} \text { and } n=1,2,3, \ldots, N, \\
\bar{\sigma}_{m}+\sum_{j=1}^{N} \int_{\xi_{j}-L_{j} / l_{c}}^{\xi_{j}+L_{j} / l_{c}} w_{j}^{f}\left(\xi^{\prime}\right) p_{k_{t}-k_{j}}^{m}\left(\xi_{t}-\xi^{\prime}\right) d \xi^{\prime}+\sum_{i=1}^{M} w_{i}^{m} q_{k_{t}-k_{i}}^{m}\left(\xi_{t}-\xi_{i}\right)=\sigma_{s^{\prime}} \\
\text { fort }=1,2,3, \ldots, M,
\end{gathered}
$$

where $l_{c}=\sqrt{\frac{W E_{m}}{K}} ; E_{\mathrm{m}}$ are the Young's modulus of the matrix and $K$ is the shear-lag parameter associated with fiber/matrix interfaces. By solving the integral eqs. (9-11) one can obtain the shear yield length, $L_{\mathrm{n}}$, the coefficient functions $w_{j}^{f}(j=1,2, \ldots, N)$ and coefficients $w_{i}^{m}(i=1,2, \ldots, M)$. Physically $w_{j}^{f}$ and $w_{i}^{m}$ represent the fiber displacement and the elongation of the narrow matrix yield zone.

It is worthy to emphasize that the stress analysis method mentioned above is exact under the framework of shear-lag argument. In that sense, moreover, the mechanical interactions between the multiple damage-plasticity events have been fully and exactly taken into account since the governing equations of shear lag model, the boundary conditions and the plastic yield conditions have been fully satisfied.

\subsection{Stress solution in unloading phase}

There are two possible cases. If the unloading is completely elastic, the solutions for the stresses and the displacements at the state ' $\mathrm{B}$ ' can be attained by superposing the elastic solutions between $A$ and $B$ onto the solutions of the state ' $\mathrm{A}$ '. Otherwise, if the load range is sufficiently large, the local reverse yield of the interface and matrix will takes place before the thermomechanical load reaches state ' $\mathrm{B}$ '. We consider an unloading increment, during which the interface reverse yielding occurs in $N^{\prime}\left(N^{\prime} \leq N\right)$ lengths, $2 L_{n}^{\prime}\left(n=1,2, \ldots, N^{\prime}\right)$, and matrix reverse yielding takes place in $M^{\prime}\left(M^{\prime} \leq M\right)$ narrow matrix zones. The interfacial shear stress within the reverse yield zone $0 \leq\left|\xi-\xi_{\mathrm{n}}\right| \leq L^{\prime} \mathrm{n} / l_{\mathrm{c}}$ has to be equal to the reverse yield shear stress, i.e.

$$
\tau\left(k_{n}, \xi\right)=-\tau_{s}, \text { for } 0 \leq 1 x-x_{\mathrm{n}} \mid \leq L_{\mathrm{n}}^{\prime} / l_{\mathrm{c}} \text { and } n=1,2,3, \ldots, N^{\prime},
$$


Within the interface reverse yield region the fiber stress can be easily obtained from the equilibrium condition of fiber as follows

$$
\sigma^{f}\left(k_{n}, \xi\right)=-\frac{2 \tau_{s}}{D}\left|l_{c}\left(\xi-\xi_{n}\right)\right| \text {, for } 0 \leq\left|x-x_{\mathrm{n}}\right| \leq L_{\mathrm{n}}^{\prime} / l_{\mathrm{c}} \text { and } n=1,2,3, \ldots, N^{\prime},
$$

Matrix stress in the narrow matrix reverse yield zone is

$$
\sigma^{m}\left(k_{t}, \xi_{t}\right)=-\sigma_{s}, t=1,2,3, \ldots, M^{\prime},
$$

The incremental stress solutions for fibers and matrix regions in composite for the thermomechanical unloading increment can be obtained by using the superposition method mentioned in the previous section. The solutions for the total stresses after the thermomechanical unloading increment, then, can be obtained by superposing the incremental solutions onto the solutions before the unloading increment, that is,

$$
\begin{gathered}
\sigma^{f}(k, \xi)=\sigma_{0}^{f}(k, \xi)+\sum_{j=1}^{N^{\prime}} \int_{\xi_{j}-L_{j}^{\prime} / l_{c}}^{\xi_{j}+L_{j}^{\prime} / l_{c}} \bar{w}_{j}^{f}\left(\xi^{\prime}\right) p_{k-k_{j}}^{f}\left(\xi-\xi^{\prime}\right) d \xi^{\prime}+\sum_{i=1}^{M^{\prime}} \bar{w}_{i}^{m} q_{k-k_{i}}^{f}\left(\xi-\xi_{i}\right), \\
\sigma^{m}(k, \xi)=\sigma_{0}^{m}(k, \xi)+\sum_{j=1}^{N^{\prime}} \int_{\xi_{j}-L_{j}^{\prime} / l_{c}}^{\xi_{j}+L_{j}^{\prime} / l_{c}} \bar{w}_{j}^{f}\left(\xi^{\prime}\right) p_{k-k_{j}}^{m}\left(\xi-\xi^{\prime}\right) d \xi^{\prime}+\sum_{i=1}^{M^{\prime}} \bar{w}_{i}^{m} q_{k-k_{i}}^{m}\left(\xi-\xi_{i}\right), \\
\tau(k, \xi)=\tau_{0}(k, \xi)+\sum_{j=1}^{N^{\prime}} \int_{\xi_{j}-L_{j}^{\prime} / l_{c}}^{\xi_{j}+L_{j}^{\prime} / l_{c}} \bar{w}_{j}^{f}\left(\xi^{\prime}\right) s_{k-k_{j}}\left(\xi-\xi^{\prime}\right) d \xi^{\prime}+\sum_{i=1}^{M^{\prime}} \bar{w}_{i}^{m} t_{k-k_{i}}\left(\xi-\xi_{i}\right),
\end{gathered}
$$

where the coefficients $\bar{w}_{j}^{f}\left(j=1,2, \ldots, N^{\prime}\right)$ and $\bar{w}_{i}^{m}\left(i=1,2, \ldots, M^{\prime}\right)$ are to be determined which belong to the incremental unloading. The subscript ' 0 ' represents the state before an incremental unloading. The undetermined interface reverse yield lengths $2 L^{\prime} \mathrm{n}$ are governed by the interfacial shear stress at the end of reverse yield zone, $\bar{\xi}_{n}=\xi_{\mathrm{n}}+L_{\mathrm{n}}^{\prime} / l_{c}$, and it follows from eqs. (12) and (17) that

$$
\tau_{0}\left(k_{n}, \bar{\xi}_{n}\right)+\sum_{j=1}^{N^{\prime}} \int_{\xi_{j}-L_{j}^{\prime} / l_{c}}^{\xi_{j}+L_{j}^{\prime} / l_{c}} \bar{w}_{j}^{f}\left(\xi^{\prime}\right) s_{k_{n}-k_{j}}\left(\xi_{n}+L_{n}^{\prime} / l_{c}-\xi^{\prime}\right) d \xi^{\prime}+\sum_{i=1}^{M^{\prime}} \bar{w}_{i}^{m} t_{k_{n}-k_{i}}\left(\xi_{n}+L_{n}^{\prime} / l_{c}-\xi_{i}\right)=-\tau_{s^{\prime}},
$$

for $n=1,2,3, \ldots, N^{\prime}$

Substitution of eqs. (15-16) into eqs. (13-14) leads to

$$
\begin{aligned}
& \sigma_{0}^{f}\left(k_{n}, \xi\right)+\sum_{j=1}^{N^{\prime}} \int_{\xi_{j}-L_{j}^{\prime} / l_{c}}^{\xi_{j}+L_{j}^{\prime} / l_{c}} \bar{w}_{j}^{f}\left(\xi^{\prime}\right) p_{k_{n}-k_{j}}^{f}\left(\xi-\xi^{\prime}\right) d \xi^{\prime}+\sum_{i=1}^{M^{\prime}} \bar{w}_{i}^{m} q_{k_{n}-k_{i}}^{f}\left(\xi-\xi_{i}\right)=-\frac{2 \tau_{s}}{D}\left|l_{c}\left(\xi-\xi_{n}\right)\right|, \\
& \text { for } 0 \leq 1 x-x_{\mathrm{n}} \leq L_{\mathrm{n}}^{\prime} / l_{\mathrm{c}} \text { and } n=1,2,3, \ldots, N^{\prime} \\
& \sigma_{0}^{m}\left(k_{t}, \xi_{t}\right)+\sum_{j=1}^{N^{\prime}} \int_{\xi_{j}-L_{j}^{\prime} / l_{c}}^{\xi_{j}+L_{j}^{\prime} / l_{c}} \bar{w}_{j}^{f}\left(\xi^{\prime}\right) p_{k_{t}-k_{j}}^{m}\left(\xi_{t}-\xi^{\prime}\right) d \xi^{\prime}+\sum_{i=1}^{M^{\prime}} \bar{w}_{i}^{m} q_{k_{t}-k_{i}}^{m}\left(\xi_{t}-\xi_{i}\right)=-\sigma_{s}, \text { for } t=1,2,3, \ldots, M^{\prime}
\end{aligned}
$$


By solving the integral eqs. (18-20) one can obtain the coefficient functions $\bar{w}_{j}^{f}(j=1,2, \ldots$, $\left.N^{\prime}\right)$, coefficients $\bar{w}_{i}^{m}\left(i=1,2, \ldots, M^{\prime}\right)$ and the reverse shear yield lengths $L^{\prime}{ }_{n}\left(n=1,2, \ldots, N^{\prime}\right)$.

\subsection{Local cyclic plasticity and debonding}

The cyclic shear plasticity of the interface will take place only if $L_{n}^{\prime}>0$. Otherwise there is no alternating plastic shear and interface undergoes cyclic elastic deformation over the whole length after initial yielding of length $L_{n}$, that is, shakedown. Since the elastic, perfect-plastic constitutive relation has been assumed for the interface shear and matrix, the solutions for all cycles are the same before the new debonding takes place. The alternating plastic shear strain range $\Delta \gamma_{p}=\gamma_{p}^{A}-\gamma_{p}^{B}$ can be derived from displacement solutions for loading and unloading condition. The total interface shear strain is evaluated by eq. (21a)

$$
\begin{aligned}
& \gamma=2 \frac{u^{m}-u^{f}}{W+D},(a), \\
& \gamma_{p}=\gamma-\frac{\tau}{G_{c}}
\end{aligned}
$$

where $u^{\mathrm{f}}$ is the axial displacement of broken fiber and $u^{\mathrm{m}}$ is the axial displacement of matrix adjacent to the broken fiber. The plastic shear strain can be easily computed by eq. (21b).

Debonding is an important mechanism that stimulates stress redistribution in composites. Since plastic strain is dominant in the low cyclic fatigue regime (Llorca, 2002), the cyclic plastic shear strain range $\Delta \gamma_{p}$ may be used to predict the debonding (Zhang et al., 2002) along with Coffin-Manson fatigue equations $\Delta \gamma_{\mathrm{p}} / 2=\gamma_{\mathrm{f}} \cdot\left(2 N_{\mathrm{f}}\right)^{\mathrm{c}}$, where $N_{\mathrm{f}}$ is the cycles to interface debonding under the constant shear plastic strain range $\Delta \gamma_{\mathrm{p}}$, material constants $\gamma_{\mathrm{f}}$ and $c$ stand for the fatigue ductility coefficient and the fatigue ductility exponent, respectively. The debonding growth law based on Coffin-Mason equations represented by plastic shear strain range provides a means to account for the stable growth of debonding as the number of cycles increases, which may be relevant for the strong interface and/or the low applied load where the static debonding criterion fails to predict a further growth of debonding. The parameters in the debonding growth law are taken to the same as matrix since we assumed that the interface shear yield is governed by the shear yield of matrix in a very thin layer. This criterion for debonding based upon the shear-lag model ignores the effect of the interface tensile stress. This simplification is reasonable for the growth-dominated fatigue failure since the shear stress contributes to its growth and propagation along the fiber length. However, the interface tensile stress component could be important for the cause of the initiation of fiber/matrix debonding. Therefore, this simplification would predict a delayed debonding, leading to an overestimated fatigue lifetime for the initiation-dominated fatigue failure (high load). Although the composite stress range and the temperature range for both the in-phase and out-of-phase TMF loading conditions do not change with cycles, the local plastic shear strain ranges will change, due to new debonding, after some number of cycles. Therefore, one needs a damage accumulation fatigue rule to describe the debonding due to local varying 
amplitude fatigue. For simplicity, we assume that the fatigue life for interface debonding is governed by the linear damage accumulation rule $\sum N_{i} /\left(N_{\mathrm{f}}\right)_{i}=1$, where $N_{\mathrm{i}}$ is the number of the applied loading cycle leading to the constant plastic strain range $\left(\Delta \gamma_{\mathrm{p}}\right)_{i}$ and $\left(N_{\mathrm{f}}\right)_{i}$ is the cycles to interface debonding for the constant shear plastic strain range $\left(\Delta \gamma_{\mathrm{p}}\right)$ i. When the sum of the fractions from each step equals one, debonding is predicted (Liu, 2001)

\subsection{Stress distribution due to debonding}

After interfacial debonding, there can remain a residual shear sliding resistance across the fiber/matrix interface due to friction. For tractability, an assumption is that a constant frictional shear force, $\tau$, governed by Coulomb's law, exists within the debonded interface of the length, $L \mathrm{~d}$. In new loading phase after debonding, the interfacial shear stress within the interface debonding length and the shear yield zone has to be equal to

$$
\tau\left(k_{n}, \xi\right)=\left\{\begin{array}{cc}
\tau_{f} & 0 \leq\left|\xi-\xi_{\mathrm{n}}\right| \leq L_{d} / l_{c} \\
\tau_{s} & L_{d} / l_{c} \leq\left|\xi-\xi_{\mathrm{n}}\right| \leq\left(L_{n}-L_{d}\right) / l_{c}
\end{array} \quad, \text { for } n=1,2,3, \ldots, N^{\prime}\right.
$$

where $N$ denotes the number of broken fibers which are accompanied by interface debonding and yielding. According to the continuity conditions between debonding and yield segments $\left.\sigma^{f}\right|_{z=L_{d}^{-}}=\left.\sigma^{f}\right|_{z=L_{d}^{+}}$, and the equilibrium condition for the fiber, the fiber stress within these two length has the form

$$
\sigma^{f}\left(k_{n}, \xi\right)=\left\{\begin{array}{cc}
2 \tau_{f} \cdot\left|l_{c}\left(\xi-\xi_{n}\right)\right| / D & 0 \leq\left|\xi-\xi_{n}\right| \leq L_{d} / l_{c} \\
2\left(\tau_{s} \cdot\left|l_{c}\left(\xi-\xi_{n}\right)\right|+L_{d}\left(\tau_{f}-\tau_{s}\right)\right) / D & L_{d} / l_{c} \leq\left|\xi-\xi_{n}\right| \leq\left(L_{n}-L_{d}\right) / l_{c}
\end{array}, \text { for } n=1,2,3, \ldots, N^{\prime}\right.
$$

In subsequent TMF unloading, the reverse shear plasticity may occur in $N^{\prime}$ lengths $2 L^{\prime} \mathrm{n}(n=$ $\left.1,2,3, \ldots, N^{\prime}\right)$. In this case the interface shear stress is given by

$$
\tau\left(k_{j}, \xi\right)=\left\{\begin{array}{cc}
-\tau_{f} & 0 \leq\left|\xi-\xi_{j}\right| \leq L_{d} / l_{c} \\
-\tau_{s} & L_{d} / l_{c} \leq\left|\xi-\xi_{j}\right| \leq\left(L_{j}^{\prime}-L_{d}\right) / l_{c}
\end{array} \quad \text { for } j=1,2,3, \ldots, N^{\prime}\right.
$$

It follows for the fiber stress,

$$
\sigma^{f}\left(k_{j}, \xi\right)=\left\{\begin{array}{cc}
-2 \tau_{f} \cdot\left|l_{c}\left(\xi-\xi_{j}\right)\right| / D & 0 \leq\left|\xi-\xi_{j}\right| \leq L_{d} / l_{c} \\
2\left(-\tau_{s} \cdot\left|l_{c}\left(\xi-\xi_{j}\right)\right|+L_{d}\left(\tau_{s}-\tau_{f}\right)\right) / D & L_{d} / l_{c} \leq\left|\xi-\xi_{j}\right| \leq\left(L_{j}^{\prime}-L_{d}\right) / l_{c}
\end{array} \quad \text { for } j=1,2,3, \ldots, N^{\prime}\right.
$$

The stresses in composite with debonding can be obtained again by using the developed superposition method along with eqs. (22-25).

\section{Statistical modelling}

The catastrophic failure of fiber-reinforced composites is primarily dominated by the failure of fibers (Talrejia, 1995). The fibers typically exhibit variability in strength due to microflaws 
distributed randomly along the length. The variation of the fiber strength $\sigma_{\mathrm{f}}$ for length $L$ can be characterized by the probability density function, $F\left(\sigma_{\mathrm{f}}\right)$, which is assumed to follow a two-parameter Weibull distribution, that is,

$$
F\left(\sigma_{f}\right)=1-\exp \left\{-\left(\frac{L}{L_{0}}\right)\left(\frac{\sigma_{f}}{\sigma_{0}}\right)^{\beta}\right\},
$$

where $\sigma_{0}$ represents the scale parameter, or characteristic quantity of the material. $L_{0}$ is the reference length when the characteristic fiber strength $\sigma_{0}$ is measured. The Weibull modulus or shape parameter $\beta$ controls the scatter of the fiber tensile strength in the distribution, experimentally found to describe a variety of materials. This scatter will become large with decreasing $\beta$. The parameters $\beta$ and $\sigma_{0}$ can be calculated by statistical method

$$
\begin{gathered}
\bar{\sigma}_{c}=E\left(\sigma_{c}\right)=\sigma_{0} \Gamma\left(1+\frac{1}{\beta}\right) \\
S^{2}=D\left(\sigma_{c}\right)=\sigma_{0}^{2}\left\{\Gamma\left(1+\frac{2}{\beta}\right)-\left[\Gamma\left(1+\frac{1}{\beta}\right)\right]^{2}\right\},
\end{gathered}
$$

where $E\left(\sigma_{c}\right)$ and $D\left(\sigma_{c}\right)$ are the mean and variance of random variable, respectively.

In the approach we use here, we assume the total length of composite specimen to be modeled, $2 L_{\mathrm{T}}$, is divided into $N_{\mathrm{L}}$ segments of equal length $\Delta x$ such that $\Delta x=2 L_{\mathrm{T}} / N_{\mathrm{L}}$. The composite specimen to be modeled contains $N_{\mathrm{f}}$ fibers. For a given failure probability $F$ the strength of the fiber segments with length $\Delta x$ can be derived from the inversion of eq. (26)

$$
\sigma_{f}=\sigma_{0}\left\{\left(\frac{L_{0}}{\Delta x}\right) \ln \left(\frac{1}{1-F}\right)\right\}^{1 / \beta},
$$

in which the failure probability $F$ is a random number taken from the uniform distribution in the range $[0,1]$, then the strength of each fiber segment can be obtained. By introducing the normalized fiber segment length $\hat{L}=\Delta x / D$, eq. (29) can be written as

$$
\sigma_{f}=\sigma^{*}\left(\frac{1}{\hat{L}}\right)^{1 / \beta}\left[\ln \left(\frac{1}{1-F}\right)\right]^{1 / \beta},
$$

where $\sigma^{*}$ acts as the scale parameter and $\sigma^{*}=\sigma_{0} \cdot\left(L_{0} / D\right)^{1 / \beta}$.

The Monte-Carlo simulation technique coupled with the proposed analytical model is executed to simulate the mechanical failure process in fiber-reinforced composites under TMF loads. At the beginning of each simulation, all fiber segments are assumed to be intact. Their status changes from intact to fractured if the fiber stresses satisfy the failure criteria. To employ the overall method described above, the algorithm is follows, 
a. Randomly assign each fiber element a strength according to a two-parameter Weibull distribution.

b. From the displacement and the boundary condition, the stress for each segment of fiber, matrix, and interface are then obtained by using the stress analysis.

c. Determine whether the fiber elements will break up or not, whether the matrix and interface around broken fibers will yield or not for each incremental load. If no new damage occurs at this loading step, we calculate the composite stresses. Otherwise, we recalculate the stress field by taking account of the new breakage, and repeat this step until no more damage occurs at the load level.

d. Taking unloading. For the case except of complete elastic unloading, the cyclic plastic strain range along with Coffin-Manson equation is be used to predict the debonding

e. Increase a new loading by a small increment and repeat step (b) and (c) and (d), until the stress-strain curve up to failure for the composite is obtained.

For each loading step at which the applied composite strain is given the overall composite stress can be evaluated by

$$
\sigma_{\text {comp }}=V_{f} \tilde{\sigma}_{f}+\left(1-V_{f}\right) \tilde{\sigma}_{m}
$$

where $V_{f}$ denotes the fiber volume fraction; the symbols $\tilde{\sigma}_{f}$ and $\tilde{\sigma}_{m}$ are used for the average fiber and matrix stress, respectively.

The average fiber stress is computed by

$$
\tilde{\sigma}_{f}=\frac{1}{2 N_{f} L_{T}} \sum_{k=1}^{N_{f}}\left[\int_{-L_{T} / l_{c}}^{L_{T} / l_{c}} \sigma_{f}(k, \xi) d \xi\right],
$$

and the average matrix stress is evaluated by

$$
\tilde{\sigma}_{m}=\frac{1}{2 N_{m} L_{T}} \sum_{k=1}^{N_{m}}\left[\int_{-L_{T} / l_{c}}^{L_{T} / l_{c}} \sigma_{m}(k, \xi) d \xi\right],
$$

The fatigue failure of the fiber reinforced ductile composites occurs as a result of accumulation of the large amounts of damage-plasticity events under cyclically thermomechanical loading. As much more fiber breaks and the associated local thermoplasticity are accumulated, the composite as a whole will be unable to carry additional load and fail will ensue.

\section{Predictions and concluding remarks}

For illustrations, the Boron/Al continuous fiber reinforced composite is examined. The properties of the constituents are given below

$$
\begin{array}{r}
E_{\mathrm{f}}=400 \mathrm{GPa}, E_{\mathrm{m}}=70.2 \mathrm{GPa}, \alpha_{f}=6.3 \mu \varepsilon /{ }^{\circ} \mathrm{C}, \quad \alpha_{m}=23.9 \mu \varepsilon /{ }^{\circ} \mathrm{C}, \\
D=0.14 \mathrm{~mm}, V_{\mathrm{f}}=0.48, \quad c=-0.65, \quad \gamma_{\mathrm{f}}=0.42
\end{array}
$$


Fig. 4. shows a simulated failure process of the fiber-reinforced composite under in-phase condition. In this figure, $4 \mathrm{a}-\mathrm{f}$ indicate the damage configurations under some instantaneous stage, respectively. Because of large variability for fiber strength, the fiber element with the lowest strength is firstly broken at the early loading stage, see Fig. 4a. The high stress concentrations are generated in the matrix or the interface due to the fiber breakages, so that plastic yield appears for matrix or interface. As the applied load increases, more fiber breakages occur in the whole specimen, see Fig. $4 \mathrm{~b}$-c. Stress redistribution in the composite is caused by debonding after some cycles, shown in Figure $4 \mathrm{~d}$. Accumulation of severe damages can be observed until the composite completely fails, in Fig. 4e-f.

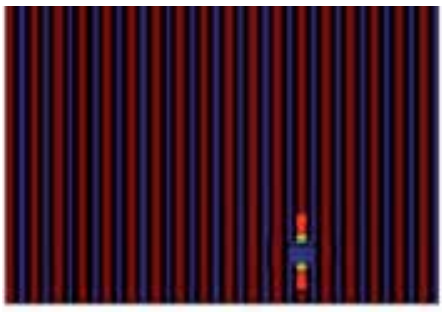

(a)

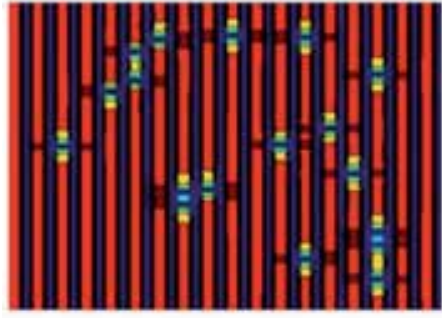

(c)

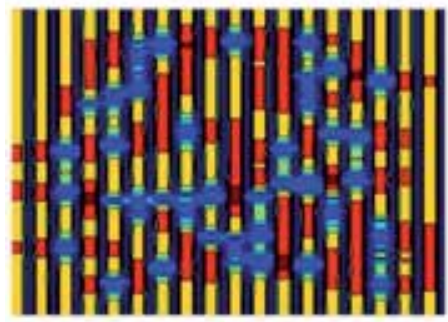

(e)

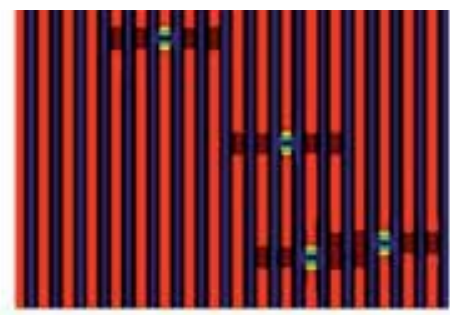

(b)

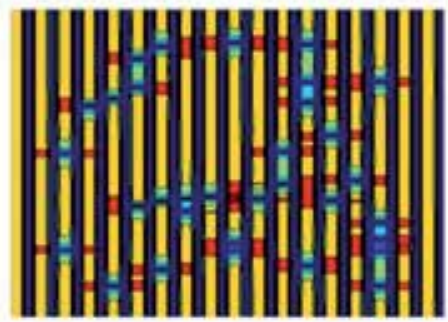

(d)

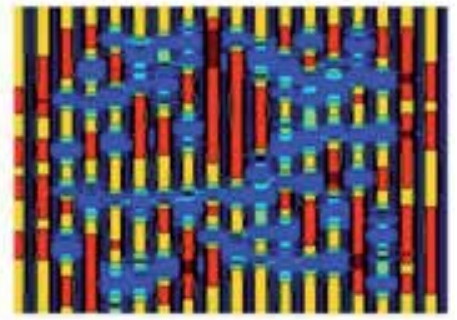

(f)

Figure 4. Progressive damage of advanced fiber composites.

Fig. 5. illustrates the cyclic stress/strain response corresponding to the example in Fig. 4. Plastic yield onset in unloading and reloading is represented by the ' + ' symbols. Due to plastic deformation, the curve deviates from linear behavior beyond the elastic range at unloading and reloading state. It is distinctly seen that the composite strain accumulates with cycles under the constant stress amplitude. This attributes to the microdamage 
mechanism that a dominant 'critical cluster' of breaks is shaped, which leads to the failure of the rest of fibers.

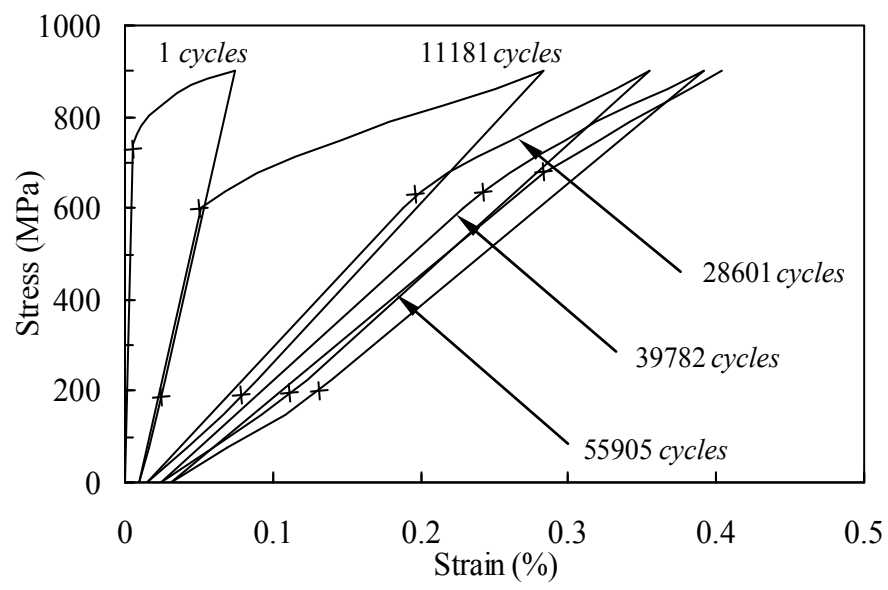

Figure 5. Predicted cyclic nonlinear stress-strain response.

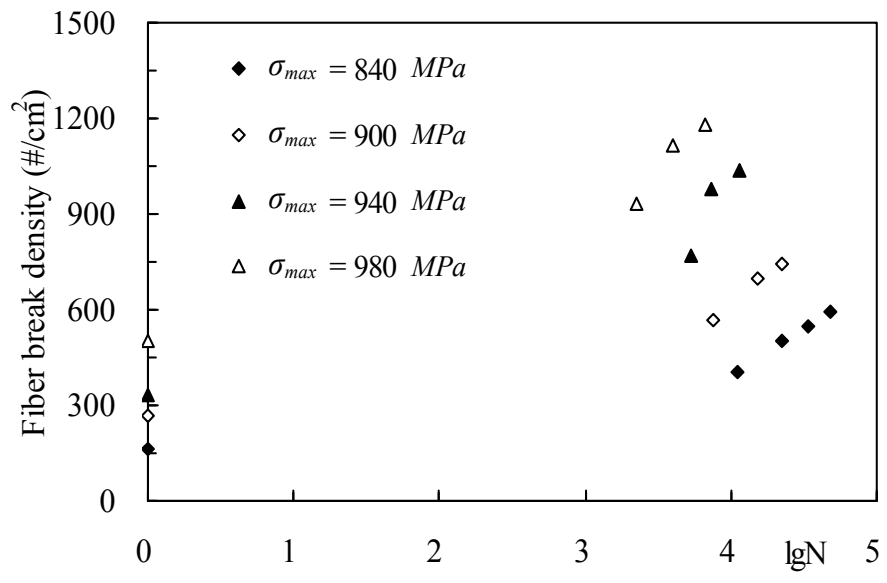

Figure 6. Evolution of fiber breakage with cycles under different load amplitude.

In order to show accumulation of damage-plasticity in the composite, Figs. 6-8. illustrate the evolution of fiber break density, matrix yield density, and the total debonding length with TMF cycles, respectively. It is obvious that the deformation behavior is dependent on the load amplitude: the degree of damage exhibits a more aggravation with cycle times under higher amplitude.

Fig. 9. shows the evolution of fiber cracking in the composites during reloading. Initial breaking takes place at about $750 \mathrm{MPa}$ in the first cycles. The density of broken fiber increases as the number of load cycles increases. It should be emphasized that this is due to the stress redistribution induced by progressive debonding. 
Fig. 10. is a plot of fatigue life by logarithm coordinate against maximum stress. The predicted S-N curves for the in-phase and out-of-phase TMF loads with a temperature range from 250 to $350{ }^{\circ} \mathrm{C}$ are plotted along with the experimental S-N curves tested by others (Nicholas, 1995; Liu \& He, 2001).

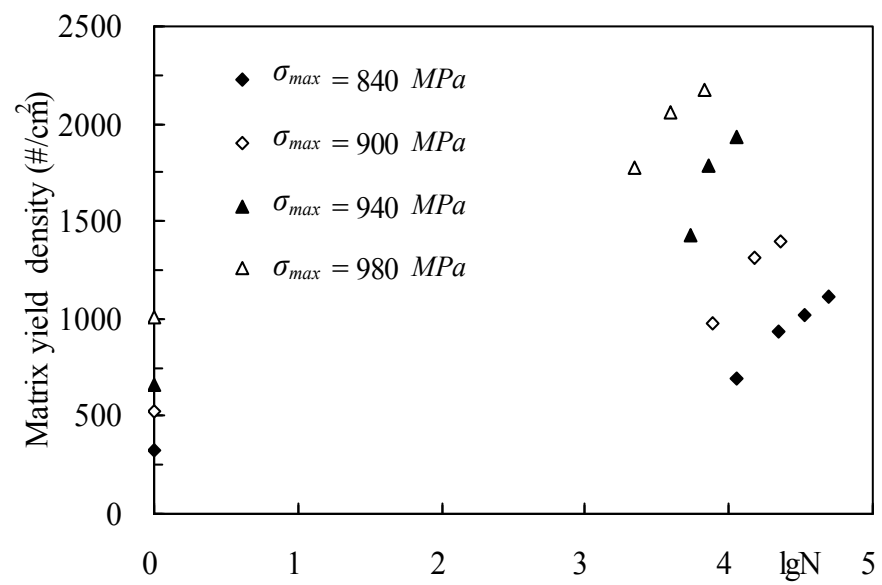

Figure 7. Evolution of matrix tensile yielding with cycles under different load amplitude.

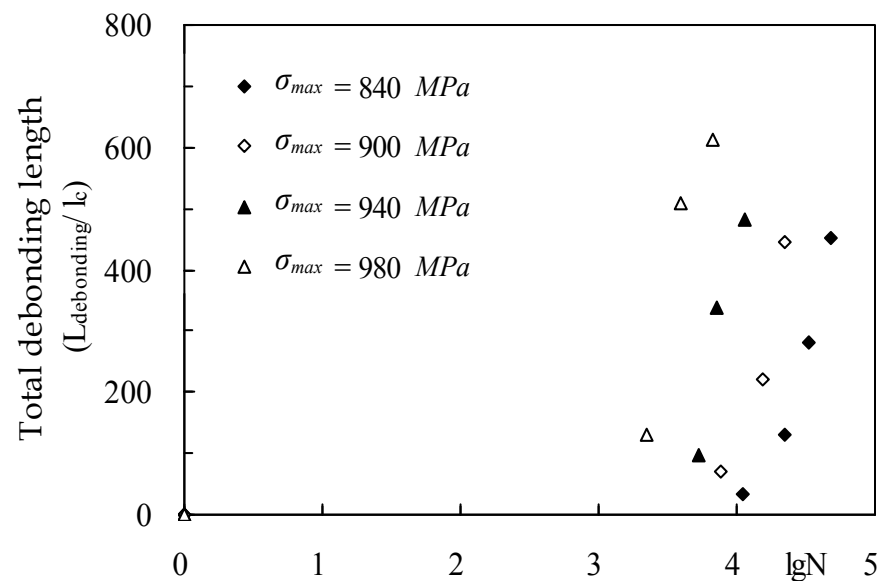

Figure 8. Evolution of debonding with cycles under different load amplitude.

In high stress level, the fatigue life for in-phase TMF conditions is considerably shorter than the life for the out-of-phase TMF conditions. On the other hand, in low stress level, the fatigue life for in-phase conditions is seen to be longer considerably than the life for out-ofphase conditions. This cross-over behavior is due to the difference of the dominated failure mode for this two TMF conditions. A micro-mechanism is presented in Figs. 11-12. Micro failure mechanism associated with the in-phase TMF is characterized by a fiber-dominated fracture with evidence of smaller matrix yielding. Evidence of larger matrix plasticity and less fiber fracture, suggestive of a matrix-dominated failure under the out-of-phase TMF, has been observed in the model simulation. 


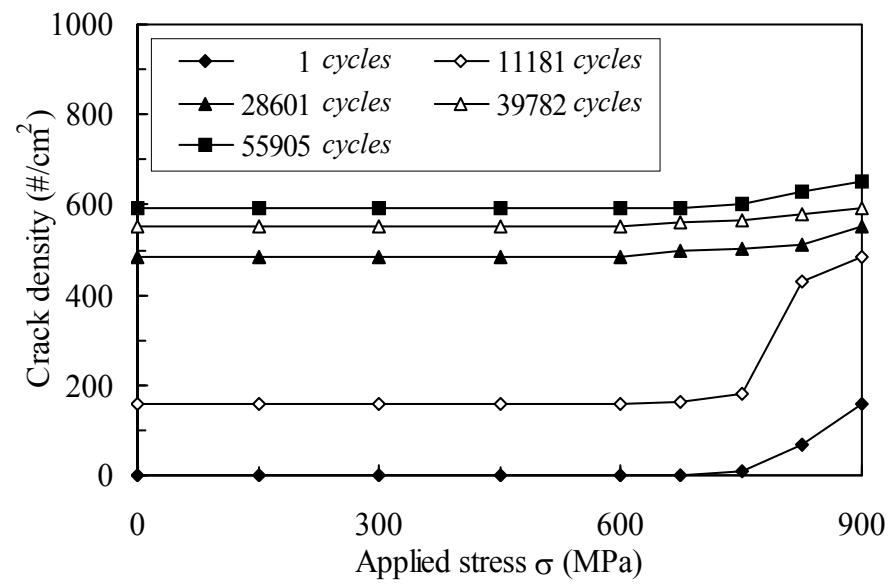

Figure 9. Evolution of fiber cracking in the composites during reloading.

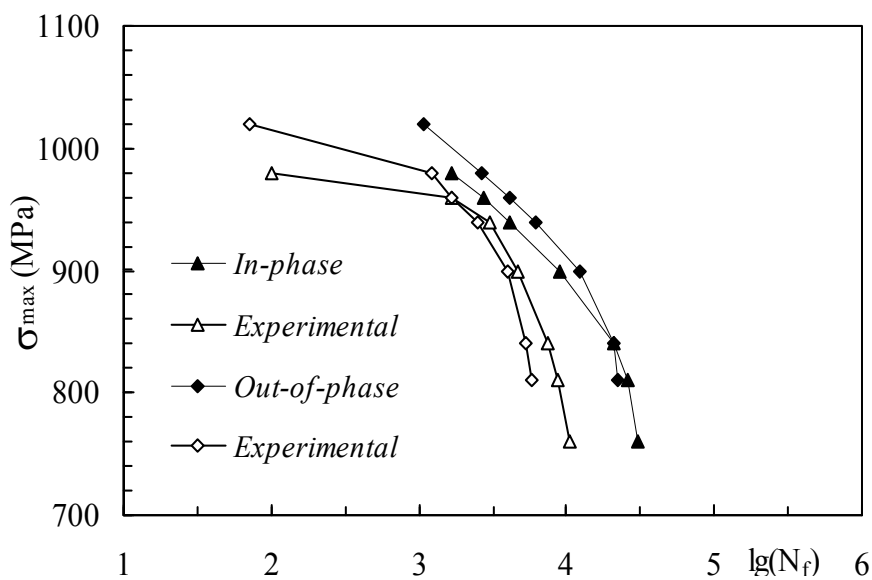

Figure 10. Comparison of model predictions with experimental results for TMF loads.
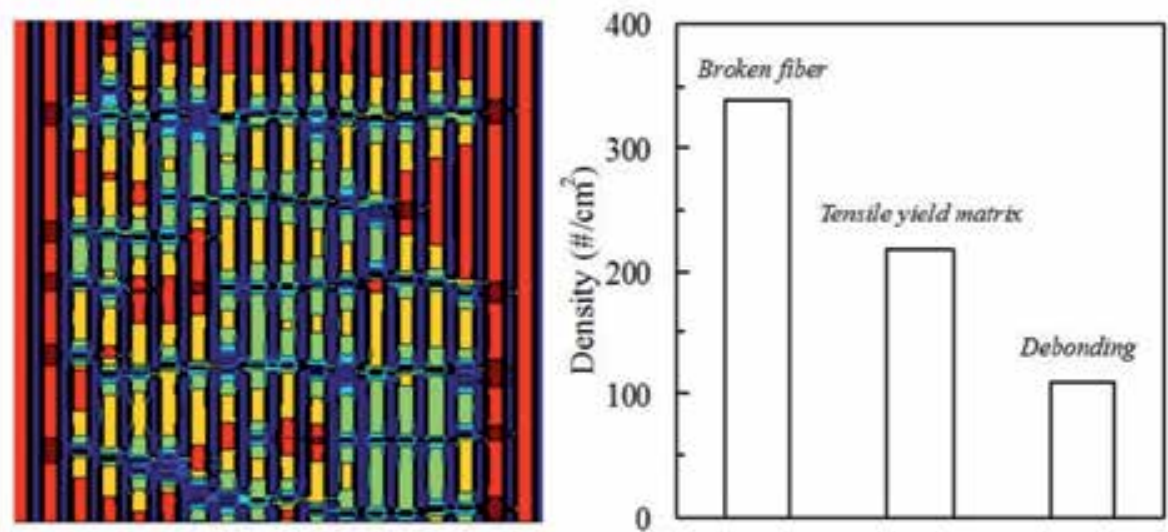

Figure 11. Damage-plasticity configuration when the composite completely fails under in-phase condition. 

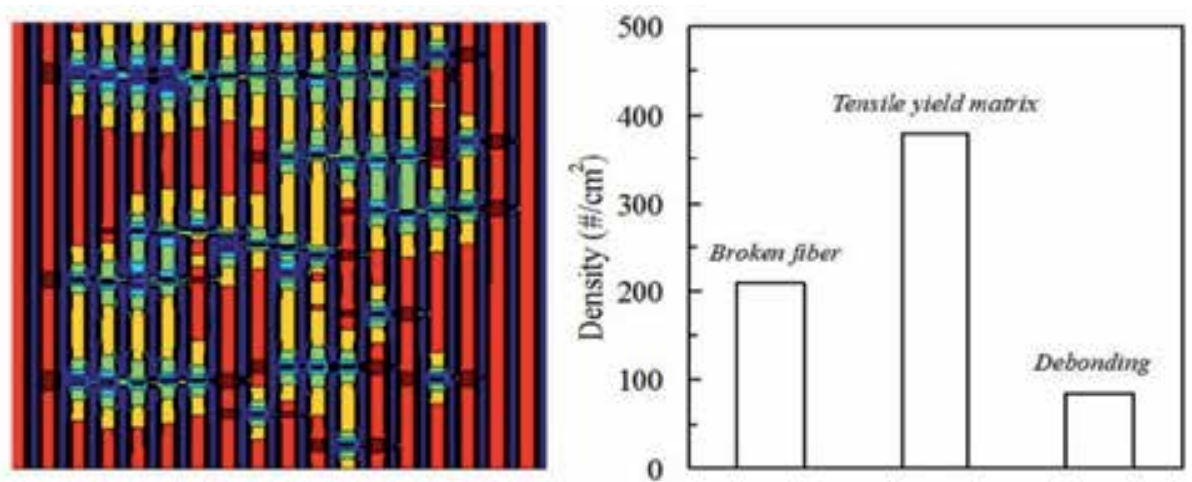

Figure 12. Damage-plasticity configuration when the composite completely fails under out-of-phase condition.

\section{Conclusions}

Modelling of the mechanical behavior of fibrous composite under progressive damages attracts much attention for a long time. The methodology developed in this paper provides an engineering tool to investigate damage evolution in fiber-reinforced composites, especially under cyclical thermomechanical loading. It has to be point out that we do not intend to make the quantitative comparison of the model with the experimental data. The predicted lifetime of fibrous composite is substantially higher than the measured, especially for the regime of high load level. There are several factors that may cause the quantitative discrepancy between the predicted and measured fatigue life. First, the model ignored the interface normal stress that dominates the initiation of debonding, leading to an estimate of delayed debonding and a longer lifetime of the composite. Second, the interface properties have been assumed to be the same as those of matrix; however, interfaces may contain inherent flaws or defects. This assumption will result in a longer lifetime of debonding growth.

As a final remark, it should be mentioned that the 2D modeling presented here does generalize the governing equations to include interactions with multiple damage events and develop the basic mechanics that are necessary to understanding failure mode of the composite produced by the failure of one or more of its components from a micromechanics perspective. The difference between predicted values and measured ones suggests, however, that consideration of the effect of variations in fiber strengths alone is not sufficient for predicting the variability of composite strength. A more precise calibration of the model, capable of explaining such effects as 3D fiber arrays and fiber/matrix interface sliding on composite strength, is the subject of our further study.

\section{Author details}

\section{F. Wang}

Southwest University, China

J.Q. Zhang

Shanghai University, China

Shanghai Key Laboratory of Mechanics in Energy Engineering, China 


\section{Acknowledgement}

The chapter was written with the financial support of the National Science Foundation of China under Grant No.11172159, 11102169, 10772105 and 10372120, and Natural Science Foundation Project of CQ CSTC, 2009BB4290.

\section{References}

Beyerlein, I.J. \& Landis, C.M. (1999). Shear-lag model for failure simulations of unidirectional fiber composites including matrix stiffness. Mechanics of Materials, Vol.31, No.5, (April, 1999), pp. 331-350, ISSN 0167-6636.

Beyerlein, I.J. \& Phoenix, S.L. (1996). Stress concentrations around multiple fiber breaks in an elastic matrix with local yielding or de-bonding using quadratic influence superposition, Journal of the Mechanics and Physics of Solids, Vol.44, No.12, (August, 1996), pp. 1997-2039, ISSN 0022-5096.

Blassiau, S., Thionnet, A. \& Bunsell, A.R. (2008). Micromechanisms of load transfer in a unidirectional carbon fiber-reinforced epoxy composite due to fiber failures: part 3. Multiscale reconstruction of composite behavior, Composite Structures, Vol.83, No.3, (May, 2007), pp. 312-323, ISSN 0263-8223.

Bussiba, A., Kupiec, M., Ifergane, S., Piat, R. \& Böhlke, T. (2008). Damage evolution and fracture events sequence in various composites by acoustic emission technique, Composites Science and Technology, Vol.68, No.5, (August, 2007), pp. 1144-1155, ISSN 0266-3538.

Curtin, W.A. (1999). Stochastic damage evolution and failure in fiber-reinforced composites, In: Advances in Applied Mechanics, E.V.D. Giessen \& T.Y. Wu, (Eds.), pp. 163-253, Academic Press, ISBN 978-012-0020-36-2, San Diego, USA.

González, C., Segurado, J. \& LLorca J. (2004). Numerical simulation of elasto-plastic deformation of composites: evolution of stress microfields and implications for homogenization model, Journal of the Mechanics and Physics of Solids, Vol.52, No.7, (January, 2004), pp. 1573-1593, ISSN 0022-5096.

Hanan, J.C., Üstündag, E., Beyerlein, I.J., Swift, G.A., Almer, J.D., Lienert, U. \& Haeffner, D.R. (2003). Microscale damage evolution and stress redistribution in Ti-SiC fiber composites, Acta Materialia, Vol.51, No.14, (April, 2003), pp. 4239-4250, ISSN 1359-6454.

Hanan, J.C., Mahesh, S., Üstündag, E., Beyerlein, I.J., Swift, G.A., Clausen, B., Brown, D.W. \& Bourke, M.A.M. (2005). Strain evolution after fiber failure in a single-fiber metal matrix composite under cyclic loading, Materials Science and Engineering A, Vol.399, No.(1-2), (February, 2005), pp. 33-42, ISSN 0921-5093.

Kabir, M.R., Lutz, W., Zhu, K. \& Schmauder, S. (2006). Fatigue modeling of short fiber reinforced composites with ductile matrix under cyclic loading. Computational Materials Science, Vol.36, No.4, (September, 2005), pp. 361-366, ISSN 0927-0256.

Kruch, S., Carrere, N. \& Chaboche, J.L. (2006). Fatigue damage analysis of unidirectional metal matrix composites, International Journal of Fatigue, Vol.28, No.10, (February, 2006), pp. 1420-1425, ISSN 0142-1123. 
Landis, C.M., Beyerlein, I.J. \& McMeeking, R.M. (2000). Micromechanical simulation of the failure of fiber reinforced composites. Journal of the Mechanics and Physics of Solids, Vol.48, No.3, (January, 2000), pp. 621-648, ISSN 0022-5096.

Liu, S.L. \& He, Y.H. (2001). Thermomechanical fatigue behavior of a unidirectional B/Al metal matrix composite, Proceedings of 13th International Conference on Composite Materials, pp. 326, ISBN 7-6023-3825-X, Beijing, CHINA, June 25-29, 2001.

Liu, Y.F. (2001). A 3-d micromechanical model of cyclic plasticity in a fiber-reinforced metal matrix composite. Journal of Materials Science Letters, Vol.20, No.5, (October, 2000), pp. 415-417, ISSN 0261-8028.

Llorca, J. (2002). Fatigue of particle- and whisker-reinforced metal-matrix composites. Progress in Materials Science, Vol.47, No.3, (June, 2000), pp. 283-353, ISSN 0079-6425.

Mall, S. \& Schubbe, J.J. (1994). Thermo-mechanical fatigue behavior of a cross ply SCS-6/Ti15-3 metal matrix composite. Composites Science and Technology, Vol.50, No.1, (February, 1993), pp. 49-57, ISSN 0266-3538.

Miracle, D.B. (2005). Metal matrix composites-From science to technological significance. Composites Science and Technology, Vol.65, No.(15-16), (May, 2005), pp. 2526-2540, ISSN 0266-3538.

Mishnaevsky, L. \& Brøndsted, P. (2008). Three-dimensional numerical modelling of damage initiation in unidirectional fiber-reinforced composites with ductile matrix. Materials Science and Engineering A, Vol.498, No.(1-2), (September, 2007), pp. 81-86, ISSN 0921-5093.

Nicholas, T. (1995). An approach to fatigue life modeling in titanium-matrix composites. Materials Science and Engineering A, Vol.200, No.(1-2), (December, 2004), pp. 29-37, ISSN 0921-5093.

Talrejia, R. (1995). A conceptual frame work for interpretation of MMC fatigue. Materials Science and Engineering A, Vol.200, No.(1-2), (December, 2004), pp. 21-28, ISSN 0921-5093.

Williams, T.O. \& Pindera, M.J. (1995). Thermo-mechanical fatigue modeling of advanced metal matrix composites in the presence of microstructural details. Materials Science and Engineering A, Vol.200, No.(1-2), (May, 1995), pp. 156-172, ISSN 0921-5093.

Xia, Z., Curtin, W.A. \& Peters, P.W.M. (2001). Multiscale modeling of failure in metal matrix composites. Acta Materialia, Vol.49, No.2, (January, 2001), pp. 273-287, ISSN 1359-6454.

Xia, Z., Okabe, T. \& Curtin, W.A. (2002). Shear-lag versus finite element models for stress transfer in fiber-reinforced composites. Composites Science and Technology, Vol.62, No.9, (October, 2001), pp. 1141-1149, ISSN 0266-3538.

Zhang, F., Lisle, T., Curtin, W.A. \& Xia, Z. (2009). Multiscale modeling of ductile-fiberreinforced composites. Composites Science and Technology, Vol.69, No.(11-12), (April, 2009), pp. 1887-1895, ISSN 0266-3538.

Zhang, J.Q., Wu, J. \& Liu, S.L. (2002). Cyclically thermomechanical plasticity analysis for a broken fiber in ductile matrix composites using shear lag model. Composites Science and Technology, Vol.62, No.5, (February, 2002), pp. 641-654, ISSN 0266-3538.

Zhang, J.Q. \& Wang, F. (2009). Modeling of progressive failure in ductile matrix composites including local matrix yielding. Mechanics of Advanced Materials and Structures, Vol.16, No.7, (March, 2009), pp. 522-535, ISSN 1537-6494.

Zhang, J.Q. \& Wang, F. (2010). Modeling of damage evolution and failure in fiber reinforced ductile composites under themomechanical fatigue loading. International Journal of Damage Mechanics, Vol.19, No.7, (January, 2010), pp. 851-875, ISSN 1056-7895. 


\title{
Molecular Simulations on Interfacial Sliding of Carbon Nanotube Reinforced Alumina Composites
}

\author{
Yuan Li, Sen Liu, Ning Hu, Weifeng Yuan and Bin Gu \\ Additional information is available at the end of the chapter
}

http://dx.doi.org/10.5772/48816

\section{Introduction}

With remarkable physical and mechanical properties [1, 2], carbon nanotube (CNT), either single-walled carbon nanotube (SWCNT) or multi-walled carbon nanotube (MWCNT), has prompted great interest in its usage as one of the most promising reinforcements in various matrices (e.g., polymers, metals and ceramics) [3-11]. However, the dramatic improvement in mechanical properties has not been achieved so far. The reason can be attributed to several critical issues: (1) insufficient length and quality of CNT, (2) poor CNT dispersion and alignment, and (3) weak interface between CNT and matrix. Although great progress has been made to improve the first two issues by developing newly cost-effective CNT synthesis methods and exploring specific fabrication methods of composites (e.g., spark plasma sintering [12], sol-gel process [13]), the proper control of interfacial properties is still a challenge as the inherent characteristics is unclear.

Up to date, large amounts of investigations have been focused on the interfacial properties of polymer-based composites by using direct pull-out experiments with the assistance of advanced instruments (e.g., transmission electronic microscopy (TEM) [14,15], atom force microscope (AFM) [16,17], Raman spectroscopy [18], scanning probe microscope (SPM) [19]), or theoretical analysis based on continuum mechanics (e.g., cohesive zone model [20], Cox's model [21], shear lag model [22,23] and pull-out model [24,25]), or atomic simulations [26-33]. However, in contrast, much less work has been focused on the interfacial properties of alumina-based composites [34-38]. For example, it has been reported that there are three hallmarks of toughening behavior demonstrated in CNT-reinforced alumina composites (CNT/Alumina) as below [34]: crack deflection at the CNT/Alumina interface; crack bridging by CNT, and CNT pull-out on the crack plane, which is consistent with that in conventional 
micron-scale fiber reinforced composites. Therefore, a fundamental understanding on the interfacial sliding between CNT and alumina matrix (i.e., CNT pull-out from alumina matrix) is important for clarifying the interfacial properties, and therefore the mechanical properties of bulk CNT/Alumina composites.

Current experimental works have reported two common sliding behaviors in CNT/Alumina composites: the pull-out of SWCNT [35] and sword-in-sheath mode [36, 37] of MWCNT (i.e. "pull-out of the broken outer walls of CNT with matrix", or "pull-out of inner walls of CNT with matrix after the breakage of the outer walls" in relativity). Therefore, clarifying the above two distinguished pull-out behaviors is of critical importance for understanding the interfacial properties of CNT-reinforced composites.

In this Chapter, a series of pull-out simulations of either SWCNT or MWCNT from alumina matrix are carried out based on molecular mechanics (MM) to investigate the corresponding interfacial sliding behaviors in CNT/Alumina composites. By systematically evaluating the variation of potential energy increment during the pull-out process, the effects of grain boundary (GB) structures of alumina matrix, nanotube length, nanotube diameter, wall number and capped structure of CNTs are explored for the first time.

\section{Computational model}

As experimentally identified, CNTs are generally located in the GB of alumina [34-37, 39], which can be schematically illustrated in Fig. 1. Note that the GB structure is generally characterized by a multiplicity index $\Sigma$ based on the geometrical concept of threedimensional (3D) coincidence between two crystals named the coincidence site lattice (CSL) model [40], which is defined as the ratio of the crystal lattice sites density to the density of the two grain superimposed lattices.The corresponding computational model by using the commercial software of Materials Studio (Accelrys) can be constructed as follows:

1. Building a hexagonal primitive cell of neutral alumina;

2. Cleaving the required GB planes and joining them together;

3. Inserting a CNT into the GB;

4. Relaxing the constructed model to obtain the equilibrated configuration.

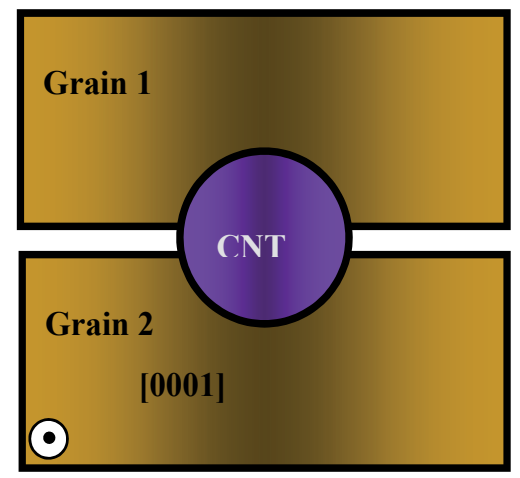

Figure 1. Schematic GB with CNT 


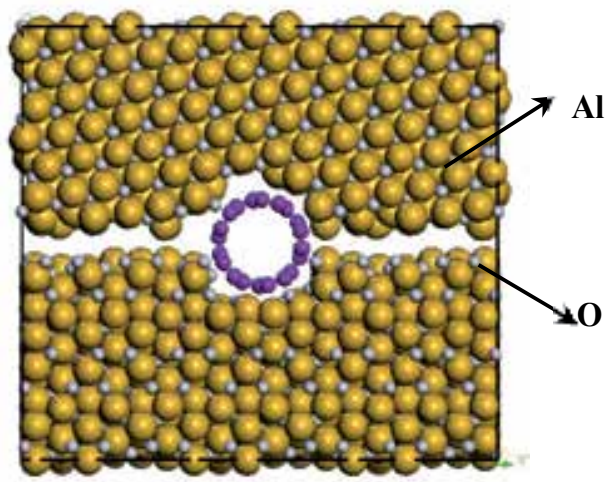

Figure 2. Simulation cell of GB with CNT

As an example, the equilibrated model of $\Sigma 7(14 \overline{5} 0) / /(41 \overline{5} 0)$ [41] GB is shown in Fig. 2, in which the inserted open-ended SWCNT $(5,5)$ has the length of $l=5.17 \mathrm{~nm}$ and diameter of $D=0.68 \mathrm{~nm}$.

The pull-out process of CNT is schematically given in Fig.3, which is mainly divided into the following two steps:

1. Applying the fixed boundary conditions to the left end of alumina matrix;

2. Pulling out the CNT gradually along its axial (x-axis) direction with a constant displacement increment $\Delta x$ of $0.2 \mathrm{~nm}$.

After each pull-out step, the structure should be relaxed in order to obtain the minimum systematic potential energy $E$.
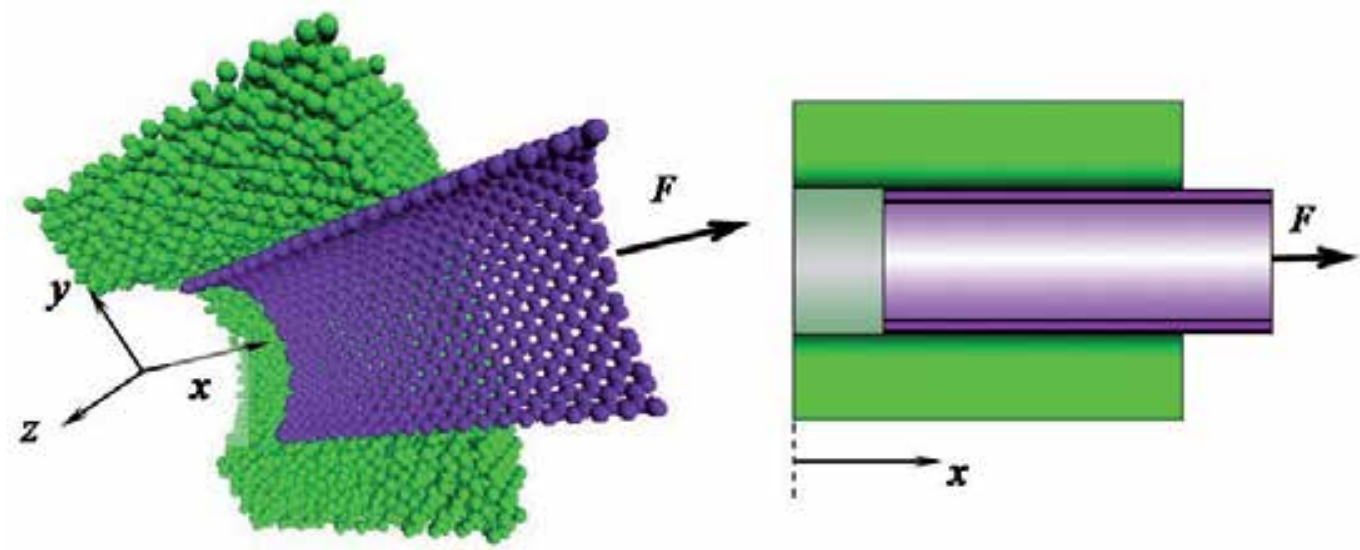

Figure 3. Schematic pull-out process of CNT from alumina matrix (green balls: atoms in alumina matrix, purple balls: atoms of CNT) 


\section{Pull-out simulations of open-ended SWCNTs from alumina matrix}

\subsection{Effect of GB structure}

To a large extent, GBs play a significant role on the microstructure formation and properties control of polycrystalline materials. To explore the influence of GB structure on the interfacial siding behavior between CNT and alumina matrix, three representative GB structures with a common rotation axis of [0001] ( $\Sigma 3(11 \overline{2} 0) / /(11 \overline{2} 0)[42], \Sigma 7(14 \overline{5} 0) / /(41 \overline{5} 0)$ $[41,43,44]$, and $\Sigma 31(47 \overline{11} 0) / /(74 \overline{11} 0)[44])$ are modeled. Note that the same fragment of $\operatorname{SWCNT}(5,5)$ with the length of $l=5.17 \mathrm{~nm}$ and diameter of $D=0.68 \mathrm{~nm}$ is employed.

The obtained variations of energy increment $\Delta E$ between two consecutive pull-out steps are plotted in Fig. 4, where three distinct stages can be clearly seen for each case. In the initial ascent stage $I, \Delta E$ increases sharply until the pull-out displacement $x$ reaches up to about $1.0 \mathrm{~nm}$. After that $\Delta E$ undergoes a long platform stage $I I$ followed by the quick descent stage III until the complete pull-out. It is noticeable that both stages $I$ and III have the same range corresponding to the pull-out displacement of approximately $a=1.0 \mathrm{~nm}$, which is very close to the cut-off distance of vdW interaction (i.e., $0.95 \mathrm{~nm}$ ). This feature of $\Delta E$ is similar to that for the pull-out process of CNT from polymer matrix [33] and that for the sliding among nested walls in a MWCNT [45].

Moreover, $\Delta E$ in these three curves are almost identical in stages $I$ and $I I I$, and have the same average value at stage $I I$ although $\Sigma 7 \mathrm{~GB}$ results in a slightly higher $\Delta E$. This suggests that the GB structure of alumina matrix has only a limited effect on the energy increment $\Delta E$ between two adjacent pull-out steps.

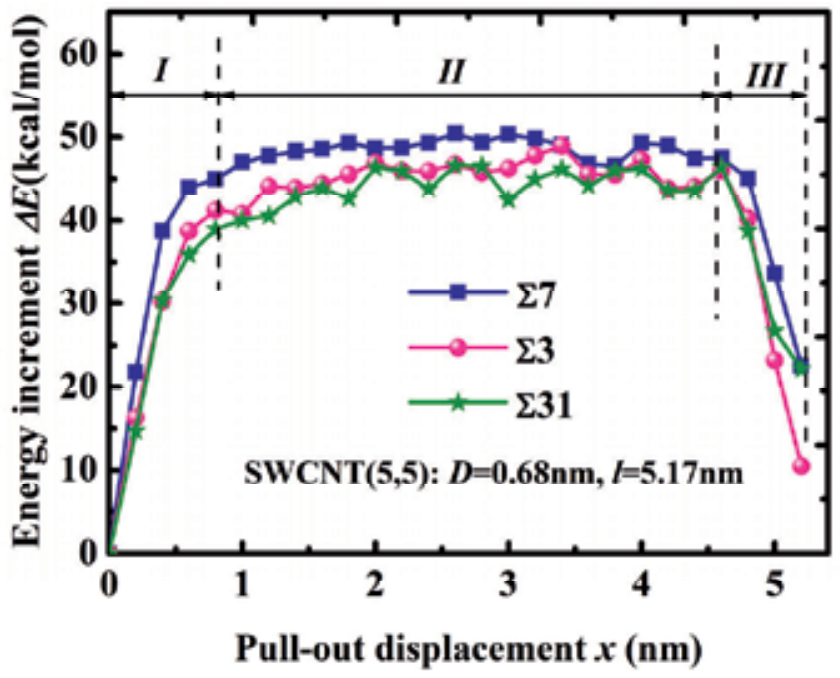

Figure 4. Effect of GB structure on the variation of energy increment during the pull-out process

Here, as discussed in Refs. $[33,45]$, the stable pull-out stage II is focused on, in which the average energy increment in stage $I I$ is referred to as $\Delta E_{I I}$ hereinafter. Obviously, $\Delta E_{I I}$ is 
independent of GB structure of alumina matrix. Therefore, in the following simulations, the $\Sigma 31$ GB structure is employed to investigate the effect of nanotube length and diameter on the pull-out process.

\subsection{Effect of nanotube length}

To investigate the effect of nanotube length on the pull-out process, three SWCNTs $(5,5)$ with different lengths are embedded in the same $\Sigma 31$ GB of alumina matrix, respectively. The obtained variations of energy increment $\Delta E$ between two adjacent pull-out steps are given in Fig.5, in which the same trend is clearly observed for each case as that in Fig. 4. Moreover, the identical $\Delta E_{I I}$ of three cases indicates its independence of nanotube length. Therefore, in the following simulations, CNTs with the same length of $5.17 \mathrm{~nm}$ are employed.

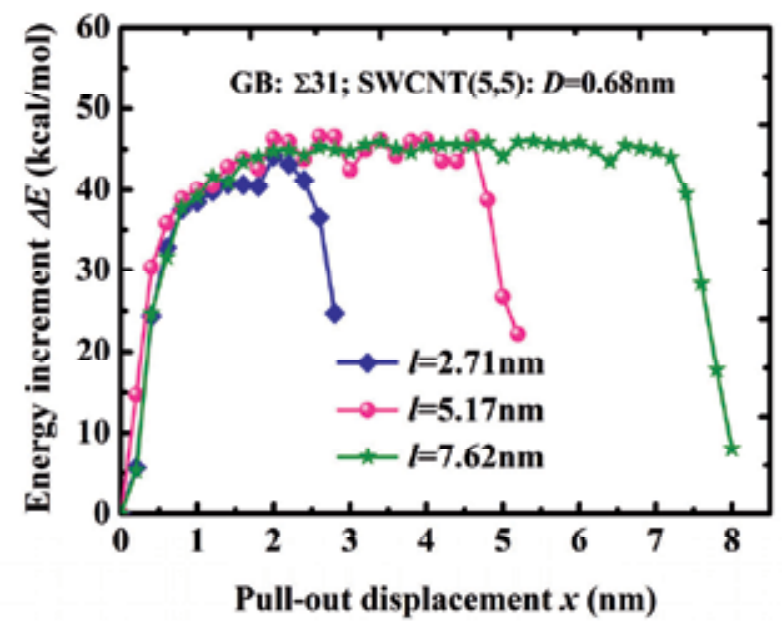

Figure 5. Effect of nanotube length on the variation of energy increment during pull-out process

\subsection{Effect of nanotube diameter}

Based on the above length-independent behavior, four SWCNTs (i.e., $(5,5),(10,10),(15,15)$, $(20,20))$ with the same length of $5.17 \mathrm{~nm}$ but different diameters are embedded into alumina matrix with $\Sigma 31 \mathrm{~GB}$ structure. The corresponding relationship between energy increment $\Delta E$ and pull-out displacement $x$ is shown in Fig.6a. Unlike the length-independent behavior, $\Delta E_{\text {II }}$ increases linearly with nanotube diameter as fitted in Fig.6b with the following formula

$$
\Delta E_{I I}=52.04 \times D+9.04
$$

where $\Delta E_{I I}$ is in $\mathrm{kcal} / \mathrm{mol}$, and $D$ is in $\mathrm{nm}$.

This phenomenon can be attributed to the number of atoms in circumferential direction, which increases linearly with nanotube diameter. For a CNT with larger diameter, there will be stronger vdW interactions needed to be overcome for the possible pull-out, which subsequently induces the higher energy increment in the same pull-out displacement. 
(a)

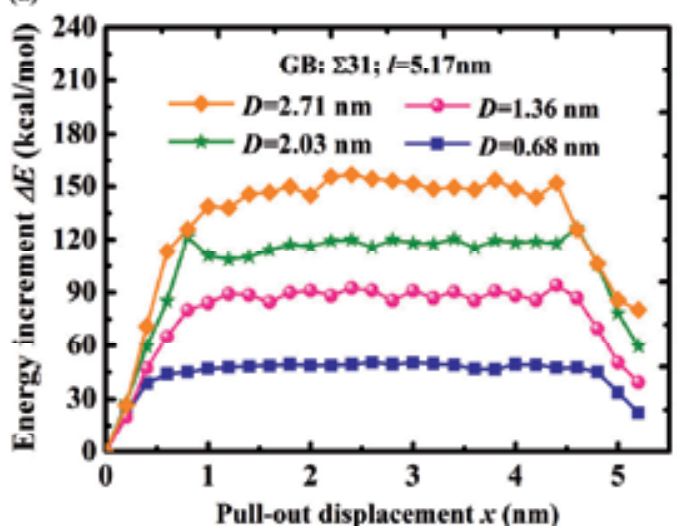

(b)

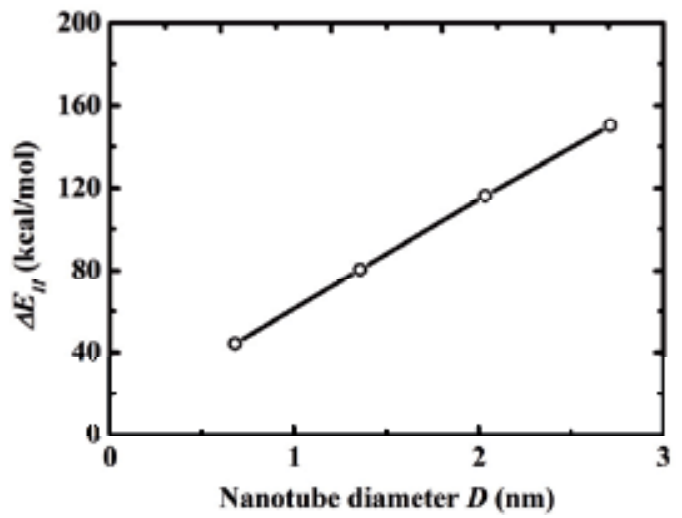

Figure 6. Effect of nanotube diameter on the variation of energy increment during the pull-out process (a) Relationship of energy increment and pull-out displacement; (b) Relationship of $\Delta E_{\text {II }}$ and nanotube diameter

\subsection{Pull-out force and surface energy density}

As discussed above, for the pull-out of a SWCNT, the corresponding average energy increment in stage $I I$, i.e., $\Delta E_{I I}$, is independent of GB structure and nanotube length, but is proportional to nanotube diameter. In view of that the work done by the pull-out force is equal to the energy increment in each pull-out step by neglecting some other minor energy dissipations, the pull-out force can be approximately calculated as

$$
F_{I I}=\frac{\Delta E_{I I}}{\Delta x}
$$

On this sense, we can conclude that the pull-out force of CNT from alumina matrix related to energy increment is also independent of GB orientation and nanotube length, but is proportional to nanotube diameter. From Eqs. (1) and (2), the corresponding empirical formula to predict the pull-out force is proposed as

$$
F_{I I}=1.81 \times D+0.31
$$

where $F_{I I}$ is in the unit of $\mathrm{nN}$, and $D$ of $\mathrm{nm}$.

It should be noted that two new surface regions are generated at two ends of CNT after each pull-out step (i.e., the inner surface of the matrix at the left side of $\mathrm{CNT}$, and the outer surface of $\mathrm{CNT}$ on the right side). Therefore, the corresponding surface energy should be equal to the energy increment. Therefore, the surface energy density can be calculated as

$$
\gamma_{I I}=\frac{\Delta E_{I I}}{2 \pi D \Delta x}=\frac{F_{I I}}{2 \pi D}
$$

Initially, this value is dependent on the diameter of SWCNT. However, as nanotube diameter increases, it will decrease gradually and then saturate to a constant. The converged 
value of surface energy density is approximately $0.3 \mathrm{~N} / \mathrm{m}$. Note that this surface energy density is newly reported for the interface of SWCNT and alumina matrix, although there have been some reports about that for the interface of SWCNT and polymer matrix with the value of $0.09 \sim 0.12 \mathrm{~N} / \mathrm{m}[26,30]$ or for sliding interface among nested walls in a MWCNT with the value of $0.14 \mathrm{~N} / \mathrm{m}$ [45]. It can be found that the surface interface density in CNT/Alumina composites is much higher than those of CNT/Polymer composites or CNT walls, implying its stronger interface.

\subsection{Interfacial shear stress}

Based on the above discussion, the corresponding interfacial shear stress is analyzed in the following.

The pull-out force is equilibrated with the axial component of vdW forces which induces the interfacial shear stress. Conventionally, if we employ the common assumption of constant interfacial shear stress with uniform distribution along the whole embedded region of CNT, the pull-out force $F_{I I}$ will vary with the embedded length of $\mathrm{CNT}$, which is obviously in contradiction to the above length-independent behavior of average energy increment $\Delta E_{I I}$ in stage II. For the extreme case of a CNT with an infinite length, the interfacial shear stress tends to be zero, which is physically unreasonable. This indicates that the conventional assumption of interfacial shear stress is improper for the perfect interface of CNT/Alumina composites with only consideration of vdW interactions.

For this problem, the interfacial shear stress should be analyzed according to the different stages in the variation of energy increment $\Delta E$. In stage $I$, the interfacial shear stress exists within a region of the length $a=1.0 \mathrm{~nm}$ at each end of CNT as described in Ref. [45] since the length of CNT in the model is equal to that of alumina matrix. In stage II, the situation may be different since the left end of CNT is deeply embedded into the alumina matrix with the pull-out displacement $x$ much larger than $a=1.0 \mathrm{~nm}$. To address the interfacial shear stress in this stage $I I$, a simple simulation is performed here.

As shown in Fig. 7a, a SWCNT(5,5) with only a half repeat unit is completely embedded in the middle position of alumina matrix. Then this SWCNT fragment is pulled out gradually with a constant increment of $\Delta x=0.2 \mathrm{~nm}$ to obtain the variation of systematic energy increment $\Delta E_{\omega}$, and the corresponding pull-out force $F_{\omega}$. As this SWCNT fragment is very short, the obtained pull-out force $F_{\omega}$, which is equilibrated by the shear force induced by the interfacial shear stress, can be used to characterize the distribution of interfacial shear stress. The obtained distribution of pull-out force $F_{\omega}$ at various pull-out steps is shown in Fig.7b. At the initial stage of the pull-out, the pull-out force keeps value at zero. When the CNT unit cell moves into the range of $a=1.0 \mathrm{~nm}$ measured from the right end of alumina matrix, the pull-out force increases sharply. It reaches the maximum when the CNT unit cell is just located on the right end of the matrix. As the CNT unit cell is further pulled out, it decreases gradually to zero. In virtue of the above results, as shown in Fig. 7c, the interfacial shear stress is solely distributed within the region of $2 a$ centered by the right end of matrix in 
stage II. The pull-out force during the pull-out process is further averaged within the range of $2 a$, i.e., $F_{\omega}^{*}=0.09 \mathrm{nN}$ in Fig. $7 \mathrm{~b}$.
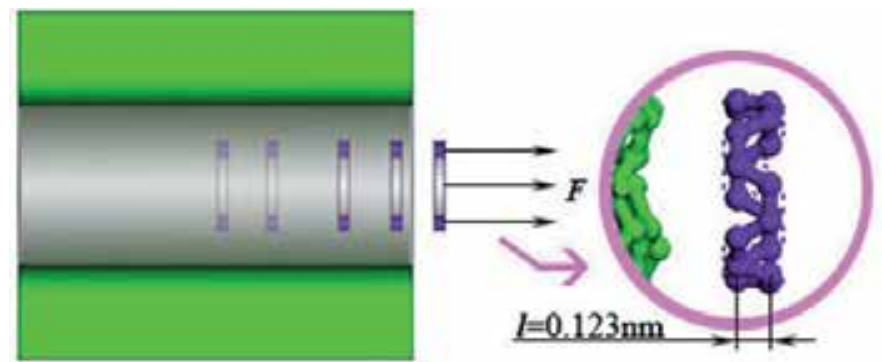

(a) Model for the pull-out of a simple CNT unit cell

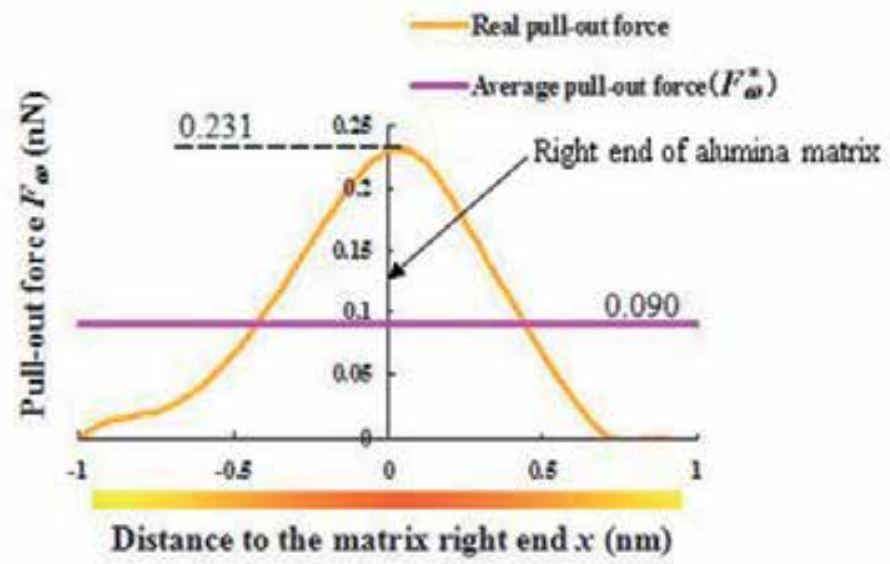

(b) Variation of pull-out force $F_{\text {send average pull-out force }} F_{\text {: }}$

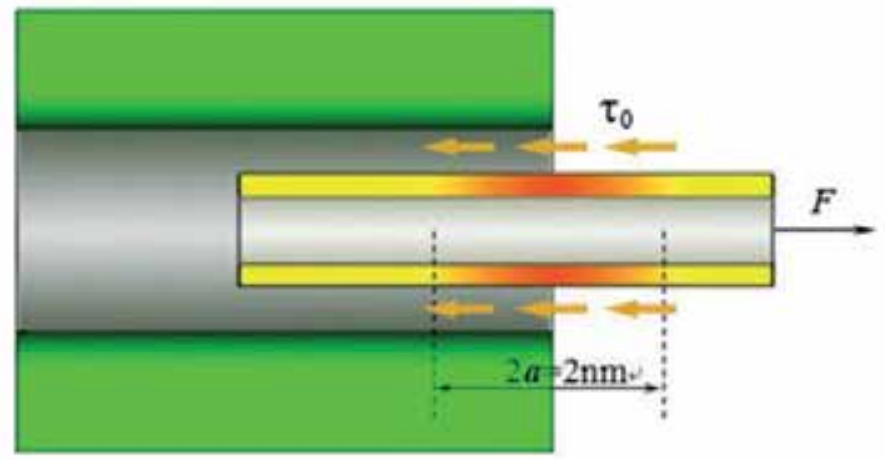

(c) Distribution of average interfacial shear stress

Figure 7. Analysis of interfacial shear stress 
By assuming that the interfacial shear stress is uniform within the above defined region for simplicity, the average of interfacial shear stress $\tau$ in stage II can be defined from the predefined average pull-out force( i.e., $F_{\omega}^{*}$ ) as

$$
\tau_{0}=\frac{F_{\omega}^{*}}{2 \pi D a}
$$

Obviously, $\tau_{0}$ is dependent on the diameter $D$ of SWCNT. However, it tends to be a constant as nanotube diameter increases gradually. The obtained converged interfacial shear stress $\tau_{0}$ from various unit cells of SWCNT with different diameters is $303 \mathrm{MPa}$. Note that it only exists within the range of $2 a$ centered by the right end of the matrix.

\section{Pull-out simulations of open-ended MWCNTs from alumina matrix}

Usually, there are two typical sliding behavior for MWCNT-reinforced composites: one is the complete pull-out of MWCNT, while the other is the so-called sword-in-sheath mode, e.g., in which the broken outer walls are pulled out (i.e., sheath) leaving the intact inner walls (i.e., sword) in the matrix. Therefore, based on the above information, two simple typical cases are firstly investigated: Case 1: pull-out of the whole MWCNT (e.g., Fig. 8a); Case 2: pull-out of only the outermost wall of MWCNT (e.g., Fig. 8b).

In view of the extremely high computational cost, several double-walled carbon nanotubes (DWCNTs) with wall number $n=2$ and triple-walled carbon nanotubes (TWCNTs) with wall number $n=3$ are discussed in the present simulation. The obtained average energy increment $\Delta E_{I I}$ in stage II related to the pull-out force is also found to be proportional to the diameter of the outermost wall of MWCNT $D_{o}$, which can be fitted as

Case 1

$$
\left\{\begin{array}{l}
\Delta E_{I I}=57.54 \times D_{o}+4.36, F_{I I}=2.00 \times D_{o}+0.15(n=2) \\
\Delta E_{I I}=58.26 \times D_{o}+6.50, F_{I I}=2.03 \times D_{o}+0.23(n=3)
\end{array}\right.
$$

Case 2

$$
\left\{\begin{array}{cc}
\Delta E_{I I}=93.61 \times D_{o}+10.17, & F_{I I}=3.26 \times D_{o}+0.35 \quad(n=2) \\
\Delta E_{I I}=96.60 \times D_{o}+10.50, & F_{I I}=3.33 \times D_{o}+0.37 \quad(n=3)
\end{array}\right.
$$

For Case 1, the relationship of $\Delta E_{I I}$ and nanotube diameter for the complete pull-out of SWCNT (Eq. 3), DWCNT (Eq. 6a), and TWCNT (Eq.7a) are plotted in Fig. 9, which indicates the effect of wall number from some aspect. The slope for DWCNT is about $9.56 \%$ higher than that for SWCNT, which highlights the contribution of the first adjacent inner wall to $\Delta E_{I I}$. However, the slope of TWCNT is only about $1.24 \%$ higher than that for DWCNT, which implies that the contribution of the second inner wall is gradually weakened as the distance from the sliding interface increases. Therefore, it can be concluded that the pull-out of MWCNT from alumina matrix is mostly affected by its two adjacent walls from the sliding interface, which indicates that for the whole pull-out of any 
MWCNT with more walls over 3, $\Delta E_{I I}$ can be approximately assumed to be equal to that of TWCNT (i.e., Eq. 6b).
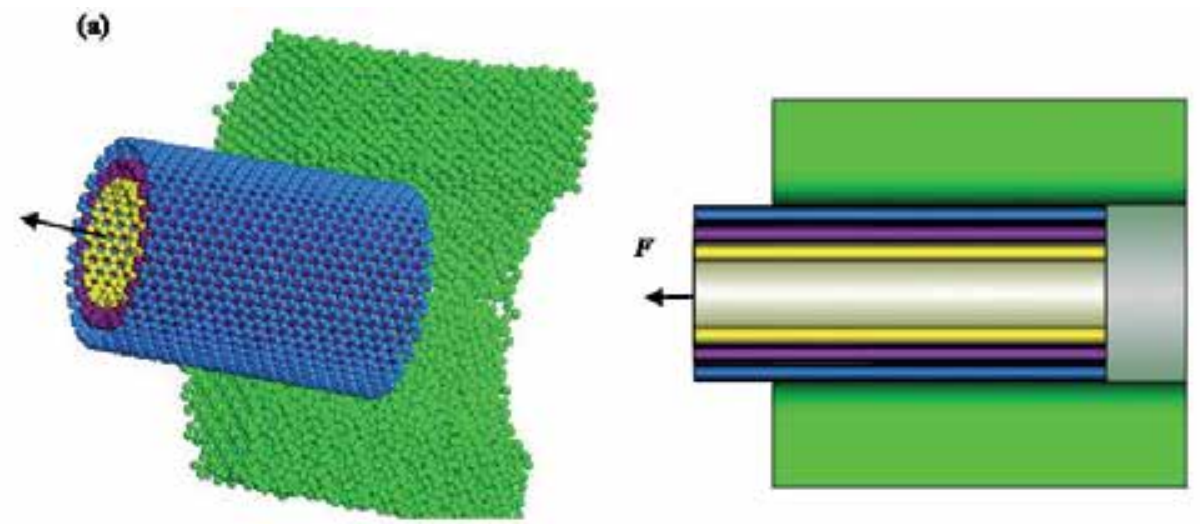

(b)
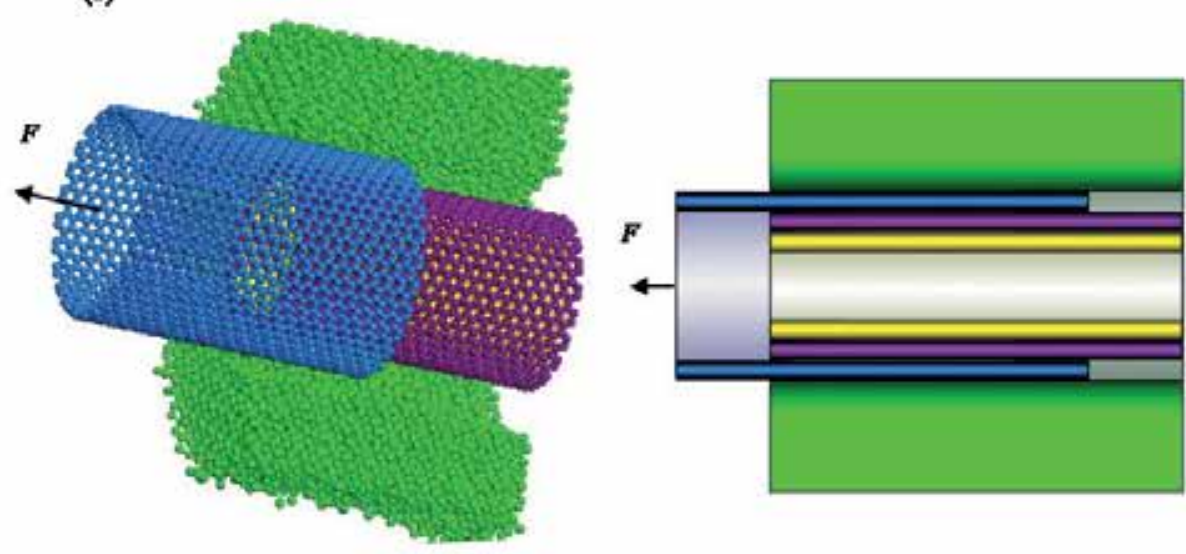

(c)
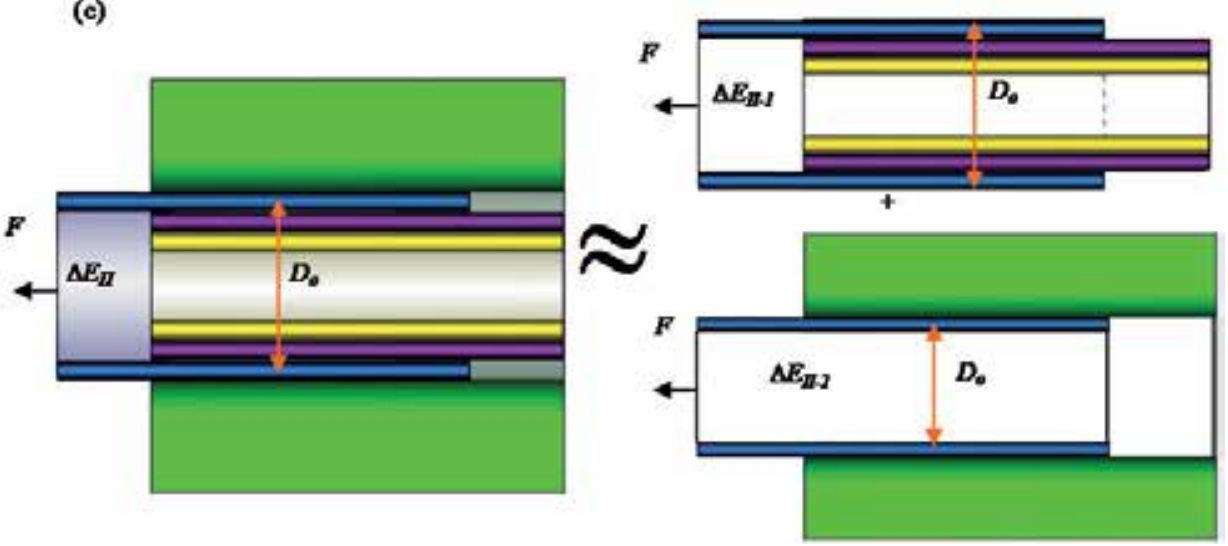

Figure 8. Two typical pull-out cases for an open-ended TWCNT

(a) Case 1: pull-out of the whole MWCNT; (b) Case 2: pull-out of the outmost wall of MWCNT;

c) Decomposition of Case 2 into two independent sub-problems 


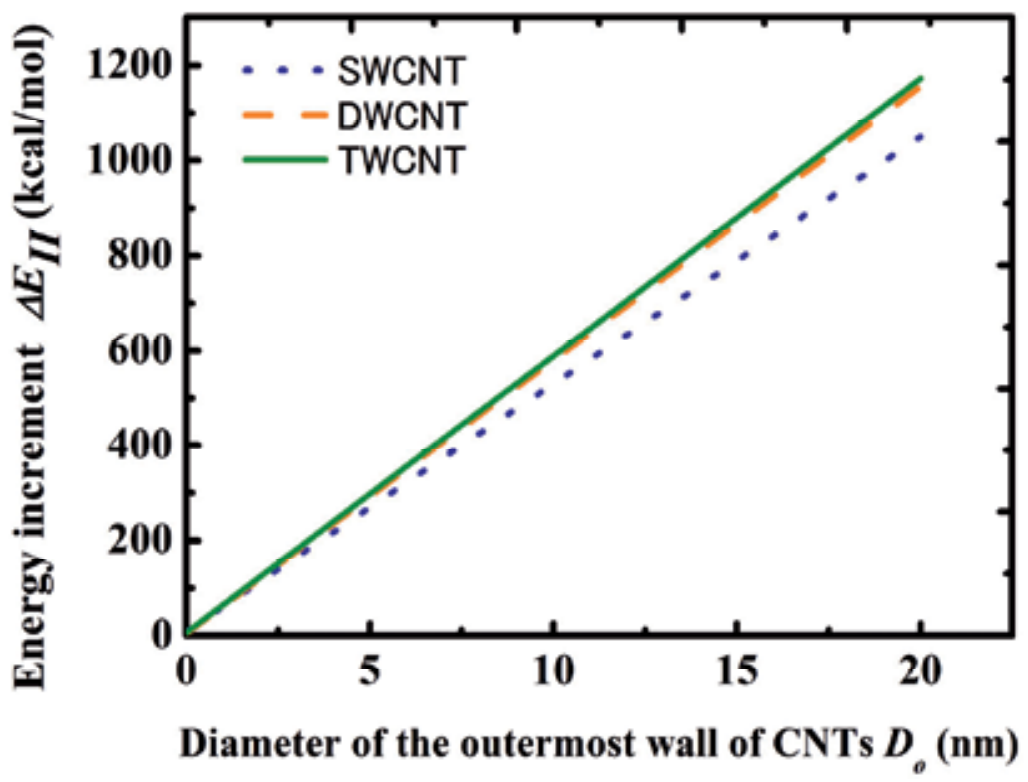

Figure 9. Effect of wall number on the energy increment for the pull-out of whole MWCNT

For Case 2, taking $\operatorname{TWCNT}(5,5) /(10,10) /(15,15)$ as an example, it is surprising to find that the corresponding $\Delta E_{I I}$ is approximately equal to the sum of those of two sub-problems, i.e., the pull-out of a SWCNT $(15,15)$ from alumina matrix, and the pull-out of the outermost wall in the TWCNT. Therefore, the corresponding $\triangle E_{I I}$ for the pull-out of any TWCNT from alumina matrix (i.e., Eq. $7 \mathrm{~b}$ ) can be approximately decomposed into the following two items as given in Fig. 8c: $\Delta E_{I I-1}$ for the pull-out of the outermost wall of TWCNT against the other two inner walls (i.e., Eq. 5 in Ref. [45]), and $\triangle E_{I I-2}$ for the pull-out of a SWCNT from alumina matrix (i.e., Eq. 3) whose diameter is equal to the outermost wall of the TWCNT.

It should be noted that for the real sword-in-sheath fracture mode, there are more than 3 walls pulled out. For example, as shown in Fig. 10a, several purple outer walls of a MWCNT are pulled out leaving the yellow inner walls within the matrix. Here, there are two sliding interfaces: one is between CNT and matrix, the other is between outer walls and inner wall. According to the above discussion, it can also be thought of as the superimposition of the following two sub-problems in Fig. 10b: one is the pull-out of the TWCNT which is composed of the outer three walls (i.e., Eq. 6b), and the other is the pull-out of outer three walls in a MWCNT with five walls (i.e., Eq. 6 in Ref. [45]). It indicates that the corresponding $\Delta E_{I I}$ and pull-out force $F_{I I}$ can be calculated as

$$
\begin{aligned}
\Delta E_{I I} & =58.26 \times D_{o}+37.56 \times D_{c}-4.00 \\
F_{I I} & =2.04 \times D_{o}+1.31 \times D_{c}-0.14
\end{aligned}
$$

Here, $D_{o}$ is the diameter of the outermost wall of MWCNT, and $D_{c}$ is the diameter of the green critical wall in Fig. 10 (i.e., the immediate outer wall at the sliding surface between outer walls and inner walls). 


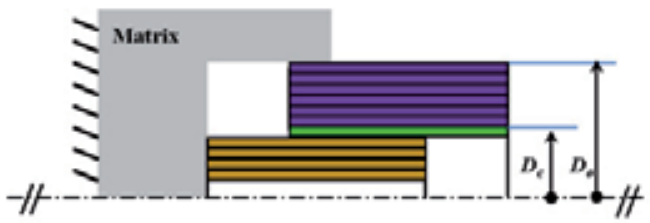

(a)

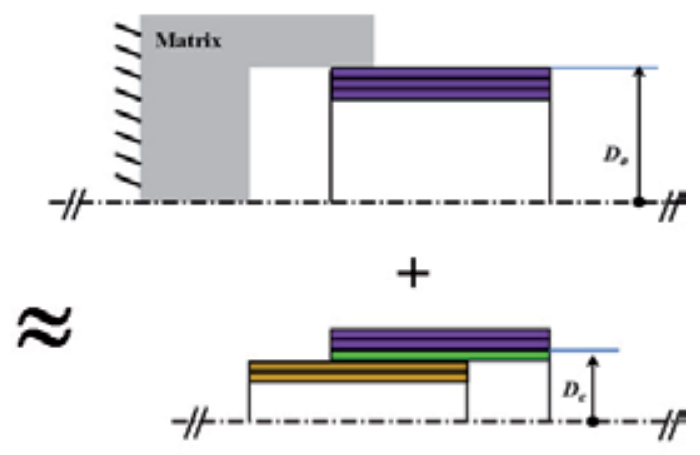

(b)

Figure 10. Real case of sword-in-sheath mode

\section{Pull-out simulations of a capped MWCNT from alumina matrix}

It is noted that open-ended CNTs are employed in the above simulations. On the other hand, it has been reported that CNT cap makes great effect on its field emission properties [46], load transferring ability among nested walls of MWCNT [45, 47, 48]. However, to our best knowledge, there is no any detailed report on the effect of CNT caps on the interfacial properties of CNT-reinforced composites. Therefore, the pull-out of capped MWCNTs from alumina matrix in a sword-in-sheath mode is discussed here.

The schematic model is given in Fig. 11. By using the principal of superimposition, this pullout process can be decomposed into the following three parts: pull-out of outer walls against matrix (i.e., part I); pull-out of inner walls against outer walls, which can be further decomposed into the open-ended part of inner/outer walls (i.e., part II) and the capped part of inner/outer walls (i.e., part III) as each wall in a MWCNT is composed of open-ended part and capped part.

Generally, the number of broken outer walls and intact inner walls are more than 3 . Therefore the corresponding pull-out forces for the above three parts are analyzed as below.

i. Pull-out of outer walls against matrix (i.e., part I in Fig. 11): According to Eq. 2, the corresponding pull-out force $F_{1}$ can be predicted by using Eq. 6 b, i.e.,

$$
F_{1}=2.03 \times D_{o}+0.23
$$

ii. Pull-out of open-ended part of inner walls against outer walls (i.e., part II in Fig. 11): According to Eq. 6 in Ref. [45], the corresponding pull-out force $F_{2}$ can be predicted as

$$
F_{2}=1.31 \times D_{c}-0.37
$$

iii. Pull-out of capped part between inner walls and outer walls (i.e., part III in Fig. 11): This part can be transferred as the interfacial sliding among nested walls in a capped MWCNT. 

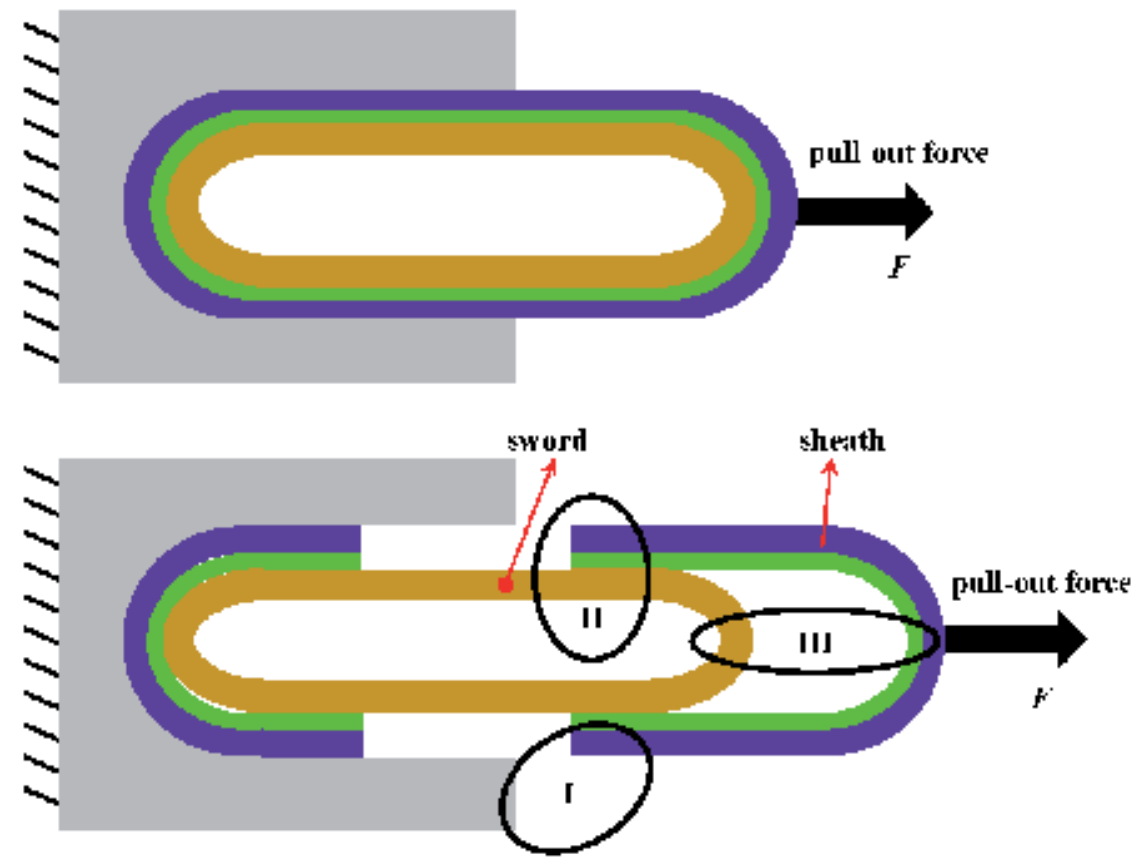

I: onter walls'matix: Il: open-ended part of inneriouter walls; Jls: cappel part if inneriouter walls

Figure 11. Schematic model for the pull-out of a capped MWCNT from alumina matrix

As illustrated in Fig. 12a, after fixing the atoms of the outer cap, the inner wall is pulled out along its axial direction by applying a constant displacement increment of $\Delta x_{2}=0.01 \mathrm{~nm}$ on the atoms of the right end of inner wall. Note that the present displacement increment $\Delta x_{2}$ is smaller than the above $\Delta x$ of $0.2 \mathrm{~nm}$, which is used for making the effect of CNT caps on energy increment clearly. After each pull-out step, the structure is relaxed to obtain the minimum potential energy E. As discussed in Ref. [45], the pull-out force of an open-ended CNT is only proportional to nanotube diameter, and independent of nanotube length. For this reason, five DWCNTs with different diameters but same length are built up to investigate the effect of CNT cap. The calculated energy increments between two consecutive pull-out steps of three DWCNTs are shown in Fig. 13a, where $D_{c}$ is the diameter of critical wall (i.e., the outer wall of DWCNT). It can be seen that for each DWCNT the energy increment $\Delta E$ increases rapidly up to a peak value at a specified displacement, and then decreases. The same feature is also observed in the simulations of two other DWCNTs with larger diameters, i.e., $(54,54) /(59,59)$ with $D_{c}=8.0 \mathrm{~nm}$ and $(83,83) /(88,88)$ with $D_{c}=11.93 \mathrm{~nm}$. The maximum energy increment (i.e., $\Delta E_{\max }$ ) for the five DWCNTs is shown in Fig. $13 \mathrm{~b}$. The relationship between $\Delta E_{\max }$ and $D_{c}$ can be perfectly fitted into a quadratic function of

$$
\Delta E_{\text {max-DWCNT }}=2.09 \times D_{c}^{2}-2.15 \times D_{c}+0.94
$$


(a)

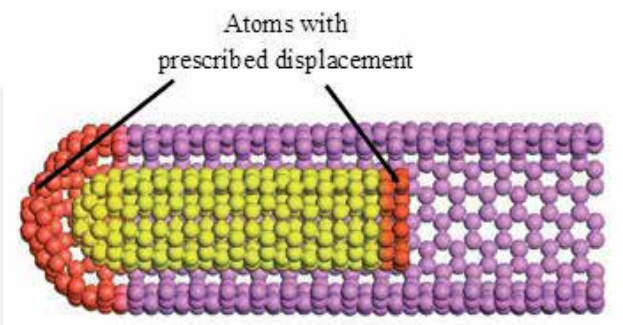

(b)

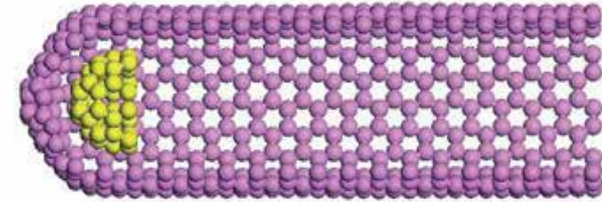

(c)

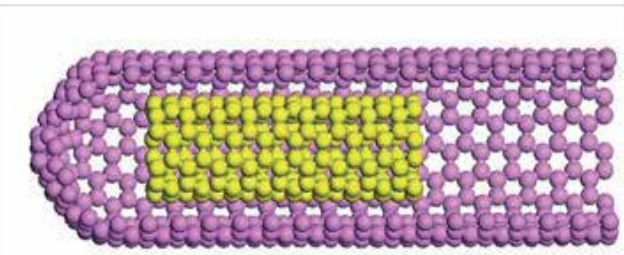

(d)

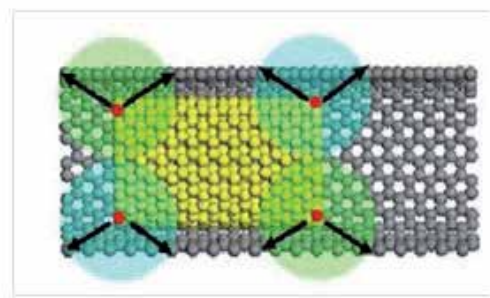

(e)

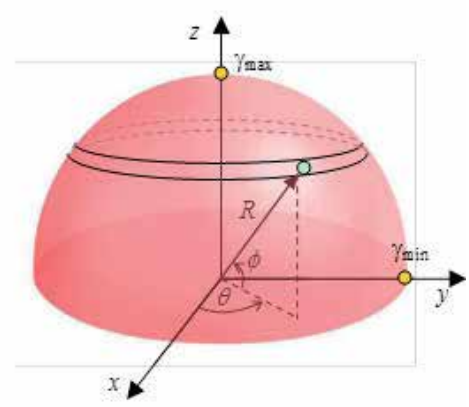

(f)

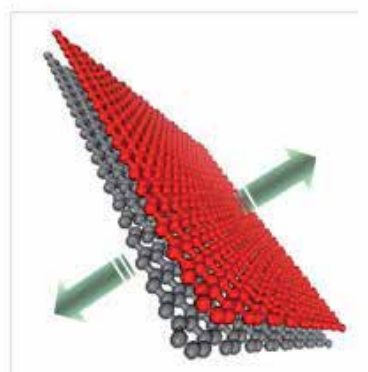

Figure 12. Interfacial sliding in a capped DWCNT

(a) Schematic model of a capped DWCNT; (b) Pull-out of the capped part;

(c) Pull-out of the open-ended part;

(d) Force state of open-ended part of CNT; (e) Estimation of energy variation of a cap;

(f) Pull-out of graphite sheets

To understand this potential energy increment in detail, we further divided the inner wall (Fig. 12a) into two parts, i.e., the capped (Fig. 12b) and the open-ended part (Fig. 12c). The corresponding pull-out forces for these two parts are $F_{3}{ }^{1}$ and $F_{3}{ }^{2}$, which means $F_{3}=F_{3}{ }^{1}+F_{3}{ }^{2}$. The pull-out of open-ended part (Fig. 12c) does not cause any change of the potential energy, i.e., $F_{3}{ }^{2}=0$. It means that the contribution of capped part, i.e., $F_{3}{ }^{1}$ dominates the total pull-out force $F_{3}$. The reason can be explained using Fig. 12d-12e. 


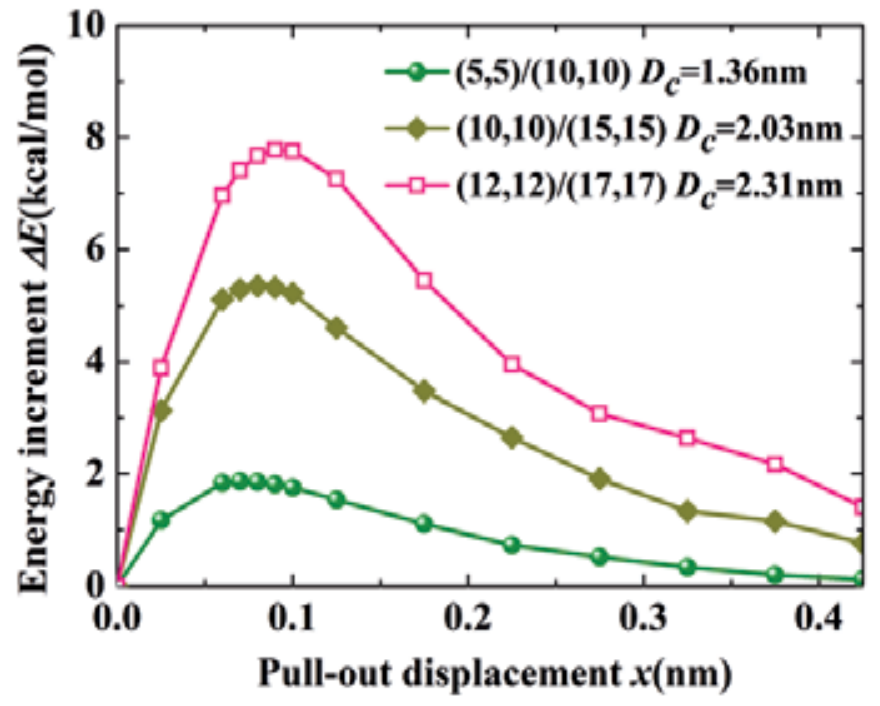

(a) Energy increment versus pull-out displacement for model of Fig.12a

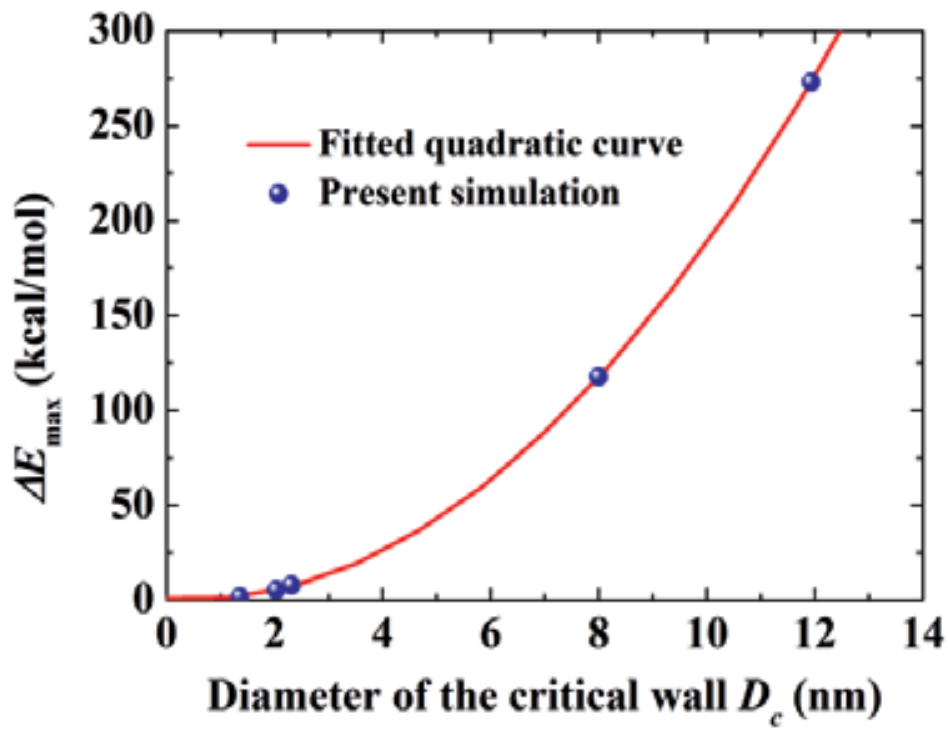

(b) Relationship between maximum energy increment and nanotube diameter

Figure 13. Variation of energy increment for the interfacial sliding in a capped DWCNT 
First, in Fig. 12d, if the length of the outer wall of DWCNT is long enough, the carbon atoms of the inner wall are always in force equilibrium. For example, in Fig. 12d, the atoms in red are balanced by the symmetrical horizontal forces form the atoms of the outer wall, which are within the cut-off distance of Lennard-Jones potential $[49,50]$. During a pull-out process, the relative motion of the atoms between the inner wall and outer wall creates repetitive breaking and reforming of the vdW interactions and no resultant resistance force can be generated on the inner wall, i.e., $F_{3}^{2}=0$.

The quadratic form of the energy increment in Eq. 12 due to the capped effect is associated with the surface energy density. Considering a cap model shown in Fig. 12e, the bottom edge is just located on the boundary between the capped and open-ended part. If we use $\gamma_{\text {max }}$ and $\gamma_{\text {min }}$ to represent the maximum and minimum surface energy density (i.e., potential energy variation per unit area) under a specified separation displacement, $\gamma_{\max }$ is at the top of the cap while $\gamma_{\text {min }}$ appears at the bottom of the cap. Then, the surface energy density is assumed to vary from the top to the bottom of the cap in the function of $\gamma(\phi)=\gamma_{\max } \cos \left(90^{\circ}-\phi\right)=\gamma_{\max } \sin \phi .$, which implies that $\gamma_{\min }=0$. This is reasonable as $F 3^{2}=0$. Then the total surface energy variation of the cap can be calculated as

$$
U_{\text {cap }}=\int_{0}^{2 \pi}\left(\int_{0}^{\frac{\pi}{2}} \gamma(\phi) R \cos \phi \times R d \varphi\right) d \theta=\frac{\pi D_{c}^{2}}{4} \gamma_{\max }
$$

From Eq. 13, regardless of the function of $\chi(\phi)$, the surface energy is always proportional to $\pi D_{c}^{2}$. As a result, the energy increment induced by the pull-out of the cap can be described by a quadratic function of $D_{c}$, which is consistent with Fig. 13b and Eq. 12. Approximately, the $\gamma_{\max }$ at the small top flat area of the cap during the pull-out process can be predicted in the same way by simulating the separation of two flat graphite sheets in Fig. 12f. It is confirmed by the displacement-energy increments curves obtained from the simulation of two graphite sheets which is quite similar with those in Fig. 13a. The corresponding $\gamma_{\text {max-cap }}$ is around $0.03 \mathrm{~N} / \mathrm{m}$ under $0.01 \mathrm{~nm}$ separation displacement in the normal direction of two graphite sheets. Substituting this value into Eq. 13 leads to the total surface energy change as: $U_{c a p}=\frac{\pi D_{c}^{2}}{4} \gamma_{\text {max-cap }}=2.76 D_{c}^{2}$, which is approximately equivalent to Eq. 12 . Therefore, it indicates the quadratic form of Eq. 12 is appropriate from the other aspect.

After validating the effectiveness of Eq. 12, the corresponding maximum pull-out force can be simply evaluated by equaling the work done by the pull-out force to the $\Delta E$ max-DWCNT with the formula of

$$
F_{3-D W C N T}=1.45 \times D_{c}^{2}-1.49 D_{c}+0.65
$$

It should be noted that the above analysis is for a capped DWCNT. For the case of MWCNT, a simplified model in Fig. 14 is developed, as only the immediate two outer and inner walls from the sliding interface can affect the corresponding pull-out interface [45]. The evaluated 
pull-out force is found to be approximately $29 \%$ higher than that for DWCNT due to the contribution of the immediate two outer and inner walls, which means

$$
F_{3-\mathrm{MWCNT}}=1.87 \times D_{c}^{2}-1.92 D_{c}+0.84
$$

Therefore, for the pull-out of a capped MWCNT from alumina matrix in a sword-in-sheath mode, the corresponding pull-out force can be assumed to be the sum of those for the above three parts (i.e., Eq. 10 for part I, Eq. 11 for part II, Eq. 15 for part III):

$$
F=F_{1}+F_{2}+F_{3-\mathrm{MWCNT}}=1.87 \times D_{c}^{2}+2.03 D_{o}-0.61 \times D_{c}+0.7
$$

For the pull-out of a MWCNT numbered as sample 14 in Ref. [37] which has the outermost wall with diameter of $D_{0}=94 \mathrm{~nm}$ and the critical wall at the sliding interface with diameter about $D_{c}=90 \mathrm{~nm}$, the calculated pull-out force using Eq. 16 is $15.28 \mu \mathrm{N}$, which is in the same scale of experimental value of $19.7 \mu \mathrm{N}$ [37].

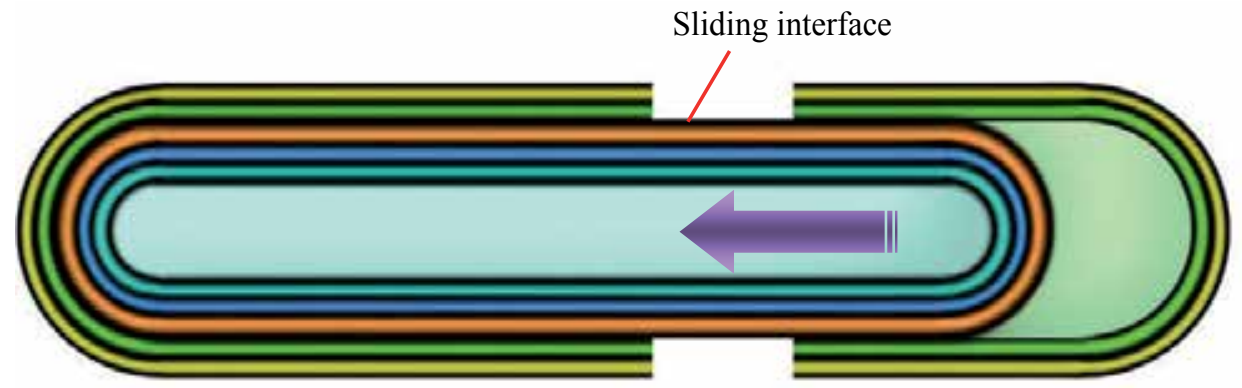

Figure 14. Schematic model for interfacial sliding in a capped MWCNT in sword-in-sheath mode

\section{Conclusions}

We systematically investigate the pull-out process of open-ended and capped CNTs from alumina matrix using MM simulations, aiming at clarifying the interfacial sliding behavior in CNT/Alumina composites. The effects of grain boundary structure of alumina matrix, nanotube length, nanotube diameter, wall number and capped structure of CNTs are explored systematically.

A set of universal formulae with the newly obtained surface energy density is proposed to approximately predict the pull-out force from nanotube diameter. The philosophy behind these simple empirical formulae is that the pull-out force is only proportional to nanotube 
diameter, and independent of nanotube length and GB structure of alumina matrix. The detailed interfacial shear stress is studied in this work, which indicates that the conventional definition of the interfacial shear strength is inappropriate in CNT/Alumina composites. Moreover, there are at most two adjacent walls at each side of the sliding interface which will affect this interfacial sliding in CNT/Alumina composites. Furthermore, it also indicates that CNT caps play a very important role in the pull-out process. These findings will be helpful for clarifying the toughening mechanism for mechanical properties of bulk CNT/Alumina composites and providing useful insight into the design of ideal materials.

\section{Author details}

Yuan Li

Department of Nanomechanics, Tohoku University, Aramaki-Aza-Aoba, Aoba-ku, Sendai, Japan

Sen Liu

Department of Mechanical Engineering, Chiba University, Yayoi-cho, Inage-ku, Chiba, Japan

Ning $\mathrm{Hu}$

Corresponding Author

Department of Mechanical Engineering, Chiba University, Yayoi-cho, Inage-ku, Chiba, Japan

Weifeng Yuan and Bin Gu

School of Manufacturing Science and Engineering, Southwest University of Science and Technology, Mianyang, P.R.China

\section{Acknowledgement}

The authors are grateful to be partly supported by Tohoku Leading Women's Jump Up Project for 2013 (J1 10002158) and Grand-in-Aids for Scientific Research (No. 19360045 and No. 22360044) from the Ministry of Education, Culture, Sports, Science and Technology (MEXT) of Japan. The authors acknowledge Prof. C.B. Fan (Beijing Institute of Technology, China) for kindly providing the computational resources.

\section{References}

[1] Dresselhaus MS, Dresselhaus G, Avouris P. (2001) Carbon Nanotubes: Synthesis, Structure, Properties, and Applications. Springer-Verlag Berlin Heidelberg.

[2] Dresselhaus MS, Dresselhaus G, Charlier JC, Hernandez E. (2004) Electronic, Thermal and Mechanical Properties of Carbon Nanotubes. Philosophical Transactions of the Royal Society A. 362:2065-2098. 
[3] Thostenson ET, Ren ZF, Chou TW (2001) Advances in the Science and Technology of Carbon Nanotubes and their Composites: A Review. Composites Science and Technology. 61(13): 1899-1912.

[4] Breuer O, Sundararaj U. (2004). Big Returns from Small Fibers: A Review of polymer/carbon nanotube composites. Polymer Composites. 25(6): 630-645.

[5] Coleman JN, Khan U, Blau WJ, Gunko YK. (2006) Small but Strong: A Review of the Mechanical Properties of Carbon Nanotube-Polymer Composites. Carbon. 44:16241652.

[6] Moniruzzaman M, Winey KI. (2006) Polymer Nanocomposites Containing Carbon Nanotubes. Macromolecules. 39(16): 5194-5205.

[7] Coleman JN, Khan U, Gun'ko YK. (2006) Mechanical Reinforcement of Polymers Using Carbon Nanotubes. Advanced Materials. 18(6): 689-706.

[8] Peigney A, Laurent C, Flahaut E, et al. (2000) Carbon Nanotubes in Novel Ceramic Matrix Nanocomposites. Ceramics International. 26(6): 677-683.

[9] Curtin WA, Sheldon BW. (2004) CNT-reinforced Ceramics and Metals. Materials Today. $7(11): 44-49$.

[10] Samal SS, Bal S. (2008) Carbon Nanotube Reinforced Ceramic Matrix Composites- a Review. Journal of Minerals \& Materials Characterization \& Engineering. 7(4): 355370.

[11] Cho J, Boccaccini AR, Shaffer M. (2009) Ceramic Matrix Composites Containing Carbon Nanotubes. Journal of Materials Science. 44(8): 1934-1951.

[12] Zhan GD, Kuntz JD, Wan J, Mukherjee Ak. (2003) Single-wall Carbon Nanotube as Attractive Toughening Agents in Alumina-based Nanocomposites. Nature Materials. 2:38-42.

[13] Chan BM, Cha SI, Kim KT, Lee KH \& Hong SH. (2005) Fabrication of Carbon Nanotube Reinforced Alumina Matrix Nanocomposite by Sol-gel Process. Materials Science and Engineering A. 395: 124-128.

[14] Qian D, Dickey EC, Andrews R, Rantell T. (2000) Load Transfer and Deformation Mechanisms in Carbon Nanotube-Polystyrene Composites. Applied Physics Letters. 76(20):2868-2870.

[15] Deng F. (2008) Investigation of the Interfacial Bonding and Deformation Mechanism of the Nano Composites Containing Carbon Nanotubes. Tokyo University, PhD Dissertation.

[16] Barber AH, Cohen SR, Kenig S, Wagner HD. (2003) Measurement of Carbon NanotubePolymer Interfacial Strength. Applied Physics Letters. 82(23): 4140-4142.

[17] Barber AH, Cohen SR, Kenig S, Wagner HD. (2004) Interfacial Fracture Energy Measurements for Multi-walled Carbon Nanotubes Pulled from a Polymer Matrix. Composite Science and Technology. 64:2283-2289.

[18] Schadler LS, Giannaris SC, Ajayan PM. (1998) Load Transfer in Carbon Nanotube Epoxy Composites. Applied Physics Letters. 73(26):3842-3844. 
[19] Cooper CA, Cohen SR, Barber AH, Wagner HD. (2002) Detachment of Nanotubes from a Polymer Matrix. Applied Physics Letters. 81(20):3873-3875.

[20] Jiang LY, Huang Y, Jiang H, Ravichandran G, Gao H, Hwang KC, et al. (2006) A Cohesive Law for Carbon Nanotube/Polymer Interfaces based on the van der Waals Force. Journal of the Mechanics and Physics of Solids. 54: 2436- 2452.

[21] Xiao KQ, Zhang LC. (2004) The Stress Transfer Efficiency of a Single-walled Carbon Nanotube in Epoxy Matrix. Journal of Materials Science. 39:4481-4486.

[22] Gao XL, Li K. (2005) A Shear-lag Model for Carbon Nanotube-Reinforced Polymer Composites. International Journal of Solids and Structures. 42: 1649-1667.

[23] Tsai J, Lu T. (2009) Investigating the Load Transfer Efficiency in Carbon Nanotubes Reinforced Nanocomposites. Composite Structures. 90:172-179.

[24] Lau K. (2003) Interfacial Bonding Characteristics of Nanotube/Polymer Composites. Chemical Physics Letters. 370:399-405.

[25] Natsuki T, Wang F, Ni QQ, Endo M. (2007) Interfacial Stress Transfer of Fiber Pullout for Carbon Nanotubes with a Composite Coating. Journal of Materials Science. 42:41914196.

[26] Lordi V, Yao N. (2000) Molecular Mechanics of Binding in Carbon-Nanotube-Polymer Composites. Journal of Materials Research. 15(12):2770-2779.

[27] Liao K, Li S. (2001) Interfacial Characteristics of a Carbon Nanotube-Polystyrene Composite System. Applied Physics Letters. 79(25):4225-4227.

[28] Frankland SJV, Caglar A, Brenner DW, Griebel M. (2002) Molecular Simulation of the Influence of Chemical Cross-links on the Shear Strength of Carbon Nanotube- Polymer Interfaces. Journal of Physical Chemistry B. 106:3046-3048.

[29] Gou J, Minaie B, Wang B, Liang Z, Zhang C. (2004) Computational and Experimental Study of Interfacial Bonding of Single-walled Nanotube Reinforced Composites. Computational Materials Science. 31:225-236.

[30] Zheng Q, Xia D, Xue Q, Yan K, Gao X, Li Q. (2009) Computational Analysis of Effect of Modification on the Interfacial Characteristics of a Carbon Nanotube- Polyethylene Composites System. Applied Surface Science. 255: 3524-3543.

[31] Al-Ostaz A, Pal G, Mantena PR, Cheng A. (2008) Molecular Dynamics Simulation of SWCNT-Polymer Nanocomposite and its Constituents. Journal of Materials Science. 43:164-173.

[32] Chowdhury SC, Okabe T. (2007) Computer Simulation of Carbon Nanotube Pull-out from Polymer by the Molecular Dynamics Method. Composites Part A. 38:747-754.

[33] Li Y, Liu Y, Peng X, Yan C, Liu S, Hu N. (2011) Pull-out Simulations on Interfacial Properties of Carbon Nanotube-reinforced Polymer Nanocomposites. Computational Material Science. 50: 1854-1860.

[34] Xia Z, Riester L, Curtin WA, et al. (2004) Direct Observation of Toughening Mechanisms in Carbon Nanotube Ceramic Matrix Composites. Acta Materialia. 52(4):931-944. 
[35] Fan, JP, Zhuang DM, Zhao DQ, et al. (2006) Toughening and Reinforcing Alumina Matrix Composite with Single-wall Carbon Nanotubes. Applied Physics Letters B. 89:121910(3).

[36] Yamamoto G, Omori M, Hashida T, Kimura H. (2008) A Novel Structure for Carbon Nanotube Reinforced Alumina Composites with Improved Mechanical Properties. Nanotechnology. 19:315708(7).

[37] Yamamoto G, Shirasu K, Hashida T, et al. (2011) Nanotube Fracture during the Failure of Carbon Nanotube/Alumina Composites. Carbon. 49: 3709-3716.

[38] Li L, Xia Z, Curtin W, Yang Y. (2009) Molecular Dynamics Simulations of Interfacial Sliding in Carbon-Nanotube/Diamond Nanocomposites. Journal of the American Ceramic Society. 92(10): 2331-2336.

[39] Vasiliev AL, Poyato R, and Padture NP. (2007) Single-wall Carbon Nanotubes at Ceramic Grain Boundaries", Scripta Materialia. 56(6): 461-463.

[40] Bollmann W. (1970) Crystal Defects and Crystalline Interfaces. Springer, Berlin.

[41] Matsunaga K, Nishimura H, Hanyu S, et al. (2005) HRTEM Study on Grain Boundary Atomic Structures Related to the Sliding Behavior in Alumina Bicrystals. Applied Surface Science. 241: 75-79.

[42] Ikuhara Y. (2001) Grain Boundary and Interface Structure in Ceramics. Journal of the Ceramic Society of Japan. 109(7): S110-S120.

[43] Nishimura H, Matsunaga K, Saito T, et al. (2003) Atomic Structures and Energies of $\Sigma 7$ Symmetrical Tilt Grain Boundaries in Alumina Bicrystals. Journal of the American Ceramic Society. 86(4): 574-580.

[44] Nishimura H, Matsunaga K, Saito T, et al. (2003) Grain Boundary Structures and High Temperature Deformations in Alumina Bicrystals. Journal of the Ceramic Society of Japan. 111(9): 688-691.

[45] Li Y, Hu N, Yamamoto G, Wang Z, et al. (2010) Molecular Mechanics Simulation of the Sliding Behavior between Nested Walls in a Multi-walled Carbon Nanotube. Carbon. 48:2934-2940.

[46] Wang MS, Wang JY, Peng LM. (2006) Engineering the Cap Structure of Individual Carbon Nanotubes and Corresponding Electron Field Emission Characteristics. Applied Physics Letters. 88(24): 243108(1-3).

[47] Shen GA, Namilae S, Chandra N. (2006) Load Transfer Issues in the Tensile and Compressive Behavior of Multi-walled Carbon Nanotubes. Materials Science and Engineering A. 429(1-2):66-73.

[48] Xia Z, Curtin WA. (2004) Pullout Forces and Friction in Multiwall Carbon Nanotubes. Physical Review B. 69:2333408(1-4).

[49] Hu N, Fukunaga H, Lu C, Kameyama M, Yan B. (2005) Prediction of Elastic Properties of Carbon Nanotube Reinforced Composites. Proceedings of the Royal Society A. 461:1685-1710. 
[50] Hu N, Nunoya K, Pan D, Okabe T, Fukunaga H. (2007) Prediction of Buckling Characteristics of Carbon Nanotubes. International Journal of Solids and Structures. 44:6565-6550. 


\section{Design, Processing, and Manufacturing Technologies}





\title{
Advanced Composite Materials by Resin Transfer Molding for Aerospace Applications
}

\author{
Susanna Laurenzi and Mario Marchetti \\ Additional information is available at the end of the chapter
}

http://dx.doi.org/10.5772/48172

\section{Introduction}

Competitiveness drives the aerospace industries to investigate new technology solutions to address market pressure and high-tech demands. The global objective is to reduce to half the amount of fuel by 2020 and at least $70 \%$ less by 2025 with respect to the Boeing 777, one of the most efficient aircraft, which is made entirely of carbon fiber. The weight saving to increase payload and the reductions of the cost/time of the production cycle are imperative targets. For these reasons, aerospace companies, which are traditionally based on the use of metal alloys, have been focusing for past decade on composite materials. The main advantages of composites with respect to metals, that are resistance to corrosion and fatigue and high performance/weight ratios, are a set of qualities for winning the current and future aerospace applications. Obviously, this is possible only through the development of economically competitive technologies.

The Resin Transfer Molding (RTM) is one of the most promising technology available today. RTM is capable of making large complex three-dimensional part with high mechanical performance, tight dimensional tolerance and high surface finish. A good design by RTM leads to fabricate three-dimensional near-net-shape complex parts, offering production of cost-effective structural parts in medium-volume quantities using low cost tooling. In addition to these advantages, the problems of the joints, typical of the metal structures, can be eliminated by integration of inserts.

The final performances of a composite depend not only on the choice of the matrix and the fiber but also on the manufacturing process by which they are made. Since the starting of the composite life, the presence of imperfections due to manufacturing must be considered. Such imperfections can be already damage for the manufactured composite piece or lead to the damage quickly. The damage for composites can be defined as a change in the microstructure of the material that causes deterioration in the structural behavior of the 
component and sometimes its collapse. The damage in a composite structure can occur at the level of fibers and matrix as well.

The most common damage to the fibers is the interruption of their continuity. For example, fibers that are subject to load tend to align again inducing states of compression and tension on the matrix. These states may cause, in addition to a local decrease of the properties of the lamina, the breaking of the fibers themselves and the gap between fiber and matrix. In the assumption of an optimal stratification, fiber misalignment is caused during the manufacturing process by a bad balance of the process parameters that leads to the deformation of the fiber bundles. The fibers may also be distributed unevenly in the volume of the composite, and this generates intra-laminar shear stresses under operating conditions.

In the matrix, the damage is essentially correlated to the presence of porosity. The formation of microvoids between fibers and dry spots are potential starting points for propagating cracks or delaminations. The response of the component to delamination depends not only on the compatibility and surface tension between fibers and matrix but mainly on the compaction, and therefore also on the impregnation phases during the manufacturing process.

Comparing RTM with traditional manufacturing process applied in the aerospace industries (i.e. autoclave), the RTM technique results in a suitable alternative to the prepreg approach permitting high finish quality and controlled fiber directions. RTM reduces voids compared to hand lay-up so increasing component mechanical properties. In addition, component design by RTM process can compete with metal one when prepreg cannot be applied to manufacture a product. Hand lay-up requires a low initial investment, but it becomes more expensive (cost per product) than the other techniques due to recurring costs associated with direct labor and material waste. Compared with compression molding (CM), RTM requests lower tooling costs because of the absence of a press system to compact the preform. RTM seems to meet both low cost/high volume requirements of the automotive industry (500 to 50,000 parts per year) and low number/high performances (50 to 500 part per year) of the aerospace industry. In fact, RTM can guarantee the demanded performances for the aeronautical production: reduction of the mass, increase of the operating life, aimed design, reduction of the production times. Today an increasingly number of parts realized using RTM is observed due to the development of new resins and the preforming technology. In addition, such technique is suitable, with only small adjustments, for the realization of large, complex and thick-walled structures for use in infrastructures and military applications.

\section{Overview of RTM process}

The RTM is a process with a rigid closed mold. Figure 1 summarizes the main steps for a simple case. The lamination sequence (preform) is draped in a half mold, then the mold is closed and the preform compacted. After that, the resin is injected using a positive gradient pressure through the gate points replacing the air entrapped within the preform. Usually, 
vacuum is applied at dedicated vents in order to favorite the air escape from the mold. When the resin reaches the vents, the gates are clamped and the preform is impregnated. At this point, the cure phase is considered to start. Finally, the mold is opened and the part removed. Especially for aerospace structures, an additional free-mold post-curing phase can be necessary in order to guarantee the polymerization of the matrix and release the internal thermal stress.

The closing mold step is characterized by the compaction of the fiber reinforcement, which permits to reach the desired thickness and design fiber volume fraction. The compaction changes the microstructure and the dimensions of the preform, producing large deformations and nonlinear viscoelastic effects. These effects are accompanied by a change in energy within the material, which causes the residual stresses due to the viscoelastic behavior of the fibers. However, during the impregnation phase a release of stress, probably due to the balance, occurs.

The injection phase must guarantee the complete impregnation of the preform: a bad impregnation of the fibers results in dry spot areas with missing adhesion between the layers, which makes the surface rough and irregular. If partial impregnation occurs in the proximity of a connecting zone among elements, it can cause a bad integration with a consequent loss of mechanical properties.

\section{MAIN STEPS OF RTM PROCESS}

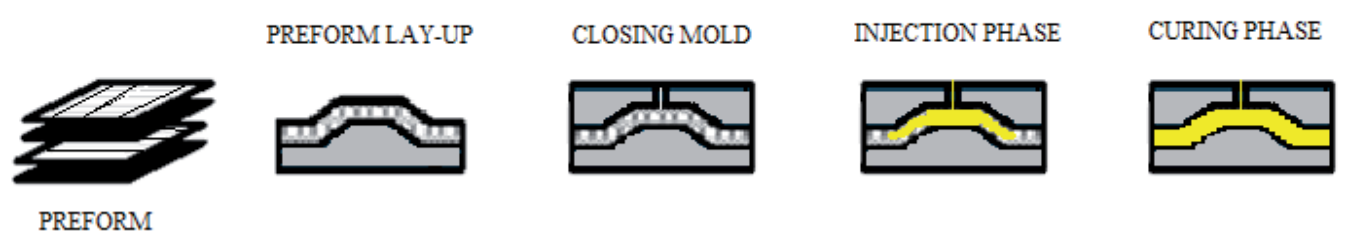

Figure 1. Sequence of the main steps of RTM process. From left to right: realization of the preform, deposition and draping of the preform in the mold cavity, closing mold, injection phase and curing phase; after the curing phase, the mold is opened and manufacred element is demolded.

\subsection{Process parameters}

The RTM process is governed by variables and parameters that are dependent on each other. Their combination affects the process and the quality of the finished product. Consequently, they need to be carefully determined. The most important parameters, which can not be neglected in the design, are pressure, temperature, viscosity, permeability, volume fraction, and filling time of the process. There are also a multitude of parameters that must be considered independently, such as the angle of attack of the nozzle, the orientation of the fibers, the paths of flow and shear rates, the stratification. In fact, the resin tends to flow more quickly in the fiber direction, thus the flow dynamic depends mainly on the type of fabric used and the number of overlapped layers. Sometimes it may be necessary to have a certain number of skins, not for structural reasons, but to obtain a homogeneous 
distribution of the resin. The thickness of the part to be manufactured can also affect the flow progress and the impregnation of the fibers, causing a high percentage of voids and dry spots. The thickness becomes a critical design constraint especially in the case of the inclusion of reinforcements and ribs.

The injection pressure determines the injection velocity of the resin into the mold, the hydraulic pressure and the holding and closing forces of the mold. Consequently, the injection velocity defines the filling time, which should not be too short to ensure an adequate impregnation of the fibers and, at the same time, the filling must be such as to avoid the risk of incurring in premature gelation of the resin. The injection pressure adjusts the distribution of the resin on the preform, which affects the formation of air voids in the matrix, the appearance surface and the mechanical properties of the finished product. Another phenomenon in which this parameter is relevant, together with the viscosity, is the so-called "fiber wash", i.e. the movement of the reinforcement inside the mold during the injection phase. In this case, the surface treatment of the fibers and especially the choice of the binder play a fundamental role. If the binder dissolves too quickly in contact with the resin, then fibers under the injection pressure can move freely.

The temperature is an extremely important process parameter and it is strictly related to the injection pressure and the viscosity of the resin. When the temperature increases, the filling time decreases and the working pressures are lower. When the temperature is low, the viscosity of the resin increases and it is necessary to increase the pressure to ensure the transfer of the resin itself.

\section{Preform technology}

As previously mentioned, the diffusion of RTM process in the aerospace industries is strictly related to the possibility of building net-shape 3D-complex structures. This possibility is given by the development of the preform technology. Preform is prepared separately and constitutes the skeleton of the final product, greatly simplifying the molding operations and reducing the time and cost of processing. When the production volumes is medium-high, an industrialized preform permits to amortize in a relatively short time the cost of equipment, even in a limited production, as required in the aerospace industry.

The textile methods are numerous. The choice of one method over the other depends on several factors: the processability, the feasibility of the geometry, the desired mechanical properties of the molded part, and the cost of production. Obviously, the preform affects strongly the performance required by the final application. The choice of the architecture of the fiber reinforcement depends on the required performance of the composite structure and the characteristics related to the process, such as permeability, compressibility and drape.

The preforms are formed weaving yarns or rovings. The terms used for the fiber comes from the textile tradition: a single fiber is a filament; a set of fibers produced simultaneously is called strand. Several parallel strands can be rolled up like a ribbon, called "roving" or "tows", or twisted and united as a strand, which is named "yarn". Generally, the yarns are 
typically not too complex because the excessive torque reduces the possibility of penetration of the resin in the cavities. Besides, a slight twist of the yarn compacts and raises the formation of composites with high fiber content.

Strands and yarns can be processed as woven roving and cloth. The first type uses effectively the resistance of the fibers, but it can produce composites with high resin content as the roving is not compacted. Woven roving is used to rapidly produce thick composites, because resin can fill them easily. Cloth is slightly less resistant than the roving because of the slight damage that is produced by rolling the fibers and also due to the twisting of the fibers themselves. The cloth is well impregnated from the resin and, as it is compact, high fiber content is obtained.

The weaving process can produce a wide variety of forms. The ratio between the number of filaments in the transverse direction (weft) and the number of filaments in the longitudinal direction (warp) may vary from nearly pure unidirectional, in which the number of filaments of the weft is the minimum required to hold together the fabric, up to the case of equal number of filaments in the warp and weft. In addition, there are hybrid fabrics with carbon fibers in the warp and glass fibers as weft. The weaving method influences the properties of the composite. In flat woven, warp and weft overlap in an alternating manner. In particular, weaving the warp passes through a number of fibers of the weft. These fabrics are better suitable for complex shapes, and their strength and stiffness are slightly higher because the fibers are straight on average. There are also more complex forms of weaving, with an angle of intersection fibers different from $90^{\circ}$. These methods are used to produce biaxial and multi-axial weaves. The complex fiber architectures can be obtained with the weaving method by interlacing and knitting the fibers along the three spatial directions; an example is shown in Figure 2. Different bi-axial layers can be also stitched. The stitching method consists on darning the layers with fibers (Figure 2). These are automated techniques that realize complex shapes and 3-D junctions in place of bolts and rivets. From the mechanical point of view, these methods can increase the resistance to fracture of the structure and reduce the progress of cracks by adopting high strength fibers in the $\mathrm{z}$ direction. On the other hand, the stitching seams can produce local defects induced in the preform as a result of penetration of the wire and the needle. Further, the robotic system is very expensive and sometimes damage due to misalignment of fibers can occur.

The choice of the fiber reinforcement is carried out according to the mechanical requirements of the manufactured component. The types of fibers that can be used are many and with variable characteristics. During machining, the preform can be damaged, particularly when using glass fibers that are fragile and have a high friction coefficient (about 1 in the contact glass on glass). For this reason, immediately after production, the fibers are protected with a coating, "finish" or "size", which performs several functions: it acts as a binder to hold the fibers together, as a lubricant to reduce the coefficient of friction between fibers, and it improves the wettability of the fiber and the adhesion of the resin. Purpose of sizing is also to protect the fibers from the environment, mainly by moisture. These protective sizes are specific to each type of resin, since they must be dissolved from the resin itself. In the case of carbon fibers, the epoxy resin is also used as a protective finish. 
Braiding method is another interesting textile process for aerospace structures. The braiding has high level of conformability to complex shapes and it is especially suitable for conical and cylindrical geometry. It regards 2D and 3D preforms. The high level of process automation reduces scrap and labour costs. More advantages of the braiding technique compared to standard tape and fabrics are strength in third dimension, improving fatigue resistance, more efficient distribution of mechanical stresses and the possibility to consider inserts during the preform realization. Despite of these characteristics, the braiding process requires the use of a mandrel and high initial cost for the preform engineering.

An important target of any textile process is obtaining the desired fiber volume fraction that is given by the design. It is very difficult to determine precisely the volume of reinforcement that must match the structural design with the manufacturing aspects. If the fibers are too compacted or their content is excessive, there is no sufficient space for the passage of the resin and the filling time becomes longer. Generally, the optimum volume of fibers is $60 \%$ of the product. An increase with respect to this value may cause a bad distribution of the resin and a dramatic drop of the mechanical properties of the manufactured component.

3D WEAVING

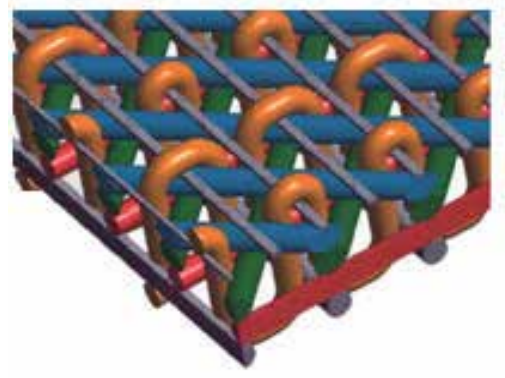

STITCHING

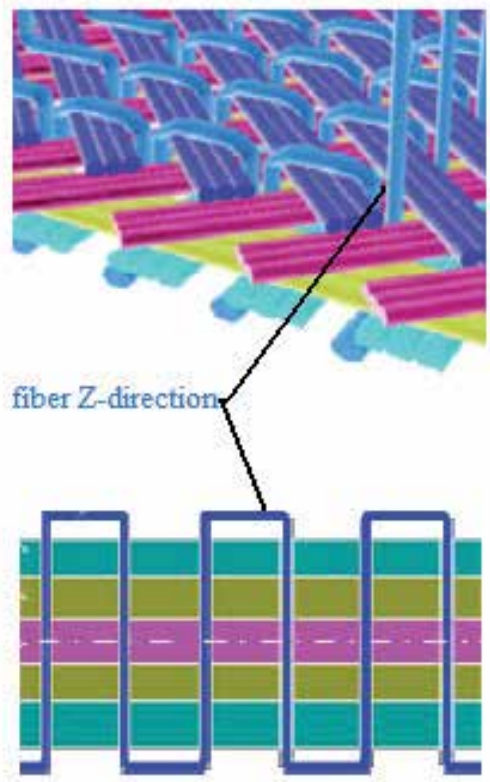

Figure 2. Examples of 3D weaving and stitching textile processes. 

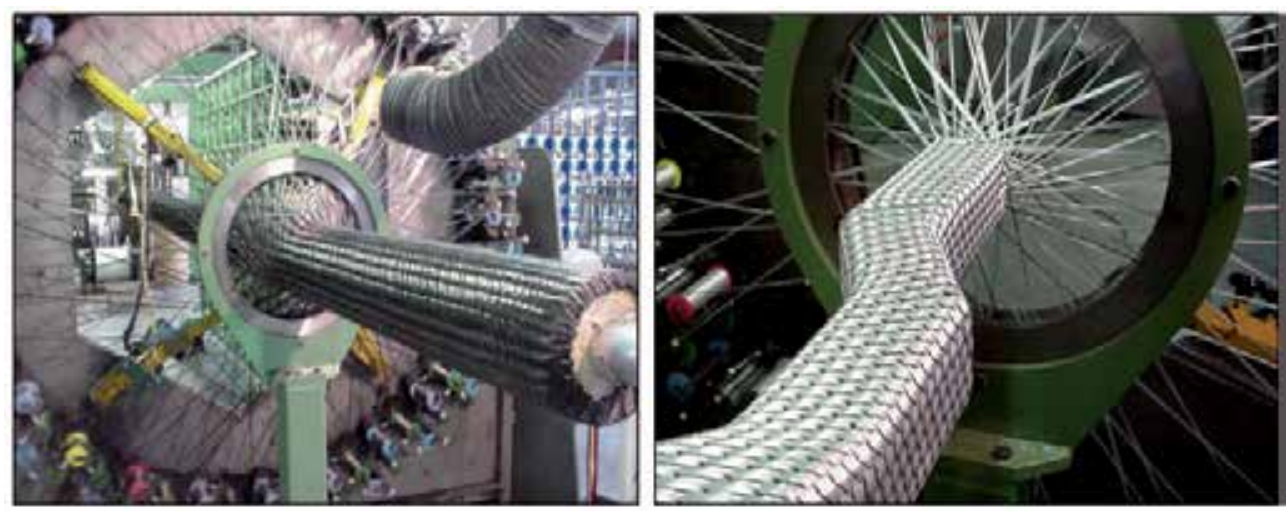

Figure 3. Braiding technology

\section{Resins in aerospace composites}

Thermosetting resins are the matrix used in RTM because of their low viscosity during the process. They are very rigid materials consisting of cross-linked polymers in which the motion of polymer chains is strongly limited by the high number of existing crosslinks. During the polymerization phase, thermosetting undergoes irreversible chemical change.

The selection of a reactive thermosetting resin for RTM application forces to deal with a large number of choices of chemical engineering, mainly due to a strong relationship between the chemistry and process engineering. In fact, the process parameters, such as temperature and pressure, cannot be selected without considering the chemistry of the resin to be used. Factors to take in consideration for a RTM system can be divided into two broad categories: processing and performance. Initial viscosity and molding life are function of the temperature, and they determine the operational temperature range of a process. The molding time is a function of the rate at which the reaction occurs between the resin and the curing agent and the rate is directly proportional to the temperature. The viscosity depends on the chemical-physical characteristics of the matrix. Viscosity may change over time because of both temperature variations and as consequence of chemical reactions that occur in the liquid state. The knowledge of the rheological behavior of systems is essential for a proper setting of process parameters. In fact, the values of the viscosity during the phases of the process must be such as to guarantee both the simultaneous removal of dissolved gases and moisture entrapped in the matrix, and the compaction of the fibers, before reaching the gel point. However, the viscosity of the resin must not be too high, especially in the case in which the fiber volume fraction is higher than $40-50 \%$.

Within thermosetting resins, several classes are suitable for aerospace applications of RTM, such as epoxy, phenolic, cyanate and bismaleimide. Phenolic resins are produced from the reaction of formaldehyde with phenol to give the condensation products. The reaction is always conducted in the presence of catalysts: these can be both acid and bases and their choice has a determinant role, together with the molar ratio of the reactants, and the nature of the reaction products. There are mainly two types of phenolic resins: resole and the novalacs. 
The base resins and formulated systems are available from Borden and Georgia Pacific. Approximately 91,000 tons of molded materials and phenolic are produced every year, but only a relatively small amount is used in composites for structural applications. A large number of industrial applications of phenolic resins are based on the excellent adhesive properties and strength. Especially when combined with appropriate reinforcements, phenolic resins have good chemical and thermal resistance, good dielectric strength and good dimensional stability over a wide range of temperatures. Materials produced with these resins have a very low flammability, are very resistant to creep, have low moisture absorption and have a remarkable resistance to degradation from a variety of lubricating fluids. Their low viscosity and the high char yield of these materials are used in many structures forming composite carbon/carbon. Phenolic resins are polymers that generate volatiles (mostly aqueous vapor) during the treatment phase. The volatile substances have a strong impact on the processability of these composite materials resulting in structural voids where the content can have a dramatic effect on the mechanical properties of the part. In addition, these polymers are generally brittle, characterized by a low elongation and a low tensile strength. Despite the relatively poor mechanical behavior and the difficulty of processability, these resins are becoming more applied in the structural field due to the low flammability and low smoke production. The only resins comparable to the phenolic for the properties of high fire resistance and low smoke generation are the bismaleimide resins, but they are at least an order of magnitude more expensive. The nature of the RTM process (closed molding) makes it difficult the implementation of this method due to the development of volatile substances typical of phenol in the curing phase. In order to overcome this problem clever methods have been developed. A particularly interesting approach is the use of a catalyst that allows gelation of the resin at temperatures below $100{ }^{\circ} \mathrm{C}$. So if the water is formed during the RTM process, but the temperature is maintained below the boiling point $\left(100{ }^{\circ} \mathrm{C}\right)$, the water will remain in the liquid phase and will act as a plasticizer. If the temperature increases above $100{ }^{\circ} \mathrm{C}$ in the initial stage of gelation in the curing phase, the water will evaporate and will leave the part producing a massive degradation. A high temperature cure achieved with a ramp rather slow through the $100{ }^{\circ} \mathrm{C}$ will lead the water out from the gelled structure and the cross-linking of the polymer occurs without degradation of the piece. Another issue to consider when phenolic resins are used in RTM is the acidic nature of many catalysts, which can cause corrosion in some materials for molds.

Epoxy resins are well known in the production of composite aerospace materials. The vast variety of epoxy and cure agents makes these systems very versatile in terms of manufacturing process and obtainable physical properties. Although in the last twenty years, a large innovative work in developing new formulations of epoxy resins was done, only a limited marketing of new resins was realized. When liquid epoxy resins such as DGBA and DGBF are used in RTM, they are usually part of a two-component systems. In this case the selection of the curing agent is really important. In the polyester and vinyl ester resins, the catalysts alter the cure time but do not have substantial effect on the viscosity and on the final properties of the polymer. In an epoxy system, the selection of the curing agent is crucial because it determines the thermal and mechanical properties of the matrix and defines the dependence of viscosity from the temperature, thus controlling the processability of the system. Epoxy resins 
polymerize with many materials such as polyamines, polyamides, phenol-formaldehyde, urea-formaldehyde, and acid anhydrides. The reactions taking place can be coupling or condensation reactions. DETDA is a liquid aromatic amine which is widely accepted as the primary hardener in many RTM formulations. DETDA is liquid at room temperature and provides good processability in RTM, both in a single-component system and bi-component one. The slow curing of epoxy system with DETDA allows these systems to be processed in a wide temperature range. The inclusion of a catalyst in the formulation of a DETDA significantly increases or decreases the reaction rate and lowers the cure temperature. The DETDA produces a polymer with high glass transition temperature (generally $\mathrm{Tg}>177^{\circ} \mathrm{C}$ ) when fully cured. The Young's modulus is usually less than $3.1 \mathrm{Gpa}$. Therefore, the use of a liquid aromatic amine such as DETDA provides an excellent processability with high glass transition temperature but with a very low value of the elastic modulus for space applications.

The aliphatic and cycloaliphatic amines are useful agents for treatment of many epoxy resins. These materials are almost all liquids with low viscosity (as DACH, IPDA, PACM, etc.) that are readily soluble in the formulation of epoxy resins. In these cases, the thermal and mechanical properties are inferior to those obtained with the aromatic amine. With these agents, the glass transition temperature is in the range $121^{\circ} \mathrm{C}-177^{\circ} \mathrm{C}$, and the elastic modulus of the polymer is in the range $2.4 \mathrm{GPa}-3.1 \mathrm{GPa}$. Anhydrides were widely used for many years as agents for epoxy polymerization in applications of filament winding. This class of catalysts has not received much attention in RTM applications. The liquid nature of most of the commonly used anhydrides (MTHPA, NMA, etc.) and their good solubility with the epoxy indicate that they could be used in RTM monocomponent and bicomponent systems. The availability of a large range of hardeners for epoxy-anhydride makes them available for systems that can meet specific process requirements. Generally, the formed polymers have a glass transition temperature of approximately $140{ }^{\circ} \mathrm{C}-150{ }^{\circ} \mathrm{C}$ and a modulus of $3.45 \mathrm{GPa}$, tensile elongation of $3 \%-5 \%$ and moisture absorption of $1.5 \%$. The curing phase with an anhydride is relatively complex. The mechanism of cure can have several important consequences, such as low moisture absorption and in many cases a high final operative temperature. When anhydrides are exposed to moisture, usually over a long period of storage in a humid environment, they form acids. These acidic components interact with the basic catalysts inhibiting the polymerization, lowering the values of the modulus and the glass transition temperature, and increasing the absorption of moisture. Epoxy systems that have the greatest success in the aerospace market are the monocomponent ones. There are substantial reasons for the success of these systems, such as ease of use, quality control of both process and materials and the excellent thermal and mechanical properties. In general, epoxy resins combine incomparable properties of flexibility, adhesion and chemical resistance.

The cyanate resins have a relatively small niche in the production of composites. The very high glass transition temperature and the excellent mechanical properties of the polymer are the primary guides to their use. The relatively high cost of these systems (150 - 500 U.S. \$ per kilogram) prevents the entry into any market that is not closely related to their required application. This resin is generally cured with the addition of heat and transition metals as catalysts [Co(III), $\mathrm{Cu}(\mathrm{II})$, etc.]. The gelation occurs at about $50 \%-60 \%$ conversion, similarly to 
the chemistry of an epoxy resin. The coefficient of thermal expansion is relatively small, about $50 \mathrm{ppm} /{ }^{\circ} \mathrm{C}$. Cyanate resins are often formulated with epoxy resins or maleimides in order to modify the processability and properties of the resulting polymer. Generally, the formulations include salts of transition metals and phenolic species as catalysts. There are very few formulations originally formulated for RTM. Usually cyanate resins produce a polymer with high transition temperature, low moisture absorption, good mechanical properties and excellent electrical properties. The use of them in the structures and radomes is driven by the needs of a high transition temperature coupled with a low dielectric constant and low dissipation factor to prevent degradation by high energy radiation transmitted and received through the structure. Some formulations have a flat response for both dielectric characteristics and dissipation in a wide range of temperatures and wavelengths of electromagnetic radiation. For these reasons, cyanate resins are widely used in aerospace fields. In particular, composites made by carbon fibers and cyanate resins are used in satellites for very rigid structures, with a high transition temperature and low absorption of moisture that can withstand to repeated thermal cycles without failure due to internal stresses.

Bismaleimide resins (BMI) currently provide a market niche in the manufacture of composite structures, i.e., for those parts which require a very high glass transition temperature, good stability and thermal oxidation and low flammability. The relative high cost of BMI resins (44-260 U.S. \$ per kg) limits the applications to advanced ones. The BMI are produced by a reaction of aromatic bi-ammine precursor with maleic anhydride. The resulting resins are cured with heat and in general without additional catalysts. The unmodified BMI are fragile materials with failure strain less than $2 \%$. In order to make these systems more resistant, modified mixtures with amines, monomers, vinyl or epoxy resins are formulated. The polymers formed in this way are reasonably resistant, but with the increasing of post-cure temperatures the fragility increases. A typical BMI resin is produced by the reaction of methylene dianiline (MDA) with maleic anhydride, using heat to remove the water produced and push the reaction to completion. A variety of process features can be obtained by changing the properties of the molecular backbone of BMI resin and adding co-reactants. The use of BMI is driven primarily by their exceptional performance at high temperatures, in particular by their ability to maintain mechanical properties at more than $149^{\circ} \mathrm{C}$ to below the saturation moisture. The other characteristics that lead to the use of BMI are their good electrical capacity, long term stability to the thermal oxidation for higher temperatures up to $177{ }^{\circ} \mathrm{C}$ and the exceptional capacity to not generate smoke when exposed to high heat fluxes. These characteristics at high temperatures push to use these resins in the aerospace field, although at present their main application is the manufacture of electrical circuits in high temperatures. One disadvantage that seems to be common to all BMI resins is the very long time of cure. The desired properties at high temperatures are achieved only by using a high temperature post-cure. The high temperature cure and postcure cycles lead to somewhat brittle polymers with a significant amount of residual stresses. The high temperatures of curing, accompanied by a significant shrinkage, often lead to the formation of microcracks. To minimize the problems of residual stress, slow processing rates especially during the cooling phase are used. 


\section{Effects of manufacturing on final product}

It is well known that the manufacturing process influences the quality and therefore the performance of the product. For instance, a good surface finish quality plays an important role in the mechanism of composite degradation upon exposure to the operative environment. The surface finish prevents the penetration of elements, such as dust, that produce and enhance micro-cracks within the structure. As consequence, a loss of mechanical properties occurs. Figure 4 shows the stages of the process that influence the behavior of the material and summarizes the relationship among process, material behavior and final performance. The compaction and impregnation phases govern the imperfections due to voids and dry spots. Following sections of this paragraph describe the compaction and impregnation phases.

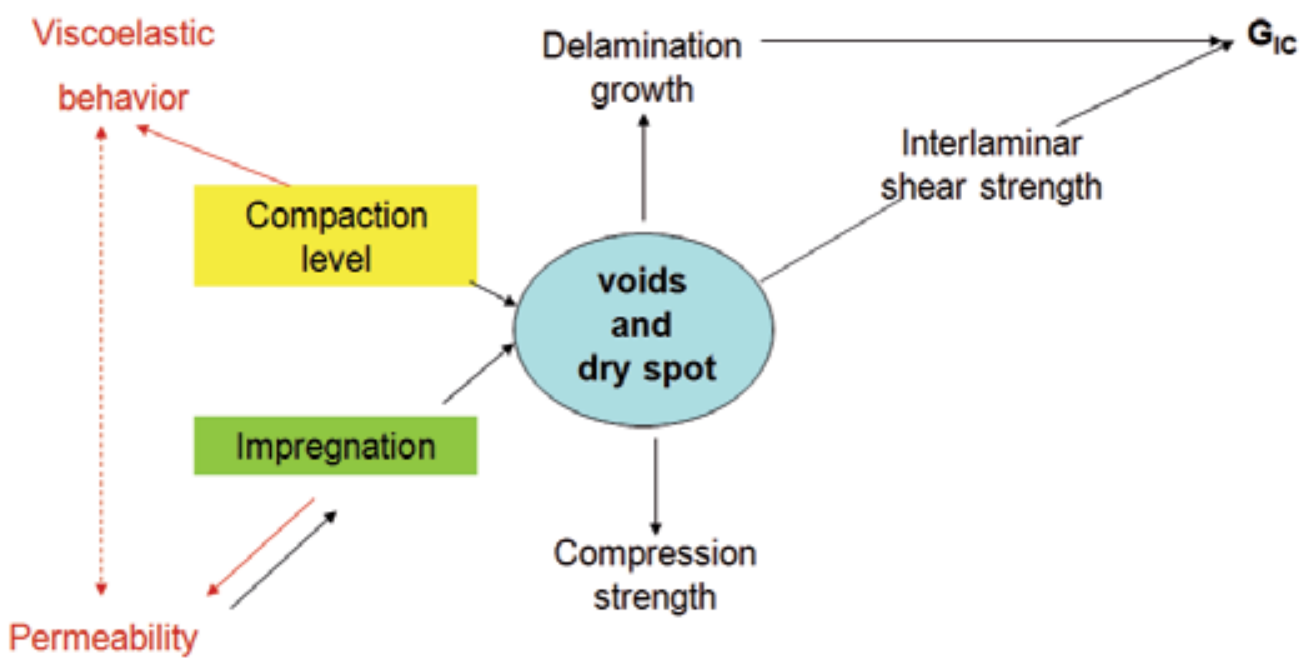

Figure 4. Relations between manufacturing phases and material behavior.

\subsection{Compaction phase}

The compaction phase is an important step that occurs in any production process for the manufacture of advanced composite structures. This phase usually takes place through the application of an external pressure, which produces a new arrangement of the fibers and changes of microstructure. In RTM, the compaction occurs when the mold is closed and the preform reaches the expected fiber volume fraction. Compaction produces large deformations and nonlinear viscoelastic effects in the preform. These effects are accompanied by a change in energy within the material. Some authors have modeled the phenomenon, introducing the function of free energy, but with limited success, because this hypothesis does not take into account permanent deformations of the preform. For instance, in case of fabric textile, when a multilayer preform is compacted, the fibers tend to be squashed with other fibers, producing interlayer packing or nesting phenomena depending on the deformation mode and the fibers architecture. If the compression load is removed, fibers tend to not return to their original position inside the preform. 
Nesting is a phenomenon that produces irreversible mechanical and geometric changes of the preform modifying the permeability and mechanical properties of the composite material. In particular, the nesting influences the rigidity of the piece and even more the resistance. In particular, the stiffness depends on the positioning of the layers. If the layer is positioned out of phase without reducing the thickness (interlayer packing), there is no change of the mechanical properties. On the other hand, if two layers are in phase, i.e. in case of existing nesting, the difference of stiffness between the two configurations is reduced approximately of $10-20 \%$. Nesting creates the so-called bridging between layers preventing delamination and increasing the resistance considerably, up to $10 \%$ for the layers out of phase. Moreover, the compaction induces residual stresses within the material due to the viscoelastic behavior of the fibers. The stress concentrations, also localized, can act as nucleation of cracks after the curing phase. However, during the impregnation phase a release of stress, probably due to the balance, occurs.

From a manufacturing point of view, it is evident that compaction changes the spatial arrangement of the fiber bundles, modifying substantially the morphology of the porosity. These variations alter the permeability of the preform and so the impregnation phase. Furthermore, compaction affects the adhesion between the layers, the material failure modes and the magnitude interlaminar shear stress more than the impregnation.

\subsection{Impregnation phase}

In RTM applications, the defects induced by the resin flow such as voids and dry spots are known as the biggest source of problems for production quality and reproducibility. The damage of the matrix is essentially related to the presence of porosity. The formation of micro-voids among fibers and dry spots are potential starting points for the propagation of cracks and delamination.

The resin flow front faces two resistance levels through the preform: the resistance between the fiber bundles and the resistance inside the fiber bundles. This means that the preforms are characterized by two different permeabilities: the permeability between the fiber bundles and the permeability inside the fiber bundles. These can be considered as a resin flow in macro-scale and micro-scale. The type of flow scale determines the potential void formation.

In the macro-scale, the formation of macro-voids can be formed when the air displaced from the resin remains trapped, or when pressure is insufficient to overcome the resistance of the preform or the viscosity is too high. In micro-scale, the porosity is given by micro-voids. Figure 5 shows the mechanism of void formation in the fiber bundle: the flow front runs around the tows and continues to impregnate the tow even after the passage of the front. If the air is not evacuated at the beginning of the filling process, it remains trapped in the tow and micro-voids occur at the center. Micro-voids formation can be also due to the macroscopic pressure drop. The pressure drop produces an apparent change in the permeability along the preform. The pressure drop can be explained by assuming the sink 
effect: the fiber bundles act as fluid sinks. The concept is based on the dual scale porous media. The individual fibers of the bundle are separated by a distance much smaller than that existing between two bundles. Consequently, the resin flows more easily between the fiber bundles rather than inside one of them. Therefore the liquid continues to impregnate the bundle even when the front part of the flow has passed. This means that a part of the injected fluid penetrates in a package of fiber, rather than push forward the front face of the flow. The result is that the pressure profile is influenced by the relative flow rate of the resin inside a bundle relative to the flow between bundles.
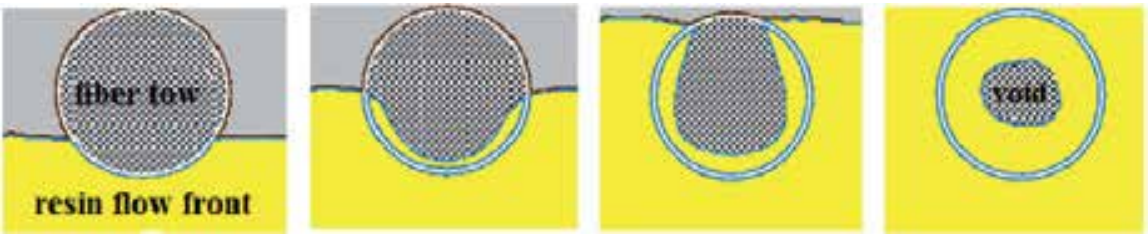

Figure 5. Mechanism of void formation in the intra-bundle.

\section{Modeling of RTM process}

In the RTM process, the resin flow is usually modeled with the assumption of isothermal flow, ignoring the exothermic nature of the thermosetting resin. Basing on this hypothesis, the resin flow is described by the conservation equation of mass and momentum with the boundary conditions. In order to complete the equation system, the constitutive equations of the fluid must be considered. The following section concerns the continuity and momentum equations of a resin flow within a region consisting of fibers and porosity (Figure 6).

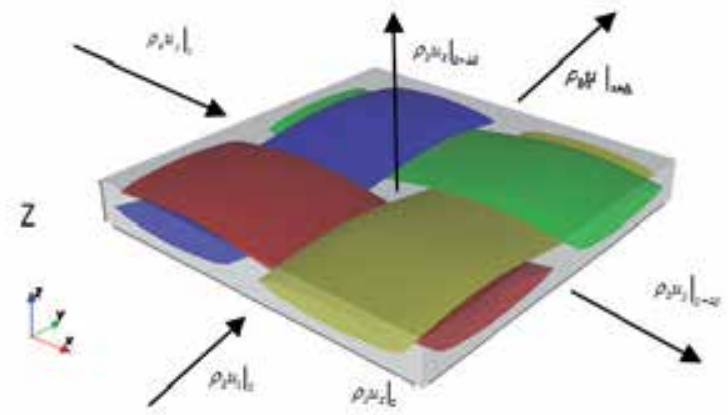

Figure 6. Control volume of porous media.

\subsection{Flow model}

The fiber reinforcement is a dual-scale porous material and to apply the conservation of momentum is an unrealistic approach. In fact, determining the pressure profile during the impregnation means to apply the Navier-Stocks equation for each channel of fibers network and solve the equation systems in their surroundings. This would allow to know the flow 
rate and the pressure within each channel. Anyway, a simple piece of preform may have millions of channels. This approach is impractical in a real case. Furthermore we have no interest in knowing the exact value of the pressure during the manufacturing process, but we need to know the relationship between pressure drop and flow rate during the progress of the flow front in the preform at a macroscopic level. For these reasons, the resin flow front is modeled by the Darcy law, considering that Reynolds number relative to the pore size is $\leq$ 1. Darcy equation (Eq. 1) describes the relationship between the flow and the pressure gradient that drives the flow through the porous medium, using the permeability parameter. This parameter, as discussed after, characterizes the fluidity of the resin through the average porosity of the fibers.

$$
\boldsymbol{u}=-\frac{\boldsymbol{K} \cdot \nabla P}{\mu}
$$

where $u$ is the speed mediated on the volume, $\mu$ is the viscosity of the fluid, $\nabla P$ is the pressure gradient and $K$ is the permeability tensor of the preform (according to the fact that fiber arrangement generally is anisotropic in porous medium).

Rewriting Eq. 1 in expanded form:

$$
\left(\begin{array}{l}
u_{x} \\
u_{y} \\
u_{z}
\end{array}\right)=-\frac{1}{\mu}\left(\begin{array}{lll}
K_{x x} & K_{x y} & K_{x z} \\
K_{y x} & K_{y y} & K_{y z} \\
K_{z x} & K_{z y} & K_{z z}
\end{array}\right)\left(\begin{array}{l}
\frac{\partial P}{\partial x} \\
\frac{\partial P}{\partial y} \\
\frac{\partial P}{\partial z}
\end{array}\right)
$$

In most RTM applications, the flow can be approximated in two-dimensional domain, since the dimensions in the plane are two or three orders of magnitude larger than the thickness. The approximation of a 3D geometry with a 2D one gives significant saves in terms of computational time and reduces the number of material parameters that need to be measured or calculated. This assumption can be considered valid since the values of permeability in the plane of the preform (in the plane in which the layers are stacked to form the thickness) are approximately of the same order of magnitude. Boundary conditions are imposed on three segments:

- injection gates

- free surface of the resin flow (i.e. on the flow front)

- $\quad$ walls of the mold.

The resin is injected into the mold at constant pressure or at constant flow rate, for which the gate conditions are

$$
P=\text { constant }=P_{i n j}
$$

or

$$
A\left(-\frac{\boldsymbol{K} \cdot \nabla P}{\mu}\right) \cdot \boldsymbol{n}=-Q_{i n j}=\text { constant }
$$


Some injection systems allow to vary the values of pressure or flow rate during the same injection. In such case, $P_{i n j}$ and $Q_{i n j}$ must be considered as a function of the time. This change does not alter the technique of solution, because the problem is solved as a stationary one for each time.

The pressure at the resin front flow $P_{f f}$ can assume the value of the atmospheric and vacuum depends on the type of process, generally:

$$
P_{f f}=P_{\text {vent }}
$$

Inserts or multiple injection points can form several different resin flow fronts. When these fronts meet and they are not connected to a vent, the air moved by them remains trapped in the regions surrounded by the resin flow fronts. In this case, the boundary conditions on the front can be modified to consider that the pressure increases on the front due to the void pressure. The trapped air can be considered as an ideal gas for which the following relation applies:

$$
\frac{P_{\text {void }} V_{\text {void }}}{T_{\text {void }}}=\text { constant }
$$

However, this would require knowing a plot of the vacuum created during the impregnation of the fronts and calculate the volume at each instant of filling. In the real case, a part of this air is dissolved in the resin, reducing the pressure of the vacuum. In order to take into account this phenomenon, the system may include an equation of the resin diffusing at the mass-to-air interface and the diffusion coefficient has to be determined. But this is not a significant factor and researchers ignore this aspect and assume that the air will dissolve in the resin.

The boundary condition on the wall of the mold is the no-slip condition:

$$
u_{n}=-\frac{1}{\mu}\left(K_{n n} \frac{\partial P}{\partial n}+K_{n t} \frac{\partial P}{\partial t}\right)=0
$$

where $n$ and $t$ are the directions normal and tangential to the mold wall.

The continuity equation of resin flow through fibrous arrangement can be determined assuming that:

- $\quad$ fibers are incompressible

- variations of resin velocity and stress-strain are small in the volume control

- $\quad$ process is quasi-steady

- body forces, such as weight, are negligible.

The derivation is quite similar to that for any fluid within a region, except for the expression of the resin density $\rho_{b}$ that is the mass of the resin divided the control volume containing both resin and fiber. In this case, $u$ is the interstitial resin velocity within the composite. Within a fibrous region partially filled is $\rho_{b}=\varepsilon S \rho$ where $\varepsilon$ is the porosity of the control volume available to be occupied by the resin, $\varepsilon=$ (pore volume/volume control), which can be expressed as $\varepsilon=1-V_{f}$, where $V_{f}$ is the volume fraction of the fiber. $S$ is the fraction of 
saturation of a porous space filled by the resin, and $\rho$ is the true density of the resin (mass of the resin/volume of resin). The pore volume within the volume control is completely empty when $S=0$, and completely full when $S=1$.

The mass balance for the volume control can be expressed as:

$$
\text { (net flow of mass) }=(\text { inflow })-(\text { outflow }) \text { - (losses) }
$$

The term of the losses is due to an internal volume $\mathrm{V}$ that absorbs fluid mass in a quantity equal to $(x, y, z, t)$ per unit volume and unit time (i.e. the fiber volume). Dividing both sides by $\Delta x, \Delta y$ and $\Delta z$ and then doing the limit for $\Delta x, \Delta y$ and $\Delta z \rightarrow 0$, the mass balance becomes:

$$
\frac{\partial(\varepsilon S \rho)}{\partial t}+U \cdot \nabla(\varepsilon S \rho)+(\varepsilon S \rho) \nabla \cdot U=S
$$

It is noted that even if the resin density $\rho$ is constant, the porosity or saturation of the fibrous medium may not be constant within the whole region. Therefore, it cannot be zero everywhere.

\section{Experimental determination of permeability}

The permeability $K$ is in general a symmetric tensor, which for an isotropic material, as random mat, is a scalar number. For a given stationary porous medium, it is necessary to know 6 scalar values $K_{j i}$ to completely determine the tensor $K$. If the selected directions of the reference system are along the principal directions of the preform, the matrix becomes diagonal. Therefore, choosing the coordinate system along the main axes of the preform, the principal values of the permeability can be measured. Then, not diagonal terms can be calculated using a coordinate transformation system.

$$
\boldsymbol{K}=\left(\begin{array}{lll}
K_{x x} & K_{x y} & K_{x z} \\
K_{y x} & K_{y y} & K_{y z} \\
K_{z x} & K_{z y} & K_{z z}
\end{array}\right)
$$

Permeability must be determined experimentally. There are basically two methods for this: radial flow and linear flow methods. The simplest way to determine the permeability is the use of 1D version of Darcy's equation (linear method). For a 1D flow in the direction of the axis (assumed to be the x-axis) Darcy's equation can be written as

$$
\frac{d x}{d t}=-\frac{K_{x}}{\Phi \mu} \frac{\partial P}{\partial x}
$$

Where $\Phi=1-\mathrm{V}_{\mathrm{f}}$ is the porosity of the material and $\mathrm{V}_{\mathrm{f}}$ is the fiber volume fraction, $K_{x}$ is the permeability in the $x$ direction, $\mu$ is the fluid viscosity and $\partial P / \partial x$ is the pressure gradient between the injection point and the flow front. Since the injection pressure and the vent pressure are constant, we can transform the partial derivative in a finite difference, so we have: 


$$
\frac{d x}{d t}=-\frac{K_{x}}{\Phi \mu} \frac{\Delta P}{x}
$$

Integrating between the position of the injection point $(x=0)$ at time $t=0$ and the position of the flow front $(x=x)$ at time $t=t$, the expression that allows to determine the permeability along a main direction of the preform is:

$$
K_{x}=\frac{\mu \text { Фslope of }\left[x^{2}{ }_{f}\left(t_{f}\right)\right]}{2 \Delta P}
$$

The slope of the line is calculated by plotting the position of the flow front at different times; the pressure gradient is equal to the injection pressure. In the case of orthotropic laminates, the permeability has different values in the two principal directions, and then two experiments sets must be performed.

For typical composites, the thickness is on average $2-3 \mathrm{~mm}$. As a consequence, the transverse permeability $\mathrm{Kz}$ is considered negligible. This means to assume that the resin flow advances uniformly on the two external surfaces (top and bottom) and that flow velocity is an average through the thickness. However, as the thickness increases, the gradient along $\mathrm{z}$ (i.e. the thickness) becomes greater, due to the fact that the resin flows quickly on the upper surface, while it continues to impregnate the lower levels. Advani et al. have developed a theoretical model to calculate an average effective permeability for flat rectangular preform with thickness $H$ formed by $n$ layers.

$$
\begin{gathered}
\bar{K}=\frac{\bar{l}}{H} \sum_{j=1}^{n} \frac{K_{j} h_{j}}{l_{j}} \\
\bar{l}=\frac{1}{H} \sum_{j=1}^{n} \frac{l_{j} h_{j}}{n}
\end{gathered}
$$

where $h_{j}$ is the thickness of the single layer, $l_{j}$ is the length of the impregnation of the single layer in the injection direction, $\bar{l}$ is the average length of impregnation in the entire laminate, $K_{j}$ the permeability of the single layer.

\section{Numerical analysis approach}

The advantage of the numerical simulations is to help the process engineer to understand the behavior of the resin inside the mold, especially when the part geometry is complex and presents variations in permeability and fiber volume fraction. This knowledge improves the design tools and optimization of the injection scheme. General method is to use a simplified model of the resin flow reducing the problem from 3-D to 2-D and using a finite element approach and control volume $(\mathrm{FE} / \mathrm{CV})$ that does not require a re-meshing at each step. The geometry is discretized as a thin shell using triangular elements or rectangular ones. The material properties such as thickness, permeability and volume fraction of fibers can be 
assigned individually to each element, such as the non-uniform material properties and thickness. A linear pressure profile is assumed among the nodes of an element:

$$
P^{e}=\sum_{i=1}^{n} N_{i} P_{i}
$$

where $\mathrm{n}$ is the number of nodes of the element, $\mathrm{P}^{e}$ is the pressure inside the element, and $\mathrm{P}_{i}$ is the nodal pressure that is unknown and a function of interpolation. The Galerkin finite element method can be used to convert the governing equations to the partial derivatives in a system of algebraic equations that minimizes the error at the nodes. It can be expressed as a linear system of equations

$$
\left[\left[S^{e}\right]\right][P]=[f]
$$

where $S$ is the stiffness matrix whose components are:

$$
S_{i j}^{e}=\int_{\Omega}\left(\frac{K_{x x}}{\mu} \frac{\partial N_{j}}{\partial x} \frac{\partial N_{i}}{\partial x}+\frac{K_{y y}}{\mu} \frac{\partial N_{j}}{\partial y} \frac{\partial N_{i}}{\partial y}+\frac{K_{x y}}{\mu} \frac{\partial N_{j}}{\partial x} \frac{\partial N_{i}}{\partial y}+\frac{K_{x y}}{\mu} \frac{\partial N_{j}}{\partial y} \frac{\partial N_{i}}{\partial x}\right) d \Omega
$$

This set of equations can be solved in every moment during the filling process. The $i$-th vector component is the amount of mass generated per unit time at the $i$-th node. The pressures are calculated at nodes by solving the set of algebraic equations described by the system.

The next step is the determination of the resin flow front progress. The approach by the control volume consists of dividing the geometry under consideration into control volumes first, and then associating one of them to each node. The flow between two control volumes is calculated by multiplying the average speed for the area connected between two control volumes. For example, the equation of the flow associated with the node $i$ and the one associated to node $\mathrm{j}$ is

$$
q_{i j}=-\int_{s_{i j}}\left(\frac{h}{\mu} \boldsymbol{n} \cdot[\boldsymbol{K}] \cdot \nabla P\right) d s
$$

where $s_{i j}$ is the boundary between two control volumes, $h$ is the thickness of the mold cavity at the contour and $n$ is the normal to the contour of the plane lying of the preform.

The nodal fill factor is used to track the movement of the flow front. This factor is associated with each node and represents the fraction of the volume occupied by the control fluid. The pressures are therefore determined only at the filled nodes and empty nodes are ignored. The nodes are considered partially filled close to the flow front. The progress of the flow front is considered at any instant of time $t$, updating the fill factor of the control volume by means of the flow between the connected nodes. Consequently, the amount of the masses is strictly taken into account. This technique can simulate the resin flow in thin cavities with high geometric complexity in 3-D. Usually the simulation process is assumed to be isothermal, because of the disadvantages in conducting non-isothermal numerical simulations. First of all, the computational efficiency and speed of convergence decrease drastically when the equations that govern the flow are solved simultaneously with the energy equation, especially with the increasing of the number of nodes. Research in the simplification of non-isothermal analysis reduced the time, but it still requires a CPU time of 
two or three orders of magnitude higher than the resolution of isothermal problems. Moreover, to obtain the results non-isothermal analysis requires a large number of material parameters, such as dispersion coefficient, thermal conductivity and kinetics of resin cure. For high temperature resins, such as in case of aerospace application, a good compromise would be to fill the mold under isothermal conditions, and at least considering the kinetics of cure. From a numerical point of view, this solution is very efficient because diffusive and convective problems are not faced.

\section{Aerospace applications}

Developing of a new composite product requires a synergy among different disciplines and sometimes entities, in other terms a Concurrent Engineering (CE) approach. This section shows some aerospace components manufactured by RTM process in CE method. The prototypes presented in the following sections are the results of years of collaboration between the School of Aerospace Engineering (currently DIAEE) of Sapienza University of Rome and Italian aerospace industries (AgustaWestland and Aermacchi).

\subsection{Concurrent engineering approach}

The need of high quality production, due to market pressure and high-tech demands of the aerospace field, drives to the faster and most effective design of the product. To this aim, engineers adopt new methods based on concurrent approaches to optimize the part before manufacturing the final piece. Especially from 1990s, with the explosion of the composite materials market in several industrial fields, involved people from industry and universities are working to determine the best way to apply the concurrent engineering as a systematic approach for product development. Some works are addressed to improve the management team and communication among members; others go toward building a virtual environment based on CAD systems.

The software systems for automation design tools are powerful. They can help any company to develop products of superior quality, faster and at lower cost. This implies clear advantages in terms of competitiveness. The empirical methods "test and fix" still dominate the field of molding technologies for composite materials, but this approach produces deleterious effects in terms of cost and time. Simulation studies lead to the complete optimization of the product before creating the prototype. The objective is to establish the optimal characteristics of the preform and tooling, optimizing the process parameters.

The targets that can be obtained with the use of a code are:

- reduction of design time

- reduction of the costs and consumption of materials

- $\quad$ optimization of the design parameters in relation to the process

- reduction of the times of modification and tuning of the molds

- integration between production process and optimization of the component with respect to constraint and loading given by specification. 
About the entities involved in a project, concurrent-development teams typically exhibit the following characteristics:

- $\quad$ they include no more ten members

- members choose to serve on the team

- members serve from the beginning to the end of the project

- members participate in the team fulltime

- members report solely to the team leader and the leader reports to general management

- $\quad$ key functions - at least marketing, engineering, and manufacturing - are included in the team

- members are co-located within conversational distance of each other

The plan should give specific information about the qualification programs regarding all the entities involved in the RTM process: materials, process, tooling, tests.

Figure 7 shows the flowchart related to the CE approach set by the DIAEE of Sapienza University of Rome during the development of several RTM helicopter components. The flowchart highlights the structural and manufacturing couplings. Process parameters have been considered at the early stage of the design as a discrimination of the material selection. The resin flow behavior and the filling time of the mold were the process criteria. These parameters are directly dependent on the preform characteristics such as the textile structure, the laminate orientation and the fiber volume fraction. The preform characteristics are the first features that determine the mechanical performance of the component. In this sense, the optimized project is a compromise between structural and process optimizations and requires a concurrent engineering design and manufacturing.

\subsection{Strake}

The strake is a typical aerodynamic surface used in both supersonic and subsonic vehicles to improve the stability and/or reduce the drag. The thickness of the structure is around 2-3 $\mathrm{mm}$ and usually made in aluminum alloy. In this case, the selected resin was a monocomponent benzoxazine polymer (Henkel Epsilon 99110). The recommended process conditions were typical infusion temperature at $90{ }^{\circ} \mathrm{C}$ and curing at $180{ }^{\circ} \mathrm{C}$ for $90 \mathrm{~min}$. The fiber reinforcement used was a carbon textile HEXCEL G0926 5H Satin. The preforms were realized manually overlaying several layers adopting a predefined lamination sequences. The lamination sequences were determined by structural analysis. The injection equipment was a commercial Hyperject machine for mono-component polymers. The Hyperject injects the resin at constant pressure and it is provided with heated dispending resin. The resin was loaded, degassed and heated inside the Hyperject. When both the temperatures of the resin and the mold were reached, the resin was pumped inside the mold until the resin came out from the vent. Molds were studied to be multi-components in order to make easily the demolding. 


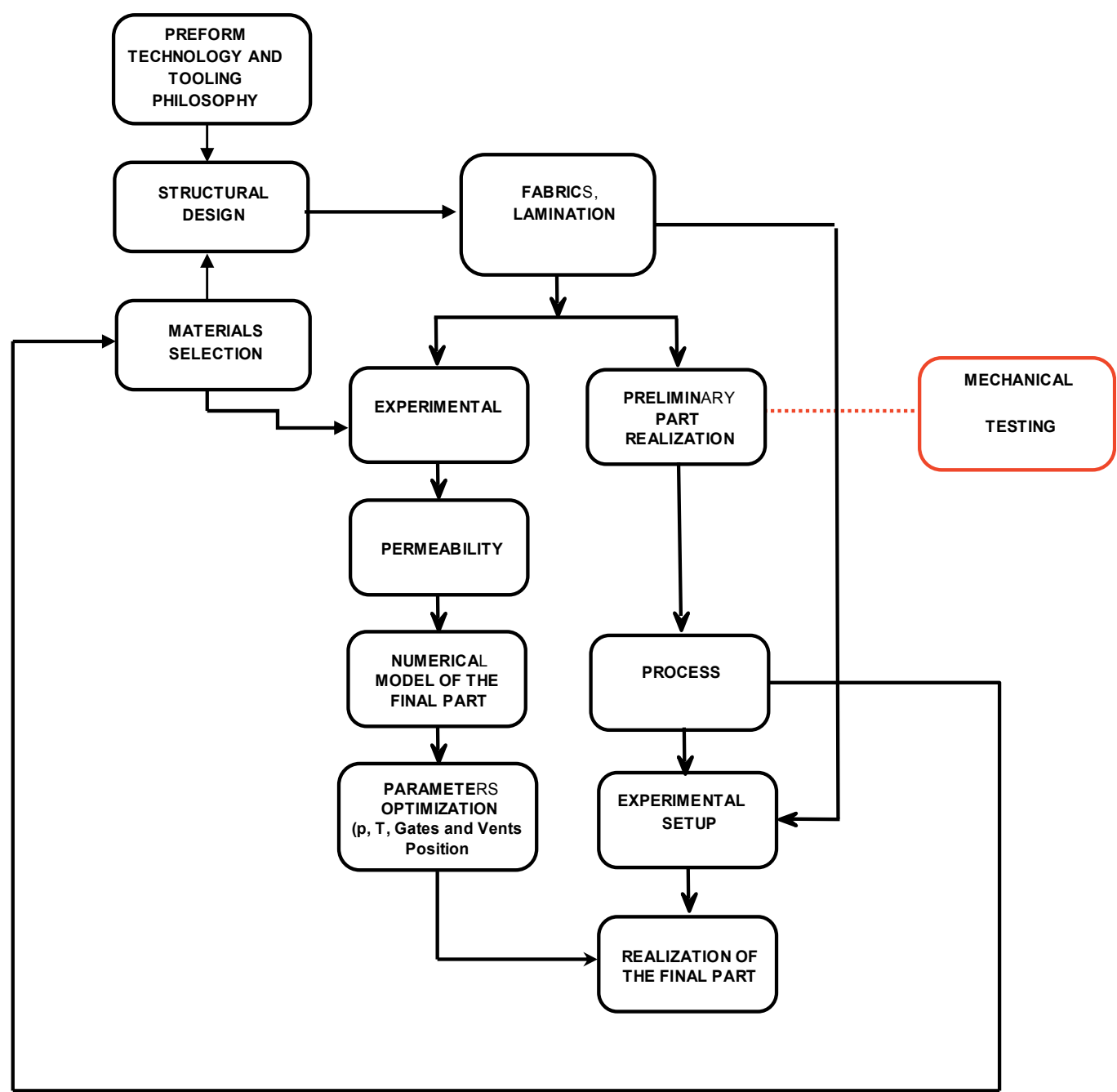

Figure 7. Flowchart of concurrent design and manufacturing

The numerical analysis was performed by commercial codes based on a finite element analysis-control volume (FEM-CV). Modelling of the flow was to allow investigating the resin impregnation process and strategically designing gates and vents and injection scheme in order to optimally fill the composite part without any dry spot. The analysis took into account the most relevant process parameters such as injection pressure and material features like the resin viscosity and permeability of the preform. The FEM-CV analysis was isothermal and provided detailed information about the pressure field, flow front patterns, and strategic injection scheme. The different choice of gate positions affects very much the filling time and the quality of the finished part. For these reasons, different injection schemes were considered. All simulations were performed using the resin viscosity value at the mold temperature. 
Figure 8 shows the simulation result of the best injection scheme. In particular, the simulation represents the trend of the filling time. Figure 9 shows the prototype of the strake after demolding.

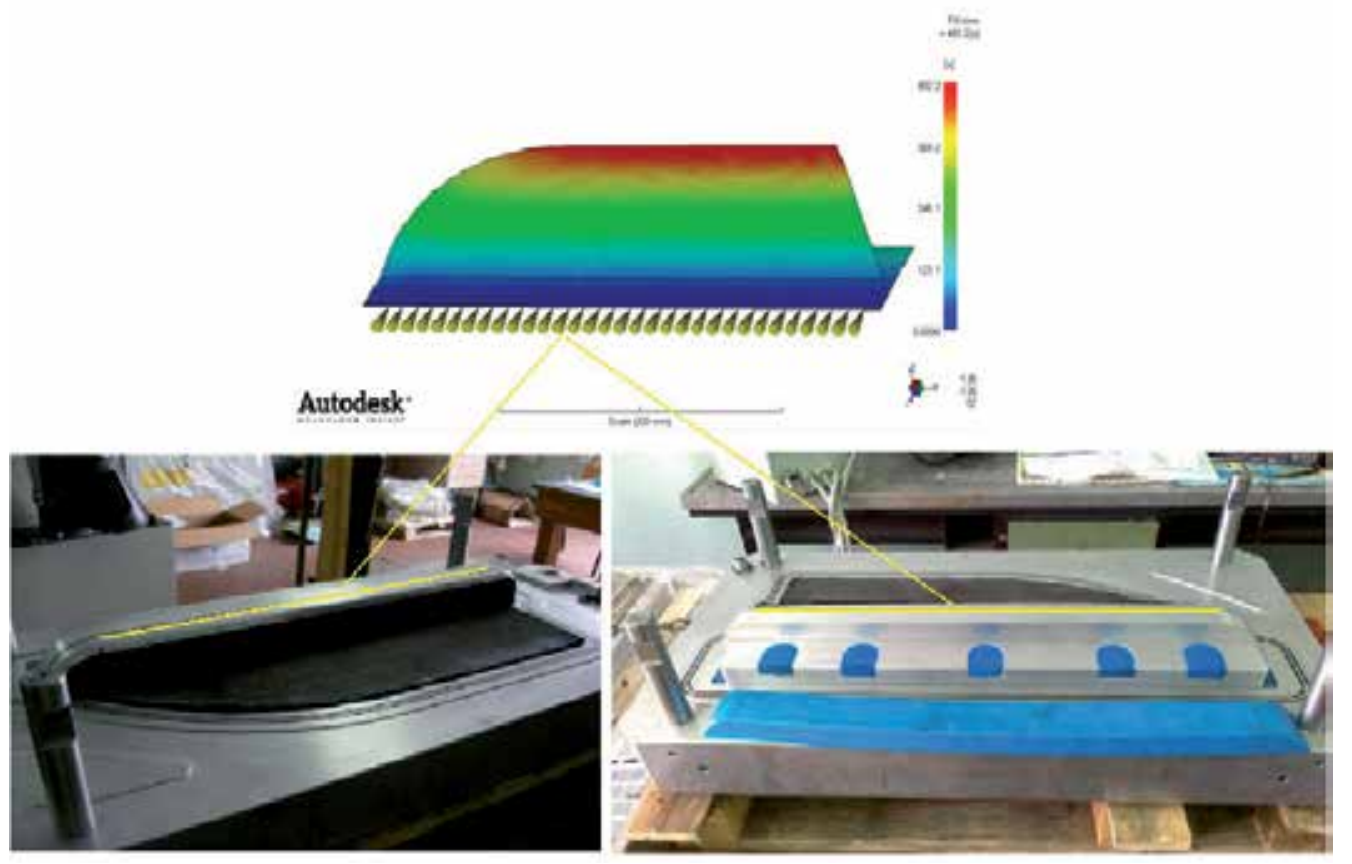

Figure 8. Injection scheme of the mold as determined by numerical simulation. On the top: filling time trend through the preform. On the bottom at left: carbon fiber reinforcement before closing mold.

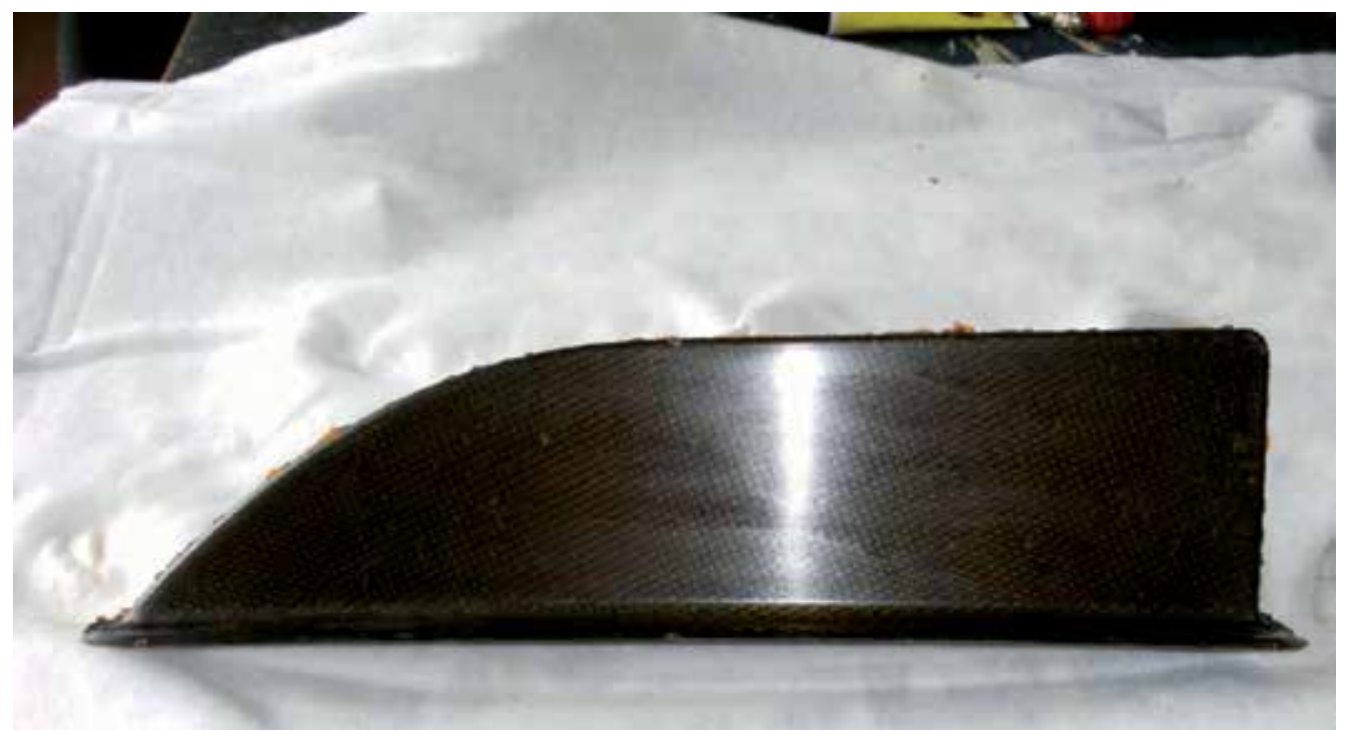

Figure 9. Strake of Aermacchi nacelles after demolding. 


\subsection{Inboard flaperon}

The aim of the work was to realize a primary structure of a helicopter by RTM process in order to compare its properties with those of the parts made with classical hand lay-up technique. The chosen component was the inboard flaperon of the BA609 (Bell Agusta), which is an aerodynamic control surface that presents different critical features. In fact, the inboard flaperon is a primary component so the reliability must be nearly absolute. Further, it is a large part and involved a complicated lamination sequence that can affect the manufacturing process. It is evident in Figure 10 the presence of three different lamination sequences that correspond to different permeability, which was determined experimentally.
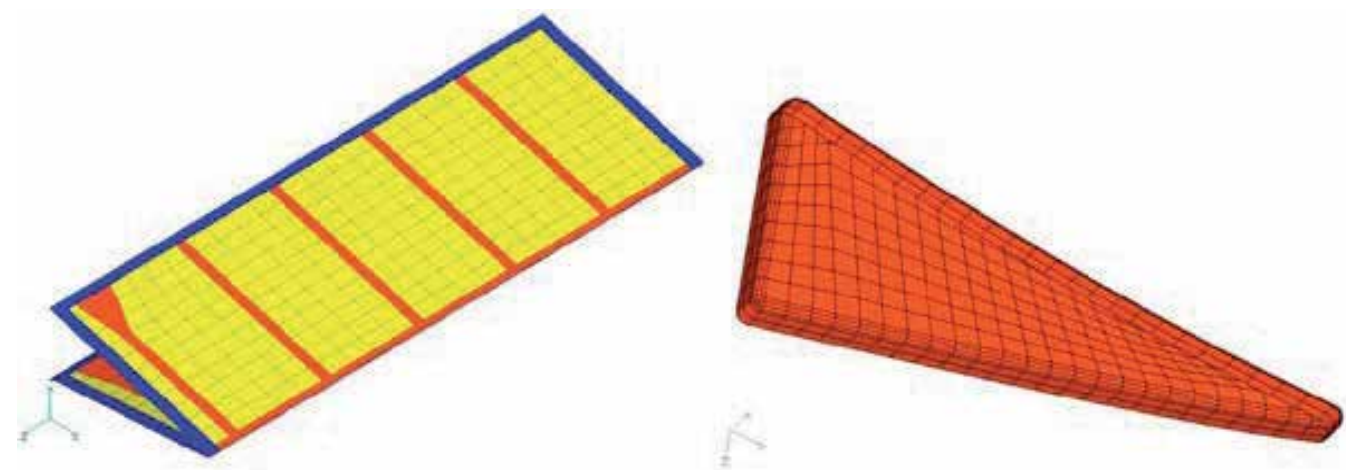

\begin{tabular}{|c|c|}
\hline Lay-up Sequence & \multirow{2}{*}{ Permeability } \\
\cline { 1 - 2 } $\mathbf{1}$ & $0.124 \mathrm{E}-11 \mathrm{~m}^{2}$ \\
\hline $\mathbf{2}$ & $0.5365 \mathrm{E}-11 \mathrm{~m}^{2}$ \\
\hline 3 & $0.744 \mathrm{E}-11 \mathrm{~m}^{2}$ \\
\hline
\end{tabular}

Figure 10. Finite element model of the inboard flaperon with a particular on a rib (red component on the right). The different colors individuate the different permeability values associated to the lamination sequence.

In the first scheme, the injection points were placed on the two leading edge borders of the skin in correspondence of the rib reinforces and the venting points on the trailing edge line. As shown in Figure 11, the total filling time given by this configuration is very long (more than $1.5 \mathrm{~h}$ ) and comparable with the resin pot life limit.

A second scheme has been analyzed. In this case five injection points were placed in the middle of each skin side and between the rib reinforces. The venting points were located both on the trailing and leading edge lines. With this second scheme the filling time was considerably reduced down to $20 \mathrm{~min}$ in good agreement with the resin processability. From the results of the previous simulation it was noted that much time was needed to fill a very small area near the outbord side. For this reason a further optimization was carried out including a sixth injection point in this area. With this simple modification the total filling resulted to be less than half of the previous simulation (Figure 12). 

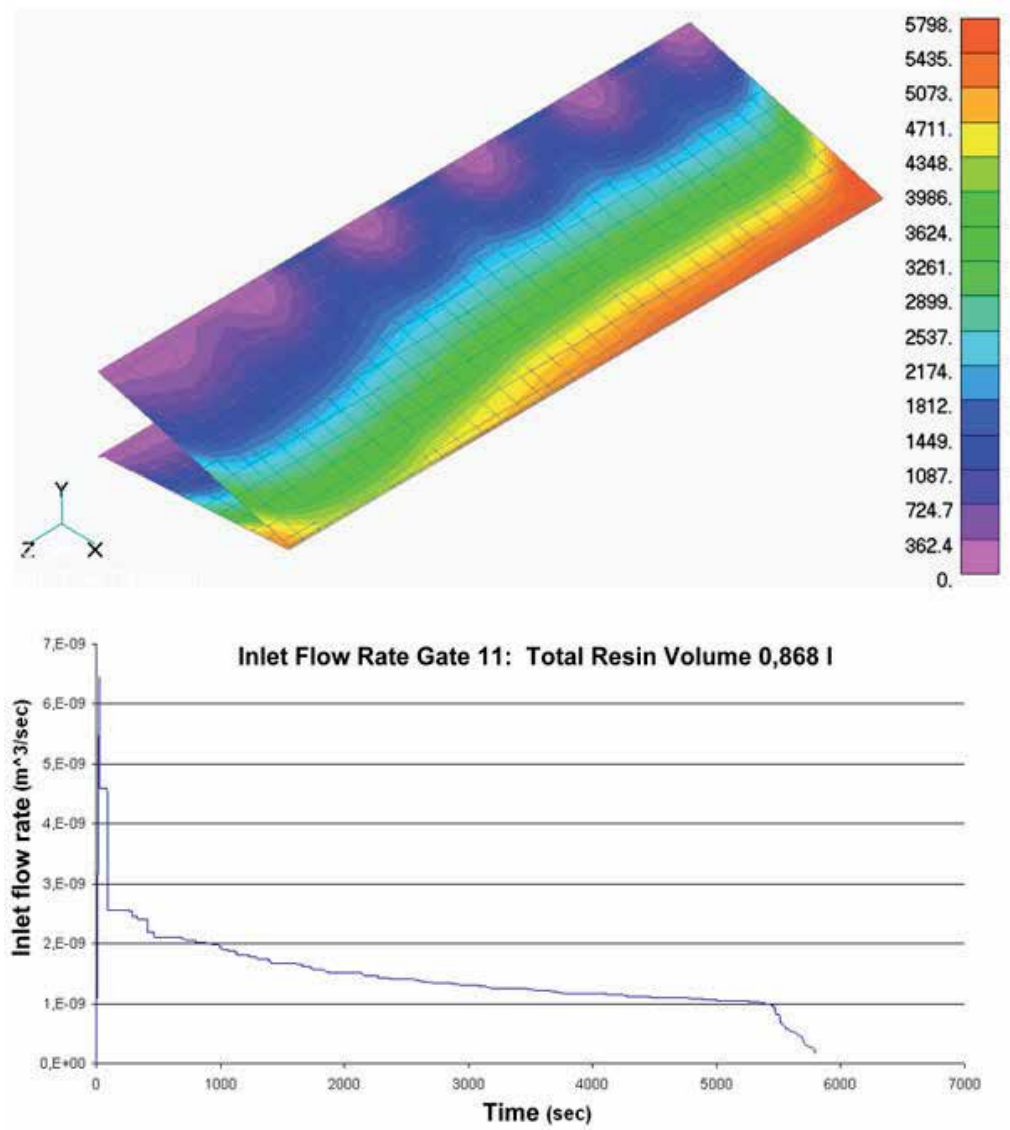

Figure 11. Filling time with the first injection scheme and predicted resin flow rate during the injection with the first scheme. 


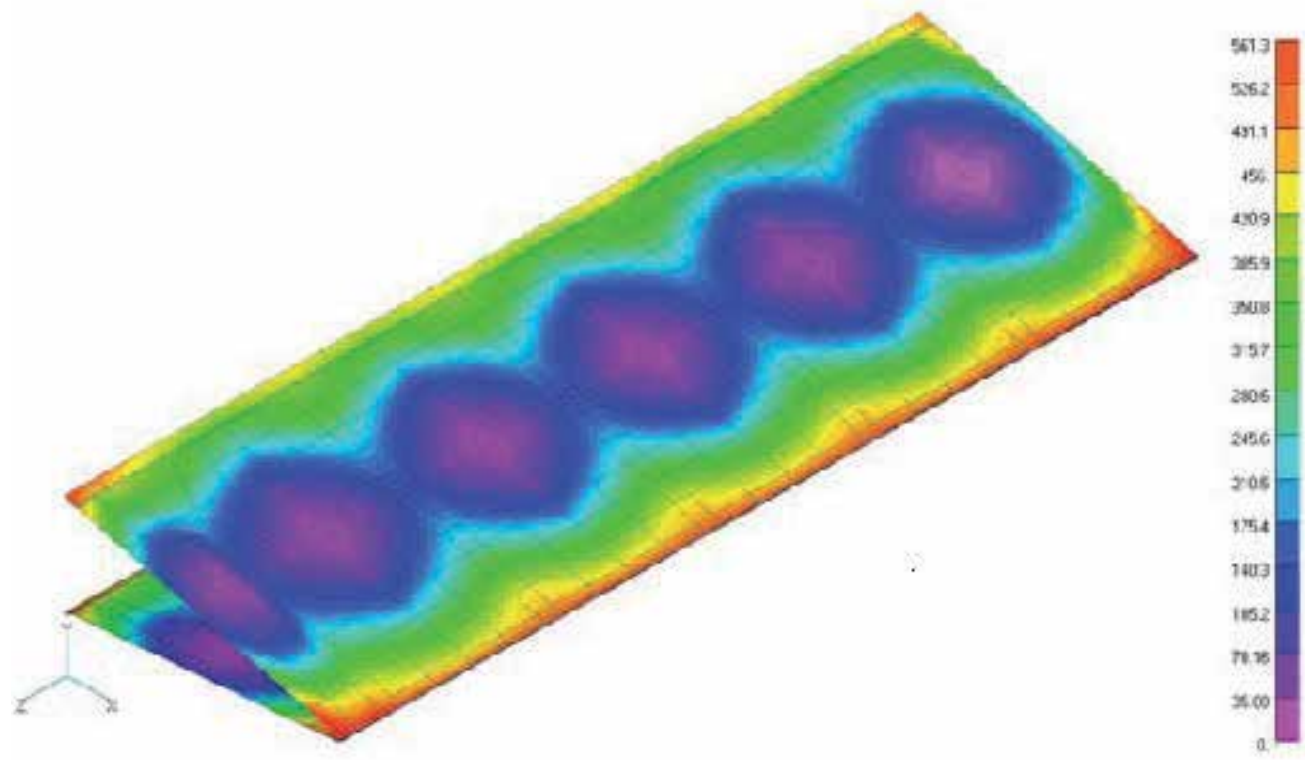

Figure 12. Filling time trend for the injection scheme selected for the mold.

The dry fabric plies for the construction of the preform were cut with the GFM ultrasonic cutting machine using a multilayer method. In this way some areas of the preform were cut directly in the final lamination sequence allowing for considerable time saving in the preform assembly phase. The different plies and sequences were sticked together on a flat surface using an adequate agent (binder). It was used the same cut program and operative cycle of the original part. In Figure 13, the glass layers used to replace the syntactic foam can be seen. The use of the glass mat strongly improves the permeability of the preform allowing very much shorter injection times. Further, the syntactic foam was incompatible with the RTM process.
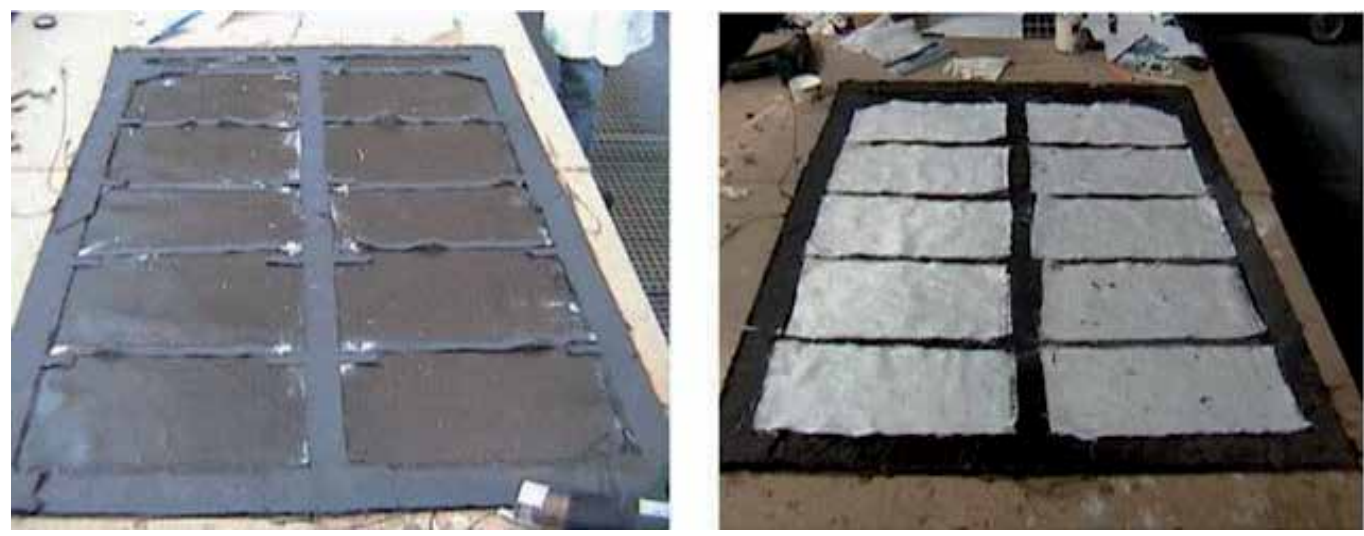

Figure 13. Particular of preform preparation. On the right: glass layers used to replace the syntactic foam of the original component. 

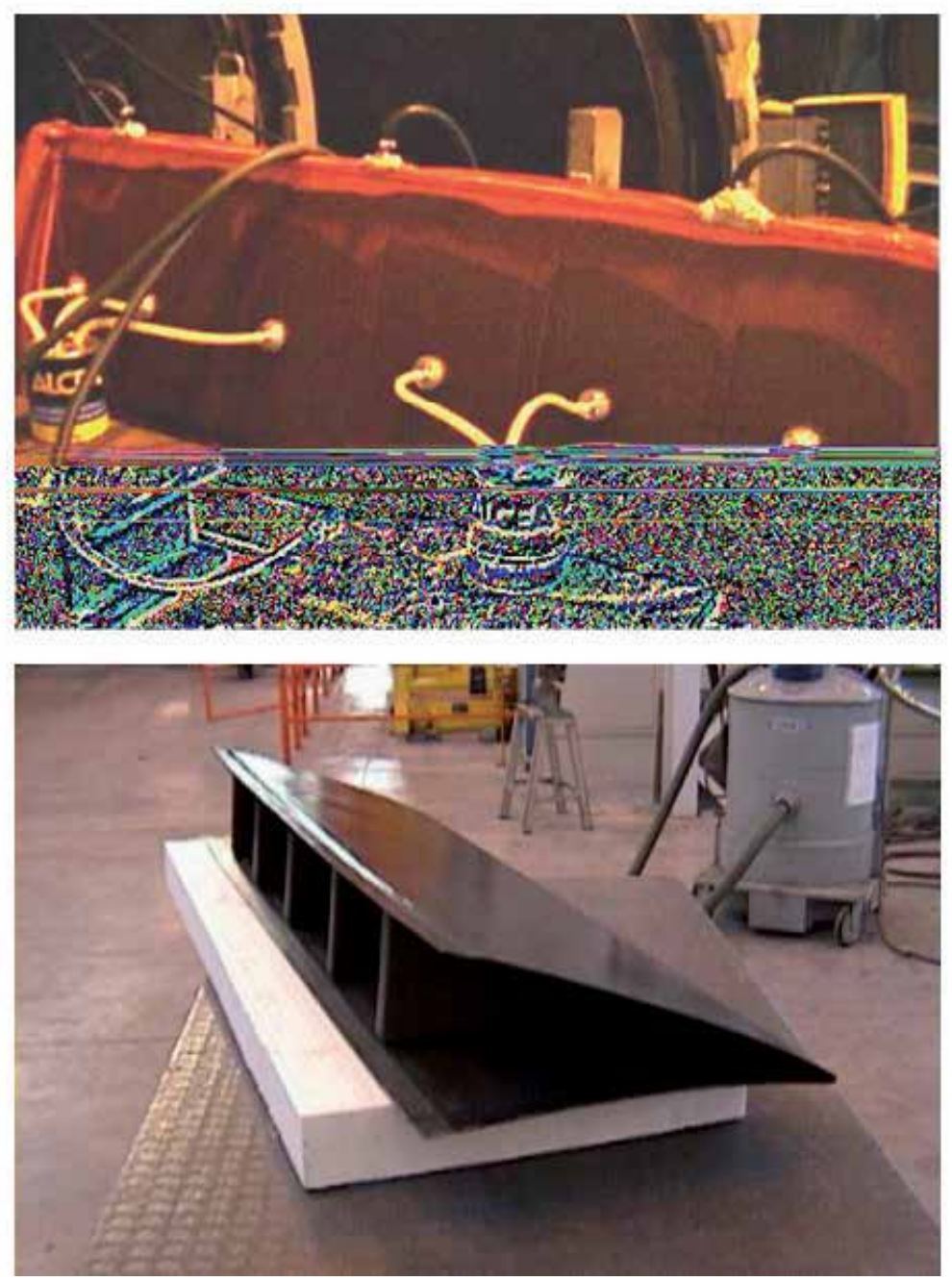

Figure 14. On the top: moment of the impregnation phase. On the bottom: the final component after demolding.

\subsection{Bracket}

The bracket is a secondary element that is present in large numbers in a helicopter. Usually it is made by metal alloy. The aim to re-design the bracket in composite materials is to reduce the weight and time of production. In this case, a consistent saving can be reached adopting an automatic textile process, which avoids the long time due to the lamination. The stitching was the selected preform technique. Figure 15 shows the cut of the layers and the preform after the assembling. Figure 16 shows the realization of the component during the impregnation phase and the part demoded after the curing step. In this case, the use of RTM process permits to save $30 \%$ weight with respect to its equivalent in metal alloy and reduces drastically the production cycle. 

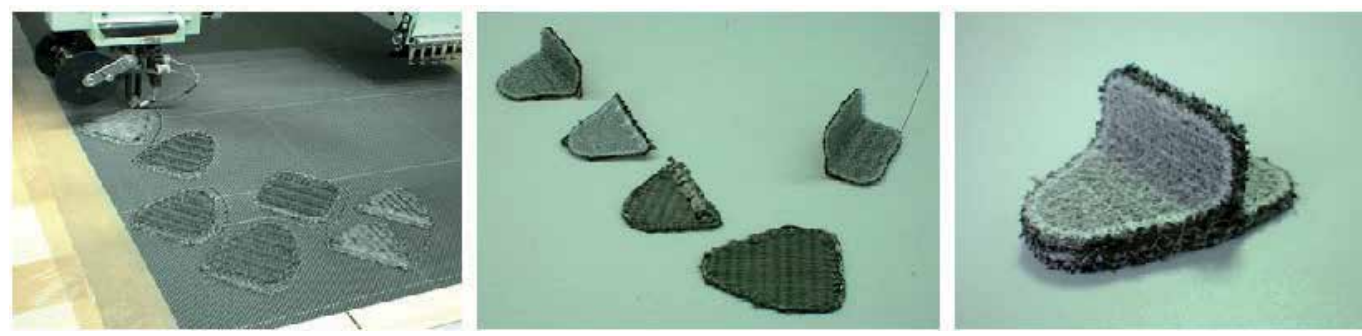

Figure 15. Realization of the preform: cut and stitching.
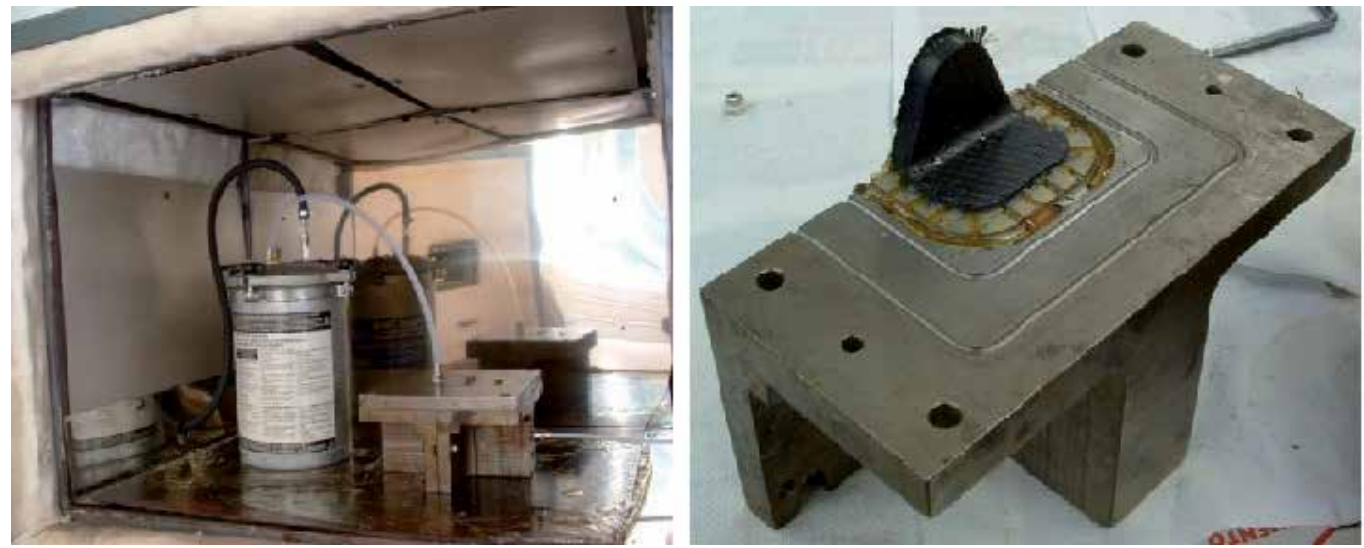

Figure 16. Realization of the bracket by RTM process: resin injection (left), bracket demolded (right).

\section{Author details}

Susanna Laurenzi and Mario Marchetti

Department of Astronautic Electrical and Energy Engineering, Sapienza Università di Roma, Italy

\section{References}

Acheson JA, Simacek P, Advani SG. The implications of fiber compaction and saturation on fully coupled VARTM simulation. Composites Part A: Applied Science and Manufacturing. 2004;35(2):159-169.

Amico S, Lekakou C. An experimental study of the permeability and capillary pressure in resin-transfer moulding. Composites Science and Technology. 2001;61(13):1945-1959.

Baker AA, Callus PJ, Georgiadis S, Falzon PJ, Dutton SE, Leong KH. An affordable methodology for replacing metallic aircraft panels with advanced composites. Composites Part A: Applied Science and Manufacturing. 2002;33(5):687-696.

Barlow D, Howe C, Clayton G, Brouwer S. Preliminary study on cost optimisation of aircraft composite structures applicable to liquid moulding technologies. Composite Structures. 2002;57(1-4):53-57. 
Belov EB, Lomov SV, Verpoest I, Peters T, Roose D, Parnas RS, et al. Modelling of permeability of textile reinforcements: lattice Boltzmann method. Composites Science and Technology. 2004;64(7-8):1069-1080.

Bickerton S, Abdullah MZ. Modeling and evaluation of the filling stage of injection/compression moulding. Composites Science and Technology. 2003;63(10):13591375.

Bickerton S, Buntain MJ, Somashekar AA. The viscoelastic compression behavior of liquid composite molding preforms. Composites Part A: Applied Science and Manufacturing. 2003;34(5):431-444.

Bickerton S, Buntain MJ. Modeling forces generated within rigid liquid composite molding tools. Part B: Numerical analysis. Composites Part A: Applied Science and Manufacturing. 2007;38(7):1742-1754.

Brouwer WD, van Herpt ECFC, Labordus M. Vacuum injection moulding for large structural applications. Composites Part A: Applied Science and Manufacturing. 2003;34(6):551-558.

Buntain MJ, Bickerton S. Modeling forces generated within rigid liquid composite molding tools. Part A: Experimental study. Composites Part A: Applied Science and Manufacturing. 2007;38(7):1729-1741.

Cairns DS, Humbert DR, Mandell JF. Modeling of resin transfer molding of composite materials with oriented unidirectional plies. Composites Part A: Applied Science and Manufacturing. 1999;30(3):375-383.

Calado VnMA, Advani SG. Effective average permeability of multi-layer preforms in resin transfer molding. Composites Science and Technology. 1996;56(5):519-531.

Chen B, Chou T-W. Compaction of woven-fabric preforms: nesting and multi-layer deformation. Composites Science and Technology. 2000;60(12-13):2223-2231.

Chen B, Lang EJ, Chou T-W. Experimental and theoretical studies of fabric compaction behavior in resin transfer molding. Materials Science and Engineering: A. 2001;317(12):188-196.

Chen Z-R, Ye L, Kruckenberg T. A micromechanical compaction model for woven fabric preforms. Part I: Single layer. Composites Science and Technology. 2006;66(16):32543262.

Chen Z-R, Ye L. A micromechanical compaction model for woven fabric preforms. Part II: Multilayer. Composites Science and Technology. 2006;66(16):3263-3272.

Correia NC, Robitaille F, Long AC, Rudd CD, Šimáček P, Advani SG. Analysis of the vacuum infusion moulding process: I. Analytical formulation. Composites Part A: Applied Science and Manufacturing. 2005;36(12):1645-1656.

Dobyns A, Rousseau CQ, Minguet P. 6.12 - Helicopter Applications and Design. In: Editorsin-Chief: Anthony K, Carl Z, editors. Comprehensive Composite Materials, Oxford: Pergamon; 2000. p. 223-242.

Gutowski TG, Cai Z, Kingery J, Wineman SJ. Resin flow/fiber deformation experiments. Journal Name: SAMPE Q; (United States); Journal Volume: 17:4. 1986:Medium: X; Size: Pages: 54-58. 
Haider M, Hubert P, Lessard L. An experimental investigation of class A surface finish of composites made by the resin transfer molding process. Composites Science and Technology. 2007;67(15-16):3176-3186.

J.M. Lawrence PS, S. Laurenzi, S. G. Advani. Flow modelling of the compression resin transfer molding process. The 8th International Conference on Flow Processes in Composite Materials, Douai, France2006.

Kruckenbrg T. Paton R. Resin Transfer Moulding for Aerospace Structures: Kluwer Academic Publishers.

Laskoski M, Dominguez DD, Keller TM. Synthesis and properties of a liquid oligomeric cyanate ester resin. Polymer. 2006;47(11):3727-3733.

Laurenzi S, Di Nallo D, Marchetti M, Lalia Morra E, Anamateros E. Manufacturing approach to realize a prototype of a helicopter transmission component by composite materials. 31st European Rotorcraft Forum, Florence, Italy2005.

Laurenzi S, Di Nallo D, Valente F, Marchetti M, Lalia Morra E, Anamateros E. Re-design of a helicopter component transmission by composite material. 45st Israel Annual Conference on Aerospace Sciences, Haifa, Israel2005.

Laurenzi S, Griccini M, Lalia Morra E, Anamateros E, Marchetti M. Processability analysis of thick braided composites manufactured with $\mathrm{rtm}$ technology. 7th International Conference on Flow Processes in Composite Materials, Newark, Delaware, USA2004.

Laurenzi S, Marchetti M, Anamateros E. Liquid Composite Molding for aeronautical application. International Council of the Aeronautical Sciences Hamburg, Germany2006.

Laurenzi S. Liquid composite molding for aerospace applications. Case studied:Helicopter A 109 gearbox. Sapienza University of Rome, School of Aerospace Engineering, 2007.

Lomov SV, Verpoest I, Peeters T, Roose D, Zako M. Nesting in textile laminates: geometrical modelling of the laminate. Composites Science and Technology. 2003;63(7):993-1007.

Luo Y, Verpoest I, Hoes K, Vanheule M, Sol H, Cardon A. Permeability measurement of textile reinforcements with several test fluids. Composites Part A: Applied Science and Manufacturing. 2001;32(10):1497-1504.

Marchetti M, Cutolo D. Tecnologie dei materiali composite: ESA Grafica; 1991.

Merotte J, Simacek P, Advani SG. Resin flow analysis with fiber preform deformation in through thickness direction during Compression Resin Transfer Molding. Composites Part A: Applied Science and Manufacturing. 2010;41(7):881-887.

Mouritz AP, Bannister MK, Falzon PJ, Leong KH. Review of applications for advanced three-dimensional fibre textile composites. Composites Part A: Applied Science and Manufacturing. 1999;30(12):1445-1461.

Ngo ND, Tamma KK. Microscale permeability predictions of porous fibrous media. International Journal of Heat and Mass Transfer. 2001;44(16):3135-3145.

Noor AK, Venneri SL, Paul DB, Hopkins MA. Structures technology for future aerospace systems. Computers \&amp; Structures. 2000;74(5):507-519.

Park CH, Lee WI, Han WS, Vautrin A. Simultaneous optimization of composite structures considering mechanical performance and manufacturing cost. Composite Structures. 2004;65(1):117-127. 
Parseval YD, Pillai KM, Advani SG. A Simple Model for the Variation of Permeability due to Partial Saturation in Dual Scale Porous Media. Transport in Porous Media. 1997;27(3):243-264.

Potter KD. The early history of the resin transfer moulding process for aerospace applications. Composites Part A: Applied Science and Manufacturing. 1999;30(5):619621.

Reduced cost, higher performance RTM. Reinforced Plastics. 1997;41(9):48-54.

Reia da Costa EF, Skordos AA. Modelling flow and filtration in liquid composite moulding of nanoparticle loaded thermosets. Composites Science and Technology. 2012;72(7):799805.

S. G. Advani EMS. Process Modeling in Composites Manufacturing: Marcel Dekker press; 2002.

S. G. Advani MVB, R. Parnas. Resin Transfer Molding. Flow and Rheology in Polymeric Composites Manufacturing, Amsterdam: Elsevier Publishers; 1994.

Saunders RA, Lekakou C, Bader MG. Compression and microstructure of fibre plain woven cloths in the processing of polymer composites. Composites Part A: Applied Science and Manufacturing. 1998;29(4):443-454.

Saunders RA, Lekakou C, Bader MG. Compression in the processing of polymer composites 1. A mechanical and microstructural study for different glass fabrics and resins. Composites Science and Technology. 1999;59(7):983-993.

Simacek P, Advani SG. A numerical model to predict fiber tow saturation during liquid composite molding. Composites Science and Technology. 2003;63(12):1725-1736.

Šimáček P, Advani SG. Desirable features in mold filling simulations for Liquid Composite Molding processes. Polymer Composites. 2004;25(4):355-367.

Slade J, Pillai KM, Advani SG. Investigation of unsaturated flow in woven, braided and stitched fiber mats during mold-filling in resin transfer molding. Polymer Composites. 2001;22(4):491-505.

Smith P, Rudd CD, Long AC. The effect of shear deformation on the processing and mechanical properties of aligned reinforcements. Composites Science and Technology. 1997;57(3):327-344.

Soutis C. Carbon fiber reinforced plastics in aircraft construction. Materials Science and Engineering: A. 2005;412(1-2):171-176.

Tan H, Roy T, Pillai KM. Variations in unsaturated flow with flow direction in resin transfer molding: An experimental investigation. Composites Part A: Applied Science and Manufacturing. 2007;38(8):1872-1892.

Tari MJ, Bals A, Park J, Lin MY, Thomas Hahn H. Rapid prototyping of composite parts using resin transfer molding and laminated object manufacturing. Composites Part A: Applied Science and Manufacturing. 1998;29(5-6):651-661. 


\title{
Netcentric Virtual Laboratories for Composite Materials
}

\author{
E. Dado, E.A.B. Koenders and D.B.F. Carvalho \\ Additional information is available at the end of the chapter
}

http://dx.doi.org/10.5772/48705

\section{Introduction}

Physical laboratory-based experiments and testing has been a way to develop fundamental research and learning knowledge for many areas of (civil) engineering education, science and practice. In the context of education, it has particularly enriched engineering education by helping students to understand fundamental principles and by supporting them to understand the link between the theoretical equations of their text books and real world applications. In the context of science, physical laboratory experiments have been used to scrutinize particular phenomenon in a real-life setting or to verify and validate scientific computational models over a longer period of time. In both the educational and research context, conducting physical laboratory experiments are generally governed by complex and expensive lab-infrastructures and require significant allocation of resources from the educational and research institutes. Besides, results most frequently have a limited range of exposure and are available for a relatively small audience (i.e. high costs versus relatively low benefits) [1]. In the context of practice, physical tests are often performed to validate performances of products. With the increasing regulations from national governments and European Union (EU) concerning quality, safety and environmental properties of products, the number of physical tests performed in laboratories of certificated (research) institutes have increased recently. For example, the European labels of conformity, known as CE markings, are a guarantee of quality and safety for products produced and sold in the EU. According to the CE conformity standards, products will have undergone a series of performance tests before they can be sold on the EU market. However, these performance tests entail additional costs which may result in financial difficulties stemming from these additional costs and in the long run result in competitive disadvantages for Small and Medium Enterprises (SMEs) in Europe [2].

To improve this situation, initiatives have been launched where research and development $(R \& D)$ projects could be conducted in so-called 'virtual laboratories'. In civil engineering, 
the National Institute of Standards and Technologies (NIST) in the United States were the first who set the standard for R\&D projects conducted in a virtual laboratory for composite materials [3]. From this initiative and the promises of emerging information and communication technologies a whole new realm of possibilities for developing virtual laboratories has become available. Based on these observations and developments, the authors of this book chapter have initiated a number of R\&D projects which main focus was to explore the concept of virtual laboratories for cement-based materials in real-life settings. A number of (journal) papers have been published about the findings of these projects in the past [4-7]. The main focus of this book chapter will be on a relatively new concept for establishing virtual laboratories which is based on a 'netcentric' approach.

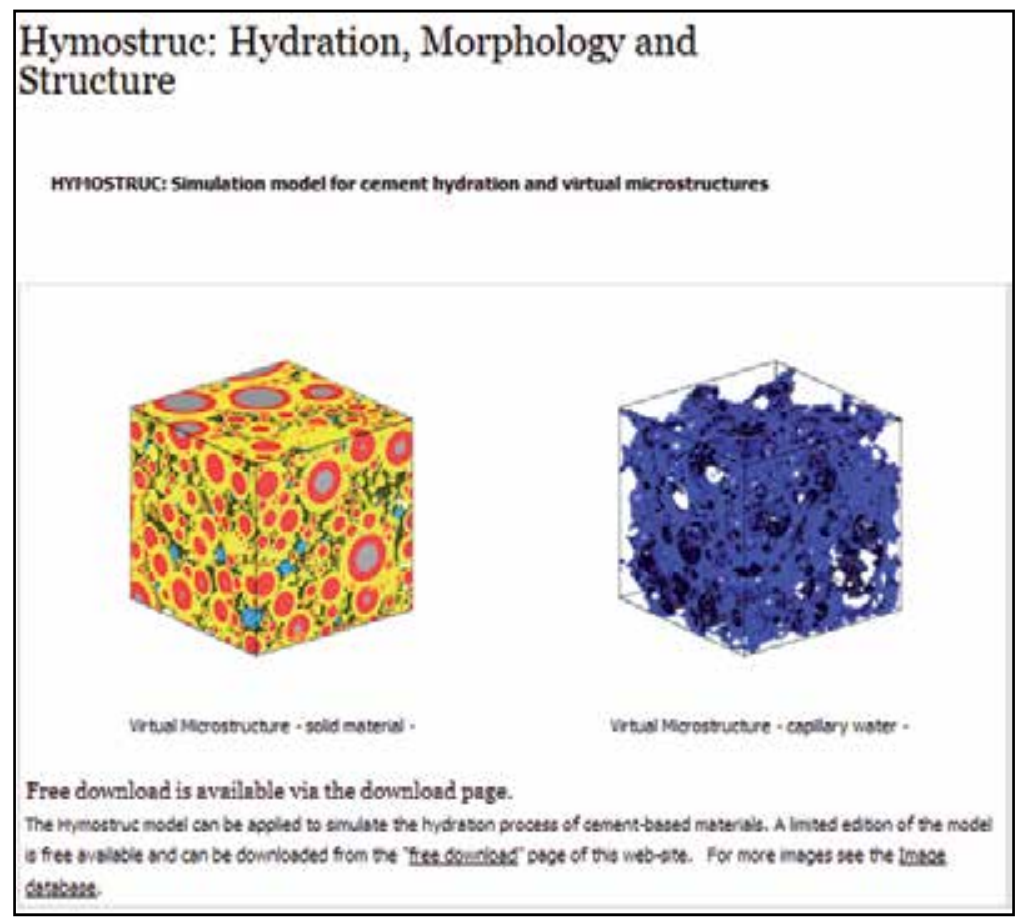

Figure 1. One of the results of the R\&D projects conducted by the authors in the past.

\section{The concept of netcentric virtual laboratories}

Virtual experiments (or testing) are rapidly emerging as a key technology in civil engineering. Although some applications of virtual experiments other than related to materials and components have been reported by a number of researchers, most effort has been put into the development of virtual laboratories for composite materials and components. In this respect, virtual experiments are often defined as a concept of making use of high performance computers in conjunction with high quality models to predict the properties and/or behavior of composite materials and components. Consequently, virtual laboratories are often seen as a new terminology for computer simulation, which is a wrong 
assumption. Although it is true that computer simulation is an important tool for virtual experiments, it is only one of key components that constitute a virtual laboratory. This can be explained by the limitation of the existing composite material models. As discussed by Garboczi et al, an ideal model of a composite material or structure should be one that starts from the known chemical composition of the composite material [8]. Beginning with the correct proportioning and arrangement of atoms, the modeling effort would build up the needed molecules, then the nanostructure and microstructure, and would eventually predict properties at the macroscale level. Such fundamental and multi-scale material model, however, is still a long way off. Each existing material model has its range of applicability and its own restrictions. Corresponding computational models and supporting computer tools are developed at a number of different research institutes worldwide. However, most existing virtual laboratories are setup as web applications that only provide access to a 'closed' virtual laboratory that contains a number of (integrated) computational models and supporting computer tools.

A good example of a closed virtual laboratory is the Virtual Cement and Concrete Testing Laboratory (VCCTL) from the National Institute of Standards and Technologies (NIST) in the United States. The main goal of the VCCTL project was to develop a virtual testing system, using a suite of integrated computational models for designing and testing cementbased materials in a virtual testing environment, which can accurately predict durability and service life based on detailed knowledge of starting materials, curing conditions, and environmental factors. In 2001, an early prototype (version 1.0) of the VCCTL became public available and accessible through the Internet. The core of this prototype was formed by the NIST 3D Cement Hydration and Microstructure Development Model (CEMHYD3D). Using the web-based interface of the VCCTL, one can create an initial microstructure containing cement, mine mineral admixtures, and inert fillers following a specific particle size distribution, hydrate the microstructure under a variety of curing conditions and evaluate the properties (e.g. chemical shrinkage, heat release, and temperature rise) of the simulated microstructures for direct comparison to experimental data. As the VCCTL project proceeded, the prediction of rheological properties (viscosity and yield stress) of the fresh materials and elastic properties (elastic modulus, creep, and relaxation) of the hardened materials were incorporated into the VCCTL resulting in the release of version 1.1 (latest) of the VCCTL in 2003 [3].

In order to cope with this particularity of distributed material (computational) models, the authors adopted a relatively new concept for establishing virtual laboratories which is based on a 'netcentric' approach. In this respect, a netcentric virtual laboratory is considered as a part of an evolutionary, complex community of people (users), devices, information (i.e. experimental data) and services (i.e. computational models and supporting computer tools) that are interconnected by the Internet. Optimal benefit of the available databases containing experimental data, computational models and supporting computer tools, that replace the physical laboratory equipment, is achieved via a distributed virtual laboratory environment and is assessable for students and researchers (see Figure 2). 


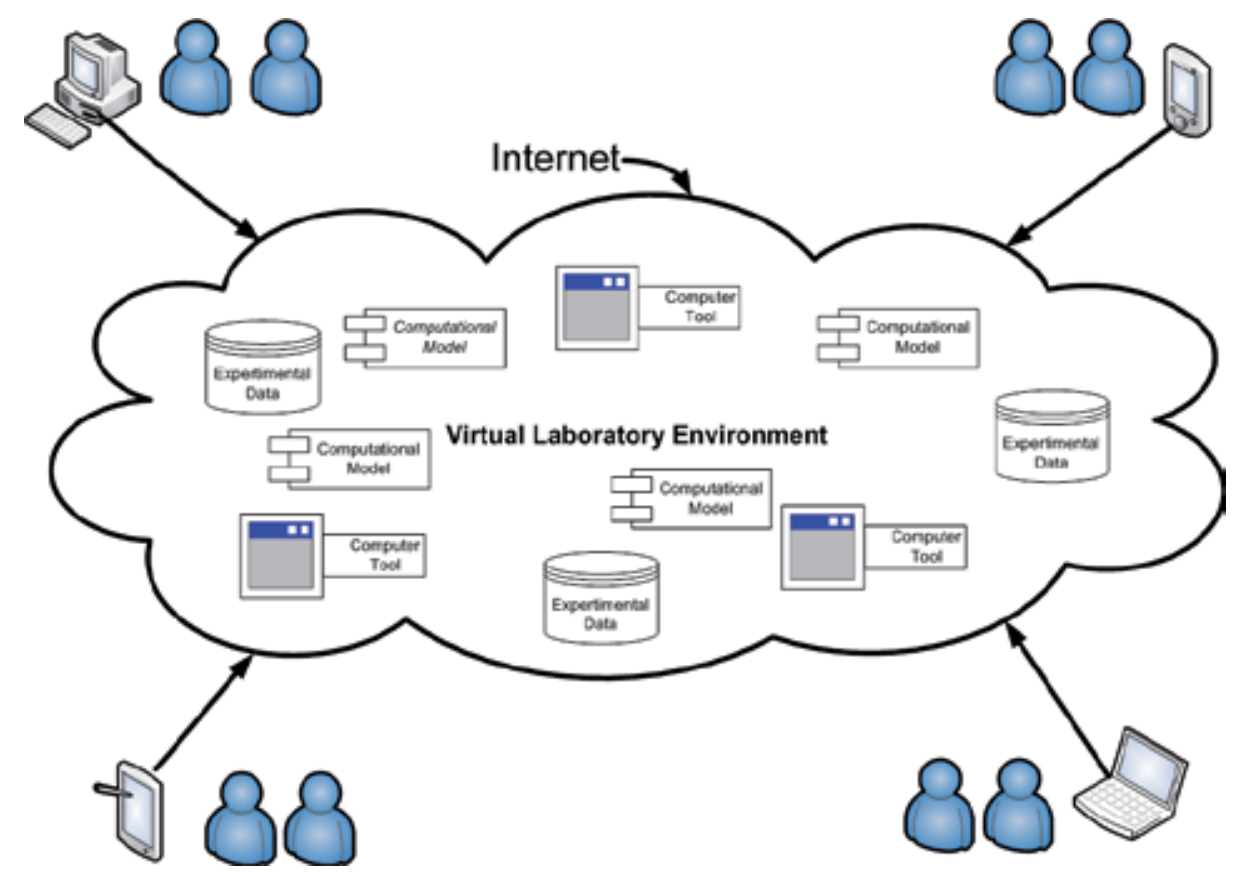

Figure 2. Virtual Laboratory Environment populated by devices, users, computational models and computer tools and databases with experimental data which are interconnected by the Internet.

\section{Overview of emerging and enabling technologies}

As discussed in the previous paragraph, a virtual laboratory is no longer regarded as an isolated web-based application, but as a set of integrated devices (and supported infrastructures), computational models and supporting computer tools and databases containing experimental data that, used together, form a distributed and collaborative virtual laboratory environment for virtual experiments. Multiple, geographically dispersed (research) institutes will use this virtual laboratory environment to establish their own virtual laboratory to perform experiments as well as share their result from their $R \& D$ projects. As discussed in [5], emerging and enabling technologies should fundamentally concern about the integration (or interoperability), connecting devices, computational models, computer tools and data stores. In order to structure the discussion in this section, a conceptual scheme of the different levels of 'integration' is presented in Figure 3.

\subsection{Integrated infrastructure}

Concerning the issue of the 'integrated infrastructure' two enabling and emerging technologies should be mentioned: Cloud Computing and Grid Computing. According to Foster et al. [9], Grid Computing and Cloud Computing are closely related paradigms that share a lot of commonality in their goals, architecture, and technology. According the National Institute of Standards and Technology (NIST) Cloud Computing (and to a large extend Grid Computing) can be defined as "a model for enabling convenient, on-demand 


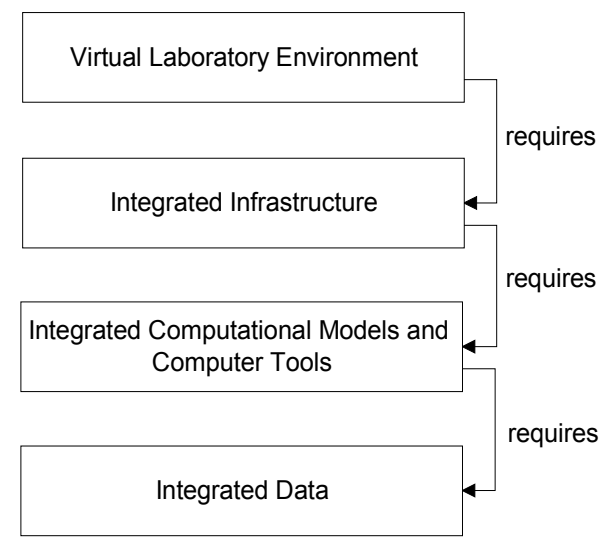

Figure 3. A Virtual Laboratory Environment requires an Integrated Infrastructure which on its turn requires respectively Integrated Computational Models and Computer Tools and Integrated Data.

network access to a shared pool of configurable computing resources (e.g., networks, servers, storage, applications, and services) that can be rapidly provisioned and released with minimal management effort or service provider interaction" [10]. In addition, Grid Computing technology adds the concept of 'virtual enterprise'. A virtual enterprise is a dynamic collection of institutes together in order to share hardware and software resources as they tackle common goals. As discussed in [3], a shared access to resources and the use of these resources is an inevitable condition for making multi-scale modeling successful.

Cloud Computing and Grid Computing also share some limitations, namely the inability to provide intelligent and autonomous services, the incompetency to address the heterogeneity of systems and data, and the lack of machine-understandable content [11]. Mika and Tummarello (2008) identified the root cause of these limitations as the lack of 'Web semantics' [12]. These limitations should be addressed at service and data levels as discussed in the next sections.

\subsection{Integrated computational models \& computer tools}

Traditionally, the programming environment at research institutes is dominated by (programming) languages such SQL for storing, retrieving and manipulating data, Fortran, $\mathrm{C}$, and $\mathrm{C}++$ and Java for implementing computational models and HTML for developing web-based interfaces for end-users. Web Services fundamentally concern about the integration of computer programs, especially when the computer programs concerned are developed using different programming languages and computer operating platforms. Web Services standards and technologies offer a widely adopted mechanism for making computer programs work together.

Currently, the two main players on the web services market are Oracle (by the ownership of Sun), with their Java platform and Microsoft with the .NET platform, where both agree on the core standards (e.g. SOAP, WSDL, UDDI and XML), but disagree on how to deliver the 
potential benefits of Web Services to their customers. Simple Object Access Protocol (SOAP) is the standard for web services messages. Based on XML, SOAP let web services exchange information over HTTP. In addition, WSDL (Web Services Description Language) is an XML-based language for describing web services and the way how to access them. UDDI (Universal Description, Discovery, and Integration) is an XML-based registry for web services to list themselves on the Internet. XML (EXtensible Markup Language) has replaced HTML as de defacto standard for describing defining and sharing data on the Internet. The most important advantages of XML are: (1) its separation of definition (content) and representation (mark-up), and (2) its ability to support the development and use of domain specific XML vocabularies. Using the web service standards SOAP, WSDL and UDDI make computational models and supporting computer tools web services that can be accessed, described and discovered. Using XML as a basis for sharing data on the Internet will solve the interoperability problems at data level as described in the next section.

\subsection{Integrated data}

As discussed earlier, one of the main causes that Cloud Computing and Grid Computing share some limitations is the lack of 'web semantics'. Using XML will not solve the problem entirely; it has also its own limitations. The limitations of XML were solved by the introduction of the Semantic Web in 2004. Driving the 'Semantic Web' is the organization of content specialized vocabularies, referred to as 'ontologies'. In this respect, ontology is a collection of concepts (or terms) and constructs used to describe a particular knowledge domain. Build upon RDF (Resource Description Framework) and XML and derived from DAML/OIL, OWL (Web Ontology Language) has become the default standard for creating ontologies.

Building ontologies for describing and exchanging data between computational models and supporting computer tools in virtual laboratory environment will result in a number of different ontologies. In this respect, three different types of ontologies can be distinguished: (1) high-level (reference) ontologies that hold common concepts which can be applied for all knowledge domains and hold high-level constructs for defining the relationships between these knowledge domains, (2) knowledge domain (reference) ontologies which hold the concepts and constructs that are common within one specific knowledge domain (i.e. referring to the different macro-, meso-, micro- and nano-scale levels that exist in material research) and (3) application ontologies that hold detailed information about concepts and constructs which form the basis for sharing data between a 'group' of computational models and supporting computer tools on the Internet. From a modeling point of view, each application ontology is an extension of one or more knowledge domain ontologies, while each knowledge domain ontology is an extension of one or more high-level ontologies. Together, they form a so-called 'ontology network' that evolutionary changes over time. 


\section{A multi-scale modeling approach for concrete materials}

The development of a virtual laboratory for construction materials requires many years of research to find out the basic principles of which a virtual laboratory should comply with, and also to find out the conditions at which a virtual laboratory would be attractive to researchers, students and people from the industry [13]. At the Delft University of Technology, first trials were focused on the virtual testing of a concrete compressive strength test, where the hydration conditions and the fracture behavior where evaluated with emphasis on the upscaling of the simulation model results. In physical-based concrete laboratories, the compressive strength is determined with an experimental device where a concrete cube is positioned in between two steel plates and compressed using a hydraulic force (Figure 4, left). The force imposed to the concrete cube is increased until failure occurs. Later, the focus was widened to other concrete properties such as tensile strength (Figure 4, right), elastic modulus, hydration temperature, etc.
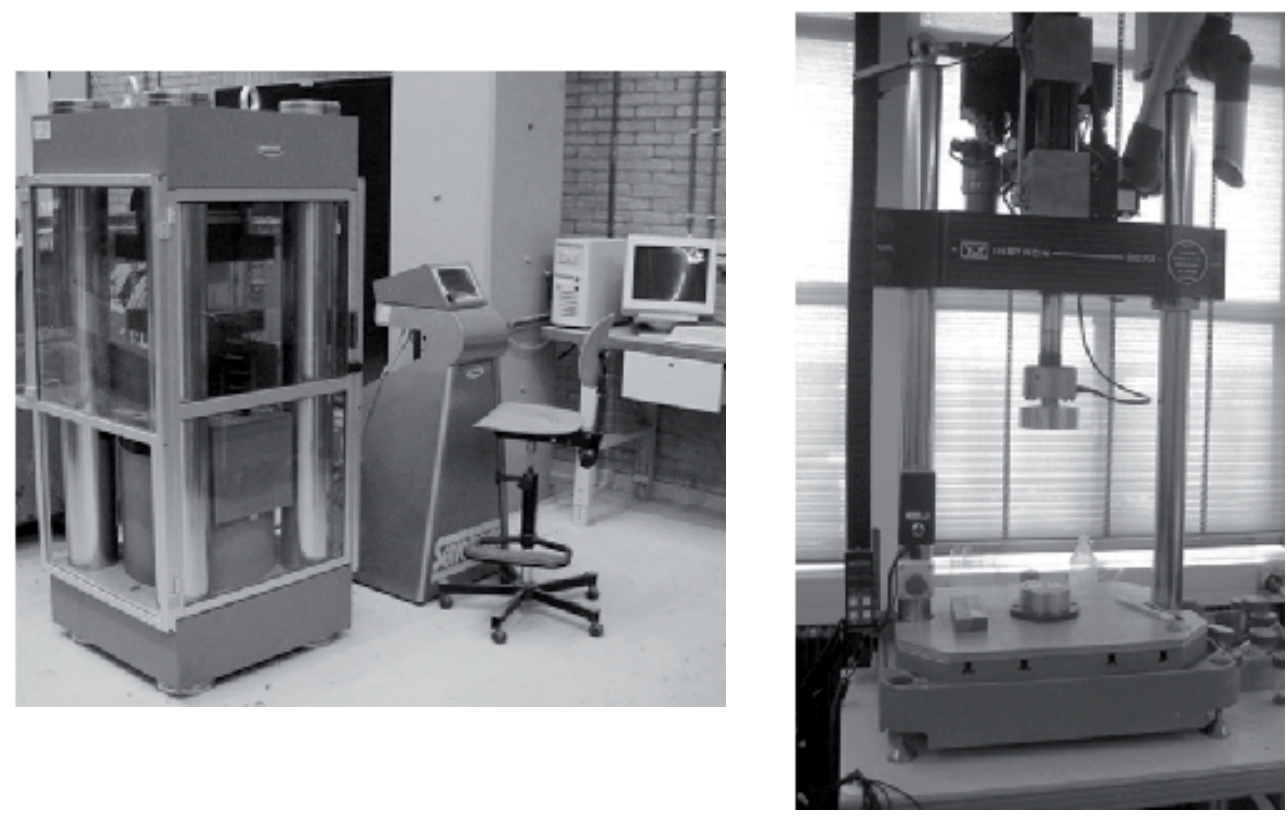

Figure 4. Experimental testing device for concrete compression (Left) and tension (Right).

In a virtual laboratory, testing procedures and methods have to be mimicked using computer simulation models. These computer models are applied to simulate the different characteristics of cement-based materials and produce results that may be validated with experimental data. Especially for heterogeneous materials such as concrete materials, characteristics are modeled at different scale-levels requiring the modeling approach to be multi-scale. This means that models, operating at scale length, have to communicate with each other and exchange information by means of parameters passing which require upscaling algorithms. In general the following scale levels can be distinguished that associate with the characterization of specific materials properties (Table 1). 


\begin{tabular}{|l|l|l|}
\hline Scale & Property & Size range [m] \\
\hline \hline Macro & $\begin{array}{l}\text { Rheology / Mechanical / Cracking / Volume stability / } \\
\text { Durability }\end{array}$ & $10^{-1}-10^{2}$ \\
\hline Meso & $\begin{array}{l}\text { Compressive and Tensile strength / Fracture energy and } \\
\text { Toughness }\end{array}$ & $10^{-5}-10^{-1}$ \\
\hline Micro & Hydration / Chemistry / Pore pressure / Permeability & $10^{-6}-10^{-5}$ \\
\hline Nano & $\begin{array}{l}\text { C-S-H analysis, Calcium leaching / CH, Ca/Si -ratio / Al/Si }- \\
\text { ratio }\end{array}$ & $10^{-10}-10^{-6}$ \\
\hline
\end{tabular}

Table 1. Overview of modeling scales, properties and size ranges.

The input for the different scale level models consists either of direct user input or of input achieved from a lower or higher scale level. This will lead to a refinement of the simulation predictability and will lead to a system with an increased synergy. A virtual laboratory can help to facilitate this linking of models and provides the opportunity to allow other partners to link their models as well, leading to an overall modeling platform for computational materials design (see Figure 5).

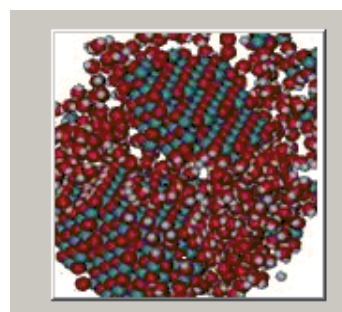

Nano

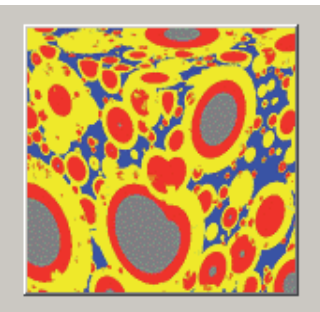

Micro

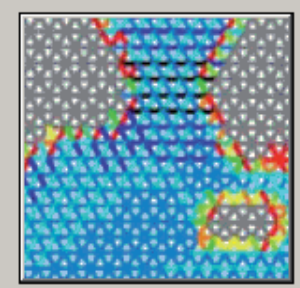

Meso

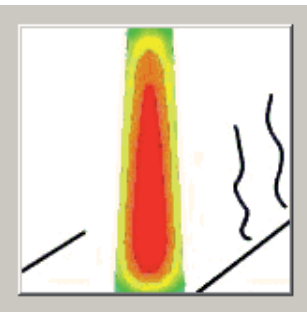

Macro

Figure 5. Schematic representation of the modeling levels.

\subsection{Macro-scale level}

The macro-scale level is the level at which full-scale structures are being designed and calculated. Relevant concrete related issues that have to be known for this particular scale level are listed in Table 1. For the macro-scale level, Finite element models (FEM) are very often used to simulate the structural respond of systems under static and/or dynamic actions, but also to simulate the early age hardening behavior of freshly cast material. Simulation models are used to avoid cracking during hardening. Practical problems are often related to the mix design and to the climatic conditions under which hardening takes place. Design and engineering of concrete structures, therefore, can be conducted with macro-scale FEM models like DIANA [14], ANSYS [15], ABAQUS [16] or FEMMASSE [17], where the latter is especially designed for early age analysis of concrete structures. In particular when considering the early age behavior, the development of the materials properties becomes very relevant. FEM models need to receive this information as input, or sometimes simple mathematical formulas or empirical functions are developed that can be fitted to experimental data and, with this, are able to predict the materials behavior. The 
disadvantage of this approach is that still expensive and time consuming physical laboratory experiments have to be conducted for the fitting and validation of the models. With these FEM models the stress and strength development can be calculated for full-scale concrete structures during the early stage of hardening. For accurate assessments, the model requires input from the structure's geometry and formwork characteristics, the ambient conditions and the thermo-mechanical properties of the hardening mix. From these inputs, the model calculates the temperature field and, from this, the development of the tensile stresses that occur as a result of the internal and external restraint (Figure 6). Important material properties herein are the development of the tensile strength, the elastic modules and the relaxation coefficient. All these properties are depending on the mix composition and on the hardening conditions, and can be expressed as a function of the degree of hydration.
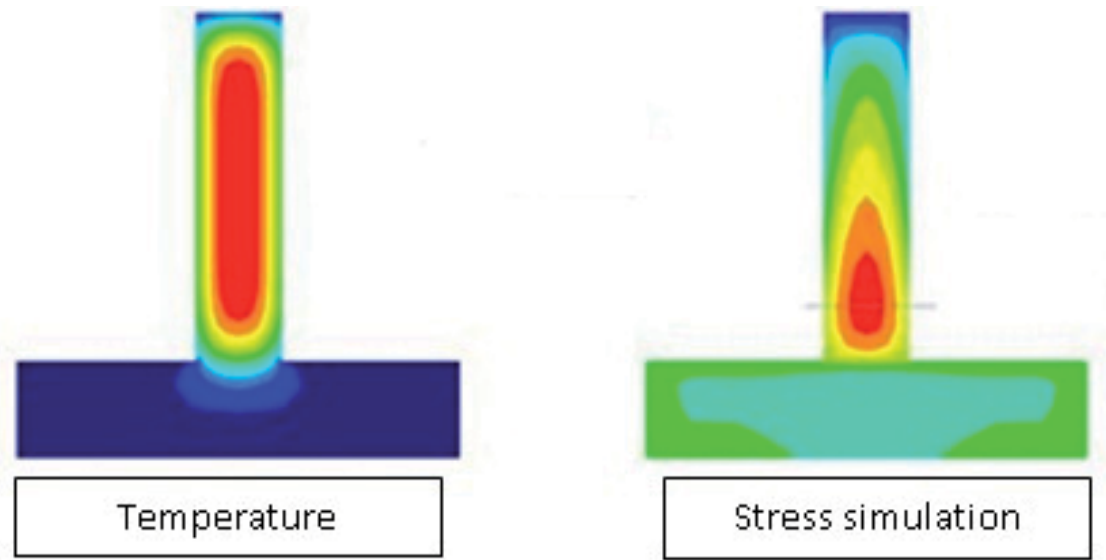

Figure 6. Temperature and stress field during early hydration of a hardening concrete wall cast on an already hardened slab [17].

With the calculated stress field and the tensile strength development known at any location in a structure, the probability of (macro) crack occurrence can be calculated using statistical methods. Cracking will occur if the calculated stress exceeds the strength while accounting for the scatter of the material properties. The accuracy of the crack predictions also requires an accurate description of the material properties. Experienced-based models, databases or numerical simulation models operating at a more detailed scale level can be used for this. More fundamental models, based on thermo-mechanical-physical mechanisms operating at an increased level of detail, can also be applied. In the next sections, models operating at the meso, micro and nano-scale level will be discussed with emphasis on the increased level of detail for the simulations while aiming to improve the prediction accuracy of the models that operate at the macro-scale level.

\subsection{Meso-scale level}

Increasing the level of detail of numerical schemes with the objective to simulate fracture propagation processes in concrete, lattice models can be applied. In order to be able to 
predict the ultimate capacity of a virtual concrete sample by simulating its cracking pattern, a fracture mechanics model is required which can handle the crack propagation of the material while loaded. A model that can be adopted in this respect is the Delft Lattice model, which has originally been developed by Schlangen and Van Mier in 1991 [18]. The model simulates fracture processes by means of mapping a framework of beams to a materials meso structure. The basic principles of the Lattice model are schematically shown in Figure 7. In this figure (a) is a schematization of a regular type of framework mesh that can be used to simulate the meso-level structure of brittle materials, such as concrete.

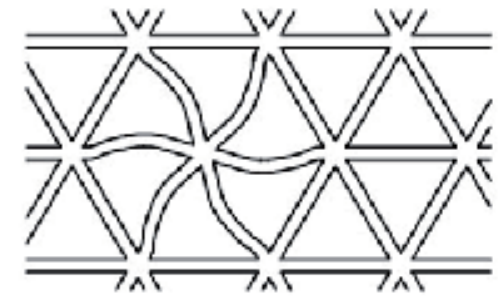

(a)

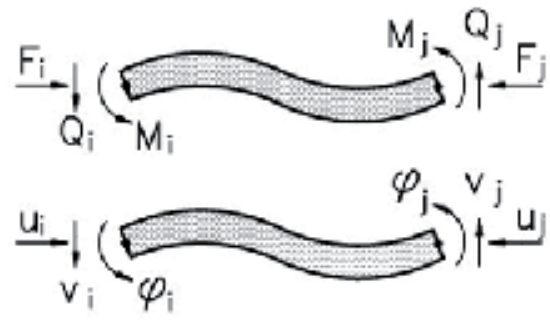

(b)

Figure 7. Principle of the Lattice model [18]; a) Lattice framework, b) Lattice beams with action forces and displacements indicated.

For composite materials in particular, the meso-level structure reflects the schematization of the paste phase, the Interfacial Transition Zone (ITZ) and the aggregate explicitly. This approach of schematization fits very well with the level that is required for modeling the compressive stress calculations inside a virtual laboratory. The model should be able to detect failure paths through the material (weakest links) and to calculate the accompanying ultimate strength of the building material from it. Once this failure path has been initiated, the inner structure of the material starts to disintegrate and the strength capacity will reach its maximum. After having reached this maximum strength level, a descending branch will follow that indicates the post-peak behavior of the material. The Lattice model is capable to calculate this part of the failure traject and to quantify the fracture energy of the failure behavior as well. For conventional concretes, the Interfacial Transition Zone (ITZ, weak bonding zone around the aggregates) is almost always the weakest part of the material that initiates and contributes to the failure paths (Figure 8). For higher quality concretes, the failure paths might cross through the aggregate particles which implicitly affect the brittleness of the material. A proper compressive strength model within a Virtual Laboratory should therefore implicitly deal with these different kinds of failure mechanisms related to the mix composition in general and the inner microstructure of the material in particular.

\subsection{Micro-scale level}

For the simulation of the evolving cementitious microstructure that forms the fundamental basis for the development of the material properties the hydration model Hymostruc can be used $[19,20]$. After mixing, hardening commences and the material properties start to develop. This process leads to a set of properties that is unique for every particular type of 

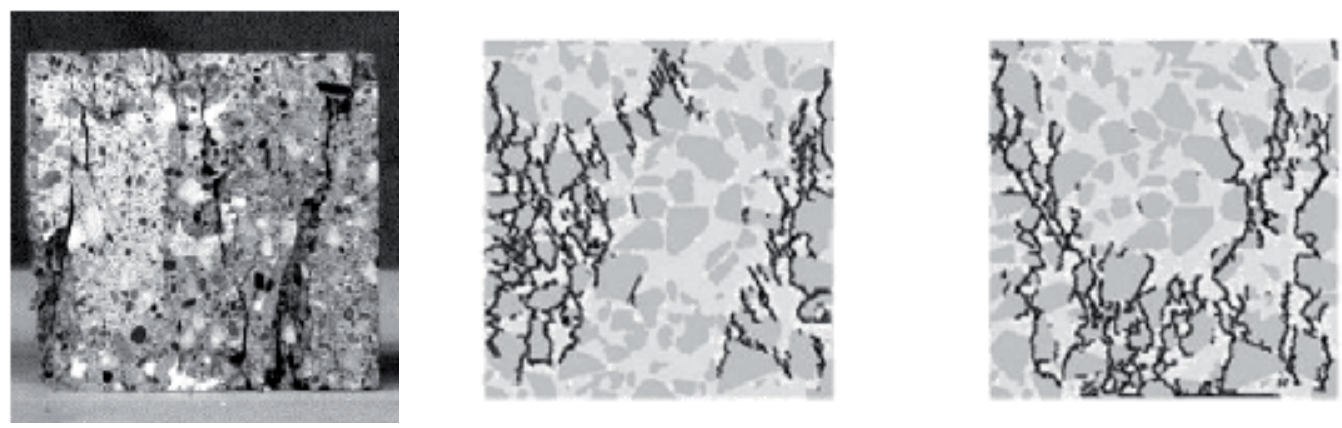

Figure 8. Left: Concrete crack pattern after loading. Right: Lattice simulation [18].

cement-based material. The Hymostruc model (Figure 9, left) can be applied to predict the actual state of the material properties with the degree of hydration as the basic parameter. The model calculates the hardening process of cement-based materials as a function of the water-cement ratio, the reaction temperature, the chemical cement composition and the particle size distribution of the cement. The model calculates the inter-particle contacts by means of the 'interaction mechanism for the expanding particles' (Figure 9, right) where hydrating particles are embedded in the outer shell of larger hydrating particles. This mechanism provides the basis of the formation of a virtual microstructure which, on its turn, can be considered as the backbone of the evolving strength capacity of the material. When considering the virtual laboratory, the Hymostruc model will operate at the micro-scale level and will be used to calculate the internal microstructure that is necessary to simulate the compressive and tensile strength development, the development of the elastic modulus and other microstructure related properties. The microstructure of the material can be considered as the morphology-based inner structure of the paste, i.e. the "glue", that tightens together the aggregate particles and/or other composite phases such as fibers, fillers, etc, inside a composite material. Failure of the paste structure, therefore, strongly depends on the strength characteristics of the internal bondings in the microstructure of the paste. Modeling the morphologies of these bondings in terms of their chemo-physical nature has to be resolved at the nano-scale level. The development of the properties of the C-S-H gel, therefore, is a scale-level that has to be considered as well.

\subsection{Nano-scale level}

Nano-scale modeling has benefit from an enormous increase in the attention of the research community with the aim to model the chemical and physical based processes of the Calcium-Silicate-Hydrates (C-S-H gel) that forms the fundamental elements of the hydration products of cementitious materials [21]. Characterizing the materials performance at this particular scale level asks for modeling the fundamental processes using molecular dynamics principles. For cement-based materials in particular, emphasis has to be on the characterization of the basic building blocks of the C-S-H nano-structure that operate at the sub-micro scale level. This intermediate scale-level between the nano and micro-scale level enables a modeling approach that bridges the gap between the micro and nano level and that enables an exchange of fundamental materials properties (Figure 10). 

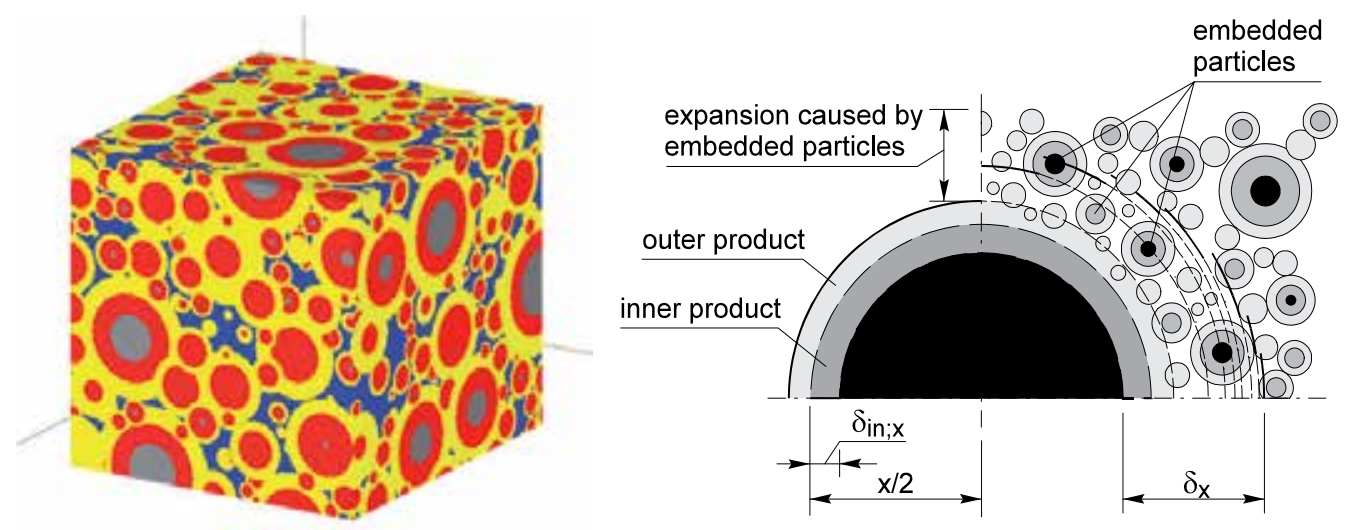

Figure 9. Left: 3D virtual microstructure simulated with Hymostruc. Right: Hymostruc interaction mechanism for expanding particles representing the formation of structure of the virtual microstructure $[19,20]$.

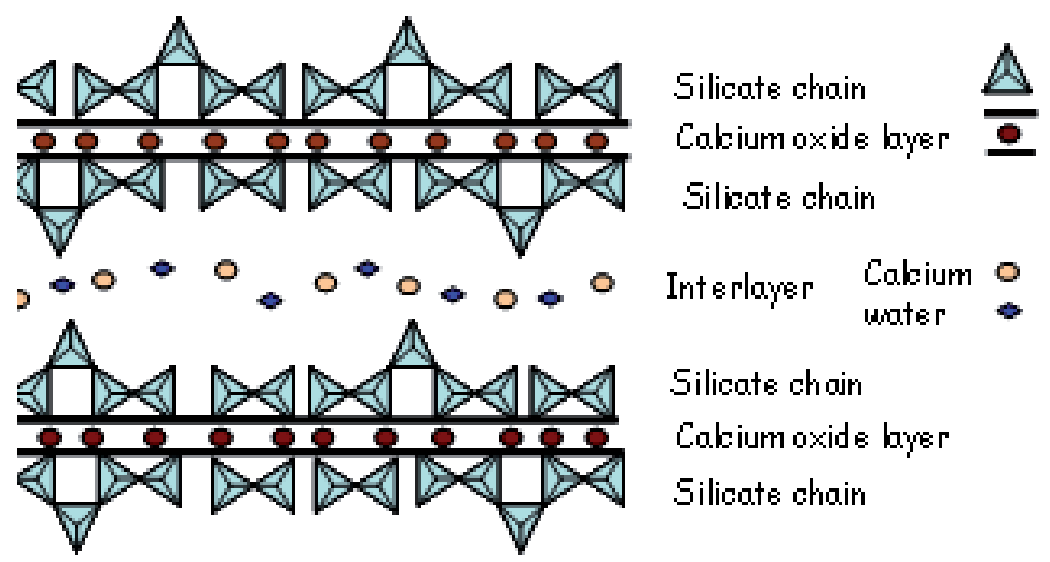

Figure 10. Structural model that describes the atomic scale of the C-S-H gel [21].

\subsection{Up-scaling}

The development of numerical algorithms that allows for particular scale-level information to be used at other scale levels is the most challenging part of multi-scale modeling. Bridging the length scales between the nano scale level and the macro-scale level requires a upscale models that enable to span of 10 orders of magnitude (see Table 1). In this approach the nano scale level forms the basis of the multi-scale framework. The output properties calculated at a particular scale level forms the input at a higher scale level. This approach enables the analysis and design of composite materials starting from the fundamental nanoscale level and evaluates the results at the full-scale macro-level. It opens the door for tailor made design of composite materials and is a first step towards property defined modeling approach. A virtual laboratory is an excellent vehicle to achieve this. 


\section{Virtual laboratory prototype}

In 2012, the first prototype of a virtual laboratory has been developed at Delft University of Technology. In this paragraph, the system development rationale and architecture are presented, including screenshots of the prototype created to demonstrate the idea of a webbased virtual laboratory. Due to the fact that existing computation models and supporting computer tools have been implemented in the traditional way in the past, the demonstrated prototype not fully operates according the netcentric approach as discussed above. The main purpose of this prototype is to support the approach of multi-scale modeling for concrete materials in an integrated web-based environment. From this, it can be derived that prototype should provide a complete environment for multi-scale experimentation easing the study of composite materials.

From the point of view of the final users, the prototype should aid and support them on their experiments relying on a fast execution of the best simulation models existing. Since the system's user interface is based on the functionalities available in the computational (simulation) models and supporting computer tools, the final user needs to know about the computational models to be able to work with them. However, this user does not want to care where these computational models are running - they want to focus on their educational and research applications instead of the technology required providing their needs. For instance, the system should relief the users from the burden of looking for simulation module availability, its installation and execution; take care of the issues regarding combining modules to perform multi-scale experiments and carry out other system administration duties too. From the point of view of the computational model creators, the virtual laboratory should be open in a way to be able to support new computational models to be plugged-in adding new services and functionalities to the existent ones. The computational models must exchange data, making possible the execution of a multi-scale experiment based on different computational models developed independently (i.e. mashup). Therefore, there must exist a platform that works as an open ecosystem environment, populated with simulation models, created and executed independently, but that are coordinated by the final users through an interface that offers multi-scale experiments of composite materials. With this purpose, the system architecture was created based on two principal modules, the user interface module (frontend) and the simulation modules (backend). This modular organization provides decoupling of the user interface from the simulation modules. The deployment diagram of the virtual laboratory system is presented in Figure 11. showing the fronted module as the Graphical User Interface (GUI) component and the backend module as the model execution controller, which relies on a Grid infrastructure to execute the computational models.

The virtual modelling laboratory contains a rich interface web application that has been developed using cutting-edge technology, such as HTML5, CSS3 and JavaScript. The backend is defined as a set of web services which are developed as a Restful API and which is accessed through AJAX calls. An extensive set of toolkits and frameworks is used in the 
final implementation. The GUI foundation is based on the Twitter Bootstrap library for UI building and on the Backbone.js toolkit for organizing the JavaScript code, and being fully based on the jQuery library. The backend was developed using the Play framework. Although the basic application architecture is defined as a two-layered system - frontend and backend, the backend layer is composed by a set of different computational modules that can be seen as independent web services. The modules executions are managed coordinated by the overall controller service, but the composition architecture is much more complex than what is exposed. Different researchers and developers, distributed over the Internet, can make their computational models available through different interfaces. In addition, the computational models can also use results from other computational models.

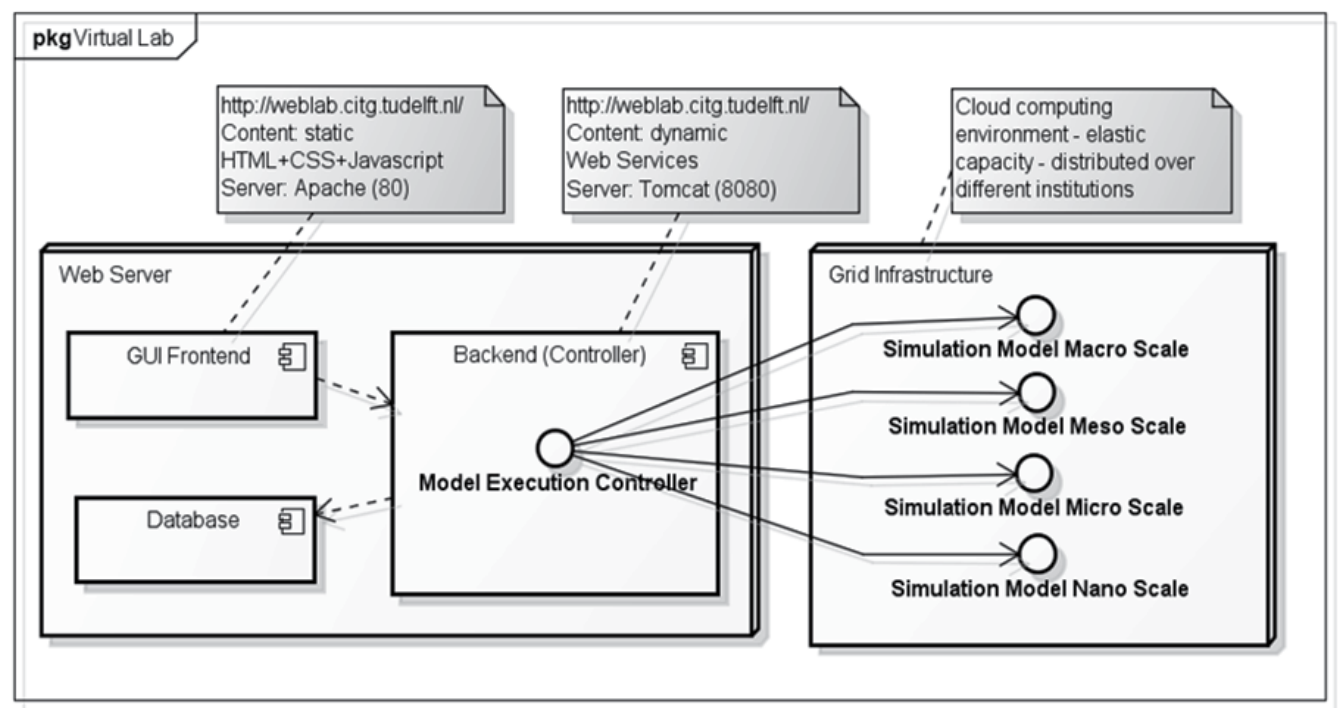

Figure 11. The deployment diagram of the virtual laboratory at Delft University of Technology.

As discussed earlier, the prototype relies on a Grid infrastructure to execute the computational models. This infrastructure is distributed over different institutions, because each computational model owner should be responsible for the model execution and availability as a web service. For this purpose, a Grid infrastructure should be available to support the whole platform. As discussed earlier, other new emerging technologies such as Cloud Computing can supply on-demand computational resources to run the computational models.

In order to be able to transfer data between the different modelling scale levels, multi-scale modelling principles have to be considered as well. Apart from how the data is transferred from one scale level to another, the most challenging part is how to connect the levels from a modelling point of view. Bridging the scale levels can go along with transfer of data only by means of parameters passing or by means of a more complicated integration of models that operate at different scale levels or a possible combination. In either way, bridging the scale levels is an intensive modelling work that requires significant effort both from materials properties as well as from a modelling point of view. With the virtual modelling lab, the 
user can choose at which level he/she wants to start the numerical experiments (Figure 12). It is organized in such a way that data can be calculated in a certain scale level and, when the user decides to proceed with an analysis at another scale level, the data can be taken.

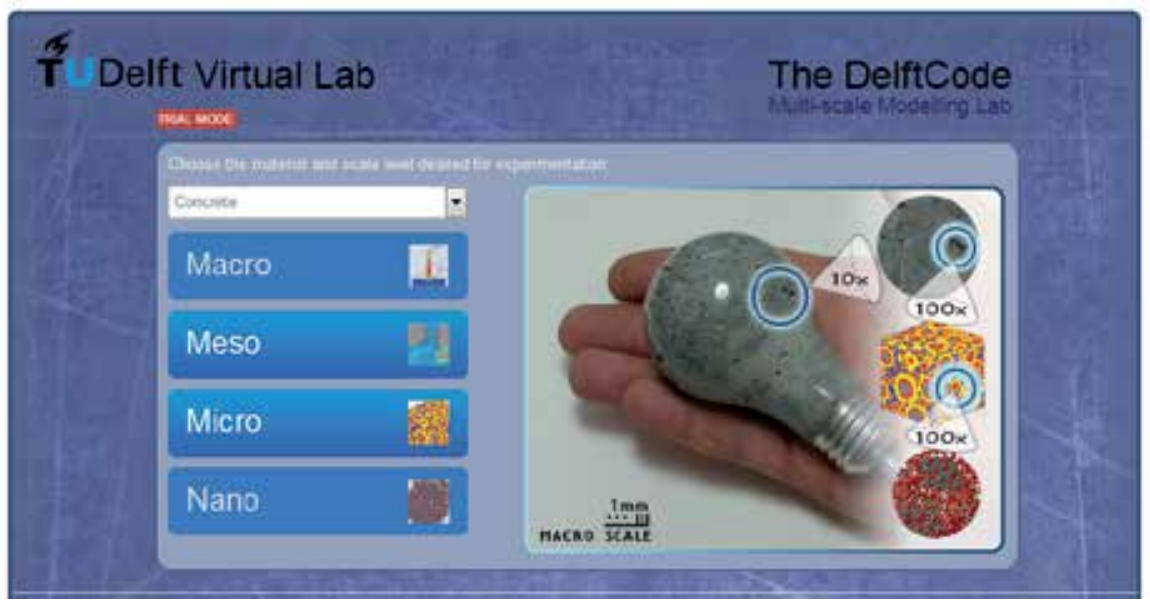

Figure 12. DelftCode: Selection of scale level.

The pilot version of the virtual laboratory at the Delft University of Technology (referred to as DelftCode) provides output (results) which are represented as graphs in the GUI. In addition, each simulation of computational model at a certain modelling scale produces specific parameter output that is managed by the DelftCode framework in such a way that it can be used as input for computational models at other scale levels (Figure 13). In this way active multi-scale modelling can be conducted and the results can be reused at other scale levels. The way how it is implemented in the DelftCode framework is that after conducting numerical experiments the user can switch to other scale levels. For the other scale levels the same procedure is followed. Since data will be stored and available for all scale levels, the user can access data of previous numerical experiments and reuse it for other experiments.
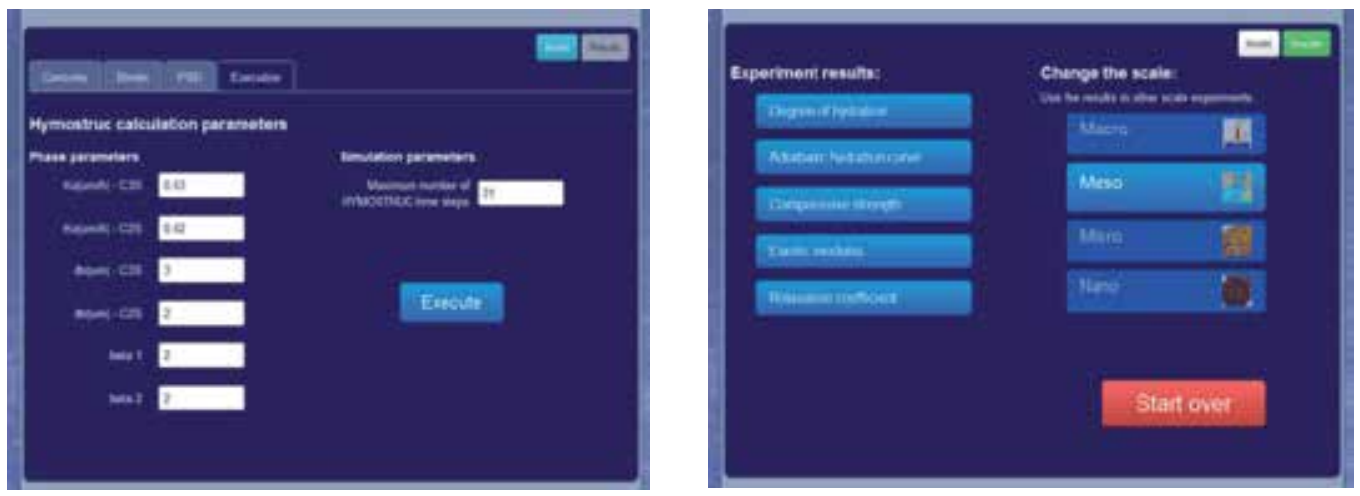

Figure 13. DelftCode: Model setup and result outputs. 
At time of writing, the prototype only supports computational models at micro and meso scales, but the prototype has been designed and implemented to support all scale levels. Figure 14 show all the proposed user interactions in the prototype. These interactions show the possibilities of the developed prototype that allows multi-scale modeling platform. The architecture of the prototype is designed in such a way that future extensions in terms of new model additions or adding another scale level can easily be achieved.

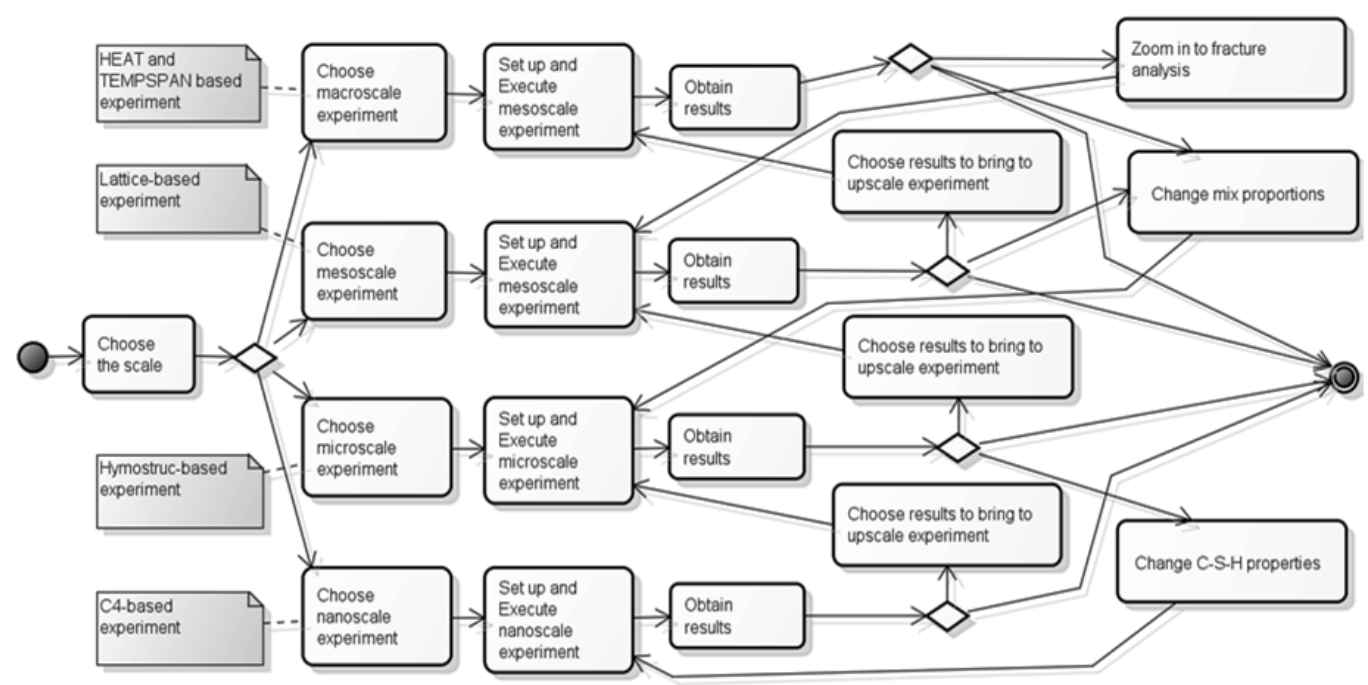

Figure 14. The activity diagram of the user experiments of all scales.

\section{Conclusion and discussion}

This paper depicts the development the concept of so-called virtual laboratories that are based on a netcentric approach. In this context, a netcentric virtual laboratory is considered as a part of an evolutionary, complex community of people (users), devices, information (i.e. experimental data) and services (computational models and supporting computer tools) that are interconnected by the Internet. A large number of emerging and enabling technologies are discussed which form technological basis for establishing such netcentric virtual labs. Although the developed prototype is hampered by the fact that most computational models and supporting computer tools have been developed using traditional programming languages and platforms, it showed the enormous potential of the netcentric approach advocated in this paper.

From the perspective of the application domain, a virtual laboratory can be considered as a most appropriate way to interact with users and developers of computational material models at different scale levels. In this paper the approach of multi-scale modeling approach for concrete materials is explained. This approach is based on numerical (computational) models developed for cementitious materials that operate at different 'geometrical' scale levels. With the ability to use the output generated at any particular scale level as input for models that run at other scale levels, the web-based virtual laboratory acts as a real multi- 
scale modeling platform. The architecture of the proposed virtual laboratory as provided in this chapter allows the models to be exchangeable and merge-able leading to an integrated approach.

The numerical models for simulating the materials performance operate at the different scale-levels with the Hymostruc model as the main microstructural model at the micro-scale level. With this model connecting to the nano-scale model for inputting detailed information on C-S-H gel properties, the microstructural information can be used as input for the mesolevel Lattice model to simulate the fracture behavior of composite materials submitted to internal actions (drying, autogenous shrinkage, etc) or external actions (loads, thermal imposed loading, etc). These models can generate input data for the macro-scale models to simulate the full scale performance of structural elements. With the multi-scale approach, the consequences of changing parameters that act as input for lower scale models (nano, micro, meso) can directly be made visible by upscaling. Therefore, from this approach the following conclusions can be drawn:

- The netcentric virtual laboratory is a most appropriate tool for the assessment of composite materials performance using a multi-scale modeling approach;

- The web-based approach enables the communication between models that operate at different geometrical scale-levels using an integrated computational modeling system;

- The prototype shows the huge potential of web-based modeling and provides an exchangeable and scalable system for multi-scale modeling;

- The future perspective of virtual web-based modeling shows to be a very powerful alternative for vast computational models that run over different time and length scales.

\section{Author details}

E. Dado

Netherlands Defence Academy, Breda, The Netherlands

E.A.B. Koenders

Delft University of Technology, Delft, The Netherlands, COPPE-UFRJ, Programa de Engenharia Civil, Rio de Janeiro, Brazil

D.B.F. Carvalho

(PUC-Rio), Rio de Janeiro, Brazil

\section{References}

[1] Kuester, F. and Hutchinson, T. A virtualized laboratory for earthquake engineering education, ASEE Journal of Engineering Education, 15:1, 2007.

[2] ECWINS consortium. The Road to Standardized Window Production. Collective Research Projects for SMEs, Vol. 3, European Union, 2007.

[3] Bullard, J. et al. Virtual Cement, Innovations in Portland Cement Manufacturing, USA, 2004. 
[4] Dado, E., Koenders, E. and Mevissen, S. Towards an advanced virtual testing environment for concrete materials, published in the proceedings of the MS2010 conference, 2010.

[5] Dado, E, Koenders, E. and Beheshti, R. Theory and Applications of Virtual Testing Environments in Civil Engineering, International Journal of Design Sciences and Technology, 16:2, 2009.

[6] Koenders, E., Schlangen, E. and Dado, E. Virtual testing of compressive strength of concrete, published in the proceedings of the ISEC-4, Conference, 2007.

[7] Koenders, E., Dado, E and van Breugel, K. A Virtual Environment for Multi-Aspect Modeling, published in the proceedings of the SCI2004 conference, 2004.

[8] Garboczi, E., Bullard, J. and Bentz, D. Virtual Testing of Cement and Concrete, Concrete International, No. 12, United States, 2004.

[9] Foster, I., Zhao, Y., Raicu, I. and Lu, S. Cloud Computing and Grid Computing 360Degree Compared. Proc. Grid Computing Environments Workshop, p.1-10, 2008.

[10] Mell, P. and Grance, T. The NIST Definition of Cloud Computing, NIST special publication 800-145, United States, 2011.

[11] $\mathrm{Wu}, \mathrm{Z}$. and Chen, H. From Semantic Grid to Knowledge Service Cloud, Journal of Zhejiang University, 13:4, China, 2012.

[12] Mika, P. and Tummarello, G. Web semantics in the clouds. IEEE Intelligent Systems Magazine, 23-5, United States, 2008.

[13] Koenders, E, Schlangen, E. and Dado, E. Virtual Testing of Compressive Strength of Concrete, Proceedings of the ISEC-4 Conference, Australia, 2007.

[14] DIANA, http://tnodiana.com/.

[15] ANSYS, www.ansys.com/.

[16] ABAQUS, www.simulia.com.

[17] FEMMASSE, www.femmasse.nl.

[18] Schlangen, E. Experimental and Numerical Analysis of Fracture Processes in Concrete, PhD thesis, Delft University of Technology, The Netherlands, 1993.

[19] Breugel, van, K. Simulation of Hydration and Formation of Structure in Hardening Cement-based Materials, PhD. Thesis, Delft University of Technology, Delft, The Netherlands, 1991.

[20] Koenders E. Simulation of Volume Changes in Hardening Cement-Based Materials, PhD-Thesis, Delft University of Technology, Delft, The Netherlands, 1997.

[21] Dolado J. S., Hamaekers J. and Griebel M. A Molecular Dynamic study of cementitious Calcium Silicate Hydrate (C-S-H) gels, Journal of American Ceramic Society. 


\section{Mechanical and Physical Properties of Composites}





\title{
Characterizations of Environmental Composites
}

\author{
Ali Hammood and Zainab Radeef \\ Additional information is available at the end of the chapter
}

http://dx.doi.org/10.5772/50494

\section{Introduction}

Recently, environmental preservation issues have been critical between the chemical pollution matters and the development technology requirements. However, the renewable and friendly materials come to use.

Numerous researches have richly studies the natural fiber reinforcement polymer composites. This fact, based on both fibers and matrixes are derived from renewable resources. Therefore, the formed composites have more compatibility with the environmental preservation issues. [1] Isabel investigated of the most natural fibers are used such as palm, cotton, silk, coconut, wool and wood fibers. A significant development in the lignocelluloses fiber in thermoplastics realized the distinct researches presented by [2-5] Composite-reinforcing fibers can be categorized by chemical composition, structural morphology, and commercial function. Natural fibers, such as kenaf, ramie, jute, flax, sisal, sun hemp and coir are derived from plants that used almost exclusively in PMCs. Aramid fibers [6] are crystalline polymer fibers are mostly used to reinforce PMCs. The compounds percentage of composite have the essential role for verify the designed values according to applications, therefore the mechanical properties of PMCs predicated by Mohamed (2007).

The primary function of a reinforcing fiber is to increase the strength and stiffness of a matrix material. The fibers reinforced composite have the essential role in this investigation for its significant property advantages as high stiffness, lightweight, easily recycled material, availability, low manufacturing cost, the environment effect and lifetime rupture behavior. Various types of natural fibers are available to combine with other mineral fiber for construct composite material. Essentially, the fiber can be classified as vegetable, animal, and man-made fibers. The main disadvantages of natural fibers are their high level of moisture absorption, poor and interfacial adhesion, relatively low heat resistance. [7-8] investigated high speed impact events using (PKV, PRM) composites. This research was indicated significant improvements in the penetration resistance. This fact comes from the improvement of target geometry structure. Numerous researches have been carried out on 
the ballistic impact on high strength fabric structures [9-11]. In the airport and marine applications, the dynamic loads effect and the chemo interactions were attracted the researchers and many methods are employed for computing the surface topography parameters, thereby numerous estimations were covered the erosion-corrosion behavior of PMCs [12-13]. The most impinging parameters focused on environment effects and impinging angle [14-17]. There were many instruments and electronic microscopes developed with the time for measuring the roughness parameters and drawing the surface topography [19]. The Erosion and corrosion of composite must be determent for the accelerator objects, whereby this values will be indicator for measuring the life time rupture of composite [18].

\section{Important}

The automotive and aerospace industries have both shifted for using natural fiber reinforced composites as a factor to reduce the weight and getting significant properties of composite components. As matter of fact, the impinging liquids of the naval and aerospace applications have a direct effect on surface topography. Therefore, advanced studies focused on corrosion and erosion behavior. The impinging angle, velocity versus time, composite morphology represented the essential parameters of this field of study. In this investigation, surface roughness versus time was the indicator for erosion and corrosion effects.

\section{Experiment Procedure and Samples Preparation}

In order to develop new composite material with high impact resistance and high erosion resistance, characterization study for two sets of composites materials have been computed. The specific composites materials in this research are: polyester resin-matrix and Kevlar reinforced fiber (PKV) with $\mathrm{V}_{\mathrm{f}}(42 \%)$, polyester resin-matrix and ramie reinforced fiber (PRM) with $V_{f}(42 \%)$. Experimental program was carried out to study the erosion and corrosion behavior by computing the surface roughness parameters of (PKV, PRM) before and after impinging operation. Hence, Polymer Matrix Composites (PMCs) were examined by impingement using water jets when the aqueous solution was $3.5 \mathrm{wt} \% \mathrm{NaCl}$. Erosion and corrosion tests were impinging at 90 angles at velocity $30 \mathrm{~m} / \mathrm{s}$ and the impinging period was 12 hours.

The composite subject study consists from five Kevlar layers and five ramie layers were impregnated with unsaturated polyester. The layers aligned alternatively according to the expectation performance. The synthetic ramie fiber was weaved as shown in Figure 1(a) \& (b).

\section{Tensile testing of composites}

Tensile specimen $(145 \times 15 \times 7)$ mm3 are caught according to ASTM D 3039 /D 3039M-95M standard (ASTME D 3039, 2003). Tensile test has been conducted and the data acquired digitally. Tensile stress, tensile strain and Young modulus of PKV with Vf (42\%) and PRM with $\mathrm{Vf}(42 \%)$ were calculated and the test was performed by instron machine $10 \mathrm{KN}$, series 


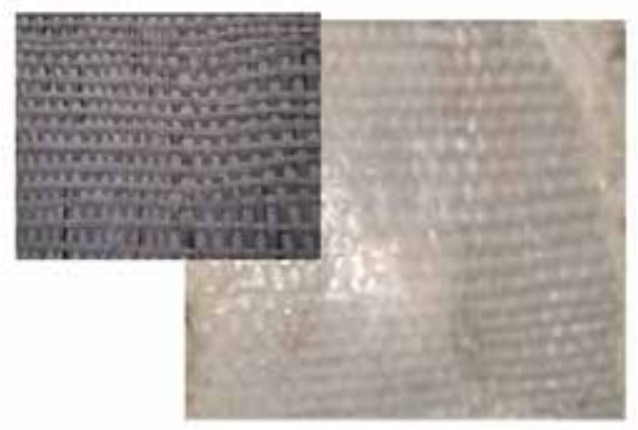

(a) Ramie Woven and PRM

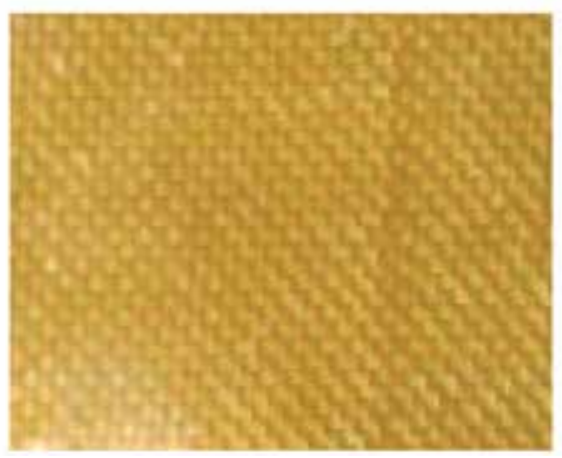

(b) Kevlar Woven

Figure 1. Ramie and Kevlar Woven

2716 and 2736 under stable speed rate $2 \mathrm{~mm} / \mathrm{min}$. The test has verified all the specific test conditions to determine the tensile properties of specimen according to the ISO 527. Tensile test has been performed to estimate the yield stress, young modulus and tensile extension at yield point. Additionally, Poisson's ratio has been calculated by evaluate transverse strain and longitudinal strain of composite. Hence, the transverse strain calculated by using strain gage that supplied from Tokyo Sokki Kenkyuio co, Ltd. The used gage type was BFLA-2-8 with gage resistance $120 \pm 0.3 \Omega$. Then, the strain is connected to DAQ bridge system for reading the transverse strain.

The composite behavior under test was an important subject for this investigation and the specimen geometry was constructed from five layers Ramie fiber and five layers of Kevlar as clarified in the Figure 2.

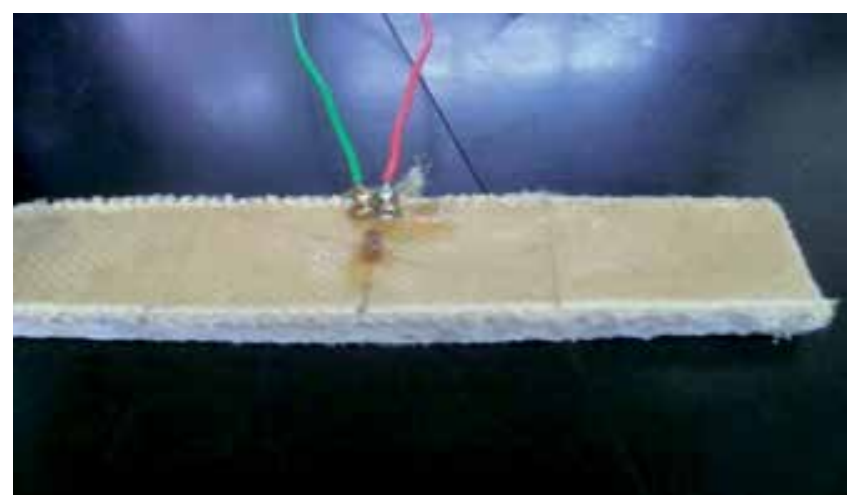

Figure 2. Tensile Test Specimen with Strain Gage

\section{Ballistic limit and number of layers}

The ballistic limit is commonly defined as a $50 \%$ probability of penetrating a target at a given impact velocity. The energy absorption is related to the impact velocity, interpreted by the effect of the striking velocity on the amount of kinetic energy that is absorbed by the 
composite material. Hence, the energy absorbed by the fabric is equal to the residual energy amount subtracted from total impact energy.

In this test, the target impinged by using gas gun machine supported by a high speed camera for record the impact event at 30,000 frames per second with image size $512 \times 64$ pixels per image [7]. Figure 3 (a) shown the gas gun machine. Thus, The velocity before the target and the residual velocity after the target were estimated. All the targets were impinged by Semi-conical bullets as shown in the Figure 3(b).

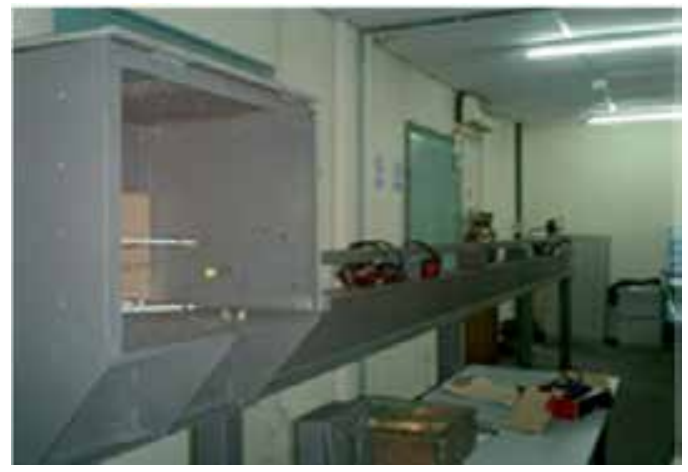

(a) Ballistic Panel

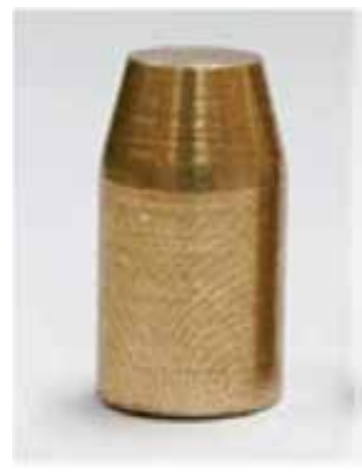

(b) Semi-conical bullets

Figure 3. Gus gun device of University Putra Malaysia $[7,8]$

\section{Erosion test and corrosion}

Accurate estimation is carried out for calculating the erosion and corrosion percentage of (PKV, PRM) samples. The tests were conducted using a jet at velocity $30 \mathrm{~m} / \mathrm{s}$ and impinging angles $90^{\circ}$. Essentially, the exposure period was the major factor of erosion estimation. Hence, the impinging periods were from 3 to 6 and from 6 to 12 hours under room temperature. Samples roughness data were recorded before and after the tests by Image processing software for scanning probe microscope

\section{Result and discussions}

\subsection{Tensile test}

The tensile test of this composite was conducted for specifying the mechanical properties of the composite. Generally, the test results recorded high tensile strength. The brittle manner was the first stage of composite under test due to the low elongation ability of matrix. The second stage was the ductile behavior that embodied of high elongation ability of Kevlar layer. From stress - strain curve topography, the specimen have extension at maximum load up to $2.49 \mathrm{~mm}$ was recorded. There are continuously reductive of the curve as a result to the elongation of Kevlar layers. The extension at maximum tensile strain observed at $43.25 \mathrm{~mm}$ that was evident in the ductile behavior at front face of the specimen. Practically, through 
the extension test, the back face of the specimen that contented from ramie layers reinforced polyester was separated gradually as result to the brittle behavior of ramie - polyester matrix. Automatic Young's modulus in this composite is $4930.5 \mathrm{MPa}$. This fact plotted in stress - strain curve in the Figure 4 . The composite properties depend at compounds types and volume fraction. Therefore, the volume fraction of fiber or matrix could be calculated as it is clarified in the following equation.

$$
V_{m}=\frac{v_{m}}{v c}
$$

Where

$V_{m}=$ volume fraction of matrix

$v_{m}=$ volume of matrix

$v_{c}=$ total volume of the composite

Volume fraction of fiber could be evaluated from.

$$
V_{m}=1-V_{f}
$$

Where

$V_{f}=$ volume fraction of fiber

The volume of ramie fiber could be calculated from the following equation.

$$
V_{f}=V_{K}+V_{R}
$$

Where

$V_{k}=$ volume fraction of Kevlar

$V_{R}=$ volume fraction of ramie

Fiber density can be determined experimentally by weighting the Kevlar and ramie fibers and calculated the volume fraction for the fibers and resin. Therefore, from the following expression could be calculated the density.

$$
\delta_{f}=\frac{\delta_{c}-V_{m} \delta_{m}}{V_{f}}
$$

Where

$\delta_{f}=$ Fiber density

$\delta_{c}=$ Composite density

$\delta_{m}=$ Matrix density

The Poisson coefficient represents the contraction in the transverse direction and could be calculated by using the follow expression. 


$$
\begin{gathered}
V_{l t}=v_{f} V_{f}+v_{m} V_{m} \\
V_{l t}=0.34
\end{gathered}
$$

$V_{l t}=$ Poisson ratio

Where the Kevlar Poisson ratio is equivalent to 0.34 [6], ramie Poisson ratio is equal to 0.3 [20] and 0.4 for unsaturated polyester resin [21].

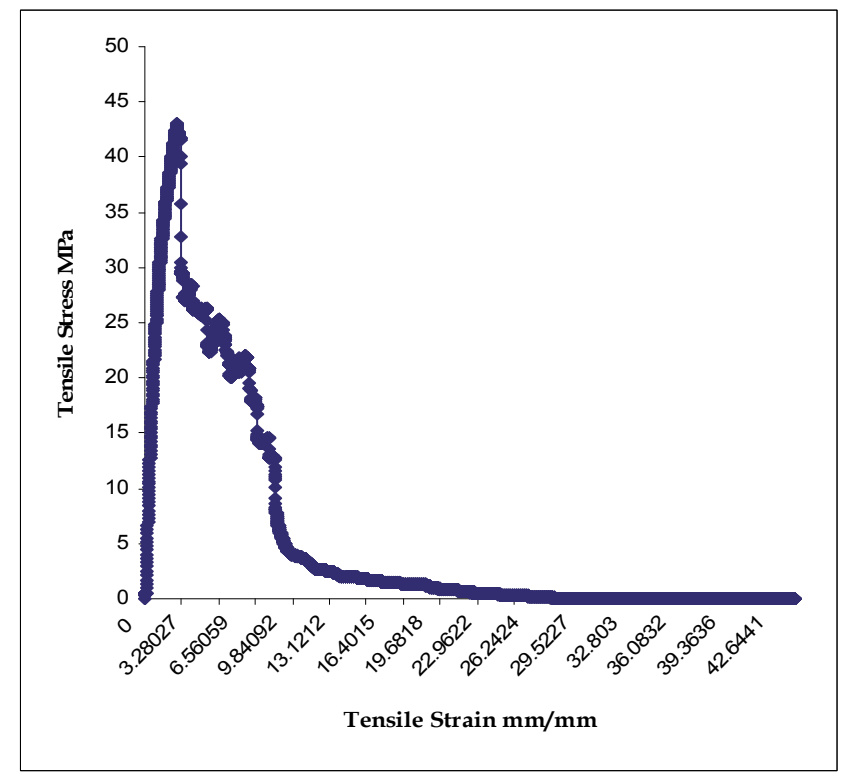

Figure 4. Stress - Strain Curve of PKV +PRM Composite

Modulus of elasticity (E11) can be calculated in (7).

$$
\mathrm{E}_{11}=\frac{\sigma_{\mathrm{L}}}{\varepsilon}
$$

$\sigma_{\mathrm{L}}=$ longitudinal tensile stress

$\varepsilon=$ strain

(E22) is calculated by using gage strain that recorded the shrinking displacement and more than 550 data point recorded load and displacement. The transverse Young's Modulus is the initial slope of $\sigma$ tra. $\varepsilon_{2}$ curve.

The Young's modulus can be calculated by using the retrieved data from Figure 5 as clarified in the following equation.

$$
\mathrm{E}_{22}=\frac{\sigma_{\text {tra }}}{\varepsilon_{2}}
$$




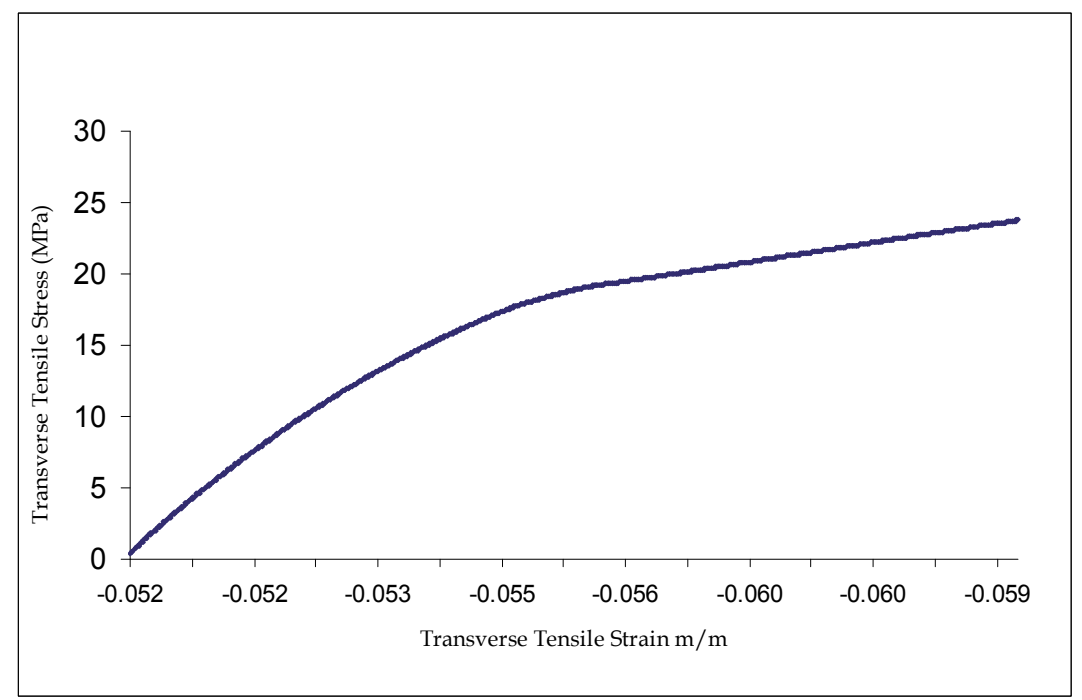

Figure 5. Stress - Transverse Tensile Stress and Strain Curve of Composite

\section{Where}

$\mathrm{E}_{22}=$ Transverse Young's Modulus

$\sigma_{\text {tra }}=$ Transverse tensile stress

$\varepsilon_{2}=$ Transverse strain

The maximum shear stress can be obtained from the following equation.

$$
\tau_{\max }=\frac{\sigma_{y}}{2}
$$

Where

$\sigma_{y}=$ Stress in the yield point

$\tau_{\max }=$ Maximum shear stress

Shear modulus $\left(\mathrm{G}_{12}\right)$ can be calculated from the load data that plotted in the shear stress curve.

$$
G_{12}=\frac{\tau}{\gamma}
$$

Where

$\tau=$ shear stress

$\gamma=$ shear strain

In addition, the Poisson ratio can be estimated from the following equation and the result seems equivalent to the Poisson ratio that was calculated according to the compounds volumes fraction. Table 1 presents the longitudinal and transverse averages of data. 


$$
\gamma_{12}=\frac{\varepsilon_{\text {lat }}}{\varepsilon_{\text {long }}}
$$

Where

$\varepsilon_{\text {lat }}=$ Transverse strain

$\varepsilon_{\text {long }}=$ Longitudinal strain

\begin{tabular}{|c|c|c|c|c|}
\hline \multicolumn{5}{|c|}{ The average of longitudinal data for Kevlar - Ramie -Polyester composite } \\
\hline $\begin{array}{c}\text { E11 } \\
(\mathrm{GPa})\end{array}$ & $\begin{array}{c}(\sigma 1) \text { ult } \\
(\mathrm{MPa})\end{array}$ & $\left(\varepsilon_{1}\right)$ ult & $\begin{array}{c}\zeta_{\max } \\
(\mathrm{MPa})\end{array}$ & $\begin{array}{c}\text { Poisson's } \\
\text { Ratio }\end{array}$ \\
\hline $3.9489 \pm 0.5$ & $66.75 \pm 5.4$ & $0.125 \pm 0.04$ & $33.37 \pm 3$ & 0.37 \\
\hline
\end{tabular}

\begin{tabular}{|c|c|c|c|c|}
\hline \multicolumn{5}{|c|}{ The average of transverse data for Kevlar - Ramie -Polyester composite } \\
\hline $\mathrm{E}_{22}(\mathrm{MPa})$ & $\left(\sigma_{2}\right) \mathrm{ult}(\mathrm{MPa})$ & $\left(\varepsilon_{2}\right) \mathrm{ult}$ & $\gamma_{12}$ & $\mathrm{G}_{12}(\mathrm{MPa})$ \\
\hline $244.74 \pm 2.5$ & $22.77 \pm 1.3$ & $0.0735 \pm 0.03$ & $0.257 \pm 0.05$ & $132.65 \pm 15$ \\
\hline
\end{tabular}

Table 1. Tensile Test Data

\subsection{High speed impact results}

Understanding the impact response of composites has be come an area of great academic and practical interest. The major advantages of composite materials are their high strength and stiffness, light weight, corrosion resistance, crack, fatigue resistance and flexibility. Ramie - Kevlar reinforced polyester resin present high resistance. The high level from resistance could be realized by increasing of Kevlar layers.

$$
\left[E_{a b s}=\frac{1}{2} m\left(V_{i m p}^{2}-V_{r e s}^{2}\right)\right]
$$

Where

$E_{a b s}=$ Energy absorption

$m=$ projectile mass

$V_{\text {imp }}=$ Impact velocity

$V_{\text {res }}=$ Residual velocity

Ballistic limit can be identified as the limit between the penetration and the fully arrested. Thus, the composite with five layers (PKV) and five layers (PRM) couldn't meet the specific requirements of ballistic resistance. In fact, the absorption of energy will be increased with increase the number of layers $[7,8]$.

In the event when no perforation occurs, the energy absorbed by the target will be equal to the initial impact energy. The high speed impact data for PKV \& PRM are shown in the table 2. The following equation has been verified to the ballistic limit or fully arrested action. 


$$
\left[E_{a b s}=\frac{1}{2} m V_{i m p}^{2}\right]
$$

Where

Eabs = absorption of energy

$\mathrm{V}_{\text {imp }}=$ impact velocity

In this prospective must be mention the most high speed impact parameters that represented rich fields of studies are, target geometry, projectile type, target thickness, composite compounds.

\begin{tabular}{|l|l|}
\hline Humanity:53\% & Bullet type: Semi-conical \\
\hline Specimen type: TSP & Camera temperature: $40^{\circ} \mathrm{C}$ \\
\hline Target area: $15 \times 10 \mathrm{~mm}$ & Temperature: $32{ }^{\circ} \mathrm{C}$ \\
\hline Material: PKV\& PRM & Camera resolution: $512 \times 48$ \\
\hline
\end{tabular}

\begin{tabular}{|c|c|c|c|c|}
\hline \multirow{2}{*}{ Layer No. } & $\begin{array}{c}\text { Gas gun } \\
\text { Pressure } \\
\text { Psi }\end{array}$ & $\begin{array}{c}\text { Initiation } \\
\text { Velocity } \\
\mathbf{m} / \mathbf{s}\end{array}$ & $\begin{array}{c}\text { Residual } \\
\text { velocity } \\
\mathbf{m} / \mathbf{s}\end{array}$ & $\begin{array}{c}\text { Absorption } \\
\text { of Energy } \\
\text { J }\end{array}$ \\
\hline \multirow{3}{*}{$5 \mathrm{~K}-5 \mathrm{R}$} & 300 & 273.9 & 125.9 & 147.926 \\
\cline { 2 - 5 } & 250 & 275.255 & 150.17 & 133.035 \\
\cline { 2 - 5 } & 250 & 255.07 & 120.9 & 126.109 \\
\hline
\end{tabular}

Table 2. High Speed Impact of PKV \& PRM

\subsection{Erosion result}

The surface roughness of engineering applications has interacted with the environment. Therefore, the studies pay attention for estimating the surfaces roughness of materials with respect to essential parameters to limit the wear mechanism of materials. Roughness value can either be calculated on a profile or on a surface $\mathrm{Rz}, \mathrm{Rq}$, and $\mathrm{Sa}$ is the arithmetic average of the $3 \mathrm{D}$ roughness. Hence, the impinging test conduct through specific periods is illustrated in the Table (3).

\begin{tabular}{|c|c|c|c|c|c|c|c|}
\hline \multicolumn{4}{|c|}{ Kevlar roughness by $(\mathrm{nm})$} & \multicolumn{4}{c|}{ Ramie roughness by $(\mathrm{nm})$} \\
\hline 0 hours & 3 hours & 6 hours & 12 hours & 0 hours & 3 hours & 6 hours & 12 hours \\
\hline 8.92 & 11 & 9.36 & 9.94 & 12.9 & 11 & 6.71 & 10.8 \\
\hline 16.7 & 8.25 & 8.06 & 10.4 & 7.37 & 9.82 & 6.85 & 10.4 \\
\hline 15.6 & 8.44 & 7.94 & 11 & 10 & 8.32 & 7.78 & 10.3 \\
\hline 7.53 & 9.26 & 6.35 & 8.78 & 9.12 & 11 & 7.82 & 8.43 \\
\hline 6.06 & 8.69 & 6.24 & 11.6 & 10.6 & 10.9 & 8.25 & 8.8 \\
\hline 6.44 & 12.9 & 9.45 & 10.4 & 11.3 & 8.31 & 9.23 & 9.18 \\
\hline 6.28 & 12.9 & 8.81 & 14 & 10.6 & 11.3 & 9.97 & 9.57 \\
\hline
\end{tabular}

Table 3. Roughness values 
The roughness parameters rates, such as (Amplitude parameters, Hybrid Parameters and Functional Parameters) were estimated by all morphology images with image size (100.00nm X 80.00nm) Figure 6 illustrate the morphology images before PKV tests.
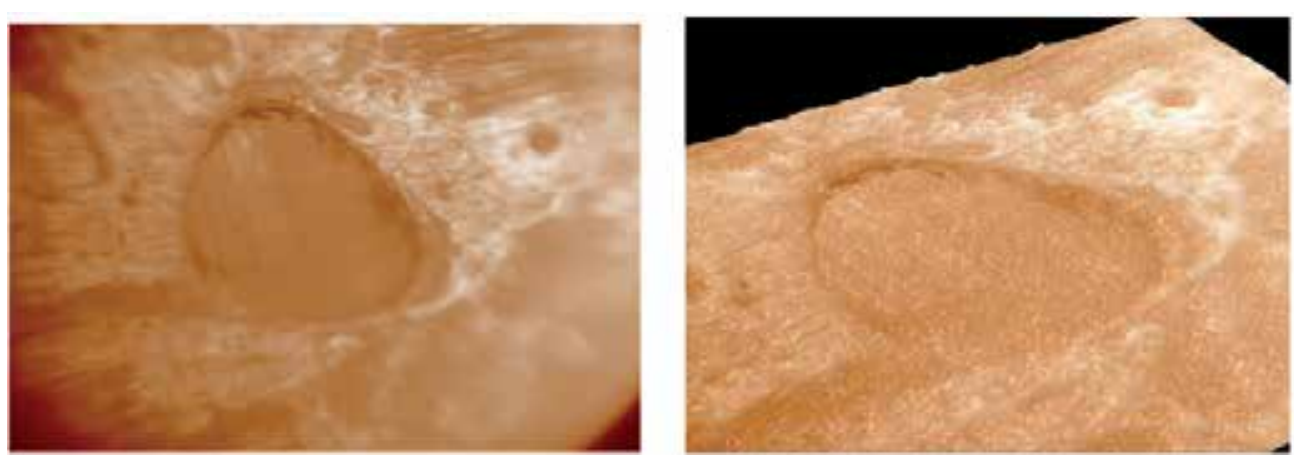

Figure 6. 3D Morphology Images after Tests of PKV.

The erosion wear loss was determined using probe microscope. Typically, scanning probe microscope image process software provided reliable means to evaluate the erosion volume lose. This technique characterizes and quantifies the surface roughness parameters, the surface profile and topographical features in three-dimension using high precision range observed at $100 \mathrm{~nm}$. All measurements were made with an effective magnification of X 12.5. Excellent data were recorded for average size and average height for surface grains. Hence, the average volume of grains can be computed by the grain size analyzer. Randomly, erosion areas were elected for scanning morphologic image. The erosion volume loss was derived from analyzing the erosion surface at three dimensions. Therefore, the erosion volume loss (Vloss) can be expressed as the average volume of surface at zero erosion time (V0) subtracted from the average volume of surface after specific time (Vt). Eroded area was randomly measured at seven locations. Then, the average of erosion volume was calculated.

$$
\mathrm{V}_{\text {loss }}=\mathrm{V}_{0}-\mathrm{V}_{\mathrm{T}}
$$

According to the Vloss formula, the averages of erosion volume loss were illustrated in Figure 7.

Ramie reinforced polyester matrix present higher value of volume erosion loss than Kevlar reinforced polyester. This fact derives from the poor of adhesion between Kevlar filaments and polyester resin. Table 4 illustrates the grains size versus time. 


\begin{tabular}{|c|c|c|c|c|c|c|}
\hline \multirow{2}{*}{ volume loss } & \multicolumn{3}{|c|}{ Kevlar } & \multicolumn{3}{c|}{ Ramie } \\
\cline { 2 - 7 } & $\begin{array}{c}\text { avg. size } \\
\left(\mathrm{nm}^{2}\right)\end{array}$ & $\begin{array}{c}\text { avg. height } \\
(\mathrm{nm})\end{array}$ & $\begin{array}{c}\text { avg. } \\
\text { volume }\end{array}$ & $\begin{array}{c}\text { avg. size } \\
\left(\mathrm{nm}^{2}\right)\end{array}$ & $\begin{array}{c}\text { avg. height } \\
(\mathrm{nm})\end{array}$ & $\begin{array}{c}\text { avg. } \\
\text { volume }\end{array}$ \\
\hline Zero Time & 1.13 & 7.14 & 8.21 & 1.24 & 8.97 & 11.18 \\
\hline after 3 hours & 1.11 & 10.334 & 10.44 & 0.82 & 9.36 & 7.846 \\
\hline after 6 hou & 1.72 & 8.91 & 11.55 & 0.9 & 5.41 & 4.25 .003 \\
\hline
\end{tabular}

Table 4. Grains size versus time

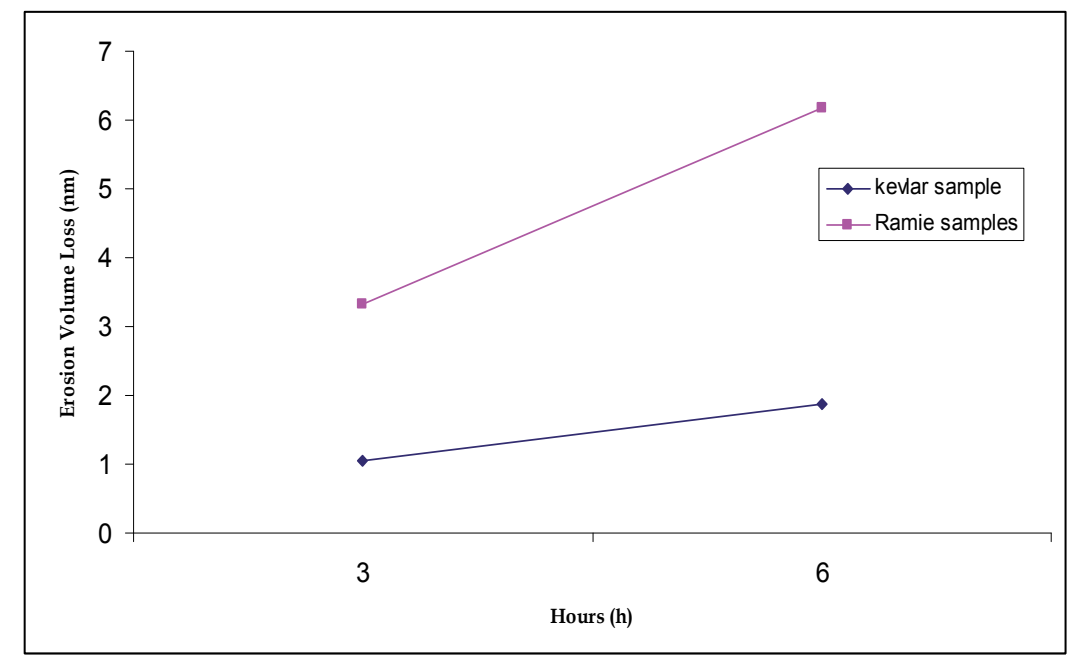

Figure 7. Erosion volume loss concomitant with time

Transforming image to 3D topography with 200\% zoom area for PKV sample at zero time (before test) illustrated accurate 3D surface profile with 243 peaks number and the maximum height $46 \mathrm{~nm}$ as shown in the Figure 8 and Figure 9 shown the PKV after 12 hours illustrated in 3D surface with 238 peaks number and maximum height $73.6 \mathrm{~nm}$. 

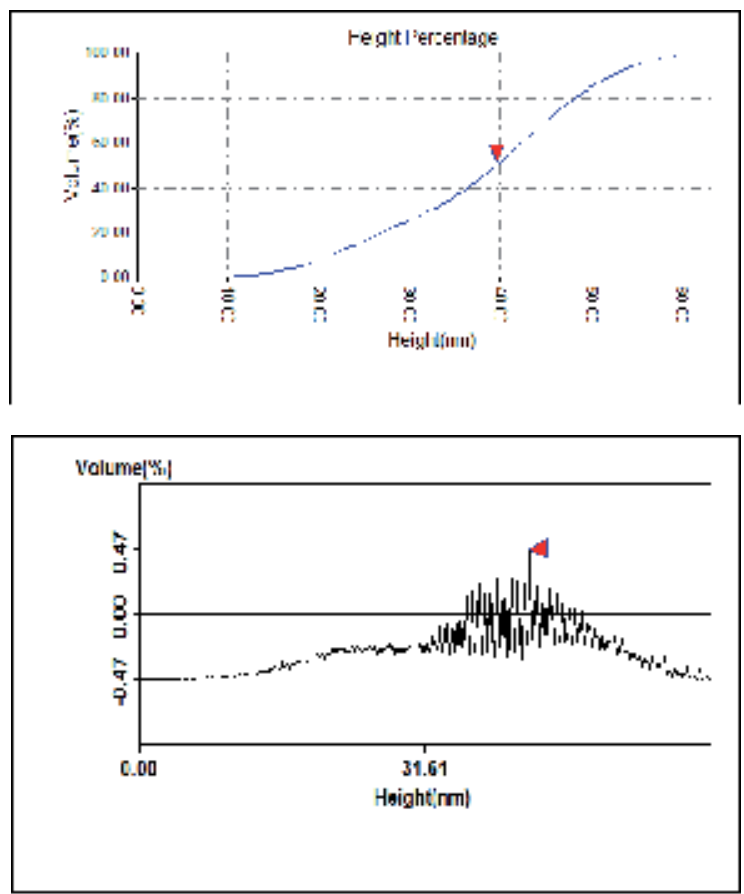

Figure 8. PKV at Zero Time (before test) in 3D Image

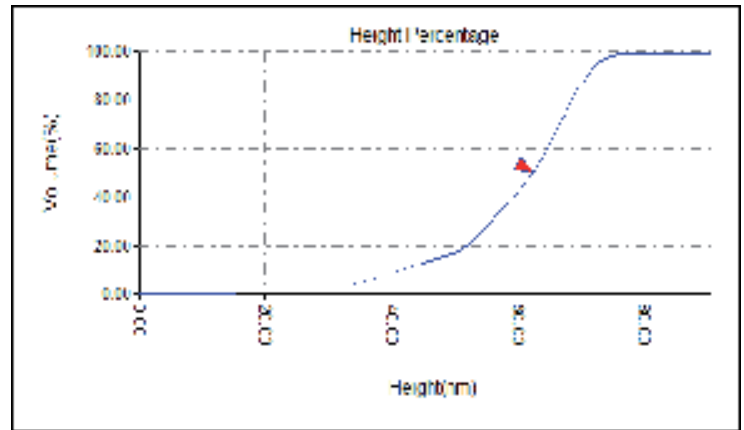

Volume|Wu|

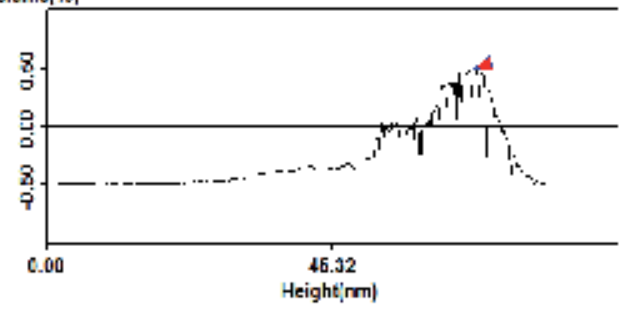

Figure 9. PKV after 12 Hours in 3D Image
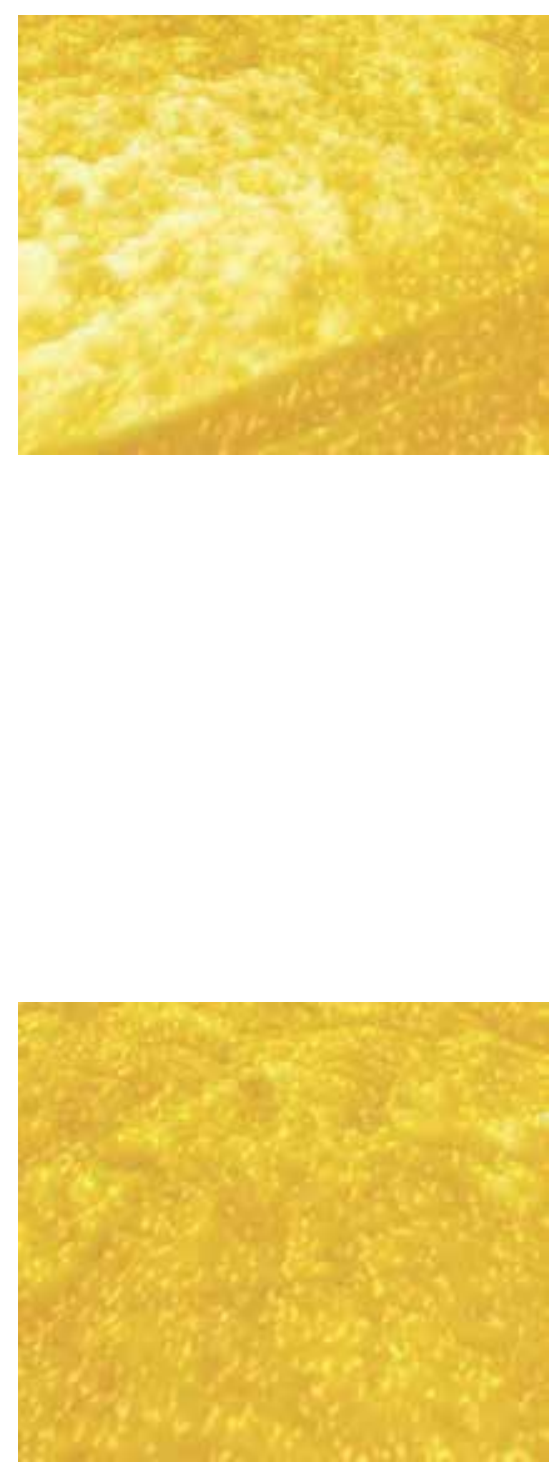
Accurate roughness parameters were established for PKV and PRM versus the time before and after test as illustrates in the table 5. PKV \& PRM morphologies images versus time were illustrated in Figure 10 \& 11.

\begin{tabular}{|c|c|c|c|c|c|}
\hline \multicolumn{6}{|c|}{ Image size:100.00nm $X$ 80.00nm (PKV) at zero time } \\
\hline Amplitude parameters: & Area 1 & Area 2 & Area 3 & Area 4 & Area 4 \\
\hline Sa(Roughness Average) [nm] & 6.06 & 7.53 & 8.92 & 15.6 & 16.7 \\
\hline Sq(Root Mean Square) [nm] & 9.1 & 10.7 & 12.3 & 18.8 & 20.2 \\
\hline Ssk(Surface Skewness) & -2.32 & -1.67 & -1.58 & -0.451 & -0.386 \\
\hline Sku(Surface Kurtosis) & 12.7 & 8.92 & 6.75 & 2.41 & 2.44 \\
\hline Sy(Peak-Peak) [nm & 87.8 & 90.2 & 89.5 & 90.3 & 91.4 \\
\hline Sz(Ten Point Height) [nm] & 85.2 & 89 & 85.8 & 89.9 & 88.5 \\
\hline \multicolumn{6}{|l|}{ Hybrid Parameters: } \\
\hline Ssc(Mean Summit Curvature) [1/nm] & -155 & -161 & -146 & -146 & -149 \\
\hline Sdq(Root Mean Square Slope) [1/nm] & 10.1 & 10.7 & 8.12 & 8.69 & 8.02 \\
\hline Sdr(Surface Area Ratio) & $3.29 \mathrm{E}+03$ & $3.62 \mathrm{E}+03$ & $2.17 \mathrm{E}+03$ & $2.44 \mathrm{E}+03$ & $2.08 \mathrm{E}+03$ \\
\hline \multicolumn{6}{|l|}{ Functional Parameters: } \\
\hline Sbi(Surface Bearing Index) & 0.624 & 1.05 & 0.779 & 2.45 & 14.1 \\
\hline Sci(Core Fluid Retention Index) & 0.983 & 1.18 & 0.949 & 1.34 & 1.32 \\
\hline Svi(Valley Fluid Retention Index) & 0.192 & 0.17 & 0.211 & 0.103 & 0.112 \\
\hline Spk(Reduced Summit Height) [nm] & 4.79 & 7.41 & 4.61 & 7.98 & 0.355 \\
\hline Sk(Core Roughness Depth) [nm] & 16.4 & 20.1 & 22.4 & 44.4 & 55.1 \\
\hline Svk(Reduced Valley Depth) [nm] & 21.5 & 20.8 & 25.2 & 19.9 & 19.6 \\
\hline $\begin{array}{l}\text { Sdc } 0-5(0-5 \% \text { height intervals of } \\
\text { Bearing Curve) [nm] }\end{array}$ & 14.6 & 10.2 & 15.7 & 7.67 & 1.43 \\
\hline $\begin{array}{l}\text { Sdc 5-10(5-10\% height intervals of } \\
\text { Bearing Curve)[nm] }\end{array}$ & 1.72 & 3.08 & 1.31 & 4.32 & 0.803 \\
\hline $\begin{array}{l}\text { Sdc } 10-50(10-50 \% \text { height intervals } \\
\text { of Bearing Curve) [nm] }\end{array}$ & 7.63 & 9.69 & 10.3 & 19.4 & 26.9 \\
\hline $\begin{array}{l}\text { Sdc } 50-95(50-95 \% \text { height intervals } \\
\text { of Bearing Curve) [nm] }\end{array}$ & 16.6 & 19.9 & 26 & 36.1 & 35.7 \\
\hline \multicolumn{6}{|l|}{ Spatial Parameters: } \\
\hline Sds(Density of Summits) [1/um2] & $4.28 \mathrm{E}+06$ & $4.23 \mathrm{E}+06$ & $6.02 \mathrm{E}+06$ & $5.67 \mathrm{E}+06$ & $5.56 \mathrm{E}+06$ \\
\hline Fractal Dimension & 3 & 3 & 2.81 & 2.37 & 2 \\
\hline
\end{tabular}

Table 5. The roughness parameters rates 


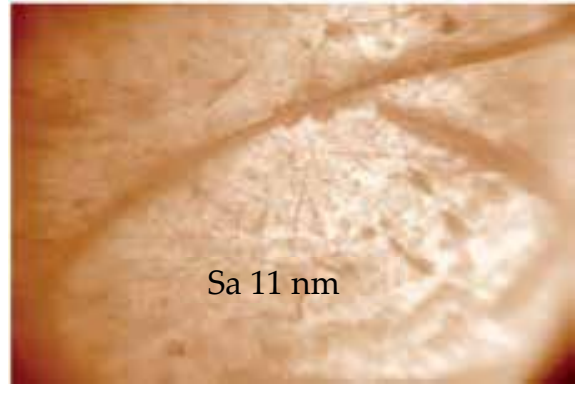

(a) After 3 hours

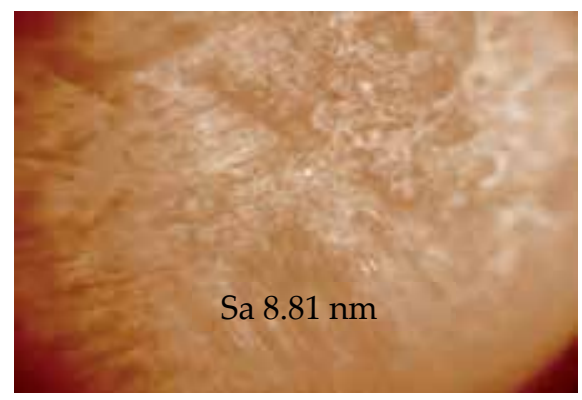

(b) After 6 hours

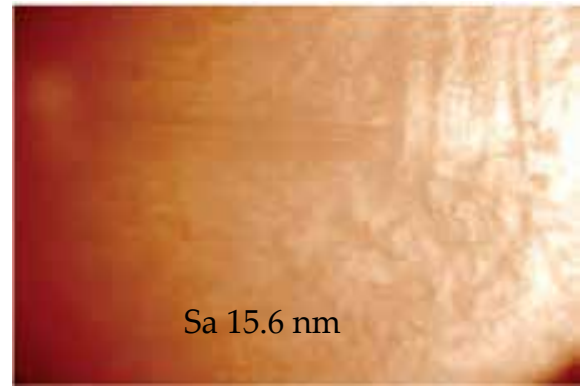

(c) Before test
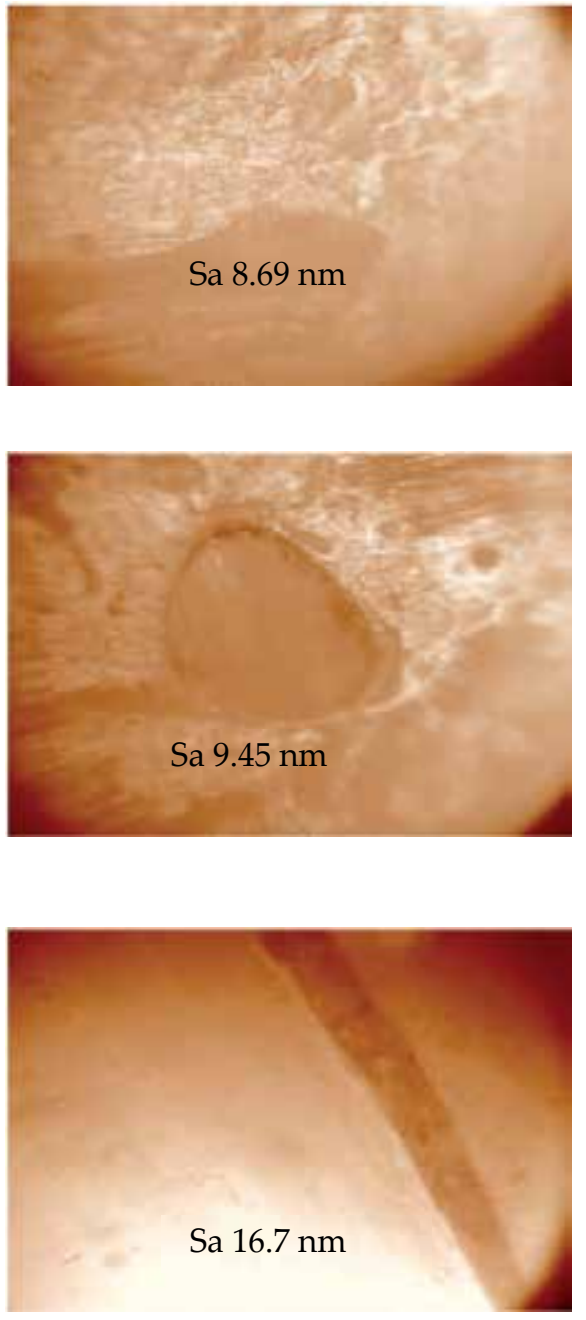

Figure 10. Erosion and corrosion test for Kevlar - polyester composite morphology and Sa(Roughness Average) 

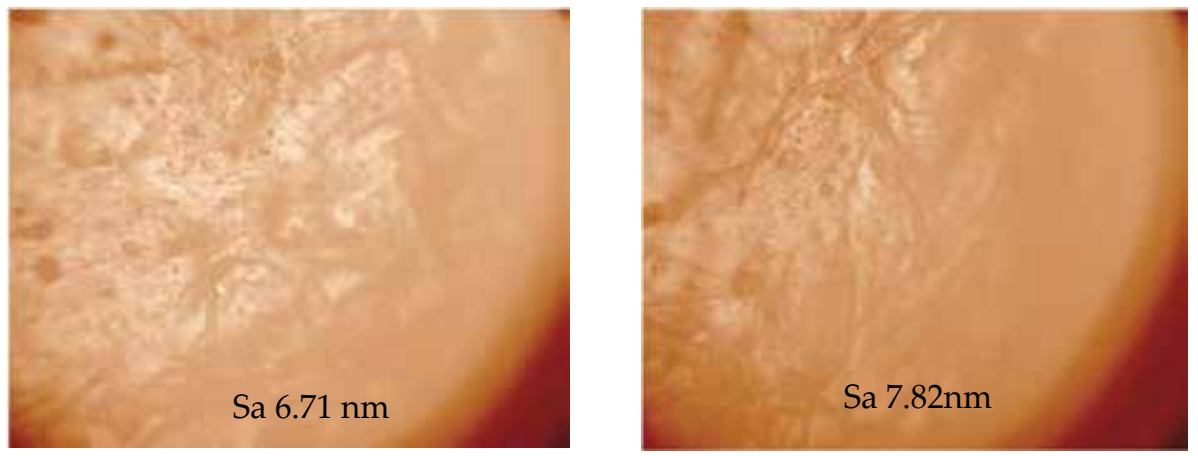

(a) After 6 hours
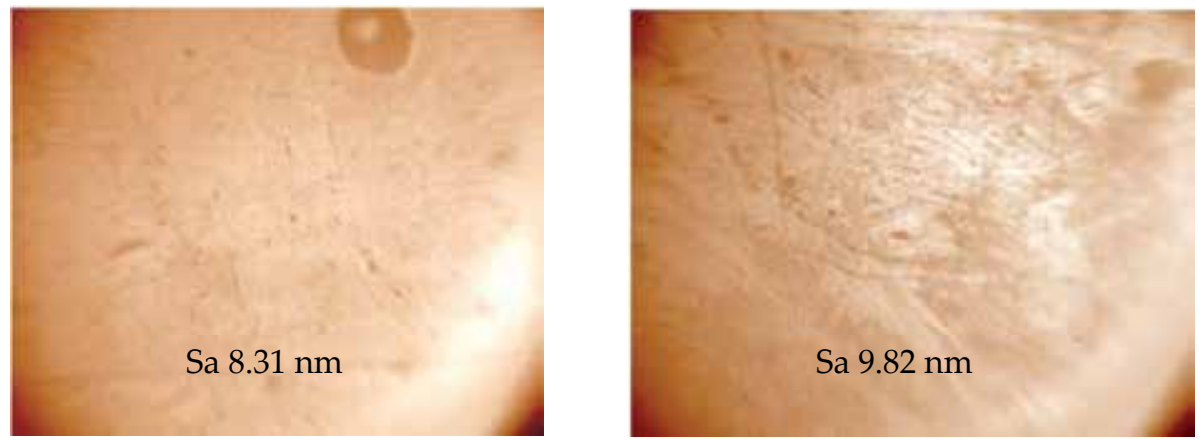

(b) After 3 hours
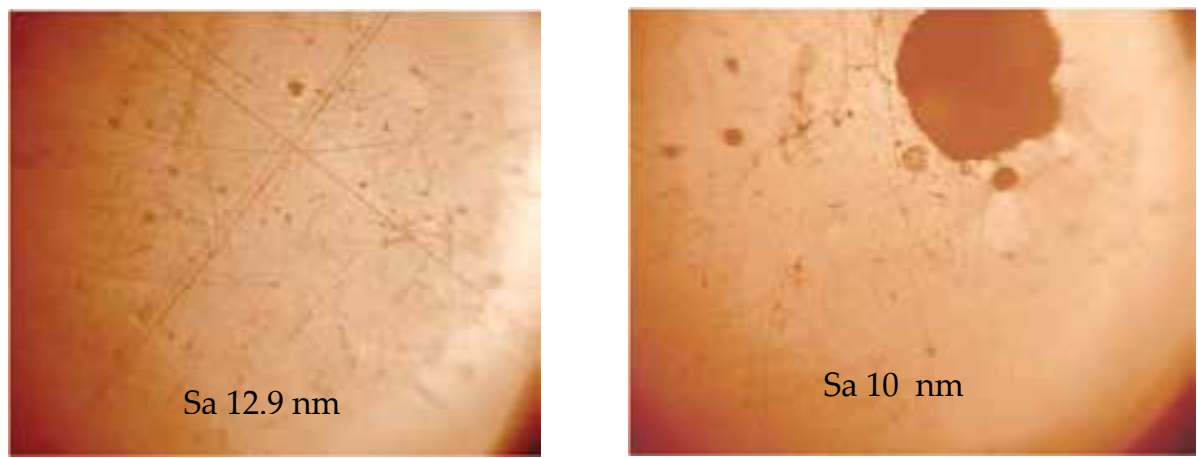

(c) Before test

Figure 11. Ramie - polyester composite morphology and Sa(Roughness Average) after erosioncorrosion test 


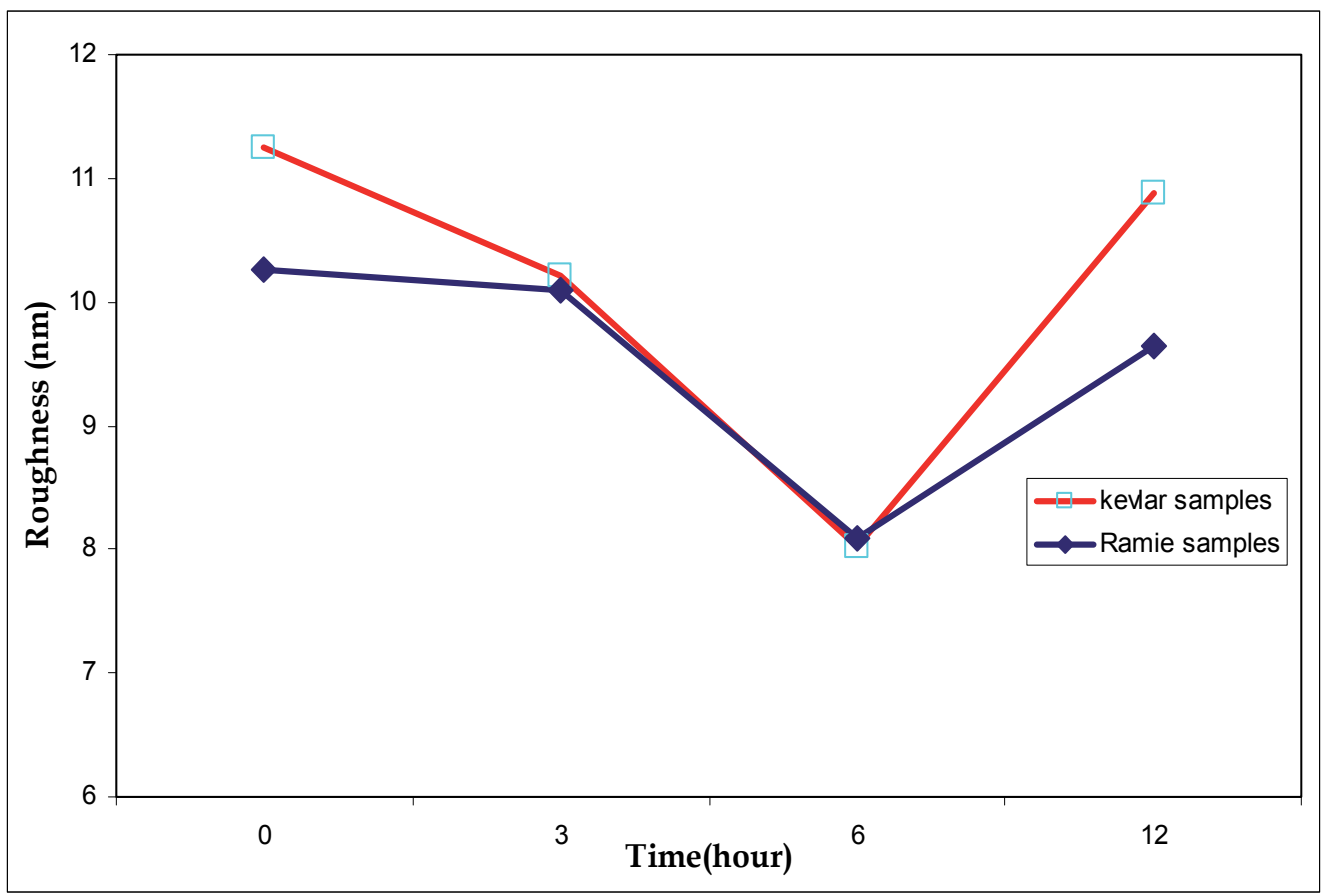

Figure 12. The Roughness Rates Versus Time

The roughness rate of the Kevlar-polyester and ramie-polyester concomitant time tented to be equivalent values. Really, the polyester reinforced faced the impinged water has the major role in the erosion and corrosion resistance. The Kevlar and ramie fibers have specific interfacial adhesion with the matrix. The ramie fibers presented high interfacial adhesion with resin as a result to plant fiber nature. On the other hand, the Kevlar presented a poor linkage with polyester resin.

All the fiber layers were protected by the matrix mass. Figure12 illustrated a declining in the polyester surface roughness versus time. The roughness will be the function for estimating the erosion rate. Therefore, within the period, from 3 until 6 hours the impinging effect assisted of soften the surface, but within period from 6 until 12 hours the erosion rate will be increase and the surface tent to be rough. Electrochemical test have been conducted and there are no significant corrosion data recorded, this finding derived from the compound nature of composite.

\section{Conclusions}

Generally, plain weave represented the most common fabric, due to the significant properties that embodied their tensile strength to weight ratio. The study of the stress strain response, high speed impact evaluation, and erosion corrosion behavior leads to the following conclusion: 
1. The Kevlar -polyester composites behave ductile manner but the ramie-polyester composites behave brittle manner.

2. The roughness rate of the Kevlar-polyester and ramie-polyester decreases through $3-6$ hours but there is an increase in the roughness rate through 6-12 hours due to the increasing in erosion rate of polyester matrix.

3. All roughness parameter computed accurately.

4. PKV and PRM with five layers PKV and five layers PRM present fully penetration.

5. No significant corrosion has been recorded.

\section{Author details}

Ali Hammood and Zainab Radeef

Department of Materials Engineering-University of Kufa, Iraq

\section{Acknowledgement}

The authors would like to express their gratitude and sincere appreciation to the department of Mechanical and Manufacturing Engineering of the University Putra Malaysia and Material Engineering Department- College of Engineering-University of Kufa for scientific assistance and support.

\section{References}

[1] Isabel B Wingate (1976) Textile fabrics and their selection. Library of congress cataloging in publication data.

[2] Woodhams R T, Thomas G, Rodgers D K (1984) wood Fiber as Reinforcing Fillers forpolyolefins.polym.eng.Sci. $\operatorname{Vol}(24): 1166$.

[3] Klason C, Kubat J (1989) Cellulose in polymer composites. In composite Systems from Natural and synthetic polymers, Salmen L, de Ruvo, A Sefe-ris, J C Stark, E B Eds; Elsevier Science: Amsterdam

[4] Myers G E, Clemons C M, Balatinecz J J, Woodhams R T(1992) Effects of composite and polypropylene Melt Flow on polypropylene-Waste Newspaper Composites. proceedings on the Annual Technical Coneference; Society of plastics Industry p 602

[5] Kokta, B V, Raj R. G, Daneault C (1989) Use of Wood Flour as Filler in Polypropylene; Studies on Mechanical Properties. Polymplast. Tecnol.Eng. 28, 247.

[6] Yang H H (1993) Kevlar Aramid Faiber. west Sussex PO191UD,England.

[7] Aidy A, Shaker Z R, Kahalina A (2010) Development of anti-ballistic board, fiber polymer-plastics technology and engineering VOL.(50):622-634.

[8] Zainab Shaker Radif, Aidy Ali \& Khalina Abdan(2010) DEVELOPMENT OF A GREEN combat armour from rame-kevlar- polyester composite, Pertanika Journal of Science and Technology. Vol(19) : PP 339-348,.

[9] Lee B L, Song J W, Ward J E J (1994) Compos Mater.VOL (28):PP 1202 - 1226. 
[10] Goldsmith W, Dharan CK.,Chang H (1995) Quasi-static and ballistic perforation of carbon fiber laminates .Int J Solid Struct VOL(32):89-103.

[11] Almohandes AA, Abdel - Kader MS, Eleiche, AM (1996) Experimental investigation of the ballistic resistance of steel-fiberglass reinforced polyester laminated plates. Composites: Part B 27:447-58.

[12] Bardal E, Eggen T G, Rogne T, Solem T (1995) The erosion and corrosion properties of thermal spray and other coatings, in: Proceedings of the Int. Therm. Spray. Conf., Kobe, Japan.

[13] Puget Y, Tretheway K R,Wood R J K (1998) The performance of cost effective coatings in aggressive saline environments, NACE Corrosion PP 688.

[14] Burstein G T, Sasaki K (2000) Effect of impact angle on the slurry erosion-corrosion of 304 L stainless steel, Wear VOL (240): 80-94.

[15] Dawson J L, Shih C C, John C C, Eden D A (1987), Electrochemical testing of differential flow induced corrosion using jet impingement rigs, NACE Corrosion, Paper no. 453,

[16] Clark H M, Wong K K, Impact angle (1995), particle energy and mass loss in erosion by dilute slurries, Wear 186-187 454-464.

[17] Stack M M, Zhou S, Newman R C, Identifications of transitions in erosion-corrosion regimes in aqueous environments, Wear 186 (1995) 523-532.

[18] Sherrington.(1988), modern measurement techniques in surface metrology, wear VOL(125):271-288.

[19] Matsuno Y., Yamada H., Harada M. and Kobayashi A. (1975), The microtopography of the grinding wheel surface with SEM, Ann.CIRP VOL(24):PP 237-242.

[20] Nakamura, Y et al (2003). Neurosci. Abst. 608.5

[21] Girardi M. A and Phill M. G. (1993), Microstructure and properties of polyester/urethane acrylate thermosetting blends, and their use as composite matrices, Journal of Materials Science, Volume 28. 3116-3124, DOI: 10.1007/BF00354718.

[22] Mohamed Thariq. (2007), High velocity Impact analysis of glass epoxy-laminate plates. Thesis, university Putra, Malaysia, Malaysia. 


\title{
The Chosen Aspects of Materials and Construction Influence on the Tire Safety
}

\author{
Pavel Koštial, Jan Krmela, Karel Frydrýšek and Ivan Ružiak
}

Additional information is available at the end of the chapter

http://dx.doi.org/10.5772/46506

\section{Introduction}

Security of the road transport depends on the quality of basic and applied research concerning materials and internal construction of tires. The design shapes and material properties characterized by low hysteretic losses as well as a construction have an influence on the driving comfort, adhesion, wear resistance and fatigue resistance. Thick fibre reinforced composites are used extensively in rubber products such as tires and conveyer belts. Generally, the reinforced parts of rubber products on a sub macroscopic level are highly heterogeneous and anisotropic because they are composed of rubber compounds, and textile and steel cords. Rubber compounds consist of natural or synthetic rubber, carbon black, curing agents, cure accelerators, plasticizers, protective agents and other ingredients.

First, the properties of the different parts of the studied tire will be outlined. A bead is a part of the tire, which fixes to the rim. The bead consists of a steel bead wire, a core, a bead filling and a carcass. They help to transmit loading and breaking.

Particularly we will focus our attention on the influence of breaker angle on tire deformation and potential risks resulting from improper breaker construction.

Experimental results of tread and side wall deformation (influenced by rubber blend as well as a breaker construction) measured independently by both line laser and Aramis system are compared with those obtained by computer simulation in Abaqus environment. The tire tread contributes to a good road grip and water expulsion, the multi-ply steel belt optimizes the directional stability and rolling resistance, the steel casing substantially determines the driving comfort, the inner-liner makes the tire airtight, the sidewall protects from lateral scuffing and the effects of the weather, the bead core ensures the tire sits firmly on the rim, and bead reinforcement promotes directional stability and a precise steering response. 
The high security and long life of a tire can be assured only by its correct assignment to the particular type of vehicle and automobile as show Figure 1 (tires only for road operation, for off-road, combined operation as well as for summer or winter conditions). Tires are divided by type of tire-casing on radial, diagonal, bias-belted and special tire.

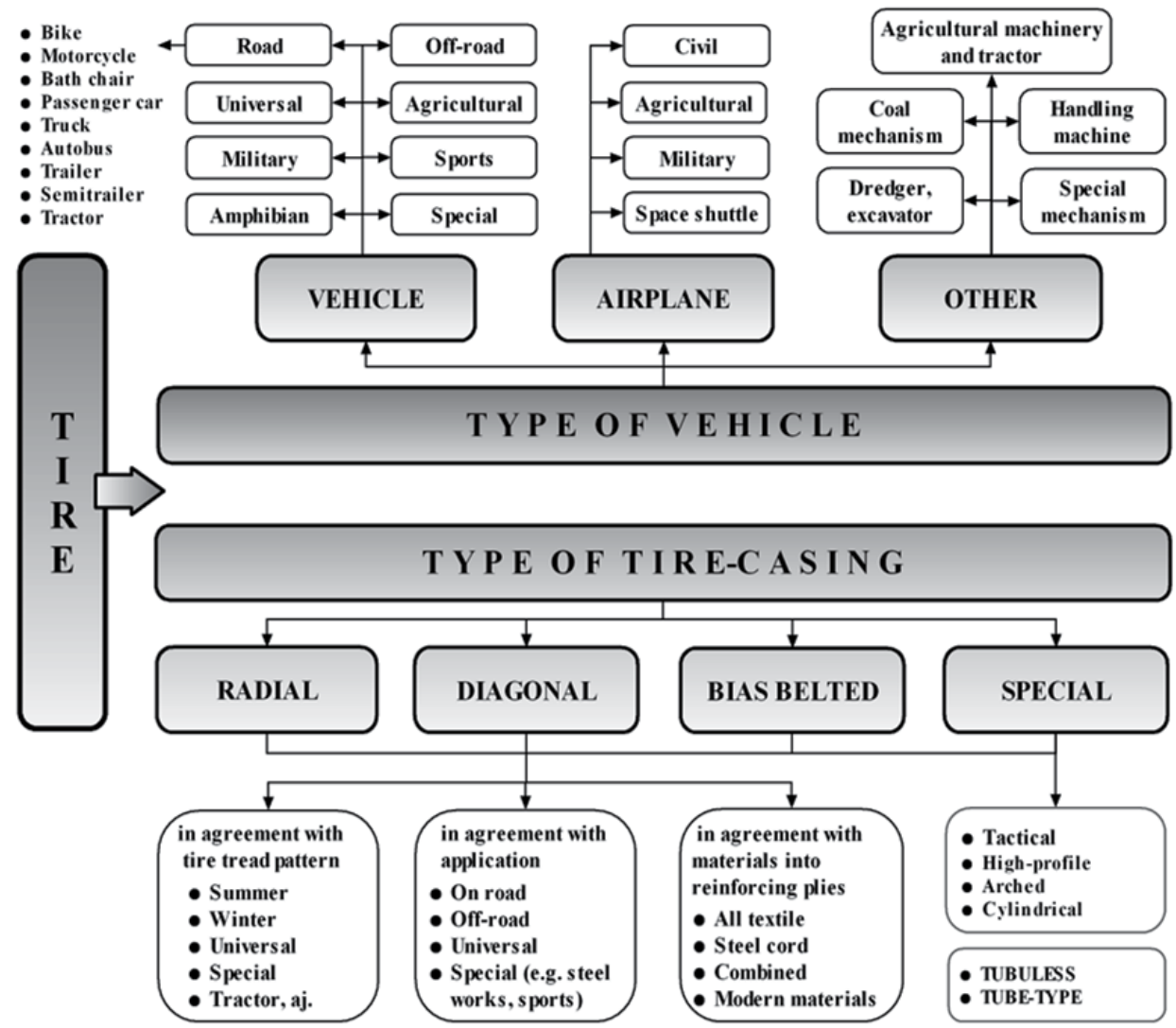

Figure 1. Type of tire by vehicle and construction

Radial tires can be considered as pressure vessels with a maximum pressure given by the particular type of tire. A tire can be generally considered as a statically and dynamically loaded automobile element. The structured view of a tire is apparent from the Figure 2.

The function of wheels with tires is not only to align a car reliably. As can be seen in more detail from the Figure 3 there are more requirements on tires. The main operating requirements on car tires are that car wheels should be as light as possible and at the same time tough, statically and dynamically balanced.

The main requirements on tires are, apart from other things, high wear resistance, optimal deformation characteristics, low rolling resistance, high operational life and safeness, etc. Wheels with tires must meet particular functional requirements given by parameters of tires which affect the running properties of the car, i.e. affect their dynamic behavior (car maneuverability, stability, acceleration, deceleration, driving comfort, etc.). 


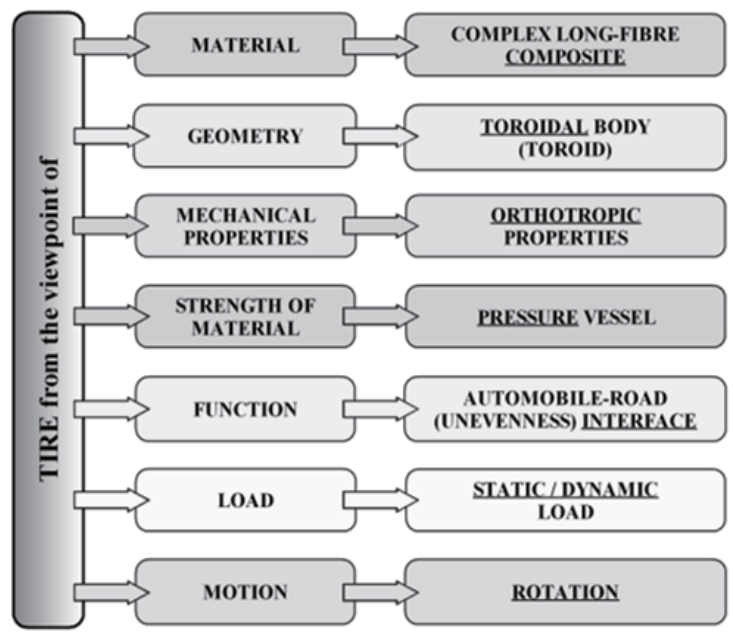

Figure 2. Definition of tire from various viewpoints

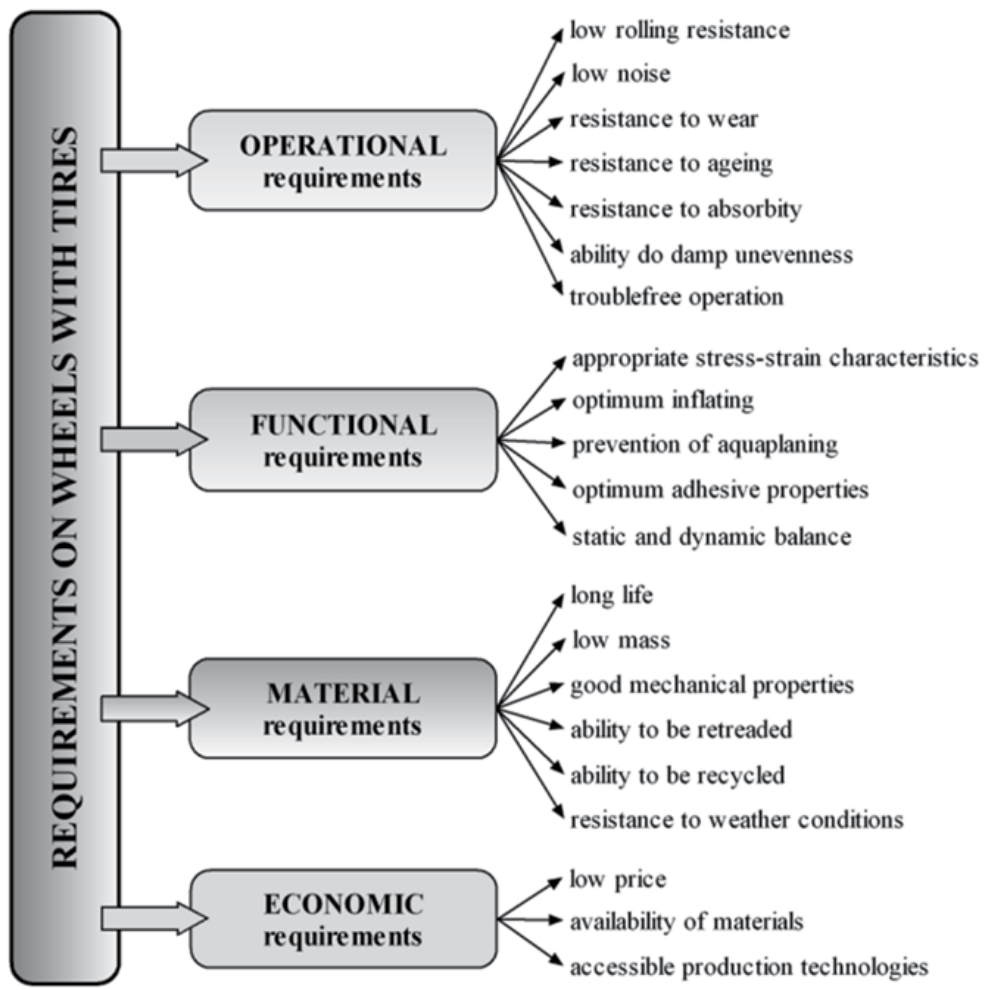

Figure 3. Basic requirements of wheels with tires

Tire safety is passive and active - see Figure 4. Passive safety depends on the quality of the production of a tire casing, the applied technology and used materials and in the case of computational modeling also on the accuracy of the performed calculations and appropriate choice of the computing algorithm. 


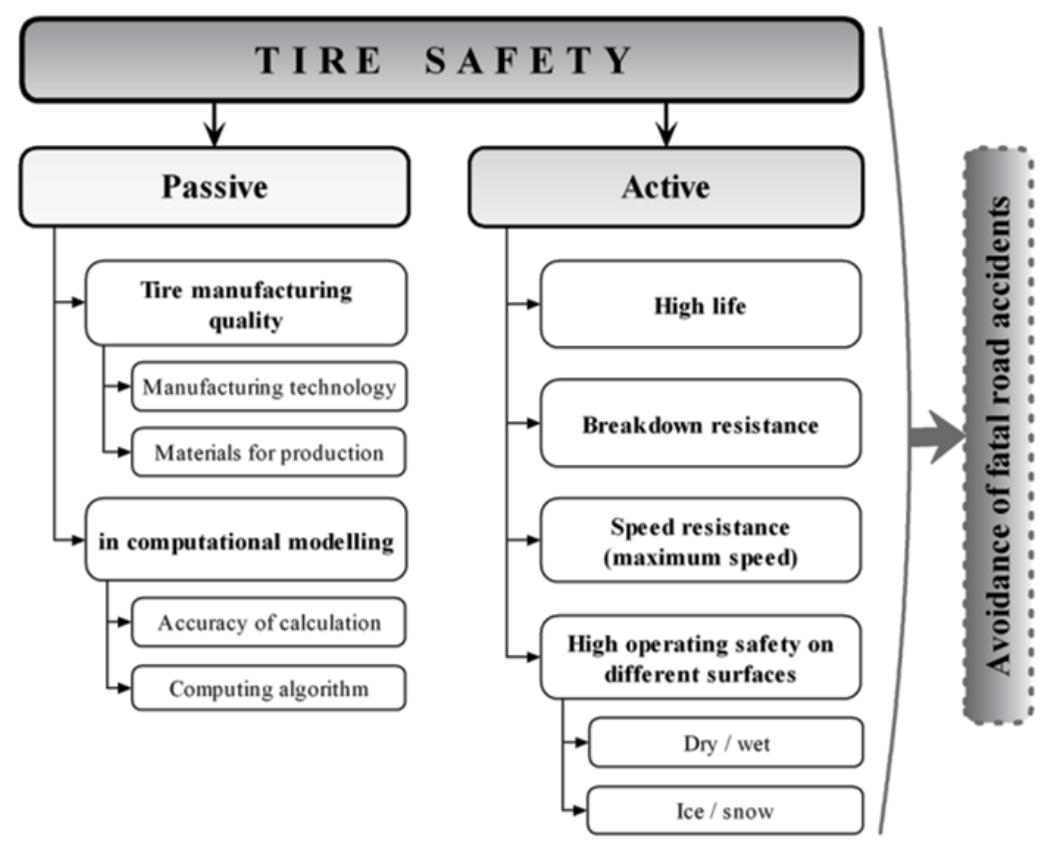

Figure 4. Viewpoints of tire safety

Requirements on active safeness are particularly high running safety on various types of road surfaces, breakdown resistance, speed resistance and high life of materials used for the production of tires, namely reinforcing materials.

Tire life is affected by many factors (e.g. manufacturing way of a tire, its operation and handling, storage conditions of base materials for tire production etc.) while it's assumed an ideal adhesive bond among the rubber elements in matrix (e.g. an interface between tire tread and textile overlap belt) and the cord reinforcing and rubber drift inside tire carcass, and belts is assumed in this cases. For long life tires must resist during operating to surrounding effects, to negative effects of operation and to other effects, which could lead to wear and degradation processes as are e.g. delaminations. The aim is to avoid fatal road accidents which might be caused by tire casing defects either by neglecting operating conditions of tires (depth of tire tread pattern, tire inflation pressure, use of inappropriate tires with a different structure, etc.) or by bad vulcanization during the manufacturing process creating delaminations.

The tire is during the operation exposed to combined loading as from a mechanical (statical, dynamic) as a temperature point of view (local heating in subzones, overall heating in the tire-tread area permeating into the tire during breaking). Also this has to be considered in defining tire safety at high speeds. For this reason tires and wheels as a unit are modified from the structural point of view, particularly for special army vehicles where even a sudden drop of pressure does not put an end to the operating capability of the vehicle (system with a central collar providing circular indexing of the casing with respect to the wheel rim). 
New features are introduced for high speeds, e.g. electronic systems which warn the drivers in the case a gradual drop of the tire pressure or adjusting systems for inflation based on the temperature load of the tire casing. Each manufacturer protects the results of his developments and patents considering them as private "know-how". Consequently all new information is only very scarcely available.

In the material point of view a rubber blend could be considered as a composite material which consists of a matrix, a filler and a mesophase.

The dynamic - mechanical properties of such composites can also be described by an elastic, viscous modulus and a loss factor, (Simek 1987, Sepe 1998, Wang 1998, Schaefer 1994, Murayama 1978, Ferry 1980, Jančíková 2006, Jančíková \& Švec 2007 and Jakubíková et al 2007). Such properties depend, in turn, on the operating temperature and frequency of the external excitation. Biodegradability of polymers has been studied in (Jakubíková et al 2007).

In a molecular scale, the mechanical properties of rubber blends are influenced mainly by the structure of the blend. The interaction between a matrix and filler plays the most important role and this role is closely connected with dependency of $\mathrm{E}$ and $\mathrm{G}$ modulus to an applied load or a frequency (both functions have falling tendency and this phenomenon is called Payne effect) (Payne 1965). The polymer in the network loses its identity, and behaves like a filler. The loss factor $E^{\prime \prime}$ depends on the dissolution and regeneration speed of the network. It is reflected on the decreasing trend of complex Young's modulus dependence versus increasing sample loading. The values of elastic modulus ( $\left.E^{\prime}\right)$ for vulcanizates without fillers are not changed with the increasing of dynamic deformation (Payne 1965, Medalia 1978 and Maier \& Gand Göritz 1996).

The blend properties are characterized by the following parameters: $T_{g}$ is a glass transition temperature, it is influenced by the silica filler, and for not filled rubber it is approximately $40^{\circ} \mathrm{C}$. The phase shift between stress and strain is the loss angle. It is postulated that the loss factor, represented by $\tan \delta$, in the temperature span $-10^{\circ} \mathrm{C}$ to $5^{\circ} \mathrm{C}$ (frequency $10000 \mathrm{~Hz}$ ) characterizes the adhesion of the tire on a wet road. In the span $60^{\circ} \mathrm{C}$ to $80^{\circ} \mathrm{C}$ (at the frequency $100 \mathrm{~Hz}$ ) the course of $\tan \delta$, characterizes the rolling resistance (http://ao4.ee.tut.fi/pdlri).

The breaker angle (see below) also influences the security of a tire as well as the driving comfort and stiffness of a tire. The driving properties of the tire as a whole could be substantially improved by optimizing such angle of steel wires of the breaker.

\section{The tire description}

The work of the authors over a long period of time is devoted to radial tires. The automobile radial tire consists (e.g. cross-sections of selected tire 165 R13 Matador are on the Figures 5 and 6, in detail on the Figures 6 below and 7) of rubber parts and composite structure parts (Figure 8) with textile cords (especially PA 6.6 and PES textile fibers are used) and steelcords into tire tread as reinforcements. 


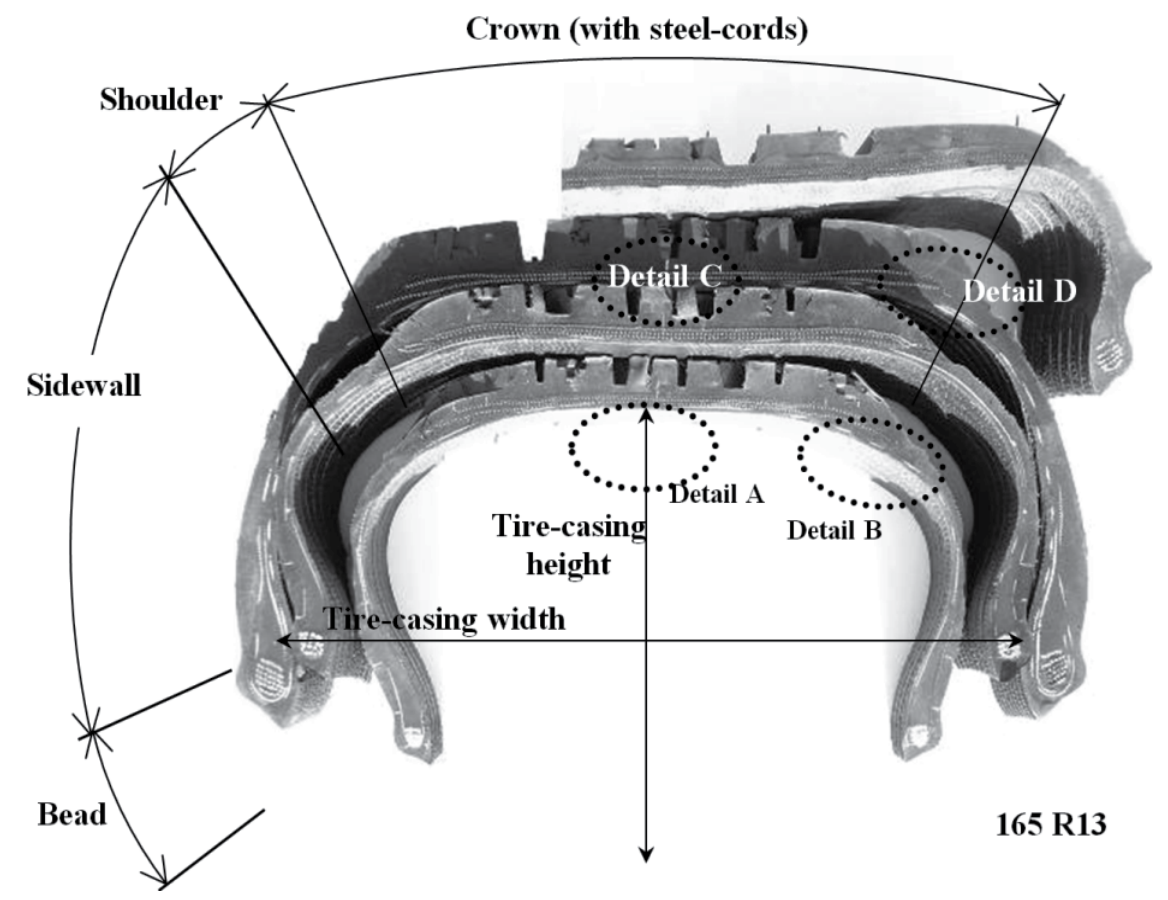

Figure 5. Cross sections of tire-casing

The composite structure parts applied into radial tires (Figure 8) are:

- Textile tire carcass;

- Textile overlap belt;

- $\quad$ Steel-cord belt.

These structures of tire have got:

- $\quad$ Different cord-angle (e.g. for steel belt applied angle $21-27^{\circ}$ into radial tire for passenger car);

- Material of cords (steel, textile, Kevlar, combine);

- Shape and construction of cord (wire, wire strand);

- Numbers of layer (single-layer or multi-layer).

So a tire has got characteristic specific deformation properties.

One construction of tire is used for passenger cars, other constructions for trucks, offhighway cars and sports cars. The tires for air transportation, agricultural vehicle, mining machine and other vehicles have got complicated structured in comparison radial tires for passenger cars. The tire structures are differentiated by numbers of reinforcing plies into belt tire, construction of belts, materials and cord-angles, geometry parameters of tire, width of belts etc. These aspects are influenced on final behavior of tires, namely deformation characteristics of tires. It is possible increase of resistance of tire to some degradation processes by suitable tire construction. 

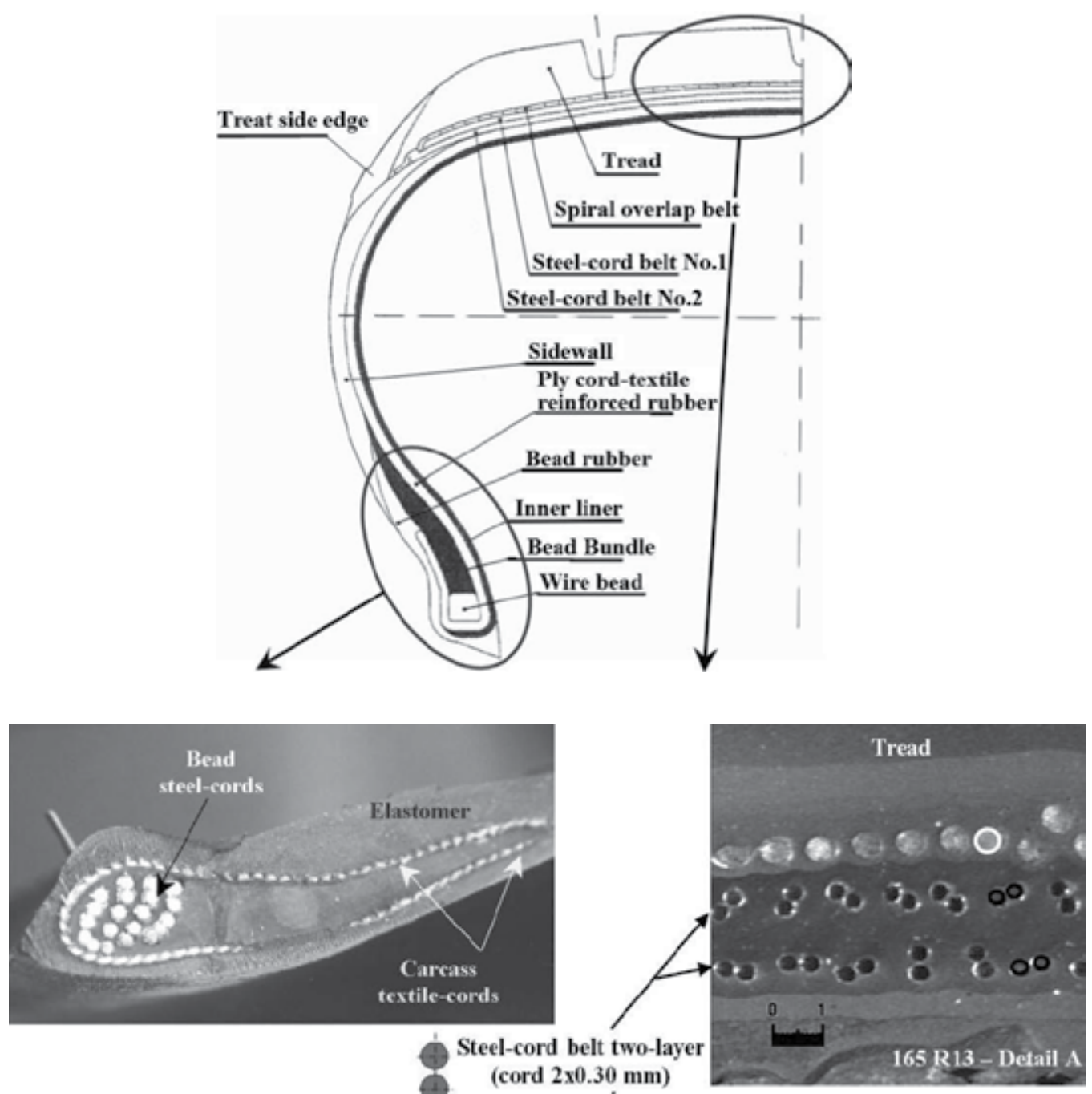

Steel-cord belt two-layer (cord $2 \times 0.30 \mathrm{~mm})$

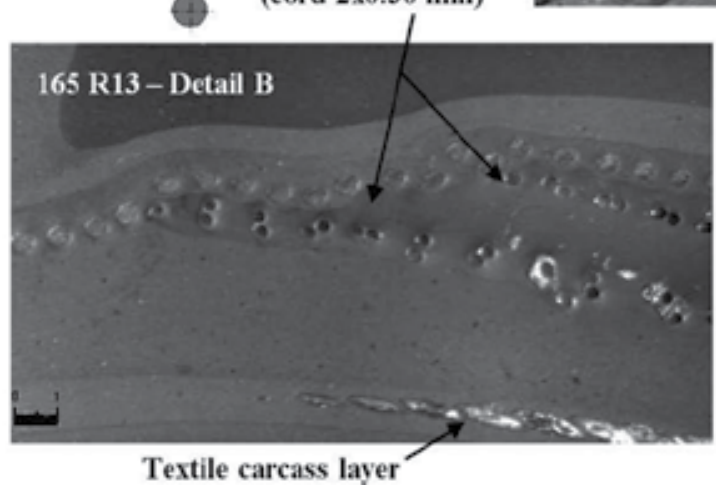

Figure 6. Structure of the tire $165 \mathrm{R} 13$ [based on Matador] with microstructure of reinforcing plies detail $\mathrm{A}$ in the area of tire crown and detail B at the end of steel-cord belt (below) 

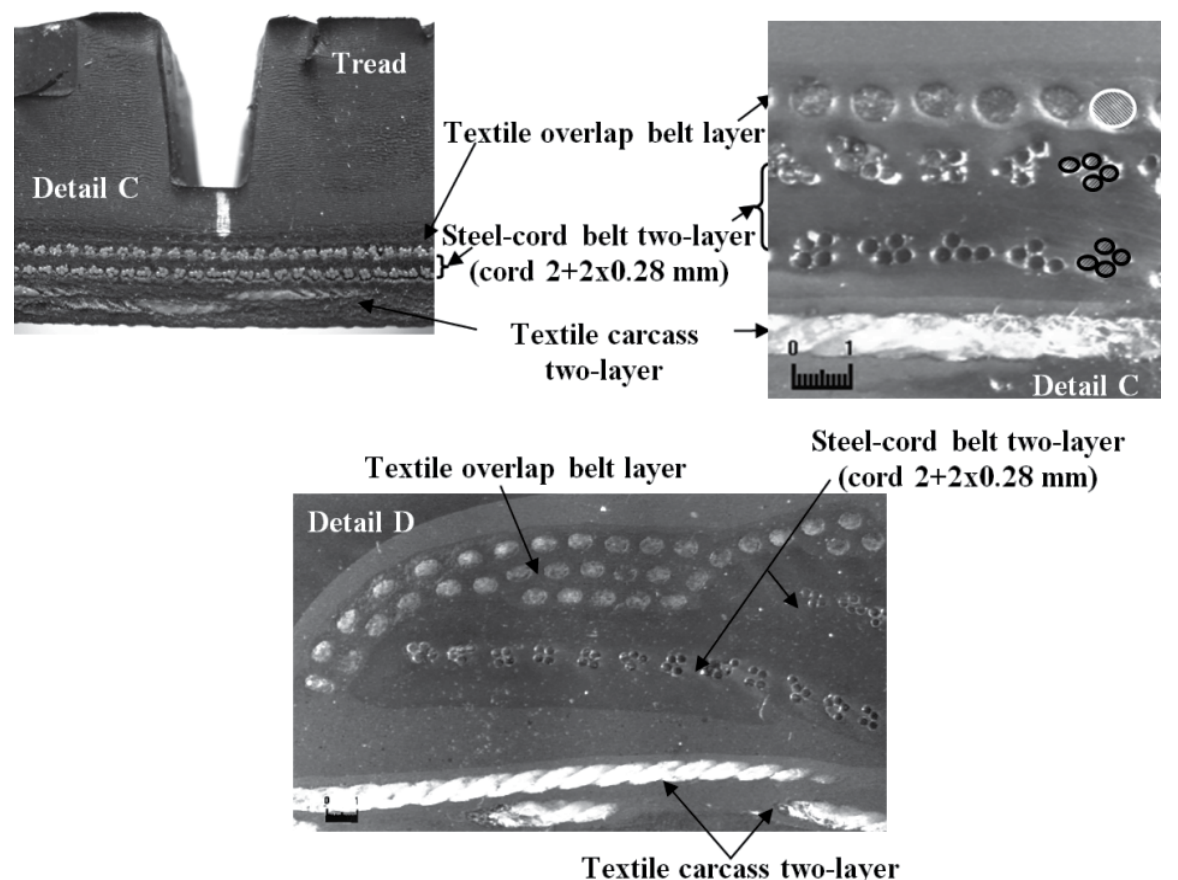

Figure 7. Detail C in the middle of tire crown and detail D at the end of the belt layers (below) of different radial tire

\section{COMPOSITE STRUCTURES of tire carcass}

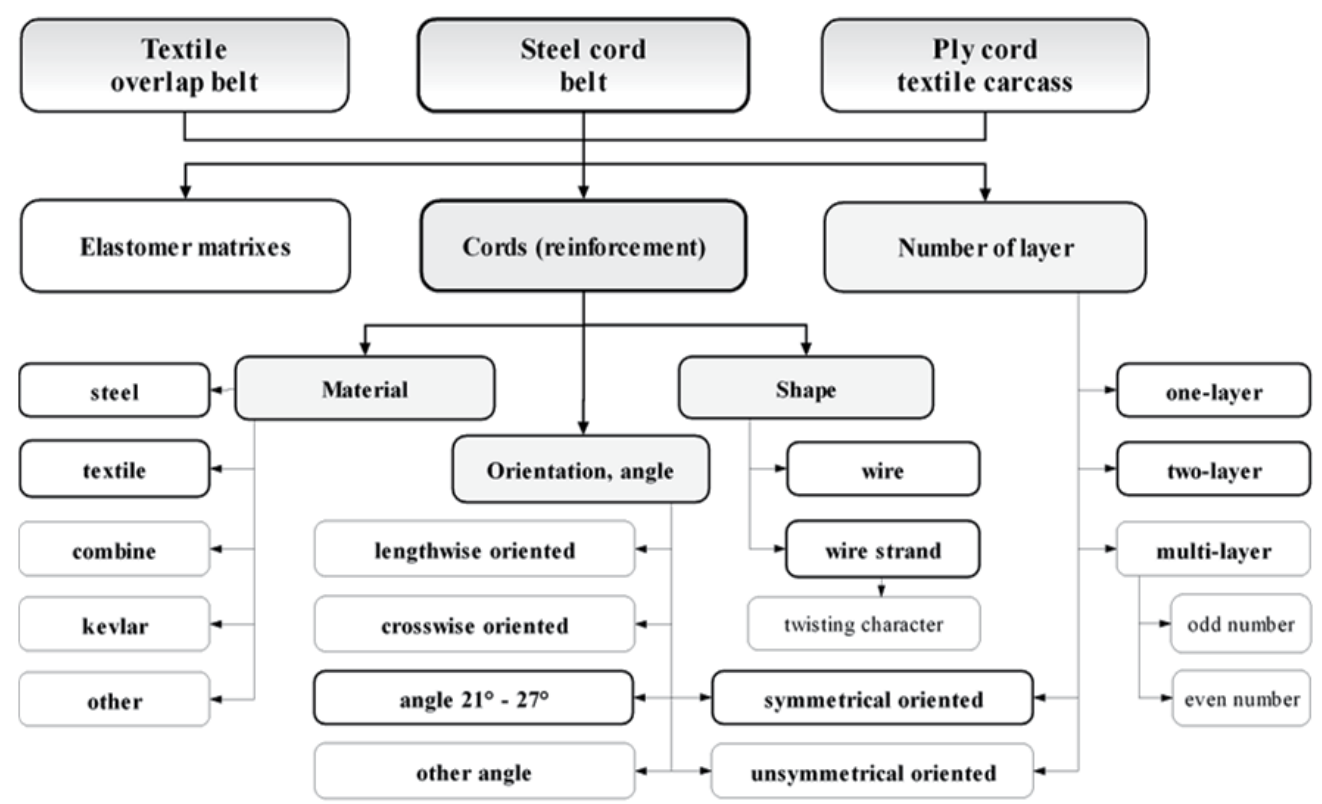

Figure 8. Composite structures used in tire 
Two-layer steel-cord belt is used in radial tire 165 R13 Matador with construction of cord $2 x 0.30 \mathrm{~mm}$ with texture 961 (number of cord over meter width of belt). The cord angle is $23^{\circ}$, the layers are symmetrical.

Structure - cord orientations of radial tire 22.5" for truck vehicles presented in the Figure 9 as an example.

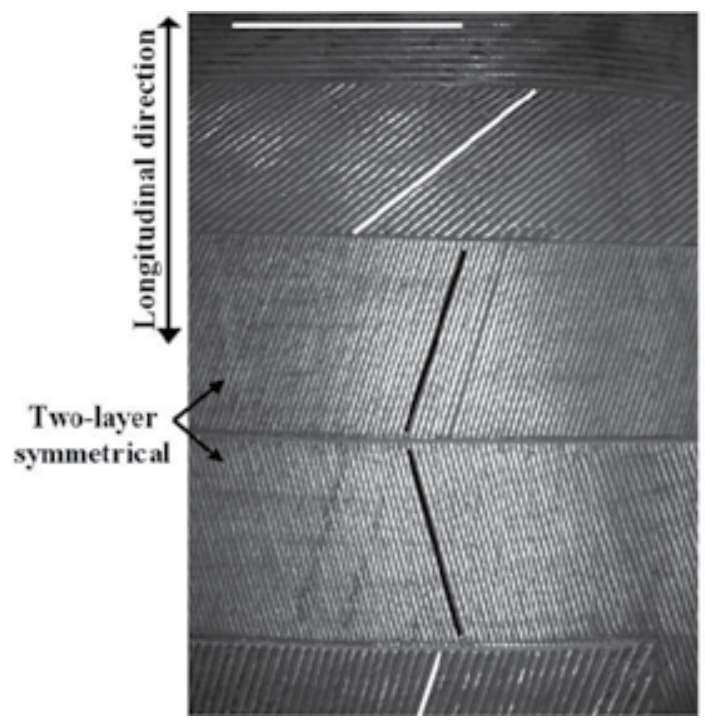

\author{
Bottom layer $90^{\circ}$ \\ Steel-cord carcass \\ $+50^{\circ}$ (Belt No.1) \\ $+18^{\circ}$ (Belt No.2) \\ $-18^{\circ}$ (Belt No.3) \\ Top layer $+18^{\circ}$ \\ (Belt No.4)
}

Figure 9. Structure of truck tire in the middle of tire crown

Steel-cords can be in form of thin wire or wire strand with different constructions. Highstrength steels are used exclusively for steel-cord production and good adhesive bond between rubber and cords required. Steel-cord surfaces are modified by chemical-thermal treatment (braze or copperier, Figure 10) to achieve the best adhesive bond of a steel cord and rubber and get it corrosion resistant. The substantial factor, which expressive influence on coherence of whole tire, is good adhesive bonds between reinforcement materials and rubber parts of tire.

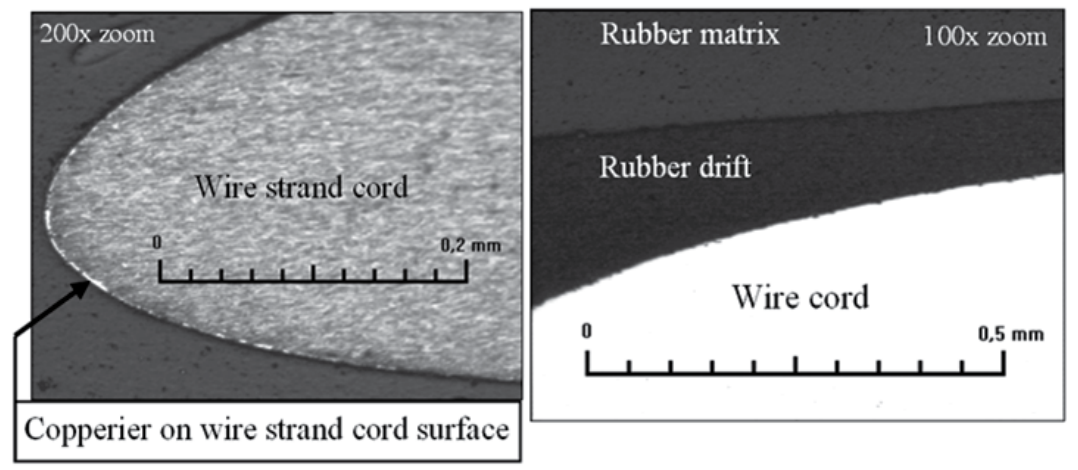

Figure 10. Steel-cord surface and interface between cord-rubber drift-rubber matrix 
The tire steel-cords are exposed to various chemical and thermal influences (Figure 11) during cyclic loading states by tensile-compression in tire loading processes. Account on this the adhesive bond is more exposed to be damaged than the basic materials (steel, textile and rubber). The aggressive environment (e.g. action of salts in winter) activates the corroding process on steel-cord surfaces that can lead to decreasing of the adhesion between reinforcement-and-matrix, which demonstrates itself by negative changes in material properties of steel-cord belts and such of whole tire too.

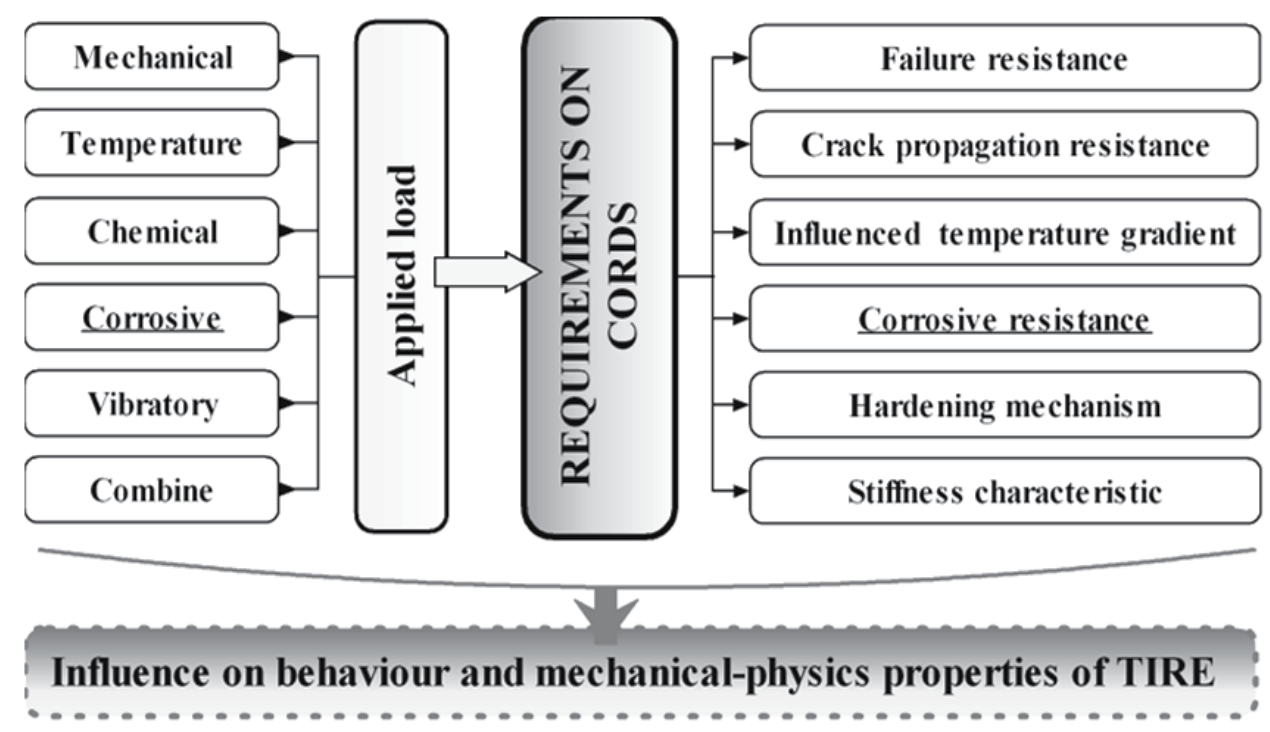

Figure 11. Requirements on reinforcing tire cords

In addition if the tire is in use is defected in tire crown (e.g. defect caused by sharp object as a nail and after the repair is placed back into operation) the initiation of corrosion with faster process is being assumed. Consequently this can lead to gradual or sudden failure of the steel-cords and bonds of steel-cord and rubber with a serious car accident as a final consequence.

Any damage in the area of tire crown, namely into steel-cord belt, is perilous.

\section{Degradation processes of tires}

Tires are subject to internal and external effects which can more or less cause limit states leading to degradation processes Figure 12. Ones of them marked as very dangerous and unacceptable tire casing damage are so-called separations and delaminations (Figure 13 left). Breakdown or damage is not necessary only at the border of single layer e.g. between the layers of steel-cord belt plies in the tread of tire casing, but also between rubber matrix and reinforcing cords. Delamination between rubber drift-rubber matrix on Figure 13 right. 


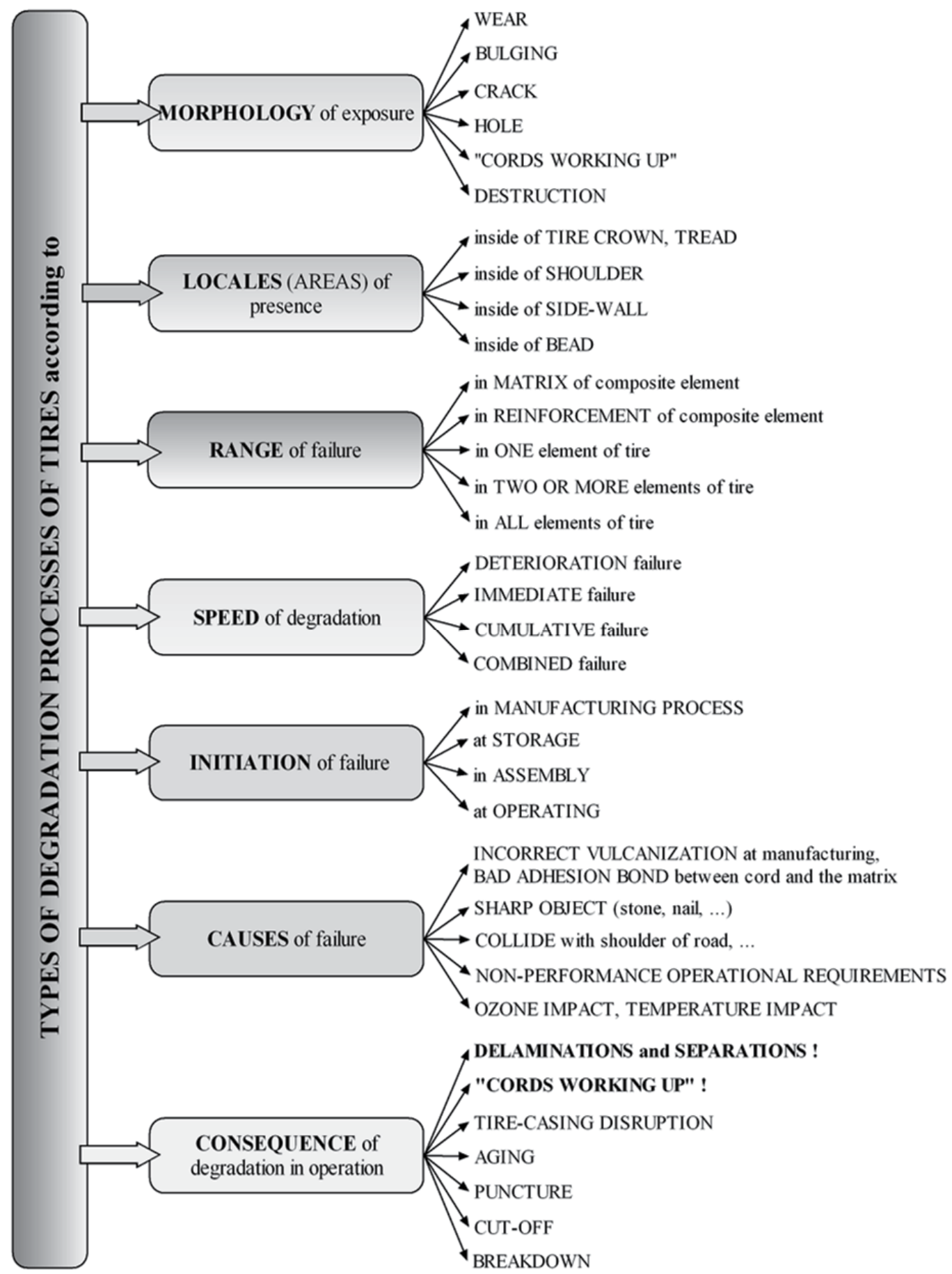

Figure 12. Degradation processes of tire 

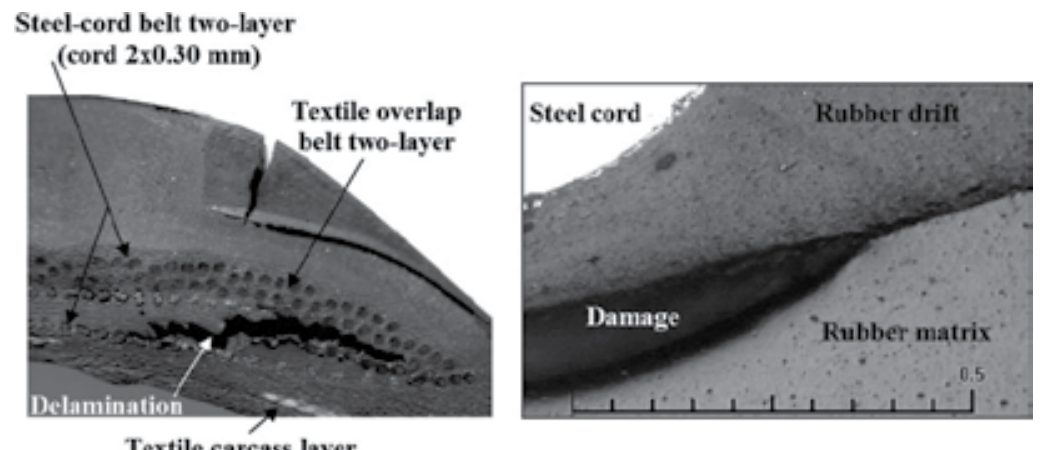

Textile carcass layer

Figure 13. Factitious delamination between belt plies and damage between rubber drift-rubber matrix (right)

Root cause of mentioned degradation processes can be caused by using of low quality materials for the tire in manufacturing process, their incorrect storage leading to early aging especially at rubber compositions, not keeping optimal manufacturing conditions vulcanization, as well as by the influence of incorrectly pressurized tire and damaged adhesive bond between cords-matrix and belt plies etc. Damaged adhesive bond is greatly decreasing of tire safety during the operation of a vehicle at high speeds. This has a significant influence to the quality of the tire casing expressing by lowering the level of usage (decreasing speed index) or leading to the catastrophic situations. In every case it is mandatory to avoid these premature limiting states.

In tires can be caused:

- $\quad$ Cords release leading to the cords working-up;

- Separations - delaminations;

- Combined wear;

- Total breakdown.
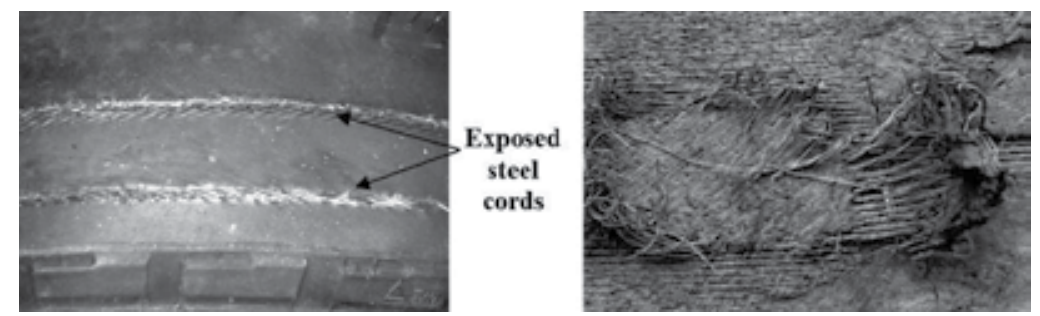

Figure 14. Extreme wear of tire on tread surface; and "cords working-up" (right-photo by Prof. Janíček, VÚT Brno, Czech Republic)

Results of wear due to adverse change (Figure 14) of tire casing surfaces are gave in impairment of mechanical-physics properties of whole tire. This will be influenced the incoming behavior of tire in operation and related interfaces between of tire and surroundings. Particularly dangerous is creation of failure in such places where initiation is not assumed to be caused by impairment of the surface. Structural changes in a part of tire as composites are not only responsible for the impairment of its properties but also of its 
geometry which can initiate vibrations leading to loss of the part's functional ability of whole vehicles (automobiles).

Wear can be of various character (development, place, form, appearance) and leads to the failure of the tire (Figure 15a and 15b). The task of prediction is to find ways how to reduce wear and to postpone initiation of dangerous degradation processes such as delamination and separation and to focus on the removal of initiators of these degradation processes.

Wear due to adverse changes of surfaces results in impairment of properties and behavior of parts. Particularly dangerous is failure in such places where initiation is not assumed to be caused by impairment of the surface. Structural changes in a part are not only responsible for the impairment of its mechanical properties but also of its geometry which can initiate vibrations leading to loss of the part's functional ability. All this resulted in environmental and economical losses.

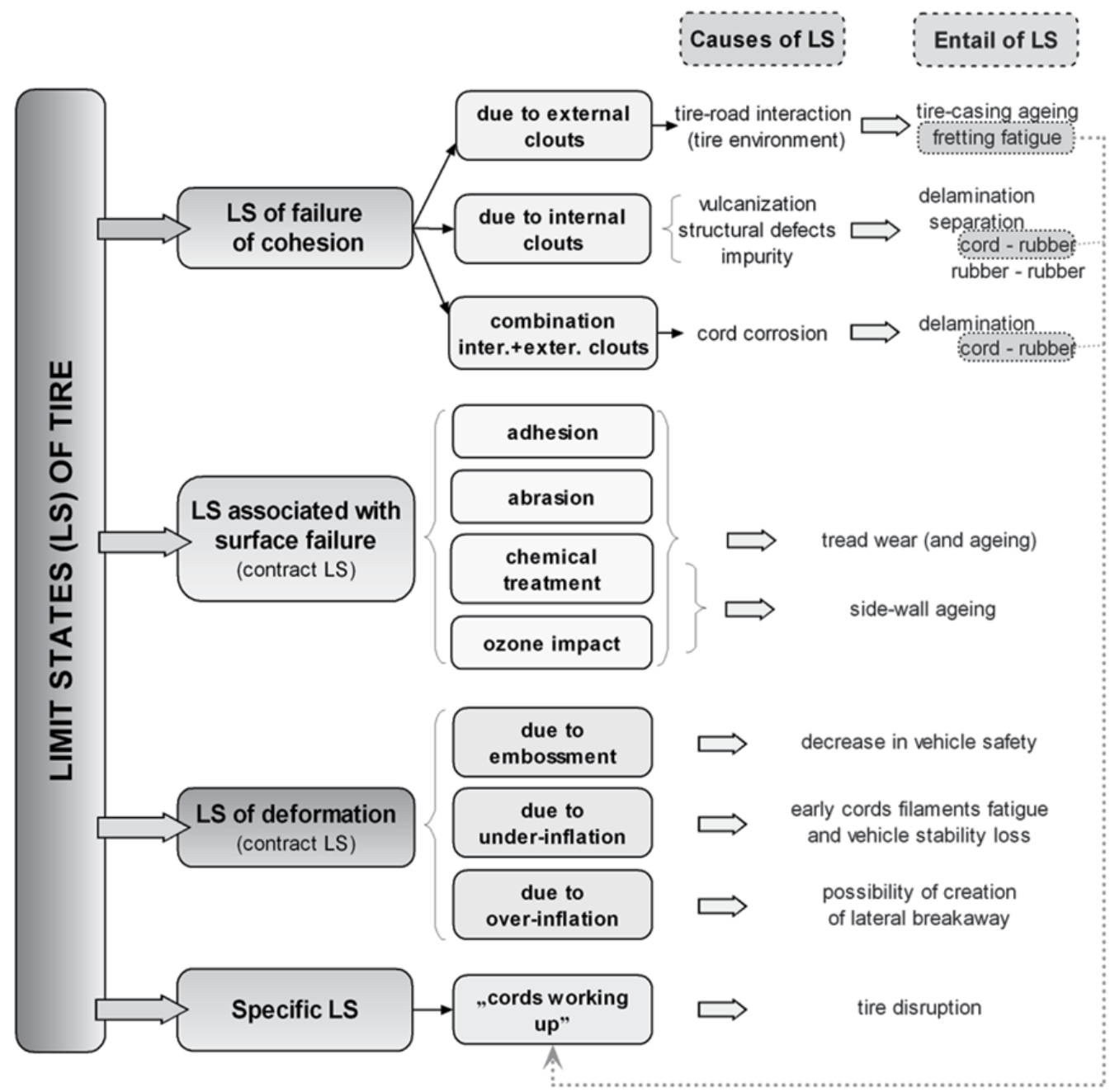




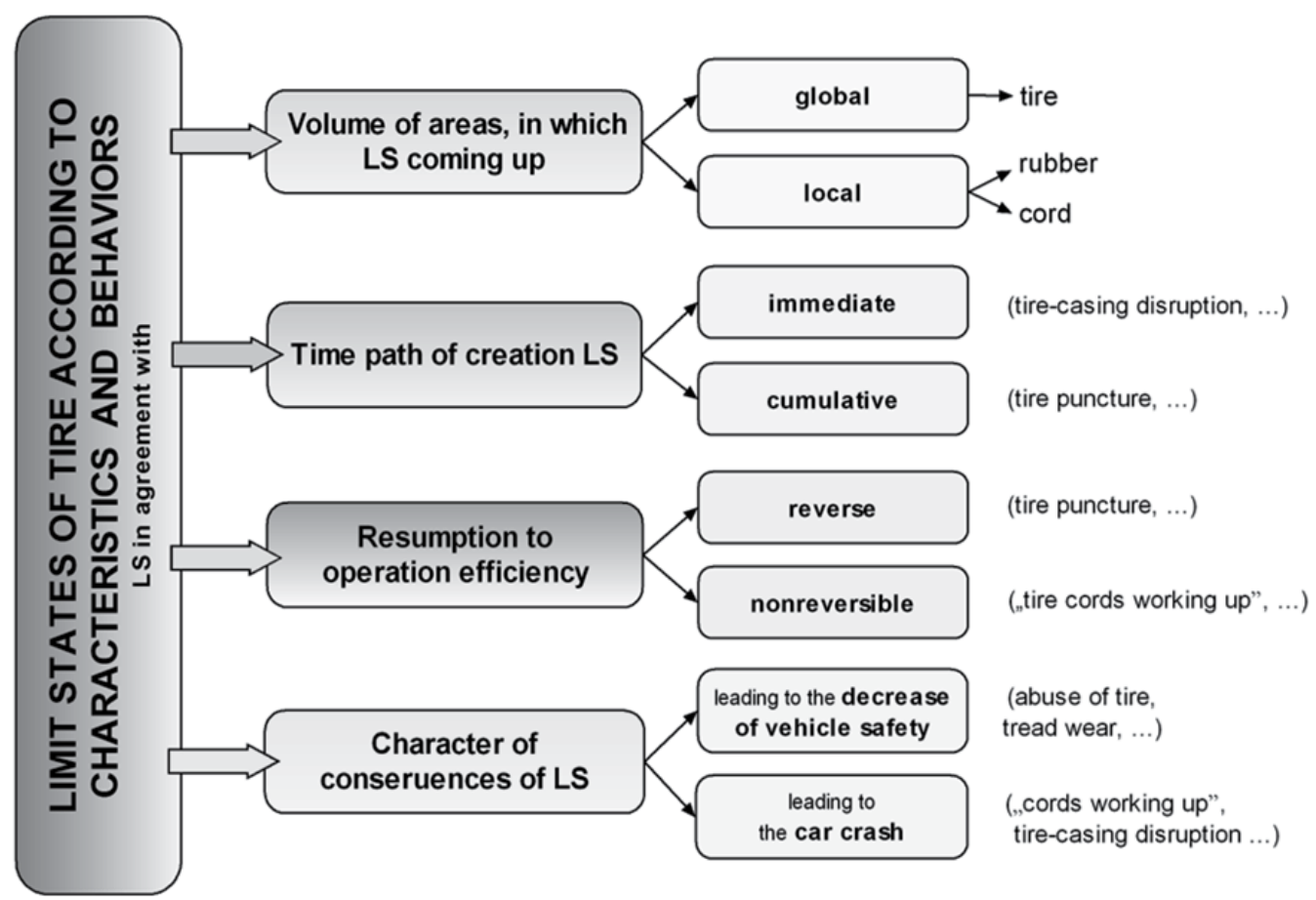

Figure 15. a. Limit states of tire; b. Limit states of tire

Tires must resist during operating to surrounding effects, to negative effects of operation and to other effects, which could lead to wear and degradation processes as are e.g. delamination. Resistance to the following effects is considered (Figure 16):

- $\quad$ Puncture - capability of tires to resist puncture by sharp objects;

- Cut-through - capability of tires (especially of the tread and sidewall) to resist contact with sharp objects;

- Breakdown - capability of tires to resist damage during short-term loading by concentrated forces;

- Fatigue - capability of tires to resist material fatigue and defects in consequence of repeated loading cycles;

- Separation and delamination - capability of structural tire components to maintain integrity of the system during operation;

- Humidity - tire elements must be able to resist degradation by contact with water;

- Ozone influence - capability of tires and of theirs components to resist degradation caused by ozone present in atmosphere;

- Temperature - tire components must be able to resist high and low ambient temperatures and also consequences of contact with the road;

- Chemicals - capability of tires and theirs components to resist degradation caused by chemicals (in winter - influence of salt solutions);

- Corrosion processes - capability of tire reinforcing cords to resist corrosion, etc. 


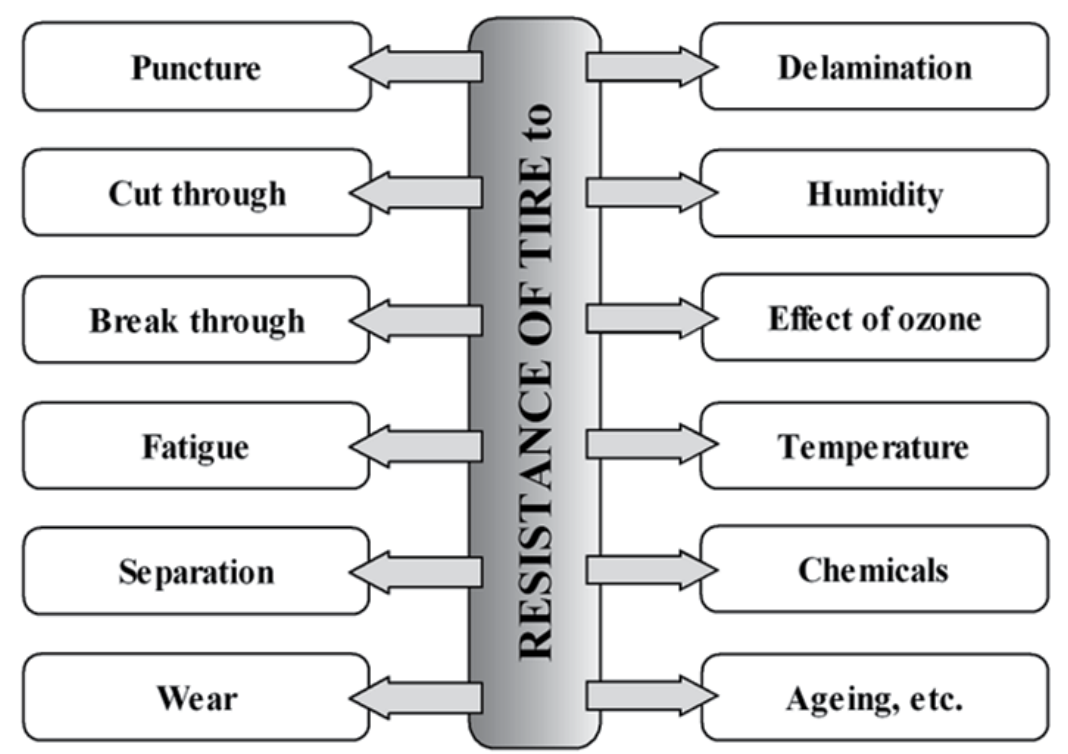

Figure 16. Basic requirements on the tire resistance

An appropriate design will help to increase resistance of the tire to certain degradation processes such as e.g. corrosive attacks initiated by local damage of the tire in cases where damaged are the steel cord reinforcements. Design optimization aimed at resistance to degradation and at achievement of longer life can be well performed by computer modelling. The computer modelling has reached such a level that it can work with a great amount of input data which represent the initiation of degradation effects on such a complicated technical object as a tire.

It is important to design such structure that the tire would be as much resistant to any degradation type as possible. These are required complex approach to experiments and computation of tire from macrostructure and microstructure too. It is necessary to have a good knowledge about:

- $\quad$ Structure of tire-casing;

- Material parameters of matrixes and reinforcements (steel-belt);

- Adhesive bonds cord-rubber, which obtained by metallography observation of reinforcements-matrix transit;

- Influence of degradation processes - corrosion effect on composite materials from micro and macrostructure point of view.

\section{Testing of tires}

It is necessary to run tests of tires as a whole, as shown in the Figure 17, and tests of individual tire casing components, purposely separated parts etc. This is how an overview which structural modifications can lead to an increase of the level of safety criteria, increase of resistance, life etc. can be obtained. 


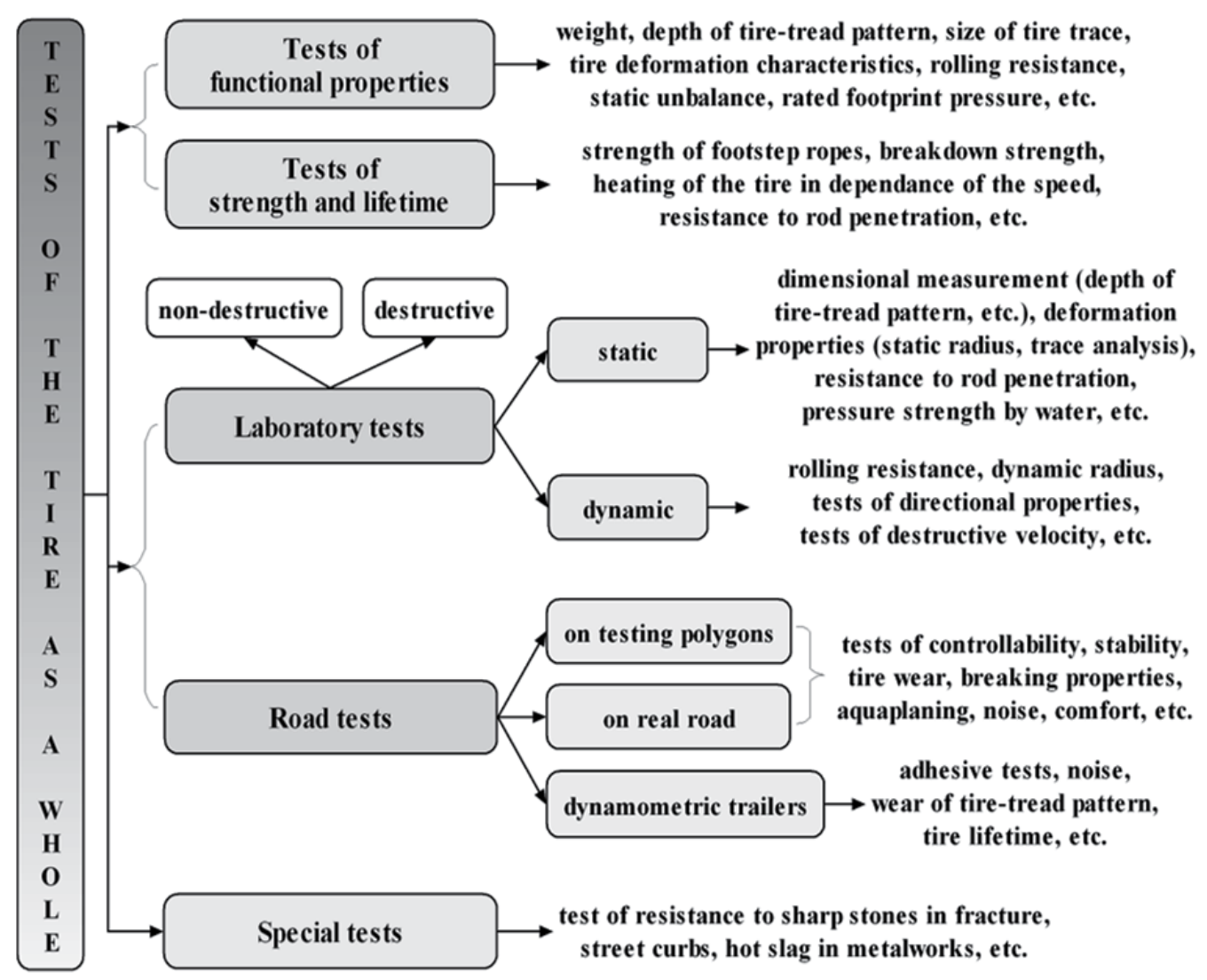

Figure 17. Tests of the tire as a whole

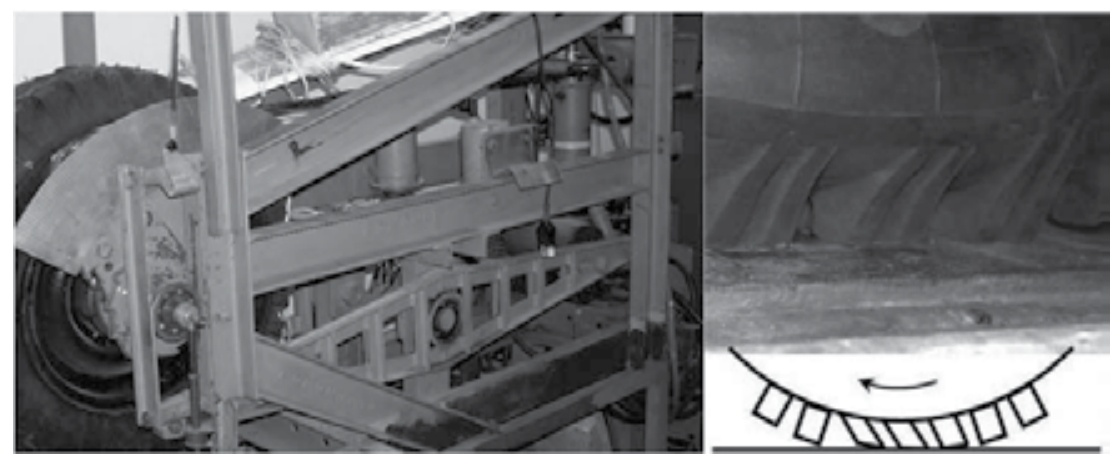

Figure 18. Static adhesor with detail of contact patch

Also basic statical deformation characteristics of tires can be obtained from a device called statical adhesor (Figure 18), which is available to author. The statical adhesor also enables measurement of data from the contact surface under defined conditions - shape of obstacles, vertical loading and inflation pressure. It is possible to obtain outputs from experiments on statical adhezor: 
- $\quad$ Radial deformation characteristic (by vertical tire force loading);

- Torsion deformation characteristic (slip curve by twist moment);

- $\quad$ Size and shape of contact area and distribution of contact pressure;

- at following conditions:

- $\quad$ Loading (vertical);

- $\quad$ Tire pressure (under-inflation, overinflated tire, specified pressure);

- Size of radial deformation;

- Shape of obstacle etc.

\section{Test of tire structure}

Material parameters of long-fiber composite structural parts as the tire steel-belt are necessary input data for tire computational models (e.g. steel-cord belt) and for subsequent comparison of computational models with experiments. Knowledge is necessary of the behaviour of composites as belts under mechanical load. These data are obtained by experimental modelling of composite specimens and composite structural parts (matrixes and reinforcement) by static tensile, compression, shear and bending tests. The behaviour of such materials as tire belts under mechanical loading is in many ways different from the behaviour of commonly used technical materials such as steels. In composites, compared with metals, final mechanical properties can be controlled e.g. in the direction of the orientation of fibers-cords. Composites of tire also have elevated fatigue life, by one order higher material damping and are resistant to failure due to their ability to stop growth or decelerate propagation of cracks on the rubber matrix-cord interface. Tests of specific long-fiber composite materials with hyperelastic matrixes (namely steel.-cord belt test sample) are not standardized and neither are the shapes and dimensions of test samples, namely for tensile tests, which are for the observation of mechanical behaviour absolutely essential. For determination of material parameters of rubber matrixes and cord-reinforcements are necessary make experiments in agreement with standard specifications.

In composite samples or in samples with a certain content of composite layers of concern are the configurations of cords with respect to the direction of loading which results in a change of the stiffness characteristics. Therefore is necessary to design the geometrically parameters and shapes of one or multi-layer tested samples before experiments. The samples must have different:

- Angle of cord (with respect of the direction of loading - not only longitudinal and transverse orientated samples) - see figure 19;

- Material of cord (surface treatment);

- $\quad$ Form of cord (wire, thin wire);

- $\quad$ Number of layers (single-layer, two-layer - Figure 19, multi-layer);

- Specimen width, shape etc.

The author Krmela was designed multi-layer test samples with different wide 10, 15 and 25 $\mathrm{mm}$ and of length $120 \mathrm{~mm}$. The cord-angle orientations in single-layer specimens are $0^{\circ}, 22.5^{\circ}$, 
$45^{\circ}, 67.5^{\circ}$ and $90^{\circ}$. Two-layer specimens (Figure 19) are symmetrically orientated between top/bottom layer $\pm 22.5^{\circ}, \pm 67.5^{\circ}, \pm 45^{\circ}$ and asymmetrically orientated with cord-angles $+0^{\circ}$ $45^{\circ}$ and $+67.5^{\circ} \% 22.5^{\circ}$ (it is $+22.5^{\circ}-112.5^{\circ}$, specimen D) with thickness $4 \mathrm{~mm}$. Real single and two-layer specimens are presented Figure 20 as an example.
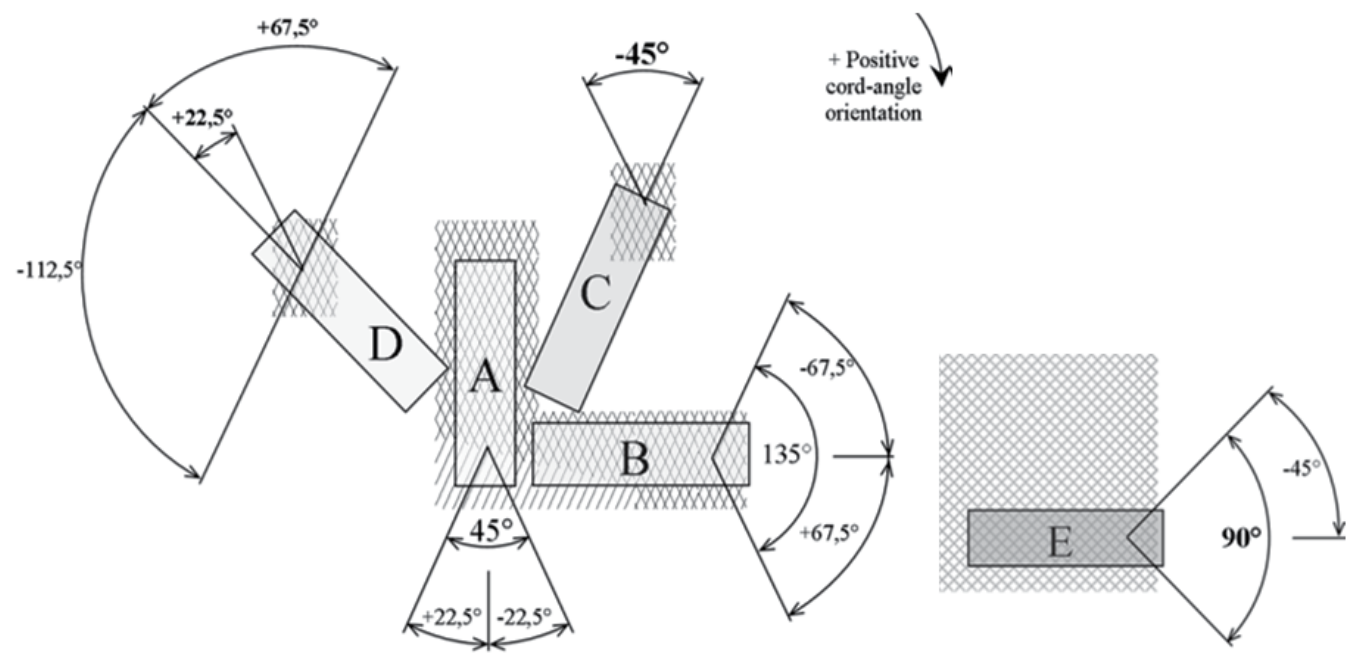

Figure 19. Two-layer specimens from plates with cord orientations $45^{\circ}$ (left): A - lengthwise symmetrical specimen with $22.5^{\circ}$; B - transverse symmetrical specimen with $67.5^{\circ}$; C - asymmetrical specimen with $+0^{\circ} /-45^{\circ}$; D - asymmetrical specimen with $+67.5^{\circ} /+22.5^{\circ}$; Specimens from plates with cord orientations $90^{\circ}$ (right): E - symmetrical specimen with $45^{\circ}$
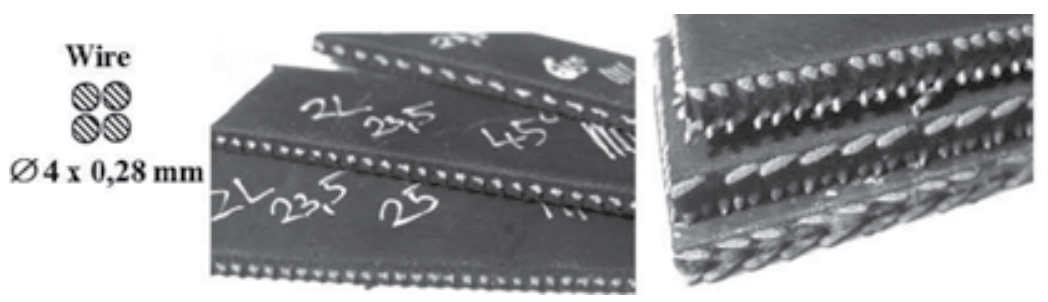

Thin-wire

$\varnothing 0,94 \mathrm{~mm}$

Figure 20. Single-layer specimens of steel-cord belt with wire cord and two-layer specimens with thinwire cord (right)

Also must be determined conditions for individual type of tests, namely:

- Statically tensile tests (uniaxial and biaxial);

- Statically bend tests;

- Statically tests of composites under combined loading states (combinations tensile with bend) - that are to be approximated tire real state during tire operational loading (predicate about real deformation behaviors of steel-cord belt plies);

- Corrosion tests in a corrosion chamber (exposition time);

- Dynamically test etc. 
Statical tensile tests of steel-belt samples are important for obtaining knowledge about stiffness characteristics and material parameters. The conditions of the tensile tests are:

- Initial length between the jaws of the testing machine is $92 \mathrm{~mm}$.

- Elongation measured on the same length and also measured on 50 (or $25 \mathrm{~mm}$ ) in centre of specimens.

- Rate of test is 10 or $25 \mathrm{~mm} / \mathrm{min}$.

As output example from tensile test of two-layer belt for different cord-angle and cord-type Figures 21 and 22 give tensile force-elongation and stress-strain dependences (elongation measured on the length between the jaws).

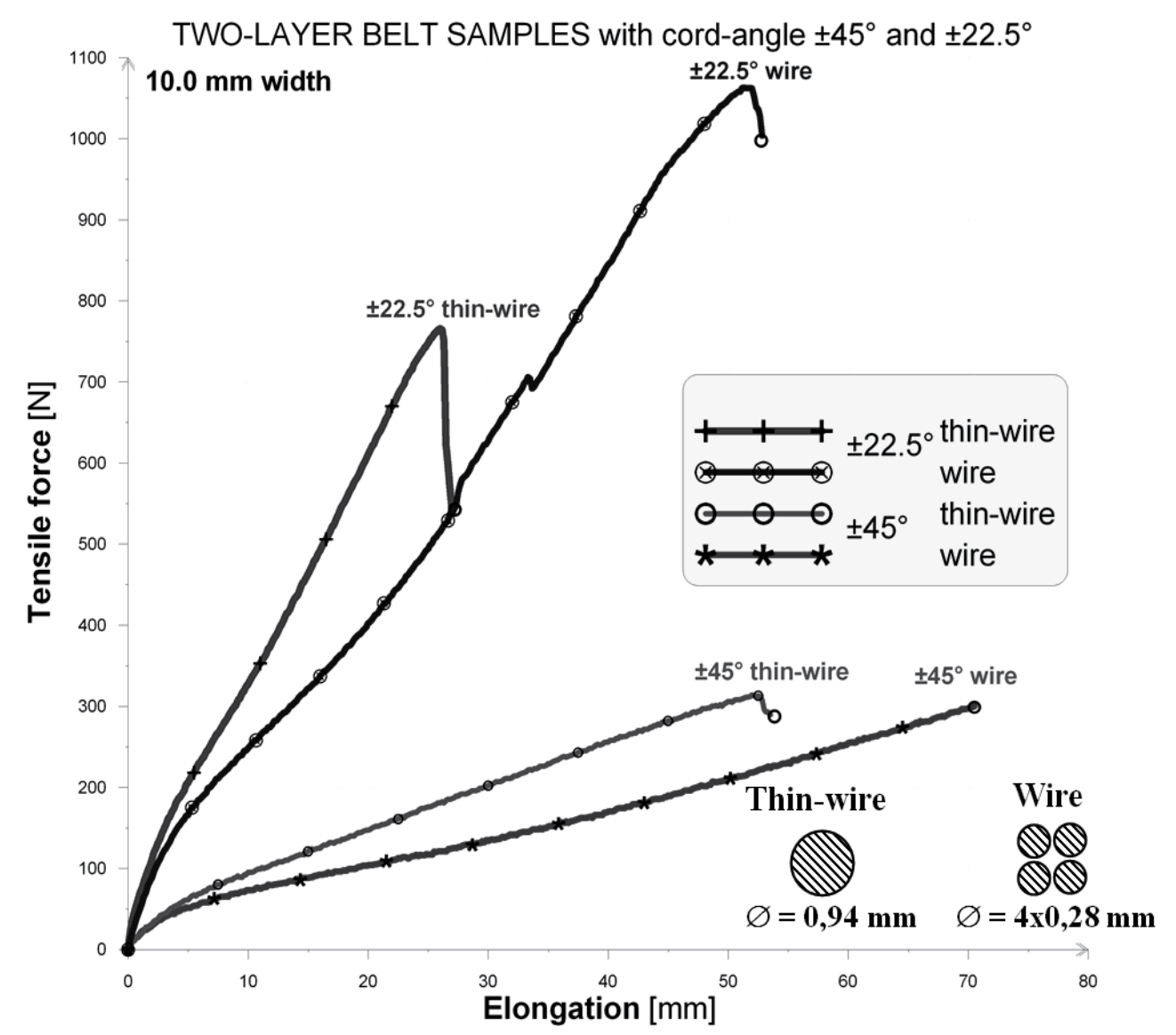

Figure 21. Outputs from tensile test of steel-cord belt samples - force-elongation dependences 


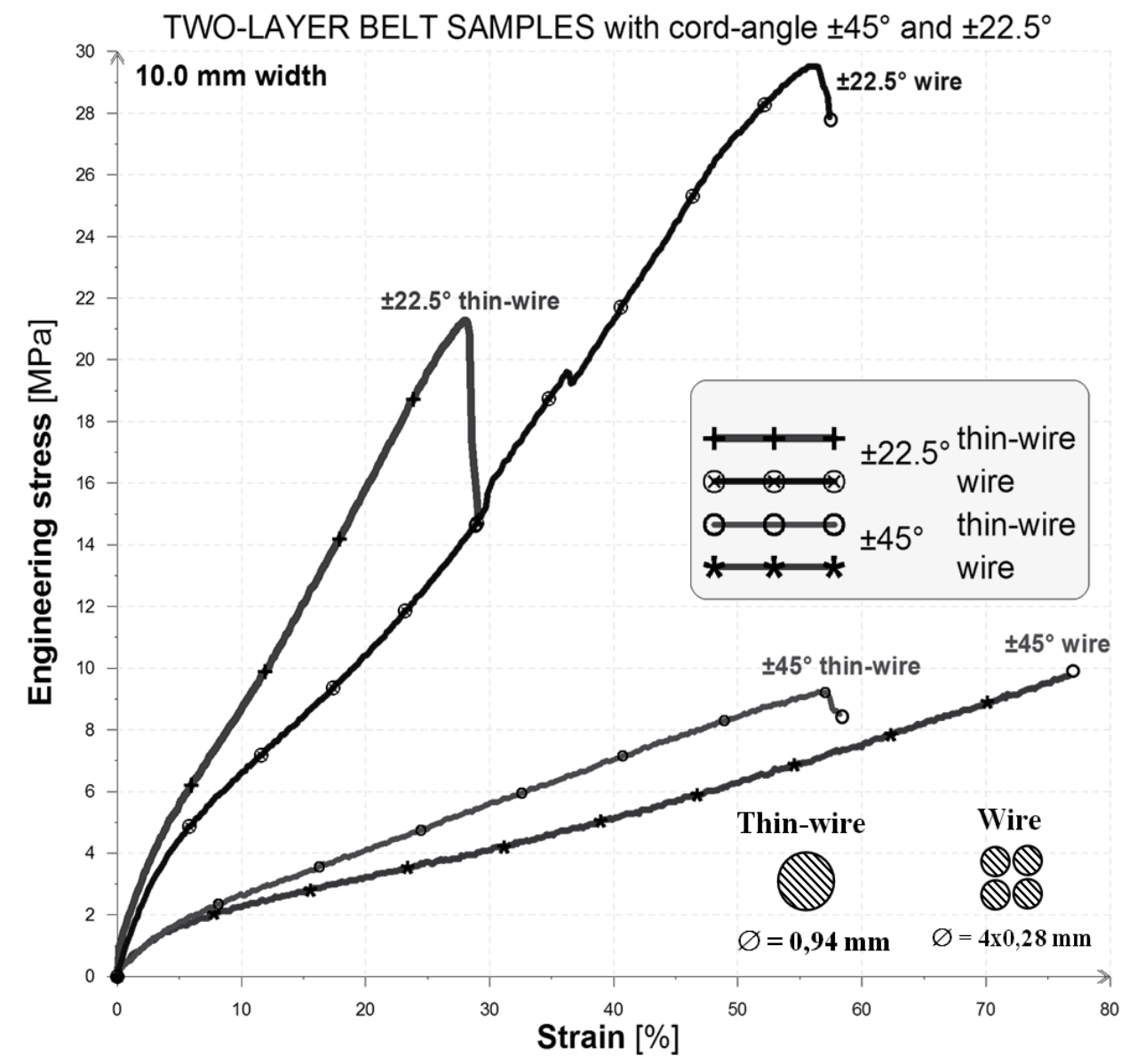

Figure 22. Outputs from tensile test of steel-cord belt samples - stress-strain dependences

Figure 23 presents some examples of specimens' failure after tensile test.
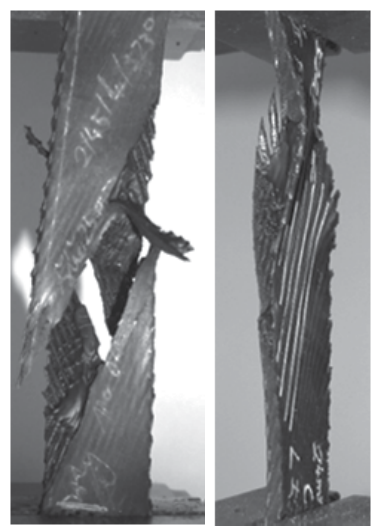

Figure 23. Failure of two-layer symmetrical $\pm 22.5^{\circ}$ and asymmetrical specimens $+67.5^{\circ} / 22.5^{\circ}$ (right) after tensile test 
The selected specimens were subject to statical tensile, also compression, shear and bending tests. Also testing conditions have been designed. Tests is necessary perform not only at ambient temperature $20^{\circ} \mathrm{C}$ but also at lowered and elevated temperature (from $-30^{\circ}$ into $180^{\circ}$ Celsius).

\section{Corrosion test of steel-cord belt}

It will be possible uniform statically test conditions for samples affected by corrosion and samples without corrosion.

Selected single and two-layer composite test specimens are exposed to corrosion tests in a corrosion chamber Gebr. Liebisch S 400 M TR (for 500 or 265 hours in saline application by temperature at $70^{\circ} \mathrm{Celsius} \mathrm{-} \mathrm{authors} \mathrm{note:} \mathrm{such} \mathrm{extreme} \mathrm{conditions} \mathrm{should} \mathrm{not} \mathrm{ever} \mathrm{appear} \mathrm{in}$ tire operations if proper conditions are kept) and to static tensile tests till the failure. The aim of these tests is to find the influence of the degrading process on the stiffness characteristics of the composite structures. Also will be investigated an influence of degree of degradation on the adhesive bond matrix-reinforcement.

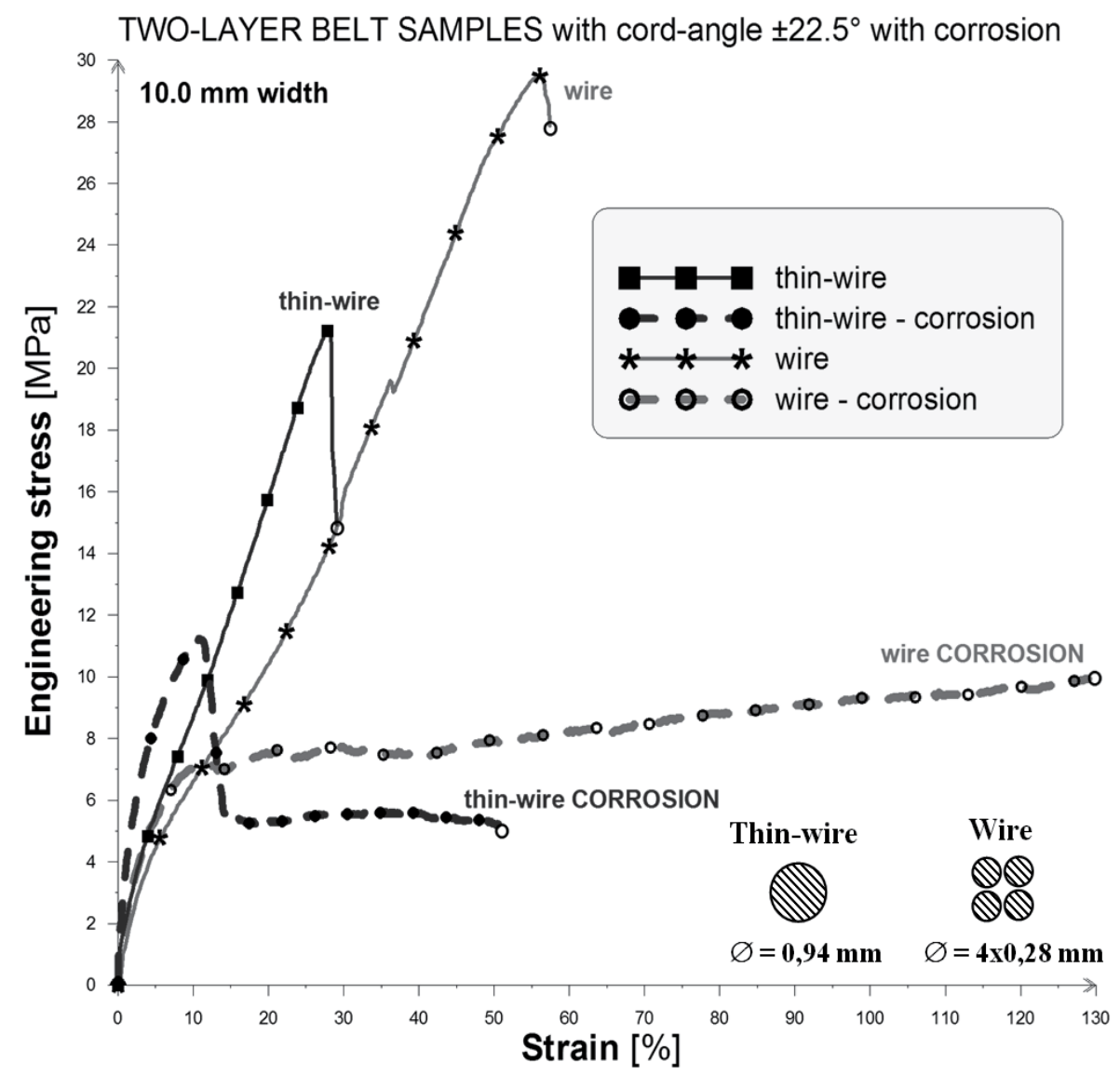

Figure 24. Outputs from tensile test of steel-cord belt samples - stress-strain dependences 
The results obtained from tensile test will be compared with results obtained from tensile test on samples without corrosion. The experimental results of tensile tests of undamaged (non-corrosion) steel-cord belt ply (two-layer with cord-angle $\pm 22.5^{\circ}$ ) for comparison analyses with belt ply after corrosion tests are shown in Figure 24 as dependences stress on strain.

The influence of corrosion on the stiffness and tensile force-elongation or engineering-stress dependences is sizable. The oxide film is strongly affected on failure of adhesive bond.

The fracture characters of test specimen after tensile and corrosion test in a corrosion chamber were accounted. The fracture of test specimen with $22.5^{\circ}$ angle and thin wire cord is on Figure 25 as an example. The corrosion processes on cord surfaces is very dangerous. Therefore, it will be important to study also adhesive bond.

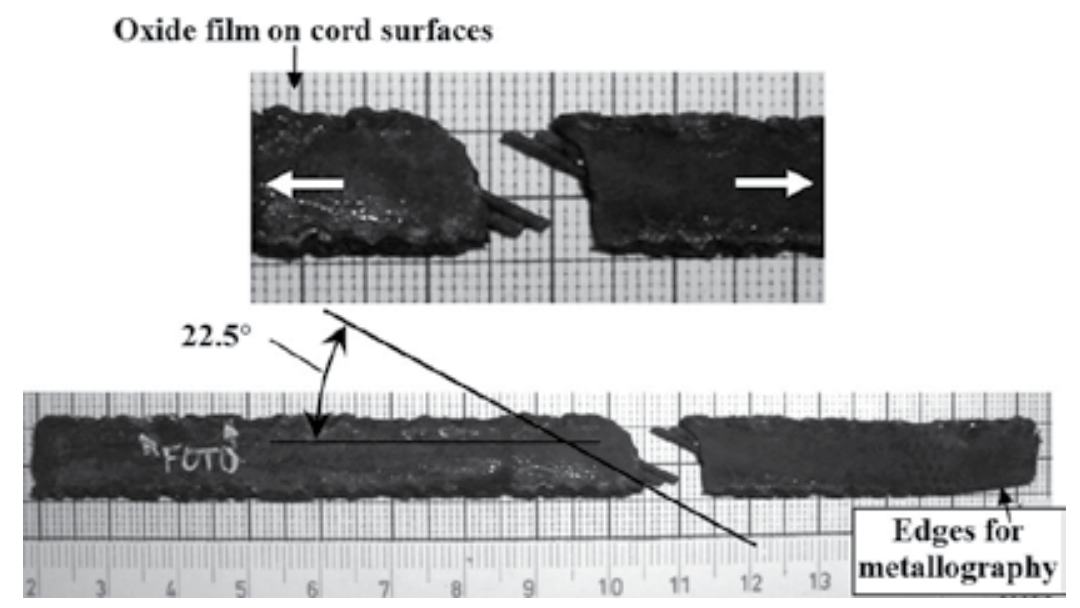

Figure 25. Fracture character of specimen with corrosion after tensile test

\section{Metallography of Interface between Cord-Rubber}

The light microscope is used for metallography observations of the adhesive bond between steel-cord and rubber after failure after corrosion test in corrosion chamber and statically tensile test and without corrosion too. The edges of steel-cord belt specimens were observed in detail - (see Figures 25 and 26).

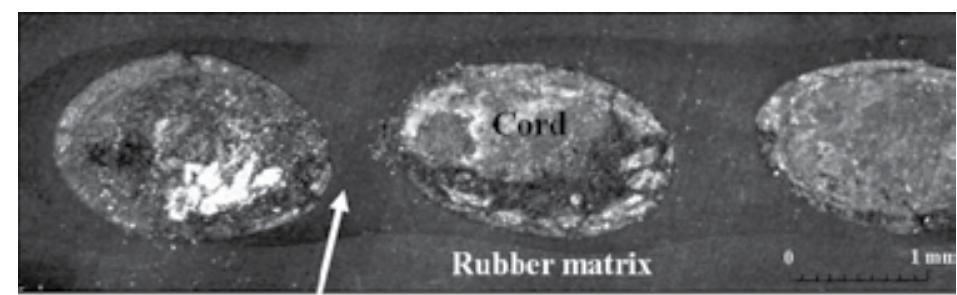

Rubber drift

Figure 26. Interface between thin wire steel-cord/rubber drift/rubber matrix after corrosion and tensile tests 
Microscopy with 100x-200x zoom will be used for the evaluation of adhesive bonds from level of failure point of view. It appears that sufficient zoom from setting of failure level point of view (detection of delaminations, separations).It is necessary to prepare of samples for microscopy observation of structures so that the samples included different:

- $\quad$ Form of cord;

- Geometrical configurations and number of layers;

- Level of corrosion impact of steel-cords - adhesive bonds (without corrosion, easy corrosion, after corrosion test behind extremely conditions).

For selected cords were accounted:

- Uniformity of layer of rubber drift on cord surfaces;

- Surface treatment of cords;

- Interface between cord-rubber matrix after tire production;

- Structural change into microlocality of cord-rubber after corrosion.

The corrosion processes on cords are shown Figure 27. The results from microscopy observation are presented in Figures 28-33.

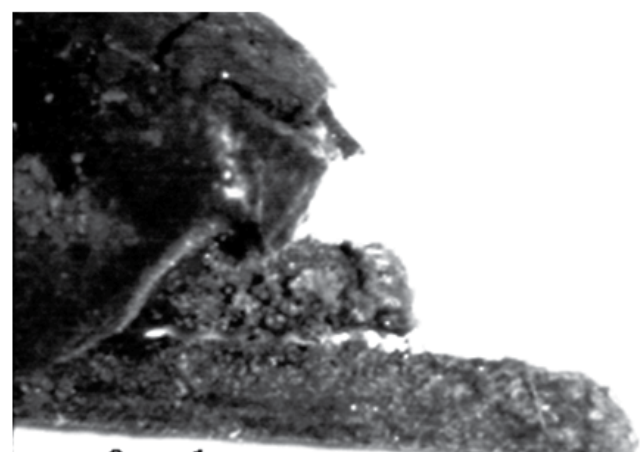

$0 \quad 1 \mathrm{~mm}$ |unIIIII

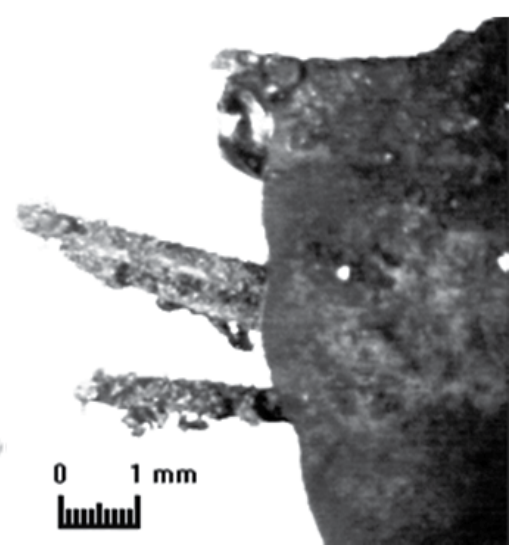

Lmuluml

Figure 27. Corrosion processes on steel-cord surfaces - thin wire versus wire (right)
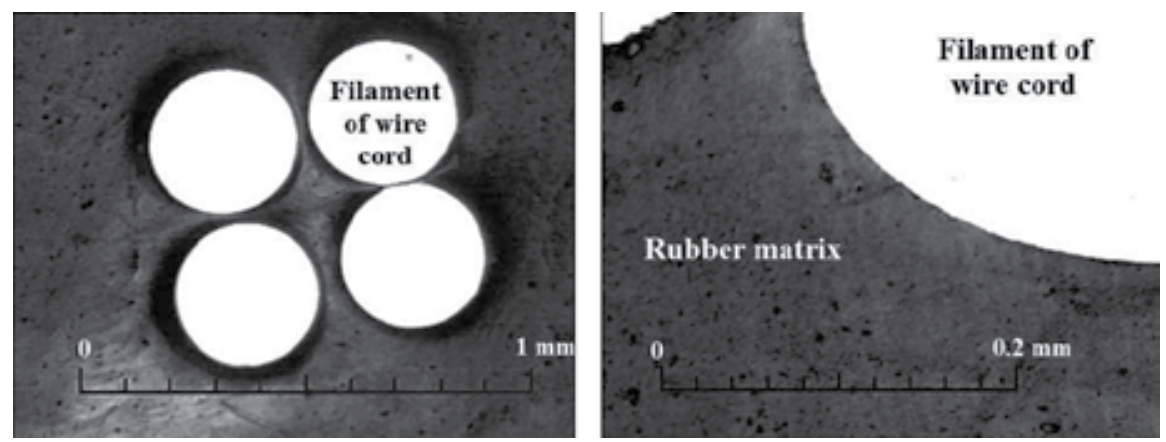

Figure 28. Good adhesive bond between wire steel cord $2+2 \times 0.28 \mathrm{~mm}$ (cord consists of 4 filaments) and rubber after tire production (without corrosion) 

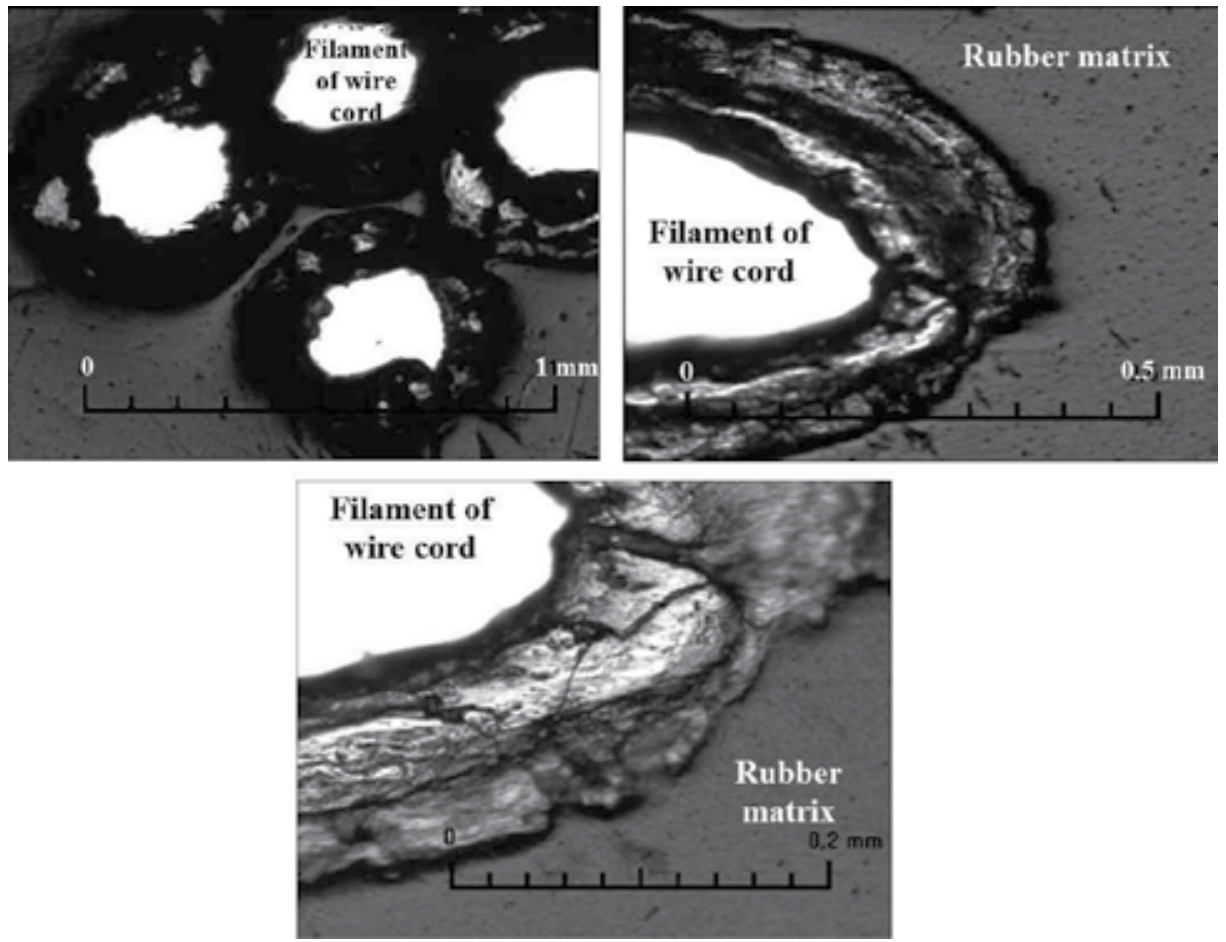

Figure 29. Damaged adhesive bond between wire steel cord $2+2 \times 0.28 \mathrm{~mm}$ and rubber after corrosive attack (with extreme corrosion and tensile loading) with detail of oxide on filament surfaces

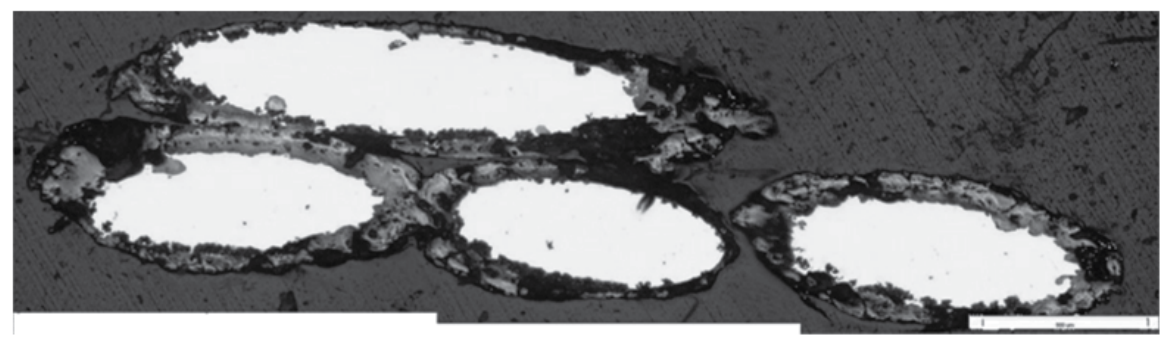

Figure 30. Damaged whole wire steel cord $2+2 \times 0.28 \mathrm{~mm}$
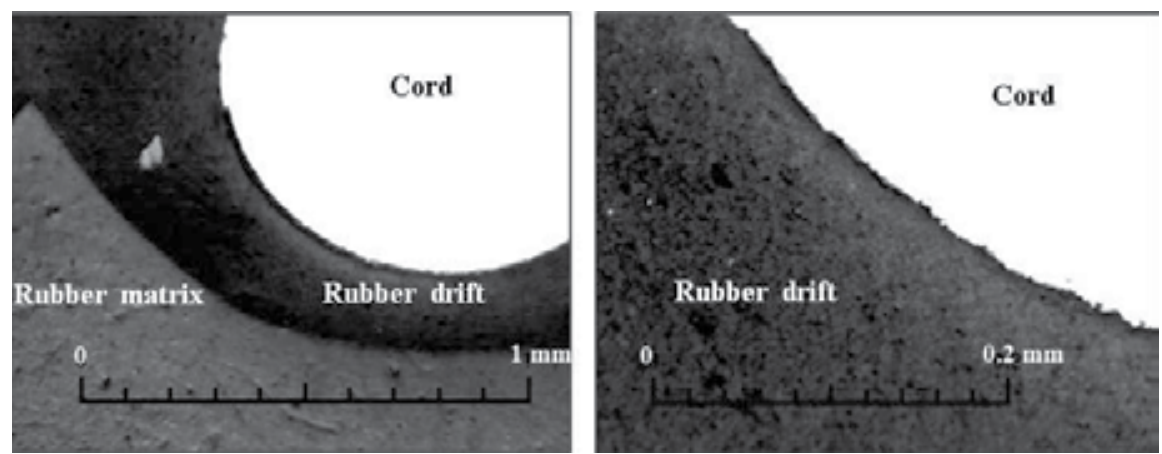

Figure 31. Good adhesive bond between thin-wire steel cord $0.94 \mathrm{~mm}$ and rubber drift after tire 

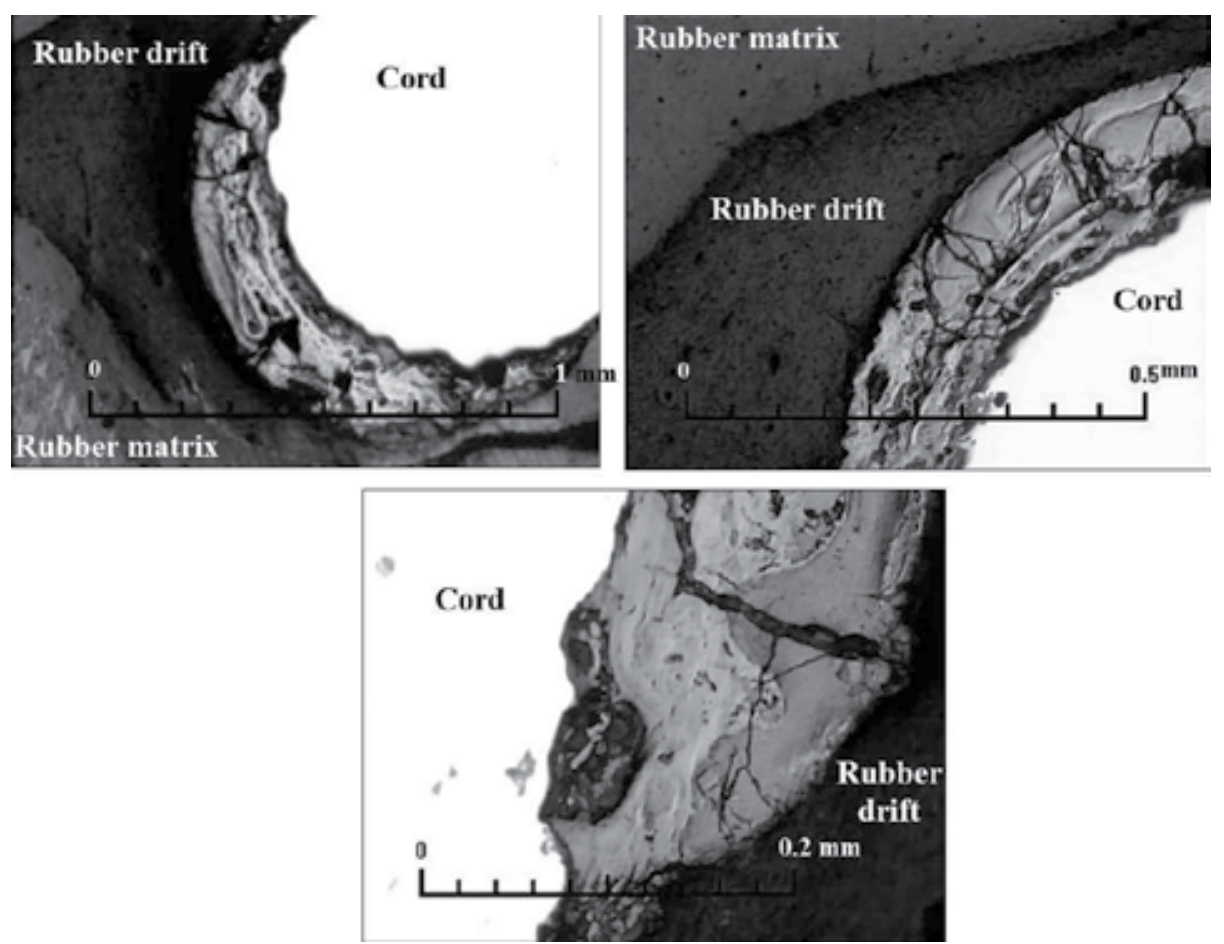

Figure 32. Damaged adhesive bond between thin-wire steel cord $0.94 \mathrm{~mm}$ and rubber drift after corrosive attack (with extreme corrosion and tensile loading) with detail of oxide on cord surfaces

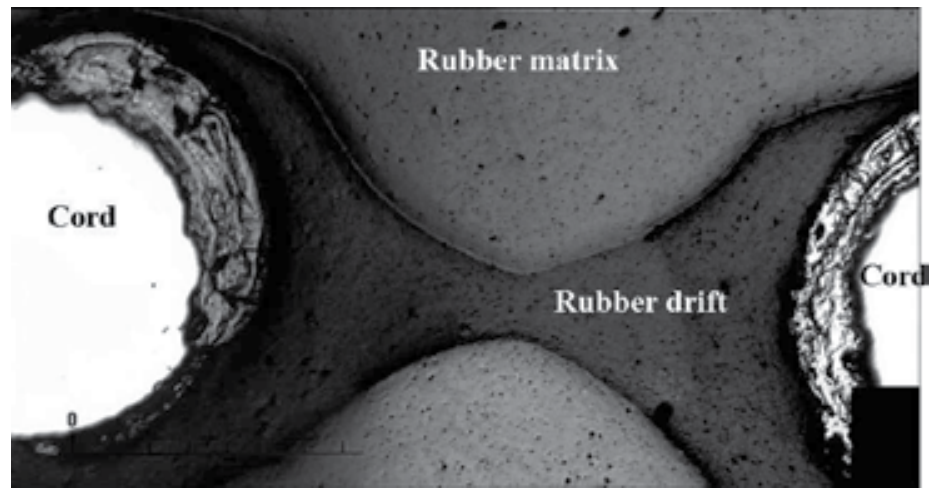

Figure 33. Damaged whole thin-wire steel cord $0.94 \mathrm{~mm}$

On the base of corrosion tests is possible note:

- $\quad$ Arise of uniform surface corrosion;

- Fragility and hardness of corrosive layer;

- Quicker grow of oxides near cord surfaces;

- Fracture of oxide layer;

- Quality of cord-surfaces and surface treatment of cords with respect to corrosion attack;

- Decreased of material characteristics of steel-cord belt on the basic of tensile tests. 
The adhesive bonds are influenced internal impacts (inserted during production, mounting) and external impacts (operating conditions, surrounding conditions etc.) or their interaction. It can be caused degradation on reinforcement-matrix adhesive bond, when its effect is failure into whole macro volume of tire which isn't permissible from safety aspect of vehicle.

- $\quad$ Any damage in the area of tire crown, namely into steel-cord belt plies, is perilous.

- If extreme corrosion on cords then cord surface treatment lost function of corrosive protection.

- If cords are with corrosion then adhesive bonds between cord-rubber matrix are damaged and safety of steel-cord belt plies and also tire is decreased.

- For predication of damaged belt ply is possible used combination of computational with experimental modeling.

- Corrosive attacks on reinforcing cords in whatever form can reduce the quality and operating safety of the whole tires.

\section{Dynamic testing}

In this part we continue with a description of dynamic materials behavior as well as a dynamic tire testing.

The tread displacement changes caused by the breaker angle changes were measured by an apparatus presented in Figure 34. The apparatus consists of a line laser, CCD camera and a computer with an appropriate measuring software. The CCD camera records the changes of the line laser spot, which copies the tire dimension changes. This system measures the main dimension of the rotating tire (the illuminated part of tread) at constant velocity. For more details see the work (Koštial et al 2006).

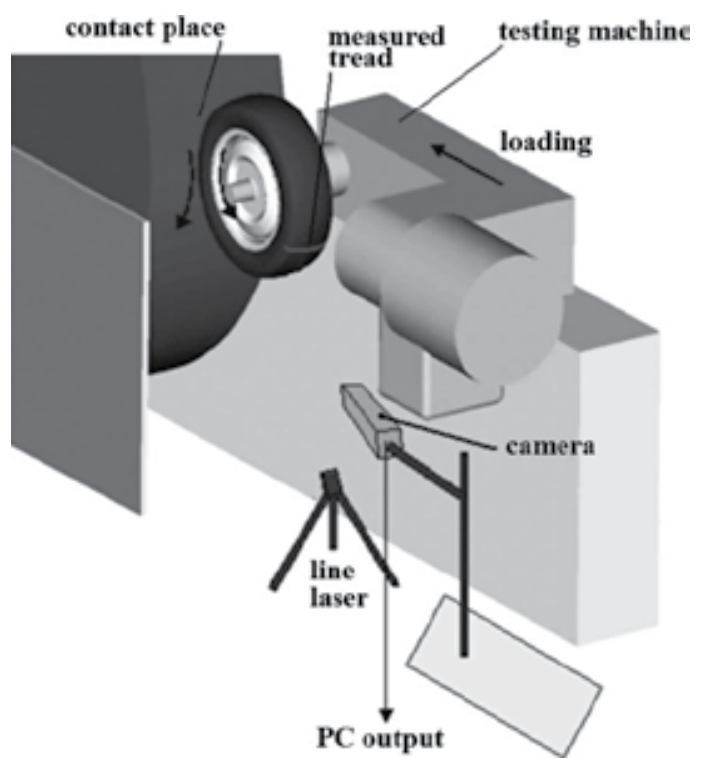

Figure 34. The measuring system for a tread deformation 


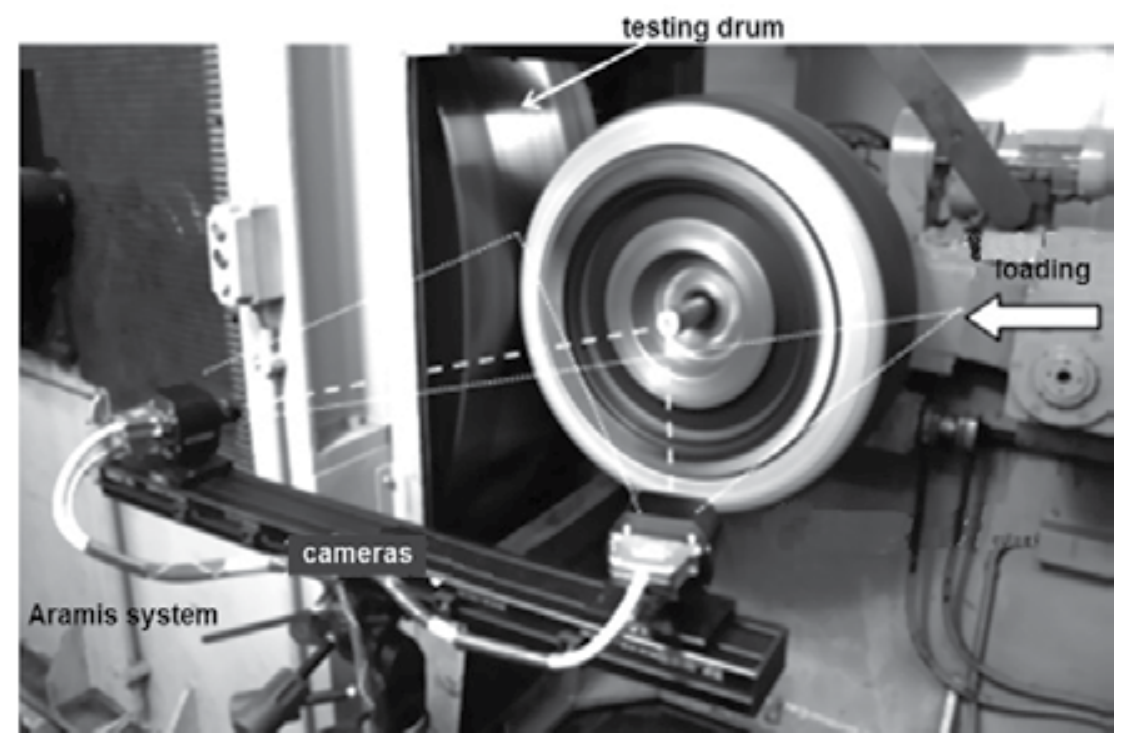

Figure 35. The measuring system for a sidewall displacement measurement

\begin{tabular}{|c|c|c|c|c|c|}
\hline \multicolumn{2}{|l|}{ I. mixture } & Properties & $S$ & $\mathrm{~T}$ & $\mathrm{D}$ \\
\hline Hardness of vulcanizate [ShA] & 73 & Strength , LOP, [MPa] & 19.23 & 17.08 & 23.27 \\
\hline Modulus 300\% [MPa] & 15 & Elongation [\%] & 565 & 495 & 440 \\
\hline Strength [MPa] & 16.2 & Modulus 300\% [MPa] & 7.8 & 9.2 & 17.4 \\
\hline Elongation [\%] & 340 & Hardness [ShA] & 58 & 66 & 72 \\
\hline \multicolumn{2}{|l|}{ II. mixture } & Elasticity [\%] & 35 & 18 & 48 \\
\hline Hardness of vulcanizate [ShA] & 85 & \multirow{3}{*}{$\begin{array}{l}\text { Tear resistance }\left[\mathrm{kNm}^{-1}\right] \\
\text { at } 20^{\circ} \mathrm{C} \\
\text { at } 90^{\circ} \mathrm{C}\end{array}$} & \multirow{3}{*}{$\begin{array}{l}57.1 \\
43.4\end{array}$} & \multirow{3}{*}{$\begin{array}{l}37.5 \\
29.9\end{array}$} & \multirow{3}{*}{$\begin{array}{l}74.8 \\
74.3\end{array}$} \\
\hline Modulus 300\% [MPa] & 12 & & & & \\
\hline Strength $[\mathrm{MPa}]$ & 16.5 & & & & \\
\hline Elongation [\%] & 285 & & & & \\
\hline \multicolumn{2}{|l|}{ III. mixture } & & & & \\
\hline Hardness of vulcanizate [ShA] & 60 & & & & \\
\hline Modulus 300\% [MPa] & 7.2 & & & & \\
\hline Strength [MPa] & 17.9 & & & & \\
\hline Elongation [\%] & 570 & & & & \\
\hline
\end{tabular}

Table 1. The physical parameters of bead core blends (left) and other part of the tire (right part).

The sidewall displacement changes caused by both, bead core and the breaker angle, were measured by a contactless system Aramis. This system is able to measure changes of the displacements (radial and axial) during the rotation of the tire. More experimental details about the apparatus are in the work (Koštial et al 2005) (see Figure 35.) The statistically evaluated precision of both described equipments at the actual arrangement of the apparatus was $0.05 \mathrm{~mm}$ (the result of ten independent measurements on the same tire). The radial loading was $7360 N$ (that is $80 \%$ of the maximum available load) with a tire inflation 
of $290 \mathrm{kPa}$. After the tire conditioning (30 min at the velocity $80 \mathrm{~km} / \mathrm{h}$ ) the tire inflation increased (due to heat generation at the tire movement) to a pressure of 310-320 $\mathrm{kPa}$. The considered testing velocities were $10,50,80,120,150$ and $180 \mathrm{~km} / \mathrm{h}$. The reference velocity was $10 \mathrm{~km} / \mathrm{h}$ (zeroth stage). The image of a sidewall obtained at $10 \mathrm{~km} / \mathrm{h}$ is compared with other images obtained at different velocities. The difference between those images determines a displacement.

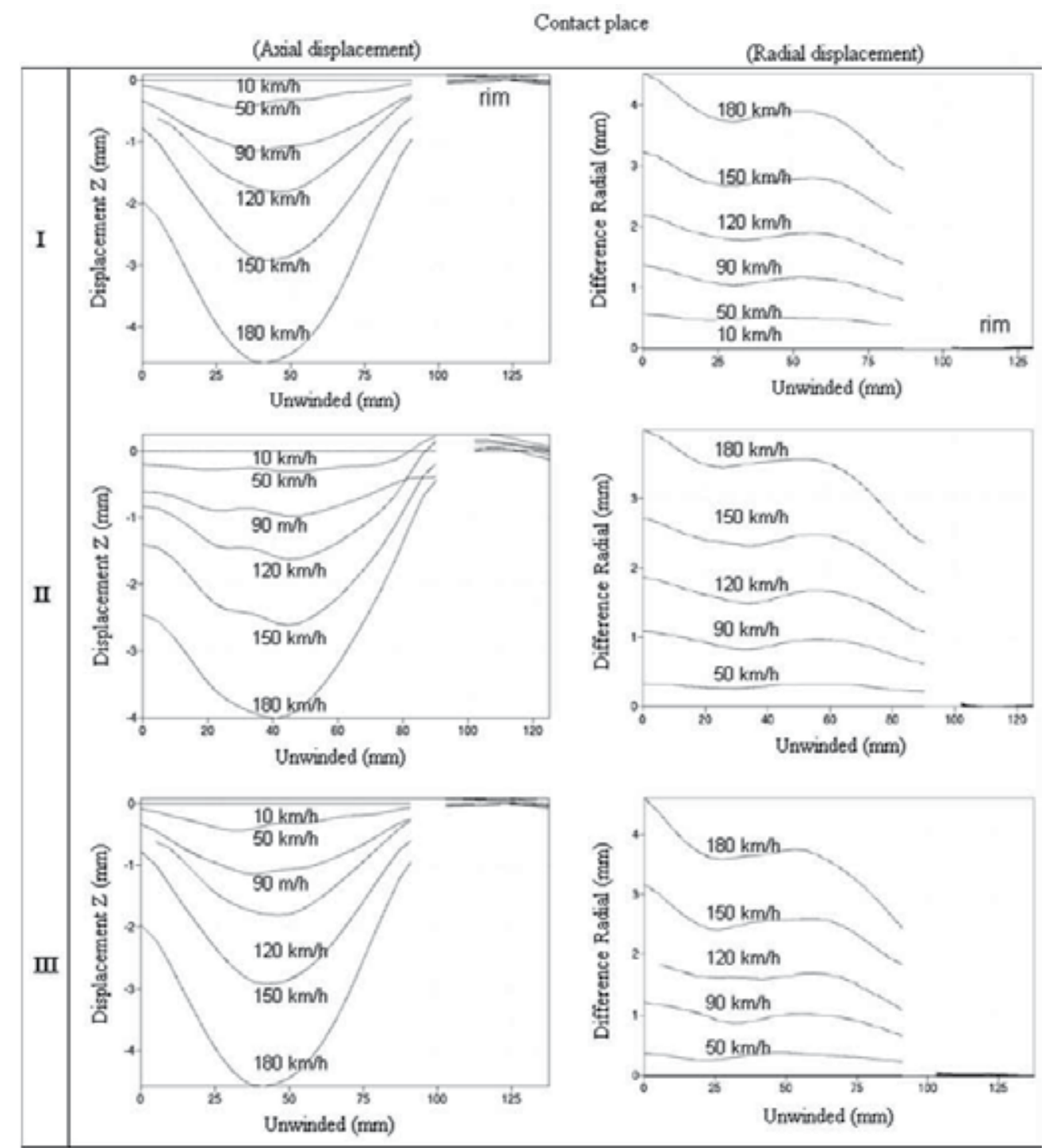

Figure 36. The axial displacement changes at the contact (left side) and radial displacement (right side) for three different bead core blends. Unwinded means a length of a given sidewall part.

The physical parameters of the bead core blends are described in the left part of Table 1 . The characteristic physical parameters blends used for the construction of the other part of a tire 
are collected in Table 1 - right part. The abbreviations used in Table 1 mean: $S$ - sidewall rubber mixture, $T$ - tread rubber mixture and $D$ - depositional rubber mixture on the steel cord.

The measurements of the sidewall displacement at the contact of the driving drum and tire presented in Figure 36 also show the smallest radial and axial value for the sidewall containing the blend II. The upper line is for the reference velocity $10 \mathrm{~km} / \mathrm{h}$. The minimum showing at the bottom of every picture (for axial displacement) corresponds to the velocity of $180 \mathrm{~km} / \mathrm{h}$. According to the presented results it is possible to conclude that in the current case the blend II has the best properties (the smallest displacement of a sidewall means higher mechanical stiffness and smaller rolling resistance, for instance) for the sidewall construction. Further we will analyze the changes of the tread and sidewall displacement caused by changes of breaker angle with unchanged bead core blend. The tread and sidewall materials were also the same according to the description presented above (Table 1).

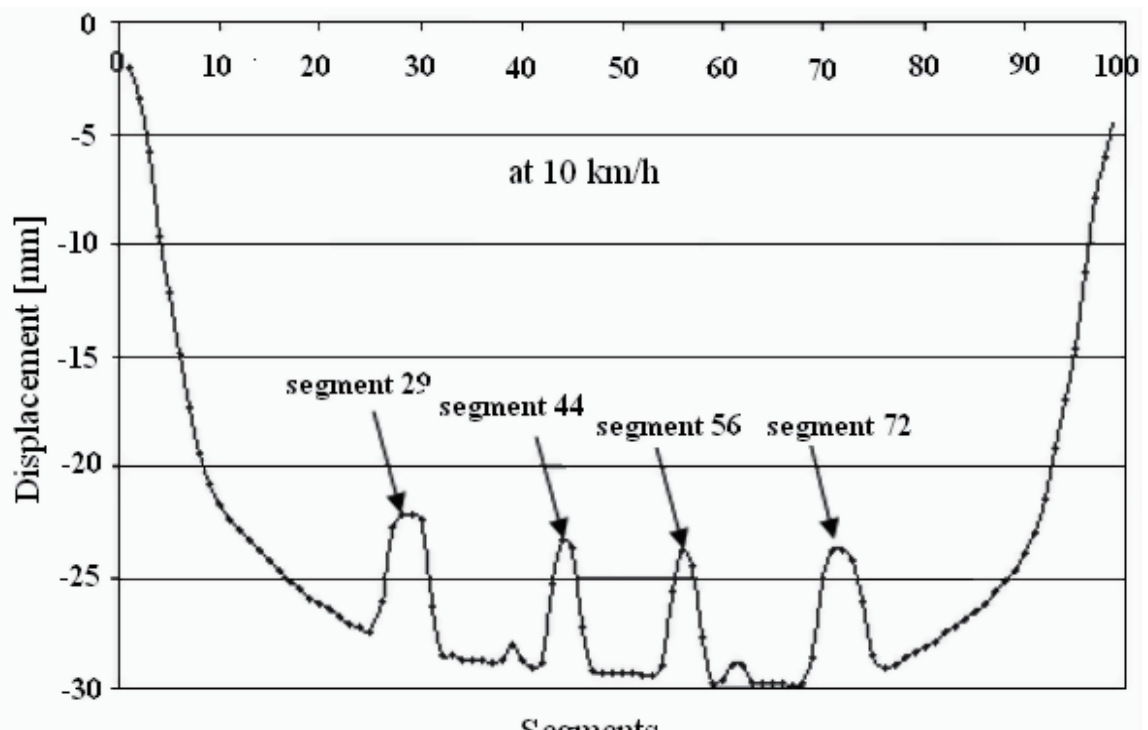

Segments

Figure 37. The electronic picture of a tread with characteristic points marked as "segments"

Figure 37 shows the electronic picture of the tread with characteristic points marked as "segments" obtained by an optical system with a line laser. The tread displacement changes in radial direction (at above defined velocities) caused by a breaker angle change are visible in Figure 38.

Rising of the breaker angle changes the shape of the tread deformation from convex to concave. The best solution was obtained for the breaker angle equals $27^{\circ}$, where practically the full profile of the tread is in contact with the road. In order to study the influence of breaker angle changes on "sidewall displacement dynamic" at different velocities we also tested the sidewall displacement changes (measured by ARAMIS, breaking angle equal to 
$20^{\circ}$ and $27^{\circ}$ ). The experimental results of both the axial and radial displacement in this case were compared with those obtained by the FEM simulation in ABAQUS environment. It is possible to see a good agreement between both simulated and measured curves. Differences occur at higher velocities for radial displacement. Both, the experimental and simulated axial displacements for different velocities and chosen breaker angles $\left(20^{\circ}\right.$ and $\left.27^{\circ}\right)$ are displayed in Figures 39 and 40. The corresponding simulation and experimental results obtained for radial displacement also show the best results (the highest axial displacement) for breaker angle $27^{\circ}$.

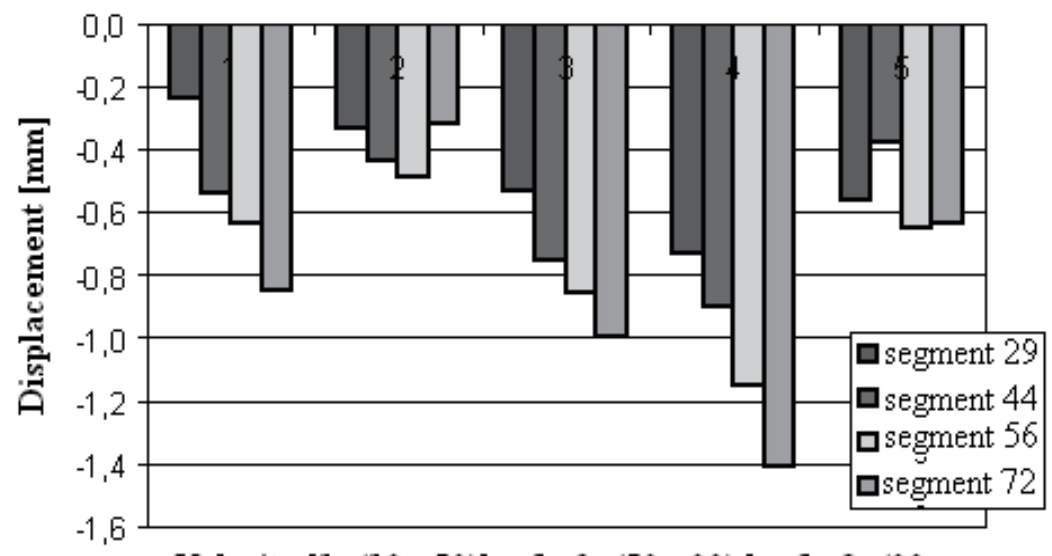

Velocity [1: $(10-50) \mathrm{km} / \mathrm{h} ; 2:(50-90) \mathrm{km} / \mathrm{h} ; 3:(90-$

120) $\mathrm{km} / \mathrm{h} ; 4:(120$ - 150) km/h; $5:(150-190) \mathrm{km} / \mathrm{h}]$

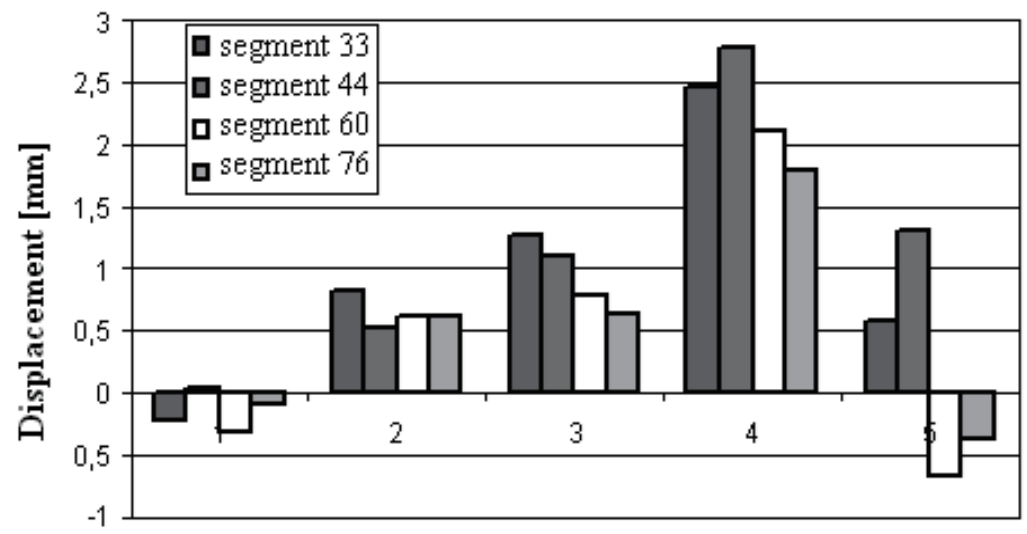

Velocity [1: $(10-50) \mathrm{km} / \mathrm{h} ; 2:(50-90) \mathrm{km} / \mathrm{h} ; 3:(90-$ 120) $\mathrm{km} / \mathrm{h} ; 4:(120$ - 150) km/h; 5: (150 - 190) km/h]

Figure 38. The displacement differences for the breaker angle $20^{\circ}$ (variant 1 - left) and the displacement differences for the breaker angle $27^{\circ}$ (variant 4 - right)

On the basis of these results it is possible to state that the higher the breaker angle the higher the displacement is in both axial and radial directions. In other words, the tire "grows" with rising of the breaker angle.). These results support the highest values of elongation and a relatively high value of strength and elasticity which provide also the so called driving 
comfort. On the other hand, the highest tear resistivity, $300 \%$ modulus and hardness of the depositional rubber mixture on a steel cord provide all together good tire safety (see parameters in Table 1).
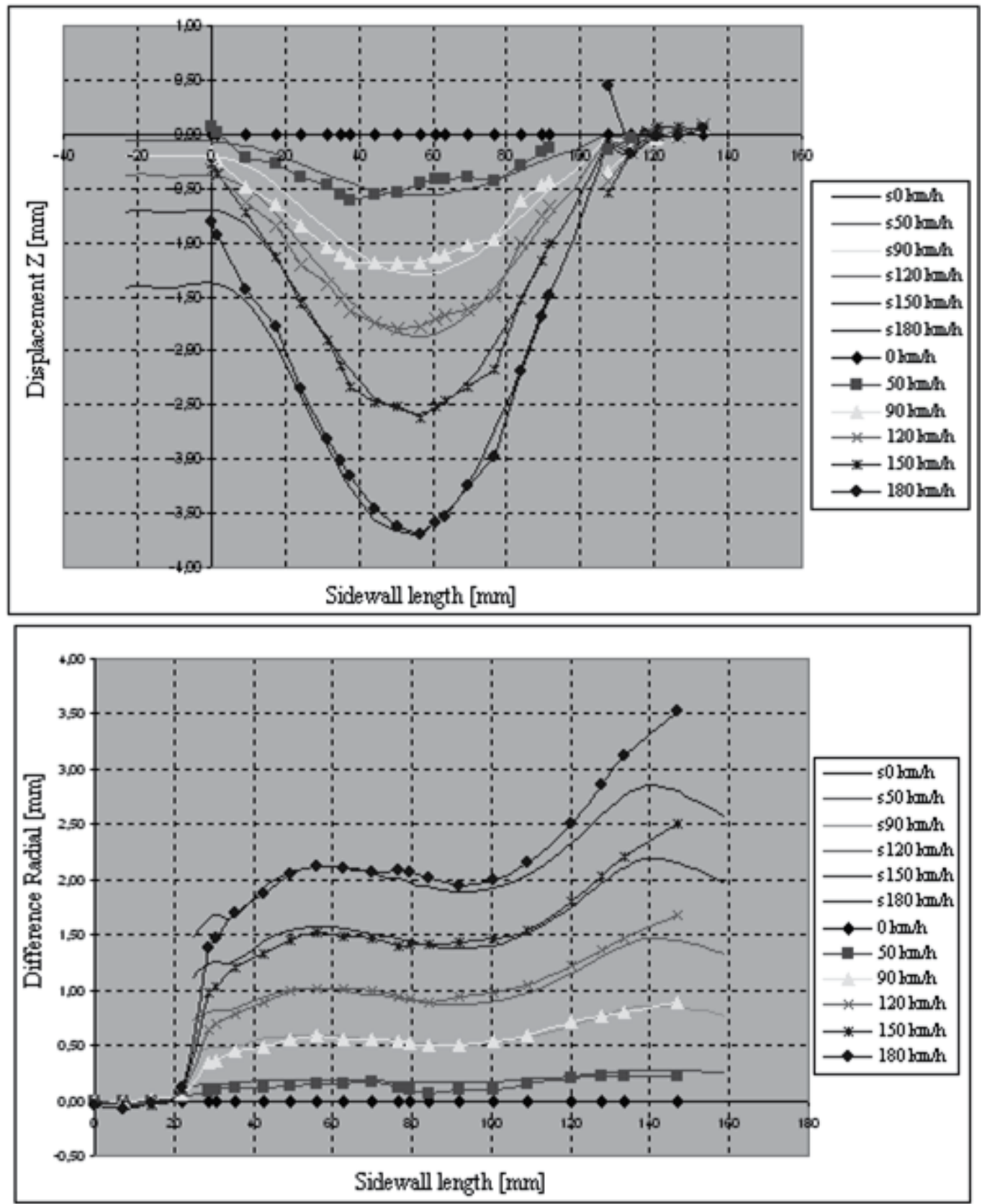

Figure 39. The axial (left) and radial (right) displacement of the sidewall for $20^{\circ}$ breaker angle (s-simulation) 

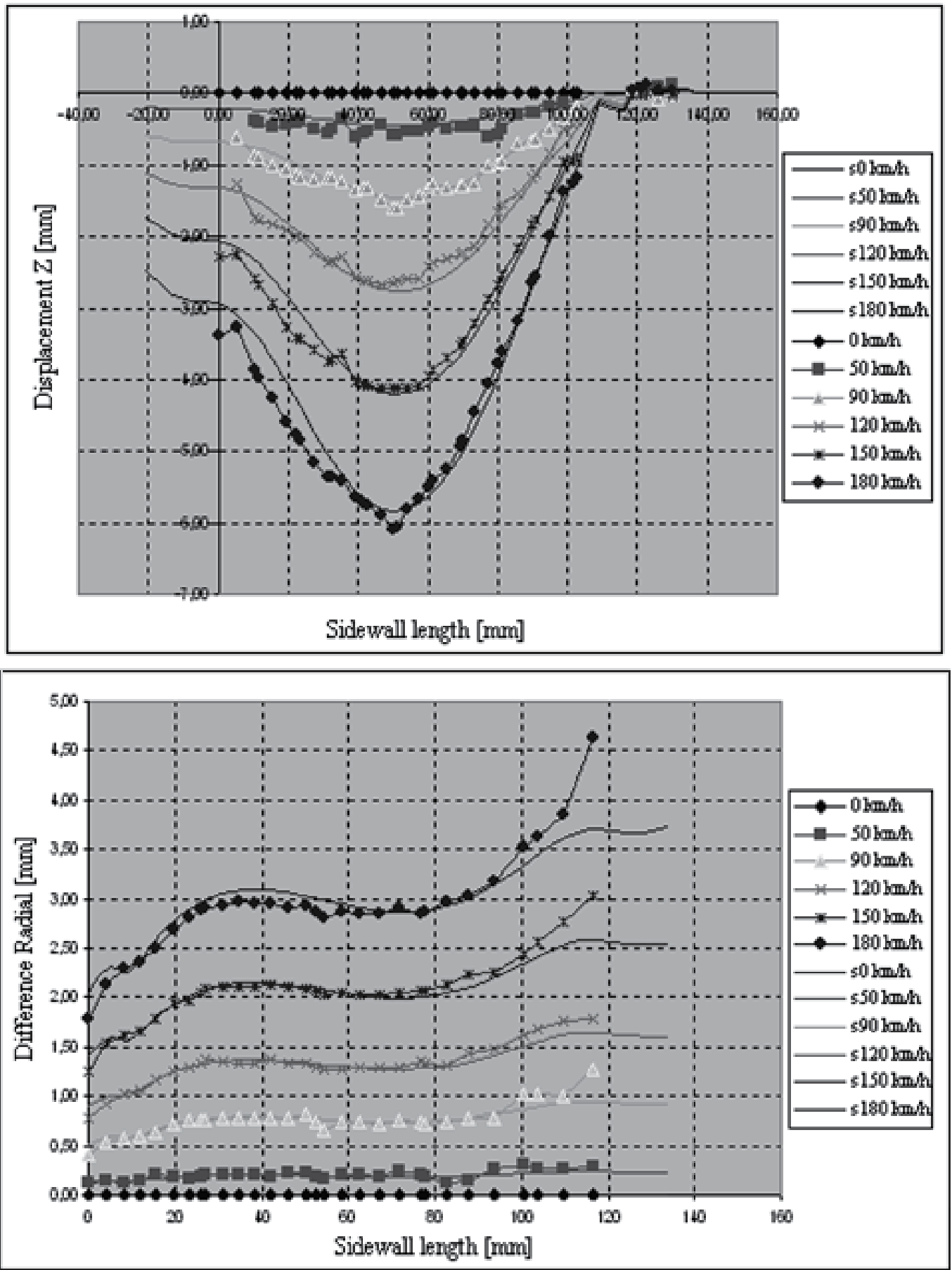

Figure 40. The axial displacement of the sidewall for $27^{\circ}$ breaker angle (s-simulation) 


\section{Conclusion}

The chapter presents the large scale view on the problem of tire safety concluding materials aspects, damage and experimental testing of tires and tire components materials. For more information about the solution of tyres, see reference (Krmela 2008).

\section{Author details}

Pavel Koštial and Ivan Ružiak

VŠB - Technical University of Ostrava, Faculty of Metalurgy and Material Engineering,

Department of Material Engineering, Ostrava, Czech Republic

Jan Krmela

University of Pardubice, Jan Perner Transport Faculty,

Department of Transport Means and Diagnostics, Pardubice, Czech Republic

Karel Frydrýšek

ING-PAED IGIP, VŠB - Technical University of Ostrava, Faculty of Mechanical Engineering,

Department of Mechanics of Materials, Ostrava, Czech Republic

\section{Acknowledgement}

The work has been supported by the Czech grant projects MPO FR-TI3/818 (sponsored by the Ministry of Industry and Trade of the Czech Republic) and by the Slovak-Czech grant project 7AMB12SK126 (sponsored by the Ministry of Education, Youth and Sports of the Czech Republic).

\section{References}

Ferry, J. D. (1980). Viscoelastic properties of Polymers; John Wiley\&Sons, New York, 1980

Jakubíková, Z.; Skalková, P.; Mošková, Z. (2007). Mechanical, Thermal Characterization of low Density Polyethylene (LDPE)/Carboxymethylstarch (CMS) Blends, In: $1^{\text {st }}$ Bratislava Young Polymer Scientists workshop BYPoS. 2007, Bratislava, Slovakia

Jančíková, Z. (2006). Umělé neuronové sítě v materiálovém inženýrství, GEPARTS Ostrava, Czech Republic, 2006, written in Czech language

Jančíková, Z., Švec, P. (2007). Acta Metallurgica Slovana, 2007, 13, 5

Koštial, P., Mokryšová, M., Klabník, M., Žiačik, P.; Kopal, I.; Hutyra, J. (2006). Rubber World. 2006, 233, 4, 18-20

Koštial, P., Mokryšová, M., Kopal, I.; Žiačik, P., Rusnáková, S., Klabník, M. (2005). 12th International metrology congress. 2005, Lyon, Collége Francais de Métrologie, France

Krmela, J. (2008). Systems Approach to the Computational Modelling of Tyres - I. Part, Czech Republic, 2008, pp.1-102, ISBN 978-80-7399-365-8, book written in Czech language

Maier, P., Gand Göritz, D. (1996). Kautschuk Gummi Kunststoffe. 1996, 49, 18 http://ao4.ee.tut.fi/pdlri 
Medalia, A. I. (1978) Rubber Chem. Technol., 1978, 51, 437

Murayama, T. (1978). Elsevier scientific publishing company Amsterdam-Oxford-New York, 1978

Payne, A. R. (1965), Wiley Interscience, New York, 1965

Schaefer, R. J. (1994). Rubber World. 1994, 11, 17

Sepe, M. P. (1998). Plastics Design Library Norwich, New York, USA, 1998

Simek, I. (1987). Fyzika polymérov; SVST, Bratislava, Czechoslovakia, 1987, written in Slovak language

Wang, M. J. (1998). Rubber Chem. and Technol., 1998, 71, 3 


\title{
Friction and Wear of Polymer and Composites
}

\author{
Dewan Muhammad Nuruzzaman and Mohammad Asaduzzaman Chowdhury
}

Additional information is available at the end of the chapter

http://dx.doi.org/10.5772/48246

\section{Introduction}

Polymer and its composites are finding ever increasing usage for numerous industrial applications in sliding/rolling components such as bearings, rollers, seals, gears, cams, wheels, piston rings, transmission belts, grinding mills and clutches where their self lubricating properties are exploited to avoid the need for oil or grease lubrication with its attendant problems of contamination [1]. However, when the contact between sliding pairs is present, there is the problem of friction and wear. Yamaguchi [2], Hooke et al. [3] and Lawrence and Stolarski [4] reported that the friction coefficient can, generally, be reduced and the wear resistance increased by selecting the right material combinations.

Several researchers [5-7] observed that the friction force and wear rate depend on roughness of the rubbing surfaces, relative motion, type of material, temperature, normal force, stick slip, relative humidity, lubrication and vibration. The parameters that dictate the tribological performance of polymer and its composites also include polymer molecular structure, processing and treatment, properties, viscoelastic behavior, surface texture etc. [8-11]. There have been also a number of investigations exploring the influence of test conditions, contact geometry and environment on the friction and wear behavior of polymers and composites. Watanabe [12], Tanaka [13] and Bahadur and Tabor [14] reported that the tribological behavior of polyamide, high density polyethylene (HDPE) and their composites is greatly affected by normal load, sliding speed and temperature. Pihtili and Tosun $[15,16]$ showed that applied load and sliding speed play significant role on the wear behavior of polymer and composites. They also showed that applied load has more effect on the wear than the speed for composites. Several authors [17-22] observed that the friction coefficient of polymers and its composites rubbing against metals decreases with the increase in load though some other researchers have different views. Stuart [23] and other researchers [2426] showed that value of friction coefficient increases with the increase in load. Friction coefficient and specific wear rate values for different combinations of polymer and its composite were obtained and compared [27]. For all material combinations, it was observed 
that the coefficient of friction decreases linearly with the increase in applied pressure values. Unal et al. $[28,29]$ reported that the applied load exerts greater influence on the sliding wear of polymer and its composite than the sliding velocity.

Friction and wear behavior of glass fiber reinforced polyster composite were studied and results showed that in general, friction and wear are strongly influenced by all the test parameters such as applied load, sliding speed, sliding distance and fiber orientations [30]. Moreover, it was found that applied normal load, sliding speed and fiber orientations have more pronounced effect on wear rate than sliding distance. Wang and $\mathrm{Li}$ [31] observed that the sliding velocity has more significant effect on the sliding wear as compared to the applied load and variations of wear rate with operating time can be distinguished by three distinct periods. These periods are running-in period, steady-state period and severe wear period, respectively. Tsukizoe and Ohmae [32] showed that reinforcement of fiber or filler significantly improve the tribological behavior of polymeric material but this is not necessarily true for all cases. Suresha et al. [33] showed that there is a strong interdependence on the friction coefficient and wear loss with respect to the applied loads for steel-composites contact.

Friction process with vibration is an important practical phenomenon because the influence of vibration can cause significant change in this process. It is known that vibration and friction are dependent on each other. Friction generates vibration in various forms, while vibration affects friction in turns. Some explanations [34-38] are given in order to justify the decrease in the friction coefficient under vibration condition though some of the researchers have different views. Skare and Stahl [39] claimed that mean friction force increases as well as decreases depending on the vibration parameters.

Friction may be increased or decreased depending on the sliding pairs and operating parameters. In this chapter, friction coefficient and wear rate of different types of polymer and composite materials sliding against steel counterface are described. Effects of duration of rubbing, normal load, sliding speed, vertical vibration, horizontal vibration, natural frequency of vibration on friction coefficient are discussed. Some correlations of friction coefficient and wear rate are also incorporated in this chapter.

\section{Effect of duration of rubbing on friction coefficient}

In sliding contacts, friction coefficient varies with duration of rubbing and these variations are different at different normal loads and sliding velocities. Research works were carried out to investigate the friction coefficient with duration of rubbing for different types of composite and polymer materials. Figure 1 shows the variation of friction coefficient with the duration of rubbing at different normal loads for gear fiber [40]. For normal load $10 \mathrm{~N}$, curve 1 shows that during initial stage of rubbing, friction coefficient is low which remains constant for few minutes then increases very steadily up to a maximum value over a certain duration of rubbing and after that it remains constant for the rest of the experimental time [40].

At initial stage of rubbing, friction force is low due to contact between superficial layer of pin and disc and then, friction coefficient increases due to ploughing effect which causes 


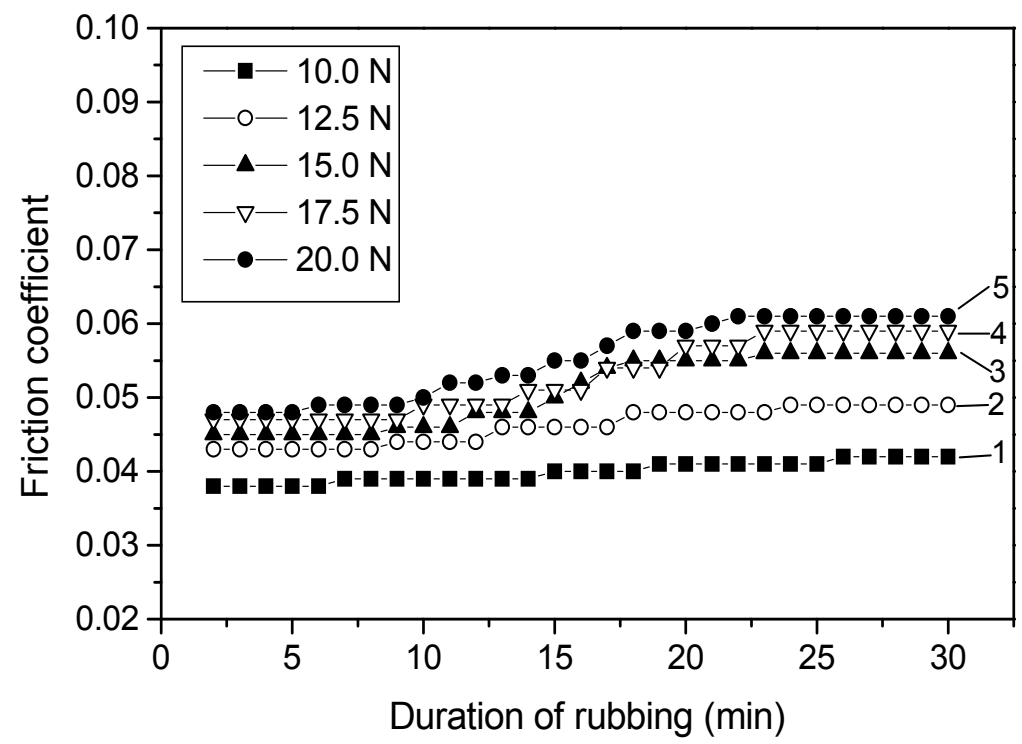

Figure 1. Friction coefficient as a function of duration of rubbing at different normal load, sliding velocity: $1 \mathrm{~m} / \mathrm{s}$, relative humidity: $70 \%$, test sample: gear fiber.

roughening of the test disc surface. For normal load $12.5 \mathrm{~N}$, curve 2 shows similar trend as that of curve 1. For normal loads 15, 17.5 and $20 \mathrm{~N}$, curves 3, 4 and 5 show the friction results respectively. The increase in friction coefficient with the increase in normal load is due to the detachment and removal of worn materials and more contact with reinforced cloth fibers and the higher the normal load, time to reach constant friction is less [40]. This is due to the fact that the surface roughness and other parameters attain a steady level at shorter period with the increase in normal load [40].

Figure 2 shows the effect of the duration of rubbing on the value of friction coefficient at different normal loads for glass fiber. For normal load $10 \mathrm{~N}$, curve 1 shows that during initial stage of rubbing, friction coefficient rises for few minutes and then decreases very steadily up to a certain value over some duration of rubbing and then it becomes steady for the rest of the experimental time. Almost similar trends of variation are observed for loads $12.5,15,17.5$ and $20 \mathrm{~N}$ respectively and these results show that friction coefficient decreases with the increase in applied load [40]. It is known that tribological behavior of polymers and polymer composites can be associated with their viscoelastic and temperature-related properties. Sliding contact of two materials results in heat generation at the asperities and hence increases in temperature at the frictional surfaces of the two materials which influences the viscoelastic property in the response of materials stress, adhesion and transferring behaviors [27]. From these results, it can also be seen that time to reach constant friction is different for different normal loads and higher the normal load, glass fiber takes less time to stabilize [40]. Figure 3 shows the variation of friction coefficient with the duration of rubbing at different normal loads for nylon. For $10 \mathrm{~N}$ load, curve 1 indicates that during starting of the rubbing, the value of friction coefficient is low which increases for few minutes to a certain value and then decreases almost linearly over some duration of 


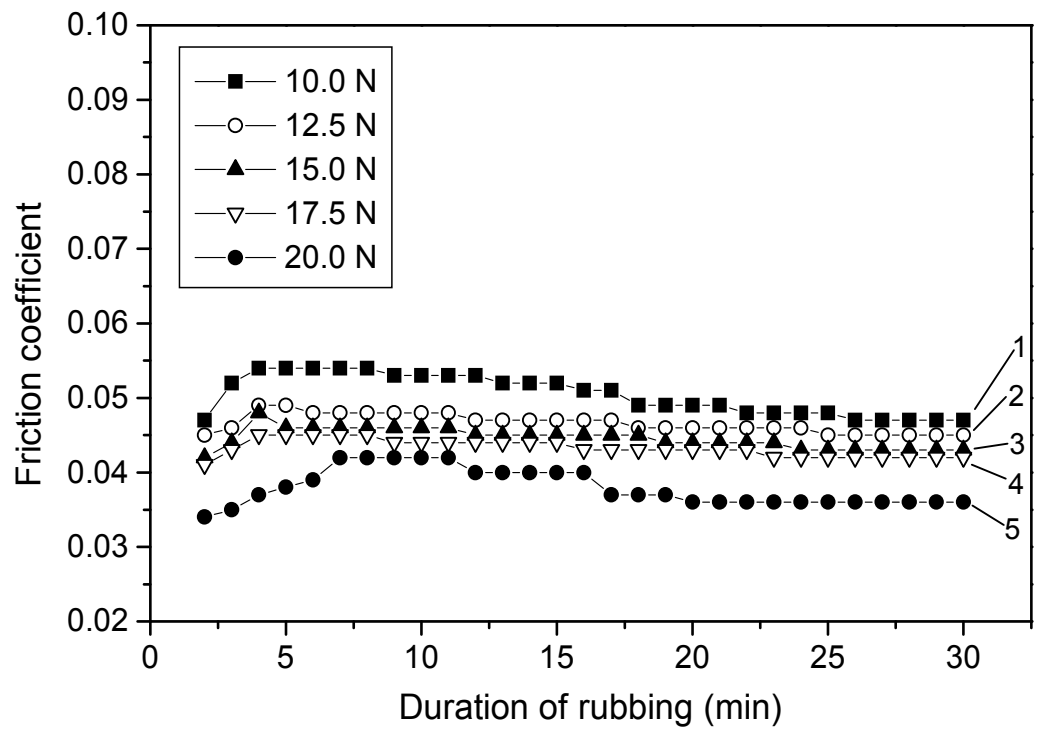

Figure 2. Friction coefficient as a function of duration of rubbing at different normal load, sliding velocity: $1 \mathrm{~m} / \mathrm{s}$, relative humidity: $70 \%$, test sample: glass fiber.

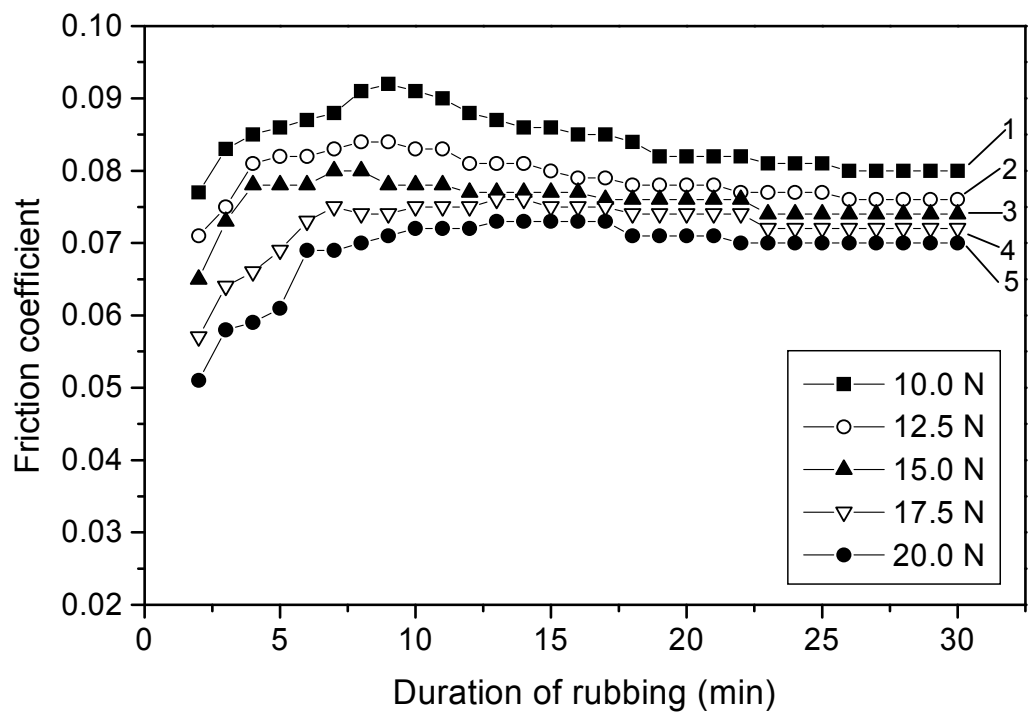

Figure 3. Friction coefficient as a function of duration of rubbing at different normal load, sliding velocity: $1 \mathrm{~m} / \mathrm{s}$, relative humidity: $70 \%$, test sample: nylon.

rubbing and after that it remains constant for the rest of the experimental time. Similar trends of variation are observed for normal loads 12.5, 15, 17.5 and $20 \mathrm{~N}$. In these cases, transfer film formed on the stainless steel couterface and the transfer film has important effects on the tribological behavior of a material [22, 40-42]. Friction and wear behavior of polymer sliding against a metal is strongly influenced by its ability to form a transfer film on the counterface [42]. The transfer film formed on a non-polymer counterface is controlled by the counterface material, roughness, and sliding conditions [2]. 


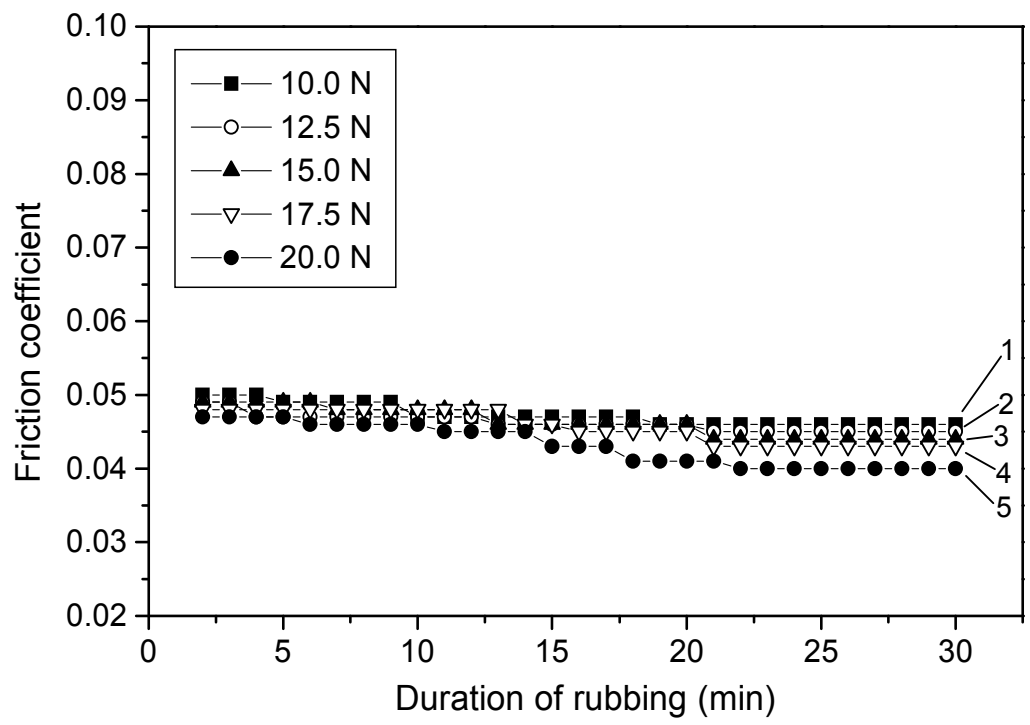

Figure 4. Friction coefficient as a function of duration of rubbing at different normal load, sliding velocity: $1 \mathrm{~m} / \mathrm{s}$, relative humidity: $70 \%$, test sample: PTFE.

Figure 4 for PTFE shows that friction coefficient decreases almost linearly up to certain value over some duration of rubbing and after that it remains constant for the rest of the experimental time. It can be noted that transfer film of PTFE formed on the steel counterface due to the strong adhesion across the interface [40,43].

Friction coefficient varies with duration of rubbing at different sliding speeds for different composite and polymer materials [44]. These results are presented in Figs. 5-8.

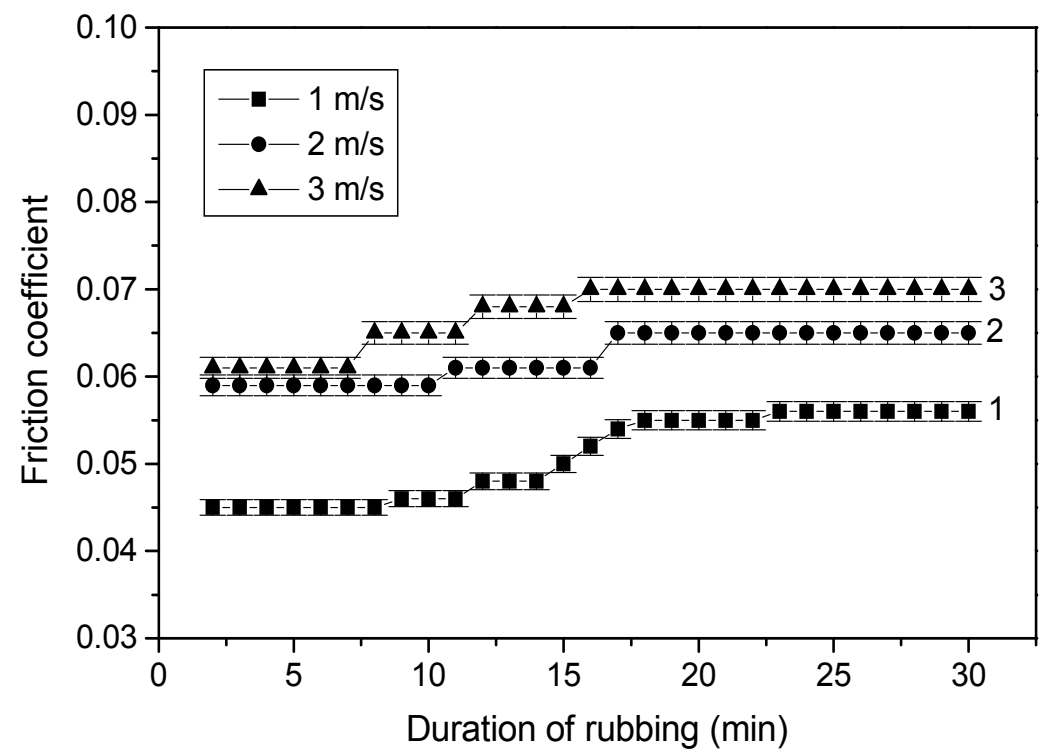

Figure 5. Friction coefficient as a function of duration of rubbing at different sliding speeds, normal load: $15 \mathrm{~N}$, relative humidity: $70 \%$, test sample: gear fiber. 


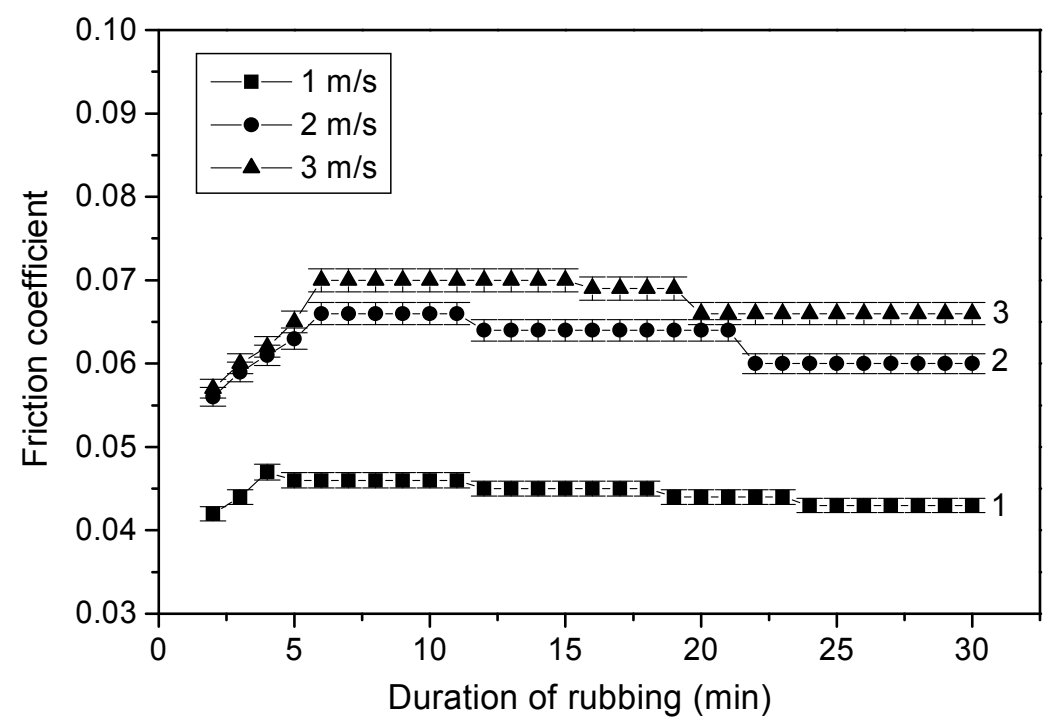

Figure 6. Figure 6. Friction coefficient as a function of duration of rubbing at different sliding speeds, normal load: $15 \mathrm{~N}$, relative humidity: $70 \%$, test sample: glass fiber.

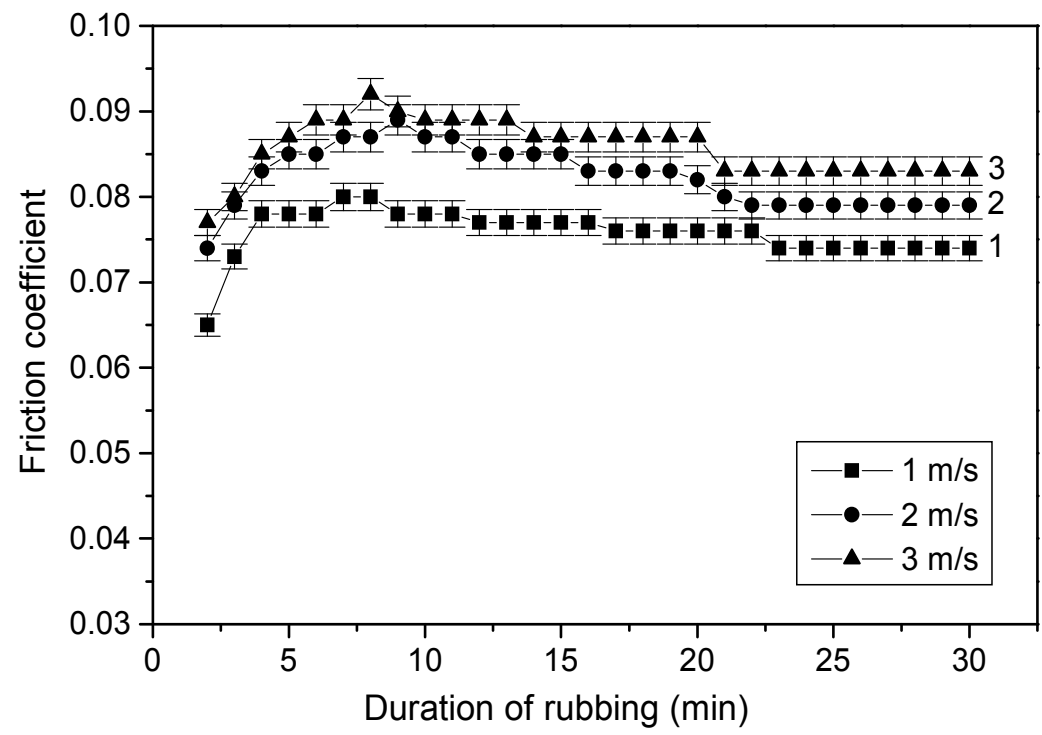

Figure 7. Friction coefficient as a function of duration of rubbing at different sliding speeds, normal load: $15 \mathrm{~N}$, relative humidity: $70 \%$, test sample: nylon. 


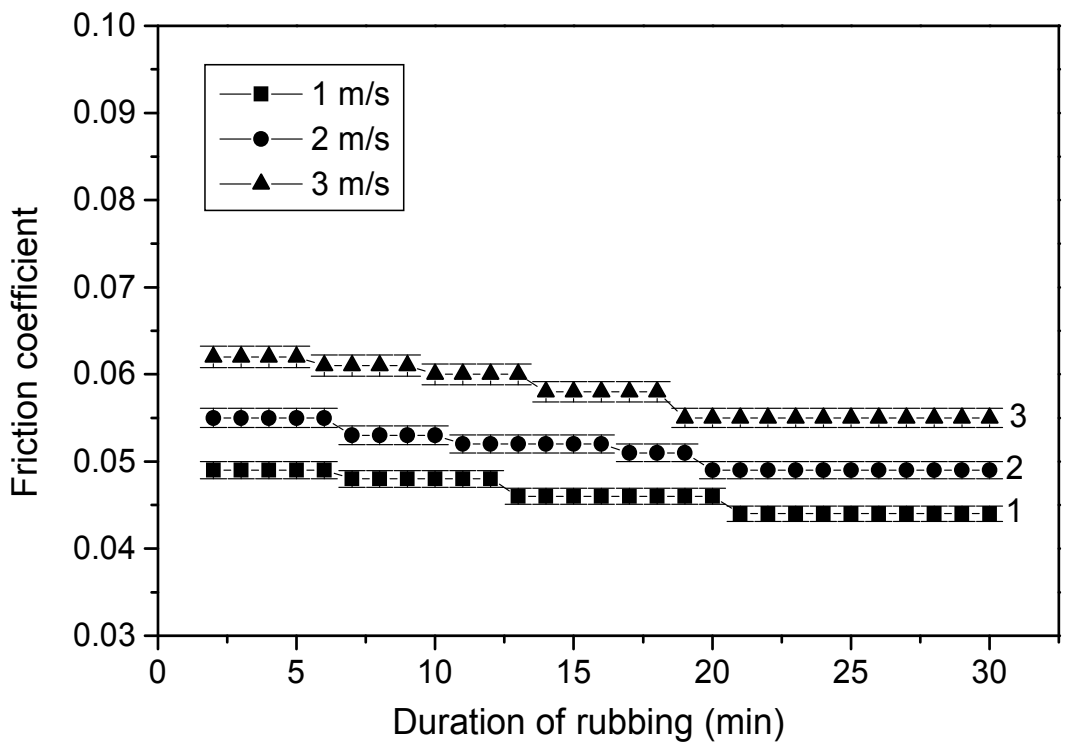

Figure 8. Friction coefficient as a function of duration of rubbing at different sliding speeds, normal load: $15 \mathrm{~N}$, relative humidity: $70 \%$, test sample: PTFE.

\section{Effect of normal load on friction coefficient and wear rate}

In this section, a comparison of the variation of friction coefficient with normal load for different materials has been discussed. Wear rates of different materials are also compared. Results of Fig. 9 show that friction coefficient decreases with the increase in normal load for glass fiber, PTFE and nylon. Different behavior is observed for gear fiber that is, friction coefficient of gear fiber increases with the increase in normal load. Some factors such as high ploughing, surface damage and breakage of reinforced fibers are responsible for higher friction with higher normal load [40]. Variations of wear rate with normal load for gear fiber, glass fiber, nylon and PTFE are shown in Fig. 10. This figure indicates that wear rate increases with the increase in normal load for all types of materials investigated. The shear force and frictional thrust are increased with the increase in applied load and these increments accelerate the wear rate. Figure 10 also shows the comparison of the variation of wear rate with normal load for gear fiber, glass fiber, nylon and PTFE. The highest values of wear rate for PTFE and lowest values for nylon are obtained among these materials. In case of composite materials, the values of wear rate are higher for gear fiber compared to that of glass fiber. For plastic materials, higher values are observed for PTFE compared to nylon [40]. 


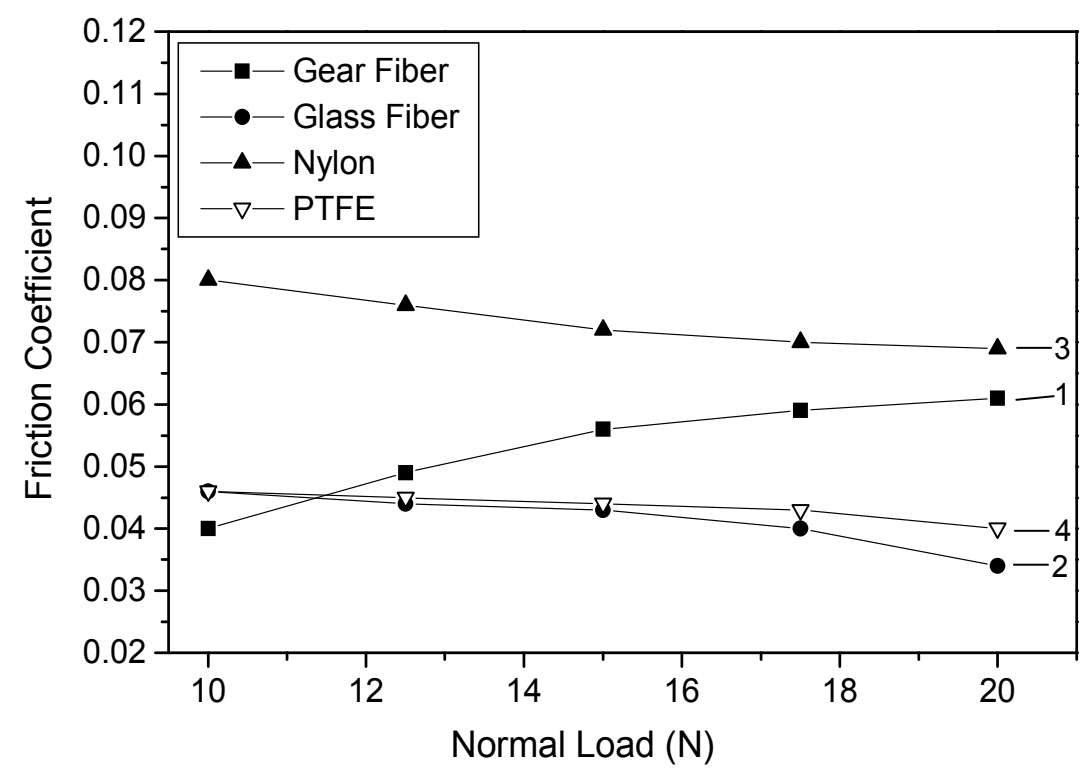

Figure 9. Friction coefficient as a function of normal load for different materials, sliding velocity: $1 \mathrm{~m} / \mathrm{s}$, relative humidity: $70 \%$.

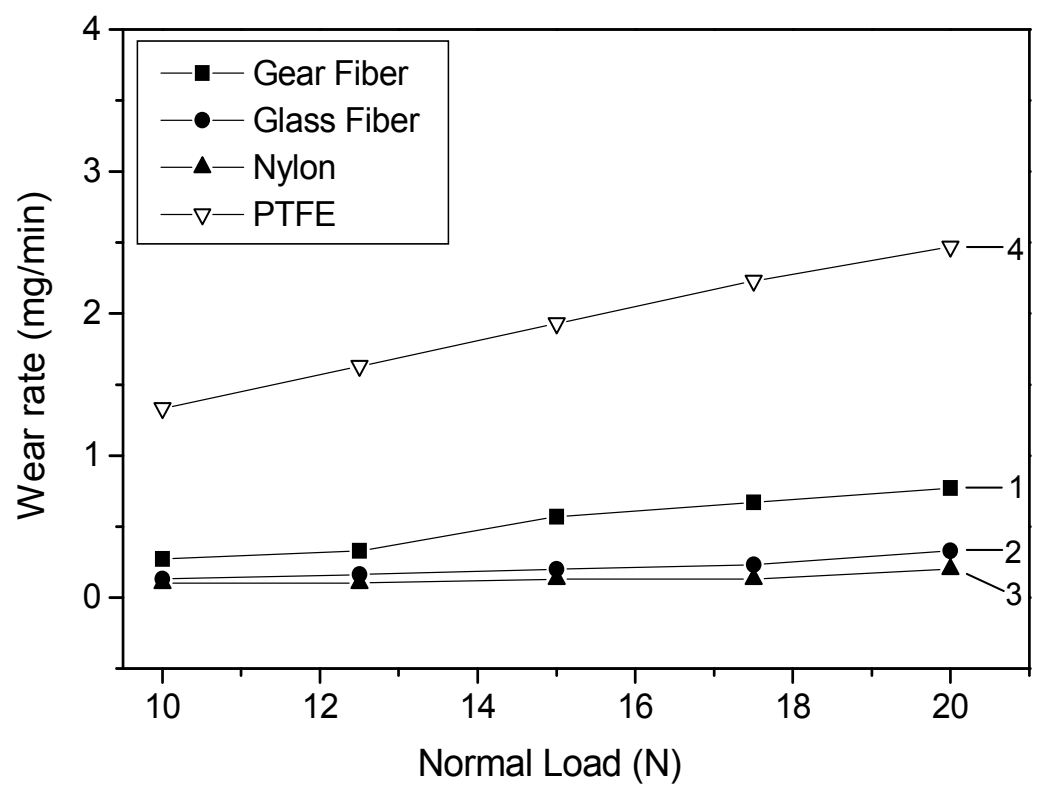

Figure 10. Wear rate as a function of normal load for different materials, sliding velocity: $1 \mathrm{~m} / \mathrm{s}$, relative humidity: 70\%. 


\section{Effect of sliding speed on friction coefficient and wear rate}

In sliding contacts, sliding speed has an important role on friction and wear of different polymer and composite materials. Figure 11 shows the comparison of the variation of friction coefficient with sliding speed for different materials. Results show that friction coefficient increase almost linearly with sliding speed [44]. These findings are in agreement with the findings of Mimaroglu et al. [27] and Unal et al. [45]. With the increase in sliding speed, the frictional heat may decrease the strength of the materials and high temperature results in stronger or increased adhesion with pin [27,43]. The increase of friction coefficient with sliding speed can be explained by the more adhesion of counterface pin material on disc. Figure 11 indicates that nylon shows the highest friction coefficient within the observed range of sliding speed. Results also reveal that PTFE shows the lowest friction coefficient among these four materials except at sliding speed $1 \mathrm{~m} / \mathrm{s}$. At sliding speed $1 \mathrm{~m} / \mathrm{s}$, glass fiber shows slightly lower friction coefficient than PTFE but at a sliding speed $3 \mathrm{~m} / \mathrm{s}$, glass fiber exhibits much higher friction coefficient than PTFE. This may be due to the breakage of reinforced glass fibers with the increase in sliding speed. Results also show that friction coefficient of gear fiber is higher than that of glass fiber and PTFE. This is due to ploughing effect and breakage of the exposed reinforced cloth fiber of the fracture material [44]. Variations of wear rate with sliding speed for gear fiber, glass fiber, nylon and PTFE are presented in Fig. 12. This figure shows that wear rate increases with the increase in sliding speed for all types of materials investigated. These findings are in agreement with the findings of Mimaroglu et al. [27] and Suresha et al. [33]. The shear force, frictional heat and

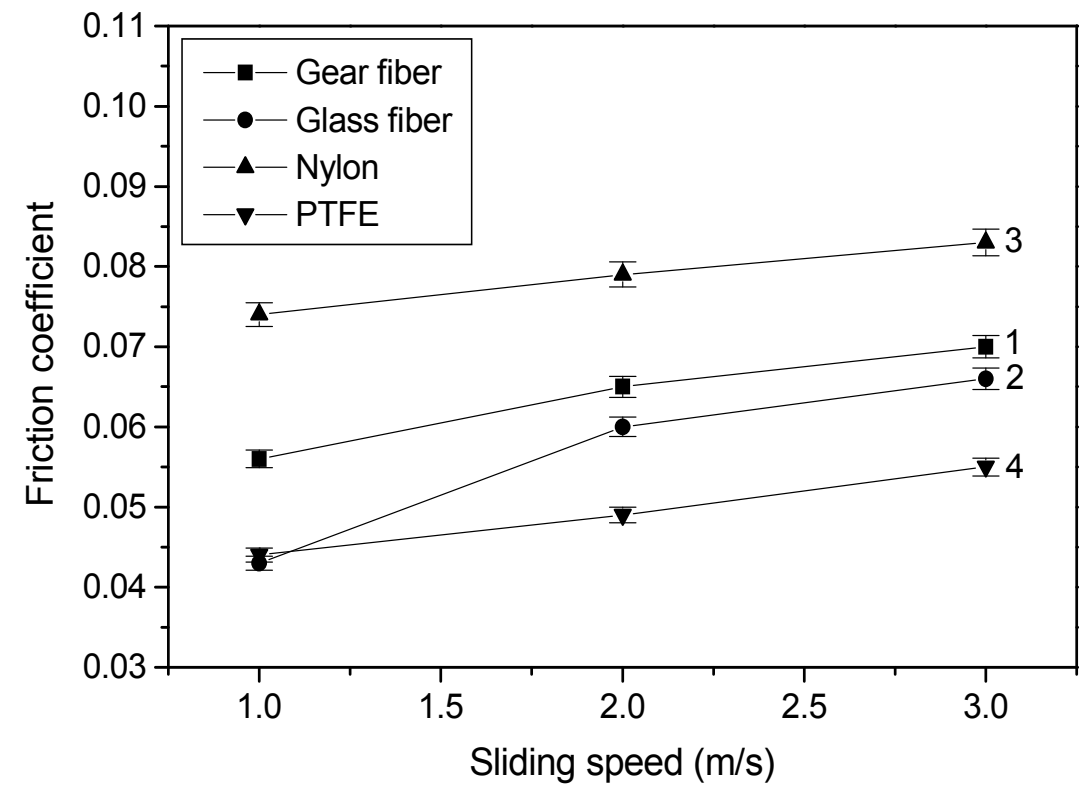

Figure 11. Friction coefficient as a function of sliding speed for different materials, normal load: $15 \mathrm{~N}$, relative humidity: $70 \%$. 


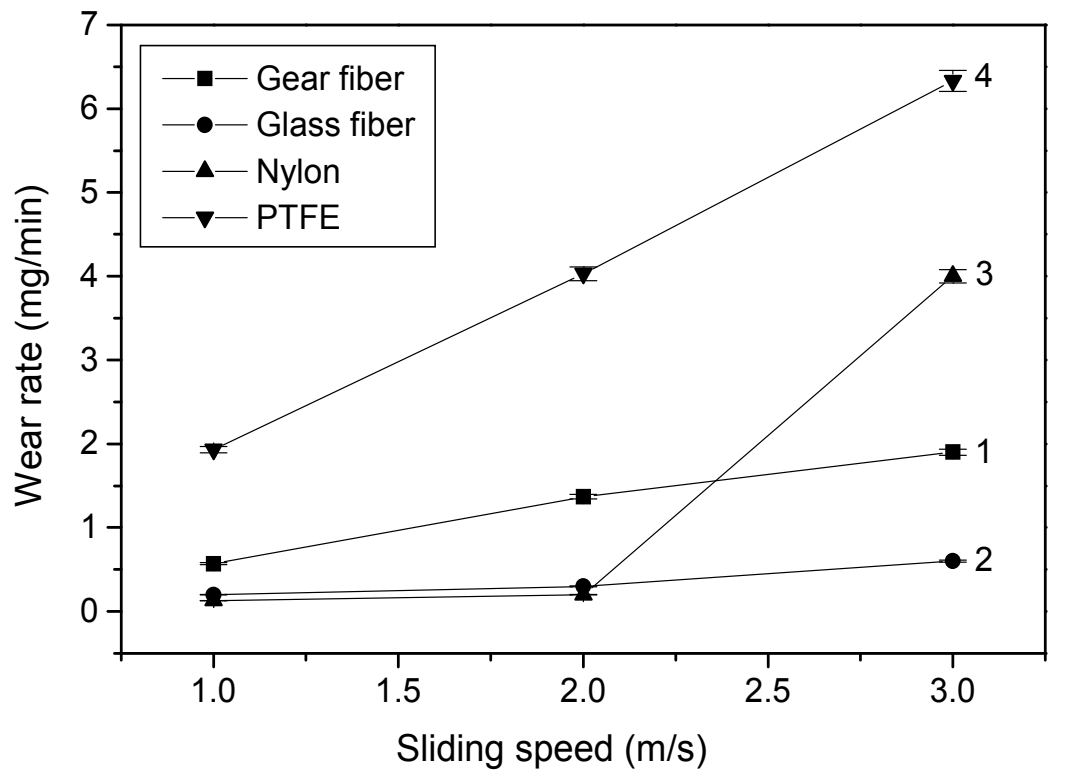

Figure 12. Wear rate as a function of sliding speed for different materials, normal load: $15 \mathrm{~N}$, relative humidity: $70 \%$.

frictional thrust are increased with the increase in sliding speed and these increments accelerate the wear rate. Figure 12 also shows the comparison of the variation of wear rate with sliding speed for gear fiber, glass fiber, nylon and PTFE. From this figure it is observed that PTFE has the highest wear rate among these four materials within the observed range of sliding speed. It is also observed that nylon has the lowest wear rate among these four materials except at sliding speed $3 \mathrm{~m} / \mathrm{s}$. At sliding speed $3 \mathrm{~m} / \mathrm{s}$, wear rate of nylon is higher than that of gear fiber and glass fiber. Because of higher sliding speed, loss of strength is higher for nylon [43] compared to that of other materials. In case of composite materials, the values of wear rate are higher for gear fiber compared to that of glass fiber [44].

\section{Effect of vertical vibration}

Figure 13 shows the pin-on-disc set-up with vertical vibration arrangement [46]. For generating vertical vibration, there are two circular plates near the bottom end of the shaft. The upper circular plate fitted with the bottom end of the shaft has a spherical ball in such a way that the ball is extended from the lower surface of this plate. On the top surface of the lower circular plate there are a number of slots. When the shaft rotates, the ball of the upper circular plate slides on the slotted surface of the lower circular plate and due to the spring action, the shaft along with the rotating plate vibrates. The direction of vibration is vertical, i.e. perpendicular to the sliding direction of the pin. By varying the shaft rotation and the number of slots of the lower circular vibration generating plate, the frequency of vibration is varied. By adjusting the height of this slotted plate, the amplitude of the vibration is varied. 


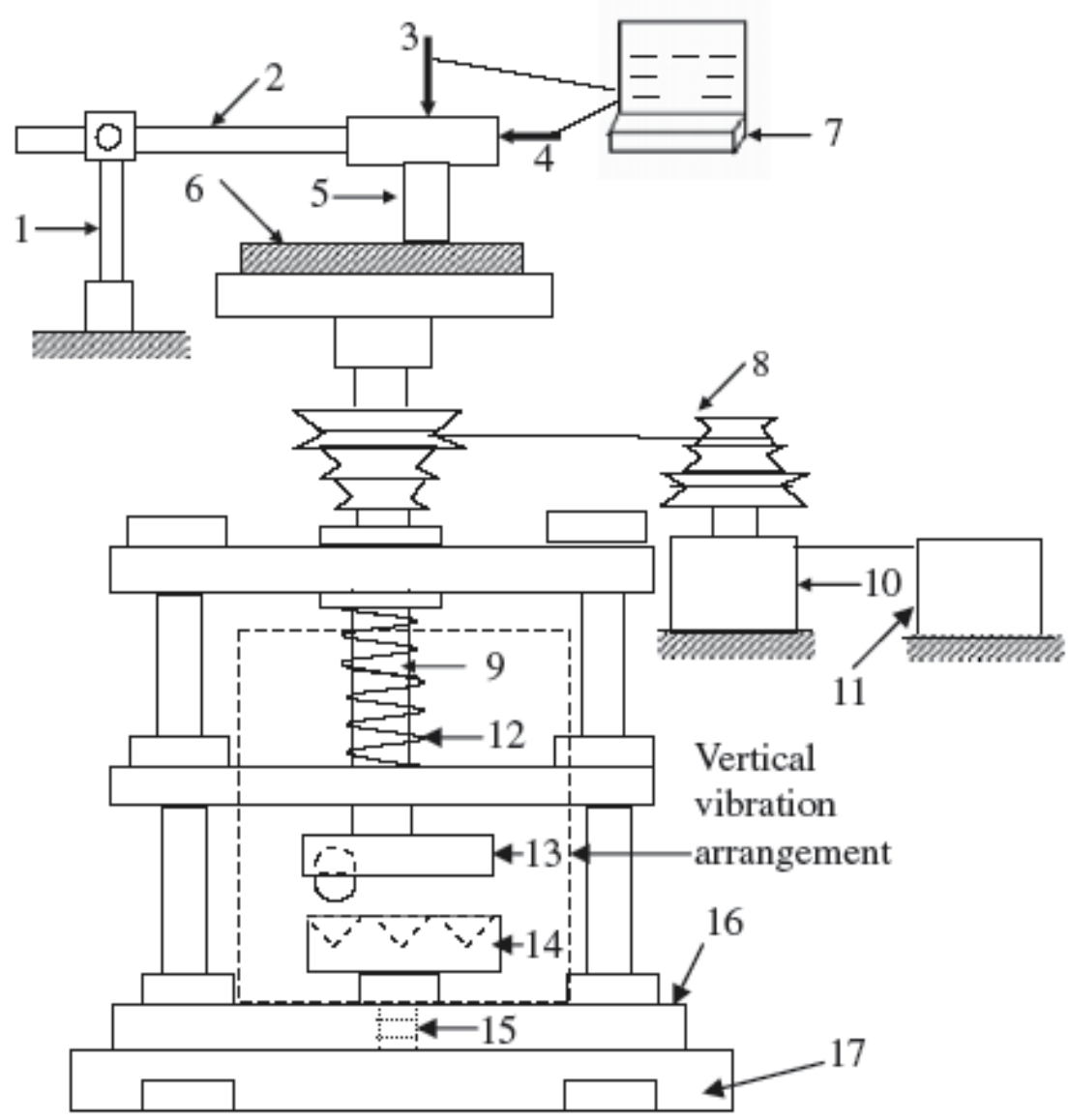

Figure 13. Schematic diagram of the experimental set-up for vertical vibration (1) Load arm holder (2) Load arm (3) Normal load (4) Horizontal load (5) Pin sample (6) Test disc with rotating table (7) Computer (8) Belt and pulley (9) Main shaft (10) Motor (11) Speed control unit (12) Compression spring (13) Upper plate with ball (14) Lower plate with V-slots (15) Height adjustable screw (16) Base plate (17) Rubber block

\subsection{Friction coefficient with duration of rubbing at different amplitudes of vibration}

The presence of external vertical vibration affects the friction force of different materials considerably which is discussed in this section. Figures 14-17 show the variation of friction coefficient with the duration of rubbing and amplitude of vibration for different types of material. Variations of friction coefficient with time of rubbing for glass fiber reinforced plastic (glass fiber), cloth reinforced ebonite (gear fiber), PTFE and rubber are presented in Figs. 14-17, respectively. These figures indicate that time to reach steady-state value is different for different materials. 
Research works carried out for different materials at different frequencies of vibration and these results show that time to reach constant friction is same for these materials [46]. Results also reveal that friction coefficient decrease with the increase in amplitude of vibration. This is due to the fact that the greater the amplitude of vibration, the higher the

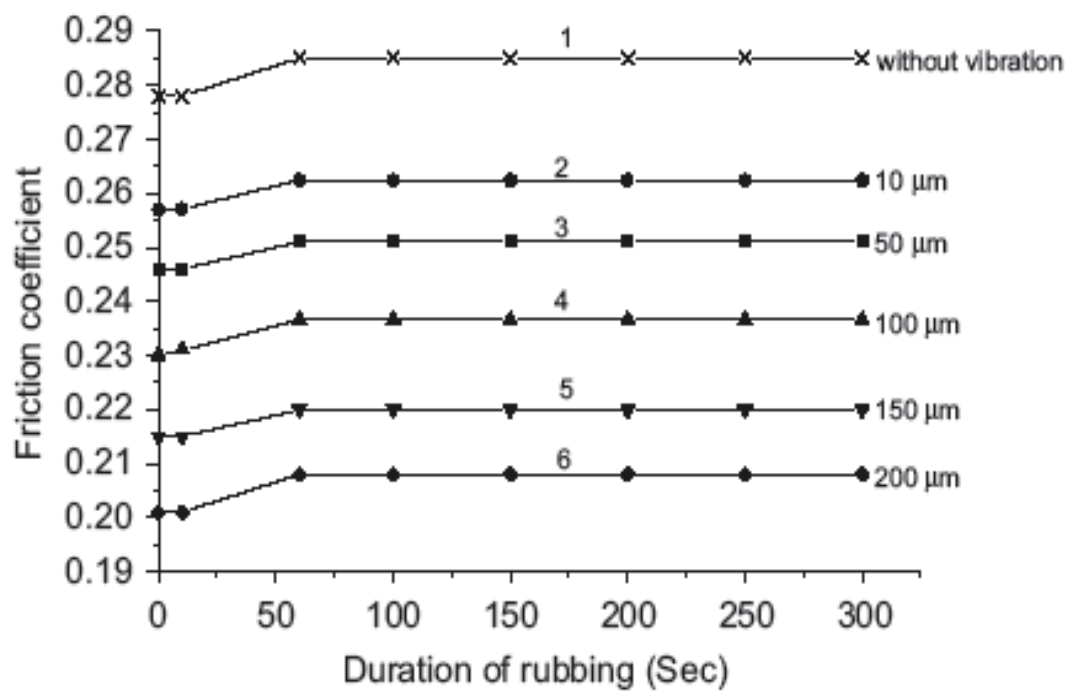

Figure 14. Variation of friction coefficient with the variation of duration of rubbing at different amplitude of vibration (sliding velocity: $0.785 \mathrm{~m} / \mathrm{s}$, normal load: $10 \mathrm{~N}$, frequency of vibration: $500 \mathrm{~Hz}$, roughness: $0.2 \mu \mathrm{m}$ (RMS), relative humidity: $50 \%$, test sample: glass fiber reinforced plastic).

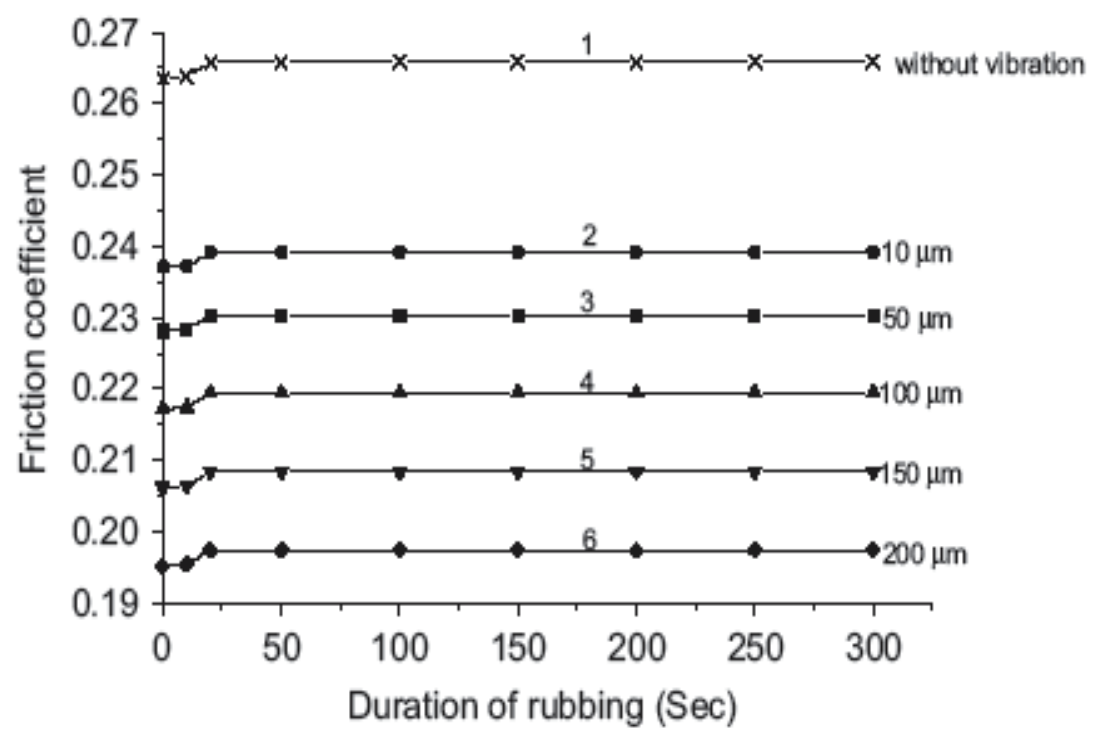

Figure 15. Variation of friction coefficient with the variation of duration of rubbing at different amplitude of vibration (sliding velocity: $0.785 \mathrm{~m} / \mathrm{s}$, normal load: $10 \mathrm{~N}$, frequency of vibration: $500 \mathrm{~Hz}$, roughness: $0.2 \mu \mathrm{m}$ (RMS), relative humidity: $50 \%$, test sample: cloth reinforced ebonite). 


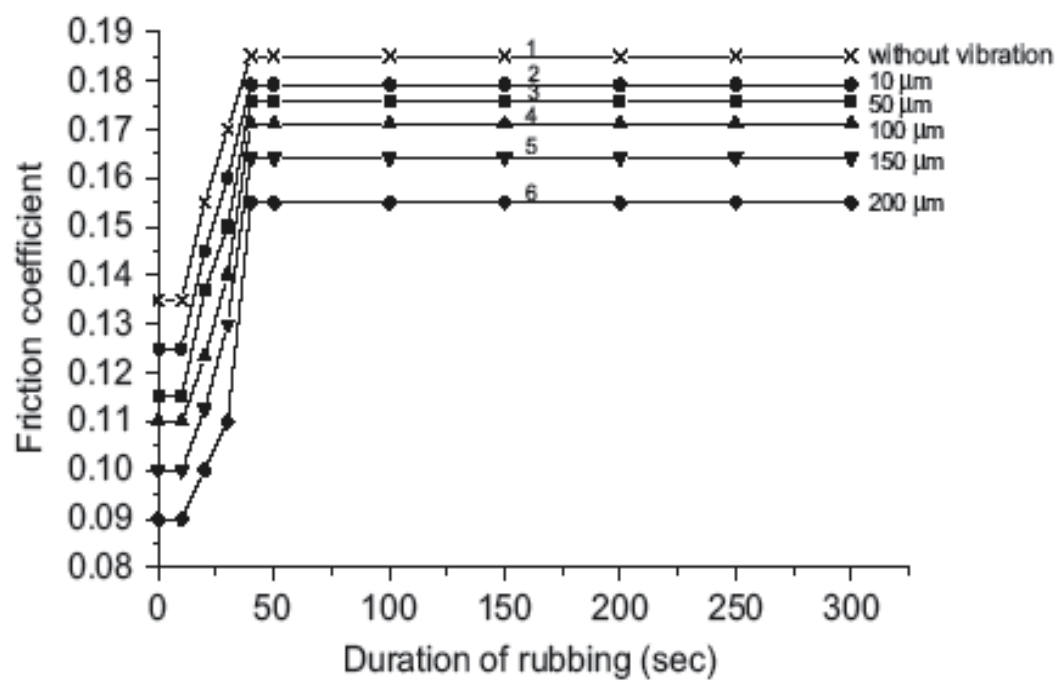

Figure 16. Variation of friction coefficient with the variation of duration of rubbing at different amplitude of vibration (sliding velocity: $0.785 \mathrm{~m} / \mathrm{s}$, normal load: $10 \mathrm{~N}$, frequency of vibration: $150 \mathrm{~Hz}$, roughness: $1.5 \mu \mathrm{m}$ (RMS), relative humidity: $50 \%$, test sample: PTFE).

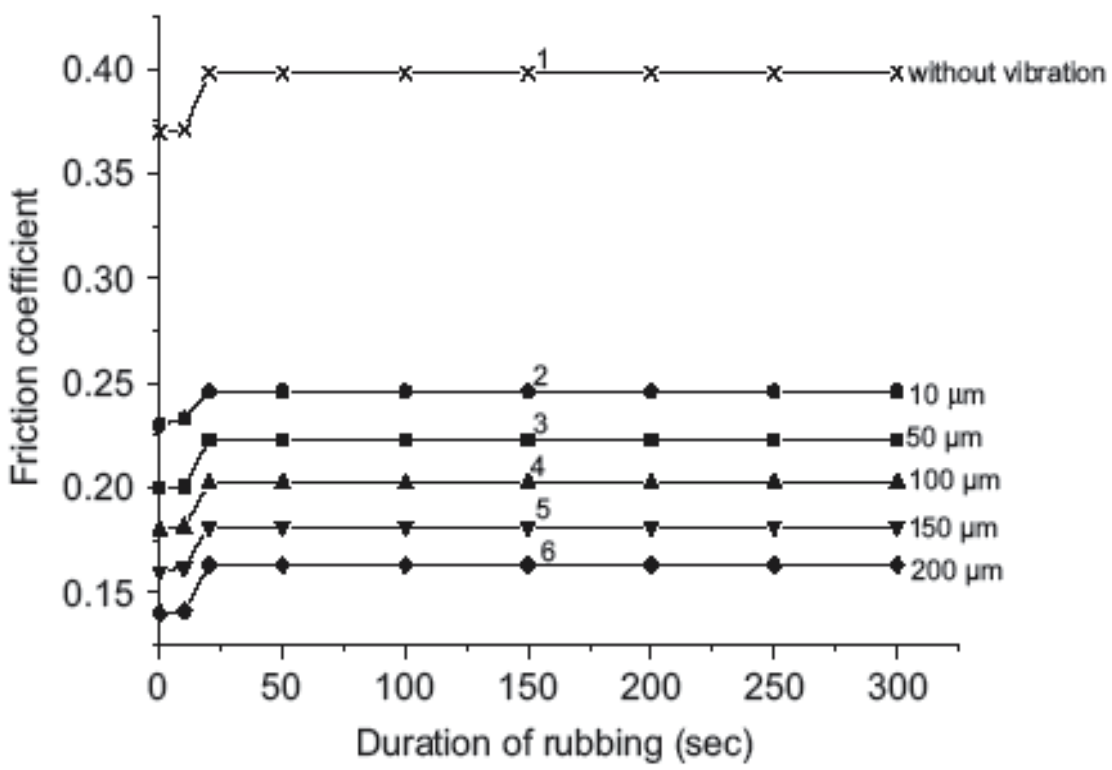

Figure 17. Variation of friction coefficient with the variation of duration of rubbing at different amplitude of vibration (sliding velocity: $0.0393 \mathrm{~m} / \mathrm{s}$, normal load: $10 \mathrm{~N}$, frequency of vibration: $50 \mathrm{~Hz}$, roughness: $1.5 \mu \mathrm{m}$ (RMS), relative humidity: 50\%, test sample: rubber).

actual rubbing time, because there is always more separation between the rubbing surfaces due to reduction in the mean contact area of the two sliding objects for vibration [38]. Therefore, the reduction of friction coefficient for the increase in amplitude of vibration is 
due to the separation of contact surfaces as the higher the amplitude the higher the separation of rubbing surfaces. In fact the higher the separation, the higher the time of contact between the rubbing surfaces is required. As the amplitude increases, keeping the frequency of vibration constant, the acceleration of vibration will also increase that might cause momentary vertical load reduction, which causes the reduction of effective normal force resulting reduction of friction coefficient with the increase of amplitude of vibration. The factors responsible for this momentarily load reduction are: (i) superposition of static and dynamic force generated during vibration, (ii) reversal of the friction vector, (iii) local transformation of vibration energy into heat energy, and (iv) approaching excitation frequency to resonance frequency, etc.

\subsection{Friction coefficient at different amplitudes and frequencies of vibration}

Amplitude and frequency of vibration have a major role on friction coefficient which is discussed in this section. The effects of amplitude of vibration on the friction coefficient at different frequencies for different materials are shown in Figs. 18-21. Results represent that friction coefficient decreases with the increase in amplitude of vibration at different frequencies of vibration at different rates for different materials. This decrease in friction coefficient is nearly linear for glass fiber, gear fiber and rubber but that for PTFE is nonlinear and its rate is increasing with higher amplitude of vibration [46]. Results also reveal that friction coefficient decreases with the increase in frequency of vibration. These findings are in agreement the findings for mild steel [7].

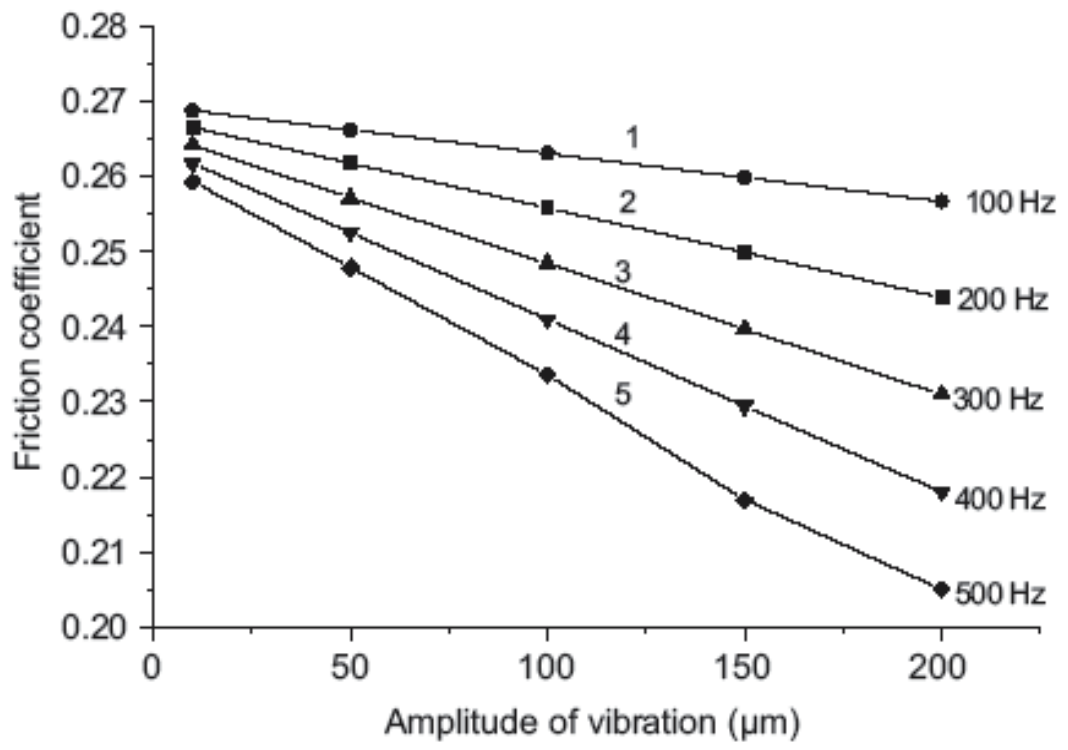

Figure 18. Variation of friction coefficient with the variation of amplitude of vibration at different frequency of vibration (sliding velocity: $1.17 \mathrm{~m} / \mathrm{s}$, normal load: $10 \mathrm{~N}$, roughness: $0.2 \mu \mathrm{m}$ (RMS), relative humidity: 50\%, test sample: glass fiber reinforced plastic). 


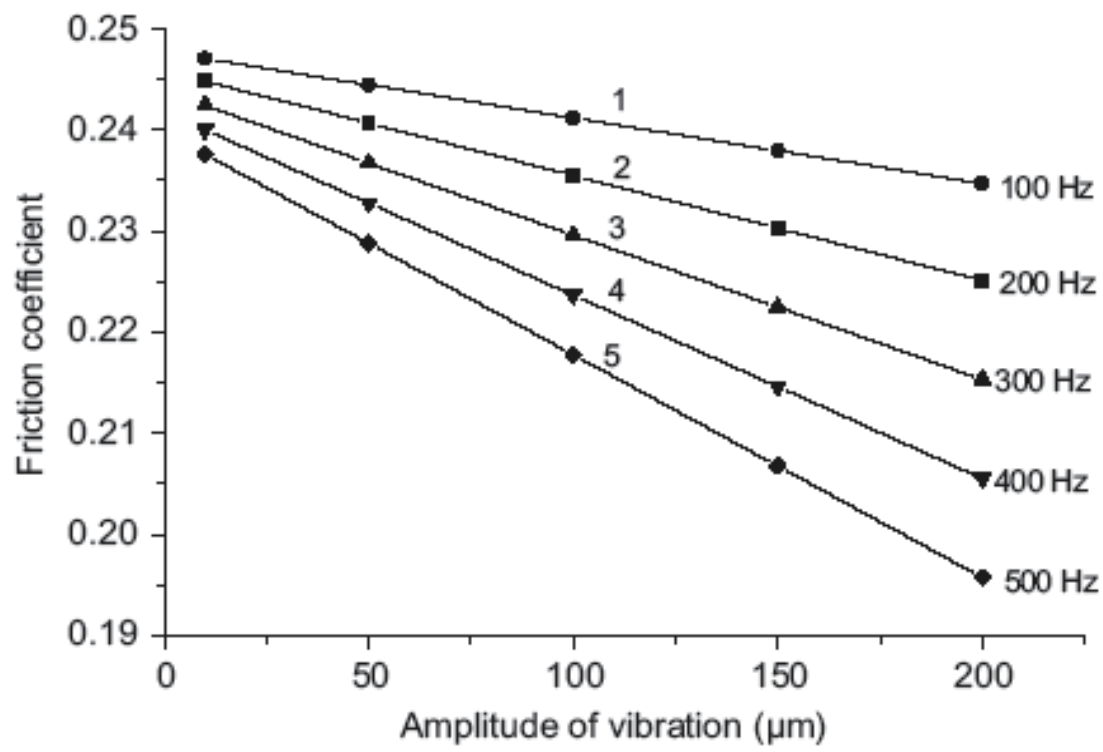

Figure 19. Variation of friction coefficient with the variation of amplitude of vibration at different frequency of vibration (sliding velocity: $1.17 \mathrm{~m} / \mathrm{s}$, normal load: $10 \mathrm{~N}$, roughness: $0.2 \mu \mathrm{m}$ (RMS), relative humidity: $50 \%$, test sample: cloth reinforced ebonite).

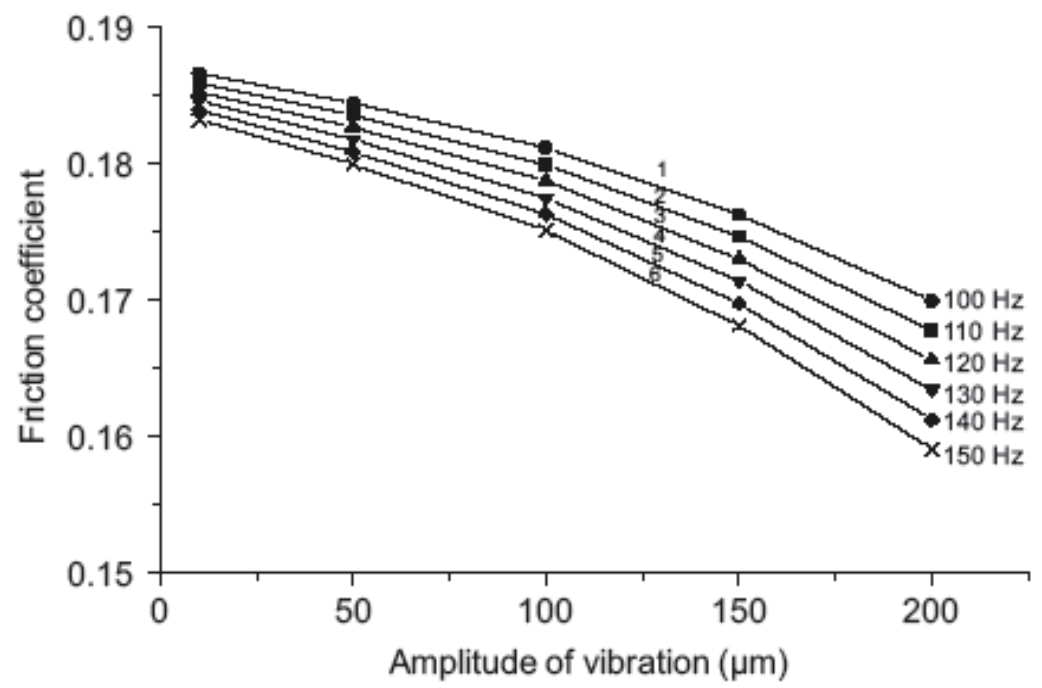

Figure 20. Variation of friction coefficient with the variation of amplitude of vibration at different frequency of vibration (sliding velocity: $1.17 \mathrm{~m} / \mathrm{s}$, normal load: $10 \mathrm{~N}$, roughness: $1.5 \mu \mathrm{m}$ (RMS), relative humidity: $50 \%$, test sample: PTFE). 


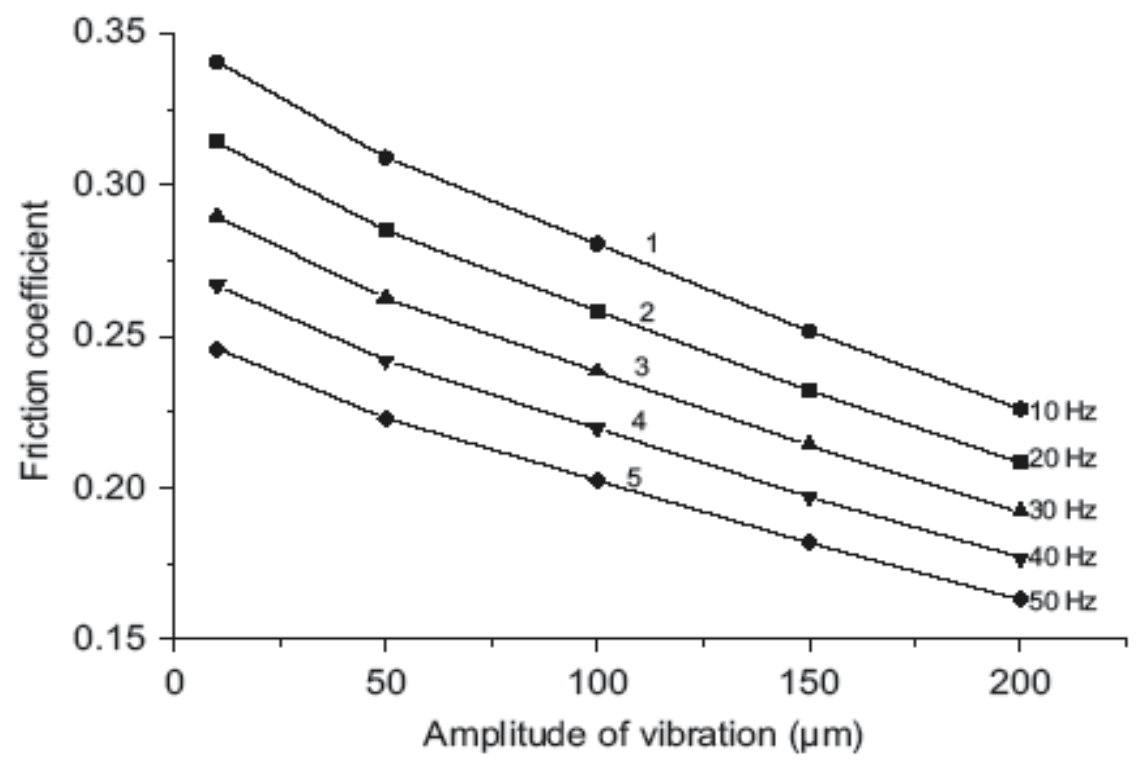

Figure 21. Variation of friction coefficient with the variation of amplitude of vibration at different frequency of vibration (sliding velocity: $0.0393 \mathrm{~m} / \mathrm{s}$, normal load: $10 \mathrm{~N}$, roughness: $1.5 \mu \mathrm{m}$ (RMS), relative humidity: $50 \%$, test sample: rubber).

Friction coefficients of GFRP, mild steel and ebonite are compared for different conditions of vibration (frequency: 100 to $500 \mathrm{~Hz}$ and amplitude: 0 to $200 \mu \mathrm{m}$ ) of similar hardness range [47]. These results are presented in Figs. 22-26. Results show that the magnitude and the slope of line of friction coefficient of mild steel are higher than that of GFRP and ebonite. This might be due to the lack of rigidity and strength of the asperities of ebonite and GFRP than mild steel. The variation of friction coefficient with the variation of materials also depends on different physical properties of mating materials and adhesion which depends on inter-atomic force, surface free energy, van der Waals forces, interface condition and chemical interaction due to different types of bonding [43]. It can be noted that at lower frequency $(100 \mathrm{~Hz})$, the magnitude of friction coefficient of mild steel is varied significantly than GFRP and ebonite. This variation decreases with the increase in frequency of vibration and at higher frequency $(500 \mathrm{~Hz})$, the values friction coefficient of mild steel are almost similar to the friction values of GFRP and ebonite. Under similar conditions, the values of friction coefficient of GFRP are higher than that of ebonite. 


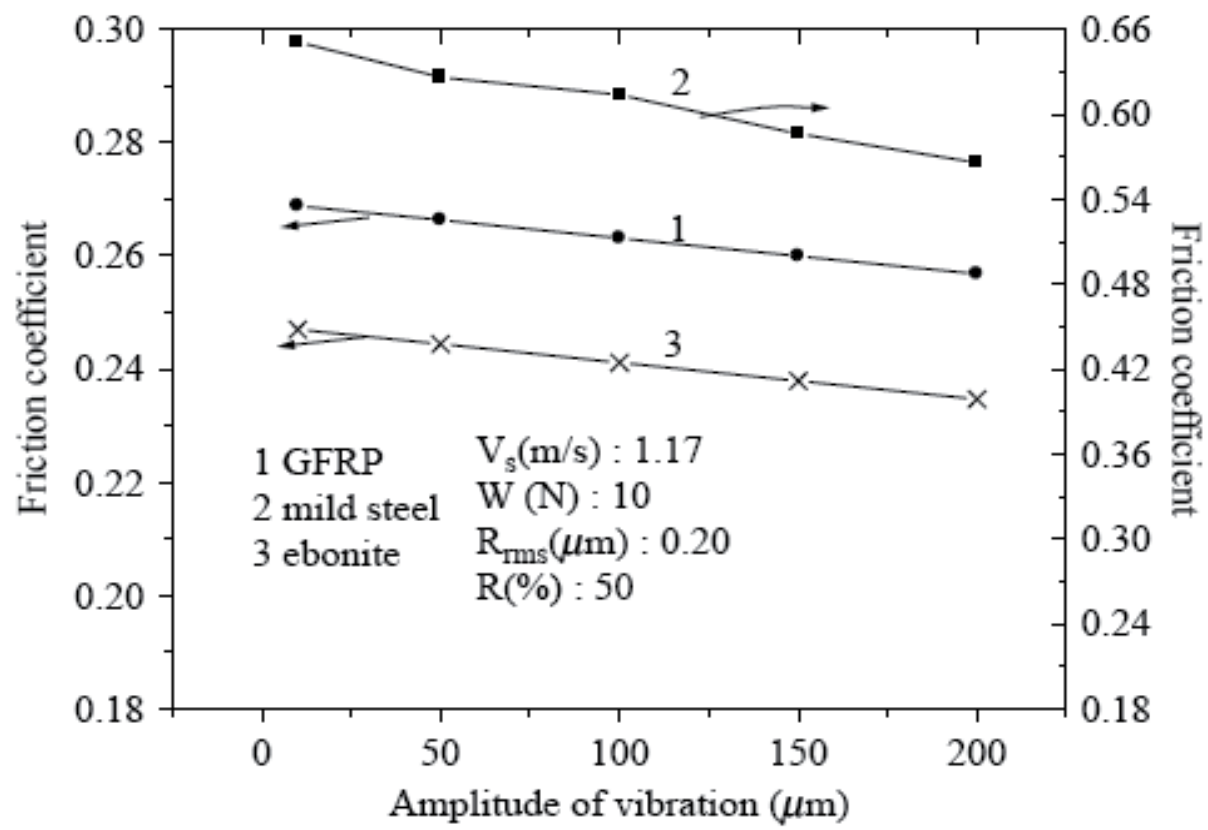

Note: $f(H z): 100$

Figure 22. Variation of friction coefficient with the variation of amplitude of vertical vibration

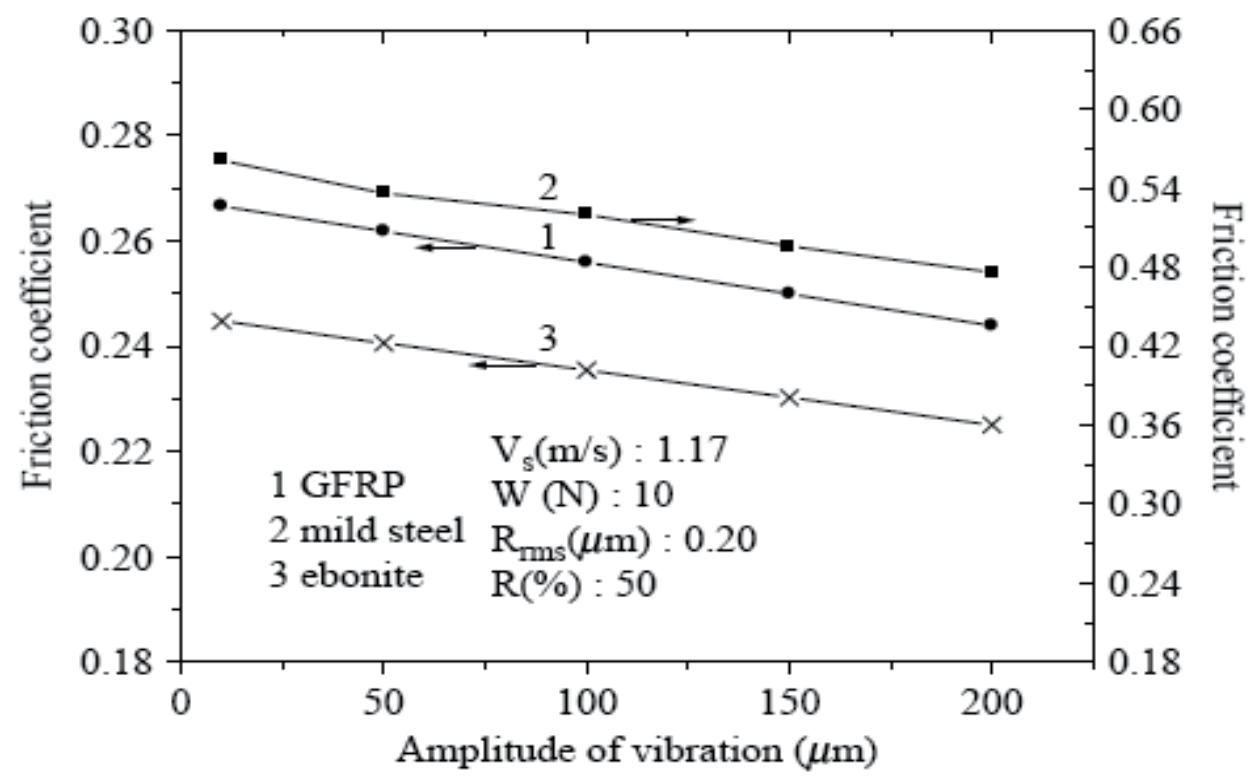

Note: $f(\mathrm{~Hz}): 200$

Figure 23. Variation of friction coefficient with the variation of amplitude of vertical vibration 


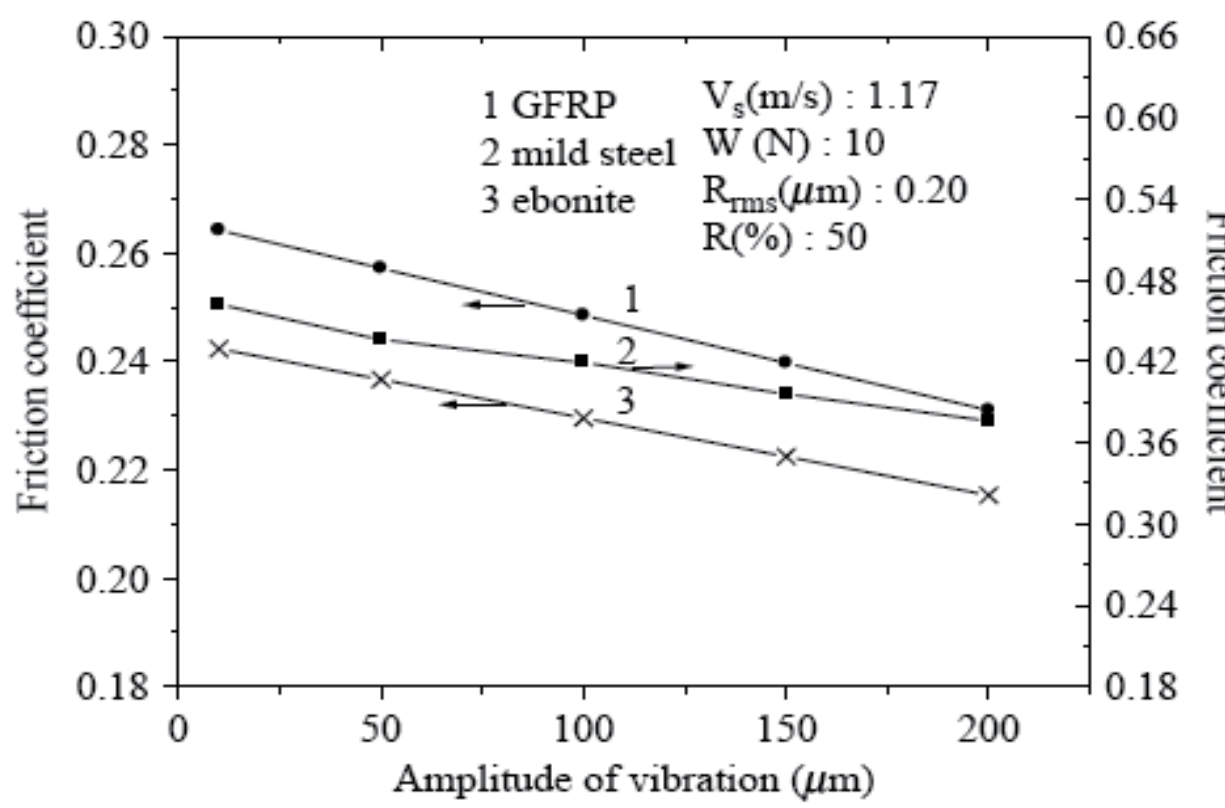

Note: $f(H z): 300$

Figure 24. Variation of friction coefficient with the variation of amplitude of vertical vibration

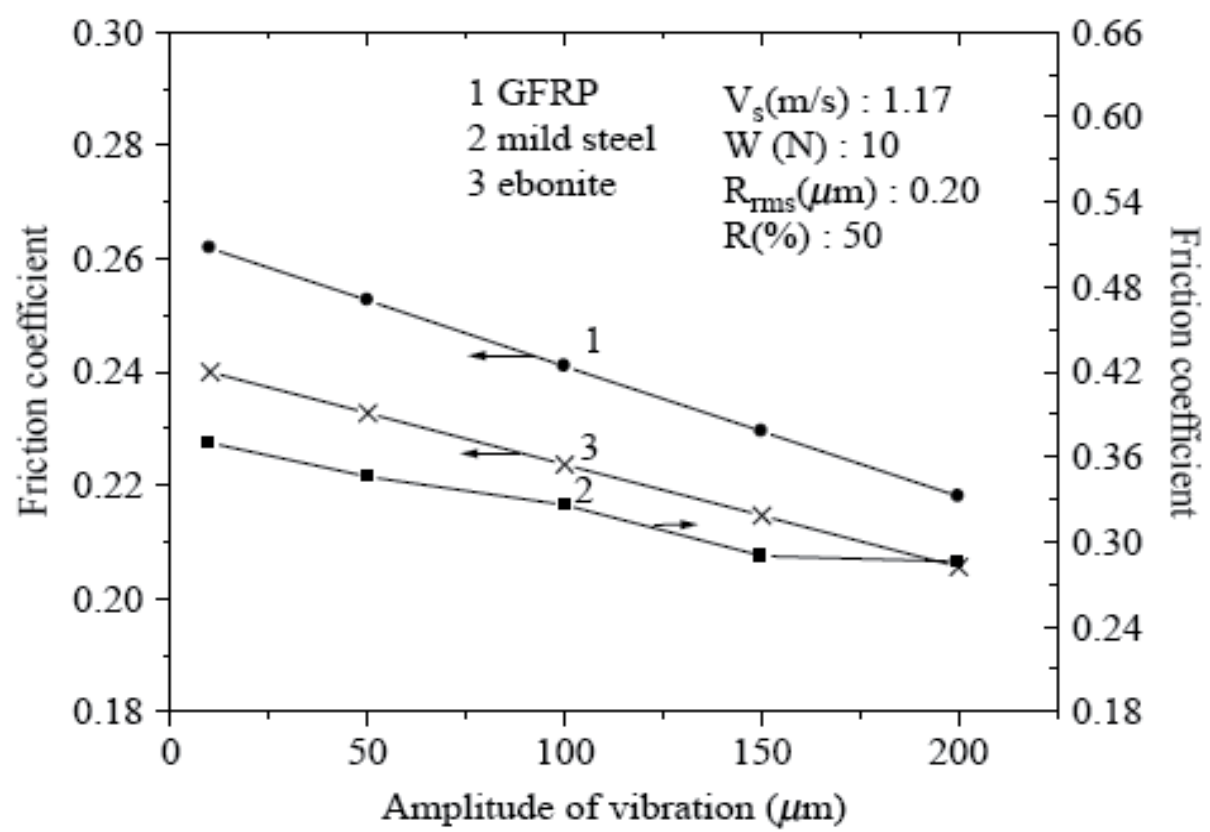

Note: $\mathrm{f}(\mathrm{Hz}): 400$

Figure 25. Variation of friction coefficient with the variation of amplitude of vertical vibration 


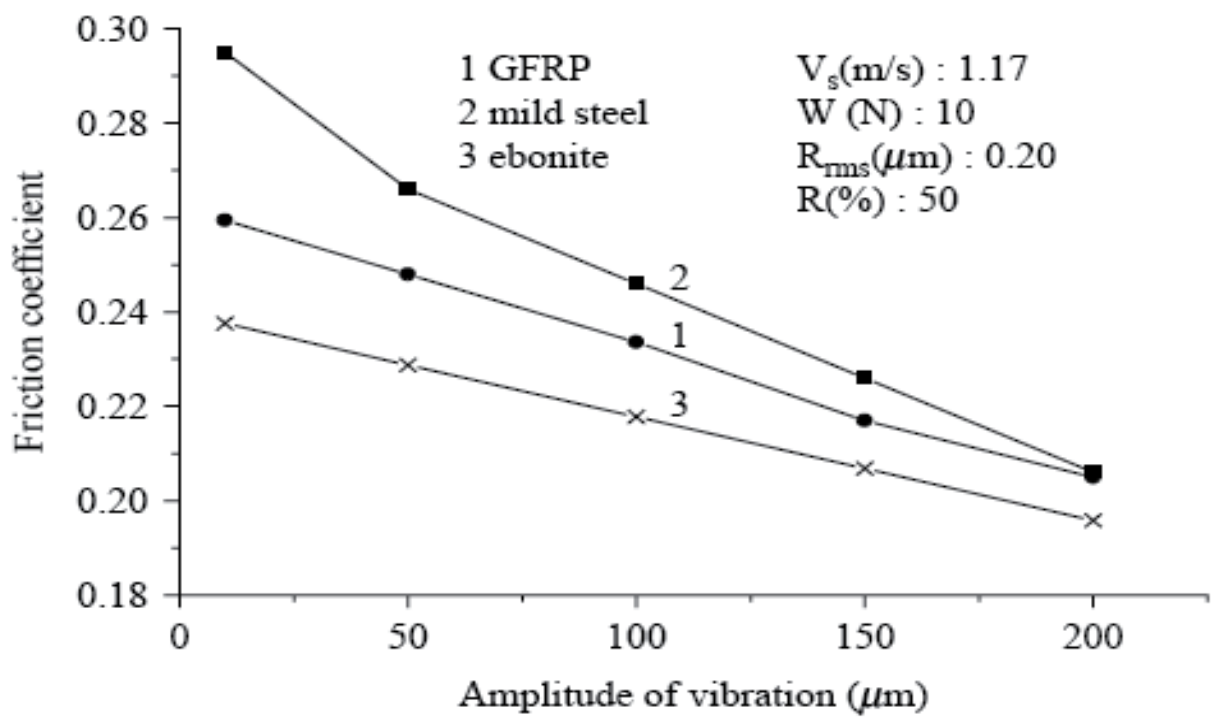

Note: $f(H z): 500$

Figure 26. Variation of friction coefficient with the variation of amplitude of vertical vibration

\section{Effect of horizontal vibration}

The pin-on-disc set-up (Fig. 27) used for generating horizontal vibration [48] in which one end of a coil spring is fixed with the rotating shaft and other end of the spring is fixed with the Vslotted rotating table. An adjusting rigid barrier with spherical tip is fixed with the basic structure of the set-up. Owing to spring action and rotation, the table vibrates horizontally. The direction of vibration is either longitudinal (along the direction of sliding velocity) or transverse (along perpendicular to the direction of sliding velocity) depending on the position of sliding pin on the rotating vibrating table. By varying rotation of the shaft and the number of slots of the rotating table, the frequency of vibration is varied. By adjusting the depth of penetration of the adjustable barrier, the amplitude of the vibration is varied.

The frictional behavior of composite materials under external horizontal vibration is presented in this section. Friction coefficients of GFRP, mild steel and ebonite under longitudinal horizontal vibration are shown in Figs. 28-32. Results show that the friction coefficient increases almost linearly with the increase in amplitude of horizontal vibration for these materials. The increase of friction coefficient might be due to the increase of length of rubbing with the increase of amplitude of vibration. In addition to this the increase of friction coefficient $[39,43]$ is also associated with: (i) Fluctuation of inertia force along the direction of friction force (positive and negative). (ii) More sliding causes more abrasion resistance. Higher abrasion results more shearing due to penetration and ploughing of the asperities between contacting surfaces that might have some effect on the increment of friction force. (iii) Microwelding, reversal of friction vector, and mechanical interlocking. (iv) Formation and enhance an electrically charge layer at the interface. (v) Increase of solubility due to high temperature. 




Figure 27. Block diagram of the experimental set-up for horizontal vibration

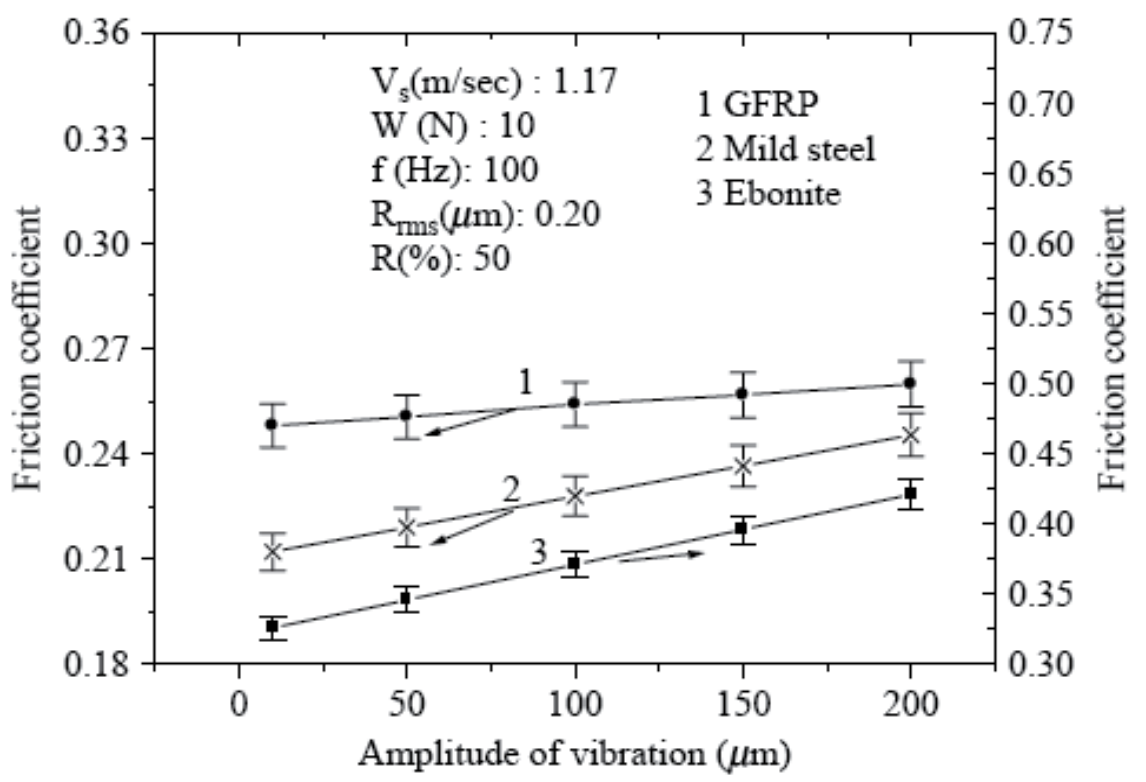

Figure 28. Variation of friction coefficient with the variation of amplitude of longitudinal vibration for frequency $100 \mathrm{~Hz}$ 


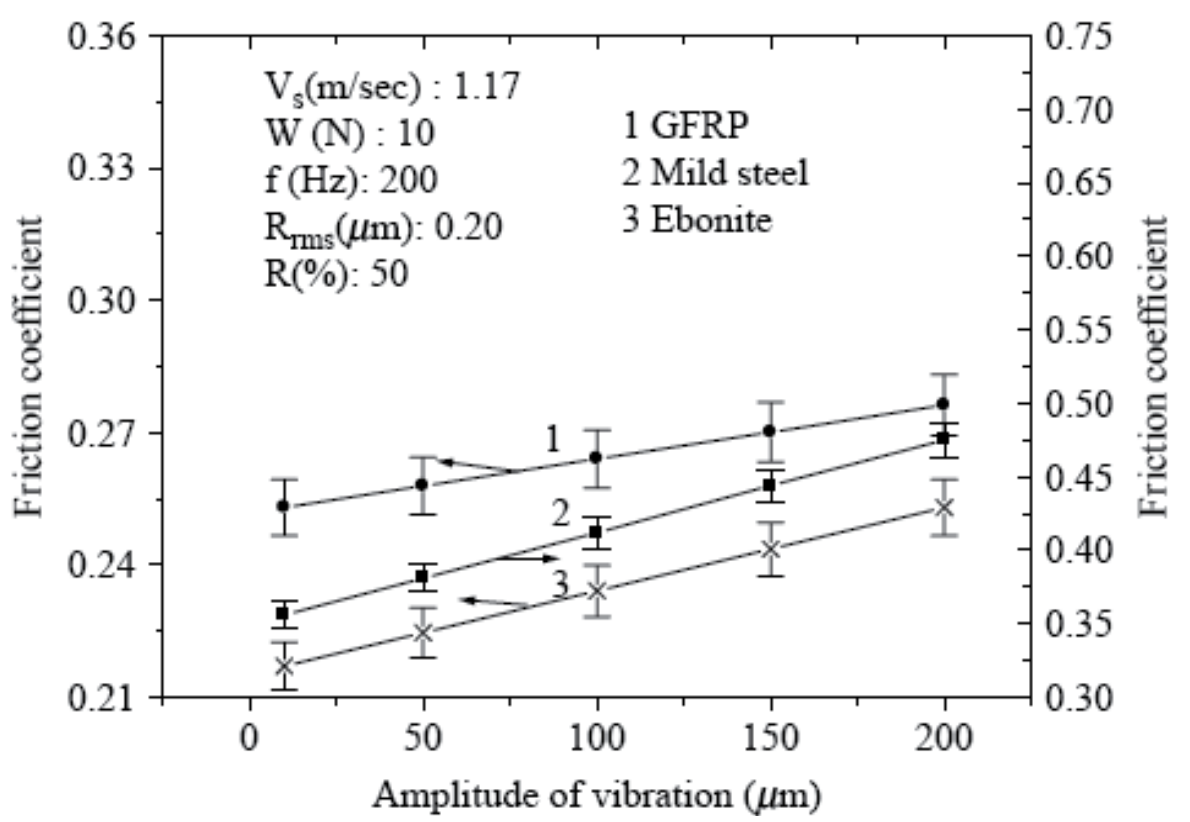

Figure 29. Variation of friction coefficient with the variation of amplitude of longitudinal vibration for frequency $200 \mathrm{~Hz}$

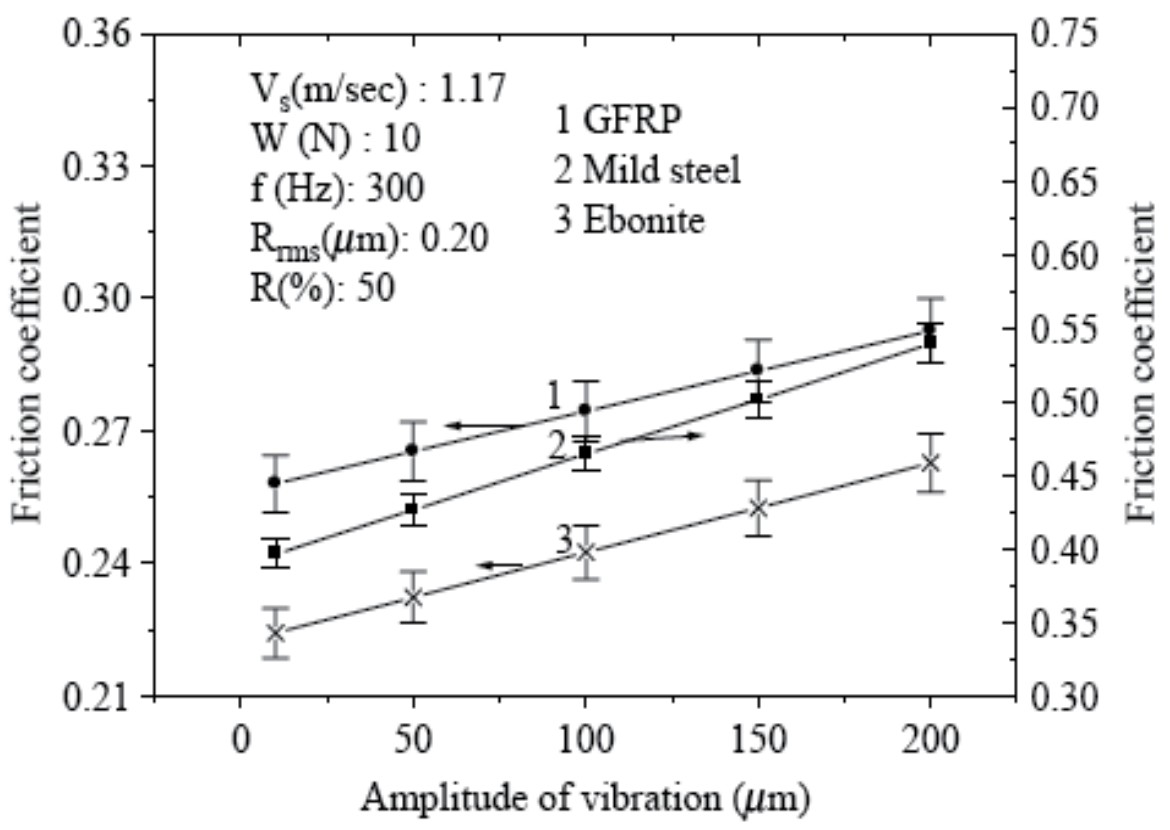

Figure 30. Variation of friction coefficient with the variation of amplitude of longitudinal vibration for frequency $300 \mathrm{~Hz}$ 


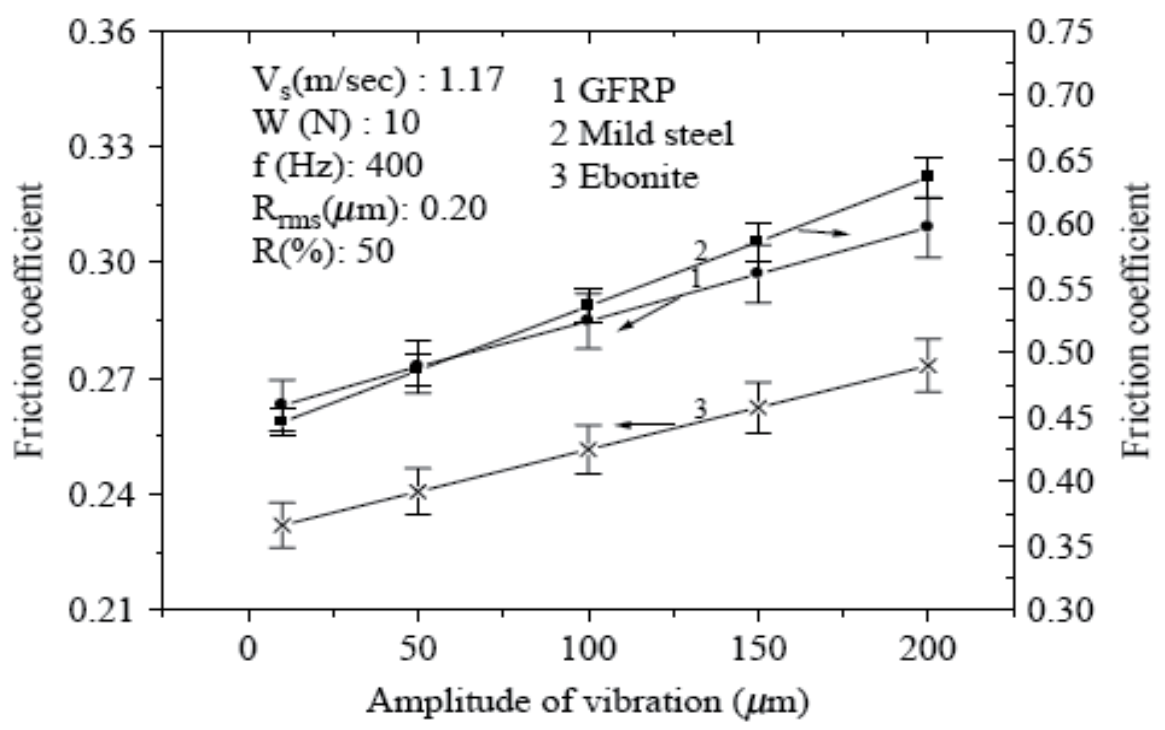

Figure 31. Variation of friction coefficient with the variation of amplitude of longitudinal vibration for frequency $400 \mathrm{~Hz}$

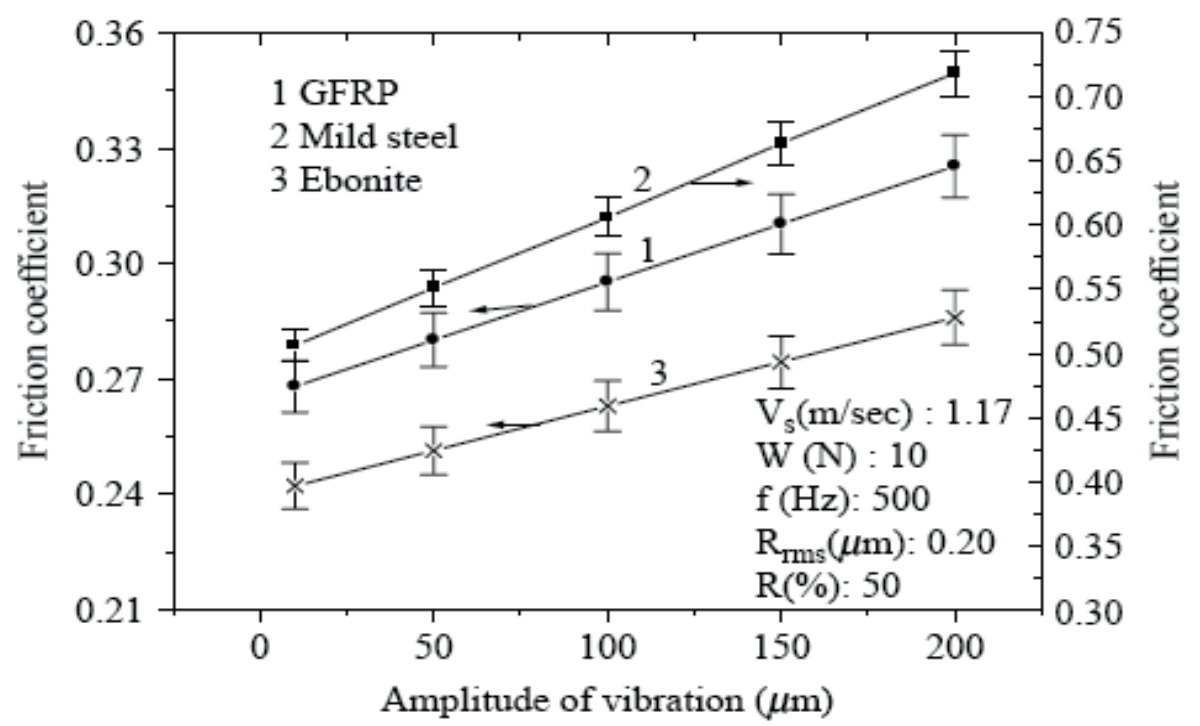

Figure 32. Variation of friction coefficient with the variation of amplitude of longitudinal vibration for frequency $500 \mathrm{~Hz}$

Results indicate that the magnitude and the slope of line of friction coefficient of mild steel under vibration condition are higher than that of GFRP and ebonite. It can be noted that the values of friction coefficient of mild steel are almost twice the values of ebonite and GFRP within the observed range of frequency of horizontal vibration. Under similar vibration conditions, the values of friction coefficient of GFRP are higher than that of ebonite [48]. Results also show that the friction coefficients obtained under transverse vibration are 
slightly higher than those of longitudinal vibrations. Changing of direction of inertia forces of the vibrating body and the effect of length of sliding path may be responsible for higher friction under transverse vibration [48].

\section{Effect of natural frequency on friction coefficient and wear rate}

The effects of natural frequency of the experimental set-up on the friction and wear of glass fiber are presented in this section. Figure 33 shows the variation of friction coefficient with the duration of rubbing at different natural frequencies of vibration for glass fiber. Results in Fig. 34 show that friction coefficient increases with the increase in natural frequency of vibration. If a body (either static or dynamic) is in contact with another moving (either rotation or translation) body, where the second body is vibrating, the contact of those two bodies takes place at some particular points of the second body instead of continuous contact. When the natural frequency of vibration of second body is more, for a constant length of contact, the contact points as well as the area of contact between two bodies will be more (Figure 35 (b)) compared to the situation when the natural frequency of second body is less (Figure 35 (a)). As the area of contact or the points of contact between two bodies are more, they experience more frictional resistance for a constant length of contact. Hence, the friction factor between the two bodies will increase with increased natural frequency [49].

The variation of wear and corresponding friction coefficient with the variation of natural frequency of the experimental set-up for glass fiber is presented in Fig. 36. Results show that wear rate as well as friction coefficient increases with the increase in natural frequency of vibration. The shear force and frictional thrust is increased with the increase in natural frequency of vibration and these increased values may accelerate the wear rate. The other possible causes are (i) high ploughing; and (ii) surface damage and breakage of reinforced fibers [50].

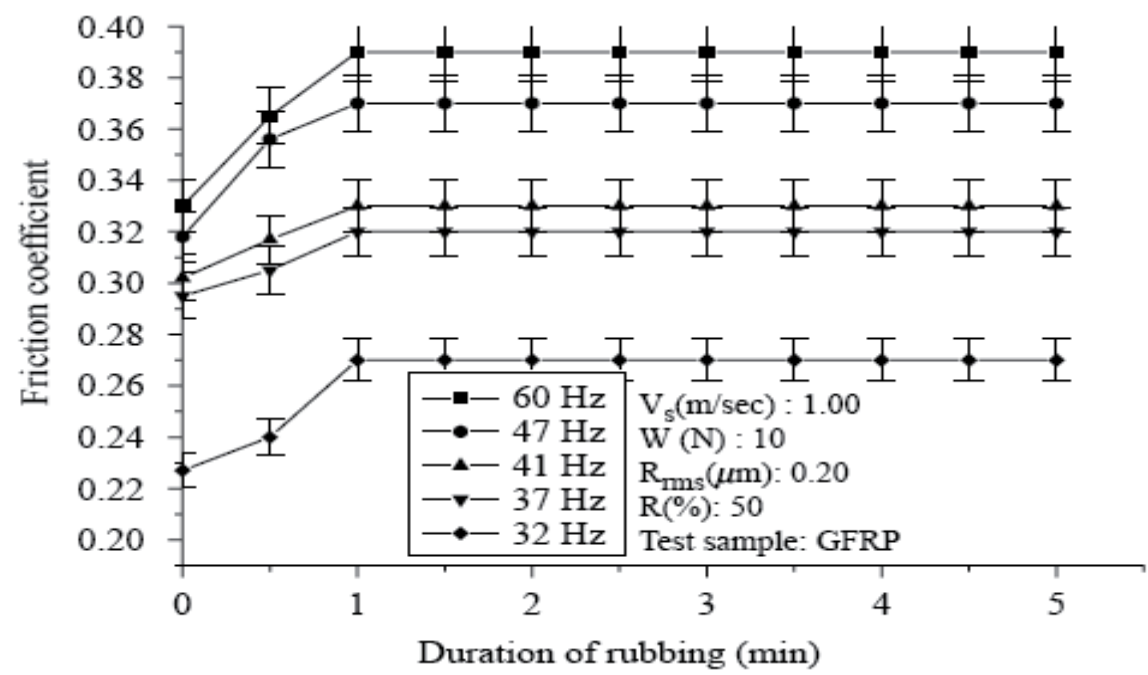

Figure 33. Variation of friction coefficient with the variation of natural frequency of the experimental set-up 


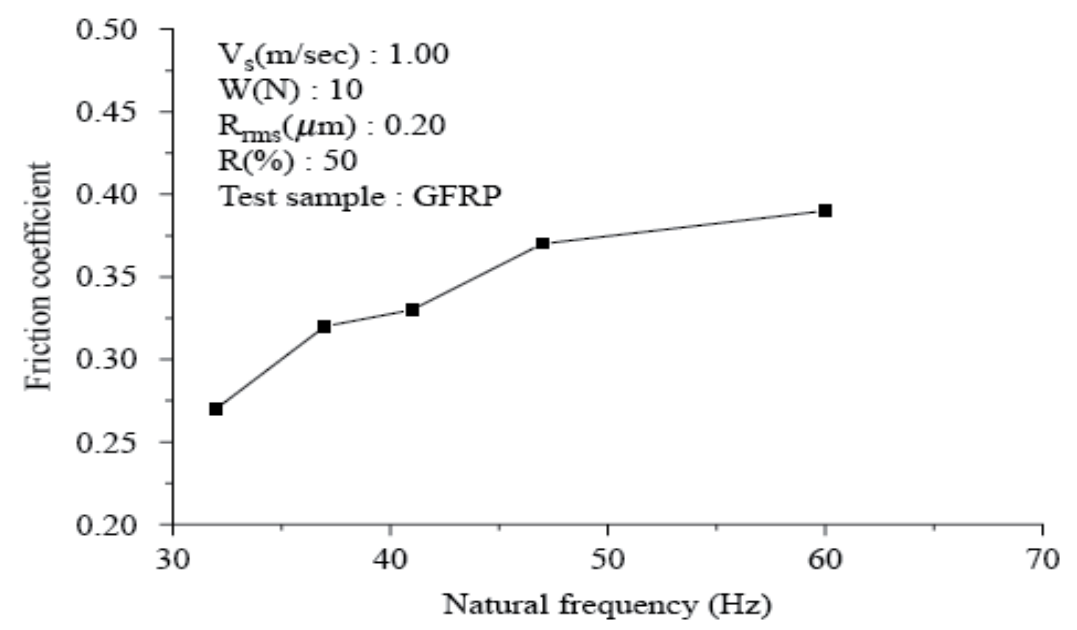

Figure 34. Variation of friction coefficient with the variation of natural frequency of the experimental set-up for GFRP

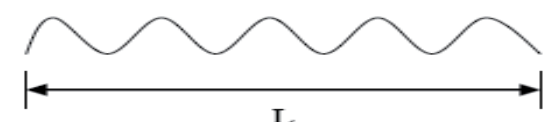

(a)

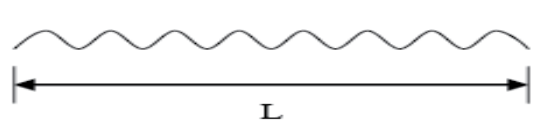

(b)

Figure 35. (a) The points of contact of a body with low natural frequency; (b) the points of contact of a body with high natural frequency for constant length of contact (L)

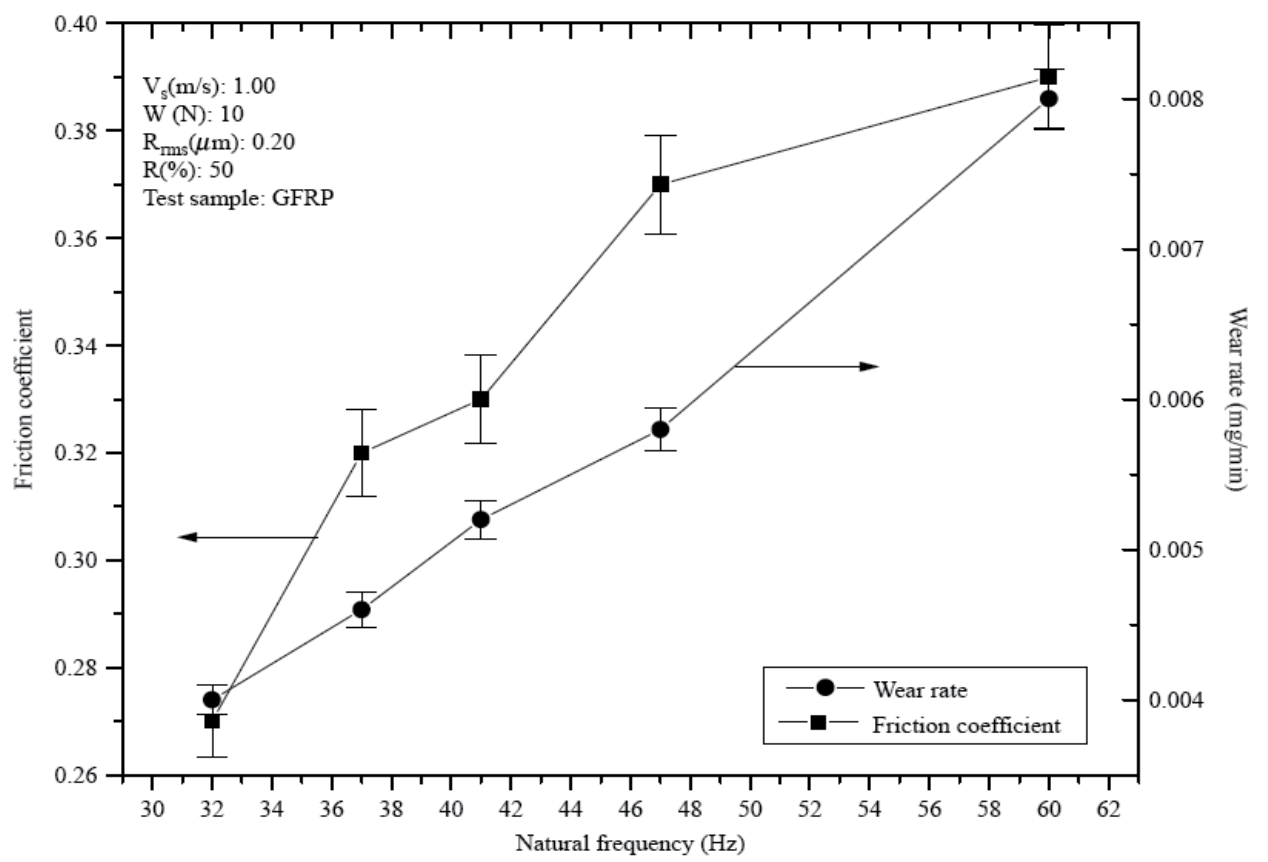

Figure 36. The variation of wear and corresponding friction coefficient with the variation of natural frequency of the experimental set-up for GFRP 


\section{Dimensionless relationship for friction and wear}

\subsection{Vertical vibration: Friction coefficient as a function of sliding velocity, amplitude and frequency of vibration}

The empirical formula of friction coefficient is derived from the dimensionless analysis to correlate the friction coefficient with sliding velocity, frequency and amplitude of vibration is expressed as [47]:

$$
\mu_{f}=k\left[\frac{A f}{V}\right]^{a}
$$

Where,

$\mu_{\mathrm{f}}=$ Friction coefficient

A = Amplitude

$\mathrm{V}=$ Sliding velocity

$\mathrm{f}=$ Frequency

' $\mathrm{a}$ ' and ' $\mathrm{k}$ ' are arbitrary constants

The dimensional friction parameter $\left[\frac{A f}{V}\right]$ is called 'Zaman Number' and abbreviated as Zn No.

Figures 37 and 38 show the plot of friction coefficient $\mu \mathrm{f}$ versus $\mathrm{Zn}$ no. for glass fiber and ebonite, respectively. Figures show that $\mu$ decreases linearly with the increase of $\mathrm{Zn}$ no. and are represented by the equations[47]:

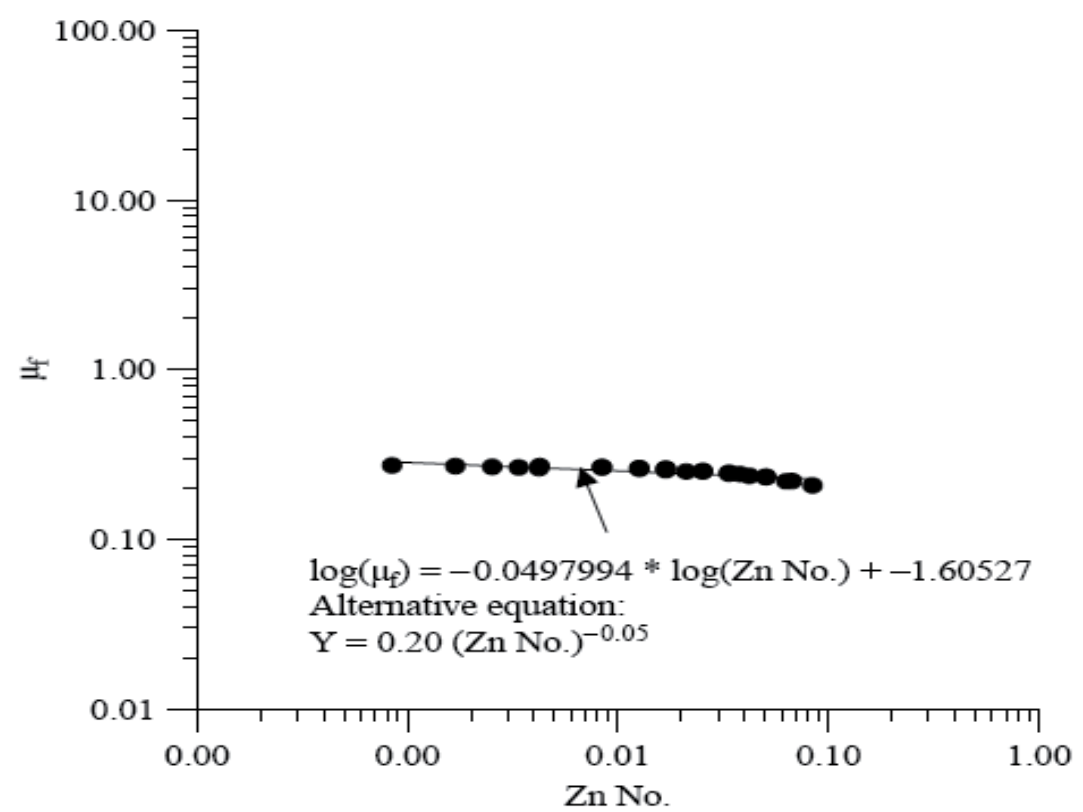

Figure 37. Friction coefficient as a function of $\mathrm{Zn}$ no. for GFRP 


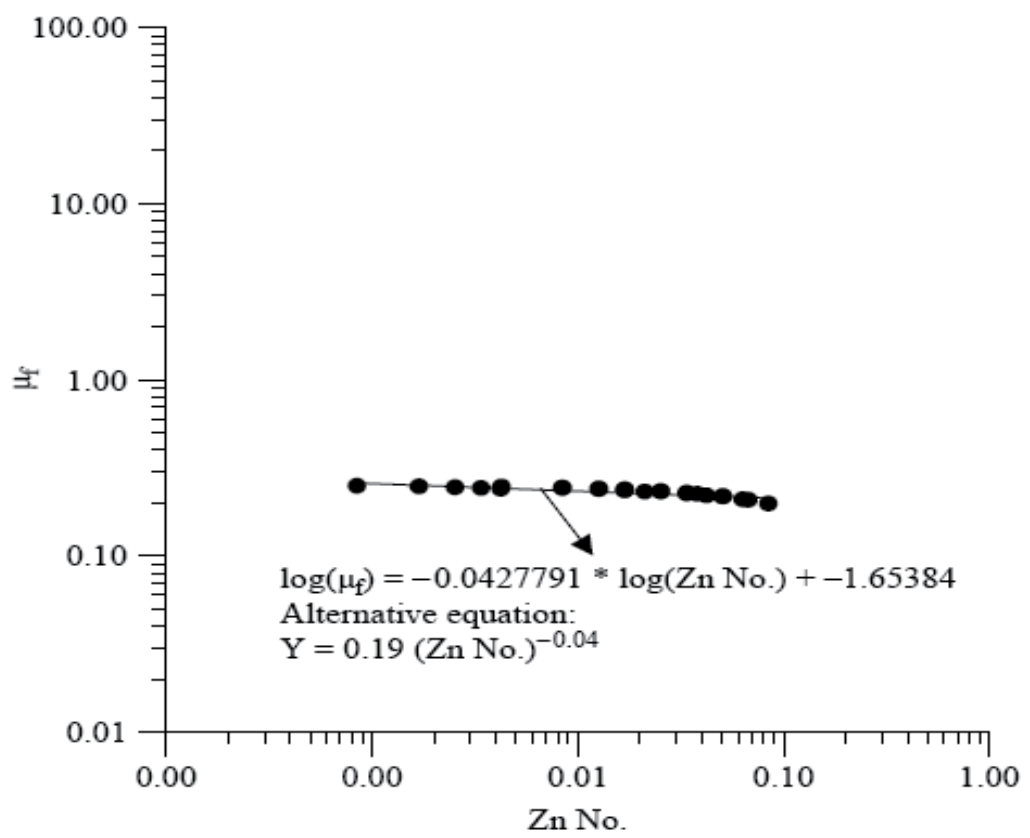

Figure 38. Friction coefficient as a function of $\mathrm{Zn}$ no. for ebonite

$\mu_{f}=0.20\left[\frac{A f}{V}\right]^{-0.05} \quad$ for glass fiber;

$\mu_{f}=0.19\left[\frac{A f}{V}\right]^{-0.04} \quad$ for ebonite

The maximum percentage variation between experimental and theoretical results for GFRP and ebonite are almost $\pm 5 \%$ within the observed range of $\mathrm{Zn}$ no. The coefficient of determination of GFRP and ebonite are $72 \%$ and $75 \%$ respectively. This indicates that experimental results are in good agreement with the theoretical calculations.

\subsection{Horizontal vibration: Friction coefficient as a function of sliding velocity, amplitude and frequency of vibration}

Figures 39 and 40 show the plot of friction coefficient $\mu$ versus $\mathrm{Zn}$ no. for GFRP and ebonite, respectively. Results show that $\mu$ increases linearly with the increase of $\mathrm{Zn}$ no. and are expressed by the equations for external horizontal vibration [48]:

$\mu_{f}=0.34\left[\frac{A f}{V}\right]^{0.05} \quad$ for glass fiber;

$\mu_{f}=0.31\left[\frac{A f}{V}\right]^{0.06} \quad$ for ebonite 


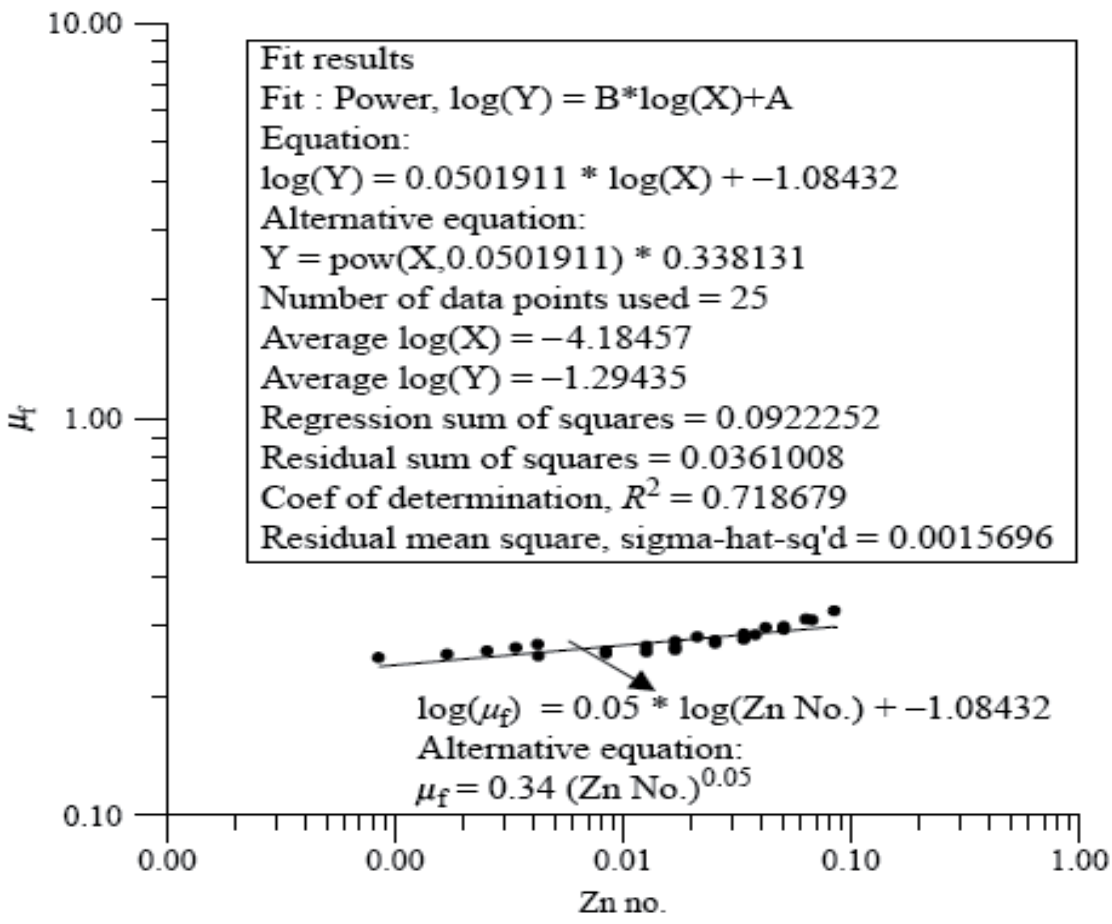

Figure 39. Friction coefficient as a function of $\mathrm{Zn}$ no. for GFRP

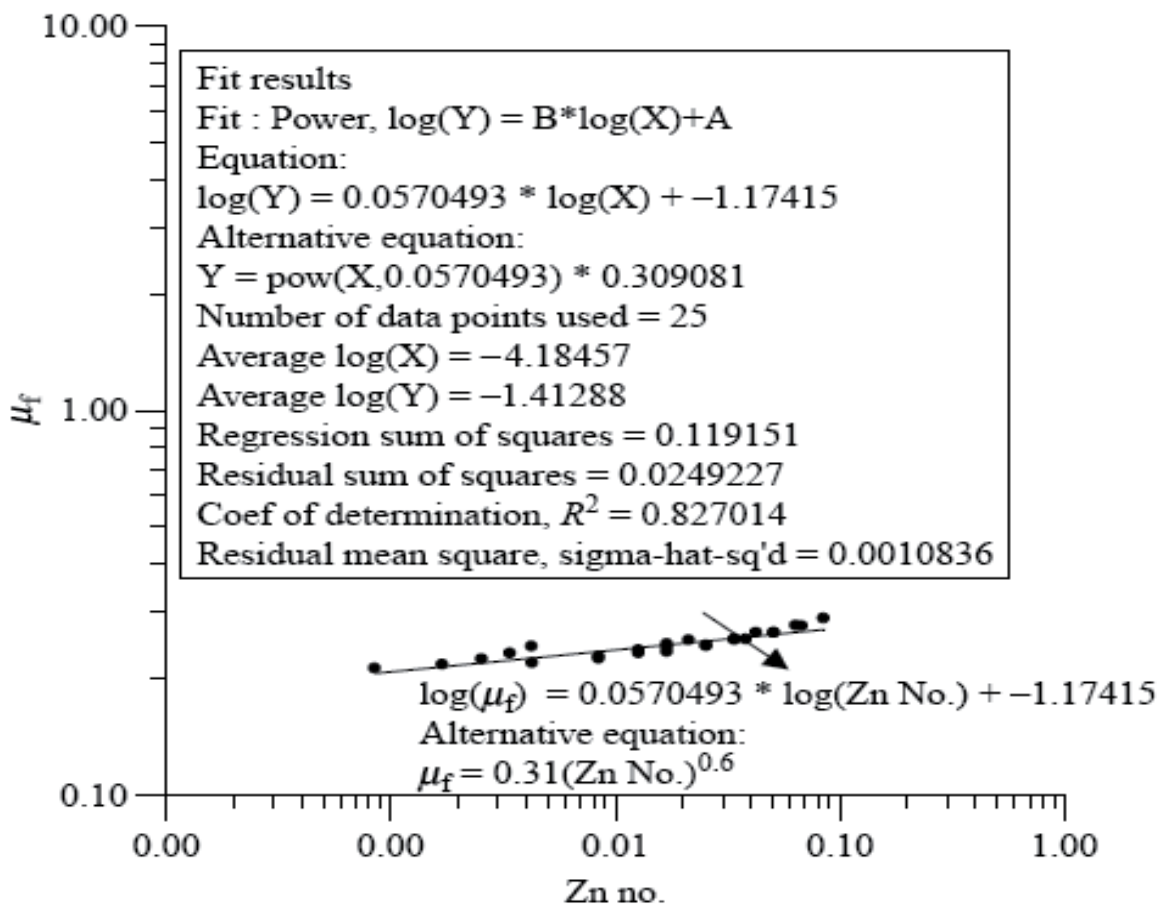

Figure 40. Friction coefficient as a function of $\mathrm{Zn}$ no. for ebonite 


\subsection{Wear rate as a function of natural frequency, sliding velocity, normal load and surface roughness}

The empirical formula of wear rate is derived from the dimensional analysis to correlate wear rate with natural frequency, roughness and sliding velocity is expressed as [50]:

$$
W_{r}=\frac{k N}{f_{n} R}\left[\frac{f_{n} R}{V}\right]^{-b}
$$

Where,

$\mathrm{W}_{\mathrm{r}}=$ Wear rate $=\mathrm{Mt}^{-1}$

$\mathrm{f}_{\mathrm{n}}=$ Natural frequency $=\mathrm{t}^{-1}$

$\mathrm{V}=$ Sliding velocity $=\mathrm{Lt}^{-1}$

$\mathrm{N}=$ Normal load $=$ MLt $^{-2}$

$\mathrm{R}=$ Root mean square roughness of the tested surface $=\mathrm{L}$

' $\mathrm{b}$ ' and ' $\mathrm{k}$ ' are arbitrary constants

The dimensional wear parameter $\frac{f_{n} R}{V}$ is called 'Asad Number' and abbreviated as Ad No.

Figure 41 shows the plot of wear rate $W_{r}$ versus Ad No. Results indicate that $W_{r}$ increases linearly with the increase of Ad. No. and is represented by the equation:

$$
\mathrm{Wr}=-6.52579 \mathrm{E}-4+711.092\left[\frac{f_{n} R}{V}\right]
$$

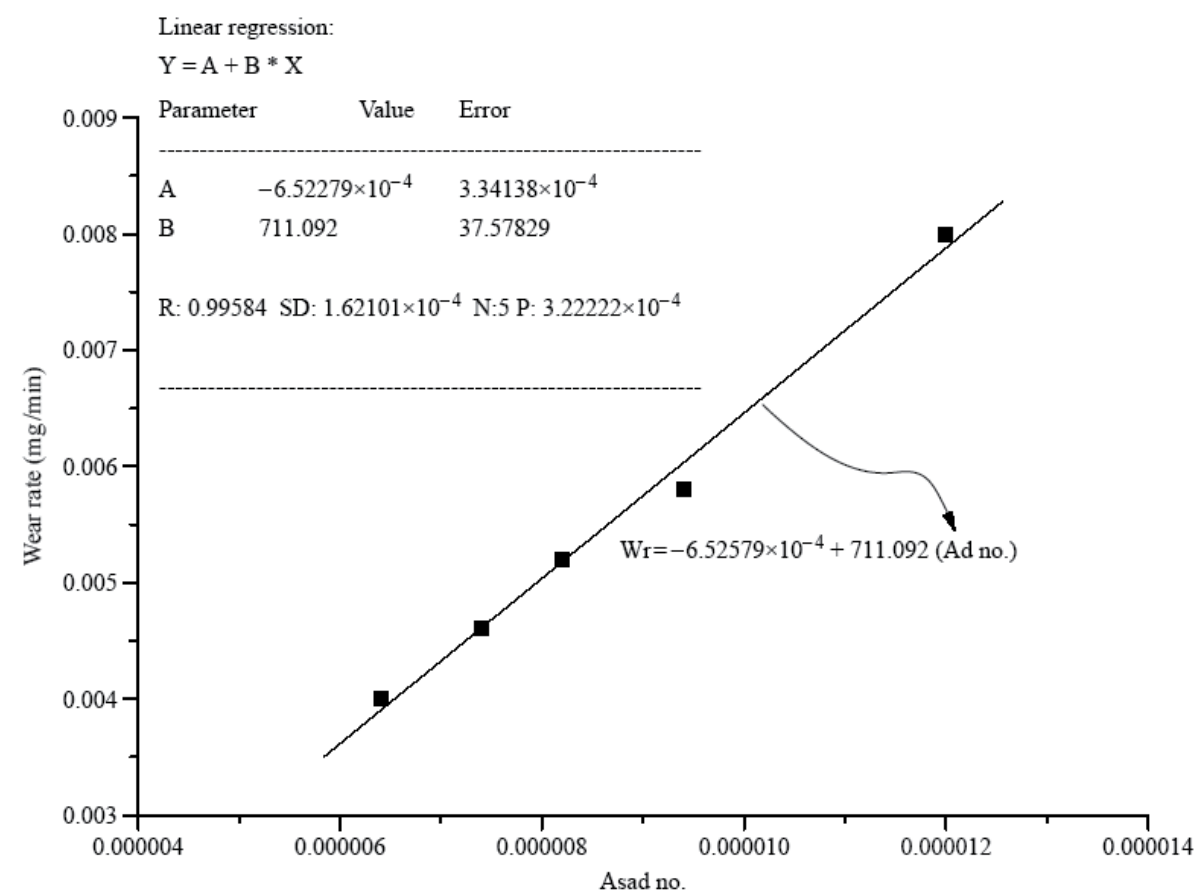

Figure 41. Wear rate as a function of Asad number 
The coefficient of determination for the relationship between wear rate and Ad No. is almost $99 \%$. That is, trend line or Ad. No. can explain $99 \%$ of the variation in wear rate. This means that experimental results are in good agreement with the theoretical calculations [50].

\section{Summary}

Friction and wear of polymer and composites are significantly influenced by normal load, sliding velocity, amplitude of vibration, frequency of vibration, direction of vibration and natural frequency. Friction coefficient also depends on duration of rubbing and it is different for different materials. Friction coefficient can be increased or decreased depending on sliding pairs and operating parameters. There are also some correlations between friction/wear and other influencing parameters. The current trends of these experimental and analytical results can be used in future to design different tribological and mechanical components. The researchers can use these results to innovate some design strategies for improving different concerned mechanical processes. It is expected that the research findings of tribological behavior of polymer and composites discussed in this chapter will also be used for future research and development.

\section{Author details}

Dewan Muhammad Nuruzzaman* and Mohammad Asaduzzaman Chowdhury Department of Mechanical Engineering, Dhaka University of Engineering and Technology (DUET), Gazipur, Gazipur, Bangladesh

\section{References}

[1] Zhang, S. W. State-of-the-art of polymer tribology. Tribology International, 1998; 31 (13): 49-60.

[2] Yamaguchi Y., Tribology of plastic materials: their characteristics and applications to sliding components. Amsterdam: Elsevier 1990.

[3] Hooke, C. J., Kukureka, S. N., Liao, P., Rao, M., and Chen, Y. K. The friction and wear of polymers in non-conformal contacts. Wear 1996; 200: 83-94.

[4] Lawrence, C. C., and Stolarski, T. A. Rolling contact wear of polymers: a preliminary study. Wear 1989; 132: 83-91.

[5] J.F. Archard, Wear theory and mechanisms. Wear Control Handbook, ASME, New York, 1980.

[6] D. Tabor, Friction and wear - developments over the last 50 years, keynote address, Proceedings, International Conference of Tribology - Friction, Lubrication and Wear, 50 years on, London, Institute of Mechanical Engineering (1987) 157-172.

[7] Chowdhury MA, Helali MM. The effect of frequency of vibration and humidity on the coefficient of friction. Tribol Int 2006;39:958-62.

" Corresponding Author 
[8] N.S.M. El-Tayeb, I.M. Mostafa, The effect of laminate orientations on friction and wear mechanisms of glass reinforced polyester composite. Wear 195 (1996) 186-191.

[9] N.S.M. El-Tayeb, R.M. Gadelrab, Friction and wear properties of E-glass fiber reinforced epoxy composites under different sliding contact conditions. Wear 192 (1996) 112-117.

[10] S. Bahadur, Y. Zheng, Mechanical and tribological behavior of polyester reinforced with short glass fibers. Wear 137 (1990) 251-266.

[11] S. Bahadur, V.K. Polineni, Tribological studies of glass fabric-reinforced polyamide composites filled with $\mathrm{CuO}$ and PTFE. Wear 200 (1996) 95-104.

[12] Watanabe, M. The friction and wear Properties of nylon. Wear 1968; 110: 379-388.

[13] Tanaka, K. Transfer of semicrystalline polymers sliding against smooth steel surface. Wear 1982; 75: 183 - 199.

[14] Bahadur, S., and Tabor, D. Role of fillers in the friction and wear behavior of highdensity polyethylene. In: Lee $\mathrm{LH}$, editor. Polymer wear and its control. ACS Symposium Series, Washington DC 1985; 287: 253-268.

[15] Pihtili, H., and Tosun, N. Investigation of the wear behavior of a glass fiber-reinforced composite and plain polyester resin. Composites Science and Technology, 2002; 62:367370.

[16] Pihtili, H., and Tosun, N. Effect of load and speed on the wear behavior of woven glass fabrics and aramid fiber-reinforced composites. Wear, 2002; 252:979-984.

[17] Santner, E., and Czichos, H. Tribology of polymers. Tribology International 1989; 22(2): 103-109.

[18] Tevruz, T. Tribological behaviours of bronze-filled polytetrafluoroethylene dry journal bearings. Wear 1999; 230: 61-69.

[19] Tevruz, T. Tribological behaviours of carbon-filled polytetrafluoroethylene dry journal bearings. Wear 1998; 221: 61-68.

[20] Anderson, J. C. The wear and friction of commercial polymers and composites. In: Friction and wear and polymer composites. Friedrich K, editor. Composite materials series, vol. 1. Amsterdam: Elsevier 1986; 329-362.

[21] Unal, H., Mimaroglu, A., Kadioglu, U., and Ekiz, H. Sliding friction and wear behaviour of polytetrafluoroethylene and its composites under dry conditions. Materials and Design 2004; 25: 239 - 245.

[22] Sirong, Yu, Zhongzhen, Yu, and Yiu-Wing, Mai. Effects of SEBS-g-MA on tribological behavior of nylon 66/organoclay nanocomposites. Tribology International 2007; 40: 855 -862 .

[23] Stuart, B. H. Tribological studies of poly(ether ether ketone) blends. Tribology International 1998; 31(11): 647-651.

[24] Unal, H., and Mimaroglu, A. Influence of test conditions on the tribological properties of polymers. Industrial Lubrication and Tribology 2003; 55(4): 178-183.

[25] Unal, H., and Mimaroglu, A. Friction and wear behavior of unfilled engineering thermoplastics. Material Design 2003; 24: 183-187.

[26] Suresha, B., Chandramohan, G., Prakash, J.N., Balusamy, V., and Sankaranarayanasamy, K. The role of fillers on friction and slide wear characteristics in 
glass-epoxy composite systems. Journal of Minerals \& Materials Characterization \& Engineering 2006; 5 (1): 87 - 101.

[27] Mimaroglu, A., Unal, H., and Arda, T. Friction and wear performance of pure and glass fiber reinforced Poly-Ether-Imide on polymer and steel counterface materials. Wear 2007; 262: 1407 - 1413.

[28] Unal, H., Mimaroglu, A., Kadioglu, U., and Ekiz, H. Sliding friction and wear behaviour of polytetrafluoroethylene and its composites under dry conditions. Materials and Design 2004; 25: 239 - 245.

[29] Unal, H., Sen, U., and Mimaroglu A. An approach to friction and wear properties of polytetrafluoroethylene composite. Materials and Design, 2006; 27: 694-699.

[30] El-Tayeb, N. S. M., Yousif, B. F., and Yap, T. C. Tribological studies of polyester reinforced with CSM 450-R-glass fiber sliding against smooth stainless steel counterface. Wear, 2006; 261:443-452.

[31] Wang, Y.Q., and Li, J. Sliding wear behavior and mechanism of ultra-high molecular weight polyethylene. Materials Science and Engineering, 1999; 266:155-160.

[32] Tsukizoe, T., and Ohmae, N. Friction and wear of advanced composite materials. Fiber Science and Technology, 1983; 18 (4): 265-286.

[33] Suresha, B., Chandramohan, G., Samapthkumaran, P., Seetharamu, S., and Vynatheya, S. Friction and wear characteristics of carbon-epoxy and glass-epoxy woven roving fiber composites. Journal of Reinforced Plastics and Composites 2006; 25: 771-782.

[34] Godfrey, D. (1967), "Vibration reduces metal to metal contact and causes an apparent reduction in friction", ASME Transactions, Vol. 10, pp. 183-92.

[35] Tolstoi, D.M., Borisova, G.A. and Grigorova, S.R. (1973), "Friction reduction by perpendicular oscillations", Soviet Physics-Doklady, Vol. 17, pp. 907-9.

[36] Lenkiewicz, W. (1969), "The sliding process - effect of external vibrations", Wear, Vol. 13, pp. 99-108.

[37] Hess, D.H. and Soom, A. (1991), "Normal vibrations and friction under harmonic loads: Part I - Hertzian contacts", Journal of Tribology, Vol. 113, pp. 80-6.

[38] Budanov, B.V., Kudinov, V.A. and Tolstoi, D.M. (1980), "Interaction of friction and vibration", Soviet Journal of Friction and Wear, Vol. 1, pp. 79-89.

[39] Skare, T. and Stahl, J. (1992), "Static and dynamic friction processes under the influence of external vibrations", Wear, Vol. 154, pp. 177-92.

[40] Nuruzzaman, D.M., Chowdhury, M.A., and Rahaman, M.L. Effect of Duration of Rubbing and Normal Load on Friction Coefficient for Polymer and Composite Materials. Industrial Lubrication. and Tribology 2011; 63: 320 - 326.

[41] Cho M.H., Bahadur, S. and Pogosian A.K. Friction and wear studies using Taguchi method on polyphenylene sulfide filled with a complex mixture of MoS2, Al2O3, and other compounds. Wear 2005; 258: 1825-1835.

[42] Bahadur, S. The development of transfer layers and their role in polymer tribology Wear 2000; 245: 92-99.

[43] Bhushan, B. Principle and Applications of Tribology. John Wiley \& Sons, Inc., New York, 1999. 
[44] Nuruzzaman, D.M., Rahaman, M.L. and Chowdhury, M.A. Friction coefficient and wear rate of polymer and composite materials at different sliding speeds. International Journal of Surface Science and Engineering 2012 (in press).

[45] Unal, H,. Sen U and. Mimaroglu A. Dry sliding wear characteristics of some industrial polymers against steel counterface Tribology International 2004; 37: 727-732.

[46] Chowdhury M.A.and Helali M.M. The Effect of Amplitude of Vibration on the Coefficient of Friction for Different Materials Tribology International 2008;41: 307- 314.

[47] Chowdhury MA and Helali MM. The frictional behavior of materials under vertical vibration. Industrial Lubrication. and Tribology 2009;61:154-160.

[48] Chowdhury MA and Helali MM. The frictional behavior of composite materials under horizontal vibration. Industrial Lubrication. and Tribology 2009;61:246-253.

[49] Chowdhury MA, Ali M and Helali MM. The effect of natural frequency of the experimental set-up on the friction coefficient Industrial Lubrication. and Tribology 2010;62:78-82.

[50] Chowdhury MA. The effect of natural frequency of the experimental set-up on the wear rate Industrial Lubrication. and Tribology 2010;62:356-360. 


\title{
Frequency-Dependent Effective Material Parameters of Composites as a Function of Inclusion Shape
}

\author{
Konstantin N. Rozanov, Marina Y. Koledintseva and Eugene P. Yelsukov \\ Additional information is available at the end of the chapter
}

http://dx.doi.org/10.5772/48769

\section{Introduction}

Electromagnetic composite materials have a number of promising applications in various radio-frequency $(\mathrm{RF})$, microwave and high-speed digital electronic devices, and allow for solving problems related to electromagnetic compatibility (EMC) and electromagnetic immunity (EMI) [1]. For this reason, study and prediction of frequency-dependent radiofrequency RF and microwave properties of materials currently attract much attention. The problem of interest is the analytical description of wideband RF/microwave permittivity and permeability behavior of materials. This is necessary, in particular, to numerically optimize wideband electromagnetic performance of materials and devices at the design stage.

This chapter discusses frequency dependences of effective material parameters (permittivity and permeability) of different types of composites. The chapter consists of three sections. Section I presents a review of approaches for predicting effective material parameters of composites, such as mixing rules, the Bergman-Milton spectral theory, and the percolation theory. Section II suggests on how to select the most appropriate mixing rule for the analysis of properties of a particular composite. Section III considers the dielectric microwave properties of composites filled with fiber-shaped inclusions.

\section{Approaches to describe effective material parameters of composites}

\subsection{Basic mixing rules}

In most studies, two-component mixtures are considered, where identical inclusions are imbedded in a homogeneous host matrix. Effective properties of such a composite depend on the intrinsic properties of the inclusions and the host matrix, as well as on the 
morphology of the composite. The morphology is a characterization of the manner, in which inclusions are distributed in the composite, including their concentration, shape, and correlations in the location. Therefore, the morphology determines how inclusions are shaped and distributed, whether they are mutually aligned/misaligned in the composite, and what concentrations of inclusion phases and a matrix material are.

A conventional approach to describe the properties of composites employs mixing rules, i.e., equations that relates the intrinsic properties of inclusions and the host matrix with the effective properties of composite based on a simple idealized model considering an ellipsoidal-shaped inclusion. Typically, the characterization of the concentration and the shape of inclusions are included explicitly in the mixing rules, and the account for other morphological characteristics is attempted by a proper selection of the mathematical form of mixing rules.

A number of mixing rules are found in the literature. The basic mixing rules are the Maxwell Garnet equation (MG) [2], Bruggeman's Effective Medium Theory (EMT) [3], and the Landau-Lifshitz-Looyenga mixing rule (LLL) [4, 5]. The MG mixing rule,

$$
\frac{\chi_{\text {eff }}}{1+n \chi_{\text {eff }}}=p \frac{\chi_{\text {incl }}}{1+n \chi_{\text {incl }}}
$$

is equivalent to the Clausius-Mossotti approximation, and also complies with the EwaldOseen extinction theorem [6]. Bruggeman's EMT,

$$
-(1-p) \frac{-\chi_{\text {eff }}}{\chi_{\text {eff }}+1-n \chi_{\text {eff }}}=p \frac{\chi_{\text {incl }}-\chi_{\text {eff }}}{\chi_{\text {eff }}+1+n\left(\chi_{\text {incl }}-\chi_{\text {eff }}\right)},
$$

is often referred to as the Polder-van Santen mixing rule in the theory of magnetic composites [7]. The Landau-Lifshitz-Looyenga mixing rule (LLL) is written as

$$
\left(\chi_{\text {eff }}+1\right)^{1 / 3}-1=p\left(\left(\chi_{\text {incl }}+1\right)^{1 / 3}-1\right) \text {. }
$$

Eqs. (1)-(3) are written for the generalized susceptibilities of inclusions, $\chi_{\text {incl, }}$ and the effective susceptibility, $\chi$ eff, both normalized to the susceptibility of the host matrix, since all susceptibilities of a certain composite, including the effective permittivity and permeability, are governed by the same mixing rule [8], with a possible correction for the tensor nature of the susceptibilities. If permittivity $\varepsilon=\varepsilon^{\prime}-\mathrm{i} \varepsilon^{\prime \prime}$ is under consideration, then $\chi_{\text {incl }}=\varepsilon$ incl $/ \varepsilon_{\text {host }}-1$ and $\chi_{\text {eff }}=\varepsilon_{\text {eff }} / \varepsilon_{\text {host }}-1$. For the permeability $\mu=\mu^{\prime}-\mathrm{i} \mu \prime \prime, \chi_{\text {incl }}=\mu_{\text {incl }}-1$ and $\chi_{\text {eff }}=\mu_{\text {eff }}-1$, because most magnetic composites are based on a non-magnetic host matrix. In Eqs. (1)-(3), $n$ is the form factor, i.e., either depolarization or demagnetization factor, and $p$ is the volume fraction of inclusions.

Starting from the basic mixing rules, simple empirical models of a composite may be suggested.

The MG mixing rule considers the total polarizability of inclusions represented by the right part of Eq.(1) and assumes that this polarizability is acquired to a homogeneous medium. As 
a consequence, this mixing rule defines the weakest possible cooperative phenomena between neighboring inclusions that are feasible for the given volume fraction of inclusions. The MG mixing rule is an accurate result for the case, when excitation of inhomogeneous fields due to multiple scattering on inclusions and the effect of neighboring inclusions are negligible. Therefore, it coincides with the lower Hashin-Shtrikman limit [9] that provides the smallest possible material parameter in the case, when loss is negligible. The MG mixing rule is believed to be valid for regular composites, i.e., those comprising regularly arranged inclusions, and for the case of conducting inclusions covered with an isolating shell [10, 11].

The physical model for the EMT assumes that the host matrix consists of particles having the same shape that inclusions have, and both the inclusions and the host matrix particles, are embedded in an effective medium with the material constant equal to the effective material constant the composite. The sum of the polarizabilities of these two types of particles must be zero, which corresponds to a homogeneous medium. Though a practical realization of the EMT involves a very special morphology of a composite [12], this mixing rule is widely used, because it incorporates the percolation threshold when modeling a metal-dielectric mixture. The percolation threshold, $p_{c}$, is the lowest concentration, at which a macroscopic conductivity appears in the mixture. From the standpoint of the mathematics, the EMT is reduced to a quadratic equation for the effective permittivity. Below and above the percolation threshold, different solutions of the equation must be selected according to the physical selection rules. Equation (2) yields $p_{c}=n$. In the MG mixing rule (1), the percolation threshold is $p_{\mathrm{c}}=1$.

The LLL mixing rule is built up by an iterative procedure starting from a homogeneous material of inclusions and replacing small amount of this material by the material of the host matrix. After that, the resulting "effective" material is regarded as the homogeneous component for the succeeding substitution step, and so on, which results in Eq. (3). The mixing rule obtained by the same iterative procedure starting with the homogeneous host matrix is referred to as the asymmetric Bruggeman approximation. The result of the LLL mixing rule is independent of the form factor of inclusions. For a metal-dielectric composite, the LLL mixing rule always provides a conductive mixture, so that $p_{\mathrm{c}}=0$.

The LLL mixing rule is known to be an accurate result for the case when the material parameter of inclusions differs slightly from that of the host matrix. In particular, this mixing rule is valid for all material parameters of composites at very high frequencies, because any intrinsic susceptibility of any material approaches zero with the frequency tending to infinity. Agreement of both the MG and EMT mixing rules with the LLL mixing rule in the case of the susceptibility of inclusions slightly differing from zero is attained only if $n=1 / 3$.

When the volume fraction of inclusions is small, $p<<p_{c}$, and the interaction between the inclusions is negligible, all three theories are reduced to the small perturbation limit,

$$
\chi_{\text {eff }}=\frac{p \chi_{\text {incl }}}{1+n \chi_{\text {incl }}} .
$$


Strictly speaking, Eqs. (1), (2), and (4) are valid for the case of inclusions of perfectly spherical shape, which have the shape factor equal to $1 / 3$. Otherwise, the equations are not consistent with the LLL mixing rule in the limiting case of high frequencies. For non-spherical particles, the polarizability of inclusions must be averaged over all three principal axes of the inclusion [13]. Two particular cases of non-spherical inclusions are of practical interest - nearly spherical inclusions and highly elongated inclusions (long fibers or platelets). For nearly spherical inclusions, the composites are conventionally described by Eqs. (1), (2), (4) with averaged form factor involved, which is found empirically and may differ from $1 / 3$. For elongated inclusions, the form factor along the shorter axis (in the platelet case), or the sum of two form factors along the shorter axes (in the fiber case) is close to unity, and the polarization of inclusion is these directions can be neglected. In this case, the above equations are valid again, with a randomization factor, $\kappa$, included in the right-hand part of the equations to account for an alignment of non-spherical inclusions. For a fiber-filled composite, $k=1 / 3$, when the fibers are randomly oriented in space, and $\kappa=1 / 2$, when the fibers are randomly oriented in plane and the wave vector in perpendicular to the plane. For composites filled with platelet-shaped inclusions, $\kappa=2 / 3$ for the $3 \mathrm{D}$ isotropic orientation. For the permeability of composites filled with non-spherical particles, possible anisotropy of magnetic moment, associated with crystallographic anisotropy of particle material, should be also taken into account, see, e.g., [14].

\subsection{The Bergman-Milton theory}

A generalization of mixing rules may be made with the use of the Bergman-Milton spectral theory (BM) [8]. The theory expresses the effective material parameter of a composite as

$$
\chi_{\text {eff }}=p \int_{0}^{1} \frac{\chi_{\text {incl }} b(n) d n}{1+n \chi_{\text {incl }}},
$$

where the spectral function, $b(n)$, is introduced as a quantitative characterization of the composite morphology. As is seen from Eq. (5), the BM theory accounts for a distribution in effective form factors of inclusions in a composite. This distribution may be associated with the following statistical parameters and processes within the composite: a spread in shapes of individual inclusions comprising the composite; possible agglomeration of inclusions to clusters; and the effects of multiple scattering and inhomogeneous fields excited by neighboring inclusions. Again, the spectral function is the same for all susceptibilities of a particular composite.

The sum rules,

$$
\int_{0}^{1} b(n) d n=1 \text { and } \int_{0}^{1} n b(n) d n=\frac{(1-p)}{D},
$$

relate the spectral function $b(n)$ to the volume fraction of inclusions $p$ for a macroscopically isotropic composite in $\mathrm{D}$ dimensions. The practically important cases are $D=3$ (an isotropic 3D composite with non-aligned randomly distributed inclusions, the shape of which is arbitrary in the general case) and $D=2$ (an assembly of infinitely long cylinders). The sum rules provide an agreement of the spectral theory with the LLL mixing rule at $\chi_{\text {incl }} \rightarrow 0$. 
The spectral theory provides a complete characterization of the frequency dependence of the effective material parameters. The concentration dependence of effective material parameters is implicit in the spectral theory, with the volume fraction involved in the spectral function as a parameter. The analysis of concentration dependences is a powerful tool for understanding properties of composites. However, application of the spectral function approach is not convenient for such analysis.

Another reason that prevents the BM theory from the wide use for the analysis of measured data is that the theory exploits an unknown function, which is difficult to find from the experiment. There are just a few published examples of how to apply the BM theory to the measured data analysis and predicting frequency characteristics of composites [15]. A conventional approach is to accept a functional dependence $b(n)$ as a function of some parameters and to search for these parameters from the measured data [16-18].

Figure 1 shows the calculated spectral functions $b(n)$ for some mixing rules. The spectral function for the MG mixing rule is a delta-function, as is shown in Fig. 1a. The spectral function for the EMT mixing rule presented in Fig. $1 b$ is a semi-circle when plotted as $n b(n)$ against $n$. Plots $d, e$, and $f$ show the spectral functions for the McLachlan, Sheng, and MusalHahn mixing rules, correspondingly, which are discussed in Subsection 1.4. The latter two plots are composed of several distinct peaks of spectral function even at $n=1 / 3$. Other examples of calculated spectral functions for mixing rules are found in [19]. In case of elongated inclusions, or if a composite is composed of inclusions with significantly different aspect ratios, the spectral function may consist of two or larger number of separated peaks. Also, several distinct peaks of the spectral function are found to appear due to the interaction between inclusions in periodical composite structures [8].

\subsection{The percolation theory}

A different approach is provided by the percolation theory, see, e.g., [17]. The percolation theory considers the quasi-static permittivity of a metal-dielectric mixture at concentrations close to the percolation threshold. The main assumption of the theory is that the properties of the material are due to statistical properties of large conductive clusters in this case, rather than due to individual properties of inclusions. The theory predicts a power dependence of static permittivity of the mixture on the difference between the concentration, $p$, and the percolation threshold, $p_{\mathrm{c}}$ :

$$
\begin{aligned}
& \varepsilon_{\text {eff }}^{\prime} \propto\left(p_{c}-p\right)^{-s}, \quad p<p_{c^{\prime}} \quad s \approx 0.7 \\
& \varepsilon_{\text {eff }}^{\prime \prime} \propto\left(p-p_{c}\right)^{t}, \quad p>p_{c}, \quad t \approx 1.8
\end{aligned}
$$

The values of critical indices, $s$ and $t$, are believed to be universal, i.e., independent of detailed structure of composite.

A consequence of Eqs. (7) is a power dependence of the real and imaginary permittivity on frequency $f$ : 


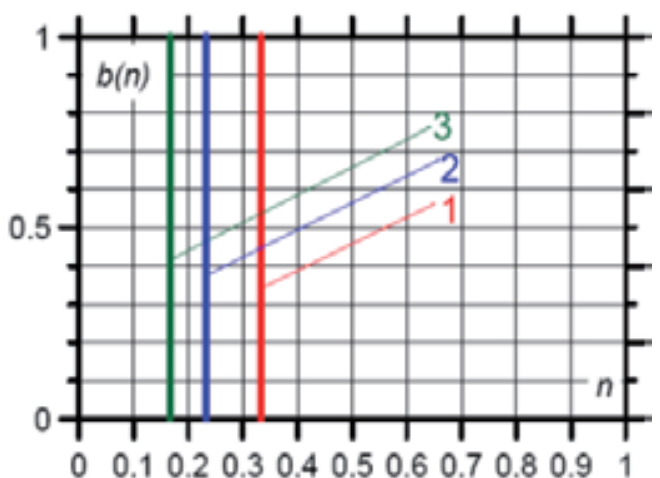

(a)

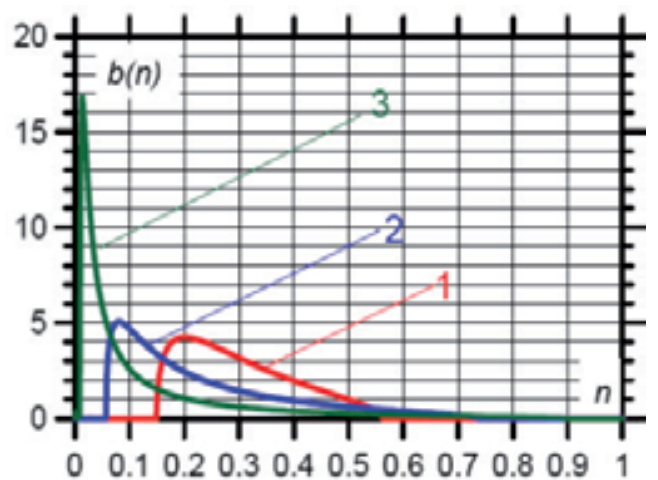

(c)

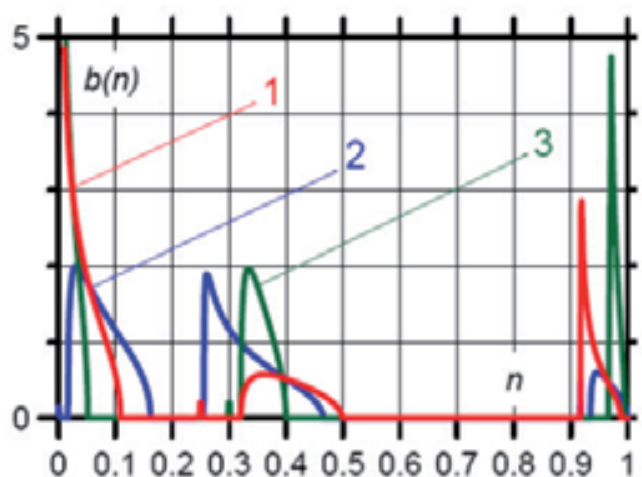

(e)

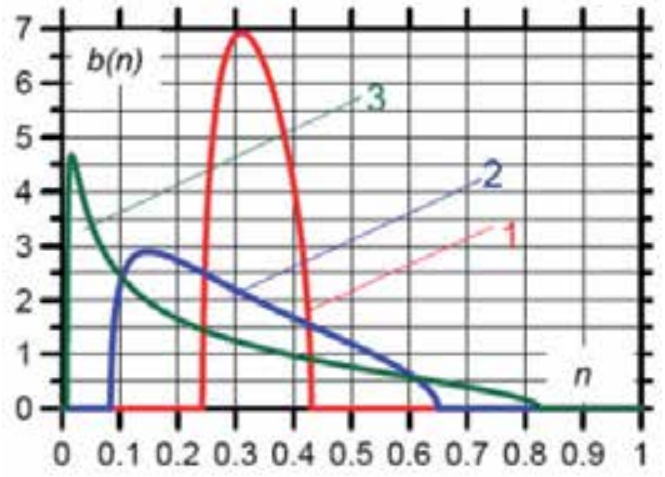

(b)

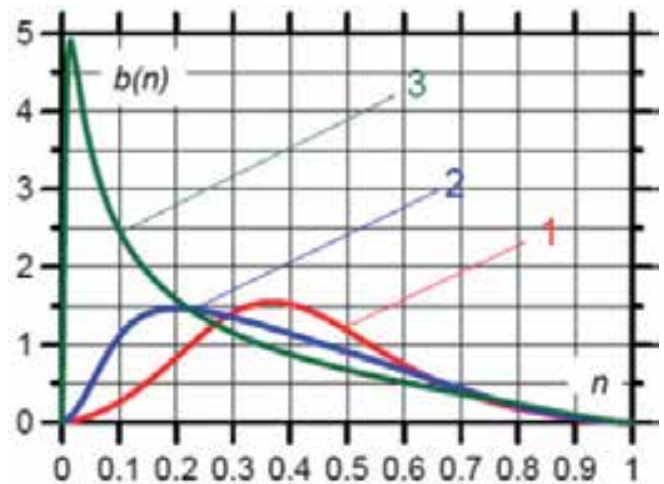

(d)

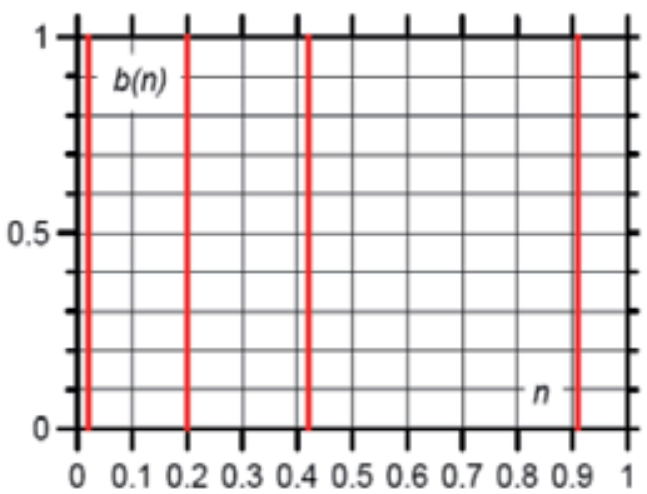

(f)

Figure 1. The spectral function, $b(x)$, calculated for various mixing rules: (a) MG, $n=1 / 3: 1-p=0.1,2-p=$ $0.2,3-p=0.5$; (b) EMT, $n=1 / 3: p=0.01,2-p=0.1,3-p=0.25$; (c) Asymmetric Bruggemann's mixing rule: 1 $-p=0.1,2-p=0.3,3-p=0.6$, (d) McLachlan's theory, $n=1 / 3, s=1.8, t=0.7: p=0.01,2-p=0.1,3-p=0.25$; (e) Sheng theory, $n=1 / 3: 1-p=0.25, F=0.25$, $2-p=0.1, F=0.25,3-p=0.25, F=0.75$; (f) Musal-Hahn theory: $n=1 / 3, p=0.4, F=0.2$. 


$$
\varepsilon_{\text {eff }}^{\prime}-\varepsilon_{\text {host }}^{\prime} \propto f^{-Y}, \quad \varepsilon_{\text {eff }}^{\prime \prime} \propto f^{-Y}
$$

The equality of critical indices for the real and imaginary permittivity established by Eq. (8) follows from the Kramers-Krönig relations, if the frequency dependence of permittivity is governed by law (8) within the whole frequency range. In this case, the dielectric loss tangent, $\varepsilon^{\prime \prime} / \varepsilon^{\prime}$, is independent of frequency and equal to $\tan (\pi Y / 2)$. It follows from the percolation theory that $Y=s /(s+t) \approx 0.28$. In practice, observed values of $Y$ are typically closer to zero [20].

The physical reason for the powder dependence of the permeability on frequency may be understood as follows. Assume that the frequency response of an individual inclusion in the composite is governed by the Debye frequency dispersion law,

$$
\varepsilon(f)=\varepsilon_{\infty}+\frac{\varepsilon_{0}-\varepsilon_{\infty}}{1+\mathrm{i} f \tau}
$$

where $\varepsilon_{0}$ is the static permittivity, $\varepsilon_{\infty}$ is the optical permittivity, and $\tau$ is the characteristic relaxation time, which is reciprocal to the linear relaxation frequency $f_{\text {rel: }} \tau=1 / f_{\text {rel. }}$. The Debye dispersion law governs the frequency dependence of composites filled with conducting inclusions in most cases. When individual inclusions form large clusters of various sizes, a spread of the characteristic relaxation times $\tau$ appears. In this case, the total permittivity is written as:

$$
\varepsilon(\omega)=\varepsilon_{\infty}+\int_{0}^{\infty} \frac{B(y) d y}{1+\mathrm{i} \omega y},
$$

where $B(y)$ is the distribution function of the relaxation times, and

$$
y=\tau / \tau_{0} \text { and } \int_{0}^{\infty} B(y) d y=1 .
$$

The cumulative dispersion curve becomes more gently sloping. With a special form of the distribution,

$$
B(y)=\frac{y}{2 \pi} \frac{\sin \beta \pi}{\cosh (1-\beta) y-\cos \beta \pi},
$$

the Cole-Davidson frequency dispersion, see, e.g., [21], is obtained,

$$
\varepsilon(f)=\varepsilon_{\infty}+\frac{\varepsilon_{0}-\varepsilon_{\infty}}{1+(\mathrm{i} f \tau)^{1-\alpha}} .
$$

Dispersion law (13) involves a frequency region governed by a power frequency dependence of the permittivity. The form of the distribution does not significantly affect the result provided that the distribution is wide, which can be a kind of justification for the percolation theory. 
When the property under consideration is the permeability, the percolation behavior is not readily observed [22].

\subsection{Complex mixing rules}

There are many practical scenarios when none of the simple mixing laws agree with the measured data on a practical composite. A classical example is related to carbonyl iron composites. Despite almost perfect spherical shape of carbonyl iron particles, the form factor restored from the volume fraction dependence of the permittivity or permeability frequently differs greatly from $1 / 3[18,23]$. The reason is an agglomeration of the inclusions.

Another example is the percolation threshold study in composites composed of the same carbon black and different polymer host matrices [24]. Depending on a polymer, the percolation threshold may vary from 5 to $50 \%$. Polymerization with different polymers results in different morphology of the composites. The reason is agglomeration or deagglomeration of inclusions, which depends on the properties of the interface between inclusions and the host matrix. The importance of spatial distribution of inclusions in a composite for validity of mixing rules is discussed in [25].

Practically, in describing properties of composites, many other factors must be accounted for. Among these factors, there are the distribution of inclusions in shape [26-28] and size [29]; the presence of an oxide layer on the surface of conducting particles, statistical spread of intrinsic material parameters of inclusions, e.g., their conductivities [30], as well as possible cones of orientations, if elongated particles are aligned or randomly oriented [31]. For these reasons, fitting parameters are typically unavoidable in accurate description of material properties of composites.

Therefore, taking into account peculiarities of a composite morphology may be crucial for accurate description of composite performance, especially in the case of permittivity of metal-dielectric mixtures, where the intrinsic permittivity of inclusions is infinity in the quasi-static case, and the effective permittivity of composite is determined solely by the shape of inclusions.

Conventionally, a composite morphology is accounted for using more complex mixing rules, which involve some fitting parameters. Three examples of such mixing rules are discussed below. These theories combine the above mentioned basic approaches, and allow for introducing appropriate fitting parameters.

A well known example of such combination is the Lichtenecker mixing rule [32], which is written for the case of the effective permittivity as

$$
\varepsilon_{\mathrm{eff}}^{k}=p \varepsilon_{\mathrm{incl}}^{k}+(1-p) \varepsilon_{\mathrm{host}}^{k} .
$$

In Eq. (14), $k$ has a physical meaning of a critical exponent, which is conventionally treated as a fitting parameter to obtain an agreement with measurements. Equation (14) may be considered as an empirical combination of the LLL mixing rule and the percolation theory. 
A combination of the EMT mixing rule and the percolation theory is MsLachlan's Generalized Effective Medium Theory [33]:

$$
p \frac{\varepsilon_{\text {incl }}^{1 / t}-\varepsilon_{\text {eff }}^{1 / t}}{\varepsilon_{\text {eff }}^{1 / t}+n\left(\varepsilon_{\text {incl }}^{1 / t}-\varepsilon_{\text {eff }}^{1 / t}\right)}+(1-p) \frac{\varepsilon_{\text {host }}^{1 / s}-\varepsilon_{\text {eff }}^{1 / s}}{\varepsilon_{\text {eff }}^{1 / s}+n\left(\varepsilon_{\text {host }}^{1 / s}-\varepsilon_{\text {eff }}^{1 / s}\right)}=0 .
$$

In this equation, which is also written for the case of permittivity, the EMT equation (2) is supplemented with percolation indices $s$ and $t$. These indices, together with the effective depolarization factor of inclusions $n$, are also treated as the fitting parameters.

Another approach for developing complex mixing rules is to divide inclusions in composite into two groups (e.g., a part of inclusions are considered as isolated and the other part are assumed to compose dense clusters [34], or any other way of subdivision into groups), and then to mix these groups with different mixing rules. The value of $F, 0<F<1$, a fraction of inclusions attributed to one of the groups, provides a fitting parameter. An example of this approach is Sheng's theory [35]:

$$
F P_{1}+(1-F) P_{2}=0,
$$

where $P_{1}$ and $P_{2}$ are the effective polarizabilities of the two groups of particles; for the case of spherical inclusions, $P_{1}$ and $P_{2}$ are found in Sheng's theory as

$$
\begin{gathered}
P_{1}=\frac{\left(\varepsilon_{\text {eff }}-\varepsilon_{\text {host }}\right)\left(\varepsilon_{\text {incl }}+2 \varepsilon_{\text {host }}\right)+\left(\varepsilon_{\text {host }}-\varepsilon_{\text {incl }}\right)\left(\varepsilon_{\text {eff }}+2 \varepsilon_{\text {host }}\right) p}{\left(2 \varepsilon_{\text {eff }}+\varepsilon_{\text {host }}\right)\left(\varepsilon_{\text {incl }}+2 \varepsilon_{\text {host }}\right)+2\left(\varepsilon_{\text {eff }}-\varepsilon_{\text {host }}\right)\left(\varepsilon_{\text {host }}-\varepsilon_{\text {incl }}\right) p}, \\
P_{2}=\frac{\left(\varepsilon_{\text {eff }}-\varepsilon_{\text {incl }}\right)\left(2 \varepsilon_{\text {incl }}+\varepsilon_{\text {host }}\right)+\left(\varepsilon_{\text {incl }}-\varepsilon_{\text {host }}\right)\left(\varepsilon_{\text {eff }}+2 \varepsilon_{\text {host }}\right)(1-p)}{\left(2 \varepsilon_{\text {eff }}+\varepsilon_{\text {incl }}\right)\left(2 \varepsilon_{\text {incl }}+\varepsilon_{\text {host }}\right)+2\left(\varepsilon_{\text {eff }}-\varepsilon_{\text {incl }}\right)\left(\varepsilon_{\text {incl }}-\varepsilon_{\text {host }}\right)(1-p)} .
\end{gathered}
$$

Equation (17) describes the polarizability of a spherical particle consisting of an inclusion material and then coated by a shell of the host matrix material. Equation (18) represents the inverse structure, with the shell made of the inclusion material and the core made of the host matrix material. Both Eqs. (17) and (18) are consistent with the MG formalism. The effective structures are mixed with each other according to the EMT equation (16). An analogous approach is suggested by Musal and Hahn [36], with the only difference that the EMT equation (16) describing a mixture of the two groups is substituted in the MG equation (1). Doyle and Jacobs [34, 37] suggested the model, where the two groups of inclusions comprise isolated inclusions and clusters of closely packed inclusions.

The complex mixing rules are suggested and provide rather good/reasonable agreement with measured data mostly for the concentration dependences of the permittivity in metaldielectric mixtures. However, these theories may fail when describing frequency dependences of material parameters. The reason is that the complex mixing rules have the spectral function consisting of several isolated peaks even in the case of nearly-spherical inclusions, as is seen in Fig. $1 e$ and $f$. A physical meaning can hardly be attributed to these 
peaks in case of a random composite filled with spherical inclusions, because, as is shown below, the appearance of isolated peaks of spectral function generally results in the appearance of several isolated regions of frequency dispersion of material parameters.

Another approach to the problem of the permittivity dependence on concentration for metal-dielectric mixtures has been suggested by Odelevskiy [38]. He was the first who noticed the analogy between the MG and EMT equations, in which the concentration dependence of the permittivity for conducting inclusions are written as

$$
\varepsilon_{\text {eff }}=1+\frac{1}{n} \frac{p}{1-p}
$$

and

$$
\varepsilon_{\mathrm{eff}}=1+\frac{1}{n} \frac{p}{1-p / n}
$$

respectively. Odelevskiy suggested an equation that generalizes these two theories in the case of a metal-dielectric mixture:

$$
\varepsilon_{\text {eff }}=1+\frac{1}{n} \frac{p}{1-p / p_{\mathrm{c}}} .
$$

In Eq. (21), the form factor $n$ and percolation threshold $p_{\mathrm{c}}$ are the two fitting parameters. With these fitting parameters, the equation demonstrates an excellent agreement with measured data for a variety of different metal-dielectric mixtures [39], if the concentration of inclusions is not very close to the percolation threshold. Equation (21) cannot be considered as an independent mixing rule, because it does not leave a room for the permittivity of inclusions different from infinity.

\section{Frequency-dependent behavior of composites and validity of mixing rules}

Effective properties of composites in the majority of mixing rules and theories are considered in the quasi-static approximation. Because of this, the frequency dependence of effective material parameter appears due to the difference in frequency dependences of material parameters of constituents.

Frequency dispersion of permittivity in a composite frequently appears due to the different frequency behavior of its dielectric host matrix and of conducting inclusions. Host matrices are typically considered as non-dispersive over a frequency range of interest, while the permittivity of metallic inclusions is imaginary and reciprocal to frequency. There are other dielectric materials possessing dielectric dispersion at microwaves, e.g., water, some ferroelectrics [40], some lossy polymers, but necessity of accounting for this dispersion is a fairly rare. 
In contrast, a multitude of magnetic materials exhibit frequency dispersion of permeability at microwaves. The reason is that all magnets lose their magnetic properties at frequencies below several gigahertz, as is shown in Subsection 2.2. These are the microwaves, or even lower frequencies, where the permeability changes from large static permeability to unity. Notice that the intrinsic permeability of magnetic powders is generally unknown. It depends not only on the composition of the material, but also on manufacturing and treatment technology, and the latter dependence may be essential.

In the first-order approximation, the frequency dependence of material parameters may be considered as an assembly of loss peaks accompanied by corresponding frequency dispersion of the real part, according to the Kramers-Kronig relations. In many cases, the Lorentzian (resonance) dispersion law,

$$
\chi(f)=\sum_{i=1}^{m} \frac{\chi_{\mathrm{st}, i}}{1+\mathrm{i} f / f_{\mathrm{rel}, i}-\left(f / f_{\mathrm{res}, i}\right)^{2}},
$$

provides a good fitting of measured dependences of susceptibility $\chi$ on frequency $f$. In Eq. (22), $m$ is the number of the resonance terms involved in the dispersion law, and $\chi_{\mathrm{st}, i}, f_{\mathrm{rel}, i}$, and $f_{\text {res }, i}$ are the static susceptibility, relaxation frequency, and resonance frequency attributed to $i$-th resonance term, respectively.

\subsection{Frequency-dependent behavior of composites}

Almost all mixing rules deduce the effective material parameters from the polarizability,

$$
P=\frac{\chi_{\text {incl }}}{1+n \chi_{\text {incl }}}
$$

embedded in either a host matrix or the effective medium. From Eq. (23), two limiting cases are clearly seen, $n \chi_{\text {incl }}<<1$ and $n \chi_{\text {incl }}>>1$.

In the case of $n \chi_{\text {incl }}<<1$, the LLL mixing rule (3) is a rigorous result. For majority of practical cases, Eq. (3) may be rewritten just as the perturbation limit given by Eq. (4). In this case, the effective material parameter is just the intrinsic material parameter multiplied by the volume fraction of inclusions. This means that the effect of interaction between inclusions is negligible. The morphology of the composite, including the shape of inclusions, does not affect the effective material parameter. This case is typical for the microwave permeability of composites filled with either fibrous or platelet inclusions, as well as for all effective susceptibilities at very high frequencies.

In the other limiting case, $n \chi_{\text {incl }}>>1$, the effective material parameter depends on the morphology only. Here, the effective static susceptibility increases non-linearly with the concentration of inclusions, according to the percolation behavior and Odelevskiy equation (21). It is the case, for which most of the complex mixing rules have been developed. The case is related to the permittivity of metal-dielectric mixtures, since the imaginary part of the 
permittivity of metal inclusions is so high that the absolute value of the microwave permittivity can be considered as infinite. As to the permeability, this case may be observed in some composites filled with ferromagnetic inclusions of spherical shape, or for lowfrequency magnetic materials, whose permeability may be very high.

Let the effective material parameter be considered in a wide frequency range. Assume that the host matrix of the composite is lossless and non-dispersive. Then, the frequency dispersion in the composite is due to the frequency dispersion of inclusions. It is well known that a material parameter of any medium approaches unity with the frequency tending to infinity. Because of that, the case of $n \chi_{\text {incl }}<<1$ is always observed at very high frequencies, where the LLL mixing rule (3) describes material parameters of composites.

If the intrinsic susceptibility of inclusions is low, the LLL mixing rule is valid for low frequencies as well. In this case, the frequency dependence of any effective material parameter is just proportional to the dependence for the intrinsic material parameter, and the volume fraction of inclusions is the coefficient of proportionality. The loss peak in the composite and the loss peak of inclusions are located at the same frequency. The concentration dependence of the effective parameter is linear over the entire frequency range.

Another possibility is when the inequality $n \chi_{\text {incl }}>>1$ holds at low frequencies. In this case, the frequency dispersion in the composite appears, when the absolute value of $n \chi$ incl is about unity. The loss peak in the composite is shifted towards higher frequencies as compared to the loss peak of inclusions. As the concentration of inclusions increases, the loss peak is shifted to the lower frequencies. At frequencies above the peak, the effective susceptibility is again proportional to the intrinsic susceptibility. At frequencies below the peak, the effective permeability depends mostly on the composite morphology and is independent of the intrinsic susceptibility. The concentration dependence of the effective susceptibility is nonlinear.

This case is typical for metal-dielectric mixtures. However, the conductivity of metals is usually too high to provide a loss peak of permittivity at microwaves. The microwave permittivity for most metal-dielectric composites may be considered as non-dispersive and low-loss. An exception is the percolation behavior, which will be discussed in Section 3.

If the frequency dependence of the intrinsic material parameter is Lorentzian (22) with $m=1$, and the mixing rule describing the composite is the MG, then the frequency dependence of the effective material parameter is Lorentzian as well. The parameters of the dispersion law for the effective material parameters are given by the simple equations [41]

$$
\begin{gathered}
\chi_{\text {st,eff }}=\frac{\chi_{\text {st,incl }} p}{\chi_{\text {st,incl }} n(1-p)+1}, \\
f_{\text {rel, eff }}=f_{\text {rel,incl }}\left(\chi_{\text {st,incl }} n(1-p)+1\right),
\end{gathered}
$$




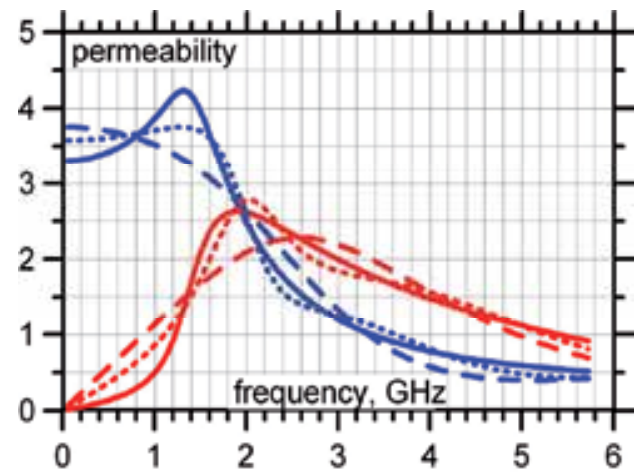

Figure 2. The frequency dependence of effective permeability of a composite calculated by the EMT (2) with $p=0.25$ and $n=1 / 3$ (solid curves, blue curve for real permeability and red curve for imaginary permeability). Inclusions in the composite exhibit the Lorentzian frequency dispersion (22) with $m=1$, $\chi_{\mathrm{st}}=100, f_{\mathrm{res}}=1 \mathrm{GHz}$, and $f_{\mathrm{rel}}=2 \mathrm{GHz}$. The dashed curves are the best fit of the solid line with the Lorentzian dispersion law with $m=1$, the dotted curves - with a sum of two Lorentzian terms, $m=2$.

and

$$
f_{\text {res,eff }}=f_{\text {res,incl }} \sqrt{\chi_{\text {st,incl }} n(1-p)+1},
$$

where subscript "incl" indicates the Lorentzian parameters of the intrinsic permeability of inclusions and subscript "eff" is related to the Lorentzian parameters of the effective susceptibility of composite. It is clearly seen from the equations that the non-linear concentration dependence of static susceptibility is accompanied by a low-frequency shift of both the characteristic frequencies. A general validation of this fact is given in the next subsection.

As is seen from Eqs. (25) and (26), the MG mixing rule retains the shape of effective susceptibility loss peak characteristic for the intrinsic susceptibility of inclusions. From the standpoint of the BM spectral theory, the reason is that additional loss due to mixing may arise over the entire range of effective form factors, where the spectral function has non-zero values. The spectral function for the MG mixing law is a delta-function, therefore, additional loss, which may distort the loss peak, does not appear.

Other mixing rules are characterized by a spectral function of a finite width and may therefore result in distorted shape of the loss peak. Figure 2 shows the frequency dependence of effective permeability of a composite calculated by the EMT (2). Inclusions in the composite are assumed to exhibit the Lorentzian frequency dispersion (22) with $m=1$. In the figure, the dashed curves are the best fit of the calculated permeability with the Lorentzian dispersion law with $m=1$, the dotted curves are obtained for the sum of two Lorentzian terms, $m=2$. It is seen that the EMT produces a large distortion of the Lorentzian dispersion curve, when the concentration is close to the percolation threshold. The distortion has a form of the increased loss at the high-frequency slope of the loss peak, because the spectral function peak for the EMT is extended to the region of large arguments, see Fig. $1 b$. 


\subsection{Integral relations for the frequency dependences in composites}

The low-frequency shift of the loss peak appearing with increasing volume fraction and accompanied by non-linear concentration dependence of static susceptibility is a general rule. Let us consider two integrals,

$$
I_{1}=\frac{2}{\pi} \int_{0}^{\infty} \chi^{\prime \prime} f d f \text { and } I_{2}=\frac{2}{\pi} \int_{0}^{\infty} \chi^{\prime} d f,
$$

which are analogous to the well-known sum rule for the Kramers-Kronig relations,

$$
\chi_{\mathrm{st}}^{\prime}=\frac{2}{\pi} \int_{0}^{\infty} \frac{\chi^{\prime \prime} d f}{f} .
$$

The difference between (28) and (27) is that the values of $I_{1}$ and $I_{2}$ are determined by the high-frequency asymptote of the susceptibility, rather than by the low-frequency asymptote, which defines the value of integral (28). In composites, this asymptotic behavior is governed by the LLL mixing law. Therefore, integrals $I_{1}$ and $I_{2}$ for any composite are equal to the corresponding values for the bulk material of inclusions multiplied by the volume fraction of inclusions $[42,43]$

$$
I_{i, \text { composite }}=p I_{i, \text { inclusions }} \text {. }
$$

Consideration of Eq. (29) makes sense if the integrals are convergent and have a non-zero value. For $I_{1}$, this is true for the Lorentzian dispersion law (22) that has the high-frequency asymptote given by:

$$
\chi(f) \approx-\chi_{\text {st }}\left(\frac{f_{\text {res }}}{f}\right)^{2}+\mathrm{i} \chi_{\text {st }}\left(\frac{f_{\text {res }}}{f}\right)^{3} \frac{f_{\text {res }}}{f_{\text {rel }}} .
$$

For $I_{2}$, the convergence is provided by the Debye dispersion law (9), which is the limiting case of (22) at $f_{\text {res } \rightarrow \infty}$ and has the high-frequency asymptote represented as:

$$
\chi(f) \approx \mathrm{i} \chi_{\mathrm{st}}\left(\frac{f_{\text {rel }}}{f}\right)+\chi_{\text {st }}\left(\frac{f_{\text {rel }}}{f}\right)^{2} .
$$

In the theory of magnetic material, these integrals are employed to validate ultimate values of high-frequency permeability. The corresponding constants for magnetic materials depend on the saturation magnetization of the material, $M_{\mathrm{s}}$. If the frequency dependence of effective permeability is either single-term Lorentzian or Debye, then the values of the integrals are related to the static magnetic susceptibility and the resonance frequency

$$
I_{1}=p \kappa\left(\gamma M_{s}\right)^{2} \approx \chi_{\text {st,eff }} f_{\text {res,eff }}^{2},
$$




$$
I_{2}=p \kappa\left(\gamma M_{s}\right) \approx \chi_{\text {st,eff }} f_{\text {rel,eff }} .
$$

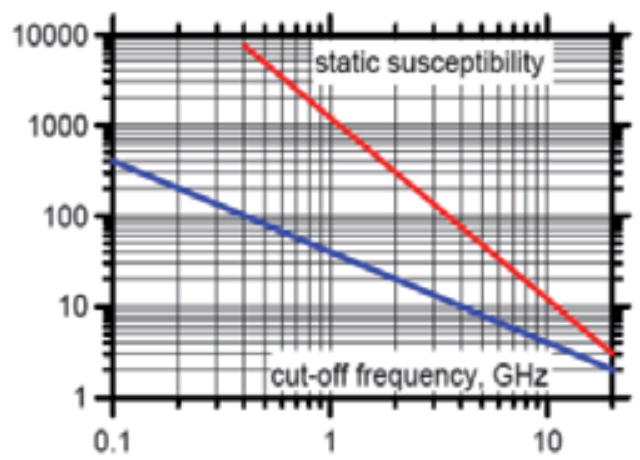

Figure 3. The static susceptibility as a function of cut-off frequency calculated with Acher's law (32) (red line, $\kappa=1 / 3$ ) and Snoek's law (33) (blue line, $\kappa=2 / 3$ ). In both the cases, $M_{s}=2.15 \mathrm{~T}, p=1$.

In Eqs. (32) and (33), $\gamma \approx 3 \mathrm{GHz} / \mathrm{kOe}$ is the gyromagnetic ratio, and $\kappa$ is the randomization factor. For $I_{2}$, typically $\kappa=2 / 3$; for $I_{1}$, different possibilities are discussed in [14]. Equations (32) and (33) represent the well known Acher's law [44] and Snoek's law [45], respectively. Then $\mathrm{I}_{1}$ has a meaning of Acher's constant, and $\mathrm{I}_{2}$ is Snoek's constant. For most materials, Snoek's law is valid, which involved Debye frequency dependence and integral $I_{2}$. For some materials, such as hexagonal ferrites and thin ferromagnetic films, Acher's law is valid, so that integral $I_{1}$ is calculated as Eq. (32), and much larger high-frequency permeability values can be obtained. The laws (32) and (33) are used for estimating high-frequency magnetic behavior of materials. A magnetic material may have high permeability value at frequencies below the cut-off frequency, which is the least of $f_{\text {res }}$ and $f_{\text {rel, }}$ where the permittivity falls to values close to unity. As the saturation magnetization of magnetic materials is typically below approximately $2 \mathrm{~T}$, it follows from (32) and (33) that magnetic materials with high static permeability are permeable at frequencies of microwave range or lower.

Figure 3 shows the ultimate values of the static magnetic susceptibility as a function of the cut-off frequency calculated with Acher's law (32), red line, and Snoek's law (33), blue line. In both cases, $M_{s}=2.15 \mathrm{~T}$ and $p=1$, which corresponds to a homogeneous sample of pure iron. For Snoek's law, $\kappa=2 / 3$; for Acher's law, $\kappa=1 / 3$ is accepted, which corresponds to random distribution of thin platelets. It is seen from the figure that with low values of the cut-off frequency, below $1 \mathrm{GHz}$, Acher's law enables a large advantage over Snoek's law in feasible values of the static permeability. At higher frequencies, this advantage eliminates, and both the laws permits rather small ultimate values of static permeability with cut-off frequencies of several dozen gigahertz.

For the permittivity of a metal-dielectric mixture, the frequency dependence is of Debye type, and an analogue of Snoek's law may be introduced. As $\varepsilon^{\prime \prime}$ incl $=2 \sigma / f$, where $\sigma$ is the conductivity of inclusions, the analogue of Snoek's constant for permittivity would be just the doubled conductivity of inclusions,

$$
I_{2}=2 p \sigma .
$$



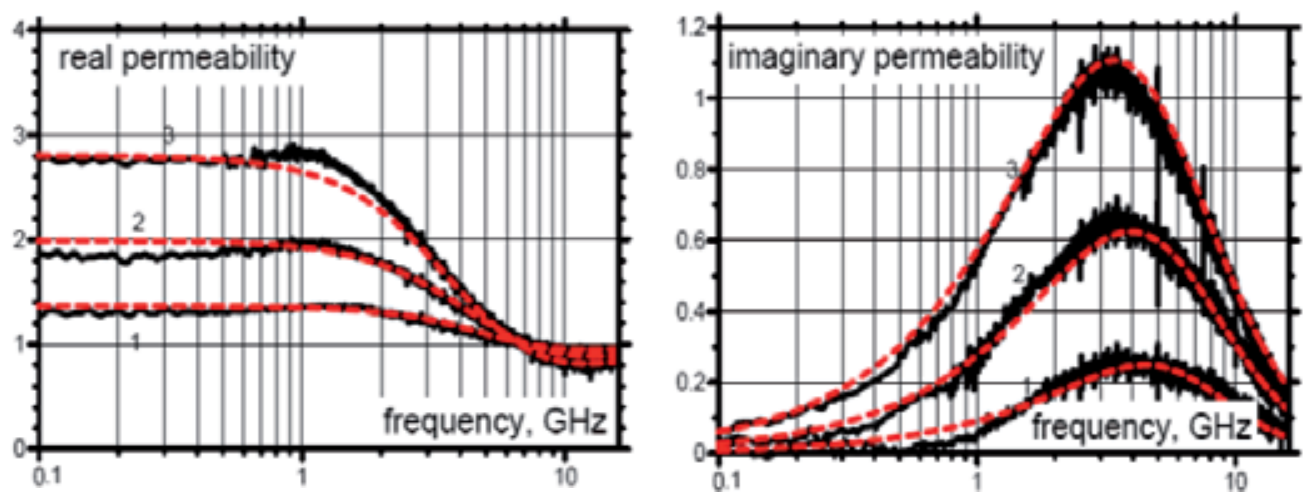

Figure 4. The measured frequency dependence of permeability of hexagonal ferrite composites (black curves, left: the real part, right: the imaginary part) for the $\mathrm{Co} Z \mathrm{Z}$ composites. The volume fractions of ferrite are: $1, p=0.1 ; 2, p=0.3$; and $3, p=0.5$. The red curves show the results of fitting the measured data with the Lorentzian dispersion law (22) with $m=1$ [41].
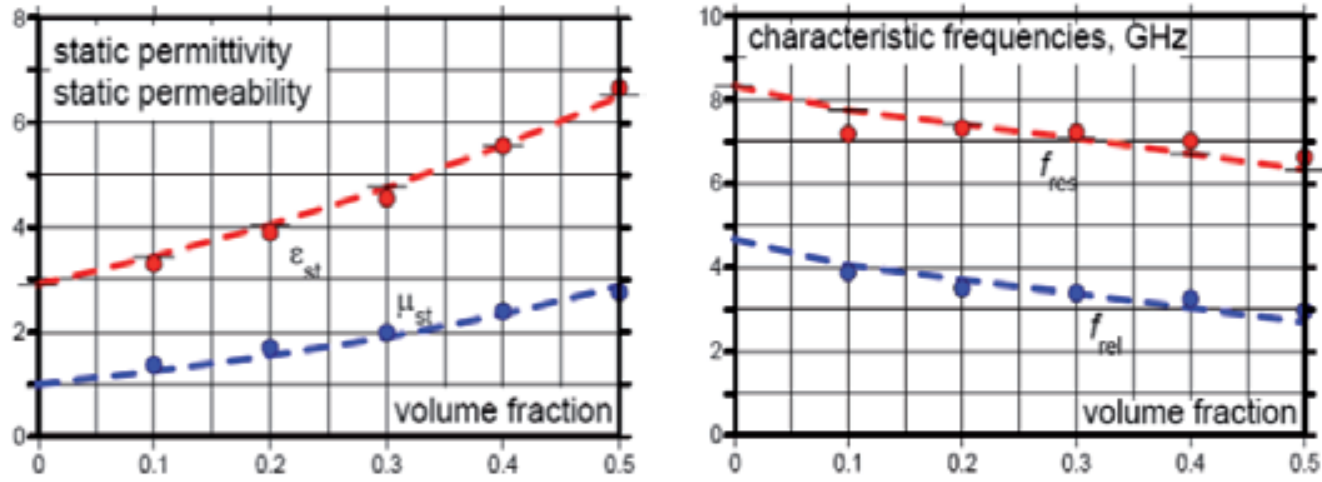

Figure 5. Left: the static permittivity (red dots) and static permeability (blue dots); right: the resonance frequency (red dots) and relaxation frequency (blue dots). The data are obtained for hexagonal ferrite composites by fitting the measured frequency dependences of permeability with the Lorentzian dispersion law (22) with $m=1$. The curves are the best fit of corresponding dots with Eqs. (24-26) [41].

\subsection{Applicability of the MG mixing rule}

The MG mixing rule usually agrees closely with the measured data, when $n \chi_{\text {incl }} \sim 1$. This is a frequent occasion for the microwave permeability of magnetic composites. The intrinsic permeability of magnetic materials does not exceed several units at microwaves due to the fast decrease with frequency, according to Snoek's and Acher's laws. With these relatively low intrinsic permeability values, the dependence of the effective material parameters on the shape of inclusions appears. In particular, a low-frequency shift of the loss peak is observed as $p$ increases. However, the dependence is still weak and may therefore be characterized by an averaged demagnetization factor $n$. 


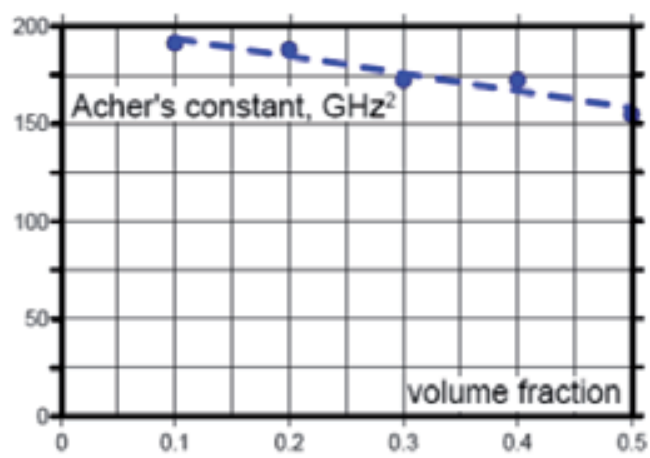

Figure 6. The measured ratio of Acher's constant to the volume fraction plotted against the volume fraction (dots). The line show the linear fit of the measured data [41].

An example of measured data having a good agreement with the MG mixing rule is taken from [41], where composites filled with powders of hexagonal ferrite have been studied. Figure 4 shows the measured microwave permeability for three of the samples. Application of the single-term Lorentzian dispersion law (22) provides a good agreement with the measured data for all volume fractions. This is seen from Fig. 5, where the static permittivity and the Lorentzian characteristic frequencies, obtained by the best fits of the measured magnetic dispersion curves, are plotted as functions of volume fraction. The curves in the figure are obtained by fitting the experimental points (dots) with Eqs. (24-26). For the bulk hexagonal ferrite, the retrieved static values are $\varepsilon_{\text {st,incl }}=16$ and $\mu_{\text {st,incl }}=11$, and $n \approx 0.33$, which indicates that the ferrite particles are of nearly spherical shape. Therefore, $n \chi_{\text {st,incl }}$ is the range from 3 to 5, and is reasonably close to unity. The measured data on the microwave material parameters of the composites under study agree with the MG mixing rule calculations, which is evidenced by close agreement of the dots and the fitting curves in Fig. 5.

However, an accurate analysis of the data reveals some disagreement. Acher's constant of the composites, calculated from the data for different volume fractions does not agree with Eq. (32). As Fig. 6 shows, Acher's constant depends on the volume fraction of inclusions, which should not be the case. The reason could be a distribution of shapes of individual inclusions that may result in deviation of the morphology from that postulated in the MG approach. This problem is discussed in more details in the next Subsection.

\subsection{Account for the distribution in shapes of inclusions}

A case, which may require a sophisticated mixing rule, is a composite filled with conducting ferromagnetic inclusions, whose both permittivity and permeability must be predicted, for example, to describe electromagnetic performance of the composite. In this case, two products of the form factor and the static susceptibility are involved, for the dielectric and magnetic susceptibility, which enlarges the range of variation of this value with a result of necessity for a more sophisticated theory to obtain better agreement between the measured data and theory. 

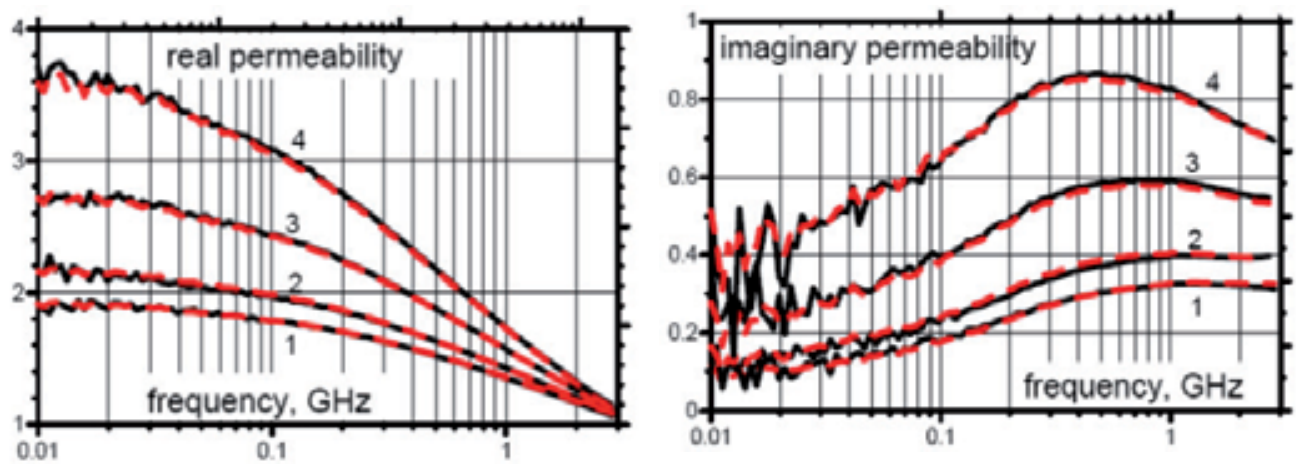

Figure 7. Black curves: the measured frequency dependencies of permeability of composites filled with milled iron powder (black lines), left - real permeability, right - imaginary permeability. Red curves fitting of the measured data with theory (35). Volume fractions of inclusions are $15.0 \%$ (1), $17.7 \%$ (2), $23.6 \%$ (3), $30.3 \%$ (4) [39].

Recently, a new theory, which unites the MG and EMT approaches, has been proposed [46]. This theory allows for introducing the percolation threshold through a general quadratic equation, the same as the EMT, postulating two requirements to the solution. The On the one hand, the solution must be consistent with the LLL mixing rule (3) for the case of low intrinsic material parameter; on the other hand, it should satisfy the Odelevskiy equation (21) for the case of intrinsic material parameter tending to infinity. This produces a unique solution for the equation, which can be considered as a new mixing rule, which generalizes the EMT and MG mixing rules,

$$
p \frac{\chi_{\text {eff }}}{\chi_{\text {incl }}}+\frac{1-p}{D p n} \frac{1}{1 / p-1 / p_{c}-1 / n / \chi_{\text {eff }}}=1
$$

where $D$ is the dimensionality of composite. Mixing rule (35) involves two fitting parameters: the effective form factor of inclusions, $n$, and the percolation threshold, $p_{\mathrm{c}}$, that can be found from the concentration dependence of the effective permittivity. In fact, these parameters are related to peculiarities of morphology of composites, such as the distribution of inclusions in shape.

The derivation of Eq. (35) is based on the assumption that the spectral function has a single wide peak. It is shown [46] that Eq. (35) allows for a variation of these parameters over the ranges,

$$
\begin{gathered}
\sqrt{4 / D}-1<n<1 / D \\
\frac{1}{2}\left(D n(1+n)-n \sqrt{D} \sqrt{D(1+n)^{2}-4}\right)<p_{\mathrm{c}}<1 .
\end{gathered}
$$

These conditions correspond to the case of nearly-spherical inclusions. Derivation of similar approach for composites filled with highly-elongated inclusions, such as thin platelets or fibers, must incorporate a spectral function comprising two separated peaks, which would require more sophisticated mathematical approaches. However, to develop such approach is 
not a challenging problem. As is shown above, the dilute limit approximation is sufficient for the analysis of microwave magnetic performance of such composites.

With the fitting parameters retrieved from the concentration dependence of permittivity, the intrinsic permeability of inclusions may be found from the measured effective permeability at each volume fraction of inclusions in the composite, as is described in [39]. An agreement of the data on the intrinsic permeability of inclusions found from different concentrations of inclusions provides an additional test for the validity of the mixing rule. It is found that the theoretical predictions agree closely with the measured microwave permittivity and permeability of composites filled with milled Fe powders [39], see Fig. 7. In the figure, the intrinsic permeability of inclusions was calculated for each concentration of inclusions, after which the average value was used to calculate the theoretical curve for each concentration. This is the reason for the noise observed in the theoretical curves in Fig. 7.

\section{Composites with fibrous inclusions}

\subsection{Measured microwave permittivity of fiber-filled composites}

Frequency dispersion of permittivity typically is not observed in composites over the microwave range. One of the rare examples of microwave dielectric dispersion is provided by composites filled with carbonized organic fibers. The conductivity of such fibers is much lower that that of metals. The thickness of the fibers is about a few microns, and their length can be on the order of several millimeters. The form factor of the fiber is very low, and the region of frequency dispersion may be at microwaves, as is seen in Fig. 8 [47].

Figure 8 shows the measured frequency dependence of permittivity for a composite filled with carbon fibers with length $l=1.5 \mathrm{~mm}$, thickness $d=8 \mu \mathrm{m}$, and resistivity of 10000 $\mathrm{Ohm} \times \mathrm{cm}$. The volume fraction of the fibers in the composite is $p=0.01 \%$. The sample is a sheet polymer-based composite of thickness of less than $1 \mathrm{~mm}$. Fibers are parallel to the sheet material plane, and they are distributed and oriented randomly in this plane. Experimental details are given in [47]. The frequency dependence of permittivity is of the Debye type. The low-frequency permittivity varies linearly with the volume fraction. The measured frequency and concentration dependences of permittivity agree well with the dilute limit approximation (4), written for the case under consideration as

$$
\varepsilon_{\text {eff }}=\varepsilon_{\text {host }}\left(1+p \kappa \frac{2 \mathrm{i} \sigma / f-\varepsilon_{\text {host }}}{\varepsilon_{\text {host }}+n\left(2 \mathrm{i} \sigma / f-\varepsilon_{\text {host }}\right)}\right),
$$

where $\sigma$ is the conductivity of the fibers, $\kappa$ is a factor describing the averaged polarizability of inclusions, and $n$ is the depolarization factor of the fibers,

$$
n=\frac{d^{2}}{l^{2}} \ln \frac{l}{d} .
$$




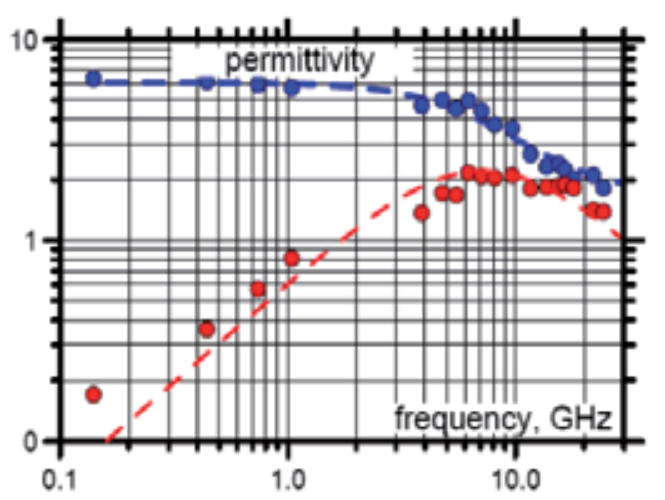

Figure 8. The measured frequency dependence of real (blue dots) and imaginary (red dots) permeability of a composite filled with carbon fibers of $1.5 \mathrm{~mm}$ in length with the resistivity of 10000 $\mathrm{Ohm} \times \mathrm{cm}$. The volume fraction of the fibers is $0.01 \%$ [47]. Curves are the result of fitting of measured data with the Debye dispersion law.

The value of $\kappa=1 / 3$ in Eq. (37) for the case under consideration, that is a product of the value of $1 / 2$, which accounts for the isotropic in-plane orientations of the fibers in a sheet sample, and the value of $2 / 3$, which accounts for cylindrical shape of fibers instead of ellipsoidal shape considered by the theories.

The type of the frequency dependence observed in fiber-filled composites is determined by the conductivity of fibers. In the general case, the dielectric dispersion curve is of the Lorentzian type with the parameters written as [48]

$$
\chi_{\mathrm{st}}=\frac{1}{3} \frac{\varepsilon_{\mathrm{host}} p}{\ln l / d}\left(\frac{l}{d}\right)^{2}, f_{\mathrm{rel}} \approx \frac{2 \sigma}{\varepsilon_{\mathrm{host}}}\left(\frac{d}{l}\right)^{2} \ln l / d, f_{\mathrm{res}} \approx \frac{c}{2 l \sqrt{\varepsilon_{\mathrm{host}}}}
$$

The resonance of the permittivity arises from the half-wavelength resonance excited within the fibers.

Figure 9 shows the measured frequency dependence of permittivity for a composite filled with aluminum-coated fibers of $10 \mathrm{~mm}$ long [47]. The volume fraction the fibers is $0.01 \%$. Due to high conductivity of the fibers, the frequency dependence of permittivity is of pronounced resonance (Lorentzian) type.

It is seen from Fig. 9 that the quality factor of the dielectric resonance is much lower than that predicted by Eq. (39). This is because Eq. (39) does not account for the radiation resistance of the fibers. The radiation resistance of a half-wavelength dipole is approximately $75 \mathrm{Ohm}$ in the free space, which is much larger than the ohmic resistance of the fiber, and contributes dominantly to the quality factor of the resonance.

In fact, such composites behave as a kind of a metamaterial over the frequency range near the resonance, because they contain inhomogeneities, whose characteristic dimensions are close to the wavelength, and the principal features of their dielectric dispersion depend on the resonance scattering on the fibers. This is also evidenced by the facts that the measured 
permittivity is less than that produced by the MG mixing law, and that the radiation resistance makes a dominant contribution into the quality factor of the dielectric resonance. Rigorously, metamaterials cannot be described in terms of effective material parameters. However, an experimental observation of deviation of microwave performance of the composites from Fresnel law has required special measurement conditions, see [49] for details.

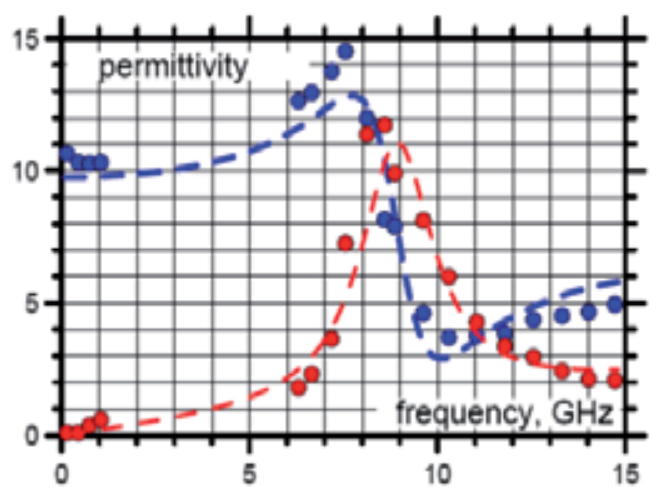

Figure 9. The measured frequency dependence of real (blue dots) and imaginary (red dots) permeability of a composite filled with aluminum-coated fibers $20 \mathrm{~mm}$ long. The volume fraction of the fibers is $0.01 \%$ [47]. Curves are the result of fitting of measured data with the Lorentzian dispersion law.

\subsection{Theories for the effective properties of fiber-filled composites}

A typical feature of fiber-filled composites is a low value of the percolation threshold: $p_{\mathrm{c}} \propto$ $d / l$, see [47] for the measured data. Although the percolation threshold is conventionally considered as a structure-dependent parameter, the dependence has been validated with composites based on a random mixture of conducting and non-conducting fibers, so that the dependence on agglomeration would be minimized. The standard EMT produces even lower values, $p_{\mathrm{c}} \propto(d / l)^{2}$, and a large disagreement with the measured permittivity values at concentrations close to the percolation threshold can be observed. Mixing rules for the fiberfilled composites were primarily aimed at obtaining proper dependence of the percolation threshold on the aspect ratio of fibers.

Historically, the first theories describing the effective properties of fiber-filled composites have been suggested in [37] and [50]. However, the theory [37] describes the case of infinite conductivity of inclusions and is not suitable for describing of frequency dependences in metal-dielectric composites. Theory [50] is a modification of the EMT. It is based on the assumption of strong anisotropy of the effective medium in the vicinity of a particular fiber, which results in the equations

$$
\frac{p}{3}\left(\frac{\varepsilon_{\mathrm{eff}, \mathrm{Il}}}{\varepsilon_{\mathrm{eff}, \mathrm{II}}+n\left(\varepsilon_{\mathrm{incl}}-\varepsilon_{\mathrm{eff}, \mathrm{II}}\right)}+\frac{2 \varepsilon_{\mathrm{eff}, \perp}}{\varepsilon_{\perp}+n_{\perp} \varepsilon_{\mathrm{incl}}\left(\varepsilon_{m}-\varepsilon_{\mathrm{eff}, \perp}\right)}\right)+\frac{(1-p) \varepsilon_{\mathrm{eff}, \perp}}{\varepsilon_{\perp}+n\left(\varepsilon_{\mathrm{host}}-\varepsilon_{\mathrm{eff}, \perp}\right)}=1,
$$




$$
\frac{p}{3}\left(\frac{\left(\varepsilon_{\mathrm{eff}, \mathrm{II}}-\varepsilon_{\mathrm{incl}}\right)}{\varepsilon_{\mathrm{eff}, \mathrm{II}}+n\left(\varepsilon_{\mathrm{incl}}-\varepsilon_{\mathrm{eff}, \mathrm{II}}\right)}+\frac{2\left(\varepsilon_{\mathrm{eff}, \perp}-\varepsilon_{\mathrm{incl}}\right)}{\varepsilon_{\perp}+n_{\perp}\left(\varepsilon_{\mathrm{incl}}-\varepsilon_{\mathrm{eff}, \perp}\right)}\right)+\frac{(1-p)\left(\varepsilon_{\mathrm{eff}, \perp}-\varepsilon_{\mathrm{host}}\right)}{\varepsilon_{\perp}+n\left(\varepsilon_{\mathrm{host}}-\varepsilon_{\mathrm{eff}, \perp}\right)}=0
$$
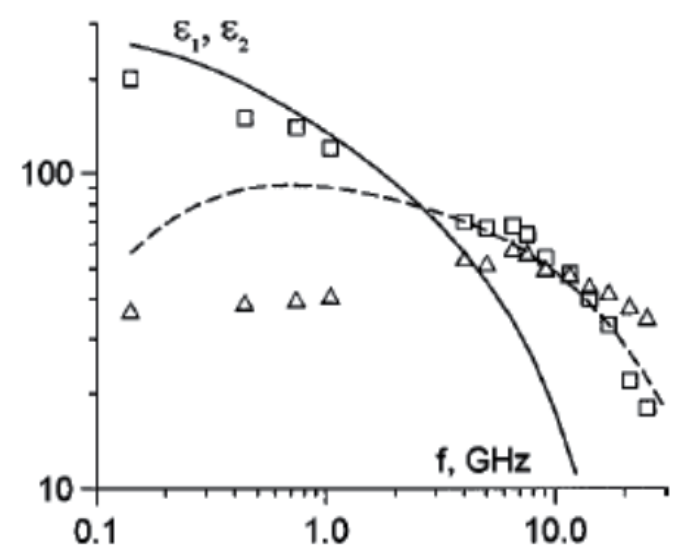

Figure 10. Dots: the measured dielectric dispersion curves for a composite filled with carbon fibers 1.8 $\mathrm{mm}$ long with the resistivity of $1400 \mathrm{Ohm} \times \mathrm{cm}$. The volume concentration of the fibers is $0.52 \%$. Curves: calculation by Eq. (42) [47].

The two equations (40) and (41) are used for the search of two values of the effective

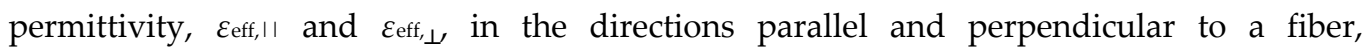
respectively; $n$ is given by Eq. (38), $n_{\perp}=(1-n) / 2$, and the observed effective permittivity of the

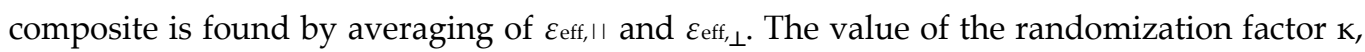
defined in the Subsection 2.1, equals to 1/3, and is substituted in Eqs. (40) and (41). The theory [50] predicts correctly the dependence of the percolation threshold on the aspect ratio of fibers, but disagrees with the dilute limit approximation, and, therefore, with the measured permittivity of composites at low concentrations of fibers.

Theory [51] allows for a better quantitative agreement over a wide range of volume fractions below the percolation threshold. The theory is based on the assumption that, in the vicinity of a particular fiber, the permittivity of effective medium $\varepsilon^{*}$ is a function of the distance from to fiber $z$ :

$$
\begin{aligned}
& \varepsilon^{*}(z)=\varepsilon_{\text {host }}+\left(\varepsilon_{\text {eff }}-\varepsilon_{\text {host }}\right)(z / a x) \text { at } \quad z<a x \\
& \varepsilon^{*}(z)=\varepsilon_{\text {host }} \text { at } z \geq a x
\end{aligned}
$$

where $x$ is a parameter of the theory. This assumption results in the EMT equation written as

$$
\frac{\varepsilon_{\text {incl }}}{3 \varepsilon_{\text {eff }}} \frac{p}{1+\left(d^{2} \varepsilon_{\text {incl }} / l^{2} \varepsilon_{\text {host }}\right) \ln \left(1+\left(l x \varepsilon_{\text {host }}\right) /\left(d \varepsilon_{\text {eff }}\right)\right)}+3 \frac{\varepsilon_{\text {host }}-\varepsilon_{\text {eff }}}{\varepsilon_{\text {host }}+2 \varepsilon_{\text {eff }}}=0,
$$

which is conventionally used in microwave studies of fiber-filled composites, see, e.g., [52]. 
There is lack of measured data on the frequency-dependent dielectric performance of fiberfilled composites near the percolation threshold in the literature; one of examples of the data is given in Fig. 10 [45]. It is seen from the figure that the EMT predicts a gradual shift of the loss peak. The measured dielectric loss peak differs from that predicted by the theory. In contrast, the measured variation of the loss peak appearing as approaching the percolation threshold looks like a rise of the low-frequency loss level, with a well-defined trace of the loss peak associated with individual fibers.

This difference between the theory and measurements may be associated not with the geometrical distribution of shapes of conducting clusters, as the percolation theory suggests, but with other low-frequency loss mechanisms near the percolation threshold. For example, imperfect electric contacts between fibers comprising a conductive cluster may contribute to the low-frequency loss [20]. The conductivity of such contacts must be much lower than the conductivity of the fibers. Therefore, imperfect contacts may result in a large low-frequency shift of dielectric loss. Because of a low value and wide distribution of the conductivity of contacts, this loss forms a very smooth dispersion curve, which is seen in Fig. 10.

The same may be true for composites filled with carbon black or carbon nanotubes, which are known to have the percolation type of frequency dispersion at microwaves, see, e.g., [20]. Dielectric loss appearing at low frequencies might be associated with very prolate conductive clusters, if the conductivity of clusters is on the same order of magnitude as the conductivity of inclusions. Account for the imperfect contacts would allow for more realistic assumptions on the shape of conducting clusters.

In principle, the effect of contacts may be understood as a presence of a comparatively lowconductive shell covering the surface of conducting inclusions. For an individual inclusion, the presence of such a shell leads to a low-frequency shift of the loss peak, without change in its shape. To get an agreement with the measured data, a distribution of these conductivities should be included in the model. By the analogy to the Cole-Cole dispersion law, such distribution would results in the power frequency dependences of the permittivity. Hence the difference between measured critical indices and universal values derived from geometrical considerations can be observed, but there is no theory explaining and quantifying such phenomena in the literature.

The available data of the microwave permeability of composites filled with magnetic fibers are consistent with the dilute limit approximation $[53,54]$.

\section{Conclusions}

The problem of describing of the effective permittivity as a function of concentration of inclusions in a metal-dielectric mixture is well studied. However, newly developed mixing rules still appear in the literature. This means that the solution for the problem is not satisfactory to some extent, and is typically related to the description of frequency dependences of material parameters. 
The above consideration allows for determining the validity limits of various mixing rules. These limits are dependent on the difference between the susceptibilities of inclusions and the host matrix, and on the elongation of inclusions.

For microwave permeability, the difference is typically not high, and the effective properties of composites are well described by the MG mixing rule. For lower frequencies, the intrinsic permeability may be high, and a more sophisticated mixing rule may be needed. For composites containing platelet and fibrous magnetic inclusions, the microwave permeability is described by the dilute limit approximation. The same is true for composites with dielectric fibers.

For microwave permittivity of a metal-dielectric mixture, the difference is typically large, and the effective properties are determined by the morphology of the composite. But fitting of measured data to the theoretical results is typically rather simple, because the frequency dispersion of permittivity is a rare occasion at microwaves. In metal-dielectric composites, the region of frequency dispersion is located at much higher frequencies, as can be estimated from typical conductivity of metals and feasible dimensions of inclusions.

For simultaneous modeling of the permittivity and permeability of composites with conducting inclusions, sophisticated mixing rules are unavoidable, with an account for a distribution of inclusions in shape. This case is the most difficult, because both concentration and frequency dependences of material parameters may be non-trivial.

\section{Author details}

Konstantin N. Rozanov

Institute for Theoretical and Applied Electromagnetics, Russian Academy of Sci., Moscow, Russia,

Marina Y. Koledintseva

Missouri University of Science and Technology, Rolla, MO, USA

Eugene P. Yelsukov

Physical and Technical Institute, Ural Branch of Russian Academy of Sci., Izhevsk, Russia

\section{Acknowledgement}

K. Rozanov acknowledges the partial financial support of the work from the RFBR, grants no. 12-02-91667 and 12-08-00954. M. Koledintseva acknowledges the partial support by the U.S. NSF Grant No. 0855878. The authors also thank Alexei Koledintsev for his assistance and valuable comments regarding technical English writing.

\section{References}

[1] Koledintseva MY, Rozanov KN, Drewniak JL (2011) Engineering, modeling and testing of composite absorbing materials for EMC applications, In: Adv. in Composite 
Materials - Ecodesign and Analysis, ed. B. Attaf, InTech, ISBN 978-953-307-150-3, Ch. 13, pp. 291-316.

[2] Garnett JCM (1904) Colours in Metal Glasses and in Metallic Films. Phil. Trans. R. Soc. Lond. 203: 385-420.

[3] Bruggeman DAG (1935) Berechnung Verschiedener Physikalischer Konstanten von Heterogenen Substanzen. Ann. Phys. (Leipzig) 24: 636-679.

[4] Landau LD, Lifshitz EM (1984) Electrodynamics of Continuous Media, Pergamon, 474 p.

[5] Looyenga H (1965) Dielectric Constants of Heterogeneous Mixtures. Physica. 31: 401-406.

[6] Born M, Wolf E (1986) Principles of Optics. 6 Ed., Pergamon, 854 p.

[7] Polder D, van Santen JH (1946) The Effective Permeability of Mixtures of Solids. Physica. 12: 257-271.

[8] Bergman DJ, Stroud D (1992) Physical Properties of Macroscopically Inhomogeneous Media. Solid. State Phys. 46: 147-269.

[9] Hashin Z, Shtrikman S (1962) A Variational Approach to the Theory of the Effective Magnetic Permeability of Multiphase Materials J. Appl. Phys., 33: 3125-3131.

[10] Sareni B, Krahenbuhl L, Beroual A, Brosseau C (1996) Effective Dielectric Constant of Periodic Composite Materials. J. Appl. Phys. 80: 1688-1696.

[11] Sihvola AH (1999) Electromagnetic Mixing Rules and Applications. IET, 284 p.

[12] Torquato S, Hyun S (2001) Effective Medium Approximation for Composite Media: Realizable Single-Scale Dispersions. J. Appl. Phys. 89: 1725-1729.

[13] Reynolds JA, Hough JM (1957) Formulae for Dielectric Constant of Mixtures. Proc. Phys. Soc. B 70: 769-775.

[14] Lagarkov AN, Rozanov KN (2009) High-Frequency Behavior of Magnetic Composites. J. Magn. Magn. Mater. 321: 2082-2092.

[15] Day AR, Grant AR, Sievers AJ, Thorpe MF (2000) Spectral Function of Composites from Reflectivity Measurements. Phys. Rev. Lett. 84: 1978-1981.

[16] Theiss W (1996) The Dielectric Function of Porous Silicon - How to Obtain It and How to Use It. Thin Solid Films. 276: 7-12.

[17] Ghosh K, Fuchs R (1988) Spectral Theory for Two-Component Porous Media. Phys. Rev. B: 38 5222-5236.

[18] Osipov AV, Rozanov KN, Simonov NA, Starostenko SN (2002) Reconstruction of Intrinsic Parameters of a Composite from the Measured Frequency Dependence of Permeability. J. Phys.: Condens. Matter 14: 9507-9523.

[19] Goncharenko AV, Lozovski VZ, Venger EF (2000) Lichtenecker's Equation: Applicability and Limitations. Optics. Commun. 174: 19-32.

[20] Liu L, Matitsine S, Gan YB, Chen LF, Kong LB, Rozanov KN (2007) Frequency Dependence of Effective Permittivity of Carbon Nanotube Composites. J. Appl. Phys. 101: 094106. 
[21] Jonscher AK (1983) Dielectric Relaxation in Solids, Chelsea Dielectrics Press, 1983, 380 p.

[22] Mattei JL, Le Floc'h M (2003) Percolative Behaviour and Demagnetizing Effects in Disordered Heterostructures. J. Magn. Magn. Mater. 257: 335-345.

[23] Pitman KC, Lindley MW, Simkin D, Cooper JF (1991) Radar Absorbers: Better by Design. IEE Proc. F - Radar and Signal Processing 138: 223-228.

[24] Miyasaka K, Watanabe K, Jojima E, Aida H, Sumita M, Ishikawa K (1982) Electrical Conductivity of Carbon-Polymer Composites as a Function of Carbon Content. J. Mater. Sci. 17: 1610-1616.

[25] Duan HL, Karihaloo BL, Wang J, Yi X (2006) Effective Conductivities of Heterogeneous Media Containing Multiple Inclusions with Various Spatial Distributions. Phys. Rev. B 73: 174203.

[26] Gao L, Gu JZ (2002) Effective Dielectric Constant of a Two-Component Material with Shape Distribution. J Phys. D - Appl. Phys. 35: 267-271.

[27] Goncharenko AV (2003) Generalizations of the Bruggeman Equation and a Concept of Shape-Distributed Particle Composites. Phys. Rev. E 68: 041108.

[28] Koledintseva MY, Chandra SKR, DuBroff RE, Schwartz RW (2006) Modeling of Dielectric Mixtures Containing Conducting Inclusions with Statistically Distributed Aspect Ratio. PIER 66: 213-228.

[29] Spanoudaki A, Pelster R (2001) Effective Dielectric Properties of Composite Materials: The Dependence on the Particle Size Distribution. Phys. Rev. B 64: 064205.

[30] Koledintseva MY, DuBroff RE, Schwartz RW, Drewniak JL (2007) Double Statistical Distribution of Conductivity and Aspect Ratio of Inclusions in Dielectric Mixtures at Microwave Frequencies. PIER 77: 193-214.

[31] Koledintseva MY, DuBroff RE, Schwartz RW (2009) Maxwell Garnett Rule for Dielectric Mixtures with Statistically Distributed Orientations of Inclusions. PIER 99 131-148.

[32] Lichtenecker K (1926) Die Dielektrizitätskonstante Natürlicher und Künstlicher Mischkörper. Physikal. Z. 27: 115-158.

[33] McLachlan DS, Priou A, Chenerie I, Isaak E, Henry F (1992) Modeling the Permittivity of Composite Materials with a General Effective Medium Equation. J. Electromagn. Waves Appl. 6: 1099-1131.

[34] Doyle WT, Jacobs IS (1990) Effective Cluster Model of Dielectric Enhancement in MetalInsulator Composites. Phys. Rev. B 42: 9319-9327.

[35] Sheng P (1980) Theory for the Dielectric Function of Granular Composite Media. Phys. Rev. Lett. 45: 60-63.

[36] Musal HM, Hahn HT, Bush GG (1988) Validation of Mixture Equations for DielectricMagnetic Composites. J. Appl. Phys. 63: 3768-3770.

[37] Doyle WT, Jacobs IS (1992) The Influence of Particle Shape on Dielectric Enhancement in Metal-Insulator Composites. J. Appl. Phys. 71: 3926-3936.

[38] Odelevskiy VI (1947) The Calculation of Generalized Conductivity of Heterogeneous Systems. Ph. D. Thes., Moscow, 110 p. 
[39] Rozanov KN, Osipov AV, Petrov DA, Starostenko SN, Yelsukov EP (2009) The Effect of Shape Distribution of Inclusions on the Frequency Dependence of Permeability in Composites. J. Magn. Magn. Mater. 321: 738-741.

[40] Li M, Feteira A, Sinclair DC, West AR (2007) Incipient Ferroelectricity and Microwave Dielectric Resonance Properties of $\mathrm{CaCu}_{2.85} \mathrm{Mn}_{0.15} \mathrm{Ti}_{4} \mathrm{O}_{12}$ Ceramics. Appl. Phys. Lett. 91: 132911.

[41] Rozanov KN, Li ZW, Chen LF, Koledintseva MY (2005) Microwave Permeability of Co2Z Composites. J. Appl. Phys. 97: 013905.

[42] Lagarkov AN, Osipov AV, Rozanov KN, Starostenko SN (2005) Microwave Composites Filled with Thin Ferromagnetic Films. Part I. Theory. Proc. Symp. R: Electromagn.. Mater., $3^{\text {rd }}$ Int. Conf. on Mater. Adv. Technol. (ICMAT 2005), Jul. 3-8, 2005, Singapore, pp. 74-77.

[43] Acher O, Dubourg S (2008) Generalization of Snoek's Law to Ferromagnetic Films and Composites. Phys. Rev. B 77: 104440.

[44] Snoek JL (1948) Dispersion and Absorption in Magnetic Ferrites at Frequencies above 1 Mc/s. Physica 14: 207-217.

[45] Acher O, Adenot AL (2000) Bounds on the Dynamic Properties of Magnetic Materials. Phys. Rev. B 62: 11324.

[46] Rozanov KN, Koledintseva MY, Drewniak JL (2012) A Mixing Rule for Predicting of Frequency Dependence of Material Parameters in Magnetic Composites J. Magn. Magn. Mater. 324: 1063-1066.

[47] Lagarkov AN, Matytsin SM, Rozanov KN, Sarychev AK (1998) Dielectric Properties of Fiber-Filled Composites. J. Appl. Phys. 84: 3806-3814.

[48] Matitsine SM, Hock KM, Liu L, Gan YB, Lagarkov AN, Rozanov KN (2003) Shift of Resonance Frequency of Long Conducting Fibers Embedded in a Composite. J. Appl. Phys. 94: 1146-1154.

[49] Vinogradov AP, Machnovskii DP, Rozanov KN (1999) Effective Boundary Layer in Composite Materials. J. Communic. Technology Electr. 44: 317-322.

[50] Lagarkov AN, Sarychev AK, Smychkovich YR, Vinogradov AP (1992) Effective Medium Theory for Microwave Dielectric Constant. J. Electromagn. Waves Appl. 6: 1159-1176.

[51] Lagarkov AN, Sarychev AK (1996) Electromagnetic Properties of Composites Containing Elongated Conducting Inclusions. Phys. Rev. B 53: 6318-6336.

[52] Makhnovskiy DP, Panina LV, Mapps DJ, Sarychev AK (2001) Effect of Transition Layers on the Electromagnetic Properties of Composites Containing Conducting Fibres. Phys. Rev. B 64: 134205.

[53] Liu L, Kong LB, Lin GQ, Matitsine S, Deng CR (2008) Microwave Permeability of Ferromagnetic Microwires Composites/Metamaterials and Potential Applications. IEEE Trans. Magn. 44: 3119-3122. 
[54] Han MG, Liang DF, Deng LJ (2011) Fabrication and Electromagnetic Wave Absorption Properties of Amorphous Fe79Si16B5 Microwires. Appl. Phys. Lett. 99: 082503. 


\title{
The Lightweight Composite Structure and Mechanical Properties of the Beetle Forewing
}

\author{
Jinxiang Chen, Qing-Qing Ni and Juan Xie \\ Additional information is available at the end of the chapter
}

http://dx.doi.org/10.5772/48807

\section{Introduction}

The development of lightweight, energy-saving structures is believed to be one key solution to current world problems, such as increasing population, resource shortages, and environmental pollution. Acquiring inspiration from living creatures is an effective approach because these forms have evolved over millions of years to adapt to the natural environment [Thompson, 1945]. Examples of evolutionary adaptations provide evidence of how nature has helped organisms to overcome their structural weaknesses [Thompson, 1945; Wainwright et al., 1976; Hepburn, 1976]. Motivated by our insight that the forewings of beetles should have both high strength and minimal weight, which are needed both for defense and for flight, the first author of this paper has been studying their architecture since 1997 [Chen et al., 2000; Chen et al., 2001a; Chen et al., 2007a; Chen et al., 2007b]. However, beetle forewings were considered nonliving materials in early work [Ishii, 1982], which may be the reason why research on beetle forewings and their biomimetic applications has been limited to two-dimensional fiber orientations [Gullan \& Cranstion, 1994; Zelazny \& Neville, 1972]. To date, we have comprehensively investigated their threedimensional structures and obtained new information on their biological morphology [Chen et al., 2007b; Chen et al., 2001a; Chen et al, 2002], and we have gained new insight into their mechanical properties [Chen et al., 2000; Chen et al., 2007a; Ni et al., 2001]. Our investigations have not only confirmed the above insights but also led us to discover a new type of lightweight biomimetic composite that is more complicated and more delicate than the presently used honeycomb structure. This new composite features a completely integrated honeycomb structure with fiber-reinforced trabeculae at the corners of the honeycomb cores [Chen et al., 2005; Chen et al., 2012].

In this chapter, we present insight into the lightweight composite structure of the beetle forewing, including its mechanical properties and practical applications. 


\section{The lightweight composite structure of the beetle forewing}

\subsection{Experiment}

Two species of adult beetles, $A$. dichotoma and $P$. inclinatus, were used as experimental samples and are shown in Fig. 1(a) and (b), respectively.

Since living samples were used, their careful treatment by specialized methods to ensure that their structures would remain unchanged during the sample preparation process was highly important. Here, three specialized treatments were used for comparison of the forewing structural damage caused by removal from the living beetle body: (1) living samples (tests are conducted within 1-3 minutes); (2) traditional mistreatment; and (3) the living samples left at room temperature for one day or, in some cases, even several months. The results show that by using method (3), which may result in drying due to loss of water and partial damage of the cell membrane, the original structural integrity of the forewing still remains [Chen et al., 2002]. In this experiment, unless otherwise noted, method (3) was used for sample preparation.

The observation positions were set using Cartesian coordinates, and the names of the different regions of the forewing are shown in Fig. 1(c). The cross sections were prepared by slicing the forewing along the $\mathrm{X}$ or $\mathrm{Y}$ line, after which the inner structure of the forewing was observed by removing a portion of the lower lamination with forceps.
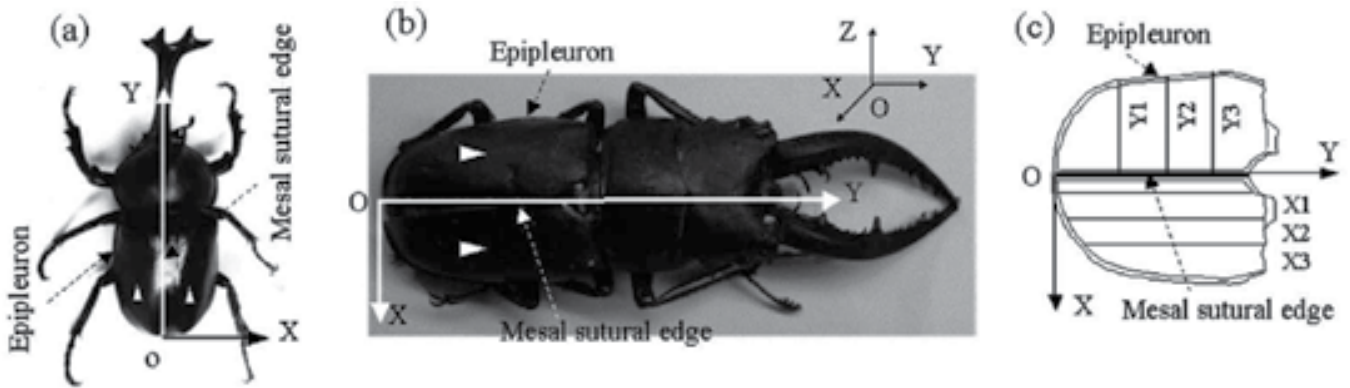

Figure 1. Two species of adult beetle and the Cartesian coordinates ( $\triangle$, the fore-wings). (a) male $A$. dichotoma, (b) male P. inclinatus, (c) observation sites.

A strong alkali can be used to examine the composition of the forewing; the chitin fibers do not readily dissolve in the alkaline solution, while the proteins dissolve easily. Once the protein matter has been removed by the alkali solution, the structures of the trabeculae can be readily observed. Therefore, some samples were prepared by boiling in a $10 \% \mathrm{KOH}$ solution [Zelazny \& Neville, 1972] for three hours. This treatment does not affect the orientation of the chitin fibers.

The main experimental instruments used in this study were an environmental scanning electron microscope (Nikon ESEM-2700 at $20 \mathrm{kV}$ and $460 \mathrm{~Pa}$ ) and a general SEM (Hitachi, S510 at $15 \mathrm{kV})$. 


\subsection{Results and discussion}

\subsubsection{General structure of the beetle forewing}

The forewing photographs of A. dichotoma are presented in Fig. 2(a-e). Fig. 2 (a, c) and Fig. 2 $(b, d, e)$ contain images captured with a camera under a penetrating light with a short focus
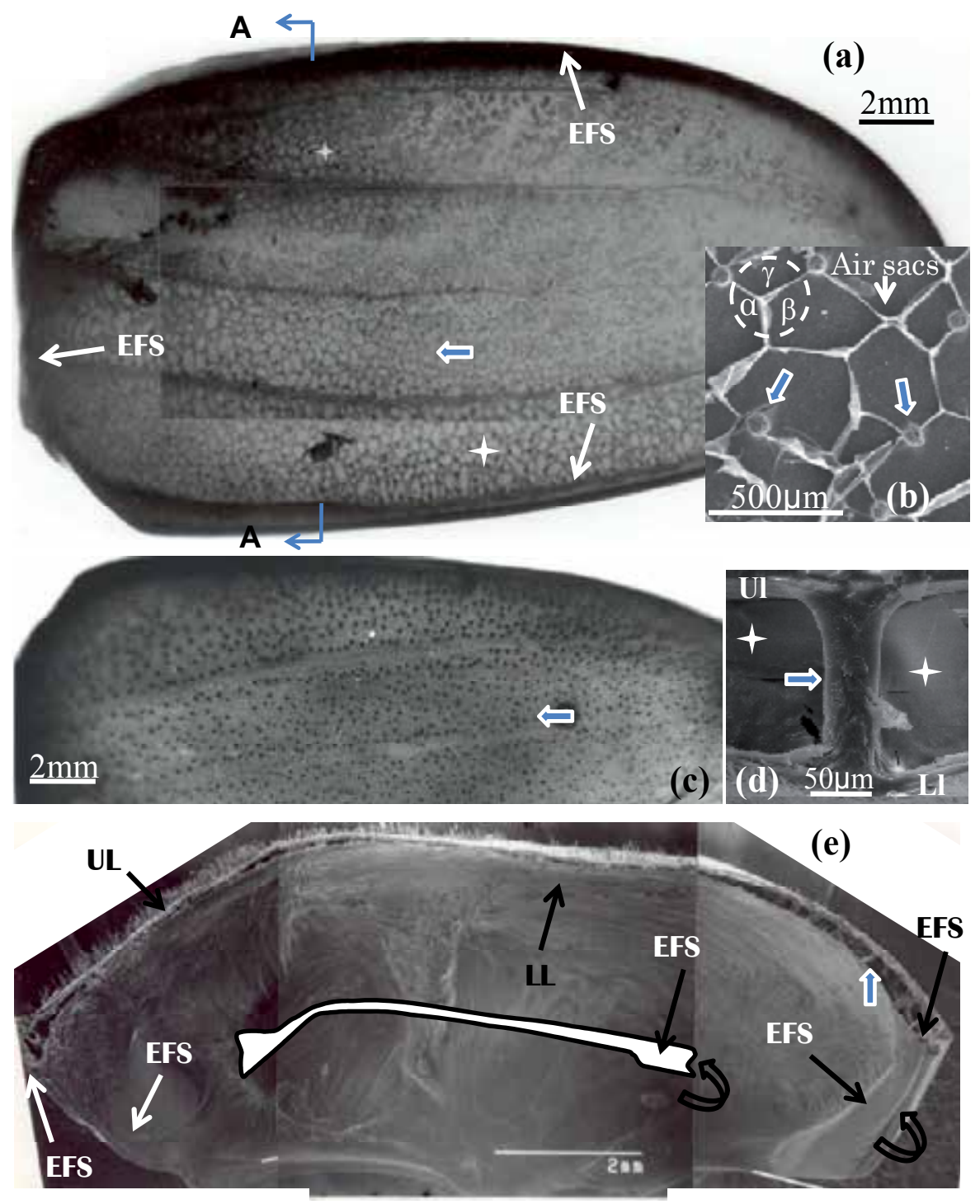

Figure 2. The integrated structure and edge sealing of the forewing of A. dichotoma. (a) Meshwork and trabeculae (dots) in a fresh forewing, (b) the internal structure and the lower lamination were eliminated by forceps, (c) distribution of trabeculae (dots) in the forewing (following $10 \% \mathrm{KOH}$ treatment), (d) a trabecula, (e) the cross sectional view of the A-A section, and the middle part of the figure is a simple sketch of the cross section. EFS: edge frame structure, asterisk: air sacs, thick arrows: trabeculae, round arrows: concavo-convex junction, Ul: upper lamination, Ll: lower lamination. 
time and with a SEM, respectively. The many lines Fig. 2(a) apparent in those photos are actually air sacs or tracheae [Chen et al., 2002]. The images in Fig. 2 show the samples after three hours of boiling in the $10 \% \mathrm{KOH}$ solution. The air sacs and the tracheae that can be observed in Fig. 2(a) have disappeared here because they were dissolved during the $\mathrm{KOH}$ treatment. However, many black solid dots (Fig. 2c) are visible, each of which represents an individual trabecula [Chen et al., 2002].

As shown in Fig. 2 (e), the entire beetle forewing is composed of a single type of frame structure. Fig. 2 (e) shows an electron micrograph of this frame structure at an X-Z plane cross section (see Fig. 1). It was found that the forewing cross section consists of three subdivisions; the upper lamination, the lower lamination and the central void, with the large voids located at both the mesal sutural and the epipleuron edges (see Fig. 1). The middle portion of the forewing is a sandwich structure containing a wide void area.

\subsubsection{Trabecular structure in the beetle forewing}

The longitudinal shapes of typical forewing trabeculae in A. dichotoma are shown in Fig. 3 (a, b), and a cross section is shown in Fig. 3(c). The trabecular size and shape can change according to location in the forewing. Both trabeculae with a straight shape (Fig. 3a) and with a curved shape (Fig. 3b) were observed, although the majority of the trabeculae were of the straight shape kind. The cross section of any trabecula is similar to the circular structure presented in Fig. 3(c). The diameter of a trabecula is larger near the locations of joints with the upper and lower laminations and is smaller in the void region between.
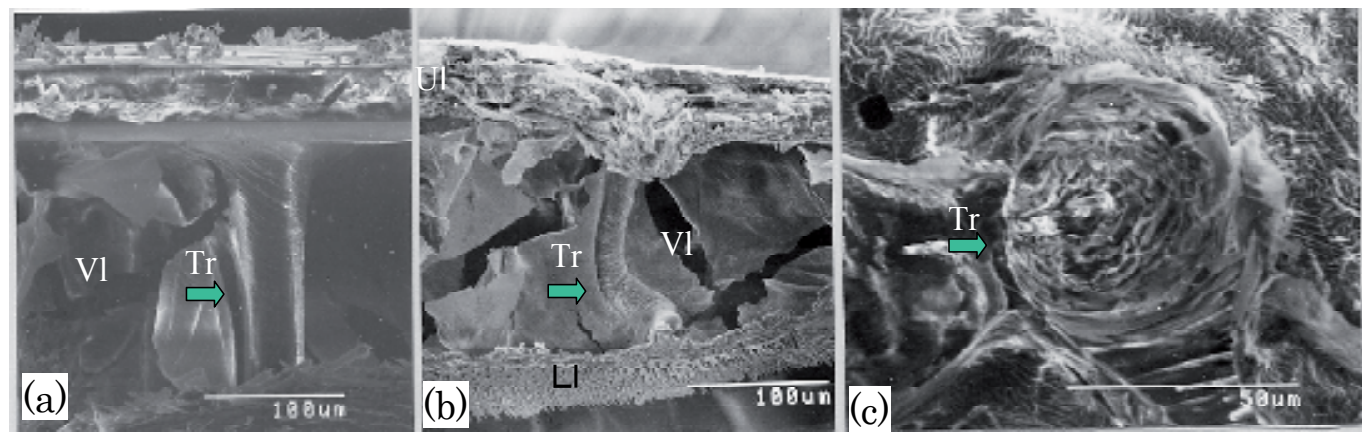

Figure 3. The trabecular ( $\mathrm{Tr}$ ) shapes. (a) Straight cylinder, (b) curve cylinder, (c) section view. Ul, upper lamination, $\mathrm{Vl}$, void lamination, $\mathrm{Ll}$ : lower lamination.

Fig. 4 shows longitudinal sections of the trabeculae, which allow for clear observation of the chitin fiber arrangement within the trabeculae. In Fig. 4(a), the chitin fibers $\mathrm{Fb}$ (or marked $t$ ) form a distinct layer within the upper lamination. It should be noted that the fiber angle within this layer is nearly perpendicular (at 90 degrees) to the paper and that the neighboring chitin fiber layers (Fl or $\searrow$ ) are instead horizontally arranged and connected with the trabecula in a curving manner. This connection between the chitin fiber layers in the upper lamination and the trabecula can also be seen clearly in Fig. 4(b). Fig. 5 shows micrographs of trabeculae after having been boiled in the $10 \% \mathrm{KOH}$ solution for three hours. 
Due to removal of the protein matter, the chitin fiber arrangement on the outside of the trabeculae can easily be observed. The chitin fibers in the left image (Fig. 5a) are in a straight arrangement, while the fibers in the right image (Fig. 5b) have a spiral arrangement on the trabecular surface. The central regions of the trabeculae become hollow (Fig. 5c) due to dissolution of the internal proteins during the $\mathrm{KOH}$ solution treatment. Thus, the central portion of the trabecular shaft is composed mainly of protein [Chen et al., 2001b].
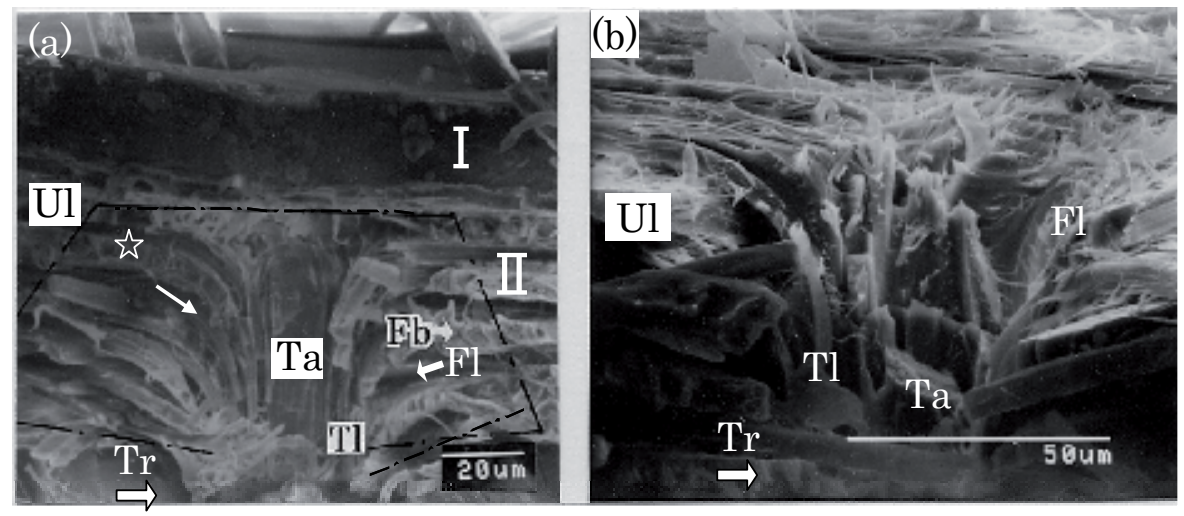

Figure 4. The trabecular structure in the forewing of A. dichotoma. (a) longitudinal section with the trabecular root marked by the lines; (b) a naturally rupture trabecula. Ul, upper lamination. $\mathrm{Fl}$, fibers from left to right. $\mathrm{Fb}$, fibers from front to back. $\mathrm{Ta}$, trabecular central part. $\mathrm{Tl}$, trabecular cylindrical layers. $\mathrm{Tr}$, trabecula.

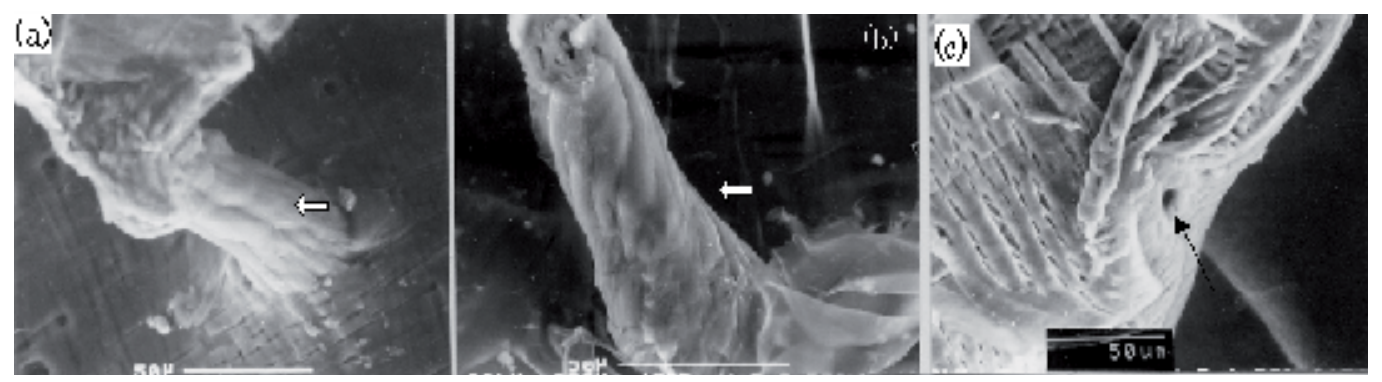

Figure 5. Trabecular structures treated with $\mathrm{KOH}$, (a) the straight orientation (b) the spiral curved orientation of the chitin fiber at the outside, $(c)$ the hollow $(\kappa)$ at the center of the trabecular root.

\subsubsection{Schematic model of the forewing structure}

Based on these observations and analyses of the beetle forewing and its inner trabecular structures, it is evident that the general forewing structure in A. dichotoma is a sandwich frame structure with a central void region. However, the details of the forewing microstructure are extremely complex. These models for the forewing and trabecula structures are proposed to assist in the development of biomimetic composite structures. According to our observations, we have developed a sandwich plate structure model with a central void layer and trabecular struts [Chen et al., 2005]. It is well known that sandwich plates with a central void layer are typical of lightweight structures and already have been applied in many fields [Jung \& Aref, 
2005; Matsunaga, 2002; Nguyen et al., 2005; Mania, 2005; Hosur et al., 2005]. The presence of the sandwich plate structures with trabeculae in the beetle forewings indicate that the beetle wings have been evolutionarily optimized for competition in nature.

Fig. 6 shows the sandwich plate model that was inspired by the forewings of the beetles. The plate consists of upper and lower lamination layers and a core layer with trabeculae and honeycomb walls (Fig. 6a). There are many trabeculae positioned throughout the honeycomb, and they are located at the corners of the honeycomb units (Fig. 6b).
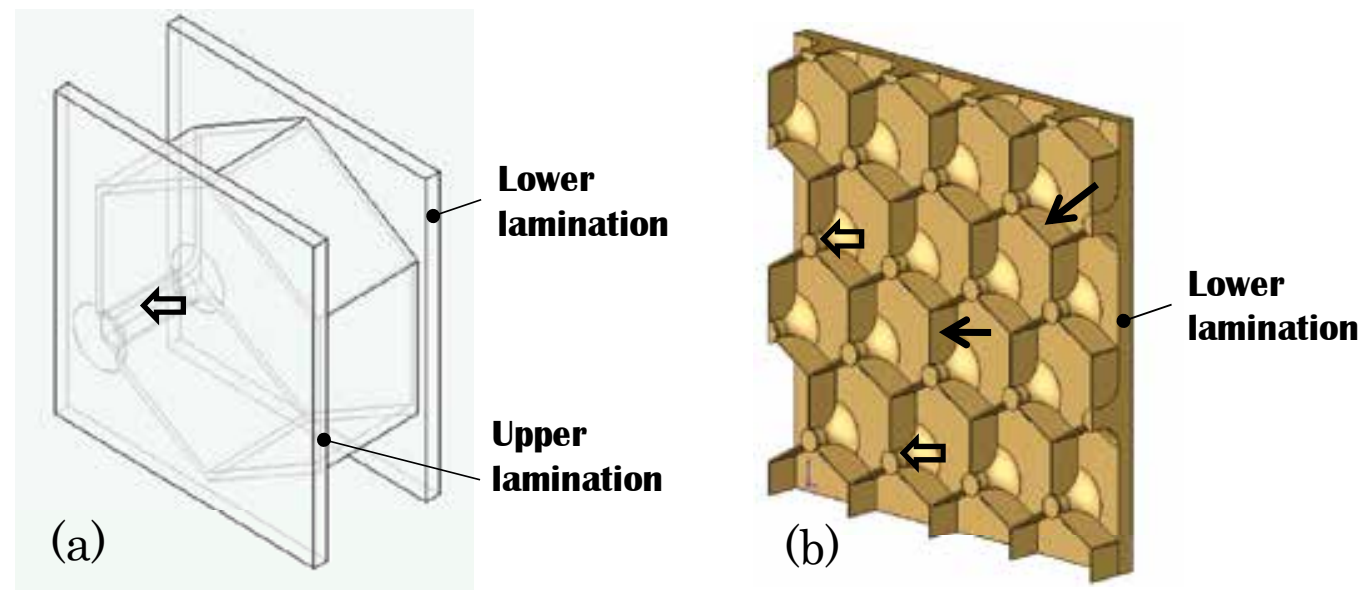

Figure 6. Three-dimensional sketch of a trabecula-honeycomb cell. (a) A cell. (b) the assembly of cells without an upper lamination layer. The thin and thick arrows indicate the honeycomb wall and the trabeculae, respectively.

\subsubsection{Schematic model of the trabecular structure}

Based on our observations, a schematic structural model of the trabeculae in A. dichotoma was generated and is shown in Fig. 7. This model indicates the following: (1) the substance in the central part of the trabecula is protein (see thin arrow in Fig. 7a); (2) The upper and lower laminations are comprised of chitin fiber layers that either extend into the trabeculae continuously in a curved shape (see Fl in Fig. 4a and Fig. 7) or connect to the trabeculae by surrounding the circumference of the trabecular shaft in a spiral fashion (see Fb in Fig. $4 \mathrm{a}$ and Fig. 7). Through peeling tests, we have confirmed that, when comparing the trabeculae of the upper and lower laminations, the peeling resistance of trabeculae in a general region is approximately three times greater and in a local region is approximately thirty times greater [Ni et al., 2001]. Thus, the use of the trabecular structures to join the upper and lower laminations effectively increases the interlaminar strength of the composite structures.

\subsection{Conclusions}

To develop lightweight biomimetic composite structures, the forewing structures of $A$. dichotomais were investigated. Structural models for both the beetle forewings and the forewing trabeculae were also established, and the results obtained are as follows: 

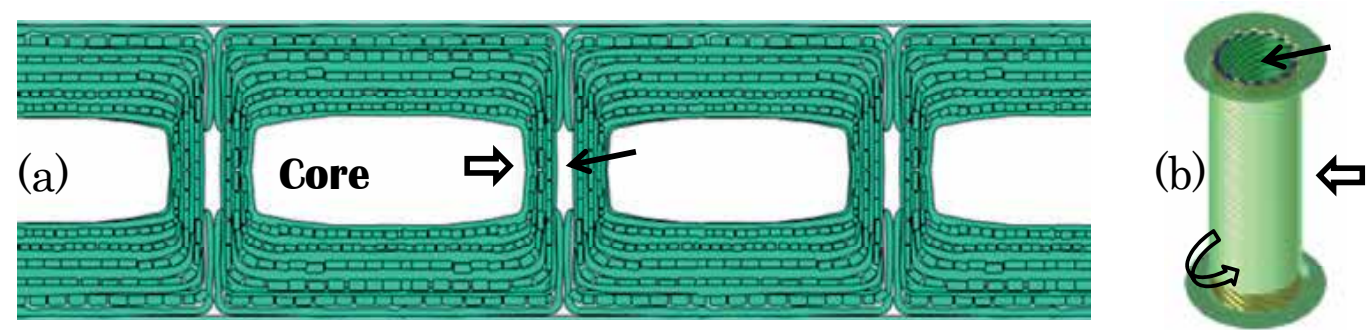

Figure 7. Schematic view of reinforcing fibers. a, Cross section of a forewing. $b$, Three-dimensional view of a trabecula. The thin and thick straight arrows show a void (protein) inside a trabecula and itself, respectively. The circular arrow shows a spiral fiber surrounding the trabecula (the lower part shows the structure of a trabecula without a protein).

1. It was found that both species of beetle forewings have a sandwich plate structure and a frame structure with a central void layer and interconnecting trabeculae. These characteristics are typical of lightweight composite structures.

2. The chitin fibers in the trabeculae connect with a curved shape continuously to the chitin fibers on the upper and lower laminations, and it is clear that this structure greatly increases the peeling resistance of the laminated composite structures.

\section{Interlaminar reinforcement mechanism of the beetle forewing}

The fracture toughness of fiber-reinforced composite materials used in engineering applications mainly depends on the interlaminar and/or intralaminar fracture toughness values, with the interlaminar and/or intralaminar interface tending to be the critical location of weakness. Investigations of the interfacial behavior have been undertaken by many researchers, such as evaluation of the interlaminar and/or intralaminar fracture toughness [Iwamoto et al., 1999a], the interlaminar delamination resistance [Turss et al. 1997], and the role of bridging fibers in crack propagation [Sekine \& Kamiya, 1987; He \& Cox, 1998]. In recent years, 3D textile-reinforced composites have been developed toward improving the interlaminar and/or intralaminar fracture toughness values. On the other hand, many examples of composite structures already exist in nature, and their structures have already been optimized by evolutionary pressures over the course of a deep ecosystem history.

This section focuses on the mechanical properties and the three-dimensional interlaminar reinforcement mechanism of the $A$. dichotoma beetle forewing.

\subsection{Experiment}

The specimen utilized was the living forewing of the adult male $A$. dichotoma beetle (Fig. 1a, $\Delta$ arrowhead). The width of the fresh specimen was $2.1 \mathrm{~mm}$. A tensile device (SHIMAZDU, AUTOGRAPH DSC-10T) with a custom low load cell was used for the interlaminar peeling test, which was conducted with zipper forceps, as developed by the authors. The tensile loading direction was vertical to the peeling surface, as shown in Fig. 8(a). To prevent sliding of the specimen at the zippers, the inner surface of the forceps was adhered to the specimen 
with glue tape. The peeling schematic is indicated in Fig. 8(b). In the present study, 10 specimens were tested at a tensile speed of $5 \mathrm{~mm} / \mathrm{min}$ and a peeling distance of $7 \mathrm{~mm}$.

(a)

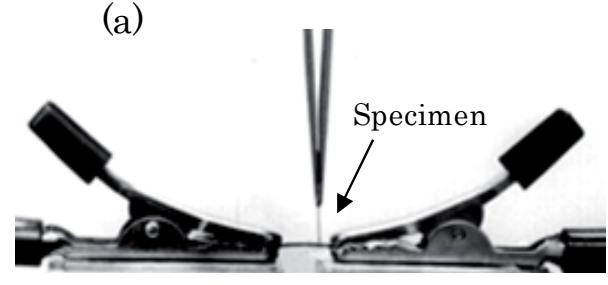

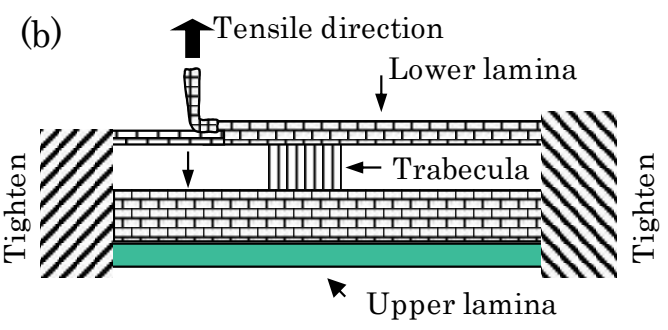

Figure 8. A peeling experiment; (a) peeling device, (b) a peeling schematic.

\subsection{Results and discussion}

\subsubsection{Peeling characteristics of the forewing of $A$. dichotoma}

Micro Fractograph of Peeling Surface Fig. 9 presents a micrograph of a forewing after the peeling test by illuminating the specimen from behind with penetrating light, which is a new technique developed for observation of the forewing structure. Many black points and ducts can be observed in this image, where the black points can be interpreted as trabeculae and the ducts, as tracheas. Fig. 10 (a) is a micrograph taken at the $\Delta$ arrowhead mark in Fig. 9 and shows the fracture characteristics of the first trabecula during the peeling process. The peeling test was started by applying pulling force to the lower lamina of the forewing. When the delamination crack tip met the first trabecula (Fig. 10a, $\Delta$ arrowhead), a portion of the lower lamina tore, while the remaining portion was held in tension. In this manner, the peeling tip continued to develop and propagate between the chitin fiber laminae. A fracture micrograph illustrates a peeling surface in the region of the $\boldsymbol{\Delta}$ arrowhead marked in Fig. 9 and has been shown in Fig. 10(b). The resulting fracture patterns on the peeling surface had an appearance similar to that of a tree root (Fig. 10b, $\Delta$ arrowheads) with details of this phenomenon presented in Fig. 11(b) and Fig. 13.

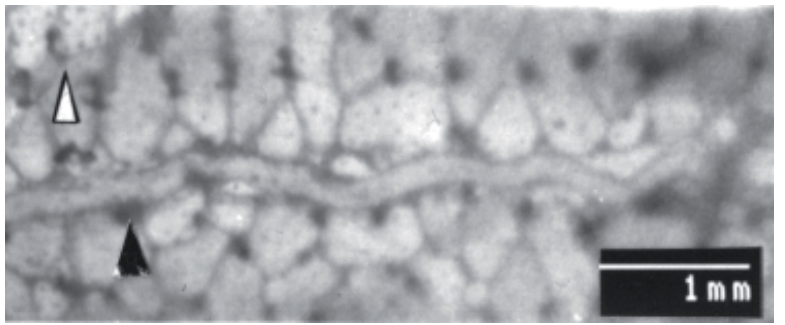

Figure 9. A photograph of a fore-wing after peeling test and black points are trabeculae.

Fig. 11(a) illustrates the fracture pattern of the peeling surface without any trabeculae present. Although the chitin fibers are arranged in rows ( $\Delta$ arrowhead), some of have torn ( $\Delta$ arrowhead) during the peeling process. Fig. 11(b) shows a fracture micrograph of the chitin fibers at a trabecular root. It was that the chitin fibers ( $\Delta$ arrowhead) formed very thin 


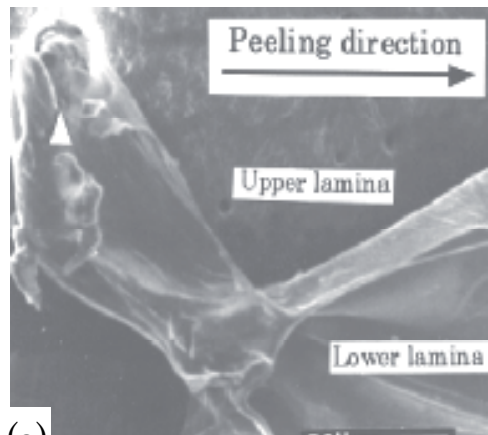

(a)

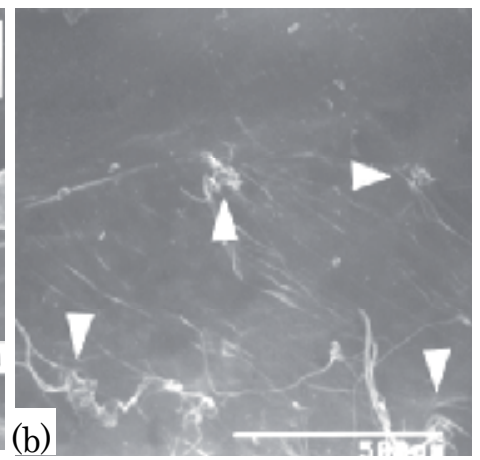

Figure 10. Fractographs of a forewing; (a) fracture of first trabecula, $\Delta$ : trabecula, (b) a peeled chitin fiber lamina, $\Delta$ : roots of the trabeculae.

layers here, with Fig. 11(c) illustrating a side view of the peeling process. When the peeling tip had propagated about half of the thickness of the lower lamina, a few bridging fibers can be seen between the lower and upper peeling surfaces.

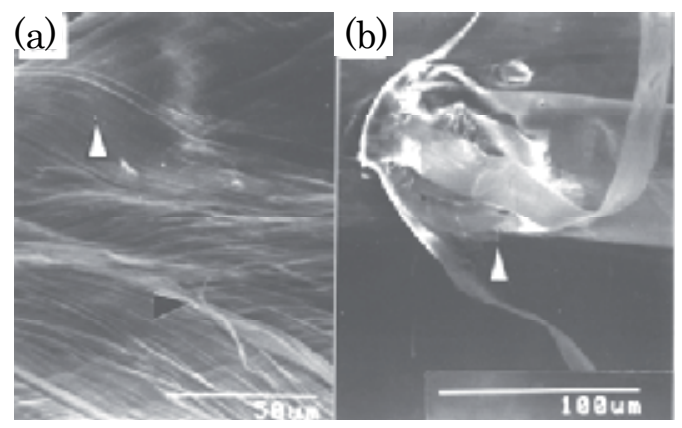

(c)

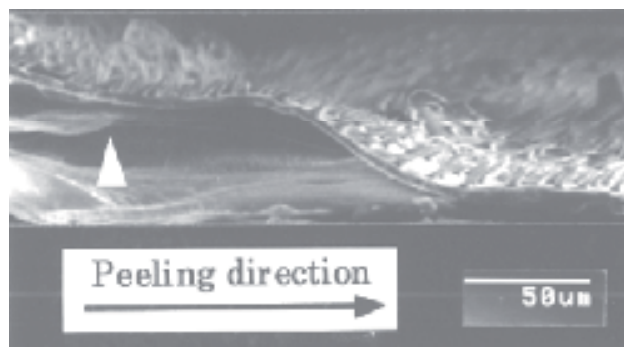

Figure 11. Fractographs of a forewing; (a) peeled surface without a trabecula, (b) trabecula root and thin chitin fiber layers, (c) a peeling process from a side view. $\Delta$ : bridging fibers.

\subsubsection{Load peaks and trabeculae in a peeling test}

A representative load-displacement curve, with load $F$ and displacement $\delta$, is presented for a forewing peeling test in Fig. 12(a). The force, $F(\delta)$, was proportional to the resistance force because the tensile direction was held vertical to the peeling surface, as described above. Here, the $F-\delta$ curve is named the "peeling test" and the curve is given by $F(\delta)$. Many individual load peaks were present in the peeling curve, and their shapes were similar to the standard load peak shape, although their peak values were quite different. In the peeling test, the minimum value of each ravine was not equal to zero but instead held a nearly constant value during the peeling process. Here, the $\mathrm{j}^{\text {th }}$ values of the load peak and the ravine minimum are indicated by $F_{\mathrm{j} 1}(\delta)$ and $F_{\mathrm{j} 0}(\delta)$, respectively.

The number of individual load peaks in the $F-\delta$ peeling test was compared to the number of trabecular roots, which were observed by SEM, and these values were found to be nearly equal [Ni et al., 2001]. Accordingly, it was determined that each trabecula contributed a 
single load peak in the peeling curve. In other words, the load peaks resulted from breakage of the individual trabeculae during the peeling process.
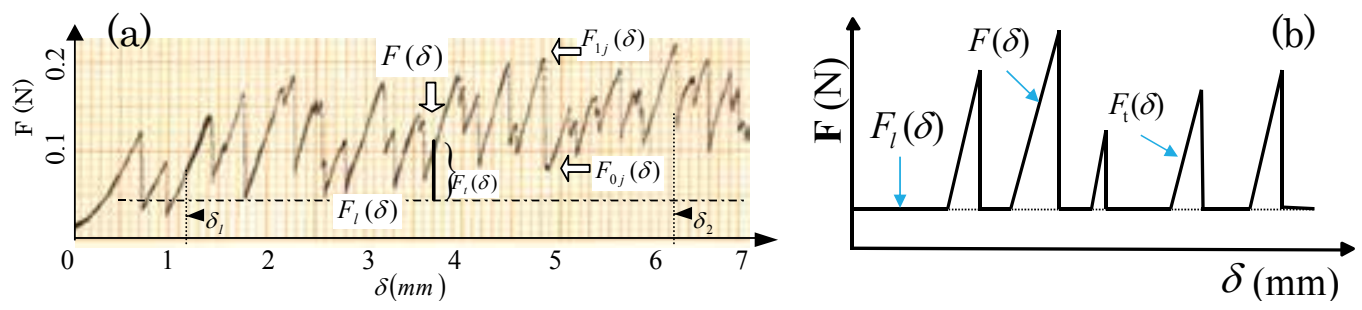

Figure 12. A representative load-displacement curve (a) and its model (b) in a forewing peeling test.

Peeling Curve As mentioned above, the load peaks in the $F-\delta$ peeling curve were attributed to specific contributions from the individual trabeculae. Based on this fact, the peeling curve, $F(\delta)$, was considered to be comprised of two components, i.e., $F_{l}(\delta)$ (Fig. 12a) due to interlaminar delamination of the chitin fiber laminae and $F_{t}(\delta)$ due to trabecula failure. Thus, $F(\delta)$ may be represented by equation (1):

$$
F(\delta)=F_{l}(\delta)+F_{t}(\delta)
$$

$F_{l}(\delta)$ depends mainly on the properties of the chitin fibers, the protein matter and fiber adhesion. If the interlaminar debonding force, $F_{l}(\delta)$, was constant during the peeling process and the trabeculae were distributed along only one line in the propagation direction, then a schematic of the peeling curve can be represented as Fig. 12(b). In other words, each load peak, $F_{t}(\delta)$, results from the fracture of one trabecula, and the line, $F_{l}(\delta)$, at the ravine minimum results from the delamination resistance between chitin fiber layers without the trabeculae.

However, the horizontal line, $F_{l}(\delta)$, cannot be observed in the $F-\delta$ peeling curve as indicated in Fig. 12. This is attributed to the trabeculae being distributed intermittently throughout the plane (Fig. 11c) with the bottom of a ravine, $F_{\mathrm{j} 0}(\delta)$, generated from the delamination of laminar fibers that are piled upon each other. In comparing Fig. 12 (b) with Fig. 12 (b), it was not possible to fully distinguish $F_{l}(\delta)$ and $F_{t}(\delta)$. However, the value of $F_{l}(\delta)$ should be less than or close to the minimum of all ravine bottoms, $F_{\min 0}$, i.e.,

$$
F_{l}(\delta) \leq F_{\min 0}=\operatorname{Min}\left\{F_{\mathrm{j} 0}(\delta)\right\}
$$

where $F_{\min 0}$ is the maximum of $F_{l}(\delta)$ in the following calculation.

\subsubsection{D Reinforcement mechanism of the beetle forewing}

Fracture Type of Trabecular Roots Three representative fracture modes of the trabecular roots were observed during the forewing peeling test (Fig. 13). Fig. 13(a) presents type A, which possesses a convex shape, and also shows a schematic of the longitudinal section shown in the fractograph. In type $\mathrm{A}$, trabecular roots in the lower laminae remained 
following peeling such that the setae ( $\boldsymbol{\Delta}$ arrowhead) could be observed. In type $\mathrm{C}$, a smooth surface (Fig. 13c) has had the trabecular roots in the lower laminae completely removed. In contrast to this, type B was between type A and type C (Fig. 13b). Although three types of failure shape for trabecular roots were observed, the load peaks in the peeling curve were similar to each other. In the following section, possible reasons for this are considered.
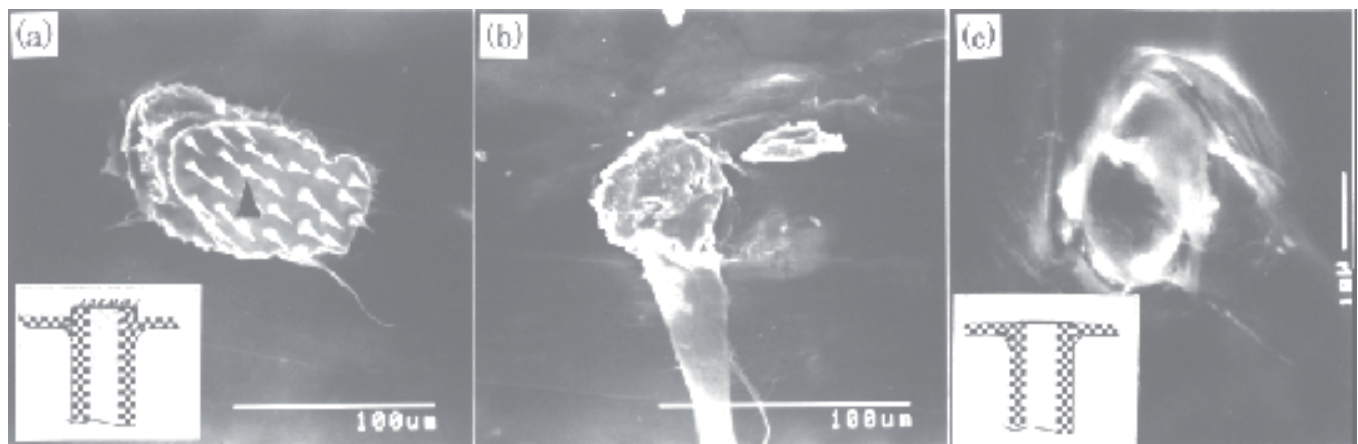

Figure 13. Fractographs of three typical trabecular root.

According to the trabecular structure, the chitin fibers within the trabeculae are thin, continuous, and curved (Fig. 14, $\mathbf{\Delta}$ arrowhead) and interconnected with each other between the chitin fiber laminae. During the peeling process, the peeling tip continues to propagate horizontally (see Fig. 11c) in the absence of a trabecula. When the peeling tip encounters a trabecula, however, its progress is impeded by the chitin fibers of the trabecular; meanwhile, the peeling tip around the outside of the trabecular root continues to propagate, which results in a stress concentration at the trabecular root itself. That is, the chitin fibers of the trabecula initially withstand the higher stress and then become stretched into a straight line until the trabecula root fails (Fig. 14a). Therefore, the chitin fibers within the trabecular root

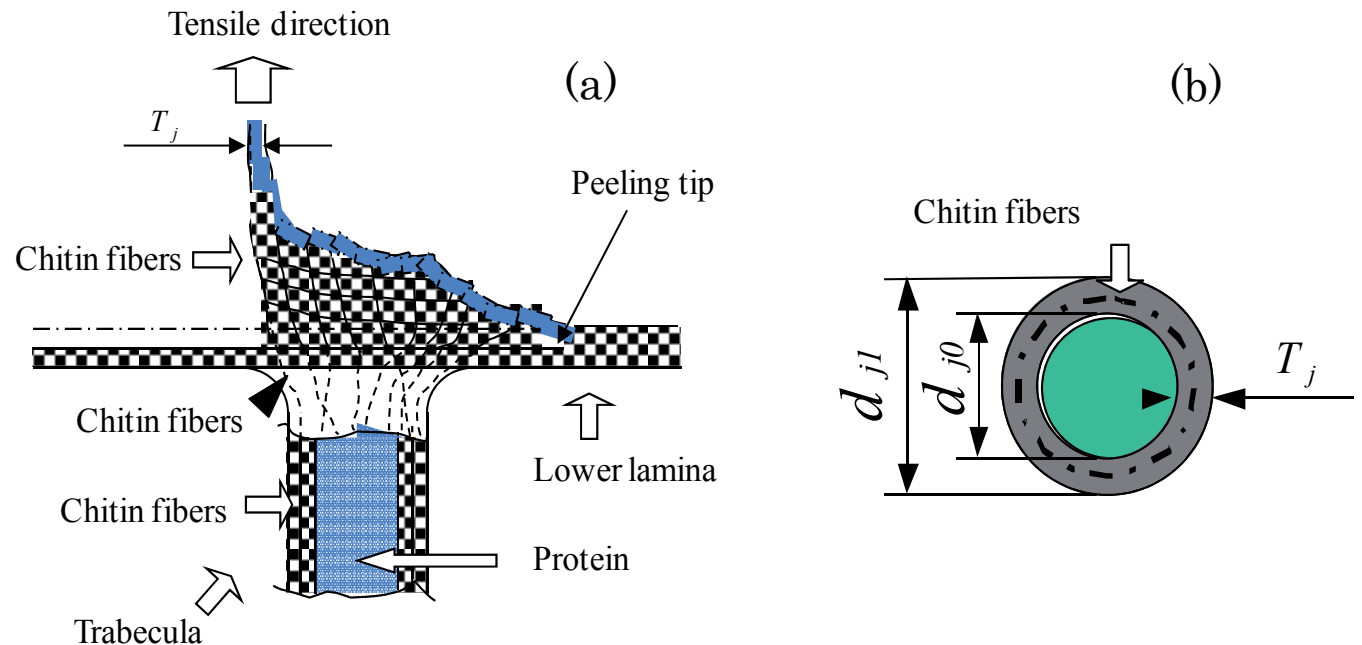

Figure 14. A chitin fiber reinforcement model (a) and a cross sectional trabecular model (b). 
fracture in tension. Thus, the chitin fibers in the trabeculae have a high resistance to the applied load, and their failure results in the load peaks noted in the $F-\delta$ peeling curves as shown in Fig. 12. This also results in similar peeling curve peak shapes for the three fracture types (Fig. 13).

Failure Strength of Chitin Fibers in the trabecular root The failure strength of chitin fibers in the trabecular root has been discussed using the proposed models (Fig. 14). Using equation (1), the load peaks, $F_{j 1}(j=1,2, \ldots, \mathrm{n}$, the number of trabeculae), and the resistance force, $F_{l}(\delta)$, of the interlaminar delamination in the peeling curve, the average failure strength, $\sigma_{b}$, of the chitin fibers in the trabeculae was calculated as follows:

$$
\overline{\sigma_{b}}=\frac{\sum_{j=1}^{\mathrm{n}}\left[F_{\mathrm{j} 1}-F_{l}(\delta)\right] / n}{\sum_{j=1}^{\mathrm{n}} S_{j} / n}
$$

where $S_{j}$ is the failure area of the chitin fibers and was calculated using the thin ring area of chitin fiber layers within the trabecular root (Fig. 14b). The thickness of the thin ring was considered to be equal to the chitin fiber lamina thickness, $T_{j}$, and $S_{j}$ was given by equation (4)

$$
S_{j}=T_{j}\left(d_{j 1}-T_{j}\right) \pi
$$

The justification for using the cross section of the thin ring area is given as follows: first, the trabecula consists of two sections, which include the central part that is mainly protein and the outside that is chitin fiber laminae. This result was confirmed by treating the forewing with $10 \% \mathrm{KOH}$ at $100^{\circ} \mathrm{C}$ for 3 hours [Iwamoto et al., 1999b]. After treatment, an empty space was observed in the central region of the trabecula (see Fig. 5c, arrowhead part), which was attributed to dissolution of the protein in the $\mathrm{KOH}$ solution. Therefore, it is clear that the central region of each trabecula contains no chitin fibers and is instead mainly composed of protein. Second, considering the fracture of chitin fibers during the peeling process (see Fig. 14a), when the peeling tip of the chitin fibers progresses around the outside of the trabecular root, the chitin fibers at the remaining intact side bear a higher applied load than those in the delaminated side. However, in the cross section of the trabecular root thin ring, the stress is uniformly accommodated; in particular, this is true after the outside chitin fiber laminae of the trabecula are peeled apart (delaminated) because the chitin fiber layer is very thin. This hypothesis was supported by the fact that the load peaks in the peeling curve dropped when a trabecular root fractured during the peeling process (Fig. 12). Third, the trabecular diameter in the forewing of A. dichotoma is very small and only on the order of tens of microns. In light of the above reasons, and for simplicity, equation (4) was thus adopted.

Moreover, for equation (3), only data collected in the region between $1 \mathrm{~mm}$ and $6 \mathrm{~mm}$ of displacement were used because the peeling front had not always progressed through the full specimen width in the initial and final failure regions. Thirty load peaks in the region of 
$1 \mathrm{~mm}$ to $6 \mathrm{~mm}$ were used for the calculation, although there were 44 load peaks in the total peeling curve as shown in Fig. 12. As a result, the trabecular root failure load and the resistance force, $F_{l}(\delta)$, of interlaminar delamination were found to be $0.156 \pm 0.029 \mathrm{~N}$ and $0.049 \mathrm{~N}$, respectively. The thickness of the peeled chitin fiber laminae, $T_{j}$, was $4.5 \mu \mathrm{m}$, which remained nearly constant in the entire peeling region from $1 \mathrm{~mm}$ to $6 \mathrm{~mm}$.

Next, the external diameter of the peeled trabecular root, $d_{j 1}$, was determined. In practice, the cross section of the trabecular root is not circular but instead is closer in shape to an ellipse. Thus, the average of the long and short axes of the ellipse was used as $d_{j 1}$. The results of $d_{j 1}$ as measured by SEM indicated the average external diameter of 30 trabecular roots was $55 \mu \mathrm{m}$. Using all of the results obtained above, the failure strength, $\sigma_{b}$, of the chitin fibers within the trabecular root was calculated as approximately $149 \mathrm{MPa}$. This result is close to the tensile strength of $147 \mathrm{MPa}$ in the same $0^{\circ}$ direction of the chitin fibers. Therefore, during the peeling test, the failure strength of the chitin fibers within the trabecular root ultimately resulted from tensile fracture of the chitin fibers, as mentioned above.

Interlaminar Reinforcement Mechanism The chitin fibers of the forewing trabecular root were found to have the following characteristics: a curved and continuous shape; bonded to both chitin fiber laminae and to each other; with the angle of about $90^{\circ}$ between the lower laminae and the trabecula. The increment of interlaminar strength is due to the tensile failure of the chitin fibers within the trabecular root, as determined by the peeling process. These results indicate a creative approach for researchers to overcome the interfacial weakness issues currently associated with laminated composite structures.

Strengthening Effects due to the Trabeculae The strengthening effects due to the forewing trabeculae were investigated. Here, the local strengthening effect, $\lambda_{l}$, is calculated by equation (5), i.e., $\lambda_{l}$ is the ratio of the average failure load of the chitin fibers in the trabecular root due to the resistance force of the interlaminar lamination, $F_{l}(\delta)$, in units of inverse length.

$$
\lambda_{l}=\overline{\sigma_{b}} \cdot T_{j} / \frac{F_{l}(\delta)}{B} \cong 29
$$

Using the data presented in Fig. 12, the average failure load of the chitin fibers in the trabecular root, $F_{t}(\delta)$, the resistance force of the interlaminar lamination, $F_{l}(\delta)$, and the specimen width of $B=2.1 \mathrm{~mm}$ were all calculated. The local strengthening effect, $\lambda_{l}$, was found to have a value of approximately 30. Therefore, the 3D strengthening effect of the interlaminar structure is very pronounced, specifically due to the curved and continuously shaped chitin fibers, which are shown in Fig. 14.

The strengthening effect for the whole specimen that is due to the trabeculae can be evaluated from instances where the trabeculae are or are not present to provide resistance during the peeling process. The whole strengthening effect, $\lambda_{m}$, was calculated using equations (6) and (7): 


$$
\begin{gathered}
\lambda(\delta)=\frac{F(\delta)}{F_{l}(\delta)} \geq \frac{F(\delta)}{F_{\min 0}} \\
\lambda_{m} \geq \frac{\int_{\delta_{1}}^{\delta_{2}} F(\delta) \mathrm{d} \delta}{\int_{\delta_{1}}^{\delta_{2}} F_{\min 0} \mathrm{~d} \delta}=\frac{\int_{\delta_{1}}^{\delta_{2}} F(\delta) \mathrm{d} \delta}{F_{\min 0} \cdot\left(\delta_{2}-\delta_{1}\right)}
\end{gathered}
$$

where $\lambda(\delta)$ is the strengthening effect at a peeling distance, $\delta$. In this paper, the average of 10 specimens, $\overline{F(\delta)}, \overline{F_{\min 0}}, \bar{\lambda}_{m}$ and the standard deviations for $F(\delta), F_{\min 0}(\delta), \lambda_{m}$ were calculated. The value of $\overline{F(\delta)}$ is given by equation (8)

$$
\overline{F(\delta)}=\sum_{i=1}^{10}\left[\int_{\delta_{1}}^{\delta_{2}} F_{i}(\delta) \mathrm{d} \delta /\left(\delta_{2}-\delta_{1}\right)\right] / 10
$$

The entire strengthening effect of the interlaminar strength due to the trabeculae in the chitin fiber laminae was approximately 3 times as large as that in the chitin fiber laminae without the trabeculae. Therefore, the trabecular structure of the forewing is not only lightweight but also high-strength.

\begin{tabular}{|c|c|c|c|}
\hline Object & $\overline{F(\delta)}(\mathrm{N})$ & $\overline{F_{\min 0}}(\mathrm{~N})$ & $\overline{\lambda_{m}}$ \\
\hline Average & 0.148 & 0.051 & 3.2 \\
\hline Standard deviation & 0.047 & 0.019 & 1.1 \\
\hline
\end{tabular}

Table 1. Strength ratio and its standard deviation by trabecula

\subsection{Conclusions}

A peeling test between the chitin fiber laminae of the A. dichotoma forewing was conducted, and the reinforcement mechanism, especially for the 3D interlaminar reinforcement, was investigated with the following results.

1. Many load peaks were observed in the peeling curves with their number being equal to the number of trabeculae found in the chitin fiber laminae. This finding demonstrated that the fracture of each trabecula contributed to a single load peak in the peeling curve, which ultimately contributed to the interlaminar strength. Furthermore, the characteristics of the peeling curve depended on the density and 2D distribution of the trabeculae within the forewing.

2. The chitin fibers were interconnected between the chitin fiber laminae and the trabeculae, and they were curved and continuously shaped where the trabeculae were bonded to the chitin fiber laminae. The fibers were distributed in two dimensions within the plane of each chitin fiber lamina. The chitin fibers broke in tension during the peeling process and exhibited a high resistance to the applied load.

3. The amount of the interlaminar strength that was due to the trabeculae in the chitin fiber laminae was approximately 3 times greater within the whole region and about 30 
times greater in the local region compared to that in the chitin fiber laminae without any trabeculae. Based on the above results, this strong natural reinforcement mechanism was understood, and a model for the reinforcement mechanism was proposed.

\section{Optimal composite structures in the forewings of beetles}

Within the forewings of insects, both the laminated arrangement of the chitin fibers [Gullan \& Cranstion, 1994; Leopoldj et al., 1992; Banerjee, 1988; Zelazny \& Neville, 1972] and the mechanical characteristics of these equiangular laminating layers of the biomimetic composites [Tanimoto et al., 1998; Ben et al., 1998; Masuda et al., 1995] have been reported on previously. The authors have reported the presence of non-equiangular laminating structures in the forewing of A. dichotoma [Chen et al., 2001a]. It is well known that the somatotypes of beetles differ from one another due to their sex, with the larger male bearing a horn. In the present study, based upon previous work on the characteristics of somatotype beetles, the forewing, the tensile fracture force of the forewing and its suitability for biomimetic studies have been examined for male and female beetles. Both optimal structures of the fiber's cross section and the typical reticular cross-linking of twodimensional reinforced structures within the beetle's forewing are discussed.

\subsection{Experimental specimens and methods}

Two types of forewing specimens were selected from both male and female A. dichotoma, as shown in Fig. 15(a) and Fig. 1(a). The experimental bio-samples included a total of 12 males and 12 females with measurements of each beetle's weight, its forewing dimensions and its physical size.
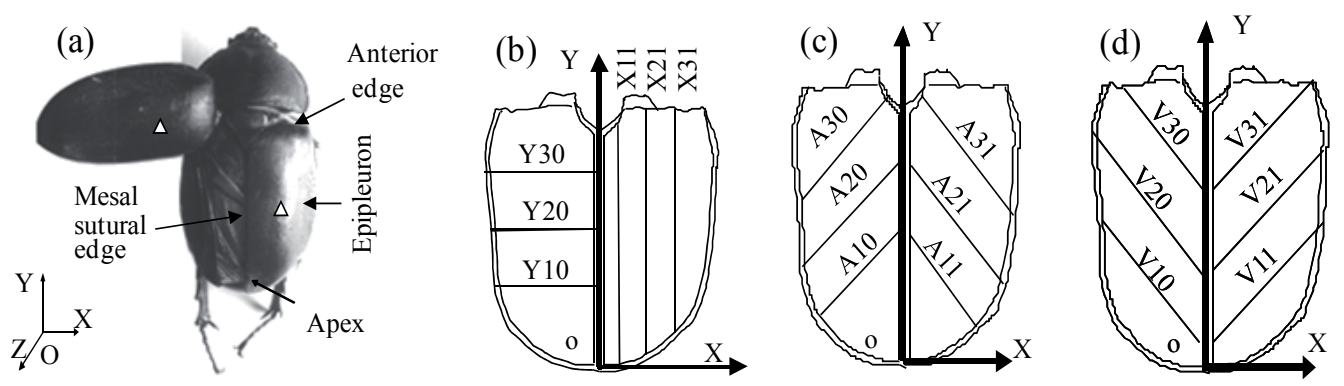

Figure 15. Female beetle (a) and example position in (b) $0^{\circ}$ or $90^{\circ}$, (c) $45^{\circ}$, (d) $135^{\circ}$ of the beetle forewing.

The different orientations of the tensile test specimens were prepared as shown in Fig. 15. The tensile test specimens measuring $4 \mathrm{~mm}$ wide were then sliced and prepared by a special parallel cutting blade [Chen et al., 2001b]. A total of 22 test specimens for both the left and right forewings were prepared. Under the conditions of constant room temperature and humidity $\left(20 \pm 2^{\circ} \mathrm{C}, 60 \% \pm 5 \% \mathrm{RH}\right)$, the tensile test was carried out on a Shimazu AUTOGRAPH DSC-10T with a special low load cell and jig at a tensile strain rate of $0.5 \mathrm{~mm} / \mathrm{min}$, where the distance between clamps was $5 \mathrm{~mm}$. Additionally, a special clamp head was attached with 
cloth tape to prevent failure. After the tensile test, the thickness of both the upper and lower lamination layers were measured at four points using the ESEM-2700, and the tensile strength of the specimens were evaluated by taking into account the average thickness of each specimen.

\subsection{Experimental results and discussions}

\subsubsection{Somatotypes}

Table 2 shows the measurement results of the somatotypes of A. dichotoma, along with the weight and size of the forewing. This also includes the mean values and the standard deviation values (STDEV).

To identify the sex-related differences between the male and female A. dichotoma, a T-test [Yoshikawa et al., 1985] was performed on these experimental results, comparing sample group A (male) to sample group B (female). The results of the T-test are shown in Table 2. When the probability value is $\mathrm{P}\left(\mathrm{t}_{0}\right) \leqq \alpha$ (generally $\alpha=0.05$ at normal significance level), there exists a significant difference and $\mathrm{a}^{*}$ is labeled in that specific cell of Table 2 . It is clear that both the weight and physical size of the male A. dichotoma are larger than the weight and size of the female. The male forewing measures $28.7 \mathrm{~mm}$ in length and $14.8 \mathrm{~mm}$ in width, whereas the female forewing measures only $28.3 \mathrm{~mm}$ in length and $13.6 \mathrm{~mm}$ in width. The significant differences indicate that the male is larger than the female; however, the forewings of the male and female beetle have a similar weight. In fact, females have a forewing weight of $0.160 \mathrm{~g}$ and males have a forewing weight of $0.151 \mathrm{~g}$; surprisingly, the female beetles weigh slightly more than the males, according to this experimental data.

Compared to female $A$. dichotoma, males have a larger somatotype and physical size. However, the male also has a lighter forewing. It is thus supposed that there exist certain physical differences between males and females in A. dichotoma forewings in features such as thickness, laminated arrangement and laminated layers. Accordingly, these differences between male and female beetles should be considered when their forewing structures and mechanical properties are investigated.

\begin{tabular}{|c|c|c|c|c|c|c|}
\hline \multirow{2}{*}{ Beetle } & \multicolumn{2}{|c|}{ Weight $(\mathrm{g})$} & \multicolumn{2}{c|}{ Length $(\mathrm{mm})$} & \multicolumn{2}{c|}{ Width(mm) } \\
\cline { 2 - 7 } & Male & Female & Male & Female & Male & Female \\
\hline \multirow{2}{*}{$\begin{array}{c}\text { AVERAGE } \\
\text { STDEV }\end{array}$} & 5.58 & 4.72 & 46.13 & 42.82 & 23.30 & 21.79 \\
\cline { 2 - 7 } & 0.83 & 0.97 & 2.96 & 2.75 & 1.14 & 1.10 \\
\hline P(t 0$)$ & 0.03 & $*$ & 0.01 & $*$ & 0.00 & $*$ \\
\hline & & & & & & \\
\hline Forewing & Male & Female & Male & Female & Male & Female \\
\hline AVERAGE & 0.151 & 0.160 & 28.70 & 28.30 & 14.80 & 13.60 \\
\hline STDEV & 0.015 & 0.022 & 1.26 & 1.23 & 0.65 & 0.56 \\
\hline P $\left(\mathrm{t}_{0}\right)$ & 0.27 & & 0.01 & $*$ & 0.00 & $*$ \\
\hline
\end{tabular}

Table 2. Measurements of the $A$. dichotoma forewing 


\subsubsection{Forewing strength and optimal forewing structure according to sex}

Table 3 shows the tensile fracture force of the 4-mm-wide male and female specimens, including the thickness of the upper and lower laminations of the forewings and the corresponding stress. The figures above are the mean values obtained for specimens in all orientations as shown in Fig. 15. The T-test results regarding male and female A dichotoma are also presented in Table 3. Although there is no difference in stress between the male and female forewing, there are significant differences in both the thickness and the tensile fracture force. The forewings of the females are thicker and exhibit a higher tensile fracture force than the males.

\begin{tabular}{|c|c|c|c|c|}
\hline \multirow{2}{*}{ Beetle } & \multirow{2}{*}{ Object } & Force & Thickness & Stress \\
\cline { 2 - 5 } & & $(\mathrm{KN})$ & $(\mu \mathrm{m})$ & $(\mathrm{MPa})$ \\
\hline \multirow{2}{*}{ Male } & Average & 28.1 & 54.0 & 130.7 \\
\cline { 2 - 5 } & STDEV & 5.94 & 7.22 & 25.84 \\
\hline \multirow{2}{*}{ Female } & Average & 35.6 & 69.7 & 127.9 \\
\cline { 2 - 5 } & STDEV & 2.32 & 8.04 & 25.20 \\
\hline \multirow{2}{*}{ T-test } & $\mathrm{P}\left(\mathrm{t}_{0}\right)$ & 0.00 & 0.00 & 0.49 \\
\cline { 2 - 5 } & & $*$ & $*$ & \\
\hline
\end{tabular}

Table 3. Tensile results of the forewing

However, in nature, beetles regularly engage in combat with each other or with insects of other species, either when searching for food or for a mate. For this reason, injured spots can often be found on beetle forewings, which are the result of tearing by the horns of competing beetles. Initially, 20 of the $A$. dichotoma died, most likely due to fighting or other natural causes. Among these beetles, 14 were male and 34 injured spots were identified among the forewings of the 12 wounded beetles, i.e., an average of 2.43 injured spots per male A. dichotoma [Chen et al., 2007a]. Meanwhile, only a single injured spot was located on the forewings of the females. Therefore, it is reasonable to assume that the differences in fighting injuries may be the result of evolutionary adaptations.

The male A. dichotoma has a heavy, large body with a light forewing (see Table 2). The beetle uses its lightweight wing to support its heavy body effectively in flight and uses its horn to protect its body. Observations have indicated that the male has a large but thin forewing with a lightweight construction. The females, who lack horns, have only the forewing to serve as protecting armor. It is thus reasonable to assume that possession of a thick forewing maximizes the female beetle's chance of survival in nature, increasing its lifespan and mating opportunities and further affording it a better opportunity to pass on its genes to the next generation. As a result of the thickened forewing, the beetle's tensile fracture force is increased, which forms a stronger barrier against external damage (see Table 3).

Furthermore, it is also hypothesized that the forewing of the female A. dichotoma is reinforced not only by an increased thickness but also by varying the orientation of its laminated layers and other features. These possible reinforcement mechanisms are areas for future work. The 
following discussion, however, primarily covers the fiber-reinforcement mechanism and the respective design techniques within both the male and female A. dichotoma.

\subsubsection{Optimum cross section of the chitin fibers and their volume fraction in the forewing}

As discussed above, the chitin fibers play a role in fiber enhancement of the forewing. Here, we discuss the "shape" (cross section) and the "amount" (density as a percentage) of reinforced fiber enhancements within the current bio-composite materials.

To discuss the cross sectional shape of the fibers and the distribution of the fiber density in the forewing, the forewing fiber cross sectional information must first be discussed. As shown in Fig. 16(a,b), the forewing consists of an upper lamination, a lower lamination and a void lamination, in which many trabeculae are distributed throughout. The inside of both the upper and lower laminations, called the endocuticle, is composed of chitin fibers (see Fig. 16a, b). The outsides of the lamination regions, called the exocuticle, are composed mainly of proteins. It was observed that the fibers terminated at the exocuticle of the epipleuron tip, and their cross sectional shapes were different from the fibers located at the endocuticle. Cross sections of the fibers that were observed in the exocuticle and the endocuticle are shown in Fig. 17, below. Also in Fig. 17(a, b), the fibers at the epipleuron tip of the forewing can be seen to have a circular cross section with a sparse distribution. Meanwhile, the fibers located primarily around the void lamination in the forewing's endocuticle are densely distributed with a rectangular cross section as seen in Fig. 17(c, d).
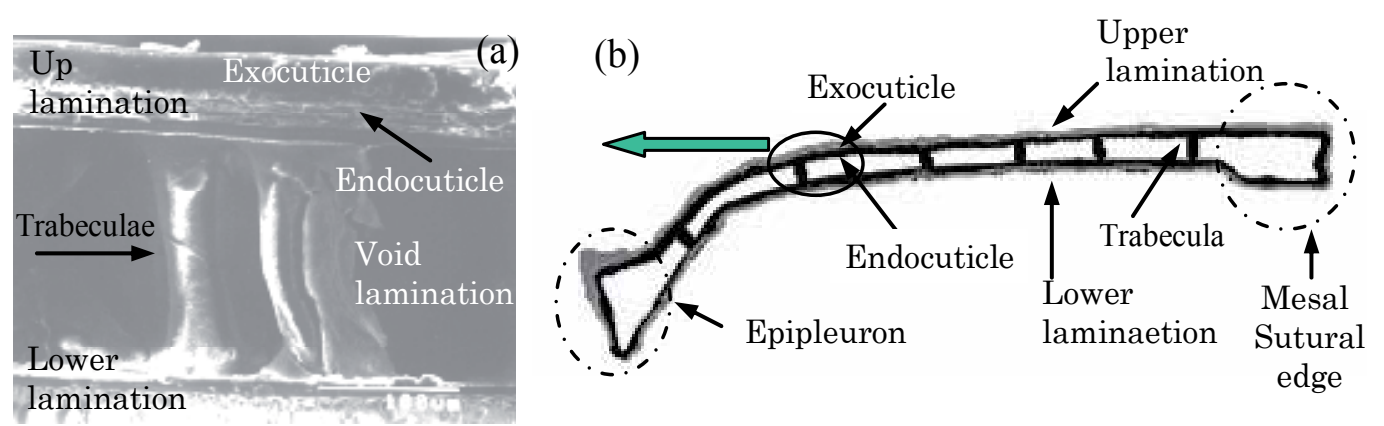

Figure 16. Schematic of section component in the forewing of beetle. (a) micrograph, (b) single model of whole section of forewing.

Fig. 18 illustrates two types of idealized chitin fibers, with either square or circular cross sections. Assuming that both the side of the square and the diameter of the circle are of the same dimension $\mathrm{D}$, then multi-layers of the chitin fibers oriented normally at $90^{\circ}$ in a dense and sparse distribution as shown in Fig. 18 (a) and (b). Under the same densely distributed condition, the chitin fiber volume fraction in the square cross section (Fig. 18a) is approximately $27 \%$ higher than that of the circular (Fig. 18b). It is thus supposed that the fiber reinforcements with rectangular cross sections result in a maximal fiber volume and the high strength of the forewing. 

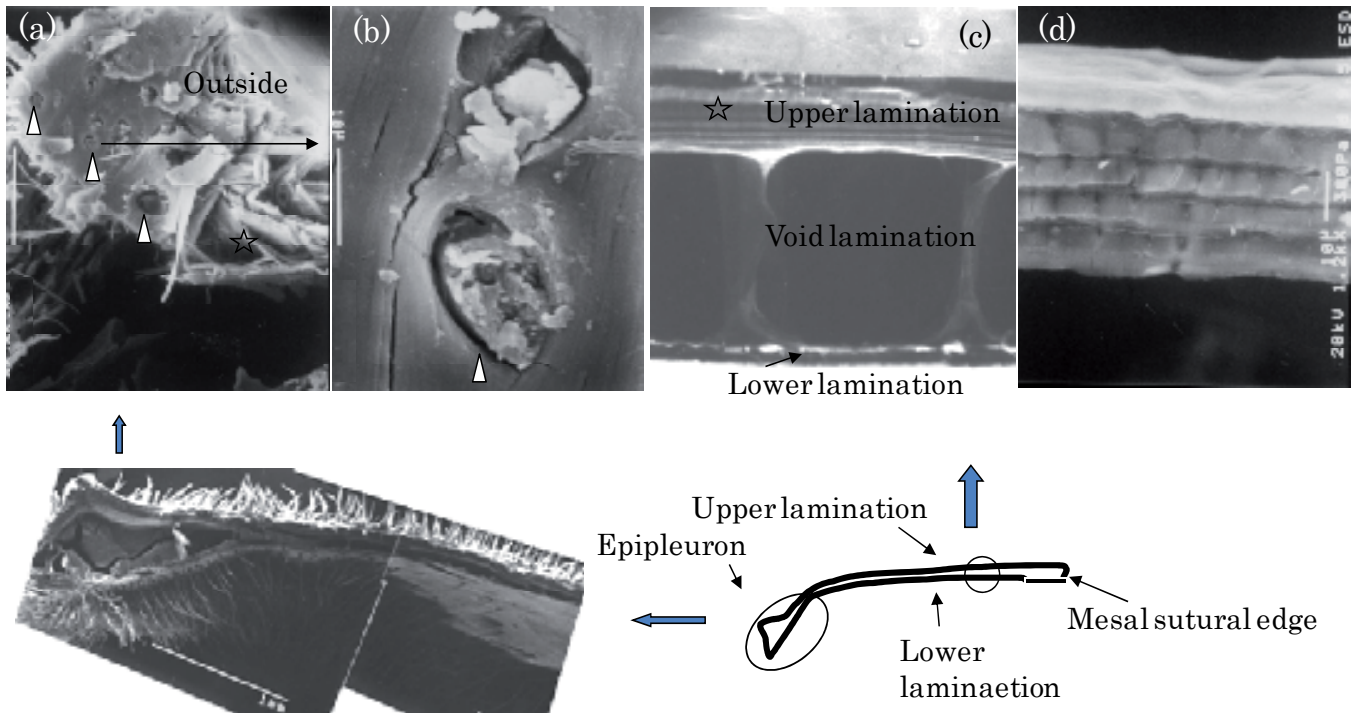

Figure 17. Typical cross sectional shape of the Chitin fibers and their positions in the forewing of $A$. dichotoma, the circular cross section $(\Delta)$ (a) and a magnified view (b) within the epipleuron tip and the rectangular cross section ( $\left(\boldsymbol{\zeta}^{2}\right)(\mathrm{c})$ around the void lamination in the endocuticle, as well as its magnified view $(\mathrm{d})$.
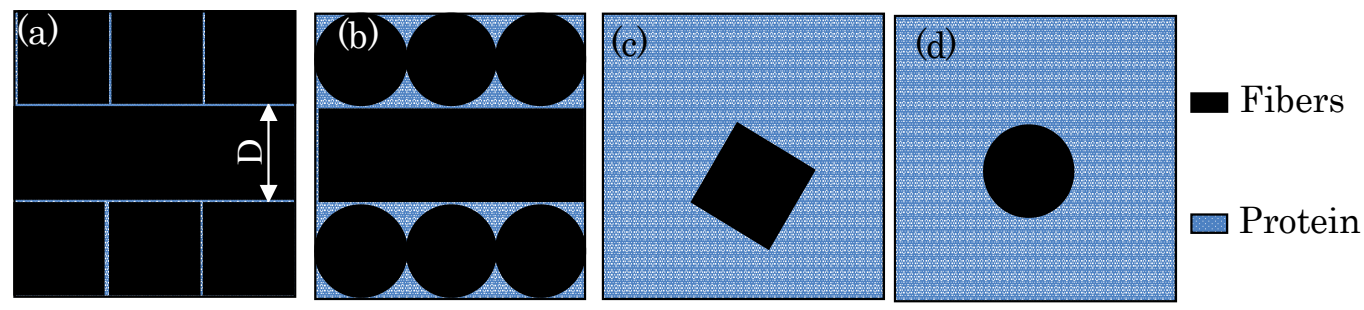

Figure 18. Model of a chitin fiber cross section - Higher density with (a), rectangular and (b) circular shapes and lower density with (c) rectangular and (d) circular shapes.

Next, the sparsely distributed samples of square and circular chitin fibers, as shown in Fig. 18 (c) and (d), have approximately only a 5\% fiber volume fraction. The circular samples have less stress concentration than either rectangular or triangular cross sections would, and they also have a more effective interface with the surrounding protein matrix. Furthermore, the circular samples measure $10 \mu \mathrm{m}$ in diameter and are twice as broad as those of the square cross section in length; its polar moment of inertia is more than ten times higher than that of the rectangular cross sections because it is proportional to the fourth power of the cross section size.

\subsubsection{Optimal structures by kind of beetles}

For comparison, photographs of $P$. inclinatus (Fig. 19 to Fig. 21) display the sections of the forewing, the trabecular distribution and their structure. Based on these, we confirmed that the fundamental structures of $P$. inclinatus, such as the frame structure (Fig. 19), the chitin 
fiber layers and their arrangement, are similar to that of A. dichotoma. The ratio of the thickness at the edge or the thickness at the central region to the total thickness of both beetles is nearly the same at any position (at the edge or center), but the void thickness in $A$. dichotoma is much larger than that of $P$. inclinatus [Chen et al., 2000].

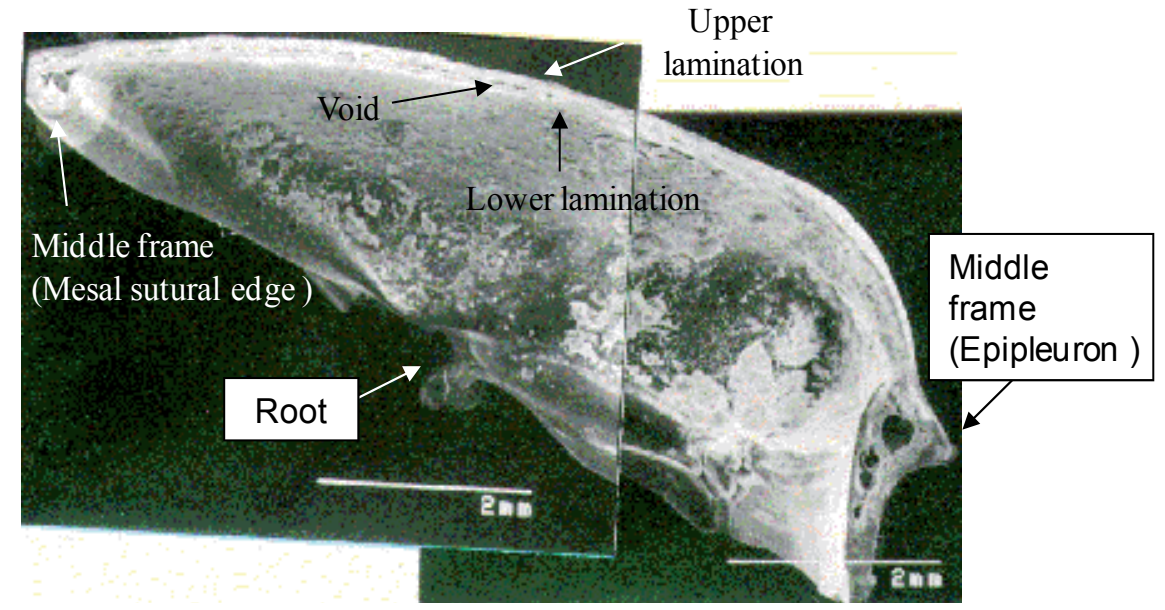

Figure 19. Structure of different sections of the forewing of $P$. inclinatus.

The average density of the trabeculae, calculated as the number of trabeculae per square of $\mathrm{mm}$, is 35 for $P$. inclinatus, and 6 for A. dichotoma, which is a difference in trabecular density of approximately six times. The trabeculae can be seen in Fig. 20(a) for A. dichotoma, and Fig. 20(b) for P. inclinatus.
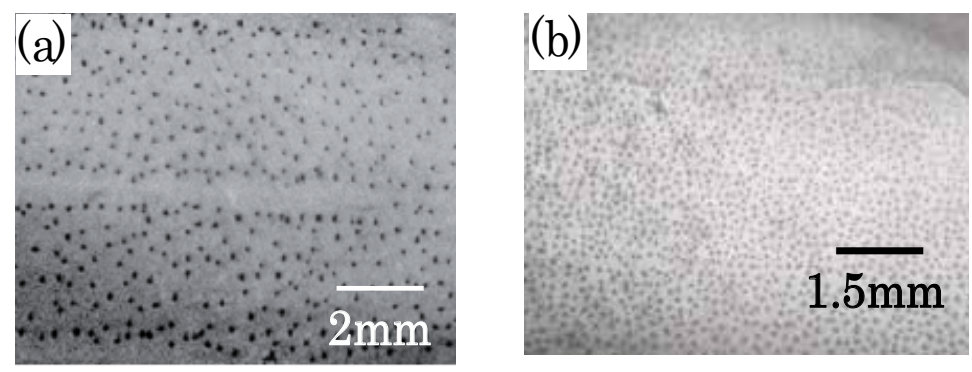

Figure 20. The forewing and trabeculae with $10 \% \mathrm{KOH}$ treatment of (a) A. dichotoma, (b) P. inclinatus.

The trabeculae in A. dichotoma are also relatively long and of a smaller diameter, whereas in $P$. inclinatus, they are relatively short with a larger diameter (Fig. 21a-c).

Here, the differences in structural details between A. dichotoma and P. inclinatus are discussed. Although both species of beetles possess a lightweight structure based upon a sandwich plate structure and frame construction with variable cross section, there are still measurable differences that exist between the two. For example, P. inclinatus has not only short and large diameter trabeculae but also a trabecular density six times greater than $A$. dichotoma. The forewing of $P$. inclinatus is twice as thick in the upper and lower laminations, 

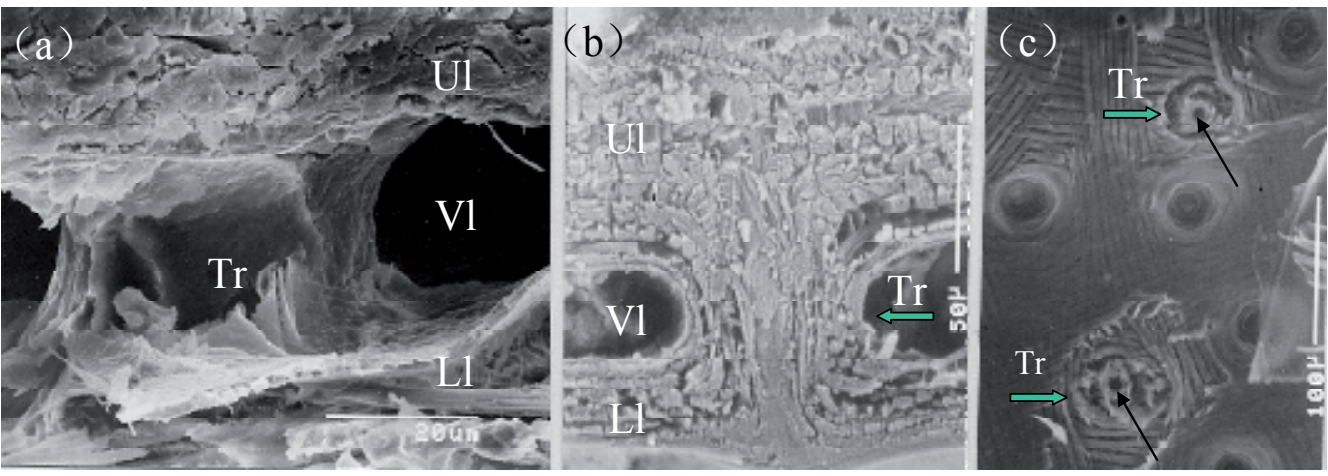

Figure 21. Microstructure of the trabeculae of $P$. inclinatus. (a) whole, (b) in longitudinal section, (c) in upper lamination following treatment with $\mathrm{KOH}$.

and its void height is $40 \%$ that of $A$. dichotoma [Chen et al.,2000]. Based on these differences, we consider that $A$. dichotoma must have a weaker forewing structure, while $P$. inclinatus possesses the higher strength structure. The forewing structure of $P$. inclinatus is optimized mainly by its lightweight structure, which is achieved by varying the quantity parameters, such as trabecular diameter, density, and thickness of lamination.

Why do these two beetles have such obvious structural differences? We have considered that these differences may be related to the lifespan of each beetle species. $P$. inclinatus has a longer lifespan and is able to survive through the winter, but A. dichotoma has a lifespan of only a few weeks and cannot survive the winter. In other words, P. inclinatus would be expected to have more durable physical characteristics than A. dichotoma because the latter has an extremely short lifespan. From a product viewpoint, the structural design of $P$. inclinatus is meant for durability and longevity, while A. dichotoma is of a more economical design with shorter lifetime. It is also noteworthy that the middle section of the lower lamination of $A$. dichotoma is extremely thin, possessing a thickness of only $1 / 3$ that of $P$. inclinatus. Because this part of the beetle's body is unexposed and thus rarely attacked by enemies in nature, the evolution of an extremely lightweight forewing in A. dichotoma resulted [Chen et al., 2007b].

\subsection{Conclusions}

In this section, the optimal composite structures in the beetle's forewing were discussed based on the beetle's sex, the tensile fracture force of the forewing, the chitin fiber shape and the fiber content. The results are as follows:

1. In comparison to the females, it is clear that the males have lighter and thinner forewings with a lower tensile fracture force; this is because male beetles have a large horn to protect them, while the females do not. The females, with their larger bodies to produce offspring, have only their thick forewings to serve as protecting armor.

2. By investigating the characteristics of the reinforced composite structures within the beetle forewing, it was found that densely distributed chitin fibers were located around 
the void lamination in the endocuticle of the forewing and that they have a rectangular cross section, possessing a maximum fiber volume fraction for reinforcement. Meanwhile, the sparsely distributed cross sections within the exocuticle at the epipleuron tip of the forewing are of a circular cross section and have a strong interfacial reinforcement within a protein matrix.

3. Both beetle species forewings are of the sandwich plate structure with a central void layer, in which there exists many distributed trabeculae. Thus, the beetle forewings are a lightweight composite frame structural design. The structure of the A. dichotoma forewing is disposable and shows an economical design, while the structure of $P$. inclinatus shows a strong and durable design.

\section{Applications of the beetle forewing structure for developing composite materials}

Honeycomb structures are typical lightweight and high-strength composites. There are numerous examples of related studies and applications: from nanomaterials [Kim, 2006; Hideki \& Kenji, 1995] to massive structures, such as those used in F4 fighters, Boeing-767 and Airbus-380 airplanes, and Boeing-360 helicopters [Sato \& Kino, 2004; Shafizadeh et al., 1999; Llorente, 1989]. Presently manufactured commercial honeycomb sandwich plates are produced by adhesively joining the plate parts and core parts, which are made separately by different processes [Leng, 2009; http://www.corecomposites.com; Chen et al., 2008; http://www.nida-core.com/english/contact.htm]. The sandwich plates made by the above processes can be readily separated at the joint of the side plates to the core, and this separation potential is a factor that limits both the strength and the side sealing effectiveness. Furthermore, the use of the adhesive glue is not only environmentally harmful but also expensive [Han et al., 2002; Gu et al., 2010]. To overcome the weaknesses associated with traditional manufacturing method, we have recently developed an integrated molding process [Chen et al., 2012] and the bionic composite sample of the first integrated honeycomb plate is shown in Fig. 22
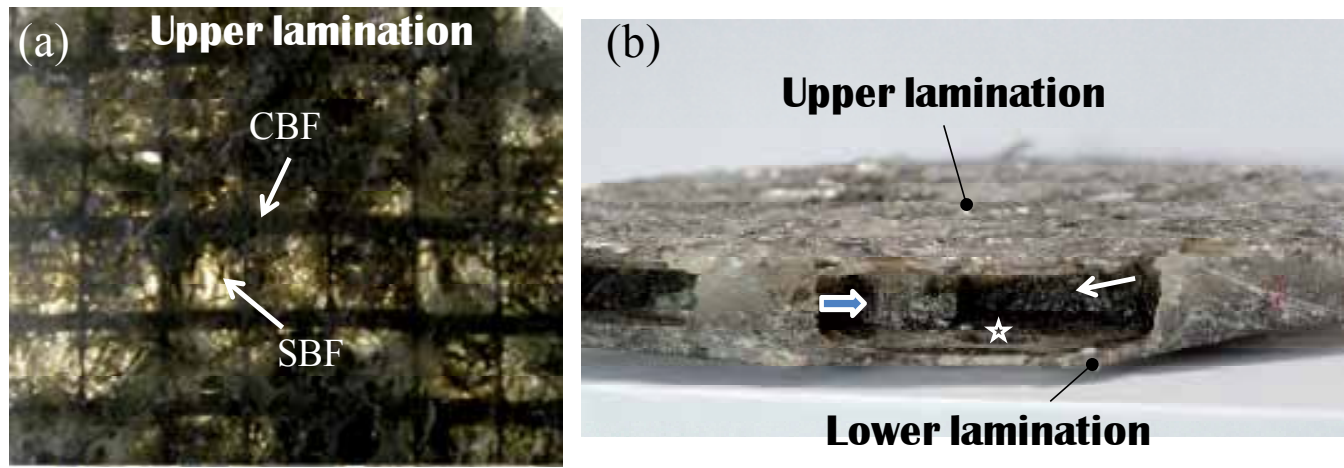

Figure 22. An example of the bionic composite. (a) Front view, (b) side view. CBF denotes continuous basalt fiber; SBF denotes short basalt fiber; the thick and thin arrows indicate the trabeculae and the honeycomb walls, respectively, and the star indicates a processing hole. 
Fig. 23 shows the flow chart of the integrated manufacturing process used to produce the honeycomb plate [Chen et al., 2012]. To improve the association between the BF (Basalt Fiber) and the epoxy resin, the surfaces of the short BFs and CBF (Continuous Basalt Fiber) geogrid were treated with a silane-coupling agent. At a mass ratio of $30 \%$, the BFs were placed in an ethanol solution with $0.75 \% \mathrm{KH} 550$ ( $\gamma$-aminopropyltriethoxysilane); the BFs were removed from the ethanol solution after $30 \mathrm{~min}$ of soaking and were allowed to dry naturally. Finally, the BFs were heat treated for 1 hour at $120{ }^{\circ} \mathrm{C}$ in an oven. At the same time, the mold tools were prepared for the integrated molding, the CBF geogrid was placed in the upper and lower layer, and then the short BFs and epoxy resin were evenly mixed. Table 4 lists the materials used for the mixture and their mass ratios [Chen et al., 2012].

\subsection{Experiment}

Fig. 23 shows the flow chart of the integrated manufacturing process used to produce the honeycomb plate [Chen et al., 2012]. To improve the association between the BF (Basalt Fiber) and the epoxy resin, the surfaces of the short BFs and CBF (Continuous Basalt Fiber) geogrid were treated with a silane-coupling agent. At a mass ratio of $30 \%$, the BFs were placed in an ethanol solution with $0.75 \%$ KH550 ( $\gamma$-aminopropyltriethoxysilane); the BFs were removed from the ethanol solution after $30 \mathrm{~min}$ of soaking and were allowed to dry naturally. Finally, the BFs were heat treated for 1 hour at $120{ }^{\circ} \mathrm{C}$ in an oven. At the same time, the mold tools were prepared for the integrated molding, the CBF geogrid was placed in the upper and lower layer, and then the short BFs and epoxy resin were evenly mixed. Table 4 lists the materials used for the mixture and their mass ratios [Chen et al., 2012].

After pouring the well-mixed fluid of fibers and resin into the mold tools, the mixture was vacuum-dried for 30 minutes at room temperature in a vacuum oven. A portion of aluminum foil and a heavy object were set on the assembly of mold tools after the tools were removed from the vacuum oven. The assembly was then cured for 10 hours at $35^{\circ} \mathrm{C}$ in an incubator. The final molded sample was obtained by removing the paraffin support at $75{ }^{\circ} \mathrm{C}$ in the incubator.

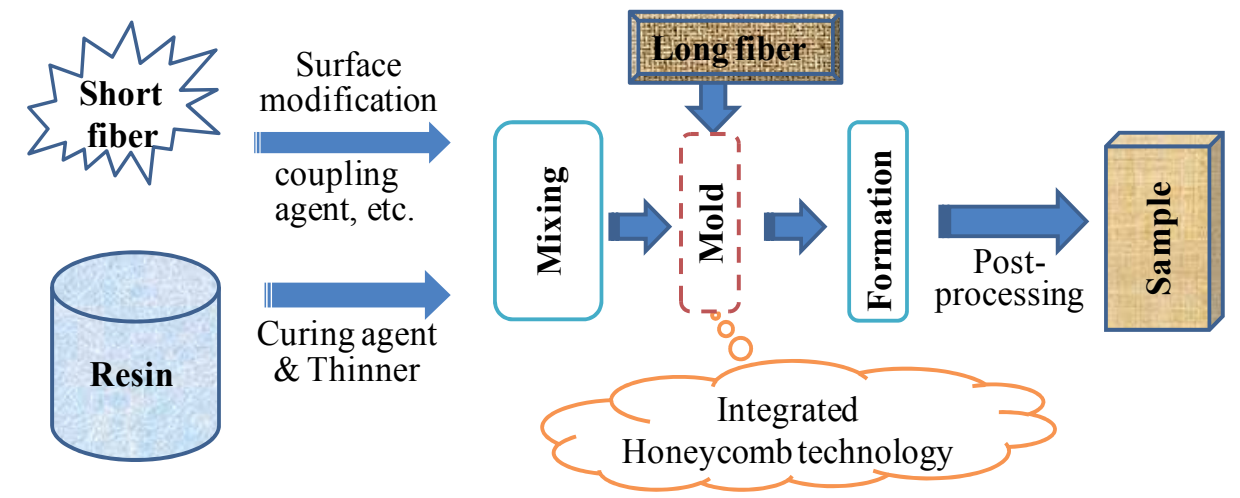

Figure 23. Flow chart of the integrated honeycomb technology. 


\begin{tabular}{|c|c|c|c|c|}
\hline Material & Epoxy resin & Curing agent & Thinner & Short basalt fiber \\
\hline $\mathrm{m} / \mathrm{g}$ & 80 & 20 & 10 & 10 \\
\hline $\mathrm{w} / \%$ & 66.7 & 16.7 & 8.3 & 8.3 \\
\hline
\end{tabular}

Table 4. Main materials and their mass ratios

\subsection{Results and discussion}

In this section, we begin with the examination of the natural edge sealing structure used in the beetle forewing, then we investigate the mechanisms of structure formation and the structure functions, and last, we propose an edge sealing technique for integrated honeycomb processing technology.

\subsubsection{Edge sealing of the forewing integrated honeycomb plates and its biomimetic application}

As mentioned above, the macroscopic structure of the beetle forewing features a sandwich plate construction with extensive internal meshwork (denoted by stars in Fig. 2 a, b) and hundreds of trabeculae (denoted by thick arrows in Fig. 2 a-e). The cross section of the forewing edge frame changes by location (denoted by EFS in Fig. 2a, e). With the exception of the concavo-convex junction between the left (denoted by round arrows, Fig. 2e) and the right forewings, the edge frame is an ingeniously integrated sealing structure without any seams (Fig. 2e). Before the bionic application of the forewing of A. dichotoma could be carried out, a search for the formation mechanisms and an analysis of the structural function were conducted, as discussed in the next section.

\subsubsection{The formation mechanisms of the forewing integrated trabecular honeycomb structure}

To understand the natural mechanisms behind the formation of the forewing integrated honeycomb structure, we first investigated the hexagonally shaped honeycomb structure inside the beetle forewings (Fig. 2a, b); specifically, we were interested in the edge sealing structure without any seams. According to Thompson's cell partitioning theory (Fig. 24) [Thompson, 1945], when a cell membrane intersects with two other cells on a plane, angles $\alpha, \beta$, and $\gamma$ are determined by the reciprocity of the three tensions $t, T$, and $T^{\prime}$ at the intersection O. For example, three scenarios are shown in Fig. 24 (a)-(c) as follows: (a) when $\mathrm{T}^{\prime}=\mathrm{T}>\mathrm{t}$, i.e., when comparing $\mathrm{T}^{\prime}$ and $\mathrm{T}, \mathrm{t}$ can be ignored, and in this case, $\alpha$ and $\beta$ are right angles; (b) when $\mathrm{T}^{\prime}=\mathrm{T}>\mathrm{t}$, i.e., $\mathrm{T}^{\prime}$ and $\mathrm{T}$ are quite large compared with $\mathrm{t}$, and thus, $\alpha$ and $\beta$ are obtuse angles; (c) $\alpha, \beta$, and $\gamma$ are all $120^{\circ}$ when $\mathrm{T}^{\prime}=\mathrm{T}=\mathrm{t}$. The mesh structures inside the forewing of the $A$. dichotoma beetle are extensively distributed, and the forewing exhibits a curved structure with thousands of trabeculae and air sacs possessing dissimilar internal stresses. Most of the cells of the forewing have essentially the same function as the branched veins of the dragonfly wing [Thompson, 1945], and the forewing air sacs are distributed in a specific manner to form a $120^{\circ}$ angle between the two adjacent membranes, i.e., they are similar to a honeycomb structure (Fig. 2b). 

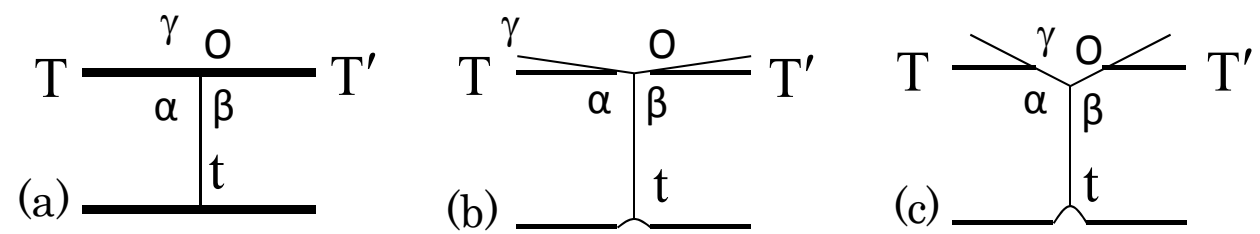

Figure 24. The theoretical cell shape and the relationship between the three tensile forces $T^{\prime}, T$ and $t:(a)$ $\mathrm{T}^{\prime}=\mathrm{T}>\mathrm{t}$, (b) $\mathrm{T}^{\prime}=\mathrm{T}>\mathrm{t}$, (c) $\mathrm{T}^{\prime}=\mathrm{T}=\mathrm{t}$ [Thompson, 1945].

In addition, to better understand why the forewing is constructed with the trabeculae and edge frame of the honeycomb structure, the functions of these structural features, the trabeculae and the edge frame, have been investigated previously [Chen et al., 2000; Chen et al., 2001a; Chen et al., 2007b; Ni et al., 2001]. These investigations have reported the following: the trabeculae and the edge frame provide bending stiffness and resistance to applied stress for the entire forewing structure [Chen et al., 2000; Chen et al., 2007b]. These structures also effectively increase the inter-laminar strength between the lamination layers of the composite structure [Chen et al., 2001a; Ni et al., 2001]. Furthermore, the structures impart the forewing with sufficient mechanical properties to enable the beetle with flight capabilities. These beneficial properties are achieved by the specific angles held between the different fiber layers in the upper lamination of the forewing [Chen et al., 2001a] and the natural design of the transitional region of the edge frame [Chen et al., 2000]. In this manner, the entire forewing of the $A$. dichotoma beetle forms the completely integrated trabecular honeycomb structure.

\subsubsection{Integrated honeycomb plates with edge sealing and the bionic imitation technique}

In the previous section, a complete schematic of a beetle forewing was included to demonstrate the structure of the integrated trabecular honeycomb plate with edge sealing (Fig. 25); in the present work, we did not consider the special functions of the edge frame,
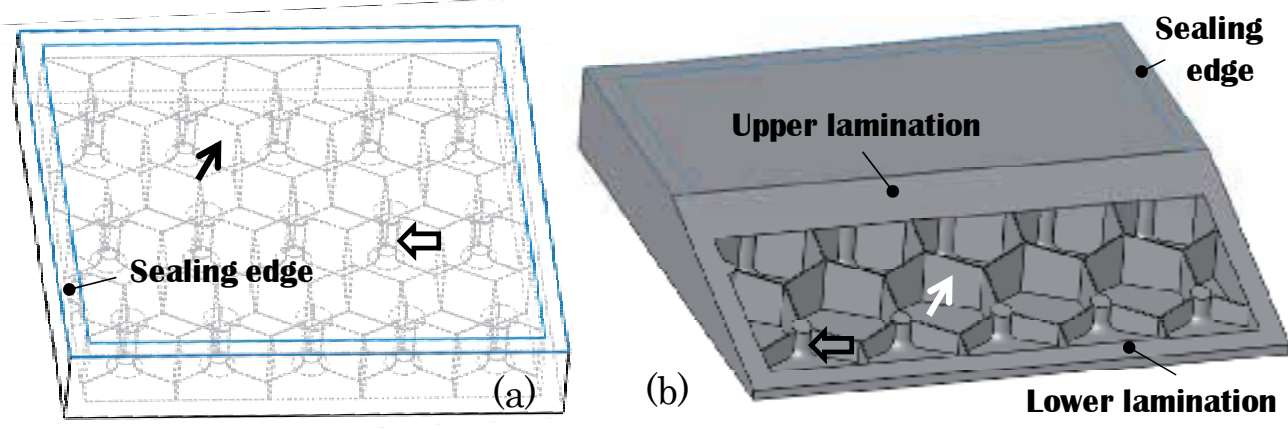

Figure 25. The edge sealing schematic developed for manufacturing the integrated honeycomb, which was inspired by the beetle forewing. (a) Entire integrated honeycomb plate, (b) oblique cross section of (a). The thick arrows indicate trabeculae; the thin arrows indicate honeycomb wall segments. 
and thus, the hollow part of the edge frame was not incorporated. This structure helped us to develop not only a new manufacturing method for producing honeycomb plates but also a new edge sealing technology. Fig. 26a shows a set of mold tools used for sealing operations. By adding extra space around the edge of the female mold [Chen et al., 2012], a sealing edge can be generated. Fig. 26b shows a cross sectional schematic of the sealing edge of the integrated honeycomb plate. This technology does not require bonding or other mechanical connections made by nail or inlay and is therefore preferred over the traditional honeycomb plates because of the simplified manufacturing process, the perfect sealing (as seams are not required) and the enhanced integration.
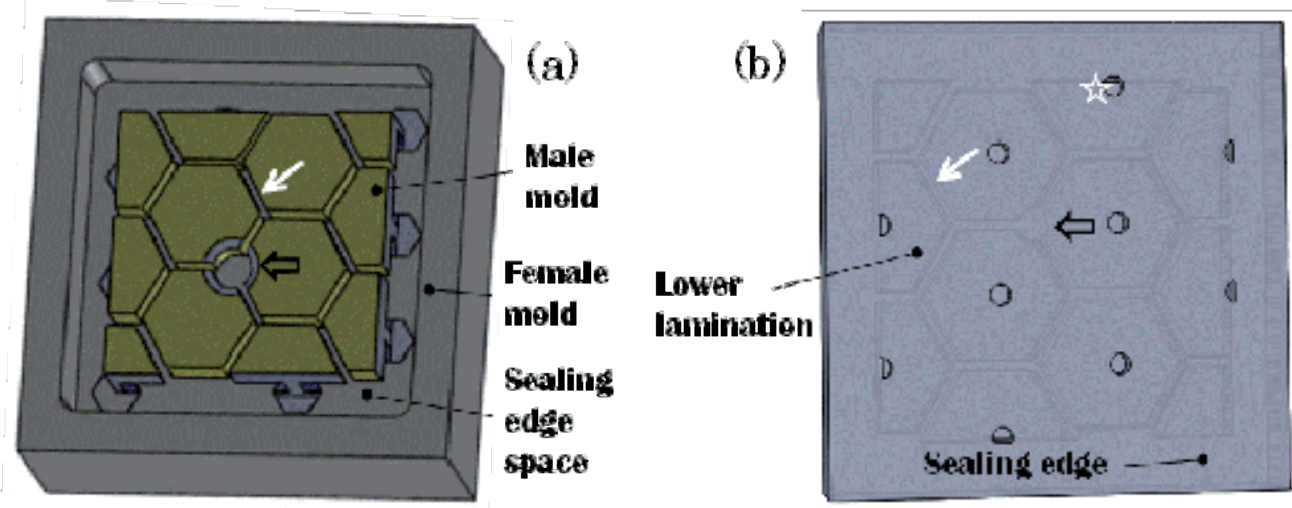

Figure 26. Schematic of the edge sealing approach for use in the integrated honeycomb technology. (a) Mold tools for bionic sealing, (b) image of the integrated honeycomb plate with the sealing edge. The thick arrows indicate trabeculae or the space used to form it; the thin arrows indicate honeycomb wall segments or the space used to form it, and the star indicates a processing hole.

\subsubsection{Processing and optimization of the integrated honeycomb technology}

Optimization of the processing hole In the integrated honeycomb plate, as shown in Fig. 26, there is a small processing hole in each core. However, the processing holes can have an adverse effect on the strength of the composite and should ultimately be minimized or eliminated. Certain approaches, such as decreasing the diameter of the holes or reducing their number by using integrated male molds (Fig. 27a), may prove effective toward reducing the impact of the processing holes on the composite strength. Increasing the interconnections between the basic male molds to integrate them into the structure can reduce the number of the processing holes needed; however, this requires special molds, as shown in Fig. 27b, c. To accomplish this goal, a thin-walled hexagonal box for each honeycomb unit can be formed and then integrated [Chen et al., 2012]. Integrating the boxes and then fusing them into the plate can help to eliminate the number of processing holes required. 

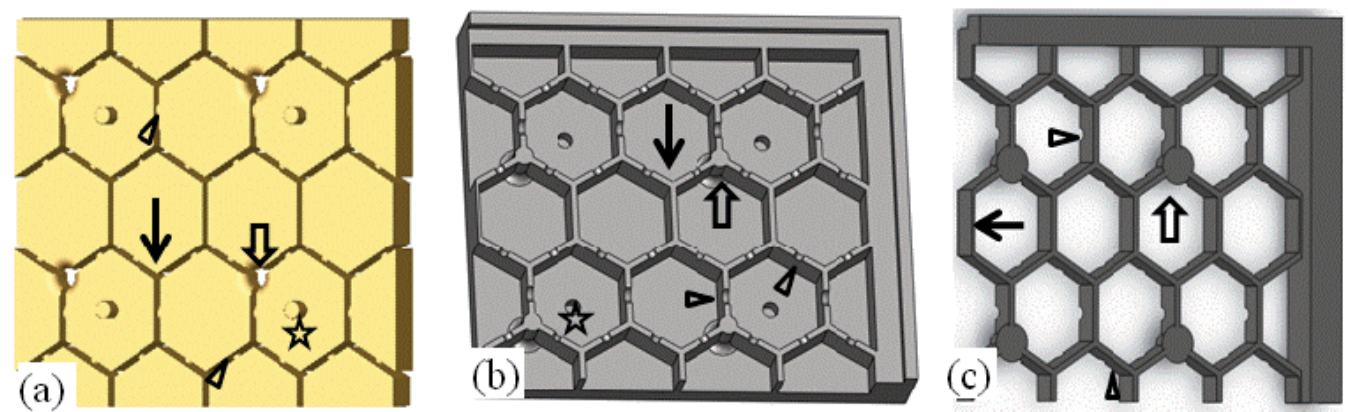

Figure 27. Schematic of the integrated male mold and its mold tools (only a quarter of the symmetric structure is shown in this figure). (a) Bottom view of the integrated male mold, (b) lower half of a mold tool assembly, (c) upper half of a mold tool assembly. The thick arrows show the trabeculae or the space to form them. The thin arrows indicate the honeycomb wall or the space to form them. The star indicates either a positioning hole or a positioning pin. The triangles indicate the processing columns or the space to form them.

Optimization of materials and manufacturing processes As discussed above, the long molding time required for the first method is a drawback. Even so, the molding time can be optimized by selecting appropriate resins or even by using the presently presented process of wax mold tools. For example, two laminating resins, TG-1001 ${ }^{\mathrm{TM}}$ and TG-1009 ${ }^{\mathrm{TM}}$, can be cured in a short period of time (such as $5 \mathrm{~min}$ ) and under a relatively low temperature (33$85^{\circ} \mathrm{C}$ ) [http://www.thermalguard-technology.com]; resin transfer molding and vacuumassisted molding are both performed in this temperature range. Such resins, which are noncombustible, exhibit high strength, are refractory, and meet our complete list of requirements. They also have potential for use in furniture manufacturing, civil engineering, transportation devices, and other applications. The disadvantages of these resins, such as the low content and uneven distribution of fibers, can still be optimized by depositing more fiber-woven roving, by prefilling a certain amount of short fibers into the resin, or by applying vacuum-assisted molding processes.

\subsubsection{Mechanical properties of the integrated trabecular honeycomb structure}

To gain knowledge of the mechanical properties of the integrated trabecular honeycomb structure, we focused on revealing whether fiber interconnections exist between the upper/lower laminations and the trabeculae; these interconnections are considered to be the main difference between the trabecular honeycomb plate and the traditional honeycomb plate. Several studies have previously investigated the mechanical properties of the trabecular honeycomb structure of the beetle forewing as well as bionic materials based on the beetle forewing. First, experiments and analyses using the finite-element method (FEM) were conducted on living forewing specimens and their peeling models; the results indicated that the forewing possesses a high inter-laminar strength value [Ni et al., 2001; Gu et al., 2010]. Second, a bionic composite specimen made from a sandwich structure with fiber-reinforced trabeculae was constructed; the experimental results demonstrated that the 
energy release rate and the maximum shear stress of the fiber-reinforced material were approximately four times greater than those of a traditional plate without fiber-reinforced trabeculae [Okazaki et al., 2005]. Third, the energy-absorbing capabilities of the original and improved models of the beetle forewing were analyzed using nonlinear FEM, and it is clear from the models that the forewing structure is both crashworthy and energy-absorbing, particularly in the improved models [Guo \& Wang, 2011]. Furthermore, the trabecular honeycomb plates that were produced by our bionic method not only achieved integrated preparation between the upper and lower lamination and the core lamination but also produced a natural seamless edge using the new sealing technology. Additionally, the reinforcing fibers were distributed between the core layer (trabeculae, honeycomb walls) and the upper and lower laminations (including the edge part); that is, the bionic materials of the real integration trabecular honeycomb plate are similar to the biological structure of the beetle forewing. Therefore, the bionic plate should exhibit excellent mechanical properties, as suggested by the aforementioned physical experiments and FEM analysis.

\subsection{Conclusions}

The processes for manufacturing integrated honeycomb plates have been outlined, and the problems associated with the integrated honeycomb technology have been discussed and resolved:

1. Each forewing of a beetle has a natural edge sealing design with a completely integrated structure consisting of honeycomb cores, including trabeculae and an edge frame. The formation mechanism of the integrated trabecular honeycomb structure and its mechanical properties were discussed. An edge sealing technique inspired by the forewing structure was proposed. This technique has numerous advantages, such as processing simplicity and complete seamless integrated.

2. For the first time, detailed manufacturing processes have been developed and presented toward achieving integrated honeycomb plates that include edge sealing. Optimization methods have been developed, such as integrating the male molds that can eliminate the influence of the processing holes and choosing a fast-curing and hightemperature resin that can shorten the molding time.

\section{Author details}

Jinxiang Chen* and Juan Xie

International Institute for Urban Systems Engineering \& School of Civil Engineering, Southeast University, China

Qing-Qing Ni

Dept. of Functional Machinery \& Mechanics, Shinshu University, Japan

" Corresponding Author 


\section{Acknowledgement}

This work was supported by the Natural Science Foundation of China (Grant No. 51173026), the Jiangsu NSF (No.BK2010015) and the National Key Technologies R\&D Program of China (2011BAB03B10).

\section{References}

Banerjee, S. (1988) Organisation of Wing Cuticle in Locusta Migratoria Linnaeus, Tropidacris Cristata Linnaeus and Romalea Microptera Beauvais (Orthoptera: Acrididae). International Journal of Insect Morphology and Embryology, Vol. 17, pp 313-326.

Ben, G.; Nishi, Y. \& Asano, S. (1998) A Discussion of Mechanical Propreties for an Optimum Stackling Sequence in Beetles. Nippon Kikai Gakkai Zairyo Rikigaku Bumon Koenkai Koen Ronbunshu, Vol. 1998-B, pp 349-350.

Chen, J.; Dai, G.; Xu, Y. \& Iwamoto, M. (2007a) Optimal Composite Structures in the Forewings of Beetles. Journal of Composite Structures, Vol. 81, pp 432-437.

Chen, J.; Gu, C.; Guo, S.; Wan, C.; Wang, X.; Xie, J. \& Hu, X. (2012) Integrated Honeycomb Technology Motivated by the Structure of Beetle Forewings. Journal of Bionic Engineering. doi:10.1016/j.msec.2012.04.067.

Chen, J.; Iwamoto, M.; Ni, Q.Q.; Kurashiki, K. \& Saito, K. (2000) Cross Sectional Structure and its Optimality of the Forewing of Beetles. Journal of the Society of Materials Science, Vol. 49, No. 4, pp 407-412.

Chen, J.; Iwamoto, M.; Ni, Q.Q.; Kurashiki, K. \& Saito, K. (2001a) Laminated Structure and its Mechanical Properties of the Forewing of Beetle. Journal of the Society of Materials Science, Vol. 50, pp 455-460.

Chen, J.; Ni, Q.Q.; Endo, Y. \& Iwamoto, M. (2001b) Fine Structure of the Trabeculae in the Forewing of Allomyrina Dichotoma (Linne) and Prosopocoilus Inclinatus, (Motschulskey) (Coleoptera: Scarabaeidae). Insect Science, Vol. 8, pp 115-123.

Chen, J.; Ni, Q.Q; Endo, Y. \& Iwamoto, M. (2002) Distribution of the Trabeculae in the Forewing of Horned Beetle, Allomyrina Dichotoma (Linne) (Coleoptera: Scarabaeidae). Insect Science, Vol. 9, pp 55-61.

Chen, J.; Ni, Q.Q.; Li, Q. \& Xu, Y. (2005) Biomimetic Lightweight Composite Structure with Honeycomb-trabecula. Acta Materiae Compositae Sinica, Vol. 22, pp 103-108.

Chen, J.; Ni, Q.Q.; Xu, Y. \& Iwamoto, M. (2007b) Lightweight Composite Structures in the Forewings of Beetles. Journal of Composite Structures, Vol. 79, pp 331-337.

Chen, J.; Xie, J.; Zhu, H.; Guan, S.; Wu, G.; Noori, M.N. \& Guo, S. (2012) Integrated Honeycomb Structure of a Beetle Forewing and its Imitation. Materials Science $\mathcal{E}$ Engineering C, Vol. 32, pp 613-618.

Chen, X.; Sun, Y. \& Gong, X. (2008) Honeycomb Textile Composites, and Experimental Analysis of 3D Honeycomb Textile Composites Part I: Design and Manufacture. Textile Research Journal, Vol. 78, No. 9, pp 771-781. 
Gu, R.; Guo, K.; Qi, C.; Zhang, J. \& Liu, Z. (2010) Properties and Bonding Mechanism of Konjak Powder-chitosan-PVA Blending Adhesive. Transactions of the CSAE, Vol. 26, No. 5, pp 373-378.

Gullan, P.J. \& Cranstion, P. (1994) The Insects: An Outline of Entomology. Chapman \& Hall, pp 22-55.

Guo, T.; Wang, Y.F.; (2011) Energy Absorbing Structures Imitating Trabecular of Beetle Cuticles. Engineering Mechanics, Vol. 28, No. 2, pp 246-251.

Han, T.S.; Ural, A.; Chen, C.S.; Zehnder, A.T.; Ingraffea, A.R.; Billington, S.L. (2002) Delamination Buckling and Propagation Analysis of Honeycomb Panels Using a Cohesive Element Approach. International Journal of Fracture, Vol. 115, pp 101-123.

He, M. \& Cox, B.N. (1998) Crack Fridging by Through-Thickness Reinforcement in Delaminating Curved Structures, Composites Part A, Vol. 29A, pp 377-393.

Hepburn, H.R. (1976) The Insect Integument. Elsevier scientific Publication Company,New York, pp 1-100.

Hideki, M. \& Kenji, F. (1995) Ordered Metal Nanohole Arrays Made by a Two-Step Replication of Honeycomb Structures of Anodic Alumina. Science, Vol. 268, No. 5216, pp 1466-1468.

Hosur, M.V.; Abdullah, M.; Jeelani, S. (2005) Manufacturing and low-velocity impact characterization of foam filled 3-D integrated core sandwich composites with hybrid face sheets. Composite Structures, Vol. 69, No. 2, pp 167-181.

http://www.corecomposites.com

http://www.thermalguard-technology.com.

http://www.nida-core.com/english/contact.htm

Ishii, S. (1982) Physiology of Insects. Baifukan press, pp 40-57.

Iwamoto, M.; Chen, J.; Ni, Q.Q.; Kurashiki, K. \& Saito, K. (1999b) Structure Optimality with Biomimetics of the Fore-wing of Beetles. The 3rd China-Japan Joint Conference on Composites, HangZhou, pp 15-20.

Iwamoto, M.; Ni, Q.Q.; Fujwara, T. \& Kurashiki, K. (1999a) Interlaminar Fracture Mechanism in Unidirectional CFRP Composites-Part I: Interlaminar Toughness and AE Characteristics. Engineering Fracture Mechanics, Vol. 64, pp 721-745.

Jung, W.Y. \& Aref, A.J. (2005) Analytical and Numerical Studies of Polymer Matrix Composite Sandwich Infill Panels. Composite Structures, Vol. 68, pp 359-370.

Kim, H.; Kim, J.; Yang, H.; Suh, J.; Kim, T.; Han, B.; Kim, S.; Kim, D.S.; Pikhitsa, P.V. \& Choi, M.; (2006) Parallel Patterning of Nanoparticles via Electrodynamic Focusing of Charged Aerosols. Nature Nanotechnology, Vol. 1, pp 117-121.

Leopoldj, R.A.; Newman, S.M. \& Helgeson, G. (1992) A Comparison of Cuticle Deposition during the Pre-and Post-eclosion Stages of the Adult Weevil, Anthonomus Grandis Boheman. International Journal of Insect Morphology and Embryoogyl, Vol. 21, pp 37-62. 
Llorente, S. (1989) Honeycomb Sandwich Primary Structure Applications on the Boeing Model 360 Helicopter. Society for the Advancement of Material and Process Engineering, Vol. 34, No. 5, pp 824-838.

Mania, R. (2005) Buckling Analysis of Trapezoidal Composite Sandwich Plate Subjected to In-plane Compression. Composite Structures, Vol. 69, pp 482-490.

Masuda, Y.; Okada, A.; Tabata, H.; Yoneda, K. \& Yamamoto, Y. (1995) Structure and mechanical properties of the elytra of pachyrhynchid weevils. Lecture Article of L,ecture Meeting of Japan Mechanical Society Department of Materials Mechanics, pp 121-122.

Matsunaga, H. (2002) Assessment of a Global Higher-order Deformation Theory for Laminated Composite and Sandwich Plates. Composite Structures, Vol. 56, pp 279291.

Nguyen, V.T.; Caron, J.F. \& Sab, K. (2005) A Model for Thick Laminates and Sandwich Plates. Composites Science and Technology, Vol. 65, pp 475-489.

Ni, Q.Q.; Chen, J.; Iwamoto, M.; Kurashiki, K. \& Saito, K. (2001) Interlaminar Reinforcement Mechanism in a Beetle Fore-Wing. JSME International Journal Series C, Vol. 44, No. 4, pp 1111-1116.

Okazaki, J.; Ni, Q.Q; Iwamoto, M. \& Kurashiki, K. (2005) Research on Design of a Composite Material Imitated from the Fore-wing of a Beetle. Third International Workshop on Green Composites, pp 91-94.

Sato, T. \& Kino, Z. (2004) Exploration for application business of honeycomb cores and composite materials. Function and Materials, Vol. 24, pp 64-73.

Sekine, H. \& Kamiya, S. (1987) Analysis of a Shear Fracture in Unidirectional FiberReinforced Composites II (For the Case That the Displacement on Boundaries is Constrained in the Direction Normal to the Shear Fracture). Japan Society Mechanical Engineers, Vol. 53, No. 489A, pp 930-934.

Shafizadeh, J.E; Seferis, J.C.; Chesmar, E.F. \& Geyer, R. (1999) Evaluation of the in-service performance behavior of honeycomb composite sandwich structures. Journal of Materials Engineering and Performance, Vol. 8, No. 6, pp 661-668.

Tanimoto, T.; Chen, Q.H. \& Taguchi, M. (1998) Damage Tolerance of biomimetic CFRP Laminate. Abstacts of 51th Annual Meeting of Textile Mechanical Society, pp 78-79.

Thompson, D. W. (1945) On Growth and Form. Cambridge University press, pp 1-131.

Turss, R.W.; Hine, P.J. \& Duckett, R.A. (1997) Interlaminar and Intralaminar Fracture Toughness of UniaxialContinuous and Discontinuous Carbon Fiber/Epocy Composites. Composites Part A: Applied Science and Manufacturing, Vol. 28, pp 627-636.

Leng, L. (2009) An Edge-sealed Table Top Board with Paper Honeycomb Core. Patent (Uk) No. 0722071.8.

Wainwright, S.A.; Biggs, W.D.; Currey, J.D. \& Gosline, J.M. (1976) Mechanical Design in Organisms. Princeton University Press, pp 158-170.

Yoshikawa, H. (1985) The Statistical Analysis Procedure by the Portable Calculator. Japan Union Science Engineering Press publisher, pp 28-32. 
Zelazny, B. \& Neville, A.C. (1972) Quantitative Studies on Fibril Orientation in Beetle Endocuticle. Insect Physiology, Vol. 18, pp 2095-2121. 


\title{
Comparative Review Study on Elastic Properties Modeling for Unidirectional Composite Materials
}

\author{
Rafic Younes, Ali Hallal, Farouk Fardoun and Fadi Hajj Chehade
}

Additional information is available at the end of the chapter

http://dx.doi.org/10.5772/50362

\section{Introduction}

Due to the outstanding properties of $2 \mathrm{D}$ and $3 \mathrm{D}$ textile composites, the use of 3D fiber reinforced in high-tech industrial domains (spatial, aeronautic, automotive, naval, etc...) has been expanded in recent years. Thus, the evaluation of their elastic properties is crucial for the use of such types of composites in advanced industries. The analytical or numerical modeling of textile composites in order to evaluate their elastic properties depend on the prediction of the elastic properties of unidirectional composite materials with long fibers composites "UD". UD composites represent the basic element in modeling all laminates or 2D or 3D fabrics. They are considered as transversely isotropic materials composed of two phases: the reinforcement phase and the matrix phase. Isotropic fibers (e.g. glass fibers) or anisotropic fibers (e.g. carbon fibers) represent the reinforcement phase while, in general, isotropic materials (e.g. epoxy, ceramics, etc...) represent the matrix phase (Figure 1).

The effective stiffness and compliance matrices of a transversely isotropic material are defined in the elastic regime by five independent engineering constants: longitudinal and transversal Young's moduli E11 and E22, longitudinal and transversal shear moduli $\mathrm{G}_{12}$ and $\mathrm{G}_{23}$, and major Poisson's ratio $v_{12}$ (Noting that direction 1 is along the fiber). The minor Poisson's ratio $v_{23}$ is related to $E_{22}$ and $\mathrm{G}_{12}$. The effective elastic properties are evaluated in terms of mechanical properties of fibers and matrix (Young's and shear moduli, Poisson's ratios and the fiber volume fraction $\mathrm{V}^{\mathrm{f}}$ ). The compliance matrix [S] of a transversely isotropic material is given as follow:

$$
[S]=\left[\begin{array}{cccccc}
1 / E_{11} & -v_{12} / E_{11} & -v_{12} / E_{11} & 0 & 0 & 0 \\
-v_{12} / E_{11} & 1 / E_{22} & -v_{23} / E_{22} & 0 & 0 & 0 \\
-v_{12} / E_{11}-v_{23} / E_{22} & 1 / E_{22} & 0 & 0 & 0 \\
0 & 0 & 0 & 1 / G_{23} & 0 & 0 \\
0 & 0 & 0 & 0 & 1 / G_{12} & 0 \\
0 & 0 & 0 & 0 & 0 & 1 / G_{12}
\end{array}\right]
$$


The stiffness matrix [C] is the invers of the compliance matrix [S].

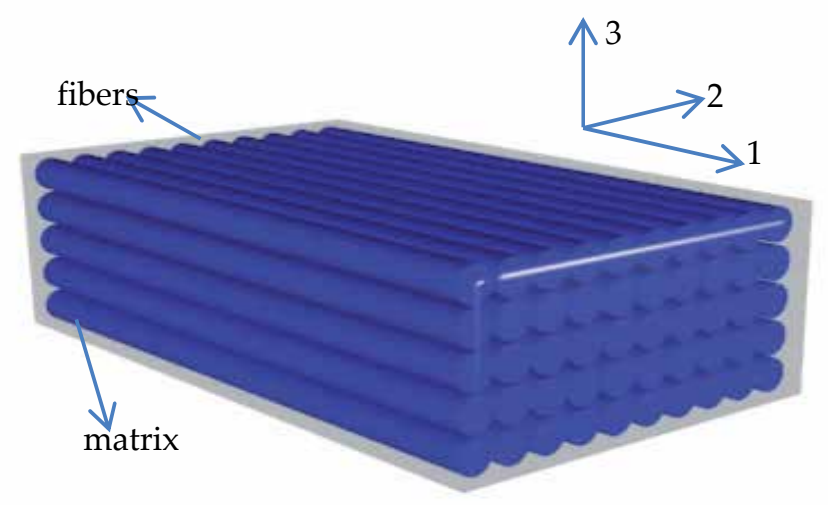

Figure 1. Unidirectional Composite.

In this chapter, a review of most known available analytical micromechanical models is presented in the second section of this chapter. Investigated models belonged to different categories: phenomenological models, semi-empirical models, elasticity approach models and homogenization models. In addition, the evaluation of elastic properties of UD composites using numerical FE method is investigated. Boundary, symmetric and periodic conditions, with different unit cells (square, hexagonal and diamond arrays), are discussed. In the third section, a comparison of the results obtained by the investigated analytical and numerical models is compared to available experimental data for different kinds of UD composites.

\section{Review}

The prediction of the mechanical properties of UD composites has been the main objective of many researches. Various micromechanical models have been proposed to evaluate the elastic properties of UD composites. These models could be divided into four categories: phenomenological models, elasticity approach models, semi-empirical models and homogenization models.

\subsection{Phenomenological models}

\subsubsection{Rule of Mixture "ROM"}

The well-known models that have been proposed and used to evaluate the properties of UD composites are the Voigt [1] and Reuss [2] models. The Voigt model is also known as the rule of mixture model or the iso-strain model, while the Reuss model is also known as the invers rule of mixture model or the iso-stress model.

Elastic properties are extracted from the two models where they are given under the rule of mixture (ROM) and the invers rule of mixture models (IROM). 


$$
\begin{gathered}
E_{11}=V^{f} \cdot E_{11}^{f}+V^{m} \cdot E^{m} \quad \text { (from Voigt model) } \\
v_{12}=V_{f} v_{11}^{f}+V_{m} v^{m} \quad \text { (from Voigt model) } \\
E_{22}=\frac{E_{22}^{f} \cdot E^{m}}{E^{m} \cdot V^{f}+E_{22}^{f} \cdot V^{m}} \quad \text { (from Reuss model) } \\
G_{12}=\frac{G_{12}^{f} \cdot G^{m}}{G^{m} \cdot V^{f}+G_{12}^{f} \cdot V^{m}} \quad \text { (from Reuss model) }
\end{gathered}
$$

\subsection{Semi-empirical models}

Semi empirical models have emerged to correct the ROM model where correcting factors are introduced. Under this category, it's noticed three important models: the modified rule of mixture, the Halpin-Tsai model [3] and Chamis model [4].

\subsubsection{Modified Rule of Mixture (MROM)}

While the investigations show that the obtained results by the ROM model for E11 and ๑12 are in good agreement with experimental and finite element data, the results for $\mathrm{E}_{22}$ and $\mathrm{G}_{12}$ do not agree well with experimental and finite element data. Corrections have been made for $E_{22}$ and $\mathrm{G}_{12}$.

$$
\frac{1}{E_{22}}=\frac{\eta^{f} \cdot V^{f}}{E_{22}^{f}}+\frac{\eta^{m} \cdot V^{m}}{E^{m}}
$$

Where factors $\eta^{f}, \eta^{m}$ are calculated as:

$$
\begin{gathered}
\eta^{f}=\frac{E_{11}^{f} \cdot V^{f}+\left[\left(1-v_{12}^{\mathrm{f}} \cdot v_{21}^{\mathrm{f}}\right) \cdot E^{m}+v^{\mathrm{m}} \cdot v_{21}^{\mathrm{f}} \cdot E_{11}^{f}\right] \cdot V^{m}}{E_{11}^{f} \cdot V^{f}+E^{m} \cdot V^{m}} \\
\eta^{m}=\frac{\left[\left(1-v^{\mathrm{m}^{2}}\right) \cdot E_{11}^{f}-\left(1-v^{\mathrm{m}} \cdot v_{12}^{\mathrm{f}}\right) \cdot E^{m}\right] \cdot V^{f}+E^{m} \cdot V^{m}}{E_{11}^{f} \cdot V^{f}+E^{m} \cdot V^{m}} \\
\frac{V^{f}}{G_{12}^{f}}+\frac{\eta^{\prime} \cdot V^{m}}{G^{m}} \\
V^{f}+\eta^{\prime} \cdot V^{m}
\end{gathered}
$$

With $0<\eta^{\prime}<1$, (it is preferred to take $\eta^{\prime}=0.6$ )

\subsubsection{Halpin-Tsai model [3]}

The Halpin-Tsai model also emerged as a semi-empirical model that tends to correct the transversal Young's modulus and longitudinal shear modulus. While for E11 and v12, the rule of mixture is used. 


$$
\begin{gathered}
E_{22}=E^{m} \cdot\left(\frac{1+\zeta \eta V_{f}}{1-\eta V_{f}}\right) ; G_{12}=G^{m} \cdot\left(\frac{1+\zeta \eta V_{f}}{1-\eta V_{f}}\right) \\
\text { with } \eta=\left(\frac{M_{f} / M_{m}-1}{M_{f} / M_{m}+\zeta}\right)
\end{gathered}
$$

with $\zeta=1$ and 2 , and $M=E$ or $G$ for $E_{22}$ and $G_{12}$ respectively.

\subsubsection{Chamis model [4]}

The Chamis micromechanical model is the most used and trusted model which give a formulation for all five independent elastic properties. It's noticed in this model that E11 and $v_{12}$ are also predicted in the same maner of the ROM model, while for other moduli, $\mathrm{V}^{\mathrm{f}}$ is replaced by its square root.

$$
\begin{aligned}
& E_{11}=V^{f} E_{11}^{f}+V^{m} E^{m} \\
& E_{22}=\frac{E^{m}}{1-\sqrt{V^{f}}\left(1-E^{m} / E_{22}^{f}\right)} \\
& v_{12}=V^{f} v_{12}^{f}+V^{m} v^{m} \\
& G_{12}=\frac{G^{m}}{1-\sqrt{V^{f}}\left(1-G^{m} / G_{12}^{f}\right)} \\
& G_{23}=\frac{G^{m}}{1-\sqrt{V^{f}}\left(1-G^{m} / G_{23}^{f}\right)}
\end{aligned}
$$

\subsection{Elasticity approach models}

Under this category, Hashin and Rosen [5] initially proposed a composite cylinder assemblage model (CCA) to evaluate the elastic properties of UD composites. Moreover, Christensen proposed a generalized self-consistent model [6] in order to better evaluate the transversal shear modulus $\mathrm{G}_{23}$.

$$
\begin{gathered}
E_{11}=V^{f} E_{11}^{f}+V^{m} E^{m}+\frac{4 \cdot V^{f} \cdot V^{m} \cdot\left(v_{12}^{f}-v^{m}\right)^{2}}{\frac{V^{f}}{K^{m}}+\frac{1}{G^{m}}+\frac{V^{m}}{K^{f}}}(\text { Hashin and Rosen [5]) } \\
v_{12}=V^{f} \cdot v_{12}^{f}+V^{m} v^{m}+\frac{V^{f} V^{m}\left(v_{12}^{f}-v^{m}\right)\left(\frac{1}{K^{m}}-\frac{1}{K^{f}}\right)}{\frac{V^{f}}{K^{m}}+\frac{1}{G^{m}}+\frac{V^{m}}{K^{f}}} \text { (Hashin and Rosen [5]) } \\
G_{12}=G^{m} \cdot \frac{G^{f} \cdot\left(1+V^{f}\right)+G^{m} \cdot V^{m}}{G^{f} \cdot V^{m}+G_{m}\left(1+V^{f}\right)}(\text { Hashin and Rosen [5]) }
\end{gathered}
$$

$G_{23}$ is the solution of the following equation: (Christensen [6])

$$
A\left(\frac{G_{23}}{G_{m}}\right)^{2}+2 B\left(\frac{G_{23}}{G_{m}}\right)+C=0
$$


With:

$$
\begin{gathered}
\mathrm{A}=3 V^{f} \cdot\left(1-V^{f}\right)^{2} \cdot\left(\frac{G_{23}^{f}}{V^{m}}-1\right)\left(\frac{G_{23}^{f}}{G^{m}}+\eta_{f}\right) \\
+\left[\frac{G_{23}^{f}}{G^{m}} \eta_{m}+\eta_{f} \eta_{m}-\left(\frac{G_{23}^{f}}{G^{m}} \eta_{m}-\eta_{f}\right) V^{f^{3}}\right] \cdot\left[V_{f} \eta_{m}\left(\frac{G_{23}^{f}}{G^{m}}-1\right)-\left(\frac{G_{23}^{f}}{G^{m}} \eta_{m}+1\right)\right] \\
\mathrm{B}=-3 V^{f} V^{m^{2}}\left(\frac{G_{23}^{f}}{G^{m}}-1\right)\left(\frac{G_{23}^{f}}{G^{m}}+\eta_{f}\right)+\frac{V^{f}}{2}\left(\eta_{m}+1\right)\left(\frac{G_{23}^{f}}{G^{m}}-1\right)\left[\frac{G_{23}^{f}}{G^{m}}+\eta_{f}+\left(\frac{G_{23}^{f}}{G^{m}} \eta_{m}-\eta_{f}\right) V^{\left.f^{3}\right]}\right. \\
+\left\{\frac{1}{2}\left[\frac{G_{23}^{f}}{G^{m}} \eta_{m}-\left(\frac{G_{23}^{f}}{G^{m}}-1\right) V^{f}+1\right] \cdot\left[\left(\eta_{f}-1\right)\left(\frac{G_{23}^{f}}{G^{m}}+\eta_{f}\right)-2\left(\frac{G_{23}^{f}}{G^{m}} \eta_{m}-\eta_{f}\right) V^{f^{3}}\right]\right\} \\
\mathrm{C}=-3 V^{f} V^{m^{2}}\left(\frac{G_{23}^{f}}{G^{m}}-1\right)\left(\frac{G_{23}^{f}}{G^{m}}+\eta_{f}\right)+\left[\frac{G_{23}^{f}}{G^{m}} \eta_{m}+\left(\frac{G_{23}^{f}}{G^{m}}-1\right) V^{f}+1\right]\left[\frac{G_{23}^{f}}{G^{m}}+\eta_{f}+\left(\frac{G_{23}^{f}}{G^{m}} \eta_{m}-\eta_{f}\right) V^{f^{3}}\right]
\end{gathered}
$$

With

$$
\eta_{m}=3-v_{m} ; \eta_{f}=3-v_{23}^{f}
$$

$K_{f}=\frac{E_{f}}{2\left(1-2 v_{f}\right)\left(1+v_{f}\right)}$ and $K_{m}=\frac{E_{m}}{2\left(1-2 v_{m}\right)\left(1+v_{m}\right)}$ are the bulk modulus of the fiber and the matrix under longitudinal strain respectively .

$$
v_{23}=\frac{K-m \cdot G_{23}}{K+m \cdot G_{23}} ; \text { with } \mathrm{m}=1+4 \mathrm{~K} \cdot \frac{v_{12}^{2}}{E_{11}}
$$

$\mathrm{K}$ is the bulk modulus of the composite under longitudinal strain

$$
\begin{gathered}
\mathrm{K}=\frac{K^{m} \cdot\left(K^{f}+G^{m}\right) \cdot V^{m}+K^{f} \cdot\left(K^{m}+G^{m}\right) \cdot V^{f}}{\left(K^{f}+G^{m}\right) \cdot V^{m}+\left(K^{m}+G^{m}\right) \cdot V^{f}} \\
E_{22}=2 \cdot\left(1+v_{23}\right) \cdot G_{23}
\end{gathered}
$$

\subsection{Homogenization models}

\subsubsection{Mori-Tanaka model (M-T)}

The Mori-Tanaka model is initially developed by Mori and Tanaka [7]. This is a well-known model which is widely used for modeling different kinds of composite materials. This is an inclusion model, where fibers are simulated by inclusions embedded in a homogeneous medium. The Benveniste formulation [8] for the Mori-Tanaka model is given by:

$$
C_{M T}=C_{m}+\left[V_{f} \cdot\left\langle\left(C_{f}-C_{m}\right) \cdot A_{\text {Eshelby }}\right\rangle\right] \cdot\left[V_{m} \cdot I+V_{f} \cdot\left\langle A_{\text {Eshelby }}\right\rangle\right]^{-1}
$$

With $\mathrm{C}_{\mathrm{m}}$ and $\mathrm{C}_{\mathrm{f}}$ are the stiffness matrices of the matrix phase and the reinforcement phase (inclusions) respectively. $\mathrm{V}_{\mathrm{f}}$ and $\mathrm{V}_{\mathrm{m}}$ are the volume fractions of the matrix phase and the reinforcement phase (inclusions) respectively. $A_{\text {Eshelby }}$ is the strain concentration tensor of the dilute solution presented by: 


$$
\mathrm{A}_{\text {Eshelby }}=\left[\mathrm{I}+\mathrm{E} \cdot \mathrm{C}_{\mathrm{m}}^{-1} \cdot\left(\mathrm{C}_{\mathrm{f}}-\mathrm{C}_{\mathrm{m}}\right)\right]^{-1}
$$

With $\mathrm{E}$ is the Eshelby tensor which depends on the shape of the inclusion and the Poisson's ratio of the matrix. More detailed information about the Eshelby tensor could be found in Mura [9]. The Eshelby tensor is then calculated for each inclusion along with the stiffness matrix.

\subsubsection{Self-consistent model (S-C)}

The self-consistent model has been proposed by Hill [10] and Budianski [11] to predict the elastic properties of composite materials reinforced by isotropic spherical particulates. Later the model was presented and used to predict the elastic properties of short fibers composites [12]. In this study the potential of the S-C model will be investigated when applied on UD composites with long fibers. The S-C model is an iterative model yielding the stiffness matrix as follows:

At the first iteration, fibers which represent the inclusions are supposed surrounded by an isotropic matrix, thus the S-C model is similar to the Eshelby dilute solution model. Then, at the second iteration, the inclusions are considered to be embedded in homogeneous medium which supposed to have the stiffness matrix similar to that of the composite calculated at the first iteration.

First iteration:

$$
\begin{aligned}
& A_{\text {Eshelby }}=\left[I+\text { E. } C_{m}^{-1} \cdot\left(C_{f}-C_{m}\right)\right]^{-1} \\
& C_{s c}=C_{m}+\left[V_{f} \cdot\left\langle\left(C_{f}-C_{m}\right) \cdot A_{\text {Eshelby }}\right\rangle\right]
\end{aligned}
$$

Second iteration:

$$
\begin{aligned}
& A_{\text {Eshelby }}=\left[I+E \cdot C_{s c}^{-1} \cdot\left(C_{f}-C_{s c}\right)\right]^{-1} \\
& C_{s c}=C_{m}+\left[V_{f} \cdot\left\langle\left(C_{f}-C_{m}\right) \cdot A_{\text {Eshelby }}\right\rangle\right]
\end{aligned}
$$

\subsubsection{Bridging model}

Recently, a new micromechanical model has been proposed by Huang et al. $[13,14]$. The model is developed to predict the stiffness and the strength of UD composites. The elastic properties by the bridging model is given as follows:

$$
\begin{gathered}
E_{11}=V_{f} \cdot E_{11}^{f}+V_{m} \cdot E_{m} \\
E_{22}=\frac{\left(V_{f}+V_{m} \cdot a_{11}\right)\left(V_{f}+V_{m} \cdot a_{22}\right)}{\left(V_{f}+V_{m} \cdot a_{11}\right)\left(V_{f} \cdot S_{11}^{f}+V_{m} \cdot a_{22} \cdot S_{22}^{m}\right)+V_{f} \cdot V_{m}\left(S_{21}^{m}-S_{21}^{f}\right) a_{12}} \\
V_{12}=V_{f} v_{11}^{f}+V_{m} v_{m} \\
G_{12}=\frac{\left(V_{f}+V_{m} \cdot a_{66}\right) G_{12}^{f} G_{m}}{V_{f} \cdot G_{m}+V_{m} \cdot a_{66} \cdot G_{12}^{f}}
\end{gathered}
$$




$$
\mathrm{G}_{23}=\frac{0.5\left(\mathrm{~V}_{\mathrm{f}}+\mathrm{V}_{\mathrm{m}} \cdot \mathrm{a}_{44}\right)}{\mathrm{V}_{\mathrm{f}}\left(\mathrm{S}_{22}^{\mathrm{f}}-\mathrm{S}_{23}^{\mathrm{f}}\right)+\mathrm{V}_{\mathrm{m}} \cdot \mathrm{a}_{44}\left(\mathrm{~S}_{22}^{\mathrm{m}}-\mathrm{S}_{23}^{\mathrm{m}}\right)}
$$

With $\mathrm{a}_{\mathrm{ij}}$ are the components of the bridging matrix $\mathrm{A},[13,14]$.

$S_{i j}^{f}$ and and $S_{i j}^{m}$ are the components of the compliance matrices of the fibers and the matrix respectively.

\subsection{Numerical FE modeling}

The numerical FE modeling is widely used in predicting the mechanical properties of composites. The numerical modeling is a reliable tool, but the time consumed on the geometrical dimensions definition and the corresponding calculation time, represent a major disadvantage against analytical models. Moreover there are many discussions and studies that deal with the appropriate boundary, symmetric and periodic conditions required to evaluate the elastic properties of UD composites. In this domain, a major work is done by S. $\mathrm{Li}$ [15]. It should be noticed that the numerical FE modeling require geometrical modeling or representation of the REV. while for UD composites, there are three types of fiber arrangements: square array, diamond array and hexagonal array (Figure 2).

In order to investigate the numerical FE modeling, the modeling of a quarter unit cell for a square array, diamond array and hexagonal array is conducted using Comsol Multiphysics software. A tetrahedral meshing is used. The resumed boundary conditions applied are given in (Table 1). Note that $\mathrm{U}, \mathrm{V}$ and $\mathrm{W}$ are the displacements along 1, 2 and 3 directions respectively applied on the $\mathrm{X+}, \mathrm{X}-, \mathrm{Y}+, \mathrm{Y}-, \mathrm{Z}+$ and $\mathrm{Z}$ - faces (with $\mathrm{X}$ faces are orthogonal to the fiber direction 1).

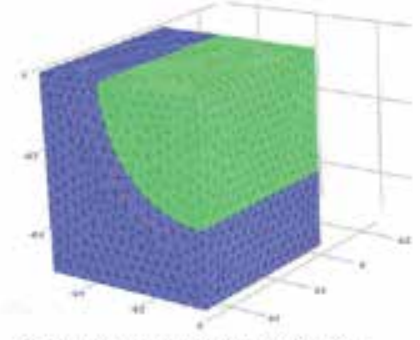

Quarter square array unit cell

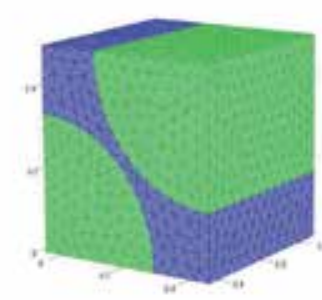

Quarter diamond array unit cell

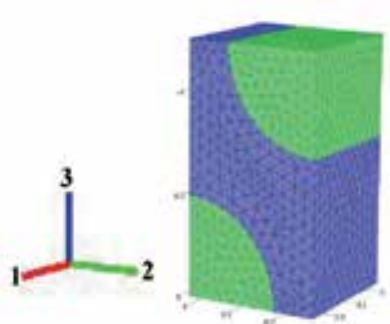

Quarter hexagonal array unit cell

Figure 2. Meshing of square, diamond and hexagonal array unit cells.

After applying boundary conditions and the displacement constant $\mathrm{K}$, the corresponding engineering constants are calculated as follow, in terms of corresponding stresses and strains $\left(\sigma_{11}, \sigma_{22}, \tau_{12}, \tau_{23}, \varepsilon_{11}, \varepsilon_{22}, \Upsilon_{12}\right.$ and $\left.\Upsilon_{23}\right)$ :

On the $\mathrm{X}+$ face:

$E_{11}=\frac{\sigma_{11}}{\varepsilon_{11}}$, where $\sigma_{11}$ and $\varepsilon_{11}$ are calculated numerically on the $\mathrm{X}+$ face 
On the $\mathrm{Y}+$ face:

$E_{22}=\frac{\sigma_{22}}{\varepsilon_{22}}$, where $\sigma_{22}$ and $\varepsilon_{22}$ are calculated numerically on the $\mathrm{Y}+$ face

On the $\mathrm{X}+$ face:

$G_{12}=\frac{\tau_{12}}{\Upsilon_{12}}$, where $\tau_{12}$ and $\Upsilon_{12}$ are calculated numerically on the $\mathrm{X}+$ face

On the Z+ face:

$G_{23}=\frac{\tau_{23}}{\Upsilon_{23}}$, where $\tau_{23}$ and $\Upsilon_{23}$ are calculated numerically on the $Z+$ face

\begin{tabular}{|c|c|c|c|c|c|c|}
\hline & \multicolumn{2}{|c|}{$\mathrm{X}$ faces } & \multicolumn{2}{c|}{$\mathrm{Y}$ faces } & \multicolumn{2}{c|}{$\mathrm{Z}$ faces } \\
\cline { 2 - 7 } & $\mathrm{X}-$ & $\mathrm{X}+$ & $\mathrm{Y}-$ & $\mathrm{Y}+$ & $\mathrm{Z}-$ & $\mathrm{Z}+$ \\
\hline E11 and $v_{12}$ & $\begin{array}{c}\mathrm{U}=0, \mathrm{~V} \\
\text { and } \mathrm{W} \text { free }\end{array}$ & $\begin{array}{c}\mathrm{U}=\mathrm{K}, \mathrm{V} \\
\text { and } \mathrm{W} \text { free }\end{array}$ & $\begin{array}{c}\mathrm{V}=0, \mathrm{U} \\
\text { and } \mathrm{W} \text { free }\end{array}$ & $\begin{array}{c}\mathrm{U}, \mathrm{V} \text { and } \mathrm{W} \\
\text { free }\end{array}$ & $\begin{array}{c}\mathrm{W}=0, \mathrm{U} \\
\text { and V free }\end{array}$ & $\begin{array}{c}\mathrm{U}, \mathrm{V} \text { and } \mathrm{W} \\
\text { free }\end{array}$ \\
\hline $\mathrm{E}_{22}$ and $v_{23}$ & $\begin{array}{c}\mathrm{U}=0, \mathrm{~V} \\
\text { and } \mathrm{W} \text { free }\end{array}$ & $\begin{array}{c}\mathrm{U}, \mathrm{V} \text { and } \mathrm{W} \\
\text { free }\end{array}$ & $\begin{array}{c}\mathrm{V}=0, \mathrm{U} \\
\text { and } \mathrm{W} \text { free }\end{array}$ & $\begin{array}{c}\mathrm{V}=\mathrm{K}, \mathrm{U} \\
\text { and } \mathrm{W} \text { free }\end{array}$ & $\begin{array}{c}\mathrm{W}=0, \mathrm{U} \\
\text { and V free }\end{array}$ & $\begin{array}{c}\mathrm{U}, \mathrm{V} \text { and } \mathrm{W} \\
\text { free }\end{array}$ \\
\hline $\mathrm{G}_{12}$ & $\mathrm{~V}=\mathrm{W}=0$ & $\begin{array}{c}\mathrm{V}=\mathrm{K} \\
\mathrm{W}=0\end{array}$ & $\mathrm{U}=\mathrm{W}=0$ & $\mathrm{U}=\mathrm{W}=0$ & $\mathrm{~W}=0$ & $\mathrm{~W}=0$ \\
\hline $\mathrm{G}_{23}$ & $\mathrm{U}=0$ & $\mathrm{U}=0$ & $\mathrm{U}=\mathrm{W}=0$ & $\mathrm{U}=\mathrm{W}=0$ & $\mathrm{U}=\mathrm{V}=0$ & $\begin{array}{c}\mathrm{U}=0 \\
\mathrm{~V}=\mathrm{K}\end{array}$ \\
\hline
\end{tabular}

Table 1. Boundary conditions on the $X, Y$ and $Z$ faces of the quarter unit cell.

\section{Comparative study, analysis and discussion}

\subsection{Results}

In this section, a comparison of analytical models and numerical models with available experimental data is presented. Three different kinds of UD composites are taken as examples: Glass/epoxy composite [16], carbon/epoxy composite [14] and polyethylene/epoxy composite [17] (Table 2). The glass fibers are isotropic fibers while the carbon and the polyethylene fibers are transversely isotropic fibers. Knowing that the epoxy matrices are assumed isotropic, it's well noticed that for the polyethylene/epoxy, the Young's modulus of the epoxy is higher than that transversal modulus of the fibers, which represent an important case to be investigated.

\begin{tabular}{|c|c|c|c|c|c|}
\hline Fibers & $E_{11}^{f}(\mathrm{GPa})$ & $E_{22}^{f}(\mathrm{GPa})$ & $G_{12}^{f}(\mathrm{GPa})$ & $v_{12}^{f}$ & $v_{23}^{f}$ \\
\hline E-Glass [16] & 73.1 & 73.1 & 29.95 & 0.22 & 0.22 \\
\hline Carbon [14] & 232 & 15 & 24 & 0.279 & 0.49 \\
\hline Polyethylene [17] & 60.4 & 4.68 & 1.65 & 0.38 & 0.55 \\
\hline Matrix & \multicolumn{2}{|c|}{$E^{m}(\mathrm{GPa})$} & $G^{m}(\mathrm{GPa})$ & \multicolumn{2}{|c|}{$v^{m}$} \\
\hline Epoxy resin [16] & 3.45 & 3.45 & 1.28 & 0.35 & 0.35 \\
\hline Epoxy [14] & 5.35 & 5.35 & 1.97 & 0.354 & 0.354 \\
\hline Epoxy [17] & 5.5 & 5.5 & 1.28 & 0.37 & 0.37 \\
\hline
\end{tabular}

Table 2. Elastic properties of the fibers and epoxy matrices. 


\subsubsection{Longitudinal Young's modulus En1}

For the longitudinal Young's modulus E11, obtained analytical and numerical results are compared to those available experimental data for carbon/epoxy and polyethylene/epoxy UD composites in terms of the fiber volume fraction Vf. Investigated analytical models belong to the ROM, the Elasticity approach model (EAM), M-T and S-C models. Please note that ROM, MROM, Chamis, Halpin-Tsai and Bridging models share the same formulation for E11.

It's well noticed that the predicted results for all investigated models are in good agreement with the experimental data for both composites with different $V^{\mathrm{f}}$ (Figure 3 and 4).

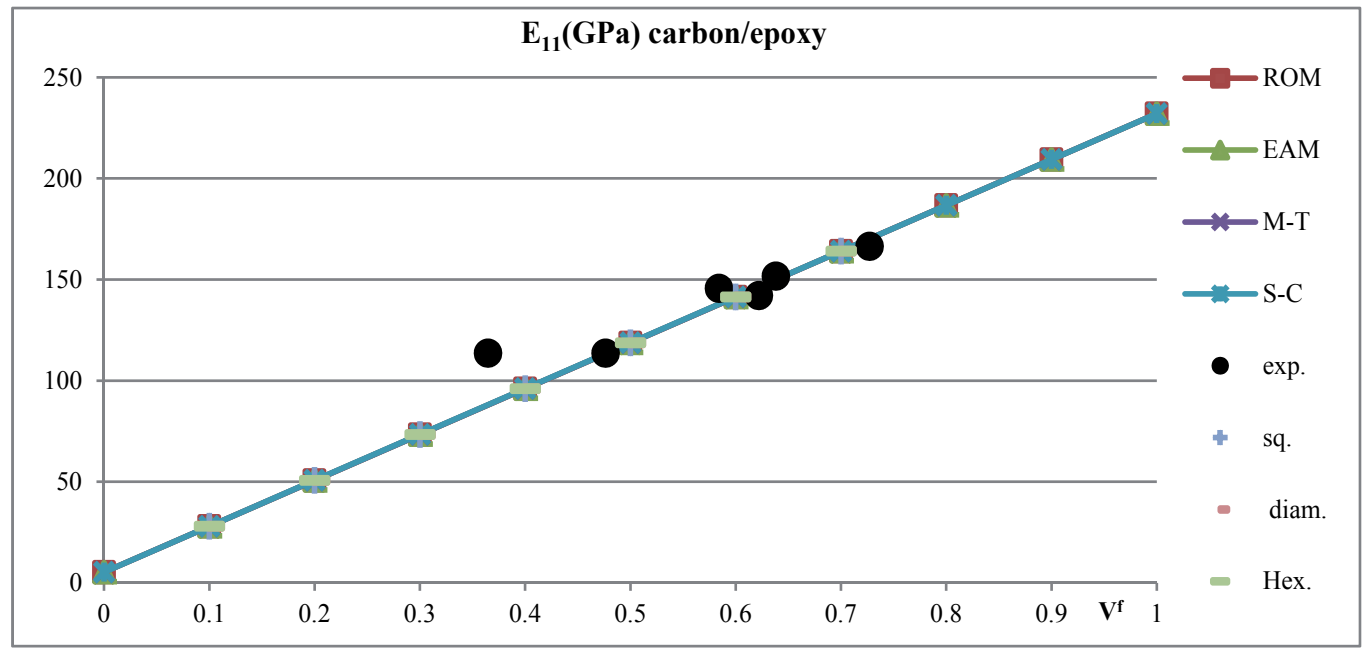

Figure 3. Predicted analytical, numerical and experimental results for E11 in terms of $\mathrm{V}^{\mathrm{f}}$.

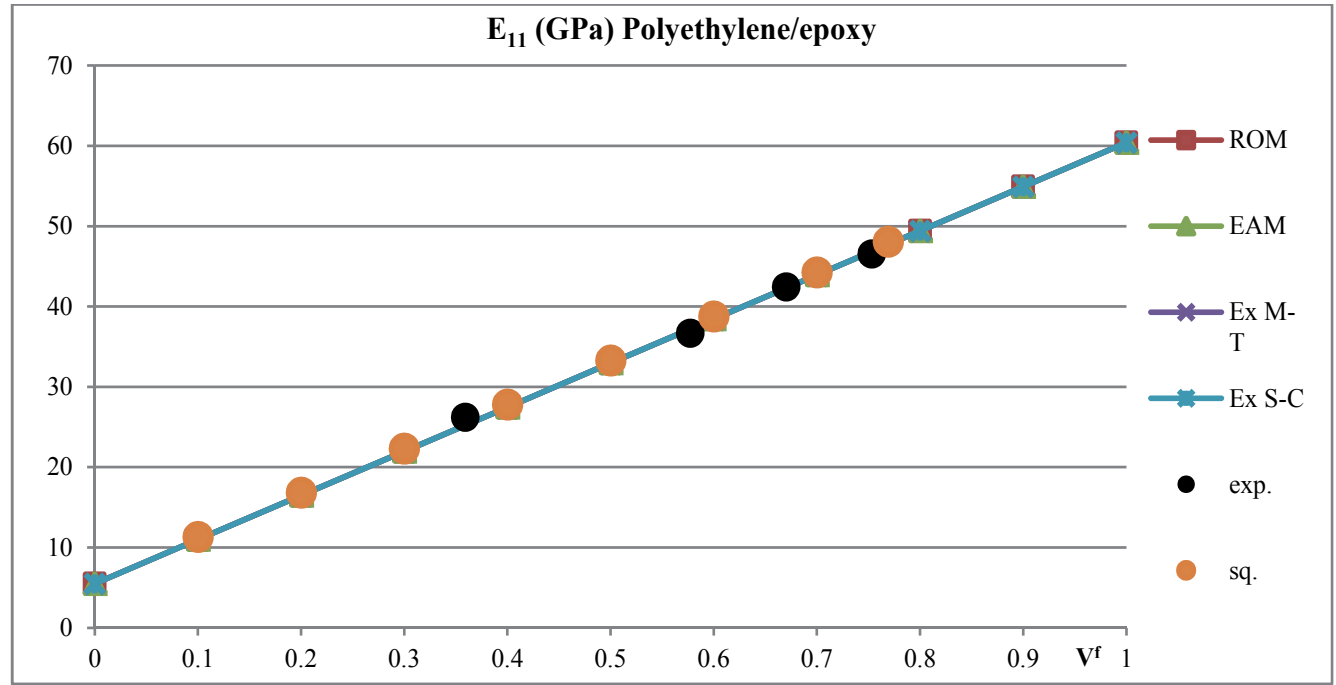

Figure 4. Predicted analytical, numerical and experimental results for E11 in terms of $\mathrm{V}^{\mathrm{f}}$. 


\subsubsection{Transversal Young's modulus E22}

The prediction of the transversal Young's modulus and in contrast with the longitudinal modulus presents a real challenge for the researchers. Thus, many analytical models are proposed belonged to different micromechanics approach. In addition, the potential of the FE element modeling is investigated. Predicted results of different analytical and numerical models for three UD composites are presented in figures (5,6 and 7)

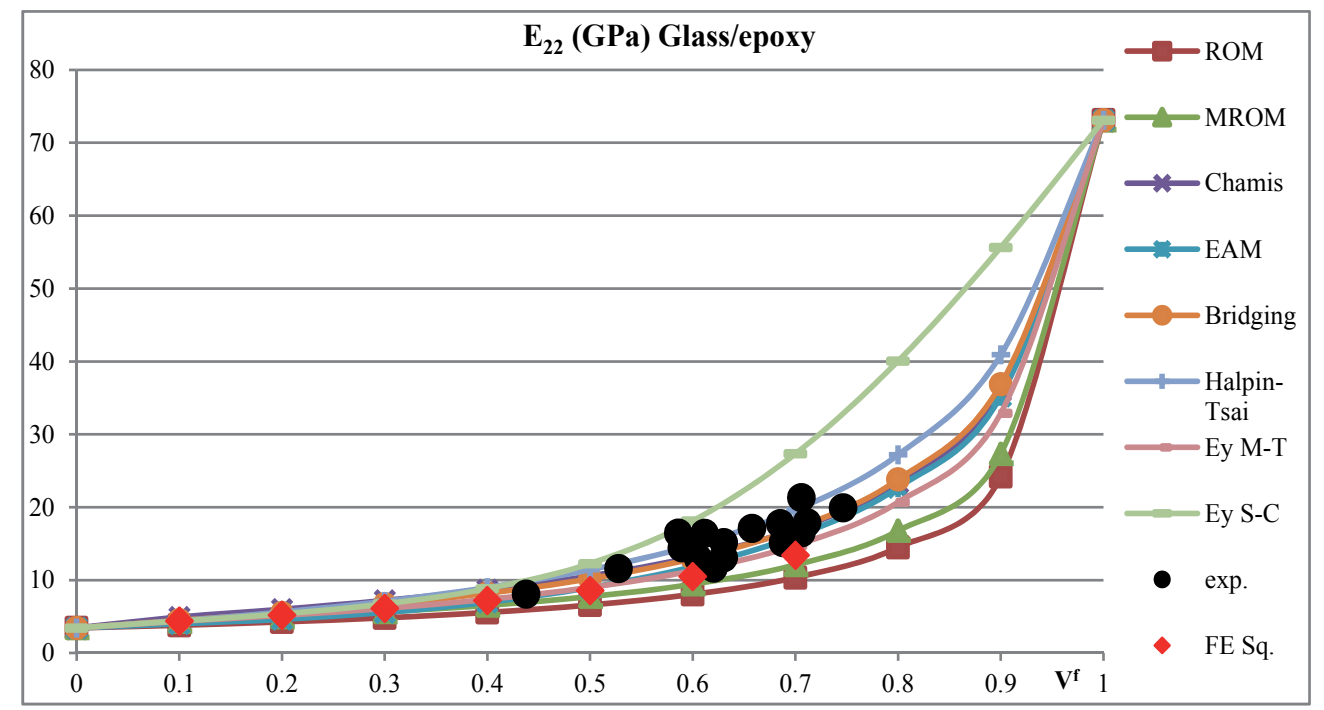

Figure 5. Predicted analytical, numerical and experimental results for $E_{22}$ in terms of Vf.

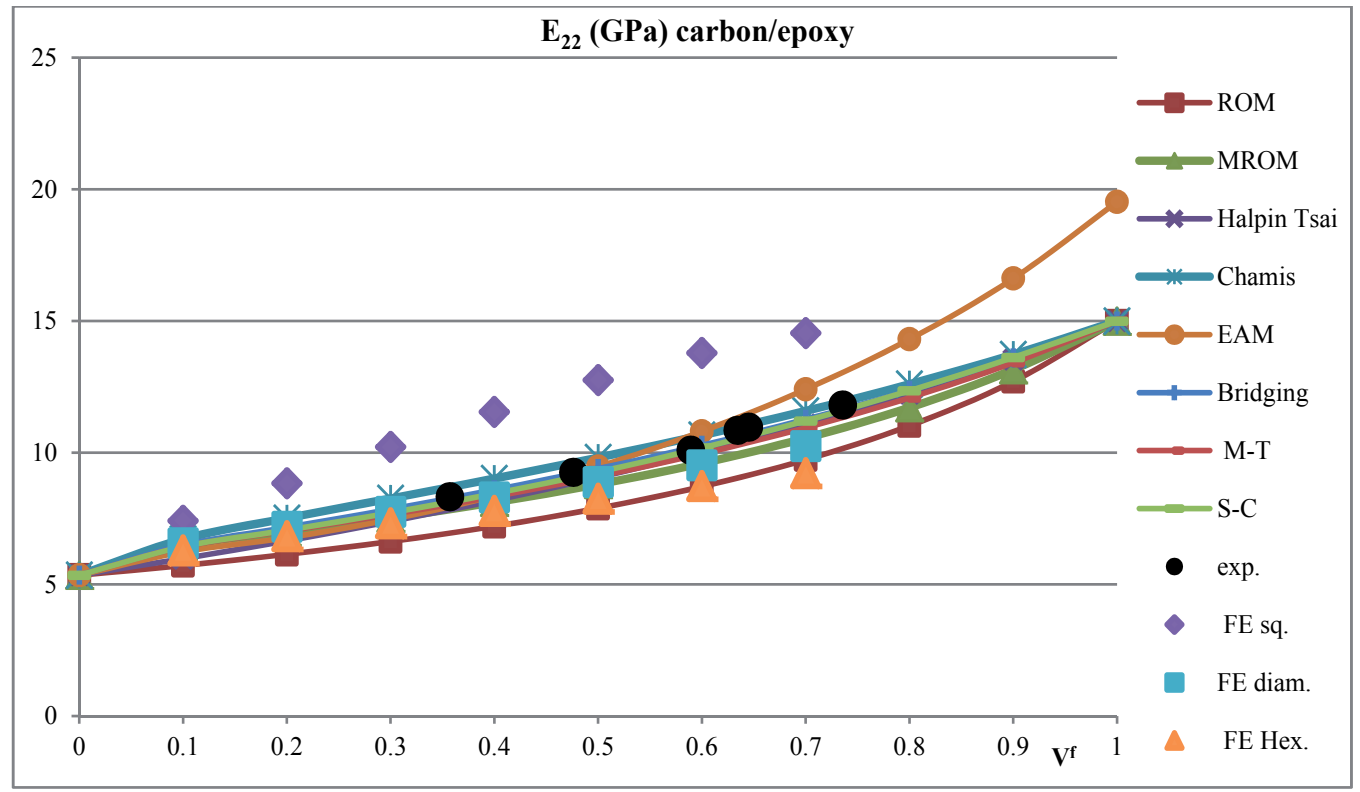

Figure 6. Predicted analytical, numerical and experimental results for $E_{22}$ in terms of $V^{f}$. 


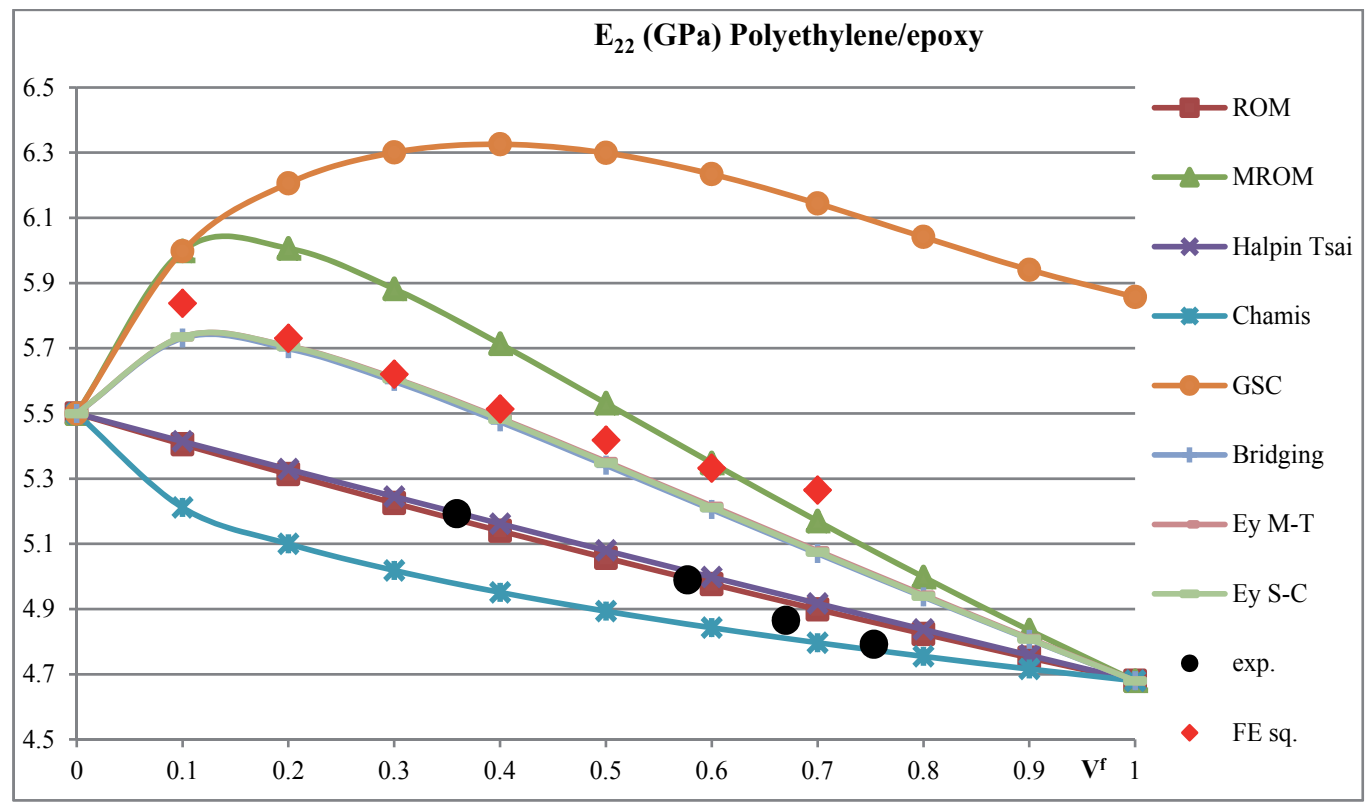

Figure 7. Predicted analytical, numerical and experimental results for $E_{22}$ in terms of $V^{f}$.

It's shown that for the glass/epoxy composite, the S-C model overestimates the experimental results, while the ROM and MROM models underestimate it. Other analytical models, especially the Chamis, Bridging and EAM models yield results that correlate well with the available experimental data for different values of Vf. Moreover, it's noticed that the FE (Square array), the Halpin-Tsai and the M-T models gives good predictions. Concerning composites reinforced with transversely isotropic fibers, it's well remarked that the EAM model well overestimates $E_{22}$ especially with the polyethylene/epoxy composite. The ROM underestimates the experimental results, while other analytical models, in addition to the numerical FE (diamond array) model, yield very good predictions for the carbon/epoxy composite. However, with the polyethylene/epoxy, it's noticed that only the results obtained from the ROM and the Halpin-Tsai models correlate well with the experimental data, while the Chamis model shows a good agreement with Vf higher than 0.6.

\subsubsection{Longitudinal shear modulus $G_{12}$}

Experimental results for two UD composites are used to be compared with. Figures 8 show clearly the MROM, EAM, Halpin-Tsai, Chamis, bridging analytical models, in addition to all numerical FE models yield very good results for the carbon/epoxy composite. However, it's remarked that the inclusion models, the $\mathrm{M}-\mathrm{T}$ and $\mathrm{S}-\mathrm{C}$ models, overestimate the longitudinal shear modulus. Concerning the polyethylene/epoxy composite, only results obtained results from the MROM and Chamis models agree well with the available experimental data (Figure 9). 


\subsubsection{Transversal shear modulus $G_{23}$}

For the transversal shear modulus $\mathrm{G}_{23}$, it's shown from Figure 10 and 11, that the bridging model yields the best results. In addition, it's remarked that the EAM, Chamis yield reasonable predictions underestimating the experimental data, while the M-T and S-C models overestimate it. Concerning the numerical modeling, predicted results always overestimate the available experimental results for the two composites.

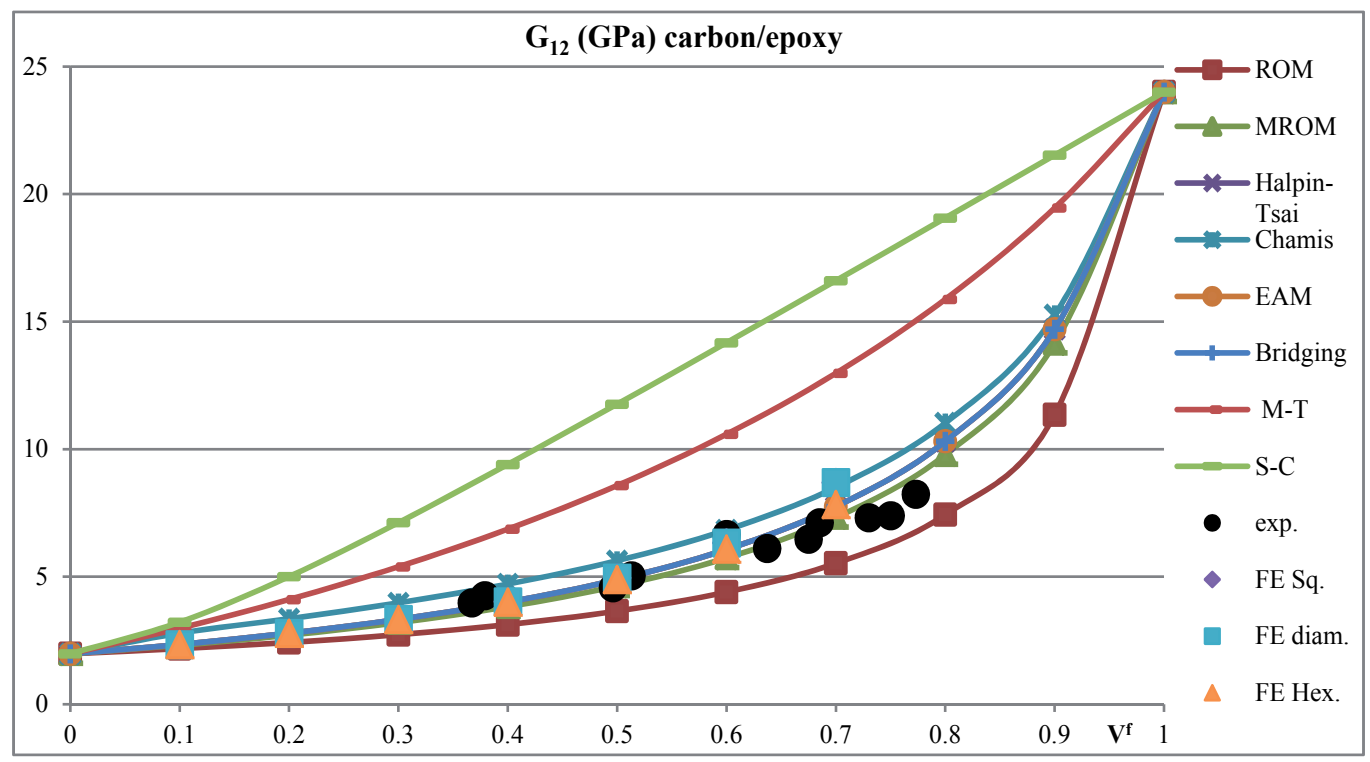

Figure 8. Predicted analytical, numerical and experimental results for $\mathrm{G}_{12}$ in terms of $\mathrm{V}^{\mathrm{f}}$.

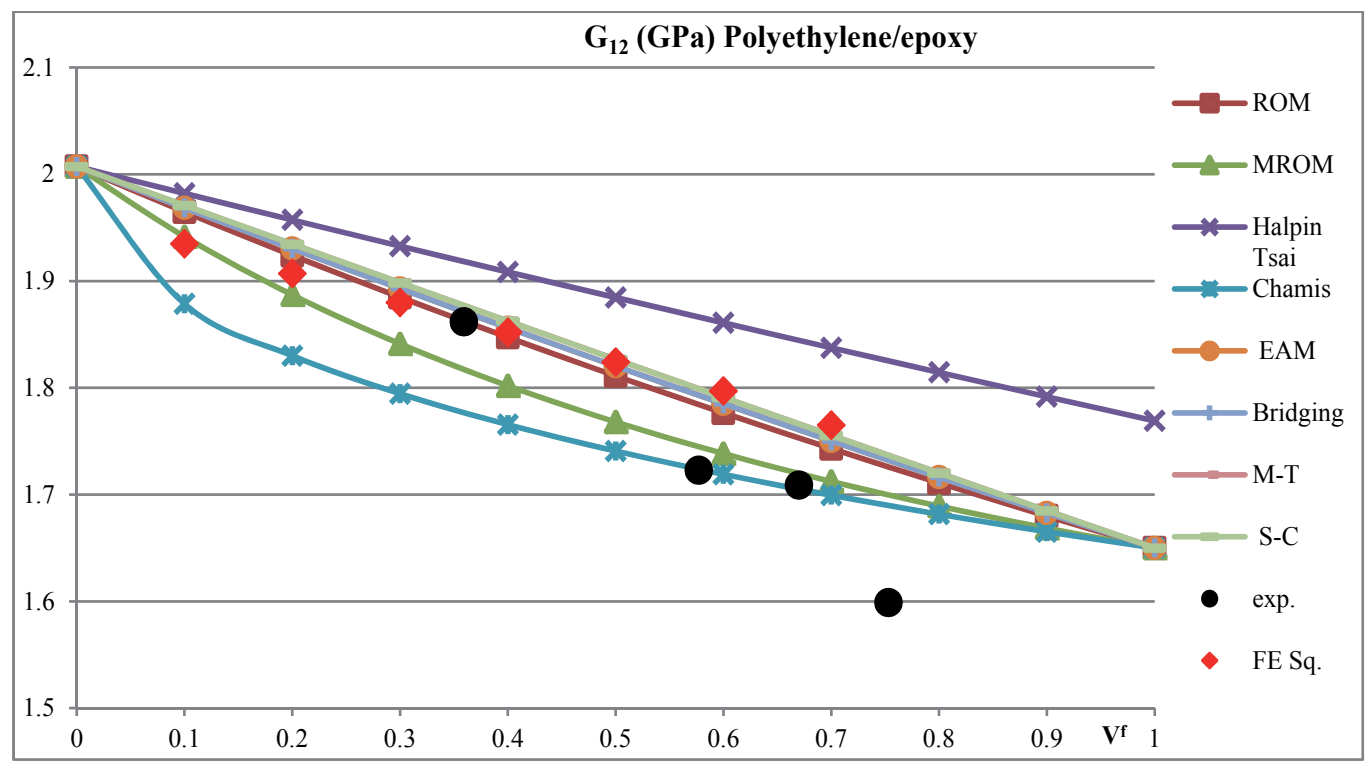

Figure 9. Predicted analytical, numerical and experimental results for $\mathrm{G}_{12}$ in terms of $\mathrm{V}^{\mathrm{f}}$. 


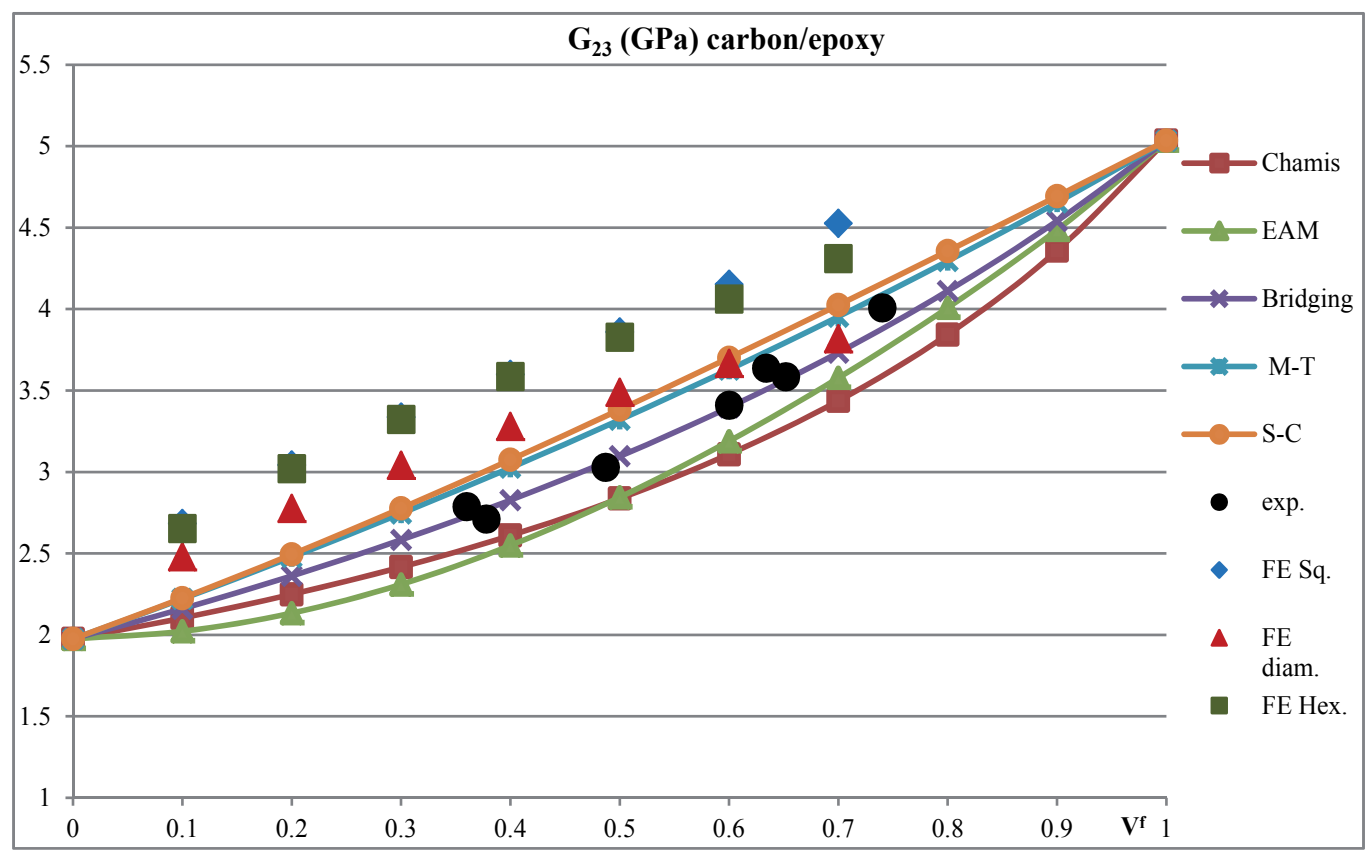

Figure 10. Predicted analytical, numerical and experimental results for $\mathrm{G}_{23}$ in terms of $\mathrm{V}^{\mathrm{f}}$.

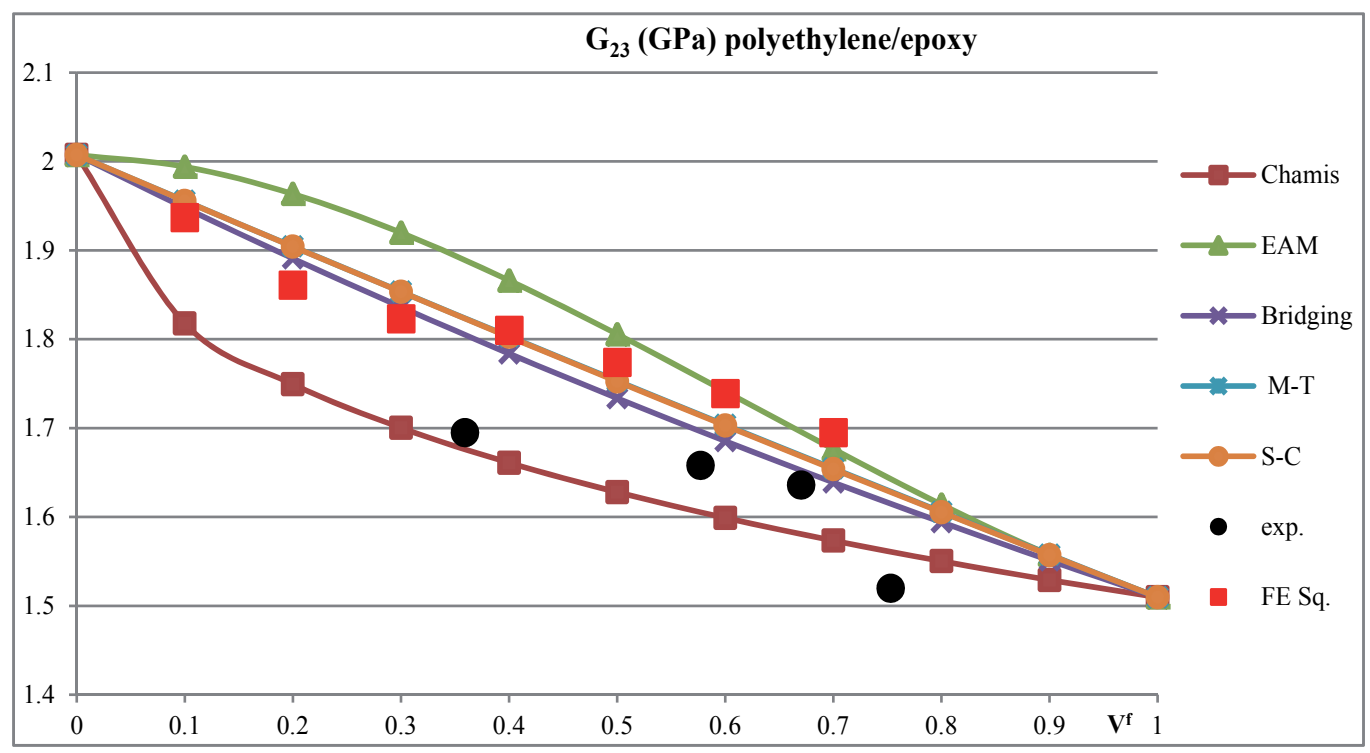

Figure 11. Predicted analytical, numerical and experimental results for $\mathrm{G}_{23}$ in terms of $\mathrm{V}^{\mathrm{f}}$. 


\subsubsection{Major Poisson's ratio $v_{12}$}

Concerning the Poisson's ratios, the obtained results of the analytical models are only compared to those numerical due the missing of experimental data for the studied UD composites. Figure 12 shows that for the major Poisson's ratio v12, all analytical and numerical models correlate well with each other.

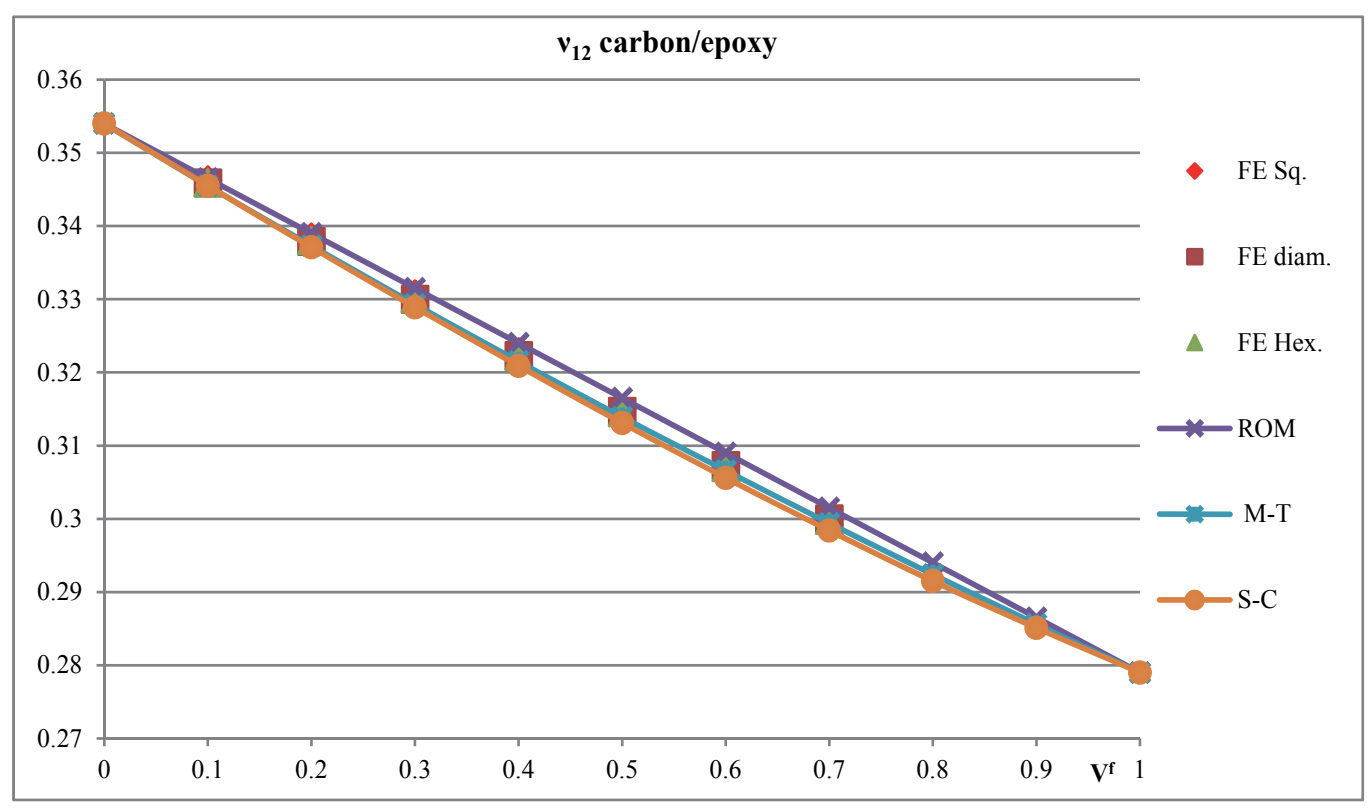

Figure 12. Predicted analytical and numerical results for $v_{12}$ in terms of $\mathrm{V}^{\mathrm{f}}$.

\subsection{Analysis and discussion}

In this section, an analysis of the predicted results for each model is presented apart. It's shown from the above results that for the phenomenological models, the Voigt and Reuss models, represented by the ROM model, show very good predictions for the longitudinal Young's modulus $E_{11}$ and major Poisson's ratio v12. However, with for the transversal Young's modulus E22 the ROM model always underestimates the experimental results 
except for the polyethylene/epoxy case where it's well agree with the available experimental data. Concerning the longitudinal shear modulus G12, the ROM model didn't yield good prediction for both studied cases the carbon/epoxy and the polyethylene/epoxy composites.

As known the semi-empirical models have been emerged and proposed in order to correct the predictions of the ROM model for the transversal Young's and longitudinal shear moduli. While the investigated models share the same formulations for E11 and $v_{12}$ with ROM model, the corrections made for $\mathrm{E}_{22}$ and $\mathrm{G}_{12}$ prove to be effective. It's shown that the Chamis model yields very good results for all studied cases, while the MROM and HalpinTsai the models only suffer with the special case of the polyethylene/epoxy with the E22 and G12 respectively.

Concerning the elasticity approach models, the proposed formulation of the E11 yields similar results for that proposed by the ROM model. While for the transversal Young's modulus E22, it's clearly noticed that with isotropic fibers, the model results correlate well with those experimental, while with the case of transversely isotropic fibers, reasonable predictions are shown for the carbon/epoxy case. However, for the polyethylene case the model well overestimates the experimental results. The reason could be conducted to that EAM models are initially proposed to deal with UD composites reinforced with isotropic fibers. For the longitudinal shear modulus $\mathrm{G}_{12}$, the elastic solution formulation agrees well with the experimental data. Concerning the transversal shear modulus, the predictions made by the generalized self-consistent model of the Christensen model [6], which is developed to enhance the predictions of this elastic property, always overestimates the experimental data.

In this study, the potential of the homogenization models is investigated. The inclusion models, the M-T and the S-C models, and the bridging model, yield good prediction for both longitudinal Young's modulus and major Poisson's ratio. However, for the transversal Young's modulus E22, reasonable agreement is shown for the glass/epoxy and carbon/epoxy cases, except with the self-consistent model which overestimates the experimental data for high $\mathrm{V}^{\mathrm{f}}$. While for the case of the polyethylene/epoxy, all three models yield almost the same results and overestimate the compared experimental data while agree with FE modeling results. The same problem is shown with the prediction of the shear moduli, where for the polyethylene/epoxy case, the models belonged to the homogenization approach give the same results overestimating the experimental data. While with the carbon/epoxy case, it's noticed that the bridging model predicts better the shear moduli, while the M-T and S-C models well overestimate the experimental data especially for the G12.

Concerning the numerical modeling, it's well noticed that there are different predicted results for different arrays. It's also remarked, that the FE numerical modeling didn't yield better results than the analytical models, except for the longitudinal Young's modulus and major Poisson's ratio where all predicted results from numerical and analytical models correlate well with available experimental data. 


\section{Conclusion}

In this study, the evaluated results, for the elastic properties, of most known analytical micromechanical models, as well as FE modeling methods, are compared to available experimental data for three different UD composites: Glass/epoxy, carbon/epoxy and polyethylene/epoxy. It should be noticed that the studied cases cover different kinds of reinforced composites by isotropic fibers (glass) and transversely isotropic fibers (carbon and polyethylene). In addition, the polyethylene/epoxy presents an interesting case study, where the matrix is stiffer than the fibers in the transvers direction.

The analyses of the compared results show clearly that all analytical and numerical models show a very good agreement for the longitudinal Young's modulus E11 and major Poisson's ratio v12. However, the other moduli, the transversal Young's modulus E22, longitudinal shear modulus $\mathrm{G}_{12}$ and the transversal shear modulus $\mathrm{G}_{23}$, represent the main challenge for the researchers. It's shown that analytical micromechanical models belonged to the semiempirical models, especially the Chamis model, predict well these elastic properties. Moreover, the bridging model proves to be a reliable model when predicting the elastic properties of carbon/epoxy composite. It's noticed that almost all models suffer with the prediction of elastic properties for the polyethylene/epoxy composite. However, models belonged to the elasticity approach and inclusion approach (M-T and S-C models) show inconsistency in predicting the elastic properties of studied UD composites. Numerical models, based on the FE method, show that using different fibers arrangements will lead to different predicted results. Moreover, the FE didn't prove that it could be more accurate than some simple and straightforward analytical model. As a conclusion from this study, the Chamis model and the bridging model could be considered as the most complete models which could give quite accurate estimations for all five independent elastic properties. Noting that the corrections proposed by the Halpin-Tsai model, prove that it well enhance the prediction of the transversal Young's modulus E22.

\section{Author details}

Rafic Younes*

LISV, University of Versailles Saint-Quentin, Versailles, France

Faculty of Engineering, Lebanese University, Rafic Hariri campus, Beirut, Lebanon

Ali Hallal

LISV, University of Versailles Saint-Quentin, Versailles, France

L3M2S, Lebanese University, Rafic Hariri campus, Beirut, Lebanon

Farouk Fardoun

L3M2S, Lebanese University, Rafic Hariri campus, Beirut, Lebanon

" Corresponding Author 
Fadi Hajj Chehade

L3M2S, Lebanese University, Rafic Hariri campus, Beirut, Lebanon

\section{References}

[1] Voigt W. Uber die Beziehung zwischen den beiden Elastizitatskonstanten Isotroper Korper. Wied. Ann, 38 (1889) 573-587.

[2] Reuss A. Berechnung der Fliessgrense von Mischkristallen auf Grund der Plastizitätsbedingung für Einkristalle. Zeitschrift Angewandte Mathematik und Mechanik, 9 (1929) 49-58.

[3] Halpin JC, Kardos JL. The Halpin-Tsai equations: A review. Polymer Engineering and Science, May, 1976, Vol. 16, No. 5.

[4] Chamis CC. Mechanics of composite materials: past, present, and future. J Compos Technol Res ASTM 1989;11:3-14.

[5] Hashin Z, Rosen BW. The elastic moduli of fiber reinforced materials. Journal of Applied Mechanics, Trans ASME, 31 (1964). 223-232.

[6] Christensen RM. A critical evaluation for a class of micromechanics models. Journal of Mechanics and Physics of Solids 1990; 38(3):379-404.

[7] Mori T, Tanaka K. Average stress in matrix and average elastic energy of materials with misfitting inclusions, Acta Mettall. 21, 1973, 571-574.

[8] Benveniste Y. A new approach to the application of Mori-Tanaka's theory in composite materials. Mechanics of Materials, 6, 1987, 147-157.

[9] Mura T. Micromechanics of Defects in Solids, 2nd edn. Martinus Nijhof Publishers, Dordrecht, 1987.

[10] Hill R. Theory of mechanical properties of fibre-strengthen materials-III. Self-consistent model. Journal of Mechanics and Physics of Solids 1965; 13: 189-198.

[11] Budiansky B. On the elastic moduli of some heterogeneous materials, J. Mech. Phys. Solids 13, 1965, 223-227.

[12] Chou TW, Nomura S, Taya M. A self-consistent approach to the elastic stiffness of short-fiber composites, J. Compos Mater, 14, 1980, 178-188.

[13] Huang ZM. Simulation of the mechanical properties of fibrous composites by the bridging micromechanics model. Composites: Part A 32 (2001) 143-172.

[14] Huang ZM. Micromechanical prediction of ultimate strength of transversely isotropic fibrous composites. International Journal of Solids and Structures 38 (2001) 4147-4172.

[15] Li S. Boundary conditions for unit cells from periodic microstructures and their implications. Composites Science and Technology 68 (2008) 1962-1974.

[16] Shan HZ, Chou TW. Transverse elastic moduli of unidirectional fiber composites with fiber/matrix interfacial debonding. Composites Science and Technology 53 (1995) 383391. 
[17] Wilczynski AP, Lewinski J. Predecting the properties of unidirectional fibrous composites with monotropic reinforcement. Composite Science and Technology 55 (1995) 139-143 


\section{Metal and Ceramic Matrix Composites}





\title{
Manufacturing and Properties of Quartz $\left(\mathrm{SiO}_{2}\right)$ Particulate Reinforced Al-11.8\%Si Matrix Composites
}

\author{
M. Sayuti, S. Sulaiman, T.R. Vijayaram, B.T.H.T Baharudin and M.K.A. Arifin
}

Additional information is available at the end of the chapter

http://dx.doi.org/10.5772/48095

\section{Introduction}

Metal matrix composites (MMC) are a class of composites that contains an element or alloy matrix in which a second phase is fixed firmly deeply and distributed evenly to achieve the required property improvement. The property of the composite varies based on the size, shape and amount of the second phase (Sayuti et al., 2010; Sulaiman et al., 2008). Discontinuously reinforced metal matrix composites, the other name for particulate reinforced composites, constitute $5-20 \%$ of the new advanced materials (Gay et al., 2003). The mechanical properties of the processed composites are greatly influenced by their microstructure. An increased stiffness, yield strength and ultimate tensile strength are generally achieved by increasing the weight fraction of the reinforcement phase in the matrix. Inspite of these advantages, the usage of particulate reinforced MMCs as structural components in some applications is limited due to low ductility (Rizkalla and Abdulwahed, 1996). Owing to this and to overcome the draw-backs, a detailed investigation on the strengthening mechanism of composites has been carried out by composite experts (Humphreys, 1987). They have found that the particle size and its weight fraction in metal matrix composites influences the generation of dislocations due to thermal mismatch. The effect is also influenced by the developed residual and internal stresses too. The researchers have predicted that the dislocation density is directly proportional to the weight fraction and due to the amount of thermal mismatch. As a result, the strengthening effect is proportional to the square root of the dislocation density. This effect would be significant for fine particles and for higher weight fractions. The MMCs yield improved physical and mechanical properties and these outstanding benefits are due to the combined metallic and ceramic properties (Hashim et al., 2002). Though there are various types of MMCs, particulate-reinforced composites are the most 
versatile and economical (Sayuti, Sulaiman, Vijayaram, et al., 2011; Sayuti, Suraya, et al., 2011).

In the past 40 years, the researchers and design experts have perceived their research to emphasis on finding lightweight, environmental friendly, low-cost, high quality, and good performance materials (Feest, 1986). In accordance with this trend, MMCs have been attracting growing interest among researchers and industrialists. The attributes of MMCs include alterations in mechanical behavior (e.g., tensile and compressive properties, creep, notch resistance, and tribology) and physical properties (e.g., intermediate density, thermal expansion, and thermal diffusivity) a change, primarily induced by the reinforced filler phase (Sayuti, et al., 2011). Even though MMCs posses various advantages, they still have limitations of thermal fatigue, thermo-chemical compatibility, and posses lower transverse creep resistance. In order to overcome these limitations, fabrication of discontinuously reinforced Al-based MMCs was carried out by standard metallurgical processing methods such as powder metallurgy, direct casting, rolling, forging and extrusion. Subsequently, the products were shaped, machined and drilled by using conventional machining processes. Consequently, the MMCs would be available in suitable quantities with desirable properties, particularly for automotive applications (Sharma et al., 1997).

In general, composite materials posses good mechanical and thermal properties, sustainable over a wide range of temperatures (Vijayaram et al., 2006). The desirable factors such as property requirements, cost factor considerations and future application prospects would decide the choice of the processing method (Kaczmar et al., 2000). In practice, composite materials with a metal or an alloy matrix are fabricated either by casting or by powder metallurgy methods (Fridlyander, 1995). They are considered as potential material candidates for a wide variety of structural applications in the transportation, automobile and sport goods manufacturing industries due to the superior range of mechanical properties they exhibit (Hashim et al., 1999). MMCs represent a new generation of engineering materials in which a strong ceramic reinforcement is incorporated into a metal matrix to improve its properties such as specific strength, specific stiffness, wear resistance, corrosion resistance and elastic modulus (Baker et al., 1987; Chambers et al., 1996; Kok, 2005). As a virtue of their structure and bonding between the matrix and the reinforcement, MMCs combine metallic properties of matrix alloys (ductility and toughness) with ceramic properties of reinforcements (high strength and high modulus), therein leading to greater strength in shear and compression as well as higher service-temperature capabilities (Huda et al., 1993). Thus, they have scientific, technological and commercial significance. MMCs, because of their improved properties, are being used extensively for high performance applications such as in aircraft engines especially in the last decade. Recently, they also find application in automotive sectors (Surappa, 2003; Therén and Lundin, 1990).

Aluminum oxide $\left(\mathrm{Al}_{2} \mathrm{O}_{3}\right)$ and silicon carbide $(\mathrm{SiC})$ powders in the form of fibers and particulates are commonly used as reinforcements in MMCs. In the automotive and aircraft industries for example, production of engine pistons and cylinder heads, the tribological properties of the materials used are considered crucial. Hence, Aluminum oxide and silicon carbide reinforced aluminum alloy matrix composites are applied in these fields (Prasad and 
Asthana, 2004). Due to their high demand, the development of aluminum matrix composites is receiving considerable emphasis in modern application. Research reports ascertain that the incorporation of hard second phase particles in the alloy matrices to produce MMCs is beneficial and economical due to its high specific strength and corrosion resistance properties (Kok, 2005). Therefore, MMCs are those materials that have higher potential for a large range of engineering applications.

\section{Metal Matrix Composites (MMCs)}

Metal matrix composites are a family of new materials which are attracting considerable industrial interest and investment worldwide. They are defined as materials whose microstructures compromise a continuous metallic phase (the matrix) into which a second phase, or phases, have been artificially introduced. This is in contrast to conventional alloys whose microstructures are produced during processing by naturally occurring phase transformations (Feest, 1986). Metal matrix composites are distinguished from the more extensively developed resin matrix composites by virtue of their metallic nature in terms of physical and mechanical properties and by their ability to lend themselves to conventional metallurgical processing operations. Electrical conductivity, thermal conductivity and noninflammability, matrix shear strength, ductility (providing a crack blunting mechanism) and abrasion resistance, ability to be coated, joined, formed and heat treated are some of the properties that differentiate metal matrix composites from resin matrix composites. MMCs are a class of advanced materials which have been developed for weight-critical applications in the aerospace industry. Discontinuously reinforced aluminum composites, composed of high strength aluminum alloys reinforced with silicon carbide particles or whiskers, are a subclass of MMCs. Their combination of superior properties and fabricability makes them attractive candidates for many structural components requiring high stiffness, high strength and low weight. Since the reinforcement is discontinuous, discontinuously reinforced composites can be made with properties that are isotropic in three dimensions or in a plane. Conventional secondary fabrication methods can be used to produce a wide range of composites products, making them relatively inexpensive compared to the other advanced composites reinforced with continuous filaments. The benefit of using composite materials and the cause of their increasing adoption is to be looked for in the advantage of attaining property combinations that can result in a number of service benefits. Among these are increased strength, decreased weight, higher service temperature, improved wear resistance and higher elastic module. The main advantage of composites lies in the tailorability of their mechanical and physical properties to meet specific design criteria. Composite materials are continuously displacing traditional engineering materials because of their advantages of high stiffness and strength over homogeneous material formulations. The type, shape and spatial arrangement of the reinforcing phase in metal matrix composites are key parameters in determining their mechanical behavior. The hard ceramic component which increases the mechanical characteristics of metal matrix composites causes quick wear and premature tool failure in the machining operations. Metal matrix composites have been investigated since the early 1960s with the impetus at that time, being the high potential structural properties 
that would be achievable with materials engineered to specific applications (Mortensen et al., 1989).

In the processing of metal matrix composites, one of the subjects of interest is to choose a suitable matrix and a reinforcement material (Ashby and Jones, 1980). In some cases, chemical reactions that occur at the interface between the matrix and its reinforcement materials have been considered harmful to the final mechanical properties and are usually avoided. Sometimes, the interfacial reactions are intentionally induced, because the new layer formed at the interface acts as a strong bond between the phases (Gregolin et al., 2002).

During the production of metal matrix composites, several oxides have been used as reinforcements, in the form of particulates, fibers or as whiskers (Zhu and lizuka, 2003). For example, alumina, zirconium oxide and thorium oxide particulates are used as reinforcements in aluminum, magnesium and other metallic matrices (Upadhyaya, 1990). Very few researchers have reported on the use of quartz as a secondary phase reinforcement particulate in an aluminum or aluminum alloy matrix, due to its aggressive reactivity between these materials (Sahin, 2003). Preliminary studies showed that the contact between molten aluminum and silica-based ceramic particulates have destroyed completely the second phase microstructure, due to the reduction reaction which provokes the infiltration of liquid metal phase into the ceramic material (Mazumdar, 2002). Previous works carried out by using continuous silica fibers as reinforcement phases in aluminum matrix showed that even at temperatures nearer to $400{ }^{\circ} \mathrm{C}$, silica and aluminum can react and produce a transformed layer on the original fiber surface as a result of solid diffusion between the phases and due to the aluminum-silicon liquid phase formation (Seah et al., 2003). The organizations and companies that are very active in the usage of MMCs in Canada and United States include the following (Rohatgi, 1993):

1. Aluminium Company of Canada, Dural Corporation, Kaiser Aluminium, Alcoa, American Matrix, Lanxide, American Refractory Corporation

2. Northrup Corporation, McDonald Douglas, Allied Signal, Advanced Composite Materials Corporation, Textron Specialty Materials

3. DWA Associates, MCI Corporation, Novamet

4. Martin Marietta Aerospace, Oakridge National Laboratory , North American Rockwell, General Dynamics Corporation, Lockheed Aeronautical Systems

5. Dupont, General Motors Corporation, Ford Motor Company, Chrysler Corporation, Boeing Aerospace Company, General Electric, Westinghouse

6. Wright Patterson Air Force Base, (Dayton, Ohio), and

7. Naval Surface Warefare Centre, (Silver Spring, Maryland)

India also has substantial activity in PM and cast MMCs. It has had world class R\&D in cast aluminium particulate composites which was sought even by western countries.

\subsection{Classification of composites}

Among the major developments in materials in recent years are composite materials. In fact, composites are now one of the most important classes of engineered materials, because they 
offer several outstanding properties as compared to conventional materials. The matrix material in a composite may be ceramic based, polymer or metal. Depending on the matrix, composite materials are classified as follows:

Metal matrix composites (MMCs)

Polymer matrix composites (PMCs)

Ceramic matrix composites (CMCs)

Majority of the composites used commercially are polymer-based matrices. However, metal matrix composites and ceramic matrix composites are attracting great interest in high temperature applications (Feest, 1986). Another class of composite material is based on the cement matrix. Because of their importance in civil engineering structures, considerable effort is being made to develop cement matrix composites with high resistance to cracking (Schey, 2000). Metal matrix composites (MMCs) are composites with a metal or alloy matrix. It has resistance to elevated temperatures, higher elastic modulus, ductility and higher toughness. The limitations are higher density and greater difficulty in processing parts. Matrix materials used in these composites are usually aluminum, magnesium, aluminum-lithium, titanium, copper and super alloys. Fiber materials used in MMCs are aluminum oxide, graphite, titanium carbide, silicon carbide, boron, tungsten and molybdenum. The tensile strengths of non metallic fibers range between $2000 \mathrm{MPa}$ to $3000 \mathrm{MPa}$, with elastic modulus being in the range of $200 \mathrm{GPa}$ to 400 GPa. Because of their lightweight, high specific stiffness and high thermal conductivity, boron fibers in an aluminum matrix have been used for structural tubular supports in the space shuttle orbiter. Metal matrix composites having silicon carbide fibers and a titanium matrix, are being used for the skin, stiffeners, beams and frames of the hypersonic aircrafts under development. Other applications are in bicycle frames and sporting goods (Wang et al., 2006). Graphite fibers reinforced in aluminum and magnesium matrices are applied in satellites, missiles and in helicopter structures. Lead matrix composites having graphite fibers are used to make storage-battery plates. Graphite fibers embedded in copper matrix are used to fabricate electrical contacts and bearings. Boron fibers in aluminum are used as compressor blades and structural supports. The same fibers in magnesium are used to make antenna structures. Titanium-boron fiber composites are used as jet-engine fan blades. Molybdenum and tungsten fibers are dispersed in cobalt-base super alloy matrices to make high temperature engine components. Squeeze cast MMCs generally have much better reinforcement distribution than compocast materials. This is due to the fact that a ceramic perform which is used to contain the desired weight fraction of reinforcement rigidly attached to one another so that movement is inhibited. Consequently, clumping and dendritic segregation are eliminated. Porosity is also minimized, since pressure is used to force the metal into interfiber channels, displacing the gases. Grain size and shape can vary throughout the infiltrated preform because of heat flow patterns. Secondary phases typically form at the fiber-matrix interface, since the lower freezing solute-rich regions diffuse toward the fiber ahead of the solidifying matrix (Surappa, 2003).

\subsection{Significance of composites}

Composites technology and science requires interaction of various disciplines such as structural analysis and design, mechanics of materials, materials science and process 
engineering. The tasks of composites research are to investigate the basic characteristics of the constituents and composite materials, develop effective and efficient fabrication procedures, optimize the material for service conditions and understanding their effect on material properties and to determine material properties and predict the structural behavior by analytical procedures and hence to develop effective experimental techniques for material characterization, failure analysis and stress analysis (Daniel and Ishai, 1994). An important task is the non-destructive evaluation of material integrity, durability assessment, structural reliability, flaw criticality and life prediction. The structural designs and systems capable of operating at elevated temperatures has spurred intensive research in high temperature composites, such as ceramic/matrix, metal/ceramic and carbon/carbon composites. The utilization of conventional and new composite materials is intimately related to the development of fabrication methods. The manufacturing process is one of the most important stages in controlling the properties and ensuring the quality of the finished product. The technology of composites, although still developing, has reached a state of maturity. Nevertheless, prospects for the future are bright for a variety of reasons. Newer high volume applications, such as in the automotive industry, will expand the use of composites greatly.

\subsection{Matrix}

Matrix is the percolating alloy/metal/polymer/plastic/resin/ceramic forming the constituent of a composite in which other constituents are embedded. If the matrix is a metal, then it is called as a metal matrix and consecutively polymer matrix, if the matrix is a polymer and so on. In composites, the matrix or matrices have two important functions (Weeton et al., 1988). Firstly, it holds the reinforcement phase in the place. Then, under an applied force, it deforms and distributes the stress to the reinforcement constituents. Sometimes the matrix itself is a key strengthening element. This occurs in certain metal matrix composites. In other cases, a matrix may have to stand up to heat and cold. It may conduct or resist electricity, keep out moisture, or protect against corrosion. It may be chosen for its weight, ease of handling, or any of many other applications. Any solid that can be processed to embed and adherently grip a reinforcing phase is a potential matrix material.

In a composite, matrix is an important phase, which is defined as a continuous one. The important function of a matrix is to hold the reinforcement phase in its embedded place, which act as stress transfer points between the reinforcement and matrix and protect the reinforcement from adverse conditions (Clyne, 1996). It influences the mechanical properties, shear modulus and shear strength and its processing characteristics. Reinforcement phase is the principal load-carrying member in a composite. Therefore, the orientation, of the reinforcement phase decides the properties of the composite.

\subsection{Reinforcing phase / Materials}

Reinforcement materials must be available in sufficient quantities and at an economical rate. Recent researches are directed towards a wider variety of reinforcements for the range of 
matrix materials being considered, since different reinforcement types and shapes have specific advantages in different matrices (Basavarajappa et al., 2004). It is to be noted that the composite properties depend not only on the properties of the constituents, but also on the chemical interaction between them and on the difference in their thermal expansion coefficients, which both depend on the processing route. In high temperature composites, the problem is more complicated due to enhanced chemical reactions and phase instability at both processing and application temperatures. Reinforcement phases in MMCs are embedded in the form of continuous reinforcement or discontinuous reinforcement in the matrix material. The reinforcing phase may be a particulate or a fiber, continuous type or discontinuous type. Some of the important particulates normally reinforced in composite materials are titanium carbide, tungsten carbide, silicon nitride, aluminum silicate, quartz, silicon carbide, graphite, fly ash, alumina, glass fibers, titanium boride etc. The reinforcement second phase material is selected depending on the application during the processing of composites (Clyne, 1996). The reinforcement phase is in the form of particulates and fibers generally. The size of the particulate is expressed in microns, micrometer. However, the discontinuous fiber is defined by a term called as 'Aspect Ratio'. It is expressed as the ratio of length to the diameter of the fiber. To improve the wettabilty with the liquid alloy or metal matrix material, the reinforcement phase is always preheated (Adams et al., 2003).

\subsection{Factors affecting reinforcement}

The interface between the matrix and the reinforcement plays an important role for deciding and explaining the toughening mechanism in the metal matrix composites. The interface between the matrix and the reinforcement should be organized in such a way that the bond in between the interface should not be either strong or weak (Singh et al., 2001).

\subsection{Matrix interface / Interphase of matrices}

Interfaces are considered particularly important in the mechanical behavior of MMCs since they control the load transfer between the matrix and the reinforcement. Their nature depends on the matrix composition, the nature of the reinforcement, the fabrication method and the thermal treatments of the composite. For particular matrix/reinforcement associations and especially with liquid processing routes, reactions can occur which change the composition of the matrix and lead to interfacial reaction products, thus changing the mechanical behavior of the composites. The interfacial phenomena in MMCs have been surveyed by several authors. Considering physical and chemical properties of both the matrix and the reinforcing material, the actual strength and toughness desired for the final MMCs, a compromise has to be achieved balancing often several conflicting requirements. A weak interface will lead to crack propagation following the interface, while a strong matrix associated with a strong interface will reveal cracks across both the matrix and the reinforcements. If however the matrix is weak in comparison with the interface and the particle strength, the failure will propagate through the matrix itself. The wettability of the 
reinforcement material by the liquid metallic matrix plays a major role in the bond formation. It mainly depends on heat of formation, electronic structure of the reinforcement and the molten metal temperature, time, atmosphere, roughness and crystallography of the reinforcement. Similarity between metallic bond and covalent bond is reflected in some metal, like titanium carbide and zirconium carbide which are more easily wetted than strong ionic bonds found in ceramics such as alumina that remains poorly wetted. Surface roughness of the reinforced material improves the mechanical interlocking at the interface, though the contribution of the resulting interfacial shear strength is secondary compared to chemical bonding. Large differences in thermal expansion coefficient between the matrix and the reinforcement should be avoided as they can include internal matrix stresses and ultimately give rise to interfacial failures. From a purely thermo dynamical point of view, a comparison of free enthalpy of formation at various temperatures shows that many metals in the liquid state are reactive toward the reinforcing materials in particular oxides or carbides. Though thermodynamically favored, some reactions are however not observed and practically the kinetics of these reactions has to be considered in conjunction with thermodynamic data in order to evaluate the real potential of the reactions. The consequences of such interfacial reactions are the chemical degradation of the reinforcing material associated with a decrease of its mechanical properties, the formation of brittle reaction products at the interface, as well as the release of elements initially part of the reinforcing material toward the matrix may generate inopportune metallurgical phases at the vicinity of the reinforcing materials. Moreover in the case of alloyed matrices, the selective reactivity and depletion of given elements from the alloy can generate compositional gradients in the matrix and may therefore alter its properties close to the interface. Though a moderate reaction may improve the composite bonding, extended reactions usually ruin the reinforcing material. The relation between interfacial reactions and interface strength depends on the materials. The elaboration of MMC requires often a very short solidification time to avoid excess interfacial reaction. During the cooling process, differences in thermal capacity and thermal conductivity between the reinforcing material and the matrix induce localized temperature gradients. Solidification of the metallic matrix is believed to be generally a directional outward process, starting from the inside of the metallic matrix while ending at the reinforcing material surface. Finally, the processing type and the parameters have to be selected and adjusted to a particular MMC system. Metals are generally more reactive in the liquid rather than in the solid state. Consequently, shorter processing time, that is, short contact time between the liquid metal and the reinforcement can limit the extent of interfacial reactions. The study of reinforcement and matrix bonding is important in composite matrix structure, which has been described by Gregolin (2002). While the load is acting on the composite, it has been distributed to the matrix and the reinforcement phase through the matrix interface. The reinforcement is effective in strengthening the matrix only if a strong interfacial bond exists between them. The interfacial properties also influence the resistance to crack propagation in a composite and therefore its fracture toughness (Dusza and Sajgalik, 1995). The two most important energyabsorbing failure mechanisms in a composite are debonding and particle pull-out at the particle matrix interface. If the interface between the matrix and reinforcement debonds, 
then the crack propagation is interrupted by the debonding process and instead of moving through the particle, the crack moves along the particle surface allowing the particle to carry a higher load (El-Mahallawy and Taha, 1993).

\subsection{Physical phenomena of wettability and application}

Wettability is defined as the extent to which a liquid will spread over a solid surface. Interfacial bonding is due to the adhesion between the reinforcement phase and the matrix. For adhesion to occur during the manufacturing of a composite, the reinforcement and the matrix must be brought into an intimate contact. During a stage in composite manufacture, the matrix is often in a condition where it is capable of flowing towards the reinforcement and this behavior approximates to that of the flow of a liquid. A key concept in this contact is wettability. Once the matrix wets the reinforcement particle, and thus the matrix being in intimate contact with the reinforcement, causes the bonding to occur (Hashim et al., 2001; Oh et al., 1987). Different types of bonding will occur and the type of bonding varies from system to system and it entirely depends on the details such as the presence of surface contaminants. The different types of bonding observed are mechanical bonding, electrostatic bonding, chemical bonding, and inter diffusion bonding (Burr et al., 1995). The bonding strength can be measured by conducting the tests like single particle test, bulk specimen test, and micro-indention test (Dusza and Sajgalik, 1995).

Poor wettability of most ceramic particulates with the molten metals is a major barrier to processing of these particulate reinforced MMCs by liquid metallurgy route. The characterization and enhancement of wettability is therefore, of central importance to successful composite processing (Asthana and Rohatgi, 1993). Wettability is shown in the Figure 1 below and it is customarily represented in terms of a contact angle defined from the Young-Dupre equation which is expressed as follows:
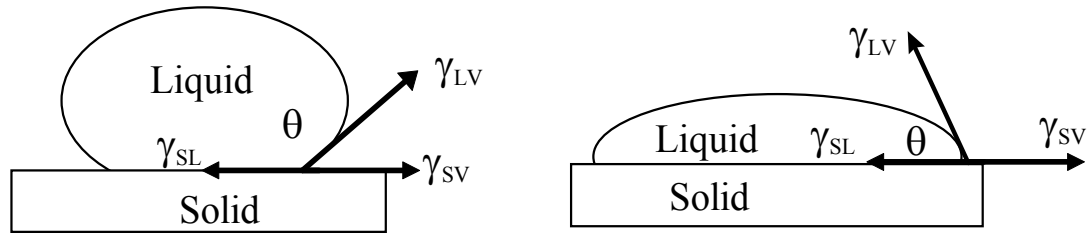

Figure 1. A sessile drop to the left is an example of poor wetting $\left(\theta>90^{\circ}\right)$ and the sessile drop to the right is an example of good wetting $\left(\theta<90^{\circ}\right)$ (Rajan et al., 1998).

$$
\gamma_{l v} \cos \theta=\gamma_{s v}-\gamma_{s l}
$$

Where $\gamma_{S V}=$ Solid/Vapor surface energy, $\gamma_{S L}=$ Solid/Liquid surface energy and $\gamma_{\mathrm{LV}}=$ Liquid/Vapor surface energy.

The wetting behavior of a liquid on a solid can be characterized by the wetting or contact angle that is formed between the liquid and the solid substrate. A "sessile drop" is a continuous drop of liquid on a flat, solid surface under steady-state conditions. To neglect the effects of gravity, the gravitational forces should be small compared to the surface tension of the drop. If 
this condition is satisfied, the drop will approach a hemispherical shape which represents its smallest area and lowest surface free energy. The sessile drop is placed on the solid substrate and the angle between the solid surface and the tangent to the liquid surface at the contact point is measured. This is known as the contact angle or wetting angle. The contact angle can vary between 0 and $180^{\circ}$ and is a measure of the extent of wetting. The conditions of good wetting $\left(\theta<90^{\circ}\right)$ and partial wetting $\left(\theta>90^{\circ}\right)$ are illustrated in Figure 1. Complete wetting (also referred to as spreading) is obtained at an angle of $0^{\circ}$ and complete non-wetting occurs at an angle of $180^{\circ}$. The contact angle is the vector sum of the interfacial surface energies between the solid/liquid $\left(\gamma_{\mathrm{sl}}\right)$, liquid/vapor $\left(\gamma_{\mathrm{lv}}\right)$, and solid/vapor $\left(\gamma_{\mathrm{sv}}\right)$ phases. Young's equation represents a steady-state condition for a solid/liquid interface in stable or metastable thermodynamic equilibrium. Temperature changes have been shown to affect the contact angle of many different systems. The temperature effect, in most cases, can be explained by a reaction at the liquid/solid interface. Thermally activated reactions can occur because many systems are not at chemical equilibrium. The reactions that contribute to wetting (decrease of the contact angle) are those that increase the driving force for wetting ( $\gamma \mathrm{sv}-\gamma \mathrm{sL})$, which is acting at the surface of the liquid drop and the solid substrate. The reactions that contribute to the driving force for wetting are the ones in which the composition of the substrate changes by dissolution of a component of the liquid. On the contrary, if the reaction results in a change of the liquid's composition by dissolution of the solid substrate, but with no change in the composition of the substrate, there is no contribution to the driving force for wetting.

As mentioned above, if the solid substrate is an active participant in the reaction, the free energy of the outer surface of the liquid drop will contribute to the driving force for wetting. As the drop expands on the substrate, the perimeter remains in contact with the unreacted solid and thus the reaction continues to contribute to the driving force for wetting. Examination of phase diagrams representing the interaction between the constituents of the liquid and solid surfaces can help to predict the wetting behavior of a system.

Moreover, measurement of wettability of powders consisting of irregular and polysized particles is extremely difficult. Several techniques have been proposed in the thermodynamic literature to measure wettability. However, these techniques have been applied mostly to non-metallic liquids and their application to metal ceramic systems with reference to pressure casting of composites has been quite limited. The engineering approaches to increasing wettability can be broadly classified into two categories. One method is the surface modification of the reinforcement phase and the other technique is melt treatment. Surface modifications of reinforcements include heat treatment of the particulates to determine surface gas desorbtion, surface oxidation and coating of particles with materials that react with the matrix. Melt treatment is usually done to promote reactivity between the metal and the particulate surface. The wetting reaction must be constrained to prevent reinforcement degradation during the fabrication of subsequent utilization (Ho and $\mathrm{Wu}, 1998)$.

\subsection{Particulate reinforcement}

The improvement in toughness due to the particulate reinforcement depends on the residual stresses surrounding the particles, the weight fraction of the particles, size and 
shape of the particles (Suery and Esperance, 1993). Particles can be spherical, disk-shaped, rod shaped, and plate shaped. Each particle forces the crack to go out of plane, and can force the crack to deflect in more than one direction and thus increase the fracture surface energy (Gogopsi, 1994). Plate and rod shaped particles can increase the composite toughness by another mechanism called as 'pullout' and 'bridging'. The residual stress around the particles results from thermal expansion mismatch between the particles and the matrix, which helps to resist the crack propagation. The term 'particulates' is used to distinguish these materials from particle and referred as a large, diverse group of materials that consist of minute particles. The second phase particle can produce small but significant increase in toughness and consequently increases its strength through crack deflection processes. The particles, sometimes given a proprietary coating can be used for improving strength. When compared to whiskers-reinforcement systems, particle reinforcement systems have less processing difficulties and should permit to add higher weight fractions of the reinforcing phase. The orientation of particles appears as flat plates (Matthew and Rawlings, 1999; Pardo et al., 2005).

\section{Experimental procedure}

\subsection{Materials selected for processing composites}

\section{Aluminum - $11.8 \%$ silicon (LM6)}

The main materials used in this project are LM6 aluminum alloy as a matrix material and $\mathrm{SiO}_{2}$-quartz as a particulate reinforced added in different percentages. Pure (99.99\%) aluminum has a specific gravity of 2.70 and its density is equals $2685 \mathrm{~kg} / \mathrm{m}^{3}$. The details of the LM6 alloy properties and composition is shown in Table 1 and Table 2.

\begin{tabular}{c|c}
\hline \multicolumn{2}{c}{ Composition LM6 } \\
\hline $\mathrm{Al}$ & 85.95 \\
\hline $\mathrm{Cu}$ & 0.2 \\
\hline $\mathrm{Mg}$ & 0.1 \\
\hline $\mathrm{Si}$ & 11.8 \\
\hline $\mathrm{Fe}$ & 0.5 \\
\hline $\mathrm{Mn}$ & 0.5 \\
\hline $\mathrm{Ni}$ & 0.1 \\
\hline $\mathrm{Zn}$ & 0.1 \\
\hline Lead & 0.1 \\
\hline Tin & 0.05 \\
\hline Titanium & 0.2 \\
\hline Other & 0.2 \\
\hline
\end{tabular}

Table 1. Composition of LM6(Sayuti, Sulaiman, Baharudin, et al., 2011) 


\begin{tabular}{l|ll}
\hline \multicolumn{1}{c|}{ PHYSICAL PROPERTIES } & \multicolumn{2}{c}{ VALUES } \\
\hline Density $(\mathrm{g} / \mathrm{cc})$ & 2.66 & \multicolumn{2}{c}{ VALUES } \\
\hline \multicolumn{1}{c|}{ MECHANICAL PROPERTIES } & \multicolumn{2}{c}{} \\
\hline Tensile strength, Ultimate $(\mathrm{MPa})$ & 290 \\
\hline Tensile Strength, Yield $(\mathrm{MPa})$ & 131 \\
\hline Elongation \%; break $(\%)$ & 3.5 \\
\hline Poisson's ratio & 0.33 \\
\hline Fatigue Strength $(\mathrm{MPa})$ & 130 \\
\hline \multicolumn{1}{c}{ THERMAL PROPERTIES } & \multicolumn{1}{c}{ VALUES } \\
\hline CTE, linear $20^{\circ} \mathrm{C}\left(\mu \mathrm{m} / \mathrm{m}-{ }^{-} \mathrm{C}\right)$ & 20.4 \\
\hline CTE, linear $250^{\circ} \mathrm{C}\left(\mu \mathrm{m} / \mathrm{m}-{ }^{\circ} \mathrm{C}\right)$ & 22.4 \\
\hline Heat Capacity $\left(\mathrm{J} / \mathrm{g}-{ }^{\circ} \mathrm{C}\right)$ & 0.963 \\
\hline Thermal Conductivity $(\mathrm{W} / \mathrm{m}-\mathrm{K})$ & 155 \\
\hline Melting Point $\left({ }^{\circ} \mathrm{C}\right)$ & 574 \\
\hline
\end{tabular}

Table 2. Physical, Mechanical and thermal properties of LM6 (Sulaiman, et al., 2008)

\section{Quartz}

Pure and fused silica is commonly called quartz. Quartz is a hard mineral which is abundantly available as a natural resource. It has a rhombohedra crystal structure with a hardness of 7 on the Mohs scale and has a low specific gravity ranging from 2.50 to 2.66. It provides excellent hardness when incorporated into the soft lead-alloy, thereby making it better suited for applications where hardness is desirable. It also imparts good corrosion resistance and high chemical stability. It is a mineral having a composition $\mathrm{SiO}_{2}$, which is the most common among all the materials, and occurs in the combined and uncombined states. It is estimated that $60 \%$ of the earth's crust contain $\mathrm{SiO}_{2}$. Sand, clays, and rocks are largely composed of small quartz crystals. $\mathrm{SiO}_{2}$ is white in color in the purest form. The properties of pure quartz are listed in the Table 3.

\begin{tabular}{c|c}
\hline \multicolumn{2}{c}{ Properties of quartz } \\
\hline Molecular weight & 60.08 \\
\hline Melting Point ${ }^{\circ} \mathrm{C}$ & 1713 \\
\hline Boiling Point ${ }^{\circ} \mathrm{C}$ & 2230 \\
\hline Density gm/cc & 2.32 \\
\hline Thermal Conductivity & $0.01 \mathrm{~W} / \mathrm{cm} \mathrm{K}$ (bulk) \\
\hline Thermal Diffusivity & $0.009 \mathrm{~cm} 2 / \mathrm{sec}$ (bulk) \\
\hline Mohs Hardness @ $20^{\circ} \mathrm{C}$ & 7 Modified Mohs \\
\hline Si $\%$ & 46.75 \\
\hline O $\%$ & 53.25 \\
\hline Crystal Structure & Cubic \\
\hline Mesh size & 230 \\
\hline Size & 65 microns $(65 \mu \mathrm{m})$ \\
\hline
\end{tabular}

Table 3. Properties of quartz 


\section{Preparation of materials}

The materials used in this work were Aluminum LM6 alloy as the matrix and $\mathrm{SiO}_{2}$ as reinforcement particulates with different weight percentages. The tensile test specimens were prepared according to ASTM standards B 557 M-94 (ASTM, 1991). Sodium silicate and $\mathrm{CO}_{2}$ gas was used to produce $\mathrm{CO}_{2}$ sand mould for processing composite casting. The aluminum alloy, LM6, was based on British standards that conform to BS 1490-1988 LM6. Alloy of LM6 is actually a eutectic alloy having the lowest melting point that can be seen from the Al-Si phase diagram. The main composition of LM6 is about $85.95 \%$ of aluminum and $11.8 \%$ of silicon.

The $\mathrm{SiO}_{2}$ particulate used as a second phase reinforcement in the alloy matrix was added on the molten LM6 by different weights fraction such as $5 \%, 10 \%, 15 \%, 20 \%, 25 \%$, and $30 \%$. The mesh size of Silicon Dioxide particulate is 230 microns and the average particle size equal to 65 microns $(65 \mu \mathrm{m})$.

\section{Fabrication of composites}

Only one type of pattern was used in this project and the procedure for making the pattern involves the preparation of drawing, selection of pattern material and surface finishing. Carbon dioxide moulding process was used to prepare the specimens as per the standard moulding procedure. Quartz-particulate reinforced MMCs were fabricated by casting technique. Six different weight fractions of $\mathrm{SiO}_{2}$ particle in the range from 5\%,10\%, 15\%, $20 \%, 25 \%$, and $30 \%$ by weight were used. In this research work, the particulates were preheated to $200{ }^{\circ} \mathrm{C}$ in a heat treatment muffle furnace for 2 hours and it was transferred immediately in the crucible containing liquid LM6 alloy.

\subsection{Testing methods}

\section{Tensile testing}

Tensile test was conducted to determine the mechanical properties of the processed $\mathrm{SiO}_{2}$ particulate reinforced LM6 alloy composites. Test specimens were made in accordance to ASTM standard B557 M-94. A $250 \mathrm{KN}$ servo hydraulic INSTRON 8500 UTM was used to conduct the tensile test. The tensile testing of the samples was performed based on the following specifications and procedures according to the ASTM standards, which of one crosshead speed of $2.00 \mathrm{~mm} /$ minute, grip distance $50.0 \mathrm{~mm}$, specimen distance $50.0 \mathrm{~mm}$ and temperature $24^{\circ} \mathrm{C}$.

\section{Hardness measurement}

The hardness testing was done on a Rockwell Hardness Tester. The hardness of composites was tested by using MITUTOYO ATK-600 MODEL hardness tester. For each sample, ten hardness readings were taken randomly from the surface of the samples. Hardness values of different types of the processed composites are determined for different weight fraction $\%$ of titanium carbide particulate containing aluminum-11.8\% silicon alloy and graphs were 
plotted between the hardness value and the corresponding type of particulate addition on weight fraction basis.

\section{Impact testing}

The impact test was conducted in accordance with ASTM E 23-05 standards at room temperature using izod impact tester. The casting processing steps and testing shows are shown in Figure 2.

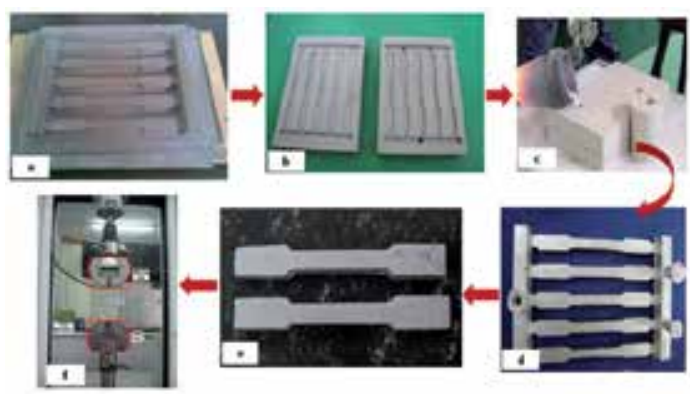

Figure 2. The casting processing steps; (a) Pattern of mould (b) sand mould : drag and copper (c) melting and pouring in the sand mould (d) tensile specimens with gating system (e) tensile specimen after removing of gating systems (f) tensile testing

\section{Density measurement}

The density of a material is defined as its mass per unit volume. A\&D-GR 200 - Analytical Balance was used to conduct the density measurement. The theoretical density of each set of composites was calculated using the rule of mixtures (Rizkalla and Abdulwahed, 1996). Each pellet was weighed in air $\left(\mathrm{W}_{\mathrm{a}}\right)$, then suspended in Xylene and weighed again $(\mathrm{W})$. The density of the pellet was calculated according to the formula:

$$
\text { Density }=\frac{W a}{(W a-W w)} \times \text { density of Xylene }
$$

\section{Thermal diffusivity measurement}

Thermal diffusivity of composite materials is measured using the photo flash method. The photoflash detection system consists of a light source, sample holder, thermocouple, low noise pre amplifier, oscilloscope, photodiode and a personal computer. The temperature rise at the back surface of the sample is detected by the thermocouple. The detected signal is amplified by a low-noise preamplifier and processed by a digital oscilloscope (Carter and Norton, 2007; Yu et al., 2002).

The voltage supplied to the camera flash is always maintained below 6 Volts before switching on the main power supply. The sample is machined to acquire flat surface to obtain better quality result and it is attached directly to the thermocouple. The camera flash is located at $2 \mathrm{~cm}$ in front of the sample holder. Before starting the equipment, the set up 
was tested using a standard material such as aluminium. Measurement was carried out every 10 minutes to allow the sample to thermally equilibrate at room temperature. The data was analyzed before running the next measurement.

Photoflash detection system is not an expensive method and the standard thermal diffusivity value for aluminum is equal to $0.83 \mathrm{~cm}^{2} / \mathrm{sec}$ for thickness greater than $0.366 \mathrm{~cm}$ (Muta et al., 2003). In the photo flash system, the excitation source consists of a high intensity camera flash. This method is well suitable for aluminum, aluminum alloys and aluminum-silicon particulate metal matrix composites (Collieu and Powney, 1973). The thermal diffusivity values can be obtained for different thicknesses of the test samples. The thermal diffusivity $\alpha$ determines the speed of propagation of heat waves by conduction during changes of temperature with time. It can be related to $\alpha$, the thermal conductivity through the following equation (Michot et al., 2008; Taylor, 1980).

The photo flash technique was originally described by Parker and it is one of the most common ways to measure the thermal diffusivity of the solid samples. The computer is programmed to calculate the thermal diffusivity, $\alpha$, using the equation:

$$
\alpha=\frac{\left(1.37 \times L^{2}\right)}{\left[(3.14)^{2} x t_{0.5}\right]}
$$

Where $\mathrm{L}=$ thickness in $\mathrm{mm}$ and $\mathrm{t} 0.5=$ half rise time in seconds.

\section{Scanning Electron Microscopy (SEM)}

LEO 1455 variable pressure scanning electron microscope with Inca 300 Energy Dispersive X-ray (EDX) was used to investigate the morphological features. Results and data obtained from the tensile tested samples were correlated with the reported mechanical properties for each volume fraction of silicon dioxide percentage addition to the LM6 alloy matrix.

\section{Results}

\section{Tensile properties}

The average value of tensile strength $(\mathrm{MPa})$ and Young's Modulus (MPa) versus weight fraction of $\mathrm{SiO}_{2}$ is shown in the Figures 3 and 4 .

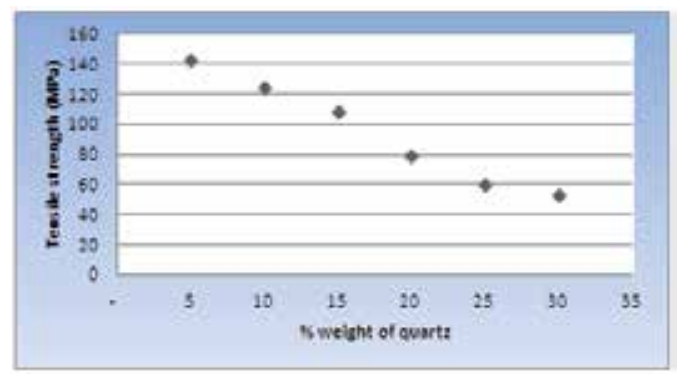

Figure 3. Tensile strength Vs \% weight 


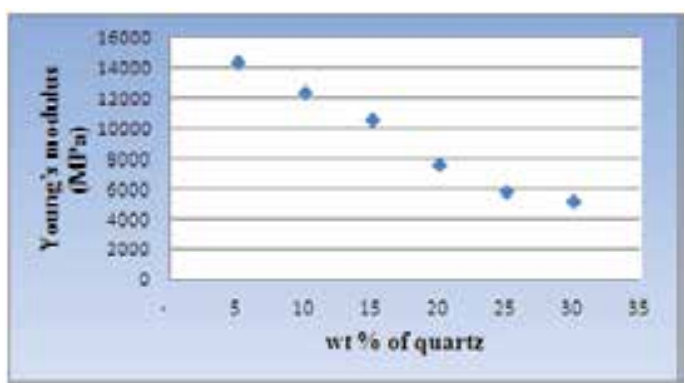

Figure 4. Young"s modulus Vs \% weight

The graph plotted between the average tensile strength and modulus or elasticity values versus variation in weight fraction of quartz particulate addition to LM6 alloy indicates that both the properties decreases with increase in the addition of the quartz particulate. The increase of closed pores content with increasing quartz particulate content would create more sites for crack initiation and hence lower down the load bearing capacity of the composite. The fluctuation maybe due to the non-uniform distribution of quartz particulates, due to experimental errors and also depends on the cooling rate of the castings (ASTM, 1991; Seah, et al., 2003). When particulates increase, particles are no longer isolated by the ductile aluminum alloy matrix, therefore cracks will be not arrested by the ductile matrix and gaps would propagate easily between the quartz particulates. This residual stress affects the material properties around it and the crack tips and the fracture toughness values would be altered. Consequently, these residual stresses would probably contribute for the brittle nature of the composites. It should be noted that the compressive strength of the quartz particulate dominates which is more than the tensile strength of the LM6 alloy matrix and hence the tensile strength decreases with more amount of addition of quartz particulate afact which is well supported is well supported and evidenced from the literature citation (Rizkalla and Abdulwahed, 1996; Seah, et al., 2003).

\section{Hardness}

Similarly, for a given $\mathrm{SiO}_{2}$ reinforcement content, some differences in the hardness values were observed depending upon the particle size of the constituents. From the Table 4, data on hardness of quartz particulate reinforced composites made in sand mold is listed. It was found that the hardness value increased gradually with the increased addition of quartz particulate by weight fraction percentage as shown in Figure 5.

The maximum hardness value obtained based on the Rockwell superficial 15N-S scale was 67.85 for $30 \%$ weight fraction addition. The EDS spectrums for $30 \%$ wt of $\mathrm{SiO}_{2}$ are shown in Figure 5. Their respective elemental analysis is shown in Table 4. It was observed that the grain-refined composite casting has higher weight percentage of Si compared with the original LM6 casting. These results indicate the interrelationship between the thermal properties and hardness.

\section{Impact strength}

Impact strength data of quartz particulate reinforced composite castings processed was determined and it is listed in the Table 4. From the plotted graph shown in the Figure 6, it is 
found that the impact strength values were gradually increased with the increased addition of quartz particulate in the alloy matrix. The maximum value of impact strength was $24.80 \mathrm{~N}$ $\mathrm{m}$ for $30 \%$ weight fraction addition of quartz particulate to the alloy matrix. A reason for the increased volume impact-abrasive wear of the $\mathrm{SiO} 2$ particle reinforced composites lies in the propensity of the carbides to fracture and spall as a result of the repeated impact from the quartzite. In the monolithic ferrous-based alloys, the matrix can absorb substantial damage in the form of plastic deformation. This plastic deformation is in fact beneficial in that, the matrix will get harder as a result, and wear, fatigue type processes ending as a material removal mechanism. In the $\mathrm{SiO}_{2}$ particle reinforced composites, however, the high weight fraction of $\mathrm{SiO}_{2}$ limits the amount of plastic deformation that the matrix can absorb. This leads more quickly to $\mathrm{SiO}_{2}$ reinforcement fracture, matrix- $\mathrm{SiO}_{2}$ particle delamination, and $\mathrm{S}_{\mathrm{i}} \mathrm{O}_{2}$ particle spalling. As a consequence, volume impact-abrasive wear increases at a more rapid rate for the composite materials as the hardness increases. However, for the very 'hardest' $\mathrm{S}_{\mathrm{i}} \mathrm{O}_{2}$ particle reinforced composites, impact-abrasion resistance is very good. The summary of mechanical properties of quartz particulate reinforced composite castings processed was determined and it is listed in the Table 4.

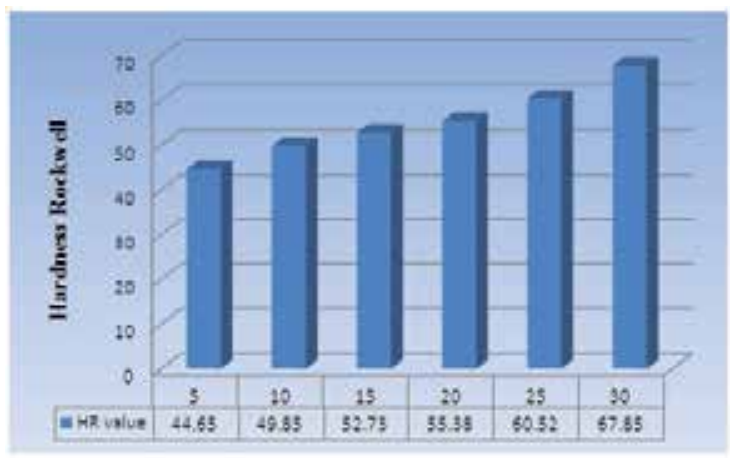

Figure 5. Hardness Vs wt \% of quartz

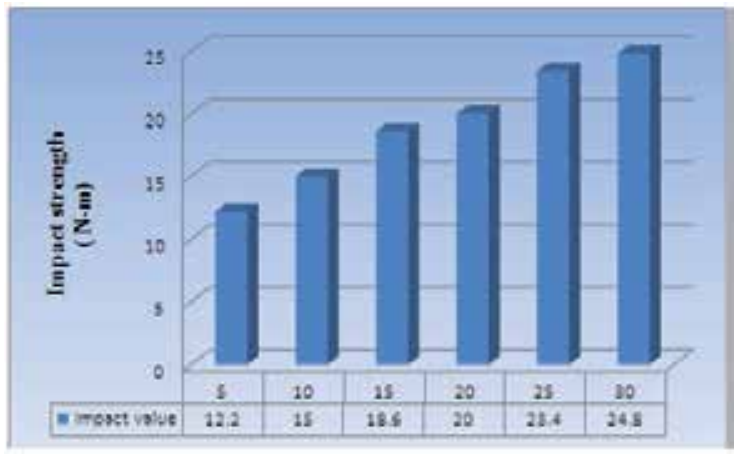

Figure 6. Impact strength Vs Weight fraction \% of quartz 


\begin{tabular}{|c|c|c|c|c|c|c|c|c|}
\hline $\begin{array}{c}\text { Wt \% of } \\
\text { quartz }\end{array}$ & $\begin{array}{c}\text { UTS } \\
(\mathrm{MPa})\end{array}$ & $\begin{array}{c}\text { Yield } \\
(\mathrm{MPa})\end{array}$ & $\begin{array}{c}\text { Young's } \\
\text { modulus } \\
\mathrm{MPa}\end{array}$ & $\begin{array}{c}\text { Fracture } \\
\text { stress } \\
\mathrm{MPa}\end{array}$ & Ductility \% & $\begin{array}{c}\text { Reduction in } \\
\text { area \% }\end{array}$ & $\begin{array}{c}\text { Rockwell } \\
\text { Hardness }\end{array}$ & $\begin{array}{c}\text { Impact } \\
(\mathrm{N}-\mathrm{m})\end{array}$ \\
\hline $5 \%$ & 142.99 & 132.00 & 14351 & 189.50 & 1.214 & 2.863 & 44.65 & 12.20 \\
\hline $10 \%$ & 124.74 & 129.60 & 12350 & 164.60 & 1.412 & 2.864 & 49.85 & 15.00 \\
\hline $15 \%$ & 108.47 & 118.50 & 10635 & 142.20 & 1.422 & 3.042 & 52.73 & 18.60 \\
\hline $20 \%$ & 78.97 & 109.60 & 7621 & 128.40 & 1.632 & 3.264 & 55.38 & 20.00 \\
\hline $25 \%$ & 59.53 & 100.50 & 5853 & 115.30 & 1.824 & 3.625 & 60.52 & 23.40 \\
\hline $30 \%$ & 52.64 & 92.65 & 5242 & 104.60 & 1.741 & 3.482 & 67.85 & 24.80 \\
\hline
\end{tabular}

Table 4. Mechanical properties of quartz particulate composites

\section{Density}

Figure 8 gives the influence of quartz addition on the density. The graph shows that as the quartz-silicon dioxide content was gradually increased, the density of the Aluminum composite decreased. Slight decrease was observed in the density because quartz-silicon dioxide has a slight lower density value than LM6 (the density of LM6 is $2.65 \mathrm{grs} / \mathrm{cc}$ and of quartz is $2.23 \mathrm{grs} / \mathrm{cc}$ ).

The investigation of the aluminum composite was well documented. The percentage of the closed pores in the sintered composites increased with increasing quartz content. This can be attributed to silica being harder than aluminum and non deformation at all under the applied compaction load. The morphological features of quartz particles were significantly different from those of Aluminum and as a result, the interparticle friction effects were different. Therefore, the increase in the amount of closed pores with increasing quartz content would justify the observed decrease in density (Rizkalla and Abdulwahed, 1996).

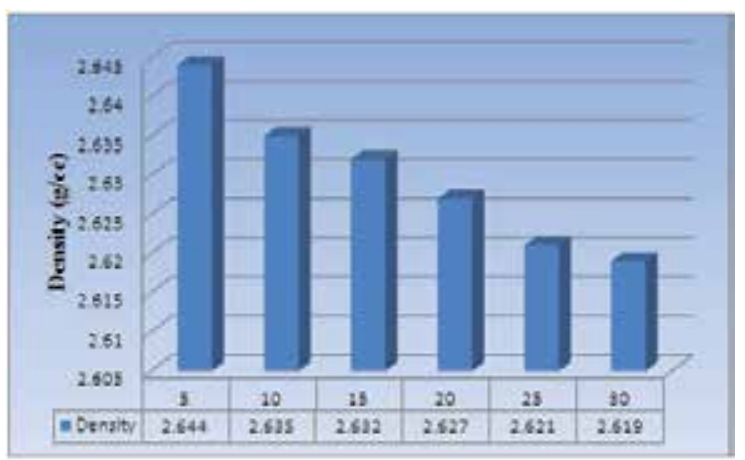

Figure 7. Graph plotted on density versus \%wt fraction of $\mathrm{SiO}_{2}$

\section{Thermal properties}

Quartz particulate reinforced composite castings made in grey cast iron mold were tested and analyzed for thermal properties. Graphs are plotted between the weight fraction \% addition of quartz and thermal diffusivity and thermal conductivity values. It is found that the thermal diffusivity of the quartz composites decreased with the increased addition in the 
alloy matrix. Reversely, the thermal conductivity of the quartz composites decreased with the increased addition of quartz particulate in the alloy matrix. Quartz particulates are a ceramic reinforcement phase and on addition of this in the alloy matrix reduces the thermal conductivity. The data for thermal diffusivity and thermal conductivity of the quartz particulate reinforced composites made in sand mold is given in the Table 4 . These are illustrated in the plotted graphs and are shown in Figure 8 and 9. The thermal diffusivity and thermal conductivity for $30 \%$ weight fraction addition of quartz are $0.2306 \mathrm{~cm}^{2} / \mathrm{sec}$ and $52.9543 \mathrm{~W} / \mathrm{mK}$ respectively and it is well supported from the literature citation (Collieu and Powney, 1973). The summary of physical properties of quartz particulate reinforced composite castings processed was determined and it is listed in the Table 5.

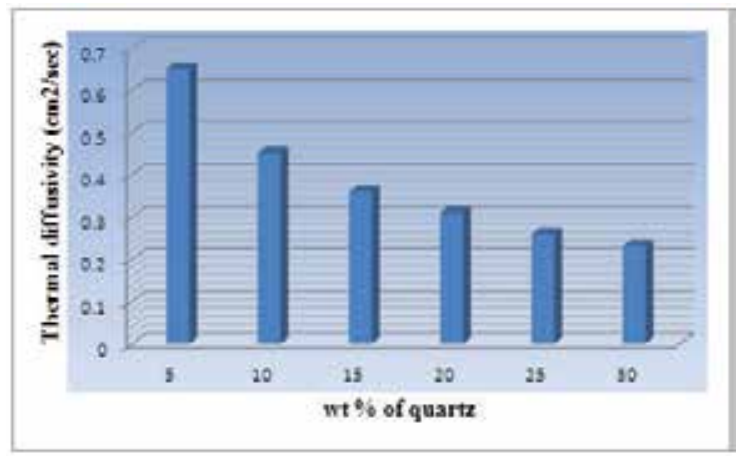

Figure 8. Thermal diffusivity Vs Wt Fraction \% of quartz

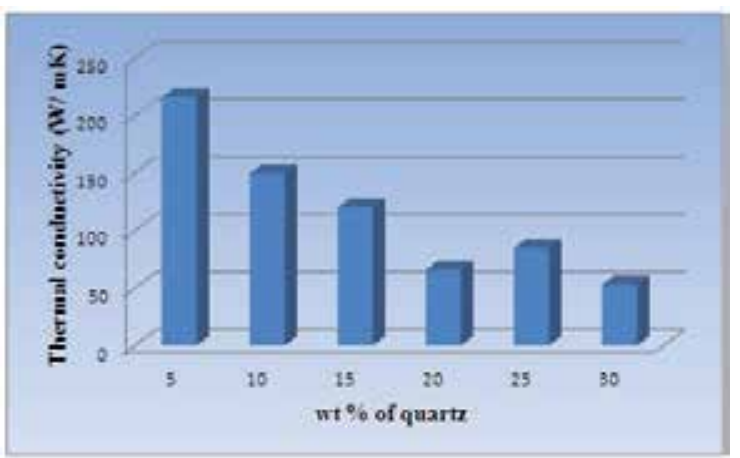

Figure 9. Thermal conductivity Vs Wt Fraction \% of quartz

\begin{tabular}{cccc}
\hline $\mathrm{Wt} \%$ of quartz & Density $(\mathrm{g} / \mathrm{cc})$ & Thermal diffusivity $\mathrm{cm}^{2} / \mathrm{sec}$ & Thermal conductivity $(\mathrm{W} / \mathrm{mK})$ \\
\hline $5 \%$ & 2.644 & 0.6513 & 215.826 \\
$10 \%$ & 2.635 & 0.4514 & 149.584 \\
$15 \%$ & 2.632 & 0.3595 & 119.933 \\
$20 \%$ & 2.627 & 0.3102 & 65.6860 \\
$25 \%$ & 2.621 & 0.2590 & 84.6830 \\
$30 \%$ & 2.619 & 0.2306 & 52.9543 \\
\hline
\end{tabular}

Table 5. Physical properties of quartz particulate composites 


\section{Scanning electron microscopy (SEM)}

Scanning Electron Microscopy and energy dispersive spectroscopy was employed to obtain some qualitative evidences on the particle distribution in the matrix and bonding quality between the particulate and the matrix. Besides this the fracture surface of the composite was analyzed by using SEM to show the detail of chemically reacted interfaces. Thus, in order to increase the potential application of MMCs, it is necessary to concentrate on the major aspects, like particle size of quartz and quartz distribution concentration.

The fracture surfaces or fractographs are shown in the Figures 10-15 after tensile testing the specimens having different weight fraction of quartz particulate. It was observed that the increase of $\mathrm{SiO}_{2}$ content would create more sites for crack initiation and would lower the load bearing capacity of MMCs. In addition the number of contacts between quartz particles would increase and more particles were no longer isolated by the ductile aluminum alloy matrix. Therefore, cracks were not arrested by the ductile matrix and they would propagate easily between quartz particulates. Decrease of $\mathrm{SiO}_{2}$ content to less than $30 \%$ in the matrix and a particle size of 230 micron could increase the tensile strength. Hence cracking on the surface is not too dominant. This phenomenon is shown in Figure 10. The problem on interfacial bonding between the particulate quartz and the matrix during the solidification of composites can be ignored because the phenomena of cracking occurs only in a small part of the surface (Seah, et al., 2003). In contrast, when the content of quartz was increased (30\%), interfacial bonding concept would be an important phenomenon because the surface cracking will be distributed on the surface of the parts. The other problem caused by the interaction between Aluminum alloy and quartz particle is not a significant one and it is removed while solidification during the pouring process and due to slip inter bonding/ inter granular movement which is illustrated with the aid of Figure 11.

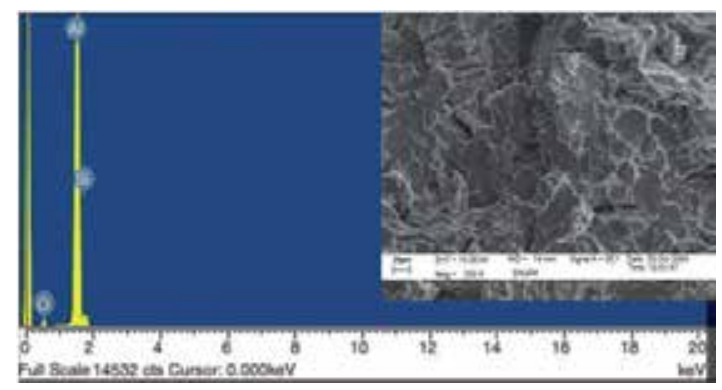

Figure 10. EDX Spectrum and Fractograph of $5 \mathrm{wt} \%$ quartz particulate reinforced in quartz -LM6 alloy matrix composite at 250X magnification by SEM after tensile testing. 


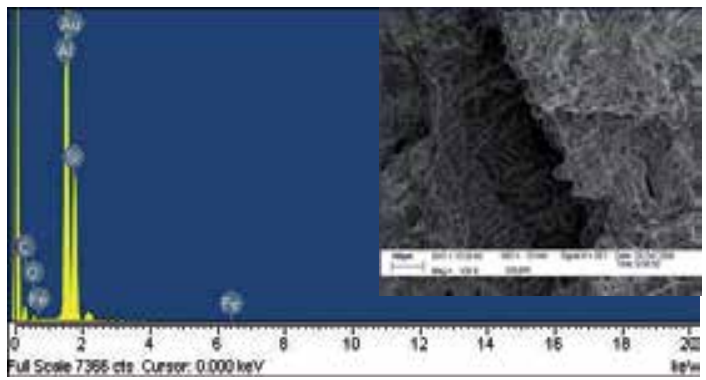

Figure 11. EDX Spectrum and Fractograph of 10wt\% quartz particulate reinforced in quartz -LM6 alloy matrix composite at 100X magnification by SEM after tensile testing.

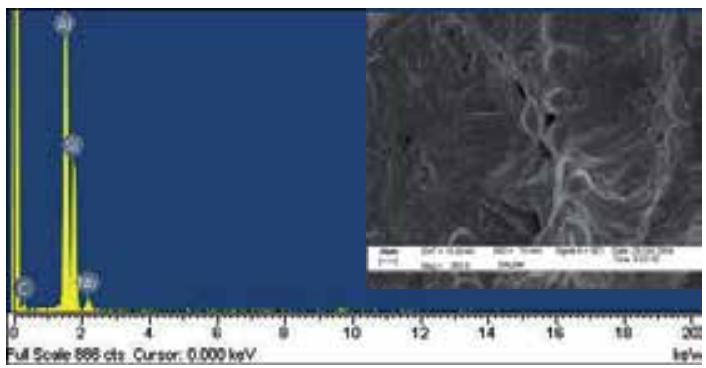

Figure 12. EDX Spectrum and Fractograph of 15wt\% quartz particulate reinforced in quartz -LM6 alloy matrix composite at 250X magnification by SEM after tensile testing.

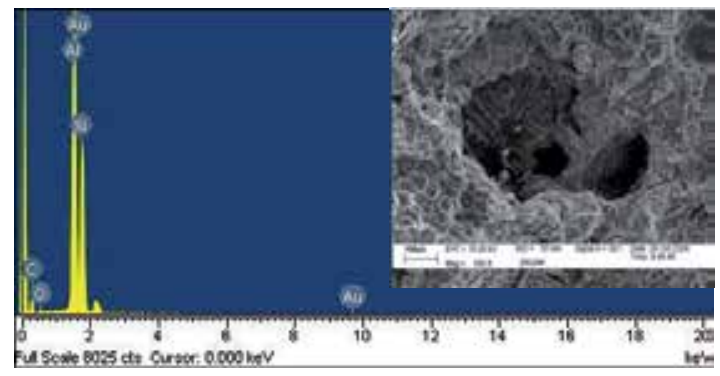

Figure 13. EDX Spectrum and Fractograph of 20wt \% quartz particulate reinforced in quartz -LM6 alloy matrix composite at 100X magnification by SEM after tensile testing

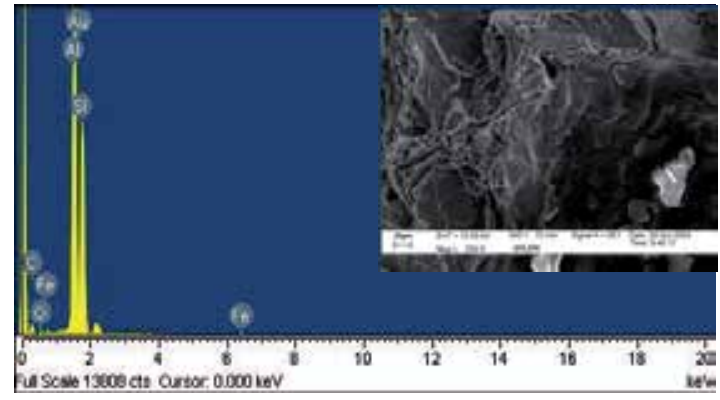

Figure 14. EDX Spectrum and Fractograph of $25 \mathrm{wt} \%$ quartz particulate reinforced in quartz -LM6 alloy matrix composite at 250X magnification by SEM after tensile testing 


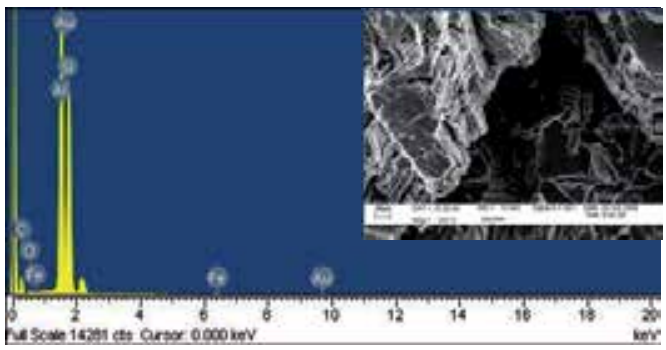

Figure 15. EDX Spectrum and Fractograph of 30wt\% quartz articulate reinforced in quartz -LM6 alloy matrix composite at 250X magnification by SEM after tensile testing

\section{Conclusions}

In this study, the compressive strength of the silicon dioxide particulate reinforcement dominates and influences more effectively than the tensile strength of the LM6 alloy matrix phase. Hence the values of tensile strength and modulus of elasticity are decreased with the increased addition of $\mathrm{SiO}_{2}$ particulate from 5 to $30 \%$ by volume fraction basis. This fact from the present experimental research is well supported and validated from the literature. The mechanical behaviour of the processed composite had a strong dependence on the volume fraction addition of the second phase reinforcement particulate on the alloy matrix. On the other hand, decreasing the $\mathrm{SiO}_{2}$ particulate content less than $30 \%$ by weight along with the particle size constraint as 230 mesh-65 microns would increase the tensile strength and cracking on the surface might not be too dominant. The hardness value of the silicon reinforced aluminum silicon alloy matrix composite is increased with the addition of quartz particulate in the matrix.

The density of these composites decreased slightly with increasing quartz content. Slight decrease was observed in the density because quartz-silicon dioxide has a slightly lower density value than LM6. For a given particle size combination, the thermal diffusivity and thermal conductivity decreases as $\mathrm{S}_{\mathrm{i}} \mathrm{O}_{2}$ wt \% of the composite increases. The particle size ratio of the constituents becomes an important factor for thermal properties, especially above 10 wt. \% $\mathrm{S}_{\mathrm{i}} \mathrm{O}_{2}$. A higher $\mathrm{Al} / \mathrm{S}_{\mathrm{i}} \mathrm{O}_{2}$ particle size ratio results in segregation of $\mathrm{S}_{\mathrm{i}} \mathrm{O}_{2}$ particles along the LM6 boundaries. This yields lower thermal conductivity with respect to the homogeneously distributed reinforcement. Therefore, a thermal conductivity value that is less than the expected one might be attributed to the micro-porosity in the segregated structure. Similar tendencies were also observed for the results of hardness tests.

In future, it is strongly recommended that tensile tests be performed by reinforcing the second phase quartz particulate addition to the LM6 alloy matrix by limiting it up to 15 $\mathrm{wt} \%$. In addition, compressive strengths testing of the processed composite samples can be done to highlight the benefits, advantages and applications of these composites. It is also worthwhile to conduct heat treatment studies of these processed composites and this will be in the scope of future research work. 


\section{Author details}

M. Sayuti

Department of Mechanical and Manufacturing Engineering, Faculty of Engineering, Universiti

Putra Malaysia, Serdang, Selangor, Malaysia

Department of Industrial Engineering, Faculty of Engineering, Malikussaleh University,

Lhokseumawe, Aceh - Indonesia

S. Sulaiman, B.T.H.T. Baharudin and M.K.A. Arifin

Department of Mechanical and Manufacturing Engineering, Faculty of Engineering, Universiti

Putra Malaysia, Serdang, Selangor, Malaysia

T.R. Vijayaram

Faculty of Engineering and Technology (FET) Multimedia University, Jalan Ayer Keroh Lama, Bukit Beruang, Melaka. Malaysia

\section{Acknowledgement}

The authors would like to express their deep gratitude and sincere thanks to the Department of Mechanical and Manufacturing Engineering, Universiti Putra Malaysia for their help to complete this work.

\section{References}

Adams, D. F., carlsson, L. A., and Pipes, R. B. (2003). Expeirmental Characterization of Advanced Composite Materials (3rd ed.). Florida, USA: CRC Press LLC.

Ashby, M. F., and Jones, D. R. H. (1980). Engineering Materials: An Introduction to their properties and applications, International series on materials science and technology. UK: Elsevier Science and Technology.

Asthana, R., and Rohatgi, P. K. (1993). A Study of Metal-Ceramic Wettability in Sic-Al Using Dynamic Melt Infiltration of SiC. Key Engineering Materials, 70-80, 47-62.

ASTM. (1991). American Society for Testing and Material, Anual Books of ASTM Standards. USA.

Baker, A. R., Dawson, D. J., and Evans, D. C. (1987). Ceramics and Composite, Materials for Precision Engine Components. Journal of Materials \& Design, 8(6), 315-323.

Basavarajappa, S., Chandramohan, G., and Dinesh, A. (2004). Mechanical Properties of $M M C^{\prime} S$-an Experimental Inversitgation. Paper presented at the International Symposium of research Students on Materials and Engineering, Chennai.

Burr, A., Yang, J. Y., Levi, C. G., and Leckie, F. A. (1995). The strength of metal-matrix composite joints. Acta Metallurgica et Materialia, 43(9), 3361-3373.

Carter, C. B., and Norton, M. G. (2007). Ceramic Material: Science and Engineering New York: Springer. 
Chambers, B. V., Seleznev, M. L., A.Cornie, J., Zhang, S., and Rye, M. A. (1996). The Strength and Toughness of Cast Aluminium Composite as a Function of Composition, Heat Treatment and Particulate. SAE International Journal, 164-169.

Clyne, T. W. (1996). Interfacial Effects in Particulate, Fibrous and Layered Composite Materials. Key Engineering Materials, 116-117, 133-152.

Collieu, A. M. B., and Powney, D. J. (1973). The Mechanical and Thermal Properties of Materials

UK, London: Edward Arnold (Publishers) Ltd.

Daniel, I. M., and Ishai, O. (1994). Engineering Mechanics of Composite Materials. USA: Oxford University Press.

Dusza, J., and Sajgalik, P. (1995). Fracture Toughness and Strength Testing of Ceramic Composites. In N. P, Cheremisinoff and P. N.Cheremisinoff (Eds.), Handbook of Advanced Materials Testing (pp. 399-435). New York, USA: Marcel Dekker, Inc.

El-Mahallawy, N. A., and Taha, M. A. (1993). Reinforcement Considerations for High Temperature Metal Matrix Composites. Key Engineering Materials, 79-80, 1-14.

Feest, E. A. (1986). Metal matrix composites for industrial application. Materials $\mathcal{E}$ Design, $7(2), 58-64$.

Fridlyander, J. N. (1995). Metal Matrix Composites. UK, London: Chapman \& Hall.

Gay, D., V.Hoa, S., and W.Tsai, S. (2003). Composite Materials: Design and Applications. USA: CRC Press LLC.

Gogopsi, Y. G. (1994). Particulate Silicon Nitride - Based Composite. Materials science, 29(4), 2541-2556.

Gregolin, Goldenstein, H., Gonçalves, M. d. C., and Santos., R. G. d. (2002). Aluminium Matrix Composites Reinforced with Co-continuous Interfaced Phases Aluminiumalumina Needles. Materials Research, 5(3), 337-342.

Hashim, J., Looney, L., and Hashmi, M. S. J. (1999). Metal matrix composites: production by the stir casting method. Journal of Materials Processing Technology, 92-93, 1-7.

Hashim, J., Looney, L., and Hashmi, M. S. J. (2001). The enhancement of wettability of SiC particles in cast aluminium matrix composites. Journal of Materials Processing Technology, 119(1-3), 329-335.

Hashim, J., Looney, L., and Hashmi, M. S. J. (2002). Particle distribution in cast metal matrix composites--Part I. Journal of Materials Processing Technology, 123(2), 251-257.

Ho, H.-N., and Wu, S.-T. (1998). The wettability of molten aluminum on sintered aluminum nitride substrate. Materials Science and Engineering: A, 248(1-2), 120-124.

Huda, D., El Baradie, M. A., and Hashmi, M. S. J. (1993). Metal-matrix composites: Manufacturing aspects. Part I. Journal of Materials Processing Technology, 37(1-4), 513-528.

Humphreys, J. (1987). Composites for automotive on-engine applications. Materials $\mathcal{E}$ Design, 8(3), 147-151.

Kaczmar, J. W., Pietrzak, K., and Wlosinski, W. (2000). The production and application of metal matrix composite materials. Journal of Materials Processing Technology, 106(1-3), 5867.

Kok, M. (2005). Production and mechanical properties of Al2O3 particle-reinforced 2024 aluminium alloy composites. Journal of Materials Processing Technology, 161(3), 381-387. 
Matthew, F. L., and Rawlings, R. D. (1999). Composite Material; Engineering and Science. UK: Imperial College of Science.

Mazumdar, S. K. (2002). Composites Manufacturing: Materials, Product and Process Engineering. USA: CRC Press Inc.

Michot, A. e., Smith, D. S., Degot, S., and Gault, C. (2008). Thermal Conductivity and Specific Heat of Kaolinite: Evolution with Thermal Treatment. Journal of the European Ceramic Society 28, 2639-2644.

Mortensen, A., Cornie, J. A., and Flemings., M. C. (1989). Solidification Processing of MetalMatrix Composites. Materials \& Design, 10(2), 68-76.

Muta, H., Kurosaki, K., Uno, M., and Yamanaka, S. (2003). Thermoelectric properties of constantan/spherical $\mathrm{SiO} 2$ and $\mathrm{Al} 2 \mathrm{O} 3$ particles composite. Journal of Alloys and Compounds, 359(1-2), 326-329.

Oh, S.-Y., Cornie, J. A., and Russell., K. C. (1987). Particulate Wetting and Metal: Ceramic Interface Phenomena. Paper presented at the Ceramic Engineering Science Proceedings.

Pardo, A., Merino, M. C., Merino, S., Viejo, F., Carboneras, M., and Arrabal, R. (2005). Influence of reinforcement proportion and matrix composition on pitting corrosion behaviour of cast aluminium matrix composites (A3xx.x/SiCp). Corrosion Science, 47(7), 1750-1764.

Prasad, S. V., and Asthana, R. (2004). Aluminium Metal Matrix Composite for Automotive Applications: Tribological Considerations. Tribology Letters, 17(3), 445-453.

Rajan, T. P. D., Pillai, R. M., and Pai, B. C. (1998). Reinforcement coatings and interfaces in aluminium metal matrix composites. Journal of Materials Science, 33(14), 3491-3503.

Rizkalla, H. L., and Abdulwahed, A. (1996). Some mechanical properties of metal-nonmetal Al---SiO2 particulate composites. Journal of Materials Processing Technology, 56(1-4), 398403.

Rohatgi, P. K. (1993). Metal Matrix Composite, Casting Processes. Science Journal, 43(4), 323349.

Sahin, Y. (2003). Preparation and some properties of $\mathrm{SiC}$ particle reinforced aluminium alloy composites. Materials E Design, 24(8), 671-679.

Sayuti, M., Sulaiman, S., Baharudin, B. T. H. T., Arifin, M. K. A., Suraya, S., and Vijayaram, T. R. (2010). Mechanical properties of particulate reinforced aluminium alloy matrix composite.

Sayuti, M., Sulaiman, S., Baharudin, B. T. H. T., Arifin, M. K. A., Vijayaram, T. R., and Suraya, S. (2011) Influence of mechanical vibration moulding process on the tensile properties of TiC reinforced LM6 alloy composite castings. Vol. 66-68 (pp. 1207-1212).

Sayuti, M., Sulaiman, S., Vijayaram, T. R., Baharudin, B. T. H. T., and Arifin, M. K. A. (2011) The influence of mechanical vibration moulding process on thermal conductivity and diffusivity of Al-TiC particulate reinforced composites. Vol. 311-313 (pp. 3-8).

Sayuti, M., Suraya, S., Sulaiman, S., Vijayaram, T. R., Arifin, M. K. H., and Baharudin, B. T. H. T. (2011) Thermal investigation of aluminium - 11.8\% silicon (LM6) reinforced SiO2 Particles. Vol. 264-265 (pp. 620-625).

Schey, J. A. (2000). Introduction to Manufacturing Processes (Vol. 3). USA: McGraw Hill. 
Seah, K. H. W., Hemanth, J., and Sharma, S. C. (2003). Mechanical properties of aluminum/quartz particulate composites cast using metallic and non-metallic chills. Materials E Design, 24(2), 87-93.

Sharma, S., Seah, K. H. W., Girish, B. M., Kamath, R., and Satish, B. M. (1997). Mechanical properties and fractography of cast lead-alloy/quartz particulate composites. Materials E Design, 18(3), 149-153.

Singh, M., Mondal, D. P., Jha, A. K., Das, S., and Yegneswaran, A. H. (2001). Preparation and properties of cast aluminium alloy-sillimanite particle composite. Composites Part A: Applied Science and Manufacturing, 32(6), 787-795.

Suery, M., and Esperance, G. L. (1993). Interfacial Reactions and Mechanical Behaviour of Aluminium Matrix Composites Reinforced with Ceramic Particles. Key Engineering Materials, 79-80, 33-46.

Sulaiman, S., Sayuti, M., and Samin, R. (2008). Mechanical properties of the as-cast quartz particulate reinforced LM6 alloy matrix composites. Journal of Materials Processing Technology, 201(1-3), 731-735.

Surappa, M. (2003). Aluminium matrix composites: Challenges and opportunities. Sadhana, 28(1), 319-334.

Taylor, R. (1980). Construction of apparatus for heat pulse thermal diffusivity measurements from 300-3000K Journal of physics E : Scientific Instruments, 13(11).

Therén, K., and Lundin, A. (1990). Advanced composite materials for road vehicles. Materials \& Design, 11(2), 71-75.

Upadhyaya, G. S. (1990). Trends in advanced materials and processes. Materials $\mathcal{E}$ Design, 11(4), 171-179.

Vijayaram, T. R., Sulaiman, S., Hamouda, A. M. S., and Ahmad, M. H. M. (2006). Fabrication of fiber reinforced metal matrix composites by squeeze casting technology. Journal of Materials Processing Technology, 178(1-3), 34-38.

Wang, J.-j., Guo, J.-h., and Chen, L.-q. (2006). TiC/AZ91D composites fabricated by in situ reactive infiltration process and its tensile deformation. Transactions of Nonferrous Metals Society of China, 16(4), 892-896.

Weeton, J. W., Peters, D. M., and Thomas, K. L. (1988) Engineers' Guide to Composite Materials. Metals Park, Ohio 44073, USA: American Society for Metals.

$\mathrm{Yu}$, S., Hing, P., and Hu, X. (2002). Thermal conductivity of polystyrene-aluminum nitride composite. Composites Part A: Applied Science and Manufacturing, 33(2), 289-292.

$\mathrm{Zhu}$, S. J., and Iizuka, T. (2003). Fabrication and mechanical behavior of Al matrix composites reinforced with porous ceramic of in situ grown whisker framework. Materials Science and Engineering A, 354(1-2), 306-314. 


\title{
Characterisation of Aluminium Matrix Syntactic Foams Under Static and Dynamic Loading
}

\author{
M. Altenaiji, G.K. Schleyer and Y.Y. Zhao \\ Additional information is available at the end of the chapter
}

http://dx.doi.org/10.5772/48560

\section{Introduction}

The resistance of engineering structures, subjected to blast and impact loads, is of great interest within the engineering community and government agencies. The interest and importance of such material stem from the need for providing protection against possible terrorist threats. Development of a light -weight, strong and ductile material capable of providing protection of vehicles and occupants against impact and blast, however, is a formidable challenge facing the materials community.

In a blast or an impact, a structure usually undergoes large plastic deformation or break. The important characteristics of structural response include: (i) mode of deformation and failure, (ii) impulse transfer, and (iii) energy absorption in plastic deformation (Hanssen et al., 2002). To characterize cellular materials, the characteristics of the base material of the foam, its relative density, type of its cells (open or closed) and the mean cell diameter, all of these must be known (Ashby et al, 1997). The characterization and testing of metallic foams require special precautions. A cellular material could be characterized by several parameters such as: the constituent raw materials, the mean cell diameter, relative density (porosity), and cell size and shape etc. The constituents can be analysed by using x-ray tomography, optical microscopy and scanning electron microscopy. The easiest way to measure porosity (relative density) is to weigh the sample of a known volume. Additionally, metal foam could be characterized by its cell topology (open cells, closed-cells). Characterizations of metal foam using optical microscopy can be carried out provide that the foam is fully impregnated with opaque epoxy resin before polishing. Scanning electron microscopy has been found to be most informative for open cells foams rather than for the closed-cell foams (Ashby et al., 2000). On the other hand, x-ray tomography technique is a good way to investigate the deformation modes of cellular solids. It depends on the low overall absorption of x-rays as such low absorption allows large specimens to be cut into small pieces. The second 
advantage of this technique is the fact that the deformation could be monitored nondestructively (Fazekas et. al, 2003).

The mechanical properties of cellular materials can be characterized by the compression test. The behaviour of the cellular material under static- compression is known to be completely different from that of the solid material without any compression. This test is also used for the study of the behaviour of material under crushing load. The compression test is useful for measuring plastic flow, ductile fracture limit and compressive fracture properties of brittle materials. In a compression, several modes of deformation can may occur as illustrated in Figure.1. These are as follows: a) buckling mode when the ratio of the sample height $(\mathrm{h})$ to its width $(\mathrm{w})$; i.e. $(\mathrm{h} / \mathrm{w})>5 ; \mathrm{b})$ shearing mode may appear when $(\mathrm{h} / \mathrm{w}) \sim 2.5$; and, c) double barrelling when ratio of length (L) and diameter (D) i.e. (L/D) $>2$ and friction is presented at contact surfaces, d) barrelling mode when $(\mathrm{h} / \mathrm{w})<2$; e) a homogenous compression mode when the ratio $(\mathrm{h} / \mathrm{w})$ lies between 2.0 and 1.5 and finally; $\mathrm{f}$ ) the possibility of compressive instability (Medlin \& Kuhn, 2000).

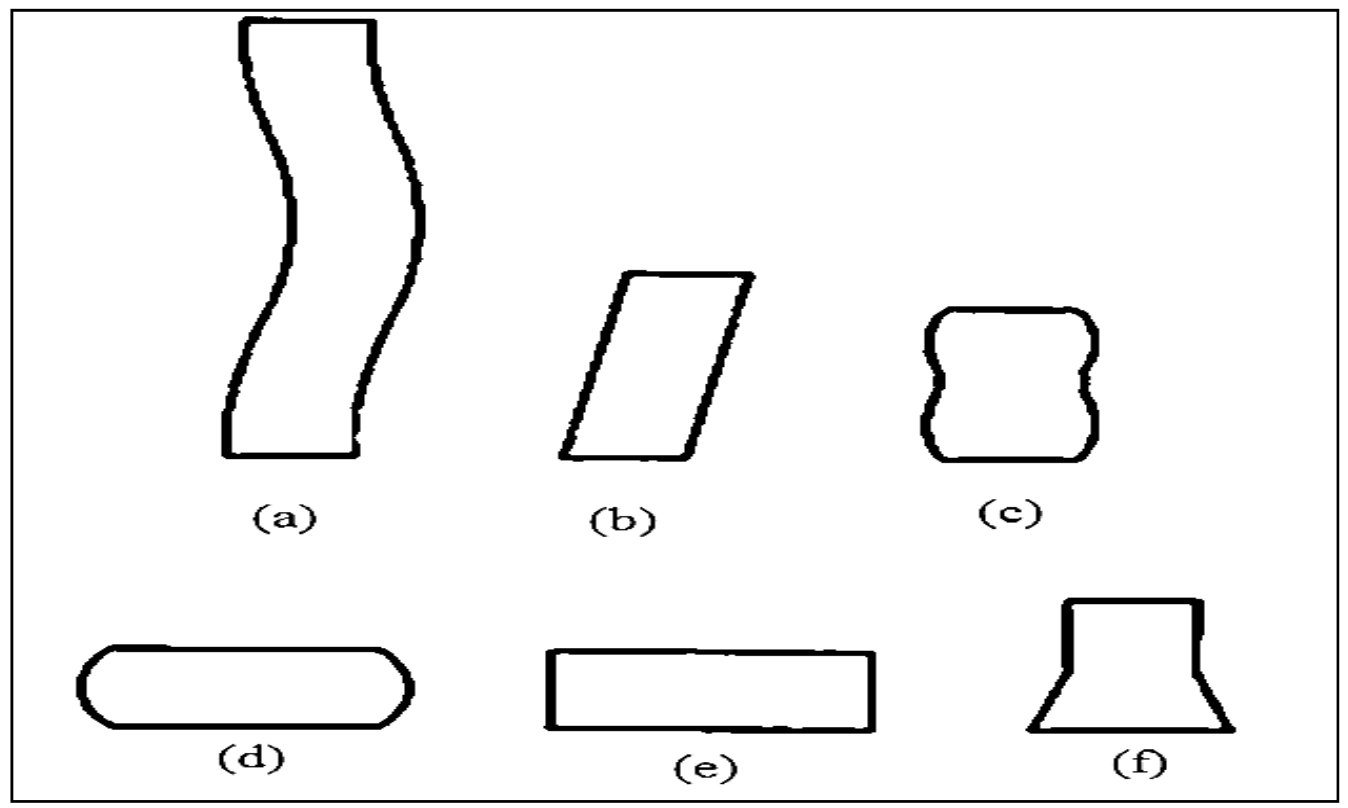

Figure 1. Modes of deformation in a compression test (Medlin \& Kuhn, 2000).

The study of the compressive behaviour of cellular materials offers the benefit of knowledge on most of the mechanical properties of the material. The mechanical properties of cellular materials are different to its base material due to the difference in structures. The following section explains the compressive properties of cellular materials and its relevance to their myriad applications.

Stress-strain curve is used to determine Young's modulus which, in turn, yields values of strength and strain densification. An example of a typical stress-strain curve (Gibson, 2000) of cellular material under uni-axial compression test condition is shown in Figure.2. Stress- 
strain curve of metallic foam shows three regimes of material behaviour. As can be seen in the graph, initially it is a linear dependence of elasticity (strain) with stress. Such dependency is governed by the strength of cell walls of the base material. As is expected, the stiffness of the metallic foam will be higher with the increase of strength of the cell wall. It has been observed that the type and grade of metallic alloy as the base material dictate the stiffness of the foam structure. It has been found that the metallic alloy which has higher yield strength, gives higher stiffness than other alloys. At this stage, the material undergoes a non-permanent deformation. Usually, the deformation is in the form of cell face stretching and consequently extending and stretching the cell edges. This, in turn, increases the stiffness which was driven by the cells edges in closed-cell foam configuration while extending and bending deformation mode in open cell foam configuration.

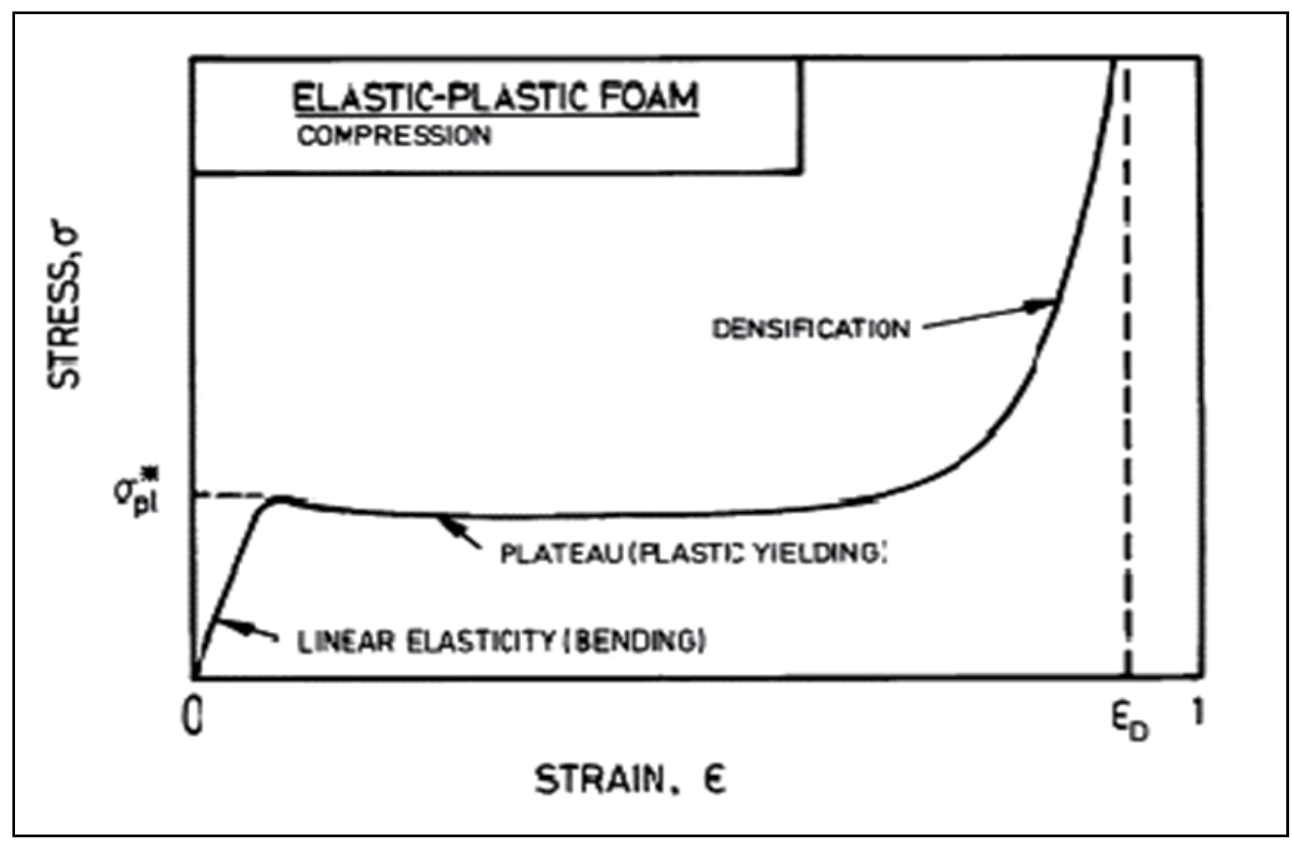

Figure 2. A typical stress-strain curve for foam showing linear elastic, stress plateau and densification regimes (Gibson, 2000).

Energy absorption is the ability of the material to convert the kinetic energy into energy of some other form such as, heat, viscosity, visco-elasticity, friction etc. The kinetic energy must be less than the maximum limit of energy absorption of the material to keep the object safe. Also, the energy absorption property must be multi-directional, i.e. allowing absorption of the impact from any direction. Cellular materials have better capacity of energy absorption than its base materials. Foams have a capability to absorb the kinetic energy by bending, buckling or fracture (plastically) of the cell walls depending on the characteristics of the base material of the foam (Ashby, 1997).

Energy absorption capability of foams depends on the stress and strain of the plateau in stress-strain curve. Foams have long, flat stress-strain curve where the cell walls collapses 
plastically to nominal strain $\varepsilon_{D}$ at a constant stress and the phenomenon is called, 'the plateau stress up'. The plateau stress must be below the value that causes damage to the object. The best energy absorbing material is that which has the longest plateau and absorbs the most energy before reaching the densification strain. The material that has a long and flat stress-strain curve is considered to be an ideal energy absorber. The energy absorber capability of the material is measured by the length and height of the flat stress-strain curve. The area under the plateau of the stress-strain curve represents energy per unit volume which can be absorbed as illustrated in Figure 3. The area under the flat part of the curves is the useful energy per unit volume, $\mathrm{W}_{\mathrm{v}}$ that can be absorbed.

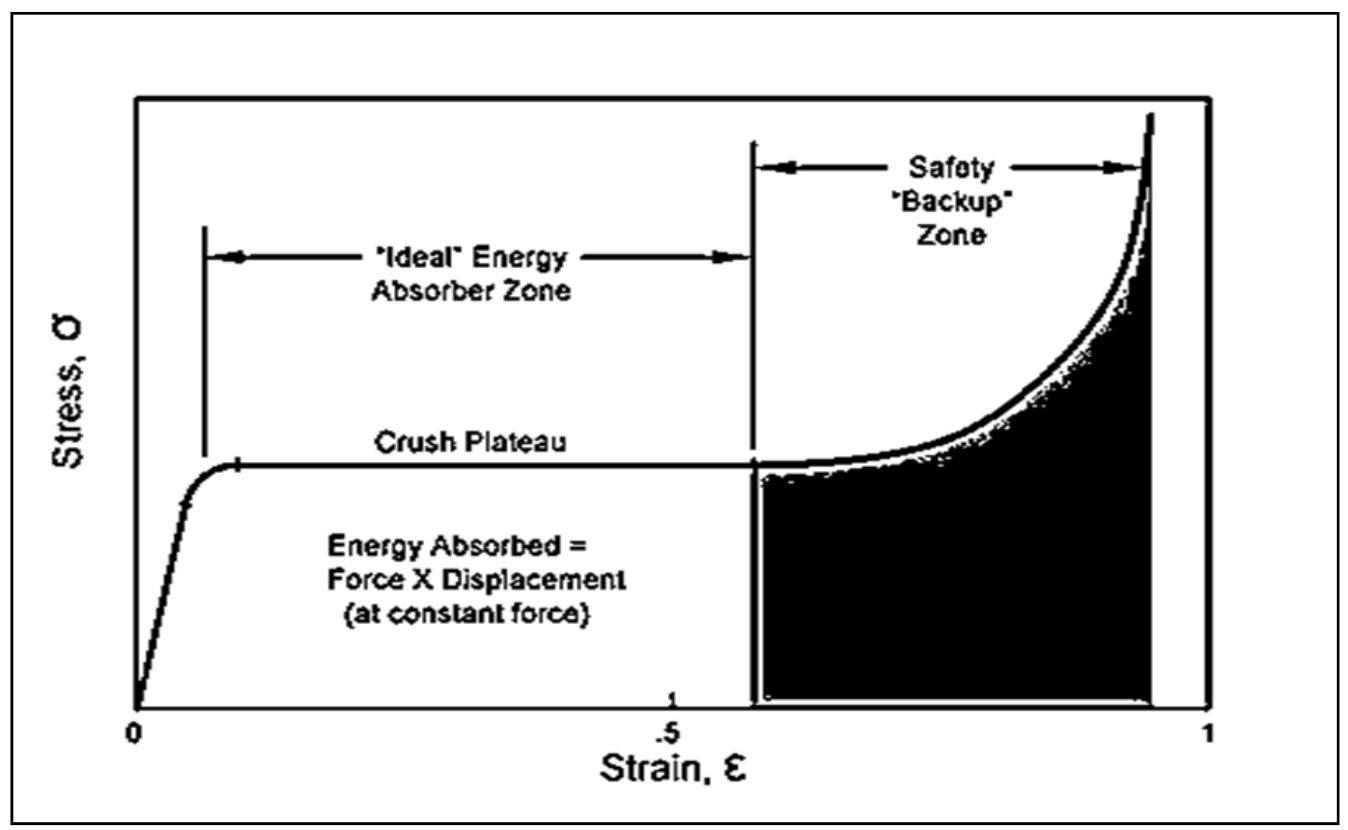

Figure 3. A typical stress-strain curve for an energy absorber.

\section{Metal matrix syntactic foam}

Matrix syntactic foams are composite materials consisting of a matrix implanted with hollow or porous ceramic particles. These foams are a new class of materials which are manufactured by a variety of metal or polymer matrices and micro-spheres ceramics. It consists of a metal matrix and micro-spheres ceramics, embedded in the matrix. A metal matrix could be of aluminium, steel, titanium or magnesium. Ceramic micro-sphere could be a porous or hollow structure, but hollow metal spheres are rarely used.

The size of the hollow ceramics micro-sphere determines the porosity of the matrix syntactic foam. In addition, it has an effect on the strength of the syntactic foam. Therefore, the matrix syntactic foam has different physical and mechanical properties than other cellular materials. Matrix syntactic foam is considered as a light material having high energy absorption capacity. It has been used in automotive, naval, aerospace and other industries 
where 'light-weight' property is an essential requirement. In addition to that it could be used for reducing shock loadings associated with mine blast on military vehicles where the high energy absorption capability and light weight property are needed.

\subsection{Fabrication process}

Metal matrix syntactic foams consist of a combination of ceramic micro-sphere and metal matrix. Most metal matrices should be light metals like aluminium, magnesium, titanium etc. Two types of micro-sphere in common use have either porous or hollow structures. Four types of cellular spheres have so far been used to fabricate metal matrix syntactic foam as follows: (i). amorphous silica, (ii). Al2O3 spheres, (iii). CMs of crystalline mullite and (vi). steel spheres (Tao, 2010).

There are two main ways to fabricate metal matrix syntactic foams as follows: i) stir casting with spray processes, and ii) infiltration casting using liquid state. In stir casting, metal matrix syntactic foams are fabricated by mixing a liquid of metal matrix with the ceramic particles followed by casting. This method is very simple and cheap, but inhomogeneous structure of syntactic foam has been found to appear due to float of ceramic spheres to the top of the melt. In infiltration casting, the metal matrix is placed above the ceramic spheres and is pressed to infiltrate into the ceramic sphere where it is solidified to produce metal matrix syntactic foam. The infiltration casting can be conducted by gas pressure or die casting. This method has the advantage that the matrix and ceramic spheres are well bonded and the micro-spheres are usually uniformly distributed.

\subsection{Porosity}

There are two classifications for porosity: open or closed types. The open porosity is defined by the ratio of the volume of void space that is accessible from exterior to bulk volume. Meanwhile, the closed porosity is defined by the ratio of the volume of void space that is not accessible from exterior to bulk volume. The porosity of foams depends on the shape and size of the pores. The porosity of metallic syntactic foams, however, is determined by the porosity of the cellular spheres. The strength of the micro-sphere of syntactic foam could be tailored through the appropriate selection of the wall thickness and the radius of microspheres (Kiser, 1999). The porosity of cellular materials controls the plateau strength and energy absorption capacity of the foams. In general, the strength of porous materials increases with the reduction of porosity.

The main parameter that has the most significant effect on the porosity of the metal matrix syntactic foam is the porosity of the ceramics micro-spheres. The radius, shell thickness and the volume fraction of the hollow spheres are the parameters that control the porosity of hollow spheres. The porosity of a ceramic sphere could be estimated by the following equation (Kiser et. al, 1999):

$$
\theta_{\mathrm{s}}=\left(1-\frac{\rho_{\mathrm{s}}}{\rho_{\mathrm{o}}}\right)
$$


Where, $\theta \mathrm{s}$ is the porosity of ceramic material, @s is its effective density and $\mathrm{Q}_{0}$ is the density of the solid part of the spheres. In principle the porosity of metal matrix syntactic foam may be calculated using the formula below (Zhang\& Zhao, 2007):

$$
\theta_{\mathrm{f}}=\left(1-\mathrm{f}_{\mathrm{al}}\right) \theta_{\mathrm{s}}
$$

Where, $\theta_{\mathrm{f}}$ is the porosity of syntactic foam and $f_{\mathrm{al}}$ is the volume fraction of metal matrix. The authors have developed a general formula that has been widely used to calculate the porosity of metallic syntactic foam for all types of spheres and is given below:

$$
\theta_{\mathrm{s}}=\frac{\rho_{\mathrm{m}}-\rho_{\mathrm{f}}}{\rho_{\mathrm{m}} \rho_{\mathrm{s}}}\left(1-\frac{\rho_{\mathrm{s}}}{\rho_{\mathrm{o}}}\right)
$$

Where, @m, @f are @o are the density of metal matrix, syntactic foam and solid part of cellular spheres respectively, and os is the effective density of the cellular spheres (Tao, 2010). It was reported earlier (Kiser et. al, 1999) that the thickness and the radius of the shell of the hollow sphere and the micro-balloon do control the composite porosity @o and developed a formula to calculate the porosity of syntactic foams with hollow spheres as follows:

$$
\rho_{\mathrm{o}}=\mathrm{f}\left(1-\frac{\mathrm{t}}{\mathrm{R}}\right)^{3}
$$

Where, $\mathrm{Q} 0$ is the porosity, $f$ is the volume percentage of the hollow spheres, and $t$ is the shell thickness and, $\mathrm{R}$ is the radius of the hollow spheres. It was also reported [Kiser et.al, 1999] that the strength of the micro-balloon could be tailored through appropriate selection of the parameter, $[t / R]$, having the strength increasing with increasing factor, $[t / R]$.

\subsection{Compressive strength of metallic matrix syntactic foam}

The compressive strength of metallic matrix syntactic foam is controlled by the strength of metal matrix and the ceramic particles. The parameters such as: volume fraction, structure and distribution of the ceramics particles have considerable effect on the properties of syntactic foams (Zhao\& Tao, 2009). It has been reported (Rohatgi et. al, 2006) that the compressive strength of the metallic syntactic foams decreases with the increase in volume fraction of the micro-spheres ceramics. In fact, the compressive strength of metal matrix has been found to be higher than that of the micro-spheres ceramics. Consequently the strength of the metallic matrix syntactic foam decreases with the increase in the volume fraction of ceramics particles.

It has been reported that the yield strength of metallic foam decreases with the decrease of the size of micro-spheres ceramic (Rohatgi et. al, 2006). Moreover, it has been noted (Altenaiji et.al, 2011) that the smaller the size of the micro-sphere ceramic, the weaker is the strength of metal matrix syntactic foam. There are also other parameters, such as the strength of the metal matrix, which effect the strength of metal matrix syntactic foam as illustrated in Figure 4 and Figure 5.

In contrast, Palmer reported (Palmer et. al, 2007) that, the lower compressive strength of metallic syntactic foams is associated with larger micro-spheres. It was however predicted 
(Zhao\& Tao, 2009) that there should be a difference in the compressive strength of different void contents in different sized ceramic spheres. It was also predicted (Sun et.al, 2007) that the thickness and the radius of micro-spheres ceramics have an effect on the compressive strength of metallic syntactic foams. The micro-sphere ceramics which have higher wall thickness to radius ratios $(t / r)$ were found to have higher compressive strength of the resultant. Earlier reports (Kiser et. al, 1999) indicated that the compressive strength of metal matrix syntactic foam increased from 70 to $230 \mathrm{MPa}$ with the increase of the factor, $(\mathrm{t} / \mathrm{r})$ from 0.12 to 0.48 , i.e. an increase by a factor of 3 for a similar factor of increase in the pressure.

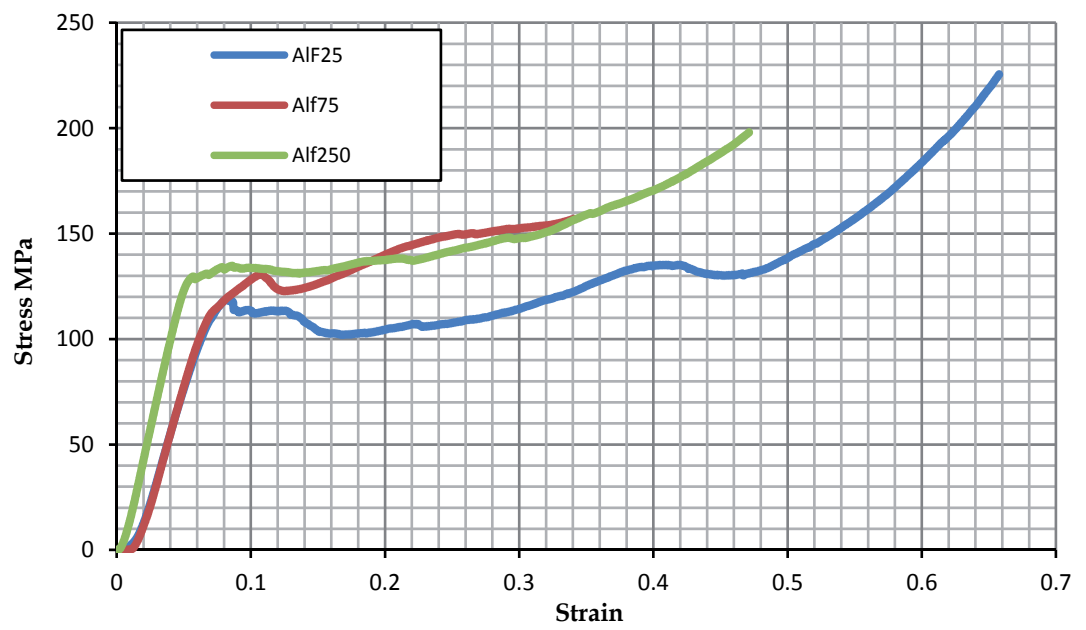

Figure 4. Comparison of stress-strain curve of aluminium syntactic foam with different ceramic microsphere size.

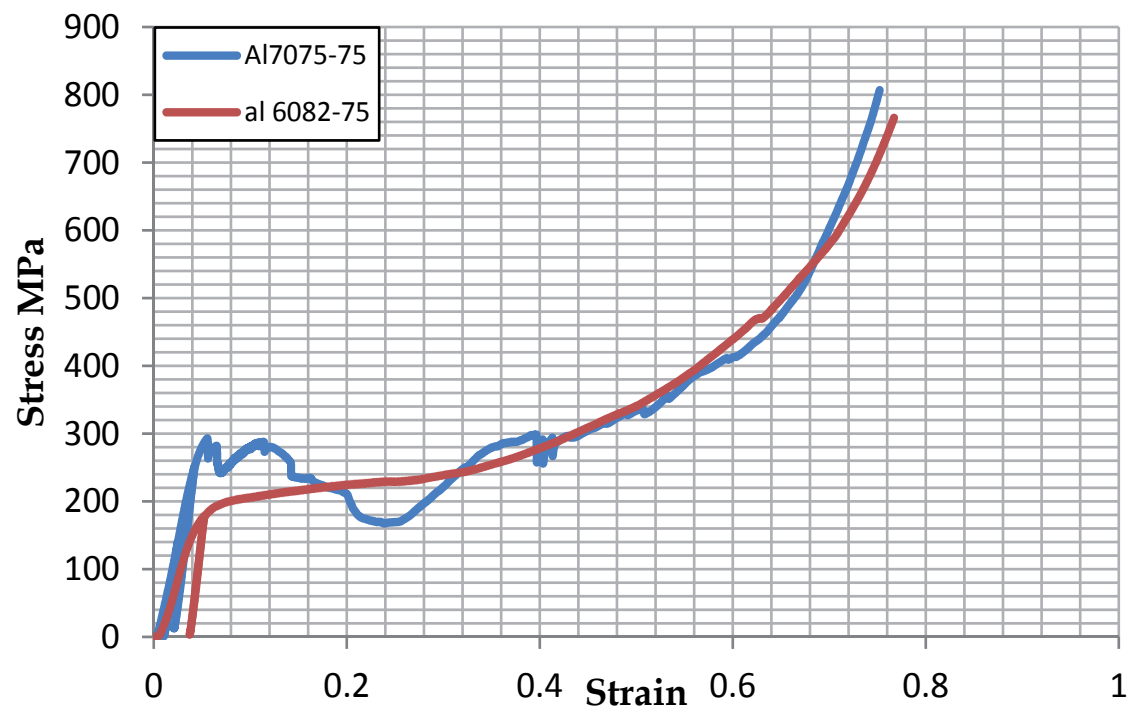

Figure 5. Comparison of stress-strain curve of aluminium syntactic foam with different aluminium matrix. 
The compressive strength of metallic matrix foams is also affected by the types of microspheres ceramics. It has been found that metallic matrix foam containing hollow ceramics spheres have much higher compressive strength than those containing porous ceramics spheres (Tao et.al, 2009). The compressive behaviour of metal matrix syntactic foams with hollow steel spheres of same thickness but different composition was studied (Vendra \& Rabiei, 2007). It was observed that the metal matrix, containing low carbon steel spheres had much lower compressive strength than the ones which had stainless steel spheres.

It has been reported that the compressive strength of the metal matrix syntactic foams is also affected by the type of metal matrix. Studies on the compressive behaviour of syntactic foams with same ceramics micro-sphere but different aluminium matrix had been conducted. It was found that the compressive strength of al7075-t is double that of al 6082-T with same method of processing. It was also indicated that when the aluminium matrix was replaced by another metal matrix the compressive strength of metallic matrix syntactic foams changed. When the al 7075 was used the compressive strength of metallic matrix syntactic foams had increased to approximately double than that containing pure aluminium (Balch\& Dunand, 2006).

\subsection{Failure of metallic matrix syntactic foam under compressive loading}

As is illustrated in Figure.2, metallic matrix syntactic foam behaved like any foam under compression loading. It has three regimes of behaviour under compression loading. Initially it starts with a linear elasticity and then follows by plateau strength and finally, ends by the densification strain. But, the failure behaviour of metal matrix syntactic foams is different due to it compositions. Its failure is controlled by different plastic characteristics. It can be either ductile, susceptible to collapse under crushing of micro-spheres ceramics or brittle, susceptible to shear failure (Balch et.al, 2005).

It was reported (Zhao\& Tao, 2009) that the three factors that affect the failure behaviour of metal matrix foam are: i) ductility of metal matrix, ii) structure of micro-spheres ceramic and, iii) volume fraction of micro-spheres ceramic and metal matrix. It is also reported that the behaviour of 'metal matrix syntactic' foams' performance failure depends on the type of the metal matrix. It was found that the aluminium alloy-T6 had two localized deformation bands of crushed materials while, the $\mathrm{CP}-\mathrm{Al}$ (commercial purity aluminium) exhibited transition into densification plateau, showing extensive barrelling and pore deformation over a large volume of the material. Additionally, the damages of these two foams were reported to be different. In the alloy-T6, damage was concentrated in two much thicker crush band where the strain was very high. In contrast, in the CP-Al foams' damage was spread over a big volume of the material, resulting in plastic deformation of the matrix along with sphere fracture (Balch et.al, 2005).

It has been reported (Tao \& Zhao, 2009) that the compressive failure of metallic matrix syntactic foam is affected by the volume fraction of metal matrix micro-spheres ceramic. High volume of metal matrix causes ductile failure in the form of collapse of the material. In contrast, low volume of the metal matrix tends to cause brittle failure in the form of shear. 
As mentioned previously, the thickness and the radius of micro-spheres ceramics have an effect on the compressive strength of metallic syntactic foams. The micro-sphere ceramics with higher wall thickness-to-radius ratios, $(t / r)$ were found to have higher compressive strength for the resultant (Sun et.al, 2007). The syntactic foam metal matrix with low values of the ratio, $(\mathrm{t} / \mathrm{r})$ failed as brittle failure form while those with higher values failed as ductile failure form also known as crushing or collapse failure form (Kiser et.al,1999) . In contrast, another report (Sun et.al, 2007) stipulated that the metal matrix syntactic foam with low values of the ratio, $(\mathrm{t} / \mathrm{r})$, failed as ductile in the form of crushing or collapse failure while that had higher values failed as brittle in the form of shear failure. It was, however, reported earlier (Gupta et.al, 2004) that different types of syntactic foams in which the matrix materials and the radius of micro-spheres were fixed while the wall thickness of the micro-spheres was varied. It had been found that failure type of different matrix syntactic foams was similar. In general, therefore, it may be concluded from the present up-to-date literature review that the value of the wall thickness to radius ratio $(t / r)$ dictates the form of the compressive failure of metal matrix syntactic foams while the value of the ratio is dependent upon the factor that affect the compressive failure. Furthermore, the strength of the micro-spheres ceramic has an effect on the strength of metal matrix syntactic foams.

\section{Dynamic compressive testing of matrix syntactic foam}

Metal matrix syntactic foams have been compressive tested under dynamic loading. Such loadings can be carried out through, either low or high speed impact. It has been studied under drop weight and split pressure Hopkinson bar test methods. It has been observed (Dou et.al, 2007) that the dynamic stress-strain curve has three regimes as the quasi-static stress strain curve. Meanwhile, the yield strength of metal matrix syntactic foams under dynamic loading is $\sim 45-60 \%$ higher than that of quasi static compression. Moreover, Zhang investigated (Zhang\& Zhao,2007) four samples of aluminium matrix syntactic foams under impact and reported that many oscillations appeared at the beginning of stress strain curve where the strain is low due to the high vertical vibration of the drop hammer. In addition, it was found that, out of four samples tested two had 10\%-30\% higher plateau stress than that of a quasi-static compression but the other two had lower plateau stress than that of the quasi-static compression. Therefore, the plateau strength is mostly determined by the volume fraction of metal matrix in the metallic syntactic foam. It was evaluated from the behaviour of aluminium matrix syntactic foams under high impact loading (Balch et al., 2005). Split pressure Hopkinson bar technique was used to characterize the material under dynamic loading. It was concluded that, the behaviour of aluminium matrix syntactic foams at high strain had higher peak strength and plateau stress than those measured during quasi-static testing. In addition, the peak strength of the stress strain curve has been found to have shifted slightly at higher strain value.

\subsection{Low velocity dynamic loading}

Low velocity dynamic loading has been conducted by using many facilities that include the Charpy and Izod pendulums, the drop weight fixture such as the Gardner as well as 
hydraulic machines, designed to conduct both in plane and out of plane testing at velocities up to $10 \mathrm{~m} \mathrm{~s}^{-1}$ (Cantwell\& Morton, 1991) The Charpy pendulum is simple to use and its design scheme is presented in Figure. 6. Usually, thick beam specimen is used which has a notch in the middle. The notch must be fixed opposite to the impact of the swing pendulum. The energy dissipated during impact is calculated by multiplication of the weight of the pendulum and the difference of the pendulum height in each side. The disadvantage of this test is that the high frequency oscillation on 'load versus time' curves due to natural frequency of the hammer does introduce error.

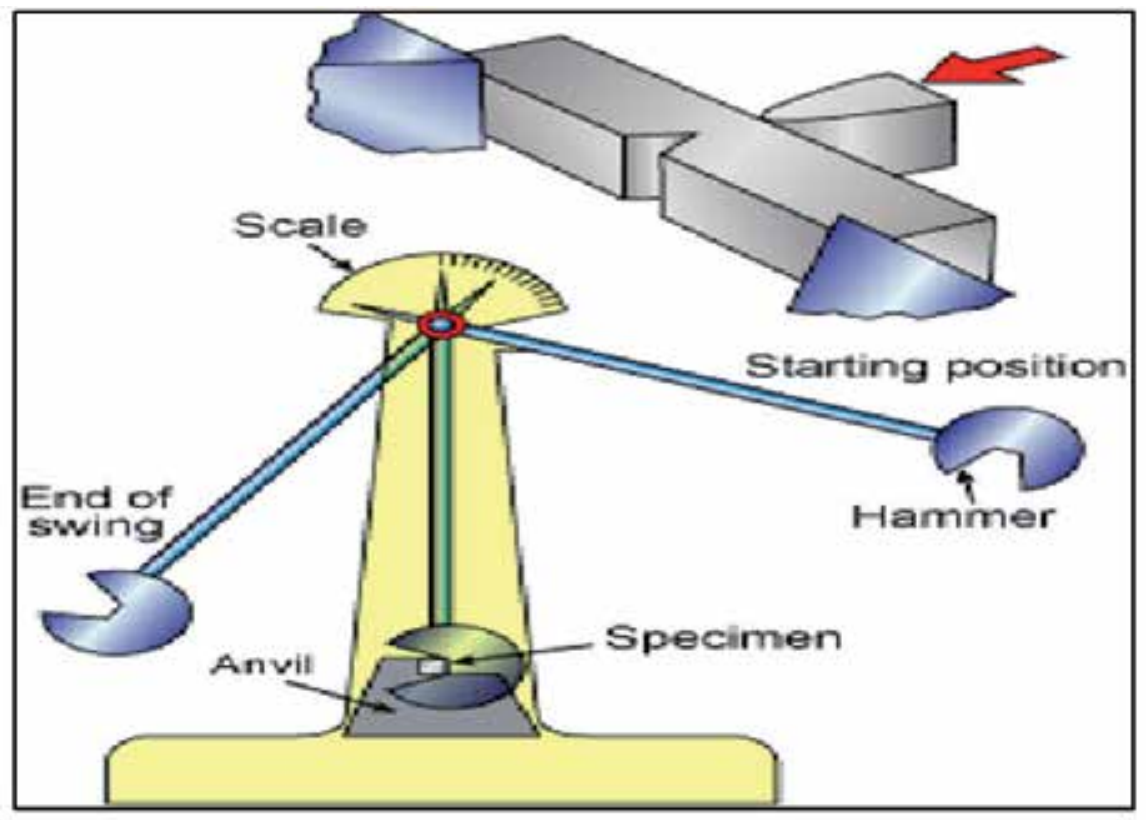

Figure 6. Schematic of the Charpy pendulum-type tester.

Comparatively, the Izod test is similar to the Charpy pendulum test except that the notch is located at the end of the specimen and the hammer impacts at the free end (Ellis, 1996). Cantwell and Morton suggested that Chapy and Izod tests both are suitable for evaluating the impact performance of the materials and a step in determining the dynamic toughness of the materials. In addition, drop-weight impact test have been used to conduct low velocity dynamics compressive testing of composites at strain rates ranging from 10 to several hundred per seconds (Hsiao et.al, 1998a, 1998b).

The heavy weight is guided by two smooth steel rails and fall from a height to strike the specimen (Fanjing, 2010). The impact of falling weight does not cause destruction of the test specimen but rebounds where the residual energy could be determined (Cantwell\& Morton, 1991) as shown in Figure.7.

A piezoelectric load cell is used to measure the variation of load with time during impact. It is located underneath the base on the test rig (Yiou, 2009). While, the incident velocity of the impactor can be determined from the equation of motion as follows: 


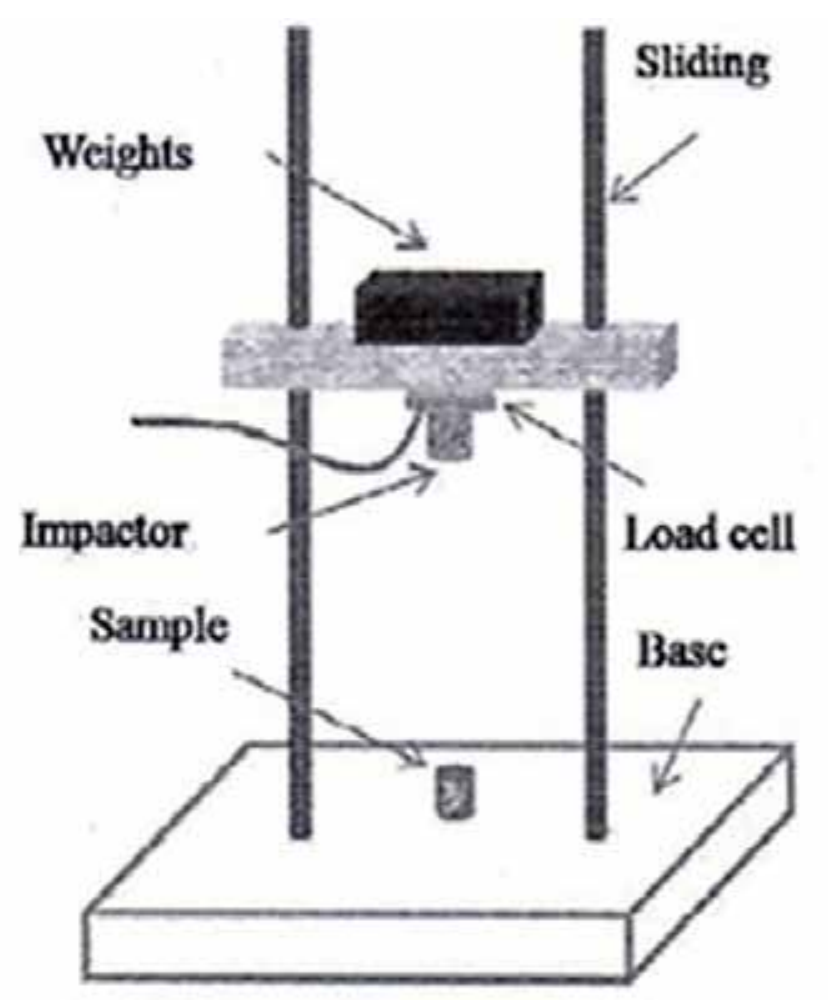

Figure 7. Schematic of the drop- hammer weight rig.

$$
\begin{gathered}
\mathrm{v}=\sqrt{ }(2 \mathrm{~g} \mathrm{~h}) \\
\frac{\mathrm{a}}{\mathrm{g}}=\frac{v \omega}{\mathrm{gD}}
\end{gathered}
$$

where $\mathrm{V}, \mathrm{w}$ and $\mathrm{D}$ are the weight velocity, natural frequency and dynamic load factor (usually equal to 1.77) respectively (Rajendran et.al, 2008).

And predicted deflection is given as:

$$
\delta=\frac{\mathrm{mgh}}{\sigma_{\rho 1} \mathrm{~A}}
$$

where, $\mathrm{m}, \mathrm{h}, \mathrm{g}, \sigma_{\mathrm{ol}}$ and $\mathrm{A}$ are the weight mass, height of drop weight, gravitational force ,plateau stress and cross section area of sample respectively. The advantage of the dropweight impact test with respect to Chapy and Izod test is that a wider range of sample geometries can be tested by changing the support size and shape. However, the stress wave reflection effect on the stress-time response of the specimen is observed in this method. Also, the limitation of strain rate which depends on the speed of falling weight was found to be directly linked to the drop height (Barre et.al, 1996). Furthermore, hydraulic test machines have been used to characterise the deformation and failure of the materials at high strain rate (Beguefin \& Barbezat, 1989). One advantage of this type of testing machine is that it practically removes the problem of vibration noise that appears when using the drop 
weight test. Additionally, the limitation of the strain rate is extended up to a value of $50 \mathrm{~s}^{-1}$ from the static strain rate value. However, caution should be exercised to ensure that mass of the load cell is as low as possible to avoid the concealment of the true material response (Beguefin \& Barbezat, 1989).

\subsection{Medium and high velocity dynamics loading}

Medium and high velocity dynamics loading has been conducted by using many facilities that include Hopkinson bar and Gas gun. In the Hopkinson bar technique, the induced wave propagation in a long elastic metallic bar has been used to measure the pressure produced during dynamic events (Kolsky, 1949). The Hopkinson bar test was used to measure the dynamic stress-strain response of materials. A high precision strain gages, signal conditioners, and high speed digital oscilloscopes are used in Hopkinson bar with high sensitivity and accuracy. The sensitivity of the pressure bar is determined by the properties of the material of the bar such as the density and the elastic wave impedance (Ramesh, 2008). In addition, the type of stain gages and the characteristics of the associated instrumentations have an effect on the sensitivity.

To determine the properties of the material under dynamics loading the procedures for the Hopkinson bar technique is similar to that of the dynamic technique mentioned earlier. These are used to provide complete stress strain data as a function of strain rate. This technique has been used to determine the dynamic properties of numerous engineering materials like ferrous and non-ferrous alloys, polymers, ceramics, and concrete (Tan et.al, 2005). Additionally, it has been used to characterise soft materials and metal foams that have low mechanical impedance, required for increasing the sensitivity of testing device (Dung et.al, 2011; Gay III et.al, 2000; Chen et.al, 1997). Split Hopkinson pressure bar has been used to measure the compressive mechanical behaviour of materials, to load samples with uniaxial tension (Klepaczco et.al, 1997) and to measure compression torsion (Gilchrist, 2009). Also, it has been designed to measure the fracture toughness of an impact loaded material (Krauthauser, 2003).

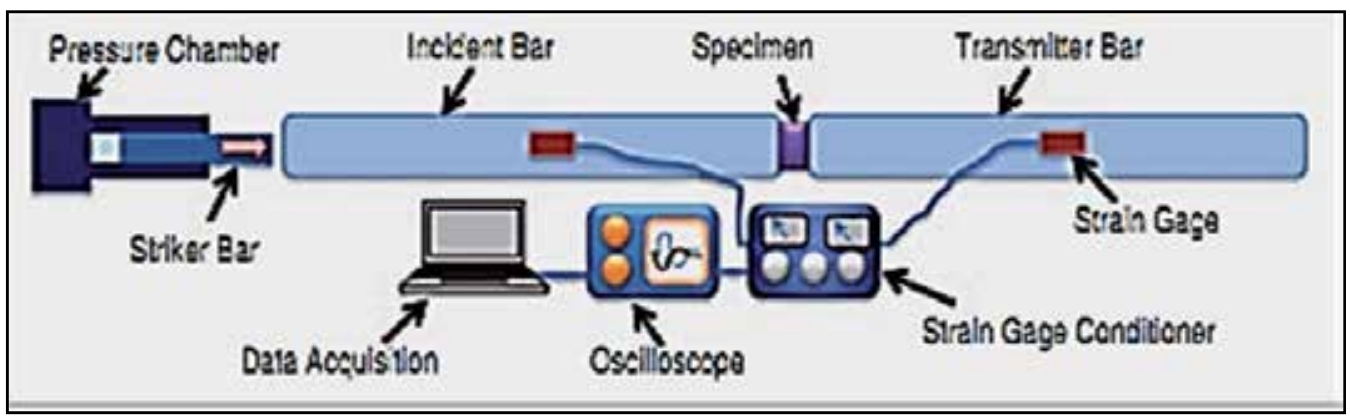

Figure 8. Schematic SHPB (Split Hopkinson pressure bar) setup for high strain rate compression testing

Before providing impact in an SHPB device the velocity of the striker was measured using an electronic velocity measurement unit. The gas gun pressure was varying that cause the 
variant impact velocities. The incident and transmitter bars in the device are made of steel having a length of $1000 \mathrm{~mm}$ and a diameter of $20 \mathrm{~mm}$. The bar's mechanical properties are: Young's modulus, $207 \mathrm{GPa}$, elastic wave speed, $5890 \mathrm{~m} / \mathrm{s}$ and Poisson's ratio, 0.29.

In the Hopkinson bar technique, the induced wave propagation in a long elastic metallic bar has been used to measure the pressure produced during dynamic events (Dung et.al, 2011). The Hopkinson device is used to measure the dynamic stress-strain response of materials. A high precision strain gage, signal conditioners, and high speed digital oscilloscopes are used in Hopkinson bar which provides high sensitivity and accuracy. The sensitivity of the pressure bar is governed by the properties of the material of the bar such as, the density and the elastic wave impedance (Gray III, 2000). In addition, the type of stain gages and the characteristics of the associated instrumentations have an effect on the sensitivity.

The stress at the output bar is found by converting strain gages data (volts) to the stress by using following relations (Harrigan, 2005)

$$
\begin{gathered}
\sigma(t)=\frac{E_{b} \cdot 2 \epsilon v(t)}{G_{g} \cdot K_{g} \cdot v_{i}\left(1+v_{B}\right)} \\
\sigma(t)=\frac{A \cdot E \cdot \varepsilon_{t}(t)}{A o}
\end{gathered}
$$

Where, $\sigma(t)$ is the stress of transmitter bar as function of time, $\varepsilon v(t)$ is the strain gage voltage as function of time, $\mathrm{Gg}$ is the amplification factor, $\mathrm{Kg}$ is stain gage factor, $\mathrm{V}_{\mathrm{I}}$ is bridge input voltage, $\mathrm{Eb}$ is the elastic modulus of the bar, $\mathrm{A}$ is cross sectional area of the bar, $\mathrm{A}_{\circ}$ is the cross sectional area of the sample, $\sigma(t)$ is the transmitted axial strain pulses and $\nu_{в}$ is Poisson's ratio of the bar material. In addition, the time dependent strain rate and strain are calculated using the following equations:

$$
\begin{array}{r}
\varepsilon^{\prime}(t)=\frac{2 C_{b \varepsilon_{\mathrm{r}}}(t)}{\text { Io }} \\
\varepsilon(t)=\int_{0}^{t} \varepsilon^{\cdot}(t) d t
\end{array}
$$

Where $C_{b}, I_{0}$ and $A_{o}$ are the sound wave velocity, length and cross sectional area of the specimen respectively, and $\varepsilon(t)$ is the reflected axial strain pulses.

The Hopkinson bar technique is used to provide completer stress strain data as a function of strain rate. This is also used to determine the dynamic properties of numerous engineering materials; like ferrous and non-ferrous alloys, polymers, ceramics, and concrete (Forrestal et.al, 2002).

Additionally, it has been widely used to characterise soft materials, metal foams that have low mechanical impedance which is required increasing the sensitivity of testing device (Klepaczco, 1997; Kiernan et.al, 2009; Lopatnikov et.al, 2003; El-Nasri, 2005; Harding, 1960).

Split Hopkinson pressure bar has been used to measure the compressive mechanical behaviour of a material, loading samples in uni-axial tension, simultaneous compression torsion (Lewis \& Goldsmith, 1973).Also, it has been designed to measure the fracture 
toughness of an impact loaded material (Taylor, 1948). Metal matrix syntactic foams are characterised by using SHPB and the experimental setup and results are shown in Table .1 and Figure.9.

\begin{tabular}{|c|c|c|c|c|c|}
\hline & Sample ID & Weight (g) & Length (mm) & $\begin{array}{l}\text { Avg. Width } \\
\text { (mm) }\end{array}$ & $\begin{array}{c}\text { Velocity } \\
\text { applied }(\mathrm{m} / \mathrm{s})\end{array}$ \\
\hline Pre & \multirow[b]{2}{*}{ SHPB \#2 } & 12.6 & 16.84 & $21.40 \pm 0.1$ & \multirow[b]{2}{*}{21.6} \\
\hline Post & & 12.6 & 14.26 & $24.00 \pm 0.2$ & \\
\hline Pre & \multirow[b]{2}{*}{ SHPB \#3 } & 12.6 & 16.84 & $21.40 \pm 0.1$ & \multirow[b]{2}{*}{$35 \pm 1$} \\
\hline Post & & 3.6 & 3.69 & $29.00 \pm 1$ & \\
\hline Pre & \multirow[b]{2}{*}{ SHPB \#4 } & 11.5 & 17.04 & $20.60 \pm 0.5$ & \multirow[b]{2}{*}{$22.35 \pm 1$} \\
\hline Post & & 7 & 7.74 & $22.15 \pm 1$ & \\
\hline Pre & \multirow[b]{2}{*}{ SHPB \#6 } & 12 & 15.2 & $20.60 \pm 0.5$ & \multirow[b]{2}{*}{35} \\
\hline Post & & 2.1 & 2.63 & $27.50 \pm 1$ & \\
\hline
\end{tabular}

Table 1. Summary of experimental results of aluminium matrix syntactic foams under dynamic loading in SHPB.

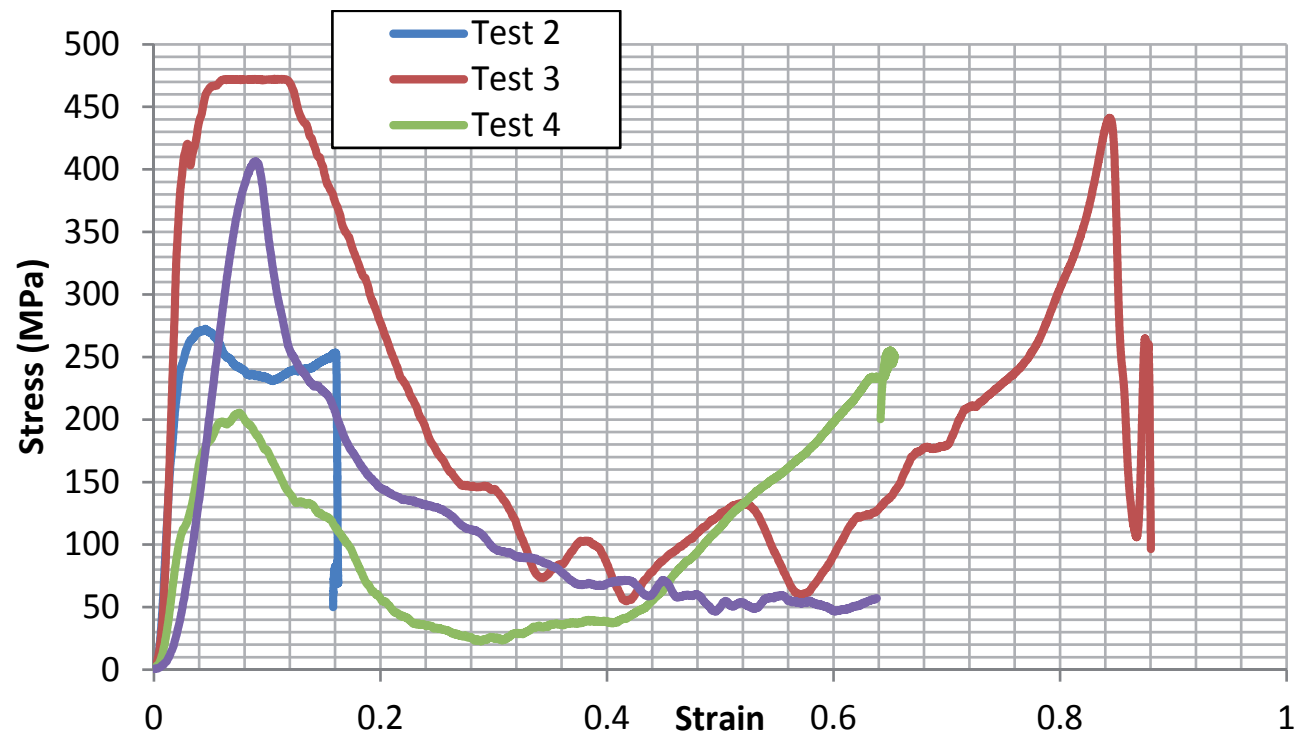

Figure 9. Stress-strain curve of aluminium matrix syntactic foam under dynamic loading of SHPB.

Dynamic compression testing showed a 10-30\% increase in peak strength compared to the quasi-static results. The strain rate sensitivities of these foams are similar to those of aluminium matrix composite materials as shown in Figure.10. These foams displayed excellent energy-absorbing capability. This suggests that these have potential for use in high loading applications or for impact protection in situations where a high plateau stress is desired (Balch et.al, 2005). 


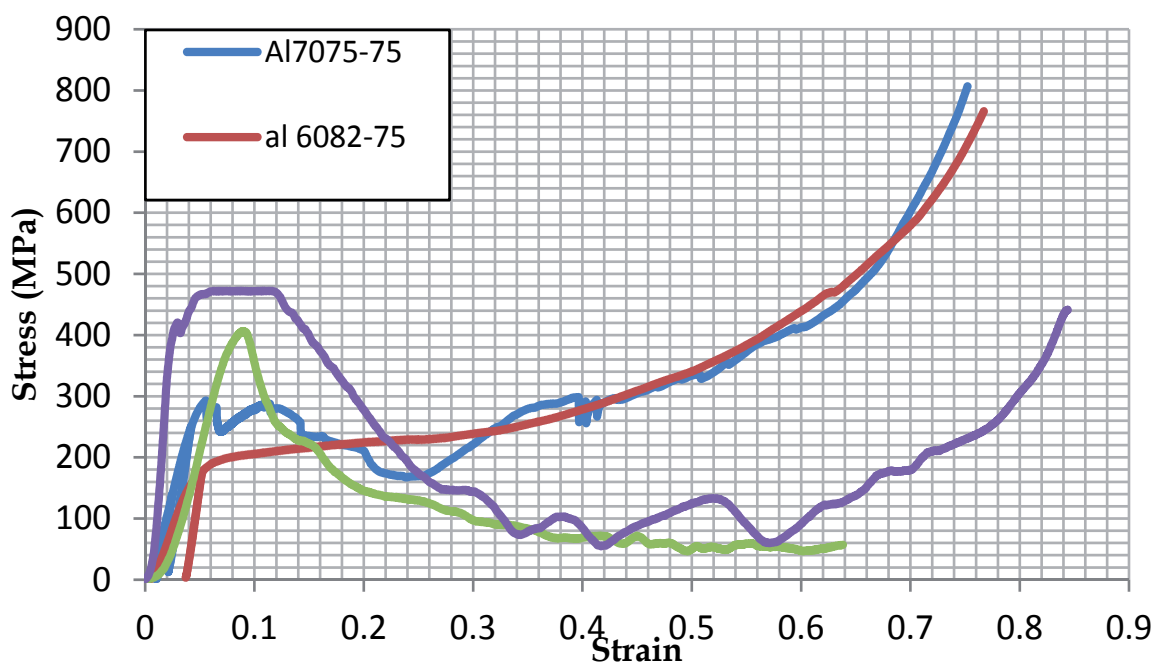

Figure 10. Stress-strain curve of aluminium matrix syntactic foam under static and dynamic loading.

High impact dynamic loading can be achieved by using high pressure gas gun (Cantwell\& Morton, 1991). Gas gun apparatus contains a breech, pump tube, transition section, launch tube and target tank as illustrated in Figure 11.

In addition to high speed camera this device can also record and analyse the moment of impact and post impact behaviour (Hazell et.al, 2010) and can monitor the behaviour of the projectile material during the impact.

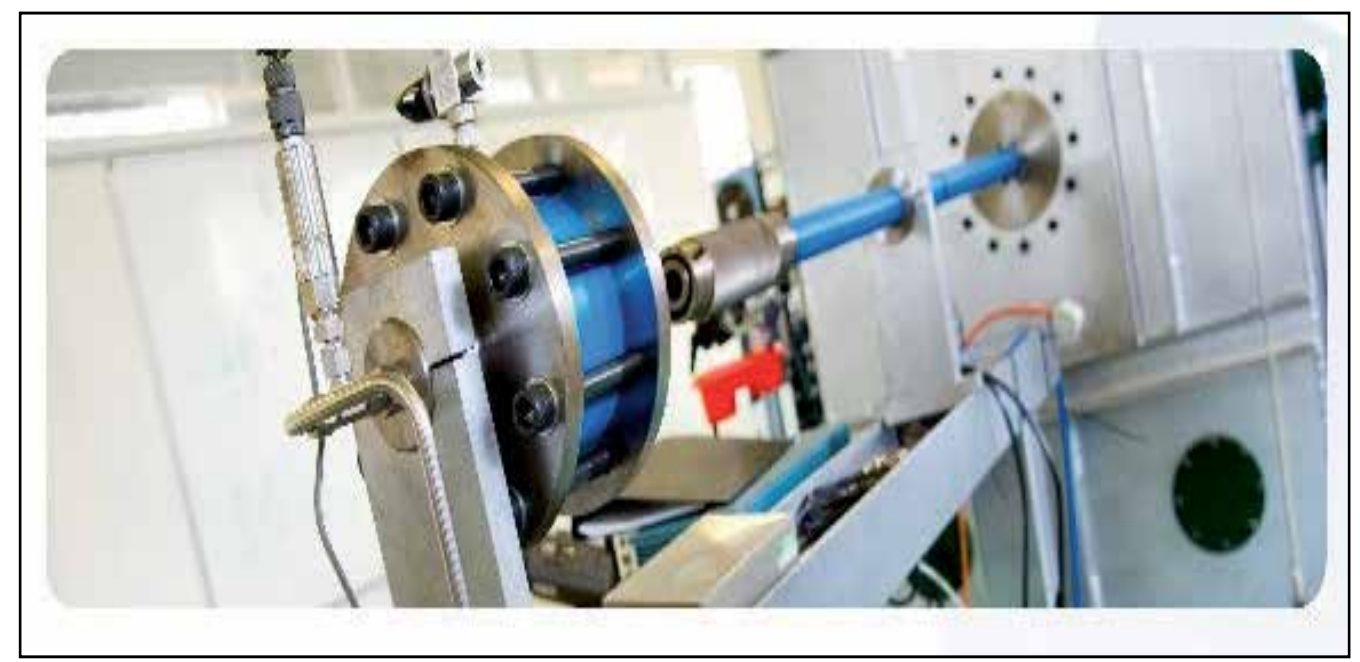

Figure 11. The gas gun testing apparatus (RMCS-Cranfield university)

In gas gun, a helium gas is used to accelerate a $12 \mathrm{~mm}$ diameter steel spheres to velocities approaching $620 \mathrm{~m} / \mathrm{s}$. The helium gas is pumped in to the breech at high pressure. An 
aluminium diaphragm blocks the release of the gas at the first stage. The diaphragm bursts and relieves the pressure when it reaches the required value. The helium gas is compressed which then heats up the volume of the gas by the polymer piston until the second diaphragm bursts. Consequently, the projectile and sabot is accelerated when the piston is at its final stage and the gas volume has expanded. The results of this test are shown in Figure. 12.

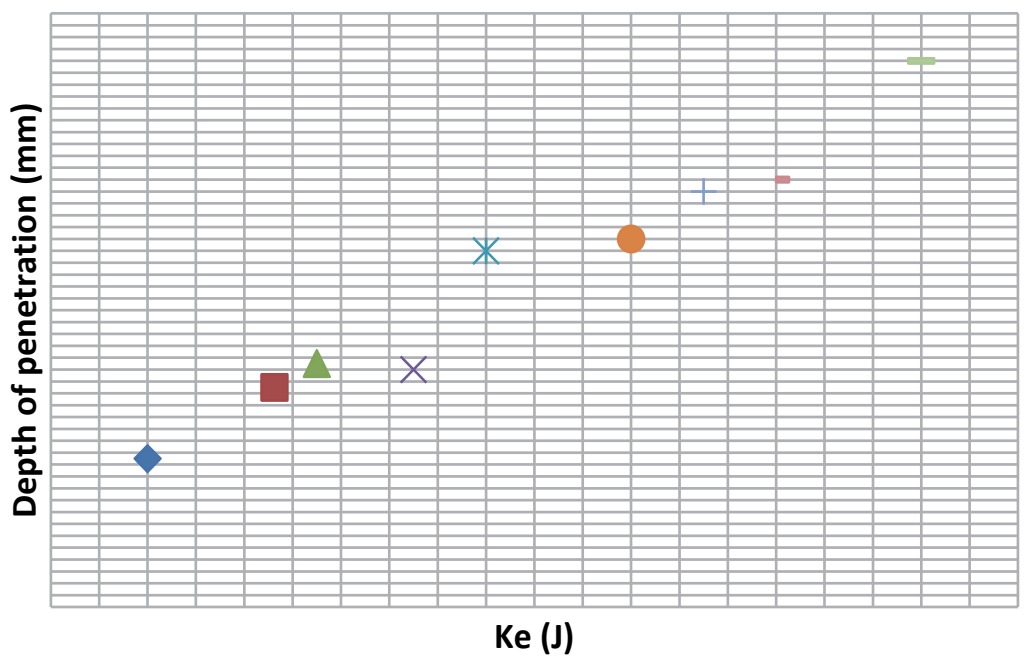

Figure 12. The kinetic energy of the impact against a depth of penetration in the material.

\section{Finite element modelling}

FEM was carried out by using ANSYS package (ANSYS, 2010). The matrix was identified by 10 nodded tetrahedron continuum elements called SOLID187 and SOLID186 in ANSYS, while for the microspheres ceramic walls SURF154 was used.

In Ansys/ workbench, the material was defined by inputting its mechanical properties that had been found by the static compression test. To define a material in Ansys/workbench, physical properties, linear elastic and plasticity of the material must be defined. The material must have a valid density defined for explicit or implicit simulation. Isotropic elasticity was used to define linear elastic material behaviour by defining Young's modulus and Poisson's ratio. However, the plastic deformation was computed by reference to Von Mises yield criterion. The multi-linear isotropic model was used to define the yield stress $\left(\sigma_{y}\right)$ as a piecewise linear function of plastic strain, $\varepsilon_{\mathrm{p}}$. The multi-linear isotropic model has been defined by introducing up to ten of stress-strain pairs.

A load of $100 \mathrm{kN}$ was imparted to aluminium matrix syntactic foam that had a dimension of $20 \mathrm{~mm} \times 20 \mathrm{~mm} \times 20 \mathrm{~mm}$. FEA is used to understand the stress distribution around the microspheres ceramics. In addition, the effect of the size of the microspheres and its volume fraction on the composite properties has been studied. Boundary condition was applied to 
the bottom of the sample that allowed the material to move freely in $\mathrm{X}$ and $\mathrm{Z}$ directions while $Y$-direction was fixed. The sample material had been defined as multi-linear isotropic hardening model.

It is found that, the Young's modulus increases with relative wall thickness, which means the particles wall thickness can be used to manage the deformation and fracture behaviour of syntactic foam. In addition, the location of the maximum stress is found on the inner surface of the particles as shown in Figure.13.

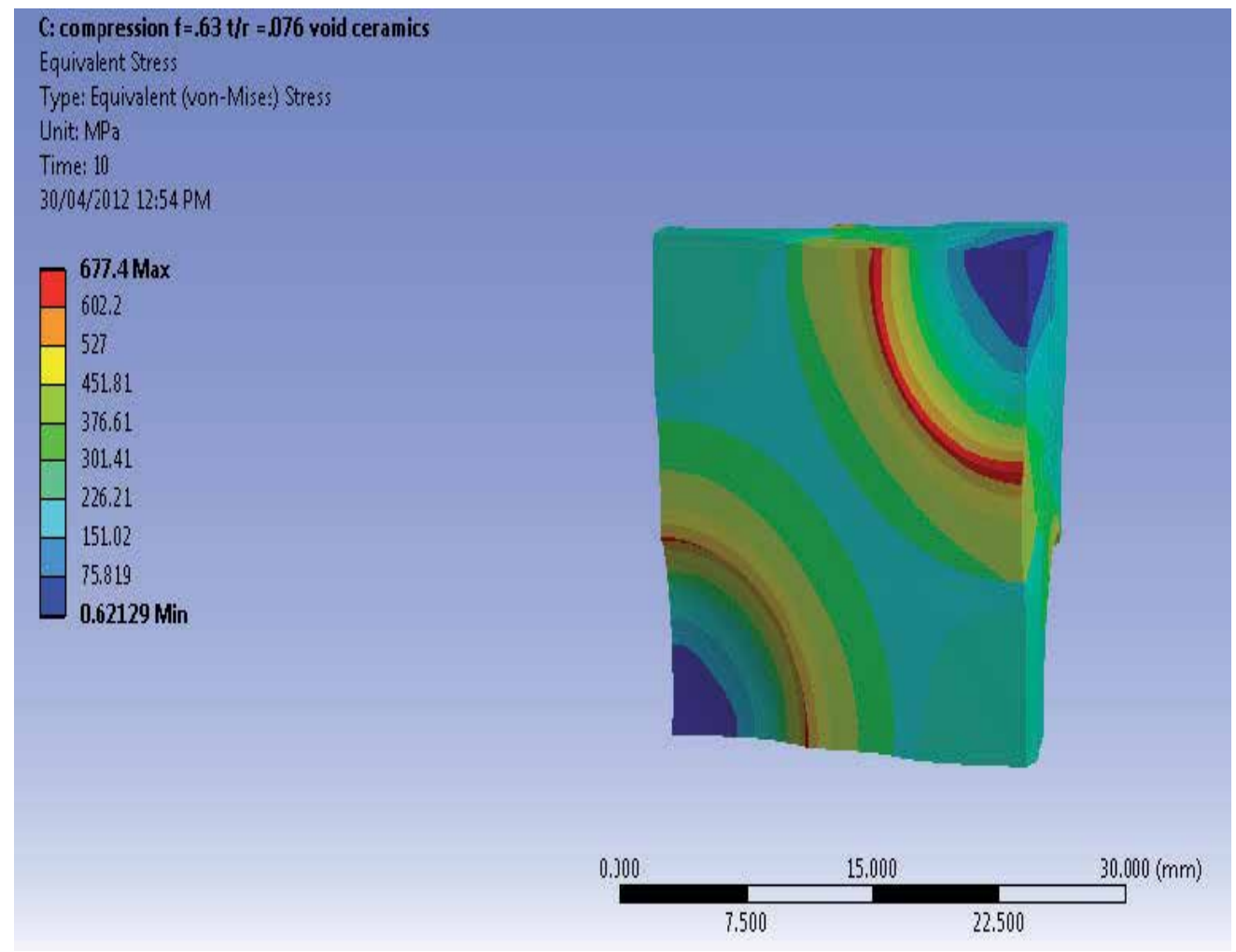

Figure 13. Maximum stress at the internal wall of the particles

\section{Summary}

- The ability to absorb high energy is the most desirable characteristic of metal matrix synthetic foam materials for use as a protective structure against dynamic loadings.

- The characterisation of the material was done by using quasi-static and dynamic loading. Static compression tests were conducted to find the stress-strain curve. The plateau stress and densification strain are found from those tests which are important properties to determine the energy absorption capability of materials.

- The effect of volume ratio of the metal matrix and the ceramic spheres on the mechanical properties of syntactic foam has, so far, not been thoroughly investigated. Therefore, the different failure modes of metal matrix syntactic foams were observed in previous studies. 
- The previous studies suggest that the simplest way to understand the failure mechanism of metal matrix syntactic foam is by comparing the failure modes of closed cell foam, in spite of the difference in the structure of the metal matrix syntactic foam that has ceramics instead of voids.

\section{Author details}

M. Altenaiji, G.K. Schleyer and Y.Y. Zhao

University of Liverpool Impact Research Centre, School of Engineering, Liverpool, UK

\section{Acknowledgement}

The authors would like to thank United Arab Emirates Government for sponsorship of this project, and also thank Mr Steve Pennington and Mr Rafael Santiago for their support during the experimental work and Dr L.P. Zhang for her assistance in preparing the syntactic foam samples, Dr. Ahmad Sheikh Rafi and Mrs Mariya Krates for her help.

\section{References}

Arnaud Fazekas, Luc Salvo, Remy Dendievel, Souhail Yossef, Peter Cloetenss, Jean Michel Letang, X-ray tomography applied to the characterization of cellular materials, related finite element modelling problems, Composite science and technology, Vol. 63, 2003, pages 2431-2443.

Balch, D.K. \& Dunand, D.C., Load partitioning in aluminium syntactic foams containing ceramic micro-spheres, Acta Materialia, Vol.54, 2006, pp. 1501-1511.

Beguefin, P. and Barbezat, M. 'Caracterisation mecanique des polymeres et composites 1 ' aide d'une machine d'essais rapide s, Proc 5th Journ e Nationale DYMAT, Bordeaux, France, 1989

Cantwell W., Morton J. Impact perforation of carbon fibre reinforced plastic, Composites science and technology, Vol.38, 1990, pp. 119-141.

Cantwell W.J. and Morton J., The impact resistance of composite materials -review, Composites, Vol. 22, Number 5, SEPT. 1991, pp.347-362.

Chen W., Lu F., Frew D., Forrestal M. Dynamic Compression Testing of Soft Materials, Journal of Applied Mechanics, Vol.69, 2002, pp. 214-223.

Dorian K. Balch, John G. O’Dwyer, Graham R. Davis, Carl M. Cady, George T. Gray III, David C. Dunand, Plasticity and damage in aluminium syntactic foams deformed under dynamic and quasi-static conditions, Material science \& Engineering, Vol.391, 2005, pp. 408-417.

Dorian K. Balch, John G. O’Dwyer, Graham R. Davis, Carl M. Cady, George T. Gray III, David C. Dunand, Plasticity and damage in aluminium syntactic foams deformed under dynamic and quasi-static conditions, Material science \& Engineering, Vol.391, 2005, pp. 408-417. 
Dou Z.Y., Jiang L.T., Wu G.H., Zhang Q., Xiu Z.Y. and Chen G.Q. High strain rate compression of ceno-sphere-pure aluminium syntactic foams, Scripta Materialia, vol. 57, 2007, pp. 945-948

Dung L., Gupta N., Rohatgi P. The high strain rate compressive response of $\mathrm{Mg}-\mathrm{Al}$ alloy/ fly ash ceno-sphere composites, JOM, 2011, pp. 48-52.

Fanjing. Y, Geometrical effects in the impact response of composite structures, $\mathrm{PhD}$ thesis, University of Liverpool, 2010.

Gibson L.J. Mechanical behaviour of metallic foams, Mater Science, Vol.30, 2000, pp. 191-227.

Goldsmith W, Sackman JL. An experimental study of energy absorption in impact on sandwich plates, International Journal of Impact Engineering, Vol.12, Part(2), 1992, Pp. 241-262.

Gray III, G. T., Classic split-Hopkinson pressure bar testing, in ASM Handbook.Vol. 8: Mechanical Testing and Evaluation, ASM International, Materials Park, Ohio, 2000, pp. 462-476.

Hanssen AG, Enstock L, Langseth M. Close-range blast loading of aluminium panels. Int. J. Impact Eng., Vol. 27, 2002, pp. 593-618.

Harding J., Wood E.O., Campbell J.D., Tensile testing of materials at impact rates of strain, journal of Mechanical Eng. Sci., Vol.2, 1960, pp. 88-96.

Hazell P., Appleby-Thomas G., Herlarr K., Painter J. Inelastic deformation and failure of tungsten carbide under ballistic loading conditions, Materials science and engineering A, Vol.527, 2010, pp.7638-7645.

Hsiao, H. M., Daniel, I. M. and Cordes, R. D., Dynamic compressive behaviour of thick composite materials, Experimental Mechanics, Vol.38- 3, pp. 172-180, 1998.

Hsiao, H. M., Daniel, Strain rate behaviour of composite materials, Composites, part B 29B, 1998, pp 521-533.

I. Irausquín, F. Teixeira-Dias, V. Miranda, J.L. Pérez-Castellanos, Numerical modelling of the dynamic compression of a closed-cell aluminium foam, Universidad Carlos III de Madrid, 28911 Leganés, España.

Kiernan S., Cui L., Gilchrist M., Propagation of a stress wave through a virtual functionally graded foam, International Journal of Non-Linear Mechanics, vol. 44, 2009, pp. 456-468.

Kiser M., He M.Y. and Zok F. W., the mechanical response of ceramic micro-balloon reinforced aluminium matrix composites under compressive loading. Actra Mater, vol. 47, No.9, 1999, pp.2685-2694.

Kohnke P. ANSYS, Inc., 'Theory reference'. ANSYS Academic research-release 13.0, 2010.

Lewis J.L. and Goldsmith W., A biaxial split Hopkinson bar for simultaneous torsion and compression, Rev. Sci. Instrum., Vol.44, 1973, pp.811-813.

Lopatnikov S., Gama B., Haque M.J., Krauthauser C., Gillespie J., Guden M., Hall I., Dynamics of metal foam deformation during Taylor cylinder-Hopkinson bar impact experiment, Composite Structures, vol.61, 2003, pp. 61-71.

Lora j, Gibson and Michael F. Ashby (1997). Cellular solids structure and properties. 2nd edition. Cambridge, UK: Cambridge University press.

M. Altenaiji, G.K. Schleyer, Y.Y. Zhao. Characterisation of aluminium matrix syntactic foams under static and dynamic loading. Applied Mechanics and Materials Vol. 82 (2011) pp 142-147. 
N. Gupta, E. Woldesenbet, Micro balloon Wall Thickness Effects on Properties of Syntactic Foams, Journal of Cellular Plastics, Vol. 40, 2004, pp. 461-480.

Novak R.C. and De Crescente M.A., in composite Materials: testing and Design (second conference), American Society for Testing and Materials, Vol. 497, 1972, pp 311-323.

Palmer, R.A., Gao, K., Doan, T.M., Green, L. \& Cavallaro, Pressure infiltrated syntactic foams process development and mechanical properties, Material science and Engineering, Vol. 464, no.1-2, 2007, pp.358-366.

Rajendran R., Prem K, Basu S., B. Chandrasekar, A. Gokhale, Preliminary investigation of aluminium foam as an energy absorber for nuclear transportation cask, Materials and design, Vol. 29, 2008, pp. 1732-1739.

Roger L. Ellis (1996). Ballistic impact resistance of graphite epoxy composites with shape memory alloy and extended chain polyethylene spectra ${ }^{\mathrm{TM}}$ hybrid component, MSC, Faculty of the Virginia Polytechnic Institute and State, Blacksburg, Virginia.

Rohatgi P.K., Kim J.K. ,Gupta,Simon Alaraj N., Daoud A. Compressive characteristics of A356/fly ash ceno-sphere composites synthesized by pressure infiltration technique, Composites: Part A 37, 2006, pp. 430-437.

S. Barre, T. Chotard, M. L. Benzeggagh, Comparative study of strain rate effects on mechanical properties of glass fibre- reinforced thermoset matrix composites, Composites, Part A - 27A, 1996, pp 1169-1181.

Tao X.F. and Zhao Y.Y. Compressive behaviour of $\mathrm{Al}$ matrix syntactic foams toughened with Al particles, Scripta Materialia, vol. 61, 2009, pp. 461-464.

Taylor G.I., The use of flat ended projectiles for determining yield stress, part I: theoretical considerations, proc. R. Soc. (London) A, vol. 194, 1948, pp.289-299.

Vendra, L.J \& Rabiei, A study on aluminium-steel composite metal foam processed by casting, Materials Science and Engineering, Vol. 465, no. 1-2, 2007, pp. 59-67.

Wu G.H., Dou Z.Y., Sun D.L., Jiang L.T. , Ding B.S. and He B.F., Compression behaviours of ceno-sphere-pure aluminium syntactic foams, Scripta Materialia, Vol. 56, 2007, pp.221-224.

Xingfu Tao, Fabrication and mechanical properties of metal matrix syntactic foams, Liverpool University, PhD thesis, 2010.

Yiou S., High performance sandwich structures based on novel metal core, PhD thesis, University of Liverpool, 2009

Zhang L.P.and Zhao Y.Y. Mechanical Response of Al Matrix Syntactic Foams Produced by Pressure Infiltration casting, Journal of Composite Materials , Vol. 41, 2007.

Zhao H., El-Nasri I., Abdennadher S., An experimental study on the behaviour under impact loading of metallic cellular materials, International Journal of Mechanical Sciences, vol. 47, 2005, pp. 757-774.

Zhao H., Gary G., Klepaczco J., on the use of a visco-elastic split Hopkinson pressure bar, International Journal of Impact Engineering, vol. 19, 1997, pp. 319-330.

Zhao Y. Y. and Tao X. F., Behaviour of metal matrix under compression, in: Proceedings of Materials Science and Technology 2009, pp. 1785-1794. 


\title{
YSZ Reinforced Ni-P Composite by Electroless Nickel Co-Deposition
}

\author{
Nor Bahiyah Baba
}

Additional information is available at the end of the chapter

http://dx.doi.org/10.5772/46496

\section{Introduction}

The importance of nickel ceramic composite in engineering applications especially for corrosion (Rabizadeh and Allahkaram 2011), wear (Lekka, Zanella et al. 2010) and thermal (Gengler, Muratore et al. 2010) resistance, and also for fuel cell anode (Pratihar, Sharma et al. 2007) has increasingly significance. Nickel ceramic composite namely Ni-P-YSZ manufactured by electroless nickel (EN) co-deposition process is investigated for its composite ratio, porosity content and electrical conductivity. The composite is the state-ofthe-art as it is the combination of nickel that is very well known for its high thermal, electronic conductivity and corrosion resistance with yttria-stabilised zirconia (YSZ) for its high ionic conductivity, impact and hardness strength. The understanding of this composite formulation and properties will increase its ability as well as expanding its application in multi-engineering disciplines.

The unique of this composite is the fabrication method via EN co-deposition process. This is a single fabrication method consists of an in-situ incorporation of inert ceramic particles in the conventional Ni-P matrix. The co-deposition of fine particle in-situ in an electroless metal-matrix is very attractive as it saves energy and time. Typical co-deposition consists of particulates in the size range of $0.1-10 \mu \mathrm{m}$ with loading of up to $40 \mathrm{vol} \%$ of the total matrix (Feldstein 1990). Common fabrications for particulate composites are limited to conventional ceramic processing, solid state powder processing and thermal spraying techniques. The incorporation of particles in EN deposit has been widely investigated and the application of ceramic YSZ in EN composite coating is the new approach (Baba, Waugh et al. 2009).

EN deposition is an autocatalytic electrochemical reaction by a chemical reduction of nickel ions near the surface of the activated substrate (Feldstein 1990). In addition to its good coating characteristics, this process can be applied to the surfaces of almost all materials. The understanding of EN chemical process as described by equations (1) - (4) is the key to 
control the EN deposition morphology, composition and properties. The conventional EN deposition processes are a direct chemical reduction of nickel ions from EN Slotonip 1850 (Schloetter 2006) solution to metallic nickel process as illustrate in equation (2) and the deposition of phosphorus in equation (3) below. This process is controlled by the EN bath composition, bath temperature, bath $\mathrm{pH}$ and soaking time, deposition rate, substrate surface and substrate orientation. Altering the process parameters cause physical changes to the deposit.

$$
\begin{gathered}
\left(\mathrm{H}_{2} \mathrm{PO}_{2}\right)^{-}+\mathrm{H}_{2} \mathrm{O} \rightarrow \mathrm{H}^{+}+\left(\mathrm{HPO}_{3}\right)^{2-}+2 \mathrm{H}_{a b s} \cdots \\
\mathrm{Ni}^{2+}+2 \mathrm{H}_{a b s} \rightarrow \mathrm{Ni}+2 \mathrm{H}^{+} \cdots \\
\left(\mathrm{H}_{2} \mathrm{PO}_{2}\right)^{-}+\mathrm{H}_{a b s} \rightarrow \mathrm{H}_{2} \mathrm{O}+\mathrm{OH}^{-}+\mathrm{P} \cdots \\
\left(\mathrm{H}_{2} \mathrm{PO}_{2}\right)^{-}+\mathrm{H}_{2} \mathrm{O} \rightarrow \mathrm{H}^{+}+\left(\mathrm{HPO}_{3}\right)^{2-}+\mathrm{H}_{2} \cdots
\end{gathered}
$$

The application of EN deposition in producing composites by in-situ incorporation of inert particles in the conventional Ni-P matrix has been extensively researched, i.e. incorporation of diamond, silicon carbide, silicon nitride, silicon oxide, boron carbide, alumina, iron oxide, titanium oxide, ceria, yttria, zirconia and PTFE particles with varying the particulate sizes (micro or nano), as listed in Table 1.

The composition of nickel and YSZ must be controlled to result in the desired properties. High ceramic composition ensures high wear, thermal and corrosion resistance. At the same time, the coefficient of thermal expansion within the coating and between coating and substrate should be compatible to avoid cracking and delamination respectively. Finally, the proportion of ceramic YSZ coating in the composite can be varied from layer to layer. The construction of a gradient of coating layers, for example, with lower ceramic content inside, at the lowest layer, increasing through subsequent layers confers the advantages of heat and corrosion resistance.

In order to effectively control the composition of Ni-YSZ composites for the best results, the conventional EN deposition process must be understood in terms of the process parameters especially the EN bath chemical and substrate conditions that affect its efficiency and quality. In addition to bath composition, conventional EN deposition is also controlled by several other bath-related factors such as bath temperature, bath $\mathrm{pH}$ and soaking time (Baudrand 1994).

The properties and affecting factors of conventional EN deposition might or might not be applicable to EN co-deposition. A study showed that the structural characteristics and phase transformation of EN co-deposition incorporating $\mathrm{Si}_{3} \mathrm{~N}_{4}, \mathrm{CeO}_{2}$ and $\mathrm{TiO}_{2}$ remained unchanged as from those of the conventional EN deposition. In general, various factors have been shown to affect the deposition of EN composites, including (1) particle catalytic inertness, (2) particle charge, (3) EN bath composition, (4) bath reactivity, (5) particle compatibility with the matrix, (6) plating rate, and (7) particle size distribution (Feldstein 1990). 


\begin{tabular}{|c|c|c|c|}
\hline Types & Particle & $\begin{array}{l}\text { Molecular } \\
\text { formula }\end{array}$ & References \\
\hline \multirow[t]{10}{*}{$\begin{array}{l}\text { Inert/ hard } \\
\text { materials }\end{array}$} & Diamond & $\mathrm{C}$ & $\begin{array}{l}\text { (Hung, Lin et al. 2008); } \\
\text { (Matsubara, Abe et al. 2007); } \\
\text { (Sheela and Pushpavanam 2002) }\end{array}$ \\
\hline & Silicon nitride & $\mathrm{Si}_{3} \mathrm{~N}_{4}$ & $\begin{array}{l}\text { (Balaraju and Rajam 2008); (Dai, } \\
\text { Liu et al. 2009); } \\
\text { (Das, Limaye et al. 2007) }\end{array}$ \\
\hline & Silicon carbide & $\mathrm{SiC}$ & $\begin{array}{l}\text { (Berkh, Eskin et al. March 1996); } \\
\text { (Kalantary, Holbrook et al. } \\
\text { 1993); (Lin, Chen et al. 2006) }\end{array}$ \\
\hline & Silicon oxide & $\mathrm{SiO}$ & (Dong, Chen et al. 2009) \\
\hline & Boron carbide & $\mathrm{B}_{4} \mathrm{C}$ & (Vaghefi, Saatchi et al. 2003) \\
\hline & Alumina & $\mathrm{Al}_{2} \mathrm{O}_{3}$ & $\begin{array}{l}\text { (Balaraju, Kalavati et al. 2006); } \\
\text { (Hazan, Reutera et al. 2008; } \\
\text { Hazan, Werner et al. 2008) }\end{array}$ \\
\hline & Ceria & $\mathrm{CeO}_{2}$ & (Necula, Apachitei et al. 2007) \\
\hline & Yttria & $\mathrm{Y}_{2} \mathrm{O}_{3}$ & $\begin{array}{l}\text { (McCormack, Pomeroy } \\
\text { et al. 2003) }\end{array}$ \\
\hline & Zirconia & $\mathrm{ZrO}_{2}$ & (Shibli, Dilimon et al. 2006) \\
\hline & Iron oxide & $\mathrm{Fe}_{3} \mathrm{O}_{4}$ & (Zuleta, Galvis et al. 2009) \\
\hline \multirow[t]{2}{*}{ Others } & Polytetrafluoroethylene & PTFE & (Ger and Hwang 2002) \\
\hline & Titanium oxide & $\mathrm{TiO}_{2}$ & (Balaraju, Narayanan et al. 2006) \\
\hline \multirow[t]{4}{*}{ Multiple } & Silicon carbide- Alumina & $\mathrm{SiC}-\mathrm{Al}_{2} \mathrm{O}_{3}$ & $\begin{array}{l}\text { (Li, An et al. 2005; Li, } \\
\text { An et al. 2005; Li, An et al. 2006) }\end{array}$ \\
\hline & Silicon carbide-Graphite & $\mathrm{SiC}-\mathrm{G}$ & (Wu, Shen et al. 2006) \\
\hline & Silicon carbide- PTFE & SiC-PTFE & (Huang, Zeng et al. 2003) \\
\hline & $\begin{array}{l}\text { Zirconia-Alumina- Zirconium } \\
\text { aluminide }\end{array}$ & $\begin{array}{l}\mathrm{ZrO}_{2}-\mathrm{Al}_{2} \mathrm{O}_{3}- \\
\quad \mathrm{Al}_{3} \mathrm{Zr}\end{array}$ & (Sharma, Agarwala et al. 2005) \\
\hline
\end{tabular}

Table 1. Types, names and formulas of the particles used in EN co-deposition

Particle stability in this case could be the charge stability of the particles in the solutions. Particle stability determines the particle dispersion in the solution and particles' tendency for agglomeration or sendimentation. Necula et al. (2007) found that particles are having good dispersion stability in deionised water but not so in the EN solution. Studies on alumina (Hazan et al. 2008b, 2008a), boron carbide (Vaghefi et al. 2003) and ceria (Necula et al. 2007) particles showed that the dispersion stability strongly depends on $\mathrm{pH}$ and the low stability caused a short sedimentation time. Studies by Hazan et al. (2008b; 2008a) on dispersion stability in Ni-P-Al $\mathrm{Al}_{2} \mathrm{O}_{3} \mathrm{EN}$ system incorporating comb-polyelectrolyte showed high particle concentrations of up to 50 vol.\% particle incorporation.

Periene et al. (1994) concluded that volume percent of co-deposition particles is dependent on powder conductivities and hydrophobic/ hydrophilic properties. Another study done on 
co-depositing boron carbide $\left(\mathrm{B}_{4} \mathrm{C}\right)$ with particle sizes ranging from 5 to $11 \mu \mathrm{m}$ in hypophosphite-reduced EN solution gave a maximum of 33 vol. $\% \mathrm{~B}_{4} \mathrm{C}$ when the $\mathrm{B} 4 \mathrm{C}$ particles were wetted with surfactant before being added into the bath (Vaghefi, Saatchi et al. 2003). The surfactant is a blend of surface active agent which contains both hydrophilic and hydrophobic groups which helps increase deposition, even at $8 \mathrm{gl}^{-1}$ particle loading. The application of surfactant in the deposition of PTFE on low carbon steel substrate showed strong adsorption (Ger and Hwang 2002).

The shape and size of the particles play an important role as they influence the deposition surface area and energy. It was found that spherical particles with smaller particle size (average $1 \mu \mathrm{m}$ boron particles and $3.4 \mu \mathrm{m}$ alumina particles) gave high particle concentration in the matrix (Apachitei, Duszczyk et al. 1998). Study done Balaraju et al. (2006a) varying alumina powder sizes of $50 \mathrm{~nm}, 0.3 \mu \mathrm{m}$ and $1.0 \mu \mathrm{m}$ showed that the highest particle incorporation occurred at $1.0 \mu \mathrm{m}$ particle size.

Another important factor is the particle loading. Particle loading is the amount of powder particles in a litre solution. Co-depositing very fine polycrystalline diamond ranging between 8 and $12 \mu \mathrm{m}$ with varying concentrations from 2-10 g/l onto aluminium substrate at $70-90^{\circ} \mathrm{C}$ for an hour yielded as high as $18.40 \mathrm{vol} . \%$ diamond powder in the deposit (Sheela and Pushpavanam 2002). The particle incorporation in a Ni-P- $\mathrm{ZrO}_{2} \mathrm{EN}$ system was found to be directly proportional to increase particle loading up to $9 \mathrm{~g} / \mathrm{l}$ as well as the deposition rate (Shibli, Dilimon et al. 2006). In Ni-P-B4C EN system, particle composition in the matrix increased from 12 to 33 vol.\% as the particle loading increased from 1 to $8 \mathrm{~g} / \mathrm{l}$ (Vaghefi, Saatchi et al. 2003). It was found that a particle loading more of than $15 \mathrm{~g} / 1 \mathrm{SiC}$ in hypophosphite-reduced solution caused extensive bath foaming which reduced the plating rate (Kalantary, Holbrook et al. 1993).

Bath conditions might be disturbed by the addition of inert particles. The addition of silicon carbide $(\mathrm{SiC})$ powders with particle size ranging from 4-7 $\mu \mathrm{m}$ in varying EN solution conditions (composition, $\mathrm{pH}$, temperature and time) showed an increase in SiC loading in the deposition but a reduction of the deposition rate and deposition weight (Kalantary, Holbrook et al. 1993). Another study showed that co-deposition of SiC (1-5 $\mu \mathrm{m})$ in sodium hypophosphite-reduced EN solution at $\mathrm{pH} 4.5-5.5,80-90^{\circ} \mathrm{C}$ with air agitation resulted in 25$30 \mathrm{vol} \% \mathrm{SiC}$ in the deposition (Li 1997). Aggressive agitation might cause substrate or deposition abrasion by other factors such as particle hardness, particle shape and size, particle loading and bath movement (Kalantary, Holbrook et al. 1993).

Substrate orientation is defined as the position of the substrate in the EN bath during the EN deposition. The variations of substrate position have shown to give an effect on the EN deposition. In a study by Sheela \& Pushpavanam (2002) on diamond EN co-deposition in hypophosphite-reduced solution showed that a vertical substrate position gave less than $20 \%$ particle incorporation compared to the horizontal position. Another study on Ni-P-SiC using hypophosphite-reduced solution with a particle loading of $25 \mathrm{~g} / \mathrm{l}$ showed that the substrate held tangentially gave the highest particle composition in the matrix and a vertical 
position leading to uniform particle incorporation and adherence provided, that uniform agitation was used (Kalantary et al. 1993).

Having discussed all the above affecting factors on EN co-deposition process, the study is concentrated on four of the factors, namely, particle size (Balaraju, Kalavati et al. 2006), agitation method (Sevugan, Selvam et al. 1993), bath $\mathrm{pH}$ (Liu, Hsieh et al. 2006), and substrate surface treatment (Teixeira and Santini 2005). The application of design of experiment (DoE) using full factorials of a two-level factor is economical and practical to reduce the number of experiments. Unlike the Taguchi method, full factorial DoE highlights the significant main and interactions effects between the factors (Anthony 2003).

Factorials design is the basis of DoE. Factorials design is the most efficient way to study the effects of two or more factors. For example, 2-levels is simplified as $2^{\mathrm{k}}$ or three levels as $3^{\mathrm{k}}$. Factorial design investigates all possible combinations of factor levels for each complete trial or replication of experiment. This is because the factors arranged in a factorial design are crossed by the Yates algorithm (Bisgaard 1998). A $2^{\mathrm{k}}$ factorial design is used to study the effects of $k$ factors with two levels for each factor. The $k$ represents a multiple-factor design with a variation of treatment designs where a set of treatments (factors) are tested over one or more sets of treatments (levels). In practice the higher-order interactions are usually not significant, thus most design are limited to 2-3 levels.

The effects of EN composite coating parameters on Ni-YSZ composition and porosity content were investigated and optimized using full factorials and ANOVA. An empirical model for prediction of $\mathrm{Ni}$ composition and porosity content were established by means of piecewise linear regression analysis. The composite response to electrical conductivity is also investigated.

\section{Experimental methods}

\subsection{Materials and preparations}

A ceramic substrate of alumina tile (Coors Ceramics, U.K.) with manufacturer standard dimensions of $50 \times 50 \times 1 \mathrm{~mm}$ as shown in Figure 1 was used as the base for composite deposition. Surfaces of the substrate sample were treated by chemical etching and mechanical blasting. Chemical etching of the substrate sample was done by immersion for 5 minutes at room temperature in a hydrofluoric (HF) etching solution comprising a mixture of 1 part hydrofluoric acid $(20 \mathrm{ml} / \mathrm{l})$ to 5 parts ammonium fluoride $\left(\mathrm{NH}_{4} \mathrm{~F}\right)(2 \mathrm{~g} / \mathrm{l})$. Then mechanical treatment of the substrate was done by blasting the substrate with brown alumina sand for 1-2 minutes. The contamination after sand blasting was cleaned ultrasonically for 30 minutes at room temperature by submerging the substrate samples individually in beakers filled with acetone.

Reinforcement ceramic particles of $8 \mathrm{~mol} \%$ YSZ (8YSZ; United Ceramics, England) were used. Yttria stabilised zirconia (YSZ) is a ceramic phase that is very well-known for high hardness, good scratch and corrosion resistance; and very high thermal resistance, but low 
toughness and high brittleness. Normally the addition of ceramic particles in composites increases the composite's mechanical properties in term of its hardness, corrosion resistance and thermal resistance. The 8 YSZ particles varied between the nominal sizes of 2 $\mu \mathrm{m}$ and $10 \mu \mathrm{m}$.

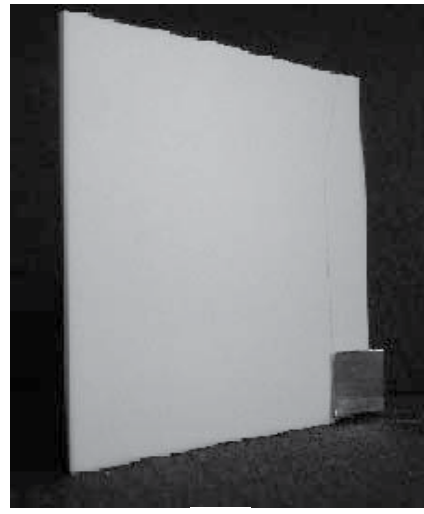

(a)

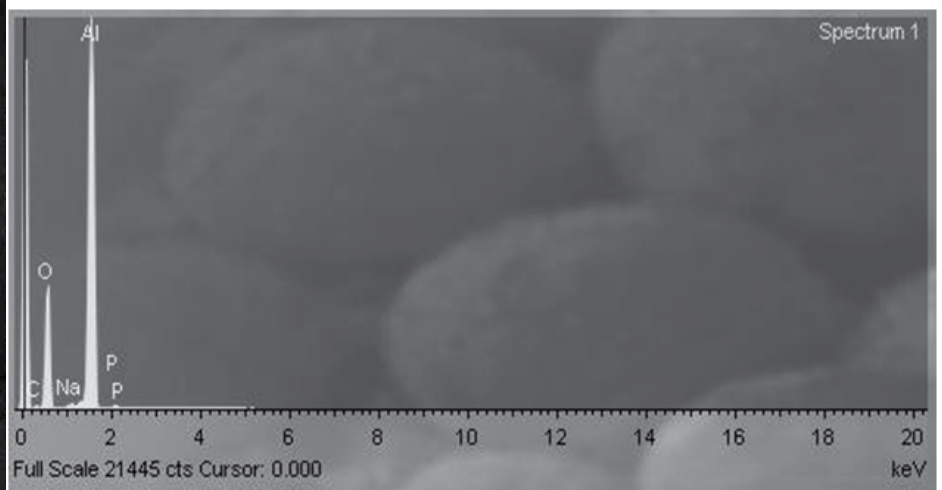

(b)

Figure 1. (a) Alumina tile as a ceramic substrate for EN co-deposition (b) Alumina substrate EDX spectrum

\subsection{EN co-deposition}

The ceramic alumina substrate requires sensitising to activate the surface. All nonproprietary solutions were prepared using AR grade chemicals and high purity deionised water. After the pre-treatment process sequence as listed in Table 2, the EN composite deposition of Ni-YSZ was performed within 3 hours to minimize effects of chemical degradation. The EN chemicals produced a bright mid-phosphorous $(6-9 \%)$ nickel deposit. The solution was heated and the temperature maintained at $89^{\circ} \mathrm{C}$ using a Jenway hotplate.

\begin{tabular}{lcc}
\hline Trade name & Soaking Time & Temperature \\
\hline Cuprolite X96DP & $15 \mathrm{~min}$ & $60^{\circ} \mathrm{C}$ \\
\hline Uniphase PHP Pre-catalyst & $15 \mathrm{~min}$ & $20^{\circ} \mathrm{C}$ \\
\hline Uniphase PHP Catalyst & $15 \mathrm{~min}$ & $40^{\circ} \mathrm{C}$ \\
\hline Niplast AT78 & $15 \mathrm{~min}$ & $40^{\circ} \mathrm{C}$ \\
\hline Electroless Nickel SLOTONIP 1850 & $60 \mathrm{~min}$ & $89^{\circ} \mathrm{C}$ \\
\hline
\end{tabular}

Table 2. EN co-deposition materials and procedure

A ceramic powder of $50 \mathrm{~g} / \mathrm{l}$ was added into the bath along with the substrate. With agitation, suspended particles near the surface are co-deposited onto the substrate surface. The $\mathrm{pH}$ of the EN solution was varied between the manufacturer standard $\mathrm{pH} 4.9$ and $\mathrm{pH}$ 5.4. The $\mathrm{pH}$ was altered to $\mathrm{pH} 5.4$ by adding $10 \%$ ammonium hydroxide. The coating time was kept constant at 60 minutes. The bath temperature was kept constant at $89 \pm 2^{\circ} \mathrm{C}$. The particles 
were kept in suspension in the EN bath by either mechanical stirring or air bubbling agitation methods. Mechanical stirring was done by Jenway hotplate with magnetic stirrer, and air bubbling was performed at1.2W air pressure.

\subsection{SEM-EDX}

Composition of Ni-P and YSZ composite in the deposition is controlled to give desired properties. It is desirable to get high ceramic to metal ratio for corrosion, thermal and wear resistance and even in the application of anode of fuel cell. Varying ceramic YSZ deposition in the composite has an advantage in which a gradient of coating layers with increasing ceramic content inside to outside for heat and corrosion resistance respectively. The influence of the process parameters in gaining high particle incorporation is analysed. The characterisation of the composite deposition was done by Hitachi field emission gun scanning electron microscope (SEM) coupled with energy dispersive $x$-ray (EDX).

The common setting of SEM is $24 \mathrm{~mm}$ working distance and $25 \mathrm{kV}$ acceleration voltage unless stated otherwise to ensure optimum condition for EDXA. Magnification at 1000 or 2000 and resolution are varied according to the requirement (3.9-6.0). The EDX expose time was kept constant at 300s and expose area was kept constant throughout the whole specimens.

SEM enables surface morphology and chemical microanalysis in conjunction with EDX. EDX stands for Energy dispersive X-ray where an x-ray emitted during electron beam targeted to the sample surfaces is detected and collected for elemental composition characterisation. The electron beam bombardment knock-out the electron near the surface and resulting electron vacancy is filled by higher energy electron level. This energy is between 10-20 eV, depending on the materials and emits x-rays to balance the energy difference between the two electron states. EDX collaborated with INCA software extends its ability for quantitative analysis, qualitative analysis, elemental mapping, and line profile analysis.

\subsection{Archimedes buoyancy}

Archimedes specific density can be used to measure the porosity fraction in a material. The basic Archimedes principle states that the amount of displaced water volume is equal to the immersed object volume. The determination of the solid substance density can be done by buoyancy or displacement methods. The true density on the other hand is the total solid density. Figure 2 (Sartorius 1991) illustrates the Archimedes density buoyancy methods. The density of a solid body is defined as a product of fluid density and fraction of solid mass over fluid mass. The apparent weight of a body in a liquid - weight reduced by buoyancy force is measured. The density of water at room temperature is assumed to be unity. The substrate alumina tile is assumed to be fully dense thus the calculation of pores of deposition will not be affected by the substrate. 


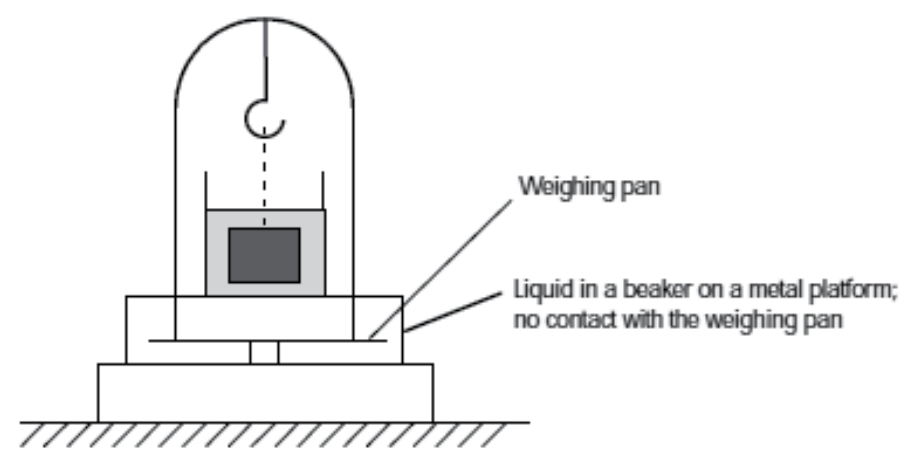

Figure 2. Schematic diagram of Archimedes density buoyancy measurement (Sartorius 1991)

\subsection{Four-point probe}

The composite fabricated via EN co-deposition was tested in term of its electrical resistivity and conductivity performance measured using four-point probe. The conductivity performance of the Ni-YSZ composite was tested in air environment up to $800^{\circ} \mathrm{C}$. Then another set of test where the nitrogen gas was purged into the furnace up to $600^{\circ} \mathrm{C}$ at $20^{\circ} \mathrm{C} / \mathrm{min}$. A four-point probe was used to measure the sheet resistance and thus the resistivity and conductivity of the anode at every $5-15^{\circ} \mathrm{C}$ increment under $50 \mathrm{~mA}$ current.

The resistance of Ni-YSZ anode was measured using four-point electrical probe as illustrate in Figure 3. A power supply provides a constant current flow between probe 1 and 4 . The current output can be obtained by an ammeter. The second set of probe (probe 2 and 3) is used for sensing and since negligible current flows in these probes - only voltage drop thus accurate resistance is measured. A resistance of the sample between probes 2 and 3 is the ratio of the voltage registering on the digital voltmeter to the value of the output current of the power supply. A simple 4-point measurement at room temperature was done at 1 $\mathrm{mA}, 50 \mathrm{~mA}$ and $90 \mathrm{~mA}$ at two different points as a trial test.

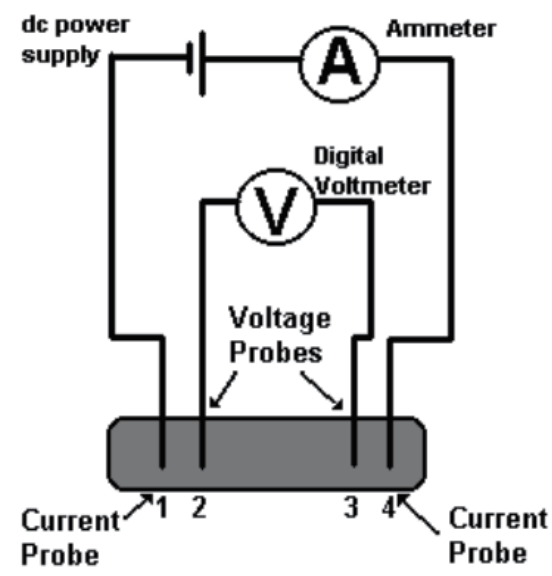

Figure 3. Schematic four-point probe set up 


\subsection{Full factorials}

As for the EN composites process, the conventional process is disturbed by the addition of $8 \mathrm{~mol} \%$ YSZ (8YSZ; United Ceramics, England) particles in the bath. Therefore the codeposition process is not only affected by the basic process parameters but also other factors including the particle stability, particle size and shape, particle loading, and bath agitation (Feldstein 1990). Few process parameters were identified to be most effective in gaining the yields namely bath agitation, bath $\mathrm{pH}$, particle size and surface treatment. These parameters were then design for experiment using 16 run full factorials with five replications.

The experimental design involved four EN co-deposition process parameters (Table 3). Both EN conventional and composite coating have been shown to be very much affected by bath $\mathrm{pH}$, bath agitation, substrate surface condition, and by particle shape and size in the composite EN coating. A full factorials DoE approach with four parameters at two levels gave $2^{4}$ full factorials of 16 runs. The DoE was repeated five times independently yielding 80 sets of experimental data.

\begin{tabular}{lccc}
\hline Parameters & Symbols & \multicolumn{2}{c}{ Level } \\
\cline { 3 - 4 } & & Low $(-1)$ & High $(+1)$ \\
\hline Particle size & A & 2 & 10 \\
\hline Bath agitation & B & Air Bubbling & Mechanical Stirring \\
\hline Bath pH & C & 4.9 & 5.4 \\
\hline Surface treatment & D & HF Etching & Mechanical Blasting \\
\hline
\end{tabular}

Table 3. EN co-deposition process parameters and their levels

\section{Results and discussions}

\subsection{Composite characterisation}

Ni-YSZ was successfully deposited on alumina substrate by EN co-deposition. The typical microstructure of EN Ni-YSZ composite deposition is shown in Figure 4. The ceramic codeposition of 10 microns (Figure $4 \mathrm{~b}$ ) ceramic powders in EN Ni-YSZ composite is higher compared to the 2 microns (Figure 4a). The coating consists primarily of ceramic YSZ powders (white areas), metallic Ni matrix (grey areas) and pores (dark areas). In general, both coating surfaces showed uniform distribution of ceramic particles. This indicates no agglomeration of YSZ particle in the coating. Uniformly distributed ceramic particles ensure constant coefficient of thermal expansion within the coating to avoid cracking due to thermal gradient.

The corresponding EDX spectrum is given in Figure 5 shows the presence of major peak of nickel $(\mathrm{Ni})$, yttria $(\mathrm{Y})$, zirconium $(\mathrm{Zr})$, oxygen $(\mathrm{O})$ and phosphorus $(\mathrm{P})$ elements with primary $\mathrm{K} \alpha$ and $\mathrm{L} \alpha$ peaks were present. These confirmed that the composites are composed of the combination of metallic nickel and ceramic YSZ. The existence of element $\mathrm{P}$ in the 
composite is due to the fact that $\mathrm{P}$ is one of the major elements in the EN hypophosphitebase bath solution. The Ni-P deposition reaction in hypophosphite-based bath involved the chemical reaction as in equation (1.3) described in section 1.0.

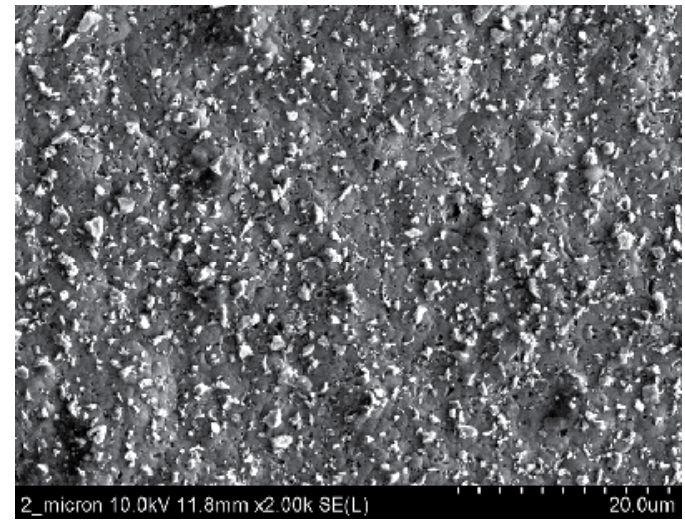

(a)

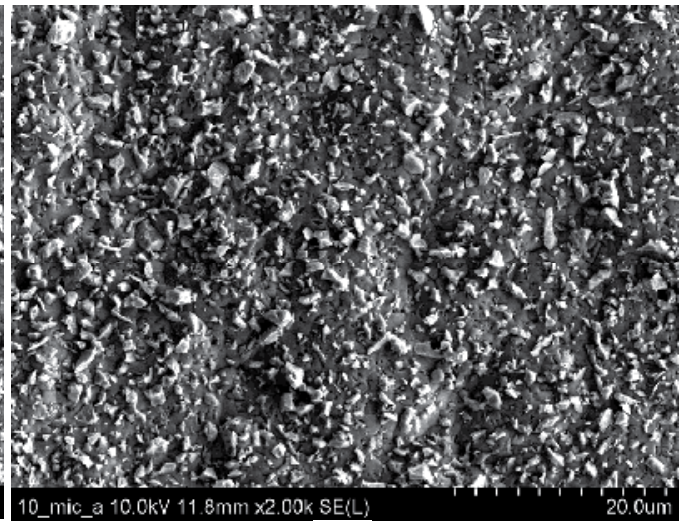

(b)

Figure 4. Comparison of surface microstructure of Ni-YSZ for ceramic particle size (a) $2 \mu \mathrm{m}$ and (b) $10 \mu \mathrm{m}$

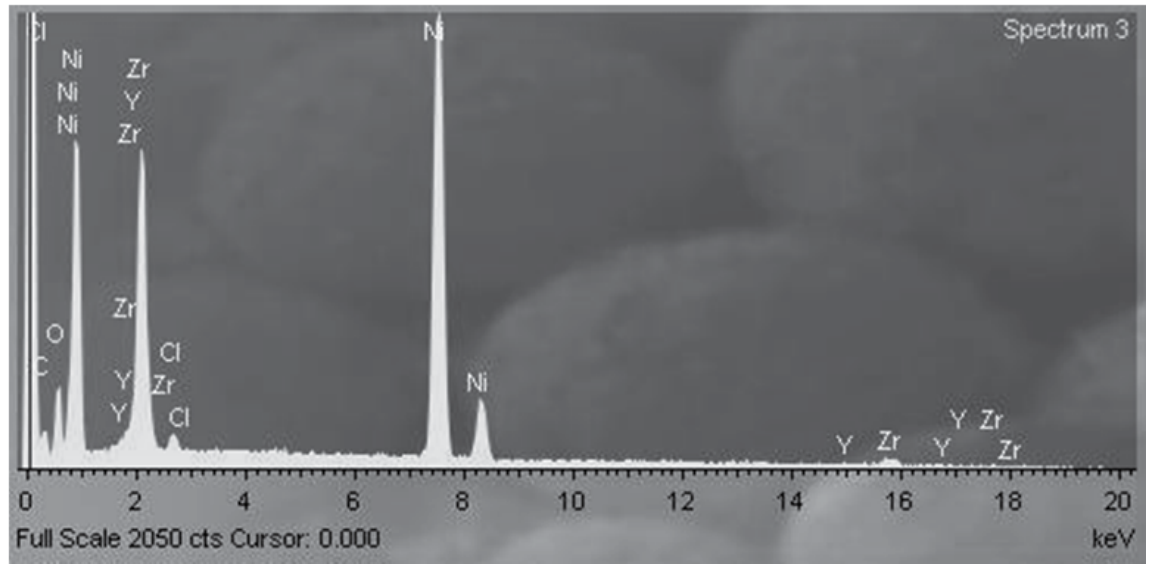

Figure 5. EDX spectrum showing presence of the corresponding EN co-deposition

In order to investigate the existence of pores at the dark areas, a higher magnification images were taken. The images show the surface morphology at various magnifications at (a) 6k, (b) 10k and (c) 20k in Figure 6. At 6k magnification image (Figure 6a) shows few dark areas which are the expected pore spots and the centre spot was focused and magnified to $10 \mathrm{k}$ magnification (Figure $6 \mathrm{~b}$ ). Clearly the images confirmed the existence of pores at higher magnification of $20 \mathrm{k}$ (Figure $6 \mathrm{c}$ ) the pores seemed to be open or connected.

It is expected that varying surface morphology of the substrate will increase the porosity formation. The images of field emission gun SEM at various magnifications from 1-20k are shown in Figure 7 compares the mechanical blasting treatment substrate surfaces (all images 
on the left represented by a, c and e) and chemical etching treatment (all images vertically on the right represented by $b, d$ and $\mathrm{f}$ ). At the $1 \mathrm{k}$ magnification, Figure $7 \mathrm{a}$ (blasted) indicated more highly populated black areas compared to Figure $7 \mathrm{~b}$ (etched). The chemically etched surface morphology is much flatter.

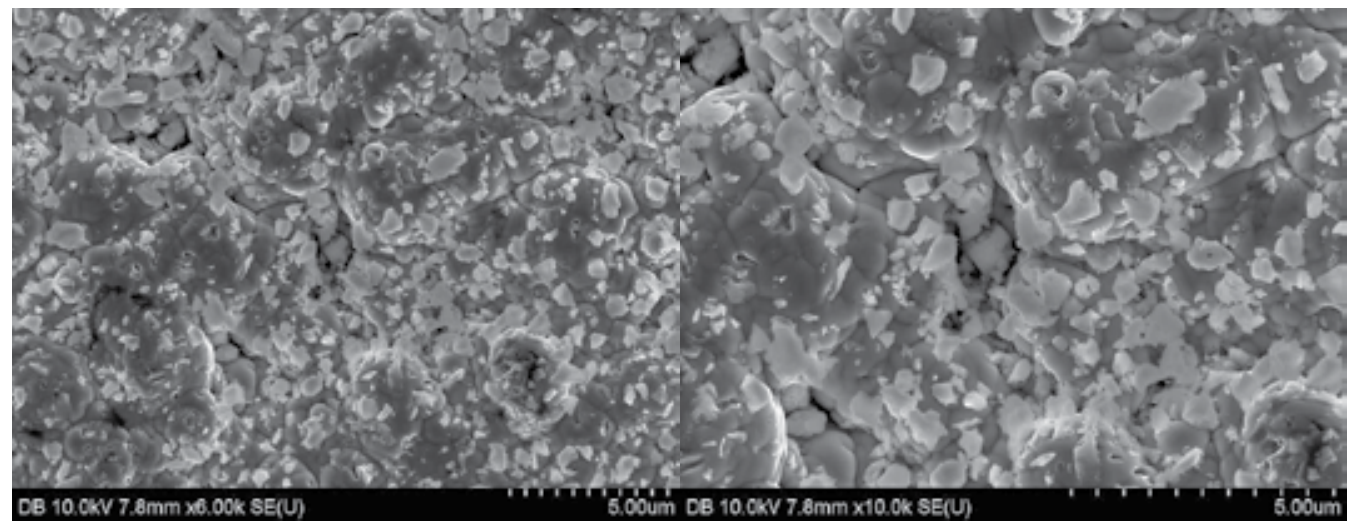

(a)

(b)

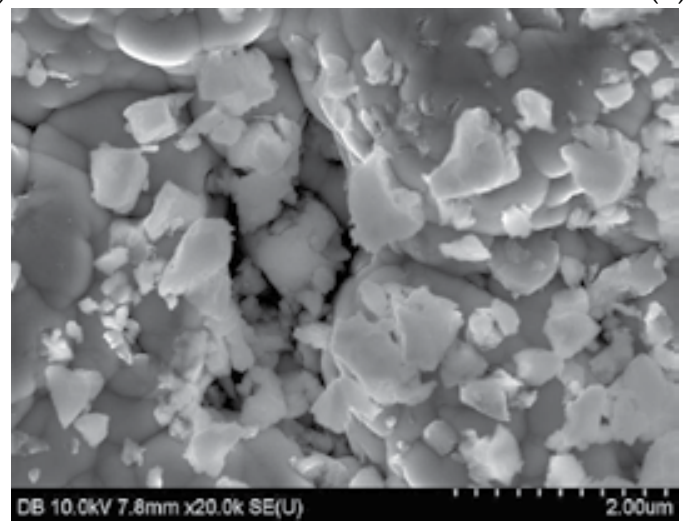

(c)

Figure 6. EN Ni- 8 YSZ (2 $\odot \mathrm{m})$ and bath pH5.4 at varying magnification (a) $6 \mathrm{k}$ (b) $10 \mathrm{k}$ and (c) $20 \mathrm{k}$

At 5k magnification, the difference is more obvious and it is confirmed that the black areas or spots were the pores. At even higher magnification (20k), the mechanically blasted deposition (Figure 7e) exhibits many open or connected pores which are highlighted with white circles compared to the chemically etched deposition (Figure 7f).

\subsection{Composite composition}

Composite composition is basically an investigation of the ratio between the metallic nickel and ceramic YSZ. The composition was investigated by an analysis of the $2^{\mathrm{k}}$ factorials design of experiment. The experiment was designed for lower nickel composition and higher ceramic incorporation in the Ni-P-YSZ composite. The main effect plot of all the four main effects namely particle size (A), agitation (B), bath $\mathrm{pH}(\mathrm{C})$ and substrate surface treatment 


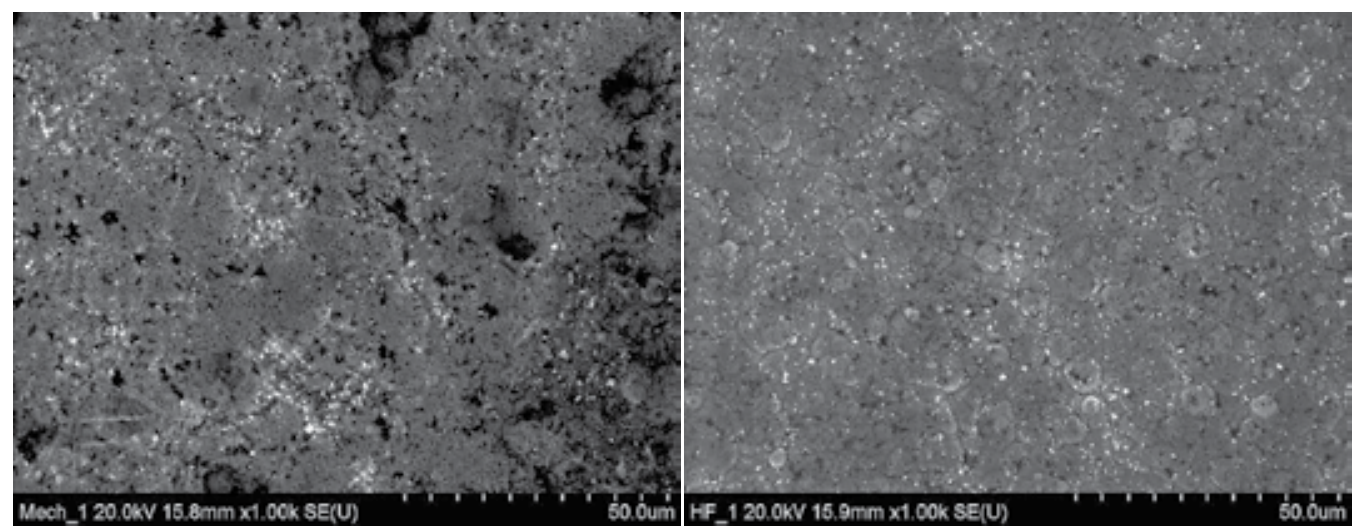

(a)

(b)

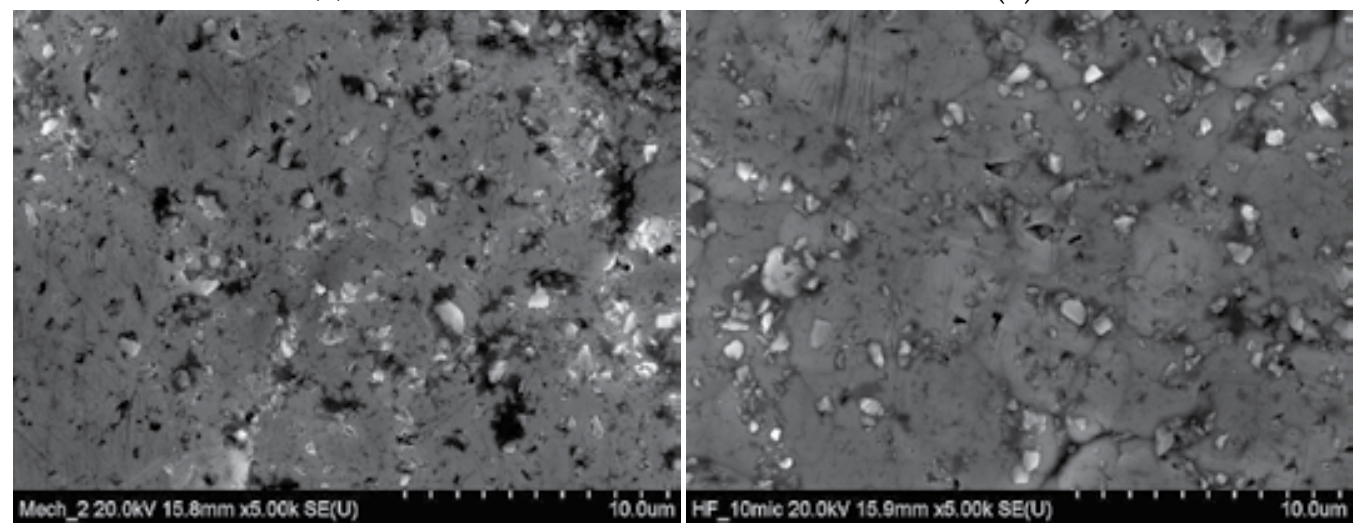

(c)

(d)

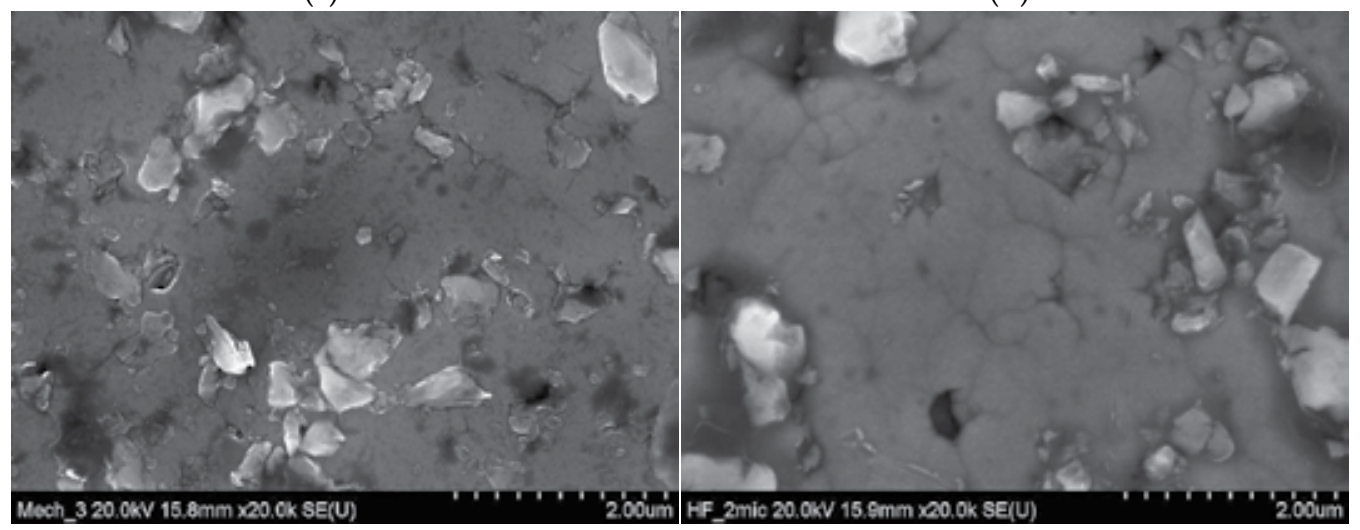

(e)

(f)

Figure 7. SEM micrographs at $1 \mathrm{k}, 5 \mathrm{k}$ and $20 \mathrm{k}$ magnification. Mechanical treated surface deposition (left: a, c, e) and chemical treated surface deposition (right: b, d, f).

(D) is illustrated in Figure 8. The main effect plot is a plot of the mean Ni content in vol.\% at each level of a design parameter. The bigger the difference between the high and low levels, the higher is the effect. Referring to Figure 8, it is clearly indicated that the most significant 
factor is A with 3.483 strength effects followed by C (1.341), then B (0.734) and lastly D (0.036). The effect of parameter $\mathrm{D}$ is almost zero, since there is almost no difference between the high and low level.

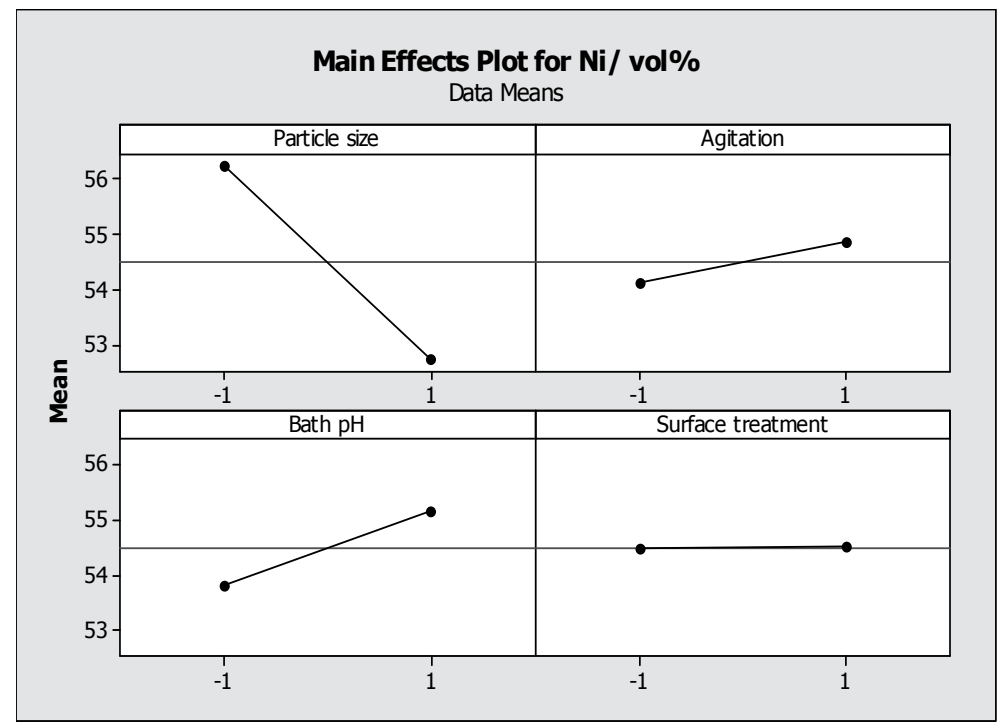

Figure 8. The main effect plots for Ni content response

Generally, by referring to the main effect plot above, particle size at high level, bath agitation at low level and bath $\mathrm{pH}$ at low level give the lower nickel content. This concluded that the main effects that influence the EN co-deposition process for lower Ni to YSZ deposition are particle size and bath $\mathrm{pH}$. Generally, particle size (A) effect at high level (10 $\mu \mathrm{m})$ and bath $\mathrm{pH}(\mathrm{C})$ at low level ( $\mathrm{pH} 4.9)$ gives lower Ni to YSZ ratio.

Based on ANOVA analysis, the three-way interaction of factor A (particle size), B (bath agitation) and $\mathrm{C}$ (bath $\mathrm{pH}$ ) was found to be significant. The investigation of the three-way interactions between factors A, B and C is referred to the contour plot given in Figure 9. The contour plot shows variation of the main effect factor $\mathrm{A}$ (particle size) and $\mathrm{C}$ (bath $\mathrm{pH}$ ) where the interaction effect B (bath agitation) was kept constant at either low level of air bubbling (Figure 9a) and high level of stirring (Figure 9b). Under low level bath agitation, low $\mathrm{Ni}$ content was obtained at high level factor A (particle size) of $10 \mu \mathrm{m}$. It is also shown that factor $\mathrm{C}$ (bath $\mathrm{pH}$ ) gives no effect on $\mathrm{Ni}$ composition at high (Figure 9a) or low level of A (Figure 9b). The low Ni content was obtained for high level bath agitation (Figure 9b) when factor $\mathrm{A}$ (particle size) at high level and factor $\mathrm{C}$ (bath $\mathrm{pH}$ ) at low level.

The influence of factor B on both main factors A and C in determining low Ni to YSZ ratio is greatly affected by factor A (particle size). Larger particle size was found easier to be codeposited in EN deposition rather than smaller particle size. Balaraju et. al. (2006) showed highest particle incorporation of largest alumina powder for a range of particle size between $50 \mathrm{~nm}, 0.3 \mu \mathrm{m}$ and $1.0 \mu \mathrm{m}$. The lowest Ni to YSZ ratio was obtained under high level bath 
agitation (mechanical stirring) with Ni content less than 52 vol.\%. The effect of factor C (bath $\mathrm{pH}$ ) on Ni content showed low level gives the lowest Ni composition in both condition of factor $\mathrm{B}$. The incorporation of ceramic powders is higher under the standard EN solution $\mathrm{pH}$ 4.9 (Schloetter 2006) ensures optimum condition. Although higher $\mathrm{pH}$ increased deposition rate in conventional EN deposition (Baudrand 1994), less ceramic particles were able to be incorporated in the EN co-deposition.

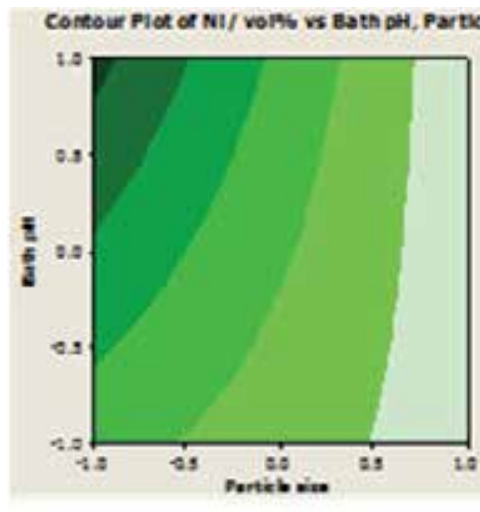

(a)

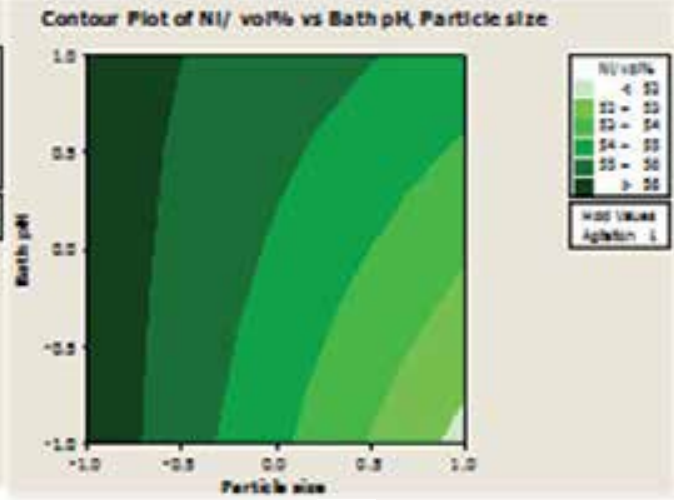

(b)

Figure 9. Contour plots for Ni content, $\mathrm{pH}$ and particle size at (a) low level agitation - air bubbling and (b) high level agitation - mechanical stirring

Thus to achieve low nickel to ceramic ratio, the conditions are (1) large particle size of 10 $\mu \mathrm{m},(2)$ mechanical stirring agitation and (3) bath $\mathrm{pH}$ of 4.9. Larger particle size was found easier to be co-deposited in EN deposition rather than smaller particle size. This was supported by Balaraju et al (2006) on alumina powder sizes of $50 \mathrm{~nm}, 0.3 \mu \mathrm{m}$ and $1.0 \mu \mathrm{m}$ that resulted in the highest particle incorporation at $1.0 \mu \mathrm{m}$ particle size. A study done by Vaghefi et al. (2003) showed 33 vol.\% of $\mathrm{B}_{4} \mathrm{C}$ particle with particle size ranges $5-11 \mu \mathrm{m}$ which indicates that larger particle sizes give higher particle incorporation in the EN composite.

In terms of bath agitation, it is crucial to keep the particles in suspension throughout the deposition (Sevugan, Selvam et al. 1993). Mechanical stirring showed higher incorporation of particles in this study compared to air bubbling. It should be noted that this is in contradiction to the finding by Vaghefi (1997) in electroless nickel-phosphorus-molybdenum disulfide which showed that air purging was better than magnetic stirring. However, the particles used in this research were ceramic YSZ which, in terms of inertness, wettability and particle stability to the substrate (Apachitei, Duszczyk et al. 1998; Necula, Apachitei et al. 2007) are different to the study by Vaghefi. As for the bath $\mathrm{pH}$, it is shown that a higher bath $\mathrm{pH}$ caused a higher deposition rate in conventional EN deposition (Baudrand 1994). Thus at a higher deposition rate, it is possible that less ceramic particles were able to be dragged along in the EN co-deposition. This resulted in the observation that the incorporation of ceramic particle is higher at lower $\mathrm{pH}$ of 4.9 than at 5.4. 
Therefore, the best deposition parameter combination for the three-way interaction based on this observation are high level particle size of $10 \mathrm{um}$, low level bath $\mathrm{pH}$ of 4.9 and high level agitation of mechanical stirring $(\mathrm{A}+1 \mathrm{~B}+1 \mathrm{C}-1)$.

\subsection{Porosity content}

Investigation of porosity level in the deposition is critical as the amount of porosity enhances thermal insulation for thermal barrier coatings (Wang, Wang et al. 2011) and gas circulation in fuel cell anode (Simwonis, Thulen et al. 1999) application. The amount of porosity should not be more than $40 \mathrm{vol} \%$ as greater amount of porosity will reduce the mechanical properties of the deposit. Thus, an adequate amount of porosity and reasonable mechanical properties should be balance. It is known that EN deposition has excellent uniformity and dense deposition with thickness less than $10 \mu \mathrm{m}$ (Das and Chin 1959). The amount of porosity in EN deposition could be induced by varying the agitation methods, deposition rate, bath $\mathrm{pH}$ and also substrate surface condition. The porosity of deposition was measured by Archimedes density measurement.

Figure 10 shows the main effect plot indicates the variation of the data mean between low and high levels for each main parameter. The most dominant parameter as already verified by the ANOVA is the substrate surface treatment (D) with bath agitation (B) closely behind. The difference between low and high levels for both parameters $\mathrm{D}$ and $\mathrm{B}$ are the largest and difficult to differentiate. Descending rank order for the strength effect is surface treatment, bath agitation, particle size and then bath $\mathrm{pH}$. The 'higher-the-better' characteristic for porosity response described that high porosity content can be achieved at low level particle size $(2 \mu \mathrm{m})$, high level bath agitation (mechanical stirring) and high level substrate surface treatment (mechanical blasting).

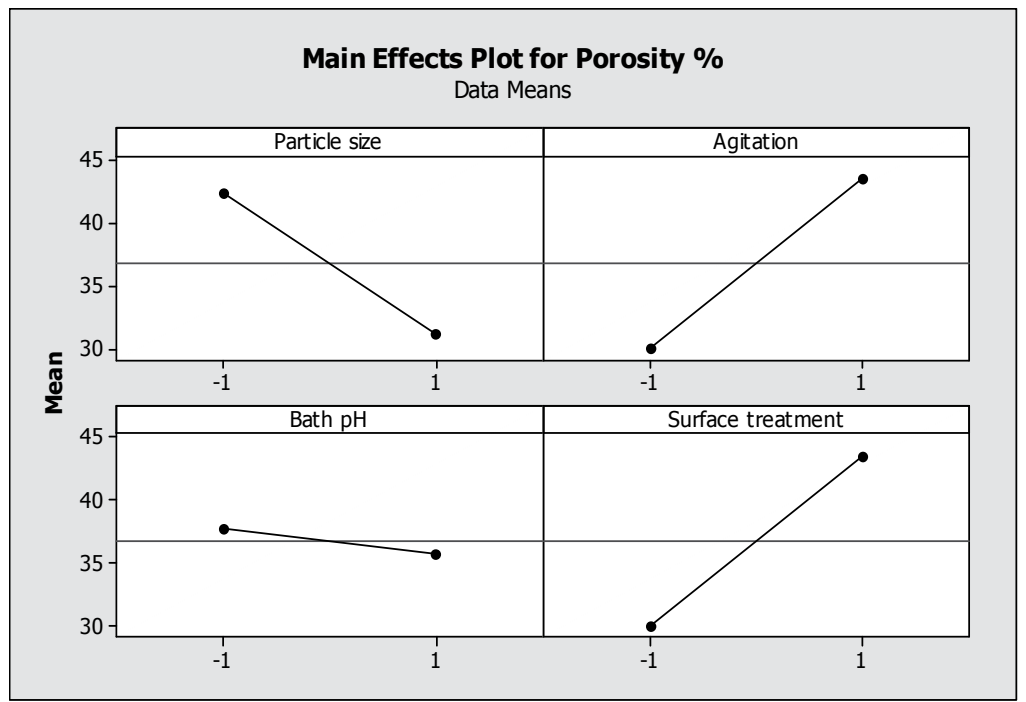

Figure 10. Main effects plot for the porosity response 
The interaction plot is a powerful graphic tool which plots the mean of response of two factors. The AD interaction plot in Figure 11 shows unparallel lines of high and low level particle size under surface treatment variation. This indicates the present of interaction between the particle size and the substrate surface treatment. The porosity content was very much affected by the substrate surface treatment. Varying substrate surface treatment from low (-1) to high (+1) level increases porosity $\%$ for both particle size at low $(-1)$ and high $(+1)$ levels. The effect of particle size on porosity \% at low level $(-1)$ is more pronounce than at high level (+1) as the substrate surface treatment changes from low (-1) to high (+1) level. At both particle size levels, mechanical blasting gave greater effect on porosity.

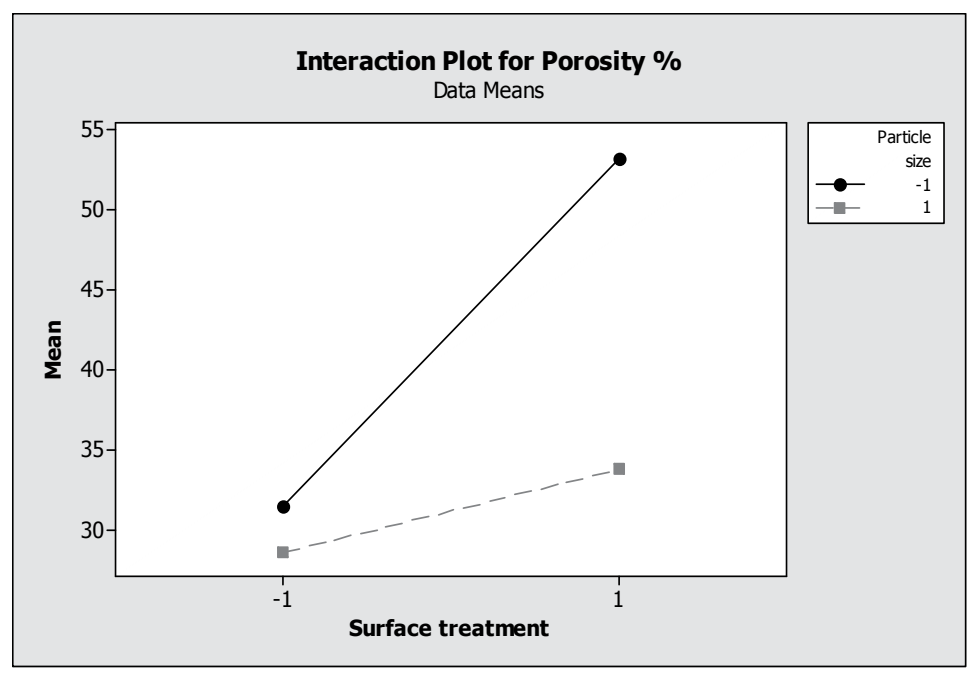

Figure 11. Figure 11: Interaction (A-D) plot for porosity

Thus to achieve high porosity content, the condition are (1) small particle size of $2 \mu \mathrm{m}$, (2) mechanical stirring agitation and (3) substrate surface treatment of mechanical blasting. Study by Wang et. al. (2006) has shown that fine particles introduced smaller size porosity. This indirectly indicates high porosity volume, i.e. like smaller pebbles in a jar has more quantity than the larger one. The mechanical stirring bath agitation gives higher porosity due to low removal of absorbed hydrogen or oxygen (Sevugan, Selvam et al. 1993).

The mechanical stirring bath agitation gives higher porosity as the agitation is not very aggressive compared to air bubbling thus most of the absorbed hydrogen or oxygen was not removed (Sevugan, Selvam et al. 1993) and trapped inside the EN deposition introducing more porosity. Mechanical blasting resulted in a rougher substrate surface. EN deposition is very well-known to follow the substrate profile rather than filling the spaces (Taheri, Oguocha et al. 2001). Therefore, rougher surface caused a rougher deposition surface and thus introduced more porosity.

Porosity measurement using Image Pro-Plus software was conducted. An SEM image of an EN co-deposition (8YSZ, $2 \mu \mathrm{m}$ in bath pH 5.4) is shown in Figure 12. This image was then analysed using colour contrast for porosity measurement. The red coloured area was the 
amount of porosity in the deposition (Figure 12b). It can be estimated that the coloured porosity area is approximately $20 \%$.

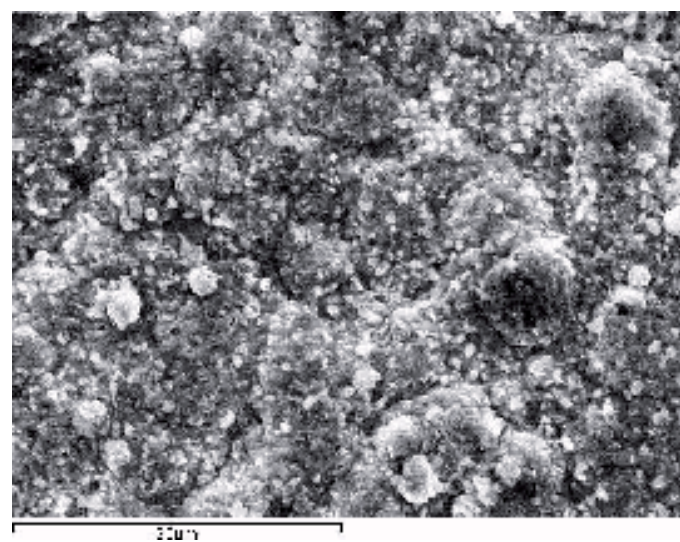

(a)

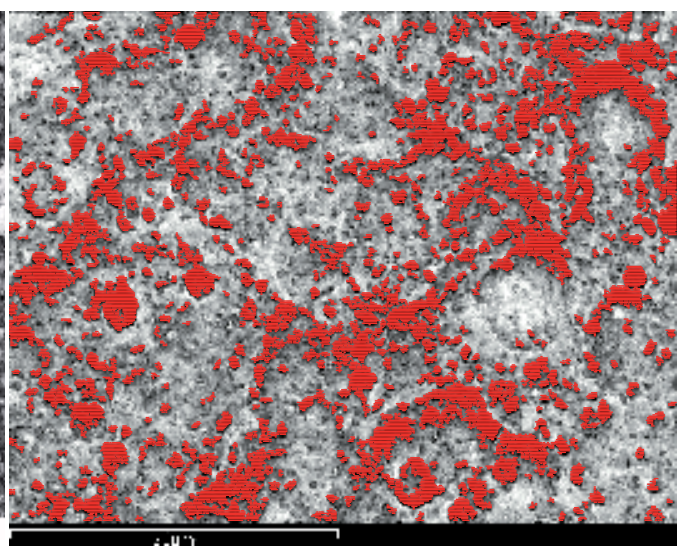

(b)

Figure 12. Image Pro-Plus porosity measurement; (a) SEM image (b) porosity area mapping

\subsection{Regression modelling}

A model to predict response function can be built by a regression model. The build model can be used to illustrate the relationship between the experimental data and the predicted data, describe the relationship between a response and a set of process parameters that affect the response. Other than that, the model can also be used to predict a response for different combinations of process parameters at their best levels. Based on the 80 sets of experimental data, the linear regression models were successfully developed for nickel to YSZ ratio response as shown in equation (5).

$$
\hat{y}=54.491-1.742 A+0.367 B+0.671 C+0.018 D+0.785 A B C \cdots
$$

where $\hat{y}$ is the nickel content, $\mathrm{A}$ is particle size $(\mu \mathrm{m}), \mathrm{B}$ is bath agitation, $\mathrm{C}$ is bath $\mathrm{pH}$ and $\mathrm{D}$ is surface treatment. The coefficient of determinations $\left(\mathrm{R}^{2}\right)$ was 0.72 , indicating a reasonable correlation between the measured and predicted values of nickel content as shown in Figure 13. This means the model is reliable in predicting the response with $28 \%$ variation. Referring to the coefficient of the developed models, it was confirmed that particle size was the most prominent parameter in minimising the nickel to YSZ ratio.

Minitab analysis showed the optimum condition for achieving a low Ni to YSZ ratio in the coating is when factors $\mathrm{A}$ and $\mathrm{B}$ are at high level and factor $\mathrm{C}$ is at low level. The minimum nickel content obtained experimentally was 51.827 vol.\% whereas the predicted content was 51.293 vol.\%. The difference between the measured value and predicted value $(0.534)$ is minimal, indicating that the model is reliable in predicting the response value at the desired parameter levels. 


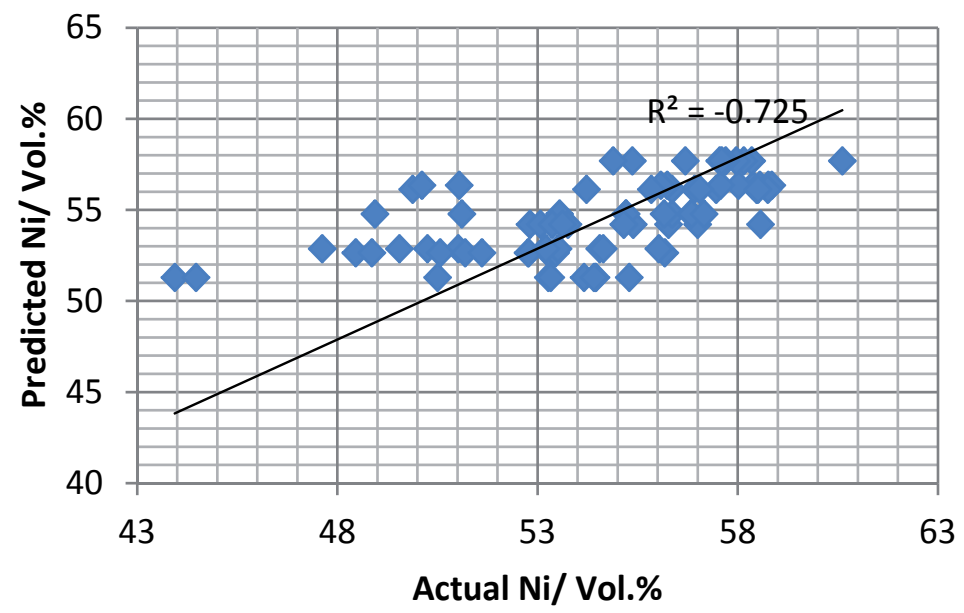

Figure 13. Comparison of experimental and predicted values of Ni content

Similarly for the Porosity \%, the analysis done by Minitab in the previous section led to a reduced model that represents the optimum condition of the process in obtaining high porosity $\%$. The reduced model equation contains main parameters A, B and D and two-way interactions of $\mathrm{AD}$ and is shown in equation (6).

$$
\hat{y}=36.734-5.614 A+6.692 B+6.776 D-4.167 A D \cdots
$$

where $\hat{y}$ is the response Porosity $\%$, A is particle size $(\mu \mathrm{m}), \mathrm{B}$ is bath agitation and D is substrate surface treatment. The coefficient of determinations $\left(R^{2}\right)$ as shown in Figure 14 is 0.47 indicating that the correlation between the actual and predicted values of Porosity \% was not very good. Referring to the coefficient of the developed model, it is confirmed that the substrate surface treatment is the most prominent parameter followed by the bath $\mathrm{pH}$ in increasing the Porosity \%.

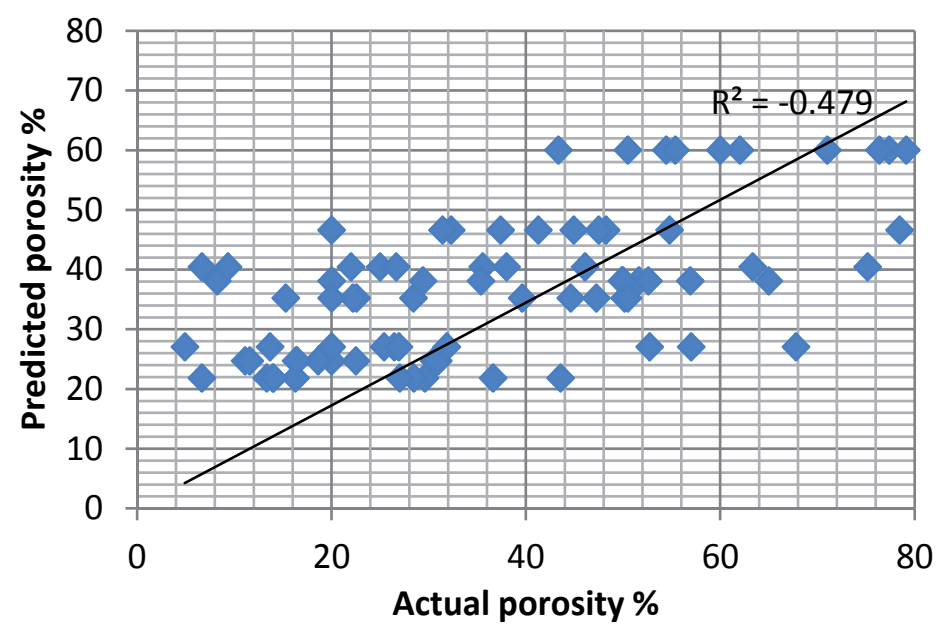

Figure 14. Comparison of experimental and predicted values for porosity content 
A study by Azmir and co-authors (2009) developed the first and second order polynomial models of their four factors Taguchi giving to a reasonably high correlation between the actual and predicted values. The coefficients of regression were determined using the same software, Minitab 15. This means this approach could be applicable in this study in order to improve the correlation coefficient of these two models for future work.

\subsection{Electrical conductivity}

Nickel-YSZ composite is the common material for fuel cell anode and thus the electrical conductivity of this composite fabricated via EN co-deposition process is also investigated. The issue of phosphorus impurity in the EN co-deposit could impede the electronic performance of the composite. The conductivity using four-point probe both in air and nitrogen environment was measured. The air environment is to simulate the oxidation environment and nitrogen for an inert environment. The measured conductivities in different environment were found to be comparable to the published data.

The Ni-YSZ co-deposition was deposited onto a ceramic substrate representing the anode. The initial sample with the thickness of $13 \mu \mathrm{m}$ Ni-YSZ co-deposition contains 48.32 vol.\% Ni. This was co-deposited with $2 \mu \mathrm{m}$ YSZ particle size. The initial electrical conductivity tests were carried out at room temperature $\left(25^{\circ} \mathrm{C}\right)$ and involved measurements at two different points on the surface of the composite sample. The tests were carried out at three different currents- $1 \mathrm{~mA}, 50 \mathrm{~mA}$ and $100 \mathrm{~mA}$. The resistance, resistivity and conductivity of the sample at the three different currents are given in Table 4.

\begin{tabular}{lcccccc}
\hline Current & \multicolumn{2}{c}{$1 \mathrm{~mA}$} & \multicolumn{2}{c}{$50 \mathrm{~mA}$} & \multicolumn{2}{c}{$100 \mathrm{~mA}$} \\
\cline { 2 - 7 } & $1^{\text {st }}$ Point & $2^{\text {nd }}$ Point & $1^{\text {st }}$ Point & $2^{\text {nd }}$ Point & $1^{\text {st }}$ Point & $2^{\text {nd }}$ Point \\
\hline Resistance $/ \Omega$ & 0.117 & 0.210 & 0.353 & 0.425 & 0.317 & 0.261 \\
Resistivity $/ 10^{-4} \Omega \mathrm{cm}$ & 1.52 & 2.73 & 4.59 & 5.52 & 4.12 & 3.4 \\
Conductivity $/ 10^{4} \mathrm{Scm}^{-1}$ & 0.66 & 0.36 & 0.22 & 0.18 & 0.24 & 0.29 \\
\hline
\end{tabular}

Table 4. Initial four-point electrical test at various current

The observed values were very encouraging. The initial test was carried out at room temperature using four-point probe measurement. Since YSZ is non-metallic and nickel is metallic, the Ni-YSZ composite behaves as biphasic composite system - having a conductivity percolation threshold at an adequate amount of nickel. The conductivity values of $50 \mathrm{vol} . \% \mathrm{Ni}-\mathrm{YSZ}$ is a factor of ten less than half the value of pure metallic nickel at room temperature $\left(11.8 \times 10^{4}(\Omega \mathrm{cm})^{-1}\right)$. Thus the obtained values for this initial test are still comparable.

A test on the Ni-YSZ fabricated via EN co-deposition was carried out at temperatures increasing from $25^{\circ} \mathrm{C}$ to $420^{\circ} \mathrm{C}$ in air. The scatter plot of the resistivity and conductivity are given in Figure 15. The trend of resistivity showed a linear relationship with the temperature. Resistivity increases as temperature increases. The conductivity is inversely proportional to resistivity thus it is expected to show opposite linear relationship to the 
resistivity. The linear decrease in conductivity with temperature is indicative of metallic conduction.

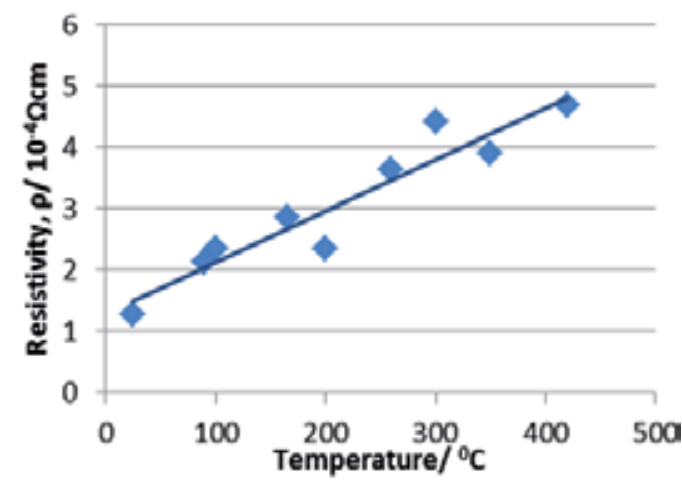

(a)

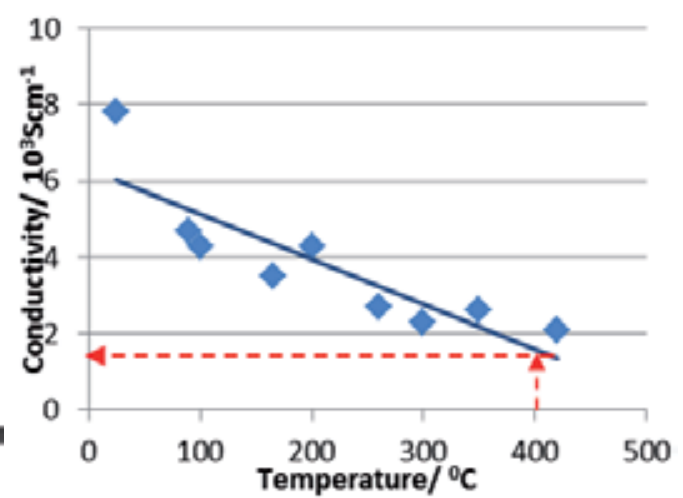

(b)

Figure 15. Scatter plot of Ni-YSZ composite (a) resistivity (b) conductivity against temperature in air

This conductivity trend was similar to the study done by Pratihar and co-authors (2005) as shown in Figure 15. The conductivity values obtained from the study at variable fabrication techniques for $40 \mathrm{vol} . \% \mathrm{Ni}$ at $400^{\circ} \mathrm{C}$ are tabulated in Table 5. Based on the best fitted linear line, at $400^{\circ} \mathrm{C}$, the conductivity value of $50 \mathrm{vol} . \% \mathrm{Ni}$ is approximately $1500 \mathrm{Scm}^{-1}$ (referring to the red dotted line in Figure 15b). This is comparable to the conductivity obtained by the composite fabricated via EN powder coating at $40 \mathrm{vol} \% \mathrm{Ni}$.

\begin{tabular}{lc}
\hline Fabrication Technique & $\boldsymbol{\sigma} / \mathbf{S c m}^{-1}$ \\
\hline Solid state & 450 \\
Liquid dispersion & 250 \\
EN powder coating & 1100 \\
\hline
\end{tabular}

Table 5. Conductivity values of Ni-YSZ composite fabricated by various techniques at $400^{\circ} \mathrm{C}$

A study on a 50 vol.\% Ni gave a conductivity of $10 \mathrm{Scm}^{-1}$ (Koide, Someya et al. 2000). Comparing these values with the one obtained for Ni-YSZ composite fabricated via EN coating, the conductivity values for EN co-deposition higher by a factor of hundred. Another study for 50 vol. $\%$ Ni-YSZ by Aruna and co-authors (Aruna, Muthuraman et al. 1998) stated a value of $2.5 \times 10^{3} \mathrm{Scm}^{-1}$ at $400^{\circ} \mathrm{C}$ which is comparable to the value obtained in this work.

Two series of electrical performance tests were conducted on another Ni-YSZ composite fabricated via EN co-deposition. These were carried out in two different environments - in air varying temperatures from $25^{\circ} \mathrm{C}$ to $800^{\circ} \mathrm{C}$ and in nitrogen varying temperatures from $25^{\circ} \mathrm{C}$ to $600^{\circ} \mathrm{C}$. Both composite samples in air and nitrogen had coating deposition thickness of 10 microns. The conductivity plots for both series are given in Figure 16. Again, the conductivity trend decreases with temperature, an indication that it has a metallic conductivity.

The conductivity values are similar in air and nitrogen environment although the former is slightly higher. This observation might be due to the high moisture content in air compared 
to nitrogen. A review by Zhu and Deevi (2003) found that the Ni-YSZ composite overpotential is significantly reduced in the presence of moisture or steam. Lowering anodic overpotential enhanced the electronic conductivity.

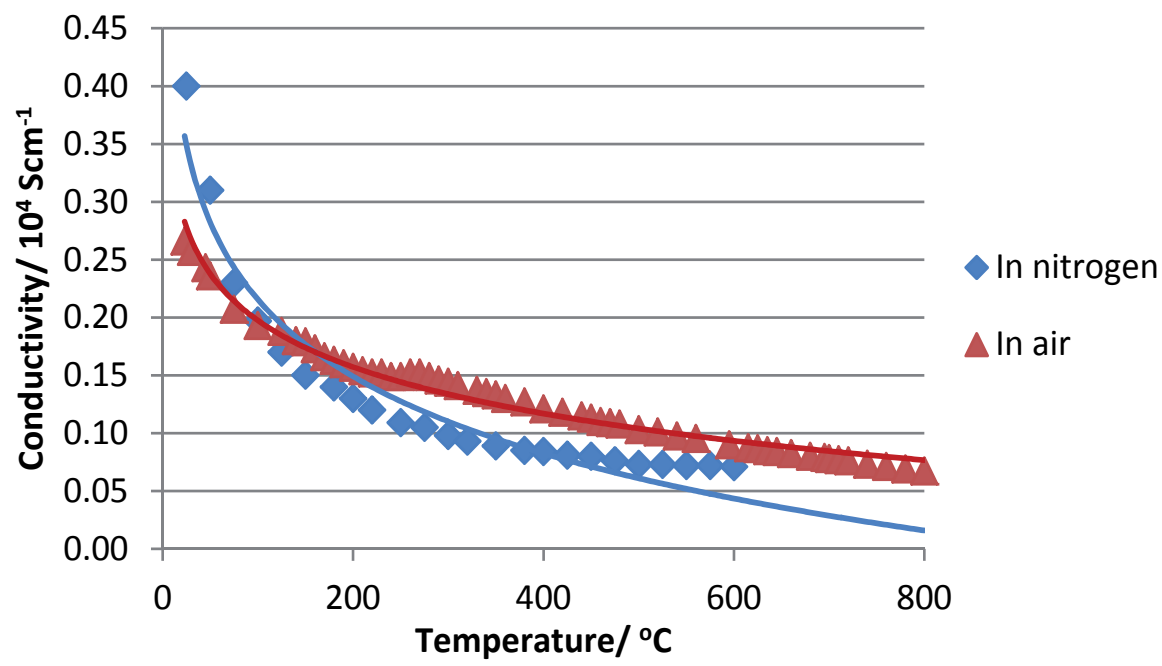

Figure 16. Scatter plot of conductivity against temperature in both nitrogen and air

In general, the conductivity at $600-800^{\circ} \mathrm{C}$ of Ni-YSZ fabricated via EN deposition ranged between $700-1000 \mathrm{Scm}^{-1}$ in both environments. These values are comparable with the published data from several studies as tabulated in Table 6. The role of phosphorus may be important. Parkinson stated that the electrical resistivity of EN deposited nickel increases with phosphorus content (Parkinson 1997). Referring to the nickel-phosphorus phase diagram, $\mathrm{Ni} 3 \mathrm{P}$ are exists at temperatures greater than $400^{\circ} \mathrm{C}$. The effect of these $\mathrm{Ni}_{3} \mathrm{P}$ crystals for porosity as it could evaporate at higher temperature in fuel cell anode application should be the subject of future work. For example, this composition might be evaporated at temperature between $1107-1517^{\circ} \mathrm{C}$ (Viksman and Gordienko 1992).

\begin{tabular}{|c|c|c|c|c|c|}
\hline Composite & $\mathrm{T} /{ }^{\circ} \mathrm{C}$ & Fabrication & Environment & $\sigma / \mathrm{Scm}^{-1}$ & Ref. \\
\hline \multirow{4}{*}{$\begin{array}{l}\text { 40vol.\% Ni- } \\
\text { YSZ }\end{array}$} & 600 & Solid state coating & $\mathrm{H}_{2}$ & 1500 & Kim \\
\hline & & Solid state mixing & & 900 & et al. (2006) \\
\hline & 800 & Solid state coating & $\mathrm{H}_{2}$ & 1400 & \\
\hline & & Solid state mixing & & 800 & \\
\hline \multirow[t]{2}{*}{$\begin{array}{l}45 w t . \% \text { Ni- } \\
\text { YSZ }\end{array}$} & $600-800$ & $\begin{array}{l}\text { Solid state with 2- } \\
\text { step calcinations }\end{array}$ & $\mathrm{H}_{2} / \mathrm{Ar}$ & 500 & $\begin{array}{l}\text { Han } \\
\text { et al. (2006) }\end{array}$ \\
\hline & & $\begin{array}{l}\text { Conventional solid } \\
\text { state mixing }\end{array}$ & & 430 & \\
\hline
\end{tabular}

Table 6. Published electrical conductivity of Ni-YSZ composite

The composite fabricated via EN co-deposition is a possibility for in-situ fabrication onto ceramic substrate. It is proven the composite of $50 \mathrm{vol} . \% \mathrm{Ni}$ has a metallic conductivity with 
highest conductivity of $1500 \mathrm{Scm}^{-1}$ at $400^{\circ} \mathrm{C}$ in air and $700 \mathrm{Scm}^{-1}$ in $\mathrm{N}_{2} ; 1000 \mathrm{Scm}^{-1}$ in air at $600^{\circ} \mathrm{C}$ and $750 \mathrm{Scm}^{-1}$ at $800^{\circ} \mathrm{C}$ in air.

\section{Conclusions}

This study investigates a method of fabricating Ni-YSZ composite via EN co-deposition approach. The work involved (i) showing that successful EN co-deposition of Ni-YSZ composite by selective combinations of process parameters (ii) investigation of physical properties of the composites.

The $2^{4}$ full factorials of 16 runs used particle sizes of 2 and $10 \mu \mathrm{m}$, bath agitation methods of air bubbling and mechanical stirring, bath $\mathrm{pH}$ of 4.9 and 5.4 and substrate surface treatment of HF etching and mechanical blasting. The design of experiment responses were nickel content and porosity content. The design of experiment were then analysed by Minitab 15 software and it was found that the optimum condition for low Ni to YSZ ratio involved a particle size of $10 \mu \mathrm{m}$, bath agitation of mechanical stirring, a bath $\mathrm{pH}$ of 4.9 and a substrate condition of HF etching. On the other hand, the porosity response optimum condition involved a particle size of $2 \mu \mathrm{m}$, a bath agitation method of mechanical stirring, a bath $\mathrm{pH}$ of 4.9 and a substrate surface treatment of mechanical blasting.

Based on the 80 sets of experimental data, the linear regression models were successfully developed for both responses. The coefficients of determinations $\left(\mathrm{R}^{2}\right)$ of nickel composition and porosity content were found to be 0.72 and 0.47 respectively. There is a reasonable correlation between the measured values and predicted value for nickel content. These models can be used in determining EN co-deposition parameters for tailored amount of nickel content.

In terms of the electrical conductivity performance, the initial electrical conductivity test carried out at room temperature showed an encouraging outcome in that the value for a 50 vol.\% Ni-YSZ composite was only a factor of ten less than the equivalent loading of pure nickel. The electrical conductivity of this composite at $400^{\circ} \mathrm{C}$ in air was comparable to published data in other studies and was superior to those recorded for composites manufactured by traditional techniques. At temperatures up to $800^{\circ} \mathrm{C}$, the electrical conductivity tests were carried out in two different environments - air and nitrogen - and results were comparable to those in the public domain.

\section{Author details}

Nor Bahiyah Baba

TATI University College (TATIUC), Terengganu, Malaysia

\section{Acknowledgement}

The author would like to express her gratitude to Mr. Alan Davidson and Prof. Tariq Muneer from School of Engineering and Build Environment, Edinburgh Napier University, United Kingdom for their technical support and great contribution of knowledge. 


\section{References}

Anthony, J. (2003). Design of Experiments for Engineers and Scientist, Butterworth-Henimen.

Apachitei, I., J. Duszczyk, et al. (1998). "Particles Co-Deposition by Electroless Nickel." Scripta Materialia 38(9): 1383-1389.

Aruna, S. T., M. Muthuraman, et al. (1998). "Synthesis and properties of Ni-YSZ cermet: anode materials for solid oxide fuel cells." Solid State Ionics 111: 45-51.

Azmir, M. A., A. K. Ahsan, et al. (2009). "Effect of abrasive water jet machining parameters on aramid fibre reinforced plastics composite." International Journal Materials Form 2: 3744.

Baba, N. B., W. Waugh, et al. (2009). Manufacture of Electroless Nickel/YSZ Composite Coatings. World Congress of Science, Engineering and Technology (WCSET) Dubai, UAE, WASET 2009 ISSN 2070-3740, Vol.37. ISSN 2070-3740, Vol.37: 715-720.

Balaraju, J. N., Kalavati, et al. (2006). "Influence of particle size on the microstructure, hardness and corrosion resistance of electroless $\mathrm{Ni}-\mathrm{P}-\mathrm{Al}_{2} \mathrm{O}_{3}$ composite coatings." Surface E Coatings Technology 200: 3933 - 3941.

Balaraju, J. N., T. S. N. S. Narayanan, et al. (2006). "Structure and phase transformation behaviour of electroless Ni-P composite coatings." Materials Research Bulletin 41: 847860.

Balaraju, J. N. and K. S. Rajam (2008). "Preparation and characterisation of sutocatalytic low phosphorus nickel coatings containing submicron nitride particles." Journal of Alloys and Compounds 459(1-2): 311-319.

Baudrand, D. W. (1994). "Electroless Nickel Plating " ASM Handbook Volume 5 Surface Engineering: 290-310.

Berkh, S. Eskin, et al. (March 1996). Properties of Electrodeposited NiP-SiC Composite Coatings. Metal Finishing: 35-40.

Bisgaard, S. (1998). A Practical Aid for Experimenters. Preliminary Edition. Madison, Starlight Press: 57.

Dai, J., X. Liu, et al. (2009). "Preparation of Ni-coated $\mathrm{Si}_{3} \mathrm{~N}_{4}$ powders via electroless plating method." Ceramics International 35(8): 3407-3410.

Das, C. M., P. K. Limaye, et al. (2007). "Preparation and characterization of silicon nitride codeposited electroless nickel composite coatings." Journal of Alloys and Compounds 436: 328-334.

Das, L. and D. T. Chin (1959). "Electrochemical Porosity Measurement of EN Coating." Plating and Surface Finishing 84: 66-68.

Dong, D., X. H. Chen, et al. (2009). "Preparation and properties of electroless $\mathrm{Ni}-\mathrm{P}-\mathrm{SiO}_{2}$ composite coatings." Applied Surface Science 255: 7051-7055.

Feldstein, N. (1990). Composites Electroless Plating. Electroless Plating: Fundamentals and Applications. G. O. Mallory and J. B. Hajdy. Orlondo, FI, AESF Publication. Chapter 11: 269-287.

Gengler, J. J., C. Muratore, et al. (2010). "Yttria-stabilized zirconia-based composites with adaptive thermal conductivity " Composites Science and Technology 70(14): 2117-2122. 
Ger, M.-D. and B. J. Hwang (2002). "Effect of surfactants on codeposition of PTFE particles with electroless Ni-P coating." Materials Chemistry and Physics 76(1): 38-45.

Han, K. R., Y. Jeong, et al. (2006). "Fabrication of NiO/YSZ anode material for SOFC via mixed NiO precursors." Materials Letters 61: 1242-1245.

Hazan, Y. d., T. Reutera, et al. (2008). "Interactions and dispersion stability of aluminum oxide colloidal particles in electroless nickel solutions in the presence of comb polyelectrolytes." Journal of Colloid and Interface Science 323: 293-300.

Hazan, Y. d., D. Werner, et al. (2008). "Homogeneous $\mathrm{Ni}-\mathrm{P} / \mathrm{Al}_{2} \mathrm{O}_{3}$ nanocomposite coatings from stable dispersions in electroless nickel baths." Journal of Colloid and Interface Science 328: 103-109.

Huang, Y. S., X. T. Zeng, et al. (2003). "Development of electroless Ni-P-PTFE-SiC composite coating." Surface \& Coating Technology 167: 207-211.

Hung, C. C., C. C. Lin, et al. (2008). "Tribological studies of electroless nickel/ diamond composite coatings on steels." Diamond \& Related Materials 17: 853-859.

Kalantary, M. R., K. A. Holbrook, et al. (1993). "Optimisation of a Bath for Electroless Plating and its use for the Production of Ni-P-SiC Coatings." Trans. Inst. Metal Finish 71(2): 5561.

Kim, S.-D., H. Moon, et al. (2006). "Performance and durability of Ni-coated YSZ anodes for intermediate temperature solid oxide fuel cells." Solid State Ionics 177: 931-938.

Koide, H., Y. Someya, et al. (2000). "Properties of Ni/YSZ cermet as anode for SOFC." Solid State Ionics 132: 253-260.

Lekka, M., C. Zanella, et al. (2010). "Scaling-up of the electrodeposition process of nanocomposite coating for corrosion and wear protection " Electrochimica Acta 55(27): 78767883.

Li, L., M. An, et al. (2005). "Model of electroless Ni deposition on $\mathrm{SiC}_{\mathrm{p}} / \mathrm{Al}$ composites and study of the interfacial interaction of coatings with substrate surface." Applied Surface Science 252: 959-965.

Li, L., M. An, et al. (2006). "A new electroless nickel deposition technique to metallise $\mathrm{SiC}_{\mathrm{p}} / \mathrm{Al}$ composites." Surface $\mathcal{E}$ Coatings Technology 200: 5102 - 5112.

Li, L. B., M. Z. An, et al. (2005). "Electroless deposition of nickel on the surface of silicon carbide/aluminum composites in alkaline bath." Materials Chemistry and Physics 94: 159164.

Li, Y. (1997). Investigation of Electroless Ni-P-SiC Composite Coatings. Plating \& Surface Finishing. November 1997: 77-81.

Lin, C. J., K. C. Chen, et al. (2006). "The cavitation erosion behavior of electroless Ni-P-SiC composite coating." Wear 261: 1390-1396.

Liu, W. L., S. H. Hsieh, et al. (2006). "Temperature and pH dependence of the Electroless NiP deposition on Silicon." Thin Solid Films 510: $102-106$.

Matsubara, H., Y. Abe, et al. (2007). "Co-deposition mechanisms of nano-diamond with electrolessly plated nickel films." Electrochimica Acta 52: 3047-3052.

McCormack, A. G., M. J. Pomeroy, et al. (2003). Journal of Electrochemical Society 150 (5): C356C361. 
Necula, B. S., I. Apachitei, et al. (2007). "Stability of nano-/microsized particles in deionized water and electroless nickel solutions." Journal of Colloid and Interface Science 314: 514522.

Parkinson, R. (1997). Properties and Application of Electroless Nickel, Nickel Development Institute: 33.

Periene, N., A. Cesuniene, et al. (1994). Codeposition of Mixtures of Dispersed Particles With Nickel-Phosphorus Electrodeposits. Plating ans Surface Finishing. October 1994: 68-71.

Pratihar, S. K., A. D. Sharma, et al. (2005). "Processing microstructure property correlation of porous Ni-YSZ cermets anode for SOFC application." Materials Research Bulletin 40: 1936-1944.

Pratihar, S. K., A. D. Sharma, et al. (2007). "Properties of Ni/YSZ porous cermets prepared by electroless coating technique for SOFC anode application." Journal of Materials Science 42: 7220-7226.

Rabizadeh, T. and S. R. Allahkaram (2011). "Corrosion resistance enhancement of Ni-P electroless coatings by incorporation of nano- $\mathrm{SiO}_{2}$ particles." Materials and Design 32(1): 133-138.

Sartorius (1991). Manual of weighing applications Part 1: Density: 1-62.

Schloetter (2006). Electroless Nickel - Solotonip 1850. Bath 18810 - PE. Worchestershire, England: 1-11.

Sevugan, K., M. Selvam, et al. (1993). Effect of Agitation in Electroless Nickel Deposition. Plating and Surface Finishing: 56-58.

Sharma, S. B., R. C. Agarwala, et al. (2005). "Development of Electroless Composite Coatings by using in-situ Co-deposition followed by co-deposition process." Sadhana 28: 475-493.

Sheela, G. and M. Pushpavanam (2002). Diamond-dispersed electroless nickel coatings. Metal finishing. January 2002: 45-47.

Shibli, S. M. A., V. S. Dilimon, et al. (2006). "ZrO2-reinforced Ni-P plate: An effective catalytic surface for hydrogen evolution." Applied Surface Science 253: 2189-2195.

Simwonis, D., H. Thulen, et al. (1999). "Properties of Ni/YSZ porous cermets for SOFC anode substrates prepared by tape casting and coat-mix process." Journal of Materials Processing Technology 92-93: 107-111.

Taheri, R., I. N. A. Oguocha, et al. (2001). "The tribological characteristics of electroless NiP coatings." Wear 249: 389-396.

Teixeira, L. A. C. and M. C. Santini (2005). "Surface Conditioning of ABS for Metallisation into the use of Chromium baths." Journal of Materials Processing Technology 170: 37-41.

Vaghefi, S. M. M., A. Saatchi, et al. (2003). "Deposition and properties of electroless Ni-P-B4C composite coatings." Surface and Coatings Technology 168: 259-262.

Vaghefi, S. M. M., A. Saatchi, et al. (1997). "The effect of agitation on electroless nickelphosphorus-molybdenum disulfide composite plating." Metal Finishing 95(6): 102.

Viksman, G. S. and S. P. Gordienko (1992). "Behavior in vacuum at high temperatures and thermodynamic properties of nickel phosphide Ni3P." Poroshkovskaya Metallurgiya 12(360): 70-72. 
Wang, L., Y. Wang, et al. (2011). "Influence of pores on the thermal insulation behavior of thermal barrier coatings prepared by atmospheric plasma spray " Materials and Design 32(1): 36-47.

Wang, Y., M. E. Walter, et al. (2006). "Effects of powder sizes and reduction parameters on the strength of Ni-YSZ anodes." Solid State Ionics 177: 1517-1527.

$\mathrm{Wu}$, Y., B. Shen, et al. (2006). "The tribological behaviour of electroless Ni-P-Gr-SiC composite." Wear 261: 201-207.

Zhu, W. Z. and S. C. Deevi (2003). "A review on the status of anode materials for solid oxide fuel cells." Materials Science and Engineering A362: 228-239.

Zuleta, A. A., O. A. Galvis, et al. (2009). "Preparation and characterization of electroless Ni$\mathrm{P}-\mathrm{Fe}_{3} \mathrm{O}_{4}$ composite coatings and evaluation of its high temperature oxidation behaviour." Surface \& Coatings Technology 203: 3569-3578. 


\title{
Carbon Nanotube Reinforced Alumina Composite Materials
}

\author{
Go Yamamoto and Toshiyuki Hashida \\ Additional information is available at the end of the chapter
}

http://dx.doi.org/10.5772/48667

\section{Introduction}

Novel materials and processing routes provide opportunities for the production of advanced high performance structures for different applications. Ceramic matrix composites are one of these promising materials. Engineering ceramics such as $\mathrm{Al}_{2} \mathrm{O}_{3}, \mathrm{Si}_{3} \mathrm{~N}_{4}, \mathrm{SiC}$ and $\mathrm{ZrO}_{2}$ produced by conventional manufacturing technology have high stiffness, excellent thermostability and relatively low density, but extreme brittle nature restricted them from many structural applications (Mukerji, 1993). Considerable attention has been adopted to improve the fracture toughness. An approach has been paid to the development of nanocrystalline ceramics with improved fracture properties. Decreasing the grain size of ceramics to the sub- and nano-meter scale leads to a marked increase in fracture strength (Miyahara et al., 1994). However, fracture toughness of nanocrystalline ceramics generally displays modest improvement or even deterioration (Miyahara et al., 1994; Rice, 1996; Yao et al., 2011). As one possible approach, incorporation of particulates, flakes and short/long fibers into ceramics matrix, as a second phase, to produce tougher ceramic materials is an eminent practice for decades (Evans, 1990). Recently, researchers have focused on the carbon nanomaterials, in particular carbon nanotubes (CNTs), which are nanometer-sized tubes of single- (SWCNTs) or multi- layer graphene (MWCNTs) with outstanding mechanical, chemical and electrical properties (Dai et al., 1996; Ebbesen et al., 1996; Treacy et al., 1996; Huang et al., 2006; Peng et al., 2008), motivating their use in ceramic composite materials as a fibrous reinforcing agent.

It is well recognized that some difficulties appear to be the major cause for the limited improvement in CNT/ceramic composites prepared to date. The first is the inhomogeneous dispersion of CNTs in the ceramic matrix. Pristine CNTs are well known for poor solubilization, which leads to phase segregation in the composite owing to the van der Waals attractive force (Chen et al., 1998). Such clustering produces a negative effect on the 
physical and mechanical properties of the resultant composites (Yamamoto et al., 2008). The second is the difficulty in controlling connectivity between CNTs and the ceramics matrix, which leads to a limited stress transfer capability from the matrix to the CNTs (Peigney, 2003; Sheldon \& Curtin, 2004; Chen et al., 2011). The strengthening and toughening mechanisms of composites by fibers are now well established (Evans, 1990; Hull \& Clyne, 1996); central to an understanding is the concept of interaction between the matrix and reinforcing phase during the fracture of the composite. The fracture properties of such composites are dominated by the fiber bridging force resulting from debonding and sliding resistance, which dictates the major contribution to the strength and toughness. Thus, the adequate connectivity with the matrix, and uniform distribution within the matrix are essential structural requirements for the stronger and tougher CNT/ceramic composites. To overcome these obstacles, various efforts, such as surface modification (De Andrade et al., 2008; Yamamoto et al., 2008; Kita et al., 2010; Gonzalez-Julian et al., 2011), heterocoagulation (Fan et al., 2006a, 2006b), extrusion (Peigney et al., 2002), and their combination, have been made to effectively achieve good dispersion of CNTs in ceramic matrix. Until now, however, most results for strengthening and toughening have been disappointing, and only little or no improvement have been reported in $\mathrm{CNT} /$ ceramic composite materials, presumably owing to the difficulties in homogeneous dispersion of CNTs in the matrix and in formation of adequate interfacial connectivity between two phases.

This chapter presents that novel processing approach based on the precursor method. The MWCNTs used in this study are modified with an acid treatment. Combined with a mechanical interlock induced by the chemically modified MWCNTs, this approach leads to improved mechanical properties. Mechanical measurements on the composites revealed that only 0.9 vol. $\%$ acid-treated MWCNT addition results in $37 \%$ and $36 \%$ simultaneous increases in bending strength $(689.6 \pm 29.1 \mathrm{MPa})$ and fracture toughness $(5.90 \pm 0.27$ $\left.\mathrm{MPa} \cdot \mathrm{m}^{1 / 2}\right)$, respectively, compared with a MWCNT-free alumina sample prepared under similar processing conditions. Structure-property relationship of present composites will be explained on the basis of the detailed nano/microstructure and fractographic analysis. We also explain why previous reports indicated only modest improvements in the fracture properties of MWCNT based ceramic composites. Here, the failure mechanism of the MWCNTs during crack opening in a MWCNT/alumina composite is investigated through transmission electron microscope (TEM) observations and single nanotube pullout tests. Achieving tougher ceramic composites with MWCNTs is discussed based on these results.

\section{A novel approach for preparation of MWCNT/alumina composites}

To disperse the MWCNTs homogeneously in the matrix and improve the connectivity between MWCNTs and matrix, we developed a novel approach with combination of a precursor method for synthesis of an alumina matrix, an acid treatment of MWCNTs and a spark plasma sintering method. The improvement on the bending strength and fracture toughness was confirmed by the fracture tests. 


\subsection{Materials and specimen preparation}

\subsubsection{Starting materials}

The MWCNT material (Nano Carbon Technologies) used in this research was synthesized by a catalytic chemical vapor deposition method followed by high temperature annealing at $2600^{\circ} \mathrm{C}$. The purity was claimed to be $99.5 \%$ by the producer. Fig. 1 shows scanning electron microscope (SEM, Hitachi S-4300) and TEM (Hitachi HF-2000) images of the pristine MWCNTs. It can be seen from Fig. $1 \mathrm{~b}$ that the pristine MWCNTs have a highly crystalline multi-walled structure with a narrow central channel. The corresponding geometrical and mechanical properties of the pristine MWCNT are listed in Table 1. The estimated diameter and length of the pristine MWCNTs from SEM and TEM measurements ranged from 33 to $124 \mathrm{~nm}$ (average: $70 \mathrm{~nm}$ ) and 1.1 to $22.5 \mu \mathrm{m}$ (average: $8.7 \mu \mathrm{m}$ ), respectively. Tensile-loading experiments with individual MWCNTs using a nanomanipulator tool operated inside SEM revealed that the tensile strengths of 10 pristine MWCNTs ranged from $\sim 2$ to $\sim 48 \mathrm{GPa}$ (average: $20 \mathrm{GPa}$ ) and the Young's modulus ranged from $~ 50$ to $1360 \mathrm{GPa}$ (average: 790 GPa) (Yamamoto et al., 2010). It seems that the average tensile strength of the pristine MWCNT used in this research were somewhat lower than that of the arc-discharge grown MWCNTs (Yu et al., 2000).
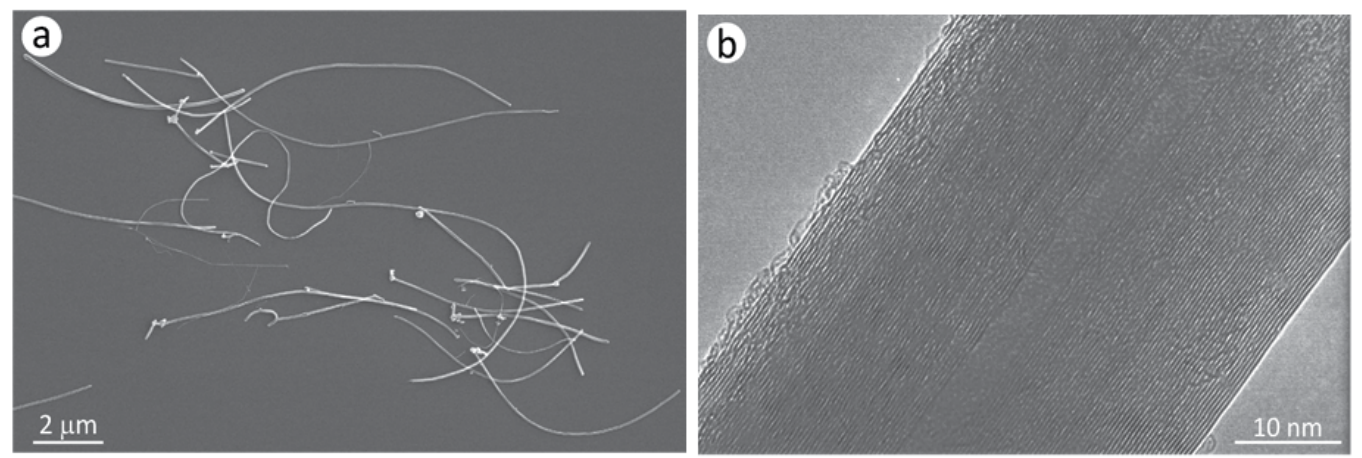

Figure 1. (a) SEM and (b) TEM image of pristine MWCNTs used in this research.

\begin{tabular}{|c|c|c|c|c|c|c|c|}
\hline $\begin{array}{c}\mathrm{ID} \\
(\mathrm{nm})\end{array}$ & $\begin{array}{c}\text { OD } \\
(\mathrm{nm})\end{array}$ & $\begin{array}{c}l \\
(\mu \mathrm{m})\end{array}$ & $\begin{array}{c}\rho \\
\left(\mathrm{Mg} / \mathrm{m}^{3}\right)\end{array}$ & $\begin{array}{c}\sigma_{\mathrm{f}} \\
(\mathrm{GPa})\end{array}$ & $\begin{array}{c}E \\
(\mathrm{GPa})\end{array}$ & $\begin{array}{c}I \\
\left(\mathrm{~nm}^{4}\right)\end{array}$ & $\begin{array}{c}E I \\
\left(\mathrm{~N} \cdot \mathrm{nm}^{2}\right)\end{array}$ \\
\hline 7 & 70 & $\begin{array}{c}8.7 \\
(1.1 \sim 22.5)\end{array}$ & 2.1 & $\begin{array}{c}20 \\
(2 \sim 48)\end{array}$ & $\begin{array}{c}790 \\
(50 \sim 1360)\end{array}$ & $1.2 \times 10^{6}$ & $9310 \times 10^{-4}$ \\
\hline
\end{tabular}

Table 1. Measured geometrical and mechanical properties of pristine MWCNTs. Shown are the nanotube inner diameter (ID), outer diameter (OD), length $(l)$, density $(\rho)$, tensile strength $(\sigma \mathrm{f})$, Young's modulus $(E)$, moment of inertia of cross sectional area $(I)$ and flexural rigidity $(E I)$, respectively.

\subsubsection{Acid treatment of MWCNTs}

The rationale behind the acid treatment is to introduce nanoscale defects and adsorb negatively charged functional groups at the MWCNT ends and along their lengths. The 
pristine MWCNTs were refluxed in 3:1 (volume ratio) concentrated $\mathrm{H}_{2} \mathrm{SO}_{4}: \mathrm{HNO}_{3}$ mixture at a temperature of $70^{\circ} \mathrm{C}$ for 1 hour, 2 hours and 4 hours, washed thoroughly with distilled water to be acid-free, and then finally dried in an air oven at $60^{\circ} \mathrm{C}$. Fig. 2 shows the typical TEM images of a series of the acid-treated MWCNTs and the corresponding distribution of nanodefect depths treated with the various conditions. It is demonstrated that with the acid treatment of the pristine MWCNTs, we have deliberately introduced nanoscale defects on the surface of the MWCNTs. The depth of the nanodefects is on the nanoscale and the average size is in the range of $4.4 \sim 7.0 \mathrm{~nm}$ for the acid treatment used in this study. We can see that the nanodefects density, i.e., the number of nanodefects per unit of a MWCNT surface area increases with the increasing treatment time. Hereafter, the number of the nanodefects per unit of the MWCNT surface area is referred to as the nanodefect density.

In addition to the nanodefects density, the average size of the nanodefect depths appears to vary with respect to the treatment time. When the treatment time increases from 1 hour to 2 hours, the average size of nanodefect depths increases from $4.4 \mathrm{~nm}$ to $6.5 \mathrm{~nm}$. Furthermore, when the treatment time further increases to 4 hours, it increases to $7.0 \mathrm{~nm}$. The aspect ratio $(\alpha)$ of the nanodefects on the MWCNT surface were estimated using the equation $\alpha=$ $L_{\text {width }} / L_{\text {depth, }}$ where $L_{\text {width }}$ is the average size of nanodefect widths on the MWCNT surface, and $L$ depth is the average size of nanodefect depths. For the acid treated products with treatment time of 1 hour, 2 hours and 4 hours was 4.4, 4.9 and 3.9, respectively. The experimental results demonstrate that the present method, which uses the acid treatment, may provide an effective route for preparation of the nanodefects on the MWCNT surface, and it may be possible to adjust and control the average size and nanodefect density by varying the treatment time. According to the current TEM observations, peel-off of a few layers in the MWCNT structure was frequently observed for the MWCNT powders acidtreated for 4 hours. Thus, reduction of $\alpha$ may be due to the decrease in the MWCNT diameter by the peel-off of a few layers in the MWCNT structure and imply that the excessive acid treatment of the MWCNT resulted in degradation of the quality and mechanical properties of MWCNTs (Yamamoto et al., 2010). As previously reported (Liu et al., 1998), SWCNTs can be cut into shorter segments by acid treatment of 3:1 (volume ratio) concentrated $\mathrm{H}_{2} \mathrm{SO}_{4}: \mathrm{HNO}_{3}$ mixture. In this study, however, when the acid treatment times are 1 hour and 2 hours, no such change in the length has been found in the acid-treated MWCNTs. The average lengths of the acid-treated MWCNTs were $8.7 \mu \mathrm{m}$ and $8.3 \mu \mathrm{m}$, respectively. In contrast, average length was decreased slightly with a further increase in the treatment time up to 4 hours, and reached about $7.2 \mu \mathrm{m}$.

The zeta potential values of the pristine MWCNTs and the acid-treated MWCNTs at different $\mathrm{pH}$ values are shown in Fig. 3a. Here, the changes in zeta potentials were measured in $1.0 \mathrm{mM} \mathrm{KCl}$ aqueous solution of varying $\mathrm{pH}$ using a zeta potential analyzer (ZEECOM ZC2000, Microtec). The $\mathrm{pH}$ value of the aqueous solution was adjusted with $\mathrm{HCl}$ and $\mathrm{NaOH}$. Zeta potential values were calculated using the Smoluchowski equation. The isoelectric point ( $\mathrm{pH}_{\mathrm{iep}}$ ) for the pristine MWCNTs is located at about 3.0, whereas the acid 
treatment process makes the surface more negatively charged at tested $\mathrm{pH}$ values. The change in the zeta potential may be mainly due to the introduction of more functional groups after the acid treatment (Esumi et al., 1996; Liu et al., 1998). These functional groups make them easily dispersed in polar solvents, such as water and ethanol. Fig. $3 \mathrm{~b}$ shows a photograph of the pristine MWCNTs and acid-treated MWCNTs suspensions at $\mathrm{pH} \mathrm{6}$, respectively. It is clear that the pristine MWCNTs are not dispersed at $\mathrm{pH}$ 6. In contrast, the dispersion of the acid-treated MWCNTs is seen to improve dramatically. Furthermore, it can be expected that the larger electrical repulsive force between the acid-treated MWCNTs will facilitate their dispersion and prevent them from tangling and agglomeration. The zeta potential of the aluminum hydroxide, which is used as the starting material for synthesis of the alumina matrix, exhibited positive values over a wide $\mathrm{pH}$ range $(\mathrm{pH}=3 \sim 9)$, while that of the acid-treated MWCNTs was negative in this $\mathrm{pH}$ range. On these two colloidal suspensions are mixed, particles of the aluminum hydroxide will bind onto the acid-treated MWCNTs because of the strong electrostatic attractive force between them, and this results in a homogeneous MWCNTs and aluminum hydroxide solution.

\subsection{Preparation of MWCNT/alumina composites}

A typical synthesis procedure for the composite preparation is as follows. The $50 \mathrm{mg}$ MWCNTs acid-treated for 2 hours or pristine MWCNTs were dispersed in $400 \mathrm{ml}$ ethanol with aid of ultrasonic agitation. $15.2 \mathrm{~g}$ aluminum hydroxide (Wako Pure Chemical Industries) was added to this solution and ultrasonically agitated. $73 \mathrm{mg}$ magnesium hydroxide (Wako Pure Chemical Industries) was added to prevent excessive crystal growth. Here, the weight loss of the hydroxides caused by the dehydration process was accounted for in the calculation of the composite composition. The weight loss of the aluminum hydroxide and the magnesium hydroxide was $34.7 \%$ and $31.9 \%$, respectively. The resultant suspension was filtered and dried in an air oven at $60^{\circ} \mathrm{C}$. Finally, the product obtained in the previous step was put into a half-quartz tube and was dehydrated at $600^{\circ} \mathrm{C}$ for $15 \mathrm{~min}$ in argon atmosphere. The composites were prepared by spark plasma sintering (SPS, SPS-1050 Sumitomo Coal Mining) (Omori, 2000) in a graphite die with an inner diameter of $30 \mathrm{~mm}$ at a temperature of $1500^{\circ} \mathrm{C}$ under a pressure of $20 \mathrm{MPa}$ in vacuum for $10 \mathrm{~min}$. For comparison, similar preparation processes were applied while using the pristine MWCNTs as the starting material. Fig. 4 shows X-ray diffraction patterns (M21Mac Science) of the (a) aluminum hydroxide-MWCNT mixture, (b) dehydrated product and (c) sintered body, respectively. It is difficult to distinguish the MWCNT peaks from all XRD patterns, probably due to the small quantity of MWCNTs. The diffraction peaks corresponding to the aluminum hydroxide and the intermediate were observed in the aluminum hydroxideMWCNT mixture. However, the diffraction peaks corresponding to the aluminum hydroxide and the intermediate disappeared completely in the sintered body, suggesting the phase transformation of the aluminum hydroxide to form $\alpha$-alumina via an amorphous phase (b). These results clearly indicate that alumina was successfully synthesized by SPS at $1500^{\circ} \mathrm{C}$ under $20 \mathrm{MPa}$ in vacuum. 

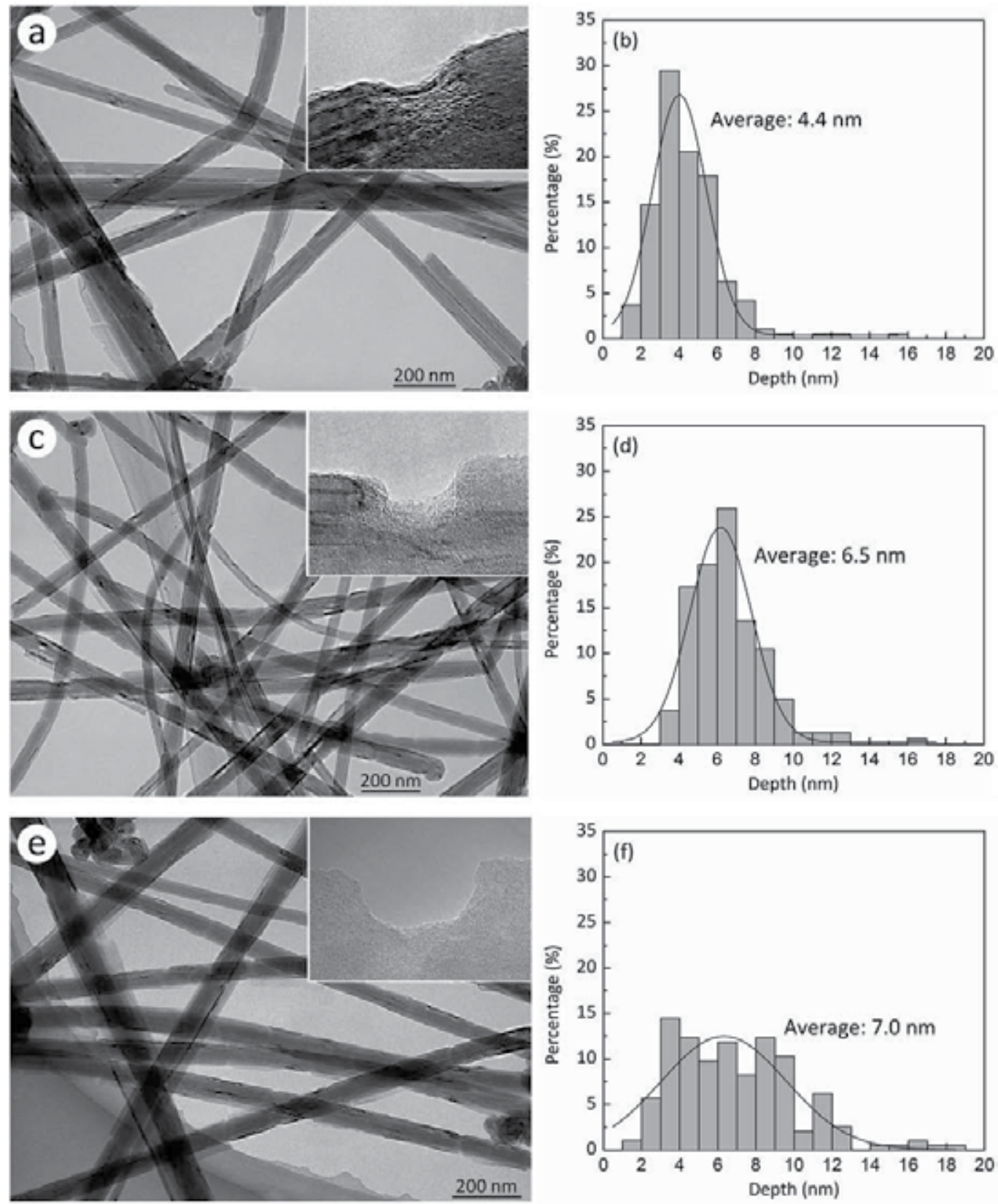

Figure 2. Typical low-magnification TEM images of acid-treated MWCNTs treated with the various conditions of (a) 1 hour, (c) 2 hours and (e) 4 hours. The insets show the high magnification images. (b), (d), (f) Corresponding depth distribution of the nanodefects in the sample (a), (c) and (e), respectively. The solid lines in (b), (d), (f) represent the Gaussian fitting curves. The observation was made for approximately 200 defects. 

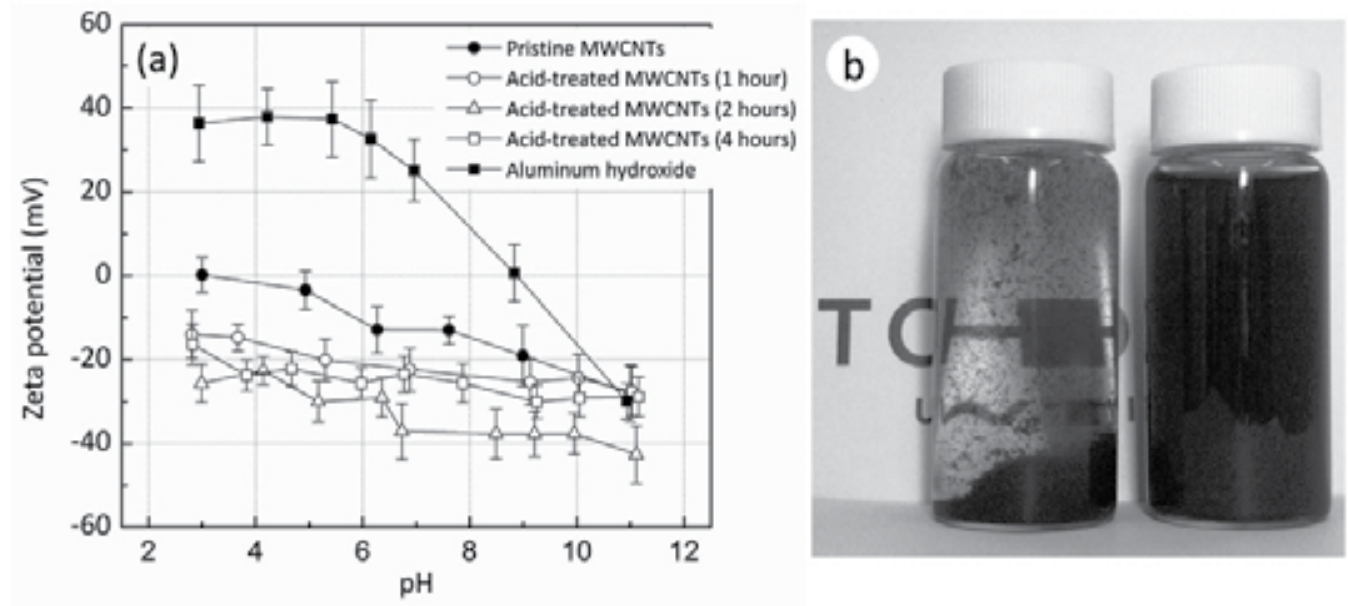

Figure 3. (a) Zeta potential values of MWCNTs and acid-treated MWCNTs at different pH. (b) Undistributed one-day old aqueous suspensions of (left) pristine MWCNTs and (right) acid-treated MWCNTs.

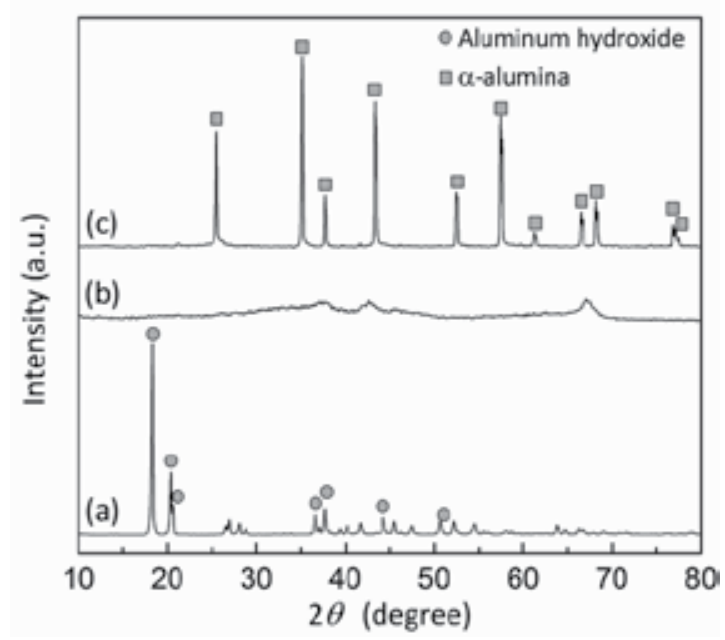

Figure 4. XRD patterns of the (a) aluminum hydroxide-MWCNT mixture, (b) dehydrated product and (c) sintered body, respectively.

\subsection{Micro- and nanostructures of MWCNT/alumina composites}

We now discuss the micro- and nanostructures of the acid-treated MWCNT/alumina composites using SEM and TEM analysis. An interesting geometric structure was observed between the individual MWCNT and the alumina matrix, as shown in Fig. 5. It is revealed that a nanodefect on the acid-treated MWCNT is filled up with alumina crystal, which may be intruding into the nanodefect during grain growth. This nanostructure is novel in that its structure resembles a nanoscale anchor with an alumina crystal spiking the surface of the MWCNT. 
From the SEM observations on the fracture surface, the following features can be noted. First, numerous individual MWCNTs protrude from the fracture surface, and the pullout of the MWCNTs can be clearly observed (Fig. 6a), which had not been obtained until now for conventional CNT/ceramic composites. Most of MWCNTs are located in the intergranular phase and their lengths are in the range $0 \sim 10 \mu \mathrm{m}$. The alumina grains have sizes in the micron range, around $1.5 \mu \mathrm{m}$ (The grain size of the composite was obtained using SEM images, and the observation was made for 224 grains.). No clear difference in the grain size is observed between the acid-treated MWCNT/alumina composites and the pristine MWCNT/alumina composites, even though the incorporation of MWCNTs seems to suppress the grain growth of the alumina. Second, in the case of the smaller amount of the acid-treated MWCNTs, no severe phase segregation was observed, whereas the composites made with the pristine MWCNTs revealed an inhomogeneous structure even for MWCNT addition as low as 0.9 vol. $\%$. In addition to the above features, some MWCNTs on the fracture surface showed a "clean break" near the crack plane, and that the diameter of MWCNT drastically slenderized toward their tip, as illustrated in Figs. $6 \mathrm{~b}$ and $6 \mathrm{c}$, respectively. As SEM cannot clearly resolve the thickness of a single MWCNT, TEM was used to determine if the fracture phenomenon of MWCNTs was indeed occurring during crack opening.

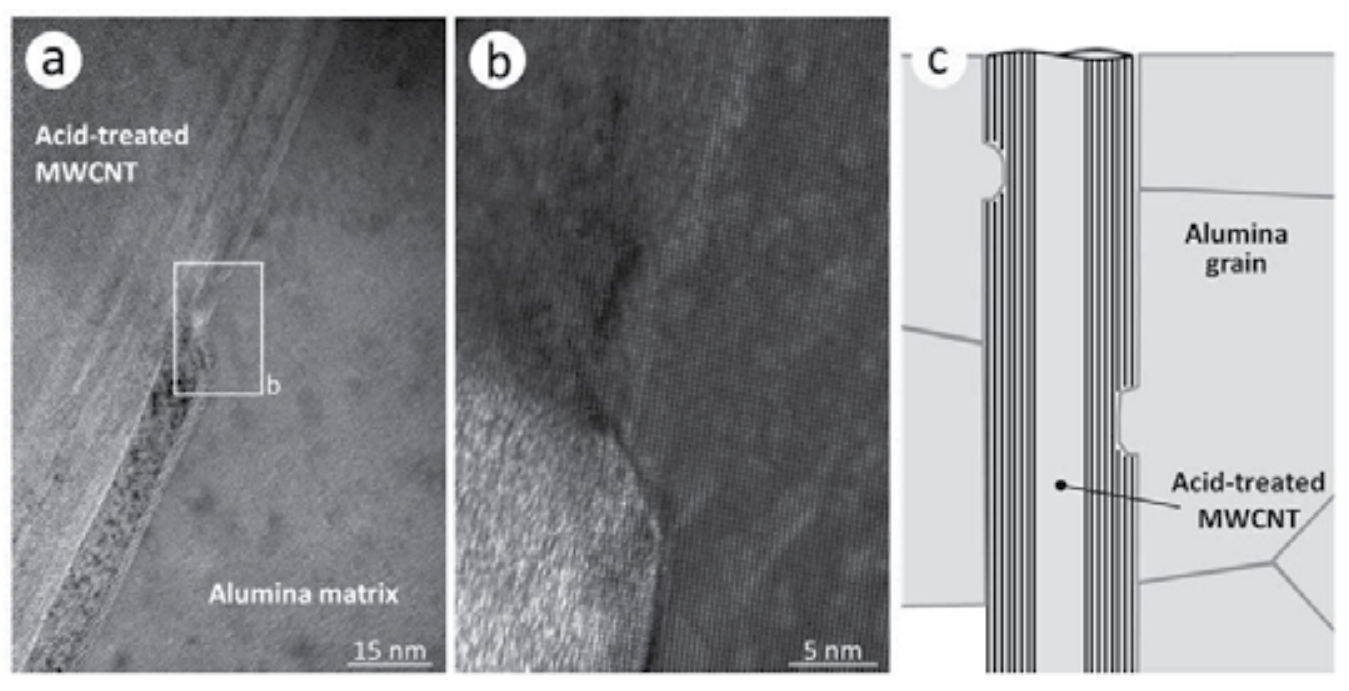

Figure 5. MWCNT morphology in the composites. (a) It is demonstrated that a nanodefect on the acidtreated MWCNT is filled up with alumina crystal. (b) Enlarged TEM image, taken from the square area. (c) Schematic description of MWCNT morphology in the composites.

TEM observations on the fracture surface demonstrated that a diameter change in the MWCNT structure was evidently observed for a certain percentage of the MWCNTs (Fig. 7a). At least, $25 \%$ MWCNT appear to have an apparent diameter change (The observation was made for 281 MWCNTs.). As shown in Fig. 7b, the high magnification TEM image clearly showed a change in diameter, and this morphology is quite similar to a "sword-insheath"-type failure (Yu et al., 2000; Peng et al., 2008; Yamamoto et al., 2010). Key features are illustrated in enlarged TEM image, taken from the square area in Fig. 7b. The inset showed 
that outer-walls having approximately 10 shells were observed to break up at the location where the MWCNT undergo failure, and that the edges of the broken outer shells were observed to be perpendicular to the cylinder axis. Since no apparent variation in the diameter of the MWCNTs has been observed along the axis in the as-received MWCNTs, these results imply that some MWCNTs underwent failure in the sword-in-sheath manner prior to pullout from the matrix. Note that MWCNT failure was also observed in fracture surfaces of alumina composites made with arc-discharge-grown and chemical vapor deposition-grown MWCNTs prepared under the same processing conditions (Yamamoto et al., 2011).
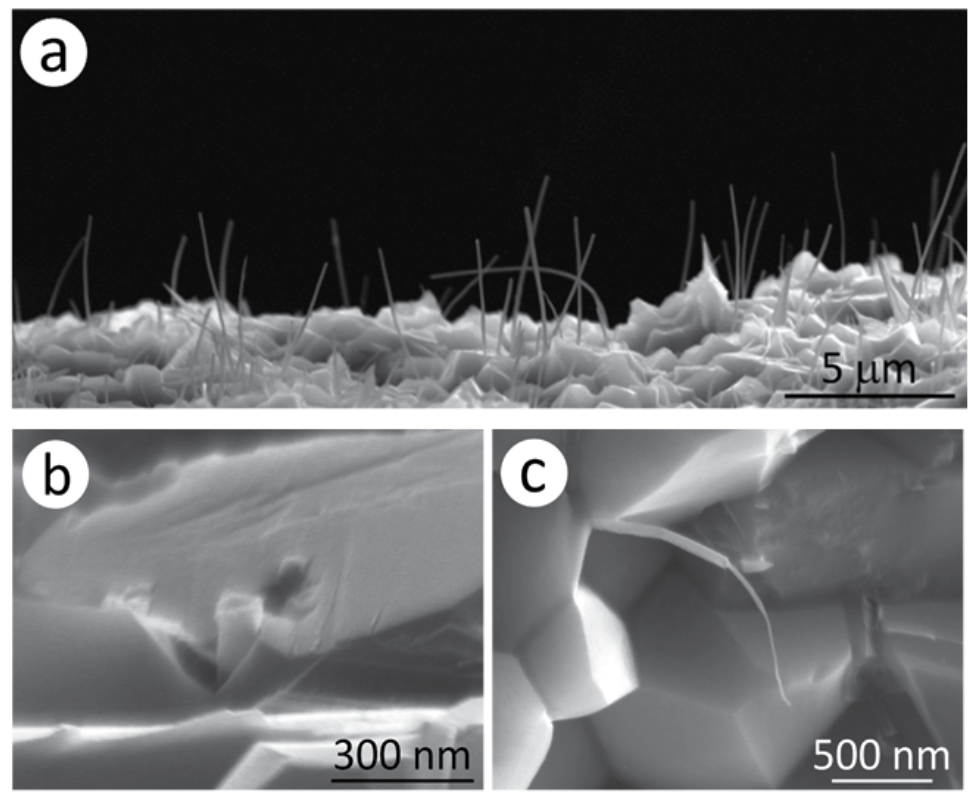

Figure 6. Fracture surface of acid-treated MWCNT/alumina composites. (a) Numerous individual MWCNTs protrude from the fracture surface. $(b, c)$ Some MWCNTs have broken in the multi-wall failure.
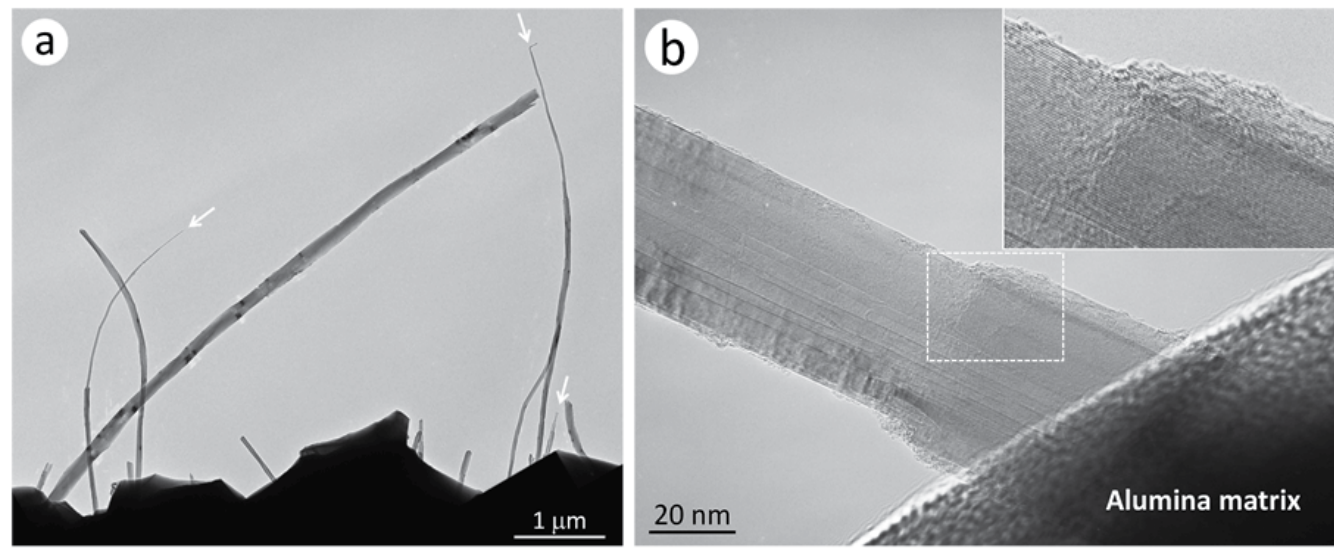

Figure 7. TEM images of the fracture surface of the composite acquired (a) low and (b) high magnification images. 


\subsection{Physical and mechanical properties of MWCNT/alumina composites}

The bending strength of the composites was measured by the three-point bending method under ambient conditions, in which the size of the test specimens was $2.0 \mathrm{~mm}$ (width) $\times 3.0$ $\mathrm{mm}$ (thickness) $\times 24.0 \mathrm{~mm}$ (length). The span length and crosshead speed for the strength tests were $20.0 \mathrm{~mm}$ and $0.83 \mu \mathrm{m} / \mathrm{s}$, respectively. The fracture toughness was measured by the single-edge notched beam (SENB) method (Japanese Industrial Standards, 1995) under ambient conditions, in which the size of test specimens was $2.0 \mathrm{~mm}$ (width) $\times 3.0 \mathrm{~mm}$ (thickness) $\times 15.0 \mathrm{~mm}$ (length). A notch with depth and width of $0.3 \mathrm{~mm}$ and $0.1 \mathrm{~mm}$ was cut in the center part of the test specimens. A span length of $12.0 \mathrm{~mm}$ and crosshead speed of $0.83 \mu \mathrm{m} / \mathrm{s}$ were applied for the toughness test. The bending strength $(\sigma \mathrm{b})$ and fracture toughness $\left(K_{\mathrm{Ic}}\right)$ are given by the following equations:

$$
\begin{gathered}
\sigma_{b}=3 P_{b} L / 2 b h^{2} \\
K_{\mathrm{I} c}=\left(3 P_{b} L / 2 b h^{2}\right) \cdot a^{1 / 2} Y
\end{gathered}
$$

where $P_{\mathrm{b}}$ is the maximum load, $L$ is the span length, $b$ is the specimen width, $h$ is the specimen thickness, $a$ is the notch depth and $Y$ is the dimensional factor. All surfaces of the specimens were finely ground on a diamond wheel, and the edges were chamfered. The indentation tests were done on a hardness tester (AVK-A, Akashi) with a diamond Vickers indenter under ambient conditions. The 0.9 vol. $\%$ acid-treated MWCNT/alumina composite with surface roughness of $0.1 \mu \mathrm{m}(R a)$ was indented using a Vickers diamond pyramid with a load of $98.1 \mathrm{~N}(P)$ applied on the surface for $15 \mathrm{~s}$. The diagonal $(d)$ and the radial crack length $(\mathrm{C})$ were measured by the SEM. The hardness $(\mathrm{Hv})$ and indentation toughness values $\left(K_{\text {Ic }}\right)$ were calculated by the following equations:

$$
\begin{gathered}
H v=0.1891 P / d^{2} \\
K_{\mathrm{I} c}=0.016\left(E / H_{v}\right)^{1 / 2}\left(P / C^{3 / 2}\right)
\end{gathered}
$$

where $E$ is the Young's modulus of the composite ( $E=362.8 \mathrm{GPa})$ measured by a pulse-echo method.

It was found that surface modification of the MWCNTs is effective in improvement of bending strength and fracture toughness of the MWCNT/alumina composites. Figs. 8a and $8 \mathrm{~b}$ show the dependence of the bending strength and the fracture toughness on MWCNT content in the composites. There are few papers which report significant improvement in the mechanical properties such as toughness (Zhan et al., 2003), and the improvement by MWCNT addition has been limited so far in previous studies (Ma et al., 1998; Sun et al., 2002; Wang et al., 2004; Sun et al., 2005; Cho et al., 2009). In our composites, however, the bending strength and the fracture toughness simultaneously increased with the addition of 
a small amount of the acid-treated MWCNTs. The bending strength and the fracture toughness of the 0.9 vol. $\%$ acid-treated MWCNT/alumina composite reached $689.6 \pm 29.1$ $\mathrm{MPa}$ and $5.90 \pm 0.27 \mathrm{MPa} \cdot \mathrm{m}^{1 / 2}$, respectively. At the same time, the bending strength and the fracture toughness of the acid-treated MWCNT/alumina composites were always higher than those of the pristine MWCNT/alumina composites with identical MWCNT content, indicating enhanced stress transfer capability from the alumina to the acid-treated MWCNTs. The Vickers indentation toughness calculated by using the Eq. (4) was $6.64 \mathrm{MPa}$. $\mathrm{m}^{1 / 2}$, which is a slightly larger value than that measured by using SENB method (5.90 MPa. $\mathrm{m}^{1 / 2}$ ). These observations revealed that the high structural homogeneity and enhanced frictional resistance of the structural components led to a simultaneous increase in the strength and the toughness of the acid-treated MWCNT/alumina composites. In contrast, for the larger amount of the MWCNTs, the degradation of mechanical properties of both the composites may be primarily attributed to the severe phase segregation. Because a bundle of segregated CNTs has poor load-carrying ability, the effect of this kind of CNT aggregate in the matrix may be similar to that of pores (Yamamoto et al., 2008a, 2008b).
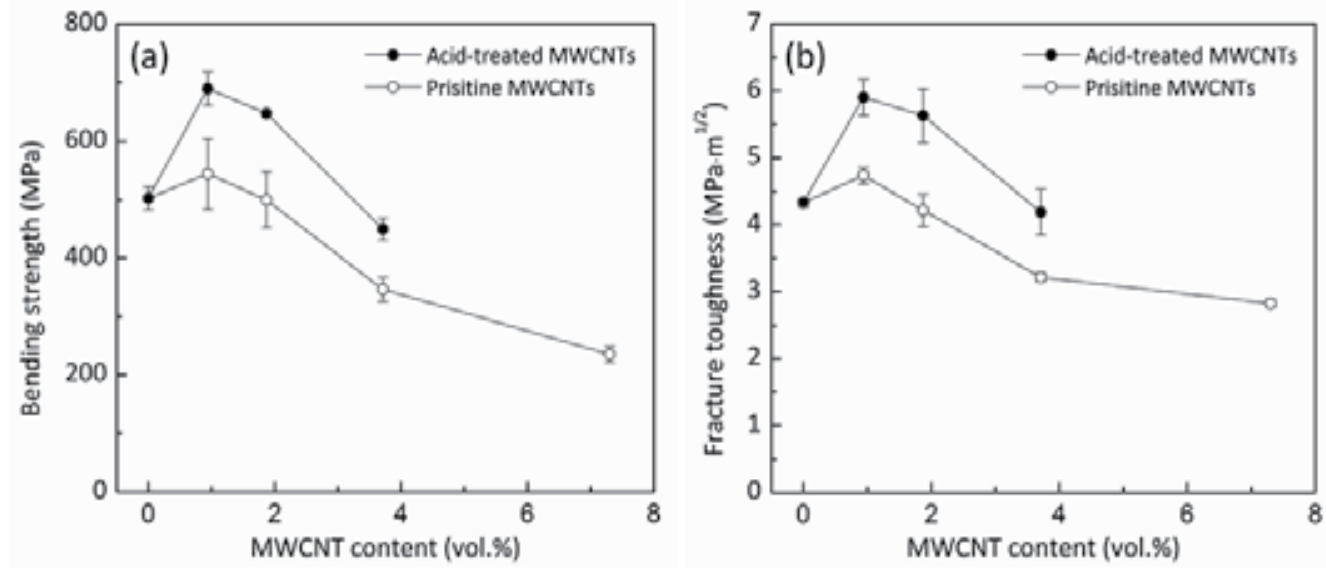

Figure 8. (a) Bending strength and (b) fracture toughness as a function of MWCNT content.

\section{Evaluation of crack bridging characteristics}

Ceramic-CNT interfacial behavior is another key factor in controlling the mechanical and physical properties of fiber reinforced composite materials (Evans, 1990; Hull \& Clyne, 1996; Chen et al., 2011). In general, strong interfacial connectivity facilitates effective load transfer effect, but it prevents CNT pull-out toughening from occurring. Weak interfacial connectivity favors CNTs pull-out but fails to toughen the ceramic matrix. Thus, a balance must be maintained between CNT pull-out and toughening mechanics. It is well recognized that improved toughness of fiber-reinforced ceramic composites is obtained under moderate fiber-ceramic interfacial connectivity. In this regard, suitable (neither too strong nor too weak) ceramic-CNT interfacial connectivity is needed to ensure effective load transfer, and to enhance the toughness and strength of ceramic-CNT composites. 
Here, the failure mechanism of the MWCNTs during crack opening in a MWCNT/alumina composite is investigated through TEM observations and single nanotube pullout tests. Achieving tougher ceramic composites with MWCNTs is discussed based on these results.

\subsection{Pullout experiment sample preparation}

The MWCNT failure during crack opening motivated our research of the crack bridging characteristics through the single nanotube pullout tests. The single nanotube pullout experiments were carried out using an in-situ SEM (Quanta 600 FEG; FEI) method with a nanomanipulator system ( $\mathrm{Yu}$ et al., 2000; Yamamoto et al., 2010). An atomic force microscope (AFM) cantilever (PPP-ZEILR, nominal force constant $1.6 \mathrm{~N} / \mathrm{m}$; NANOSENSORS) was mounted at the end of a piezoelectric bender (ceramic plate bender CMBP01; Noliac) on an $X-Y$ linear motion stage, and the composite with fracture surface (that was coated with platinum) was mounted on an opposing $\mathrm{Z}$ linear motion stage. The piezoelectric bender was used to measure the resonant frequency of each cantilever in vacuum. A single MWCNT on the fracture surface was clamped onto a cantilever tip by local electron-beam-induced deposition (EBID) of a carbonaceous material (Ding et al., 2005). As a precursor source for the EBID, we used n-docosane $\left(\mathrm{C}_{22} \mathrm{H}_{46}\right.$, Alfa Aesar), which was dissolved in toluene to make a 3 mass\% solution. A small amount of the solution was dropped on a cut-in-half copper TEM grid. After the solution evaporated, the TEM grid with paraffin source was mounted on the AFM chip, as shown in Fig. 9. The deposition rate of the EBID depends on several factors (Ding et al., 2005). Thus, the amount of the paraffin source, deposition time, and distance between the paraffin source and the cantilever tip were experimentally-optimized. The cantilevers serve as force-sensing elements and the spring constants of each were calculated in-situ prior to the pullout test using the resonance method (Sader et al., 1999). In brief, for the case of a rectangular cantilever, the force constant $(k)$ is given by following equation,

$$
k=M_{e} \rho_{c} b h L \omega_{v a c}^{2}
$$

where $\omega_{\mathrm{vac}}$ is the fundamental radial resonant frequency of the cantilever in vacuum, $h, b$, and $L$ are the thickness, width, and length of the cantilever, respectively, $\rho_{\mathrm{c}}$ is the density of the cantilever $\left(=2.33 \mathrm{Mg} / \mathrm{m}^{3}\right)$, and $\mathrm{Me}$ is the normalized effective mass which takes the value $M e=0.2427$ for $L / b>5$ (Sader et al., 1995). We measured $\omega_{\text {vac, }} h, b$ and $L$ of each cantilever in the SEM and used the measured, not the nominal provided, values to calculate $k$. The $h, b$ and $L$ are determined by counting the number of pixels in the acquired SEM images. The applied force is calculated from the angle of deflection at the cantilever tip in the acquired SEM images (Ding et al., 2006). The deflection $(\delta)$ and angle of deflection $(\theta)$ at the cantilever tip are given by

$$
\delta=P L^{3} / 3 E I
$$



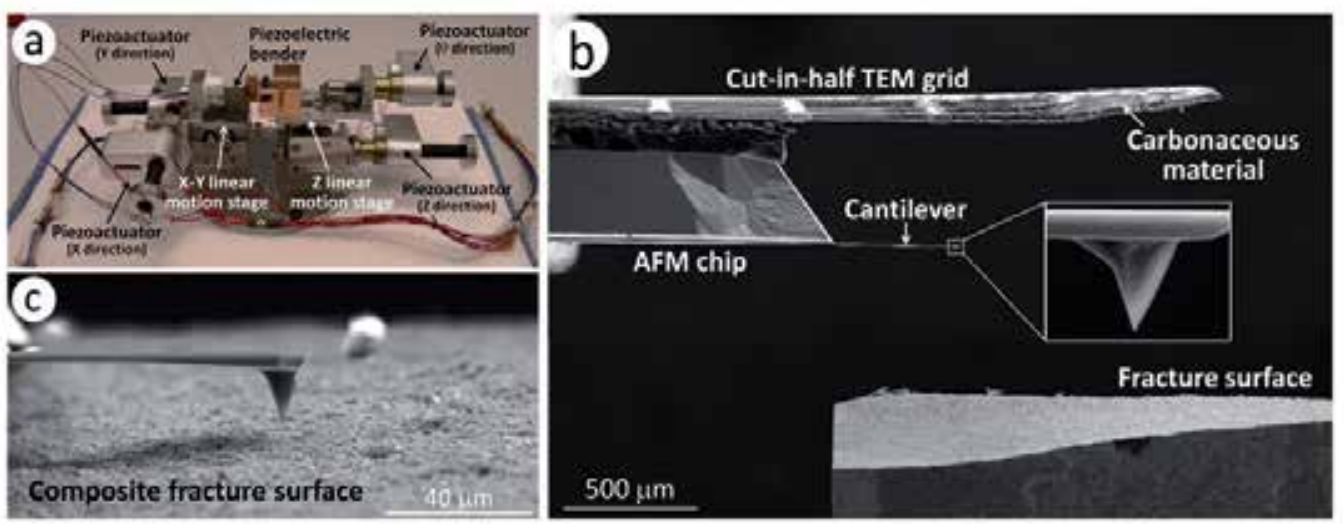

Figure 9. SEM image showing the experimental setup for pullout experiments.

$$
\theta=P L^{2} / 2 E I
$$

where $P$ is the load applied at the cantilever tip, $L$ is the cantilever length, $E$ is the elastic modulus and $I$ is the moment of inertia of the cantilever (Ding et al., 2006). Thus, the deflection at the cantilever tip can be represented by the angle of deflection with the following relationship (Ding et al., 2006):

$$
\delta=2 \theta L / 3
$$

A crosshead speed - i.e., movement rate of the cantilever - of about $100 \mathrm{~nm} / \mathrm{s}$ was applied for the pullout tests.

We fractured a composite specimen by conducting the fracture tests, which caused single MWCNT to project from the crack plane, as exemplified in Fig. 6a. This allows single MWCNT "pickup" with cantilever tip for subsequent tensile loading using the nanomanipulator. As mentioned above, however, the MWCNTs crossing the crack planes were strained during crack opening and possibly underwent failure, as shown in Figs. 6b, 6c and 7. Therefore, by observing the fracture surface on the composites, MWCNTs with no apparent damages were selected for the pullout tests. The physical and mechanical properties, and electrical conductivity of the composite used for the pullout testes are shown in Table 2.

\begin{tabular}{|c|c|c|c|c|c|c|}
\hline $\begin{array}{c}\text { Relative } \\
\text { density } \\
(\%)\end{array}$ & $\begin{array}{c}\text { Grain } \\
\text { size } \\
(\mu \mathrm{m})\end{array}$ & $\begin{array}{c}\text { Bending } \\
\text { strength } \\
(\mathrm{MPa})\end{array}$ & $\begin{array}{c}\text { Fracture } \\
\text { toughness } \\
\left(\mathrm{MPa} \cdot \mathrm{m}^{1 / 2}\right)\end{array}$ & $\begin{array}{c}\text { Hardness } \\
(\mathrm{GPa})\end{array}$ & $\begin{array}{c}\text { Young's } \\
\text { modulus } \\
(\mathrm{GPa})\end{array}$ & $\begin{array}{c}\text { Poisson's } \\
\text { ratio }\end{array}$ \\
\hline 98.9 & $1.43 \pm 0.31$ & $543.8 \pm 60.9$ & $4.74 \pm 0.12$ & $17.0 \pm 0.4$ & 358.0 & 0.20 \\
\hline
\end{tabular}

Table 2. The properties of the composite with 0.9 vol.\% pristine MWCNTs. The Young's modulus and Poisson's ratio were measured by the ultrasonic pulse echo method. 


\subsection{Nanotube fracture during the failure of MWCNT/alumina composites}

Results obtained from the pullout experiments revealed that strong load transfer was demonstrated, and no pullout behavior was observed for all 15 MWCNTs tested in this present research. Eight of these MWCNTs fractured at the composite surface and the remaining 7 MWCNTs underwent failure in the region between the fixed point on the cantilever and the crack plane, as illustrated in Fig. 10.

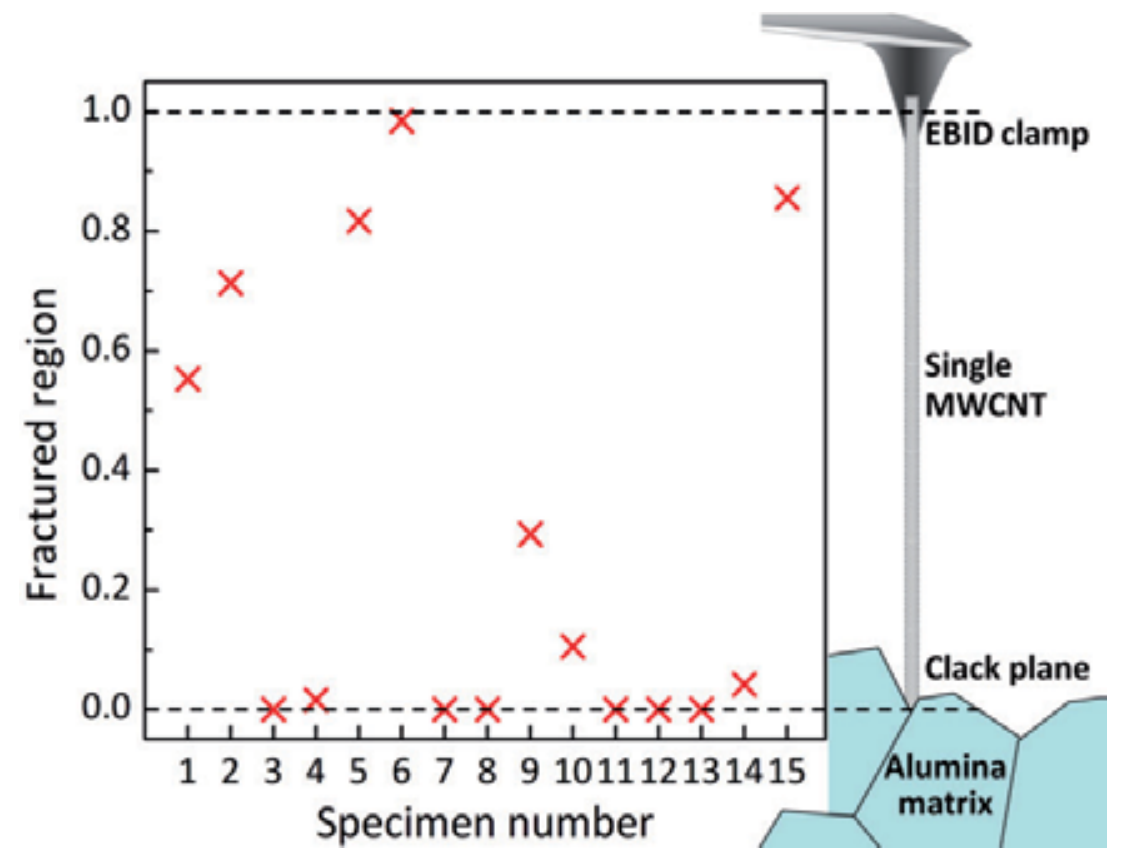

Figure 10. Fracture location of single MWCNTs under pullout loading. Of the 15 MWCNTs tested here, 8 MWCNTs fractured on the composite surface (sample numbers: $3,4,7,8,11-14$ ) and remaining 7 MWCNTs fractured in the middle (sample numbers: 1, 2, 5, 6, 9, 10, and 15).

Two series of SEM and TEM images for each of two individual MWCNTs, captured before and after their breaking, are shown in Figs. 11 and 12. In the first series (Fig. 11; sample number 14), a MWCNT projecting $5.72 \pm 0.01 \mu \mathrm{m}$ from the fracture surface (Fig. 11a) was "welded" to a cantilever tip by local EBID, and then loaded in increments until failure. The resulting fragment attached on the cantilever tip was at least $10.9 \mu \mathrm{m}$ long (Fig. 11b), whereas the other fragment remained lodged in a grain boundary of the alumina matrix (Fig. 11c), suggesting that MWCNT underwent failure in a sword-in-sheath manner. TEM images show a change in diameter at the location where the MWCNT underwent failure, and that the inner core protruding from the outer shells has a multi-walled closed-end structure, as shown in Figs. 11d and 11e, respectively. Given that uniformity of the interwall spacing of 0.34-nm-thick cylinder structure, approximately 11 shells underwent failure. There results strongly suggest that the MWCNTs broke in the outer shells and the inner core was then completely pulled away, leaving the companion fragment of the outer shells in the 
matrix. The sword-in-sheath failure did not always occur. Instead a few MWCNT failed leaving either a very short sword-in-sheath failure or a clean break. As for one example (Fig. 12; sample number 10), a MWCNT projecting $5.34 \pm 0.01 \mu \mathrm{m}$ from the crack plane (Fig. 12a) underwent failure on the composite fracture surface. The resulting fragment attached on the cantilever tip was at least $5.7 \mu \mathrm{m}$ long (Fig. 12b), and no fragment was observed at the original position on the crack plane, suggesting that in this case the MWCNT failed by breaking inside the matrix, and did not pull out. Fig. 12c shows the TEM image of the tip of the same MWCNT which underwent very short sword-in-sheath failure or clean break during crack opening.
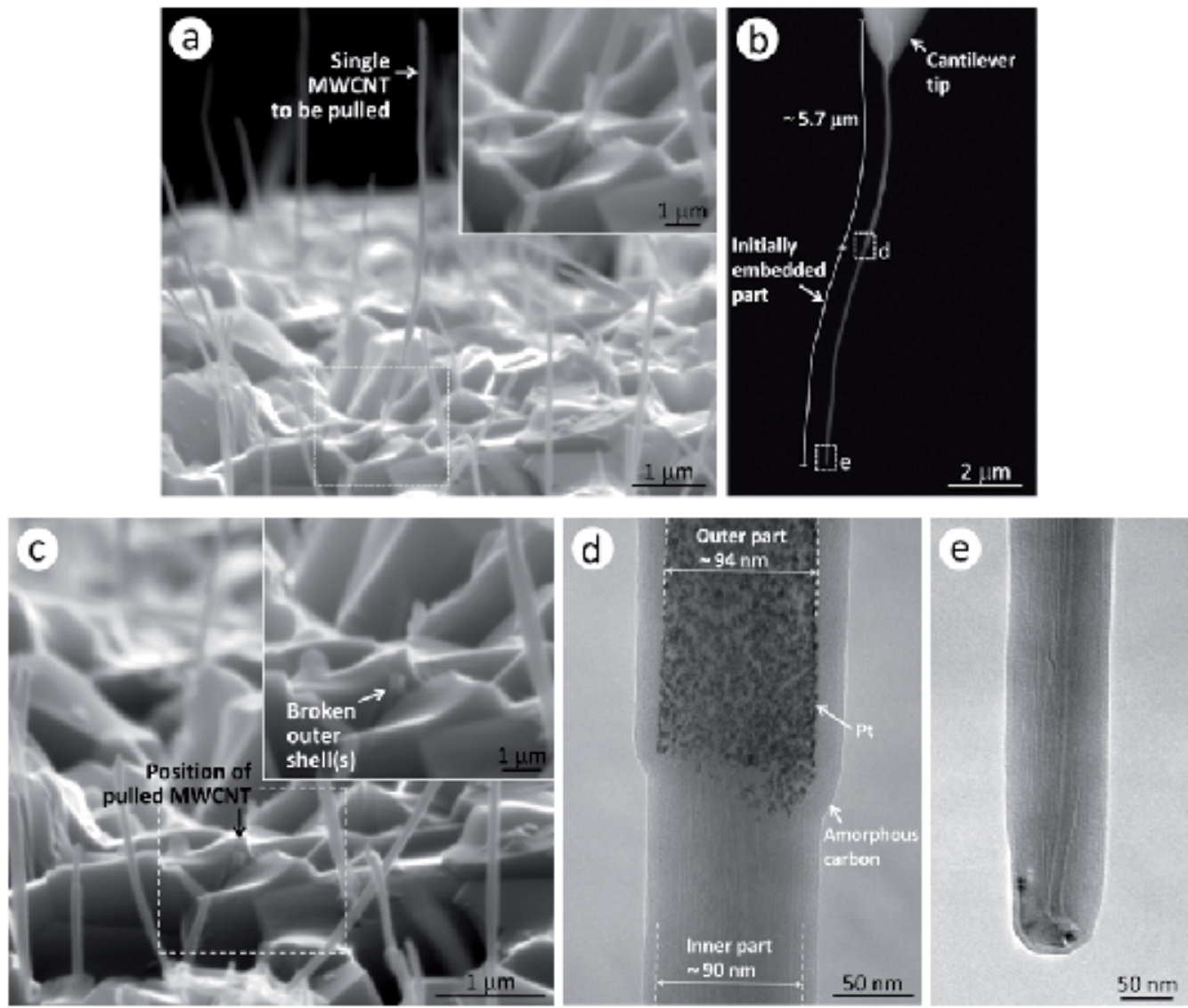

Figure 11. SEM images show (a) a free-standing MWCNT having a $5.72 \pm 0.01 \mu \mathrm{m}$-long on the fracture surface of the composite. (b) After breaking, one fragment of the same MWCNT attached on the cantilever tip had a length $\sim 10.9 \mu \mathrm{m}$. (c) The other fragment remained in the matrix. (d,e) TEM images show a change in diameter at the location where the MWCNT underwent multi-wall failure, and that it clearly has a multi-walled closed-end structure. 

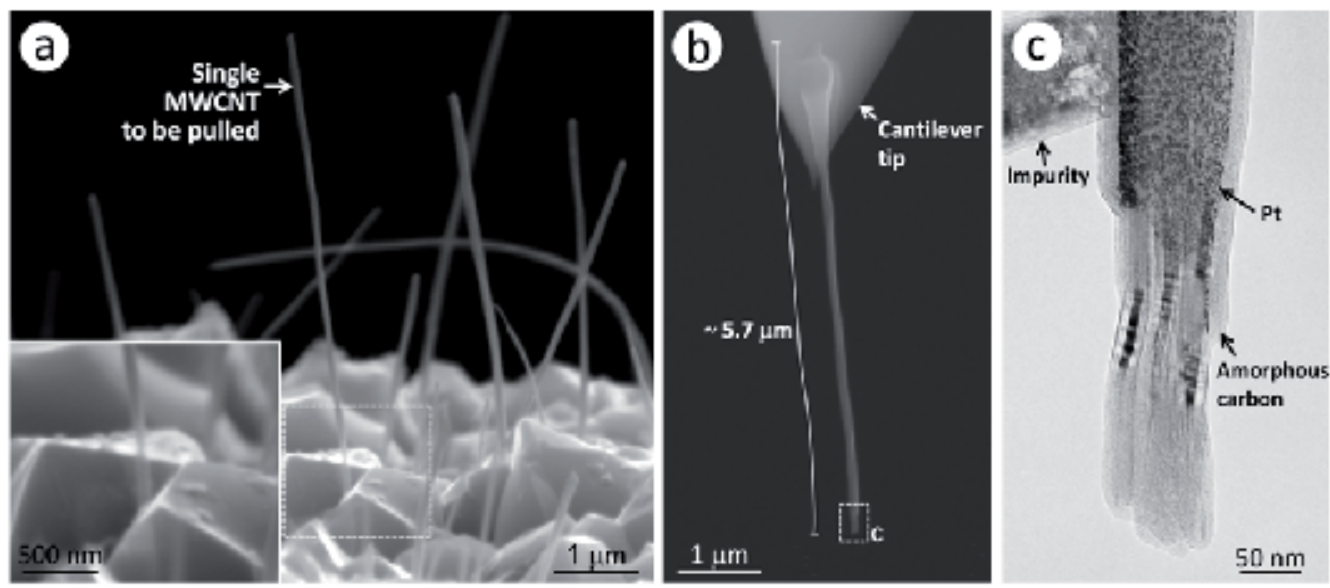

Figure 12. In the second series, (a) a tensile-loaded MWCNT with a length of $4.46 \pm 0.01 \mu \mathrm{m}$ fractured on the crack plane. (b) The resulting fragment on the cantilever tip had a length $~ 5.7 \mu \mathrm{m}$. (c) TEM image shows the MWCNT which underwent the very short sword-in-sheath failure or clean break.
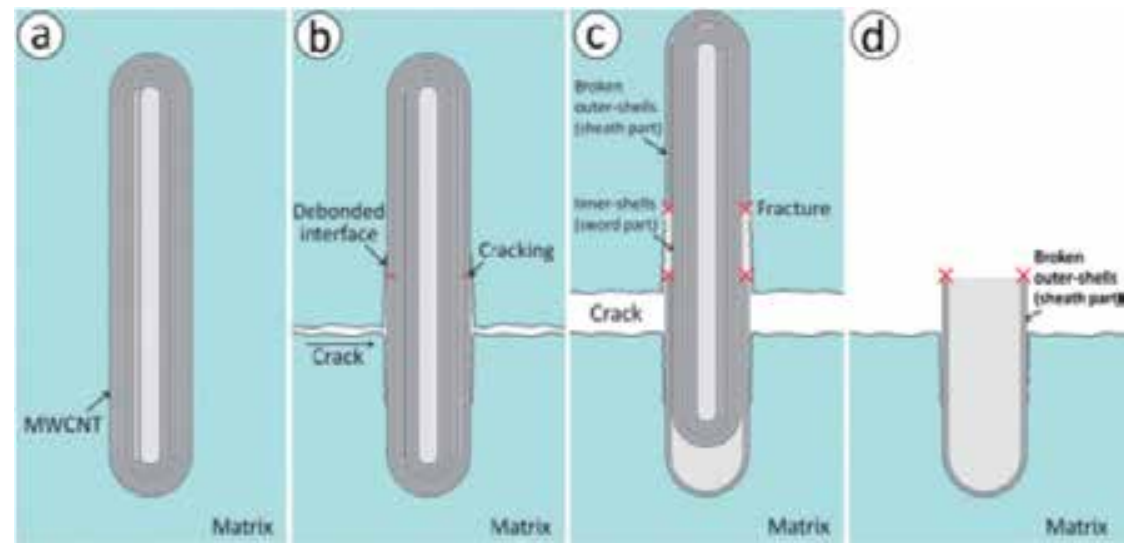

Figure 13. Schematic description of possible fracture mechanisms of the MWCNT (sample number 14). (a) Initial state of a MWCNT. (b) Tensile stresses lead to matrix crack and partial debonding formation. (c,d) As displacement increases, the MWCNTs, rather than pulling out from the alumina matrix, undergo failure in the outer shells and the inner core is pulled away, leaving the fragment of the outer shells in the matrix.

Next, we schematically describe possible processes and mechanics, explaining the MWCNT failure during crack opening (Fig. 13). As for one example, considering the sample number 14 (Fig. 11), the initial state of the MWCNT in an ideal case is a completely impregnated and isolated embedded in the matrix (Fig. 13a). Tensile stresses parallel to the axis of MWCNT length lead to matrix crack formation. Subsequently, interfacial debonding between two phases may occur (Fig. 13b), perhaps over a limited distance (but this is unlikely to make a major contribution to the fracture energy.). Since there is variability in the MWCNT strength in the debonded region on either side of the crack plane, and it is possible for the MWCNT to break at a certain position, when the stress in the MWCNT reaches a critical value. As 
displacement increases, the MWCNTs, rather than pulling out from the alumina matrix, undergo failure in the outer shells and the inner core is pulled away, leaving the fragments of the outer shells in the matrix (Figs. 13c and 13d).

\section{Conclusion}

Creating tough, fracture-resistant ceramics has been a central focus of MWCNT/ceramic composites research. In this research, the MWCNT/alumina composite with enhanced mechanical properties of $689.6 \pm 29.1 \mathrm{MPa}$ for bending strength and $5.90 \pm 0.27 \mathrm{MPa} \cdot \mathrm{m}^{1 / 2}$ for fracture toughness have been successfully prepared by a novel processing method. A combination of the precursor method for synthesis of the alumina matrix, the acid treatment of the pristine MWCNTs and the spark plasma sintering method can diminish the phase segregation of MWCNTs, and render MWCNT/alumina composites highly homogeneous. The universality of the method developed here will be applicable to a wide range of functional materials such as tribomaterials, electromagnetic wave absorption materials, electrostrictive materials, and so on. Our present work may give a promising future for the application of MWCNTs in reinforcing structural ceramic components and other materials systems such as polymer- and metal-based composites.

We have also shown from TEM observations and single nanotube pullout experiments on the MWCNT/alumina composites that strong load transfer was revealed, and no MWCNT pullout behavior was observed. It is well recognized the fracture properties of fiberreinforced composites are dominated by the fiber bridging force resulting from debonding and sliding resistance, which dictates the major contribution to the strength and toughness (Evans, 1990; Hull \& Clyne, 1996). The results reported here suggest that modest improvements in toughness reported previously may be due to the way MWCNT's fail during crack opening in the MWCNT/ceramic composites. Our finding suggests important implications for the design of tougher ceramic composites with MWCNTs. The important factor for such tougher ceramic composites will thus be the use of MWCNT having a much higher load carrying capacity (as well as a good dispersion in the matrix).

\section{Author details}

Go Yamamoto and Toshiyuki Hashida

Fracture and Reliability Research Institute (FRRI), Tohoku University, Japan

\section{Acknowledgement}

The authors thank our colleague, Dr. M. Omori, Mr. K. Shirasu, Mr. Y. Nozaka, Mr. Y. Aizawa and Ms. N. Suzuki of Fracture and Reliability Research Institute (FRRI), Tohoku University, for their helpful discussions, and Mr. T. Miyazaki of Technical Division, School of Engineering, Tohoku University, for technical assistance in the TEM analysis. The authors acknowledge Prof. R.S. Ruoff of The University of Texas at Austin for his useful guidance. This work is partially supported by Grand-in-Aids for Scientific Research (Nos. 23860004 
and 21226004) from the Japanese Ministry of Education, Culture, Sports, Science and Technology. This work is performed under the inter-university cooperative research program of the Advanced Research Center of Metallic Glasses, Institute for materials Research, Tohoku University.

\section{References}

Chen, J.; Hamon, M.A.; Hu, H.; Chen, Y.; Rao, A.M.; Eklund, P.C. \& Haddon, R.C. (1998). Solution properties of single-walled carbon nanotubes. Science, Vol. 282, pp. 95-98.

Chen, Y.L.; Liu, B.; Huang, Y.; \& Hwang, K.C. (2011). Fracture toughness of carbon nanotube-reinforced metal- and ceramic-matrix composites. Journal of Nanomaterials, Vol. 2011, Article ID 746029.

Cho, J.; Boccaccini, A.R. \& Shaffer, M.S.P. (2009). Ceramic matrix composites containing carbon nanotubes. Journal of Materials Science, Vol. 44, pp. 1934-1951.

Dai, H.J.; Wong, E.W. \& Lieber, C.M. (1996). Probing electrical transport in nanomaterials: conductivity of individual carbon nanotubes. Science, Vol. 272, pp. 523-526.

De Andrade, M.J.; Lima, M.D.; Bergmann, C.P.; Ramminger, G.D.O.; Balzaretti, N.M.; Costa, T.M.H. \& Gallas, M.R. (2008). Carbon nanotube/silica composites obtained by sol-gel and high-pressure techniques. Nanotechnology, Vol. 19, article number: 265607.

Ding, W.; Dikin, D.A.; Chen, X.; Piner, R.D.; Ruoff, R.S.; Zussman, E.; Wang, X. \& Li, X. (2005). Mechanics of hydrogenated amorphous carbon deposits from electron-beaminduced deposition of a paraffin precursor. Journal of Applied Physics, Vol. 98, article number: 014905.

Ding, W.Q.; Calabri, L.; Chen, X.Q.; Kohhaas, K.M. \& Ruoff, R.S. (2006). Mechanics of crystalline boron nanowires. Composites Science and Technology, Vol. 66, pp. 1112-1124.

Ebbesen, T.W.; Lezec, H.J.; Hiura, H.; Bennett, J.W.; Ghaemi, H.F. \& Thio, T. (1996). Electrical conductivity of individual carbon nanotubes. Nature, Vol. 382, pp. 54-56.

Esumi, K.; Ishigami, M.; Nakajima, A.: Sawada, K. \& Honda, H. (1996). Chemical treatment of carbon nanotubes. Carbon, Vol. 34, pp. 279-281.

Evans, A.G. (1990) Perspective on the development of high-toughness ceramics. Journal of the American Ceramic Society, Vol. 73, pp. 187-206.

Fan, J.P.; Zhao, D.Q.; Wu, M.S.; Xu, Z. \& Song, J. (2006). Preparation and microstructure of multiwalled carbon nanotubes-toughened composite. Journal of the American Ceramic Society, Vol. 89, pp. 750-753.

Fan J.P; Zhuang, D.M.; Zhao, D.Q.; Zhang, G.; Wu, M.S.; Wei, F. \& Fan, Z.J. (2006). Toughening and reinforcing alumina matrix composite with single-wall carbon nanotubes. Applied Physics Letters, Vol. 89, pp. 121910-1219103.

Gonzalez-Julian, J.; Miranzo, P.; Osendi, M.I. \& Belmonte, M. (2011). Carbon nanotubes functionalization process for developing ceramic matrix nanocomposites. Journal of Materials Chemistry, Vol. 21, pp. 6063-6071.

Huang, J.Y.; Chen, S.; Wang, Z.Q.; Kempa, K.; Wang, Y.M.; Jo, S.H.; Chen, G.; Dresselhaus, M.S. \& Ren, Z.F. (2006). Superplastic carbon nanotubes - conditions have been 
discovered that allow extensive deformation of rigid singlewalled nanotubes. Nature, Vol. 439, p. 281.

Hull, D \& Clyne T.W. (1996). An Introduction to Composite Materials (Second edition). Cambridge University Press, 0521388554, The Edinburgh Building, Cambridge CB2 2RU, UK.

Japanese Industrial Standards (JIS). (1995). R 1607.

Kita, J.; Suemasu, H.; Davies, I.J.; Koda, S. \& Itatani, K. (2010). Fabrication of silicon carbide composites with carbon nanofiber addition and their fracture toughness. Journal of Materials Science, Vol. 45, pp. 6052-6058.

Liu, J.; Rinzler, A.G.;Dai, H.; Hafner, J.H.; Bradley, R.K.; Boul, P.J.; Lu, A.; Iverson, T.; Shelimov, K.; Huffman, C.B.; Rodriguez-Macias, F.; Shon, Y.S.; Lee, T.R.; Colbert, D.T. \& Smalley, R.E. (1998). Fullerene pipes. Science, Vol. 280, pp. 1253-1256.

Ma, R.Z.; Wu, J.; Wei, B.Q.; Liang, J. \& Wu, D.H. (1998). Processing and properties of carbon nanotubes-nano-SiC ceramic. Journal of Materials Science, Vol. 33, pp. 5243-5246.

Miyahara, N.; Yamaishi, K.; Mutoh, Y.; Uematsu, K. \& Inoue, M. (1994). Effects of grain size on strength and fracture toughness in alumina, JSME International journal Vol. 37, pp. 231-237.

Mukerji, J. (1993) Ceramic matrix composites. Defence Science Journal, Vol. 43, pp. 385-395.

Omori, M. (2000). Sintering, consolidation, reaction and crystal growth by the spark plasma system (SPS). Materials Science and Engineering A, Vol. 287, pp. 183-188.

Peigney, A. (2003). Composite materials: tougher ceramics with nanotubes. Nature Materials, Vol. 2, pp. 15-16.

Peigney, A.; Flahaut, E.; Laurent, Ch.; Chastel, F. \& Rousset, A. (2002). Aligned carbon nanotubes in ceramics-matrix nanocomposites prepared by high-temperature extrusion. Chemical Physics Letters, Vol. 352, pp. 20-25.

Peng, B.; Locascio, M.; Zapol, P.; Li, S.Y.; Mielke, S.L.; Schatz, G.C. \& Espinosa, H.D. (2008). Measurements of near-ultimate strength for multiwalled carbon nanotubes and irradiationinduced crosslinking improvements. Nature Nanotechnology, Vol. 3, pp. 626-631.

Rice, R.W. (1996). Grain size and porosity dependence of ceramic fracture energy and toughness at $22^{\circ} \mathrm{C}$, Journal of Materials Science, Vol. 31, pp. 1969-1983.

Sader, J.E.; Chon, J.W.M. \& Mulvaney, P. (1999). Calibration of rectangular atomic force microscope cantilevers. Review of Scientific Instruments, Vol. 70, pp. 3967-3969.

Sader, J.E.; Larson, I.; Mulvaney, P. \& White, L.R. (1995). Method for the calibration of atomic force microscope cantilevers. Review of Scientific Instruments, Vol. 66, pp. 3789-3798.

Sheldon, B.W. \& Curtin, W.A. (2004). Nanoceramic composites: tough to test. Nature Materials, Vol. 3, pp. 505-506.

Sun, L.; Gao, L. \& Li, X. (2002). Colloidal processing of carbon nanotube/alumina composites. Chemistry of Materials, Vol. 14, pp. 5169-5172.

Sun, J.; Gao, L. \& Xihai Jin, X. (2005).Reinforcement of alumina matrix with multi-walled carbon nanotubes. Ceramics International, Vol. 31, pp. 893-896.

Treacy, M.M.J.; Ebbesen, T.W. \& Gibson, J.M. (1996). Exceptionally high Young's modulus observed for individual carbon nanotubes. Nature, Vol. 381, pp. 678-680. 
Wang, X.; Padture, N.P. \& Tanaka, H. (2004). Contact-damage-resistant ceramic/single-wall carbon nanotubes and ceramic/graphite composites. Nature Materials, Vol. 3, pp. 539-544.

Yamamoto, G.; Omori, M.; Yokomizo, K.; Hashida, T. \& Adachi, K. (2008). Structural characterization and frictional properties of carbon nanotube/alumina composites prepared by precursor method. Materials Science and Engineering B, Vol. 148, pp. 265-269.

Yamamoto, G.; Omori, M.; Hashida, T. \& Kimura, H. (2008). A novel structure for carbon nanotube reinforced alumina composites with improved mechanical properties. Nanotechnology, Vol. 19, article number: 315708.

Yamamoto, G.; Omori, M.; Yokomizo, K. \& Hashida, T. (2008). Mechanical properties and structural characterization of carbon nanotube/alumina composites prepared by precursor method. Diamond and Related Materials, Vol. 17, pp. 1554-1557.

Yamamoto, G.; Suk, J.W.; An, J.; Piner, R.D.; Hashida, T.; Takagi, T. \& Ruoff, R.S. (2010). The influence of nanoscale defects on the fracture of multi-walled carbon nanotubes under tensile loading. Diamond and Related Materials, Vol. 19, pp. 748-751.

Yamamoto, G.; Shirasu, K.; Hashida, T.; Takagi, T.; Suk, J.W.; An, J.; Piner, R.D. \& Ruoff, R.S. (2011). Nanotube fracture during the failure of carbon nanotube/alumina composites. Carbon, Vol. 49, pp. 3709-3716.

Yao, W.; Liu, J.; Holland, T.B.; Huang, L.; Xiong, Y.; Schoenung, J.M. \& Mukherjee, A.K. (2011). Grain size dependence of fracture toughness for fine grained alumina, Scripta Materialia, Vol. 65, pp. 143-146.

Yu, M.F.; Lourie, O.; Dyer, M.J.; Moloni, K.; Kelly, T.F. \& Ruoff, R.S. (2000). Strength and breaking mechanism of multiwalled carbon nanotubes under tensile load. Science, Vol. 287, pp. 637-640.

Zhan, G.D.; Kuntz, J.D.; Wan, J. \& Mukherjee, A.K. (2003). Single-wall carbon nanotubes as attractive toughening agents in alumina-based nanocomposites. Nature Materials, Vol. 2, pp. 38-42. 



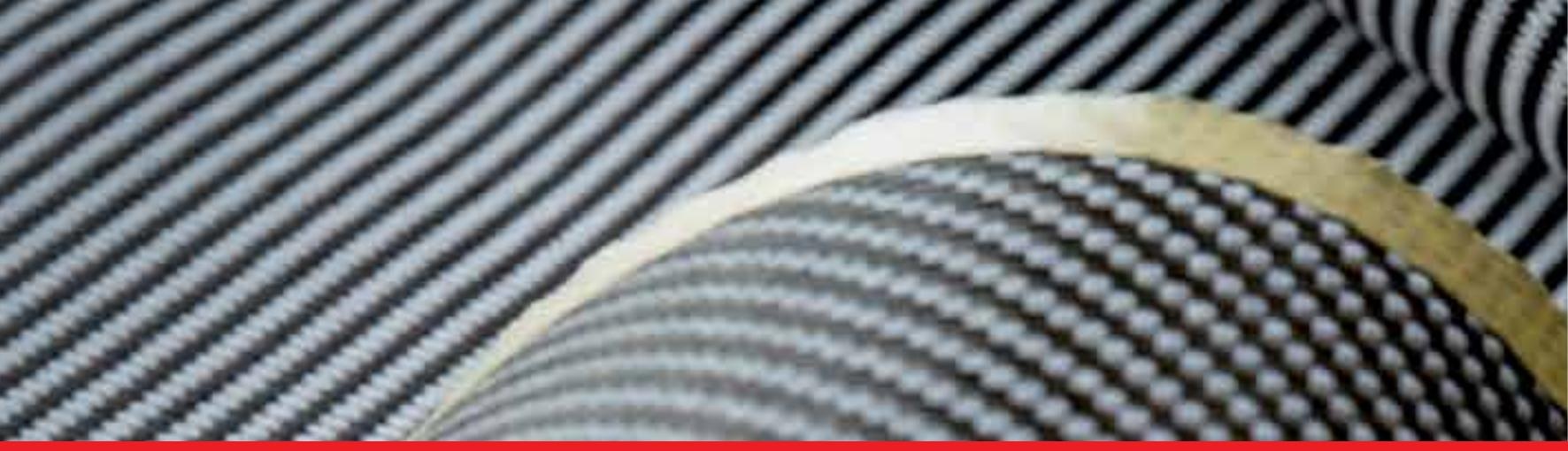

\section{Edited by Ning $\mathrm{Hu}$}

Composites are a class of material, which receives much attention not only because it is on the cutting edge of active material research fields due to appearance of many new types of composites, e.g., nanocomposites and bio-medical composites, but also because there are a great deal of promises for their potential applications in various industries ranging from aerospace to construction due to their various outstanding properties. This book mainly deals with fabrication and property characterization of various composites

by focusing on the following topics: functional and structural nanocomposites, numerical and theoretical modelling of various damages in long fiber reinforced composites and textile composites, design, processing and manufacturing technologies and their effects on mechanical properties of composites, characterization of mechanical and physical properties of various composites, and metal and ceramic matrix composites.

This book has been divided into five sections to cover the above contents. 